\title{
A Study on the Competitiveness of Eight Different Estimation Algorithms for Multinomial Logit Mode Choice Modelling Using Analytical Derivatives
}

\author{
by
}

\section{Hyuk-Jae Roh}

Bachelor of Science in Civil \& Environmental Engineering, Sung Kyun Kwan University, Korea (1993)

Master of City Planning in Graduate School of Environmental Studies, Seoul National University, Korea (2001)

\begin{abstract}
A thesis submitted to the Faculty of Graduate Studies and Research
In partial fulfillment of the requirements for the Degree of

Master of Applied Science
\end{abstract}
Department of Civil and Environmental Engineering
Carleton University
Ottawa, Ontario, CANADA

(C) Copyright April 2007

Hyuk-Jae Roh

The Master of Applied Science in Civil and Environmental Engineering Program is a Joint Program with the University of Ottawa, administered by the Ottawa-Carleton Institute for Civil Engineering 


$\begin{array}{ll}\begin{array}{l}\text { Library and } \\ \text { Archives Canada }\end{array} & \begin{array}{l}\text { Bibliothèque et } \\ \text { Archives Canada }\end{array} \\ \begin{array}{l}\text { Published Heritage } \\ \text { Branch }\end{array} & \begin{array}{l}\text { Direction du } \\ \text { Patrimoine de l'édition }\end{array} \\ \begin{array}{l}\text { 395 Wellington Street } \\ \text { Ottawa ON K1A 0N4 }\end{array} & \begin{array}{l}\text { 395, rue Wellington } \\ \text { Ottawa ON K1A ON4 } \\ \text { Canada }\end{array} \\ \end{array}$

Your file Votre référence ISBN: 978-0-494-27001-1 Ourfile Notre référence ISBN: 978-0-494-27001-1

NOTICE:

The author has granted a nonexclusive license allowing Library and Archives Canada to reproduce, publish, archive, preserve, conserve, communicate to the public by telecommunication or on the Internet, loan, distribute and sell theses worldwide, for commercial or noncommercial purposes, in microform, paper, electronic and/or any other formats.

The author retains copyright ownership and moral rights in this thesis. Neither the thesis nor substantial extracts from it may be printed or otherwise reproduced without the author's permission.
AVIS:

L'auteur a accordé une licence non exclusive permettant à la Bibliothèque et Archives Canada de reproduire, publier, archiver, sauvegarder, conserver, transmettre au public par télécommunication ou par l'Internet, prêter, distribuer et vendre des thèses partout dans le monde, à des fins commerciales ou autres, sur support microforme, papier, électronique et/ou autres formats.

L'auteur conserve la propriété du droit d'auteur et des droits moraux qui protège cette thèse. $\mathrm{Ni}$ la thèse ni des extraits substantiels de celle-ci ne doivent être imprimés ou autrement reproduits sans son autorisation.
In compliance with the Canadian

Privacy Act some supporting forms may have been removed from this thesis.

While these forms may be included in the document page count, their removal does not represent any loss of content from the thesis.
Conformément à la loi canadienne sur la protection de la vie privée, quelques formulaires secondaires ont été enlevés de cette thèse.

Bien que ces formulaires aient inclus dans la pagination, il n'y aura aucun contenu manquant. 


\section{Abstract}

The motivation of this thesis is to gain a clear understanding of underlying numerical operational procedures and to identify the differences between eight estimation algorithms adopted for the estimation of the parameters of the Discrete Mode Choice Model of Multinomial Logit (MNL) type. This model is estimated by using the Maximum Likelihood Estimator (MLE). A numerical example is designed to compare the estimation performance of eight algorithms. The data set collected for the study of mode choice decision by airport passengers travelling to airport is used. Due to the limitations of statistical packages, the eight estimation algorithms were coded by using Visual Basic Application (VBA) provided in the Microsoft EXCEL. Results show that the Newton Raphson algorithm is the best of all eight algorithms and dominates its competitors in terms of performance measures. The worst algorithm is the Steepest Ascent algorithm. Also, important factors of econometric model estimation are identified. These are convergence criterion, initial guessing of starting points, and initial Hessian matrix. It is contended that the findings of this research will be very useful to a researcher who has an interest in making a specific code for his/her own model. 


\section{Acknowledgments}

I wish to truly thank my supervisor, professor Ata M. Khan, for his kind encouragement whenever I met hurdles and his advice during writing and reviewing this thesis up to the moment of its final completion. Also, I would like to show my respect for his professional attitudes reflected in every details.

I would like to extend thanks to the thesis committee members, Dr. Yasser Hassan, Dr. Heng-Aik Khoo, and Dr. Gil A. Hartley in Department of Civil and Environmental Engineering and Dr. Rong Liu in Department of Mechanical and Aerospace Engineering; their constructive opinions from a variety of views were very useful in reviewing the results of this research. Certainly, their valuable suggestions helped me to improve the quality of this thesis.

The last, but the most important thanks go to my lovely family living here in Canada and Korea (Republic of), especially, my lovely wife (Hyun-Ju Park), my smart son (ChanMin Roh) and my only daughter in the world (Chan-Song Roh). They always cheered me up, gave me endless love and showed me their great perseverance. I would like to say to them "I have loved you and I will love you cordially to the very end of this age."

I thank God for this small ending, and for a great new start. 


\section{Table of Contents}

Abstract
Acknowledgments
List of Figures
List of Tables

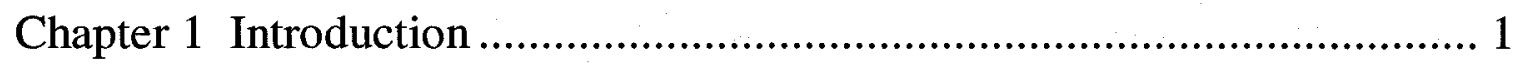

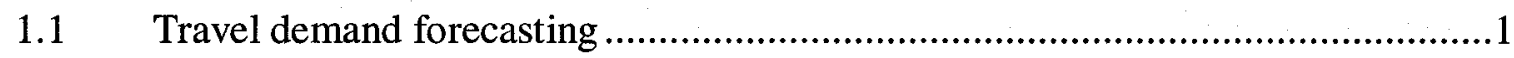

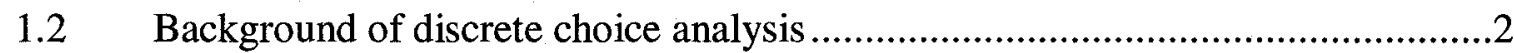

1.3 Transportation application of discrete choice analysis .......................................3

1.4 The necessity of understanding the numerical aspects of discrete choice modelling.......4

$1.5 \quad$ Research objectives and scope .....................................................................

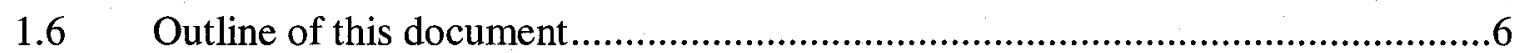

Chapter 2 Literature Review-Discrete Mode Choice Model....................... 7

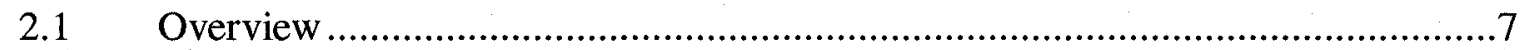

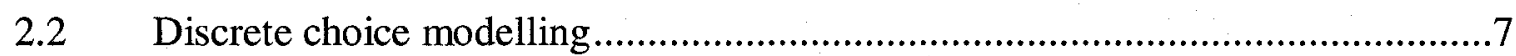

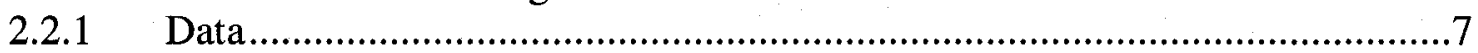

2.2.2 The decision maker ..............................................................................

2.2.3 The alternatives .................................................................................. 10

2.2.3.1 Choice set ..............................................................................10

2.2.3.2 Attributes of alternatives .............................................................11

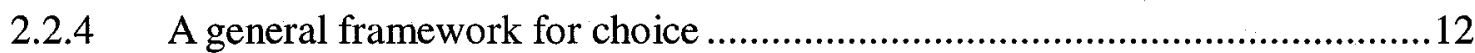

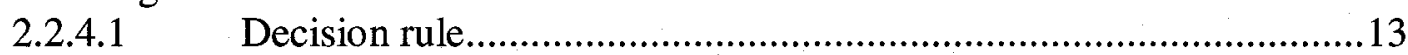

2.2.4.2 Application of utility concept to choice theory ....................................14

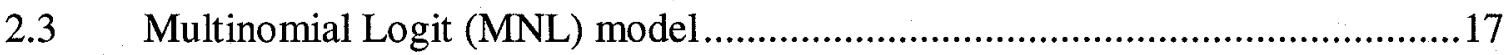

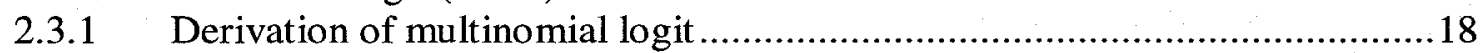

2.3.2 Linear-in-parameters part of utility functions ................................................21

2.3.3 Drawbacks of the multinomial logit model (MNL) ........................................22

2.3.3.1 Proportional substitution patterns....................................................22

2.3.3.2 Independence of irrelevant alternatives (IIA) ….............................24

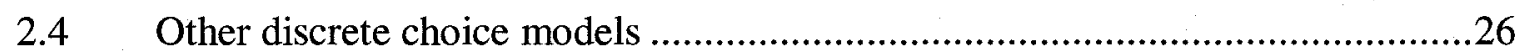

2.4.1 The Generalized Extreme Value (GEV) Model...............................................26 
2.4.1.1 Heteroskedastic extreme value model ........................................27

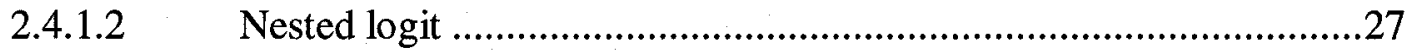

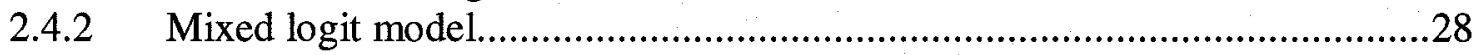

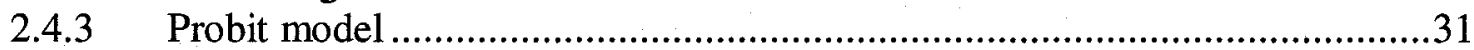

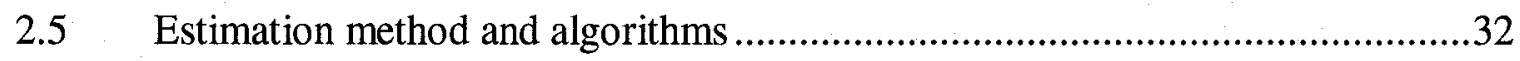

Chapter 3 The Estimation Algorithms ................................................35

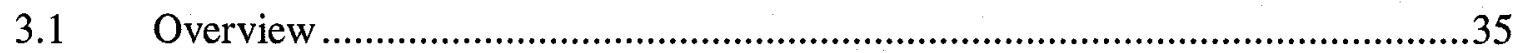

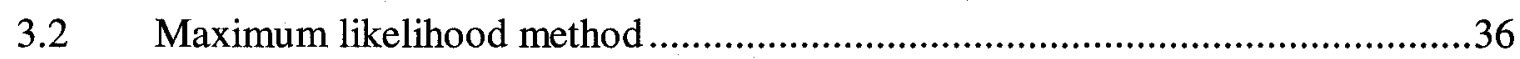

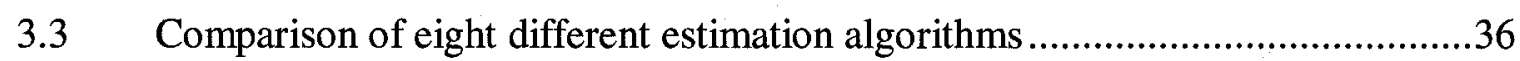

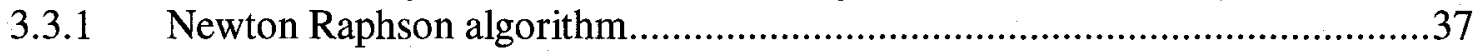

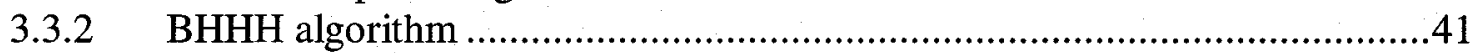

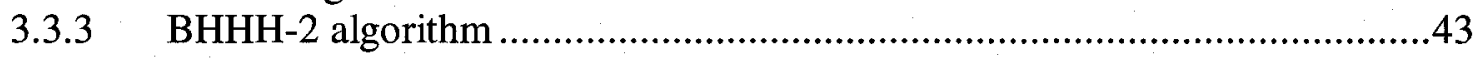

3.3.4 Steepest Ascent algorithm ...............................................................44

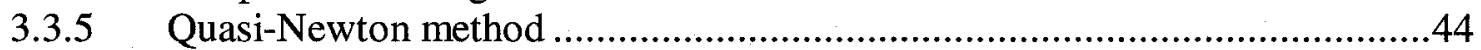

3.3.5.1 Davidon-Fletcher-Powell (DFP) algorithm................................45

3.3.5.2 Broyden-Fletcher-Goldfarb-Shanno (BFGS) algorithm .................47

3.3.6 Incorporating golden section line search method (GSM) to both DFP and BFGS algorithms .......................................................4 48

3.3.6.1 The golden section method (GSM)........................................49

3.3.6.2 Application to this research....................................................50

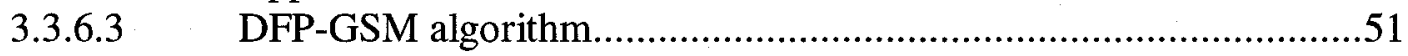

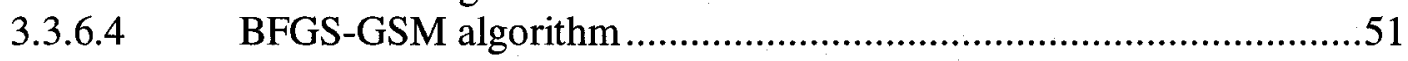

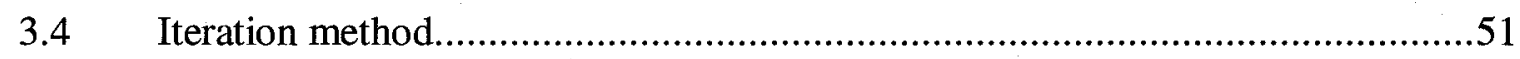

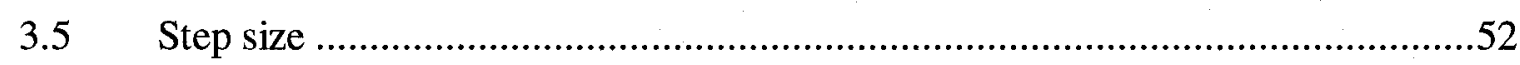

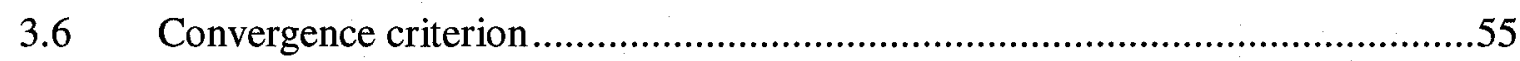

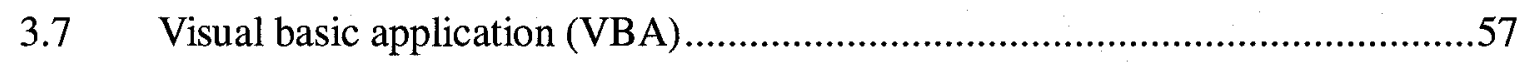

Chapter 4 Experimental Estimation Results ........................................61

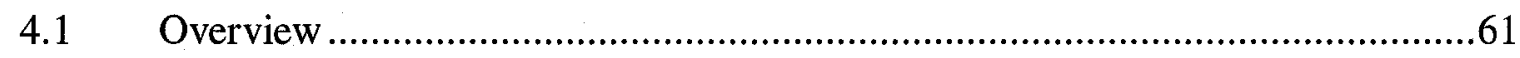

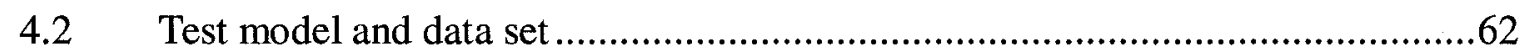

4.2.1 Assumed representative utility function ..........................................62

4.3 Analytical partial derivatives of the log-likelihood function ..........................64

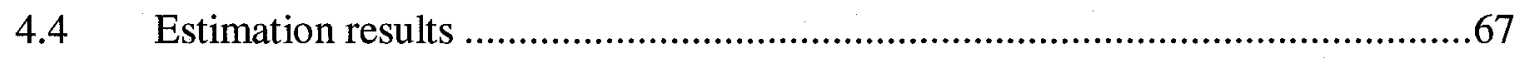

4.4.1 General tests applied in discrete mode choice model ...............................68

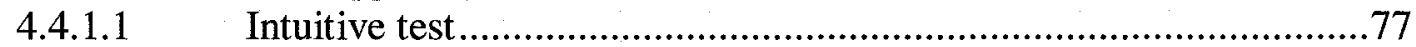

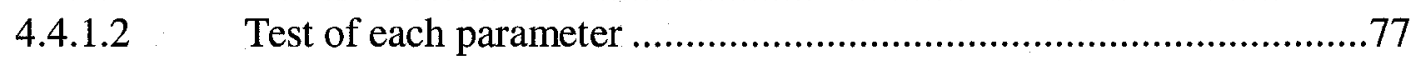

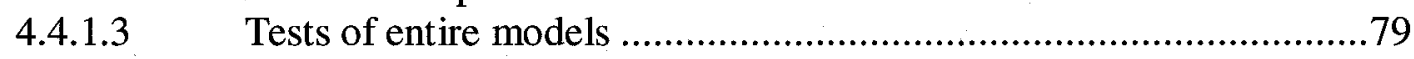

4.4.1.4 Overall goodness-of-fit measures ........................................... 81 


\section{Chapter 5 A Comparison of the Eight Estimation Algorithms in terms of} Three Performance Measures .............................................84

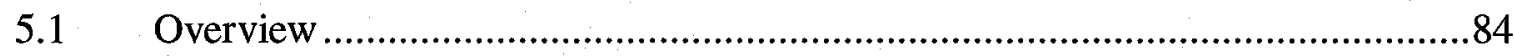

5.2 Acomparison of the variations according to the convergence criterion (experiments 1-4).....86

5.2.1 The first convergence criterion (experiment 1) .........................................86

5.2.1.1 The number of iterations and the log-likelihood values (experiment 1)......94

5.2.2 The second convergence criterion (experiment 2)................................ 117

5.2.2.1 The number of iterations and log-likelihood values (experiment 2)......123

5.2.3 The third convergence criterion (experiment 3)...................................... 144

5.2.3.1 The number of iterations and log-likelihood value (experiment 3).....149

5.2.4 The fourth convergence criterion (experiment 4) ..................................170

5.2.4.1 The number of iterations and log-likelihood value (experiment 4).... 175

5.3 A comparison of the variations responding to the starting points (experiment 5) ..196

5.4 A comparison of the variations according to the initialized Hessian (experiment 6) ...221

5.5 Comparison of estimation performance on the basis of each algorithm............251

5.5.1 Effect of convergence criterion on estimation performance ........................251

5.5.2 Effect of initial starting points on estimation performance.........................254

5.5.3 Effect of initial Hessian matrix on estimation performance .......................256

Chapter 6 Summary and Conclusions ..............................................257

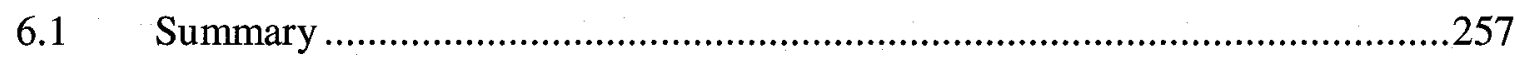

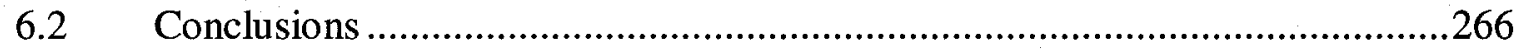

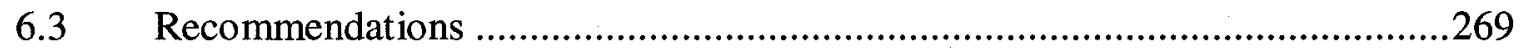

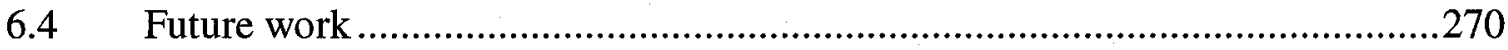

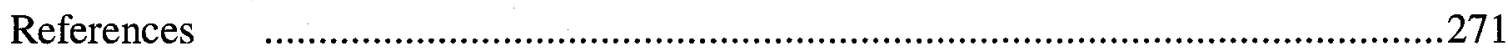

Appendix A: An Analytical Derivative of the

Log-likelihood Function ...............................................2275

A.1: $\quad$ Representative utility functions of all five alternatives ...............................276

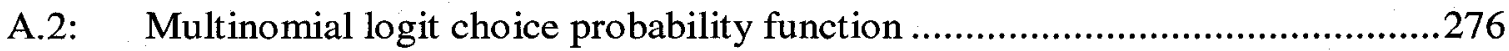

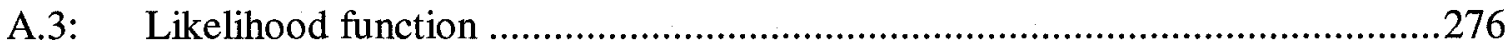

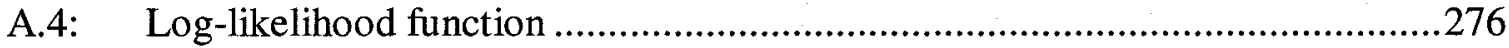

A.5: The derivation of a gradient vector formulas specified to given choice problem...276

A.6: The derivation of the Hessian matrix formulas specified to given choice problem ..288 


\section{Appendix B: Eight Estimation Algorithms}

(Visual Basic Application)

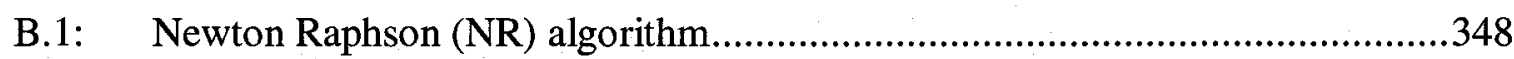

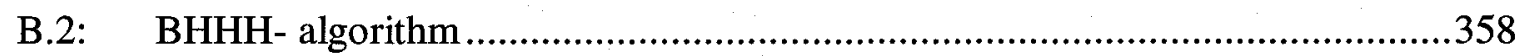

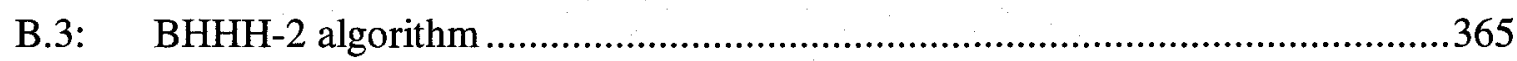

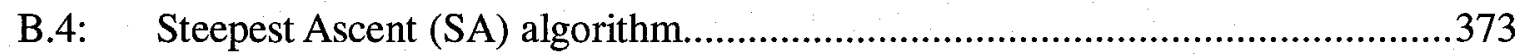

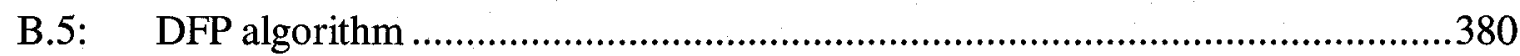

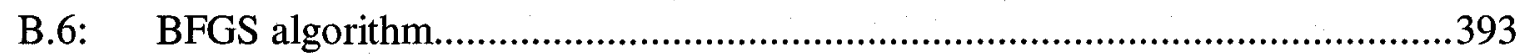

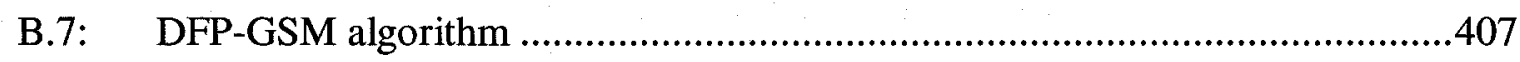

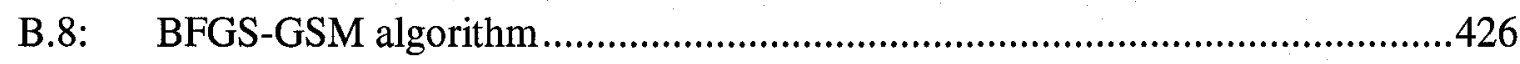

\section{Appendix C: Data Used in Experimental Estimation} (540 obsevations) .447

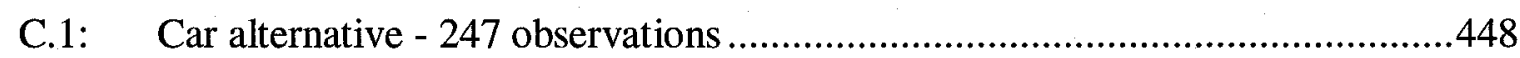

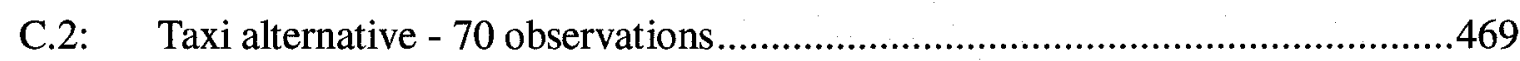

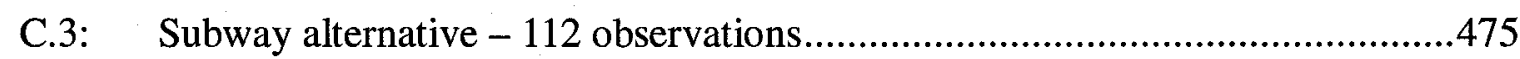

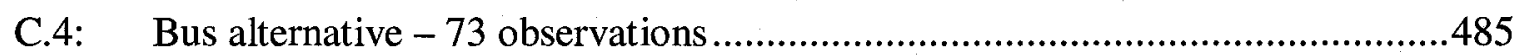

C.5: Limousine alternative -38 observations .......................................................492 


\section{List of Figures}

Figure 3-1: Direction of step follows the slope (Train, 2003) ......................................40

Figure 3-2: Step size is inversely related to curvature (Train, 2003) ..............................41

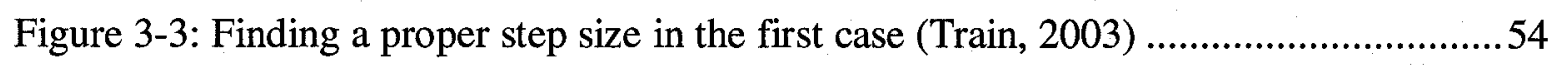

Figure 3-4: Finding a proper step size in the second case (Train, 2003) ...........................54

Figure 3-5: Coding window of Visual Basic Application (VBA) .................................58

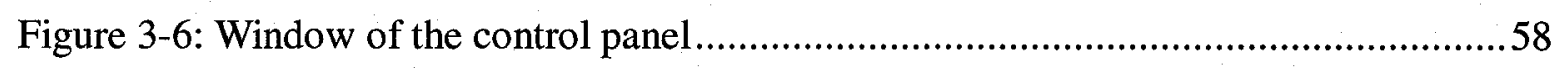

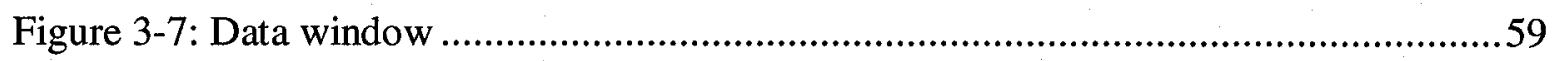

Figure 4-1: The scalar relationship of log-likelihood functions

(Koppelman and Bhat, 2006)

Figure 5-1: The final experimental results of

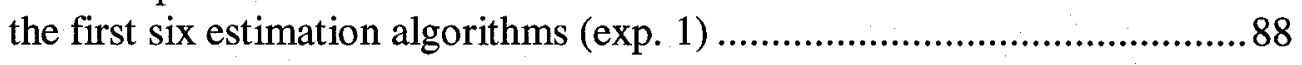

Figure 5-2: The final experimental results of

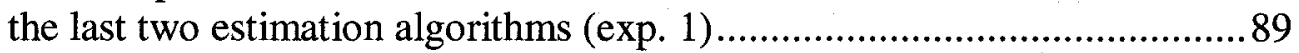

Figure 5-3: Log-likelihood values for each step size in Newton Raphson algorithm (exp. 1) ........................................... 100

Figure 5-4: Log-likelihood for the all step sizes in Newton Raphson algorithm (exp. 1) ............................................ 101

Figure 5-5: Log-likelihood values for each step size in BHHH (exp. 1)....................... 102

Figure 5-6: Log-likelihood for the all step sizes in BHHH algorithm (exp. 1)................. 103

Figure 5-7: Log-likelihood values for each step size in BHHH-2 algorithm (exp. 1)....... 104

Figure 5-8: Log-likelihood for the all step sizes in BHHH-2 algorithm (exp. 1) ............. 105

Figure 5-9: Log-likelihood values for each step size and all step sizes in Steepest Ascent algorithm (exp. 1). 106

Figure 5-10: Log-likelihood values for each step size in DFP algorithm (exp. 1)........... 107

Figure 5-11: Log-likelihood for the all step sizes in DFP algorithm (exp. 1).................. 108 
Figure 5-12: Log-likelihood values for each step size in BFGS algorithm (exp. 1) .......... 109

Figure 5-13: Log-likelihood for the all step sizes in BFGS algorithm (exp. 1) ................. 110

Figure 5-14: Log-likelihood values for each step size in DFP-GSM algorithm for the first six uncertain intervals (exp. 1)

Figure 5-15: Log-likelihood values for each step size in DFP-GSM algorithm for the last two uncertain intervals (exp, 1)

Figure 5-16: Log-likelihood for the all step sizes in DFP-GSM algorithm (exp. 1).....

Figure 5-17: Log-likelihood values for each step size in BFGS-GSM algorithm for the first six uncertain intervals (exp. 1).

Figure 5-18: Log-likelihood values for each step size in BFGS-GSM algorithm for the last two uncertain intervals (exp. 1)

Figure 5-19: Log-likelihood for the all step sizes in BFGS-GSM algorithm (exp. 1)

Figure 5-20: Final experimental results of the first six estimation algorithms (exp. 2)

Figure 5-21: Final experimental results of the last two estimation algorithms (exp. 2)

Figure 5-22: Log-likelihood values for each step size

in Newton Raphson algorithm (exp. 2)

Figure 5-23: Log-likelihood for the all step sizes

in Newton Raphson algorithm (exp. 2) 128

Figure 5-24: Log-likelihood values for each step size in BHHH algorithm (exp. 2)........ 129

Figure 5-25: Log-likelihood for the all step sizes in BHHH algorithm (exp. 2)............... 130

Figure 5-26: Log-likelihood values for each step size in BHHH-2 algorithm (exp. 2)..... 131

Figure 5-27: Log-likelihood for the all step sizes in BHHH-2 algorithm (exp. 2)

Figure 5-28: Log-likelihood values for each step size and all step sizes

in Steepest Ascent algorithm (exp. 2)

Figure 5-29: Log-likelihood values for each step size in DFP algorithm (exp. 2) 134 
Figure 5-30: Log-likelihood for the all step sizes in BHHH-2 algorithm (exp. 2) ............ 135

Figure 5-31: Log-likelihood values for each step size in BFGS algorithm (exp. 2) .......... 136

Figure 5-32: Log-likelihood for the all step sizes in BFGS algorithm (exp. 2) ............... 137

Figure 5-33: Log-likelihood values for each step size in DFP-GSM algorithm for the first six uncertain intervals (exp. 2) .......................................... 138

Figure 5-34: Log-likelihood values for each step size in DFP-GSM algorithm for the last two uncertain intervals (exp. 2)

Figure 5-35: Log-likelihood for the all step sizes in DFP-GSM algorithm (exp. 2) .......... 140

Figure 5-36: Log-likelihood values for each step size in BFGS-GSM algorithm for the first six uncertain intervals (exp. 2)

Figure 5-37: Log-likelihood values for each step size in BFGS-GSM algorithm for the last two uncertain intervals (exp. 2)

Figure 5-38: Log-likelihood for the all step sizes in BFGS-GSM algorithm (exp. 2) ....... 143

Figure 5-39: The final experimental results of the first six estimation algorithms (exp. 3).

Figure 5-40: The final experimental results of the last

two estimation algorithms (exp. 3)

Figure 5-41: Log-likelihood values for each step size in Newton Raphson algorithm (exp. 3)

Figure 5-42: Log-likelihood for the all step sizes in Newton Raphson algorithm (exp. 3)

Figure 5-43: Log-likelihood values for each step size in BHHH algorithm (exp. 3)........ 155

Figure 5-44: Log-likelihood for the all step sizes in BHHH algorithm (exp. 3).............. 156

Figure 5-45: Log-likelihood values for each step size in BHHH-2 algorithm (exp. 3)

Figure 5-46: Log-likelihood for the all step sizes in BHHH-2 algorithm (exp. 3) 158

Figure 5-47: Log-likelihood values for each step size and all step sizes in Steepest Ascent algorithm (exp. 3) 
Figure 5-48: Log-likelihood values for each step size in DFP algorithm (exp. 3)............ 160

Figure 5-49: Log-likelihood for the all step sizes in DFP algorithm (exp. 3)................... 161

Figure 5-50: Log-likelihood values for each step size in BFGS algorithm (exp. 3) .......... 162

Figure 5-51: Log-likelihood for the all step sizes in BFGS algorithm (exp. 3) ................. 163

Figure 5-52: Log-likelihood values for each step size in DFP-GSM algorithm for the first six uncertain intervals (exp. 3)

Figure 5-53: Log-likelihood values for each step size in DFP-GSM algorithm for the last two uncertain intervals (exp. 3)

Figure 5-54: Log-likelihood for the all step sizes in DFP-GSM algorithm (exp. 3)......... 166

Figure 5-55: Log-likelihood values for each step size in BFGS-GSM algorithm for the first six uncertain intervals (exp. 3)

Figure 5-56: Log-likelihood values for each step size in BFGS-GSM algorithm for the last two uncertain intervals (exp. 3) 168

Figure 5-57: Log-likelihood for the all step sizes in BFSG-GSM algorithm (exp. 3) 169

Figure 5-58: The final experimental results of the first six estimation algorithms (exp. 4)

Figure 5-59: The final experimental results of the last two estimation algorithms (exp. 4)

Figure 5-60: Log-likelihood values for each step size in Newton Raphson algorithm (exp. 4)

Figure 5-61: Log-likelihood for the all step sizes in Newton Raphson algorithm (exp. 4) 180

Figure 5-62: Log-likelihood values for each step size in BHHH algorithm (exp. 4) 181

Figure 5-63: Log-likelihood for the all step sizes in BHHH algorithm (exp. 4). 182

Figure 5-64: Log-likelihood values for each step size in BHHH-2 algorithm (exp. 4)...... 183

Figure 5-65: Log-likelihood for the all step sizes in BHHH-2 algorithm (exp. 4) 184

Figure 5-66: Log-likelihood values for each step size and all step sizes in Steepest Ascent algorithm (exp. 4) 
Figure 5-67: Log-likelihood values for each step size in DFP algorithm (exp. 4)............. 186

Figure 5-68: Log-likelihood for the all step sizes in DFP algorithm (exp. 4)................... 187

Figure 5-69: Log-likelihood values for each step size in BFGS algorithm (exp. 4) .......... 188

Figure 5-70: Log-likelihood for the all step sizes in BFGS algorithm (exp. 4)................. 189

Figure 5-71: Log-likelihood values in DFP-GSM algorithm for the first six uncertain intervals (exp. 4)

Figure 5-72: Log-likelihood values in DFP-GSM algorithm for the last two uncertain intervals (exp. 4)

Figure 5-73: Log-likelihood for the all uncertain intervals in DFP-GSM algorithm (exp. 4)

Figure 5-74: Log-likelihood values in BFGS-GSM algorithm for the first six uncertain intervals (exp. 4)

Figure 5-75: Log-likelihood values in BFGS-GSM algorithm for the last two uncertain intervals (exp. 4)

Figure 5-76: Log-likelihood for the all uncertain intervals in BFGS-GSM algorithm (exp. 4).

Figure 5-77: Performance results of the first four algorithms responding to the first set of guessing of starting points (exp. 5)

Figure 5-78: Performance results of DFP and BFGS algorithms responding to the second set of guessing of starting points (exp. 5)

Figure 5-79: Performance results of the last two algorithms responding to the second set of guessing of starting points (exp. 5)

Figure 5-80: Log-likelihood values for each step size in Newton Raphson algorithm for the first set of guessing of starting points (exp. 5)

Figure 5-81: Log-likelihood for the all step sizes in Newton Raphson algorithm for the first set of guessing of starting points (exp. 5)

Figure 5-82: Log-likelihood values for each step size in BHHH algorithm for the first set of guessing of starting points (exp. 5) 206 
Figure 5-83: Log-likelihood for the all step sizes in BHHH algorithm

for the first set of guessing of starting points (exp. 5) 207

Figure 5-84: Log-likelihood values for each step size in BHHH-2 algorithm

for the first set of guessing of starting points (exp. 5) .208

Figure 5-85: Log-likelihood for the all step sizes in BHHH-2 algorithm

for the first set of guessing of starting points (exp. 5)

Figure 5-86: Log-likelihood values for each step size and all step sizes in Steepest

Ascent algorithm for the first set of guessing of starting points (exp. 5) ...210

Figure 5-87: Log-likelihood values for each step size in DFP algorithm for

the second set of guessing of starting points (exp. 5)

Figure 5-88: Log-likelihood for the all step sizes in DFP algorithm for the second set of guessing of starting points (exp. 5).

Figure 5-89: Log-likelihood values for each step size in BFGS algorithm for the second set of guessing of starting points (exp. 5)

Figure 5-90: Log-likelihood for the all step sizes in BFGS algorithm for the second set of guessing of starting points (exp. 5)

Figure 5-91: Log-likelihood values for the second set of guessing of starting points in DFP-GSM algorithm for the first six uncertain intervals (exp. 5)

Figure 5-92: Log-likelihood values for the second set of guessing of starting points in DFP-GSM algorithm for the last two uncertain intervals (exp. 5)

Figure 5-93: Log-likelihood for the all uncertain intervals in DFP-GSM algorithm for the second set of guessing of starting points (exp. 5)

Figure 5-94: Log-likelihood values for the second set of guessing of starting points in BFGS-GSM algorithm for the first six uncertain intervals (exp. 5)......218

Figure 5-95: Log-likelihood values for the second set of guessing of starting points in BFGS-GSM algorithm for the last two uncertain intervals (exp. 5)

Figure 5-96: Log-likelihood for the all uncertain intervals in BFGS-GSM algorithm for the second set of guessing of starting points (exp. 5)

Figure 5-97: Performance results of DFP and BFGS algorithms responding to a new way of calculating the initial Hessian matrix adopted from $\mathrm{BHHH}$ algorithm (exp. 6). 225 
Figure 5-98: Performance results of DFP-GSM and BFGS-GSM algorithms responding to a new way of calculating the initial Hessian matrix adopted from BHHH algorithm (exp. 6).

Figure 5-99: Performance results of DFP and BFGS algorithms responding to a new way of calculating the initial Hessian matrix adopted from BHHH-2 algorithm (exp. 6)

Figure 5-100: Performance results of DFP-GSM and BFGS-GSM algorithms responding to a new way of calculating the initial Hessian matrix adopted from BHHH-2 algorithm (exp. 6)

Figure 5-101: Log-likelihood values for each step size in DFP algorithm responding to a new way of calculating the initial Hessian matrix adopted from BHHH algorithm (exp. 6).

Figure 5-102: Log-likelihood values for all the step sizes in DFP algorithm responding to a new way of calculating the initial Hessian matrix adopted from BHHH algorithm (exp. 6)

Figure 5-103: Log-likelihood values for each step size in DFP algorithm responding to a new way of calculating the initial Hessian matrix adopted from BHHH-2 algorithm (exp. 6)

Figure 5-104: Log-likelihood values for all the step sizes in DFP algorithm responding to a new way of calculating the initial Hessian matrix adopted from BHHH-2 algorithm (exp. 6)

Figure 5-105: Log-likelihood values for each step size in BFGS algorithm responding to a new way of calculating the initial Hessian matrix adopted from BHHH algorithm (exp. 6).

Figure 5-106: Log-likelihood values for all the step sizes in BFGS algorithm responding to a new way of calculating the initial Hessian matrix adopted from BHHH algorithm (exp. 6) .236

Figure 5-107: Log-likelihood values for each step size in BFGS algorithm responding to a new way of calculating the initial Hessian matrix adopted from BHHH-2 algorithm (exp. 6)

Figure 5-108: Log-likelihood values for all the step sizes in BFGS algorithm responding to a new way of calculating the initial Hessian matrix adopted from BHHH-2 algorithm (exp. 6) 238 
Figure 5-109: Log-likelihood values for the first six uncertain intervals in DFP-GSM algorithm responding to a new way of calculating the initial Hessian matrix adopted from BHHH algorithm (exp. 6)

Figure 5-110: Log-likelihood values for the last two uncertain intervals in DFP-GSM algorithm responding to a new way of calculating the initial Hessian matrix adopted from $\mathrm{BHHH}$ algorithm (exp. 6)

Figure 5-111: Log-likelihood values for the all uncertain intervals in DFP-GSM algorithm responding to a new way of calculating the initial Hessian matrix adopted from $\mathrm{BHHH}$ algorithm (exp. 6).

Figure 5-112: Log-likelihood values for the first six uncertain intervals in DFP-GSM algorithm responding to a new way of calculating the initial Hessian matrix adopted from BHHH-2 algorithm (exp. 6)

Figure 5-113: Log-likelihood values for the last two uncertain intervals in DFP-GSM algorithm responding to a new way of calculating the initial Hessian matrix adopted from BHHH-2 algorithm (exp. 6)

Figure 5-114: Log-likelihood values for the all uncertain intervals in DFP-GSM algorithm responding to a new way of calculating the initial Hessian matrix adopted from $\mathrm{BHHH}-2$ algorithm (exp. 6)

Figure 5-115: Log-likelihood values for the first six uncertain intervals in BFGS-GSM algorithm responding to a new way of calculating the initial Hessian matrix adopted from $\mathrm{BHHH}$ algorithm (exp. 6)

Figure 5-116: Log-likelihood values for the last two uncertain intervals in BFGS-GSM algorithm responding to a new way of calculating the initial Hessian matrix adopted from $\mathrm{BHHH}$ algorithm (exp. 6)

Figure 5-117: Log-likelihood values for the all uncertain intervals in BFGS-GSM algorithm responding to a new way of calculating the initial Hessian matrix adopted from $\mathrm{BHHH}$ algorithm (exp. 6)

Figure 5-118: Log-likelihood values for the first six uncertain intervals in BFGS-GSM algorithm responding to a new way of calculating the initial Hessian matrix adopted from BHHH-2 algorithm (exp. 6)

Figure 5-119: Log-likelihood values for the last two uncertain intervals in BFGS-GSM algorithm responding to a new way of calculating the initial Hessian matrix adopted from BHHH-2 algorithm (exp. 6) 
Figure 5-120: Log-likelihood values for the all uncertain intervals in BFGS-GSM algorithm responding to a new way of calculating the initial Hessian matrix adopted from BHHH-2 algorithm (exp. 6)

Figure 5-121: Variation of performance caused by different convergence criterion (1) ....252

Figure 5-122: Variation of performance caused by different convergence criterion (2) ....253

Figure 5-123: Variation of performance caused by different initial starting points (1)......254

Figure 5-124: Variation of performance caused by different initial starting points (2).....255

Figure 5-125: Variation of performance caused by different initial Hessian matrix .256 


\section{List of Tables}

Table 2-1: An example of cross-elasticity of the logit model in response to subsidy

(Train, 2003)

Table 3-1: Example of computation of GSM in the case of DFP-GSM

(0.3-27-26.29) .50

Table 4-1: The sample frequencies of chosen mode.

Table 4-2: Newton Raphson (NR) Algorithm

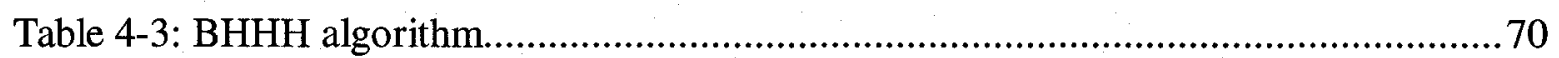

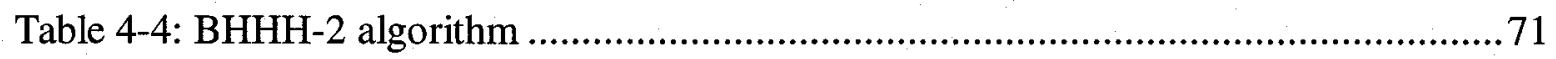

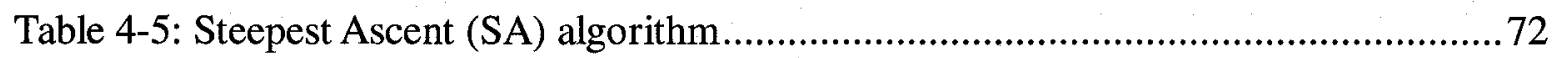

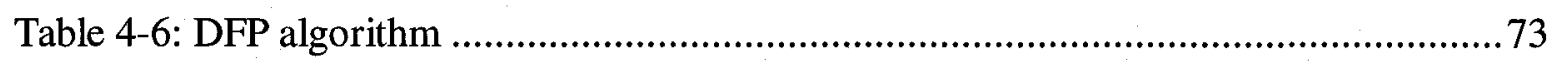

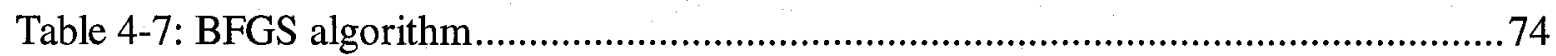

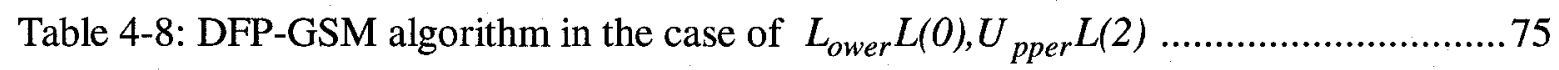

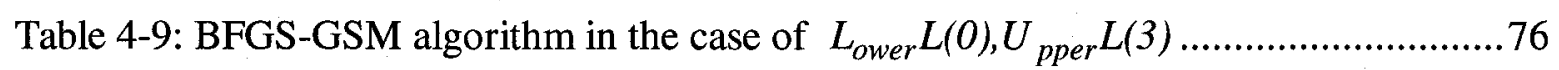

Table 4-10: Critical t-values for selected confidence levels

(Koppelman and Bhat, 2006)

Table 4-11: Critical Chi-Square $\chi^{2}$ Values (Koppelman and Bhat, 2006) .........................81

Table 5-1: The designated serial number for the eight estimation algorithms ...................85

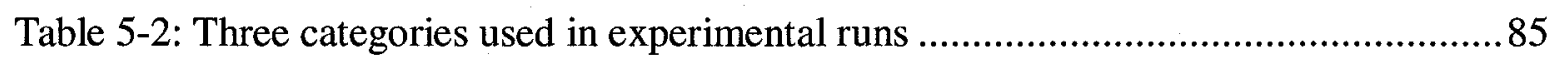

Table 5-3: The final experimental results of the eight estimation algorithms (exp. 1)........87

Table 5-4: Log-likelihood values obtained from Newton Raphson algorithm (exp. 1) .......95

Table 5-5: Log-likelihood values obtained from BHHH algorithm (exp. 1) .....................95

Table 5-6: Log-likelihood values obtained from BHHH-2 algorithm (exp. 1) ..................95

Table 5-7: Log-likelihood values obtained from Steepest Ascent algorithm (exp. 1).........95 
Table 5-8: Log-likelihood values obtained from DFP algorithm (exp. 1) ............................96

Table 5-9: Log-likelihood values obtained from BFGS algorithm (exp. 1) .........................96

Table 5-10: Log-likelihood values obtained from DFP-GSM algorithm (exp. 1) ................96

Table 5-11: Log-likelihood values obtained from BFGS-GSM algorithm (exp. 1) ..............96

Table 5-12: The final experimental results of the eight estimation algorithms (exp. 2)..... 118

Table 5-13: Log-likelihood values obtained from Newton Raphson algorithm (exp. 2) ... 124

Table 5-14: Log-likelihood values obtained from BHHH algorithm (exp. 2) .................... 124

Table 5-15: Log-likelihood values obtained from BHHH-2 algorithm (exp. 2) ................ 124

Table 5-16: Log-likelihood values obtained from Steepest Ascent algorithm (exp. 2)...... 124

Table 5-17: Log likelihood values obtained from DFP algorithm (exp. 2) ........................ 125

Table 5-18: Log likelihood values obtained from BFGS algorithm (exp. 2)...................... 125

Table 5-19: Log likelihood values obtained from DFP-GSM algorithm (exp. 2).............. 125

Table 5-20: Log likelihood values obtained from BFGS-GSM algorithm (exp. 2) ............ 125

Table 5-21: The final experiment results of the eight estimation algorithms (exp. 3) ....... 145

Table 5-22: Log-likelihood values obtained from Newton Raphson algorithm (exp. 3) ... 150

Table 5-23: Log-likelihood values obtained from BHHH algorithm (exp. 3) .................... 150

Table 5-24: Log-likelihood values obtained from BHHH-2 algorithm (exp. 3) ................ 150

Table 5-25: Log likelihood values obtained from Steepest Ascent algorithm (exp. 3) ...... 150

Table 5-26: Log-likelihood values obtained from DFP algorithm (exp. 3) ........................ 151

Table 5-27: Log-likelihood values obtained from BFGS algorithm (exp. 3) ...................... 151

Table 5-28: Log-likelihood values obtained from DFP-GSM algorithm (exp. 3) ............. 151

Table 5-29: Log-likelihood values obtained from BFGS-GSM algorithm (exp. 3)........... 151

Table 5-30: The final experiment results of the eight estimation algorithms (exp. 4) ....... 171 
Table 5-31: Log-likelihood values obtained from Newton Raphson algorithm (exp. 4) ... 176

Table 5-32: Log-likelihood values obtained from $\mathrm{BHHH}$ algorithm (exp. 4) ..................176

Table 5-33: Log-likelihood values obtained from BHHH-2 algorithm (exp. 4) ............... 176

Table 5-34: Log-likelihood values obtained from Steepest Ascent algorithm (exp. 4)...... 176

Table 5-35: Log-likelihood values obtained from DFP algorithm (exp. 4) ..................... 177

Table 5-36: Log-likelihood values obtained from BFGS algorithm (exp. 4) ................... 177

Table 5-37: Log-likelihood values obtained from DFP-GSM algorithm (exp. 4) ........... 177

Table 5-38: Log-likelihood values obtained from BFGS-GSM algorithm (exp. 4).......... 177

Table 5-39: Experimental results responding to the variation of starting points (exp. 5) .. 198

Table 5-40: Log-likelihood values obtained from Newton Raphson algorithm for the first set of guessing of starting points (exp. 5) .......................... 202

Table 5-41: Log-likelihood values obtained from BHHH algorithm for the first set of guessing of starting points (exp. 5) .................................202

Table 5-42: Log-likelihood values obtained from BHHH-2 algorithm for the first set of starting points (exp. 5)

Table 5-43: Log-likelihood values obtained from Steepest Ascent algorithm for the first set of guessing of starting points (exp. 5).

Table 5-44: Log-likelihood values obtained from DFP algorithm for the second set of guessing of starting points (exp. 5) .203

Table 5-45: Log-likelihood values obtained from BFGS algorithm for the second set of guessing of starting points (exp. 5).

Table 5-46: Log-likelihood values obtained from DFP-GSM algorithm for the second set of guessing of starting points (exp. 5). .203

Table 5-47: Log-likelihood values obtained from BFGS-GSM algorithm for the second set of guessing of starting points (exp. 5)

Table 5-48: The experimental results of DFP, BFGS and DFP-GSM, BFGS-GSM algorithms responding to a new way of calculating the initial Hessian matrix adopted from either $\mathrm{BHHH}$ or $\mathrm{BHHH}-2$ algorithm (exp. 6). 
Table 5-49: Log-likelihood values obtained from DFP algorithm responding to a new way of calculating the initial Hessian matrix adopted from $\mathrm{BHHH}$ algorithm (exp. 6)

Table 5-50: Log-likelihood values obtained from DFP algorithm responding to a new way of calculating the initial Hessian matrix adopted from BHHH-2 algorithm (exp. 6)

Table 5-51: Log-likelihood values obtained from BFGS algorithm responding to a new way of calculating the initial Hessian matrix adopted from $\mathrm{BHHH}$ algorithm (exp. 6)

Table 5-52: Log-likelihood values obtained from BFGS algorithm responding to a new way of calculating the initial Hessian matrix adopted from BHHH-2 algorithm (exp. 6)

Table 5-53: Log-likelihood values obtained from DFP-GSM algorithm responding to a new way of calculating the initial Hessian matrix adopted from BHHH algorithm (exp. 6)

Table 5-54: Log-likelihood values obtained from DFP-GSM algorithm responding to a new way of calculating the initial Hessian matrix adopted from BHHH-2 algorithm (exp. 6)

Table 5-55: Log-likelihood values obtained from BFGS-GSM algorithm responding to a new way of calculating the initial Hessian matrix adopted from BHHH algorithm (exp. 6)

Table 5-56: Log-likelihood values obtained from BFGS-GSM algorithm responding to a new way of calculating the initial Hessian matrix adopted from $\mathrm{BHHH}-2$ algorithm (exp. 6). 


\section{Glossary of Terms}

$U_{n i}(\bullet), U_{n j}(\bullet) \quad$ Utility function of alternative $\mathrm{i}$ and $\mathrm{j}$, respectively as viewed by a decision maker $n$

$A_{n i}, A_{n j}$

A vector of attribute values for alternative $i$ and $j$, respectively as viewed by decision maker $\mathrm{n}$ (e.g., total travel time, total travel cost, and other attributes for all alternativeS considered)

$P_{n}$

A vector of characteristics of the decision maker $\mathrm{n}$, which is considered to affect the choice between alternatives

(e.g., household income and size, the number of vehicles owned, the number of licensed drivers, age, education)

$\forall i \neq j$

Indicates comparson of all alternatives except for the same alternative

$i \succ j$

Indicates that the alternative $\mathrm{i}$ is preferred to the alternative $\mathrm{j}$

$i, j \in C_{n}$

Indicates that the alternatives $i, j$ are included in the choice set $C_{n}$

$C_{n}$

Actual choice set that the decision maker, $\mathrm{n}$, can consider in the given choice situation

$U_{n i}$

Representative utility of the alternative $i$ of the decision maker $n$

$S_{n i}$

Systematic (or deterministic, observable, constant) part of the representative utility and is observable to a researcher

$R_{n i}$

Random (unobservable, error) part of the representative utility and is unobservable to a researcher

$x_{n j}$

Attribute of alternative $\mathrm{j}$ when faced by decision maker $\mathrm{n}$

$\beta_{x}$

Parameters estimated

NR

Newton Raphson algorithm

BHHH

Estimation algorithm developed by Berndt, Hall, Hall, and Hausman 
BHHH-2

SA

DFP

BFGS

DFP-GSM

BFGS-GSM

$\beta_{t}$

$\beta_{t+1}$

$\lambda$

$\left(-H_{\text {tReal }}^{N R}\right)^{-1}$

$G_{t}$

$G_{t}$

$\left(-H_{\text {tReal }}^{N R}\right)^{-1} G_{t}$

$H_{t n}^{B H H H}$

$G_{t n}$

$H_{t A P}^{B H H H}$

$H_{\text {tAP }}^{B H H H-2}$

$G_{t A V E}$
Estimation algorithm improved from $\mathrm{BHHH}$

Steepest Ascent algorithm

Estimation algorithm developed by Davidon, Fletcher, and Powell

Estimation algorithm developed by Broydon, Fletcher, Goldfarb, and Shanno

Improved estimation algorithm by incorporating golden-section line search method (GSM) to DFP algorithm, tried in this thesis for the first time

Improved estimation algorithm by incorporating golden-section line search method (GSM) to BFGS algorithm, tried in this thesis for the first time

Parameter estimates after $\mathrm{t}$ iterations

Parameter estimates after $\mathrm{t}+1$ iterations

Step size (can be assumed by researcher at the time of beginning)

Inverse of the negative real Hessian in iteration $t$ in NR method

Gradient in iteration $\mathrm{t}$

Transposed gradient in iteration $\mathbf{t}$

Direction to follow in iteration process

Outer product of observation $\mathrm{n}$ in iteration $\mathrm{t}$

Gradient of observation $\mathrm{n}$ in iteration $\mathrm{t}$

Approximate Hessian of $-H_{t \text { Real }}^{N R}$ in BHHH method

Approximate Hessian of $-H_{t R e a l}^{N R}$ in BHHH-2 method

Average gradient for all observations in iteration $t$ 
$I^{S A}$

$H_{t+1(A P)}^{D F P}$

$H_{t}^{D F P}$

$\delta_{t}$

$\delta_{t}^{\prime}$

$\gamma_{t}$

$\gamma_{t}^{\prime}$

$H_{t+1(A P)}^{B F G S}$

$H_{t}^{B F G S}$

$d_{t}$

$d_{t}^{\prime}$

$v$

$L(\beta)$

$P_{n}(i)$

$y_{\text {in }}$

$P_{n}(C)$

$P_{n}(T)$
Identity matrix used in iterations instead of Hessian

Approximate Hessian of $\left(H_{t R e a l}^{N R}\right)^{-1}$ in DFP method in $\mathrm{t}+1$ iteration

Approximate Hessian in $\mathrm{t}$ iteration (if $\mathrm{t}=1$ then $H_{t}^{D F P}=I$ )

$\lambda\left(-H_{t}^{D F P}\right) G_{t}$ in iteration t

Transposed matrix of $\delta_{t}$

$G_{t+1}-G_{t}$, the difference between $G_{t+1}$ and $G_{t}$

Transposed matrix of $\gamma_{t}$

Approximate Hessian of $\left(H_{t \text { Real }}^{N R}\right)^{-1}$ in BFGS method in $\mathrm{t}+1$ iteration

Approximate Hessian in $\mathrm{t}$ iteration (if $\mathrm{t}=1$ then $H_{t}^{B F G S}=I$ )

Value of $\left(\frac{1}{\delta_{t}^{\prime} \gamma_{t}}\right) \delta_{t}-\left(\frac{1}{\gamma_{t}^{\prime} H_{t}^{B F G S} \gamma_{t}}\right) H_{t}^{B F G S} \gamma_{t}$

Transposed matrix of $d_{t}$

Value of $\gamma_{t}^{\prime} H_{t}^{B F G S} \gamma_{t}$

Likelihood function

Probability that $\mathrm{i}$ is chosen; in this thesis multinomial logit probabilistic choice function is used

Indicator, $y_{i n}$ is 1 if $\mathrm{n}$ (decision maker) choose mode $\mathrm{i}$, otherwise 0

Probability of choosing a car

Probability of choosing a taxi 
$P_{n}(S) \quad$ Probability of choosing a subway

$P_{n}(B) \quad$ Probability of choosing a bus

$P_{n}(L) \quad$ Probability of choosing a limousine

$y_{C n}, y_{T n}, y_{S n}, y_{B n}, y_{L n} \quad$ An indicator; 1 if n chooses mode car, taxi, subway, bus, limousine respectively; otherwise 0

$\beta_{k} \quad$ Estimate for $\mathrm{k}^{\text {th }}$ parameter

$\beta_{k}^{*} \quad$ Hypothesized value for $\mathrm{k}^{\text {th }}$ parameter

$S_{k} \quad$ Standard error of $\mathrm{k}^{\text {th }}$ parameter

$L L_{R} \quad$ Log-likelihood in the restricted model

$L L_{U} \quad$ Log-likelihood in the unrestricted model

$L L(0) \quad$ Log-likelihood with $\beta_{k}=0$ (results in equal probability of choosing each available alternative)

$L L(C) \quad$ Log-likelihood for constant only model

$L L(\beta) \quad$ Log-likelihood for estimated model

$L L\left(\beta_{\text {true }}\right) \quad$ Log-likelihood for the perfect prediction model

$K \quad$ Number of degrees of freedom (parameters) used in the model

$K_{C O} \quad$ Number of degrees of freedom (parameters) used in the constants only model 


\section{Chapter 1 Introduction}

\subsection{Travel demand forecasting}

Transportation demand analysis is one of the important facets in the study of transportation systems. In transportation demand analysis, an emphasis has been on studying the behaviour of transportation system users. The users are people who use given transportation services and facilities in urban, interurban, and the international transportation market (Ben-Akiva and Lerman, 1985). To analyze the users of transportation systems such as traveller and goods, their characteristics or attributes are necessary and therefore defined in measurable way. Furthermore, to identify the relationship between the transportation demand and transportation services and facilities, their characteristics and attributes also are defined, and collected.

Forecasting the behaviour of users depends heavily on econometric modelling methods. These methods are generally about choosing data types, selecting estimation methods, and constructing parameterized mathematical function, using several useful estimation algorithms frequently adopted in the commercial model estimation packages.

There are two major data types frequently used in transportation studies for extracting information through transportation demand modelling studies. The first one is aggregate data that have some level of aggregation based on geographical zones. This aggregation with respect to the value of non-homogeneous explanatory variables or independent variable leads to a decrease in the accuracy of the parameter estimates. The decreasing of the accuracy of a model is from the aggregation of data conducted generally before 
parameters estimation. The second one is disaggregate data and this type of data is considered to open a new horizon in transportation demand modelling. Disaggregate data improves the precision of model estimates significantly by using micro-level data of independent (or explanatory) variables and makes it possible to incorporate a much larger band of explanatory variables than ever (Ben-Akiva and Lerman, 1985).

Disaggregate data can be handled, in modelling works, in the frame of a discrete data analysis method. This type of data has been widely employed to describe a certain choice situation with the help of the choice modelling frameworks. Mode and route choice model in transportation network, household location decision model in land used economy can be examples. Discrete choice analysis method has been widely used to predict the choice behaviour of decision makers in a variety of researches of economists, engineers, psychologists, marketers, and planners who face a specific choice situation across disciplines (Ben-Akiva et al., 2002).

\subsection{Background of discrete choice analysis}

Utility maximization is the core theoretical background of discrete choice analysis and the concept originates from the field of economics. Within utility maximization concept, users assumed in the transportation market are to choose only one alternative among alternatives that maximizes their utility. The utility can be described with a mathematical function that includes both a systematic utility part which represents observable characteristics of both decision-makers and alternatives and a random utility part which represents unobserved characteristics of both decision-makers (or agent or individual) 
and alternatives. The systematic utility part of the representative utility has a form of linear-in parameters nature. This part is composed by observable characteristics (explanatory variables describing choice situation) of both decision makers and alternatives. The model estimation procedures can estimate the parameters of the systematic utility. Designing a perfect utility function of an alternative is actually impossible. Therefore, random utility part complementing the systematic utility is assigned to systematic utility to represent the total expected utility of individuals (or decision makers or agents) sampled from population. To summarize this concept, the representative utility consists of a systematic utility part (or deterministic component) and a random utility part (or random component). The model based on the above conceptual structure is the Random Utility Model (RUM).

\subsection{Transportation application of discrete choice analysis}

Transportation applications of discrete choice analysis have been widely used to obtain three important purposes (Ben-Akiva and Lerman, 1985). The first purpose is to obtain a value of travel time (VOT), which is a result of trade-off between travel cost and travel time of estimated discrete mode choice model. The reason for using the VOT is that researchers can assign a monetary value to travel time saving and therefore evaluate all transportation projects considered (Bhat, 1998a; Bhat, 1998b; Harvey, 1986; Hess and Polak, 2005). The second purpose of using a discrete choice analysis is to describe the choice situation faced by decision maker in transportation market or other environments. Examples of such choice problems include air cargo transshipment airport choice (Ohashi et al., 2005), an airport choice in air transport management (Harvey, 1987; Hess and 
Polak, 2005), and an access mode choice for travelling to airport (Psaraki and Abacoumkin, 2002; Harvey, 1986). Other examples are a route choice of commercial vehicles (Hunt and Abraham, 2004), household's choices of appliance (Revelt and Train, 1998), and forecasting a choice behaviour for new product (Brownstone and Train, 1999).

The third one is to study policy-sensitive mode using discrete choice analysis (Bhat 1998a; Bhat 1998b). Based on this type of study, researchers (or modellers) can support a policy decision maker by presenting a prediction on a mode share shift caused by a certain change of policy variables included in the discrete choice model.

\subsection{The necessity of understanding the numerical aspects of discrete choice modelling}

Without knowing the detailed procedures of discrete choice modelling and understanding the procedures of model's parameter estimation, which is not the subject of interest to researchers until recently, a researcher simply has been specifying his/her model specification using some possible model specification that is allowed in a certain commercial statistical packages chosen to his/her research. Moreover, this specified model's parameters are just estimated mechanically using imbedded estimation algorithm of commercial packages and employing their prefixed procedures. The mechanism of this type of modelling is the way of "the first generation of modelling." Recently, in "the second generation of modelling," a researcher is strongly encouraged to make his/her own special code for each unique modelling situation to overcome the limitations of the first generation modelling (Train, 2003). Ultimately, a researcher should try to develop the capability of unique modelling work with a self-made computer code designed only for that work. To accomplish this goal, if a researcher has a clear understanding of the 
estimation procedures focused mainly on numerical aspects such as numerical role of loglikelihood function during estimation, numerical means of the simulated log-likelihood function and calculation mechanism of critical algorithms utilized in the estimation process, this goal can be accomplished. Understanding and knowing the details of numerical procedures and applying this core knowledge to researchers' modelling works are very valuable, and are an asset. These capabilities of a researcher could open new possibilities for both developing more advanced model structure by trying to diverse experiments freely and conceiving more efficient estimation procedures or methods.

\subsection{Research objectives and scope}

In transportation planning, it is common practice to carry out modelling tasks with commercial statistical packages and results are interpreted with statistics without knowing estimation mechanism used in modelling and without understanding different operational characteristics of various estimation algorithms. The objectives of this thesis are:

(1) to gain a clear understanding of estimation mechanism in discrete mode choice modelling, and

(2) to compare the performance of a number of estimation algorithms.

The scope of this research is to compare eight different estimation algorithms that are pedagogically important in discrete choice modelling through extensive numerical experiment. More importantly, the primary interest of this research is not in model development and evaluation, but to investigate numerical calculation mechanism underlying in discrete mode choice modelling. 


\subsection{Outline of this document}

This thesis is organized as follows. Chapter 1 is introduction. Chapter 2 as a literature review provides a general description of a discrete mode choice model focusing on its theoretical background, properties of its diverse models, and applications in transportation studies. Chapter 3 introduces eight algorithms widely used in parameters estimation. The reasons for their operational differences that arise from their iteration rules are explained and the different ways for obtaining both Hessian matrix and approximate Hessian matrix are compared. More importantly, two algorithms, BFGSGSM and DFP-GSM, newly tried in this thesis by incorporating Golden Section Method (GSM) are introduced. Chapter 4 shows experimental estimation results from eight different estimation algorithms and some basic statistical tests that are frequently used in discrete choice modelling for assessing the goodness of the results of parameter estimates are presented. Chapter 5 deals mainly with the comparison of the performances between the eight estimation algorithms by means of three performance measures. Furthermore, some useful insights gained through the extensive numerical experiments provide information about what was happening in the estimation routine. Chapter 6 summarizes the experimental estimation results and covers conclusions, some useful recommendations and the future research issues. 


\section{Chapter 2 Literature Review-Discrete Mode Choice Model}

\subsection{Overview}

This chapter is designed to discuss a discrete choice model as background knowledge and to apply this specific modelling technique in making computer codes of eight different estimation algorithms. The several important elements (e.g., data, decision maker, alternatives, decision process, utility theory and so on) involved in the discrete choice modelling are introduced and discussed. Especially, as the most prominent type of discrete choice models, logit probability choice model that is used as a test model in this thesis is derived mathematically and explained by focusing on its drawbacks and also on its strengths. Also other alternate probability choice models such as generalized extreme value (GEV), probit, and mixed logit, are briefly introduced and compared with the logit model.

To the best of the author's knowledge, previous published research did not compare the eight algorithms by using a common travel survey data. Also, the level of detail and comparative analysis is original to this research.

\subsection{Discrete choice modelling}

\subsubsection{Data}

Data are fundamental and essential in modelling of real world phenomena that happen in specific situations. The data set used in analysis generally consists of huge observations and various types of variables. Only with the thorough understanding of the variables used in modelling will a researcher be able to derive the most benefit from those data. The variables can be defined on three different dimensions. Firstly, variables can be 
classified as qualitative or quantitative. A qualitative variable cannot be described as numbers, this variable can be described by verbal grouping (e.g., in case of hair color, the variable might include red, blond, brown, black). On the other hand, quantitative variables can take numerical value (e.g., price, travel time, length, etc.). Secondly, variables can be classified as continuous or non-continuous. A continuous variable is assumed to be divisible and to take any value between interval of scale on which the variable is being measured (e.g., speed, price, time, height). On the other hand, noncontinuous, sometimes called discrete variable, only can take a few distinct values (e.g., male and female for gender). Thirdly, variables can be classified as nominal scale (e.g., male and female), ordinal scale (e.g., ranking of products in order of preference), interval scale (e.g., temperature, Centigrade or Fahrenheit), ratio scale variable (e.g., dollars, comparing $\$ 1$ and $\$ 2$ ) due to their relationship between the numbers and levels assigned to variables considered to be measured (Hensher et al., 2005).

Limited to discrete choice models of transportation modelling activity, a discrete choice model is estimated with discrete outcome variables that occur in the specific research work. These types of data may be acquired for various research areas. Examples of research are travel mode choice behaviour (e.g., auto, bus, rail, transit, etc.), vehicle types owned by households (e.g., compact car, intermediate car, standard size car, full size car, etc.), and safety analysis to study vehicular accidents (e.g., run-off-road, rearend, head-on, etc.). The first two types of discrete outcome data can be considered as discrete choice outcomes. These relate to the behavioural concept of utility maximization in the choice process. On the other hand, the vehicular accident data that 
are just physical phenomena, can be modeled by using a simple probabilistic theory (Washington et al., 2003).

\subsubsection{The decision maker}

As one of common elements of the discrete choice modelling framework, the decision makers' choice among alternatives can be described as percentage that results from a discrete choice model. The decision makers that have responsibility to make the decision at hand can vary according to the specific choice situations under considerations. For example, the decision maker can be the individual in college choice, travel mode choice, etc.; can be the firms in warehouse or office choice, employee choice, branch choice, etc.; can be the households in residential area choice, heating system choice, etc.; can be the transportation institute in the choice of roadway alignments (Train, 2003; Koppelman and Bhat, 2006). An important understanding keeping in mind while doing research on discrete choice model is that the decision makers are exposed to different choice situations and moreover can recognize attributes of the alternatives differently (that is, the decision makers have different tastes). For example, in the case of travel mode choice, a different decision maker can face a different set of alternatives due to his/her geographic or residential location and also can place different importance weights on attributes such as travel time, travel cost and others. This difference in evaluating the attributes of the alternatives is important in developing and estimating a discrete choice model and thus a researcher must include variables showing differences between the decision makers (Koppelman and Bhat, 2006). More importantly, to be empirical in the framework of utility maximization concepts (or utility decision rule), which will be discussed in a later 
section, the decision maker is assumed to be rational as an economic consumer in determining his/her choice. To be like this, two fundamental rules should be satisfied in his/her choice behaviour. The first one is "consistency," which means that the decision maker makes the same choice as before if the choice environments are identical. The second one is "transitivity," which means that if alternative A is preferred to alternative B, alternative $\mathrm{B}$ is preferred to alternative $\mathrm{C}$, then alternative $\mathrm{A}$ is also preferred to alternative C (Train, 2003; Koppelman and Bhat, 2006).

\subsubsection{The alternatives}

As mentioned in the previous section, a decision maker makes a choice among alternatives (i.e., choice set). There are two types of choice sets. The first choice set is usually referred to as the universal choice set. This choice set can be constructed by knowing the decision-making context. For example, in the case of mode choice situation, let us assume that there is a light rail transit (LRT) in addition to the other travel modes in a certain city. Some people can access this mode, however the others cannot. In this situation, LRT can be considered as one alternative of the universal choice set in the assumed city but it cannot be considered as a part of the feasible choice set for people who cannot access this mode. That is, the second choice set is referred to as the feasible choice set for a specific decision maker (Koppelman and Bhat, 2006).

\subsubsection{Choice set}

Discrete choice events arise when a person (or decision maker, agent, individual) comes across the situation to choose only one alternative out of choice set, which is composed of 
all possible alternatives. Train (2003) explains the characteristics of the choice set. In discrete choice modelling, three compulsory rules should be satisfied basically when setting the choice set of alternatives. First, alternatives should be mutually exclusive, which means that the choice of one alternative does not permit the choice of another alternative. Second, exhaustiveness of the choice set, which is accomplished by including all possible alternatives in a certain specific choice modelling activity. As the last one, the alternatives' number should be finite and the counting of alternatives should be ended.

\subsubsection{Attributes of alternatives}

The alternatives in a feasible choice set are represented by their attribute values and can be postulated by parameterized function mathematically. The attractiveness of the alternatives is measured by this representative assumed mathematical function, which consists of both parameters to be estimated and the attribute variables to be observed by a researcher. The attributes variables may be generic, which apply to all alternatives and be alternative-specific, which apply to one or a subset of alternatives. For example, in the context of the mode choice, travel time and travel cost can be included as generic variables. On the other hand, if a researcher considers that the travel time is more onerous to one alternative than other alternatives, the researcher may classify the travel time as a alternative-specific variable to that one alternative and assign a different parameter. Furthermore, by adding policy-sensitive attribute variables (i.e., travel time, frequency, reliability of service, etc.) which are measures of levels of service and could be changed by a wide variety of policy actions, a researcher can evaluate the effect of those changes (Koppelman and Bhat, 2006). 


\subsubsection{A general framework for choice}

From this point, several key elements involved in a discrete choice modelling process will be introduced and discussed in the context of transportation applications. To get an operational discrete choice model, all these things should be put together following a general framework which is designed for choice theories and is constructed on classical economic consumer (or rational consumer) principle and discrete choice theories (BenAkiva and Lerman, 1985).

Ben-Akiva and Lerman (1985) proposed a general framework in which a decision-maker first defines the choice problem; second, determines the possible alternatives; third, evaluates or collects information about the attributes of every available alternative; fourth, makes a choice among available alternatives; and then implements his/her decision, using the chosen alternative. On the other hand, it is worth to know that not all decision-makers follow the structured decision process presented above. For example, a decision-maker can follow his/her habit or intuition, take some conventional behaviour patterns; a decision-maker can trust his/her friend's decision and choose the same alternative as a friend due to his/her belief that the friend is an expert (Ben-Akiva and Lerman, 1985; Koppelman and Bhat, 2006). However, whether a decision-maker is to make a choice decision within the structured decision process or not, both choice behaviours can be represented in the framework of choice in which only one alternative is the one chosen (Koppelman and Bhat, 2006). 


\subsubsection{Decision rule}

In the general framework for choice process mentioned previously, a decision-maker requires a decision rule to make a choice from a choice set including two or more alternatives. The decision rule describes the process of a choice mechanism of the decision maker. It is assumed to happen internally and it is also used to evaluate the alternatives and then based on the evaluation results, final choice is made. There exists a number of possible decision rules describing decision processes (for details, see BenAkiva and Lerman, 1985; chapter 3). Generally, utility maximization is widely employed as a decision rule in a discrete choice model due to its strong theoretical basis, extensive application history in explaining choice concepts of a decision maker, and its amenability in mathematical and statistical terms. This utility maximization decision rule assumes three important concepts. The first one is that a utility can be described by a single utility function which contains the attributes vectors characterizing each alternative and this function also is reducible to a scalar (or index). A scalar (or index) can be referred to as "utility" of that alternative. This scalar (or index) value of the utility of each alternative is possible on the assumption of the compensatory (or commensurable) relationship between a vector of attributes. That is, the decision maker makes "trade-offs" or "compensatory offsets" between a vector of attributes. The second one is that the decision makers choose only one alternative with the highest utility index among alternatives. That is, this chosen alternative has the best combination of a vector of attributes, which makes its utility to be maxima. The third one is that the utility introduced in discrete choice modelling is an ordinal utility, not cardinal utility, if it is no otherwise stated (Koppelman and Bhat, 2006). Other underlying details involved in application of the utility concept to 
choice theory are discussed in the next section.

\subsubsection{Application of utility concept to choice theory}

To formulate empirical discrete choice models, a researcher can use the utility concept to describe some principles of individual choice and to express it mathematically and statistically. Within the utility concept, a researcher can develop the probabilistic choice model, which is the most commonly employed among the empirical discrete choice models. To understand these processes precisely, a study of both the basic construct of utility and the process of application of utility theory to a discrete choice model must be covered. The utility function can be structured mathematically as:

$U_{n i}\left(A_{n i}, P_{n}\right)>U_{n j}\left(A_{n j}, P_{n}\right) \Rightarrow \forall i \neq j \quad i \succ j \quad i, j \in C_{n}$

where

$U_{n i}(\bullet), U_{n j}(\bullet) \quad$ is the utility function of alternative i and $\mathrm{j}$, respectively, as viewed by a decision maker $\mathrm{n}$,

$A_{n i}, A_{n j} \quad$ is a vector of attribute values for alternative $\mathrm{i}$ and $\mathrm{j}$, respectively, as viewed by decision maker $\mathrm{n}$ (e.g., total travel time, total travel cost, and other attributes for all alternatives considered),

$P_{n}$ is a vector of characteristics of the decision maker $n$, 
which is considered to affect the choice between alternatives (e.g., household income and size, the number of vehicles owned, the number of licensed drivers, age, education),

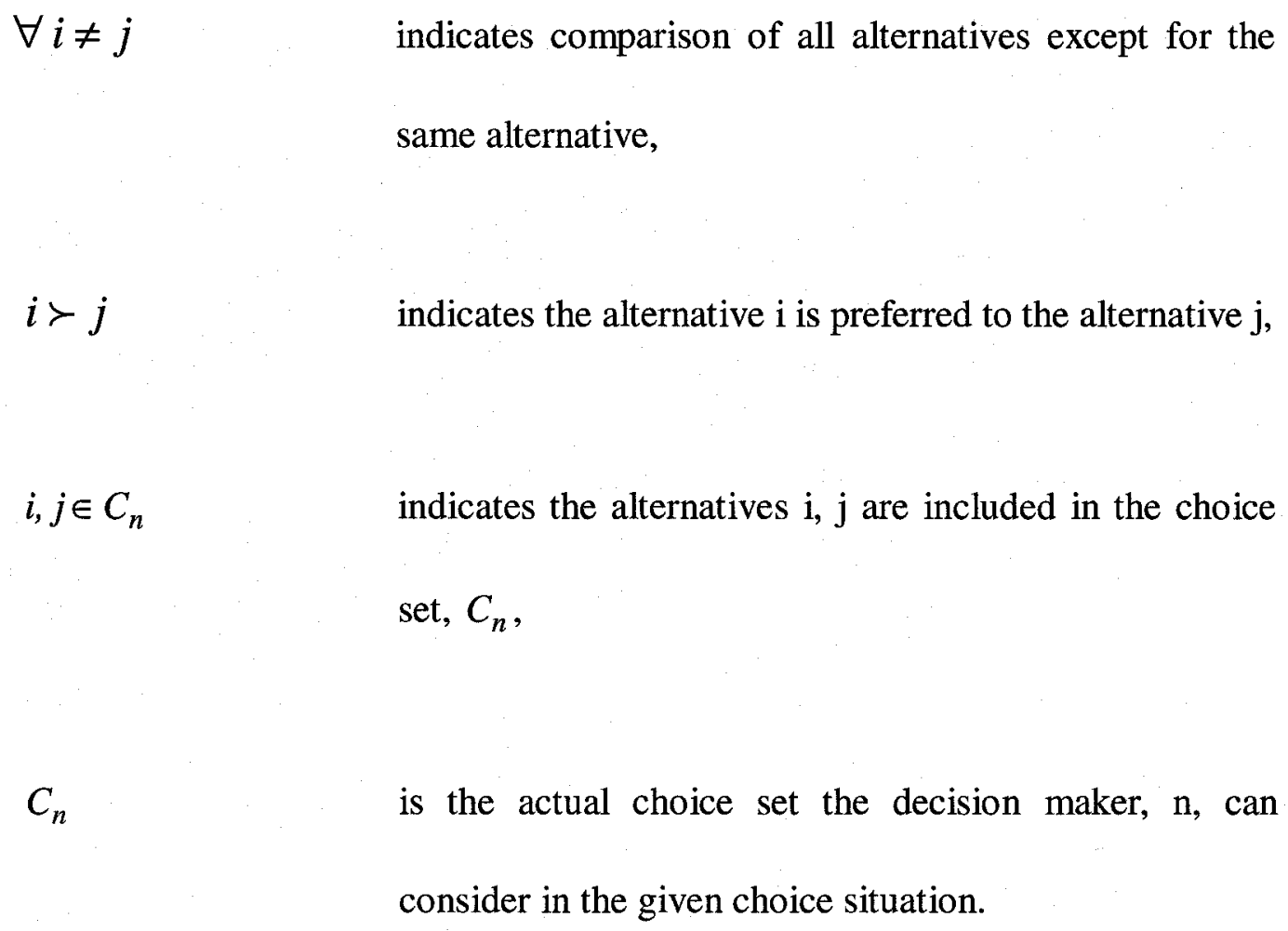

This expression means that, if the utility of alternative $i$ is greater than that of alternative $j$, alternative $i$ is preferred and therefore chosen by the decision maker within the given choice set. More importantly, a researcher should notice that there is no absolute reference value for the utilities of all alternatives. The utility has therefore a meaning when being compared between pairs of alternatives. By comparing the utility, a researcher can find the preference orderings. 
The foregoing utility function can be described with the attributes of alternatives and characteristics of decision makers and can show clearly the preference orderings between pairs of alternatives. However, this type of model, called deterministic utility model, could make a wrong prediction for the choice behaviour of decision makers.

In the real choice situations, the decision makers could choose an alternative "A" between two alternatives " $\mathrm{A}$ " and " $\mathrm{B}$ " even though the utility of a B alternative evaluated from the deterministic utility function is greater than an A alternative. That is, there exists a difference between the observed choice and the predicted choice. To accommodate the unexplained variations of the choice behaviour in a reasonable way and to account for the lack of information for a researcher in structuring utility functions, a more appropriate utility function structure is needed and thus introduced, which is called random utility or probabilistic choice models. This model can be presented as:

$$
U_{n i}\left(A_{n i}, P_{n}\right)=S_{n i}\left(A_{n i}, P_{n}\right)+R_{n i}\left(A_{n i}, P_{n}\right)
$$

where

$U_{n i} \quad$ is the representative utility of the alternative $\mathrm{i}$ of the decision maker $\mathrm{n}$,

$S_{n i} \quad$ is the systematic (or deterministic, observable, constant) part of the representative utility and is observable to a researcher,

$R_{n i} \quad$ is the random (unobservable, error) part of the representative utility and 
is unobservable to a researcher.

As shown in equation (2.2), the only difference from equation (2.1) is that the last term called random utility is assumed, and is added. $R_{n i}$ is included to accommodate the total error which is generated from a variety of sources, for example, imperfect information, measurement errors, omission of alternative attributes, omission of the characteristics of the decision maker (Koppelman and Bhat, 2006). A researcher cannot know about $R_{n i}$ and thus it is considered as a random variable. By applying the different distribution assumptions to this random variable, $R_{n i}$, the equation (2.2) can be specified by the different alternate discrete choice models. Assuming the random variable, $R_{n i}$, to have an extreme value distribution, a researcher can obtain the Multinomial Logit model (MNL) specification from the equation (2.2) after some algebraic manipulations. The mathematical processes for obtaining the final Multinomial Logit (MNL) probabilistic choice model are presented in the following section.

\subsection{Multinomial Logit (MNL) model}

The multinomial logit model was developed to predict the choice probability of decision makers from the choice set, $C_{n}$, that can consist of more than two alternatives. This model has been widely used across disciplines to predict a choice probability due to its simple probabilistic choice function, an easiness to interpret an estimation result, and clearness in the algebraic manipulations related to the derivation of the final probabilistic choice function. Derivation of multinomial logit (MNL) model will be presented in the next section. 


\subsubsection{Derivation of multinomial logit}

Being able to know the procedures of deriving the multinomial logit choice function is essential for a researcher to understand the model's limitations and to know about its characteristics. Consequently, a researcher can differentiate multinomial logit (MNL) from the other similar kinds of discrete choice models. Following Train (2003) and BenAkiva and Lerman (1985), the algebraic manipulations are described in details. Depending on the random utility or probabilistic choice theory defined in the earlier section, the probability that any alternative in $C_{n}$ is chosen by decision makers can be formulated by

$$
\operatorname{Prob}_{n}(i)=\operatorname{Pr}\left(U_{n i}>U_{n j}, \forall j \in C_{n}, i \neq j\right)
$$

As explained in the previous section, the utility of each alternative can be divided into systematic and random part:

$$
\begin{aligned}
\operatorname{Prob}_{n}(i) & =\operatorname{Pr}\left(U_{n i}>U_{n j}, \forall j \in C_{n}, i \neq j\right) \\
& =\operatorname{Pr}\left(S_{n i}+R_{n i}>S_{n j}+R_{n j}, \forall j \in C_{n}, i \neq j\right) \\
& =\operatorname{Pr}\left(R_{n j}<R_{n i}+S_{n i}-S_{n j}, \forall j \in C_{n}, i \neq j\right) .
\end{aligned}
$$

The different kinds of discrete choice models can be derived from equation (2.4) by adopting a specific assumptions on the distribution of the random parts, $R_{n i}$ and $R_{n j}$. To derive a multinomial logit (MNL), $R_{n i}$ and $R_{n j}$ are considered to be independently, identically distributed extreme value. The distribution is also called Gumbel and type I 
extreme value and Weibull. That is, the random part of the utility is assumed to be distributed following the extreme value distribution and therefore the density for each random part, $R_{n i}$ and $R_{n j}$, is

$f\left(R_{n a}\right)=e^{-R_{n a}} e^{-e^{-R_{n a}}}$,

and the cumulative distribution is

$F\left(R_{n a}\right)=e^{-e^{-R_{n a}}}$

In the equation (2.4), if $R_{n i}$ is considered given, the probability, $\operatorname{Prob}_{n}(i)$, is the cumulative distribution for $R_{n j}$ obtained at $R_{n i}+S_{n i}-S_{n j}$ and the value is, according to the equation (2.6),

$F\left(R_{n j}\right)=\exp \left(-\exp \left(-\left(R_{n i}+S_{n i}-S_{n j}\right)\right)\right)$

The $R_{n j}$ is distributed independently for all alternatives, $j \neq i$, the equation (2.4) means that the probability, $\operatorname{Prob}_{n}(i)$, is the product of the individual cumulative distributions, the equation (2.7), it can be written as given below:

$\operatorname{Prob}_{n}(i) \mid R_{n i}=\prod_{j \neq i} \exp \left(-\exp \left(-\left(R_{n i}+S_{n i}-S_{n j}\right)\right)\right)$ 
Since $R_{n i}$ is not given, to get $\operatorname{Prob}_{n}(i), \operatorname{Prob}_{n}(i) \mid R_{n i}$ should be integrated over all values of $R_{n i}$ weighted by its density, the equation (2.5):

$\operatorname{Prob}_{n}(i)=\int\left(\prod_{j \neq i} \exp \left(-\exp \left(-\left(R_{n i}+S_{n i}-S_{n j}\right)\right)\right)\right)\left(e^{-R_{n i}} e^{-e^{-R_{n i}}}\right) d R_{n i}$

To reach the final form of logit choice function, some algebraic manipulations must be made. The details are given below:

$$
\begin{aligned}
\operatorname{Prob}_{n}(i) & =\int_{R_{n i=-\infty}}^{\infty}\left(\prod_{j \neq i} e^{-e^{-\left(R_{n i}+S_{n i}-S_{n j}\right)}}\right) e^{-R_{n i}} e^{-e^{-R_{n i}}} d R_{n i} \\
& =\int_{R_{n i}=-\infty}^{\infty}\left(\prod_{j \neq i} e^{-e^{-\left(R_{n i}+S_{n i}-S_{n j}\right)}}\right) e^{-e^{-\left(R_{n i}+S_{n i}-S_{n i}\right)}} e^{-R_{n i}} d R_{n i} \\
& =\int_{R_{n i}=-\infty}^{\infty}\left(\prod_{j} e^{-e^{-\left(R_{n i}+S_{n i}-S_{n j}\right)}}\right) e^{-R_{n i}} d R_{n i} \\
& =\int_{R_{n i}=-\infty}^{\infty}\left(e^{-e^{-\left(R_{n i}+S_{n i}-S_{n 1}\right)}} e^{\left.-e^{-\left(R_{n i}+S_{n i}-S_{n 2}\right)} \cdots e^{-e^{-\left(R_{n i}+S_{n i}-S_{n i}\right)}}\right) e^{-R_{n i}} d R_{n i}}\right. \\
& =\int_{R_{n i}=-\infty}^{\infty}\left(\exp \left(-e^{-\left(R_{n i}+S_{n i}-S_{n 1}\right)}-e^{-\left(R_{n i}+S_{n i}-S_{n 2}\right)} \cdots-e^{-\left(R_{n i}+S_{n i}-S_{n i}\right)}\right) e^{-R_{n i}} d R_{n i} .\right.
\end{aligned}
$$

The equation (2.10) can be more concise as below:

$$
\begin{aligned}
\operatorname{Prob}_{n}(i) & =\int_{R_{n i}=-\infty}^{\infty}\left(\exp \left(-\sum_{j} e^{-\left(R_{n i}+S_{n i}-S_{n j}\right)}\right) e^{-R_{n i}} d R_{n i}\right. \\
& =\int_{R_{n i}=-\infty}^{\infty}\left(\exp \left(-e^{-R_{n i}} \sum_{j} e^{-\left(S_{n i}-S_{n j}\right)}\right) e^{-R_{n i}} d R_{n i} .\right.
\end{aligned}
$$


Let $e^{-R_{n i}}=Z$ and after differentiating, it becomes $-e^{-R_{n i}} d R_{n i}=d Z$. Note that if $R_{n i} \rightarrow-\infty$ then $Z \rightarrow \infty$ and if $R_{n i} \rightarrow \infty$ then $Z \rightarrow 0$. Using this new term, equation (2.11) can be written as below:

$$
\begin{aligned}
\operatorname{Prob}_{n}(i) & =\int_{\infty}^{0}\left(\exp \left(-Z \sum_{j} e^{-\left(S_{n i}-S_{n j}\right)}\right)(-d Z)=\int_{0}^{\infty}\left(\exp \left(-Z \sum_{j} e^{-\left(S_{n i}-S_{n j}\right)}\right) d Z\right.\right. \\
& =\left[\frac{\exp \left(-Z \sum_{j} e^{-\left(S_{n i}-S_{n j}\right)}\right)}{-\sum_{j} e^{-\left(S_{n i}-S_{n j}\right)}}\right]_{0}^{\infty}=\frac{1}{-\sum_{j} e^{-\left(S_{n i}-S_{n j}\right)}}[\exp (-\infty)-1] \\
& =\frac{1}{\sum_{j} e^{-\left(S_{n i}-S_{n j}\right)}}=\frac{e^{S_{n i}}}{\sum_{j} e^{S_{n j}}},
\end{aligned}
$$

Consequently the final logit function is obtained. The final form of the multinomial logit probabilistic model is composed of two parts, a denominator which is the sum of the systematic utility of all the alternatives in the choice set and a numerator which is the systematic utility of the alternative chosen by decision makers.

\subsubsection{Linear-in-parameters part of utility functions}

The functional form of $S_{n i}$, the systematic part of the utility function, can be changed. It can be linear-in-parameters or non-linear-in-parameters according to the judgment of a researcher who is interested in choosing a better specification of $S_{n i}$ to describe the given data more correctly. However, choosing non-linear-in-parameters specification might lead to a problem: a researcher cannot use the commercial statistical package and thus he should write his own code for his modelling work on hand. For this reason it is 
computationally generally preferred to limit $S_{n i}$ to the functional form of linear-inparameters (Ben-Akiva and Lerman, 1985). In this thesis, linear-in-parameters are assumed as a functional form of the utility of all the alternatives. Following this limitation on parameters, the final logit model can be written as below:

$$
\operatorname{Prob}_{n}(i)=\frac{e^{\beta^{\prime} x_{n i}}}{\sum_{j} e^{\beta^{\prime} x_{n j}}}
$$

where $S_{n i}=\beta^{\prime} x_{n i}$ and $x_{n i}$ is a vector describing the attributes of alternative $i$.

\subsubsection{Drawbacks of the multinomial logit model (MNL)}

Substitution patterns of the logit model have been recognized as drawbacks when the predictions (e.g., the ratio of choice probabilities for any two alternatives, the crosselasticity among alternatives) from the model are not consistent with the intuitive expectation of the researchers. The issue can be introduced by explaining two restrictions imposed by the logit model inherently. The first restriction is on the proportional substitution patterns of the cross-elasticity of probabilities. The second one is on the ratios of choice probabilities for any two alternatives in the given choice set (Train, 2003). The following sections cover these issues.

\subsubsection{Proportional substitution patterns}

Changing one attribute of one of the alternatives in choice set causes a change in choice probabilities throughout all alternatives. Since the sum of choice probabilities over 
alternatives is one, therefore increasing or decreasing probability of one alternative necessarily causes a decreasing or increasing of probabilities for other alternatives. To explain this result the mathematical expression of the cross-elasticity should be derived. The formula derived here is the elasticity of $\operatorname{Prob}_{n}(i)$ with respect to a variable that is included in the utility of alternative $j$. The derivation is as follows:

$$
\begin{aligned}
& \text { Cross - Elastity }{ }_{i x_{n j}}=\frac{\partial \operatorname{Prob}_{n}(i) / \operatorname{Prob}_{n}(i)}{\partial x_{n j} / x_{n j}}=\frac{\partial \operatorname{Prob}_{n}(i)}{\partial x_{n j}} \frac{x_{n j}}{\operatorname{Prob}_{n}(i)} \\
& =\frac{\partial}{\partial x_{n j}}\left(\frac{e^{\beta^{\prime} x_{n i}}}{\sum_{j} e^{\beta^{\prime} x_{n j}}}\right)\left(\frac{x_{n j}}{\operatorname{Prob}_{n}(i)}\right) \\
& =\frac{0-e^{\beta^{\prime} x_{n i}}\left(e^{\beta^{\prime} x_{n j}}\right)\left(\beta_{x}\right)}{\left(\sum_{j} e^{\beta^{\prime} x_{n j}}\right)^{2}}\left(\frac{x_{n j}}{\operatorname{Prob}_{n}(i)}\right) \\
& =-\beta_{x}\left(\operatorname{Prob}_{n}(i) \times \operatorname{Prob}_{n}(j)\right)\left(\frac{x_{n j}}{\operatorname{Prob}_{n}(i)}\right) \\
& =-\beta_{x} x_{n j} \operatorname{Prob}_{n}(j) \text {, }
\end{aligned}
$$

where $x_{n j}$ is the attribute of alternative $j$ when faced by decision maker $n$ and $\beta_{x}$ is its estimated parameter. As shown in the equation (2.14) the cross-elasticity is the same for all $i$ : an improvement in the attribute of an alternative $j$ reduces the probabilities for all other alternatives by the same percentage (Train 2003). This phenomenon is called "proportionate shifting." As an example of cross-elasticity of the logit model, consider the result presented in table 2-1, this shows the problem of the cross-elasticity predicted by the logit model. By subsidizing the small electric cars, its share increases from 0.01 to 0.1 in which the absolute increase is gained from both large gas cars' decrease by 0.06 and small gas cars' decrease by 0.03 . However, these results that come from logit cross- 
elasticity analysis are counterintuitive because generally people would expect the crosselasticity of small gas cars with respect to the improvement of small electric cars to be larger than that of large gas cars (Train 2003). In conclusion, adopting a logit model for a policy analysis might lead to a wrong prediction (e.g., overestimating for large gas cars, underestimating for small gas cars, in this specific example) and thus researchers need more flexible models to depict the reality more closely in the choice model.

Table 2-1: An example of cross-elasticity of the logit model in response to subsidy (Train, 2003) Alternatives Large gas cars Small gas cars Small electric cars

\begin{tabular}{cccc}
\hline Before subsidy & 0.66 & 0.33 & 0.01 \\
\hline After subsidy & & & 0.10 \\
(Estimated by logit cross-elasticity) & $0.60(-10 \%)$ & $0.30(-10 \%)$ & \\
\hline Mode share change & $0.06(-)$ & $0.03(-)$ & $0.09(+)$ \\
\hline
\end{tabular}

\subsubsection{Independence of irrelevant alternatives (IIA)}

Only for reason of analytical convenience, several restrictive assumptions are imposed in the process of the derivation of the multinomial logit model. The first restrictive assumption is that the random parts (disturbances) of the utilities of the all alternatives in the choice set are identically and independently distributed. "Identically" means the variances of the random parts of the utilities of the all alternatives in the choice set are equal (i.e., homoscedasticity). "Independently" means the variances of the random parts of the utilities of the all alternatives in the choice set have no correlation. The assumptions can be called identically and independently distributed (IID) assumption on the random parts. The second restrictive assumption is of the independence of irrelevant 
alternatives (IIA) which is the most widely discussed property of the multinomial logit model. The IIA property is that for a specific decision maker the ratio of the choice probabilities of any two alternatives in the choice set can be calculated with the systematic utilities of these two alternatives, and are irrelevant to any other alternatives' systematic utilities (Ben-Akiva and Lerman, 1985). The IIA is to hold in the multinomial logit model and it can be shown easily as follows:

$$
\begin{aligned}
\frac{\operatorname{Prob}_{n}(i)}{\operatorname{Prob}_{n}(k)} & =\frac{e^{S_{n i}} / \sum_{j} e^{S_{n j}}}{e^{S_{n k}} / \sum_{j} e^{S_{n j}}}=\frac{e^{S_{n i}}}{e^{S_{n k}}} \\
& =e^{S_{n i}-S_{n k}}=e^{\beta^{\prime} x_{n i}-\beta^{\prime} x_{n k}} .
\end{aligned}
$$

The IIA property originates from the assumption on the independent distribution of the random parts and the IIA assumption therefore strongly relates with the IID assumption applied in the process of the derivation of the MNL model. The MNL model might provide counterintuitive forecasts for a certain choice problem, which can be attributed to the IIA property. Consequently, the IIA property has been an assumption that is difficult to defend. The blue bus/red bus paradox can be cited as a good example to understand that this property is not always true.

A few drawbacks of the multinomial logit mentioned previously give a researcher a strong impetus to develop an advanced discrete choice model. The next section introduces briefly the other discrete choice models developed to overcome the drawbacks of the (multinomial) logit model. 


\subsection{Other discrete choice models}

The multinomial logit is the most widely used discrete choice model to date. Other discrete choice models also have been developed and applied to real choice situation. The new models other than the multinomial logit might be classified into two classes: (1) The first class is logit-extention models, the models that share the basic concept and structure with the logit model. (2) The second class is a non-logit-based model; actually the probit model is the only one used to date (Ben-Akiva and Lerman, 1985).

\subsubsection{The Generalized Extreme Value (GEV) Model}

Multinomial logit has a IIA property and it is coming from an assumption that the random parts of the utilities are independent. Of course, this assumption might be true if a researcher can make a well-specified systematic utility function that contains all variables affecting a choice (Train, 2003). Consequently the correlations among alternatives are free in this well-specified model. However, often the random parts are not independent but correlated. That is, IIA property does not hold in some cases. In these cases more flexible models are needed to reflect the fact that the random parts are correlated. A model developed to reflect this is the generalized extreme value (GEV) model, which assumes random parts for alternatives to be jointly distributed as a generalized extreme value (Train, 2003). It includes a number of discrete choice model as its sub-model and they can be derived from the GEV model according to the changes of assumption applied to the random parts of the utilities. For example, the GEV model collapses to standard logit when all correlations between alternatives are assumed to be zero (Train, 2003). Another widely used GEV model is the Nested logit model. The heteroskedastic extreme 
value model is also a type of GEV model. These models will be introduced in detail in the subsequent section.

\subsubsection{Heteroskedastic extreme value model}

As a GEV model type, Bhat (1995) proposed the heteroskedastic extreme value (HEV) model. He simply applied the hypothesis that the variance of the random parts of utilities is different for all alternatives and then showed that this specific model is more flexible in calculating the substitution pattern than other two models, the standard logit and the nested logit. Another possible model specification was presented by relaxing the limitation imposed on the random parts of the standard logit model (i.e., assumed IID: independently and identically distributed in random part). That is, by considering variations of the random parts among alternatives (i.e., to assume non-identically distributed) instead of considering the correlations of the random parts among alternatives (i.e., to assume non-independently distributed).

\subsubsection{Nested logit}

As a member of the GEV family, the nested logit model is also derived from the GEV model. It is another popular discrete choice model used to relax the drawbacks of the logit model. As implied in model's name, the alternatives are grouped by the unit, named nests. In the same nest, for any two alternatives the ratios of choice probabilities are independent of the attribute and are also independent of all other alternatives. That is, IIA property can hold only in the same nest. On the contrary, for the any two alternatives included in different nests, IIA does not hold and the ratio of the choice probabilities for 
those two alternatives can be changed when there is a change in attributes of any alternatives or when other alternatives are added or eliminated for each nest. Consequently, by allowing the proportional substitution pattern only in the same nest, one of drawbacks of the logit model can be relaxed in the nested logit model. As an example of using nested logit model in discrete choice studies, the research of the Bhat (1997) is a useful indicator for another application of the nested logit to different choice studies. He proposed COVNL model to allow heterogeneity of covariance across the decision makers for the nested alternatives with the information on observed characteristics of decision makers. In general, in standard nested logit mentioned in the earlier part of this section, the variation in the covariance among nested alternatives across the decision makers is ignored. As a result, for all the decision makers same proportional substitution is calculated and applied for each nest. Three travel modes are classified into one nest including car and train grouped as ground travel mode and one mode, the air travel mode. Two sets of parameters are estimated for both the parameters of systematic utility and the parameters representing covariance heterogeneity. By using the latter parameters he shows that there are significant differences in the correlation between car and train utilities among individuals. Three types of discrete choice models, multinomial logit, standard logit model, and COVNL are estimated. With the results he explains the possibility of conducting different policy actions that are induced based on the interpretation of the parameters of the different model.

\subsubsection{Mixed logit model}

As a model proposed most recently, it is frequently used in discrete choice analysis in 
these days because it is more convenient than probit model from the computation perspective and relaxing the limitations of the previous discrete choice models. The basic idea of this model is that all decision makers might have their own unique taste for the alternatives. The way to reflect this assumption in model specification is to assume different parameters for each decision makers in the population by adopting a density function for the parameters of the systematic utility function specified following the logit model structure. Through estimation procedures a researcher must evaluate two sets of parameters. Firstly, the parameters, $\beta$, which enter the logit choice model, should be estimated. Secondly, the parameters that describe the density function of $\beta$ should be evaluated, such as mean and covariance (Train 2003). This model also can overcome the limitations of the logit model or can show better description of choice behaviour than any other discrete choice model by using diverse density function other than normal distribution to reflect behaviour characteristics of the decision makers on given choice situations. All decisions regarding the model specification are made according to a researcher's understanding of a variety of behavioural specifications of decision makers.

Bhat (1998a) proposed a mixed logit type model that is designed to overcome a drawback of the nested logit (NL) by allowing shared random parts across the choice dimension. The drawback of the NL model is that this model restricts the sharing of random parts of utilities to only within alternatives in the same level of choice dimension, not across choice dimensions. To relax this unrealistic restriction as he asserted, he formulated the mixed logit model and estimated the model's parameters using simulation techniques. These have widely been adopted for the log-likelihood function that involves multi- 
dimensional integrals and hence that could not be evaluated analytically. By presenting empirical results of the proposed model, he asserted that the new proposed model is more flexible and thus is more realistic in presenting substitution pattern than conventional models such as MNL and NL models.

Bhat (1998b) studied another form of mixed logit model. In this research, he developed a mixed logit model form called random-coefficients logit, RCM model. The key idea involved in the model formulation is that a decision maker's responsiveness in choice situation will vary across decision makers and that this variation can be attributed to observed (to a researcher) and unobserved (to a researcher) characteristics of each decision maker. That is, all decision makers included in the population can have their own unique choice models. That is, parameters in choice model will differ from one decision maker to another. With this newly formulated model, he compared the results with those of other models (MNL, FCL) in terms of a sensitivity to level-of service variables, elasticity (self or cross) and showed its superiority in statistical terms, a possibility of changing of policy action based on the result estimated from the new model.

Bhat (2000) and Bhat and Huimin (2002) using a mixed logit model formulated a new model to accommodate geographical factors in modelling work trip mode choice. By including zonal-level (i.e., work-zone and home zone of the decision maker) attributes affecting the utility of mode, he presented more sensitive choice model and asserted the importance of including spatial factor in the discrete choice modelling. To estimate the model, he used a combination of Gaussian quadrature and quasi-Monte Carlo simulation 
techniques and maximum likelihood.

Revelt and Train (1997) used mixed logit specification for estimating parameters to predict the behaviour of household's choices of appliance efficiency level. A unique aspect of this research is that it used the data gathered in the way of repeated choices by the same decision maker. By estimating with this type of data, they showed the mixed logit's potential in diverse applications as well as the flexibility of representing substitution pattern.

Brownstone and Train (1999) and Brownstone et al. (2000) developed an automobile demand model using mixed logit specification for alternative-fuel vehicle in order to forecast the shift due to the introduction of a new product such as electric car. Brownstone et al. (2000) estimated three types of models differentiated by the data type used in their estimation. Revealed preference (RP), stated preference (SP) and joint RP/SP data were used respectively to estimate mixed logit model. All models estimated in this research were compared with other types of discrete choice models (e.g., MNL, probit) in order to demonstrate the superiority of mixed logit model.

\subsubsection{Probit model}

As mentioned in an earlier section of this thesis, the logit model uses extreme value distribution for all random parts of the utilities. The probit model differs from the logit model in terms of specification and it is the use of normal distributions for all random parts of the utilities. However, there is a problem with this model specification. In this 
case, there is no closed-form solution of choice probabilities like that of the logit model (i.e., equation (2.13)) and thus choice probabilities must be evaluated numerically using a simulation method (Train, 2003). In spite of a much more complex method for evaluation of choice probabilities, the proposed limitations in the logit model can be relaxed in the probit model. As compared to other choice models, the probit model has not frequently been used in practice due to its computational difficulties resulting from calculation of its choice probability.

Liu and Mahmassani (2000) suggest a new systematic way of guessing starting points by using a genetic algorithms and non-linear programming techniques in the context of finding a multinomial probit model's parameters. They argue that the new way can provide global maximum likelihood estimation without a failure in local maximum likelihood estimation.

A research study of Garrido and Mahmassani (2000) also used a multinomial probit model to forecast freight transportation demand by incorporating an error structure correlated spatially and temporally, representing the assumption that freight transportation demand is a highly variable process over space and time. The model was estimated with Monte-Carlo simulation to evaluate multinomial probit (MNP) likelihoods.

\subsection{Estimation method and algorithms}

The issue of estimation method has been discussed in the literature and improvements have been suggested. Generally, the standard multinomial logit (MNL) and GEV family 
model can be estimated by commercial statistical packages due to their close-form probability choice model structure. However, when the model structure becomes more complicated due to the efforts of researchers to express the choice behaviour in more realistic ways, specifically in formulating the structure of the mixed logit model, we cannot use the standard packages directly. The main reason of this is that the mixed logit model structure has a non-closed probabilistic choice function and hence it should be estimated in a numerical way, not analytically in calculating approximate value of its integrand included in its probabilistic choice function.

Bhat (2001) pointed out two reasons of why researchers are moving towards models that are analytically intractable. The first reason is that researchers more and more want to formulate and estimate a model reflecting underlying choice mechanism of decision makers with the most possible close way, especially, without imposing any prior behavioural restriction on that choice mechanism. The second reason can be attributed to dramatic development of data processing technique, making it possible to implement numerical method in evaluating multi-dimensional integrals of probabilistic choice function that is intractable analytically. Based on the background mentioned above, he compared two numerical methods, namely pseudo-random Monte Carlo estimation method (PMC) and quasi-random Monte Carlo estimation method (QMC) in terms of performance measures using mixed logit formulation. He showed that QMC out-performs the PMC with the results obtained from experimental estimation.

Sandor and Train (2004) presented experimental works to illustrate methods for 
calculating the approximate value of choice function of choice model including high dimensional integrals. As a comparison measure they used a root-mean-squared-error (RMSE) to compare the parameter estimate resulting from the different drawing methods used in simulation techniques. Bhat (2001) obtained the same result. Quasi-random draw method out-performs the pseudo-random draw in terms of RMSE.

Ben-Akiva et al. (1997) introduced another aspect of the simulation method. According to them, the simulation method can cause a problem of increasing simulation variance. That is, simulation variance is an additional variation in parameter estimation due to the use of an approximate likelihood function calculated by a finite number of random draws. They indicated that a study on finding the optimal value of random draw satisfying two ideal conditions, minimizing variations and computation time, is critical in the application of simulation methods to practical estimations.

Another important emerging topic in discrete choice modelling is the estimation algorithms. In general, researchers have not concentrated on this topic in discrete choice modelling. However, as mentioned in Ben-Akiva et al. (2002), there is a need for a study to compare alternative estimation algorithm. By doing so, researchers can have a better understanding of the estimation mechanisms underlying various algorithms. Also, they can have a prior knowledge of relative performance before initiating work. This process can be effective in determining the best candidate in discrete choice modelling contexts. The more complicated the model structures become, the more necessary it is to know of relative performance of estimation algorithms. 


\section{Chapter 3 The Estimation Algorithms}

\subsection{Overview}

In discrete choice modelling, in order to find optimum values of parameter estimates of model, it is necessary to resort to a numerical maximization or minimization process of the function developed in the given choice situation. In this thesis, the function is the loglikelihood, composed of the probabilistic choice formulas of the multinomial logit model. The main purpose of a numerical maximization or minimization process used in econometric modelling is to estimate the parameters of the given model using sample observations selected from the target population. To achieve this goal, we can use a maximum likelihood estimator and can depend on a variety of estimation algorithms frequently used in discrete choice model estimation. In the context of this thesis, eight different estimation algorithms are combined with the maximum likelihood estimator for getting parameter estimates of the given test model. The subsequent sections are devoted to the explanations of the eight algorithms adopted in this thesis, mainly focusing on their different rules for obtaining a gradient vector and the Hessian matrix. Also, other important issues related to the estimation of econometric model are explored and explained. The last part of this chapter deals with two useful algorithms (i.e., DFP-GSM and BFGS-GSM), which are tried for the first time in this thesis by combining the golden-section-line search method (GSM) with the general estimation algorithms, DFP and BFGS, to find the optimum value of the step size $\left(\lambda_{t}\right)$ which, in the context of this research, minimizes the log-likelihood function in each iteration. Relative performance of each of eight different estimation algorithms explained in this chapter will be studied with the test model using real mode choice data and will be compared with each other in terms 
of performance measures by primarily focusing on their different operational characteristics. Chapter 4 and chapter 5 present the results.

\subsection{Maximum likelihood method}

Although in some cases where the maximum likelihood method is burdensome from the computational perspective, as it is conceptually clear and thus the maximum likelihood method has been the most popular method used in discrete choice modelling (Ben-Akiva and Lerman, 1985). This method can be used for the problem of econometrically inferring model's parameters with a sample of observations, which is, in the context of this thesis, assumed to be drawn randomly from the population, otherwise, more complex estimation method are required (Train, 2003). After estimating a model using the maximum likelihood method, we can get a set of parameter estimates. This set is, as a maximum likelihood estimator, the value of the parameters for which a sample of observations is most likely to have occurred (Ben-Akiva and Lerman, 1985). In this thesis research, the combined procedures of the maximum likelihood method and the eight different estimation algorithms are used to obtain the utility functions' parameters contained in the probabilistic multinomial logit model.

\subsection{Comparison of eight different estimation algorithms}

Estimating the parameters of discrete mode choice model means finding a vector of estimates of parameters in given model specification. This process includes the optimization of a certain mathematical function. In discrete mode choice model, the loglikelihood function of the observed sample is maximized with the help of optimization algorithms. As we will see in the following sections, according to the difference in 
obtaining a column vector of gradient ( $\mathrm{K}$ dimension) and Hessian matrix $(\mathrm{K} \times \mathrm{K}$ dimension), where dimension is the number of parameters being estimated, each algorithm has a role to play. The gradient of the given function, $G_{t}=\left(\frac{\partial L L(\beta)}{\partial \beta_{k}}\right)$, is the first derivative of the target function that is maximized with respect to $\beta_{k}$, in this research, the function is log-likelihood function. Numerical value of the gradient differs in each iteration, which is evaluated and updated. The Hessian matrix, $H_{t}=\left(\frac{\partial G_{t}}{\partial \beta_{k}}\right)=\left(\frac{\partial^{2} L L(\beta)}{\partial \beta_{k} \partial \beta_{k}}\right)$, is the second derivative of the function. The gradient indicates the direction of searching for the optimum value and the Hessian matrix says how much to increase at each step in iteration procedure (Train, 2003). Detailed explanation of each specific algorithm is presented in the following sections.

\subsubsection{Newton Raphson algorithm}

Newton Raphson is the algorithm that uses the real Hessian in its updating process during estimation processes. The calculations for getting real Hessian matrix are expensive and costly. Moreover, most parts of works associated with calculation of analytical derivatives of log-likelihood function are extensive, but it is very useful. In this thesis, we got the real Hessian by means of direct analytical derivatives. The benefits of using real Hessian are demonstrated in both chapters 4 and 5 in terms of several evaluation measures.

At this point, for making a code of Newton Raphson (NR), we should understand the fundamental rule of iteration method used in parameter estimation. The iteration method 
of four algorithms described in sections 3.3.1, 3.3.2 and 3.3.3, 3.3.4 are derived with a heavy dependence on Train (2003).

The updating procedure of Newton Raphson can be derived by taking the second order Taylor's approximation of $L L\left(\beta_{t+1}\right)$ around $L L\left(\beta_{t}\right)$ and the process is as follows. For general functions, the Taylor's series expansion of $f(x)$ around $f\left(x_{0}\right)$ can be written as below:

$f(x)=\frac{f\left(x_{o}\right)}{(0) !}+\frac{f^{(1)}\left(x_{o}\right)}{(1) !}\left(x-x_{o}\right)+\frac{f^{(2)}\left(x_{o}\right)}{(2) !}\left(x-x_{o}\right)^{2}+\cdot+\frac{f^{(n)}\left(x_{o}\right)}{(n) !}\left(x-x_{o}\right)^{n}$

By taking the second-order approximation of Taylor's series, the above function can be shortened as shown below:

$$
\begin{aligned}
L L\left(\beta_{t+1}\right) & \cong \frac{L L\left(\beta_{t}\right)}{0 !}+\frac{L L^{(1)}\left(\beta_{t}\right)}{1 !}\left(\beta_{t+1}-\beta_{t}\right)+\frac{L L^{(2)}\left(\beta_{t}\right)}{2 !}\left(\beta_{t+1}-\beta_{t}\right)^{2} \\
& =L L\left(\beta_{t}\right)+G_{t}\left(\beta_{t+1}-\beta_{t}\right)+\frac{1}{2} H_{t}\left(\beta_{t+1}-\beta_{t}\right)^{2},
\end{aligned}
$$

where

$f(x)$ is $L L\left(\beta_{t+1}\right)$,

$f\left(x_{0}\right)$ is $L L\left(\beta_{t}\right)$.

Finally, we can identify the best values of $\beta_{t+1}$ that maximizes the above approximate 
function by taking $\frac{\partial L L\left(\beta_{t+1}\right)}{\partial \beta_{t+1}}=0$. The first derivatives with respect to $\beta_{t+1}$ results in

$\frac{\partial L L\left(\beta_{t+1}\right)}{\partial \beta_{t+1}}=G_{t}+H_{t}\left(\beta_{t+1}-\beta_{t}\right)$

The iteration rule of Newton Raphson is represented by the following formula:

$\beta_{t+1}=\beta_{t}+\lambda\left(-H_{t R e a l}^{N R}\right)^{-1} G_{t}$

where

$\beta_{t} \quad$ is the parameter estimates after $t$ iterations,

$\beta_{t+1} \quad$ is the parameter estimates after $\mathrm{t}+1$ iterations,

$\lambda \quad$ is the step size (can be assumed by a researcher at the initial time of model parameter estimation),

$\left(-H_{\text {tReal }}^{N R}\right)^{-1}$ is the inverse of the negative of real Hessian matrix in iteration $\mathrm{t}$ in Newton Raphson method,

$G_{t} \quad$ is a vector of gradient in iteration $\mathrm{t}$,

$\left(-H_{t R e a l}^{N R}\right)^{-1} G_{t}$ is the direction to follow on the surface of the function in iteration process.

Stated more simply, $\beta_{t+1}, \mathrm{t}+1^{\text {th }}$ parameter estimates, is equal to $\beta_{t}, \mathrm{t}^{\text {th }}$ parameter estimates, plus negative inverse Hessian multiplied by gradient. In this research, both 
$H_{t R e a l}^{N R}$ and $G_{t}$ are the values of the average value of the Hessian and gradient divided by the number of observations. The Hessian and gradient should be calculated in every iteration and continuously updated to the end of iterations. More importantly, to get an understanding of iteration rule we should know the fact demonstrated by McFadden (1974) that the log-likelihood function of logit probability is always globally concave. With the knowledge of McFadden (1974), the following explanation can be understood easily. $G_{t}$ is the slope of the log-likelihood function and $H_{t R e a l}^{N R}$ shows the changing of slope of the log-likelihood function, which means a curvature of the curve. In cases where the function is globally concave, $H_{t R e a l}^{N R}$ is always negative and thus $\left(H_{t R e a l}^{N R}\right)^{-1}$ is also negative. The negative of this negative Hessian, $-\left(H_{t R e a l}^{N R}\right)^{-1}$ is positive. Therefore, the direction of $-\left(H_{t R e a l}^{N R}\right)^{-1} G_{t}$ depends entirely on the sign of $G_{t}$. If the $G_{t}$ is positive, $\beta_{t}$ moves to the right direction (see the left side of figure 3-1), otherwise, $\beta_{t}$ moves to the left direction (see the right side of figure 3-1) according to the iteration rule seen in the equation (3.4). Its step size is given by a researcher at the initial stage of iteration and its magnitude is given by the term, $\lambda\left(-H_{t \text { Real }}^{N R}\right)^{-1} G_{t}$ included in the iteration rule of Newton Raphson. In either case, $\beta_{t}$ is moved to the best value that maximizes the loglikelihood function. This concept is shown in figure 3-1.

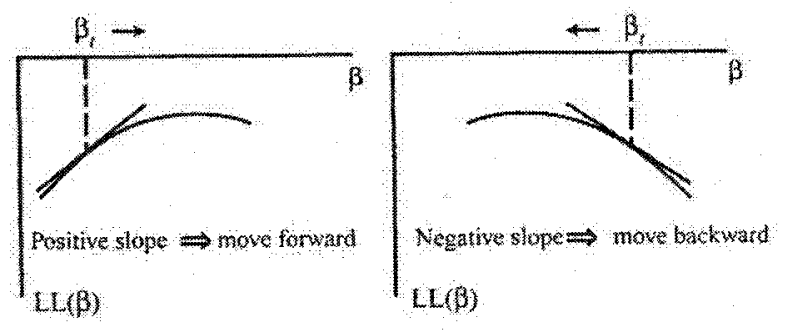

Figure 3-1: Direction of step follows the slope (Train, 2003) 
The step size implied by $\lambda\left(-H_{t \text { Real }}^{N R}\right)^{-1} G_{t}$ is decreased, if the curvature represented by $\left(-H_{\text {tReal }}^{N R}\right)^{-1}$ has a large value in its magnitude (see the left part of figure 3-2), otherwise, the step size is increased (see the right part of figure 3-2).

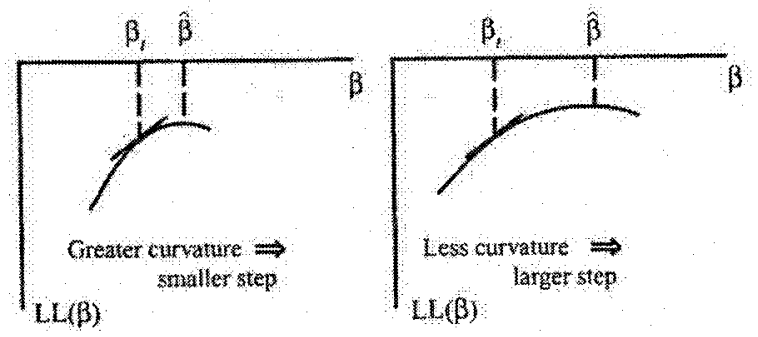

Figure 3-2: Step size is inversely related to curvature (Train, 2003)

\subsubsection{BHHH algorithm}

To avoid the difficulty of calculating the real Hessian, BHHH is suggested. The major difference between this algorithm and the Newton Raphson method is the way of obtaining Hessian matrix. The outer product of each observation's score, which is obtained from the first derivatives of the log-likelihood function for all observations, is calculated and then summed up, divided by the number of observations. This process can be described as below:

$$
H_{t n}^{B H H H}=\left(\frac{\partial L L(\beta)}{\partial \beta_{k}}\right)_{n}\left(\frac{\partial L L(\beta)}{\partial \beta_{k}}\right)_{n}^{\prime}=\left(G_{t n}\right)\left(G_{t n}\right)^{\prime}=\left(\begin{array}{ccccc}
G_{n}^{1} G_{n}^{1} & G_{n}^{1} G_{n}^{2} & G_{n}^{1} G_{n}^{3} & \cdots & G_{n}^{1} G_{n}^{k} \\
G_{n}^{2} G_{n}^{1} & G_{n}^{2} G_{n}^{2} & G_{n}^{2} G_{n}^{3} & \cdots & G_{n}^{2} G_{n}^{k} \\
G_{n}^{3} G_{n}^{1} & G_{n}^{3} G_{n}^{2} & G_{n}^{3} G_{n}^{3} & \cdots & G_{n}^{3} G_{n}^{k} \\
\vdots & \vdots & \vdots & & \vdots \\
G_{n}^{k} G_{n}^{1} & G_{n}^{k} G_{n}^{2} & G_{n}^{k} G_{n}^{3} & \cdots & G_{n}^{k} G_{n}^{k}
\end{array}\right)
$$


where

$H_{t n}^{B H H H} \quad$ is the outer product (approximate Hessian matrix) of numerical value of the first derivative of log-likelihood function for observation $\mathrm{n}$ in iteration $\mathrm{t}$,

$G_{t n} \quad$ is a vector of gradient of observation $\mathrm{n}$ in iteration $\mathrm{t}$.

Approximate Hessian of BHHH method, $H_{t A P}^{B H H H}$, is obtained by summing $H_{t n}^{B H H H}$ for all observations and dividing by the number of observations, $H_{t A P}^{B H H H}=1 / N\left(\sum_{n=1}^{N} H_{t n}^{B H H H}\right)$, and this approximate Hessian can be inserted into the iteration formula to get the next parameter estimates.

The iteration rule of $\mathrm{BHHH}$ can be written as

$\beta_{t+1}=\beta_{t}+\lambda\left(H_{t A P}^{B H H H}\right)^{-1} G_{t}$,

where

$H_{t A P}^{B H H H} \quad$ is the approximate Hessian matrix of $\mathrm{BHHH}$ algorithm, replacing the real Hessian matrix appeared as $-H_{t R e a l}^{N R}$ in Newton Raphson,

Stated simply, compared to the real Hessian used in NR, the only difference is $H_{t A P}^{B H H H}$, which replaces $-H_{t R e a l}^{N R}$ of NR. 


\subsubsection{BHHH-2 algorithm}

This method is almost similar to $\mathrm{BHHH}$, but it is slightly different in getting the approximate Hessian. For every observation gradient, the average gradient of the entire sample is deducted and then outer product of every observation is calculated for each observation. Dividing the sum of outer product of every observation by the observation number N, $H_{t A P}^{B H H H-2}$ is calculated. It is shown below:

$$
\begin{aligned}
& G_{t A V E}=\frac{1}{N}\left(\sum_{n=1}^{N} G_{t n}\right), \\
& H_{t A P}^{B H H H-2}=\frac{1}{N} \sum_{n=1}^{N}\left[\left(G_{t n}-G_{t A V E}\right)\left(G_{t n}-G_{t A V E}\right)^{\prime}\right],
\end{aligned}
$$

where

$H_{t A P}^{B H H H-2}$ is the approximate Hessian matrix of BHHH-2 method, replacing the real Hessian matrix appeared as $-H_{t \text { Real }}^{N R}$ in Newton Raphson,

$G_{t A V E} \quad$ is the average value of a vector of gradient for all observations in iteration $\mathrm{t}$.

The iteration formula of $\mathrm{BHHH}-2$ is given below

$$
\beta_{t+1}=\beta_{t}+\lambda\left(H_{t A P}^{B H H H-2}\right)^{-1} G_{t}
$$


Stated simply, compared to the real Hessian used in NR, the only difference is $H_{t A P}^{B H H H-2}$, which replaces $-H_{t \text { Real }}^{N R}$ of NR.

\subsubsection{Steepest Ascent algorithm}

Steepest ascent method presented here is the simplest to apply among the algorithms used in this research. Here simplicity is due to the use of identity matrix instead of calculating Hessian or approximate Hessian in iteration process. The rule of iteration is shown below

$\beta_{t+1}=\beta_{t}+\lambda I^{S A} G_{t}$

where

$I^{S A} \quad$ is the identity matrix used repeatedly throughout the iteration instead of the Hessian matrix.

As we will see in both chapters 4 and 5 , the performance of this method, which has a simple rule and easy to apply, is the worst of all algorithms on all measures considered in numerical experiment.

\subsubsection{Quasi-Newton method}

As the most frequently adopted in commercial statistical package, this method shows a better performance in running time and is considered to be robust in convergence properties compared to any other algorithms explained in the previous sections. More importantly, this method might be excellent in convergence properties even for problems 
that cannot be solved by other algorithms in a satisfactory manner (Greene, 2000). This unique excellence is the results of selecting different approaches to update the approximate Hessian from other methods. An important point of this method is to accept $\gamma_{t}=G_{t+1}-G_{t}$ in updating procedures, which is the difference between continuous gradient in iteration $t+1$ (i.e., $G_{t+1}$ ) and iteration $t$ (i.e., $G_{t}$ ). This means that $\gamma_{t}$ might contain more information on the shape of the function and it makes it easier to reach the optimal value that maximize the log-likelihood function (Train, 2003). There are slight differences between DFP and BFGS in updating approximate Hessian. The following sections present the explanation of updating procedures of approximate Hessian.

\subsubsection{Davidon-Fletcher-Powell (DFP) algorithm}

The two algorithms included in the two following sections depend on the description of Greene (2000). Updating rule of approximate Hessian of DFP is introduced below and it is called variable metric algorithm or rank two correction (Greene, 2000), written as

$$
H_{t+1(A P)}^{D F P}=H_{t}^{D F P}+\frac{\delta_{t} \delta_{t}^{\prime}}{\delta_{t}^{\prime} \gamma_{t}}-\frac{H_{t}^{D F P} \gamma_{t} \gamma_{t}^{\prime} H_{t}^{D F P}}{\gamma_{t}^{\prime} H_{t}^{D F P} \gamma_{t}},
$$

where

$$
\begin{aligned}
& H_{t+1(A P)}^{D F P} \text { is the approximate Hessian matrix of DFP algorithm in } t+1 \text { iteration, } \\
& \text { replacing the real Hessian matrix appeared as }\left(H_{t R e a l}^{N R}\right)^{-} \text {in Newton } \\
& \text { Raphson algorithm, }
\end{aligned}
$$


$H_{t}^{D F P} \quad$ is the approximate Hessian matrix in t iteration (if $t=I$ then $H_{t}^{D F P}=I$ ), in the context of this thesis $I$ is $9 \times 9$ dimension identity matrix,

$\delta_{t} \quad$ is equal to the value of $\lambda\left(-H_{t}^{D F P}\right) G_{t}$ in iteration $t$,

$\delta_{t}^{\prime} \quad$ is transposed matrix of $\delta_{t}$,

$\gamma_{t} \quad$ is equal to the value of $G_{t+1}-G_{t}$, the difference of value between a gradient vector in iteration $t=1, G_{t+1}$, and a gradient vector in iteration $t, G_{t}$,

$\gamma_{t}^{\prime} \quad$ is transposed matrix of $\gamma_{t}$

The iteration rule can be written as

$\beta_{t+1}=\beta_{t}+\lambda\left(-H_{t A P}^{D F P}\right) G_{t}$

The above formula looks almost the same as for other algorithms presented in previous section. The only unique feature is that as compared to NR, $H_{t A P}^{D F P}$ is used, which replaces $\left(H_{t R e a l}^{N R}\right)^{-I}$. After sufficient iterations before convergence, $H_{t A P}^{D F P}$ is assumed to approximate inverse of real Hessian of NR. In this first DFP method, we can try a variety of $\lambda$, a step size, to find the best $\lambda$ with which the iteration shows the best performance. In the following sections, we will incorporate new modules to our estimation code that find the best $\lambda_{t}$ at each iteration automatically by adopting golden-section line search method (GSM). 


\subsubsection{Broyden-Fletcher-Goldfarb-Shanno (BFGS) algorithm}

The BFGS algorithm is slightly different in terms of its updating rule of approximate Hessian compared to that of DFP. The updating formula of approximate Hessian is presented below and it shows almost the same formula as for that of DFP except the last term:

$$
H_{t+1(A P)}^{B F G S}=\left(H_{t}^{B F G S}+\frac{\delta_{t} \delta_{t}^{\prime}}{\delta_{t}^{\prime} \gamma_{t}}-\frac{H_{t}^{B F G S} \gamma_{t} \gamma_{t}^{\prime} H_{t}^{B F G S}}{\gamma_{t}^{\prime} H_{t}^{B F G S} \gamma_{t}}\right)-v d_{t} d_{t}^{\prime}
$$

where

$H_{t+1(A P)}^{B F G S} \quad$ is the approximate Hessian matrix of BFGS algorithm in $t+1$ iteration, replacing the real Hessian matrix appeared as $\left(H_{t R e a l}^{N R}\right)^{-1}$ in Newton Raphson algorithm,

$H_{t}^{B F G S}$ is the approximate Hessian matrix in $\mathrm{t}$ iteration (if $t=1$ then $\left.H_{t}^{B F G S}=I\right)$, in the context of this thesis $I$ is $9 \times 9$ dimension identity matrix

$d_{t} \quad$ is equal to the value of $\left(\frac{1}{\delta_{t}^{\prime} \gamma_{t}}\right) \delta_{t}-\left(\frac{1}{\gamma_{t}^{\prime} H_{t}^{B F G S} \gamma_{t}}\right) H_{t}^{B F G S} \gamma_{t}$,

$d_{t}^{\prime} \quad$ is transposed matrix of $d_{t}$

$v \quad$ is equal to the value of $\gamma_{t}^{\prime} H_{t}^{B F G S} \gamma_{t}$.

The additional term (i.e., $v d_{t} d_{t}^{\prime}$ ) can be calculated using the information of the first term 
already provided in parenthesis. As in the case of DFP method, $H_{t A P}^{B F G S}$ would be another approximate Hessian matrix after sufficient iteration. After inputting $H_{t A P}^{B F G S}$ into iteration formula, it has the form as below

$\beta_{t+1}=\beta_{t}+\lambda\left(-H_{t A P}^{B F G S}\right) G_{t}$

In this formula, like the iteration rule of DFP, we can employ several values to find an optimal $\lambda$ that shows the best estimation performance in terms of several measures such as one iteration time, the number of iterations, and convergence time before convergence. The following section will suggest the way of finding $\lambda$ automatically with the help of line search method. In this research, we adopted the golden-section method (GSM) as a line search tool. This method was successful in the estimation of parameter estimates.

\subsubsection{Incorporating golden section line search method (GSM) to both DFP and BFGS algorithms}

The problem of using estimation algorithm for the estimation of model's parameter is that the researchers have no preliminary knowledge of choosing step size $(\lambda)$ that guarantees the best performance of the estimation procedure. To address this problem, we put a new module of line search, which uses golden section method (GSM) between codes of both DFP and BFGS methods. Incorporating the golden-section method for line search was tried for the first time in this research. 


\subsubsection{The golden section method (GSM)}

As a line search method without using derivatives, the basic concept of golden section method is to reduce the interval of uncertainty during the search procedure to fix point for minimizing the given function. Let $L L(\beta)$ be the function to be minimized and $a \leq \beta \leq b$ is the interval of uncertainty. To find the point for minimizing the function we can exclude portions of the interval of uncertainty that do not contain the minima through the line search procedure. After a number of iterations for elimination, infinitesimally small portions are left. By averaging lower and upper bounds of the remaining portions, the minima for the given function is calculated.

The following summary of the golden section method (GSM) is of description for minimizing a strictly quasi-convex function over the interval $\left(a_{1}, b_{1}\right)$ (Bazaraa et al., 1999):

\section{- Initialization step}

We choose an final interval of uncertainty $l>0$, and designating $\left(a_{l}, b_{1}\right)$ as the initial interval of uncertainty, and let $\gamma_{1}=a_{1}+(1-\alpha)\left(b-a_{1}\right)$ and $\mu_{1}=a_{1}+\alpha\left(b_{1}-a_{1}\right)$, where $\alpha=0.618$ (i.e., reduction ratio; the interval of uncertainty is reduced by a factor of 0.618). Next we calculate $L L\left(\gamma_{1}\right)$ and $L L\left(\mu_{1}\right)$. We let $G=1$, and go to main routine.

- Main routines

Step 1. If $b_{G}-a_{G}<l$, stop. The optimal value is between $\left(a_{G}, b_{G}\right)$, Otherwise, if $L L\left(\gamma_{G}\right)>L L\left(\mu_{G}\right)$, go to step 2; and if $L L\left(\gamma_{G}\right)<L L\left(\mu_{G}\right)$, go to step 3 .

Step 2. Let $a_{G+1}=\mu_{G}$ and $b_{G+1}=b_{G}$. Furthermore, let $\gamma_{G+1}=\mu_{G}$, and let $\mu_{G+1}=a_{G+1}+\alpha\left(b_{G+1}-a_{G+1}\right)$. Calculate $L L\left(\mu_{G+1}\right)$ and go to step 4 .

Step 3. Let $a_{G+1}=a_{G}$ and $b_{G+1}=\gamma_{G}$. Furthermore, let $\mu_{G+1}=\gamma_{G}$, and let 


$$
\gamma_{G+1}=a_{G+1}+(1-\alpha)\left(b_{G+1}-a_{G+1}\right) \text {. Calculate } L L\left(\gamma_{G+1}\right) \text { and go to step } 4 \text {. }
$$

Step 4. $\quad G=G+1$ go to step 1

\subsubsection{Application to this research}

Table 3-1 shows the example computation of DFP-GSM. In this specific example, we adopt 3 as the interval of uncertainty, and use $b_{G}-a_{G}<0.01$ as stopping criterion. In table 3-1, the initial interval of uncertainty is of length 3, and after 13 iterations involving fourteen observations (see*), the interval of uncertainty is $[0.024402711,0.033719691]$, so that the minimum point can be calculated to be the midpoint of two values. This midpoint may be considered as an optimal step size $\lambda_{t}$ searched by the golden section method. Especially, in the context of this research, the log-likelihood function is globally concave (see section 3.2.1), and to apply the golden section method in a limited way to quasi-convex function, we transform the log-likelihood function symmetry to $\mathrm{x}$-axis by adding $(-)$ sign, $-L L(\beta)$.

Table 3-1: Example of computation of GSM in the case of DFP-GSM (0.3-27-26.29)

\begin{tabular}{ccccccc}
\hline Iteration & $a_{G}$ & $b_{G}$ & $\gamma_{G}$ & $\mu_{G}$ & $L L\left(\gamma_{G}\right)$ & $L L\left(\mu_{G}\right)$ \\
\hline 1 & 0 & 3 & 1.146 & 1.854 & $3493.476457^{*}$ & $5476.740529^{*}$ \\
\hline 2 & 0 & 1.854 & 0.708228 & 1.146 & $2297.447729^{*}$ & 3493.476457 \\
\hline 3 & 0 & 1.146 & 0.437772 & 0.708228 & $1595.81076^{*}$ & 2297.447729 \\
\hline 4 & 0 & 0.708228 & 0.270543096 & 0.437772 & $1206.102109^{*}$ & 1595.81076 \\
\hline 5 & 0 & 0.437772 & 0.167228904 & 0.270543096 & $1005.500045^{*}$ & 1206.102109 \\
\hline 6 & 0 & 0.270543096 & 0.103347463 & 0.167228904 & $910.8710041^{*}$ & 1005.500045 \\
\hline 7 & 0 & 0.167228904 & 0.063881441 & 0.103347463 & $871.3625899^{*}$ & 910.8710041 \\
\hline 8 & 0 & 0.103347463 & 0.039478731 & 0.063881441 & $858.3072898^{*}$ & 871.3625899 \\
\hline 9 & 0 & 0.063881441 & 0.024402711 & 0.039478731 & $856.576918^{*}$ & 858.3072898 \\
\hline 10 & 0 & 0.039478731 & 0.015080875 & 0.024402711 & $858.7347619^{*}$ & 856.576918 \\
\hline 11 & 0.015080875 & 0.039478731 & 0.024402711 & 0.03015875 & 856.576918 & $856.5491249^{*}$ \\
\hline 12 & 0.024402711 & 0.039478731 & 0.03015875 & 0.033719691 & 856.5491249 & $856.9727219^{*}$ \\
\hline 13 & 0.024402711 & 0.033719691 & 0.027961797 & 0.03015875 & $856.4520154^{*}$ & 856.5491249 \\
\hline
\end{tabular}

* Values of function evaluated newly in each iteration 


\subsubsection{DFP-GSM algorithm}

Iteration formula is nearly the same as for the DFP method, other than adopting $\lambda_{t}$ instead of $\lambda$, which is continuously having different values in every iteration. Line search has an important role of finding optimum value (i.e., $\lambda_{t}$ ) within the interval of uncertainty. In every iteration, $\lambda_{t}$ which minimizes the given log- likelihood function is founded, and used in the iteration formula shown below:

$\beta_{t+I}=\beta_{t}+\lambda_{t}\left(-H_{t A P}^{D F P}\right) G_{t}$

\subsubsection{BFGS-GSM algorithm}

In the case of BFGS-GSM, all operations are the same as applied to DFP-GSM. The approximate Hessian, $H_{t A P}^{B F G S}$, can be obtained in the same way as for the BFGS method. The iteration rule is as presented below:

$\beta_{t+1}=\beta_{t}+\lambda_{t}\left(-H_{t A P}^{B F G S}\right) G_{t}$

All the details of codes for these procedures are present in appendix B.

\subsection{Iteration method}

In most estimation models, finding parameter estimates generally depends on iteration method in which the same estimation procedures are repeated until a value of the mathematical expression given as the stopping criterion is reached as defined by the 
researcher. In the context of this thesis, we use two types of stopping criteria. More details on convergence criteria are presented in section 3.6.

\subsection{Step size}

For understanding "step size" in the context of iteration method, it is useful to know how $L L(\beta)$, which is a quadratic function in $\beta$ can be maximized and how it converges to maximum. In the case of quadratic function, the optimum value can be obtained by only one iteration (or one step). The demonstration is simple and it can be given as below (Train, 2003). The quadratic $L L(\beta)$ can be written as

$L L(\beta)=A+B \beta+C \beta^{2}$

The $\beta$ that maximizes $L L(\beta)$ is

$$
\frac{\partial L L(\beta)}{\partial \beta}=B+2 C \beta=0,
$$

thus, $\beta=-\frac{B}{2 C}$.

The $\beta$ obtained is exactly the same as the value obtained from Newton Raphson. It can be verified clearly as presented below. The gradient and Hessian are $G_{t}=B+2 C \beta$ and $H_{t}=2 C$, the Newton Raphson provides the results as follows: 


$$
\begin{aligned}
\beta_{t+1} & =\beta_{t}+\left(-H_{t}^{-1}\right) G_{t} \\
& =\beta_{t}+\left(-\frac{1}{2 c}\right)\left(B+2 C \beta_{t}\right) \\
& =\beta_{t}-\frac{B}{2 c}-\beta_{t}=-\frac{B}{2 C}
\end{aligned}
$$

In conclusion, two values obtained from different ways are the same for the case where $L L(\beta)$ is quadratic. However, the problem is that most $L L(\beta)$ are not quadratic and they need more than one step (iteration) to reach the optimum point. Based on the lesson learned form quadratic function, we can identify the role of step size to minimize the cost. That is, by choosing a good starting step size before entering the whole process, the iteration processes can be optimized in terms of iteration number and convergence time. A following section covers the topic.

- The first case

In figure 3-3, two different functions are drawn by two line types. The solid line is for actual $L L(\beta)$ and the dashed line is for the quadratic form. In this case, after one iteration $(\lambda=1)$, if the given function is quadratic, $\beta_{t+1}$ reaches maximum point. But, the actual $L L(\beta)$ is not quadratic, thus $\beta_{t+1}$ goes beyond the maximum point and as a result, $L L\left(\beta_{t+1}\right)\left\langle L L\left(\beta_{t}\right)\right.$. To make sure that $\left.L L\left(\beta_{t+1}\right)\right\rangle L L\left(\beta_{t}\right)$ and to guarantee an increasing in $L L(\beta)$, the researcher decreases $\lambda$ to $1 / 2$. After this operation, we should again compare the value between $L L\left(\beta_{t+1}\right)$ and $L L\left(\beta_{t}\right)$. If it is still $L L\left(\beta_{t+1}\right) L L\left(\beta_{t}\right)$, we decrease again $\lambda$ to $1 / 4$. We should decrease $\lambda$ to smaller values such as $1 / 8$ and $1 / 16$ repeatedly until $\left.L L\left(\beta_{t+1}\right)\right\rangle L L\left(\beta_{t}\right)$ is satisfied. After 
adjusting $\lambda$, we can guarantee that the given function converges to its maximum point.

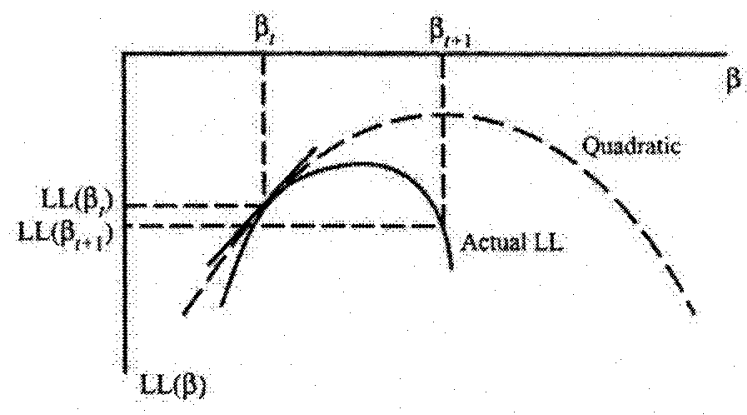

Figure 3-3: Finding a proper step size in the first case (Train, 2003)

- The second case

The second case is completely opposite to the first one. As shown in figure 3-4, the solid line is for actual $L L(\beta)$ and dashed line is for quadratic. At first iteration with $\lambda=1$, $\beta_{t+1}$ reaches to the maximum point for the quadratic case. But, the actual $L L(\beta)$ is not quadratic and the maximum point is far away and $\lambda$ should be adjusted by taking a large step in order to satisfy $\left.L L\left(\beta_{t+1}\right)\right\rangle L L\left(\beta_{t}\right)$. In figure 3-4 we can see that $\lambda=2$ is the best step size that a researcher can choose.

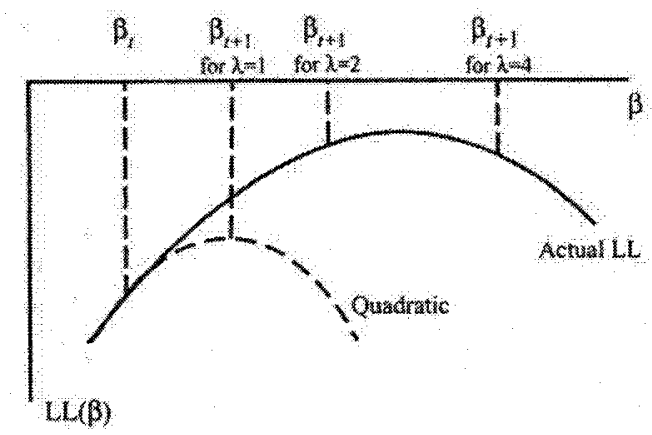

Figure 3-4: Finding a proper step size in the second case (Train, 2003) 


\subsection{Convergence criterion}

The maximum value of $L L(\beta)$ occurs when $G_{t}$ is equal to zero. However, in real calculation, $G_{t}$ can never be exactly zero, it can be close to zero (Train, 2003). Consequently, the iteration process of parameter estimation is finished when a certain convergence criterion specified by a researcher is satisfied. Three are three types of convergence criteria.

Firstly, the proportional change of parameters estimated in each iteration is calculated and evaluated in order to determine whether the termination should be stopped or go to the next iteration. The formula presented below, is called the relative-parameter-change stopping criterion (Ben-Akiva and Lerman, 1985):

$$
\left[\frac{1}{k} \sum_{k=1}^{k}\left(\beta_{t+1, k}-\beta_{t, k}\right)^{2}\right]^{1 / 2}<C R
$$

where

$k \quad$ is the number of parameter estimates,

$\beta_{t+1} \quad$ is the parameter estimates in iteration $\mathrm{t}+1$,

$\beta_{t} \quad$ is the parameter estimates in iteration $\mathrm{t}$,

CR is the stopping precision.

Secondly, the statistic, that is, named as the weighted-gradient stopping criterion is as follows (Belsley, 1979; Train, 2003) and it is considered for checking the stopping 
condition:

$G_{t}^{\prime}\left(-H_{t}^{-1}\right) G_{t}<C R$

where

$H_{t} \quad$ is the Hessian matrix in iteration $\mathrm{t}$,

$G_{t} \quad$ is a vector of gradient in iteration $\mathrm{t}$,

$G_{t}^{\prime} \quad$ is a transposed matrix of $G_{t}$.

Thirdly, the stopping criterion named as the gradient stopping criterion is given below (Belsley, 1979), but it is not used in this thesis research:

$G_{t}^{\prime} G_{t}<C R$

The researcher can specify a small value of $C R$ such as $10^{-4}, 10^{-5}$, and $10^{-6}$. In this thesis, we explore the performance variations or estimation performance with an application of the first two types of stopping criteria, and with applying variations on $C R$ of the second one. A discussion of their different operational characteristics and estimation results, such as parameter estimates and their statistics obtained from the eight different algorithms, are presented in detail in both chapters 4 and 5 . 


\subsection{Visual basic application (VBA)}

Each of the eight estimation algorithms and the maximum likelihood method explained previously were converted into computer codes by following the estimation procedures. The Visual Basic Application (VBA) provided in EXCEL, Microsoft, Inc. was utilized. By using the formulas of the eight estimation algorithms and the maximum likelihood method, visual basic computer codes were written for computations in a variety of experimental environments such as described in both chapters 4 and 5. Six experiments were organized and then executed according to the issue that is important in discrete choice model estimation. Two important points should be noted in developing computer codes. These are the codes for the log-likelihood function and the estimation algorithms. These have unique operational characteristics in the numerical calculation of both a vector of gradient and the Hessian matrix of the given log-likelihood function. Figure 3-5, figure 3-6 and figure 3-7 show the computational environments developed and used for experimental estimation runs in this thesis using Visual Basic Application (VBA) of EXCEL (Lee, 1998; Lee, 1999; Lee, 2000). All estimation runs are conducted using a personal computer (Intel Pentium 4, CPU $2.80 \mathrm{GHz}, 512 \mathrm{MB}$ Ram). 


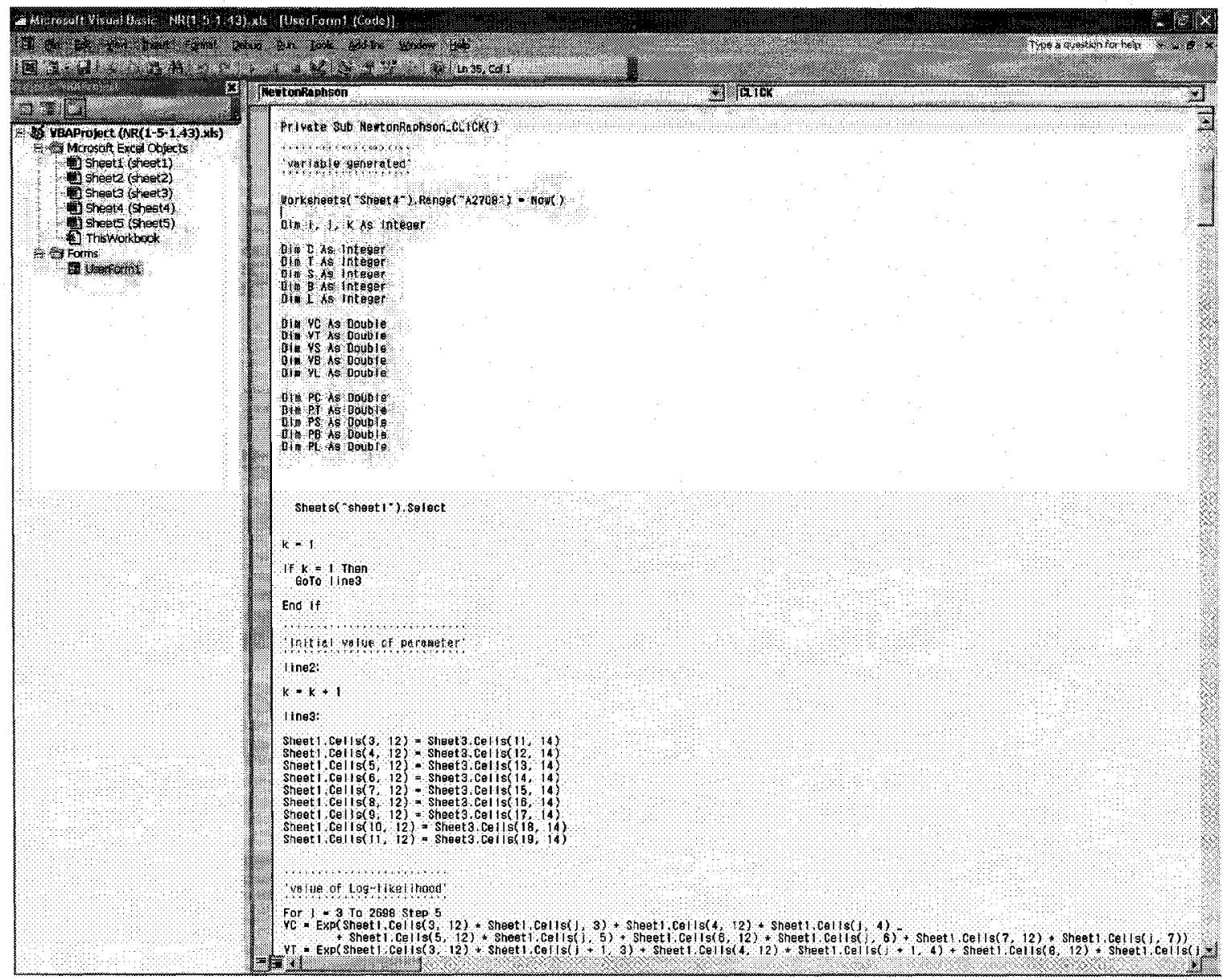

Figure 3-5: Coding window of Visual Basic Application (VBA)

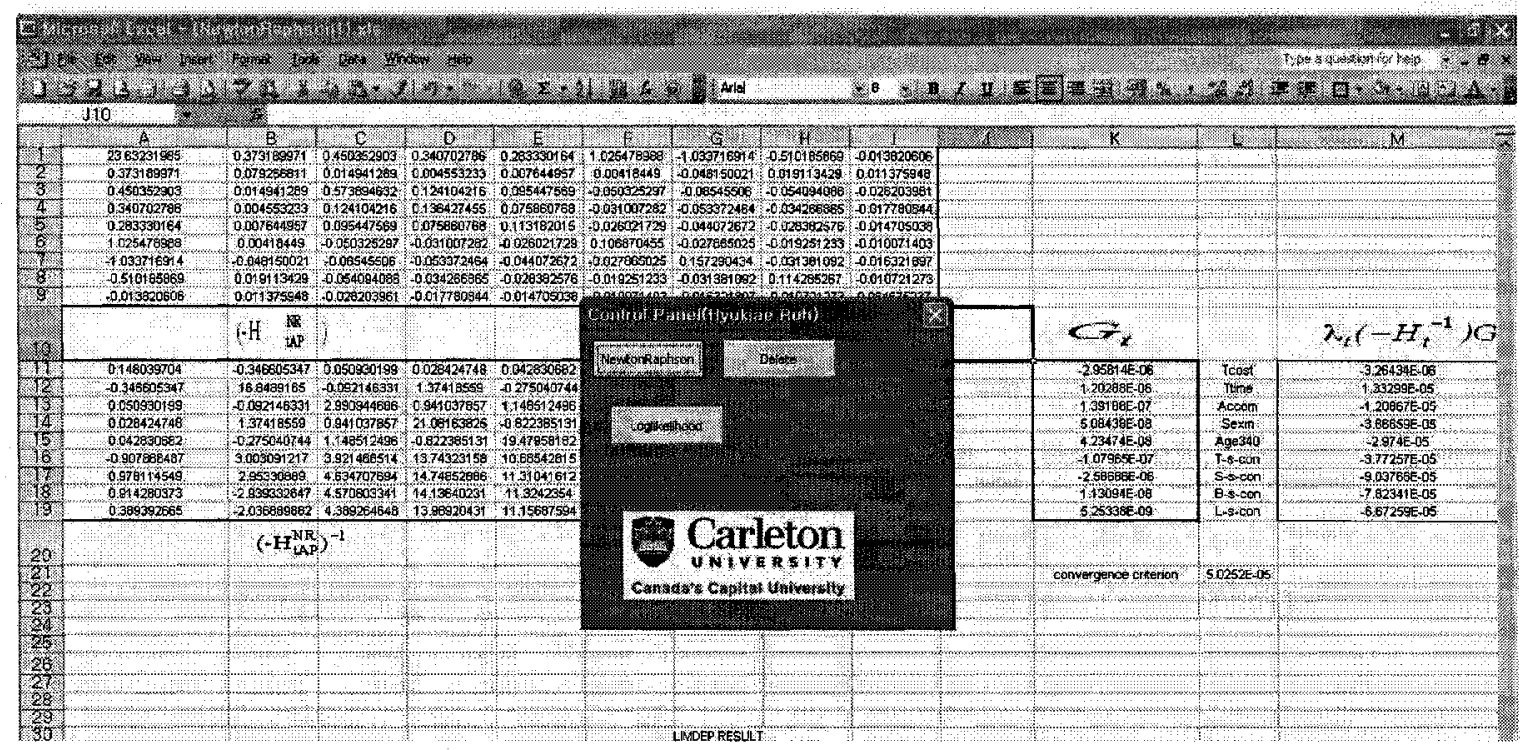

Figure 3-6: Window of the control panel 


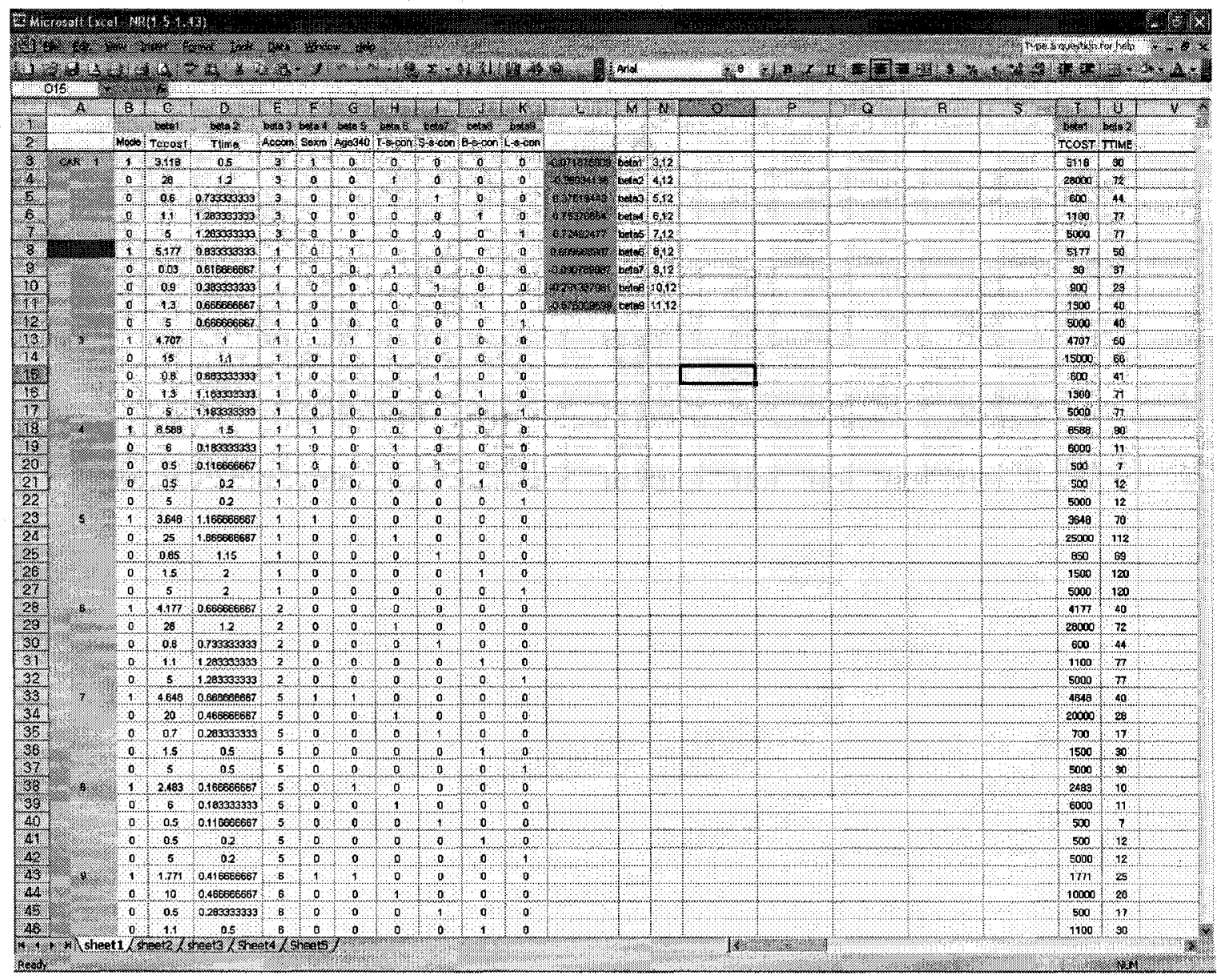

Figure 3-7: Data window

The program developed in this thesis consists of three major parts. The first part is for evaluating the log-likelihood function value of updated parameter estimates in iteration processes. The second part is intended for obtaining a vector of gradient and Hessian matrix that are core factors of the parameter estimation program. As shown in the previous sections, all elements consisting of a vector of gradient and Hessian matrix for each observation were calculated and used and updated. In the iteration method, the value was divided by the number of sample (for details, see the section 3.3 ). Third part of the program is for evaluating the stopping criterion and updating the Hessian matrix, which is used for the next iteration. These three parts are continuously repeated until the 
convergence criterion is satisfied. All procedures are programmed in Visual Basic Application provided in EXCEL. Using EXCEL as a program base to conduct discrete choice modelling provides many advantages:

- Excel as a spreadsheet can accommodate any type of data format and, furthermore, the arranged data set can simply be changed to another new format,

- The flow of application of the data set in the estimation processes are clear and thus a researcher can trace error that might be occur,

- All complex processes of discrete choice modelling can be placed under surveillance in a neat way,

- All delicate mathematical calculation processes such as matrix product, matrix inverse, and matrix transpose and so on can be carried out explicitly in the program. Therefore, diverse experimental investigations can be carried out. 


\section{Chapter 4 Experimental Estimation Results}

\subsection{Overview}

In the previous chapter, we investigate major estimation algorithms that are often employed in discrete choice modelling. More importantly, the ability to apply these practical estimation algorithms and the skills associated with real choice problems are very important to transportation researchers. In this chapter, key issues involved in discrete choice modelling will be introduced and explained. Also, multinomial logit model is estimated with real discrete choice outcome data using eight different estimation algorithms in order to compare their estimation performance within the real mode choice modelling environment. Section 4.2 considers test models and data set that are used as a basis to conduct experiments in this thesis. Section 4.3 introduces the assumed utility functions of all considered alternatives, likelihood, and log-likelihood functions composed of multinomial logit probabilistic choice function of each alternative. Mathematical processes important to make specific estimation codes are also explained in this section, more detailed mathematical manipulations are presented in appendix A. Section 4.4 presents the general results of model estimates for only one run of each eight estimation algorithms showing the best performance in terms of the convergence time, only within experiment 1 . The remainder of this chapter is devoted to an evaluation of the results obtained from experiment 1 using various tests applied in discrete choice model. 


\subsection{Test model and data set}

Test models used in this study are sourced from a previous research study (Roh, 2001), which investigated mode choice behaviour of airport passengers travelling to the airport in Korea. The five-alternative logit mode choice model was used as a test-base model to demonstrate the performance of algorithms being discussed in this study.

The estimation data are a sample of 540 trips to the airport. The travellers had five alternatives open to them in their decision-making. The data were collected from a random personal interview study carried out by the Korea Transport Institute (KOTI) at Kimpo International Passenger Terminal. The sample frequencies of chosen mode are given in table 4-1.

Table 4-1: The sample frequencies of chosen mode

\begin{tabular}{lcclll}
\hline Alternative & Car & Taxi & Subway & Bus & Limousine \\
\hline Choice (\%) & $247(46)$ & $70(13)$ & $112(21)$ & $73(13)$ & $38(7)$ \\
\hline
\end{tabular}

The primary purpose of this thesis research is to investigate numerical, operational process underlying the estimation of model parameters, not to develop a new mode choice model. To accomplish this purpose, a specific program of discrete mode choice model is developed, following the detailed steps involved in discrete choice modelling.

\subsubsection{Assumed representative utility function}

The assumed utility functions used in multinomial logit model are given below: 


$$
\begin{aligned}
& V(\text { Car })=\beta_{1}\left(T C_{C}\right)+\beta_{2}\left(T T_{C}\right)+\beta_{3}(\text { Accom })+\beta_{4}(\text { Sexm })+\beta_{5}(\text { Age 340 }) \\
& V(\text { Taxi })=\beta_{1}\left(T C_{T}\right)+\beta_{2}\left(T T_{T}\right)+\beta_{6}(\text { Tasc }=1) \\
& V(\text { Subway })=\beta_{1}\left(T C_{S}\right)+\beta_{2}\left(T T_{S}\right)+\beta_{7}(\text { Sasc }=1) \\
& V(\text { Bus })=\beta_{1}\left(T C_{B}\right)+\beta_{2}\left(T T_{B}\right)+\beta_{8}(\text { Basc }=1) \\
& V(\text { Limousine })=\beta_{1}\left(T C_{L}\right)+\beta_{2}\left(T T_{L}\right)+\beta_{9}(\text { Lasc }=1)
\end{aligned}
$$

Five alternatives (i.e., car, taxi, subway, bus, limousine) are considered as possible modes in given choice situation for all decision makers who want to travel to the airport. These five alternatives are also mutually exclusive. These represent all possible modes traveller can choose from. The mathematical form of the representative utility function is assumed to be linear-in-parameters for all five alternatives. The included variables are two generic variables, one alternative-specific socioeconomic variable, two alternative-specific dummy variables and four alternative-specific constants.

The details of variables are following. $T C_{C, T, S, B, L}$, one way total cost variable, and $T T_{C, T, S, B, L}$, one way total travel time, are included as service-level attributes of all considered modes and therefore are included in all five utility function. Accom, as an alternative-specific (to car) variable, is included in order to estimate the effect of the number of accompanied persons. To capture the effects of the difference in sex and the range of age on mode choice, demographic variables, Sexm (if male=1) and Age340 (if age range of age $30-40=1$ ) are included in the car function as mode-specific-dummy variables.

The car mode is designated to be the reference mode. Therefore, except for the function for the car mode, other functions belonging to other modes have an alternative-specific 
constant.

\subsection{Analytical partial derivatives of the log-likelihood function}

The maximum-likelihood is most often used in modelling works to estimate parameters. It works based on the concept of finding parameters maximizing the log-likelihood function value within given model specification. The estimated parameters obtained from maximum likelihood method are recognized as parameter estimates with which the sample used in modelling can be generated with the highest probability.

To use maximum likelihood estimator in model parameter estimation we should construct likelihood function with logit probabilistic function because our mode choice modelling is dependent on the multinomial logit framework. General form of the Likelihood function is shown below. Mathematical form represents the product of multinomial logit probabilistic function for all decision makers based on the mode that is actually chosen in a given choice situation.

$$
L\left(\beta_{k}\right)=\prod_{N} \prod_{i} P_{n}(i)^{y_{i n}}
$$

where

$L\left(\beta_{k}\right) \quad$ is the likelihood function,

$P_{n}(i) \quad$ is the probability that $i$ is chosen, here logit probabilistic choice function as given in equation (2.12),

$y_{\text {in }} \quad$ is an indicator, $y_{i n}$ is 1 if $n^{\text {th }}$ (decision maker) choose mode $i$, 
otherwise 0 .

In the context of this research, the specific likelihood function can be structured as follows:

$L\left(\beta_{1}, \beta_{2}, \cdots, \beta_{8}, \beta_{9}\right)=\prod_{N} \prod_{i} P_{n}(i)^{y_{i n}}=\prod_{N}\left[P_{n}(C)^{y_{C n}} \cdot P_{n}(T)^{y_{T n}} \cdot P_{n}(S)^{y_{S n}} \cdot P_{n}(B)^{y_{B n}} \cdot P_{n}(L)^{y_{L n}}\right]$,

$L L\left(\beta_{1}, \beta_{2}, \cdots, \beta_{8}, \beta_{9}\right)=\sum_{N}\left[y_{C n} \ln P_{n}(C)+y_{T n} \ln P_{n}(T)+y_{S n} \ln P_{n}(S)+y_{B n} \ln P_{n}(B)+y_{L n} \ln P_{n}(L)\right]$,

where

$P_{n}(C) \quad$ is the probability of choosing a car,

$P_{n}(T) \quad$ is the probability of choosing a taxi,

$P_{n}(S) \quad$ is the probability of choosing a subway,

$P_{n}(B) \quad$ is the probability of choosing a bus,

$P_{n}(L) \quad$ is the probability of choosing a limousine,

$y_{C n}, y_{T n}, y_{S n}, y_{B n}, y_{L n} \quad$ is an indicator, 1 if $\mathrm{n}$ chooses mode Car, Taxi, Subway, Bus, Limousine respectively, otherwise 0 .

From the mathematical point of view, obtaining a tractable formula is important. For computational convenience without losing consistency in estimating parameter, the above 
likelihood function of equation (4.3) can be transformed into log-likelihood function of equation (4.4). The function can be changed as shown below:

$$
\begin{aligned}
L L\left(\beta_{1}, \beta_{2}, \cdots, \beta_{8}, \beta_{9}\right)= & \sum_{n=1}^{N}\left[y_{C n} \ln \left(\frac{e^{V(\text { Car })}}{e^{V(\text { Car })}+e^{V(\text { Taxi })}+e^{V(\text { Subway })}+e^{V(\text { Bus })}+e^{V(\text { Limousine })}}\right)\right. \\
& +y_{T n} \ln \left(\frac{e^{V(\text { Taxi })}}{e^{V(\text { Car })}+e^{V(\text { Taxi })}+e^{V(\text { Subway })}+e^{V(\text { Bus })}+e^{V(\text { Limousine })}}\right) \\
& +y_{S n} \ln \left(\frac{e^{V(\text { Subway })}}{e^{V(\text { Car })}+e^{V(\text { Taxi })}+e^{V(\text { Subway })}+e^{V(\text { Bus })}+e^{V(\text { Limousine })}}\right) \\
& +y_{B n} \ln \left(\frac{e^{V(\text { Bus })}}{e^{V(\text { Car })}+e^{V(\text { (Taxi })}+e^{V(\text { Subway })}+e^{V(\text { Bus })}+e^{V(\text { Limousine })}}\right) \\
& \left.+y_{L n} \ln \left(\frac{e^{V(\text { Limujin })}}{e^{V(\text { Car })}+e^{V(\text { (Taxi })}+e^{V(\text { Subway })}+e^{V(\text { Bus })}+e^{V(\text { Limousine })}}\right)\right] .
\end{aligned}
$$

Log transformation applies to equation (4.3) and also all alternatives' utility function can be placed into the multinomial logit probability function. The final log-likelihood function specific to this choice situation takes the form of equation (4.5). The multinomial logit probability function derived earlier can be seen clearly in loglikelihood function. By inserting the utility function into log-likelihood function, we can have the final formula and it is used to get gradient and the Hessian through mathematical derivation. With this log-likelihood function, we can derive the formulas of first-order, second-order analytical derivatives of the log-likelihood function with respect to each parameter. The detailed derivation is described in appendix A.

For example, if there are two decision makers in given choice situation where the first person chooses car and the second one chooses taxi, the likelihood function takes the form shown below: 
$L\left(\beta_{1}, \beta_{2}, \cdots, \beta_{k}\right)=\prod_{2} \prod_{2} P_{n}(i)^{y_{i n}}$

$L\left(\beta_{1}, \beta_{2}, \cdots, \beta_{k}\right)=P_{n}(\text { Car })^{y_{C l}} \cdot P_{n}(\text { Taxi })^{y_{T 2}}$

After putting the multinomial probabilistic choice function in the same calculation line, its log-likelihood function can be simplified as below:

$L L\left(\beta_{1}, \beta_{2}, \cdots, \beta_{k}\right)=\ln P_{n}($ Car $)+\ln P_{n}($ Taxi $)$,

$\begin{aligned} L L\left(\beta_{1}, \beta_{2}, \cdots, \beta_{k}\right)= & \ln \left(\frac{e^{V(\text { Car })}}{e^{V(\text { Car })}+e^{V(\text { Taxi })}+e^{V(\text { Subway })}+e^{V(\text { Bus })}+e^{V(\text { Limousine })}}\right) \\ & +\ln \left(\frac{e^{V(\text { Taxi })}}{e^{V(\text { Car })}+e^{V(\text { Taxi })}+e^{V(\text { Subway })}+e^{V(\text { Bus })}+e^{V(\text { Limousin })}}\right)\end{aligned}$

\subsection{Estimation results}

Before discussing the results related to each estimation algorithm, it is useful to note that this study is designed to obtain an understanding, from a computational perspective, of the discrete mode choice modelling process and to compare its performance according to the eight oft-adopted algorithms. Therefore, the statistical qualities of each considered algorithm's estimation results are beyond the scope of this study. Only for general interest in discrete mode choice model estimation procedures, the representative model evaluation statistics and their calculation method will be presented and discussed in section 4.4.1 based on specific calculation environments such as stopping criterion 
$\left[\frac{1}{k} \sum_{k=1}^{k}\left(\beta_{t+1, k}-\beta_{t, k}\right)^{2}\right]^{1 / 2}<10^{-4}$, and step size $\lambda$ showing the best performance in each of eight estimation algorithms.

\subsubsection{General tests applied in discrete mode choice model}

In this section, tables 4-2 to 4-9 show the results of parameter estimates of each algorithm. All tables presented here show the results of the best performance of each algorithm in terms of step size $\lambda$, the number of iterations, and convergence time. Starting points, $\beta_{k}=o$, for all parameters, are used as initial starting points of parameters. The results are summarized following the format used frequently in discrete choice modelling. The tables include parameter estimate values of both base model (unrestricted model) and constant only model (restricted model). In addition, the t-statistics for each parameter used for the hypothesis test for each estimated parameter are presented. The values of the Log-likelihood function for each assumed unique utility specification. These are $L L(0)$ which means the novice model not containing any explanatory variables in utility function, $L L(C)$ which only includes mode-specific-constant as an explanatory variable in the utility function of each alternative, and $L L\left(\beta_{k}\right)$ which, as a full model, contains all considered explanatory variables representing characteristics of each alternative and demographics of decision makers. With these values of the log-likelihood function, overall goodness-of-fit measures and testing value used for assessing the reasonableness of model are also calculated. Detailed calculation process and explanations are presented in the following sections. 
Table 4-2: Newton Raphson (NR) Algorithm

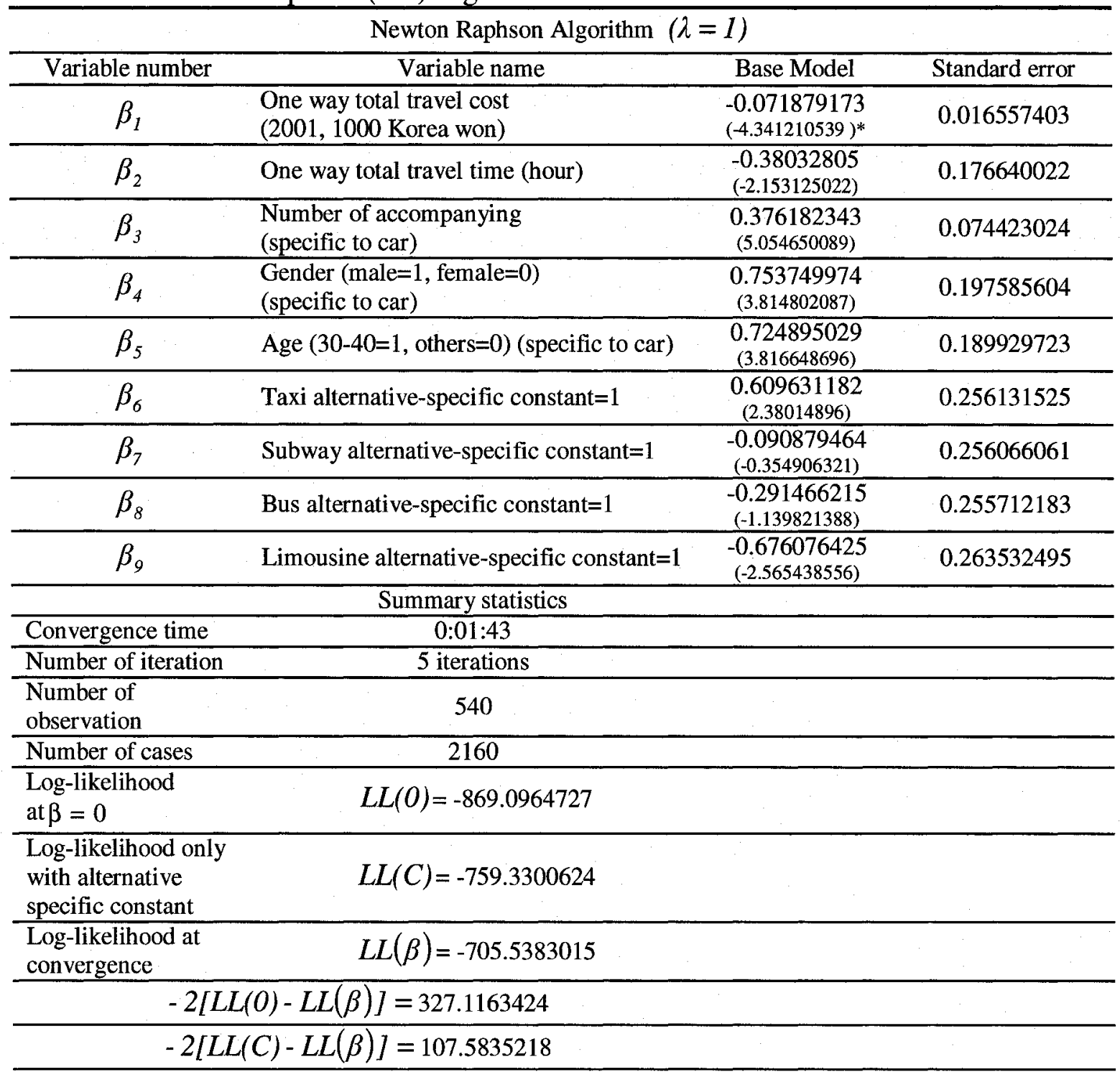

\begin{tabular}{ll}
\hline Rho-squared with respect to Zero & $\rho_{0}^{2}=0.188193343$ \\
\hline Rho-squared with respect to Constants & $\rho_{c}^{2}=0.070841078$ \\
\hline Adjusted Rho-squared with respect to Zero & $\bar{\rho}_{o}^{2}=0.177837761$ \\
\hline Adjusted Rho-squared with respect to Constants & $\bar{\rho}_{c}^{2}=0.063919611$ \\
\hline
\end{tabular}

*The value in a parenthesis is the t-statistic of the corresponding parameter

*All $\mathrm{t}$-statistics are calculated with a hypothesis that the all parameters are equal to zero, for example, the $\mathrm{t}$ statistic of $\beta_{1}$ is obtained as follows: $(-0.07189173-0) / 0.016557403=-4.341210539$ (see the equation 4.10 ) 
Table 4-3: BHHH algorithm

\begin{tabular}{|c|c|c|c|}
\hline \multicolumn{4}{|c|}{ BHHH Algorithm $(\lambda=1)$} \\
\hline Variable number & Variable name & Base Model & Standard error \\
\hline$\beta_{1}$ & $\begin{array}{l}\text { One way total travel cost } \\
(2001,1000 \text { Korea won) }\end{array}$ & $\begin{array}{c}-0.071921479 \\
(-11.9125619)^{*} \\
\end{array}$ & 0.006037448 \\
\hline$\beta_{2}$ & One way total travel time (hour) & $\begin{array}{c}-0.380273015 \\
(-3.50693788) \\
\end{array}$ & 0.108434489 \\
\hline$\beta_{3}$ & $\begin{array}{l}\text { Number of accompanying } \\
\text { (specific to car) }\end{array}$ & $\begin{array}{c}0.375342761 \\
(15.24011549) \\
\end{array}$ & 0.024628603 \\
\hline$\beta_{4}$ & $\begin{array}{l}\text { Gender (male }=1, \text { female }=0 \text { ) } \\
\text { (specific to car) }\end{array}$ & $\begin{array}{l}0.753462145 \\
(3.53263168) \\
\end{array}$ & 0.213286358 \\
\hline$\beta_{5}$ & $\begin{array}{l}\text { Age }(30-40=1, \text { others }=0) \\
\text { (specific to car) }\end{array}$ & $\begin{array}{c}0.724662342 \\
(3.476077331)\end{array}$ & 0.208471295 \\
\hline$\beta_{6}$ & Taxi alternative-specific constant $=1$ & $\begin{array}{c}0.608790967 \\
(2.63629213)\end{array}$ & 0.230926975 \\
\hline$\beta_{7}$ & $\begin{array}{l}\text { Subway alternative-specific } \\
\text { constant }=1\end{array}$ & $\begin{array}{c}-0.092286808 \\
(-0.42698705)\end{array}$ & 0.216134908 \\
\hline$\beta_{8}$ & Bus alternative-specific constant $=1$ & $\begin{array}{c}-0.292775357 \\
(-1.26671801) \\
\end{array}$ & 0.231129071 \\
\hline$\beta_{9}$ & $\begin{array}{l}\text { Limousine alternative-specific } \\
\text { constant }=1\end{array}$ & $\begin{array}{c}-0.677291974 \\
(-2.58562452)\end{array}$ & 0.261945216 \\
\hline \multicolumn{4}{|c|}{ Summary statistics } \\
\hline Convergence time & $0: 27: 44$ & & \\
\hline Number of iteration & 51 iterations & & \\
\hline Number of observation & 540 & & \\
\hline Number of cases & 2160 & & \\
\hline Log-likelihood at $\beta=0$ & $L L(0)=-869.096473$ & & \\
\hline $\begin{array}{l}\text { Log-likelihood only with } \\
\text { alternative specific } \\
\text { constant }\end{array}$ & $L L(C)=-759.3300637$ & & \\
\hline $\begin{array}{l}\text { Log-likelihood at } \\
\text { convergence }\end{array}$ & $L L(\beta)=-705.5383823$ & & \\
\hline \multicolumn{4}{|c|}{$-2[L L(O)-L L(\beta)]=327.116182$} \\
\hline \multicolumn{4}{|c|}{$-2[L L(C)-L L(\beta)]=107.5833628$} \\
\hline \multicolumn{2}{|c|}{ Rho-squared with respect to Zero } & \multicolumn{2}{|c|}{$\rho_{0}^{2}=0.188193251$} \\
\hline \multicolumn{2}{|c|}{ Rho-squared with respect to Constants } & \multicolumn{2}{|c|}{$\rho_{c}^{2}=0.070840974$} \\
\hline \multicolumn{2}{|c|}{ Adjusted Rho-squared with respect to Zero } & \multicolumn{2}{|c|}{$\bar{\rho}_{0}^{2}=0.177837668$} \\
\hline \multicolumn{2}{|c|}{ Adjusted Rho-squared with respect to Constants } & \multicolumn{2}{|c|}{$\bar{\rho}_{c}^{2}=0.063919507$} \\
\hline
\end{tabular}

*The value in a parenthesis is the t-statistic of the corresponding parameter

*All t-statistics are calculated with a hypothesis that the all parameters are equal to zero, for example, the tstatistic of $\beta_{l}$ is obtained as follows: $(-0.071921479-0) / 0.006037448=-11.9125619$ (see the equation 4.10) 
Table 4-4: BHHH-2 algorithm

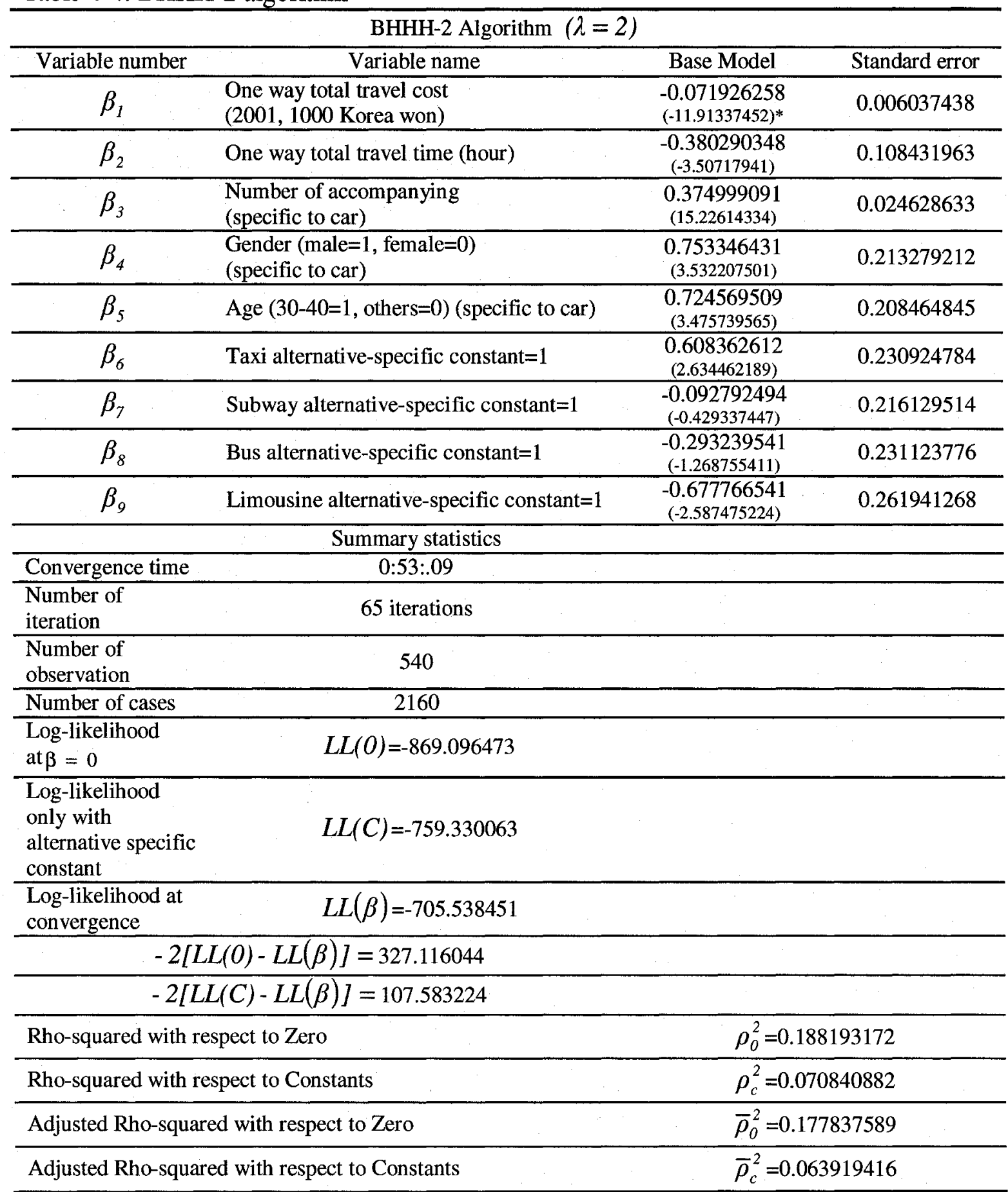

*The value in a parenthesis is the t-statistic of the corresponding parameter

*All t-statistics are calculated with a hypothesis that the all parameters are equal to zero, for example, the tstatistic of $\beta_{l}$ is obtained as follows: $(-0.071926258-0) / 0.006037438=-11.91337452$ (see the equation 4.10 ) 
Table 4-5: Steepest Ascent (SA) algorithm

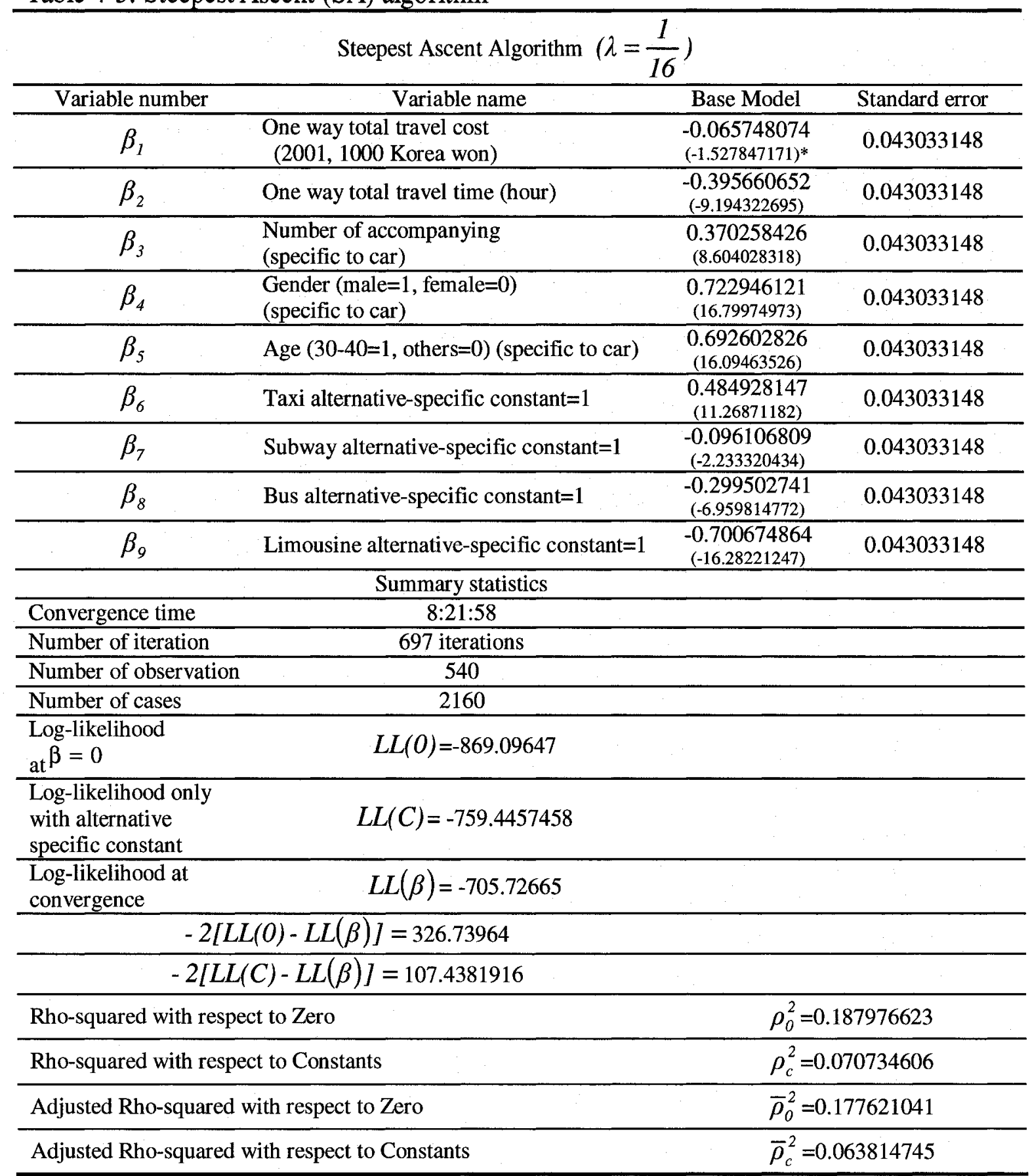

*The value in a parenthesis is the t-statistic of the corresponding parameter

*All t-statistics are calculated with a hypothesis that the all parameters are equal to zero, for example, the tstatistic of $\beta_{1}$ is obtained as follows: $(-0.065748074-0) / 0.043033148=-1.527847171$ (see the equation 4.10) 
Table 4-6: DFP algorithm

\begin{tabular}{|c|c|c|c|}
\hline \multicolumn{4}{|c|}{ DFP Algorithm $\left(\lambda=\frac{1}{2}\right)$} \\
\hline Variable number & Variable name & Base Model & Standard error \\
\hline$\beta_{1}$ & $\begin{array}{l}\text { One way total travel cost } \\
(2001,1000 \text { Korea won })\end{array}$ & $\begin{array}{l}-0.072098438 \\
(-6.947879317)^{*} \\
\end{array}$ & 0.010345484 \\
\hline$\beta_{2}$ & One way total travel time (hour) & $\begin{array}{c}-0.380926884 \\
(-7.195625156) \\
\end{array}$ & 0.052855456 \\
\hline$\beta_{3}$ & Number of accompanying (specific to car) & $\begin{array}{c}0.375606786 \\
(9.308732551)\end{array}$ & 0.040411768 \\
\hline$\beta_{4}$ & $\begin{array}{l}\text { Gender (male }=1, \text { female }=0 \text { ) } \\
\text { (specific to car) }\end{array}$ & $\begin{array}{l}0.762644547 \\
(16.73982756)\end{array}$ & 0.045027344 \\
\hline$\beta_{5}$ & Age $(30-40=1$, others $=0$ ) (specific to car) & $\begin{array}{c}0.731131563 \\
(15.69436667)\end{array}$ & 0.046188231 \\
\hline$\beta_{6}$ & Taxi alternative-specific constant $=1$ & $\begin{array}{l}0.61791948 \\
(6.153408196)\end{array}$ & 0.099072118 \\
\hline$\beta_{7}$ & Subway alternative-specific constant $=1$ & $\begin{array}{l}-0.083637206 \\
(-1.182828817)\end{array}$ & 0.076832305 \\
\hline$\beta_{8}$ & Bus alternative-specific constant $=1$ & $\begin{array}{c}-0.285910003 \\
(-3.495196147)\end{array}$ & 0.083390517 \\
\hline$\beta_{9}$ & Limousine alternative-specific constant $=1$ & $\begin{array}{l}-0.67192798 \\
(-7.852874789) \\
\end{array}$ & 0.086092857 \\
\hline \multicolumn{4}{|c|}{ Summary statistics } \\
\hline Convergence time & $0: 27: 32$ & & \\
\hline Number of iteration & 115 Iterations & & \\
\hline Number of observation & 540 & & \\
\hline Number of cases & 2160 & & \\
\hline $\begin{array}{l}\text { Log-likelihood } \\
\text { at } \beta_{k}=0\end{array}$ & $L L(0)=-869.0964727$ & & \\
\hline $\begin{array}{l}\text { Log-likelihood only } \\
\text { with alternative } \\
\text { specific constant }\end{array}$ & $L L(C)=-759.3300731$ & & \\
\hline $\begin{array}{l}\text { Log-likelihood at } \\
\text { convergence }\end{array}$ & $L L(\beta)=-705.5406805$ & & \\
\hline \multicolumn{4}{|c|}{$-2[L L(0)-L L(\beta)]=327.1115844$} \\
\hline \multicolumn{4}{|c|}{$-2[L L(C)-L L(\beta)]=107.5787852$} \\
\hline \multicolumn{2}{|c|}{ Rho-squared with respect to Zero } & \multicolumn{2}{|c|}{$\rho_{0}^{2}=0.188190606$} \\
\hline \multicolumn{2}{|c|}{ Rho-squared with respect to Constants } & \multicolumn{2}{|c|}{$\rho_{c}^{2}=0.070837959$} \\
\hline \multicolumn{2}{|c|}{ Adjusted Rho-squared with respect to Zero } & \multicolumn{2}{|c|}{$\bar{\rho}_{0}^{2}=0.177835042$} \\
\hline \multicolumn{2}{|c|}{ Adjusted Rho-squared with respect to Constants } & \multicolumn{2}{|c|}{$\bar{\rho}_{c}^{2}=0.063916507$} \\
\hline
\end{tabular}

*The value in a parenthesis is the $t$-statistic of the corresponding parameter

*All $\mathrm{t}$-statistics are calculated with a hypothesis that the all parameters are equal to zero, for example, the $t$ statistic of $\beta_{1}$ is obtained as follows: $(-0.072098438-0) / 0.010345484=-6.947879317$ (see the equation 4.10 ) 
Table 4-7: BFGS algorithm

\begin{tabular}{|c|c|c|c|}
\hline \multicolumn{4}{|c|}{ BFGS Algorithm $(\lambda=1)$} \\
\hline Variable number & Variable name & Base Model & $\begin{array}{l}\text { Standard } \\
\text { error }\end{array}$ \\
\hline$\beta_{1}$ & One way total travel cost $(2001,1000$ Korea won) & $\begin{array}{l}-0.07189141 \\
(-4.715200331)^{*} \\
\end{array}$ & 0.01524414 \\
\hline$\beta_{2}$ & One way total travel time (hour) & $\begin{array}{c}-0.380505376 \\
(-2.447144801)\end{array}$ & 0.15541706 \\
\hline$\beta_{3}$ & Number of accompanying (specific to car) & $\begin{array}{c}0.376158747 \\
(5.243009343)\end{array}$ & 0.071749318 \\
\hline$\beta_{4}$ & Gender $($ male $=1$, female $=0$ ) (specific to car) & $\begin{array}{c}0.754091182 \\
(4.830327879)\end{array}$ & 0.156045302 \\
\hline$\beta_{5}$ & Age $(30-40=1$, others $=0)$ (specific to car) & $\begin{array}{c}0.724458663 \\
(5.576440299) \\
\end{array}$ & 0.129992431 \\
\hline$\beta_{6}$ & Taxi alternative-specific constant $=1$ & $\begin{array}{c}0.609727872 \\
(2.606799059) \\
\end{array}$ & 0.233861977 \\
\hline$\beta_{7}$ & Subway alternative-specific constant $=1$ & $\begin{array}{l}-0.091078825 \\
(-0.398665442) \\
\end{array}$ & 0.227959222 \\
\hline$\beta_{8}$ & Bus alternative-specific constant $=1$ & $\begin{array}{c}-0.291558346 \\
(-1.23182074)\end{array}$ & 0.236614148 \\
\hline$\beta_{9}$ & Limousine alternative-specific constant $=1$ & $\begin{array}{l}-0.676004646 \\
(-2.807111542)\end{array}$ & 0.240844161 \\
\hline \multicolumn{4}{|c|}{ Summary statistics } \\
\hline \multicolumn{4}{|c|}{$\frac{\text { Summary statistics }}{0: 09: 16}$} \\
\hline \multicolumn{4}{|l|}{ Number of iteration } \\
\hline \multicolumn{4}{|l|}{$\begin{array}{l}\text { Number of } \\
\text { observation }\end{array}$} \\
\hline \multicolumn{4}{|l|}{ Number of cases } \\
\hline $\begin{array}{l}\text { Log-likelihood } \\
\text { at } \beta_{k}=0\end{array}$ & \multicolumn{3}{|l|}{$L L(0)=-869.0964727$} \\
\hline $\begin{array}{l}\text { Log-likelihood only } \\
\text { with alternative } \\
\text { specific constant }\end{array}$ & \multicolumn{3}{|l|}{$L L(C)=\mathrm{N} / \mathrm{A}$} \\
\hline $\begin{array}{l}\text { Log-likelihood at } \\
\text { convergence }\end{array}$ & \multicolumn{3}{|l|}{$L L(\beta)=-705.5383081$} \\
\hline \multicolumn{4}{|c|}{$-2[L L(0)-L L(\beta)]=327.1163292$} \\
\hline \multicolumn{4}{|c|}{$-2[L L(C)-L L(\beta)]=\mathrm{N} / \mathrm{A}$} \\
\hline \multicolumn{2}{|c|}{ Rho-squared with respect to Zero } & \multicolumn{2}{|c|}{$\rho_{0}^{2}=0.188193338$} \\
\hline \multicolumn{2}{|c|}{ Rho-squared with respect to Constants } & \multicolumn{2}{|c|}{$\rho_{c}^{2}=\mathrm{N} / \mathrm{A}$} \\
\hline \multicolumn{2}{|c|}{ Adjusted Rho-squared with respect to Zero } & \multicolumn{2}{|c|}{$\bar{\rho}_{0}^{2}=0.177837753$} \\
\hline \multicolumn{2}{|c|}{ Adjusted Rho-squared with respect to Constants } & \multicolumn{2}{|c|}{$\bar{\rho}_{c}^{2}=\mathrm{N} / \mathrm{A}$} \\
\hline
\end{tabular}

*The value in a parenthesis is the $t$-statistic of the corresponding parameter

*All t-statistics are calculated with a hypothesis that the all parameters are equal to zero, for example, the tstatistic of $\beta_{1}$ is obtained as follows: $(-0.07189141-0) / 0.01524414=-4.715200331$ (see the equation 4.10) 
Table 4-8: DFP-GSM algorithm in the case of $L_{\text {ower }} L(0), U_{\text {pper }} L(2)$

DFP-GSM Algorithm ( $\lambda_{t}$ : fixed automatically by golden section line search method)

\begin{tabular}{|c|c|c|c|}
\hline Variable number & Variable name & Base Model & $\begin{array}{c}\text { Standard } \\
\text { error }\end{array}$ \\
\hline$\beta_{1}$ & $\begin{array}{l}\text { One way total travel cost } \\
(2001,1000 \text { Korea won) }\end{array}$ & $\begin{array}{l}-0.071910158 \\
(-4.414462097)^{*} \\
\end{array}$ & 0.016289676 \\
\hline$\beta_{2}$ & One way total travel time (hour) & $\begin{array}{l}-0.380328681 \\
(-2.16744516)\end{array}$ & 0.175473266 \\
\hline$\beta_{3}$ & Number of accompanying (specific to car) & $\begin{array}{c}0.376022347 \\
(5.183842107)\end{array}$ & 0.072537384 \\
\hline$\beta_{4}$ & Gender (male $=1$, female $=0$ ) (specific to car) & $\begin{array}{l}0.75525721 \\
(4.525425558) \\
\end{array}$ & 0.166891975 \\
\hline$\beta_{5}$ & Age $(30-40=1$, others $=0)$ (specific to car) & $\begin{array}{c}0.722628157 \\
(6.712829499) \\
\end{array}$ & 0.107648817 \\
\hline$\beta_{6}$ & Taxi alternative-specific constant $=1$ & $\begin{array}{l}0.609371292 \\
(2.406918394)\end{array}$ & 0.253174887 \\
\hline$\beta_{7}$ & Subway alternative-specific constant $=1$ & $\begin{array}{l}-0.09164794 \\
(-0.369409934)\end{array}$ & 0.248092787 \\
\hline$\beta_{8}$ & Bus alternative-specific constant $=1$ & $\begin{array}{c}-0.292198064 \\
(-1.163700266) \\
\end{array}$ & 0.251093922 \\
\hline$\beta_{9}$ & Limousine alternative-specific constant $=1$ & $\begin{array}{r}-0.676586955 \\
(-2.584899506)\end{array}$ & 0.261745942 \\
\hline \multicolumn{4}{|c|}{ Summary statistics } \\
\hline Convergence time & $0: 26: 29$ & & \\
\hline Number of iteration & 23 Iterations & & \\
\hline $\begin{array}{l}\text { Number of } \\
\text { observation }\end{array}$ & 540 & & \\
\hline Number of cases & 2160 & & \\
\hline $\begin{array}{l}\text { Log-likelihood } \\
\text { at } \beta_{k}=0\end{array}$ & $L L(0)=-869.0964727$ & & \\
\hline $\begin{array}{l}\text { Log-likelihood only } \\
\text { with alternative } \\
\text { specific constant }\end{array}$ & $L L(C)=\mathrm{N} / \mathrm{A}$ & & \\
\hline $\begin{array}{l}\text { Log-likelihood at } \\
\text { convergence }\end{array}$ & $L L(\beta)=-705.5384214$ & & \\
\hline \multicolumn{4}{|c|}{$-2[L L(O)-L L(\beta)]=327.1161026$} \\
\hline \multicolumn{4}{|c|}{$-2[L L(C)-L L(\beta)]=\mathrm{N} / \mathrm{A}$} \\
\hline Rho-squared with re & ect to Zero & \multicolumn{2}{|c|}{$\rho_{0}^{2}=0.188193205$} \\
\hline Rho-squared with re & ect to Constants & \multicolumn{2}{|c|}{$\rho_{c}^{2}=\mathrm{N} / \mathrm{A}$} \\
\hline Adjusted Rho-squar & with respect to Zero & \multicolumn{2}{|c|}{$\bar{\rho}_{0}^{2}=0.177837$} \\
\hline Adjusted Rho-squar & with respect to Constants & \multicolumn{2}{|c|}{$\bar{\rho}_{c}^{2}=\mathrm{N} / \mathrm{A}$} \\
\hline
\end{tabular}

*The value in a parenthesis is the t-statistic of the corresponding parameter

*All t-statistics are calculated with a hypothesis that the all parameters are equal to zero, for example, the $t$ statistic of $\beta_{1}$ is obtained as follows: $(-0.071910158-0) / 0.016289676=-4.414462097$ (see the equation 4.10 ) 
Table 4-9: BFGS-GSM algorithm in the case of $L_{\text {ower }} L(0), U_{\text {pper }} L(3)$

\begin{tabular}{|c|c|c|c|}
\hline \multicolumn{4}{|c|}{ BFGS-GSM Algorithm ( $\lambda_{t}$ : fixed by golden section line search method) } \\
\hline Variable number & Variable name & Base Model & $\begin{array}{l}\text { Standard } \\
\text { error }\end{array}$ \\
\hline$\beta_{1}$ & One way total travel cost $(2001,1000$ Korea won) & $\begin{array}{l}-0.071879258 \\
(-4.319477061)^{*}\end{array}$ & 0.016640731 \\
\hline$\beta_{2}$ & One way total travel time (hour) & $\begin{array}{c}-0.38032536 \\
(-2.16691778) \\
\end{array}$ & 0.17551444 \\
\hline$\beta_{3}$ & Number of accompanying (specific to car) & $\begin{array}{c}0.376182165 \\
(5.066011699) \\
\end{array}$ & 0.074256079 \\
\hline$\beta_{4}$ & Gender $($ male $=1$, female $=0$ ) (specific to car) & $\begin{array}{c}0.753749779 \\
(3.823294511) \\
\end{array}$ & 0.197146669 \\
\hline$\beta_{5}$ & Age $(30-40=1$, others $=0)$ (specific to car) & $\begin{array}{l}0.724894588 \\
(3.814267254)\end{array}$ & 0.19004819 \\
\hline$\beta_{6}$ & Taxi alternative-specific constant $=1$ & $\begin{array}{c}0.609631135 \\
(2.386538942) \\
\end{array}$ & 0.25544571 \\
\hline$\beta_{7}$ & Subway alternative-specific constant $=1$ & $\begin{array}{c}-0.090880052 \\
(-0.356926201) \\
\end{array}$ & 0.254618606 \\
\hline$\beta_{8}$ & Bus alternative-specific constant $=1$ & $\begin{array}{c}-0.291467516 \\
(-1.136733881) \\
\end{array}$ & 0.256407872 \\
\hline$\beta_{9}$ & Limousine alternative-specific constant=1 & $\begin{array}{l}-0.676077466 \\
(-2.559976033) \\
\end{array}$ & 0.264095233 \\
\hline \multicolumn{4}{|c|}{ Summary statistics } \\
\hline Convergence time & $0: 18: 33$ & & \\
\hline Number of iteration & 21 Iterations & & \\
\hline $\begin{array}{l}\text { Number of } \\
\text { observation }\end{array}$ & 540 & & \\
\hline Number of cases & 2160 & & \\
\hline $\begin{array}{l}\text { Log-likelihood } \\
\text { at } \beta_{k}=0\end{array}$ & $L L(0)=-869.0964727$ & & \\
\hline $\begin{array}{l}\text { Log-likelihood only } \\
\text { with alternative } \\
\text { specific constant }\end{array}$ & $L L(C)=\mathrm{N} / \mathrm{A}$ & & \\
\hline $\begin{array}{l}\text { Log-likelihood at } \\
\text { convergence }\end{array}$ & $L L(\beta)=-705.5383015$ & & \\
\hline \multicolumn{4}{|c|}{$-2[L L(0)-L L(\beta)]=327.1163424$} \\
\hline \multicolumn{4}{|c|}{$-2[L L(C)-L L(\beta)]=\mathrm{N} / \mathrm{A}$} \\
\hline Rho-squared with res & ect to Zero & \multicolumn{2}{|c|}{$\rho_{0}^{2}=0.188193343$} \\
\hline Rho-squared with res & ect to Constants & \multicolumn{2}{|c|}{$\rho_{c}^{2}=\mathrm{N} / \mathrm{A}$} \\
\hline Adjusted Rho-square & with respect to Zero & \multicolumn{2}{|c|}{$\bar{\rho}_{0}^{2}=0.177837761$} \\
\hline Adjusted Rho-square & with respect to Constants & \multicolumn{2}{|c|}{$\bar{\rho}_{c}^{2}=\mathrm{N} / \mathrm{A}$} \\
\hline
\end{tabular}

*The value in a parenthesis is the $t$-statistic of the corresponding parameter

*All $\mathrm{t}$-statistics are calculated with a hypothesis that the all parameters are equal to zero, for example, the tstatistic of $\beta_{1}$ is obtained as follows: $(-0.071879258-0) / 0.016640731=-4.319477061$ (see the equation 4.10 ) 


\subsubsection{Intuitive test}

Even though model evaluation is not our primary goal in this research, but by showing that the parameters are estimated properly from either intuitive sense or from a statistical perspective, can infer that our estimation process is on the right way. Given that all tests are conducted with the results of Newton Raphson, the same tests can be applied to others in the same way. The parameter estimates obtained from eight different algorithms are almost similar in terms of parameter magnitude, excepting those of Steepest Ascent. The following three sections $(4.4 .1 .2,4.4 .1 .3,4.4 .1 .4)$ including this section present the tests, which rely both on our intuitive sense of model parameters and heavily on classical statistical tests.

All parameters have proper signs and relative magnitudes corresponding to our prior knowledge. For example, the sign of one way total cost variable and one way total travel time variable has negative $(-)$ sign, which means that these variables have a negative effect on mode choice. In other words, it is preferable for a decision-maker to experience a decrease in their value for the chosen mode. A traveller going to the airport is more sensitive to travel time than travel cost because the primary concern is to arrive at airport without delay. The person who travels to the airport for boarding a plane has a tendency to choose the car mode in proportion to the number of accompanied persons. A male person who is aging (30 40) shows a preference for using a car for trip to airport.

\subsubsection{Test of each parameter}

Statistical test for each parameter of the model can be used to evaluate the formal 
hypothesis about each individual parameter (Koppelman and Bhat, 2006). The test statistic used for this test takes the form of equation (4.10). Especially, the standard error of each parameter provides information about the magnitude of the sampling error in a parameter; the larger the standard error of a certain parameter of an estimated model, the lower the precision of that corresponding parameter (Koppelman and Bhat, 2006). We can see the term of standard error used for calculating t-statistic in the formula shown below. Table 4-10 shows critical absolute t-values. A researcher can reject the null hypothesis that a particular variable has no influence on mode choice (equivalently that the true parameter related to a particular variable is zero) at the $90 \%$ level of confidence, if that variable's absolute t-value is greater than 1.645. At $99.9 \%$ level of confidence, the corresponding t-value is 3.290 . For example, in table 4-9, a researcher can decide that the parameter $\beta_{1}$ influences significantly the utility function of each of five modes at a confidence level higher than $99.9 \%$ and thus this variable should be retained. The formula is shown below:

$$
t_{\text {statistics }}=\frac{\beta_{k}-\beta_{k}^{*}}{S_{k}}
$$

where

$$
\begin{array}{ll}
\beta_{k} & \text { is the estimate for } \mathrm{k}^{\text {th }} \text { parameter, } \\
\beta_{k}^{*} & \text { is the hypothesized value for } \mathrm{k}^{\text {th }} \text { parameter, } \\
S_{k} & \text { is the standard error of } \mathrm{k}^{\text {th }} \text { parameter. }
\end{array}
$$


Table 4-10: Critical t-values for selected confidence levels (Koppelman and Bhat, 2006) Confidence Level Critical t-value (two-tailed test)

\begin{tabular}{ll}
\hline $90 \%$ & 1.645 \\
\hline $95 \%$ & 1.960 \\
\hline $99 \%$ & 2.576 \\
\hline $99.5 \%$ & 2.810 \\
\hline $99.9 \%$ & 3.290 \\
\hline
\end{tabular}

\subsubsection{Tests of entire models}

Statistical tests used for evaluating multiple hypotheses that some parameters are zero or pairs of parameters are equal to one another can be conducted by using equation (4.11). The model before imposing a restriction is considered the unrestricted model. After imposing restrictions of multiple hypotheses mentioned above, the model becomes a restricted model. By comparing their log-likelihood value at convergence, we can get a test statistic, which takes a form as follow:

$$
-2 \times\left[L L_{R}-L L_{U}\right]
$$

where

$$
\begin{aligned}
& L L_{R} \quad \text { is the log-likelihood in the restricted model, } \\
& L L_{U} \quad \text { is the log-likelihood in the unrestricted model. }
\end{aligned}
$$


This test statistic follows chi-squared distribution, which has a different probability distribution function (PDF) in response to the number of restrictions (equivalently the degrees of freedom). The critical chi-Squared values are different when changing both the desired confidence level and the number of restrictions (Koppelman and Bhat, 2006). Some critical chi-squared values are given in table 4-11. For example, using the results of table 4-2, the test statistic can be calculated to decide whether the pre-fixed hypothesis can be rejected or not. If the null hypothesis that the variables provide no additional information except for alternative-specific constants $\left(\beta_{6} \beta_{7} \beta_{8} \beta_{9}\right)$, the restrictions for this null hypothesis are:

$H_{0}: \beta_{1}=\beta_{2}=\beta_{3}=\beta_{4}=\beta_{5}=0$

The test statistic is $-2[L L(C)-L L(\beta)]=107.5835218$ and a researcher by referring to table 4-11 (number of restriction is 5 , critical value is 20.51) can reject the null hypothesis at a confidence level higher than $99.9 \%$, in conclusion that true parameter of these five variables is not zero and it is meaningful to include these variables in model specification. 
Table 4-11: Critical Chi-Square $\chi^{2}$ Values (Koppelman and Bhat, 2006)

\begin{tabular}{|c|c|c|c|c|c|c|c|c|c|}
\hline \multirow{2}{*}{ Level of Confidence } & \multicolumn{9}{|c|}{ Number of Restrictions } \\
\hline & 1 & 2 & 3 & 4 & 5 & 7 & 10 & 12 & 15 \\
\hline $90 \%$ & 2.71 & 4.61 & 6.25 & 7.78 & 9.24 & 12.01 & 15.99 & 18.54 & 22.31 \\
\hline $95 \%$ & 3.84 & 5.99 & 7.81 & 9.49 & 11.07 & 14.06 & 18.31 & 21.02 & 25.00 \\
\hline $99 \%$ & 6.63 & 9.21 & 11.34 & 13.28 & 15.09 & 18.48 & 23.21 & 26.21 & 30.58 \\
\hline $99.5 \%$ & 7.88 & 10.60 & 12.84 & 14.86 & 16.75 & 20.28 & 25.19 & 28.30 & 32.80 \\
\hline $99.9 \%$ & 10.83 & 13.82 & 16.27 & 18.47 & 20.51 & 24.32 & 29.59 & 32.91 & 37.70 \\
\hline
\end{tabular}

\subsubsection{Overall goodness-of-fit measures}

Overall goodness-of-fit in discrete choice model can be described with $\rho^{2}$, called the rho-squared value. As shown in the following figure 4-1, by using the scalar relationship among the log-likelihood values in the case of NR (see table 4-2) for a zero parameter model (in this thesis, its value is $L L(0)=\ln (1 / 5) \times 540=-869.0964727$ ), a constant only model $(L L(C)=-759.3300624)$, the estimated model $(L L(\beta)=-705.5383015)$, and a perfect prediction model $\left(L L\left(\beta_{\text {true }}\right)=\ln (1) \times 540=0\right)$, four different rho-squared value can be calculated by using the equations (4.13) to (4.16).

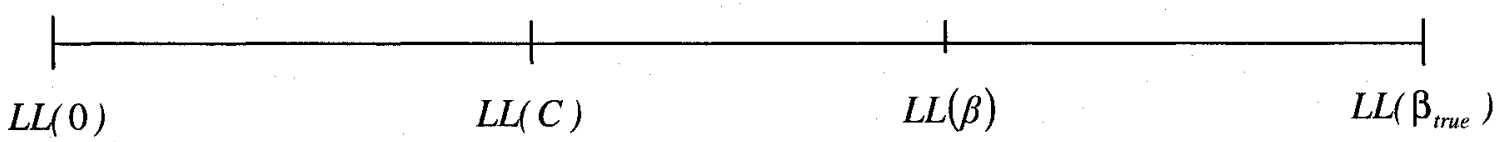

Figure 4-1: The scalar relationship of log-likelihood functions (Koppelman and Bhat, 2006)

The formulas are given below: 


$$
\begin{aligned}
& \rho_{0}^{2}=\frac{L L(\beta)-L L(0)}{L L\left(\beta_{\text {true }}\right)-L L(0)}=1-\frac{L L(\beta)}{L L(0)}, \\
& \rho_{c}^{2}=\frac{L L(\beta)-L L(C)}{L L\left(\beta_{\text {true }}\right)-L L(C)}=1-\frac{L L(\beta)}{L L(C)}, \\
& \bar{\rho}_{0}^{2}=\frac{[L L(\beta)-K]-L L(0)}{L L\left(\beta_{\text {true }}\right)-L L(0)}=1-\frac{L L(\beta)-K}{L L(0)}, \\
& \bar{\rho}_{C}^{2}=\frac{[L L(\beta)-K]-\left[L L(C)-K_{C O}\right]}{L L\left(\beta_{\text {true }}\right)-\left[L L(C)-K_{C O}\right]}=1-\frac{L L(\beta)-K}{L L(C)-K_{C O}},
\end{aligned}
$$

where

$L L(0) \quad$ is the $\log$-likelihood with $\beta_{k}=0$ (results in equal probability of choosing each available alternative, in this thesis $540 \times \ln (1 / 5)=-$ 869.0964727),

$L L(C) \quad$ is the log-likelihood for constant only model,

$L L(\beta) \quad$ is the log-likelihood for estimated model,

$L L\left(\beta_{\text {true }}\right) \quad$ is the log-likelihood for the perfect prediction model (for each observation $L L(1)=0$, thus $\left.L L\left(\beta_{\text {true }}\right)=0\right)$,

$K \quad$ is the number of degrees of freedom (parameters) used in the model,

$K_{C O} \quad$ is the number of degrees of freedom (parameters) used in the constants only model.

This rho-squared measures are widely used for evaluating the goodness of fit for discrete choice model and have generally a value between 0 and 1 . A value of zero mean that the estimated model is no better than the equally likely model (no coefficients model) and a 
value of one means that the estimated model predicts every choice perfectly. However, there are no guidelines for a "good" rho-squared value (Koppelman and Bhat, 2006). Therefore, caution should be exercised in evaluating a model using rho-squared measures. The log-likelihood value calculated from each alternative model specification can be more useful in selecting a preferred specification among alternative model specifications (Koppelman and Bhat, 2006). All rho-squared measures presented above are calculated in the context of this thesis research and the values are presented in tables 4-2 to 4-9 for the eight estimation algorithms. 


\section{Chapter 5 A Comparison of the Eight Estimation Algorithms in terms of Three Performance Measures}

\subsection{Overview}

This chapter covers the investigation of the factors affecting the performance of estimation algorithms such as one iteration time, the number of iterations and the convergence time, and experimental runs for each of the eight algorithms conducted according to three categories of experiments. After applying the different step sizes following the strategy described in chapter 3 , those experimental runs that converged successfully are chosen and summarized. The three categories of experimental runs are noted next. (1) The first category deals with different convergence criteria. These are conducted as experiments 1 to 4 . (2) The second category is about different initial guess of the parameters of the model. This is named as experiment 5. (3) The third category employs a different way of calculating the initial Hessian matrix, instead of using identity matrix. This is called experiment 6. The third category is therefore applied to only algorithms which basically use the identity matrix as an initial Hessian matrix in the model estimation procedures (i.e., DFP, BFGS, DFG-GSM, BFGS-GSM). Throughout the thesis, the eight estimation algorithms can be recognized with the unique serial number as shown in table 5-1, and three categories used to compare the estimation performance are presented in table 5-2. All mathematical symbols used to describe convergence criteria have already been introduced in chapter 3 . In the case of starting points of parameters, two types of strategies are introduced, the first strategy is that all nine parameters are assumed to have $\beta_{\text {first }}=\left[\beta_{1}=-0.1, \beta_{2}=-0.1, \beta_{3}=0, \cdots, \beta_{9}=0\right]^{\prime}$ as an initial guess for the parameters, the second strategy is that all the parameters of model 
are assumed to have $\beta_{\text {second }}=\left[\beta_{1}=-0.01, \beta_{2}=-0.1, \beta_{3}=0, \cdots, \beta_{9}=0\right]^{\prime}$ as an initial guess for the parameters. The reason of using different initial guess is that the last four algorithms did not converge with strategy one. In experiment 6 , by writing a new routine for calculating an initial Hessian matrix using the method employed in both BHHH and BHHH-2 algorithms, the possibility of showing the different estimation performance was investigated.

Table 5-1: The designated serial number for the eight estimation algorithms

Algorithms The unique serial number

\begin{tabular}{ccccc}
\hline The first four algorithms & Newton Raphson-(1) & BHHH-(2) & BHHH-2-(3) & Steepest Ascent-(4) \\
\hline The last four algorithms & DFP-(5) & BFGS-(6) & DFP-GSM-(7) & BFGS-GSM-(8) \\
\hline
\end{tabular}

Table 5-2: Three categories used in experimental runs Three categories

Experiment scheme (1) Convergence Criteria (Exp. 1)

(2) Initial guess of parameters (Exp. 2)

(3) Initial Hessian (Exp. 3)

\begin{tabular}{ccccc}
\hline $\begin{array}{c}\text { Experiment 1 } \\
\text { (applied to the all algorithms) }\end{array}$ & {$\left[\frac{1}{k} \sum_{k=1}^{k}\left(\beta_{t+1, k}-\beta_{t, k}\right)^{2}\right]^{1 / 2}<10^{-6}$} & - & - \\
\hline $\begin{array}{c}\text { Experiment 2 } \\
\text { (applied to the all algorithms) }\end{array}$ & $G_{t}^{\prime}\left(-H_{t}\right)^{-1} G_{t}<10^{-4}$ & - & - \\
\hline $\begin{array}{c}\text { Experiment 3 } \\
\text { (applied to the all algorithms) }\end{array}$ & $G_{t}^{\prime}\left(-H_{t}\right)^{-1} G_{t}<10^{-5}$ & - & - \\
\hline $\begin{array}{c}\text { Experiment 4 } \\
\text { (applied to the all algorithms) }\end{array}$ & $G_{t}^{\prime}\left(-H_{t}\right)^{-1} G_{t}<10^{-6}$ & & - & - \\
\hline $\begin{array}{c}\text { Experiment 5 } \\
\text { (applied to the all algorithms } \\
\text { with different scheme) }\end{array}$ & & $\begin{array}{c}\text { The first four algorithms } \\
\text { (the first strategy ) }\end{array}$ & The last four algorithms \\
(the second strategy) & & - \\
\hline $\begin{array}{c}\text { Experiment 6 } \\
\text { (applied to the last four } \\
\text { algorithms) }\end{array}$ & - & & BHHH \\
\hline
\end{tabular}

As previously noted, the main purpose of the thesis research is first to compare the estimation algorithms in estimating a discrete choice model (experiment 1). Next one is to identify the estimation performance due to applying the different stopping criteria 
(experiment 2, experiment 3, and experiment 4). Furthermore, with the assumption that the initial guess of parameters can be a factor for controlling the estimation performance, it is of interest to recognize the estimation performance in terms of performance measures (experiment 5). Another item of research is to show the possibility of improving the estimation performance by using the more correct approximation of initial Hessian matrix instead of using identity matrix in the estimation process (experiment 6). Through these kinds of the extensive experimental works, a researcher can find out "what factors make differences and how these factors work in the estimation procedures which are used in different algorithms?" Consequently, after identifying the factors and understanding their effects, a researcher can enhance the current estimation method faster and more efficient in terms of performance measures. All experimental studies conducted here used the tailor-made code designed for these specific experimental estimations. The following sections present results.

\subsection{A comparison of the variations according to the convergence criterion (experiments 1-4)}

\subsubsection{The first convergence criterion (experiment 1)}

A vector of the model's parameters is estimated with the first convergence criterion, as given in table 5-2, experiment 1 . This convergence criterion is sourced from Ben-Akiva and Lerman (1985). In this case, if the prefixed conditions (experiment 1) are satisfied, the iteration is terminated with $\beta_{t+1, k}$ as the solution. The precision of model's parameters can be controlled by changing $10^{-4}$ to other more strict values such as $10^{-5}, 10^{-6}$ and so on. The results of the experimental estimation for the eight different algorithms are summarized in the table 5-3 for only experimental trials that converged. 
Table 5-3: The final experimental results of the eight estimation algorithms (exp. 1)

\begin{tabular}{|c|c|c|c|c|c|c|c|c|c|}
\hline \multirow{2}{*}{\multicolumn{2}{|c|}{$\begin{array}{c}\text { Algorithms \& } \\
\text { Performance } \\
\text { measures } \\
\end{array}$}} & \multicolumn{8}{|c|}{ Step size $(\lambda)$} \\
\hline & & $1 / 32$ & $1 / 16$ & $1 / 8$ & $1 / 4$ & $1 / 2$ & 1 & 2 & 4 \\
\hline \multirow{3}{*}{ 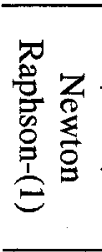 } & $\begin{array}{c}\text { One iteration } \\
\text { time }\end{array}$ & - & $0: 00: 23$ & $0: 00: 23$ & $0: 00: 22$ & 0:00:20 & 0:00:20 & - & - \\
\hline & $\begin{array}{c}\text { Number of } \\
\text { iterations }\end{array}$ & - & 99 & 54 & 29 & 14 & 5 & - & - \\
\hline & $\begin{array}{c}\text { Convergence } \\
\text { time }\end{array}$ & - & $0: 38: 44$ & $0: 20: 16$ & $0: 10: 33$ & 0:05:13 & 0:01:43 & - & - \\
\hline \multirow{3}{*}{$\begin{array}{l}\text { 罝 } \\
\text { 空 } \\
\text { 空 }\end{array}$} & $\begin{array}{c}\text { One iteration } \\
\text { time }\end{array}$ & - & - & - & $0: 00: 28$ & $0: 00: 25$ & $0: 00: 33$ & - & - \\
\hline & $\begin{array}{l}\text { Number of } \\
\text { iterations }\end{array}$ & - & - & - & 156 & 91 & 51 & - & - \\
\hline & $\begin{array}{c}\text { Convergence } \\
\text { time }\end{array}$ & - & - & - & $1: 11: 45$ & $0: 38: 15$ & $0: 27: 44$ & - & - \\
\hline \multirow{3}{*}{ 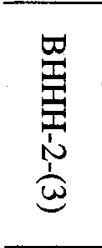 } & $\begin{array}{c}\text { One iteration } \\
\text { time }\end{array}$ & - & - & - & $0: 00: 53$ & $0: 00: 32$ & $0: 00: 32$ & $0: 00: 49$ & $0: 00: 33$ \\
\hline & $\begin{array}{c}\text { Number of } \\
\text { iterations }\end{array}$ & - & - & - & 318 & 194 & 114 & 65 & 238 \\
\hline & $\begin{array}{c}\text { Convergence } \\
\text { time }\end{array}$ & - & - & - & $4: 40: 07$ & $1: 44: 08$ & $1: 00: 57$ & 0:53:09 & $2: 11: 38$ \\
\hline \multirow{3}{*}{ 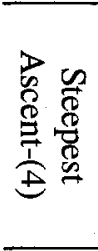 } & $\begin{array}{c}\text { One iteration } \\
\text { time }\end{array}$ & $0: 00: 36$ & $0: 00: 43$ & - & - & - & - & - & - \\
\hline & $\begin{array}{c}\text { Number of } \\
\text { iterations }\end{array}$ & 951 & 697 & - & - & - & - & - & - \\
\hline & $\begin{array}{c}\text { Convergence } \\
\text { time }\end{array}$ & $9: 34: 02$ & $8: 21: 58$ & - & - & - & - & - & - \\
\hline \multirow{3}{*}{$\frac{0}{\frac{7}{3}}$} & $\begin{array}{c}\text { One iteration } \\
\text { time }\end{array}$ & - & $0: 00: 23$ & $0: 00: 15$ & $0: 00: 15$ & 0:00:14 & - & - & - \\
\hline & $\begin{array}{c}\text { Number of } \\
\text { iterations }\end{array}$ & - & 224 & 193 & 155 & 115 & - & - & - \\
\hline & $\begin{array}{c}\text { Convergence } \\
\text { time }\end{array}$ & - & $1: 25: 04$ & $0: 47: 26$ & $0: 38: 49$ & $0: 27: 32$ & - & - & - \\
\hline \multirow{4}{*}{ 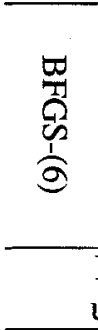 } & $\begin{array}{c}\text { One iteration } \\
\text { time }\end{array}$ & $0: 00: 16$ & $0: 00: 28$ & 0:00:39 & $0: 00: 21$ & $0: 00: 45$ & $0: 00: 13$ & - & - \\
\hline & $\begin{array}{c}\text { Number of } \\
\text { iterations }\end{array}$ & 268 & 162 & 107 & 77 & 52 & 42 & - & - \\
\hline & $\begin{array}{c}\text { Convergence } \\
\text { time }\end{array}$ & $1: 33: 28$ & $1: 15: 04$ & $1: 08: 50$ & $0: 26: 15$ & $0: 38: 52$ & $0: 09: 16$ & - & - \\
\hline & $\begin{array}{l}\text { Interval of } \\
\text { uncertainty }\end{array}$ & $\begin{array}{l}\mathrm{LL}(0) \\
\mathrm{UL}(1)\end{array}$ & $\begin{array}{l}\text { LL(0), } \\
\text { UL(2) }\end{array}$ & $\begin{array}{l}\mathrm{LL}(0) \\
\mathrm{UL}(3)\end{array}$ & $\begin{array}{l}\text { LL(0), } \\
\text { UL(4) }\end{array}$ & $\begin{array}{l}\text { LL(0), } \\
\text { UL(5) }\end{array}$ & $\begin{array}{l}\mathrm{LL}(0) \\
\mathrm{UL}(6)\end{array}$ & $\begin{array}{l}\mathrm{LL}(0), \\
\mathrm{UL}(7)\end{array}$ & $\begin{array}{l}\text { LL(0), } \\
\text { UL(8) }\end{array}$ \\
\hline \multirow{3}{*}{ 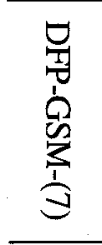 } & $\begin{array}{c}\text { One iteration } \\
\text { time }\end{array}$ & $0: 00: 44$ & 0:01:09 & 0:00:59 & 0:01:04 & 0:01:44 & 0:01:52 & 0:01:54 & 0:01:55 \\
\hline & $\begin{array}{c}\text { Number of } \\
\text { iterations }\end{array}$ & 138 & 23 & 27 & 26 & 24 & 24 & 24 & 23 \\
\hline & $\begin{array}{c}\text { Convergence } \\
\text { time }\end{array}$ & $1: 40: 55$ & $0: 26: 29$ & $0: 26: 29$ & $0: 27: 40$ & $0: 41: 38$ & $0: 44: 46$ & $0: 45: 37$ & $0: 44: 01$ \\
\hline \multirow{3}{*}{ 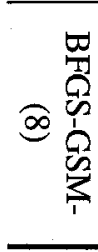 } & $\begin{array}{c}\text { One iteration } \\
\text { time }\end{array}$ & $0: 00: 48$ & $0: 00: 48$ & 0:00:53 & $0: 00: 56$ & 0:01:02 & 0:01:06 & 0:01:06 & 0:02:05 \\
\hline & $\begin{array}{l}\text { Number of } \\
\text { iterations }\end{array}$ & 38 & 25 & 21 & 20 & 20 & 19 & 19 & 19 \\
\hline & $\begin{array}{c}\text { Convergence } \\
\text { time }\end{array}$ & $0: 30: 07$ & $0: 19: 45$ & $0: 18: 33$ & $0: 18: 42$ & $0: 20: 30$ & $0: 20: 58$ & $0: 20: 55$ & $0: 39: 35$ \\
\hline
\end{tabular}



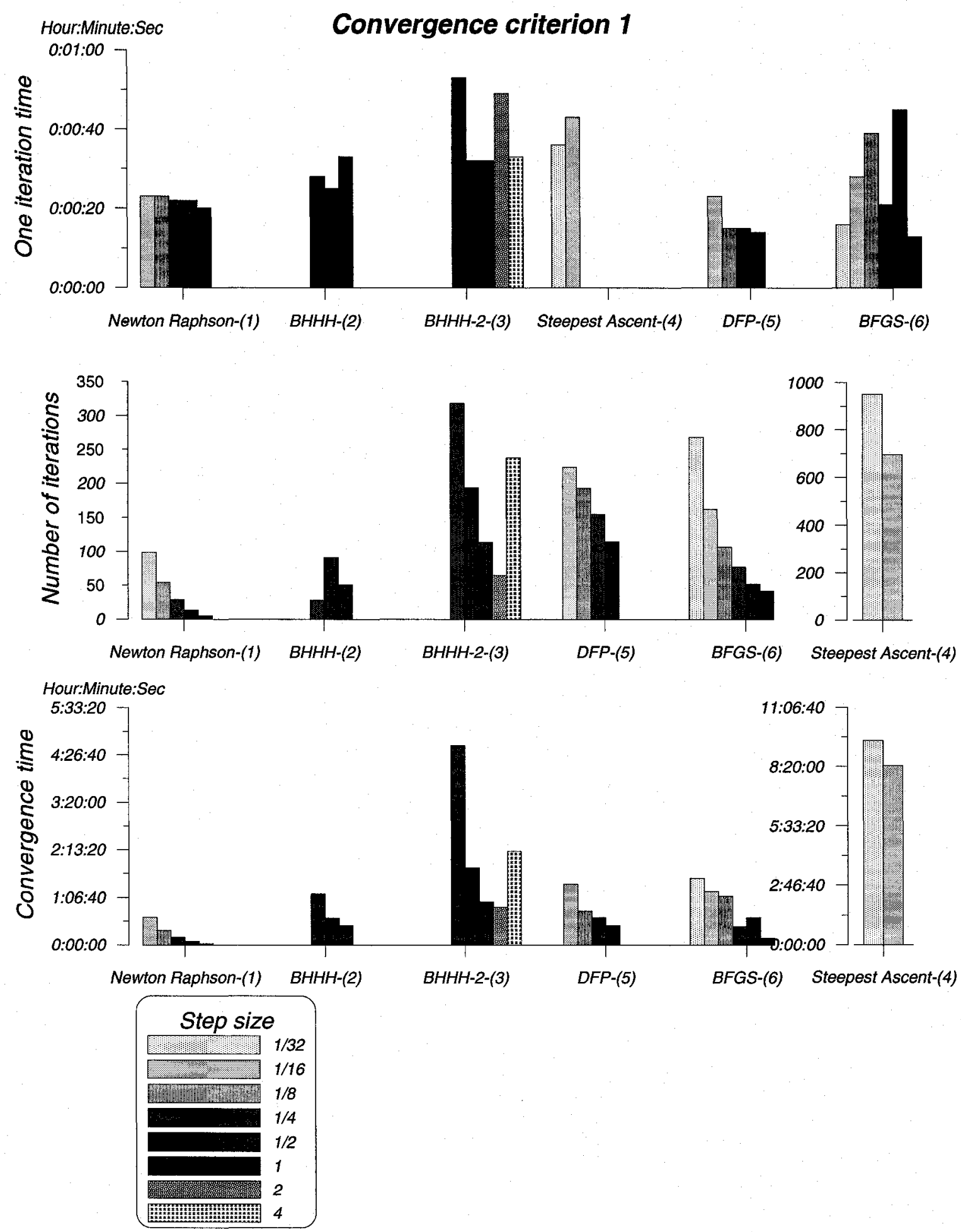

Figure 5-1: The final experimental results of the first six estimation algorithms (exp. 1) 

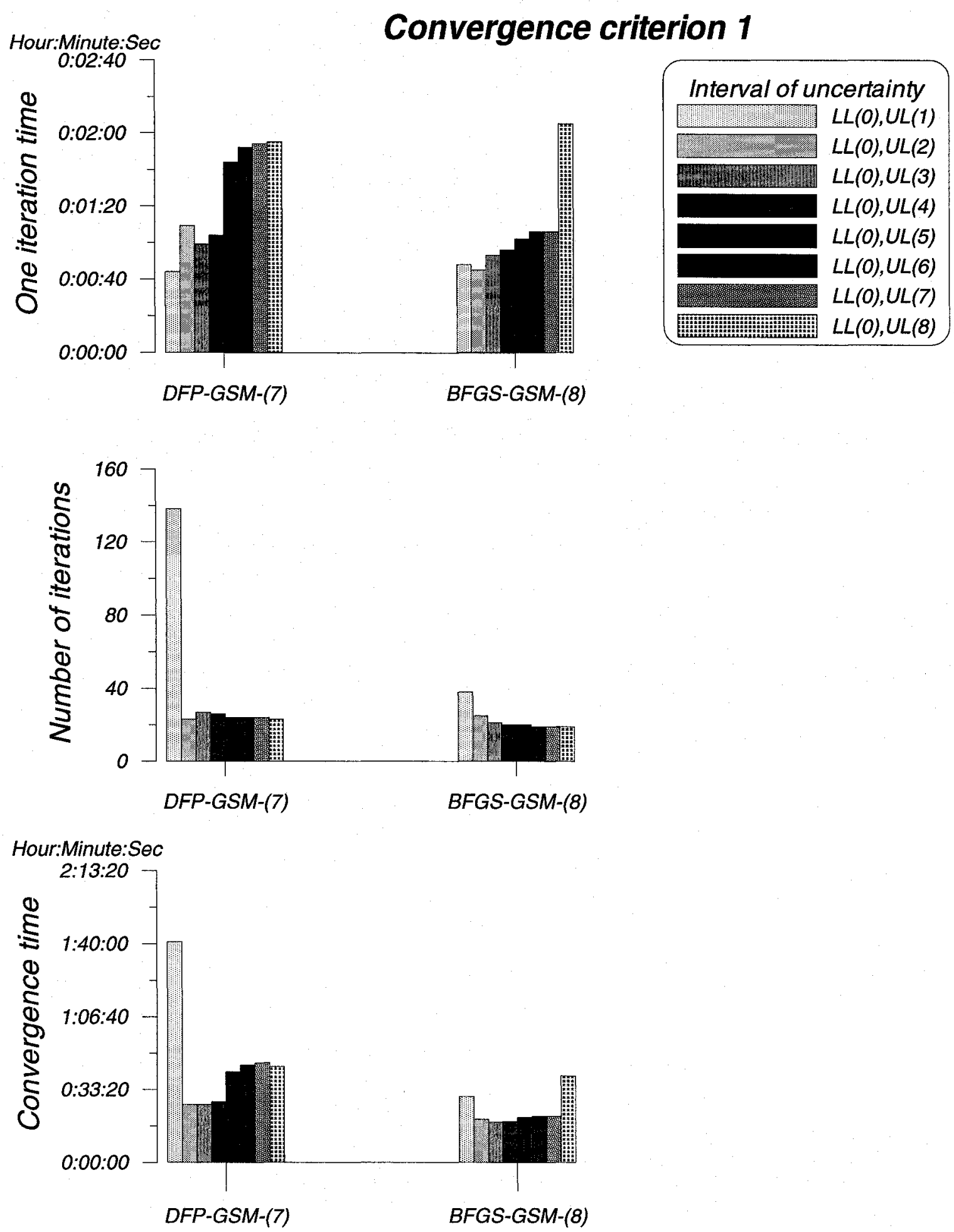

Figure 5-2: The final experimental results of the last two estimation algorithms (exp. 1) 
As shown in the table 5-3 diverse step sizes were tried for each of the eight different algorithms. The reason for trying different step size is already explained in chapter 3 . That is, it is to find the best value of step size for making the log-likelihood function to increase in the fastest way until it reaches the maximum. In the first six algorithms, the step size is fixed by a researcher by using the strategy introduced in chapter 3 . In the last two algorithms, DFP-GSM, BFGS-GSM, the step size is calculated automatically with the function which is imbedded in the general estimation routine and is operated with the golden-section line search method. Instead of choosing step size manually, a researcher must establish interval of uncertainty in which the best step size is searched by the golden section line search method. In this thesis research, eight different uncertain intervals are used and compared. Typically, there are no certain limitations in choosing the uncertain intervals, choosing the value above 0 is common (Bazaraa et al., 1999).

Figure 5-1 shows the estimation performances of the first six algorithms in terms of three performance measures and figure 5-2 shows the last two algorithms. The first six algorithms converge or do not converge in different step sizes. Only converged runs appear both in table 5-3 and figure 5-1. In contrast, the last two algorithms converge in the every uncertain interval and their results are presented in lower parts of table 5-3 and figure 5-2. For the first performance measure, one iteration time, there is a little difference between the first six algorithms. Some estimates of DFP and BFGS are slightly faster than other algorithms' one iteration time. On the other hand, there are somewhat large differences between the first six algorithms and the last two algorithms in terms of their one iteration time. This means that the last two algorithms take more one iteration time due to the procedure added for conducting a line search. It takes $1 \sim 2$ minutes for 
DFP-GSM and BFGS-GSM to finish one iteration. The values of one iteration time can be used to recognize the operational superiority or inferiority of algorithm because it dominates the overall convergence time for all algorithms.

For the second performance measure, the number of iterations, obviously there are big differences both between the step sizes in the same algorithm and between the eight different algorithms. As a result the Newton Raphson algorithm shows the best performance throughout the overall runs of the eight algorithms. It converges just after 5 iterations, compared to the Steepest Ascent algorithm which shows the worst performance, it is very fast and thus efficient. In case of Newton Raphson and DFP, BFGS, as the step size increases the number of iterations decrease. This indicates that there is a close relationship between the step size and number of iterations. Choosing a step size randomly at the initial stage of the estimation can affect the estimation performance negatively if the selection is poor or affect positively if the selection is fortunately good. To address the problem of choosing initial step size and to improve the estimation performance, in this thesis, a new systematic method, namely Golden-Section line search, is incorporated in algorithms, DFP and BFGS. As a result, DFP-GSM and BFGS-GSM algorithms show a stable estimation performance in terms of the number of iterations. The iteration numbers presented in the middle part of figure 5-2 are nearly similar for the all uncertain interval considered in experimental runs except one run using $L_{\text {ower }} L_{\text {imit }}(0), U_{\text {pper }} L_{\text {imit }}(1)$ as an uncertain interval. Stated simply, the DFP-GSM and BFGS-GSM algorithms compared to the DFP and BFGS in figure 5-1 definitely make a decrease in the number of iterations. Moreover the iteration number converges to around 
a certain value: these new algorithms are stable in terms of the number of iterations.

The third performance measure, the convergence time, is used to compare the estimation performance of the eight algorithms. This measure is the most important measure in order to decide the excellence of an algorithm because the operational time before convergence is of much interest to researchers, especially in large scale modelling it can be very critical. The Newton Raphson algorithm generally shows a good performance in terms of the convergence time for all step sizes. Particularly, in case where the step size $(\lambda)$ is 1 , the algorithm takes just 1 minute and 43 seconds to finish its calculation. This convergence time is very fast compared to any other algorithm. In contrast, the Steepest Ascent (SA) algorithm takes 9 hours, 34 minutes and 2 seconds to finish its calculation. Clearly, there is a huge difference in the convergence time between two algorithms. The difference occurred due to the use of a different way of acquiring the Hessian matrix in the estimation process as discussed in detail in chapter 3. The NR is correct and absolutely fast because it uses real Hessian matrix of the given log-likelihood function, which is calculated analytically by partial derivatives of the function to second-order. The algebraic manipulations involved are time consuming and burdensome to a researcher, and furthermore these take much time to handle. However, even though algebraic manipulations are very intensive and therefore expensive, the excellence of the estimation performance of the NR with the real Hessian dominates other algorithms which adopt a different way of calculating the approximate Hessian matrix. The NR algorithm is an excellent estimation algorithm, although until recently, it was recognized to be difficult to use. The experience acquired in this thesis research indicates that the algebraic 
manipulations are perceived to be more difficult than these actually are.

Other emerging facts in other estimation algorithms are as follows: (1) BHHH algorithm shows a better performance than BHHH-2 in terms of the convergence time. These two algorithms use almost the same estimation procedures except for using a slightly different way to calculate the Hessian matrix. (2) As quasi-newton methods, DFP and BFGS algorithms use a similar updating process for the Hessian matrix as explained in chapter 3. The BFGS algorithm takes less convergence time (e.g., $\lambda=1 / 4$ or $\lambda=1 / 16$ ) or more convergence time (e.g., $\lambda=1 / 8, \lambda=1 / 2$ ) than DFP when compared in the same step size. Key point is that, even though the number of iterations is much less in BFGS than that in DFP, the difference in convergence time between two algorithms is not significant. This phenomenon might be attributed to the updating process of the Hessian matrix: BFGS uses a more complicated process than that of DFP and it takes more time in finishing one iteration (please see chapter 3). (3) The last two algorithms used for the first time in this thesis research, namely DFP-GSM and BFGS-GSM, show stable estimation performance. In other words, the convergence times are distributed more evenly than other algorithms. The BFGS-GSM shows slightly better performance than the DFP-GSM in terms of convergence time.

In conclusion, in experiment 1 that employs the first convergence criterion, NR is the best algorithm in terms of all three performance measures in spite of its mathematical difficulty to calculate the real Hessian of the given log-likelihood function. In the remaining algorithms that use approximate Hessian matrix, DFP-GSM and BFGS-GSM 
show stable performance in terms of both the number of iterations and the convergence time irrespective of the choice of any uncertain interval. $\mathrm{BHHH}$ and $\mathrm{BHHH}-2, \mathrm{DFP}$ and BFGS show somewhat large differences in estimation performance, depending on the selection of different step sizes at the initial stage of estimation.

\subsubsection{The number of iterations and the log-likelihood values (experiment 1)}

The line graphs of the experimental estimations are drawn to show the trace of $\log$ likelihood function values during the iterations and moreover to compare clearly the different convergence behaviours between step sizes in each algorithm. Tables 5-4 to 5-9 summarize critical values in iterations such as starting log-likelihood value and convergence log-likelihood value, and number of iterations according to different step sizes. As shown in tables 5-4 to 5-9, all starting log-likelihood values are exactly the same for the all eight algorithms and for any step sizes within each algorithm because as discussed in an earlier section the initial guess of parameters is assumed to be zero, $\beta_{k}=\left[\beta_{1}=0, \beta_{2}=0, \beta_{3}=0, \cdots, \beta_{9}=0\right]^{\prime}$. On the contrary, convergence values are slightly different both between algorithms and between step sizes in each algorithm, but the differences are so small that these can be considered to be equal. Table 5-10 and table 5-11 summarize critical values for the same factors with the above noted first six algorithms for each uncertain interval. As shown in tables 5-10 and 5-11, all starting loglikelihood values are exactly the same due to the same reason as explained in the first six algorithms. In the case of the convergent log-likelihood values, slight differences exit among uncertain intervals but because these are so small, these can be ignored. 
Table 5-4: Log-likelihood values obtained from Newton Raphson algorithm (exp. 1)

\begin{tabular}{|c|c|c|c|c|c|c|}
\hline \multirow{2}{*}{\multicolumn{2}{|c|}{ Log-likelihood }} & \multicolumn{5}{|c|}{ Step size $(\lambda)$} \\
\hline & & $1 / 16$ & $1 / 8$ & $1 / 4$ & $1 / 2$ & 1 \\
\hline \multirow{3}{*}{ 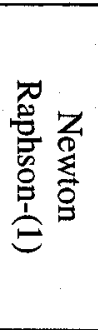 } & $\begin{array}{c}\text { Starting } \\
\text { Log- } \\
\text { likelihood }\end{array}$ & -869.0964727 & -869.0964727 & -869.0964727 & -869.0964727 & -869.0964727 \\
\hline & $\begin{array}{c}\text { Convergence } \\
\text { log- } \\
\text { likelihood }\end{array}$ & -705.5383015 & -705.5383074 & -705.5383335 & -705.5385119 & -705.5392124 \\
\hline & $\begin{array}{l}\text { Number of } \\
\text { iterations }\end{array}$ & 99 & 54 & 29 & 14 & 5 \\
\hline
\end{tabular}

Table 5-5: Log-likelihood values obtained from BHHH algorithm (exp. 1)

\begin{tabular}{ccccc}
\hline & Log-likelihood & \multicolumn{3}{c}{ Step size $(\lambda)$} \\
\cline { 2 - 5 } & $\begin{array}{c}\text { Starting } \\
\text { Log-likelihood }\end{array}$ & -869.0964727 & -869.0964727 & -869.0964727 \\
\hline \multirow{2}{*}{$\begin{array}{c}\text { Convergence } \\
\text { Log-likelihood }\end{array}$} & -705.5397332 & -705.5386333 & -705.5383823 \\
\cline { 2 - 5 } & Number of iterations & 156 & 91 & 51 \\
\hline
\end{tabular}

Table 5-6: Log-likelihood values obtained from BHHH-2 algorithm (exp. 1)

\begin{tabular}{|c|c|c|c|c|c|c|}
\hline & \multirow{2}{*}{ Log-likelihood } & \multicolumn{5}{|c|}{ Step size $(\lambda)$} \\
\hline & & $\overline{1 / 4}$ & $1 / 2$ & 1 & 2 & 4 \\
\hline \multirow{3}{*}{ 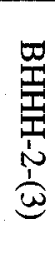 } & $\begin{array}{c}\text { Starting } \\
\text { Log-likelihood }\end{array}$ & $\begin{array}{c}- \\
869.0964727\end{array}$ & $\begin{array}{c}- \\
869.0964727\end{array}$ & $\begin{array}{c}- \\
869.0964727\end{array}$ & $\begin{array}{c}- \\
869.0964727\end{array}$ & $\begin{array}{c}- \\
869.0964727\end{array}$ \\
\hline & $\begin{array}{l}\text { Convergence } \\
\text { log-likelihood }\end{array}$ & $\begin{array}{c}- \\
705.5494505\end{array}$ & $\begin{array}{c}- \\
705.5410054\end{array}$ & $\begin{array}{c}- \\
705.5389693\end{array}$ & $\begin{array}{c}- \\
705.5384515\end{array}$ & $\begin{array}{c}- \\
705.5383019\end{array}$ \\
\hline & Number of iterations & 318 & 194 & 114 & 65 & 238 \\
\hline
\end{tabular}

Table 5-7: Log-likelihood values obtained from Steepest Ascent algorithm (exp. 1)

\begin{tabular}{|c|c|c|c|}
\hline \multirow{2}{*}{\multicolumn{2}{|c|}{ Log-likelihood }} & \multicolumn{2}{|c|}{ Step size $(\lambda)$} \\
\hline & & $1 / 32$ & $1 / 16$ \\
\hline \multirow{3}{*}{ 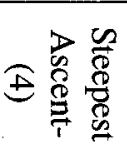 } & $\begin{array}{c}\text { Starting } \\
\text { Log-likelihood }\end{array}$ & -869.0964727 & -869.0964727 \\
\hline & Convergence log-likelihood & -705.5383015 & -705.5383074 \\
\hline & Number of iterations & 951 & 697 \\
\hline
\end{tabular}


Table 5-8: Log-likelihood values obtained from DFP algorithm (exp. 1)

\begin{tabular}{|c|c|c|c|c|c|}
\hline \multirow{2}{*}{\multicolumn{2}{|c|}{ Log-likelihood }} & \multicolumn{4}{|c|}{ Step size $(\lambda)$} \\
\hline & & $1 / 16$ & $1 / 8$ & $1 / 4$ & $1 / 2$ \\
\hline \multirow{3}{*}{$\frac{\overrightarrow{0}}{\frac{1}{3}}$} & $\begin{array}{c}\text { Starting } \\
\text { Log-likelihood }\end{array}$ & -869.0964727 & -869.0964727 & -869.0964727 & -869.0964727 \\
\hline & Convergence log-likelihood & -705.5398009 & -705.5394156 & -705.5396982 & -705.5406805 \\
\hline & Number of iterations & 224 & 193 & 155 & 115 \\
\hline
\end{tabular}

Table 5-9: Log-likelihood values obtained from BFGS algorithm (exp. 1)

\begin{tabular}{|c|c|c|c|c|c|c|c|}
\hline \multirow{2}{*}{\multicolumn{2}{|c|}{ Log-likelihood }} & \multicolumn{6}{|c|}{ Step size $(\lambda)$} \\
\hline & & $1 / 32$ & $1 / 16$ & $1 / 8$ & $1 / 4$ & $1 / 2$ & 1 \\
\hline \multirow{3}{*}{ 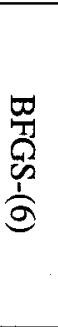 } & $\begin{array}{c}\text { Starting } \\
\text { Log- } \\
\text { likelihood }\end{array}$ & -869.0964727 & -869.0964727 & -869.0964727 & -869.0964727 & -869.0964727 & -869.0964727 \\
\hline & $\begin{array}{c}\text { Convergence } \\
\text { log- } \\
\text { likelihood } \\
\end{array}$ & -705.5387064 & -705.5384933 & -705.5384097 & -705.5383265 & -705.5383351 & -705.5383081 \\
\hline & $\begin{array}{l}\text { Number of } \\
\text { iterations }\end{array}$ & 268 & 162 & 107 & 77 & 52 & 42 \\
\hline
\end{tabular}

Table 5-10: Log-likelihood values obtained from DFP-GSM algorithm (exp. 1)

\begin{tabular}{|c|c|c|c|c|c|c|c|c|c|}
\hline \multicolumn{2}{|c|}{ Interval of uncertainty } & $\begin{array}{l}\mathrm{LL}(0), \\
\mathrm{UL}(1)\end{array}$ & $\begin{array}{l}\mathrm{LL}(0), \\
\mathrm{UL}(2)\end{array}$ & $\begin{array}{l}\text { LL(0), } \\
\text { UL(3) }\end{array}$ & $\begin{array}{l}\text { LL(0), } \\
\text { UL(4) }\end{array}$ & $\begin{array}{l}\mathrm{LL}(0), \\
\mathrm{UL}(5)\end{array}$ & $\begin{array}{l}\mathrm{LL}(0), \\
\mathrm{UL}(6)\end{array}$ & $\begin{array}{l}\text { LL(0), } \\
\text { UL(7) }\end{array}$ & $\begin{array}{l}\text { LL(0), } \\
\text { UL(8) }\end{array}$ \\
\hline \multirow{3}{*}{ 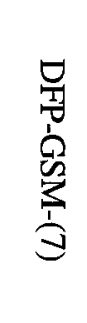 } & $\begin{array}{c}\text { Starting } \\
\text { Log- } \\
\text { likelihood }\end{array}$ & $\begin{array}{c}869.0964 \\
727\end{array}$ & $\begin{array}{c}869.0964 \\
727\end{array}$ & $\begin{array}{c}869.0964 \\
727\end{array}$ & $\begin{array}{c}869.0964 \\
727\end{array}$ & $\begin{array}{c}869.0964 \\
727\end{array}$ & $\begin{array}{c}869.0964 \\
727\end{array}$ & $\begin{array}{c}869.0964 \\
727\end{array}$ & $\begin{array}{c}869.0964 \\
727\end{array}$ \\
\hline & $\begin{array}{c}\text { Convergence } \\
\text { log- } \\
\text { likelihood }\end{array}$ & $\begin{array}{c}705.5383 \\
56\end{array}$ & $\begin{array}{c}705.5384 \\
214\end{array}$ & $\begin{array}{c}705.5383 \\
015\end{array}$ & $\begin{array}{c}705.5383 \\
015\end{array}$ & $\begin{array}{c}705.5383 \\
026\end{array}$ & $\begin{array}{c}705.5383 \\
015\end{array}$ & $\begin{array}{c}705.5383 \\
015\end{array}$ & $\begin{array}{c}705.5383 \\
015\end{array}$ \\
\hline & $\begin{array}{l}\text { Number of } \\
\text { iterations }\end{array}$ & 138 & 23 & 27 & 26 & 24 & 24 & 24 & 23 \\
\hline
\end{tabular}

Table 5-11: Log-likelihood values obtained from BFGS-GSM algorithm (exp. 1)

\begin{tabular}{|c|c|c|c|c|c|c|c|c|c|}
\hline \multicolumn{2}{|c|}{ Interval of uncertainty } & $\begin{array}{l}\text { LL(0), } \\
\text { UL(1) }\end{array}$ & $\begin{array}{l}\text { LL(0), } \\
\text { UL(2) }\end{array}$ & $\begin{array}{l}\mathrm{LL}(0), \\
\mathrm{UL}(3)\end{array}$ & $\begin{array}{l}\text { LL(0), } \\
\text { UL(4) }\end{array}$ & $\begin{array}{l}\text { LL(0), } \\
\text { UL(5) }\end{array}$ & $\begin{array}{l}\mathrm{LL}(0), \\
\mathrm{UL}(6)\end{array}$ & $\begin{array}{l}\mathrm{LL}(0), \\
\mathrm{UL}(7)\end{array}$ & $\begin{array}{l}\text { LL(0), } \\
\text { UL(8) }\end{array}$ \\
\hline \multirow{3}{*}{ 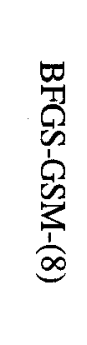 } & $\begin{array}{l}\text { Starting } \\
\text { Log- } \\
\text { likelihood }\end{array}$ & $\begin{array}{c}- \\
869.096 \\
4727\end{array}$ & $\begin{array}{c}- \\
869.096 \\
4727\end{array}$ & $\begin{array}{c}- \\
869.096 \\
4727\end{array}$ & $\begin{array}{c}- \\
869.096 \\
4727\end{array}$ & $\begin{array}{c}- \\
869.096 \\
4727\end{array}$ & $\begin{array}{c}- \\
869.096 \\
4727\end{array}$ & $\begin{array}{c}- \\
869.096 \\
4727\end{array}$ & $\begin{array}{c}- \\
869.096 \\
4727\end{array}$ \\
\hline & $\begin{array}{c}\text { Convergence } \\
\text { log- } \\
\text { likelihood }\end{array}$ & $\begin{array}{c}- \\
705.538 \\
3016\end{array}$ & $\begin{array}{c}- \\
705.538 \\
3015\end{array}$ & $\begin{array}{c}- \\
705.538 \\
3015\end{array}$ & $\begin{array}{c}- \\
705.538 \\
3016\end{array}$ & $\begin{array}{c}- \\
705.538 \\
3015\end{array}$ & $\begin{array}{c}- \\
705.538 \\
3015\end{array}$ & $\begin{array}{c}- \\
705.538 \\
3015\end{array}$ & $\begin{array}{c}- \\
705.538 \\
3015\end{array}$ \\
\hline & $\begin{array}{l}\text { Number of } \\
\text { iterations }\end{array}$ & 38 & 25 & 21 & 20 & 20 & 19 & 19 & 19 \\
\hline
\end{tabular}


Line graphs are drawn in two ways for all eight algorithms by using Grapher (2000). One way of drawing a graph is for each converged step size in experimental estimation and another way is to draw a graph for all step sizes on the same sheet. The second way is more effective for a quick comparison of different convergence behaviour of each converged step size.

In the case of the Newton Raphson algorithm where the step size is one, figures 5-3 and 5-4 and table 5-4 show the convergent log-likelihood value, -705.5392124 (which is the maximum log-likelihood value of the given log-likelihood function given in equation (4.5)), was reached just after 5 iterations. The fastest convergence is because of the steep increase of log-likelihood value in each iteration. In other words, this run has the best convergence behaviour compared to other step sizes which are slow in increasing of the $\log$-likelihood value. When taking $1 / 16$ as the step size, the line graph is the slowest of all step size in terms of slope. In conclusion, with decreasing step size, the slope also becomes low, but the number of iterations increase.

In the case of the $\mathrm{BHHH}$ algorithm where the step size is one, as shown in figures 5-5 and 5-6 and in table 5-5, the convergence log-likelihood value is -705.5383823 and it was reached after 51 iterations. This run shows the best convergence behaviour of all converged step sizes. Like a convergence behaviour of NR algorithm, with decreasing step size, the slope also becomes low, but the number of iterations increase.

In the case of the BHHH-2 algorithm where the step size is 2, as shown in figures 5-7 and 
5-8 and in table 5-6, the convergence log-likelihood value is -705.5384515 and it was reached after 65 iterations. This run shows the best performance of all step sizes. In particular, a run with step size 4 shows a good performance in the initial stage of estimation and it appears to have converged in the first place but due to the delay in the latter part of iterations, it is overtaken with step size 2. Except for the run with step size 4, the decreasing step size results in lower slope, but the number of iterations increase.

For the Steepest Ascent algorithms, only two step sizes, namely $1 / 32$ and 1/16, converged. Figure 5-9 shows its convergence behaviour and particularly the behaviour corresponding to $1 / 16$ which shows a large fluctuation without a steady increase of log-likelihood value in the initial stage of iterations relative to that using step size of $1 / 32$. This behaviour pattern is unique as compared to other algorithms. Table 5-7 gives the starting and converging value of log-likelihood.

In the case of the DFP algorithm, there is a large difference in its convergence behaviour compared to the four algorithms discussed up to this point. The most obvious difference is that the line graphs for all step sizes, except $1 / 16$, show much bigger fluctuations than that of Steepest Ascent in the initial part of iterations without a steady increase as in the first four algorithms' line graphs. This phenomenon might be assumed as a result of the mechanism used for updating the approximate Hessian matrix of this algorithm (please see chapter 3). Train (2003) indicates that this kind of algorithm, including BFGS algorithm, uses information obtained at more than one point on the log-likelihood function. Figures 5-10 and 5-11 present its convergence behaviour graphically in two 
ways. The step size of $1 / 2$ shows the best performance with 115 iterations and its critical values are presented in table 5-8.

In the case of the BFGS algorithm, like the DFP algorithm, there are large fluctuations for all step sizes except $1 / 32$ and 1/16. Moreover a similar reason used in DFP can be applied to explain this phenomenon. Overall, there is only a slight difference in the method to calculate updated approximate Hessian matrix for two algorithms, DFP and BFGS. One more term is added to BFGS algorithm as was shown in chapter 3 . A run with step size 1 outperforms the other runs, as can be seen in table 5-9. It can be noted that it experiences an extreme change in value of log-likelihood at the initial iterations.

In the last two algorithms, the use of a variety of step sizes since the the golden section line search method (GSM) takes over the role using an automatic method. Another task arises instead, making an interval of uncertainty. In this thesis research, eight different uncertain intervals are considered. It should be noted that there are no rules and no guidance is available from literature regarding the choice of intervals. Temporarily, eight different intervals characterized by an increasing of the same amount, namely 1 , were applied to simply examine the convergence behaviour that might be caused with different uncertain intervals. Figures 5-14 to 5-16 show the convergence behaviour of the DFPGSM algorithm according to various intervals. All line graphs show almost same convergence behaviour and thus are drawn in the same line irrespective of intervals used temporarily. Almost the same results were obtained from DFP-GSM and BFGS-GSM algorithm, presented in figures 5-17 to 5-19. 


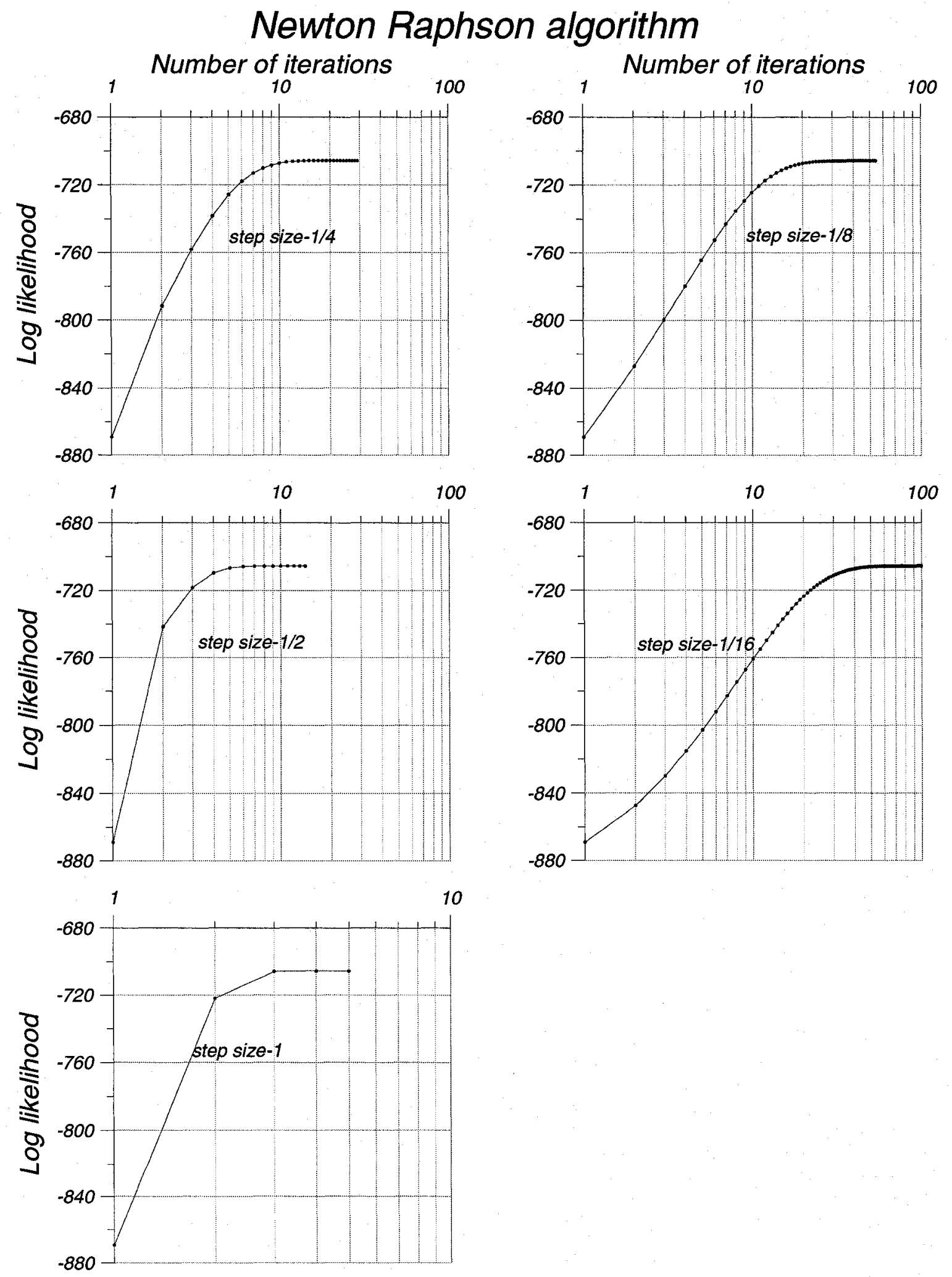

Figure 5-3: Log-likelihood values for each step size in Newton Raphson algorithm (exp. 1) 


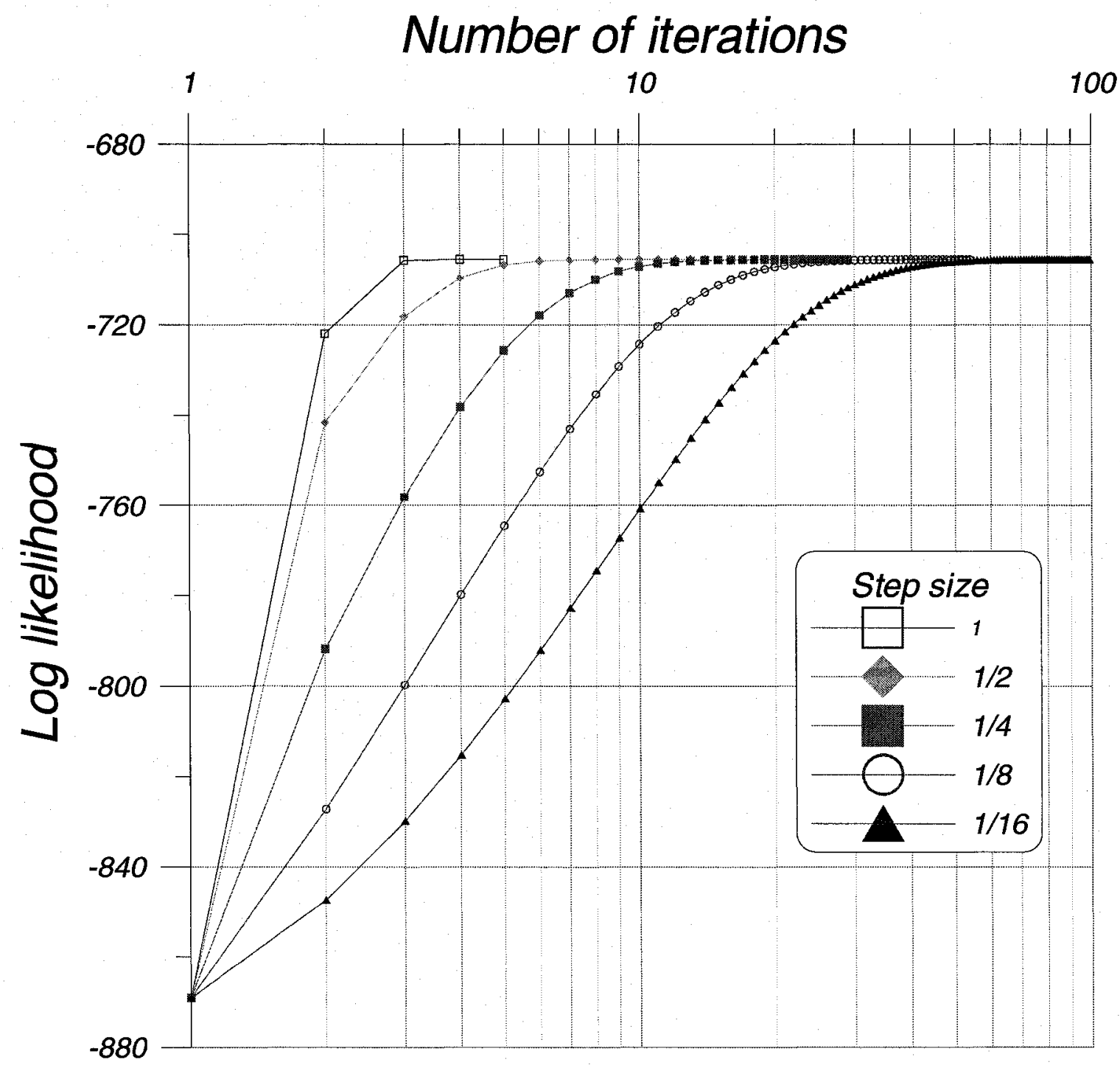

Figure 5-4: Log-likelihood for the all step sizes in Newton Raphson algorithm (exp. 1) 


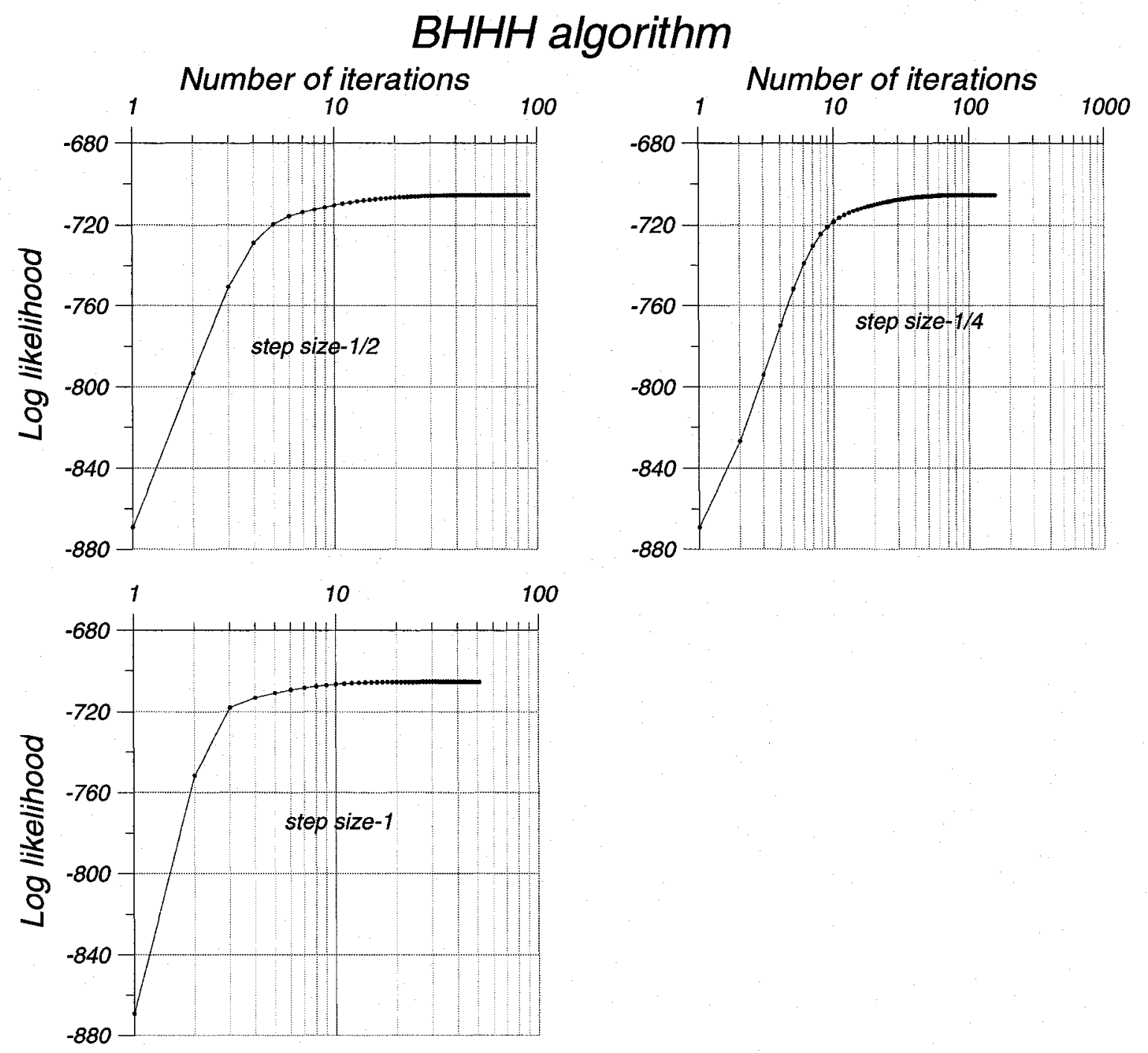

Figure 5-5: Log-likelihood values for each step size in BHHH (exp. 1) 


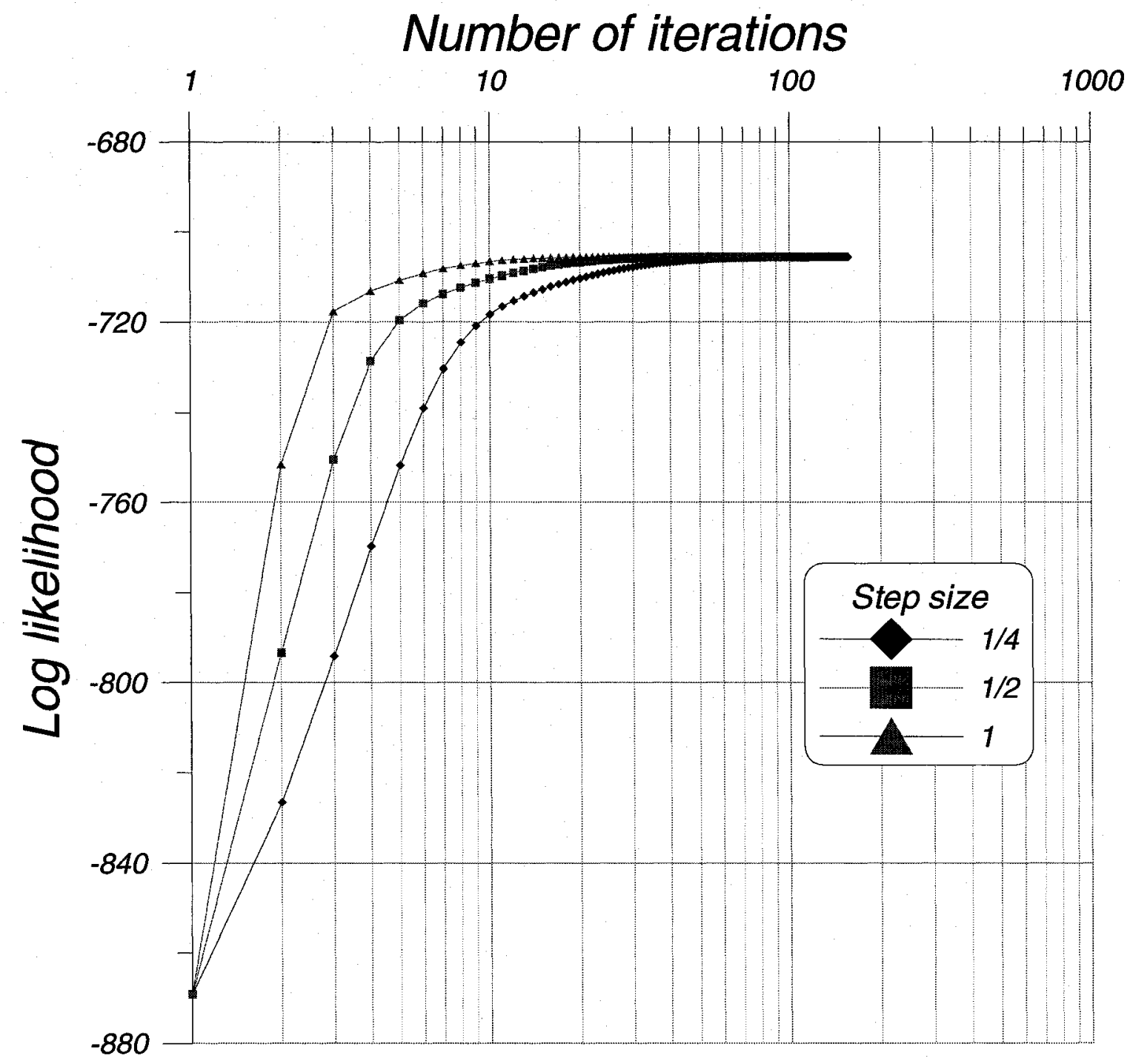

Figure 5-6: Log-likelihood for the all step sizes in BHHH algorithm (exp. 1) 


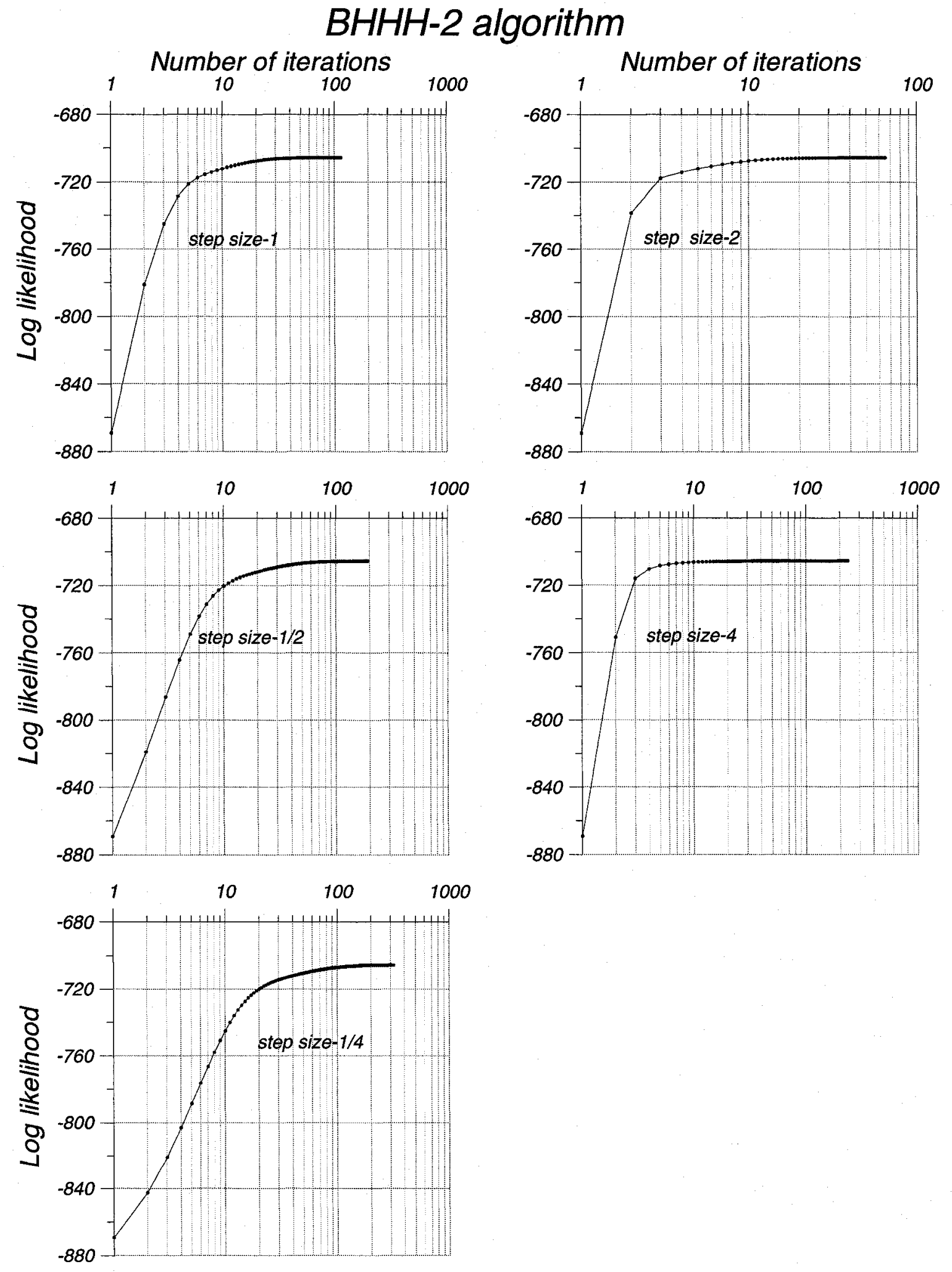

Figure 5-7: Log-likelihood values for each step size in BHHH-2 algorithm (exp. 1) 


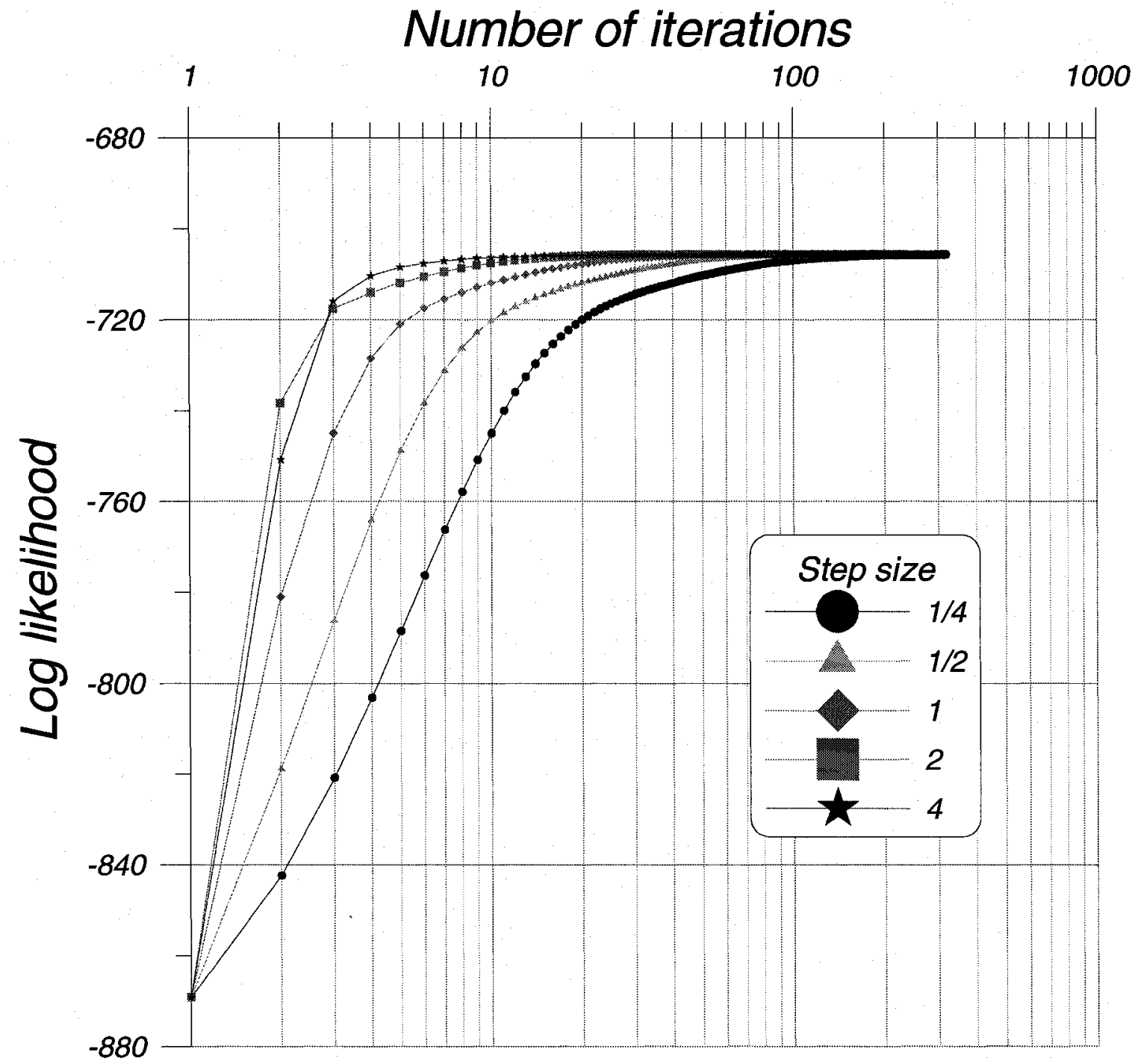

Figure 5-8: Log-likelihood for the all step sizes in BHHH-2 algorithm (exp. 1) 

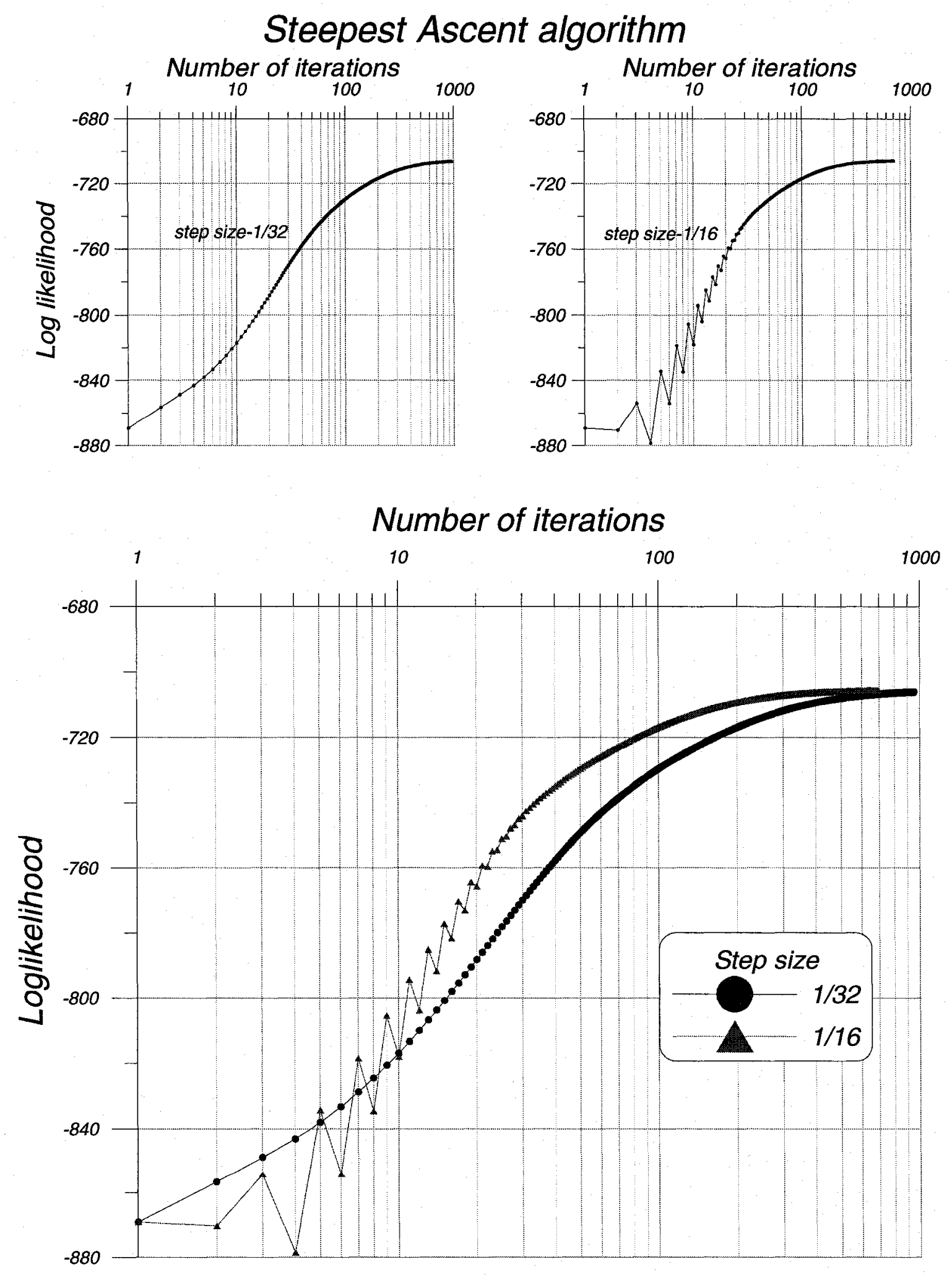

Figure 5-9: Log-likelihood values for each step size and all step sizes in Steepest Ascent algorithm (exp. 1) 


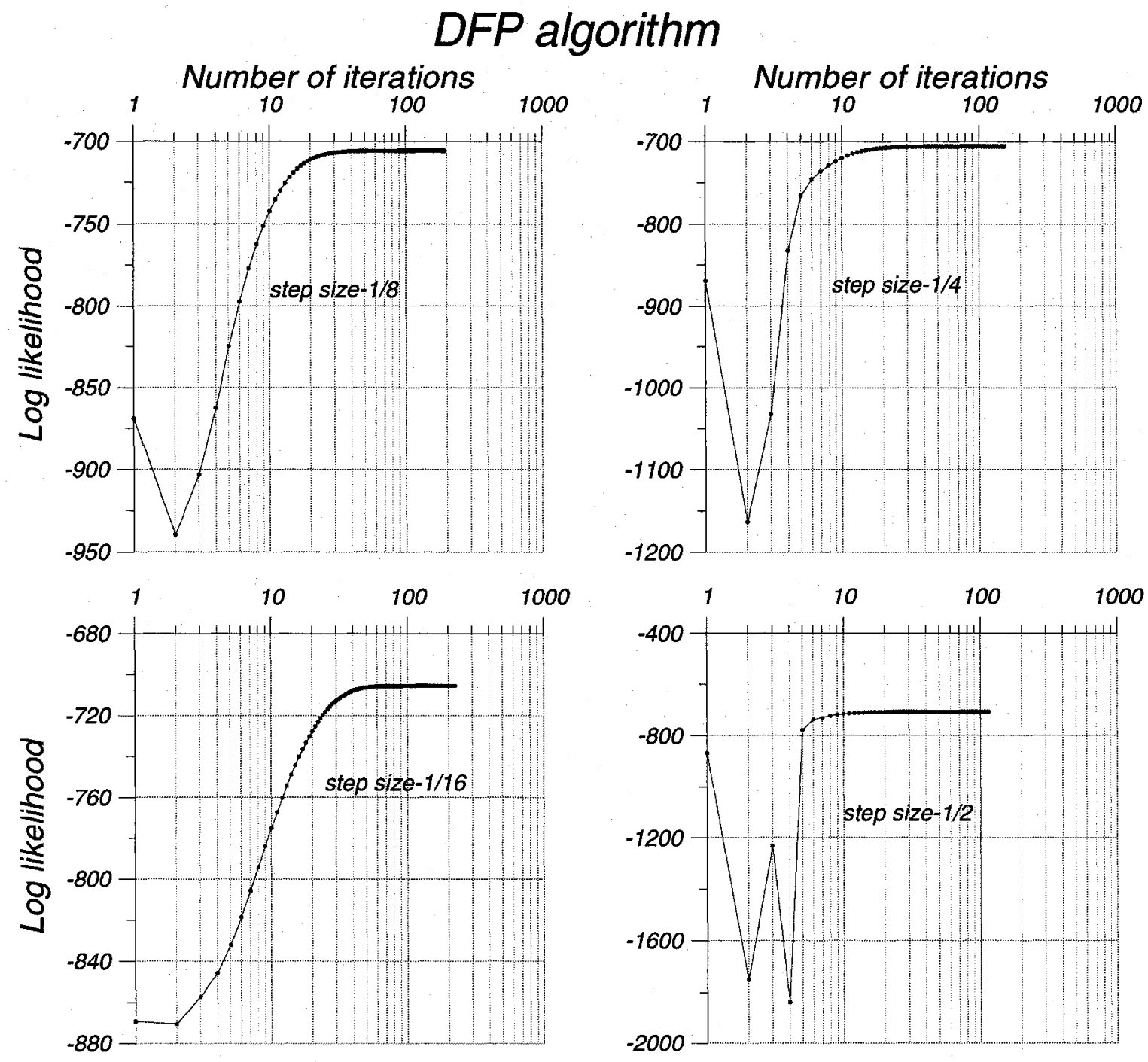

Figure 5-10: Log-likelihood values for each step size in DFP algorithm (exp. 1) 


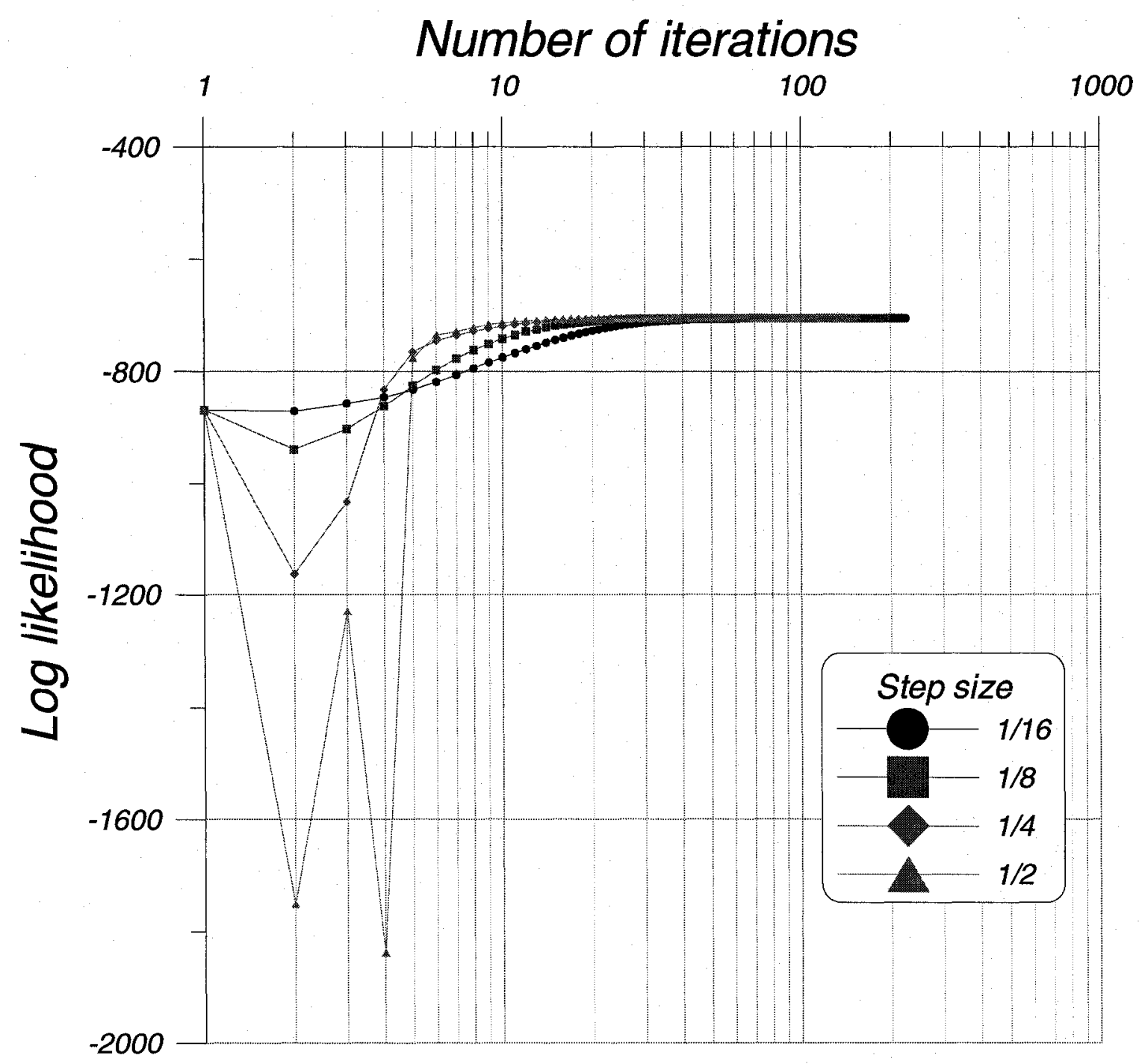

Figure 5-11: Log-likelihood for the all step sizes in DFP algorithm (exp. 1) 


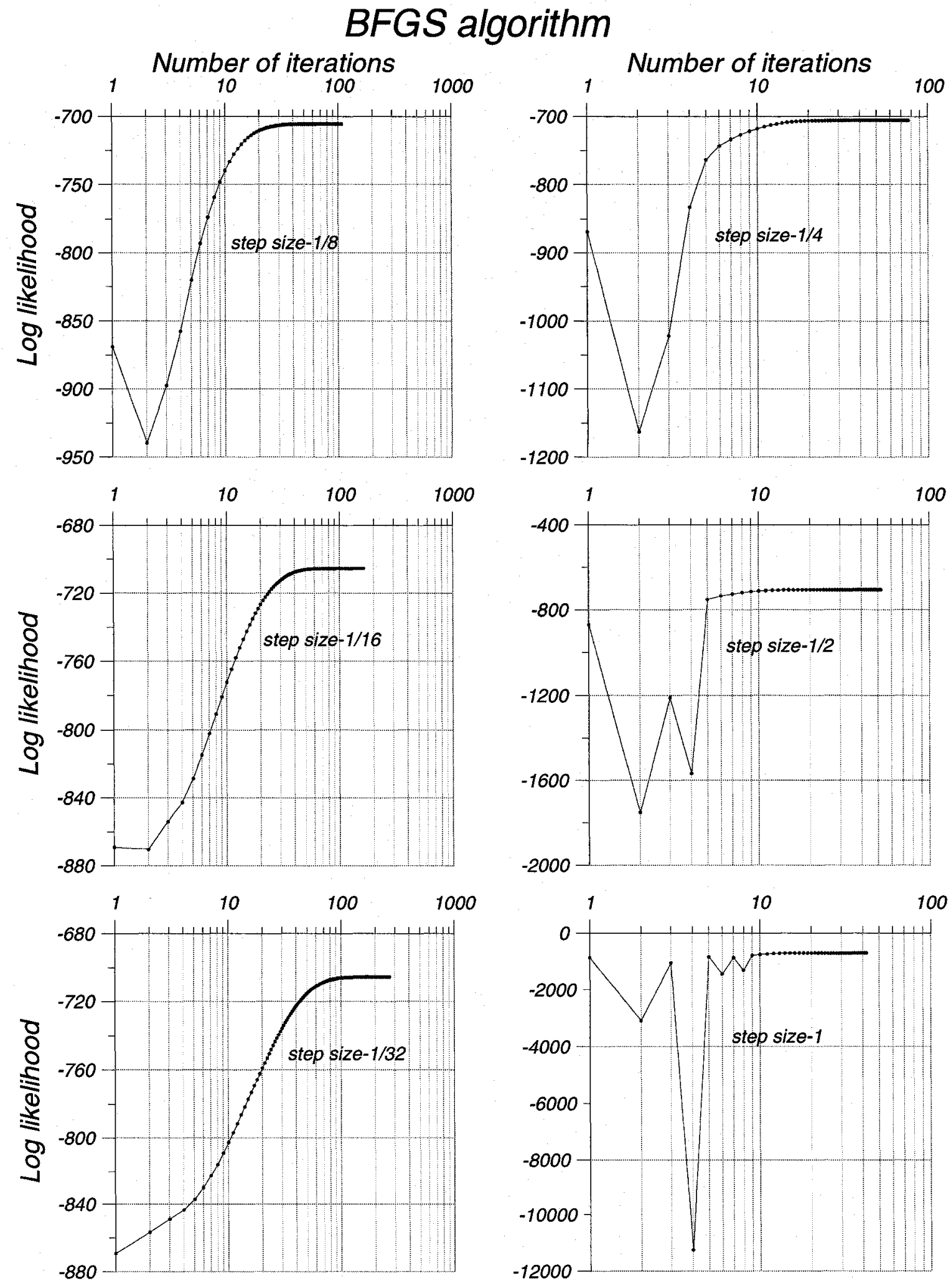

Figure 5-12: Log-likelihood values for each step size in BFGS algorithm (exp. 1) 


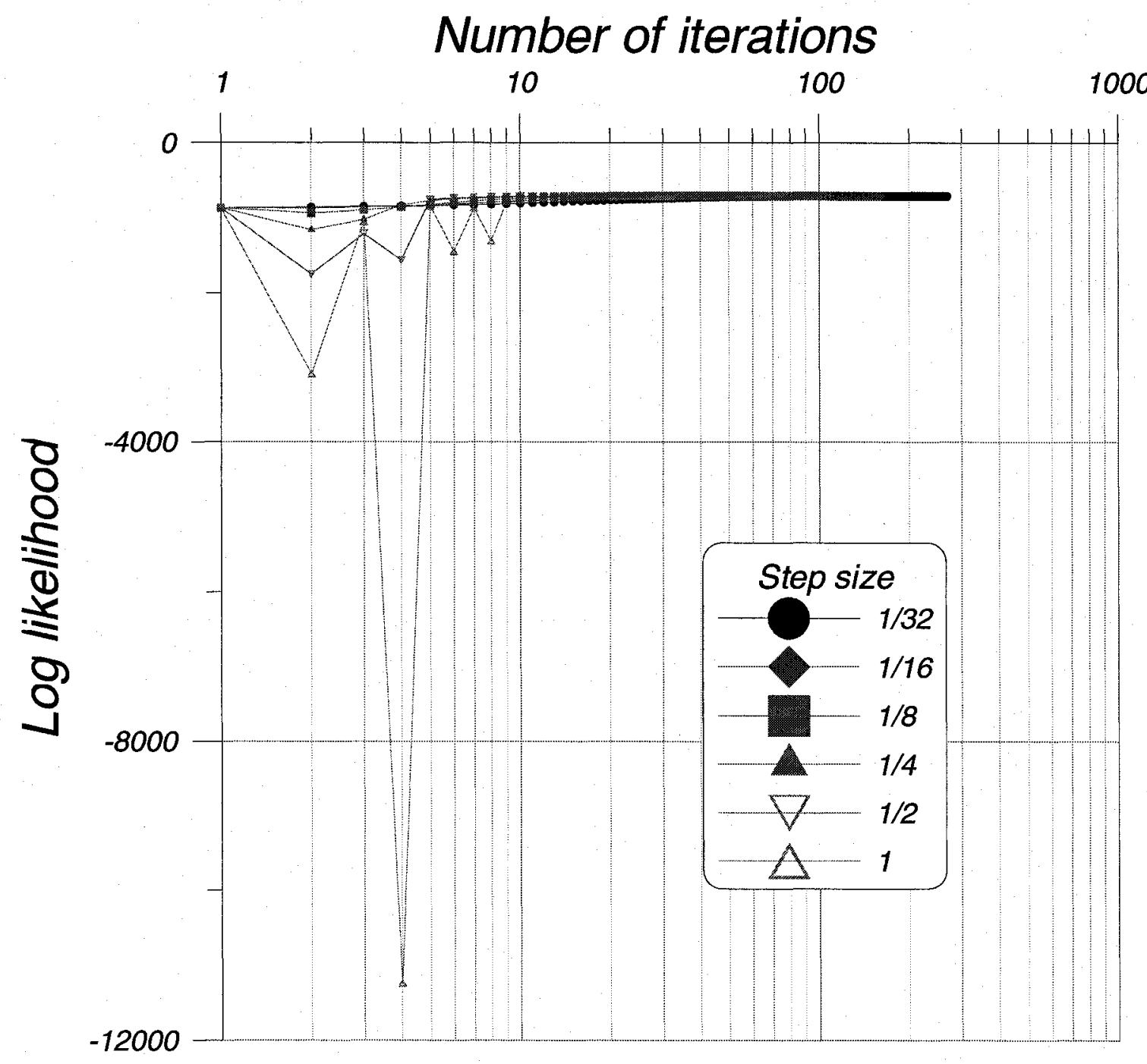

Figure 5-13: Log-likelihood for the all step sizes in BFGS algorithm (exp. 1) 


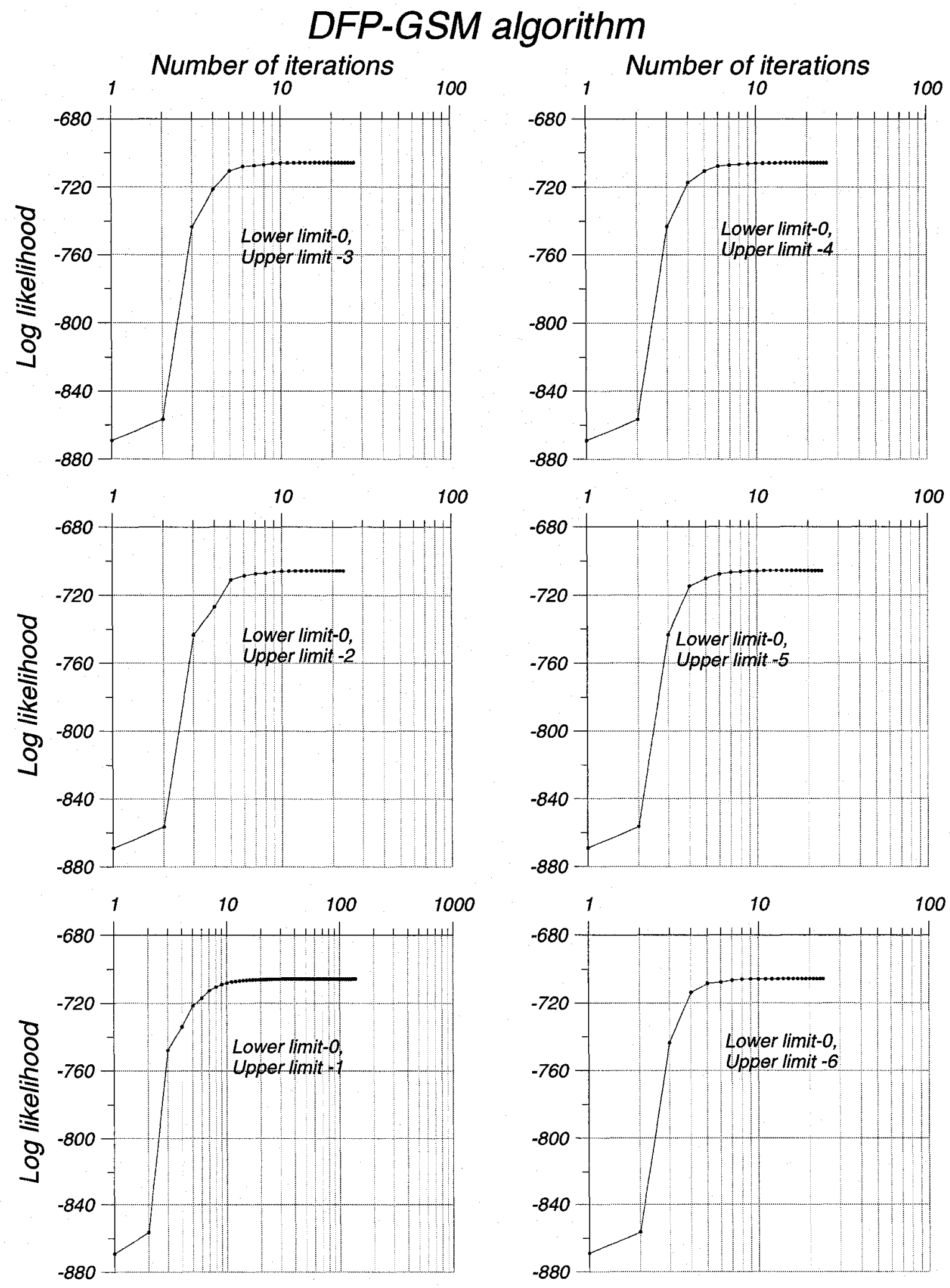

Figure 5-14: Log-likelihood values for each step size in DFP-GSM algorithm for the first six uncertain intervals (exp. 1) 


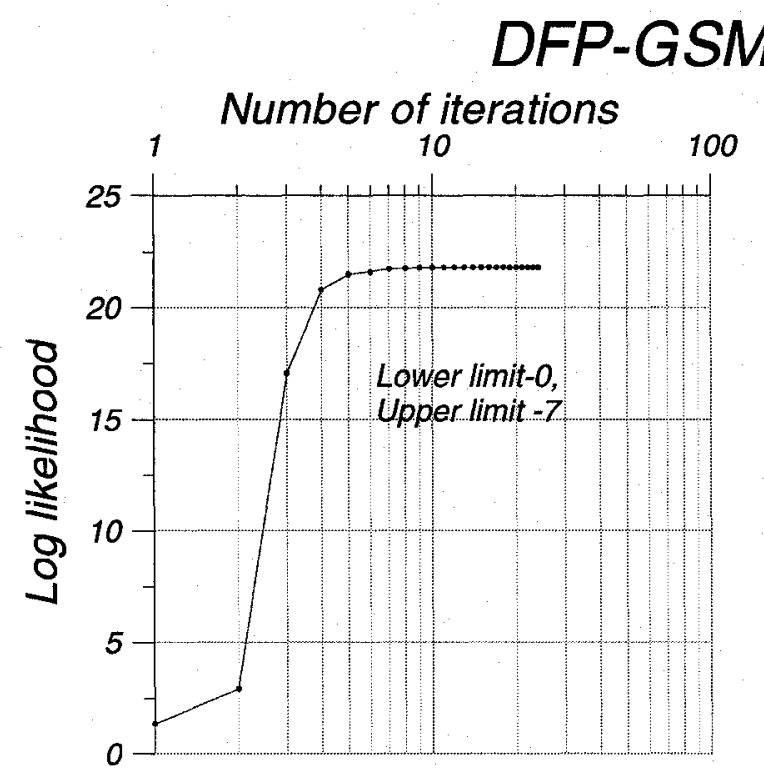

\section{algorithm}

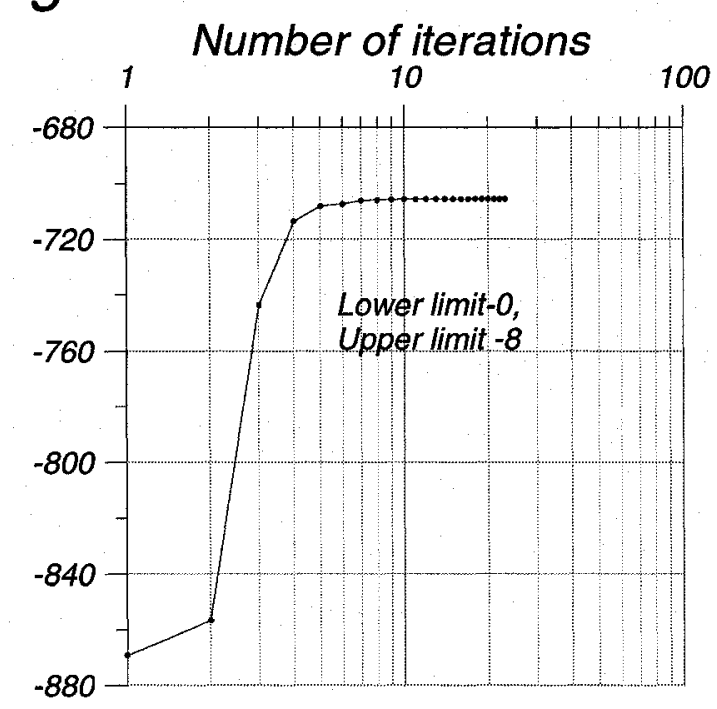

Figure 5-15: Log-likelihood values for each step size in DFP-GSM algorithm for the last two uncertain intervals (exp. 1) 


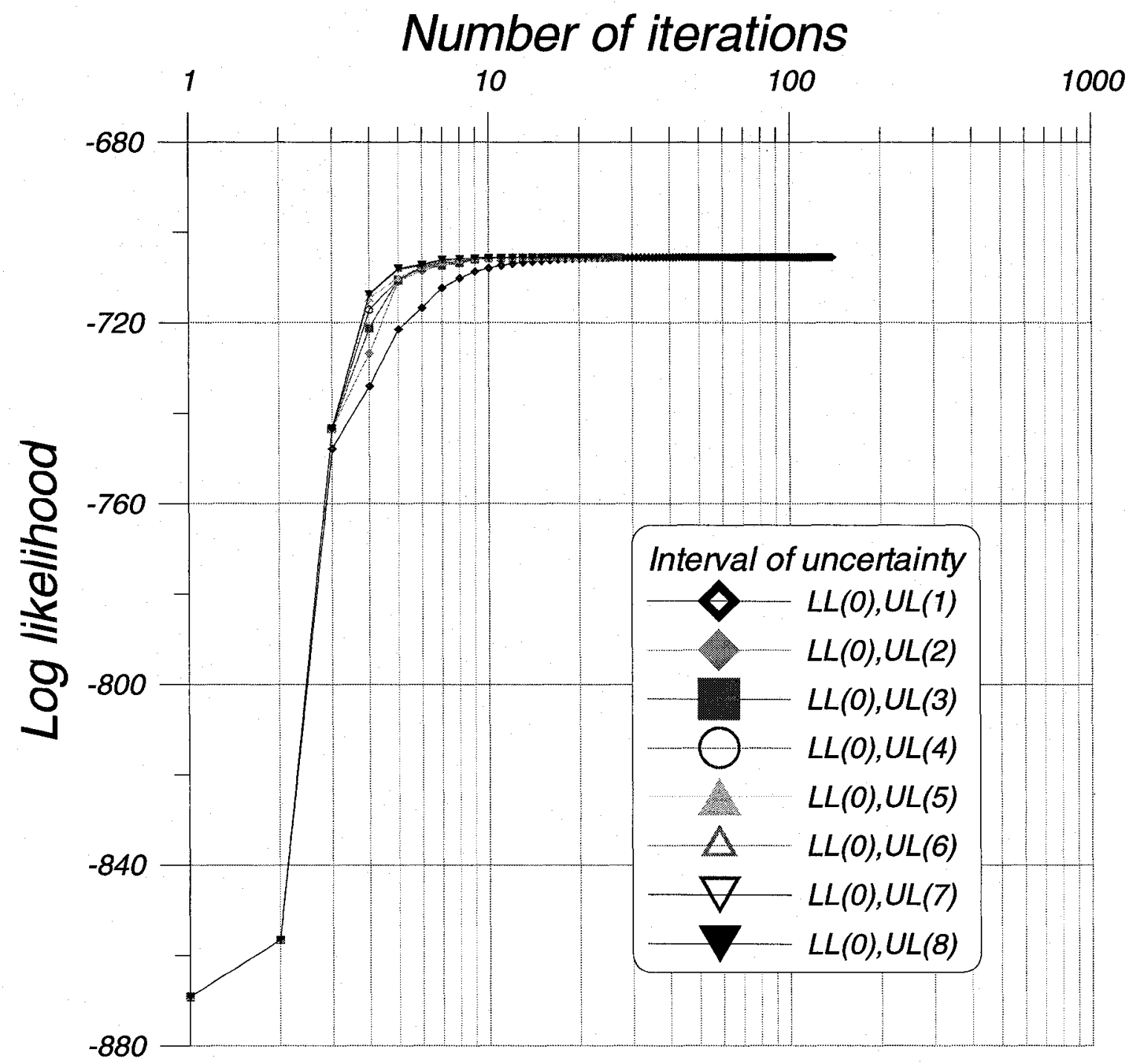

Figure 5-16: Log-likelihood for the all step sizes in DFP-GSM algorithm (exp. 1) 


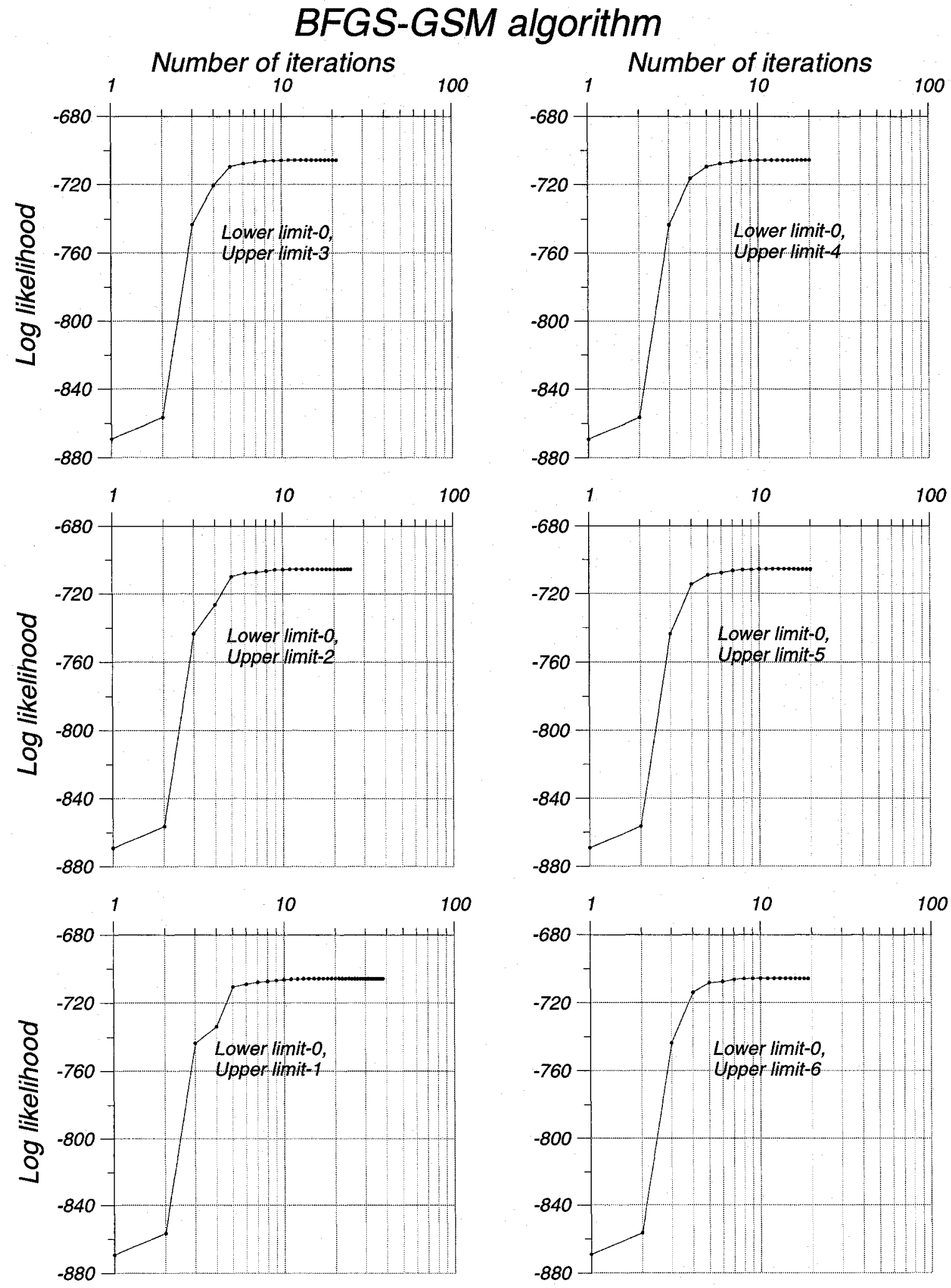

Figure 5-17: Log-likelihood values for each step size in BFGS-GSM algorithm for the first six uncertain intervals (exp. 1) 


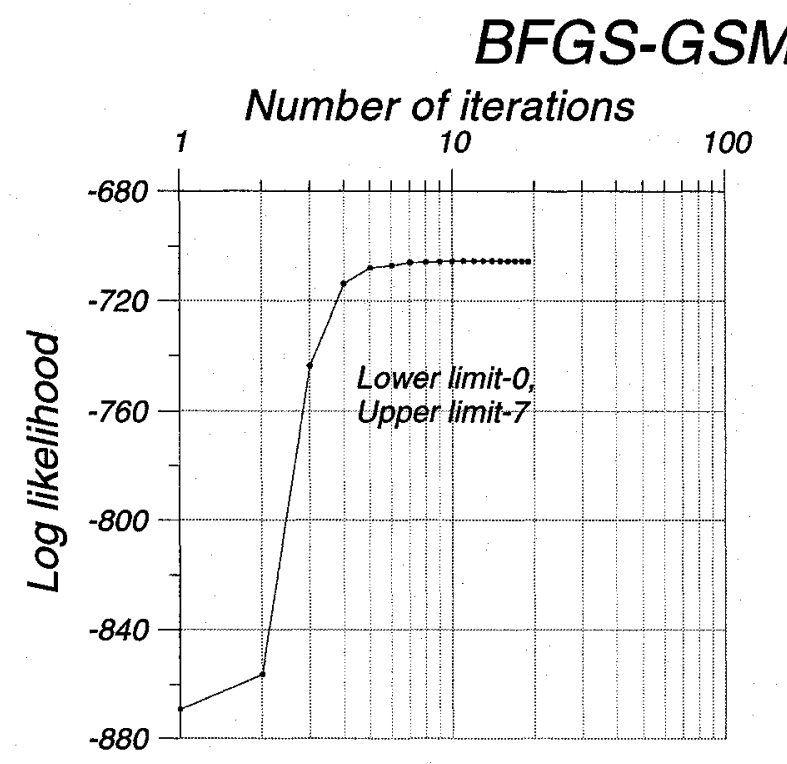

\section{algorithm}

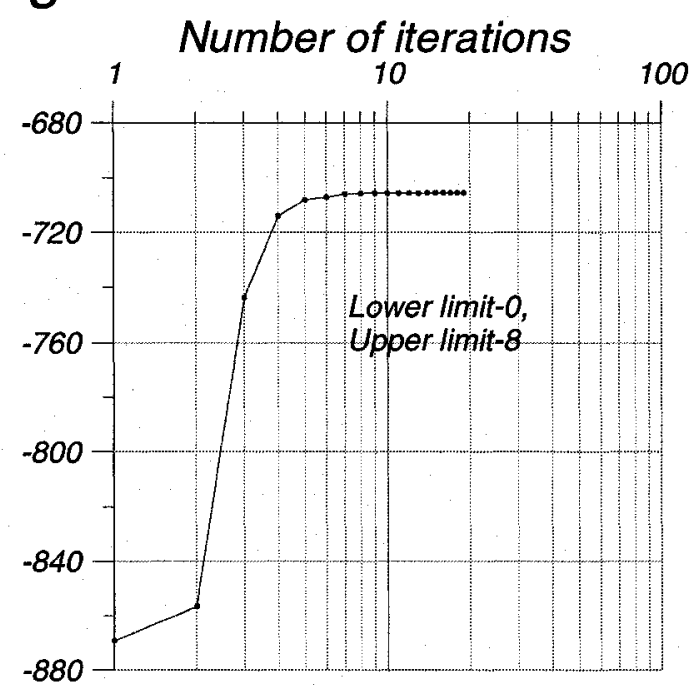

Figure 5-18: Log-likelihood values for each step size in BFGS-GSM algorithm for the last two uncertain intervals (exp. 1) 


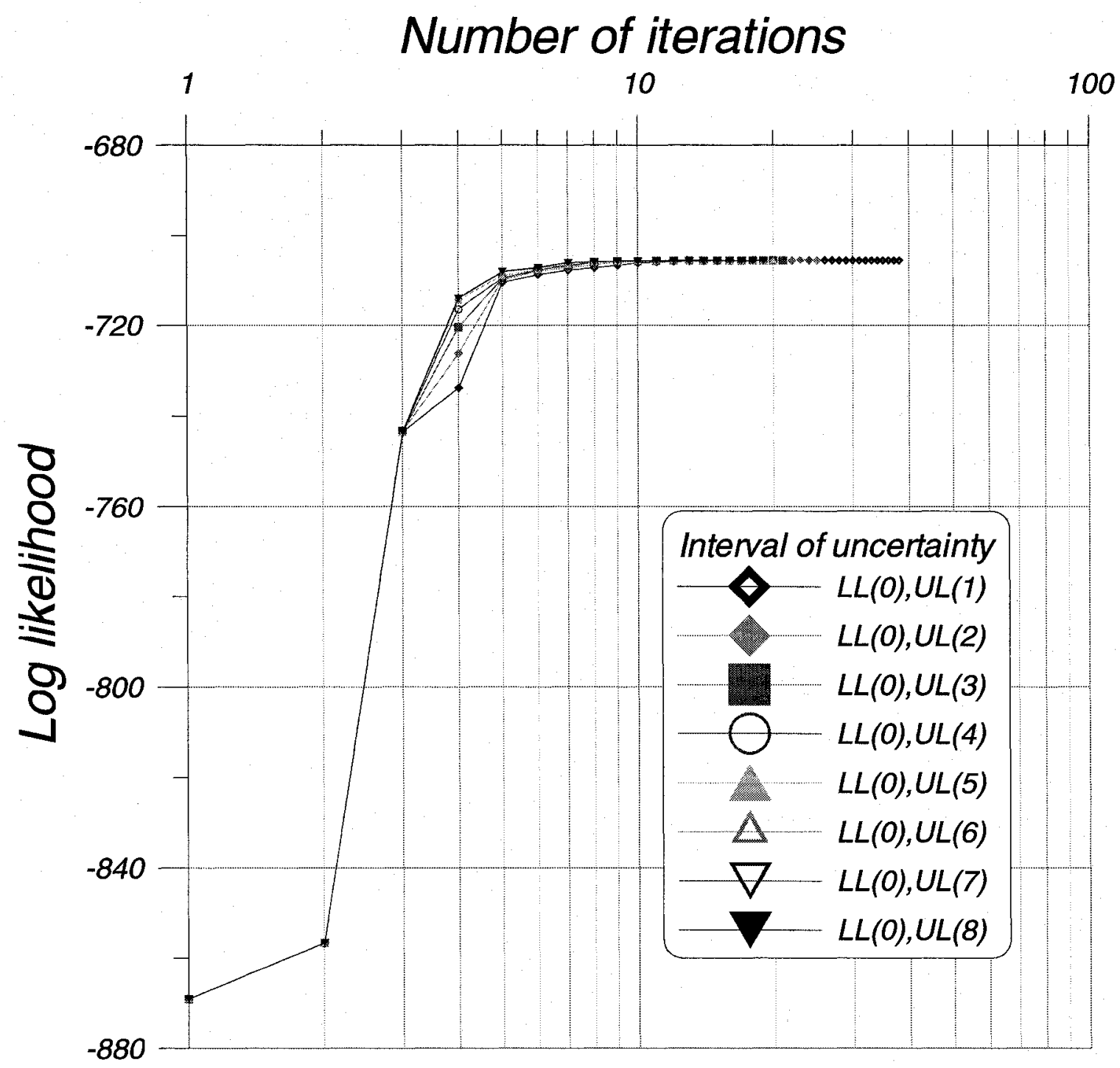

Figure 5-19: Log-likelihood for the all step sizes in BFGS-GSM algorithm (exp. 1) 


\subsubsection{The second convergence criterion (experiment 2)}

A vector of the model's parameters are estimated based on the second convergence criterion, as given in table 5-2, experiment 2 . This convergence criterion is sourced from Belsley (1979). In sections 5.2.2 to 5.2.4, the degree of precision of stopping criterion has the same mathematical expression as in experiment 2 . It is changed gradually in order to examine the impact of applying different convergence criteria on the convergence behaviour in discrete choice model estimation. The results of the experimental estimation for the eight different algorithms are summarized in table 5-12 for experimental trials that converged. The results are compared graphically in figures 5-20 and 5-21 in terms of three performance measures. 
Table 5-12: The final experimental results of the eight estimation algorithms (exp. 2)

\begin{tabular}{|c|c|c|c|c|c|c|c|c|c|}
\hline \multirow{2}{*}{\multicolumn{2}{|c|}{$\begin{array}{c}\text { Algorithms \& } \\
\text { Performance } \\
\text { measures } \\
\end{array}$}} & \multicolumn{8}{|c|}{ Step size } \\
\hline & & $1 / 32$ & $1 / 16$ & $1 / 8$ & $1 / 4$ & $1 / 2$ & 1 & 2 & 4 \\
\hline \multirow{3}{*}{ 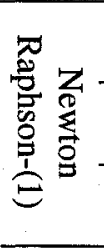 } & $\begin{array}{c}\text { One iteration } \\
\text { time }\end{array}$ & - & 0:00:22 & 0:00:24 & $0: 00: 21$ & $0: 00: 22$ & $0: 00: 17$ & - & - \\
\hline & $\begin{array}{c}\text { Number of } \\
\text { iterations }\end{array}$ & - & 73 & 36 & 18 & 8 & 4 & - & - \\
\hline & $\begin{array}{c}\text { Convergence } \\
\text { time }\end{array}$ & - & $0: 27: 12$ & 0:14:06 & 0:06:15 & $0: 02: 53$ & $0: 01: 10$ & - & - \\
\hline \multirow{3}{*}{ 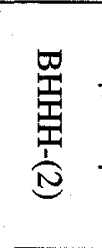 } & $\begin{array}{l}\text { One iteration } \\
\text { time }\end{array}$ & - & - & $0: 00: 27$ & $0: 00: 25$ & $0: 00: 24$ & $0: 00: 24$ & - & - \\
\hline & $\begin{array}{c}\text { Number of } \\
\text { iterations }\end{array}$ & - & - & 132 & 66 & 33 & 16 & - & - \\
\hline & $\begin{array}{l}\text { Convergence } \\
\text { time }\end{array}$ & - & - & $0: 58: 40$ & $0: 27: 20$ & $0: 13: 18$ & $0: 06: 28$ & - & - \\
\hline \multirow{3}{*}{ 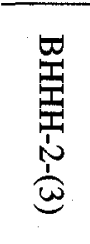 } & $\begin{array}{l}\text { One iteration } \\
\text { time }\end{array}$ & - & - & - & $0: 00: 30$ & 0:00:29 & 0:00:27 & $0: 00: 28$ & $0: 00: 30$ \\
\hline & $\begin{array}{c}\text { Number of } \\
\text { iterations }\end{array}$ & - & - & - & 94 & 47 & 24 & 12 & 27 \\
\hline & $\begin{array}{c}\text { Convergence } \\
\text { time }\end{array}$ & - & - & - & $0: 46: 32$ & $0: 22: 53$ & $0: 10: 58$ & 0:05:35 & $0: 13: 37$ \\
\hline \multirow{3}{*}{ 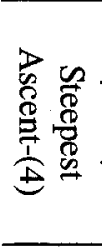 } & $\begin{array}{c}\text { One iteration } \\
\text { time }\end{array}$ & $0: 00: 16$ & $0: 00: 16$ & - & - & - & - & - & - \\
\hline & $\begin{array}{c}\text { Number of } \\
\text { iterations }\end{array}$ & 927 & 460 & - & - & - & - & - & - \\
\hline & $\begin{array}{c}\text { Convergence } \\
\text { time }\end{array}$ & $4: 02: 18$ & $2: 01: 28$ & - & - & - & - & - & - \\
\hline \multirow{3}{*}{$\frac{9}{0}$} & $\begin{array}{c}\text { One iteration } \\
\text { time }\end{array}$ & - & $0: 00: 17$ & $0: 00: 16$ & $0: 00: 23$ & $0: 00: 22$ & - & - & - \\
\hline & $\begin{array}{c}\text { Number of } \\
\text { iterations }\end{array}$ & - & 79 & 56 & 42 & 29 & - & - & - \\
\hline & $\begin{array}{c}\text { Convergence } \\
\text { time }\end{array}$ & - & $0: 22: 03$ & $0: 14: 35$ & $0: 15: 56$ & $0: 10: 30$ & - & - & - \\
\hline \multirow{3}{*}{$\begin{array}{l}\underset{⿴ 囗 十}{T} \\
\stackrel{0}{0} \\
\frac{1}{0}\end{array}$} & $\begin{array}{c}\text { One iteration } \\
\text { time }\end{array}$ & $0: 00: 21$ & $0: 00: 16$ & $0: 00: 22$ & $0: 00: 21$ & $0: 00: 21$ & $0: 00: 21$ & - & - \\
\hline & $\begin{array}{l}\text { Number of } \\
\text { iterations }\end{array}$ & 150 & 78 & 49 & 32 & 22 & 20 & - & - \\
\hline & $\begin{array}{c}\text { Convergence } \\
\text { time }\end{array}$ & $0: 52: 58$ & $0: 20: 54$ & $0: 17: 25$ & $0: 10: 56$ & $0: 07: 23$ & 0:06:50 & - & - \\
\hline \multicolumn{2}{|c|}{$\begin{array}{l}\text { Interval of } \\
\text { uncertainty }\end{array}$} & $\begin{array}{l}\text { LL(0), } \\
\text { UL(1) }\end{array}$ & $\begin{array}{l}\mathrm{LL}(0), \\
\mathrm{UL}(2)\end{array}$ & $\begin{array}{l}\text { LL(0), } \\
\mathrm{UL}(3)\end{array}$ & $\begin{array}{l}\text { LL(0), } \\
\text { UL(4) }\end{array}$ & $\begin{array}{l}\text { LL(0), } \\
\text { UL(5) }\end{array}$ & $\begin{array}{l}\text { LL(0), } \\
\text { UL(6) }\end{array}$ & $\begin{array}{l}\text { LL(0), } \\
\text { UL(7) }\end{array}$ & $\begin{array}{l}\text { LL(0), } \\
\text { UL(8) }\end{array}$ \\
\hline \multirow{3}{*}{$\begin{array}{l}\frac{0}{7} \\
0 \\
0 \\
3 \\
3 \\
\frac{1}{3}\end{array}$} & $\begin{array}{c}\text { One iteration } \\
\text { time }\end{array}$ & 0:03:02 & $0: 03: 11$ & $0: 03: 36$ & 0:01:27 & 0:01:07 & $0: 01: 34$ & 0:01:34 & $0: 01: 34$ \\
\hline & $\begin{array}{c}\text { Number of } \\
\text { iterations }\end{array}$ & 23 & 11 & 11 & 10 & 10 & 8 & 8 & 8 \\
\hline & $\begin{array}{c}\text { Convergence } \\
\text { time }\end{array}$ & $1: 09: 47$ & $0: 34: 55$ & $0: 39: 24$ & $0: 14: 26$ & 0:11:03 & $0: 12: 27$ & $0: 12: 27$ & $0: 12: 26$ \\
\hline \multirow{3}{*}{ 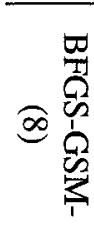 } & $\begin{array}{c}\text { One iteration } \\
\text { time }\end{array}$ & 0:01:05 & $0: 01: 13$ & 0:01:13 & 0:01:09 & 0:01:09 & 0:01:03 & 0:01:13 & $0: 01: 12$ \\
\hline & $\begin{array}{l}\text { Number of } \\
\text { iterations }\end{array}$ & 15 & 11 & 11 & 10 & 10 & 10 & 9 & 9 \\
\hline & $\begin{array}{l}\text { Convergence } \\
\text { time }\end{array}$ & $0: 16: 17$ & $0: 13: 17$ & $0: 13: 21$ & $0: 11: 26$ & $0: 11: 25$ & $0: 12: 07$ & $0: 10: 48$ & $0: 10: 45$ \\
\hline
\end{tabular}



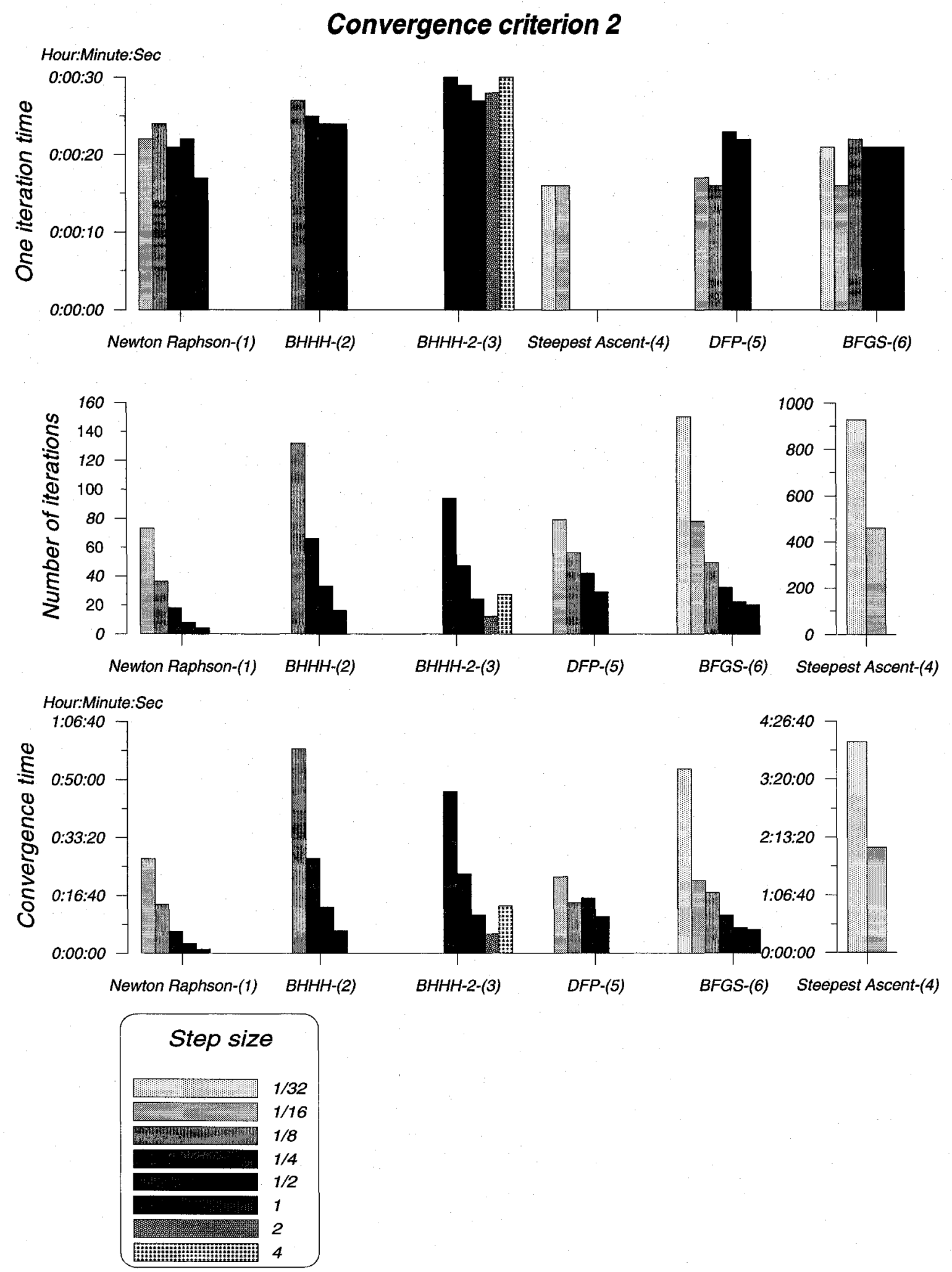

Figure 5-20: Final experimental results of the first six estimation algorithms (exp. 2) 

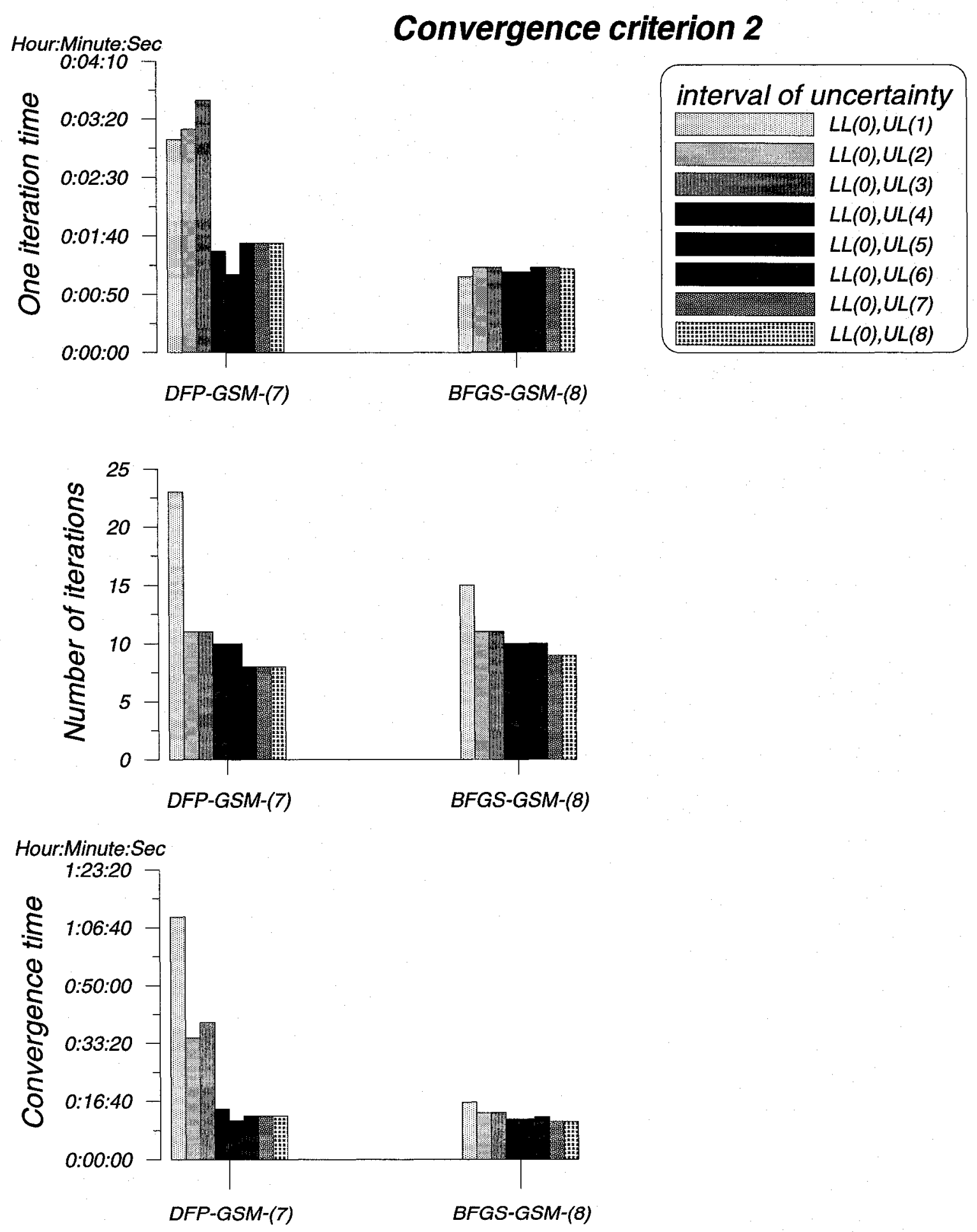

Figure 5-21: Final experimental results of the last two estimation algorithms (exp. 2) 
All conditions and environments applied in the experimental estimation are exactly the same as experiment 1 except the convergence criterion, $G_{t}^{\prime}\left(-H_{t}\right)^{-1} G_{t}<10^{-4}$. For this reason, a detailed explanation for the estimation performance either of each algorithm or of each step size applied in each algorithm will not be presented. Detailed information have already been presented in the previous section, and these can be used to interpret all the experimental estimates included in the following sections.

For all algorithms the estimation performance is improved as compared to experiment 1 in terms of three performance measures. This phenomenon can be seen in table 5-12. There are significant improvements throughout the first six algorithms for all step sizes and for all performance measures considered. For example, in the case of Steepest Ascent algorithm, the convergence time of experiment 2 decreases significantly (from 9:34:02, to 4:02:18) although the difference of the number of iterations is not significant. Consequently, this pattern of improvements occurs commonly throughout the first six algorithms.

Particularly, in the case of BHHH algorithm, one estimation run using a step size 1/8 did not converge in experiment 1 but the same run converged in experiment 2 . The reason of this different convergence behaviour is not certain in this research study. For the last two algorithms, all uncertain intervals except both LL(0)-UL(2) and LL(0)-UL(3) show an improvement of performance in terms of convergence time. Another important point is that the last two algorithms show a better performance in terms of both the number of iterations and the convergence time as the uncertain intervals increase gradually by an 
amount of unit, 1 .

In conclusion, when the second convergence criterion is applied as a stopping measure, all algorithms show an improvement in terms of three performance measures compared the case of experiment 1 . Other general operational characteristics discussed and compared among algorithms in an earlier section can also be applied, and explained in the same manner with experiment 1. 


\subsubsection{The number of iterations and log-likelihood values (experiment 2)}

As in the case of experiment 1, the graphs are drawn for each estimation results in order to show a variation in the log-likelihood function values following the iterations and moreover to compare clearly the different convergence behaviours between step sizes used in each algorithm. Tables 5-13 to 5-18 summarize critical values such as starting log-likelihood value and convergence log-likelihood value, and the number of iterations due to the different step sizes. As shown in tables 5-13 to 5-18, all calculated starting loglikelihood values are exactly the same for the all eight algorithms and for any step sizes used within each algorithm due to $\beta_{k}=\left[\beta_{1}=0, \beta_{2}=0, \beta_{3}=0, \cdots, \beta_{9}=0\right]^{\prime}$. The same condition imposed as an initial guess of starting points in experiments $1-4$, and 6 . On the contrary, convergence values are slightly different among the step sizes used in each algorithm and the differences are so small that it can be considered as being equal. The only different feature as compared with experiment 1 is that the convergence loglikelihood value obtained in both BHHH-2 and Steepest Ascent is somewhat different from that for those algorithms used in experiment 1. Tables 5-10 and 5-11 summarize critical values for the same factors with the above first six algorithms for the each different uncertain intervals. As shown in tables 5-19 and 5-20, all starting log-likelihood values are exactly the same due to the same reason explained for the first six algorithms. In case of the convergence log-likelihood values, slight differences exit among uncertain intervals but these are also so small that these can be considered to be converged at the same value even though it is not exactly the same. 
Table 5-13: Log-likelihood values obtained from Newton Raphson algorithm (exp. 2)

\begin{tabular}{|c|c|c|c|c|c|c|}
\hline \multirow{2}{*}{\multicolumn{2}{|c|}{ Log-likelihood }} & \multicolumn{5}{|c|}{ Step size } \\
\hline & & $1 / 16$ & $1 / 8$ & $1 / 4$ & $1 / 2$ & 1 \\
\hline \multirow{3}{*}{ 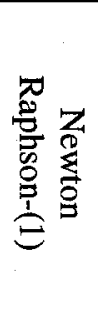 } & $\begin{array}{c}\text { Starting } \\
\text { Log- } \\
\text { likelihood }\end{array}$ & -869.0964727 & -869.0964727 & -869.0964727 & -869.0964727 & -869.0964727 \\
\hline & $\begin{array}{c}\text { Convergence } \\
\text { log- } \\
\text { likelihood }\end{array}$ & -705.5640983 & -705.5636583 & -705.5559598 & -705.5618834 & -705.5384404 \\
\hline & $\begin{array}{l}\text { Number of } \\
\text { iterations }\end{array}$ & 73 & 36 & 18 & 8 & 4 \\
\hline
\end{tabular}

Table 5-14: Log-likelihood values obtained from BHHH algorithm (exp. 2)

\begin{tabular}{cccccc}
\hline \multirow{2}{*}{ Log-likelihood } & \multicolumn{4}{c}{ Step size } \\
\cline { 2 - 6 } & $\begin{array}{c}\text { Starting } \\
\text { Log-likelihood }\end{array}$ & -869.0964727 & -869.0964727 & -869.0964727 & -869.0964727 \\
\cline { 2 - 5 } & $\begin{array}{c}\text { Convergence } \\
\text { Log-likelihood }\end{array}$ & -705.7578298 & -705.7548205 & -705.7491538 & -705.7620376 \\
\cline { 2 - 6 } & \begin{tabular}{c} 
Number of iterations \\
\cline { 2 - 6 }
\end{tabular} & 132 & 66 & 33 & 16 \\
\hline
\end{tabular}

Table 5-15: Log-likelihood values obtained from BHHH-2 algorithm (exp. 2)

\begin{tabular}{ccccccc}
\hline Log-likelihood & \multicolumn{5}{c}{ Step size } \\
\cline { 2 - 7 } & $1 / 4$ & $1 / 2$ & 1 & 2 & 4 \\
\hline \multirow{2}{*}{$\begin{array}{c}\text { Starting } \\
\text { Log-likelihood }\end{array}$} & -869.0964727 & -869.0964727 & -869.0964727 & -869.0964727 & -869.0964727 \\
\cline { 2 - 7 } & $\begin{array}{c}\text { Convergence } \\
\text { log-likelihood }\end{array}$ & -706.9983276 & -706.9910005 & -706.905528 & -706.8121611 & -705.6851269 \\
\cline { 2 - 7 } & Number of iterations & 94 & 47 & 24 & 12 & 27 \\
\hline
\end{tabular}

Table 5-16: Log-likelihood values obtained from Steepest Ascent algorithm (exp. 2)

\begin{tabular}{|c|c|c|c|}
\hline \multirow{2}{*}{\multicolumn{2}{|c|}{ Log-likelihood }} & \multicolumn{2}{|c|}{ Step size } \\
\hline & & $1 / 32$ & $1 / 16$ \\
\hline \multirow{3}{*}{ 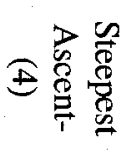 } & $\begin{array}{c}\text { Starting } \\
\text { Log-likelihood } \\
\end{array}$ & -869.0964727 & -869.0964727 \\
\hline & Convergence log-likelihood & -706.1053053 & -706.1012775 \\
\hline & Number of iterations & 927 & 460 \\
\hline
\end{tabular}


Table 5-17: Log likelihood values obtained from DFP algorithm (exp. 2)

\begin{tabular}{cccccc}
\hline \multirow{2}{*}{ Log-likelihood } & \multicolumn{5}{c}{ Step size } \\
\cline { 2 - 6 } & Starting & $1 / 16$ & $1 / 8$ & $1 / 4$ & $1 / 2$ \\
\hline \multirow{7}{*}{ 守 } & Log-likelihood & -869.0964727 & -869.0964727 & -869.0964727 & -869.0964727 \\
\cline { 2 - 6 } & Convergence log-likelihood & -705.5861209 & -705.6160738 & -705.6565252 & -705.6940834 \\
\cline { 2 - 6 } & Number of iterations & 79 & 56 & 42 & 29 \\
\hline
\end{tabular}

Table 5-18: Log likelihood values obtained from BFGS algorithm (exp. 2)

\begin{tabular}{|c|c|c|c|c|c|c|c|}
\hline \multirow{2}{*}{\multicolumn{2}{|c|}{ Log-likelihood }} & \multicolumn{6}{|c|}{ Step size } \\
\hline & & $1 / 32$ & $1 / 16$ & $1 / 8$ & $1 / 4$ & $1 / 2$ & 1 \\
\hline \multirow{3}{*}{ 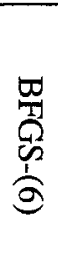 } & $\begin{array}{c}\text { Starting } \\
\text { Log- } \\
\text { likelihood }\end{array}$ & -869.0964727 & -869.0964727 & -869.0964727 & -869.0964727 & -869.0964727 & -869.0964727 \\
\hline & $\begin{array}{l}\text { Convergence } \\
\text { log-likelihood }\end{array}$ & -705.5772393 & -705.5855181 & -705.5888418 & -705.5979521 & -705.6348579 & -705.6311626 \\
\hline & $\begin{array}{l}\text { Number of } \\
\text { iterations }\end{array}$ & 150 & 78 & 49 & 32 & 22 & 20 \\
\hline
\end{tabular}

Table 5-19: Log likelihood values obtained from DFP-GSM algorithm (exp. 2)

\begin{tabular}{|c|c|c|c|c|c|c|c|c|c|}
\hline & $\begin{array}{l}\text { Interval of } \\
\text { uncertainty }\end{array}$ & $\begin{array}{l}\text { LL(0), } \\
\text { UL(1) }\end{array}$ & $\begin{array}{l}\text { LL(0), } \\
\text { UL(2) }\end{array}$ & $\begin{array}{l}\text { LL(0), } \\
\text { UL(3) }\end{array}$ & $\begin{array}{l}\text { LL(0), } \\
\text { UL(4) }\end{array}$ & $\begin{array}{l}\text { LL(0), } \\
\text { UL(5) }\end{array}$ & $\begin{array}{l}\text { LL(0), } \\
\text { UL(6) }\end{array}$ & $\begin{array}{l}\text { LL(0), } \\
\text { UL(7) }\end{array}$ & $\begin{array}{l}\text { LL(0), } \\
\text { UL(8) }\end{array}$ \\
\hline \multirow{3}{*}{ 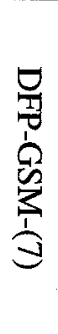 } & $\begin{array}{c}\text { Starting } \\
\text { Log- } \\
\text { likelihood }\end{array}$ & $\begin{array}{c}869.0964 \\
727\end{array}$ & $\begin{array}{c}869.0964 \\
727\end{array}$ & $\begin{array}{c}869.0964 \\
727\end{array}$ & $\begin{array}{c}869.0964 \\
727\end{array}$ & $\begin{array}{c}869.0964 \\
727\end{array}$ & $\begin{array}{c}869.0964 \\
727\end{array}$ & $\begin{array}{c}869.0964 \\
727\end{array}$ & $\begin{array}{c}869.0964 \\
727\end{array}$ \\
\hline & $\begin{array}{c}\text { Convergence } \\
\text { log- } \\
\text { likelihood } \\
\end{array}$ & $\begin{array}{c}705.7026 \\
844\end{array}$ & $\begin{array}{c}705.7337 \\
937\end{array}$ & $\begin{array}{c}705.7334 \\
852\end{array}$ & $\begin{array}{c}705.7752 \\
86\end{array}$ & $\begin{array}{c}705.7586 \\
887\end{array}$ & $\begin{array}{c}705.9187 \\
437\end{array}$ & $\begin{array}{c}- \\
705.8917 \\
489\end{array}$ & $\begin{array}{c}705.9084 \\
545\end{array}$ \\
\hline & $\begin{array}{l}\text { Number of } \\
\text { iterations }\end{array}$ & 23 & 11 & 11 & 10 & 10 & 8 & 8 & 8 \\
\hline
\end{tabular}

Table 5-20: Log likelihood values obtained from BFGS-GSM algorithm (exp. 2)

\begin{tabular}{|c|c|c|c|c|c|c|c|c|c|}
\hline & $\begin{array}{l}\text { Interval of } \\
\text { uncertainty }\end{array}$ & $\begin{array}{l}\mathrm{LL}(0), \\
\mathrm{UL}(1)\end{array}$ & $\begin{array}{l}\mathrm{LL}(0), \\
\mathrm{UL}(2)\end{array}$ & $\begin{array}{l}\text { LL(0), } \\
\text { UL(3) }\end{array}$ & $\begin{array}{l}\text { LL(0), } \\
\text { UL(4) }\end{array}$ & $\begin{array}{l}\text { LL(0), } \\
\text { UL(5) }\end{array}$ & $\begin{array}{l}\text { LL(0), } \\
\text { UL(6) }\end{array}$ & $\begin{array}{l}\mathrm{LL}(0), \\
\mathrm{UL}(7)\end{array}$ & $\begin{array}{l}\text { LL(0), } \\
\text { UL(8) }\end{array}$ \\
\hline \multirow{3}{*}{ 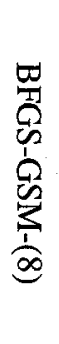 } & $\begin{array}{c}\text { Starting } \\
\text { Log- } \\
\text { likelihood }\end{array}$ & $\begin{array}{c}869.0964 \\
727\end{array}$ & $\begin{array}{c}869.0964 \\
727\end{array}$ & $\begin{array}{c}869.0964 \\
727\end{array}$ & $\begin{array}{c}869.0964 \\
727\end{array}$ & $\begin{array}{c}869.0964 \\
727\end{array}$ & $\begin{array}{c}869.0964 \\
727\end{array}$ & $\begin{array}{c}869.0964 \\
727\end{array}$ & $\begin{array}{c}869.0964 \\
727\end{array}$ \\
\hline & $\begin{array}{c}\text { Convergence } \\
\text { log- } \\
\text { likelihood }\end{array}$ & $\begin{array}{c}705.5879 \\
102\end{array}$ & $\begin{array}{c}- \\
705.6275 \\
413\end{array}$ & $\begin{array}{c}- \\
705.5961 \\
032\end{array}$ & $\begin{array}{c}- \\
705.6161 \\
016\end{array}$ & $\begin{array}{c}- \\
705.6100 \\
727\end{array}$ & $\begin{array}{c}- \\
705.6006 \\
594\end{array}$ & $\begin{array}{c}- \\
705.6241 \\
932\end{array}$ & $\begin{array}{c}- \\
705.6279 \\
414\end{array}$ \\
\hline & $\begin{array}{l}\text { Number of } \\
\text { iterations }\end{array}$ & 15 & 11 & 11 & 10 & 10 & 10 & 9 & 9 \\
\hline
\end{tabular}


In the case of the Newton Raphson algorithm where the step size is one, as shown in figures 5-22 and 5-23 and in table 5-13, only four iterations are required. One iteration decreases as compared to run of experiment 1 that used the step size 1 . The convergence behaviour is identical to experiment 1 except the fact that slight differences occur all over the three performance measures. These experimental results are clear in the graph. Overall, with decreasing step size, the slope also becomes low, but the number of iterations increase.

The same interpretation can be applied to the rest of algorithms; we can identify the best performance among the runs using various step sizes and see the convergence loglikelihood value with the help of given tables and graphs (i.e., tables 5-13 to 5-20 and graphs 5-22 to 5-38). The most important point we should note is that the last part of iteration procedures have a different profile in graph. That is, the overlap parts observed in experiment 1 disappear or simplified compared to that of experiment 1 . It means that a researcher can control the termination behaviour by changing the convergence criterion within a scientific way, of course, if or only if the new given convergence criterion guarantees the degree of accuracy of parameter estimates up to the level which a researcher expects it must be. 


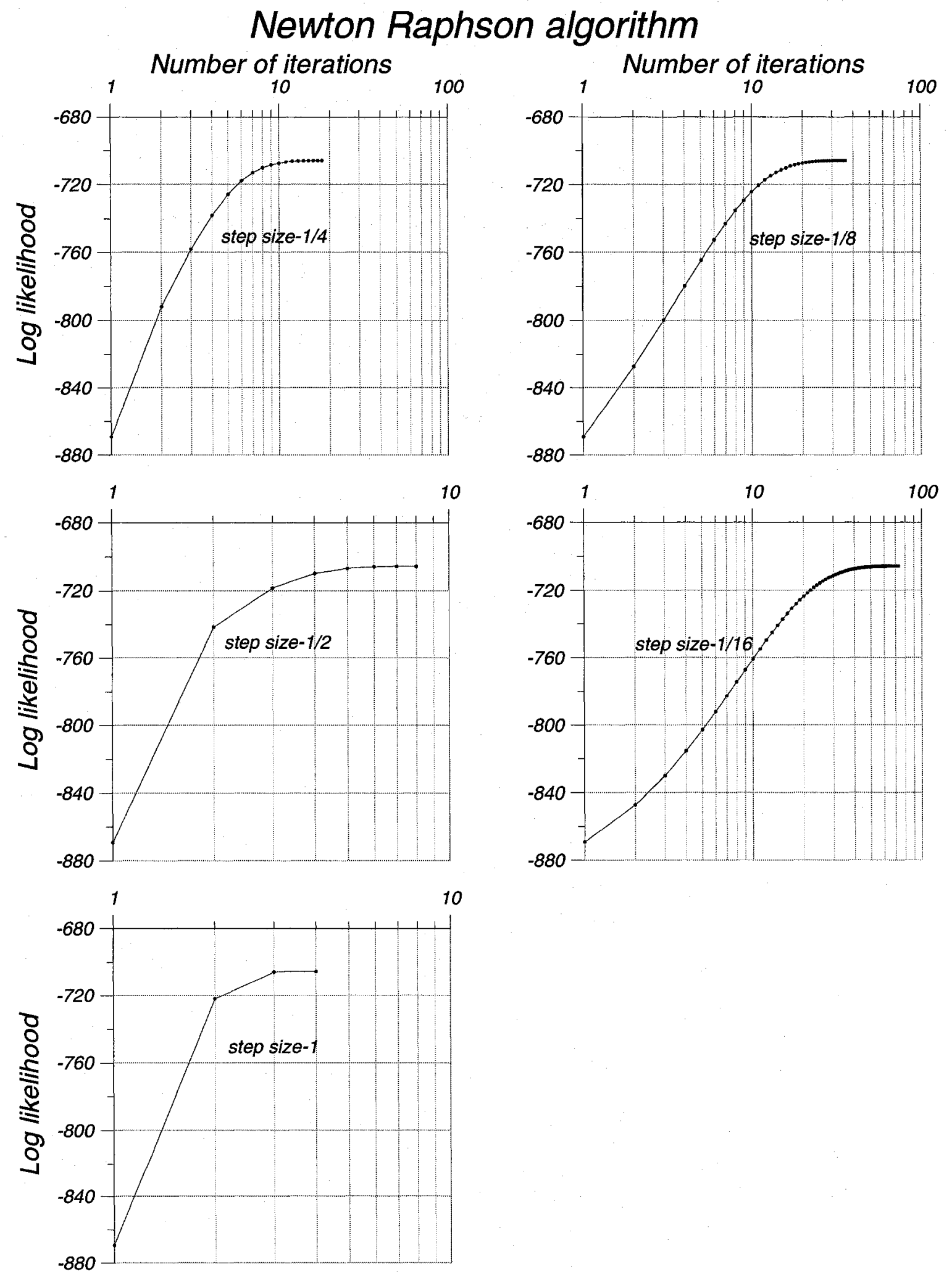

Figure 5-22: Log-likelihood values for each step size in Newton Raphson algorithm (exp. 2) 


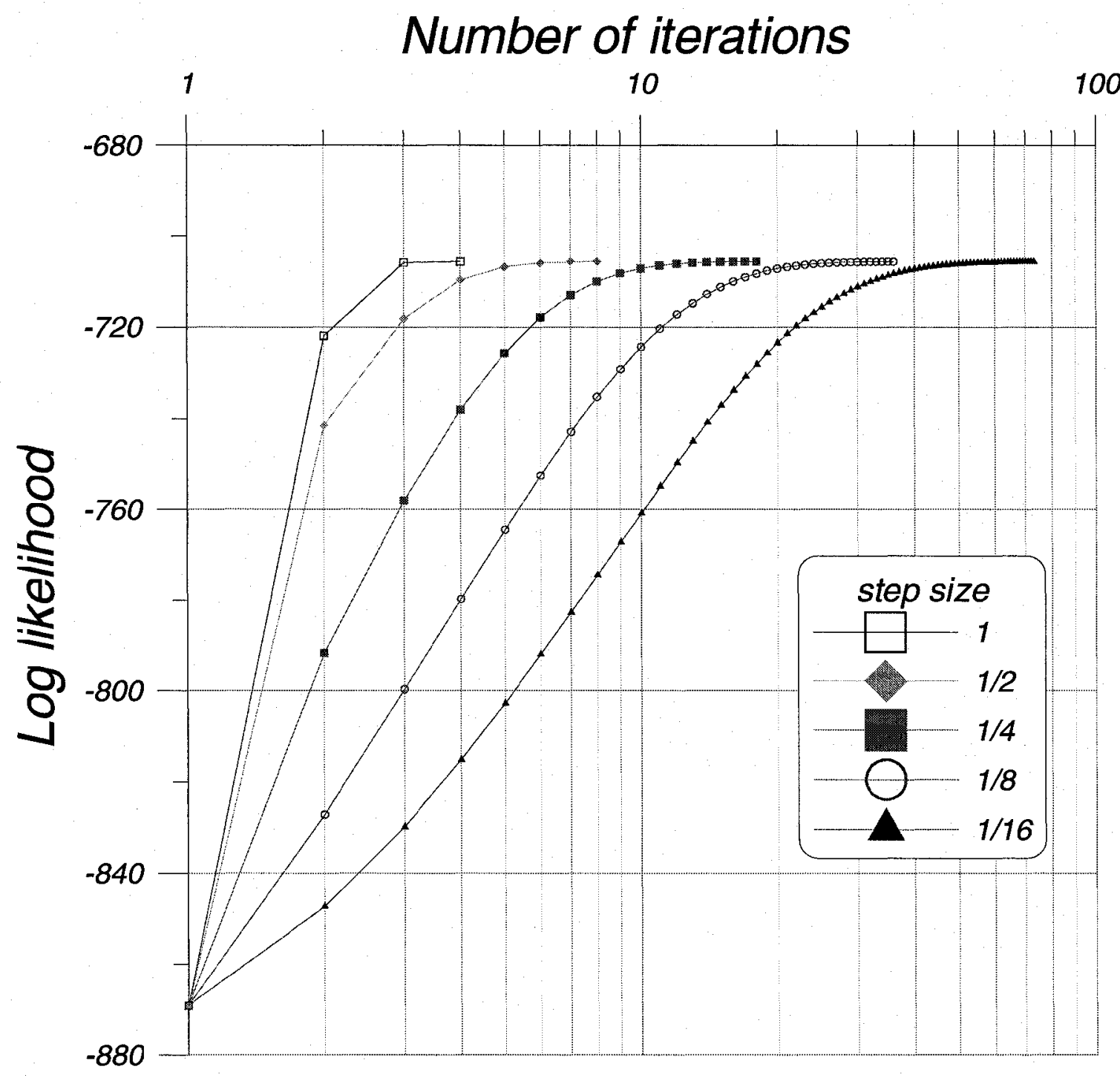

Figure 5-23: Log-likelihood for the all step sizes in Newton Raphson algorithm (exp. 2) 


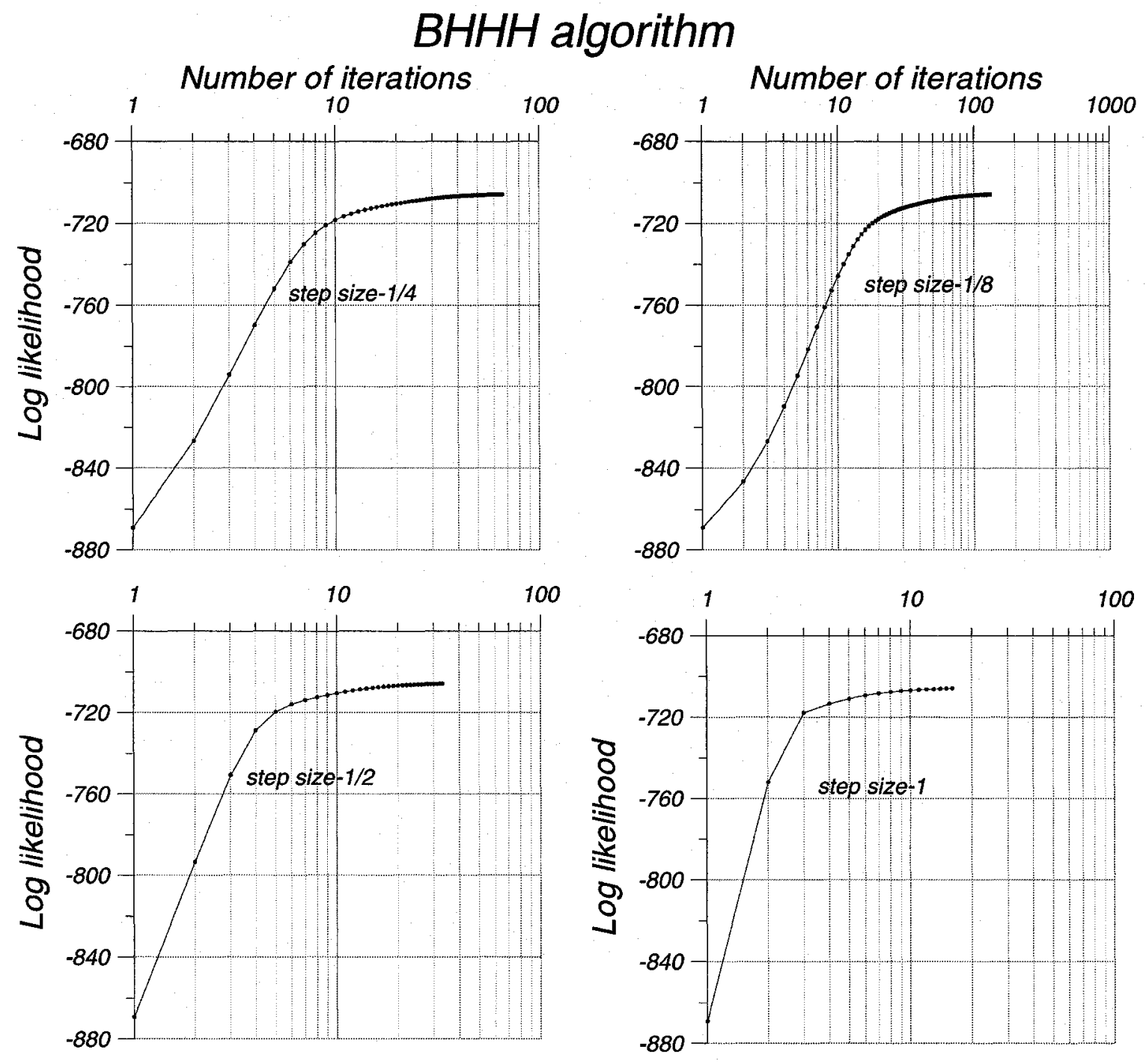

Figure 5-24: Log-likelihood values for each step size in BHHH algorithm (exp. 2) 


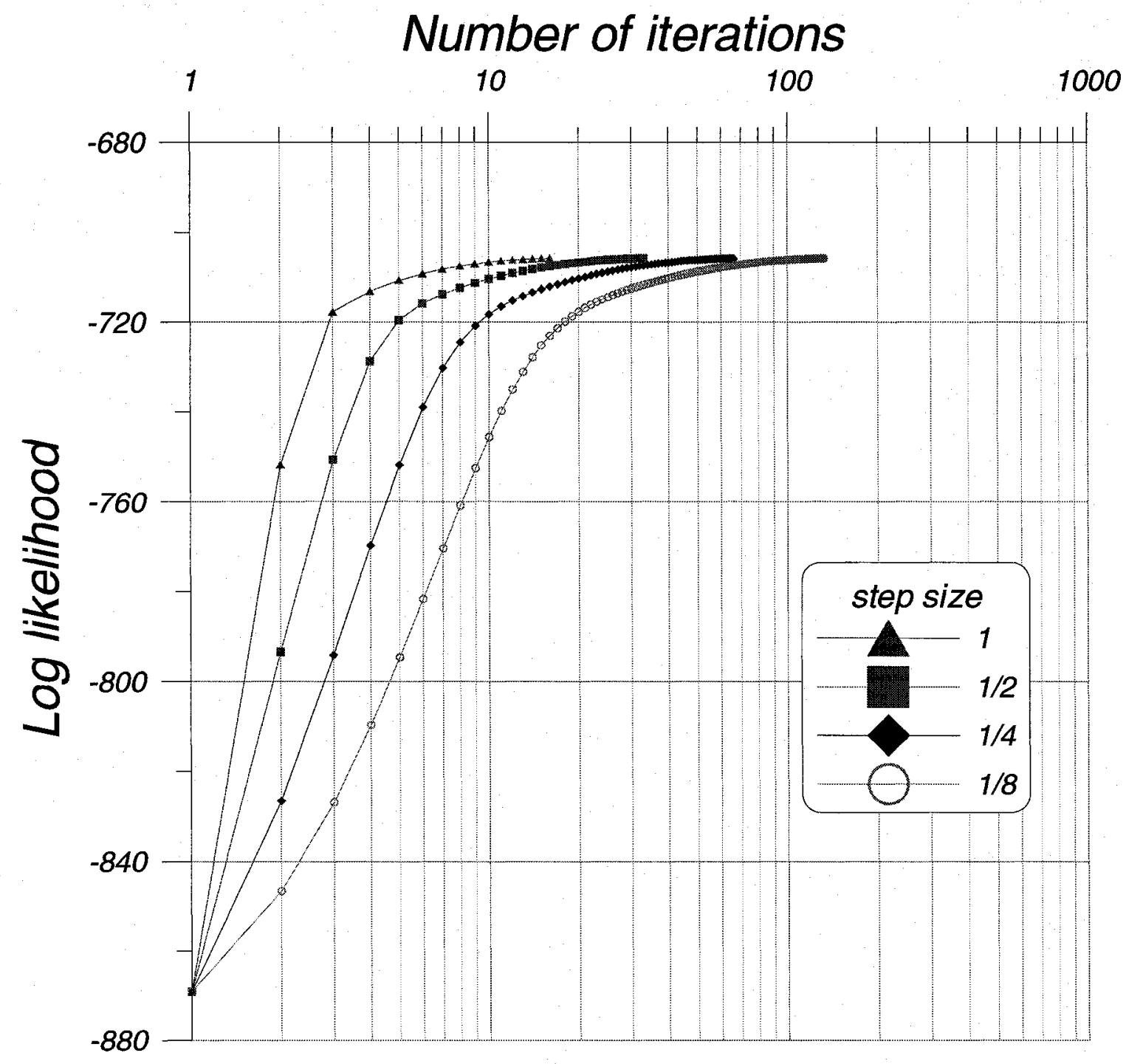

Figure 5-25: Log-likelihood for the all step sizes in BHHH algorithm (exp. 2) 


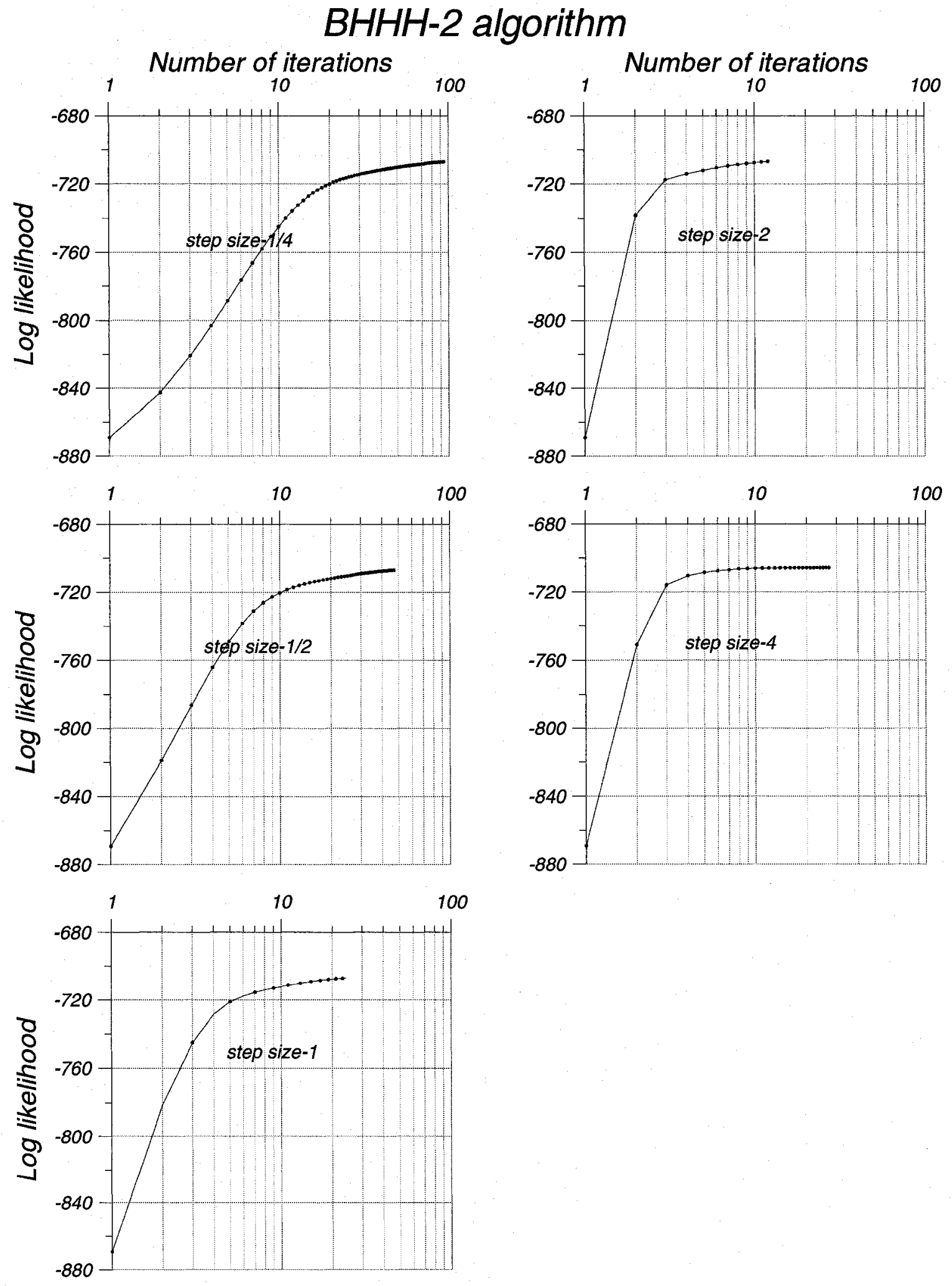

Figure 5-26: Log-likelihood values for each step size in BHHH-2 algorithm (exp. 2) 


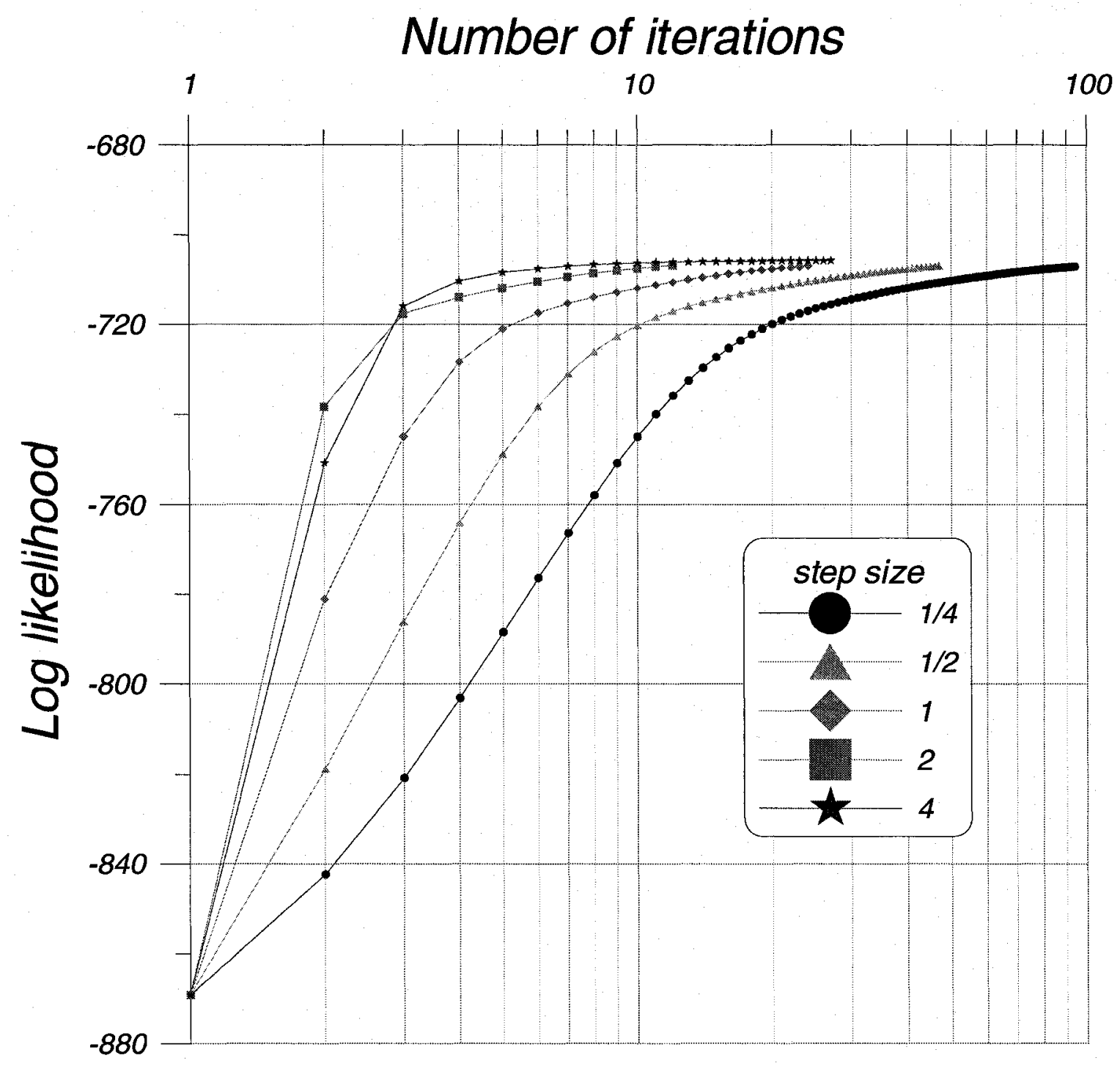

Figure 5-27: Log-likelihood for the all step sizes in BHHH-2 algorithm (exp. 2) 

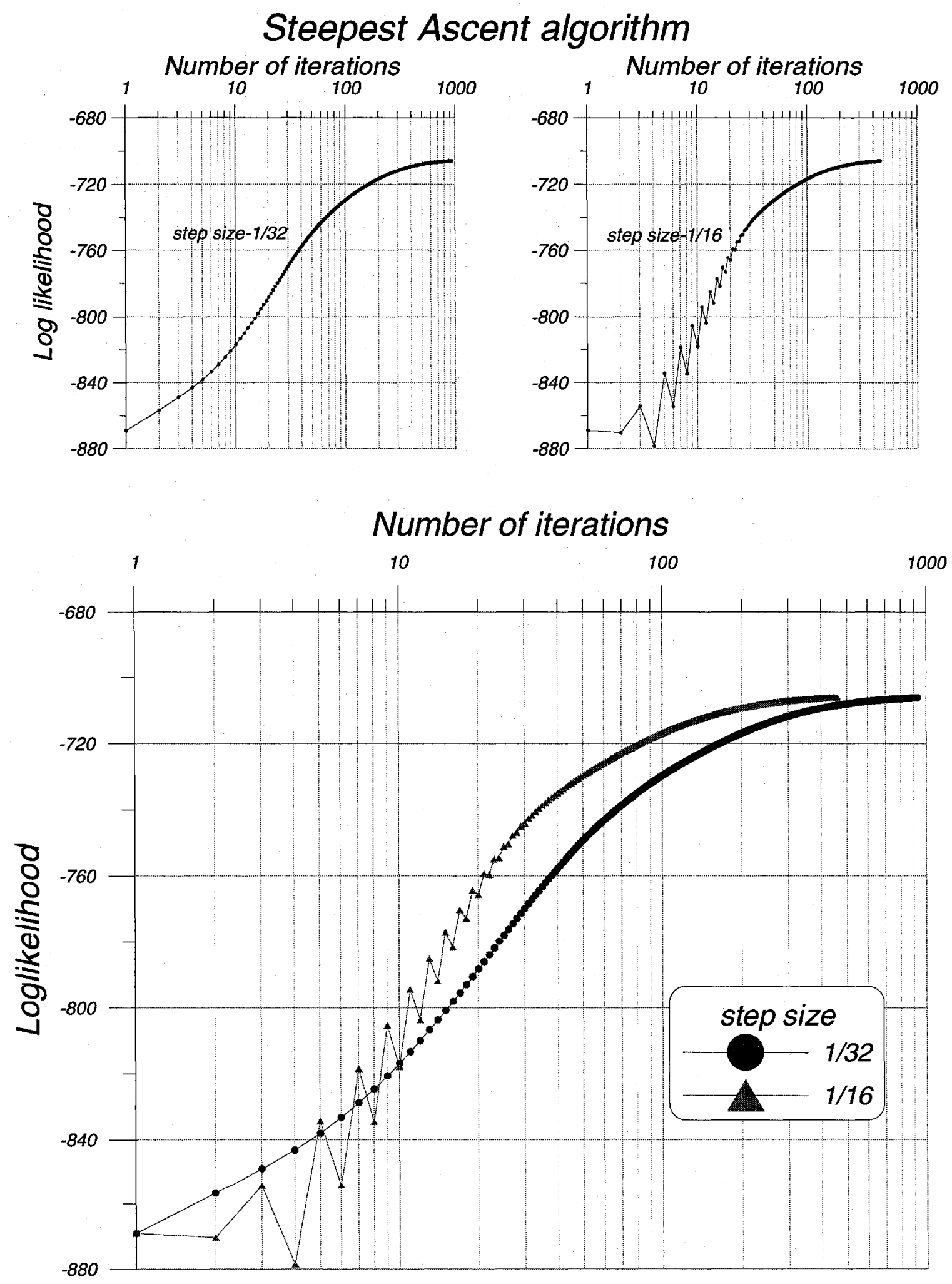

Figure 5-28: Log-likelihood values for each step size and all step sizes in Steepest Ascent algorithm (exp. 2) 


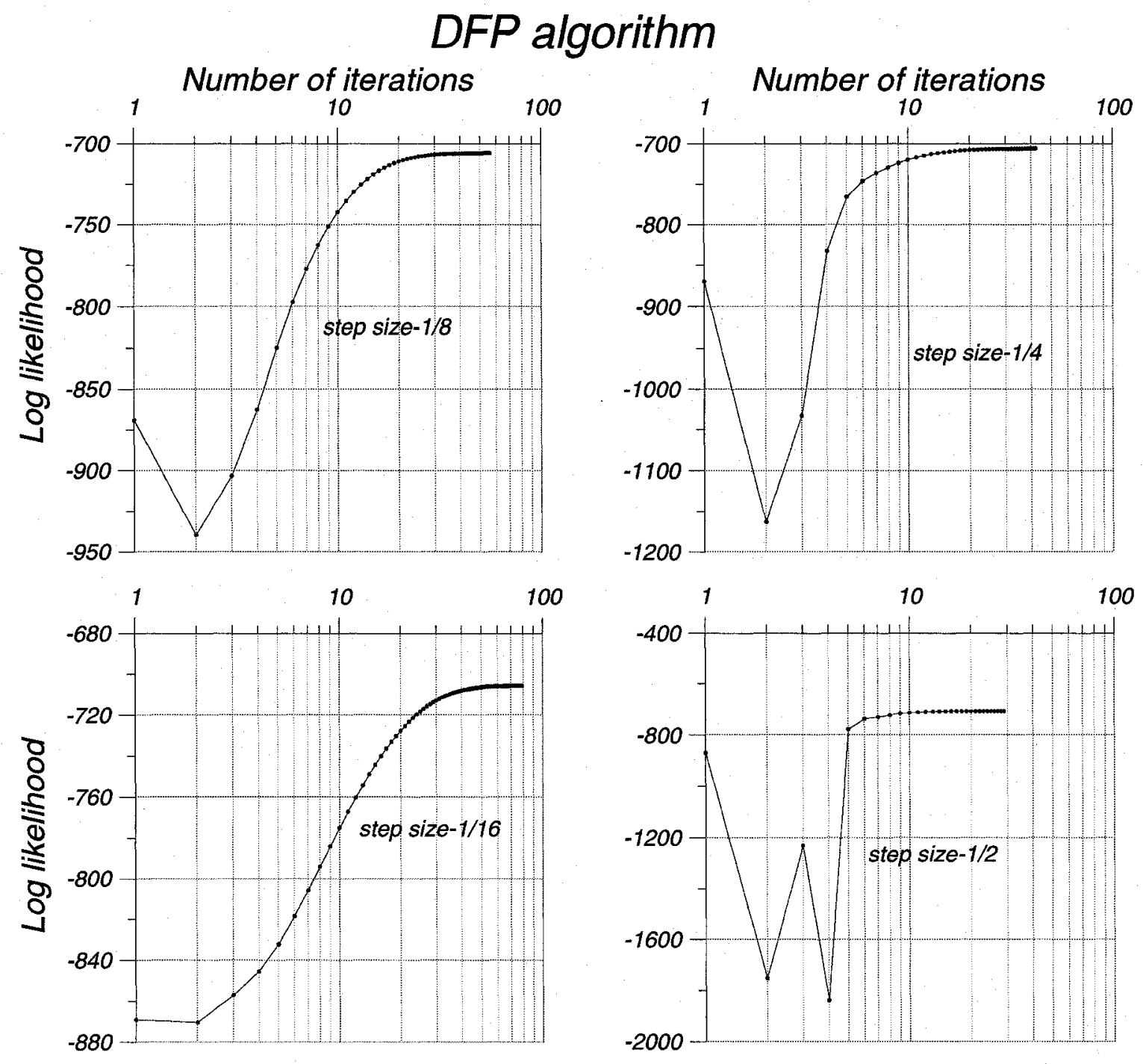

Figure 5-29: Log-likelihood values for each step size in DFP algorithm (exp. 2) 


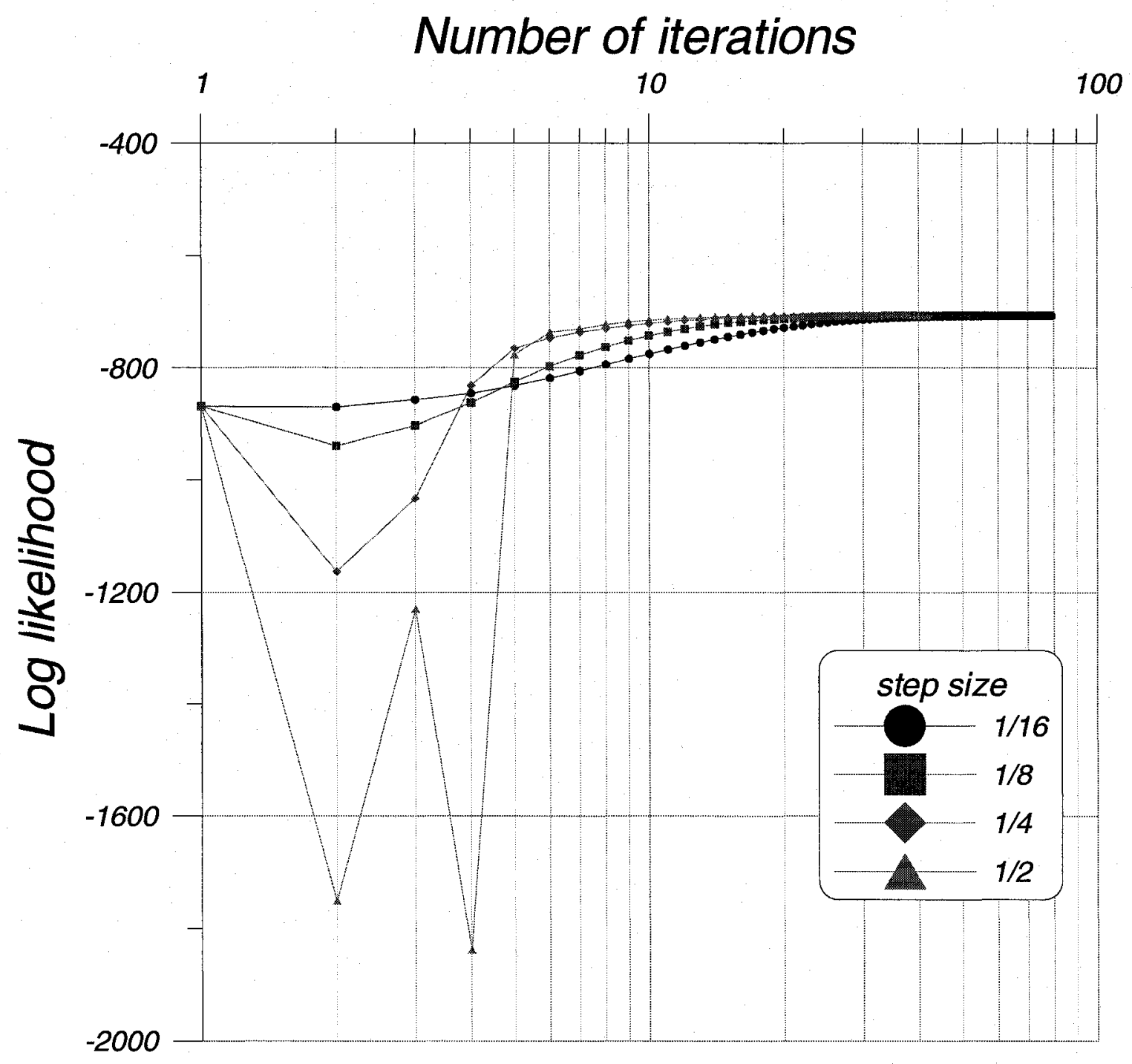

Figure 5-30: Log-likelihood for the all step sizes in BHHH-2 algorithm (exp. 2) 


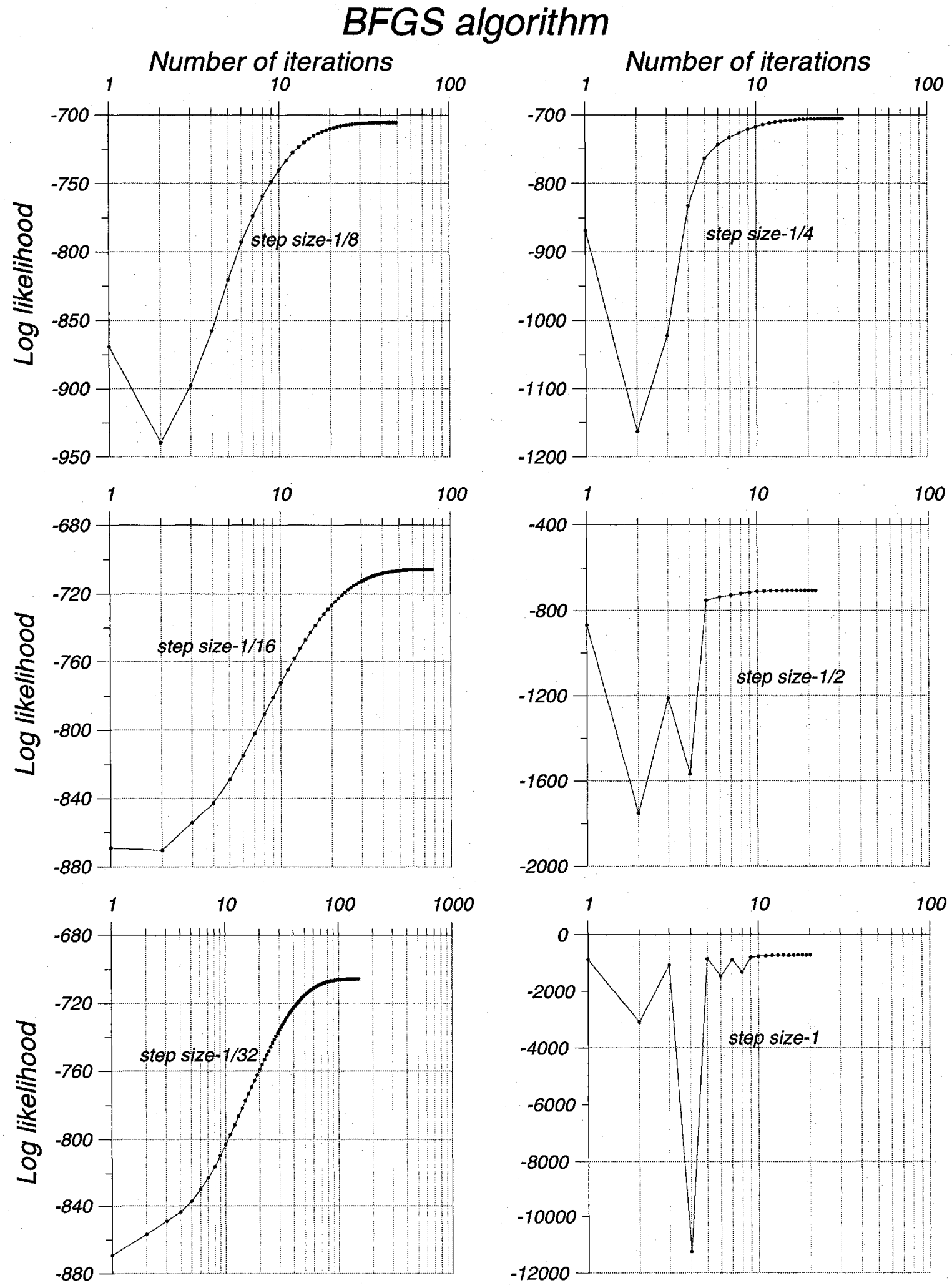

Figure 5-31: Log-likelihood values for each step size in BFGS algorithm (exp. 2) 


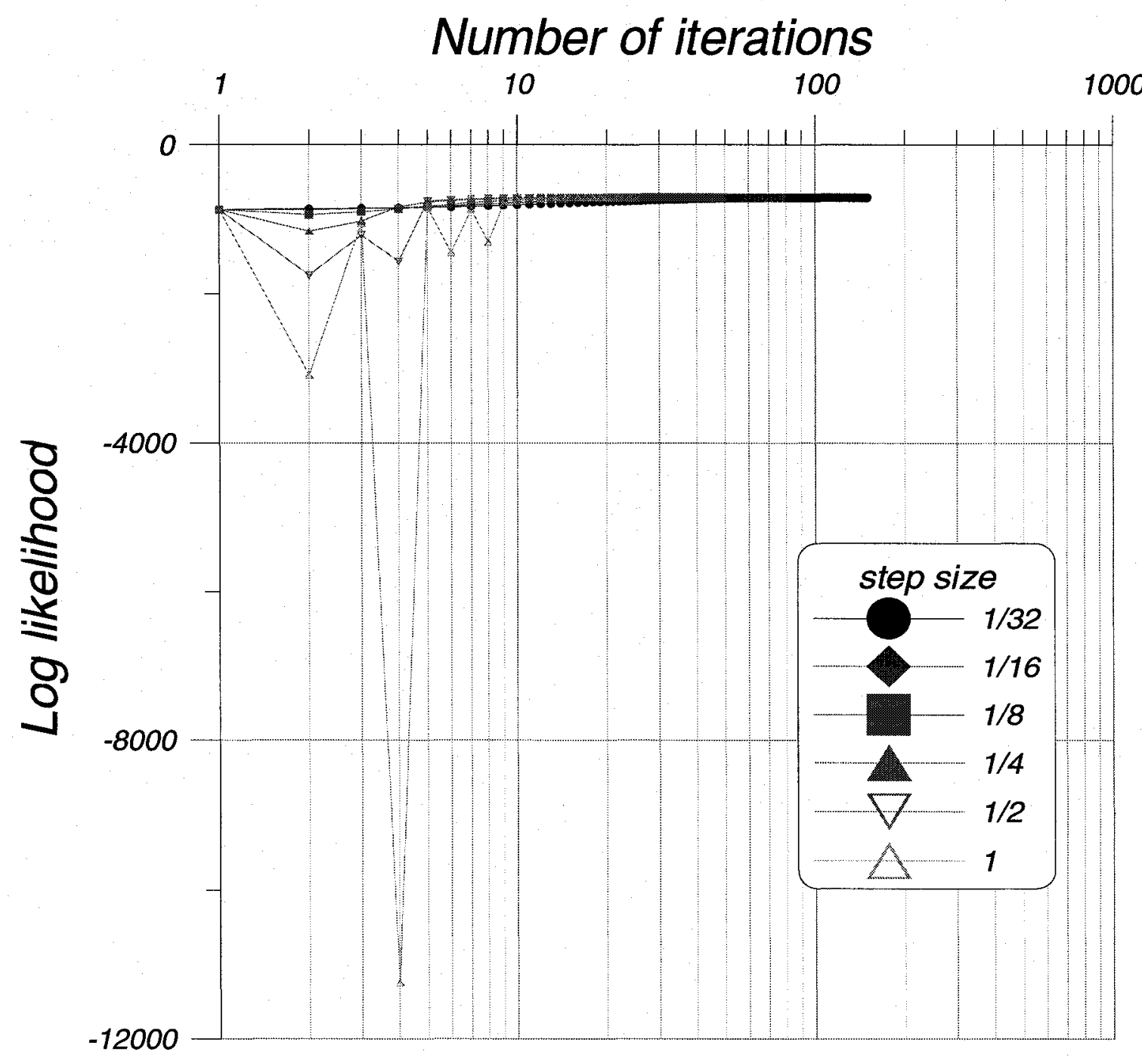

Figure 5-32: Log-likelihood for the all step sizes in BFGS algorithm (exp. 2) 


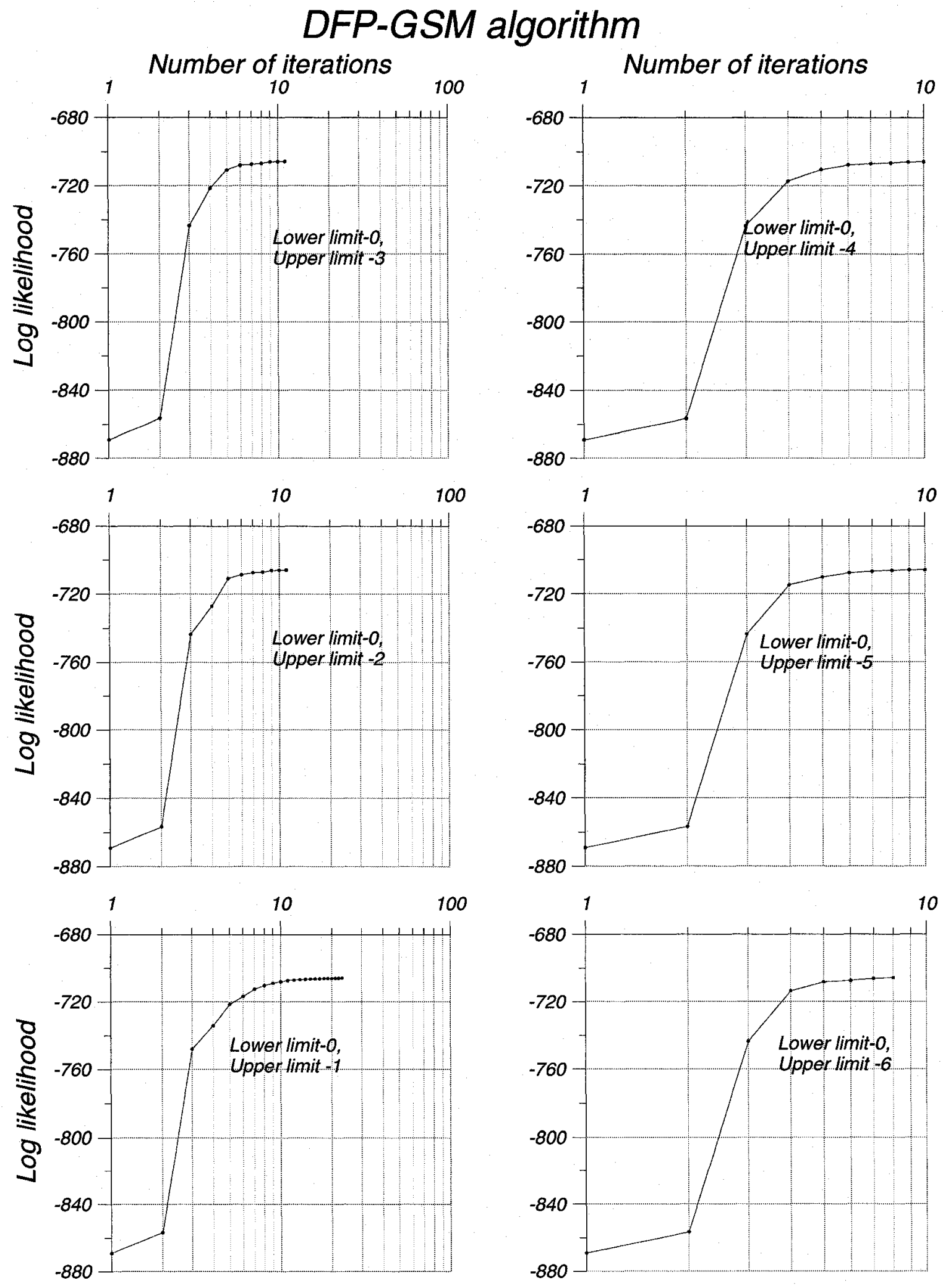

Figure 5-33: Log-likelihood values for each step size in DFP-GSM algorithm for the first six uncertain intervals (exp. 2) 

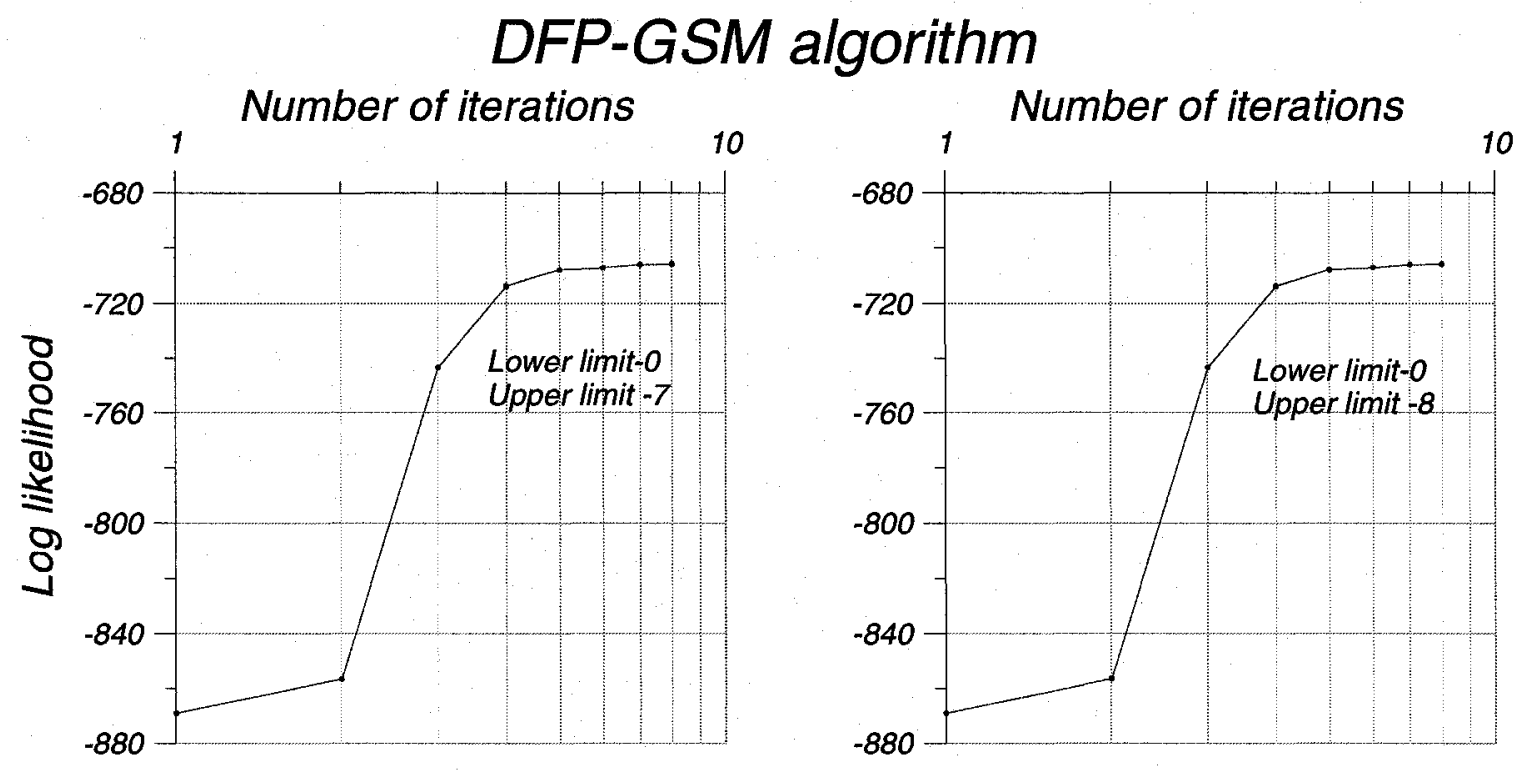

Figure 5-34: Log-likelihood values for each step size in DFP-GSM algorithm for the last two uncertain intervals (exp. 2) 


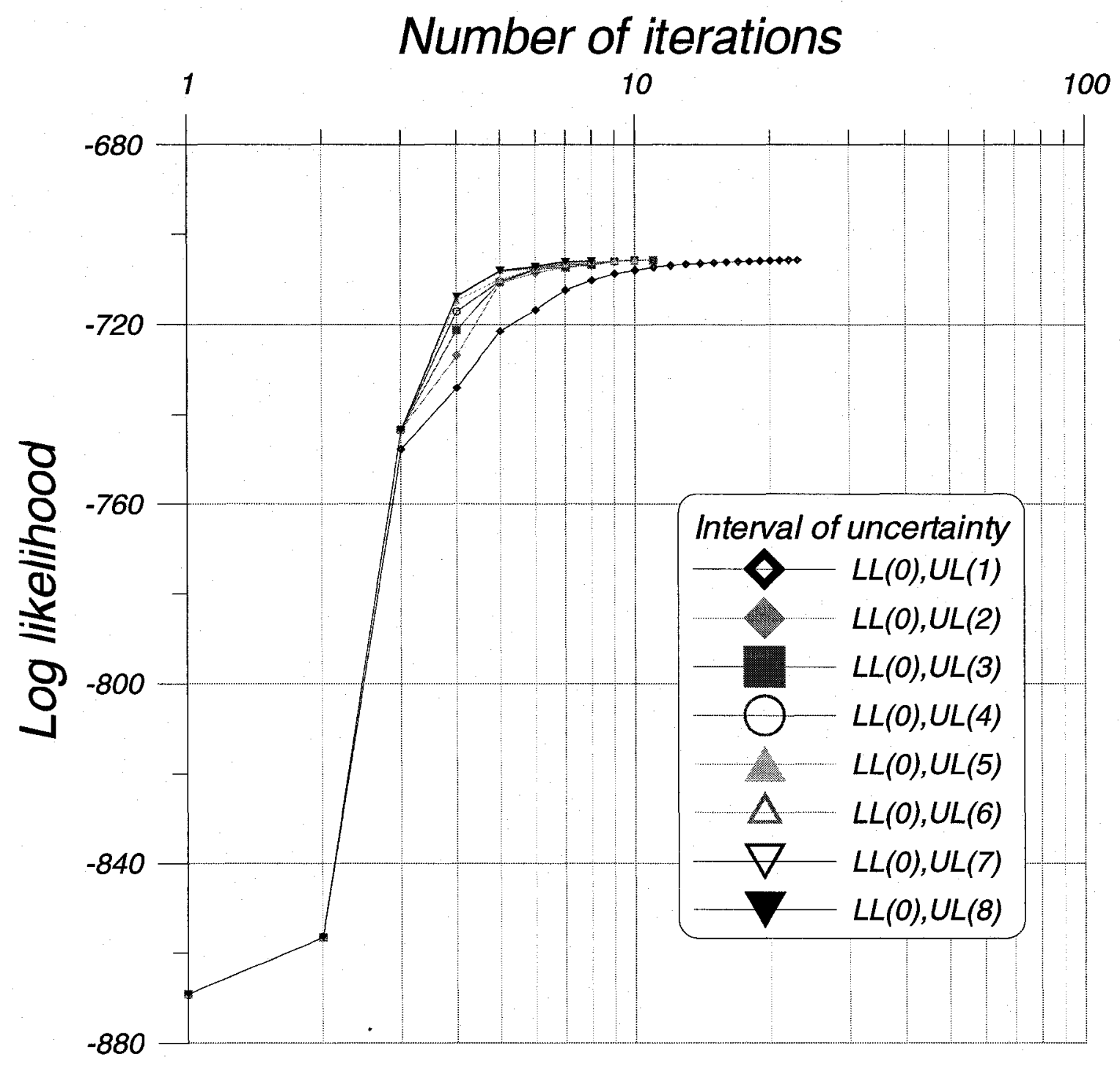

Figure 5-35: Log-likelihood for the all step sizes in DFP-GSM algorithm (exp. 2) 


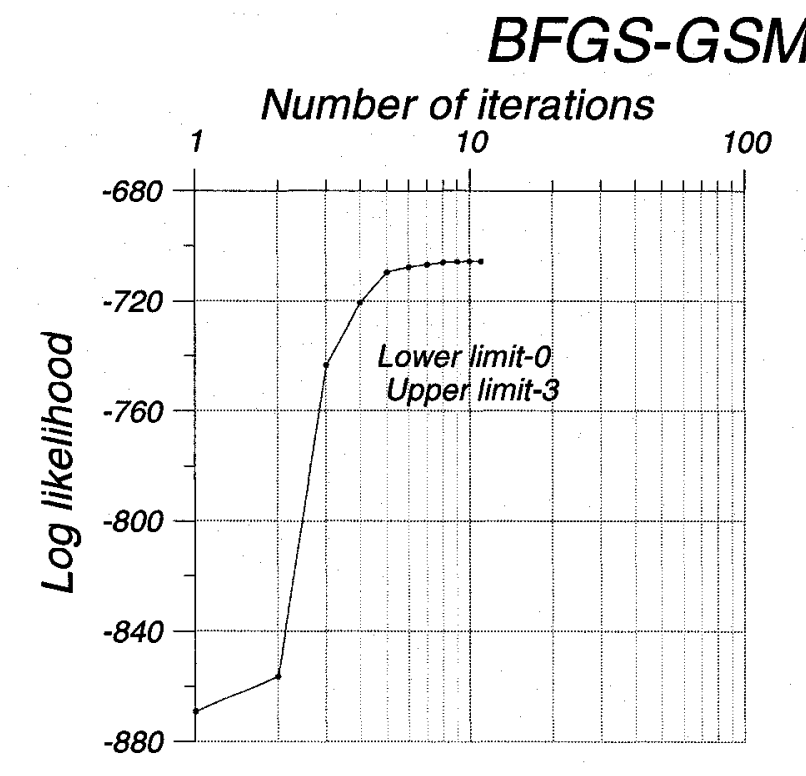

algorithm
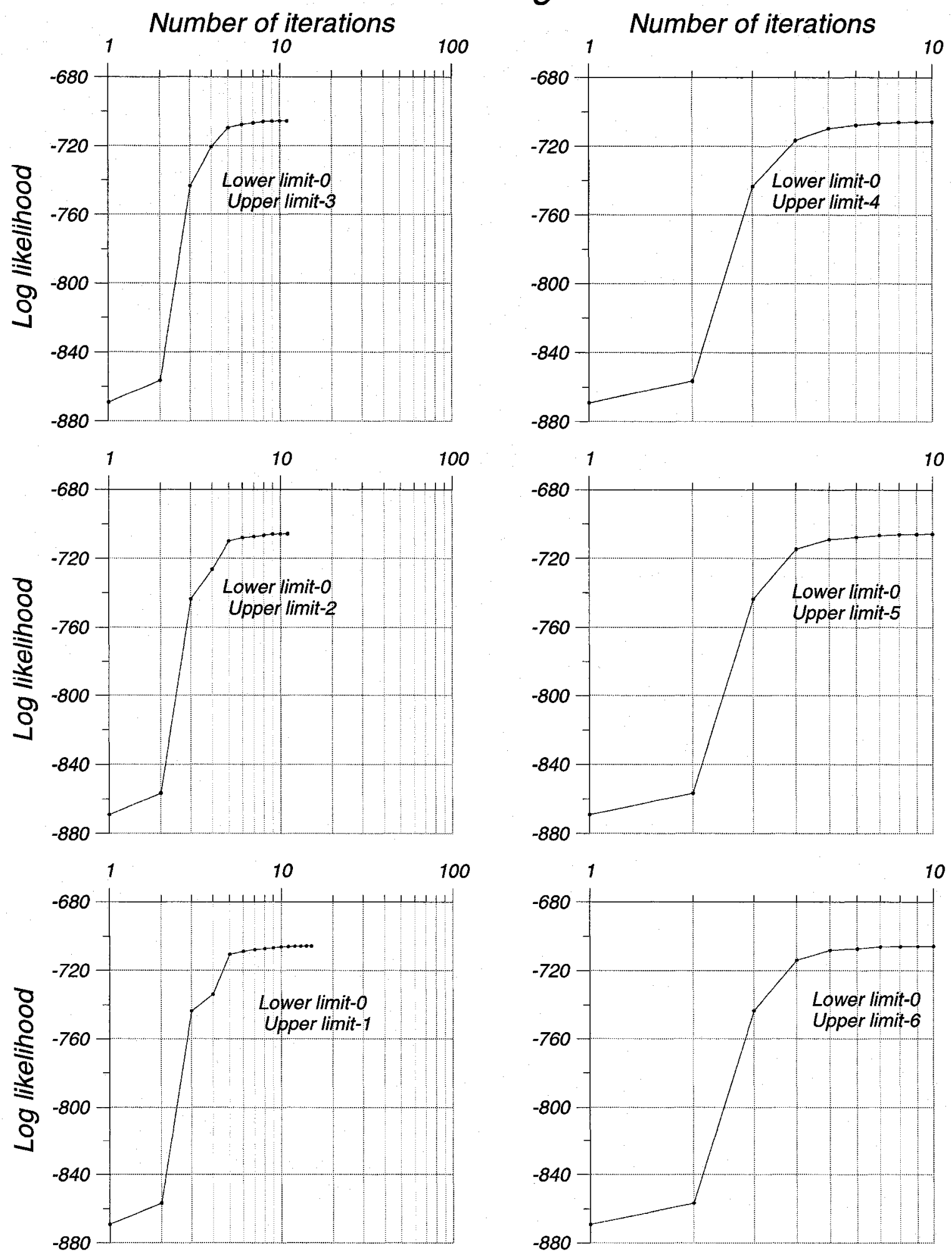

Figure 5-36: Log-likelihood values for each step size in BFGS-GSM algorithm for the first six uncertain intervals (exp. 2) 


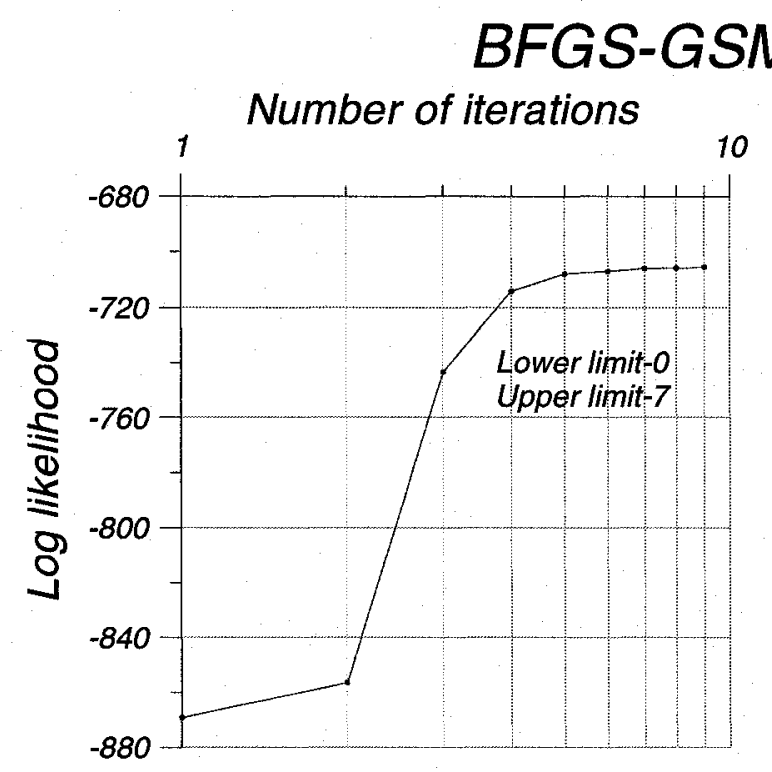

\section{algorithm}

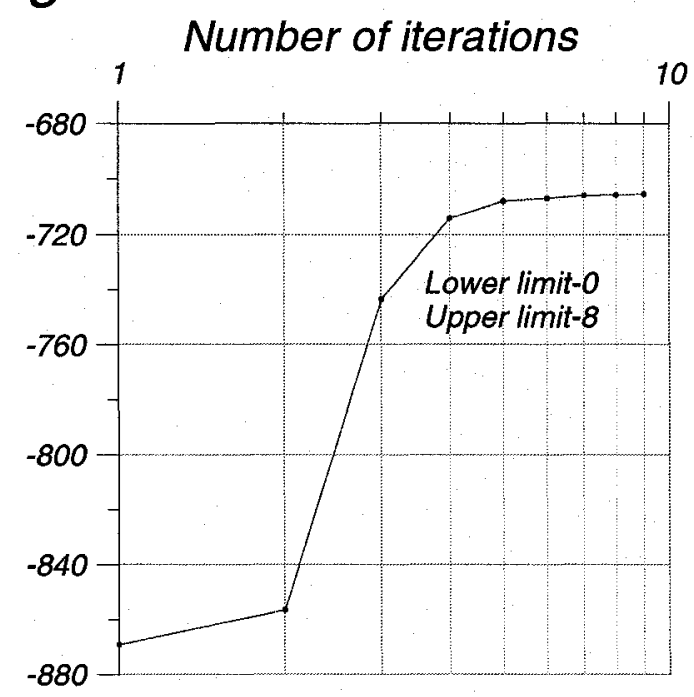

Figure 5-37: Log-likelihood values for each step size in BFGS-GSM algorithm for the last two uncertain intervals (exp. 2) 


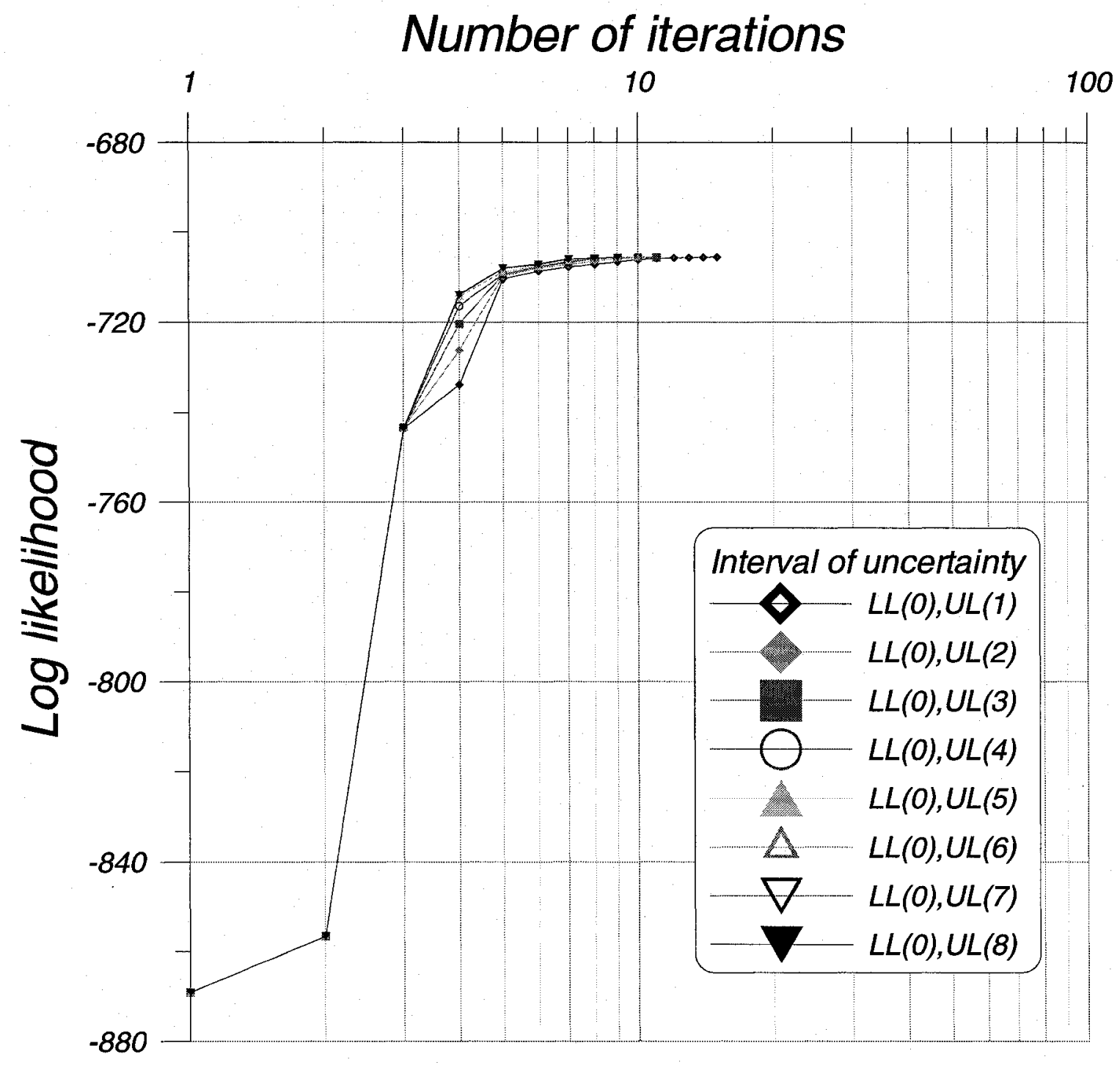

Figure 5-38: Log-likelihood for the all step sizes in BFGS-GSM algorithm (exp. 2) 


\subsubsection{The third convergence criterion (experiment 3)}

In this case, the applied convergence criterion is $G_{t}^{\prime}\left(-H_{t}\right)^{-1} G_{t}<10^{-5}$. The degree of precision of parameter estimates is changed to more stringent value, from $G_{t}^{\prime}\left(-H_{t}\right)^{-1} G_{t}<10^{-4}$ of experiment 2 to $G_{t}^{\prime}\left(-H_{t}\right)^{-1} G_{t}<10^{-5}$ of the current experiment 3. Information on this criterion was obtained from an econometric modelling study of Belsley (1979).

The results of the experimental estimation for the algorithms are summarized in table 521 in the same pattern and style as used in previous presentations. Detailed results are compared graphically in figures 5-39 and 5-40 in terms of three performance measures. 
Table 5-21: The final experiment results of the eight estimation algorithms (exp. 3)

\begin{tabular}{|c|c|c|c|c|c|c|c|c|c|}
\hline \multirow{2}{*}{\multicolumn{2}{|c|}{$\begin{array}{c}\text { Algorithms \& } \\
\text { Performance } \\
\text { measures }\end{array}$}} & \multicolumn{8}{|c|}{ Step size } \\
\hline & & $1 / 32$ & $1 / 16$ & $1 / 8$ & $1 / 4$ & $1 / 2$ & 1 & 2 & 4 \\
\hline \multirow{3}{*}{ 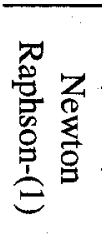 } & $\begin{array}{c}\text { One iteration } \\
\text { time }\end{array}$ & - & $0: 00: 18$ & $0: 00: 18$ & 0:00:22 & 0:00:19 & $0: 00: 16$ & - & - \\
\hline & $\begin{array}{c}\text { Number of } \\
\text { iterations }\end{array}$ & - & 91 & 45 & 22 & 10 & 4 & - & - \\
\hline & $\begin{array}{c}\text { Convergence } \\
\text { time }\end{array}$ & - & $0: 27: 50$ & $0: 13: 18$ & 0:08:10 & 0:03:07 & 0:01:05 & - & - \\
\hline \multirow{3}{*}{$\begin{array}{l}\text { 咠 } \\
\text { 夏 } \\
\text { 空 }\end{array}$} & $\begin{array}{c}\text { One iteration } \\
\text { time }\end{array}$ & - & - & $0: 0029$ & $0: 00: 28$ & $0: 00: 27$ & $0: 00: 26$ & - & - \\
\hline & $\begin{array}{c}\text { Number of } \\
\text { iterations }\end{array}$ & - & - & 209 & 104 & 52 & 26 & - & - \\
\hline & $\begin{array}{c}\text { Convergence } \\
\text { time }\end{array}$ & - & - & $1: 33: 58$ & $0: 48: 57$ & $0: 23: 45$ & $0: 11: 16$ & - & - \\
\hline \multirow{3}{*}{ 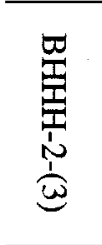 } & $\begin{array}{c}\text { One iteration } \\
\text { time }\end{array}$ & - & - & - & 0:00:39 & $0: 00: 40$ & $0: 00: 38$ & 0:00:40 & $0: 00: 40$ \\
\hline & $\begin{array}{c}\text { Number of } \\
\text { iterations }\end{array}$ & - & - & - & 188 & 94 & 47 & 23 & 64 \\
\hline & $\begin{array}{c}\text { Convergence } \\
\text { time }\end{array}$ & - & - & - & $2: 01: 37$ & $1: 02: 41$ & $0: 29: 26$ & $0: 15: 18$ & $0: 42: 45$ \\
\hline \multirow{3}{*}{ 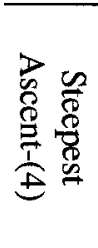 } & $\begin{array}{c}\text { One iteration } \\
\text { time }\end{array}$ & $0: 00: 20$ & 0:00:24 & - & - & - & - & - & - \\
\hline & $\begin{array}{c}\text { Number of } \\
\text { iterations }\end{array}$ & 1024 & 848 & - & - & - & - & - & - \\
\hline & $\begin{array}{c}\text { Convergence } \\
\text { time }\end{array}$ & $8: 50: 08$ & $5: 44: 44$ & - & - & - & - & - & - \\
\hline \multirow{3}{*}{$\frac{\sqrt{3}}{\frac{1}{3}}$} & $\begin{array}{c}\text { One iteration } \\
\text { time }\end{array}$ & - & $0: 00: 20$ & $0: 00: 19$ & $0: 00: 18$ & $0: 00: 20$ & - & - & - \\
\hline & $\begin{array}{c}\text { Number of } \\
\text { iterations }\end{array}$ & - & 110 & 83 & 62 & 45 & - & - & - \\
\hline & $\begin{array}{c}\text { Convergence } \\
\text { time }\end{array}$ & - & $0: 36: 37$ & $0: 26: 21$ & 0:19:03 & $0: 14: 32$ & - & - & - \\
\hline \multirow{4}{*}{$\begin{array}{l}\square \\
\frac{0}{0} \\
0 \\
\frac{1}{0} \\
0\end{array}$} & $\begin{array}{c}\text { One iteration } \\
\text { time }\end{array}$ & $0: 00: 19$ & $0: 00: 20$ & 0:00:20 & 0:00:19 & 0:00:19 & $0: 00: 19$ & - & - \\
\hline & $\begin{array}{c}\text { Number of } \\
\text { iterations }\end{array}$ & 212 & 126 & 64 & 42 & 29 & 25 & - & - \\
\hline & $\begin{array}{c}\text { Convergence } \\
\text { time }\end{array}$ & $0: 68: 41$ & $0: 41: 25$ & 0:21:00 & $0: 13: 31$ & 0:09:13 & 0:07:48 & - & - \\
\hline & $\begin{array}{l}\text { Interval of } \\
\text { uncertainty }\end{array}$ & $\begin{array}{l}\mathrm{LL}(0), \\
\mathrm{UL}(1)\end{array}$ & $\begin{array}{l}\mathrm{LL}(0), \\
\mathrm{UL}(2)\end{array}$ & $\begin{array}{l}\text { LL(0), } \\
\text { UL(3) }\end{array}$ & $\begin{array}{l}\text { LL(0), } \\
\text { UL(4) }\end{array}$ & $\begin{array}{l}\text { LL(0), } \\
\text { UL(5) }\end{array}$ & $\begin{array}{l}\text { LL(0), } \\
\text { UL(6) }\end{array}$ & $\begin{array}{l}\text { LL(0), } \\
\text { UL(7) }\end{array}$ & $\begin{array}{l}\mathrm{LL}(0), \\
\mathrm{UL}(8)\end{array}$ \\
\hline \multirow{3}{*}{ 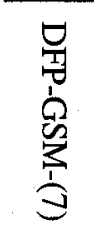 } & $\begin{array}{c}\text { One iteration } \\
\text { time } \\
\end{array}$ & 0:03:02 & $0: 03: 12$ & 0:03:16 & 0:01:16 & 0:03:23 & 0:01:53 & 0:01:53 & $0: 01: 50$ \\
\hline & $\begin{array}{c}\text { Number of } \\
\text { iterations }\end{array}$ & 33 & 15 & 15 & 14 & 14 & 13 & 13 & 13 \\
\hline & $\begin{array}{c}\text { Convergence } \\
\text { time }\end{array}$ & $1: 40: 14$ & $0: 47: 57$ & $0: 48: 48$ & $0: 17: 44$ & $0: 47: 15$ & $0: 24: 26$ & $0: 24: 27$ & $0: 23: 50$ \\
\hline \multirow{3}{*}{ ف } & $\begin{array}{c}\text { One iteration } \\
\text { time }\end{array}$ & $0: 01: 21$ & $0: 01: 26$ & $0: 01: 34$ & 0:01:30 & $0: 01: 33$ & 0:01:33 & 0:01:25 & $0: 01: 32$ \\
\hline & $\begin{array}{c}\text { Number of } \\
\text { iterations }\end{array}$ & 19 & 14 & 13 & 13 & 12 & 12 & 11 & 11 \\
\hline & $\begin{array}{c}\text { Convergence } \\
\text { time }\end{array}$ & $0: 25: 31$ & $0: 19: 55$ & $0: 20: 16$ & $0: 19: 18$ & $0: 18: 36$ & $0: 18: 27$ & $0: 15: 21$ & $0: 16: 47$ \\
\hline
\end{tabular}




\section{Convergence criterion 3}
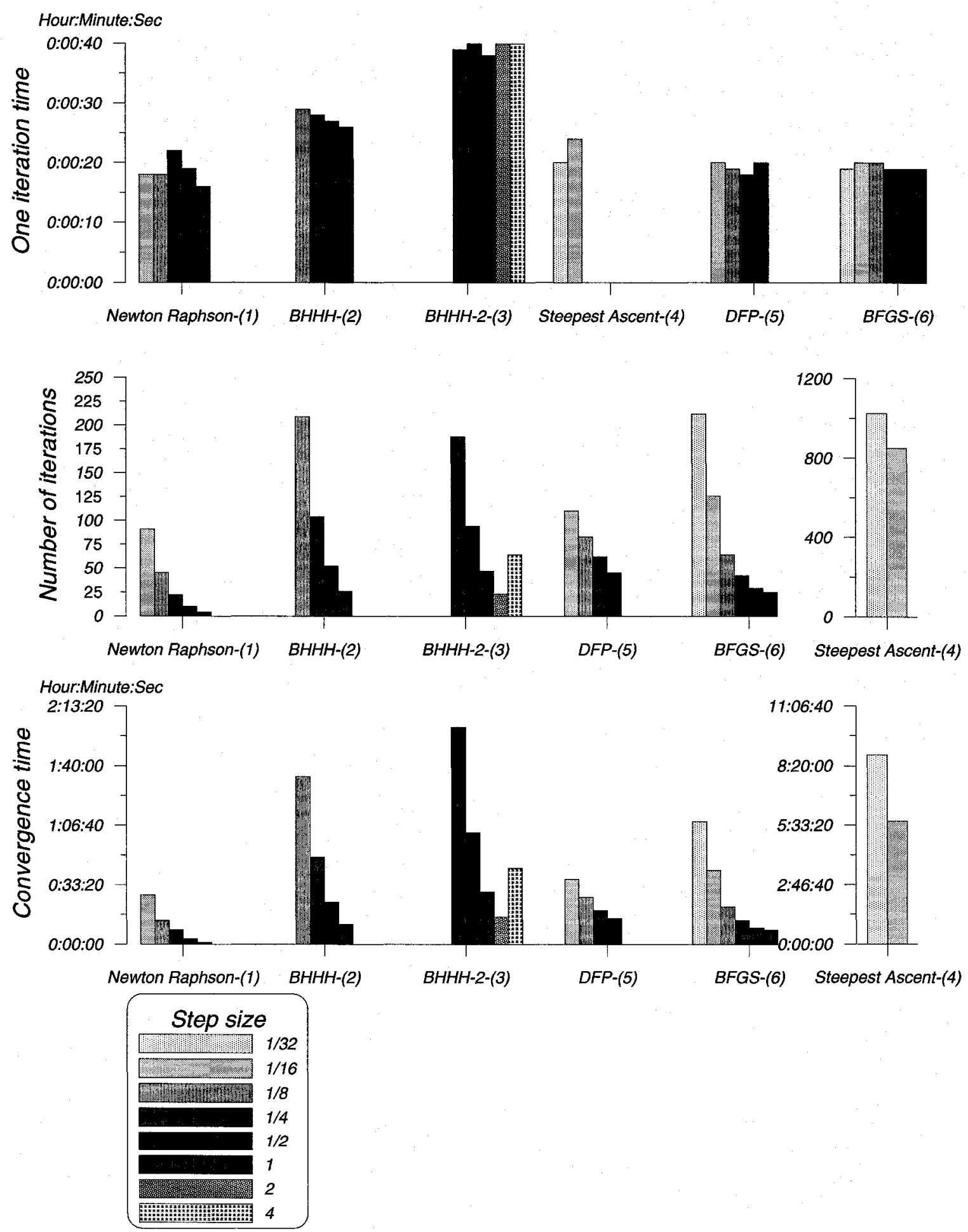

Figure 5-39: The final experimental results of the first six estimation algorithms (exp. 3) 

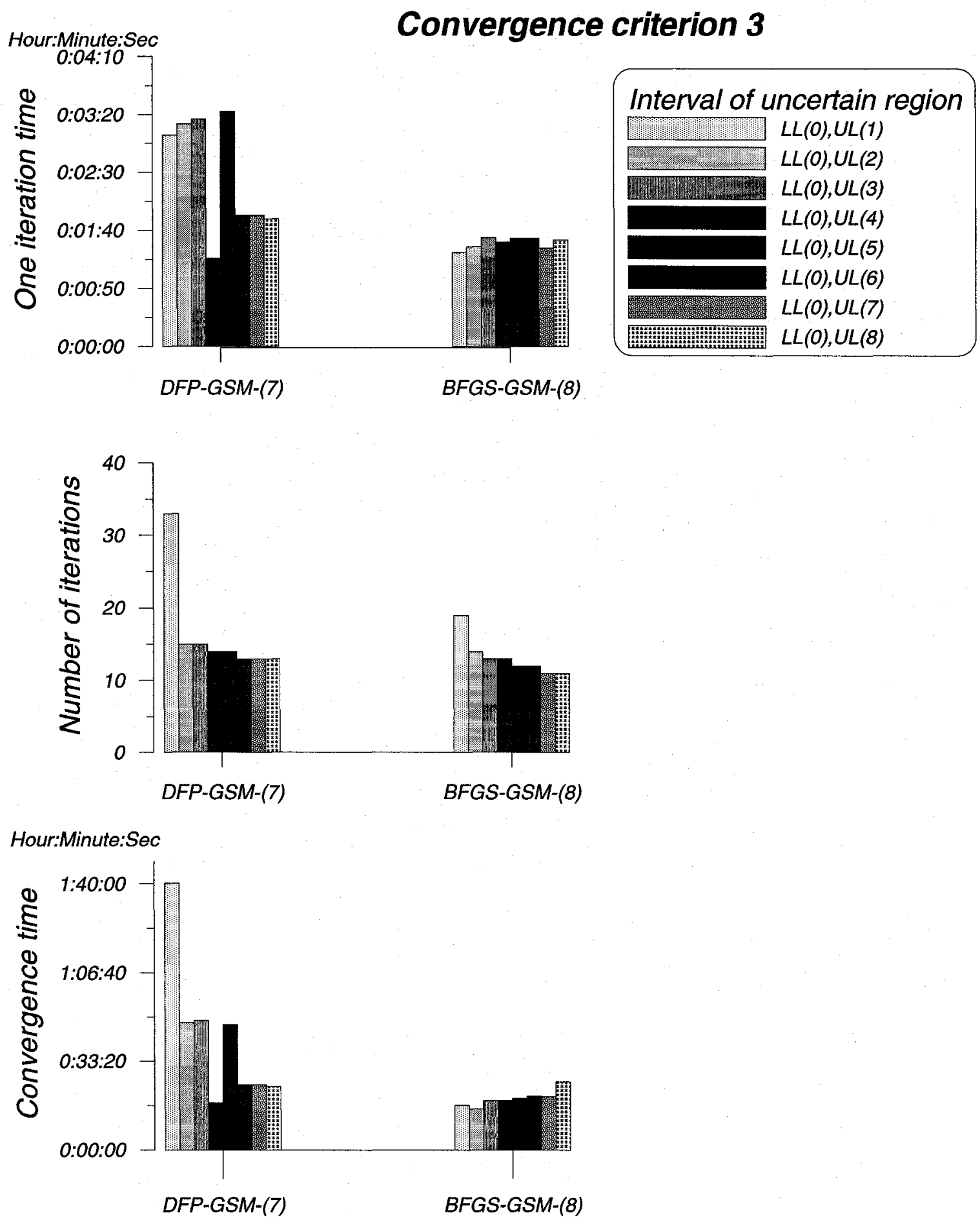

Figure 5-40: The final experimental results of the last two estimation algorithms (exp. 3) 
All conditions and calculation environments applied in the experimental estimation are exactly the same as for experiments 1 and 2, except for the convergence criterion, $G_{t}^{\prime}\left(-H_{t}\right)^{-1} G_{t}<10^{-5}$. Generally, for all eight algorithms, the estimation performance of the current experiment 3 has improved compared to that of experiment 1 , but has slightly deteriorated as compared to that of experiment 2. Although some runs in NR of experiment 3 appear to have improved in their performance, however, this phenomenon is confined only to NR, even only for some step sizes. The poor performance observed in experiment 3 can be attributed to the stopping criterion moved in the stringent direction. It must be the fact that a more stringent stopping criterion needs more calculation time to find a vector of parameter estimates satisfying the stringent stopping criterion.

In conclusion, when the third convergence criterion is applied as a new stopping measure, all algorithms show better estimation performance than experiment 1 , but present poorer performance than experiment 2. If a more stringent stopping criterion is applied, the time to convergence tends to increase. 


\subsubsection{The number of iterations and log-likelihood value (experiment 3)}

For all estimation results the graphs are drawn in order to show a variation of the loglikelihood function values following the iterations and moreover to compare clearly the different convergence behaviours between step sizes used in each algorithm. Tables 5-22 to 5-29 summarize critical values such as starting log-likelihood value and convergence log-likelihood value, and the number of iterations due to the different step sizes. As shown in tables 5-22 to 5-29, all calculated starting log-likelihood values are exactly the same for the all eight algorithms and for any step sizes used within each algorithm due to $\beta_{k}=\left[\beta_{1}=0, \beta_{2}=0, \beta_{3}=0, \cdots, \beta_{9}=0\right]^{\prime}$, which implies the same condition imposed as an initial guess of starting points in experiments 1 to 4 and 6 . On the other hands, convergence values are slightly different among the step sizes only below the decimal point and so the difference can be ignored and all runs are considered to be converged at same log-likelihood value. 
Table 5-22: Log-likelihood values obtained from Newton Raphson algorithm (exp. 3)

\begin{tabular}{|c|c|c|c|c|c|c|}
\hline \multirow{2}{*}{\multicolumn{2}{|c|}{ Log-likelihood }} & \multicolumn{5}{|c|}{ Step size } \\
\hline & & $1 / 16$ & $1 / 8$ & $1 / 4$ & $1 / 2$ & 1 \\
\hline \multirow{3}{*}{ 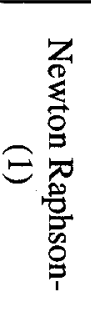 } & $\begin{array}{c}\text { Starting } \\
\text { Log- } \\
\text { likelihood }\end{array}$ & -869.0964727 & -869.0964727 & -869.0964727 & -869.0964727 & -869.0964727 \\
\hline & $\begin{array}{c}\text { Convergence } \\
\text { log- } \\
\text { likelihood }\end{array}$ & -705.5408541 & -705.54062 & -705.5400897 & -705.5398124 & -705.5384404 \\
\hline & $\begin{array}{l}\text { Number of } \\
\text { iterations }\end{array}$ & 91 & 45 & 22 & 10 & 4 \\
\hline
\end{tabular}

Table 5-23: Log-likelihood values obtained from BHHH algorithm (exp. 3)

\begin{tabular}{cccccc}
\hline \multirow{2}{*}{ Log-likelihood } & \multicolumn{4}{c}{ Step size } \\
\cline { 2 - 6 } & $1 / 8$ & $1 / 4$ & $1 / 2$ & 1 \\
\hline \multirow{2}{*}{ 荳 } & $\begin{array}{c}\text { Starting } \\
\text { Log-likelihood }\end{array}$ & -869.0964727 & -869.0964727 & -869.0964727 & -869.0964727 \\
\cline { 2 - 6 } & $\begin{array}{c}\text { Convergence } \\
\text { Log-likelihood }\end{array}$ & -705.5622106 & -705.562202 & -705.5609052 & -705.5580972 \\
\cline { 2 - 6 } & Number of iterations & 209 & 104 & 52 & 26 \\
\hline
\end{tabular}

Table 5-24: Log-likelihood values obtained from BHHH-2 algorithm (exp. 3)

\begin{tabular}{ccccccc}
\hline \multirow{2}{*}{ Log-likelihood } & \multicolumn{5}{c}{ Step size } \\
\cline { 2 - 7 } & $1 / 4$ & $1 / 2$ & 1 & 2 & 4 \\
\hline \multirow{2}{*}{$心 \begin{array}{c}\text { Starting } \\
\text { Log-likelihood }\end{array}$} & -869.0964727 & -869.0964727 & -869.0964727 & -869.0964727 & -869.0964727 \\
\hline $\begin{array}{c}\text { Convergence } \\
\text { log-likelihood }\end{array}$ & -705.7051738 & -705.702285 & -705.6965751 & -705.6908968 & -705.5530931 \\
\hline Number of iterations & 188 & 94 & 47 & 23 & 64 \\
\hline
\end{tabular}

Table 5-25: Log likelihood values obtained from Steepest Ascent algorithm (exp. 3)

\begin{tabular}{|c|c|c|c|}
\hline & \multirow{2}{*}{ Log-likelihood } & \multicolumn{2}{|c|}{ Step size } \\
\hline & & $1 / 32$ & $1 / 16$ \\
\hline \multirow{3}{*}{ 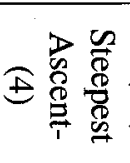 } & $\begin{array}{c}\text { Starting } \\
\text { Log-likelihood }\end{array}$ & -869.0964727 & -869.0964727 \\
\hline & Convergence log-likelihood & -705.9654962 & -705.6116978 \\
\hline & Number of iterations & 1024 & 848 \\
\hline
\end{tabular}


Table 5-26: Log-likelihood values obtained from DFP algorithm (exp. 3)

\begin{tabular}{cccccc}
\hline \multirow{2}{*}{ Log-likelihood } & \multicolumn{5}{c}{ Step size } \\
\cline { 2 - 6 } & $1 / 16$ & $1 / 8$ & $1 / 4$ & $1 / 2$ \\
\hline \multirow{3}{*}{$\begin{array}{c}\text { Starting } \\
\text { 守 }\end{array}$} & Log-likelihood & -869.0964727 & -869.0964727 & -869.0964727 & -869.0964727 \\
\cline { 2 - 6 } & Convergence log-likelihood & -705.5515777 & -705.5521877 & -705.5563912 & -705.5649595 \\
\cline { 2 - 6 } & Number of iterations & 110 & 83 & 62 & 45 \\
\hline
\end{tabular}

Table 5-27: Log-likelihood values obtained from BFGS algorithm (exp. 3)

\begin{tabular}{cccccccc}
\hline \multirow{2}{*}{ Log-likelihood } & \multicolumn{6}{c}{ Step size } \\
\cline { 2 - 7 } & $1 / 32$ & $1 / 16$ & $1 / 8$ & $1 / 4$ & $1 / 2$ & 1 \\
\hline \multirow{5}{*}{$\begin{array}{c}\text { Starting } \\
\text { Log- } \\
\text { likelihood }\end{array}$} & -869.0964727 & -869.0964727 & -869.0964727 & -869.0964727 & -869.0964727 & -869.0964727 \\
$\begin{array}{c}\text { Convergence } \\
\text { log-likelihood }\end{array}$ & -705.5433026 & -705.5435922 & -705.5504568 & -705.5505559 & -705.5514638 & -705.5638547 \\
\hline $\begin{array}{c}\text { Number of } \\
\text { iterations }\end{array}$ & 212 & 126 & 64 & 42 & 29 & 25 \\
\hline
\end{tabular}

Table 5-28: Log-likelihood values obtained from DFP-GSM algorithm (exp. 3)

\begin{tabular}{|c|c|c|c|c|c|c|c|c|c|}
\hline & $\begin{array}{l}\text { Interval of } \\
\text { uncertainty }\end{array}$ & $\begin{array}{l}\mathrm{LL}(0), \\
\mathrm{UL}(1)\end{array}$ & $\begin{array}{l}\text { LL(0), } \\
\text { UL(2) }\end{array}$ & $\begin{array}{l}\text { LL(0), } \\
\text { UL(3) }\end{array}$ & $\begin{array}{l}\text { LL(0), } \\
\text { UL(4) }\end{array}$ & $\begin{array}{l}\text { LL(0), } \\
\text { UL(5) }\end{array}$ & $\begin{array}{l}\text { LL(0), } \\
\text { UL(6) }\end{array}$ & $\begin{array}{l}\mathrm{LL}(0), \\
\mathrm{UL}(7)\end{array}$ & $\begin{array}{l}\text { LL(0), } \\
\mathrm{UL}(8)\end{array}$ \\
\hline \multirow{3}{*}{ 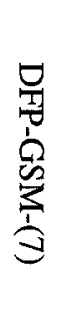 } & $\begin{array}{c}\text { Starting } \\
\text { Log- } \\
\text { likelihood }\end{array}$ & $\begin{array}{c}869.0964 \\
727\end{array}$ & $\begin{array}{c}- \\
869.0964 \\
727\end{array}$ & $\begin{array}{c}- \\
869.0964 \\
727\end{array}$ & $\begin{array}{c}869.0964 \\
727\end{array}$ & $\begin{array}{c}869.0964 \\
727\end{array}$ & $\begin{array}{c}869.0964 \\
727\end{array}$ & $\begin{array}{c}869.0964 \\
727\end{array}$ & $\begin{array}{c}869.0964 \\
727\end{array}$ \\
\hline & $\begin{array}{c}\text { Convergence } \\
\text { log- } \\
\text { likelihood }\end{array}$ & $\begin{array}{c}705.5585 \\
592\end{array}$ & $\begin{array}{c}- \\
705.5623 \\
115\end{array}$ & $\begin{array}{c}- \\
705.5614 \\
377\end{array}$ & $\begin{array}{c}- \\
705.5583 \\
746\end{array}$ & $\begin{array}{c}- \\
705.5494 \\
868\end{array}$ & $\begin{array}{c}- \\
705.5514 \\
34\end{array}$ & $\begin{array}{c}- \\
705.5497 \\
142\end{array}$ & $\begin{array}{c}705.5494 \\
614\end{array}$ \\
\hline & $\begin{array}{l}\text { Number of } \\
\text { iterations }\end{array}$ & 33 & 15 & 15 & 14 & 14 & 13 & 13 & 13 \\
\hline
\end{tabular}

Table 5-29: Log-likelihood values obtained from BFGS-GSM algorithm (exp. 3)

\begin{tabular}{|c|c|c|c|c|c|c|c|c|c|}
\hline & $\begin{array}{l}\text { Interval of } \\
\text { uncertainty }\end{array}$ & $\begin{array}{l}\mathrm{LL}(0), \\
\mathrm{UL}(1)\end{array}$ & $\begin{array}{l}\text { LL(0), } \\
\text { UL(2) }\end{array}$ & $\begin{array}{l}\mathrm{LL}(0), \\
\mathrm{UL}(3)\end{array}$ & $\begin{array}{l}\text { LL(0), } \\
\text { UL(4) }\end{array}$ & $\begin{array}{l}\mathrm{LL}(0), \\
\mathrm{UL}(5)\end{array}$ & $\begin{array}{l}\mathrm{LL}(0), \\
\mathrm{UL}(6)\end{array}$ & $\begin{array}{l}\text { LL(0), } \\
\text { UL(7) }\end{array}$ & $\begin{array}{l}\text { LL(0), } \\
\text { UL(8) }\end{array}$ \\
\hline \multirow{3}{*}{ 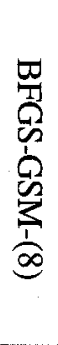 } & $\begin{array}{c}\text { Starting } \\
\text { Log- } \\
\text { likelihood }\end{array}$ & $\begin{array}{c}- \\
869.0964 \\
727\end{array}$ & $\begin{array}{c}869.0964 \\
727\end{array}$ & $\begin{array}{c}869.0964 \\
727\end{array}$ & $\begin{array}{c}- \\
869.0964 \\
727\end{array}$ & $\begin{array}{c}869.0964 \\
727\end{array}$ & $\begin{array}{c}- \\
869.0964 \\
727\end{array}$ & $\begin{array}{c}- \\
869.0964 \\
727\end{array}$ & $\begin{array}{c}869.0964 \\
727\end{array}$ \\
\hline & $\begin{array}{c}\text { Convergence } \\
\text { log- } \\
\text { likelihood }\end{array}$ & $\begin{array}{c}705.5495 \\
373\end{array}$ & $\begin{array}{c}705.5515 \\
069\end{array}$ & $\begin{array}{c}705.5498 \\
357\end{array}$ & $\begin{array}{c}705.5494 \\
556\end{array}$ & $\begin{array}{c}705.5509 \\
704\end{array}$ & $\begin{array}{c}705.5498 \\
048\end{array}$ & $\begin{array}{c}705.5505 \\
166\end{array}$ & $\begin{array}{c}705.5506 \\
81\end{array}$ \\
\hline & $\begin{array}{l}\text { Number of } \\
\text { iterations }\end{array}$ & 19 & 14 & 13 & 13 & 12 & 12 & 11 & 11 \\
\hline
\end{tabular}


In the case of the Newton Raphson algorithm where the step size is one, as shown in figures 5-41 and 5-42 and in table 5-22, four iterations were required. The number of iterations is the same as in experiment 2 , and one iteration decreases as compared to experiment 1 . The convergence behaviour is identical with experiments 1 and 2 except the fact that slight differences happen over the three performance measures. These experimental results are clearly presented in the graph. Overall, the phenomena observed are with decreasing step size, the slope also becomes small but the number of iterations rises.

The interpretation of results mentioned above can be applied to the remaining algorithms. We can identify the best performance among the runs performed using various step sizes and see the convergence log-likelihood value with the help of given tables and graphs (i.e., tables 5-22 to 5-29 and graphs 5-41 to 5-57). Also, the profile of graph at the last iteration is not as long as that of experiment 1 but as compared with experiment 2 , the overlapped part becomes longer. It means that more iterations are conducted in each algorithm to satisfy a new stringent criterion charged for stopping the iteration process. It also means that the iteration number and convergence time can be adjusted to become suitable for each specific modelling work or for maintaining the accuracy of parameters within the desired accuracy demand. 


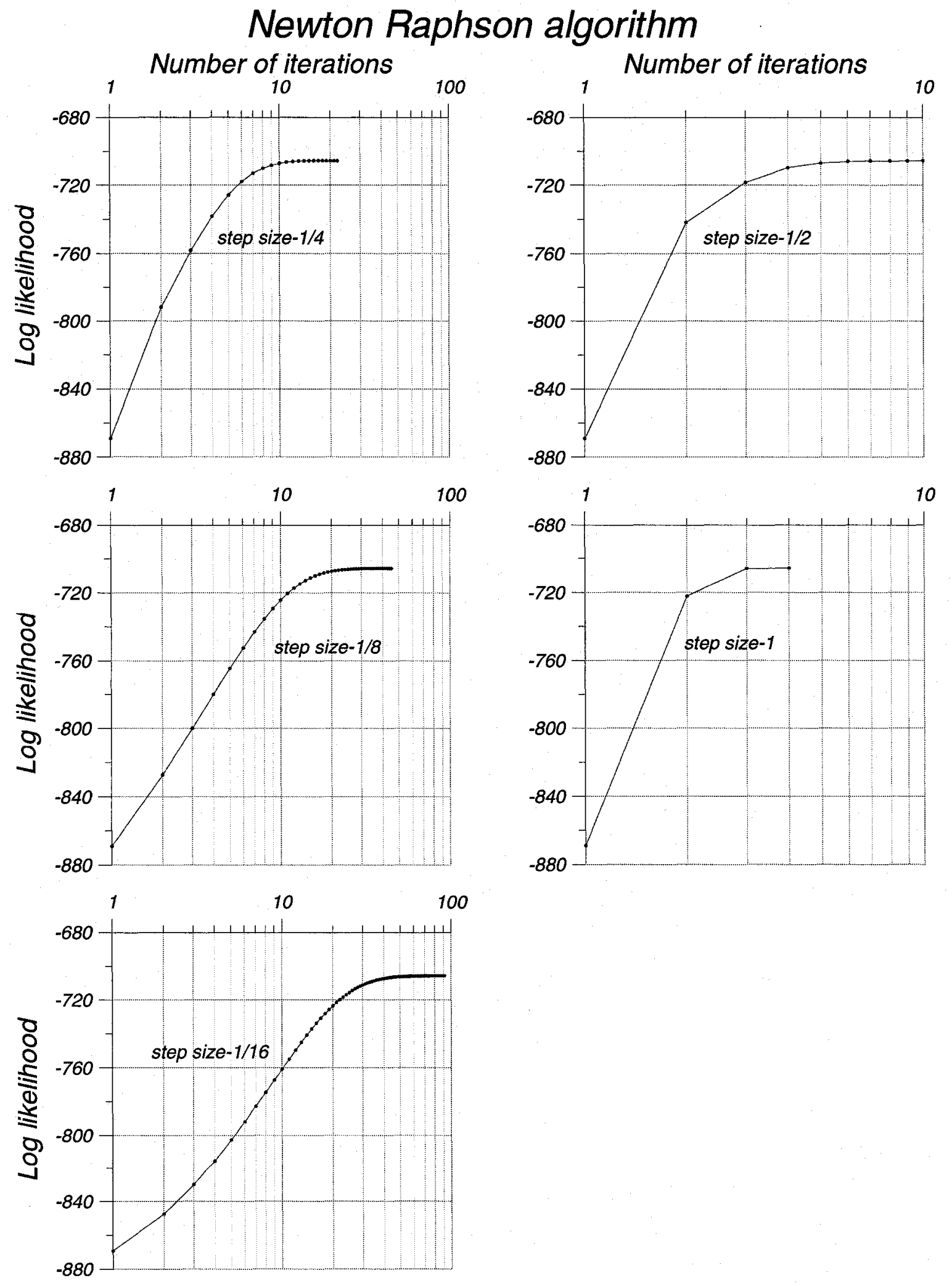

Figure 5-41: Log-likelihood values for each step size in Newton Raphson algorithm (exp. 3) 


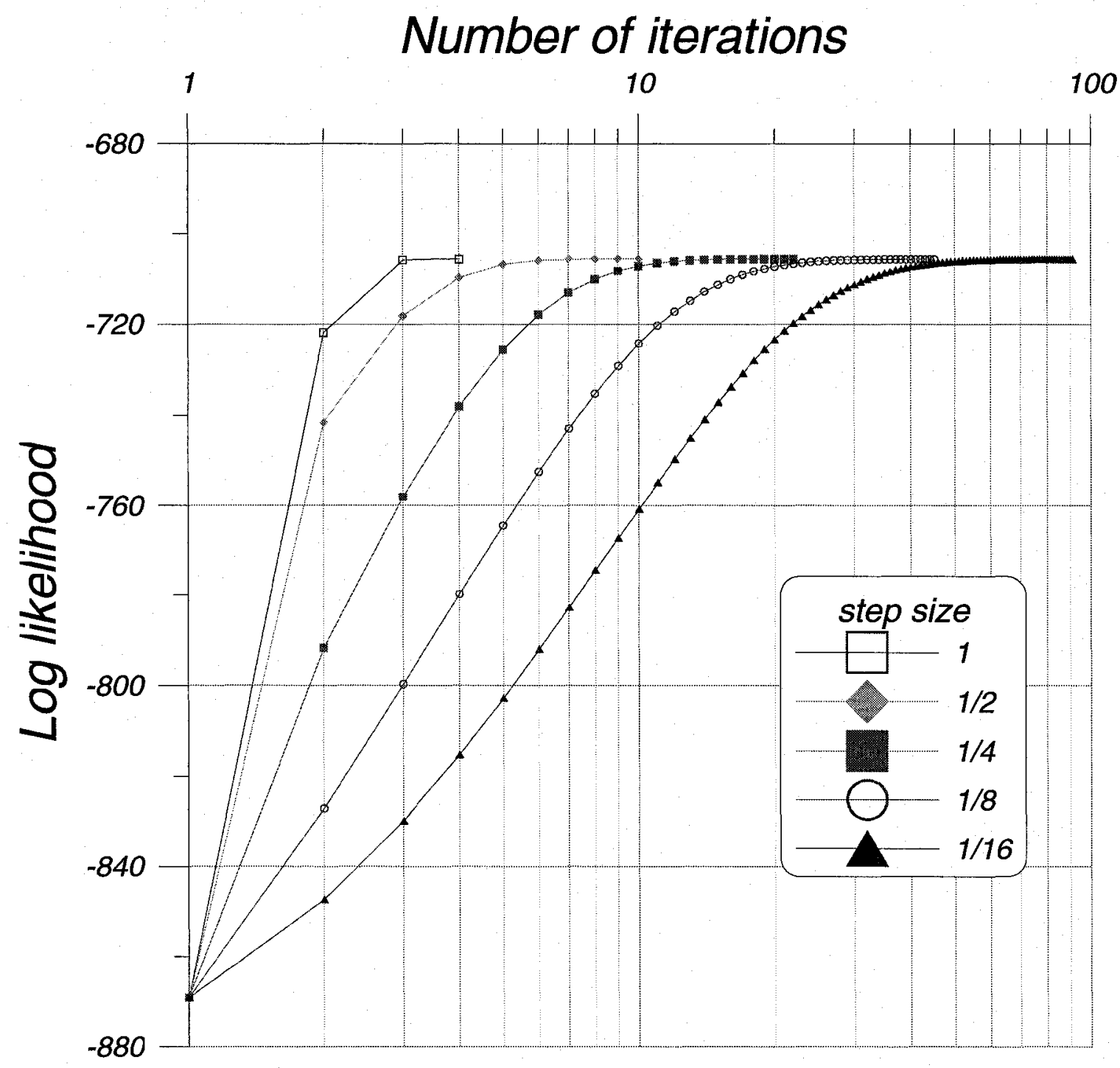

Figure 5-42: Log-likelihood for the all step sizes in Newton Raphson algorithm (exp. 3) 


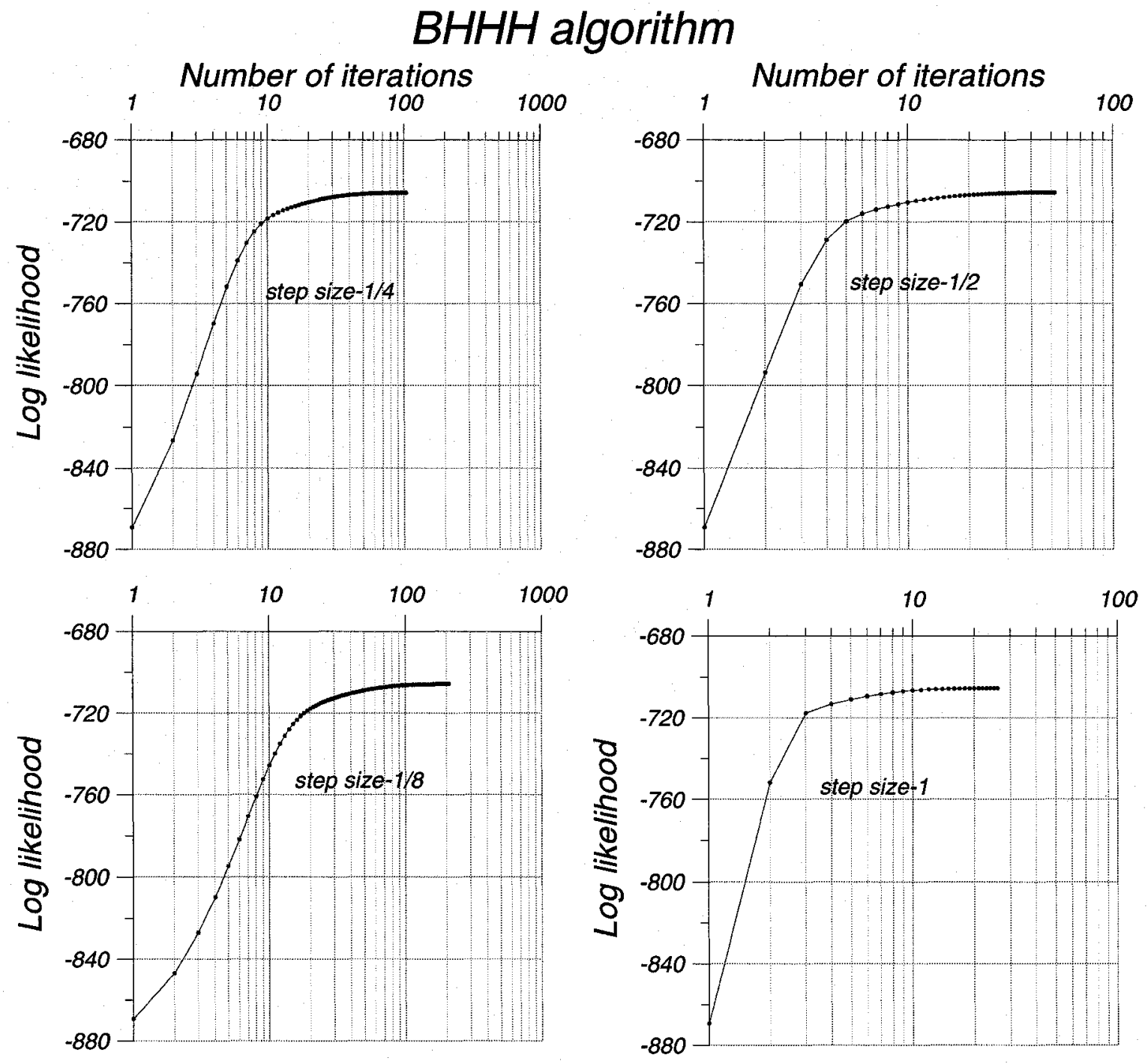

Figure 5-43: Log-likelihood values for each step size in BHHH algorithm (exp. 3) 


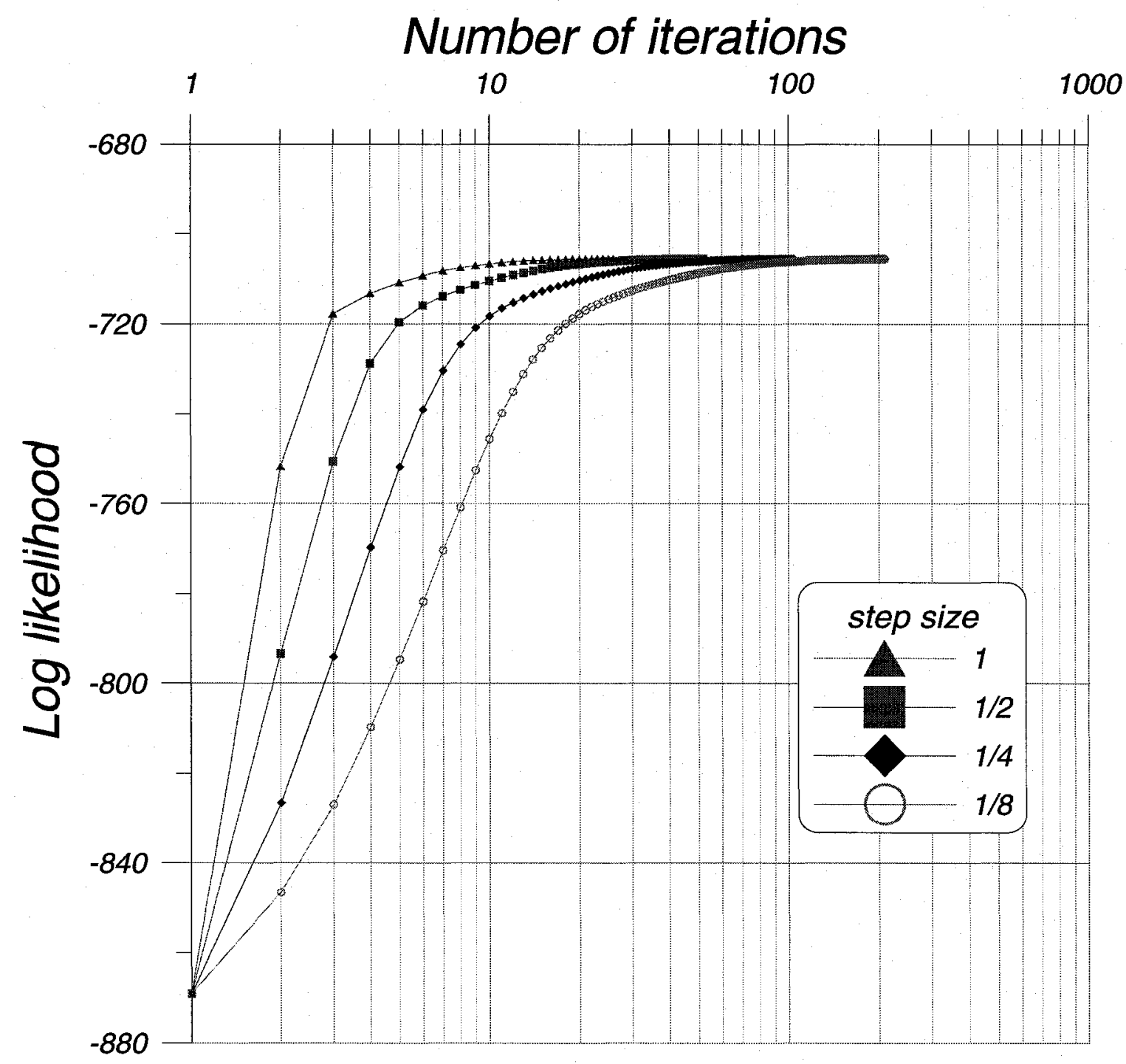

Figure 5-44: Log-likelihood for the all step sizes in BHHH algorithm (exp. 3) 


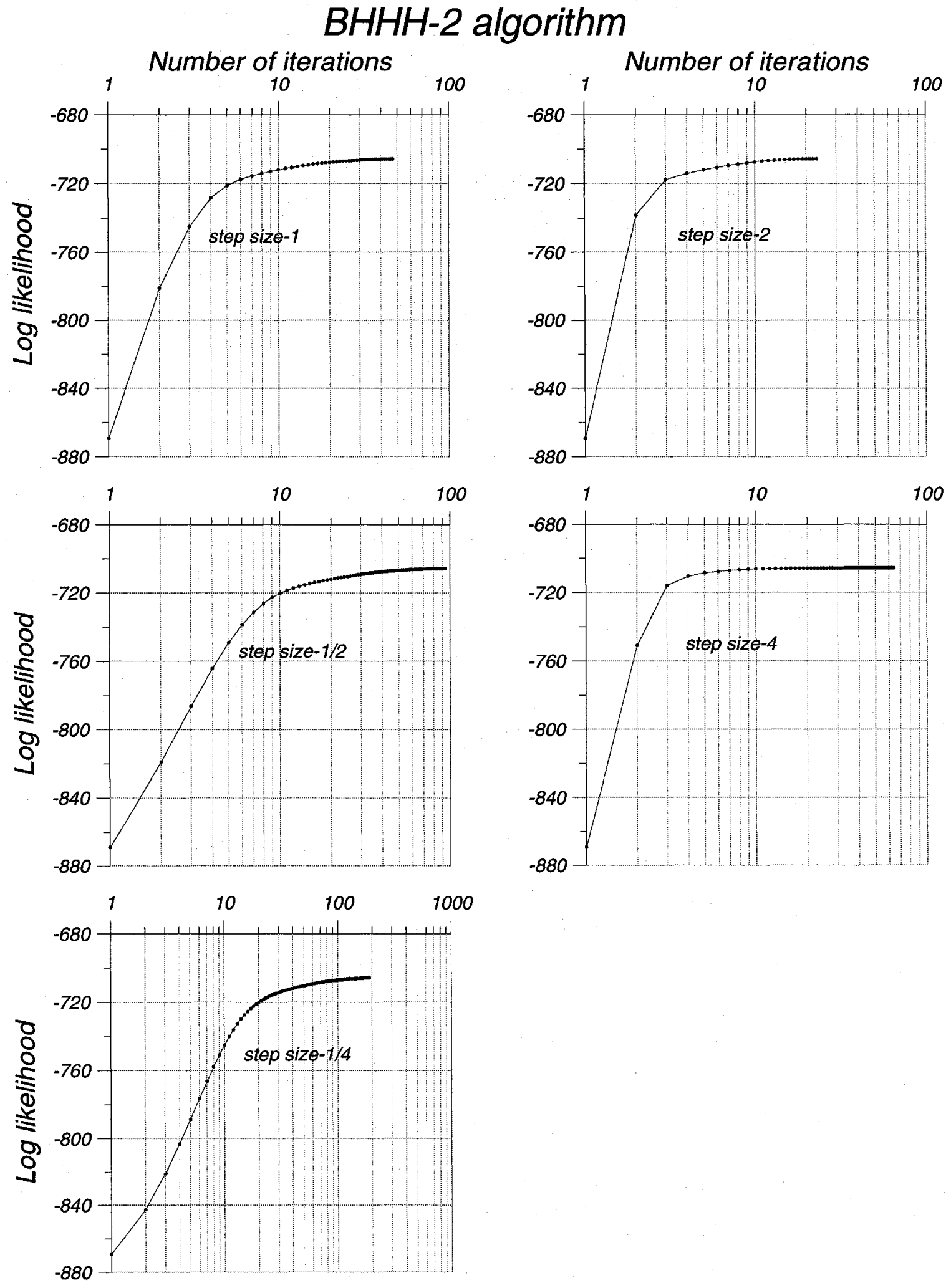

Figure 5-45: Log-likelihood values for each step size in BHHH-2 algorithm (exp. 3) 


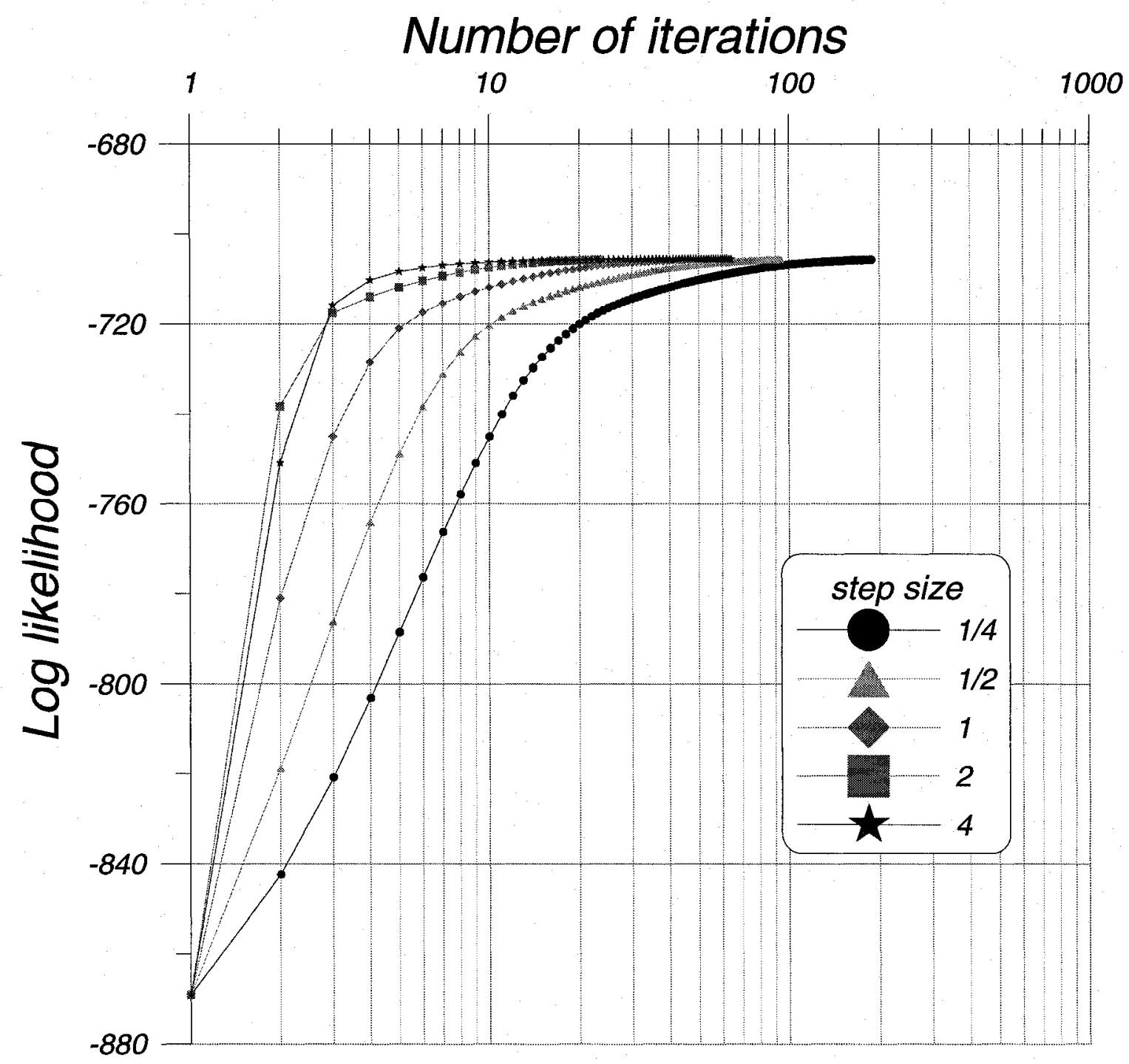

Figure 5-46: Log-likelihood for the all step sizes in BHHH-2 algorithm (exp. 3) 

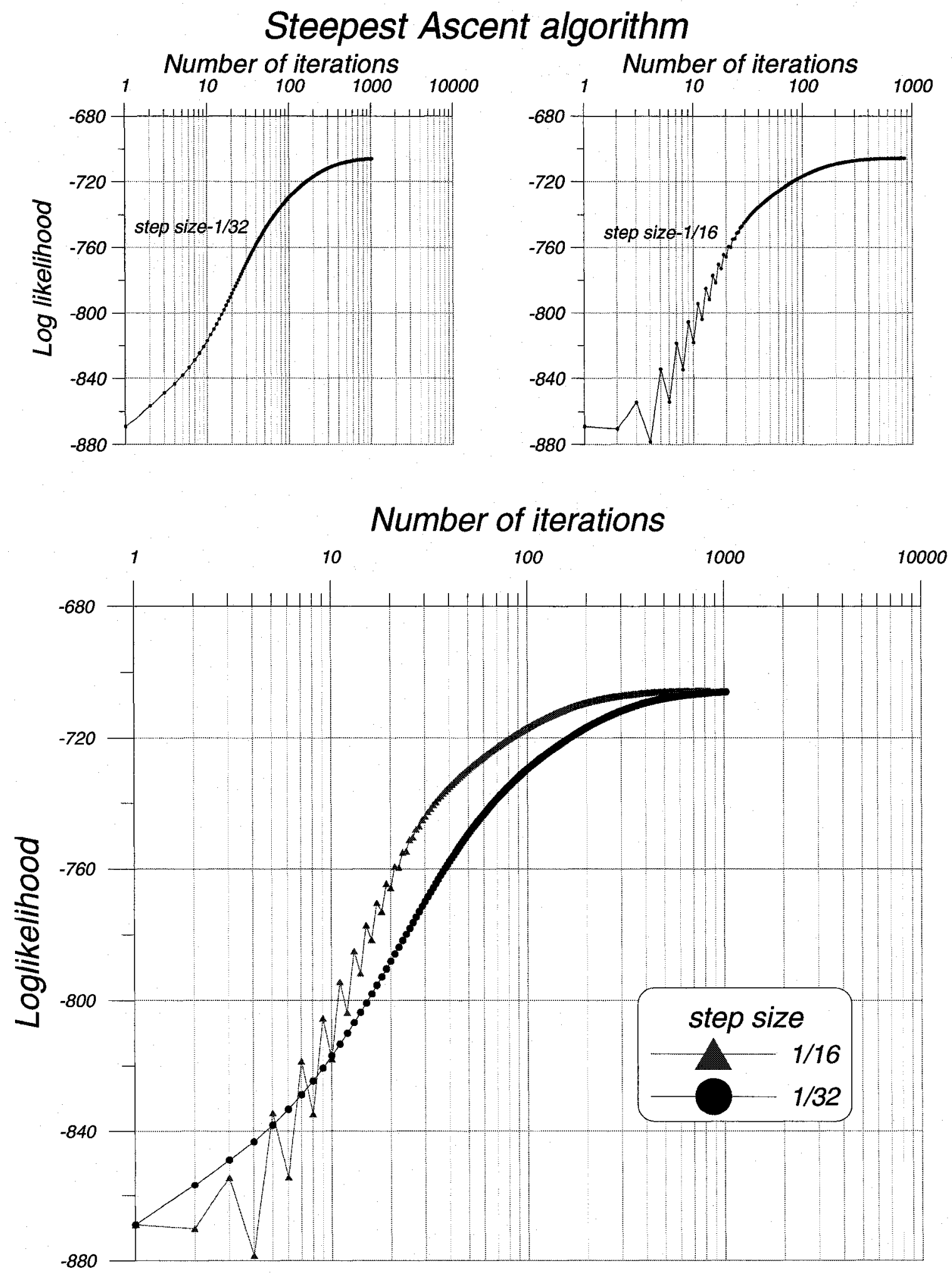

Figure 5-47: Log-likelihood values for each step size and all step sizes in Steepest Ascent algorithm (exp. 3) 


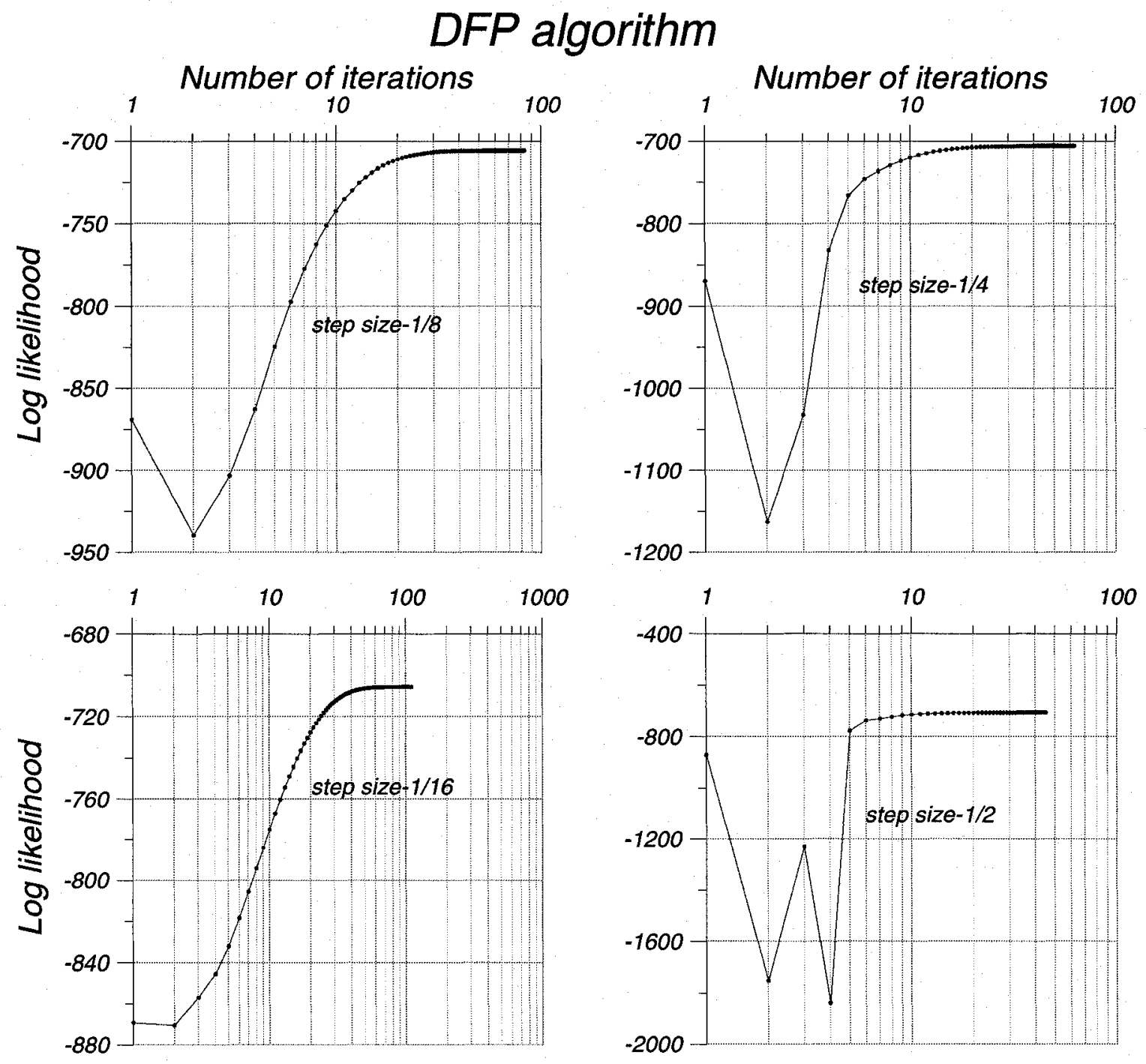

Figure 5-48: Log-likelihood values for each step size in DFP algorithm (exp. 3) 


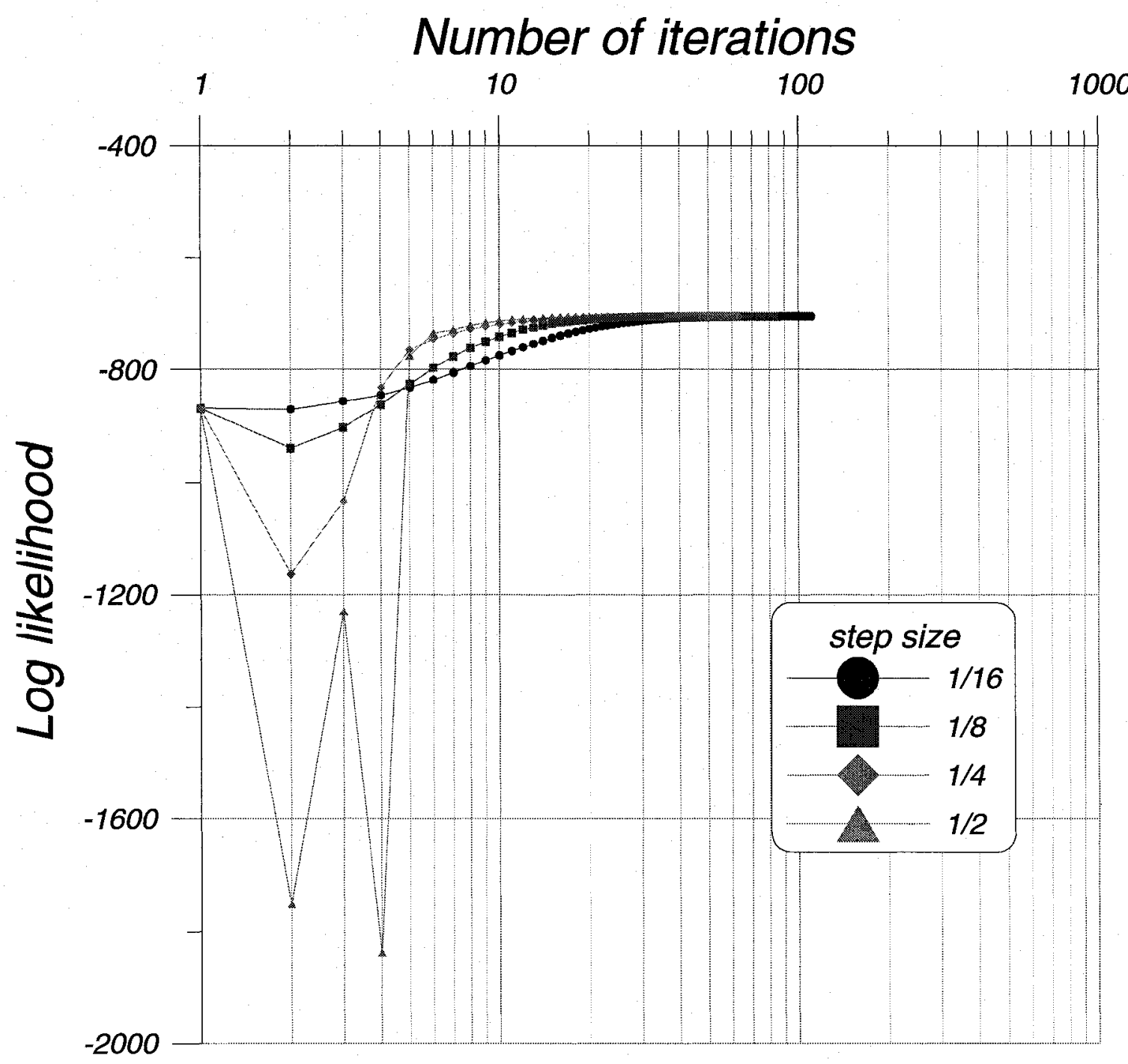

Figure 5-49: Log-likelihood for the all step sizes in DFP algorithm (exp. 3) 


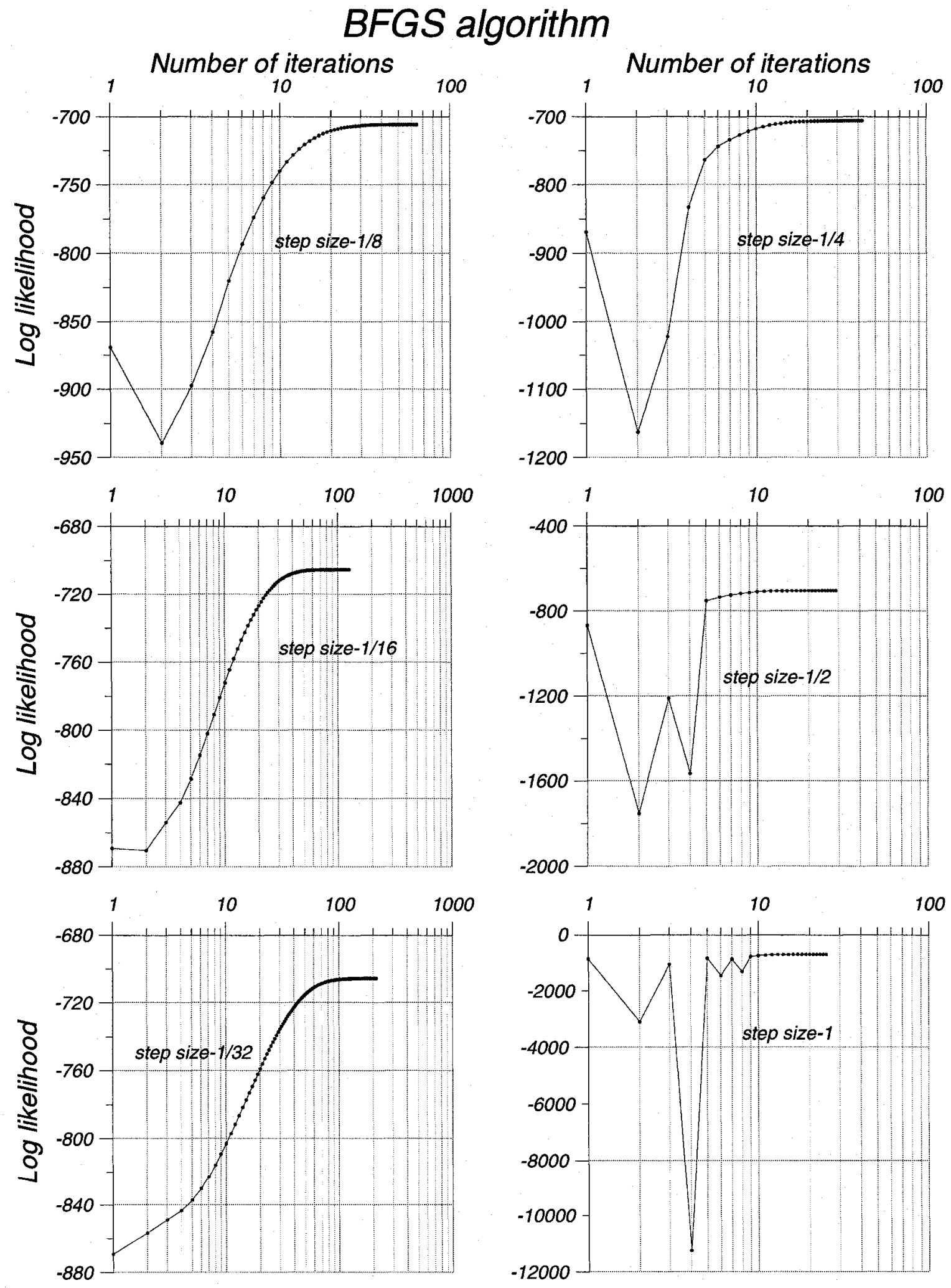

Figure 5-50: Log-likelihood values for each step size in BFGS algorithm (exp. 3) 


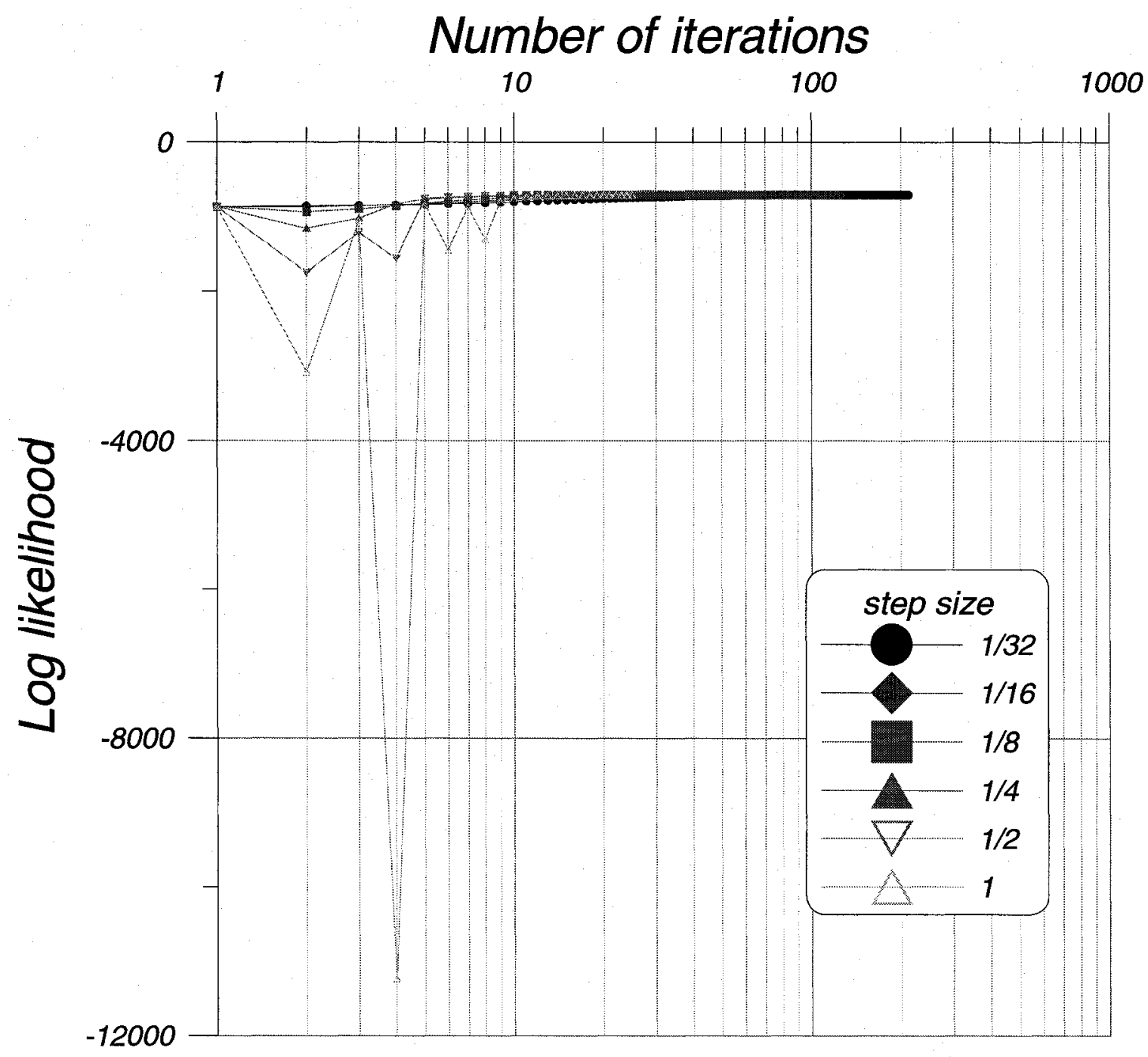

Figure 5-51: Log-likelihood for the all step sizes in BFGS algorithm (exp. 3) 


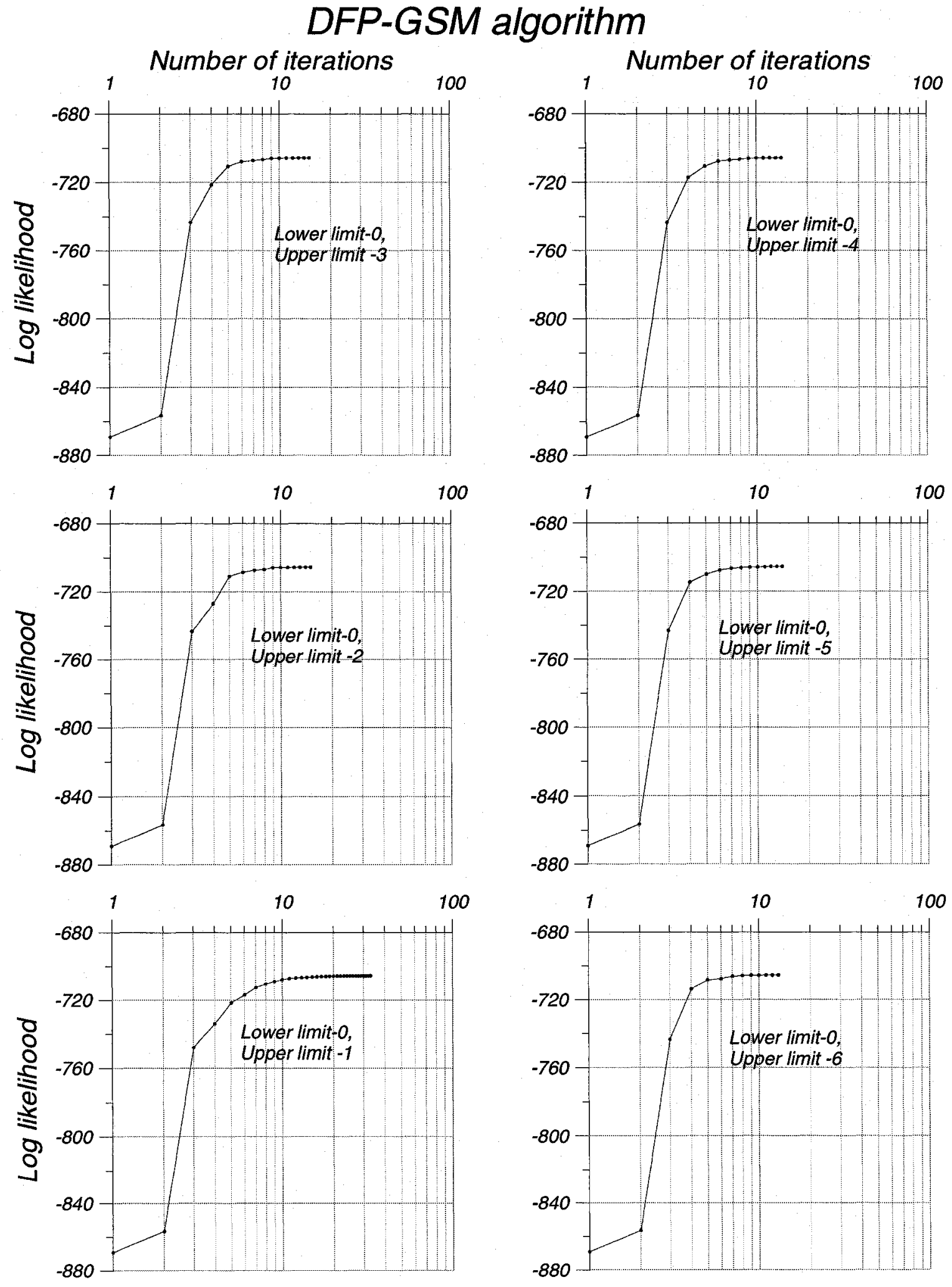

Figure 5-52: Log-likelihood values for each step size in DFP-GSM algorithm for the first six uncertain intervals (exp. 3) 


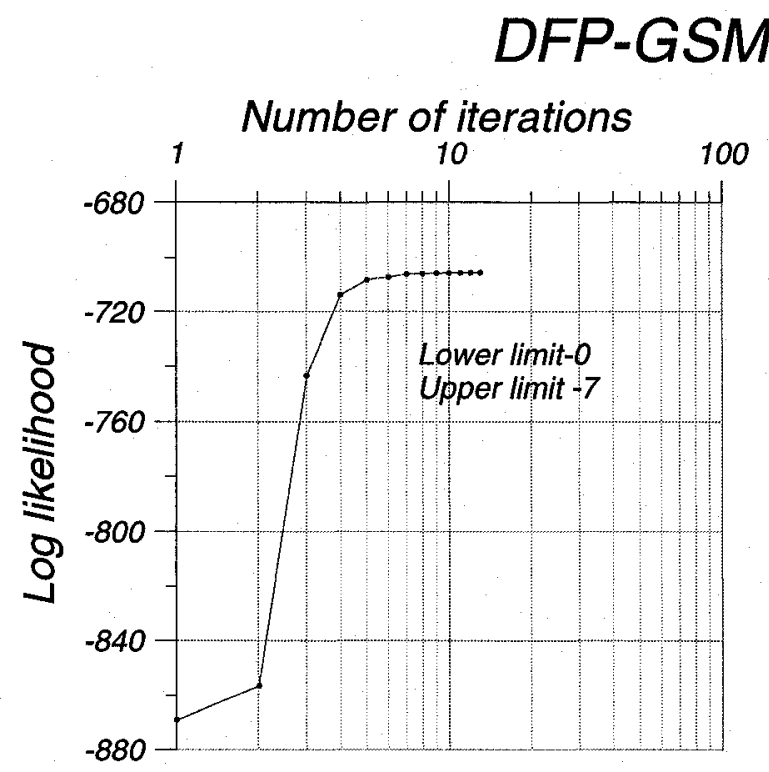

\section{algorithm}

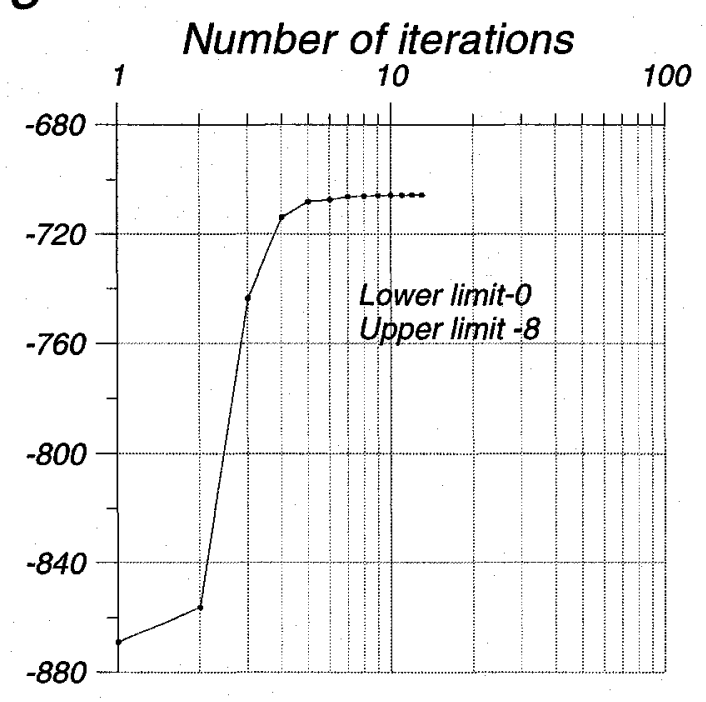

Figure 5-53: Log-likelihood values for each step size in DFP-GSM algorithm for the last two uncertain intervals (exp. 3) 


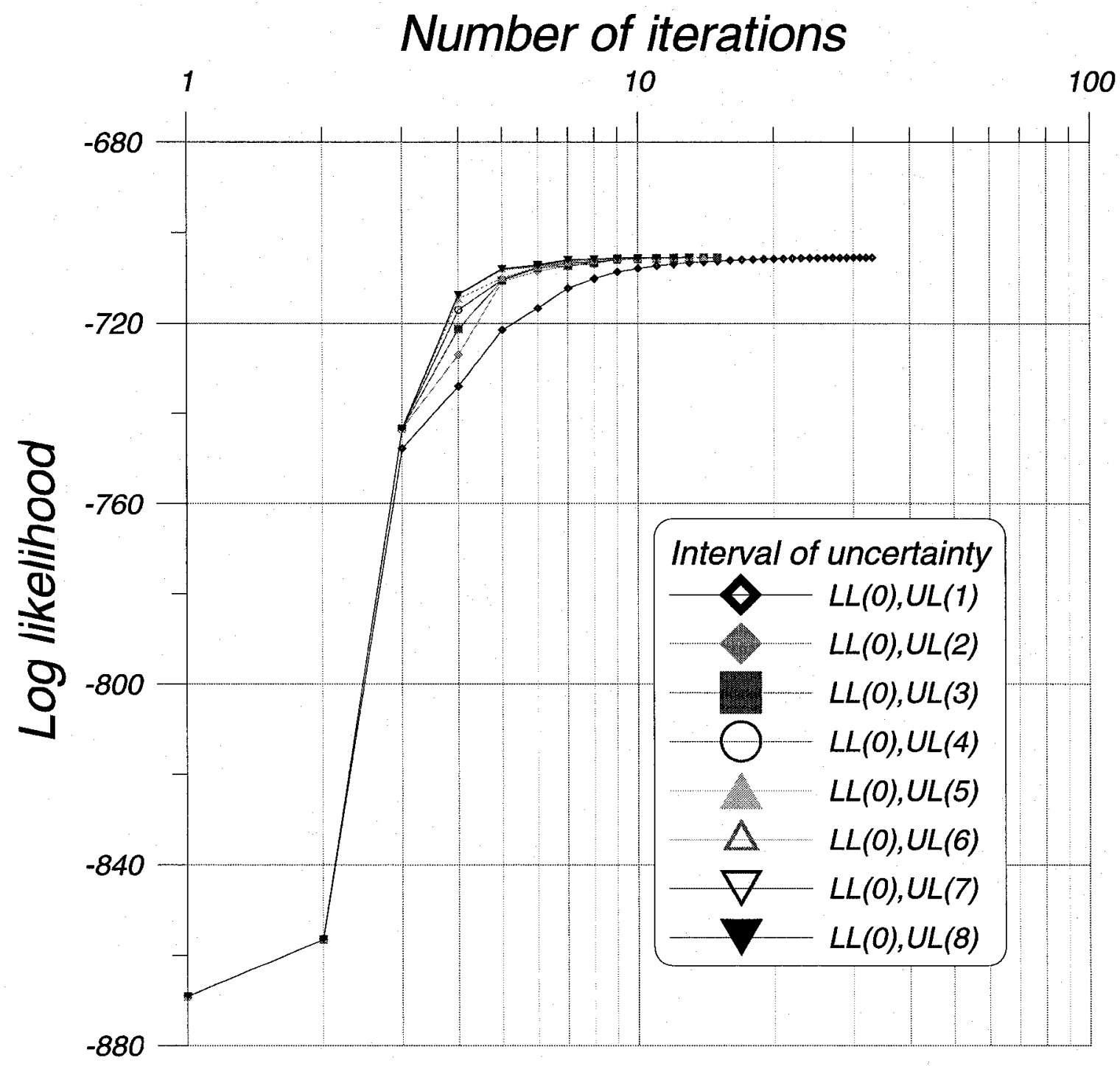

Figure 5-54: Log-likelihood for the all step sizes in DFP-GSM algorithm (exp. 3) 


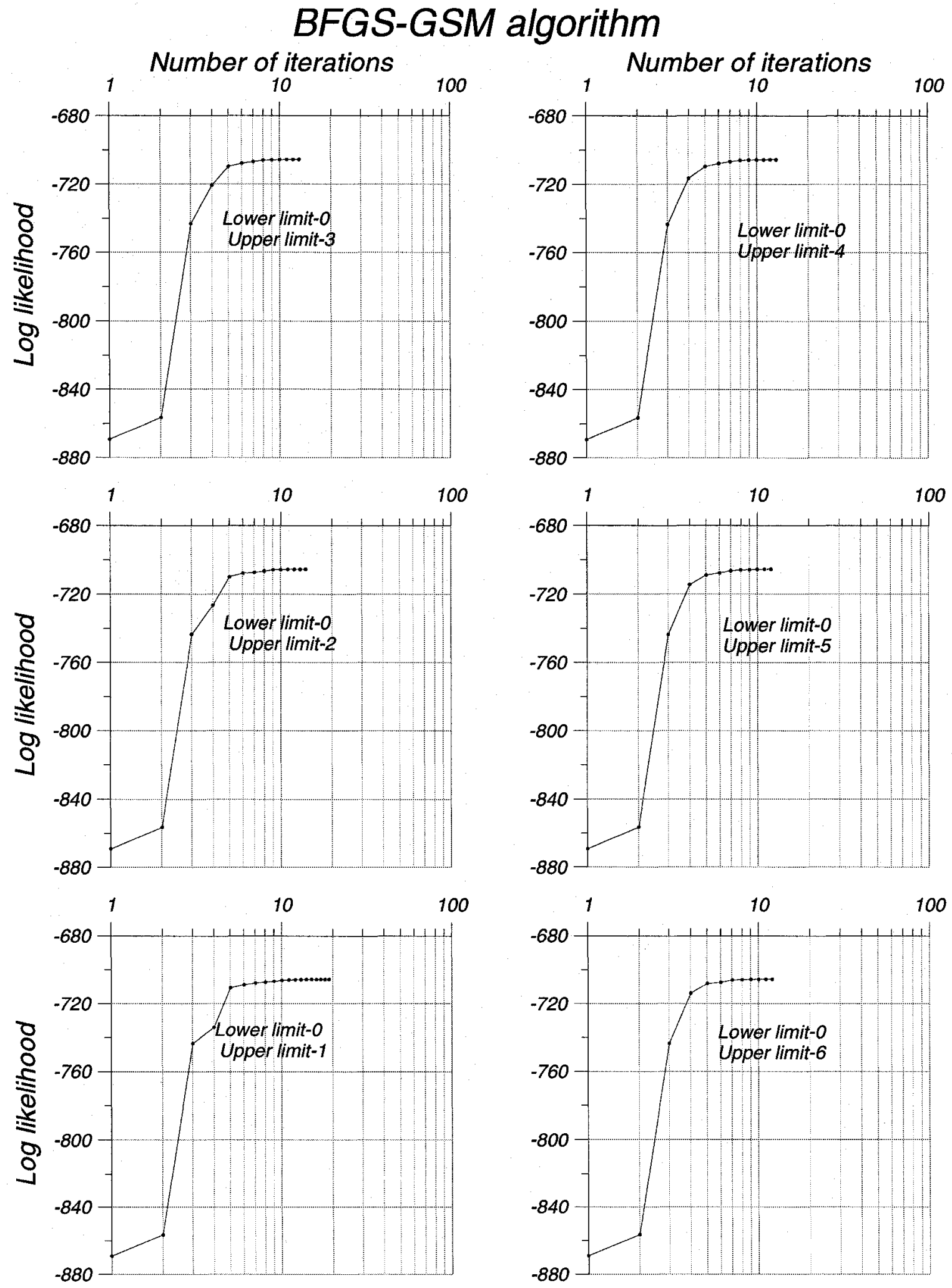

Figure 5-55: Log-likelihood values for each step size in BFGS-GSM algorithm for the first six uncertain intervals (exp. 3) 

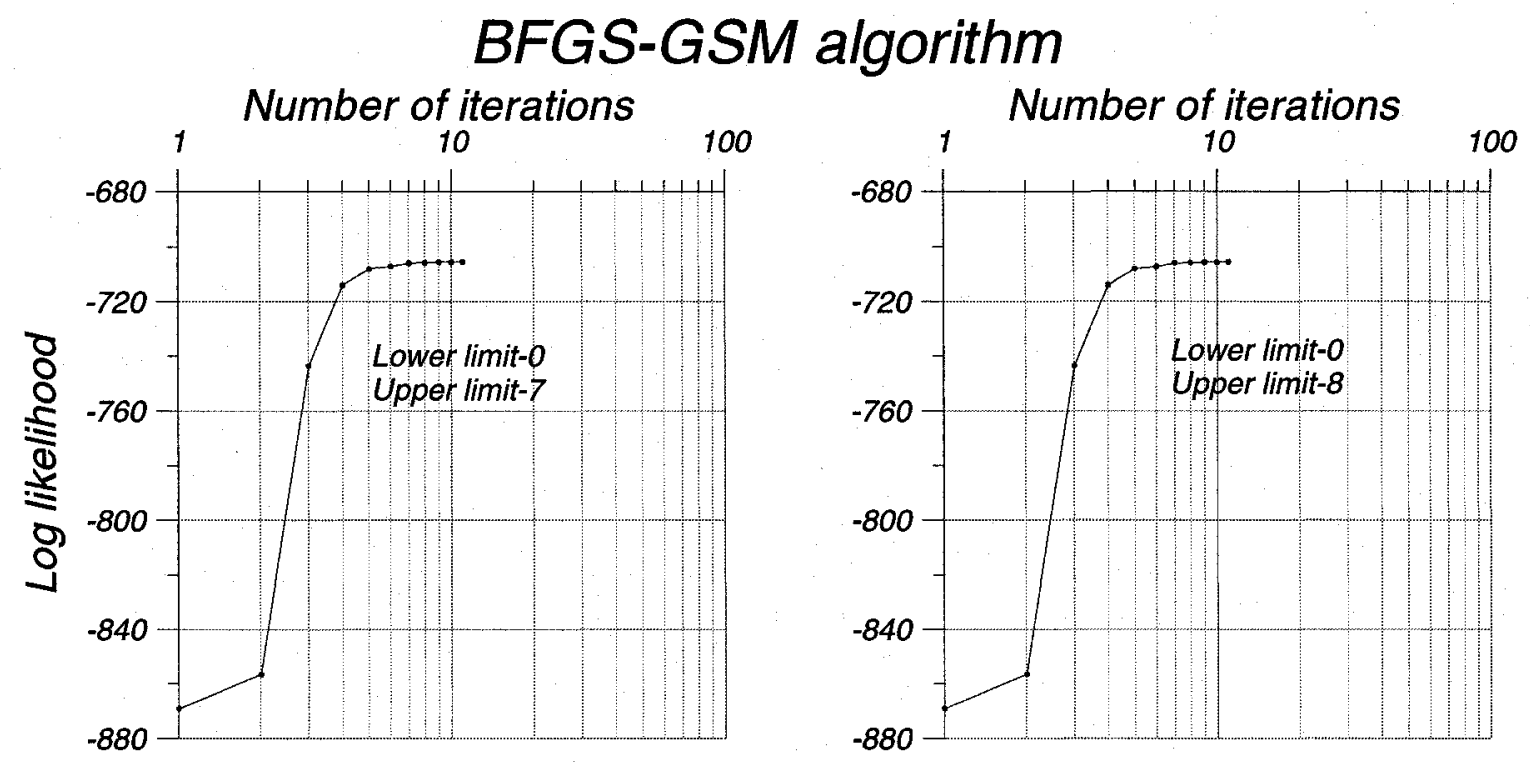

Figure 5-56: Log-likelihood values for each step size in BFGS-GSM algorithm for the last two uncertain intervals (exp. 3) 


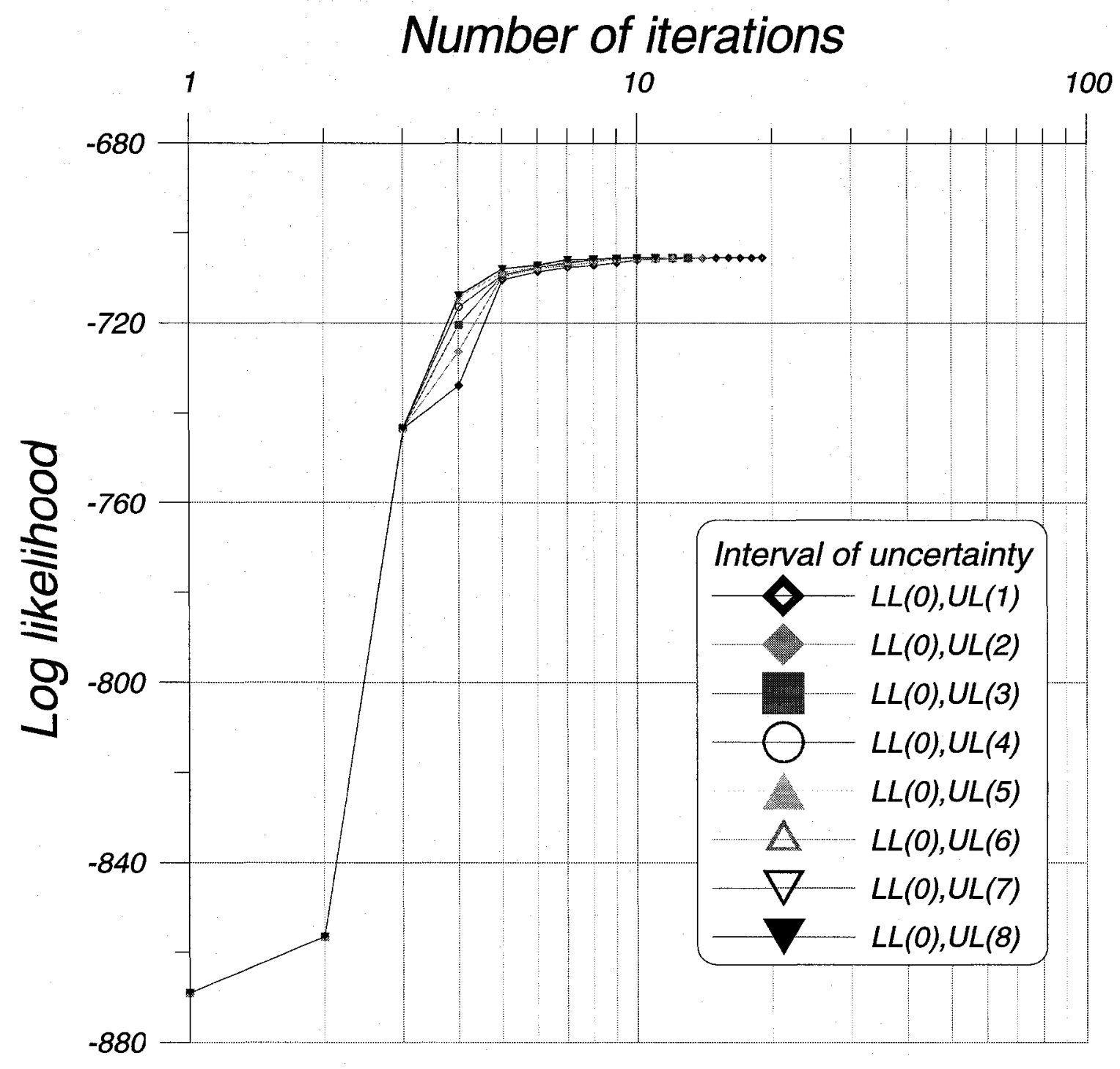

Figure 5-57: Log-likelihood for the all step sizes in BFSG-GSM algorithm (exp. 3) 


\subsubsection{The fourth convergence criterion (experiment 4)}

Here, the applied convergence criterion is $G_{t}^{\prime}\left(-H_{t}\right)^{-1} G_{t}<10^{-6}$. The degree of precision of parameter estimates is changed to more stringent value from $G_{t}^{\prime}\left(-H_{t}\right)^{-1} G_{t}<10^{-5}$ of experiment 3 to $G_{t}^{\prime}\left(-H_{t}\right)^{-1} G_{t}<10^{-6}$ of the current experiment 4. Belsley (1979) is credited for showing the benefits of using this criterion in econometric modelling.

The results obtained from the experimental estimation for the eight algorithms are summarized in table 5-30, and the detailed results are presented graphically in figures 558 and 5-59 in terms of three performance measures. 
Table 5-30: The final experiment results of the eight estimation algorithms (exp. 4)

\begin{tabular}{|c|c|c|c|c|c|c|c|c|c|}
\hline \multirow{2}{*}{\multicolumn{2}{|c|}{$\begin{array}{c}\text { Algorithms \& } \\
\text { Performance } \\
\text { measures }\end{array}$}} & \multicolumn{8}{|c|}{ Step size } \\
\hline & & $1 / 32$ & $1 / 16$ & $1 / 8$ & $1 / 4$ & $1 / 2$ & 1 & 2 & 4 \\
\hline \multirow{3}{*}{ 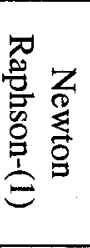 } & $\begin{array}{c}\text { One iteration } \\
\text { time }\end{array}$ & - & $0: 00: 23$ & 0:00:22 & $0: 00: 21$ & 0:00:22 & $0: 00: 23$ & - & - \\
\hline & $\begin{array}{l}\text { Number of } \\
\text { iterations }\end{array}$ & - & 109 & 54 & 26 & 12 & 4 & - & - \\
\hline & $\begin{array}{l}\text { Convergence } \\
\text { time }\end{array}$ & - & $0: 41: 07$ & $0: 20: 10$ & 0:09:18 & $0: 04: 15$ & 0:01:24 & - & - \\
\hline \multirow{3}{*}{ 萦 } & $\begin{array}{c}\text { One iteration } \\
\text { time }\end{array}$ & - & - & 0:00:29 & $0: 00: 26$ & $0: 00: 27$ & 0:00:28 & - & - \\
\hline & $\begin{array}{l}\text { Number of } \\
\text { iterations }\end{array}$ & - & - & 291 & 145 & 72 & 36 & - & - \\
\hline & $\begin{array}{c}\text { Convergence } \\
\text { time }\end{array}$ & - & - & $2: 41: 31$ & 1:01:43 & $0: 32: 19$ & $0: 16: 48$ & - & - \\
\hline \multirow{3}{*}{ 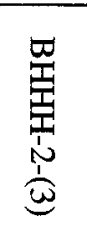 } & $\begin{array}{c}\text { One iteration } \\
\text { time }\end{array}$ & - & - & - & $0: 00: 27$ & $0: 00: 37$ & $0: 00: 37$ & $0: 00: 36$ & 0:00:39 \\
\hline & $\begin{array}{c}\text { Number of } \\
\text { iterations }\end{array}$ & - & - & - & 294 & 147 & 73 & 36 & 102 \\
\hline & $\begin{array}{c}\text { Convergence } \\
\text { time }\end{array}$ & - & - & - & $3: 30: 25$ & $1: 31: 49$ & 0:44:39 & $0: 21: 32$ & $1: 05: 58$ \\
\hline \multirow{3}{*}{ 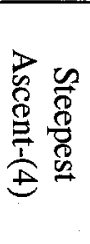 } & $\begin{array}{c}\text { One iteration } \\
\text { time }\end{array}$ & 0:00:18 & 0:00:20 & - & - & - & - & - & - \\
\hline & $\begin{array}{c}\text { Number of } \\
\text { iterations }\end{array}$ & 2662 & 1326 & - & - & - & - & - & - \\
\hline & $\begin{array}{c}\text { Convergence } \\
\text { time }\end{array}$ & $\begin{array}{c}14: 22: 1 \\
0\end{array}$ & $7: 25: 04$ & - & - & - & - & - & - \\
\hline \multirow{3}{*}{$\begin{array}{l}\frac{0}{7} \\
\frac{1}{0}\end{array}$} & $\begin{array}{c}\text { One iteration } \\
\text { time }\end{array}$ & - & 0:00:20 & $0: 00: 19$ & $0: 00: 20$ & $0: 00: 26$ & - & - & - \\
\hline & $\begin{array}{c}\text { Number of } \\
\text { iterations }\end{array}$ & - & 216 & 139 & 95 & 69 & - & - & - \\
\hline & $\begin{array}{c}\text { Convergence } \\
\text { time }\end{array}$ & - & 1:21:11 & $0: 43: 39$ & $0: 31: 45$ & $0: 29: 20$ & - & - & - \\
\hline \multirow{3}{*}{$\begin{array}{l}0 \\
0 \\
0 \\
0 \\
\frac{1}{0} \\
0\end{array}$} & $\begin{array}{c}\text { One iteration } \\
\text { time }\end{array}$ & $0: 00: 17$ & $0: 00: 19$ & $0: 00: 18$ & 0:00:19 & 0:00:19 & $0: 00: 18$ & - & - \\
\hline & $\begin{array}{c}\text { Number of } \\
\text { iterations }\end{array}$ & 274 & 158 & 100 & 66 & 48 & 36 & - & - \\
\hline & $\begin{array}{l}\text { Convergence } \\
\text { time }\end{array}$ & $1: 16: 01$ & $0: 49: 15$ & $0: 30: 39$ & $0: 20: 22$ & $0: 14: 55$ & $0: 10: 44$ & - & - \\
\hline \multicolumn{2}{|r|}{$\begin{array}{l}\text { Interval of } \\
\text { uncertainty }\end{array}$} & $\begin{array}{l}\text { LL(0), } \\
\text { UL(1) }\end{array}$ & $\begin{array}{l}\text { LL(0), } \\
\text { UL(2) }\end{array}$ & $\begin{array}{l}\text { LL(0), } \\
\text { UL(3) }\end{array}$ & $\begin{array}{l}\text { LL(0), } \\
\text { UL(4) }\end{array}$ & $\begin{array}{l}\text { LL(0), } \\
\text { UL(5) }\end{array}$ & $\begin{array}{l}\text { LL(0), } \\
\text { UL(6) }\end{array}$ & $\begin{array}{l}\text { LL(0), } \\
\text { UL(7) }\end{array}$ & $\begin{array}{l}\mathrm{LL}(0), \\
\mathrm{UL}(8)\end{array}$ \\
\hline \multirow{3}{*}{ 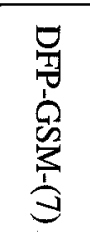 } & $\begin{array}{c}\text { One iteration } \\
\text { time }\end{array}$ & $0: 02: 27$ & $0: 02: 51$ & $0: 01: 30$ & 0:01:12 & $0: 01: 34$ & 0:01:40 & $0: 01: 38$ & $0: 01: 49$ \\
\hline & $\begin{array}{c}\text { Number of } \\
\text { iterations }\end{array}$ & 42 & 17 & 17 & 16 & 14 & 14 & 14 & 13 \\
\hline & $\begin{array}{c}\text { Convergence } \\
\text { time }\end{array}$ & 1:43:06 & $0: 48: 24$ & $0: 25: 26$ & 0:19:08 & $0: 21: 53$ & $0: 20: 10$ & $0: 22: 47$ & $0: 23: 26$ \\
\hline \multirow{3}{*}{ क人 } & $\begin{array}{c}\text { One iteration } \\
\text { time }\end{array}$ & $0: 01: 16$ & 0:01:21 & 0:01:28 & 0:01:22 & 0:01:22 & 0:01:28 & $0: 01: 29$ & $0: 01: 29$ \\
\hline & $\begin{array}{c}\text { Number of } \\
\text { iterations }\end{array}$ & 26 & 15 & 16 & 13 & 13 & 12 & 14 & 14 \\
\hline & $\begin{array}{c}\text { Convergence } \\
\text { time }\end{array}$ & $0: 32: 51$ & $0: 20: 13$ & $0: 23: 29$ & $0: 17: 40$ & $0: 17: 32$ & $0: 17: 26$ & $0: 20: 45$ & $0: 20: 32$ \\
\hline
\end{tabular}




\section{Convergence criterion 4}
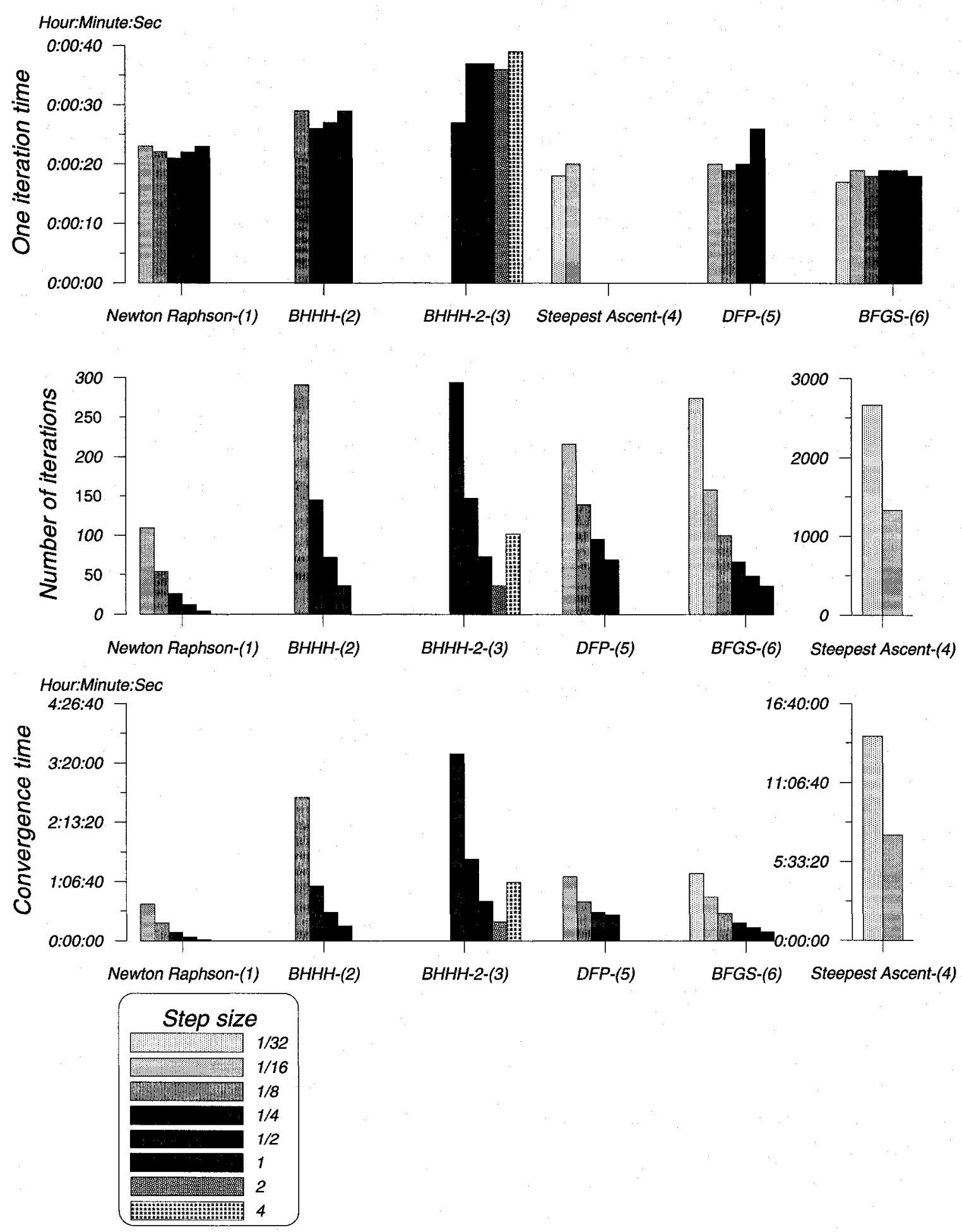

Figure 5-58: The final experimental results of the first six estimation algorithms (exp. 4) 

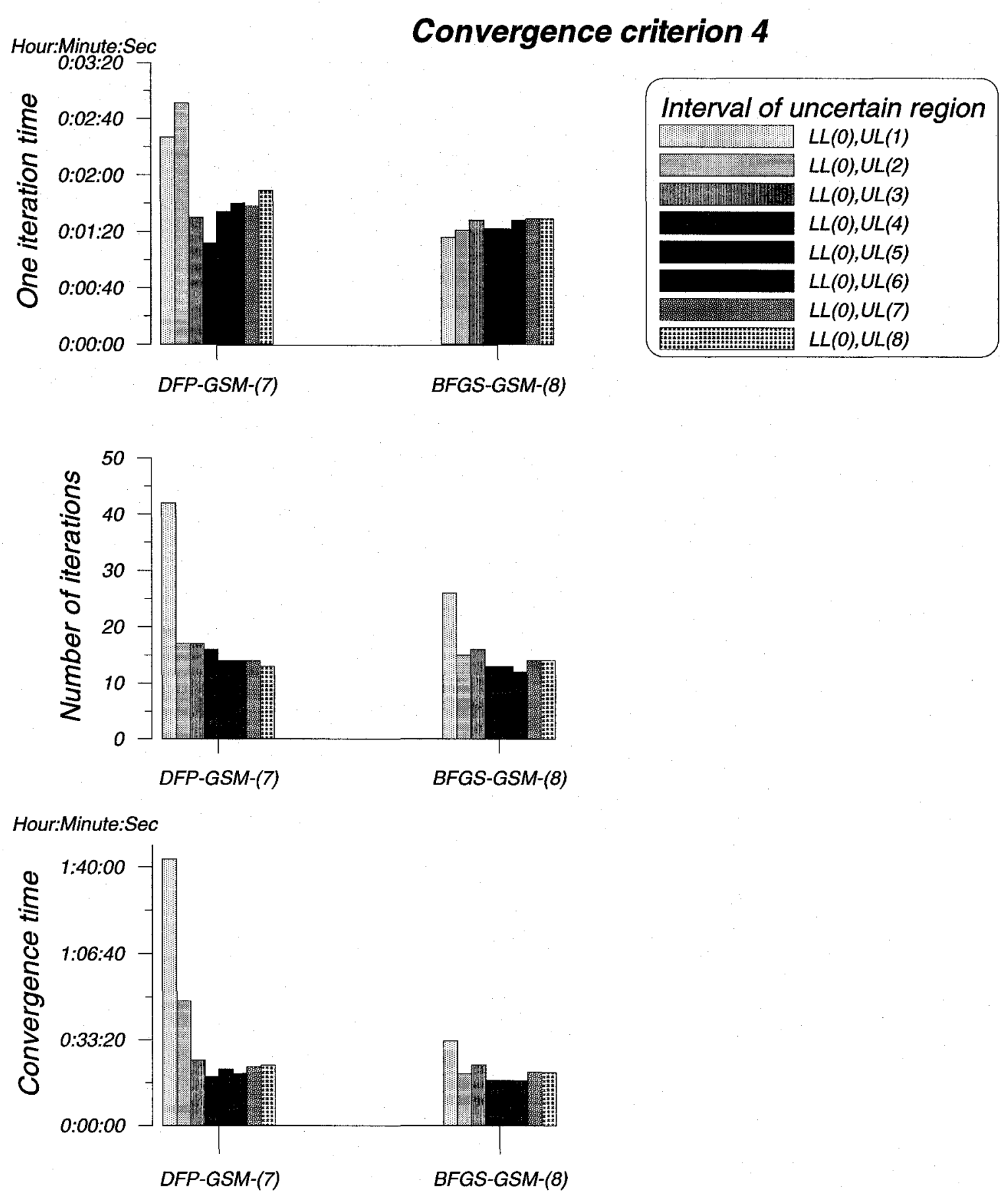

Figure 5-59: The final experimental results of the last two estimation algorithms (exp. 4) 
All conditions and calculation environments applied in the experimental estimation are exactly the same as for experiments 2 and 3 except for the fact that the convergence criterion is updated to $G_{t}^{\prime}\left(-H_{t}\right)^{-1} G_{t}<10^{-6}$. As presented in table 5-30, generally, for all eight algorithms, the estimation performance of the current experiment 4 has deteriorated as compared to that of experiments $2-3$ and even the performance is below that of experiment 1 . The reason for the worst performance witnessed in this experiment 4, as was the situation observed in experiment 3 , can be attributed to the most stringent stopping criterion applied in this experiment. The reason is the fact that the most stringent stopping criterion needs much more calculation time than for runs having a less stringent stopping criterion than used in experiment 4 . Finding a vector of parameter estimates that satisfies this stringent stopping criterion is a time consuming process and it may not be worthwhile.

In conclusion, when the fourth convergence criterion is applied as a new stopping measure, all algorithms show a worse estimation performance than all experiments tried previously. Applying a more stringent stopping criterion causes an increase in convergence time in discrete choice model estimation. 


\subsubsection{The number of iterations and log-likelihood value (experiment 4)}

For all estimation results the graphs are drawn in order to show a variation of the loglikelihood function values following the iterations and moreover to compare clearly the different convergence behaviours between step sizes used in each algorithm. Tables 5-31 to 5-38 summarize critical values such as starting log-likelihood value and convergence log-likelihood value, and the number of iterations due to different step sizes. As shown in tables 5-31 to 5-38 all, calculated starting log-likelihood values are exactly the same for all eight algorithms and for any step size used within each algorithm due to the condition applied in guessing of starting points, $\beta_{k}=\left[\beta_{1}=0, \beta_{2}=0, \beta_{3}=0, \cdots, \beta_{9}=0\right]^{\prime}$. This is the same condition imposed as an initial guess of starting points in experiments 1 to 4 and 6 . On the other hand, convergence values are slightly different among the step sizes only below one decimal point and so the difference can be ignored and all runs are considered to be converged at the same log-likelihood value. 
Table 5-31: Log-likelihood values obtained from Newton Raphson algorithm (exp. 4)

\begin{tabular}{|c|c|c|c|c|c|c|}
\hline \multirow{2}{*}{\multicolumn{2}{|c|}{ Log-likelihood }} & \multicolumn{5}{|c|}{ Step size } \\
\hline & & $1 / 16$ & $1 / 8$ & $1 / 4$ & $1 / 2$ & 1 \\
\hline \multirow{3}{*}{ 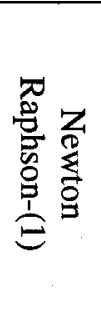 } & $\begin{array}{c}\text { Starting } \\
\text { Log- } \\
\text { likelihood }\end{array}$ & -869.0964727 & -869.0964727 & -869.0964727 & -869.0964727 & -869.0964727 \\
\hline & $\begin{array}{c}\text { Convergence } \\
\text { log- } \\
\text { likelihood }\end{array}$ & -705.5385525 & -705.5385119 & -705.5384812 & -705.5383965 & -705.5384404 \\
\hline & $\begin{array}{l}\text { Number of } \\
\text { iterations }\end{array}$ & 109 & 54 & 26 & 12 & 4 \\
\hline
\end{tabular}

Table 5-32: Log-likelihood values obtained from BHHH algorithm (exp. 4)

\begin{tabular}{|c|c|c|c|c|c|}
\hline & \multirow{2}{*}{ Log-likelihood } & \multicolumn{4}{|c|}{ Step size } \\
\hline & & $1 / 8$ & $1 / 4$ & $1 / 2$ & 1 \\
\hline \multirow{3}{*}{$\begin{array}{l}\text { 罢 } \\
\text { 空 } \\
\stackrel{1}{N}\end{array}$} & $\begin{array}{c}\text { Starting } \\
\text { Log-likelihood } \\
\end{array}$ & -869.0964727 & -869.0964727 & -869.0964727 & -869.0964727 \\
\hline & $\begin{array}{c}\text { Convergence } \\
\text { Log-likelihood }\end{array}$ & -705.5408348 & -705.5408654 & -705.5407951 & -705.540368 \\
\hline & Number of iterations & 291 & 145 & 72 & 36 \\
\hline
\end{tabular}

Table 5-33: Log-likelihood values obtained from BHHH-2 algorithm (exp. 4)

\begin{tabular}{ccccccc}
\hline & Log-likelihood & \multicolumn{5}{c}{ Step size } \\
\cline { 2 - 6 } & \multirow{2}{*}{$\underset{\omega}{*} \begin{array}{c}\text { Starting } \\
\text { Log-likelihood }\end{array}$} & -869.0964727 & -869.0964727 & -869.0964727 & -869.0964727 & -869.0964727 \\
\cline { 2 - 6 } & $\begin{array}{c}\text { Convergence log- } \\
\text { likelihood }\end{array}$ & -705.5563813 & -705.5558834 & -705.5556126 & -705.5540604 & -705.5397804 \\
\cline { 2 - 6 } & Number of iterations & 294 & 147 & 73 & 36 & 102 \\
\hline
\end{tabular}

Table 5-34: Log-likelihood values obtained from Steepest Ascent algorithm (exp. 4)

\begin{tabular}{|c|c|c|c|}
\hline & \multirow{2}{*}{ Log-likelihood } & \multicolumn{2}{|c|}{ Step size } \\
\hline & & $1 / 32$ & $1 / 16$ \\
\hline \multirow{3}{*}{ 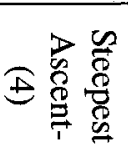 } & $\begin{array}{c}\text { Starting } \\
\text { Log-likelihood } \\
\end{array}$ & -869.0964727 & -869.0964727 \\
\hline & Convergence log-likelihood & -705.5510228 & -705.5512593 \\
\hline & Number of iterations & 2662 & 1326 \\
\hline
\end{tabular}


Table 5-35: Log-likelihood values obtained from DFP algorithm (exp. 4)

\begin{tabular}{|c|c|c|c|c|c|}
\hline \multirow{2}{*}{\multicolumn{2}{|c|}{ Log-likelihood }} & \multicolumn{4}{|c|}{ Step size } \\
\hline & & $1 / 16$ & $1 / 8$ & $1 / 4$ & $1 / 2$ \\
\hline \multirow{3}{*}{$\begin{array}{l}\frac{\pi}{7} \\
\frac{1}{3}\end{array}$} & $\begin{array}{c}\text { Starting } \\
\text { Log-likelihood }\end{array}$ & -869.0964727 & -869.0964727 & -869.0964727 & -869.0964727 \\
\hline & Convergence log-likelihood & -705.5400439 & -705.5415497 & -705.5435313 & -705.5461356 \\
\hline & Number of iterations & 216 & 139 & 95 & 69 \\
\hline
\end{tabular}

Table 5-36: Log-likelihood values obtained from BFGS algorithm (exp. 4)

\begin{tabular}{|c|c|c|c|c|c|c|c|}
\hline \multirow{2}{*}{\multicolumn{2}{|c|}{ Log-likelihood }} & \multicolumn{6}{|c|}{ Step size } \\
\hline & & $1 / 32$ & $1 / 16$ & $1 / 8$ & $1 / 4$ & $1 / 2$ & 1 \\
\hline \multirow{3}{*}{ 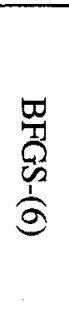 } & $\begin{array}{l}\text { Starting } \\
\text { Log- } \\
\text { likelihood }\end{array}$ & -869.0964727 & -869.0964727 & -869.0964727 & -869.0964727 & -869.0964727 & -869.0964727 \\
\hline & $\begin{array}{c}\text { Convergence } \\
\text { log- } \\
\text { likelihood }\end{array}$ & -705.5385897 & -705.5386006 & -705.5386834 & -705.5390061 & -705.5385813 & -705.5391969 \\
\hline & $\begin{array}{l}\text { Number of } \\
\text { iterations }\end{array}$ & 274 & 158 & 100 & 66 & 48 & 36 \\
\hline
\end{tabular}

Table 5-37: Log-likelihood values obtained from DFP-GSM algorithm (exp. 4)

\begin{tabular}{|c|c|c|c|c|c|c|c|c|c|}
\hline & $\begin{array}{l}\text { Interval of } \\
\text { uncertainty }\end{array}$ & $\begin{array}{l}\text { LL(0), } \\
\text { UL(1) }\end{array}$ & $\begin{array}{l}\text { LL(0), } \\
\text { UL(2) }\end{array}$ & $\begin{array}{l}\mathrm{LL}(0) \text {, } \\
\mathrm{UL}(3)\end{array}$ & $\begin{array}{l}\mathrm{LL}(0) \text {, } \\
\mathrm{UL}(4)\end{array}$ & $\begin{array}{l}\text { LL(0), } \\
\text { UL(5) }\end{array}$ & $\begin{array}{l}\mathrm{LL}(0), \\
\mathrm{UL}(6)\end{array}$ & $\begin{array}{l}\mathrm{LL}(0), \\
\mathrm{UL}(7)\end{array}$ & $\begin{array}{l}\text { LL(0), } \\
\text { UL(8) }\end{array}$ \\
\hline \multirow{4}{*}{ 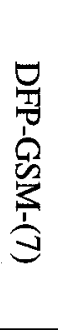 } & $\begin{array}{c}\text { Starting } \\
\text { Log- } \\
\text { likelihood }\end{array}$ & $\begin{array}{c}869.0964 \\
727\end{array}$ & $\begin{array}{c}- \\
869.0964 \\
727\end{array}$ & $\begin{array}{c}- \\
869.0964 \\
727\end{array}$ & $\begin{array}{c}- \\
869.0964 \\
727\end{array}$ & $\begin{array}{c}- \\
869.0964 \\
727\end{array}$ & $\begin{array}{c}869.0964 \\
727\end{array}$ & $\begin{array}{c}869.0964 \\
727\end{array}$ & $\begin{array}{c}869.0964 \\
727\end{array}$ \\
\hline & Convergence & & & - & & - & & & \\
\hline & $\begin{array}{c}\log - \\
\text { likelihood }\end{array}$ & $\begin{array}{c}705.5458 \\
168\end{array}$ & $\begin{array}{c}705.5482 \\
967\end{array}$ & $\begin{array}{c}705.5482 \\
987\end{array}$ & $\begin{array}{c}705.5483 \\
539\end{array}$ & $\begin{array}{c}705.5494 \\
868\end{array}$ & $\begin{array}{c}705.5491 \\
385\end{array}$ & $\begin{array}{c}705.5485 \\
776\end{array}$ & $\begin{array}{c}705.5494 \\
614\end{array}$ \\
\hline & $\begin{array}{l}\text { Number of } \\
\text { iterations }\end{array}$ & 42 & 17 & 17 & 16 & 14 & 14 & 14 & 13 \\
\hline
\end{tabular}

Table 5-38: Log-likelihood values obtained from BFGS-GSM algorithm (exp. 4)

\begin{tabular}{|c|c|c|c|c|c|c|c|c|c|}
\hline & $\begin{array}{l}\text { Interval of } \\
\text { uncertainty }\end{array}$ & $\begin{array}{l}\text { LL(0), } \\
\text { UL(1) }\end{array}$ & $\begin{array}{l}\text { LL(0), } \\
\text { UL(2) }\end{array}$ & $\begin{array}{l}\text { LL(0), } \\
\text { UL(3) }\end{array}$ & $\begin{array}{l}\text { LL(0), } \\
\text { UL(4) }\end{array}$ & $\begin{array}{l}\text { LL(0), } \\
\text { UL(5) }\end{array}$ & $\begin{array}{l}\text { LL(0), } \\
\text { UL(6) }\end{array}$ & $\begin{array}{l}\text { LL(0), } \\
\text { UL(7) }\end{array}$ & $\begin{array}{l}\text { LL(0), } \\
\text { UL(8) }\end{array}$ \\
\hline \multirow{3}{*}{ 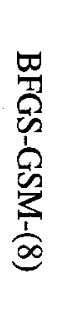 } & $\begin{array}{l}\text { Starting } \\
\text { Log- } \\
\text { likelihood }\end{array}$ & $\begin{array}{c}869.0964 \\
727\end{array}$ & $\begin{array}{c}869.0964 \\
727\end{array}$ & $\begin{array}{c}869.0964 \\
727\end{array}$ & $\begin{array}{c}869.0964 \\
727\end{array}$ & $\begin{array}{c}869.0964 \\
727\end{array}$ & $\begin{array}{c}869.0964 \\
727\end{array}$ & $\begin{array}{c}869.0964 \\
727\end{array}$ & $\begin{array}{c}869.0964 \\
727\end{array}$ \\
\hline & $\begin{array}{c}\text { Convergence } \\
\text { log- } \\
\text { likelihood } \\
\end{array}$ & $\begin{array}{c}705.5422 \\
923\end{array}$ & $\begin{array}{c}705.5485 \\
768\end{array}$ & $\begin{array}{c}705.5437 \\
015\end{array}$ & $\begin{array}{c}705.5494 \\
556\end{array}$ & $\begin{array}{c}705.5493 \\
428\end{array}$ & $\begin{array}{c}705.5498 \\
048\end{array}$ & $\begin{array}{c}705.5440 \\
254\end{array}$ & $\begin{array}{c}705.5440 \\
296\end{array}$ \\
\hline & $\begin{array}{l}\text { Number of } \\
\text { iterations }\end{array}$ & 26 & 15 & 16 & 13 & 13 & 12 & 14 & 14 \\
\hline
\end{tabular}


In the case of the Newton Raphson algorithm where the step size is one, as shown in figures 5-61 and 5-62 and in table 5-31, the iterative process stops after 4 iterations. The number of iterations is the same with experiments 2 and 3, and one iteration decreases as compared to that of experiment 1 . The convergence behaviour is identical to experiments $1-3$, except the fact that slight differences occur all over the three performance measures. These experimental results are clearly presented in the graph. Overall, observations are drawn from decreasing the step size, the lower slope and increasing number of iterations.

The same manner of interpreting results noted above can be applied to the remaining algorithms. We can identify the best performance among the runs performed using various step sizes and see the convergence log-likelihood value with the help of given tables and graphs (i.e., tables 5-31 to 5-38 and graphs 5-60 to 5-76). The profile of graph at the last iteration part also is shorter than that of experiment 1 , but as compared with experiments $2-3$, the overlapped part becomes much longer. It means more iteration are conducted for each algorithms in order to satisfy a new stringent criterion charged for stopping the iteration process. It also means that the number of iterations and convergence time can be adjusted, if desired. 


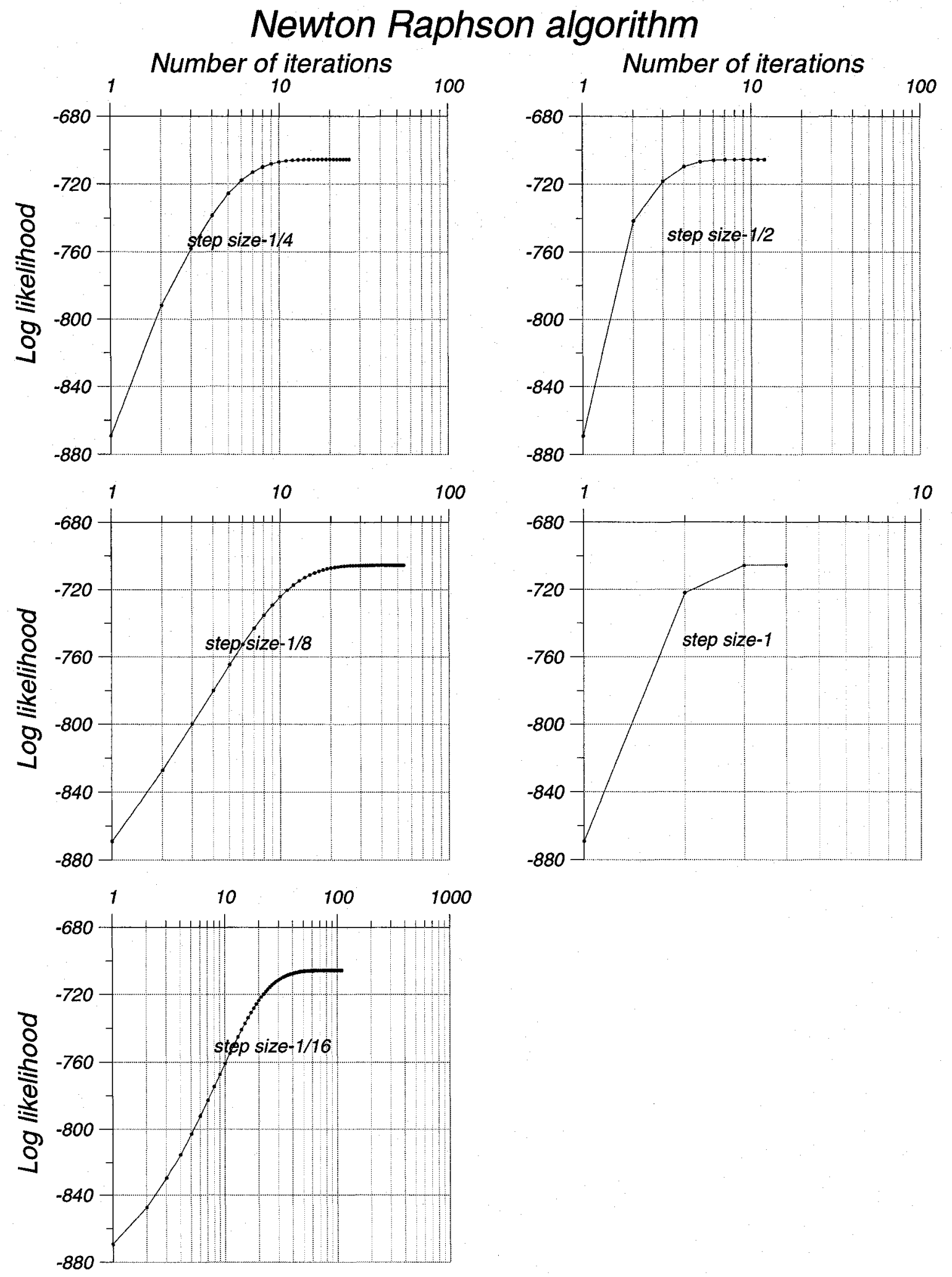

Figure 5-60: Log-likelihood values for each step size in Newton Raphson algorithm (exp. 4) 


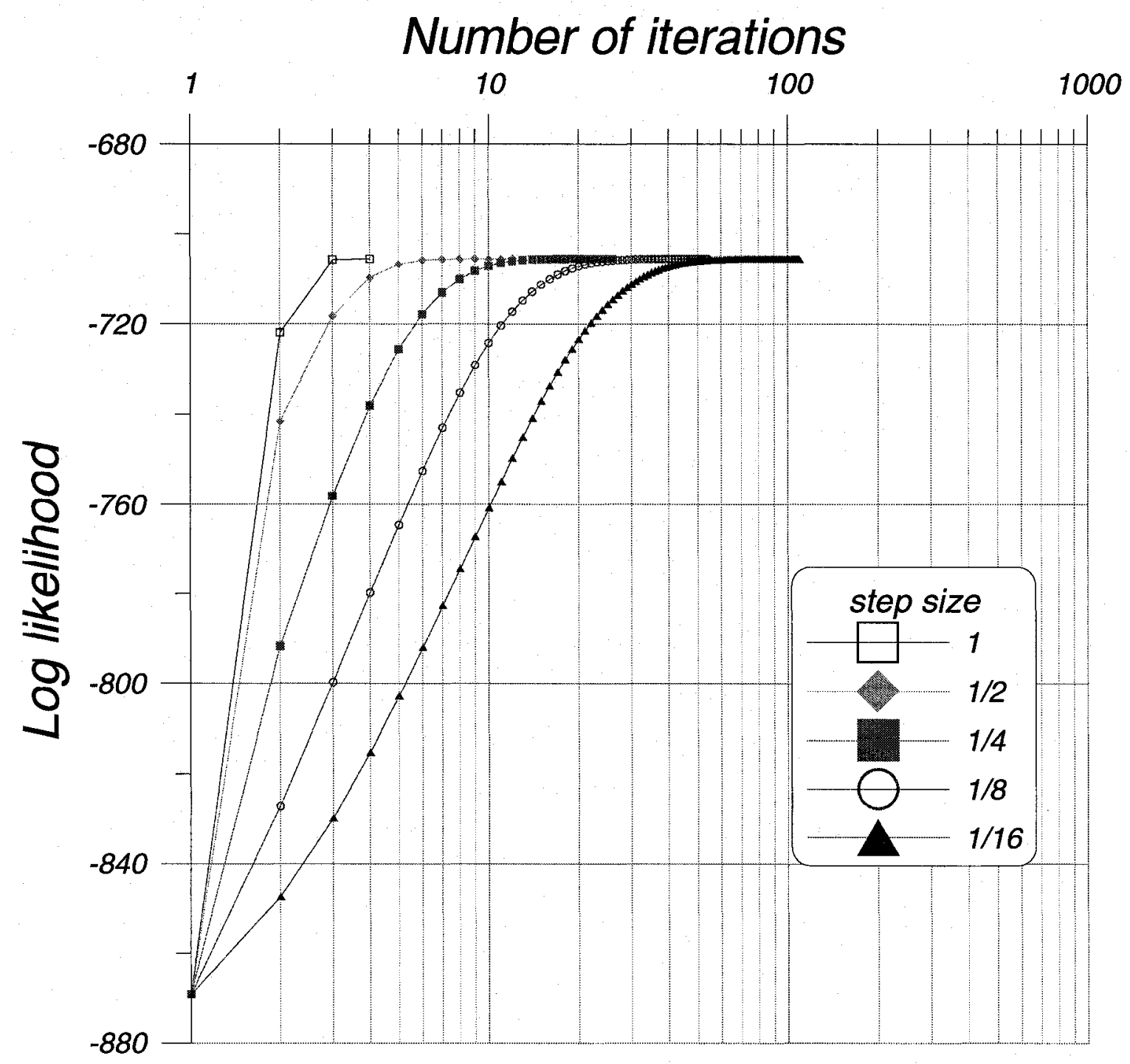

Figure 5-61: Log-likelihood for the all step sizes in Newton Raphson algorithm (exp. 4) 


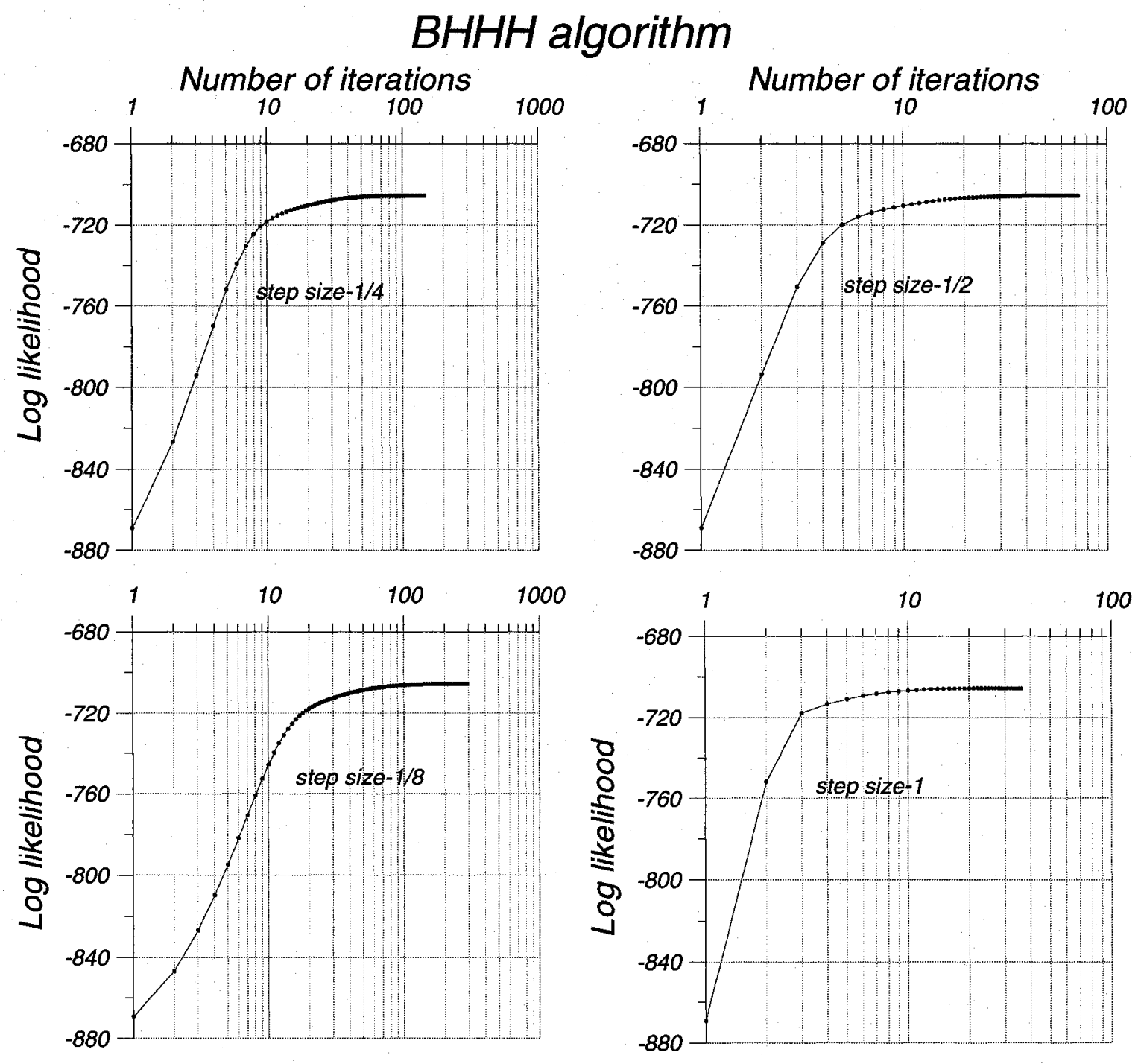

Figure 5-62: Log-likelihood values for each step size in BHHH algorithm (exp. 4) 


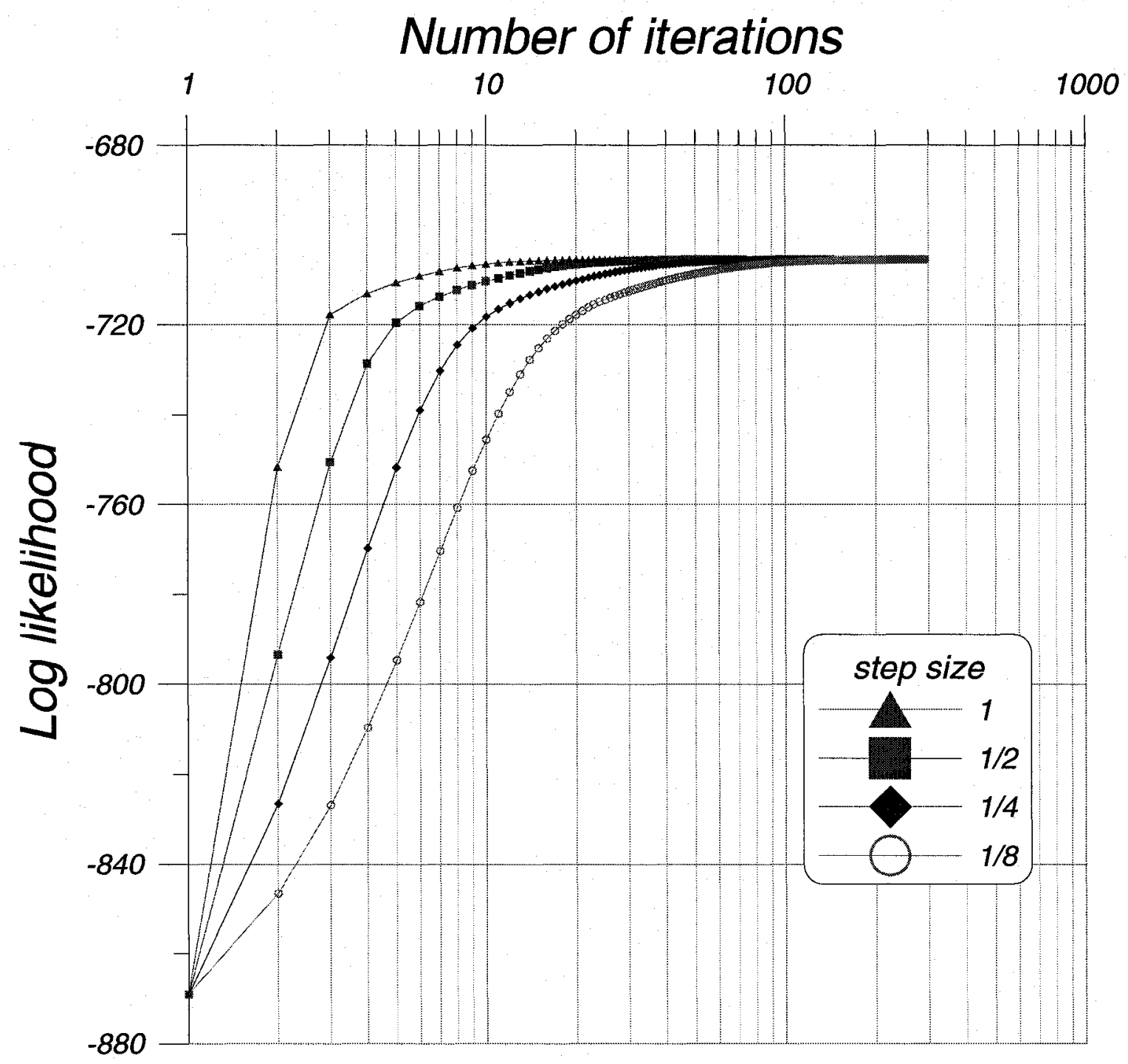

Figure 5-63: Log-likelihood for the all step sizes in BHHH algorithm (exp. 4) 


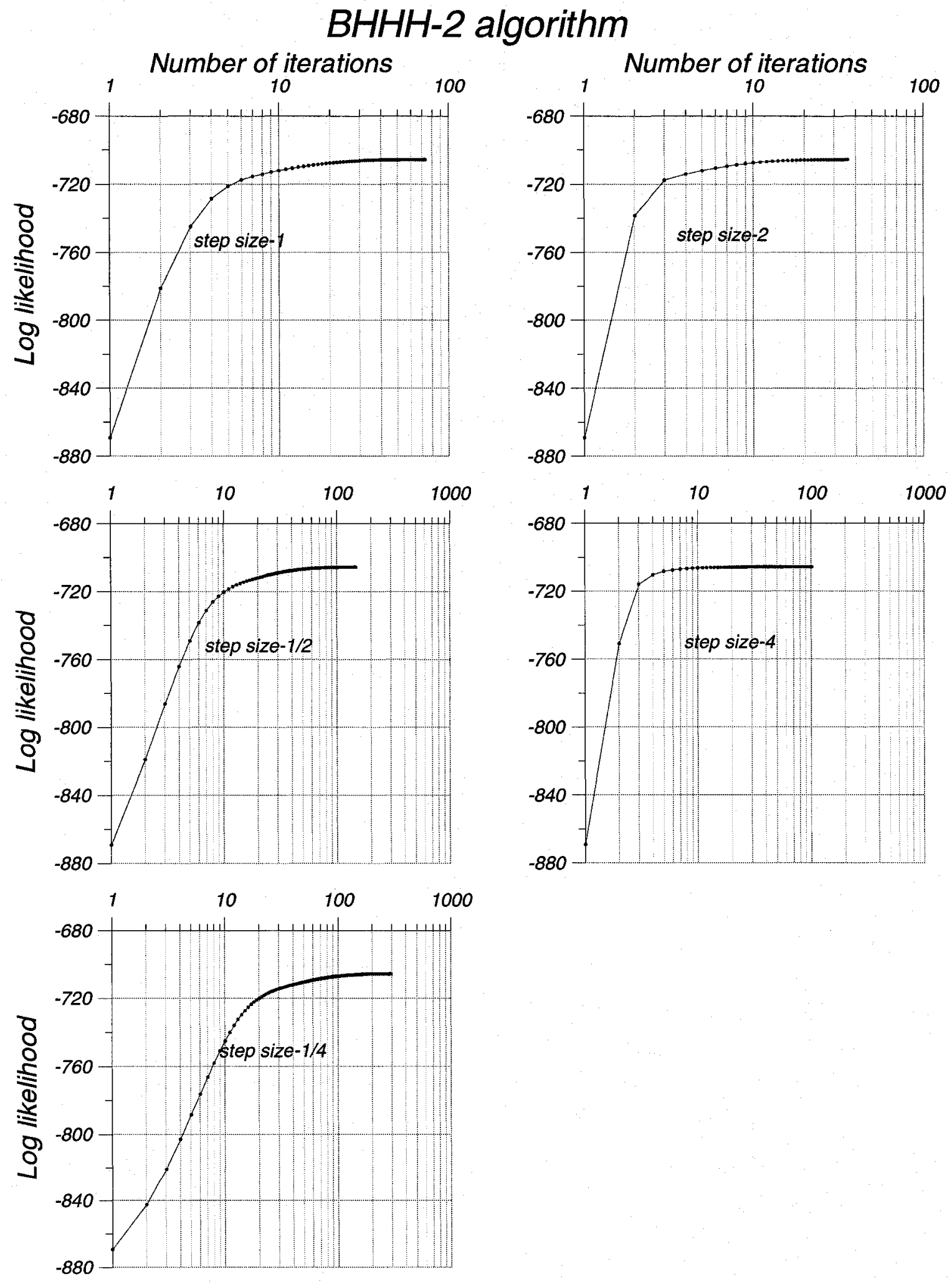

Figure 5-64: Log-likelihood values for each step size in BHHH-2 algorithm (exp. 4) 


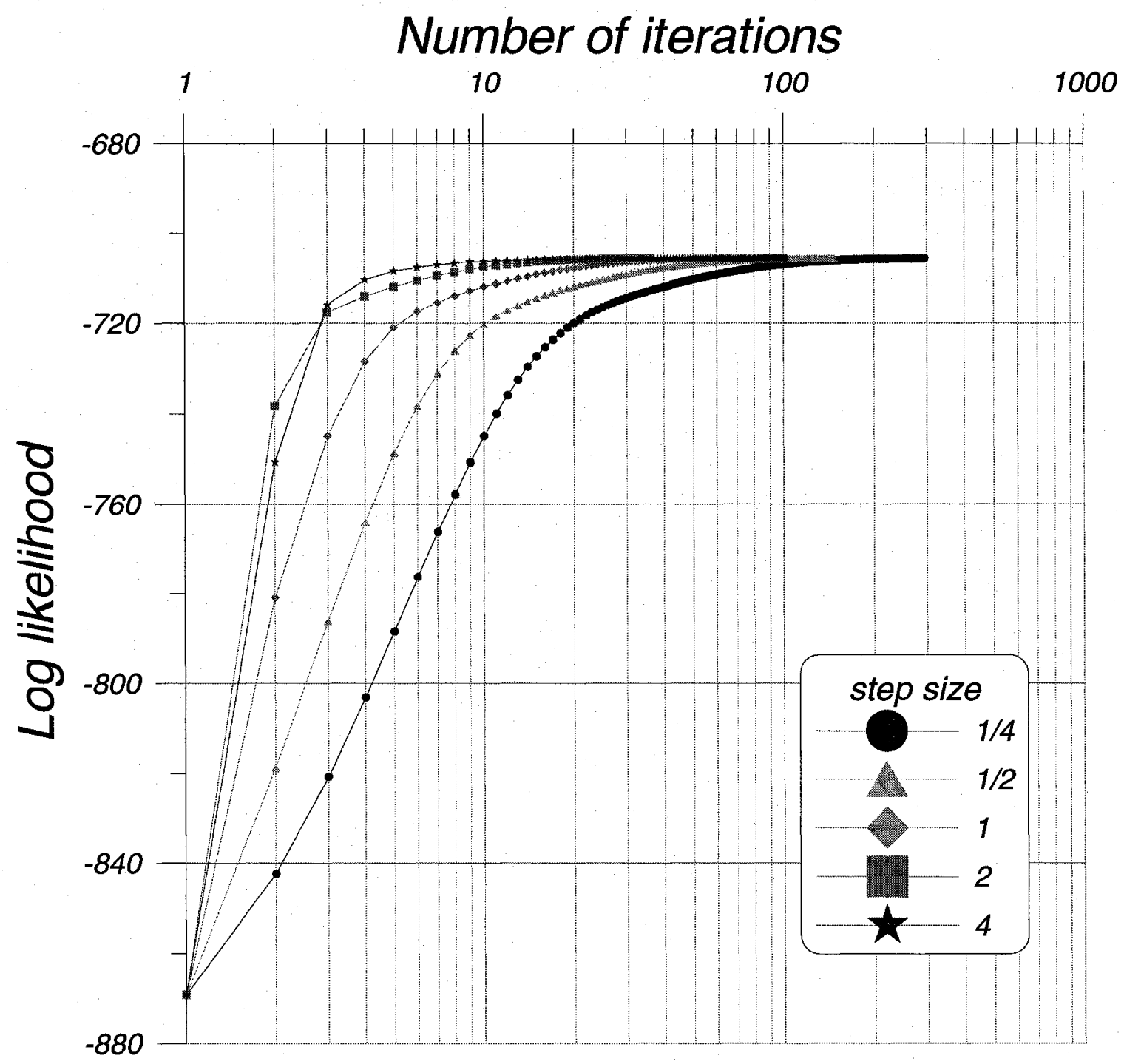

Figure 5-65: Log-likelihood for the all step sizes in BHHH-2 algorithm (exp. 4) 

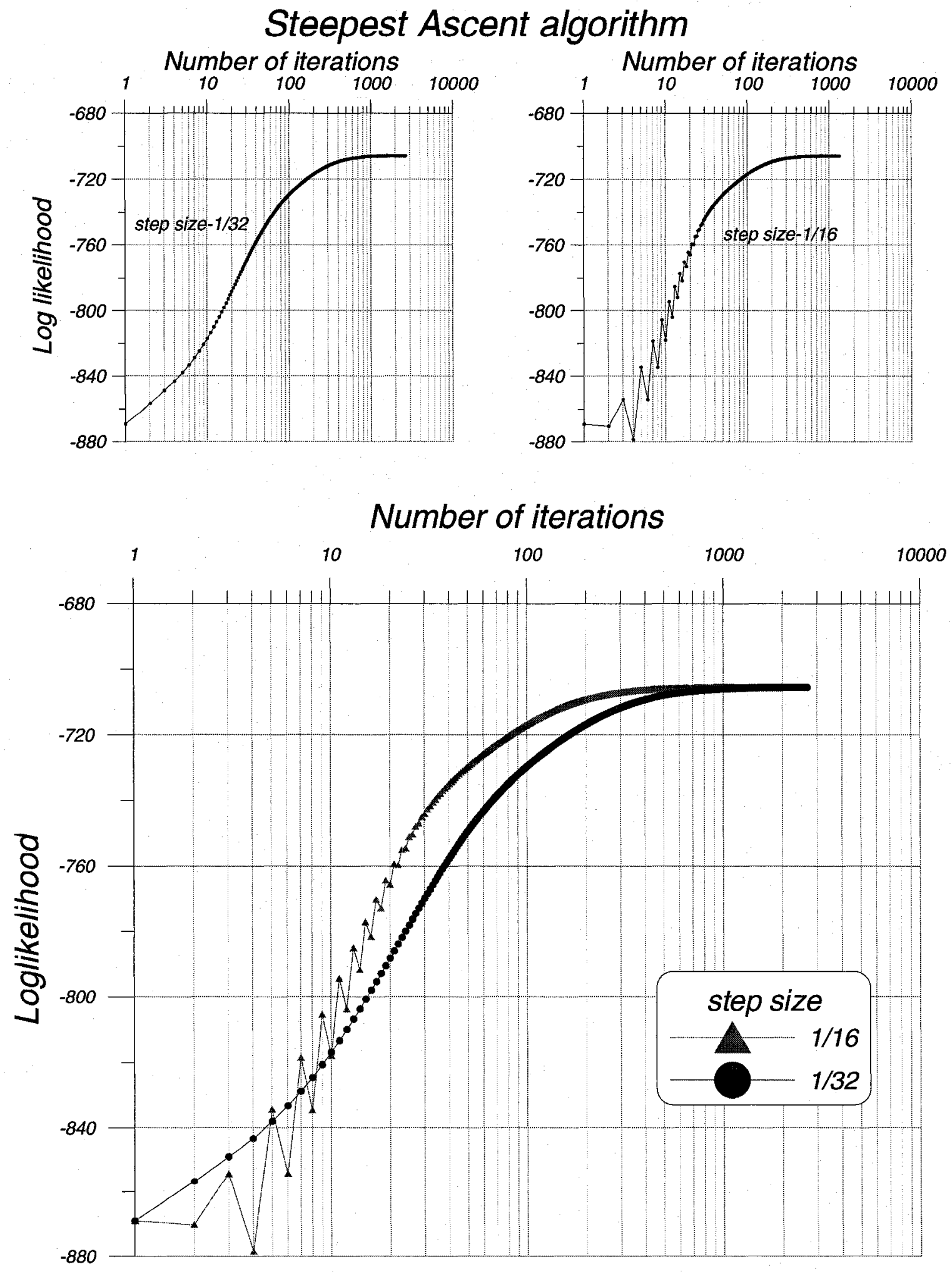

Figure 5-66: Log-likelihood values for each step size and all step sizes in Steepest Ascent algorithm (exp. 4) 


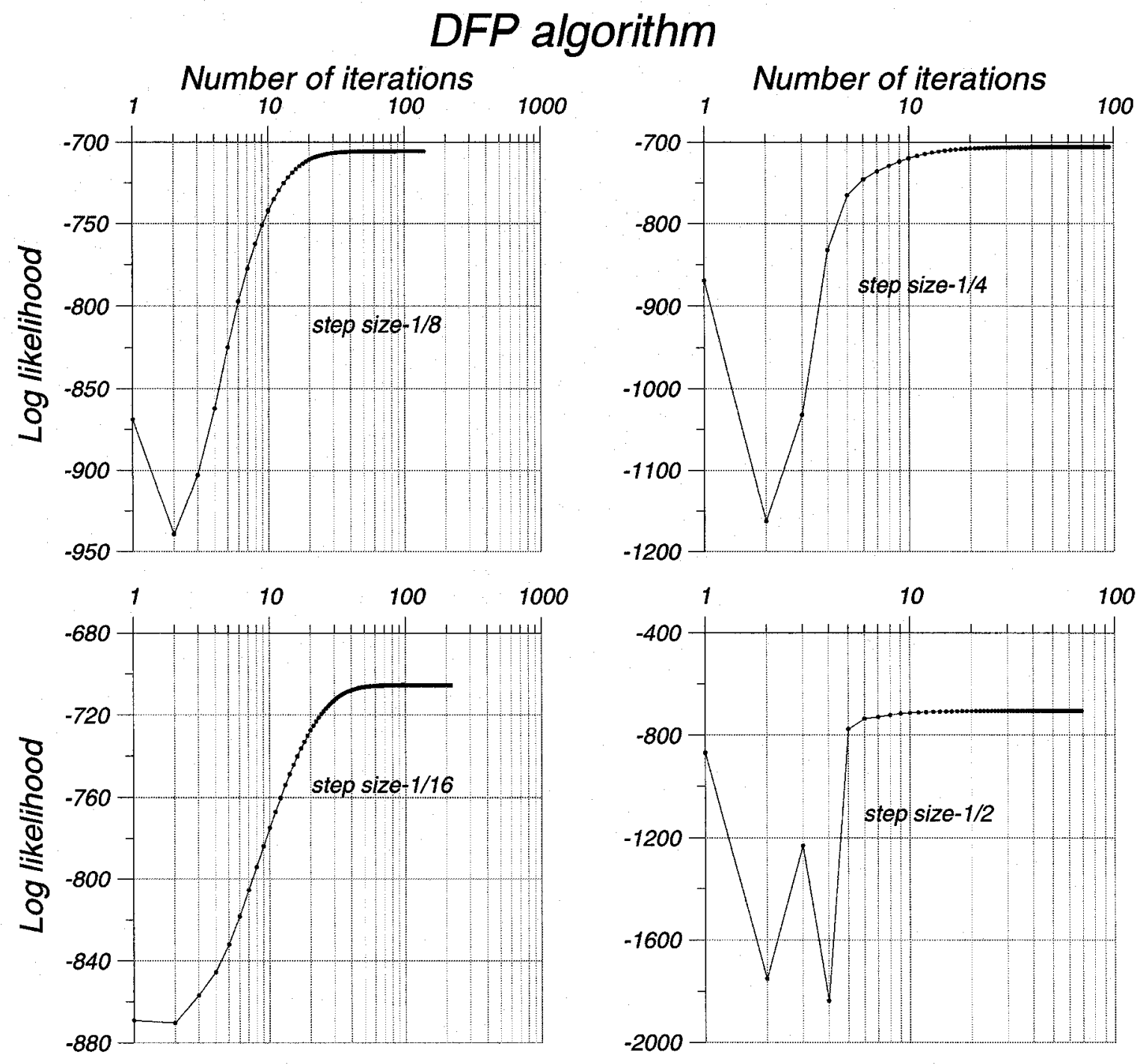

Figure 5-67: Log-likelihood values for each step size in DFP algorithm (exp. 4) 


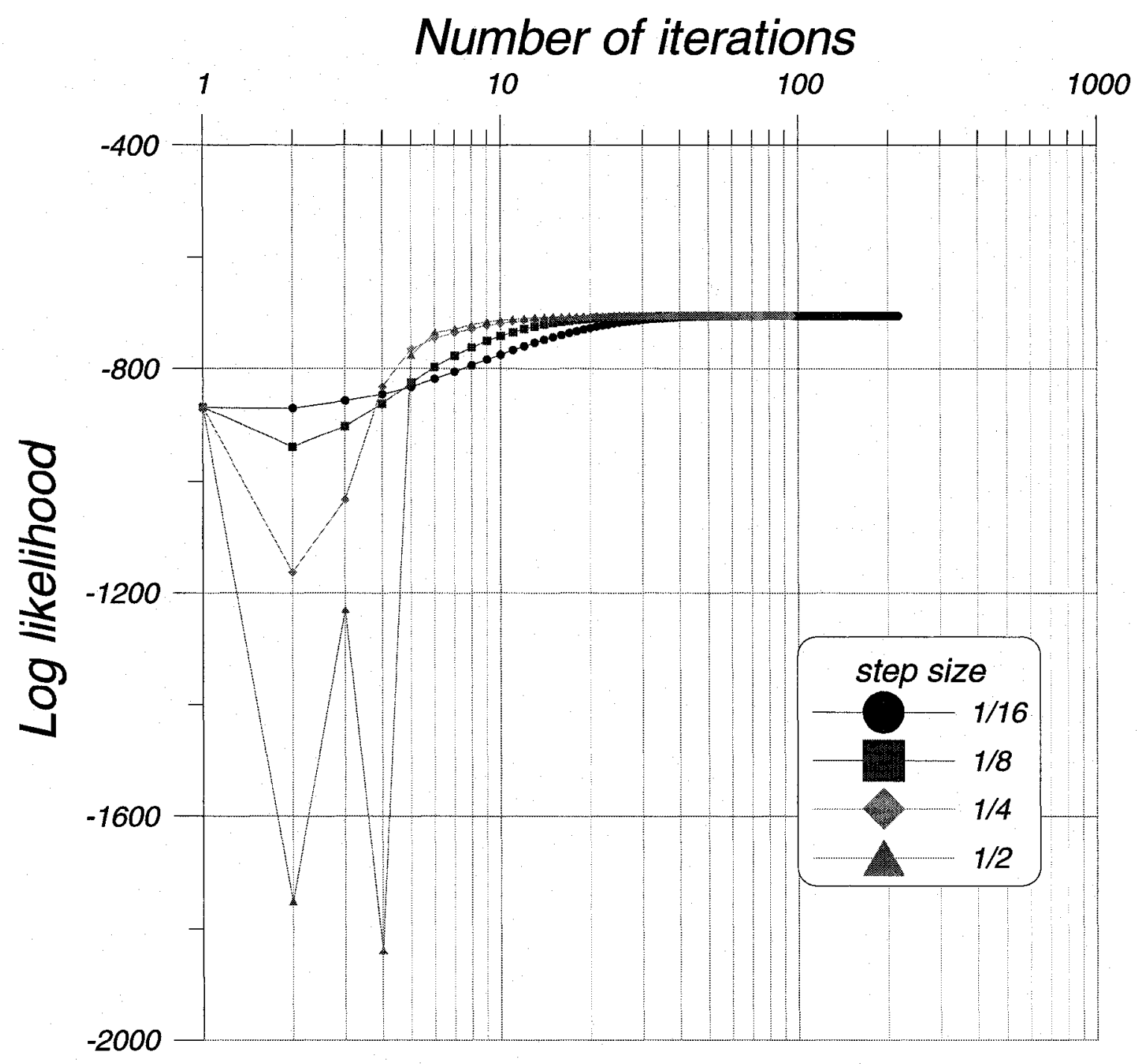

Figure 5-68: Log-likelihood for the all step sizes in DFP algorithm (exp. 4) 


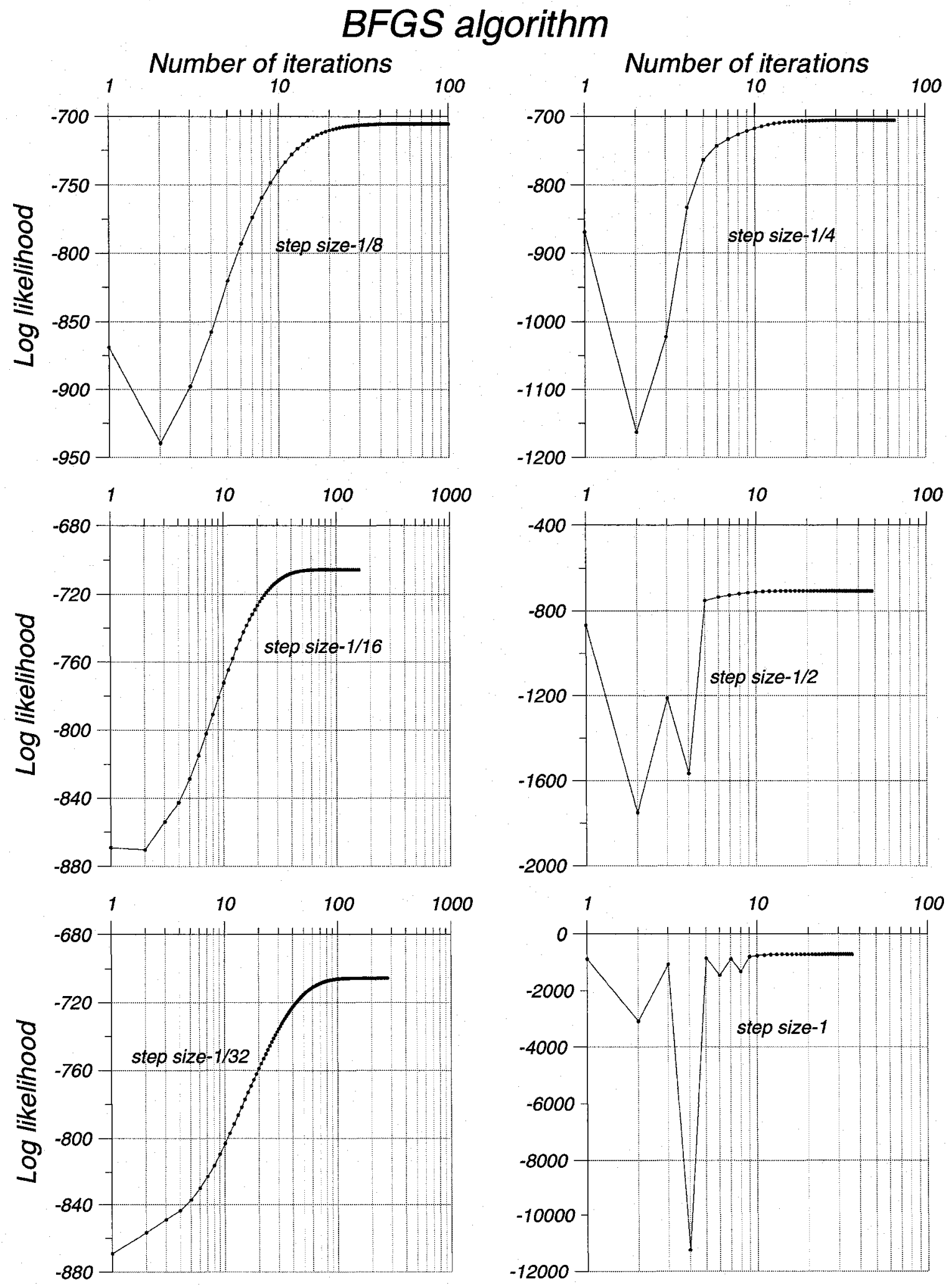

Figure 5-69: Log-likelihood values for each step size in BFGS algorithm (exp. 4) 


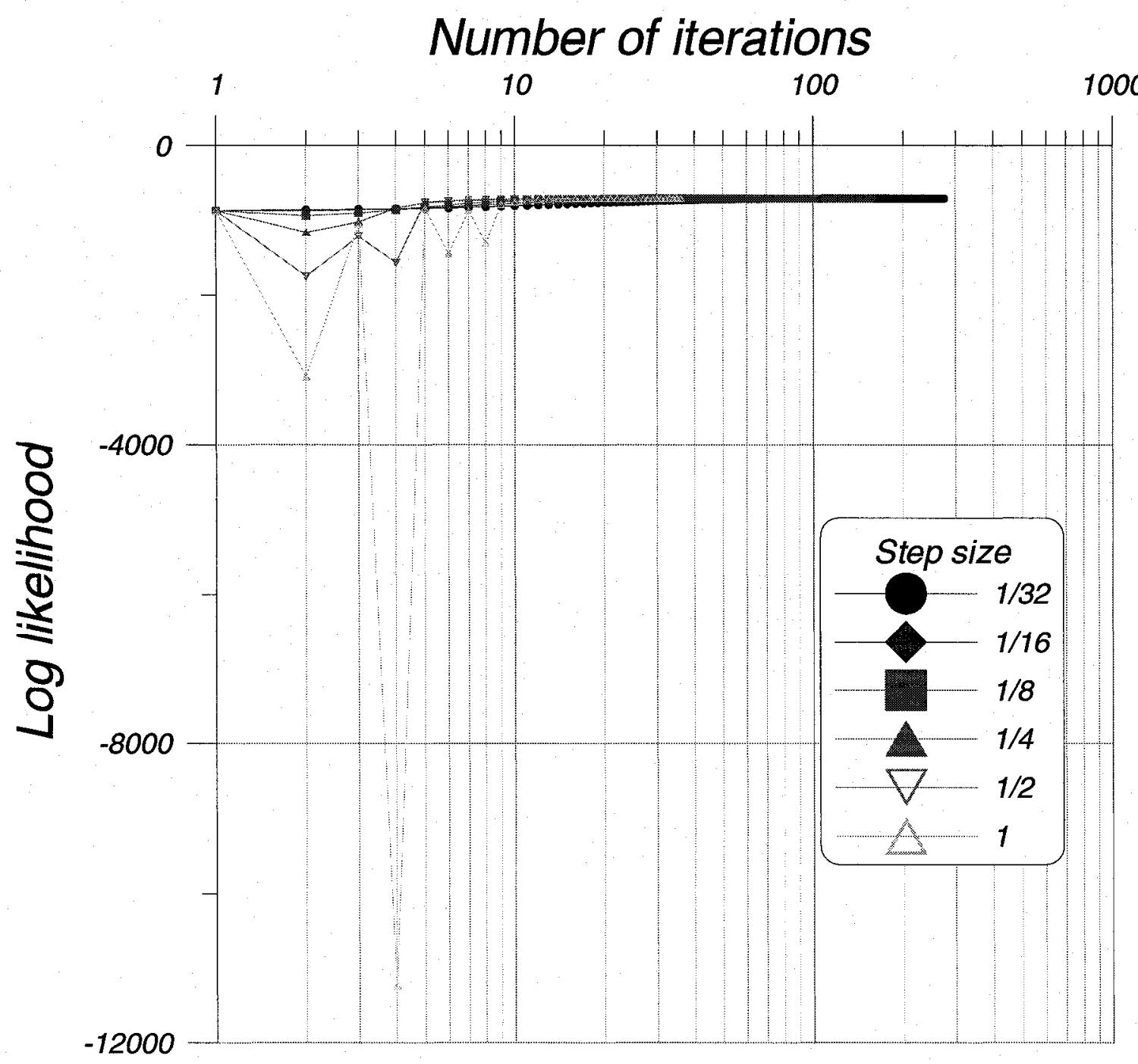

Figure 5-70: Log-likelihood for the all step sizes in BFGS algorithm (exp. 4) 


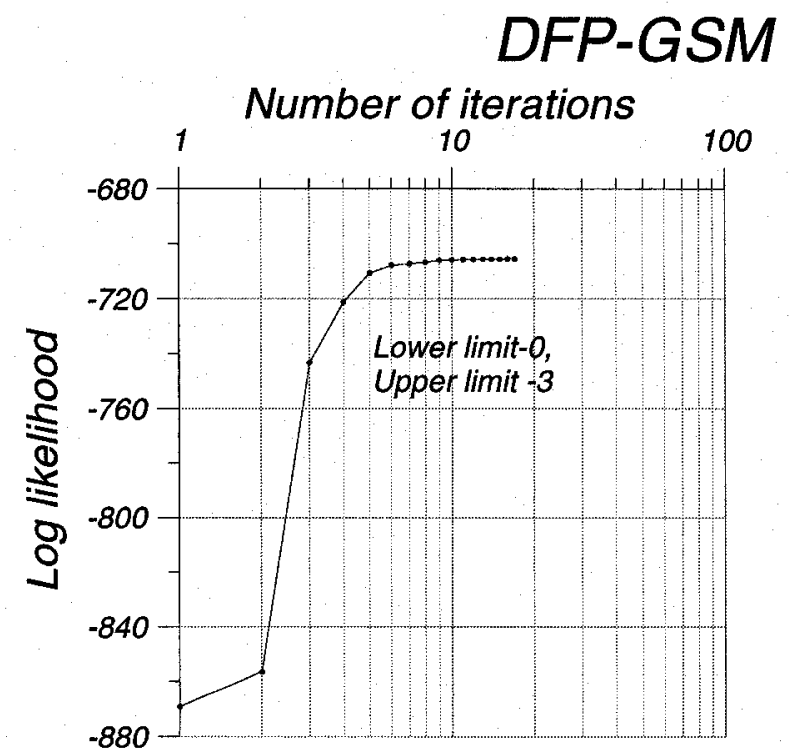

algorithm
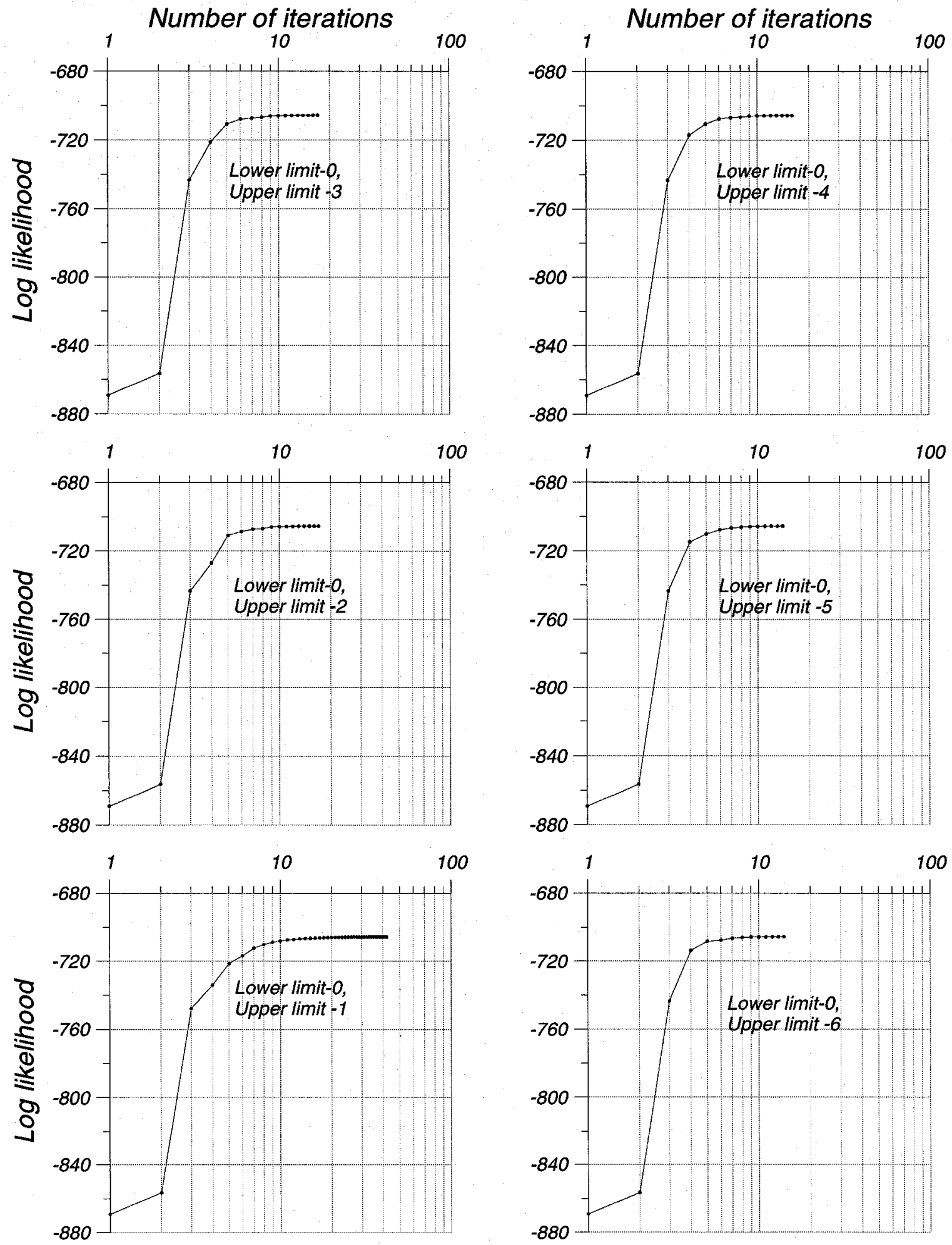

Figure 5-71: Log-likelihood values in DFP-GSM algorithm for the first six uncertain intervals (exp. 4) 

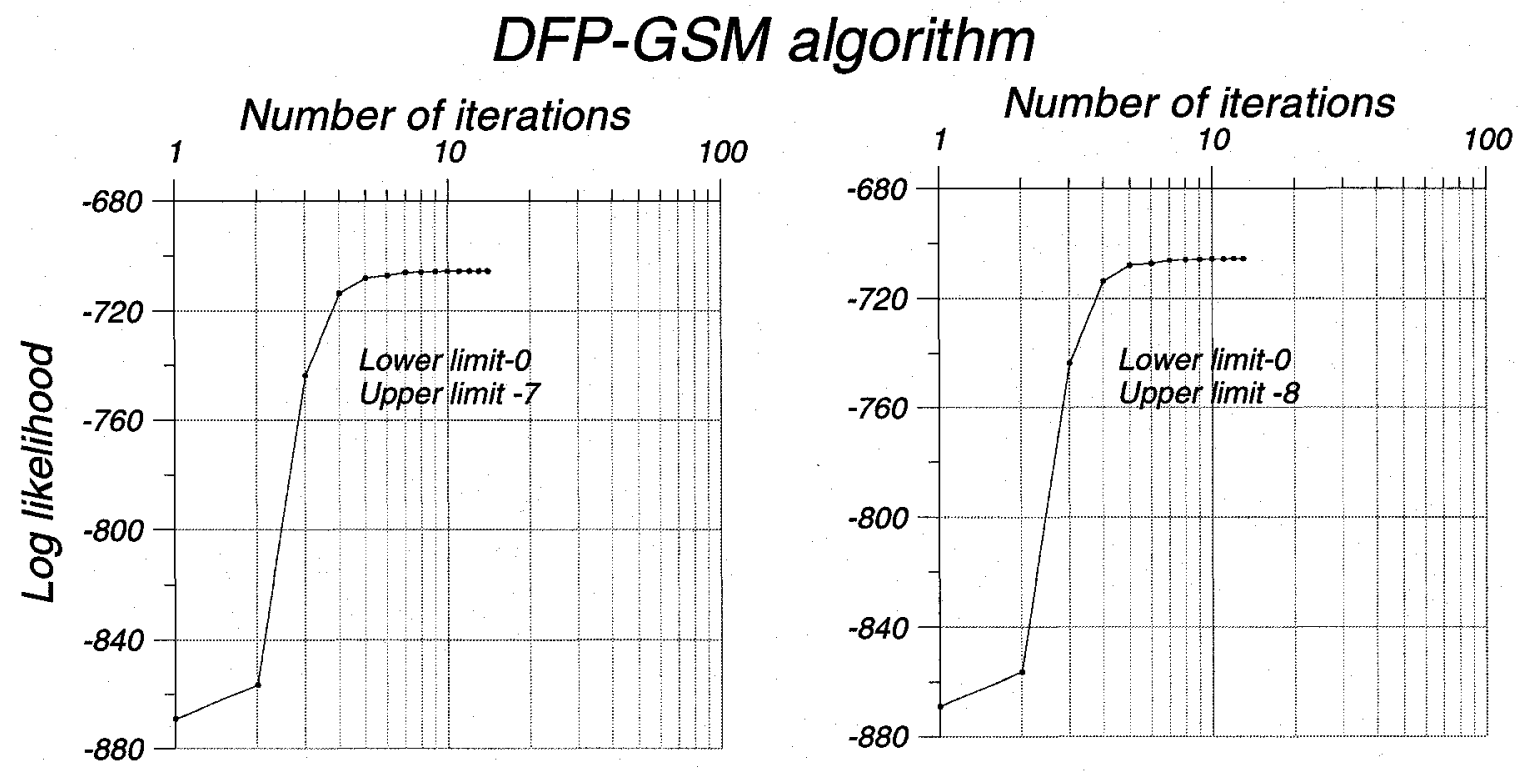

Figure 5-72: Log-likelihood values in DFP-GSM algorithm for the last two uncertain intervals (exp. 4) 


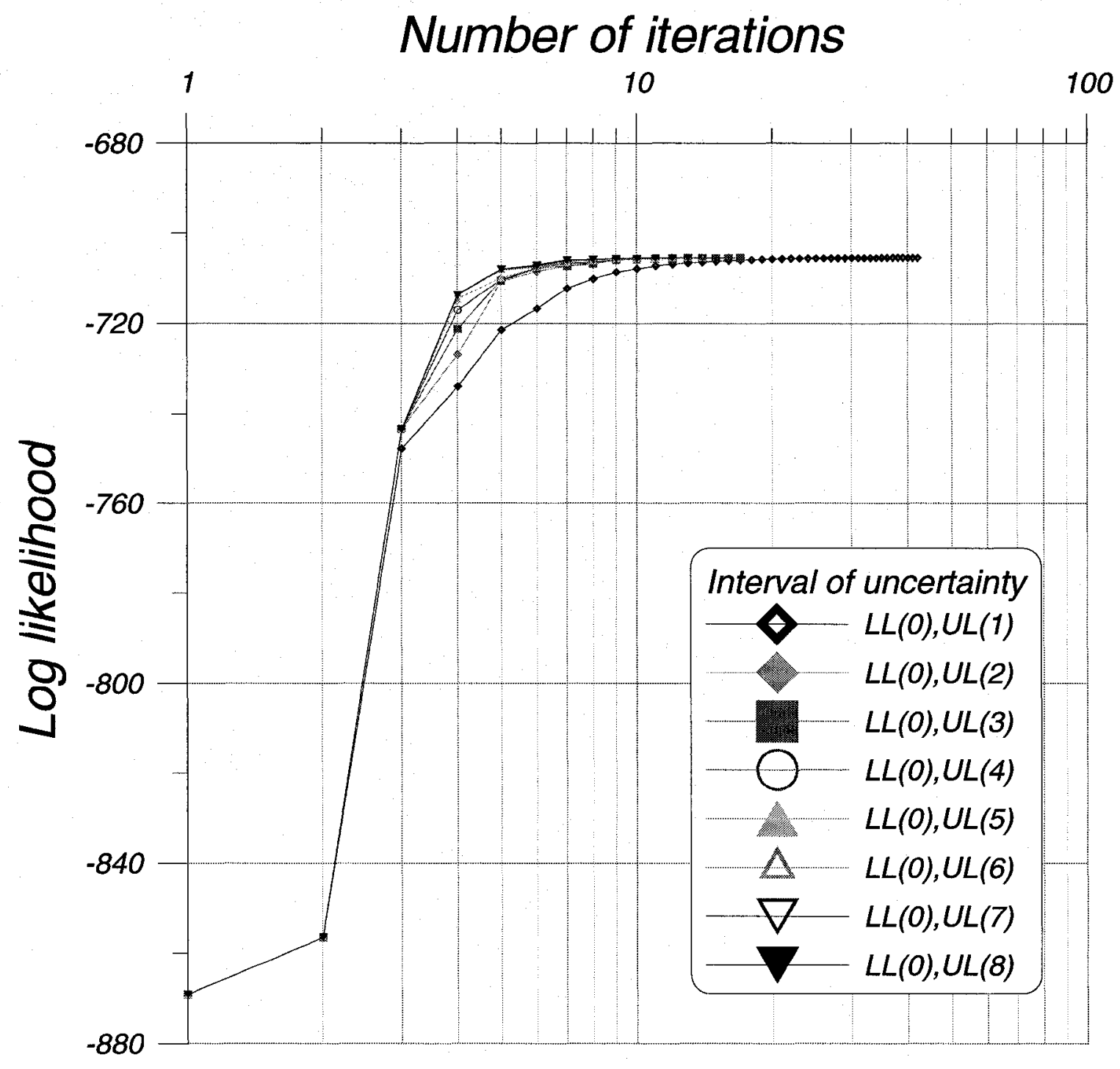

Figure 5-73: Log-likelihood for the all uncertain intervals in DFP-GSM algorithm (exp. 4) 


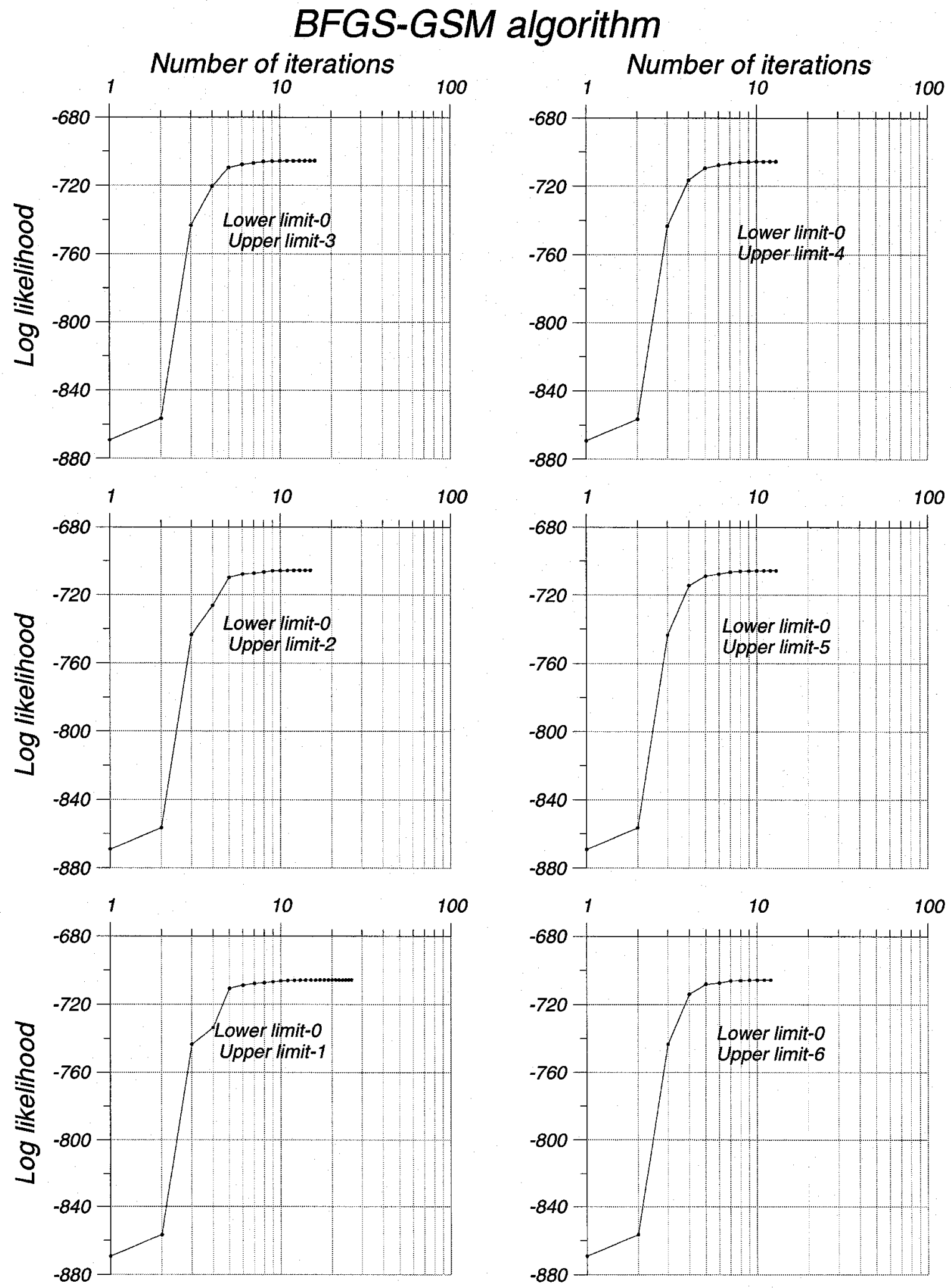

Figure 5-74: Log-likelihood values in BFGS-GSM algorithm for the first six uncertain intervals (exp. 4) 


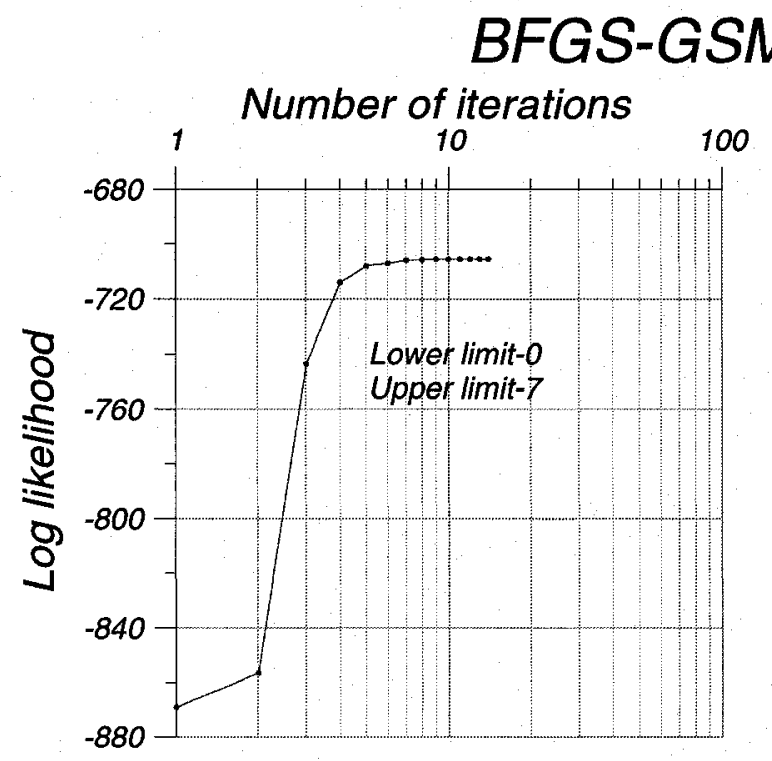

\section{algorithm}

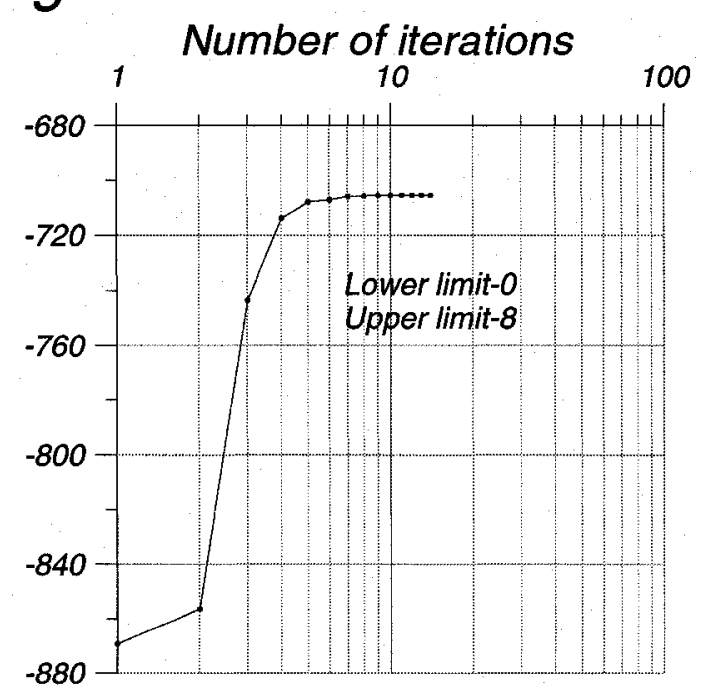

Figure 5-75: Log-likelihood values in BFGS-GSM algorithm for the last two uncertain intervals (exp. 4) 


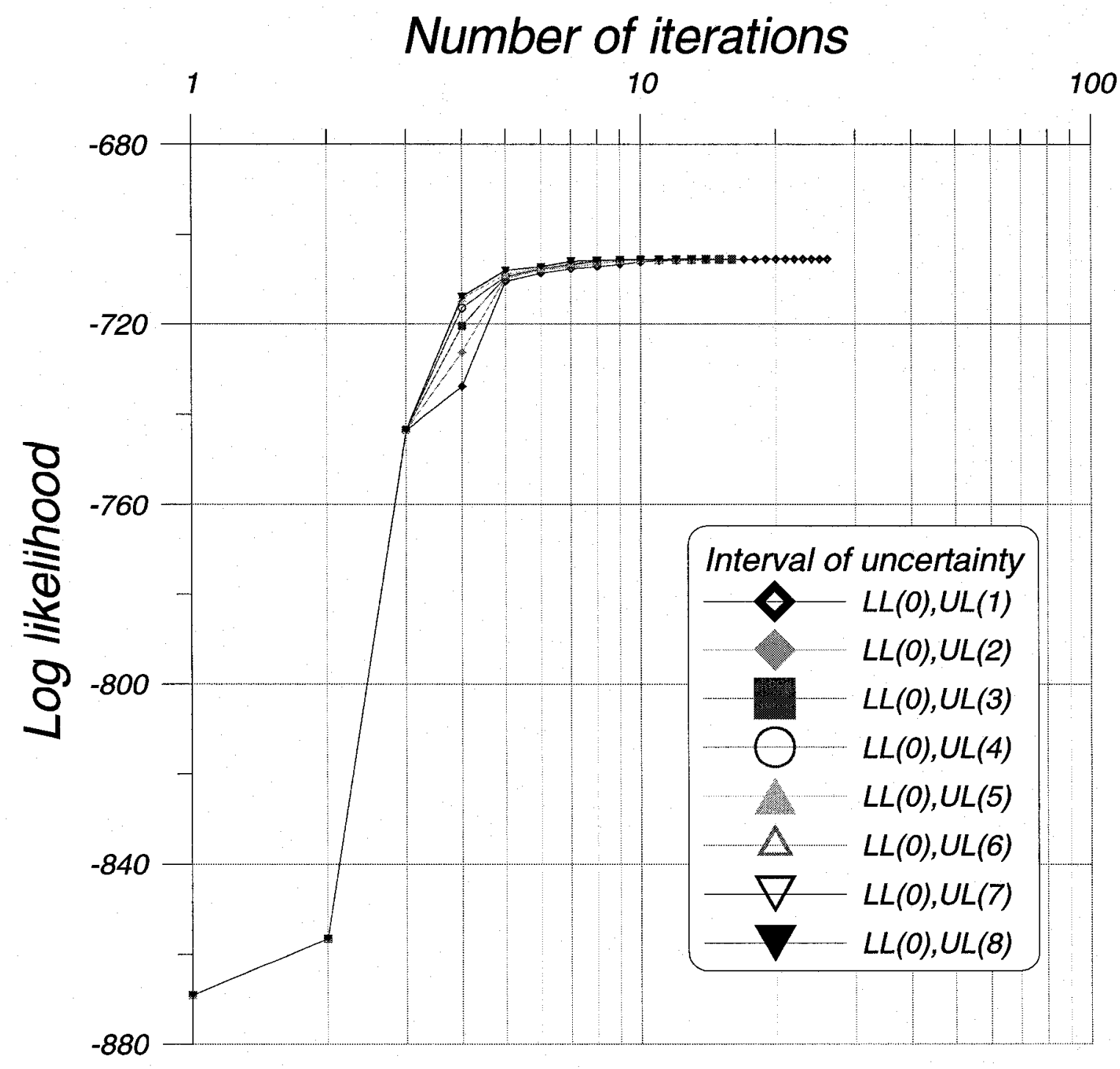

Figure 5-76: Log-likelihood for the all uncertain intervals in BFGS-GSM algorithm (exp. 4) 


\subsection{A comparison of the variations responding to the starting points (experiment 5)}

In this case, the applied convergence criterion is $G_{t}^{\prime}\left(-H_{t}\right)^{-1} G_{t}<10^{-4}$, which is the same criterion as for experiment 2. The main concern of this section is to recognize the importance of guessing starting points and exhibit different estimation performance using experimental estimation runs with different starting points. A new method or an idea of finding a good initial guess of the starting point is not the subject of this section. The point of this section is that it might be important just to know that changing initial starting points can make a difference in estimating performance and that the task of developing a new systematic way of guessing starting points that guarantee a better operational performance might be a useful future research topic. For example, see Liu and Mahmassani (2000) for additional information on this subject. To shed a light on this new interesting topic, in this section, two different vectors of initial starting points are applied as initial starting points in the estimation process of eight algorithms. A new assumed vector of parameter applied to the first four algorithms is $\beta_{k}=\left[\beta_{1}=-0.1, \beta_{2}=-0.1, \beta_{3}=0, \cdots, \beta_{9}=0\right]^{\prime}$, in which only the first two parameters are changed from 0 to -0.1 in order to reflect the case where a researcher has a good prior knowledge of the parameter estimates and can refer the sign of the first two parameters to (-). Another new assumed vector of parameter applied to the last four algorithms is $\beta_{k}=\left[\beta_{1}=-0.01, \beta_{2}=-0.1, \beta_{3}=0, \cdots, \beta_{9}=0\right]^{\prime}$. The only difference between this and the first case is that the first two parameter are replaced with new value, -0.01 and-0.1, respectively. The reason for applying a different vector of parameter is just because when applying $\beta_{k}=\left[\beta_{1}=-0.1, \beta_{2}=-0.1, \beta_{3}=0, \cdots, \beta_{9}=0\right]^{\prime}$ to the last four algorithms, the estimation runs are not to operate. The results obtained from this experimental runs are 
shown in tables 5-39 to 5-47. In addition, the detailed results are compared graphically in figures 5-77 to 5-79 in terms of three performance measures, presented separately according to each different strategy.

It is useful to know that by applying different vectors of starting points can cause diverse variations in estimation performances, that can enhance the situation or made it worse, and that trying to find an advanced way of guessing starting points guarantees improvement in the estimation performance in targeted performance measures. Therefore, this activity should be recognized as a new topic that a researcher can undertake.

Overall, results obtained from experimental estimation are as follows: (1) Starting loglikelihood values are changed due to adopting new starting points. (2) In case of SA algorithms, the convergence time is greatly improved compared to the results of other experiments and the improvement in test results means that a researcher's prior knowledge on parameter to be estimated is correct and new guessed starting points make an improvement in estimation performance. (3) For BHHH-2 and Steepest Ascent algorithms, the convergence log-likelihood values are deviation from the values of other algorithms, but the reason is not clear here. Other aspects related to their estimation performance and their performance behaviours can be observed, and compared to other experiments by using given graphs and tables. 
Table 5-39: Experimental results responding to the variation of starting points (exp. 5)

\begin{tabular}{|c|c|c|c|c|c|c|c|c|c|}
\hline \multirow{2}{*}{\multicolumn{2}{|c|}{$\begin{array}{c}\text { Algorithms \& } \\
\text { Performance } \\
\text { measures }\end{array}$}} & \multicolumn{8}{|c|}{ Step size } \\
\hline & & $1 / 32$ & $1 / 16$ & $1 / 8$ & $1 / 4$ & $1 / 2$ & 1 & 2 & 4 \\
\hline \multirow{3}{*}{ 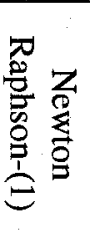 } & $\begin{array}{c}\text { One iteration } \\
\text { time } \\
\end{array}$ & - & $0: 00: 22$ & 0:00:20 & $0: 00: 21$ & $0: 00: 23$ & 0:00:19 & - & - \\
\hline & $\begin{array}{l}\text { Number of } \\
\text { iterations }\end{array}$ & - & 72 & 36 & 17 & 8 & 4 & - & - \\
\hline & $\begin{array}{c}\text { Convergence } \\
\text { time }\end{array}$ & - & $0: 26: 28$ & $0: 12: 02$ & $0: 06: 14$ & 0:03:04 & 0:01:18 & - & - \\
\hline \multirow{3}{*}{ 㝨 } & $\begin{array}{c}\text { One iteration } \\
\text { time }\end{array}$ & - & - & $0: 00: 26$ & $0: 00: 24$ & $0: 00: 23$ & $0: 00: 28$ & - & - \\
\hline & $\begin{array}{c}\text { Number of } \\
\text { iterations }\end{array}$ & - & - & 150 & 75 & 37 & 19 & - & - \\
\hline & $\begin{array}{c}\begin{array}{c}\text { Convergence } \\
\text { time }\end{array} \\
\end{array}$ & - & - & 1:03:54 & $0: 30: 07$ & $0: 14: 29$ & $0: 09: 13$ & - & - \\
\hline \multirow{3}{*}{ 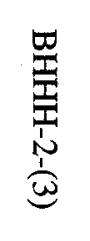 } & $\begin{array}{c}\text { One iteration } \\
\text { time }\end{array}$ & - & - & - & $0: 00: 37$ & $0: 00: 36$ & $0: 00: 27$ & $0: 00: 36$ & $0: 00: 35$ \\
\hline & $\begin{array}{l}\text { Number of } \\
\text { iterations }\end{array}$ & - & - & - & 97 & 49 & 36 & 12 & 14 \\
\hline & $\begin{array}{c}\begin{array}{c}\text { Convergence } \\
\text { time }\end{array} \\
\end{array}$ & - & - & - & $0: 59: 26$ & $0: 29: 47$ & $0: 14: 22$ & 0:07:09 & 0:08:17 \\
\hline \multirow{3}{*}{ 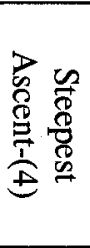 } & $\begin{array}{c}\text { One iteration } \\
\text { time }\end{array}$ & 0:00:19 & $0: 00: 13$ & - & - & - & - & - & - \\
\hline & $\begin{array}{l}\text { Number of } \\
\text { iterations }\end{array}$ & 926 & 459 & - & - & - & - & - & - \\
\hline & $\begin{array}{c}\text { Convergence } \\
\text { time }\end{array}$ & $4: 46: 23$ & $1: 39: 48$ & - & - & - & - & - & - \\
\hline \multirow{3}{*}{ 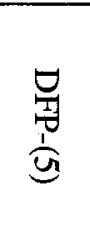 } & $\begin{array}{c}\text { One iteration } \\
\text { time }\end{array}$ & - & 0:00:19 & $0: 00: 18$ & $0: 00: 17$ & 0:00:18 & - & - & - \\
\hline & $\begin{array}{c}\text { Number of } \\
\text { iterations }\end{array}$ & - & 78 & 55 & 43 & 31 & - & - & - \\
\hline & $\begin{array}{c}\text { Convergence } \\
\text { time }\end{array}$ & - & $0: 24: 02$ & $0: 16: 46$ & $0: 12: 24$ & $0: 09: 16$ & - & - & - \\
\hline \multirow{3}{*}{ 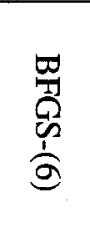 } & $\begin{array}{c}\text { One iteration } \\
\text { time }\end{array}$ & $0: 00: 18$ & $0: 00: 18$ & $0: 00: 18$ & $0: 00: 18$ & $0: 00: 18$ & $0: 00: 17$ & - & - \\
\hline & $\begin{array}{c}\text { Number of } \\
\text { iterations }\end{array}$ & 148 & 77 & 48 & 32 & 21 & 16 & - & - \\
\hline & $\begin{array}{c}\text { Convergence } \\
\text { time }\end{array}$ & 0:43:40 & $0: 22: 52$ & $0: 13: 59$ & 0:09:19 & $0: 06: 11$ & $0: 04: 24$ & - & - \\
\hline \multicolumn{2}{|r|}{$\begin{array}{l}\text { Interval of } \\
\text { uncertainty }\end{array}$} & $\begin{array}{l}\text { LL(0), } \\
\text { UL(1) }\end{array}$ & $\begin{array}{l}\text { LL(0), } \\
\text { UL(2) }\end{array}$ & $\begin{array}{l}\text { LL(0), } \\
\text { UL(3) }\end{array}$ & $\begin{array}{l}\text { LL(0), } \\
\text { UL(4) }\end{array}$ & $\begin{array}{l}\text { LL(0), } \\
\mathrm{UL}(5)\end{array}$ & $\begin{array}{l}\mathrm{LL}(0) \\
\mathrm{UL}(6)\end{array}$ & $\begin{array}{l}\mathrm{LL}(0), \\
\mathrm{UL}(7)\end{array}$ & $\begin{array}{l}\text { LL(0), } \\
\text { UL(8) }\end{array}$ \\
\hline \multirow{3}{*}{ 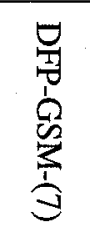 } & $\begin{array}{c}\text { One iteration } \\
\text { time }\end{array}$ & 0:03:01 & 0:03:28 & $0: 03: 33$ & $0: 01: 17$ & 0:03:39 & 0:01:46 & 0:01:46 & $0: 01: 47$ \\
\hline & $\begin{array}{l}\text { Number of } \\
\text { iterations }\end{array}$ & 23 & 11 & 9 & 10 & 10 & 9 & 8 & 8 \\
\hline & $\begin{array}{c}\text { Convergence } \\
\text { time }\end{array}$ & 1:09:07 & 0:38:08 & $0: 31: 47$ & $0: 12: 42$ & $0: 36: 23$ & $0: 15: 50$ & 0:14:05 & $0: 14: 12$ \\
\hline \multirow{3}{*}{ 䍒 } & $\begin{array}{c}\text { One iteration } \\
\text { time }\end{array}$ & 0:01:13 & $0: 01: 21$ & $0: 01: 22$ & $0: 01: 17$ & $0: 01: 17$ & $0: 01: 21$ & 0:01:22 & $0: 01: 26$ \\
\hline & $\begin{array}{c}\text { Number of } \\
\text { iterations }\end{array}$ & 15 & 11 & 11 & 11 & 10 & 9 & 10 & 9 \\
\hline & $\begin{array}{c}\text { Convergence } \\
\text { time }\end{array}$ & $0: 18: 15$ & $0: 14: 44$ & $0: 14: 58$ & $0: 14: 04$ & $0: 12: 49$ & $0: 12: 08$ & $0: 13: 33$ & $0: 12: 46$ \\
\hline
\end{tabular}




\section{Convergence criterion 5}
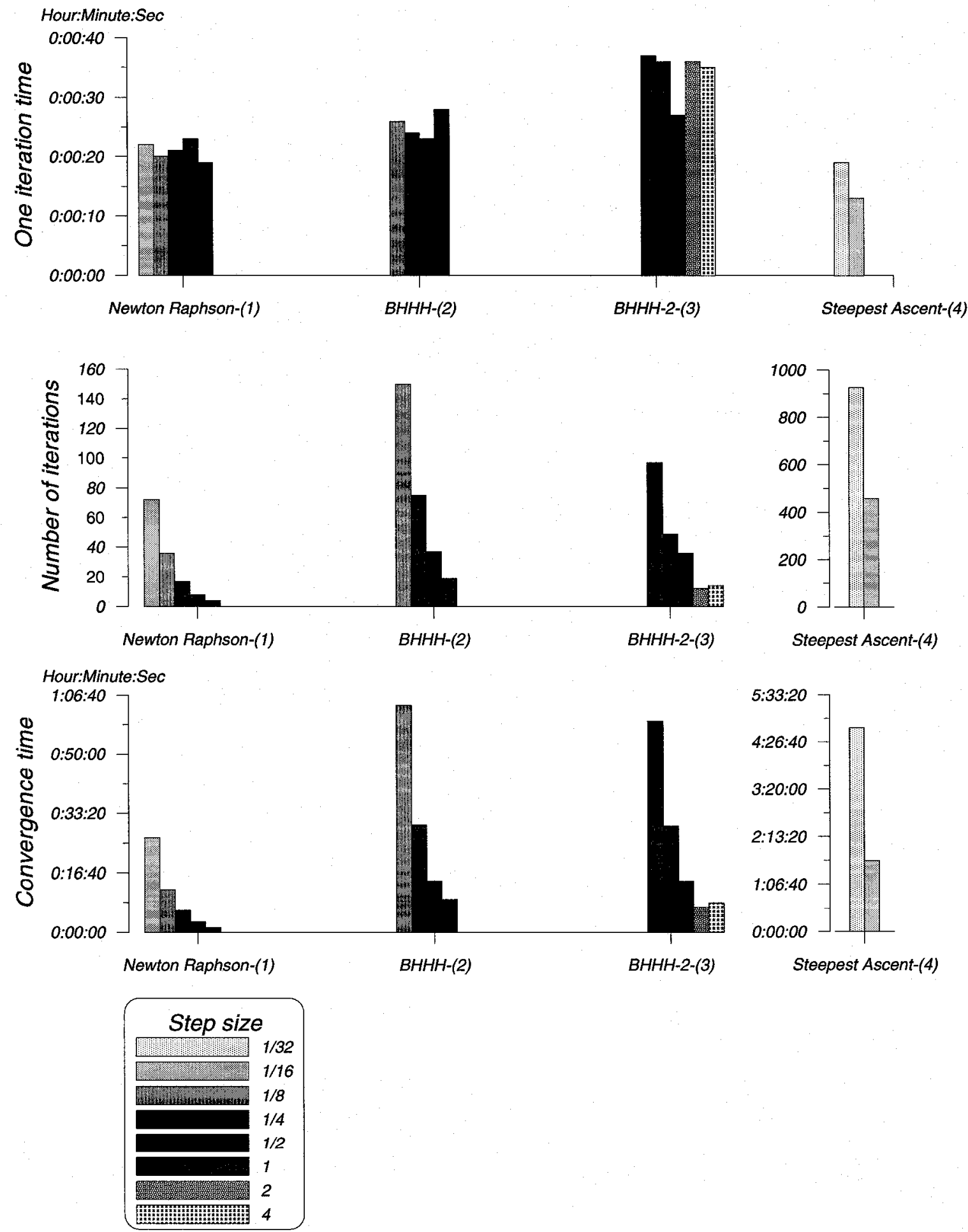

Figure 5-77: Performance results of the first four algorithms responding to the first set of guessing of starting points (exp. 5) 


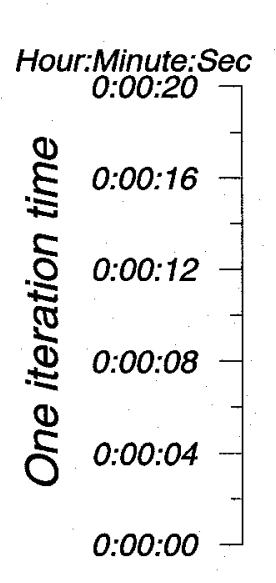

\section{Convergence criterion 5}
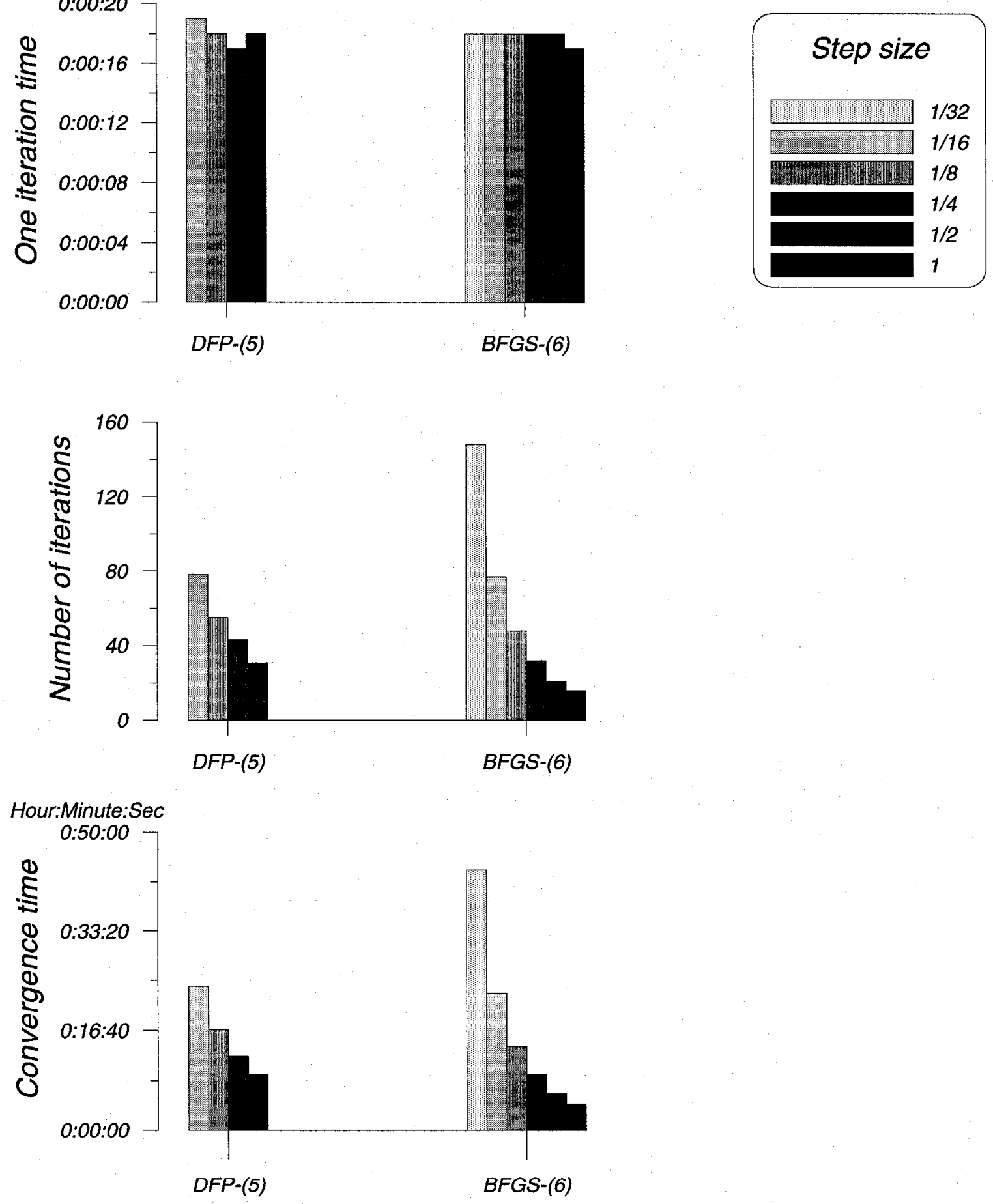

Figure 5-78: Performance results of DFP and BFGS algorithms responding to the second set of guessing of starting points (exp. 5) 

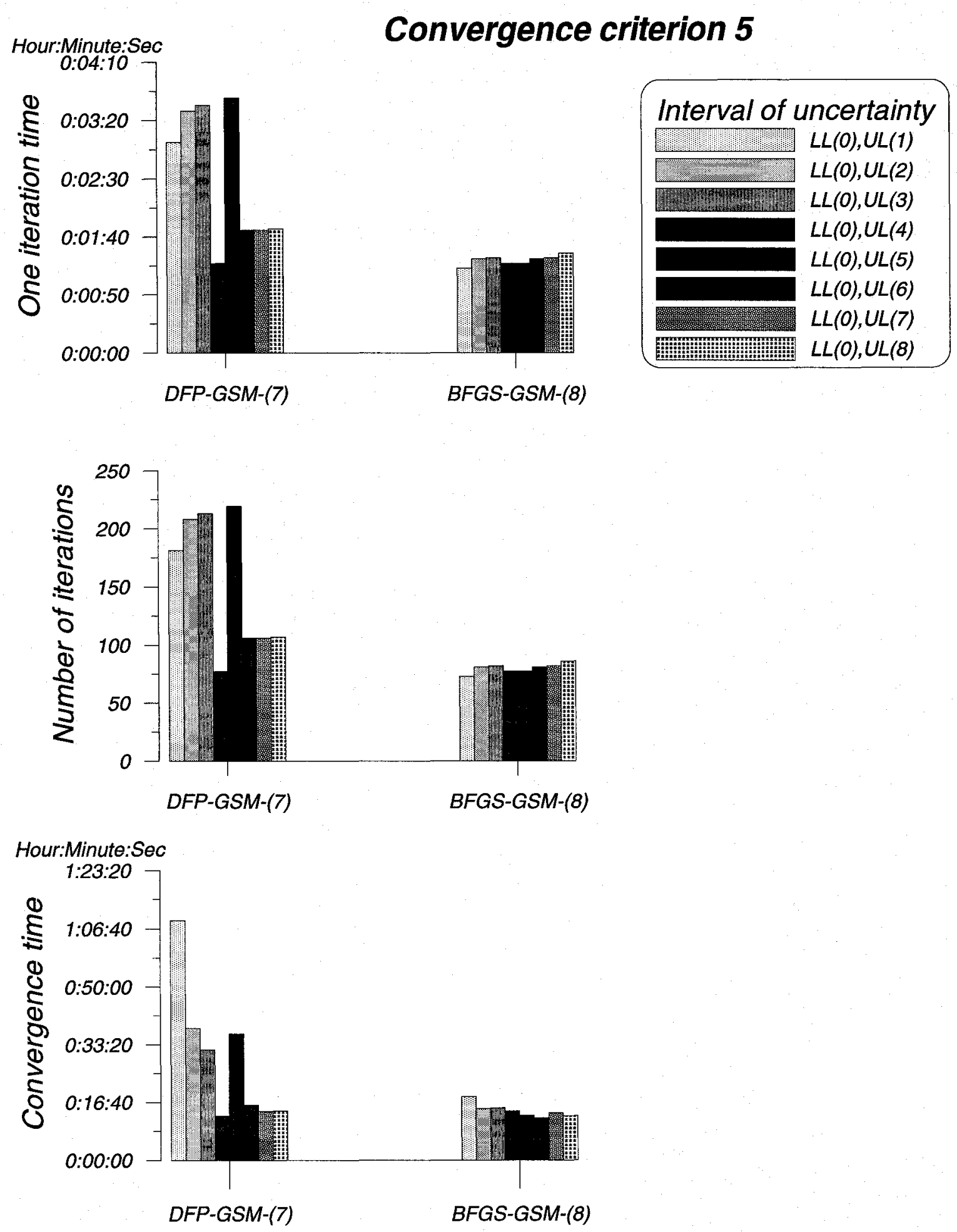

Figure 5-79: Performance results of the last two algorithms responding to the second set of guessing of starting points (exp. 5) 
Table 5-40: Log-likelihood values obtained from Newton Raphson algorithm for the first set of guessing of starting points (exp. 5)

\begin{tabular}{|c|c|c|c|c|c|c|}
\hline \multirow{2}{*}{\multicolumn{2}{|c|}{ Log-likelihood }} & \multicolumn{5}{|c|}{ Step size } \\
\hline & & $1 / 16$ & $1 / 8$ & $1 / 4$ & $1 / 2$ & 1 \\
\hline \multirow{3}{*}{ 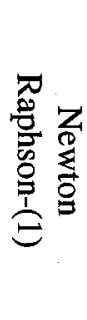 } & $\begin{array}{c}\text { Starting } \\
\text { Log- } \\
\text { likelihood } \\
\end{array}$ & -902.1892789 & -902.1892789 & -902.1892789 & -902.1892789 & -902.1892789 \\
\hline & $\begin{array}{c}\text { Convergence } \\
\text { log- } \\
\text { likelihood } \\
\end{array}$ & -705.5652393 & -705.560808 & -705.5631976 & -705.5506271 & -705.5388718 \\
\hline & $\begin{array}{l}\text { Number of } \\
\text { iterations }\end{array}$ & 72 & 36 & 17 & 8 & 4 \\
\hline
\end{tabular}

Table 5-41: Log-likelihood values obtained from BHHH algorithm for the first set of guessing of starting points (exp. 5)

\begin{tabular}{cccccc}
\hline \multirow{2}{*}{ Log-likelihood } & \multicolumn{4}{c}{ Step size } \\
\cline { 2 - 6 } & $1 / 8$ & $1 / 4$ & $1 / 2$ & 1 \\
\hline \multirow{2}{*}{$\begin{array}{c}\text { Starting } \\
\text { Log-likelihood }\end{array}$} & -902.1892789 & -902.1892789 & -902.1892789 & -902.1892789 \\
\cline { 2 - 5 } & $\begin{array}{c}\text { Convergence } \\
\text { Log-likelihood }\end{array}$ & -705.7974853 & -705.7943473 & -705.8006773 & -705.7635094 \\
\cline { 2 - 6 } & Number of iterations e & 150 & 75 & 37 & 19 \\
\hline
\end{tabular}

Table 5-42: Log-likelihood values obtained from BHHH-2 algorithm for the first set of starting points (exp. 5)

\begin{tabular}{|c|c|c|c|c|c|c|}
\hline \multirow{2}{*}{\multicolumn{2}{|c|}{ Log-likelihood }} & \multicolumn{5}{|c|}{ Step size } \\
\hline & & $1 / 4$ & $1 / 2$ & 1 & 2 & 4 \\
\hline \multirow{3}{*}{ 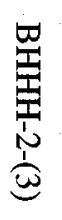 } & $\begin{array}{c}\text { Starting } \\
\text { Log-likelihood }\end{array}$ & $\begin{array}{c}- \\
902.1892789\end{array}$ & $\begin{array}{c}- \\
902.1892789\end{array}$ & $\begin{array}{c}- \\
902.1892789\end{array}$ & $\begin{array}{c}- \\
902.1892789\end{array}$ & $\begin{array}{c}- \\
902.1892789\end{array}$ \\
\hline & $\begin{array}{l}\text { Convergence log- } \\
\text { likelihood }\end{array}$ & $\begin{array}{c}- \\
707.3729016\end{array}$ & $\begin{array}{c}- \\
707.3235866\end{array}$ & $\begin{array}{c}- \\
707.3589346\end{array}$ & $\begin{array}{c}- \\
707.2846937\end{array}$ & -705.754273 \\
\hline & Number of iterations & 97 & 49 & 36 & 12 & 14 \\
\hline
\end{tabular}

Table 5-43: Log-likelihood values obtained from Steepest Ascent algorithm for the first set of guessing of starting points (exp. 5)

\begin{tabular}{|c|c|c|c|}
\hline \multirow{2}{*}{\multicolumn{2}{|c|}{ Log-likelihood }} & \multicolumn{2}{|c|}{ Step size } \\
\hline & & $1 / 32$ & $1 / 16$ \\
\hline \multirow{3}{*}{ 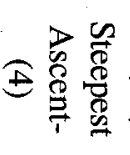 } & $\begin{array}{c}\text { Starting } \\
\text { Logl-ikelihood }\end{array}$ & -902.1892789 & -902.1892789 \\
\hline & Convergence log-likelihood & -706.1196484 & -706.1142506 \\
\hline & Number of iterations & 926 & 459 \\
\hline
\end{tabular}


Table 5-44: Log-likelihood values obtained from DFP algorithm for the second set of guessing of starting points (exp. 5)

\begin{tabular}{|c|c|c|c|c|c|}
\hline \multirow{2}{*}{\multicolumn{2}{|c|}{ Log-likelihood }} & \multicolumn{4}{|c|}{ Step size } \\
\hline & & $1 / 16$ & $1 / 8$ & $1 / 4$ & $1 / 2$ \\
\hline \multirow{3}{*}{$\begin{array}{l}\frac{6}{3} \\
\frac{1}{3}\end{array}$} & $\begin{array}{c}\text { Starting } \\
\text { Log-likelihood }\end{array}$ & -860.5995082 & -860.5995082 & -860.5995082 & -860.5995082 \\
\hline & Convergence log-likelihood & -705.5871328 & -705.6177499 & -705.6517527 & -705.6940772 \\
\hline & Number of iterations & 78 & 55 & 43 & 31 \\
\hline
\end{tabular}

Table 5-45: Log-likelihood values obtained from BFGS algorithm for the second set of guessing of starting points (exp. 5)

\begin{tabular}{|c|c|c|c|c|c|c|c|}
\hline \multirow{2}{*}{\multicolumn{2}{|c|}{ Log-likelihood }} & \multicolumn{6}{|c|}{ Step size } \\
\hline & & $1 / 32$ & $1 / 16$ & $1 / 8$ & $1 / 4$ & $1 / 2$ & 1 \\
\hline \multirow{5}{*}{$\begin{array}{l}\frac{\sigma}{\mathrm{T}} \\
\stackrel{0}{0} \\
\frac{1}{0}\end{array}$} & Starting & - & - & - & - & - & - \\
\hline & $\begin{array}{c}\text { Log- } \\
\text { likelihood }\end{array}$ & 860.5995082 & 860.5995082 & 860.5995082 & 860.5995082 & 860.5995082 & 860.5995082 \\
\hline & $\begin{array}{c}\text { Convergence } \\
\text { log- }\end{array}$ & $\frac{-}{705.5763454}$ & 7055817776 & $\frac{-}{7055869734}$ & $\begin{array}{c}- \\
7055896347\end{array}$ & $\begin{array}{c}- \\
7056084271\end{array}$ & $\begin{array}{c}- \\
7056838354\end{array}$ \\
\hline & likelihood & & & & & & \\
\hline & $\begin{array}{l}\text { Number of } \\
\text { iterations }\end{array}$ & 148 & 77 & 48 & 32 & 21 & 16 \\
\hline
\end{tabular}

Table 5-46: Log-likelihood values obtained from DFP-GSM algorithm for the second set of guessing of starting points (exp. 5)

\begin{tabular}{|c|c|c|c|c|c|c|c|c|c|}
\hline & $\begin{array}{l}\text { Interval of } \\
\text { uncertainty }\end{array}$ & $\begin{array}{l}\mathrm{LL}(0), \\
\mathrm{UL}(1)\end{array}$ & $\begin{array}{l}\text { LL(0), } \\
\text { UL(2) }\end{array}$ & $\begin{array}{l}\mathrm{LL}(0), \\
\mathrm{UL}(3)\end{array}$ & $\begin{array}{l}\text { LL(0), } \\
\text { UL(4) }\end{array}$ & $\begin{array}{l}\text { LL(0), } \\
\text { UL(5) }\end{array}$ & $\begin{array}{l}\text { LL(0), } \\
\text { UL(6) }\end{array}$ & $\begin{array}{l}\text { LL(0), } \\
\text { UL(7) }\end{array}$ & $\begin{array}{l}\text { LL(0), } \\
\text { UL(8) }\end{array}$ \\
\hline \multirow{3}{*}{$\begin{array}{l}\frac{D}{7} \\
\frac{7}{5} \\
0 \\
\frac{2}{3} \\
\frac{1}{3}\end{array}$} & $\begin{array}{c}\text { Starting } \\
\text { Log- } \\
\text { likelihood }\end{array}$ & $\begin{array}{c}- \\
860.599 \\
5082\end{array}$ & $\begin{array}{c}- \\
860.599 \\
5082\end{array}$ & $\begin{array}{c}- \\
860.599 \\
5082\end{array}$ & $\begin{array}{c}- \\
860.599 \\
5082\end{array}$ & $\begin{array}{c}- \\
860.599 \\
5082\end{array}$ & $\begin{array}{c}- \\
860.599 \\
5082\end{array}$ & $\begin{array}{c}- \\
860.599 \\
5082\end{array}$ & $\begin{array}{c}- \\
860.599 \\
5082\end{array}$ \\
\hline & $\begin{array}{c}\text { Convergence } \\
\text { log- } \\
\text { likelihood }\end{array}$ & $\begin{array}{c}- \\
705.708 \\
8334\end{array}$ & $\begin{array}{c}- \\
705.719 \\
1719\end{array}$ & $\begin{array}{c}- \\
705.855 \\
5421\end{array}$ & $\begin{array}{c}- \\
705.764 \\
4033\end{array}$ & $\begin{array}{c}- \\
705.772 \\
4656\end{array}$ & $\begin{array}{c}- \\
705.766 \\
1276\end{array}$ & $\begin{array}{c}- \\
705.878 \\
9338\end{array}$ & $\begin{array}{c}- \\
705.871 \\
8341\end{array}$ \\
\hline & $\begin{array}{l}\text { Number of } \\
\text { iterations }\end{array}$ & 23 & 11 & 9 & 10 & 10 & 9 & 8 & 8 \\
\hline
\end{tabular}

Table 5-47: Log-likelihood values obtained from BFGS-GSM algorithm for the second set of guessing of starting points (exp. 5)

\begin{tabular}{|c|c|c|c|c|c|c|c|c|c|}
\hline & $\begin{array}{l}\text { Interval of } \\
\text { uncertainty }\end{array}$ & $\begin{array}{l}\text { LL(0), } \\
\text { UL(1) }\end{array}$ & $\begin{array}{l}\mathrm{LL}(0), \\
\mathrm{UL}(2)\end{array}$ & $\begin{array}{l}\text { LL(0), } \\
\text { UL(3), }\end{array}$ & $\begin{array}{l}\mathrm{LL}(0), \\
\mathrm{UL}(4)\end{array}$ & $\begin{array}{l}\text { LL(0), } \\
\text { UL(5) }\end{array}$ & $\begin{array}{l}\text { LL(0), } \\
\text { UL(6) }\end{array}$ & $\begin{array}{l}\text { LL(0), } \\
\text { UL(7) }\end{array}$ & $\begin{array}{l}\text { LL(0), } \\
\text { UL(8) }\end{array}$ \\
\hline \multirow{3}{*}{ 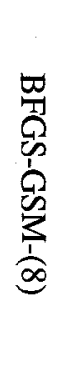 } & $\begin{array}{c}\text { Starting } \\
\text { Log- } \\
\text { likelihood }\end{array}$ & $\begin{array}{c}- \\
860.599 \\
5082\end{array}$ & $\begin{array}{c}- \\
860.599 \\
5082\end{array}$ & $\begin{array}{c}- \\
860.599 \\
5082\end{array}$ & $\begin{array}{c}- \\
860.599 \\
5082\end{array}$ & $\begin{array}{c}860.599 \\
5082\end{array}$ & $\begin{array}{c}- \\
860.599 \\
5082\end{array}$ & $\begin{array}{c}- \\
860.599 \\
5082\end{array}$ & $\begin{array}{c}860.599 \\
5082\end{array}$ \\
\hline & $\begin{array}{c}\text { Convergence } \\
\text { log- } \\
\text { likelihood }\end{array}$ & $\begin{array}{c}705.587 \\
8645\end{array}$ & $\begin{array}{c}705.608 \\
8839\end{array}$ & $\begin{array}{c}705.592 \\
4319\end{array}$ & $\begin{array}{c}- \\
705.590 \\
3149\end{array}$ & $\begin{array}{c}- \\
705.600 \\
2936\end{array}$ & $\begin{array}{c}- \\
705.618 \\
6549\end{array}$ & $\begin{array}{c}- \\
705.594 \\
8914\end{array}$ & $\begin{array}{c}- \\
705.619 \\
2299\end{array}$ \\
\hline & $\begin{array}{l}\text { Number of } \\
\text { iterations }\end{array}$ & 15 & 11 & 11 & 11 & 10 & 9 & 10 & 9 \\
\hline
\end{tabular}




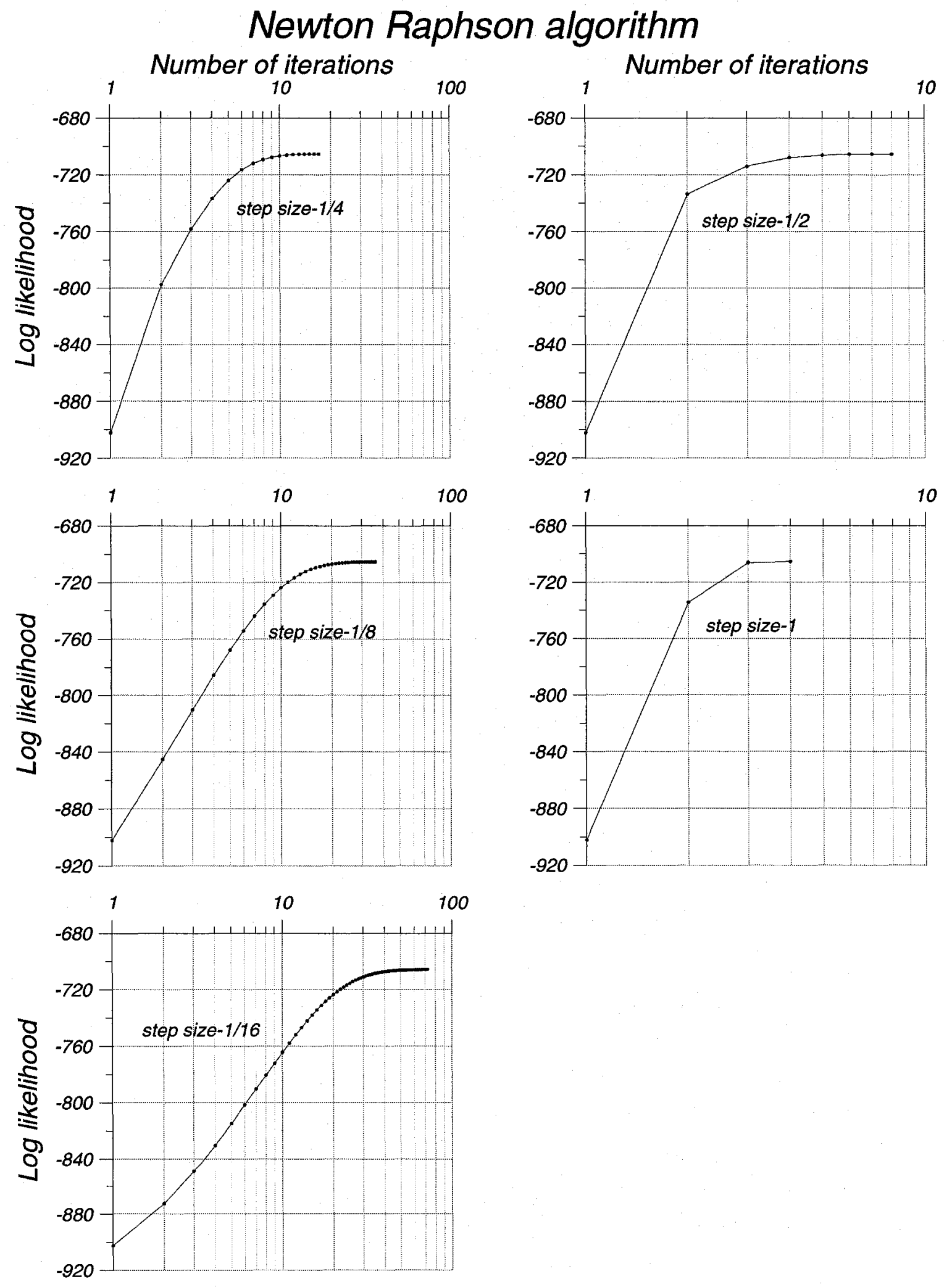

Figure 5-80: Log-likelihood values for each step size in Newton Raphson algorithm for the first set of guessing of starting points (exp. 5) 


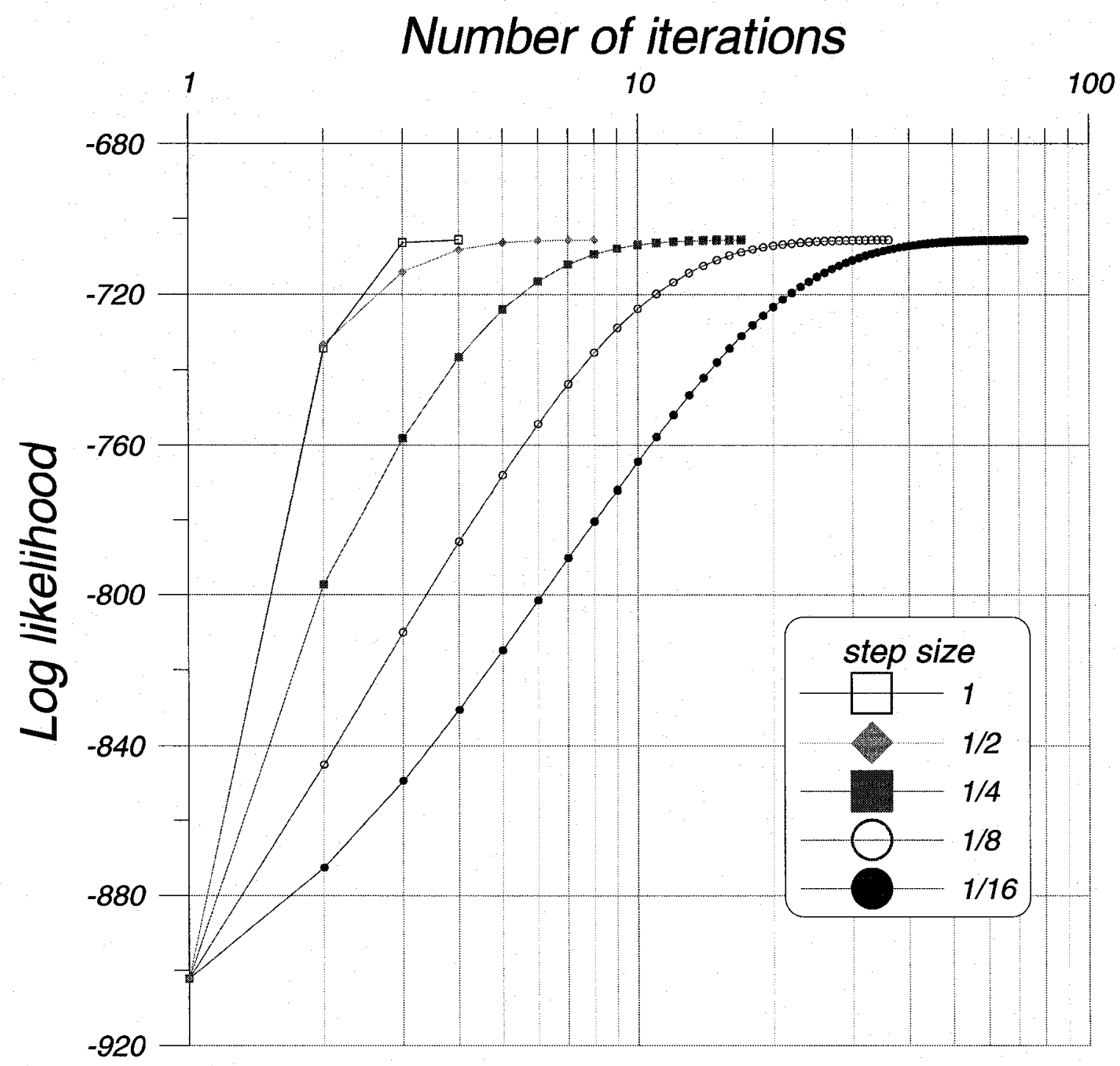

Figure 5-81: Log-likelihood for the all step sizes in Newton Raphson algorithm for the first set of guessing of starting points (exp. 5) 


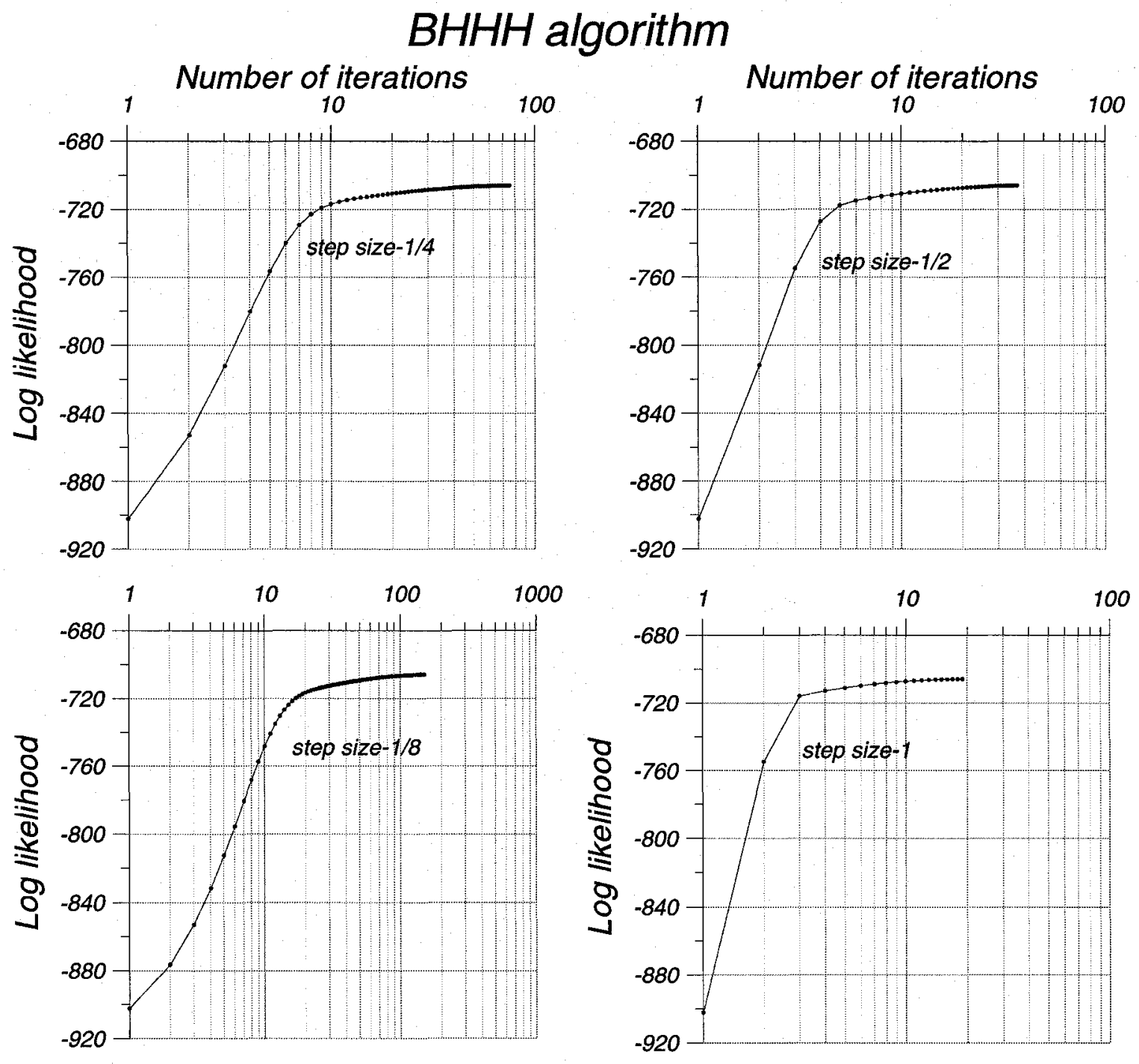

Figure 5-82: Log-likelihood values for each step size in BHHH algorithm for the first set of guessing of starting points (exp. 5) 


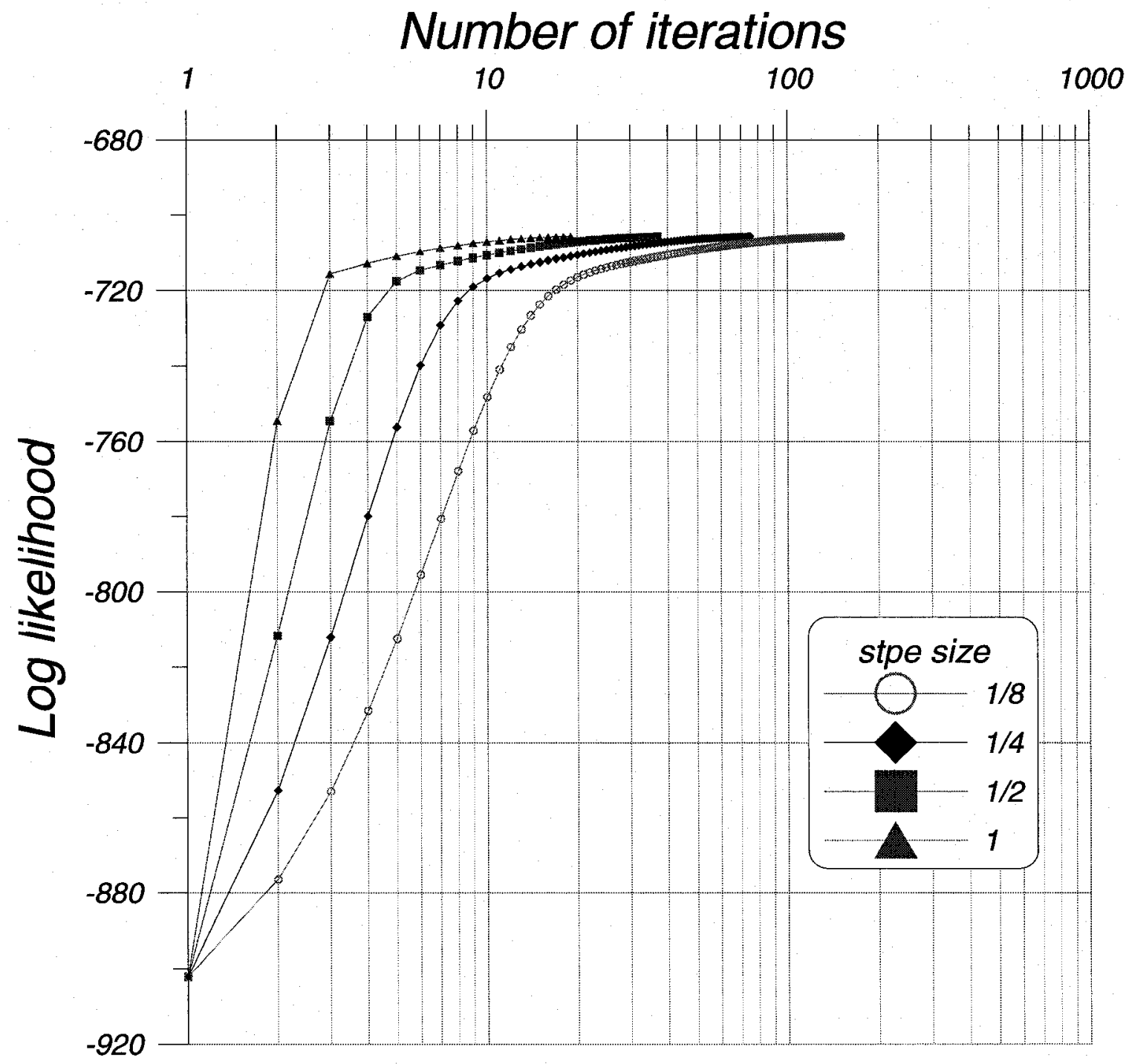

Figure 5-83: Log-likelihood for the all step sizes in BHHH algorithm for the first set of guessing of starting points (exp. 5) 


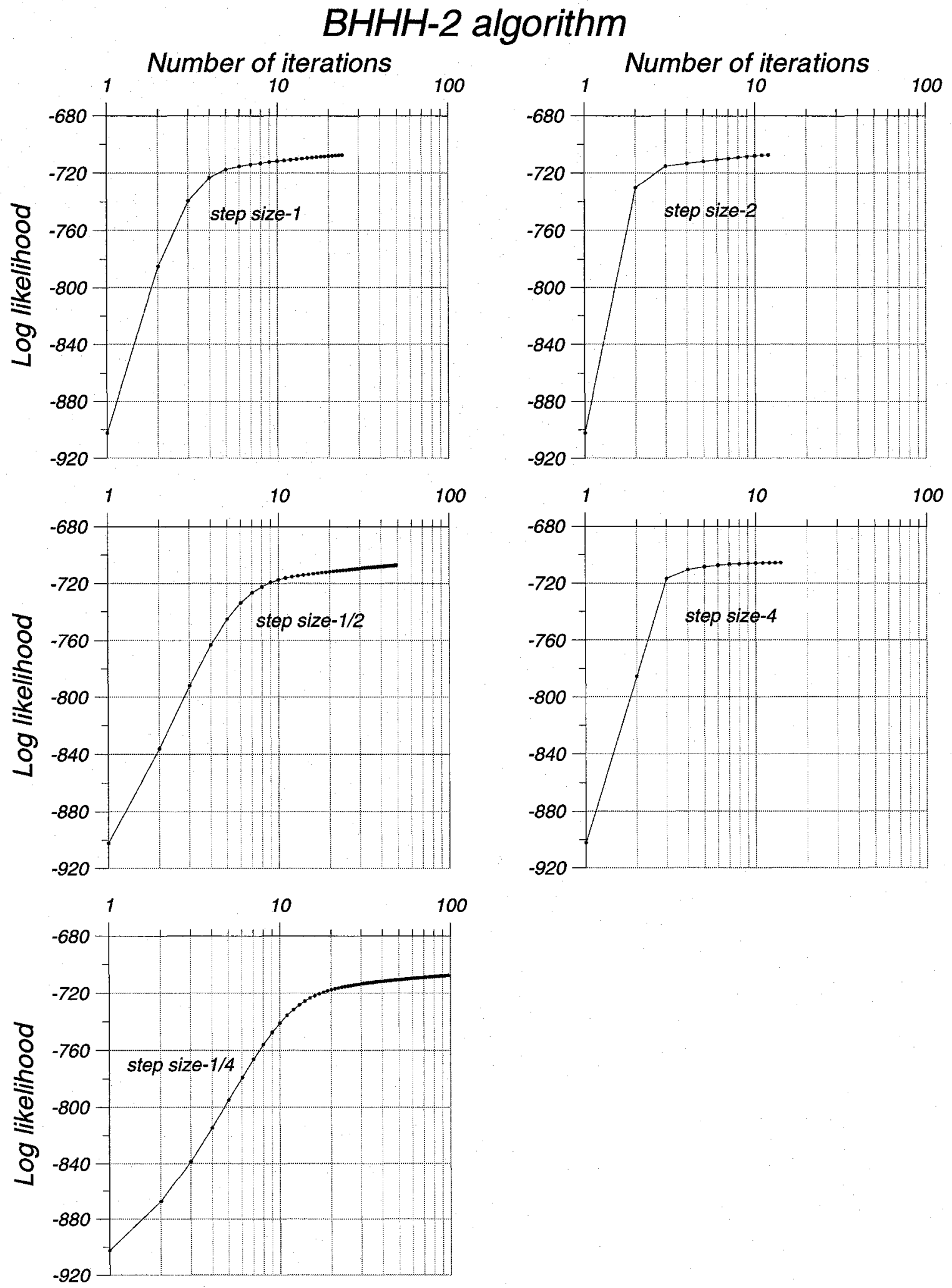

Figure 5-84: Log-likelihood values for each step size in BHHH-2 algorithm for the first set of guessing of starting points (exp. 5) 


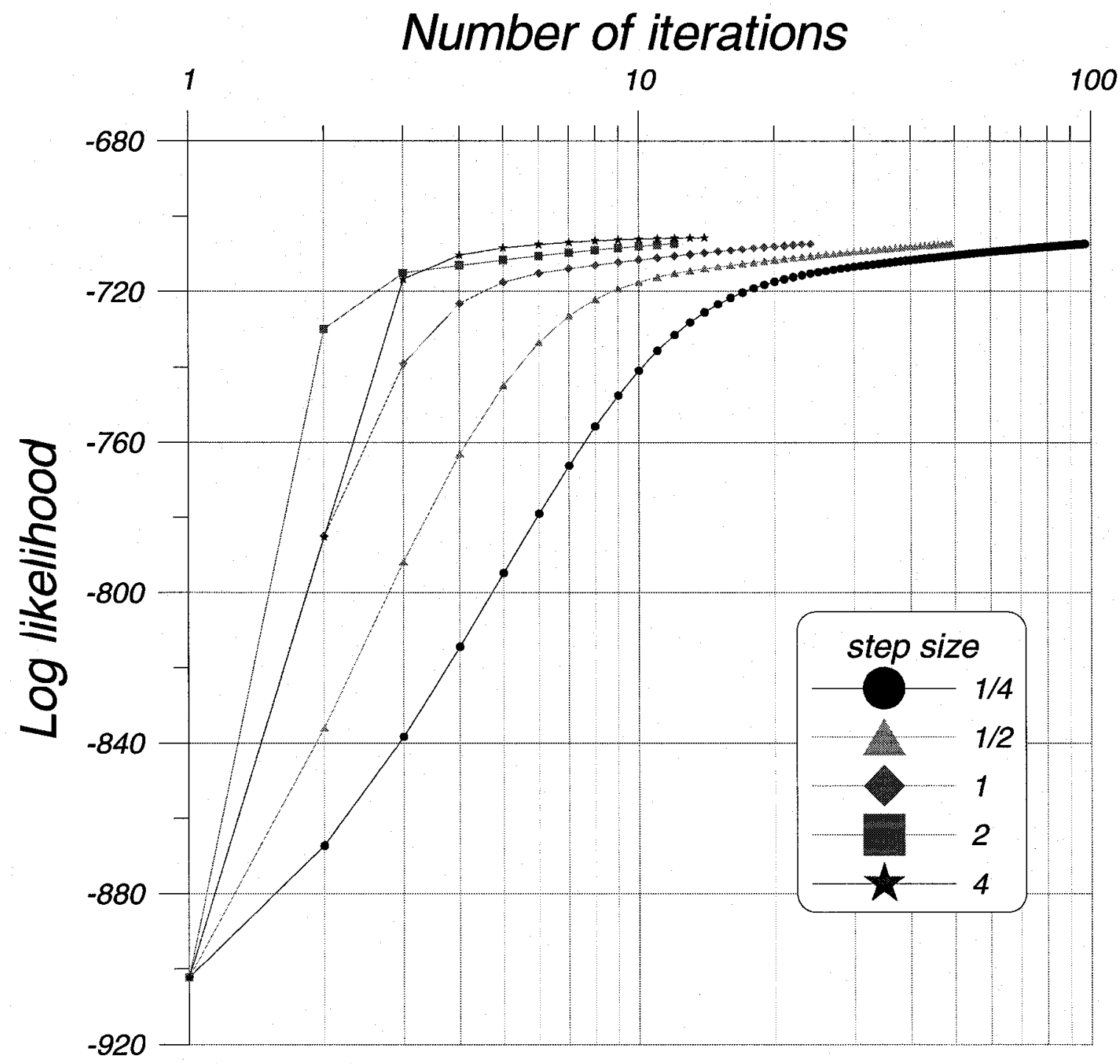

Figure 5-85: Log-likelihood for the all step sizes in BHHH-2 algorithm for the first set of guessing of starting points (exp. 5) 

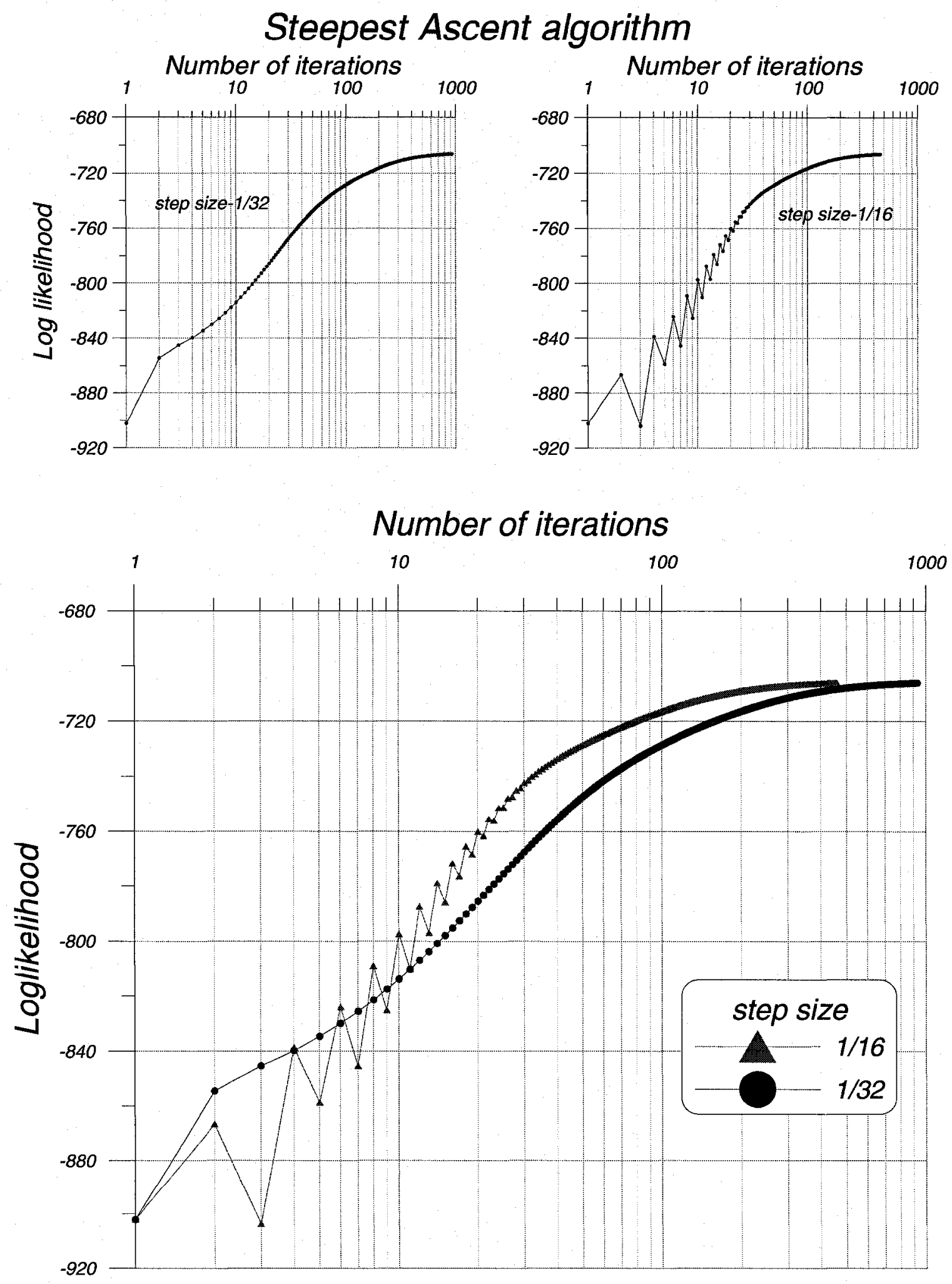

Figure 5-86: Log-likelihood values for each step size and all step sizes in Steepest Ascent algorithm for the first set of guessing of starting points (exp. 5) 


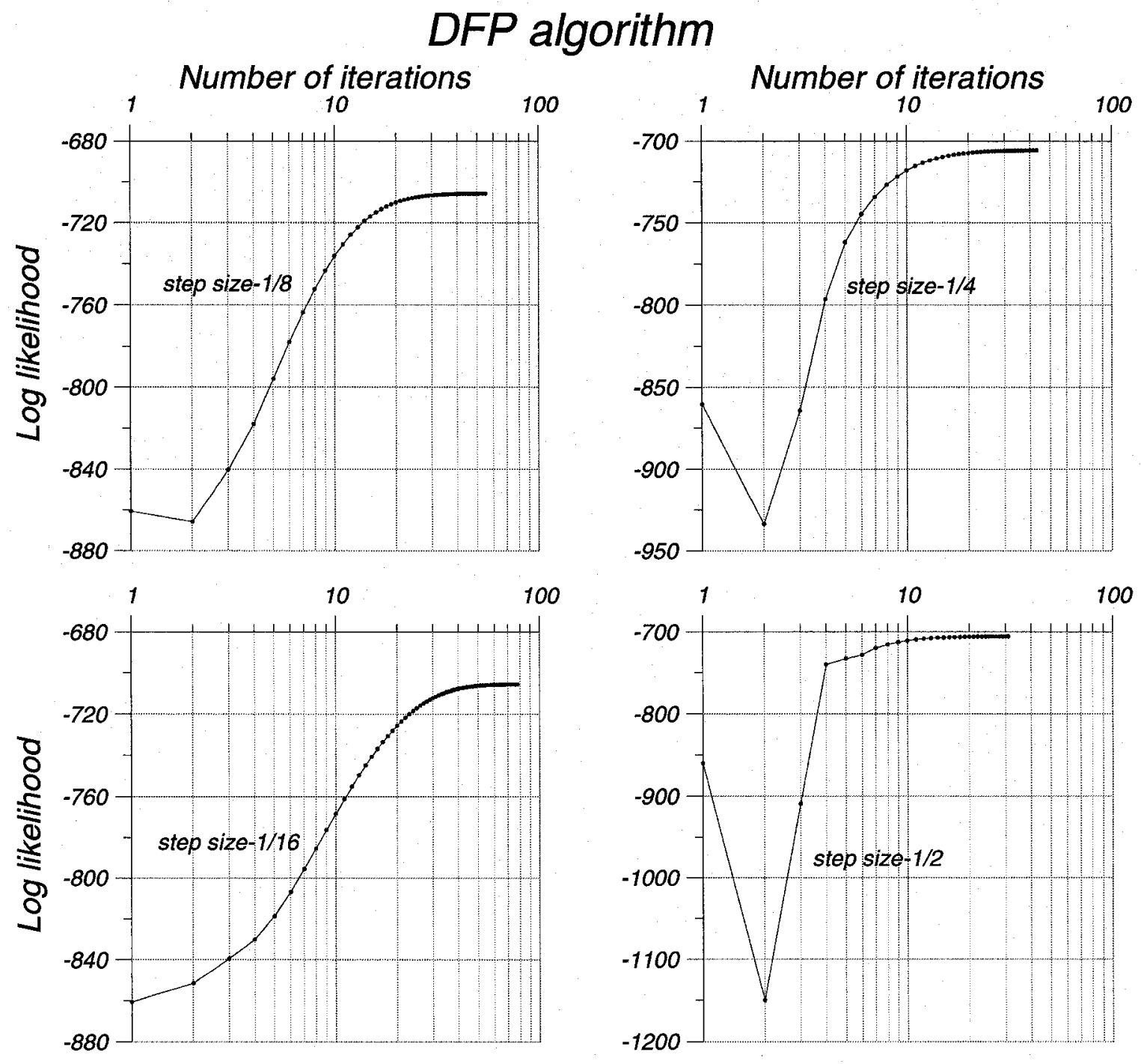

Figure 5-87: Log-likelihood values for each step size in DFP algorithm for the second set of guessing of starting points (exp. 5) 


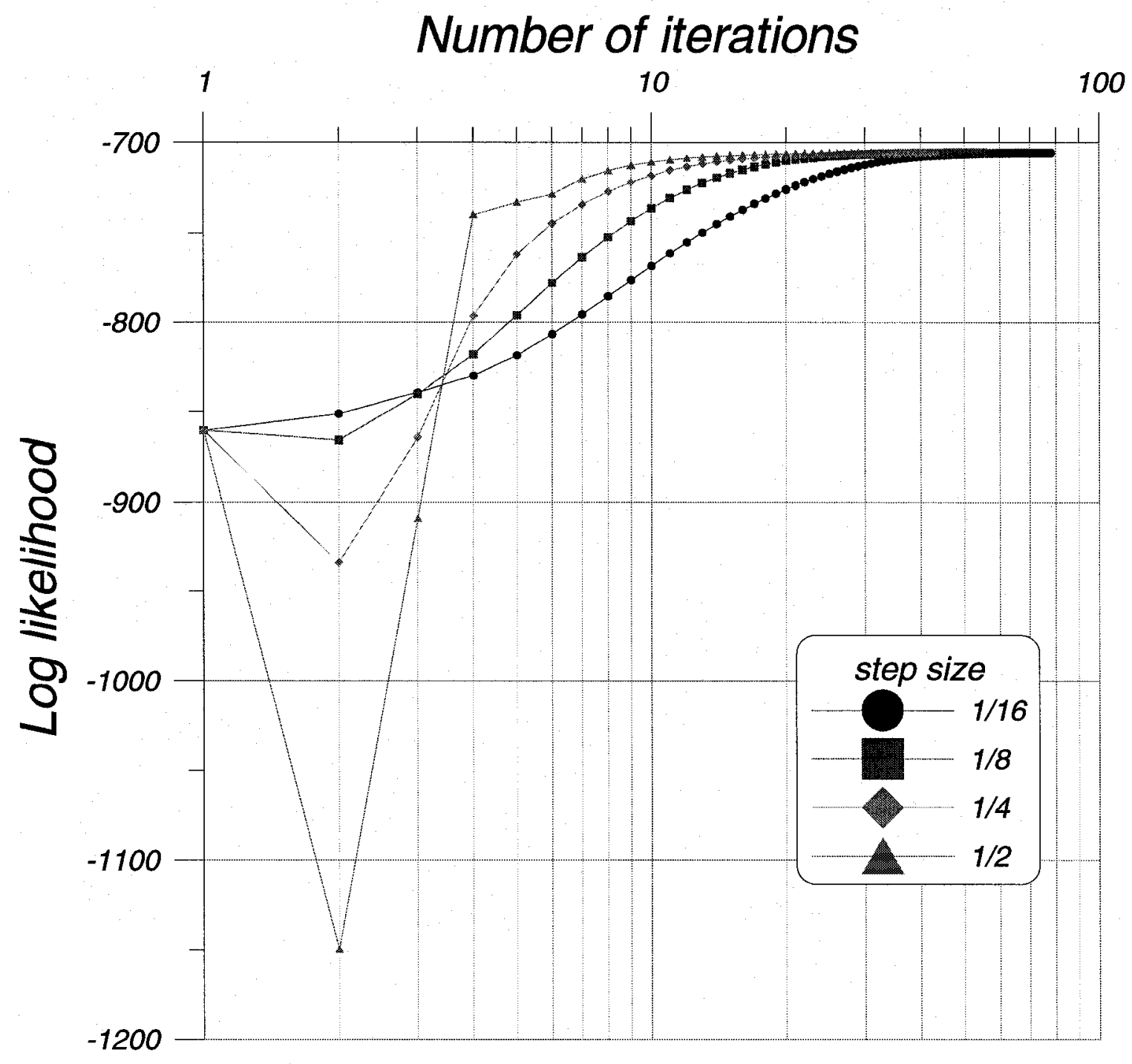

Figure 5-88: Log-likelihood for the all step sizes in DFP algorithm for the second set of guessing of starting points (exp. 5) 


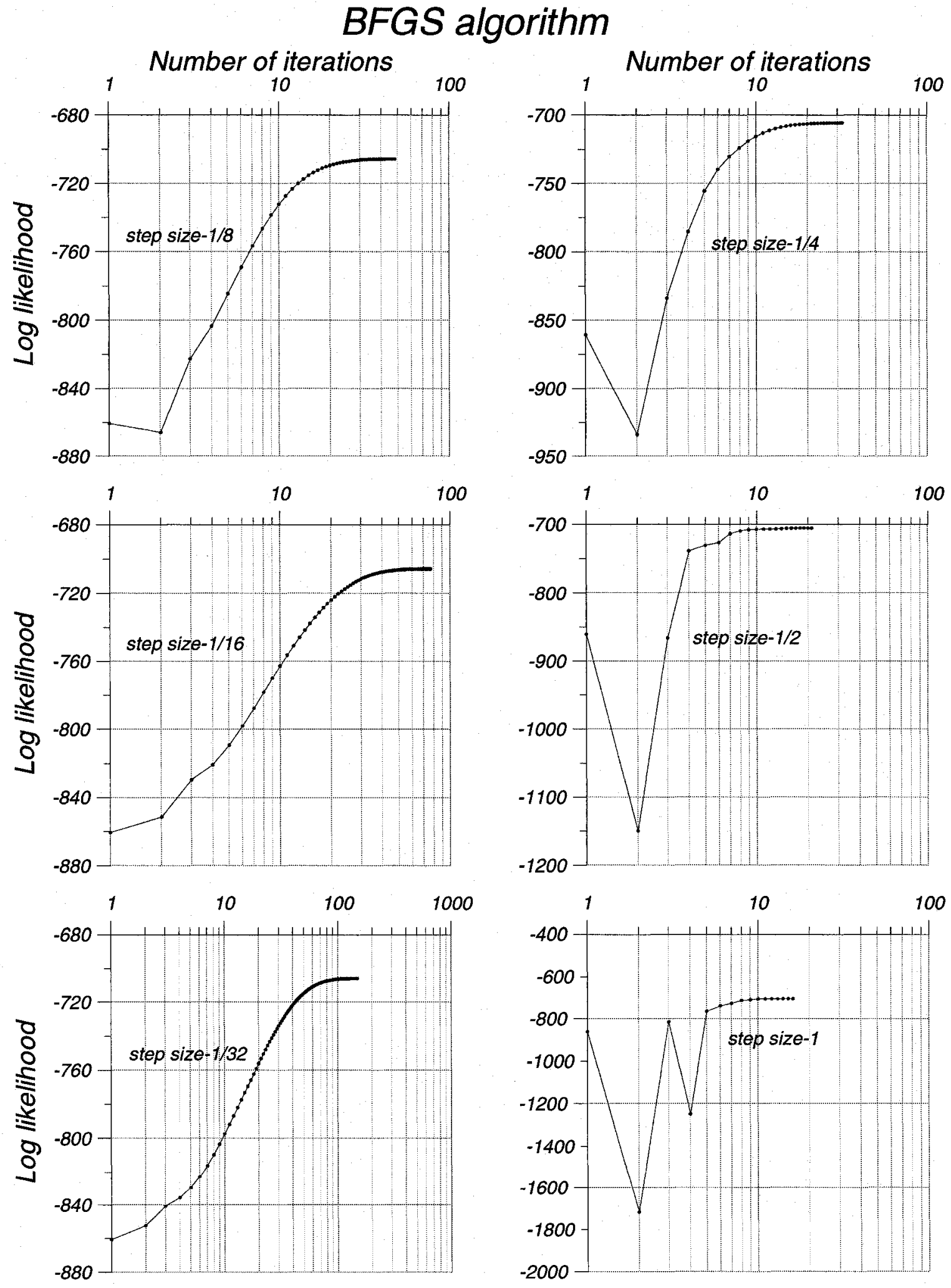

Figure 5-89: Log-likelihood values for each step size in BFGS algorithm for the second set of guessing of starting points (exp. 5) 


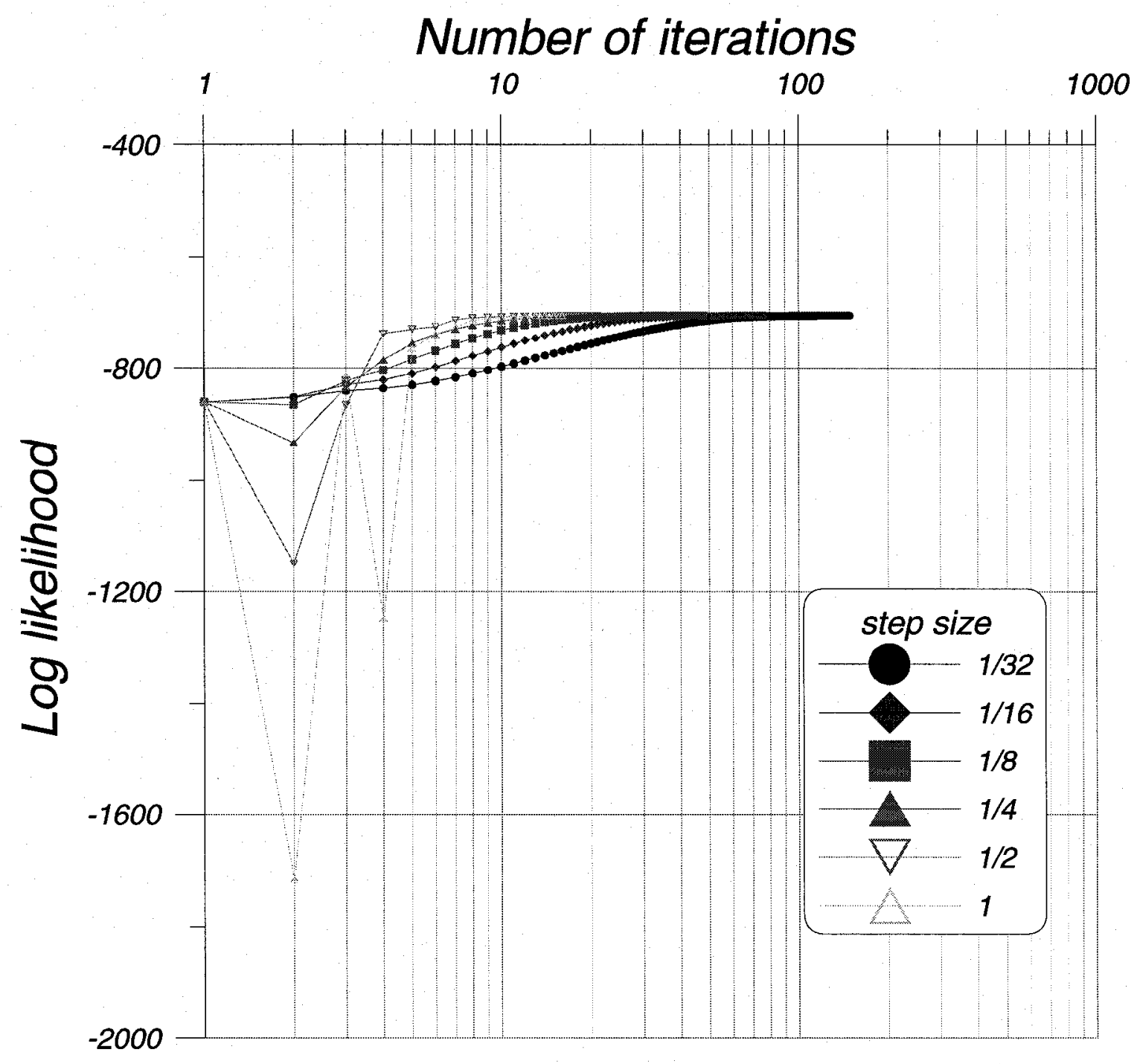

Figure 5-90: Log-likelihood for the all step sizes in BFGS algorithm for the second set of guessing of starting points (exp. 5) 


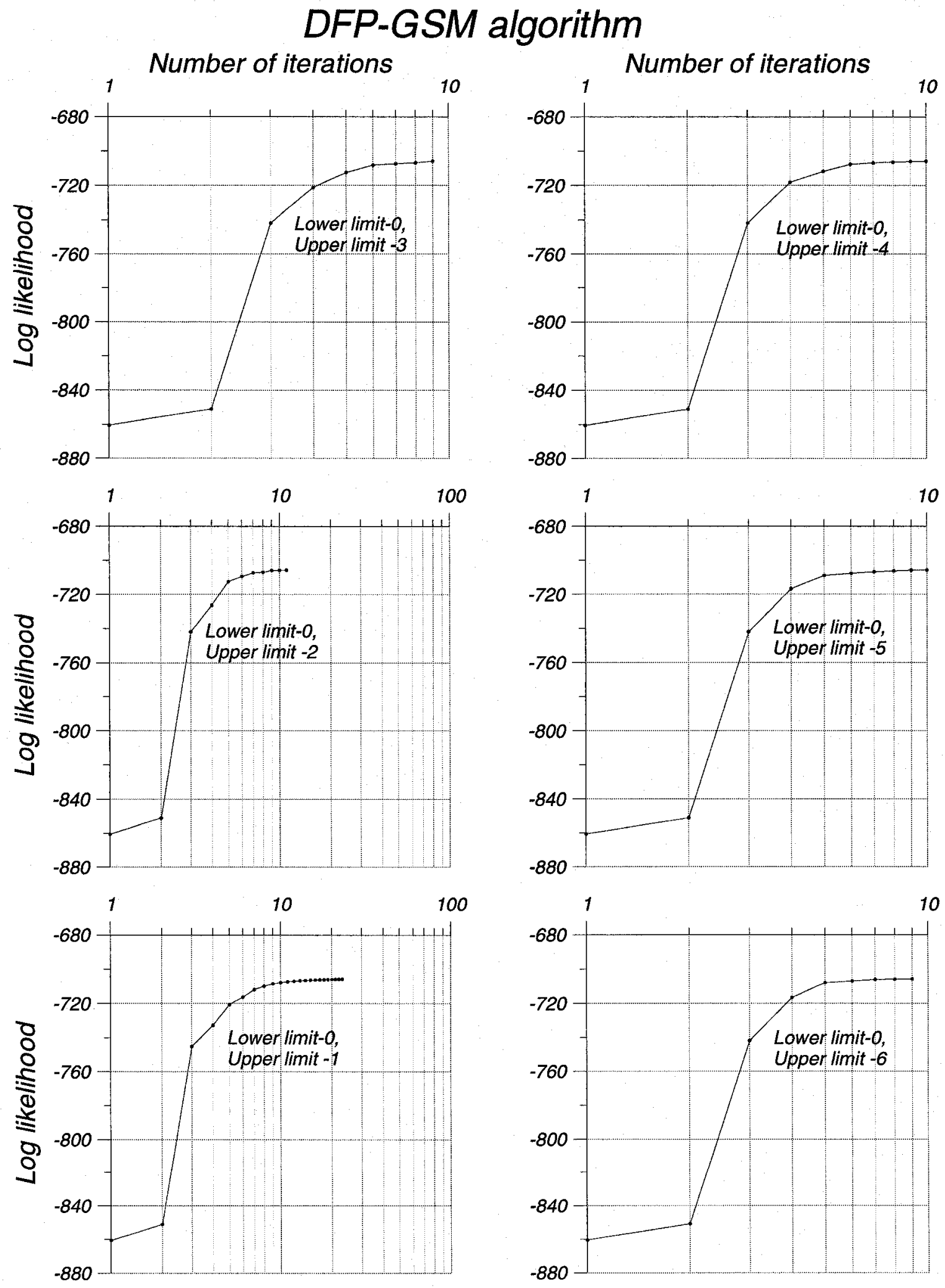

Figure 5-91: Log-likelihood values for the second set of guessing of starting points in DFP-GSM algorithm for the first six uncertain intervals (exp. 5) 


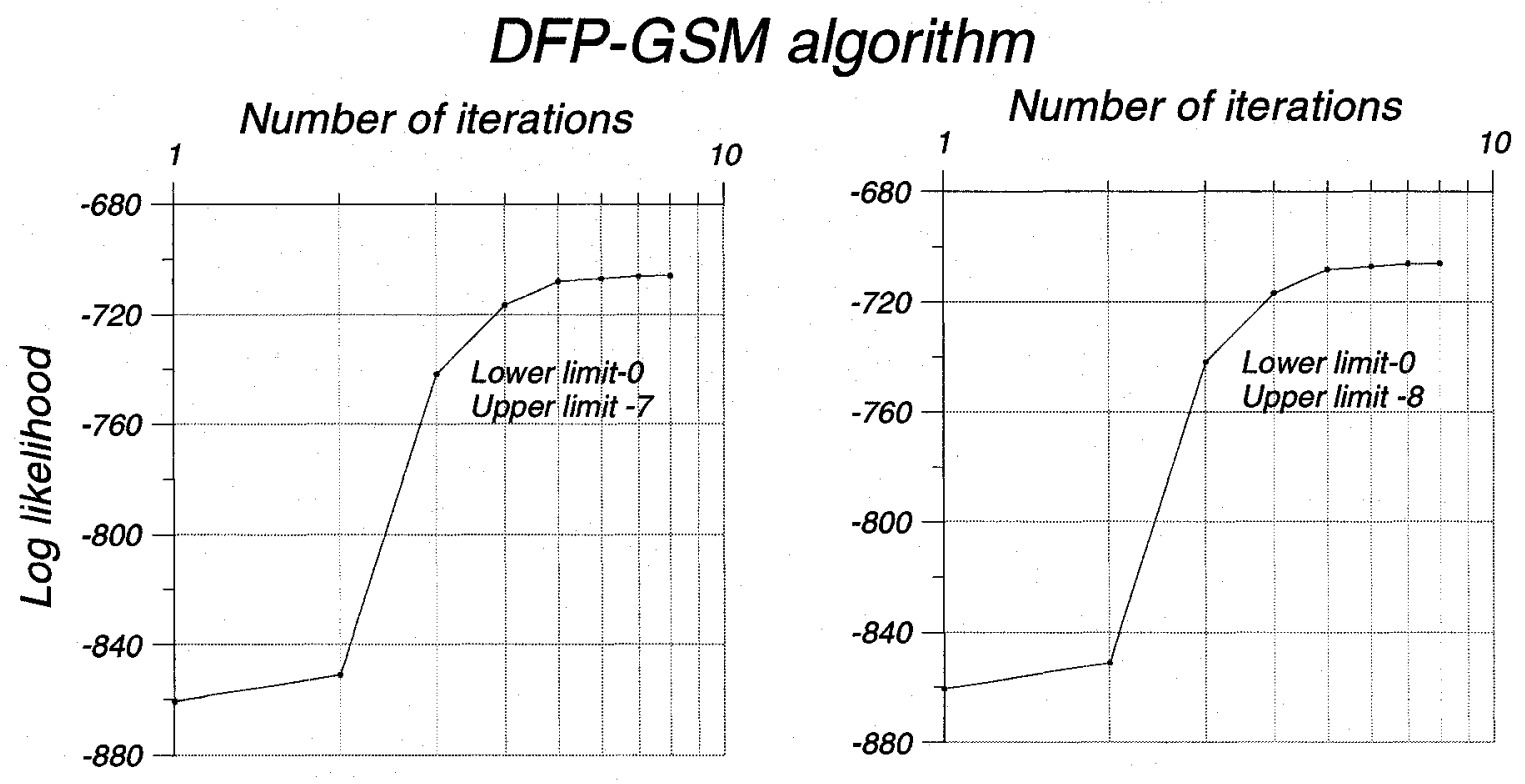

Figure 5-92: Log-likelihood values for the second set of guessing of starting points in DFP-GSM algorithm for the last two uncertain intervals (exp. 5) 


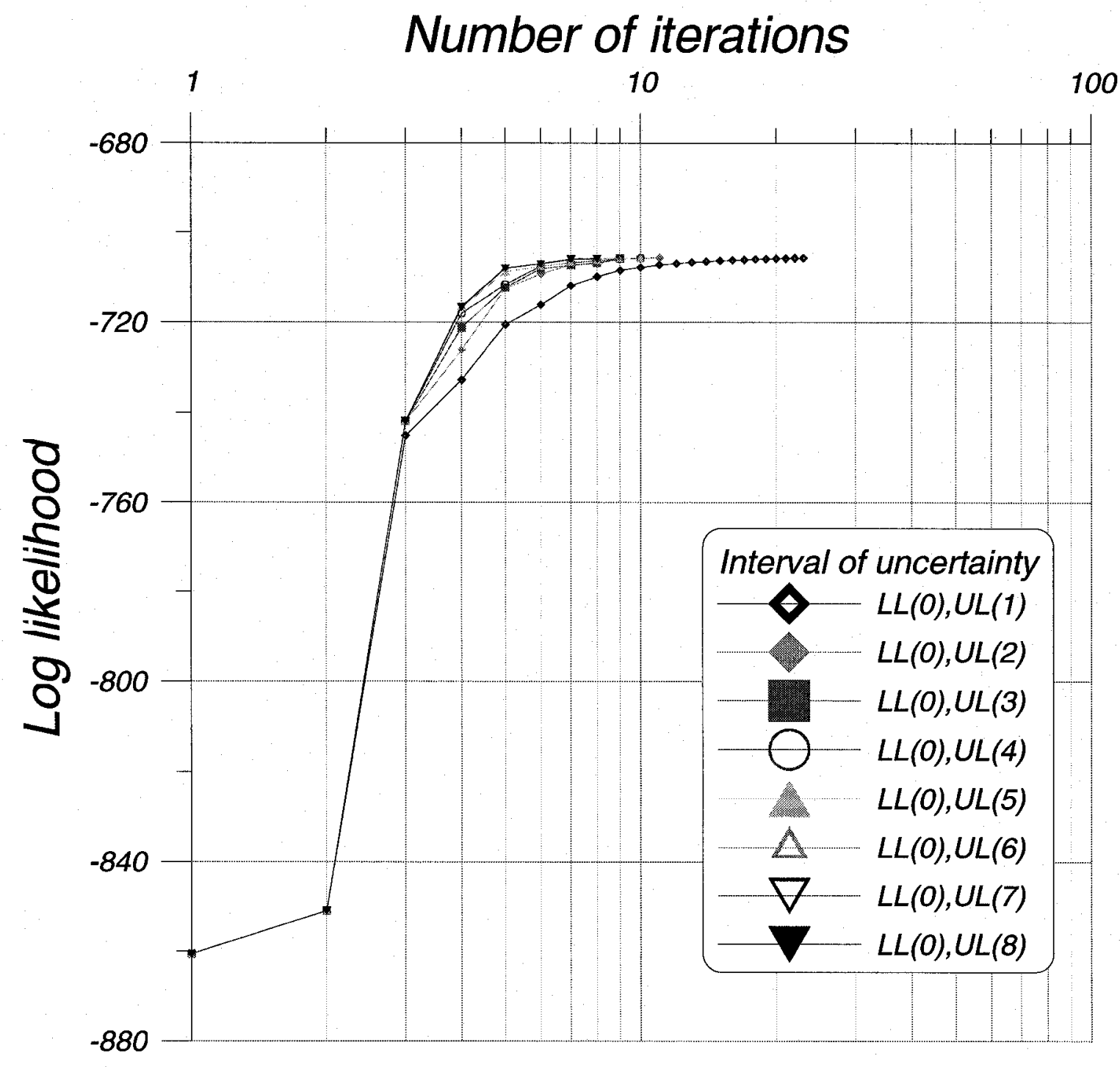

Figure 5-93: Log-likelihood for the all uncertain intervals in DFP-GSM algorithm for the second set of guessing of starting points (exp. 5) 


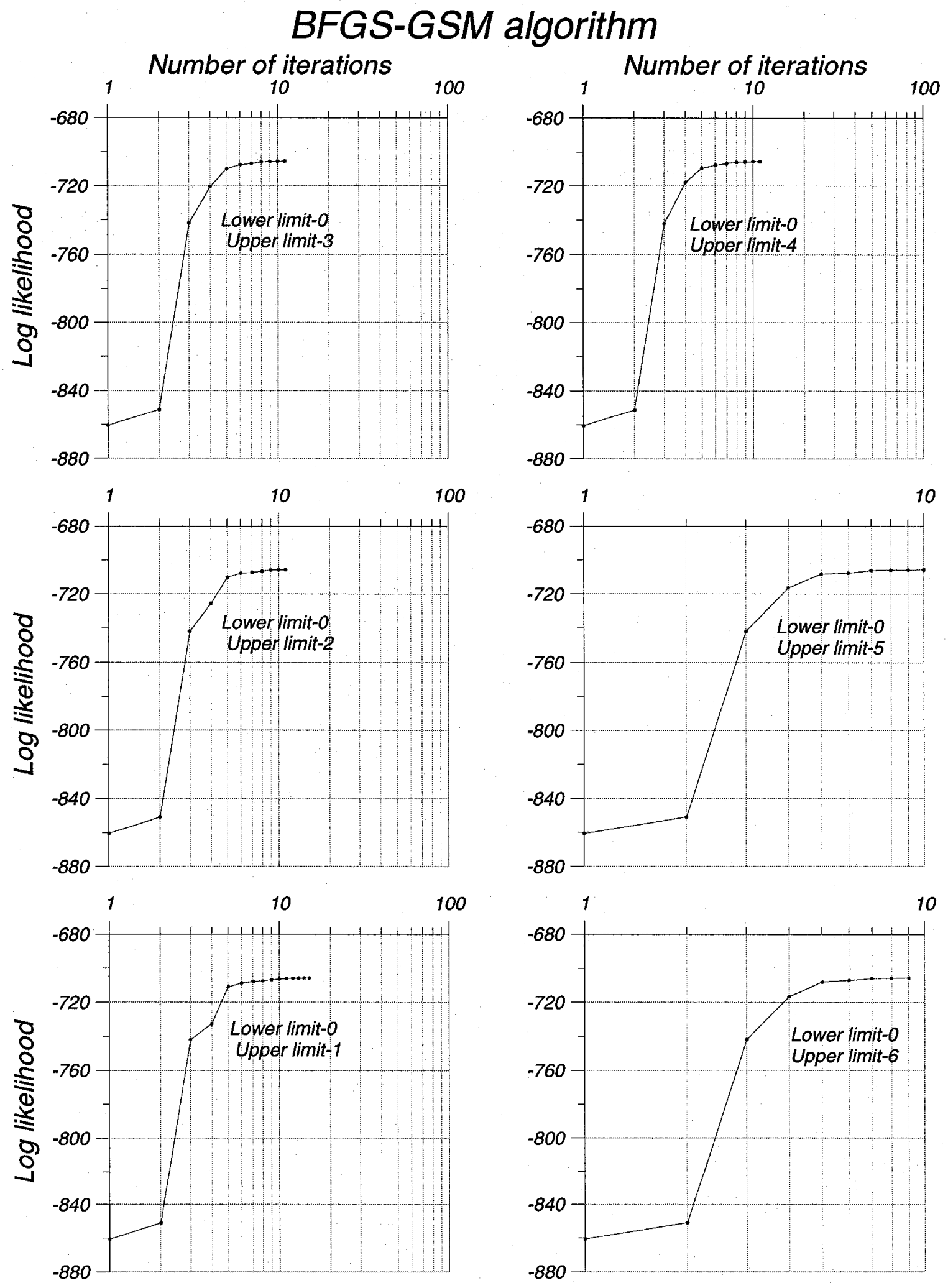

Figure 5-94: Log-likelihood values for the second set of guessing of starting points in BFGS-GSM algorithm for the first six uncertain intervals (exp. 5) 

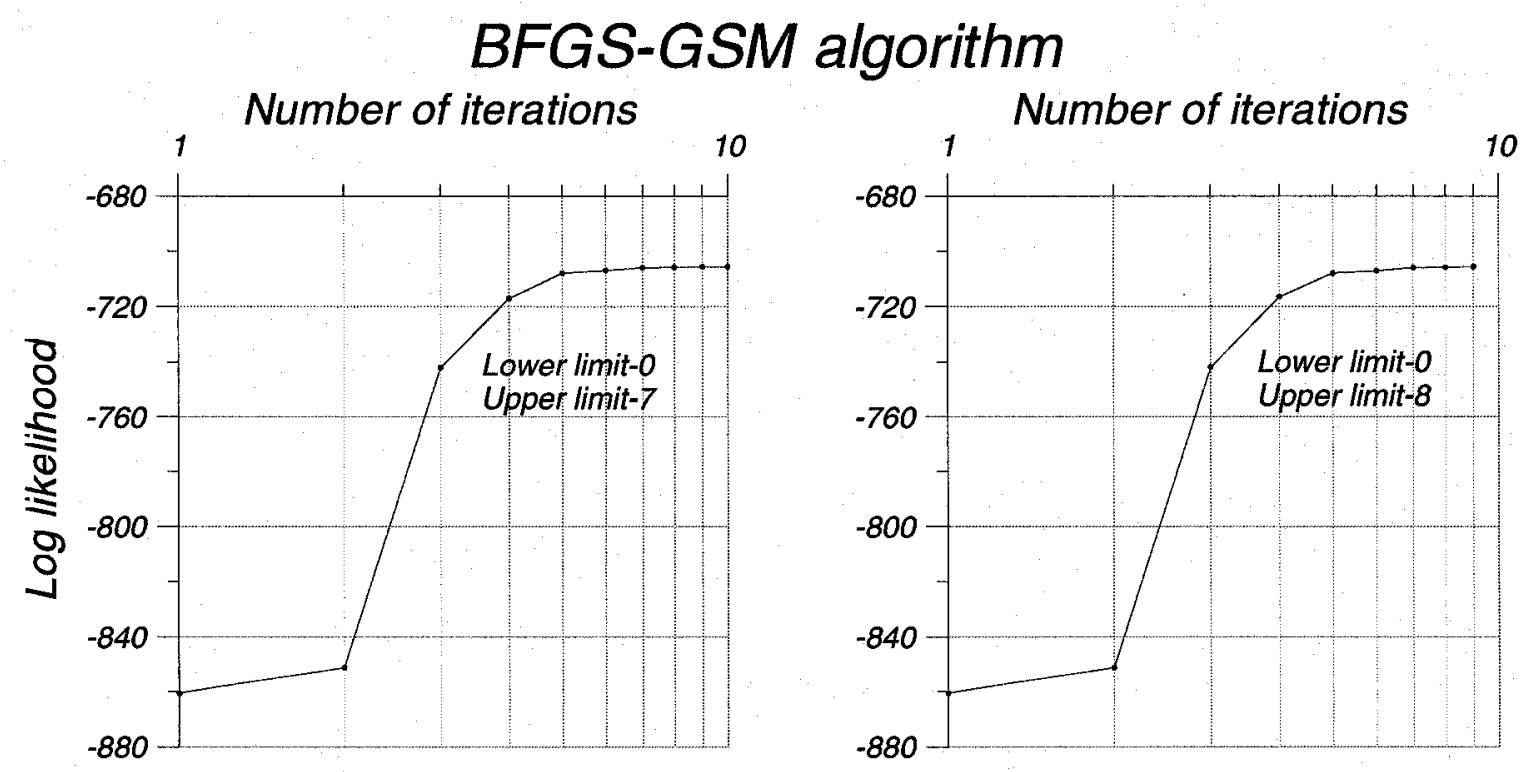

Figure 5-95: Log-likelihood values for the second set of guessing of starting points in BFGS-GSM algorithm for the last two uncertain intervals (exp. 5) 


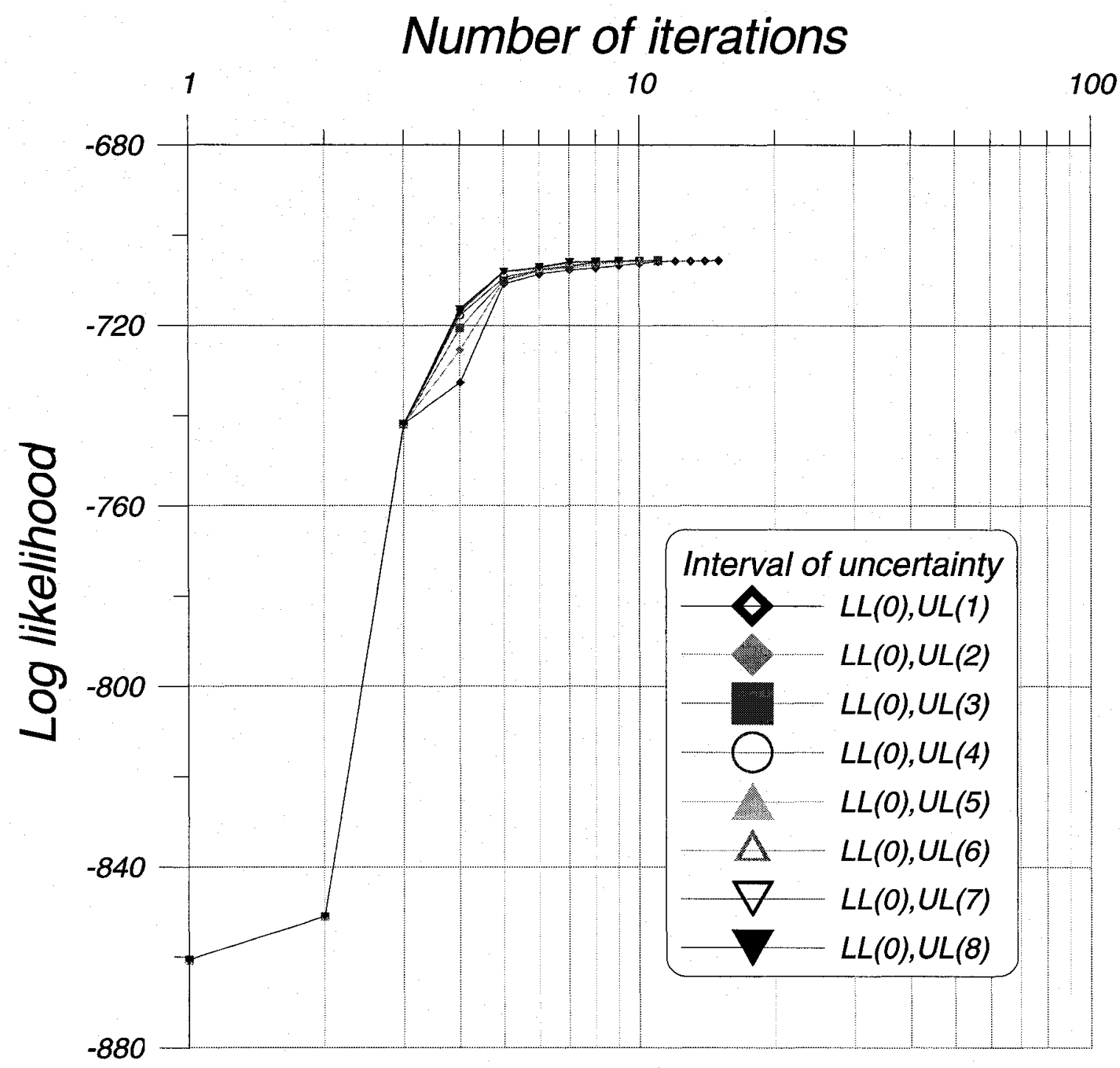

Figure 5-96: Log-likelihood for the all uncertain intervals in BFGS-GSM algorithm for the second set of guessing of starting points (exp. 5) 


\subsection{A comparison of the variations according to the initialized Hessian (experiment 6)}

In this section we consider another way of guessing the initial Hessian matrix, which is assumed to be an identity matrix before starting estimation and continuously updated as the estimation processes in the DFP, BFGS and DFP-GSM, BFGS-GSM algorithms. Another way that is tried in this section is to incorporate the method with which the initial Hessian matrix is calculated in both BHHH and BHHH-2 as a proposed way for obtaining an initial Hessian matrix instead of using an identity matrix as an initial Hessian matrix. By adding codes associated with the calculation of the initial Hessian matrix in BHHH and BHHH-2 algorithms to general estimation procedures of the above four algorithms, new estimation codes are described in this section. They are named as DFP (BHHH), BFGS (BHHH-2) and DFP-GSM (BHHH), BFGS-GSM (BHHH-2). It is important to note that the only difference between two sets of algorithms (e.g., DFP and DFP (BHHH)) is the way of obtaining the initial Hessian matrix. The first algorithm (DFP) uses an identity matrix as an its initial Hessian matrix, the second algorithm (DFP $(\mathrm{BHHH}))$ uses an advanced way presented in this section to get an initial Hessian matrix. Especially, the experimental estimations which use an advanced way of guessing initial Hessian matrix are confined to the last four algorithms out of the eight estimation algorithms used in this thesis research because the first four algorithms do not use an identity matrix as an initial Hessian matrix.

The applied convergence criterion is $G_{t}^{\prime}\left(-H_{t}\right)^{-1} G_{t}<10^{-4}$, which is the same stopping criterion used in experiment 2 and the starting points of parameters is $\beta_{k}=\left[\beta_{1}=0, \beta_{2}=0, \beta_{3}=0, \cdots, \beta_{9}=0\right]^{\prime}$, which is the same condition of the initial 
starting points applied in experiments 1-4. The estimation results obtained from this experimental runs are shown in tables 5-48 to 5-56. The detailed results are compared graphically in figures 5-97 to 5-100 in terms of three performance measures, depicted separately according to the different way of guessing the initial Hessian matrix. An important lessen being learned through a given series of experimental estimations is to know that applying a different way of guessing initial Hessian matrix can make a difference in estimation performance in terms of performance measures, which can be directed to better or worse performance. In the context of this thesis research, the experimental estimations tried in this section generally give better results than that of experiment 2 for the given performance measures. It means that a better way of guessing the initial Hessian matrix, unlike the general procedures of using an identity matrix as an initial Hessian matrix, would improve the estimation performances in the targeted performance measures. This issue can be another topic having potential to generate fruitful research results in the field of econometric modelling.

The following explanations of the results are compared to the results of the experiment 2 . The results obtained from experimental estimation are as follows: (1) In term of a single iteration time, there is no big difference between the runs using a general routine shown in figure 5-20 and 5-21 and the runs using a new routine proposed in the current section shown in figures 5-97 to 5-100. However, when using a new proposed routine, the single iteration time becomes similar to other runs irrespective of using both different step sizes (in the case of DFP (BHHH), BFGS (BHHH) and DFP (BHHH-2), BFGS (BHHH-2)) and different uncertain interval (in the case of DFP-GSM (BHHH), BFGS-GSM (BHHH) 
and DFP-GSM (BHHH-2), BFGS-GSM (BHHH-2)). (2) In term of the number of iterations, the routine proposed in this section shows a better performance, in terms of a decrease in the number of iterations. (3) In term of the convergence time, the big difference is observed between experiment 2 and experiment 6 . The experiment 6 takes much less convergence time than experiment 2 . The reason is that using a more accurate initial Hessian matrix, which can be calculated and input into the routine of estimation, instead of using an identity matrix would decrease the iteration time needed to reach the Hessian matrix of that when the estimation is converged.

Other factors related to the estimation performance and their performance behaviours can be observed and compared to the same factors of other experiments by using available graphs and tables. 
Table 5-48: The experimental results of DFP, BFGS and DFP-GSM, BFGS-GSM algorithms responding to a new way of calculating the initial Hessian matrix adopted from either BHHH or BHHH-2 algorithm (exp. 6)

\begin{tabular}{|c|c|c|c|c|c|c|c|c|c|}
\hline \multirow{2}{*}{\multicolumn{2}{|c|}{$\begin{array}{c}\text { Algorithms \& } \\
\text { Performance measures }\end{array}$}} & \multicolumn{8}{|c|}{ Step size } \\
\hline & & $1 / 32$ & $1 / 16$ & $1 / 8$ & $1 / 4$ & $1 / 2$ & 1 & 2 & 4 \\
\hline \multirow{3}{*}{ 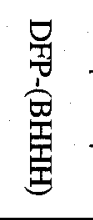 } & $\begin{array}{c}\text { One iteration } \\
\text { time }\end{array}$ & - & $0: 00: 18$ & $0: 00: 18$ & $0: 00: 24$ & $0: 00: 20$ & - & - & - \\
\hline & $\begin{array}{l}\text { Number of } \\
\text { iterations }\end{array}$ & - & 74 & 37 & 19 & 11 & - & - & - \\
\hline & $\begin{array}{c}\text { Convergence } \\
\text { time }\end{array}$ & - & $0: 22: 27$ & 0:11:05 & $0: 07: 20$ & $0: 03: 31$ & - & - & - \\
\hline \multirow{3}{*}{ 罣 } & $\begin{array}{l}\text { One iteration } \\
\text { time }\end{array}$ & - & $0: 00: 20$ & $0: 00: 20$ & $0: 00: 20$ & 0:00:19 & - & - & - \\
\hline & $\begin{array}{c}\text { Number of } \\
\text { iterations }\end{array}$ & - & 74 & 37 & 19 & 10 & - & - & - \\
\hline & $\begin{array}{l}\text { Convergence } \\
\text { time }\end{array}$ & - & $0: 24: 10$ & $0: 12: 07$ & $0: 06: 13$ & 0:03:00 & - & - & - \\
\hline \multirow{3}{*}{ 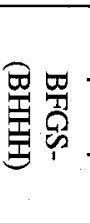 } & $\begin{array}{l}\text { One iteration } \\
\text { time }\end{array}$ & $0: 00: 18$ & $0: 00: 17$ & $0: 00: 18$ & $0: 00: 18$ & $0: 00: 20$ & $0: 00: 17$ & - & - \\
\hline & $\begin{array}{c}\text { Number of } \\
\text { iterations }\end{array}$ & 147 & 74 & 37 & 19 & 10 & 6 & - & - \\
\hline & $\begin{array}{c}\text { Convergence } \\
\text { time }\end{array}$ & $0: 42: 48$ & $0: 21: 01$ & $0: 10: 44$ & $0: 05: 33$ & $0: 03: 07$ & $0: 01: 38$ & - & - \\
\hline \multirow{3}{*}{ 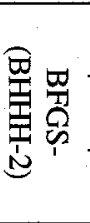 } & $\begin{array}{c}\text { One iteration } \\
\text { time }\end{array}$ & $0: 00: 18$ & $0: 00: 18$ & $0: 00: 18$ & $0: 00: 18$ & $0: 00: 19$ & $0: 00: 15$ & - & - \\
\hline & $\begin{array}{c}\text { Number of } \\
\text { iterations }\end{array}$ & 147 & 73 & 37 & 19 & 10 & 6 & - & - \\
\hline & $\begin{array}{c}\text { Convergence } \\
\text { time }\end{array}$ & $0: 44: 29$ & $0: 22: 12$ & $0: 11: 11$ & $0: 05: 42$ & $0: 03: 03$ & $0: 01: 40$ & - & - \\
\hline \multicolumn{2}{|c|}{ Interval of uncertainty } & $\begin{array}{l}\mathrm{LL}(0), \\
\mathrm{UL}(1)\end{array}$ & $\begin{array}{l}\text { LL(0), } \\
\text { UL(2) }\end{array}$ & $\begin{array}{l}\text { LL(0), } \\
\text { UL(3) }\end{array}$ & $\begin{array}{l}\text { LL(0), } \\
\text { UL(4) }\end{array}$ & $\begin{array}{l}\text { LL(0), } \\
\text { UL(5) }\end{array}$ & $\begin{array}{l}\text { LL(0), } \\
\text { UL(6) }\end{array}$ & $\begin{array}{l}\text { LL(0), } \\
\text { UL(7) }\end{array}$ & $\begin{array}{l}\text { LL(0), } \\
\text { UL(8) }\end{array}$ \\
\hline \multirow{3}{*}{ 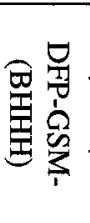 } & $\begin{array}{l}\text { One iteration } \\
\text { time }\end{array}$ & $0: 02: 40$ & $0: 01: 34$ & $0: 01: 36$ & $0: 01: 41$ & $0: 01: 41$ & $0: 01: 47$ & $0: 01: 47$ & $0: 01: 47$ \\
\hline & $\begin{array}{c}\text { Number of } \\
\text { iterations }\end{array}$ & 7 & 6 & 6 & 6 & 6 & 6 & 6 & 6 \\
\hline & $\begin{array}{c}\text { Convergence } \\
\text { time }\end{array}$ & $0: 18: 32$ & $0: 09: 13$ & $0: 09: 26$ & $0: 09: 57$ & $0: 09: 57$ & $0: 10: 32$ & $0: 10: 33$ & $0: 10: 34$ \\
\hline \multirow{3}{*}{ 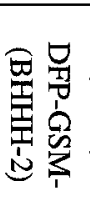 } & $\begin{array}{l}\text { One iteration } \\
\text { time }\end{array}$ & $0: 01: 21$ & $0: 01: 36$ & $0: 01: 36$ & $0: 01: 42$ & $0: 01: 42$ & $0: 01: 47$ & $0: 01: 49$ & $0: 01: 46$ \\
\hline & $\begin{array}{c}\text { Number of } \\
\text { iterations }\end{array}$ & 7 & 6 & 6 & 6 & 6 & 6 & 6 & 6 \\
\hline & $\begin{array}{c}\text { Convergence } \\
\text { time }\end{array}$ & $0: 09: 19$ & $0: 09: 25$ & $0: 09: 25$ & $0: 10: 01$ & $0: 10: 05$ & $0: 10: 31$ & $0: 10: 43$ & $0: 10: 28$ \\
\hline \multirow{3}{*}{ 兽 } & $\begin{array}{c}\text { One iteration } \\
\text { time }\end{array}$ & $0: 01: 12$ & $0: 01: 16$ & $0: 01: 16$ & $0: 01: 18$ & $0: 01: 20$ & $0: 01: 24$ & $0: 01: 25$ & $0: 01: 22$ \\
\hline & $\begin{array}{c}\text { Number of } \\
\text { iterations }\end{array}$ & 5 & 6 & 6 & 6 & 6 & 6 & 6 & 6 \\
\hline & $\begin{array}{l}\text { Convergence } \\
\text { time }\end{array}$ & $0: 07: 08$ & $0: 07: 28$ & $0: 07: 26$ & $0: 07: 37$ & $0: 07: 52$ & $0: 08: 17$ & $0: 08: 21$ & $0: 08: 05$ \\
\hline \multirow{3}{*}{ 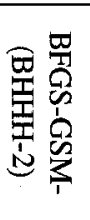 } & $\begin{array}{c}\text { One iteration } \\
\text { time }\end{array}$ & $0: 01: 06$ & $0: 01: 13$ & $0: 01: 15$ & $0: 01: 18$ & $0: 01: 18$ & $0: 01: 22$ & $0: 01: 21$ & $0: 01: 22$ \\
\hline & $\begin{array}{c}\text { Number of } \\
\text { iterations }\end{array}$ & 6 & 6 & 6 & 6 & 6 & 6 & 6 & 6 \\
\hline & $\begin{array}{l}\text { Convergence } \\
\text { time }\end{array}$ & $0: 6: 46$ & $0: 07: 30$ & $0: 07: 38$ & 0:08:00 & $0: 07: 54$ & 0:08:19 & $0: 08: 17$ & $0: 08: 32$ \\
\hline
\end{tabular}



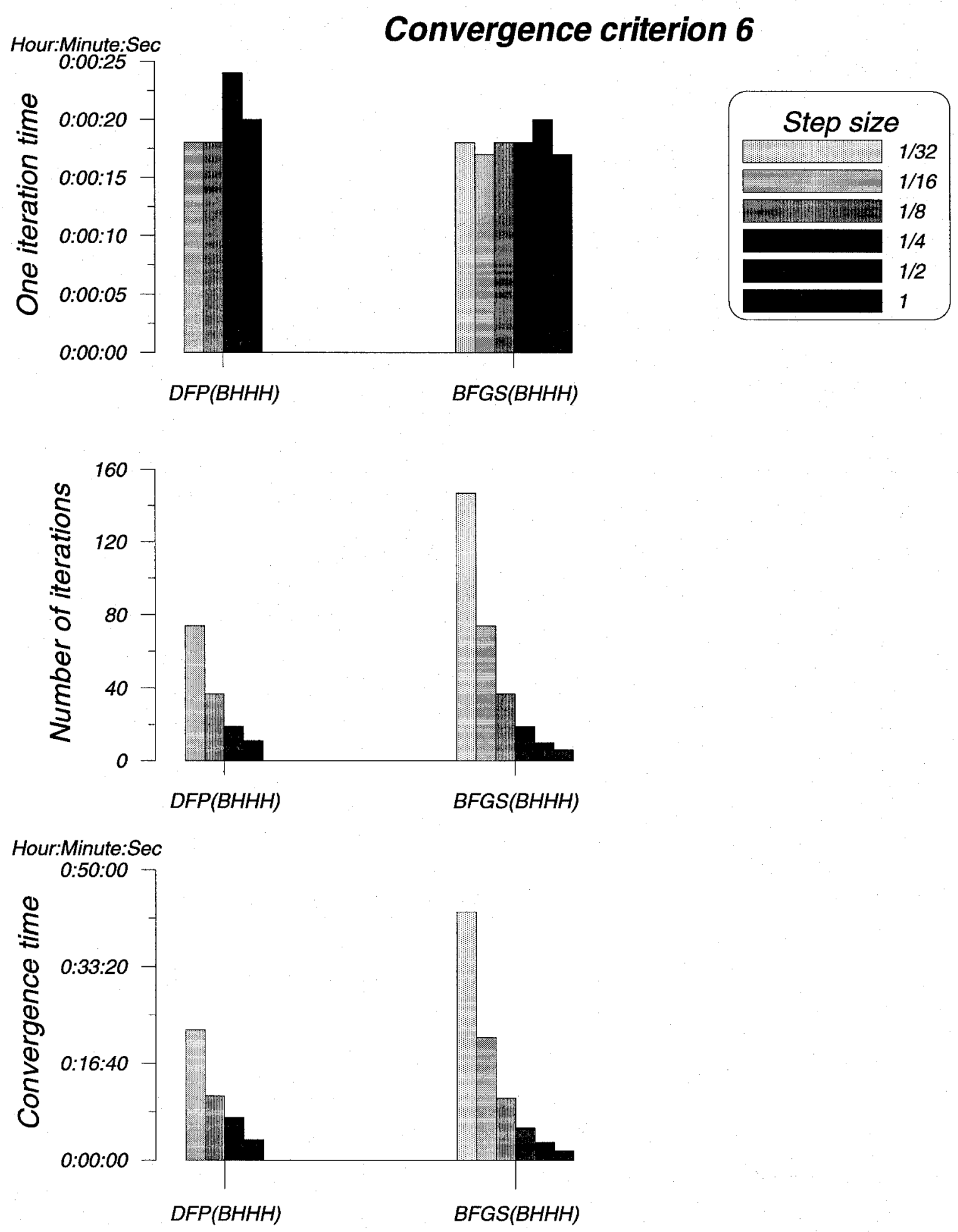

Figure 5-97: Performance results of DFP and BFGS algorithms responding to a new way of calculating the initial Hessian matrix adopted from $\mathrm{BHHH}$ algorithm (exp. 6) 

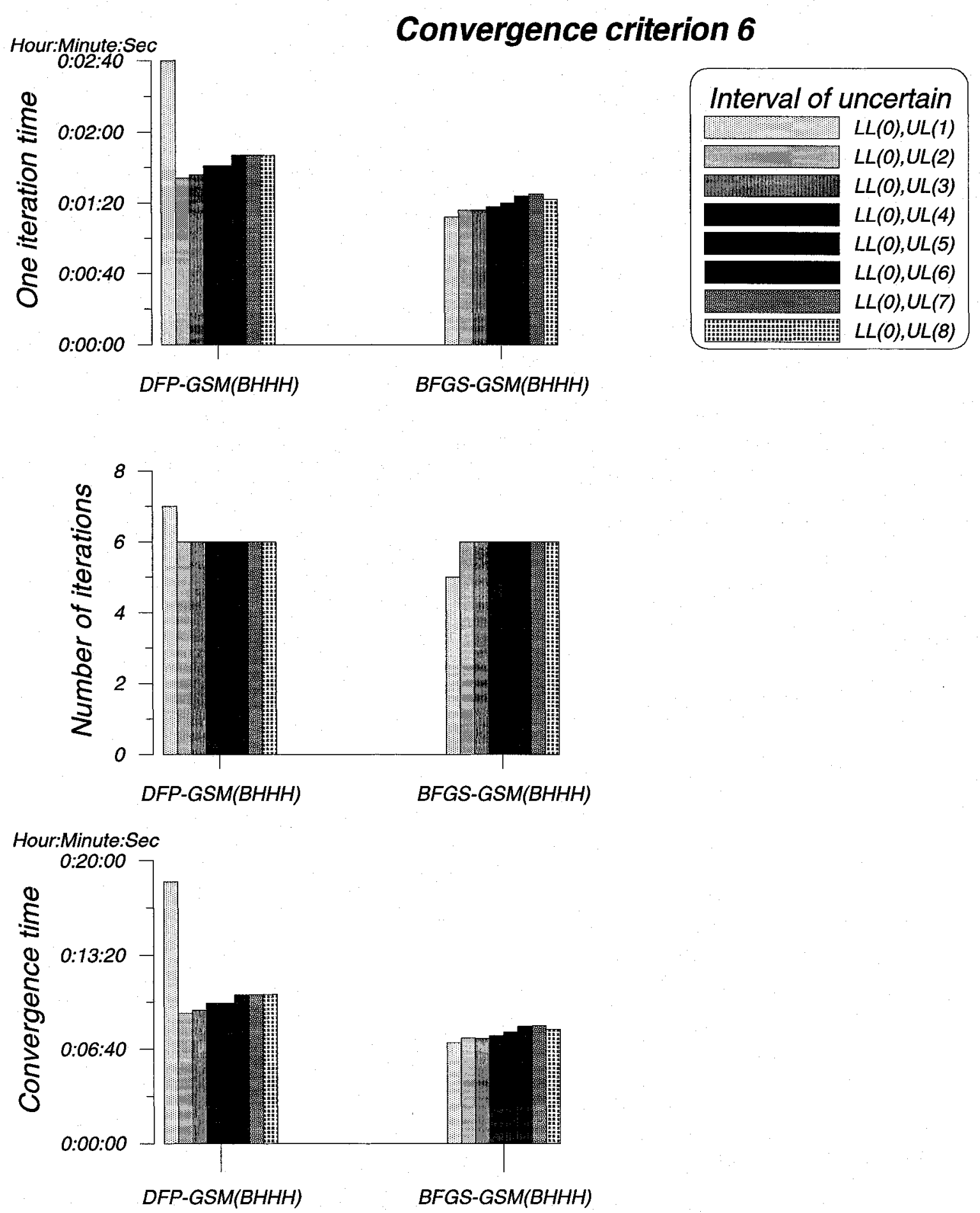

Figure 5-98: Performance results of DFP-GSM and BFGS-GSM algorithms responding to a new way of calculating the initial Hessian matrix adopted from $\mathrm{BHHH}$ algorithm (exp. 6) 

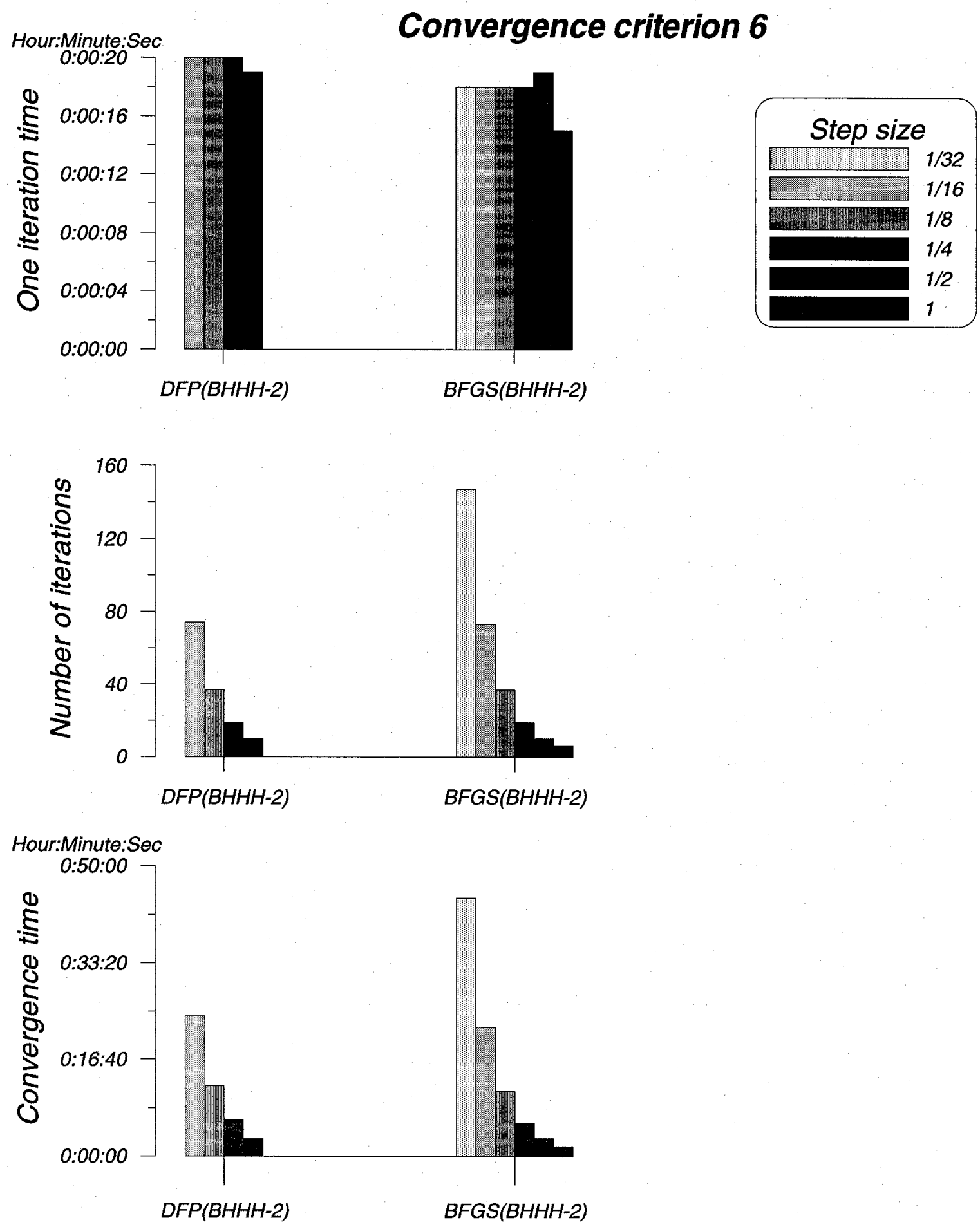

Figure 5-99: Performance results of DFP and BFGS algorithms responding to a new way of calculating the initial Hessian matrix adopted from BHHH-2 algorithm (exp. 6) 

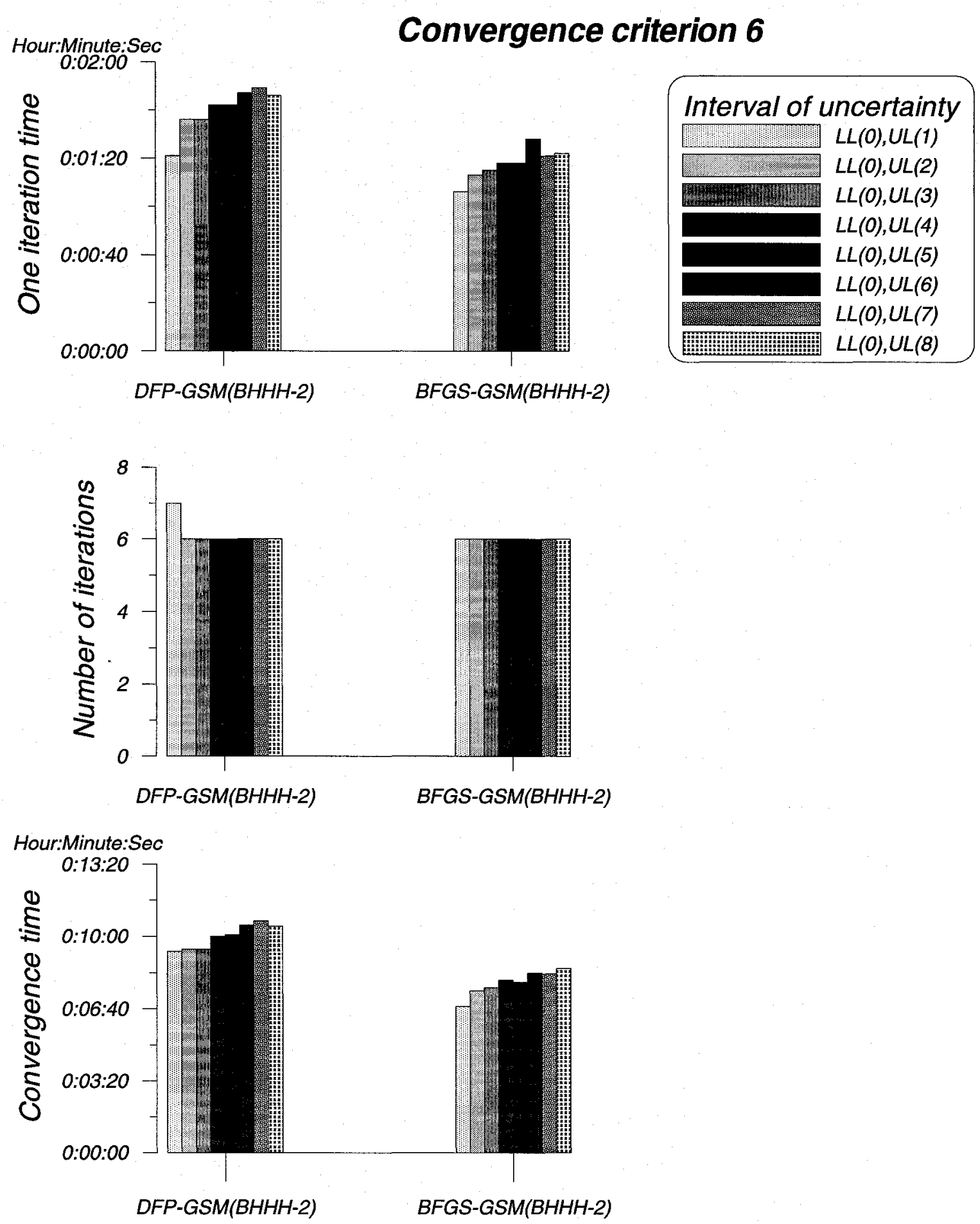

Figure 5-100: Performance results of DFP-GSM and BFGS-GSM algorithms responding to a new way of calculating the initial Hessian matrix adopted from BHHH-2 algorithm (exp. 6) 
Table 5-49: Log-likelihood values obtained from DFP algorithm responding to a new way of calculating the initial Hessian matrix adopted from BHHH algorithm (exp. 6)

\begin{tabular}{cccccc}
\hline & Log-likelihood & \multicolumn{3}{c}{ Step size } \\
\cline { 2 - 5 } & $\begin{array}{c}\text { Starting } \\
\text { Log-likelihood }\end{array}$ & -869.0964727 & -869.0964727 & -869.0964727 & -869.0964727 \\
\hline \multirow{2}{*}{$\begin{array}{c}\text { Convergence } \\
\text { Log-likelihood }\end{array}$} & -705.5637886 & -705.5640275 & -705.5628106 & -705.563426 \\
\cline { 2 - 5 } & Number of iterations & 74 & 37 & $1 / 4$ & 11 \\
\hline
\end{tabular}

Table 5-50: Log-likelihood values obtained from DFP algorithm responding to a new way of calculating the initial Hessian matrix adopted from BHHH-2 algorithm (exp. 6)

\begin{tabular}{cccccc}
\hline & Log-likelihood & \multicolumn{4}{c}{ Step size } \\
\cline { 2 - 5 } & $\begin{array}{c}\text { Starting } \\
\text { Log-likelihood }\end{array}$ & -869.0964727 & -869.0964727 & -869.0964727 & -869.0964727 \\
\hline \multirow{2}{*}{$\begin{array}{c}\text { Convergence } \\
\text { Log-likelihood }\end{array}$} & -705.5624724 & -705.5613663 & -705.5582318 & -705.5777822 \\
\cline { 2 - 6 } & Number of iterations & 74 & 37 & $1 / 2$ & 10 \\
\hline
\end{tabular}

Table 5-51: Log-likelihood values obtained from BFGS algorithm responding to a new way of calculating the initial Hessian matrix adopted from $\mathrm{BHHH}$ algorithm (exp. 6)

\begin{tabular}{|c|c|c|c|c|c|c|c|}
\hline \multirow{2}{*}{\multicolumn{2}{|c|}{ Log-likelihood }} & \multicolumn{6}{|c|}{ Step size } \\
\hline & & $1 / 32$ & $1 / 16$ & $1 / 8$ & $1 / 4$ & $1 / 2$ & 1 \\
\hline \multirow{4}{*}{ 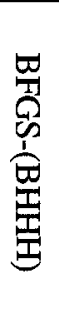 } & Starting & - & - & - & - & - & - \\
\hline & $\begin{array}{c}\text { Log- } \\
\text { likelihood }\end{array}$ & 869.0964727 & 869.0964727 & 869.0964727 & 869.0964727 & 869.0964727 & 869.0964727 \\
\hline & $\begin{array}{c}\text { Convergence } \\
\text { log- } \\
\text { likelihood }\end{array}$ & $\begin{array}{c}- \\
705.5653328\end{array}$ & 705.5632175 & $\begin{array}{c}- \\
705.5626989\end{array}$ & $\begin{array}{c}- \\
705.5594868\end{array}$ & $\begin{array}{c}- \\
705.5738092\end{array}$ & $\begin{array}{c}- \\
705.6029697\end{array}$ \\
\hline & $\begin{array}{l}\text { Number of } \\
\text { iterations }\end{array}$ & 147 & 74 & 37 & 19 & 10 & 6 \\
\hline
\end{tabular}

Table 5-52: Log-likelihood values obtained from BFGS algorithm responding to a new way of calculating the initial Hessian matrix adopted from BHHH-2 algorithm (exp. 6)

\begin{tabular}{|c|c|c|c|c|c|c|c|}
\hline \multirow{2}{*}{\multicolumn{2}{|c|}{ Log-likelihood }} & \multicolumn{6}{|c|}{ Step size } \\
\hline & & $1 / 32$ & $1 / 16$ & $1 / 8$ & $1 / 4$ & $1 / 2$ & 1 \\
\hline \multirow{3}{*}{ 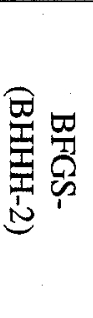 } & $\begin{array}{c}\text { Starting } \\
\text { Log- } \\
\text { likelihood }\end{array}$ & $\begin{array}{c}- \\
869.0964727\end{array}$ & $\begin{array}{c}- \\
869.0964727\end{array}$ & $\begin{array}{c}- \\
869.0964727\end{array}$ & $\frac{-}{869.0964727}$ & $\frac{-}{869.0964727}$ & $\begin{array}{c}- \\
869.0964727\end{array}$ \\
\hline & $\begin{array}{c}\text { Convergence } \\
\text { log- } \\
\text { likelihood }\end{array}$ & $\begin{array}{c}- \\
705.5647226\end{array}$ & $\begin{array}{c}- \\
705.5653491\end{array}$ & $\frac{-}{705.560551}$ & $\begin{array}{c}- \\
705.5562946\end{array}$ & $\begin{array}{c}- \\
705.5684402\end{array}$ & $\begin{array}{c}- \\
705.5903477\end{array}$ \\
\hline & $\begin{array}{l}\text { Number of } \\
\text { iterations }\end{array}$ & 147 & 73 & 37 & 19 & 10 & 6 \\
\hline
\end{tabular}


Table 5-53: Log-likelihood values obtained from DFP-GSM algorithm responding to a new way of calculating the initial Hessian matrix adopted from $\mathrm{BHHH}$ algorithm (exp. 6)

\begin{tabular}{|c|c|c|c|c|c|c|c|c|c|}
\hline & $\begin{array}{l}\text { Interval of } \\
\text { incertainty }\end{array}$ & $\begin{array}{l}\mathrm{LL}(0), \\
\mathrm{UL}(1)\end{array}$ & $\begin{array}{l}\text { LL(0), } \\
\text { UL(2) }\end{array}$ & $\begin{array}{l}\text { LL(0), } \\
\text { UL(3) }\end{array}$ & $\begin{array}{l}\text { LL(0), } \\
\text { UL(4) }\end{array}$ & $\begin{array}{l}\mathrm{LL}(0), \\
\mathrm{UL}(5)\end{array}$ & $\begin{array}{l}\mathrm{LL}(0), \\
\mathrm{UL}(6)\end{array}$ & $\begin{array}{l}\text { LL(0), } \\
\text { UL(7) }\end{array}$ & $\begin{array}{l}\text { LL(0), } \\
\text { UL(8) }\end{array}$ \\
\hline \multirow{3}{*}{ 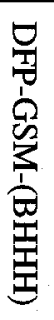 } & $\begin{array}{c}\text { Starting } \\
\text { Log- } \\
\text { likelihood }\end{array}$ & $\begin{array}{c}- \\
869.096 \\
4727\end{array}$ & $\begin{array}{c}- \\
869.096 \\
4727\end{array}$ & $\begin{array}{c}- \\
869.096 \\
4727\end{array}$ & $\begin{array}{c}- \\
869.096 \\
4727\end{array}$ & $\begin{array}{c}- \\
869.096 \\
4727\end{array}$ & $\begin{array}{c}- \\
869.096 \\
4727\end{array}$ & $\begin{array}{c}- \\
869.096 \\
4727\end{array}$ & $\begin{array}{c}- \\
869.096 \\
4727\end{array}$ \\
\hline & $\begin{array}{c}\text { Convergence } \\
\text { log- } \\
\text { likelihood }\end{array}$ & $\begin{array}{c}- \\
705.578 \\
0259\end{array}$ & $\begin{array}{c}- \\
705.580 \\
0667\end{array}$ & $\begin{array}{c}- \\
705.579 \\
3165\end{array}$ & $\begin{array}{c}- \\
705.579 \\
3036\end{array}$ & $\begin{array}{c}- \\
705.579 \\
3088\end{array}$ & $\begin{array}{c}- \\
705.579 \\
3663\end{array}$ & $\begin{array}{c}- \\
705.579 \\
2608\end{array}$ & $\begin{array}{c}- \\
705.579 \\
2724\end{array}$ \\
\hline & $\begin{array}{l}\text { Number of } \\
\text { iterations }\end{array}$ & 7 & 6 & 6 & 6 & 6 & 6 & 6 & 6 \\
\hline
\end{tabular}

Table 5-54: Log-likelihood values obtained from DFP-GSM algorithm responding to a new way of calculating the initial Hessian matrix adopted from BHHH-2 algorithm (exp. 6)

\begin{tabular}{|c|c|c|c|c|c|c|c|c|c|}
\hline & $\begin{array}{l}\text { Interval of } \\
\text { uncertainty }\end{array}$ & $\begin{array}{l}\text { LL(0), } \\
\text { UL(1) }\end{array}$ & $\begin{array}{l}\mathrm{LL}(0) \text {, } \\
\mathrm{UL}(2)\end{array}$ & $\begin{array}{l}\text { LL(0), } \\
\text { UL(3) }\end{array}$ & $\begin{array}{l}\text { LL(0), } \\
\text { UL(4) }\end{array}$ & $\begin{array}{l}\text { LL(0), } \\
\text { UL(5) }\end{array}$ & $\begin{array}{l}\mathrm{LL}(0), \\
\mathrm{UL}(6)\end{array}$ & $\begin{array}{l}\text { LL(0), } \\
\mathrm{UL}(7)\end{array}$ & $\begin{array}{l}\text { LL(0), } \\
\mathrm{UL}(8)\end{array}$ \\
\hline \multirow{6}{*}{ 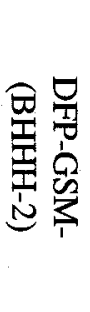 } & Starting & - & - & - & - & - & - & - & - \\
\hline & $\log _{-}$ & 869.096 & 869.096 & 869.096 & 869.096 & 869.096 & 869.096 & 869.096 & 869.096 \\
\hline & Ilkenmood & & & $472 r$ & $48<r$ & $4 / 2 r$ & & & \\
\hline & coivergence & - & $=-$ & --70 & - & - & - & - & \\
\hline & $\begin{array}{l}\text { log- } \\
\text { likelihood }\end{array}$ & 1332 & $\begin{array}{c}1271 \\
1271\end{array}$ & $\begin{array}{l}1655 \\
1659\end{array}$ & $\begin{array}{c}705.579 \\
0698\end{array}$ & $\begin{array}{c}705.579 \\
0891\end{array}$ & $\begin{array}{l}05.569 \\
119\end{array}$ & $\begin{array}{l}1455 \\
1455\end{array}$ & $\begin{array}{c}\text { cos.b79 } \\
0615\end{array}$ \\
\hline & Number of & 7 & 6 & 6 & 6 & 6 & 6 & 6 & 6 \\
\hline
\end{tabular}

Table 5-55: Log-likelihood values obtained from BFGS-GSM algorithm responding to a new way of calculating the initial Hessian matrix adopted from BHHH algorithm (exp. 6)

\begin{tabular}{|c|c|c|c|c|c|c|c|c|c|}
\hline \multicolumn{2}{|r|}{$\begin{array}{l}\text { Interval of } \\
\text { uncertainty }\end{array}$} & $\begin{array}{l}\text { LL(0), } \\
\text { UL(1) }\end{array}$ & $\begin{array}{l}\mathrm{LL}(0), \\
\text { UL(2) }\end{array}$ & $\begin{array}{l}\text { LL(0), } \\
\text { UL(3) }\end{array}$ & $\begin{array}{l}\text { LL(0), } \\
\text { UL(4) }\end{array}$ & $\begin{array}{l}\text { LL(0), } \\
\text { UL(5) }\end{array}$ & $\begin{array}{l}\text { LL(0), } \\
\text { UL(6) }\end{array}$ & $\begin{array}{l}\text { LL(0), } \\
\text { UL(7) }\end{array}$ & $\begin{array}{l}\text { LL(0), } \\
\text { UL(8) }\end{array}$ \\
\hline \multirow{5}{*}{ 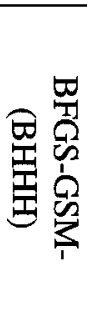 } & Starting & - & - & - & - & - & - & - & - \\
\hline & Log- & 869.096 & 869.096 & 869.096 & 869.096 & 869.096 & 869.096 & 869.096 & 869.096 \\
\hline & Convergence & ticr & 4128 & 7128 & - & 4726 & - & - & - \\
\hline & $\begin{array}{l}\text { log- } \\
\text { likelibod }\end{array}$ & $\begin{array}{c}705.771 \\
4975\end{array}$ & $\begin{array}{c}705.579 \\
4055\end{array}$ & $\begin{array}{c}705.579 \\
4037\end{array}$ & $\begin{array}{c}705.579 \\
253\end{array}$ & $\begin{array}{c}705.579 \\
2989\end{array}$ & $\begin{array}{c}705.579 \\
3014\end{array}$ & $\begin{array}{c}705.579 \\
3255\end{array}$ & $\begin{array}{c}705.579 \\
3366\end{array}$ \\
\hline & $\begin{array}{l}\text { Number of } \\
\text { iterations }\end{array}$ & 5 & 6 & 6 & 6 & 6 & 6 & 6 & 6 \\
\hline
\end{tabular}

Table 5-56: Log-likelihood values obtained from BFGS-GSM algorithm responding to a new way of calculating the initial Hessian matrix adopted from BHHH-2 algorithm (exp. 6)

\begin{tabular}{|c|c|c|c|c|c|c|c|c|c|}
\hline \multicolumn{2}{|c|}{$\begin{array}{l}\text { Interval of } \\
\text { uncertainty }\end{array}$} & $\begin{array}{l}\mathrm{LL}(0), \\
\mathrm{UL}(1)\end{array}$ & $\begin{array}{l}\text { LL(0), } \\
\text { UL(2) }\end{array}$ & $\begin{array}{l}\text { LL(0), } \\
\text { UL(3) }\end{array}$ & $\begin{array}{l}\text { LL(0), } \\
\text { UL(4) }\end{array}$ & $\begin{array}{l}\text { LL(0), } \\
\text { UL(5) }\end{array}$ & $\begin{array}{l}\text { LL(0), } \\
\text { UL(6) }\end{array}$ & $\begin{array}{l}\text { LL(0), } \\
\text { UL(7) }\end{array}$ & $\begin{array}{l}\text { LL(0), } \\
\text { UL(8) }\end{array}$ \\
\hline \multirow{6}{*}{ 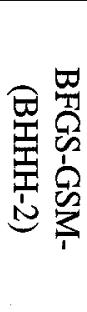 } & Starting & - & - & - & - & - & - & - & - \\
\hline & Log- $_{-}$ & $\begin{array}{c}869.096 \\
4727\end{array}$ & 869.096 & 869.096 & $\begin{array}{c}869.096 \\
4727\end{array}$ & 869.096 & 869.096 & 869.096 & 869.096 \\
\hline & Convergence & - & - & 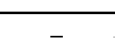 & - & 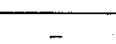 & 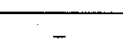 & 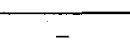 & - \\
\hline & $\log _{-}$ & 705.591 & 705.579 & 705.579 & 705.579 & 705.579 & 705.579 & 705.579 & 705.579 \\
\hline & likelihood & 1918 & 1171 & 1203 & 0991 & 1241 & 1221 & 121 & 1082 \\
\hline & $\begin{array}{l}\text { Number of } \\
\text { iterations }\end{array}$ & 6 & 6 & 6 & 6 & 6 & 6 & 6 & 6 \\
\hline
\end{tabular}




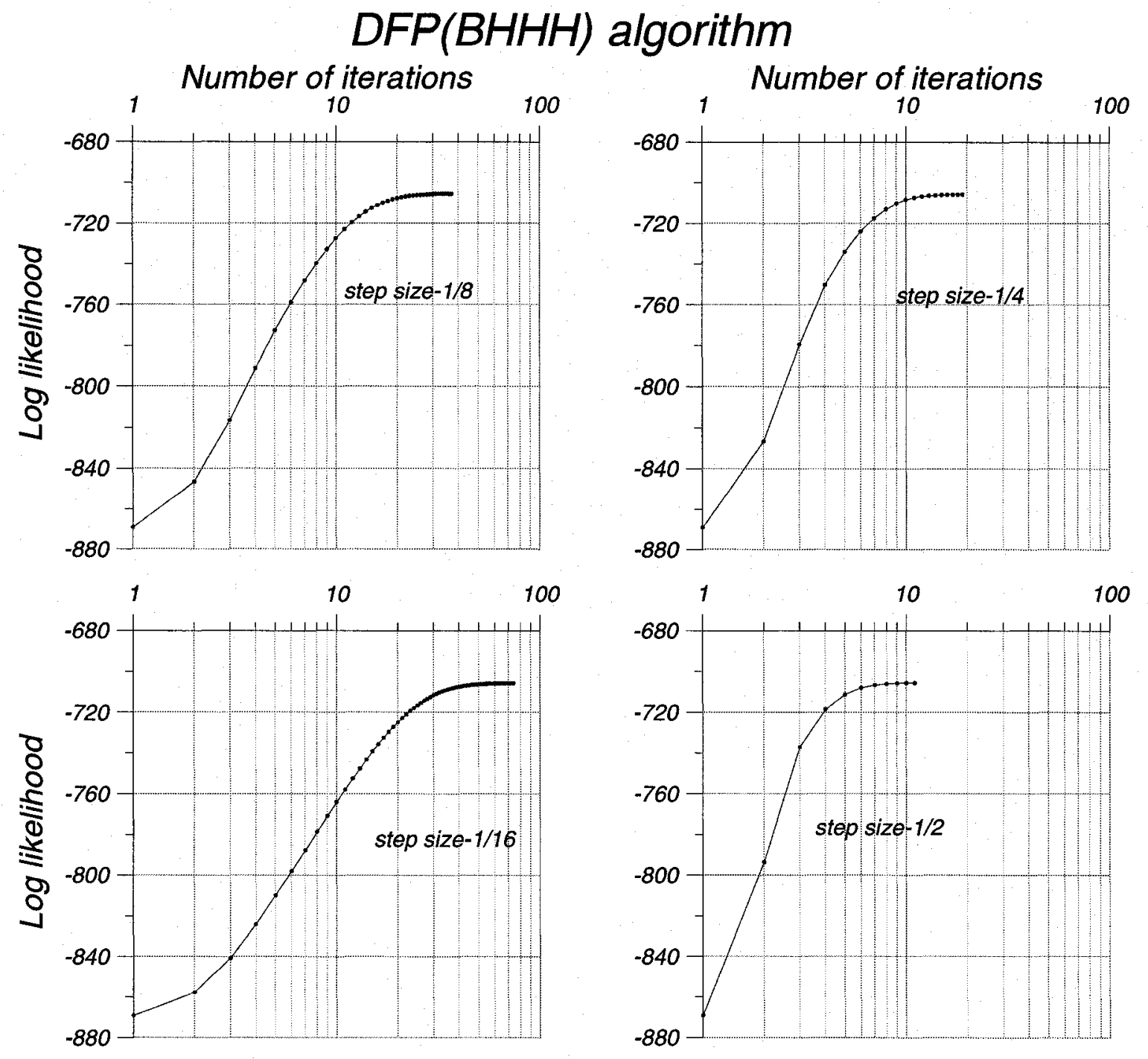

Figure 5-101: Log-likelihood values for each step size in DFP algorithm responding to a new way of calculating the initial Hessian matrix adopted from $\mathrm{BHHH}$ algorithm (exp. 6) 


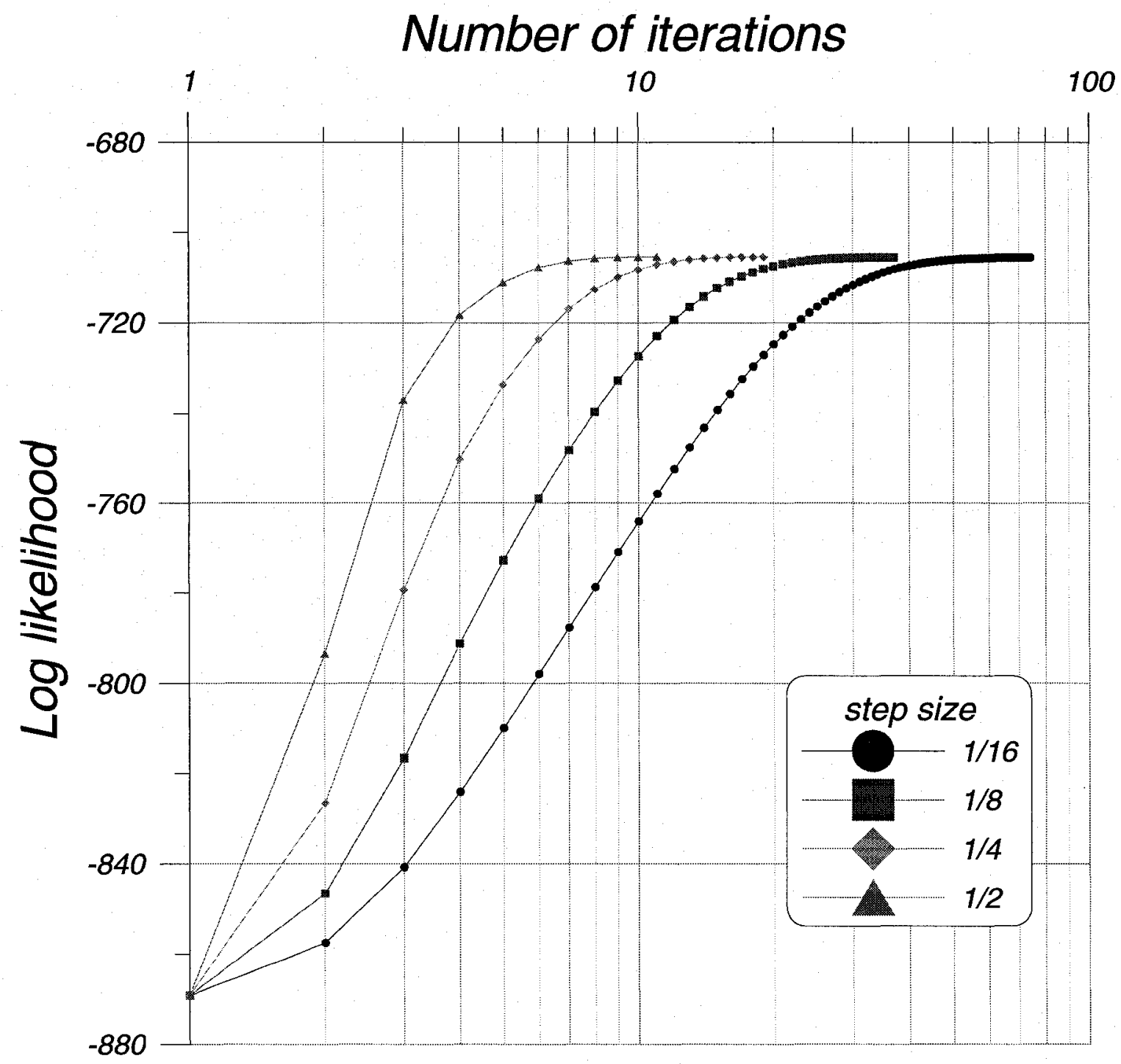

Figure 5-102: Log-likelihood values for all the step sizes in DFP algorithm responding to a new way of calculating the initial Hessian matrix adopted from BHHH algorithm (exp. 6) 


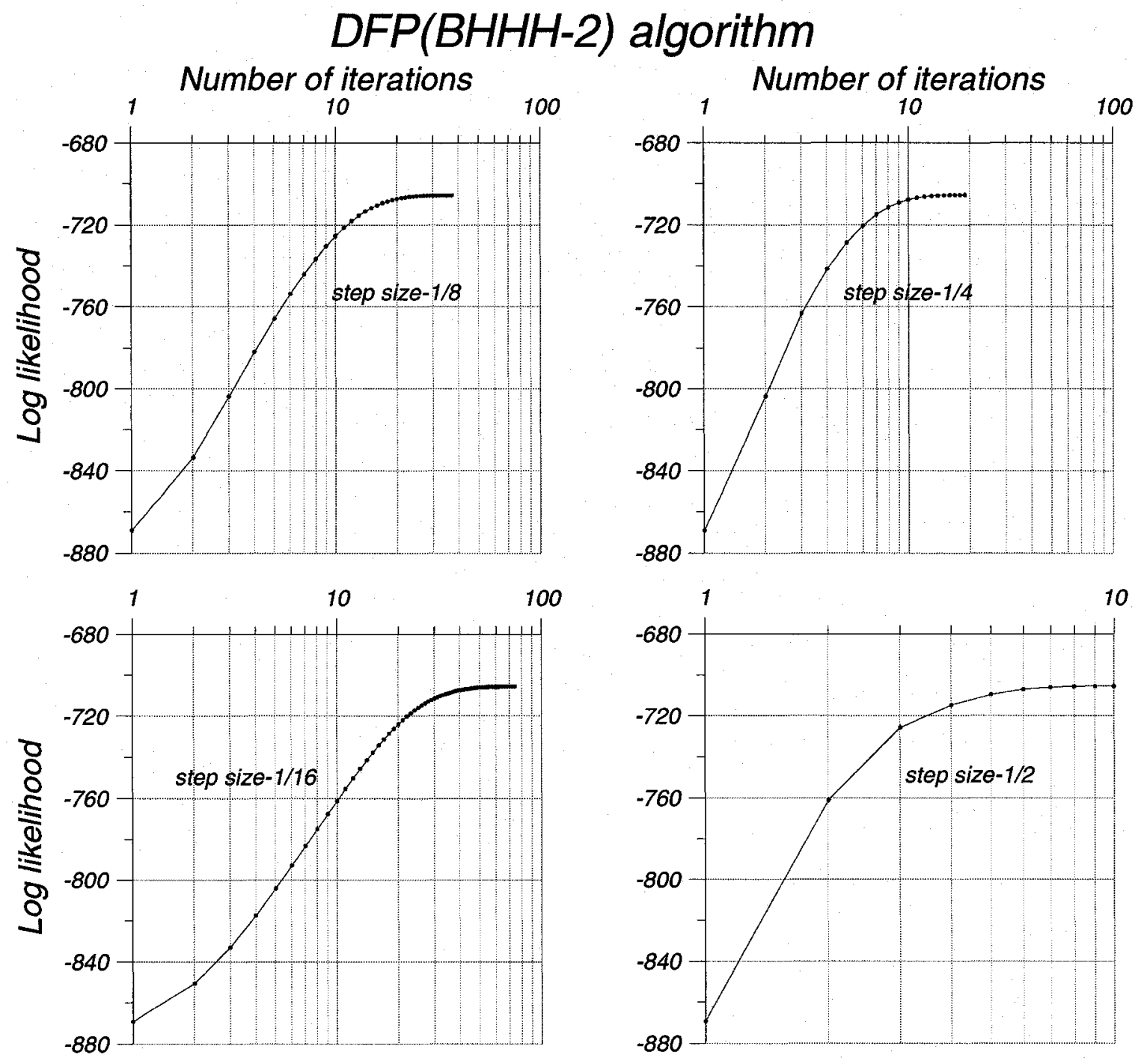

Figure 5-103: Log-likelihood values for each step size in DFP algorithm responding to a new way of calculating the initial Hessian matrix adopted from BHHH-2 algorithm (exp. 6) 


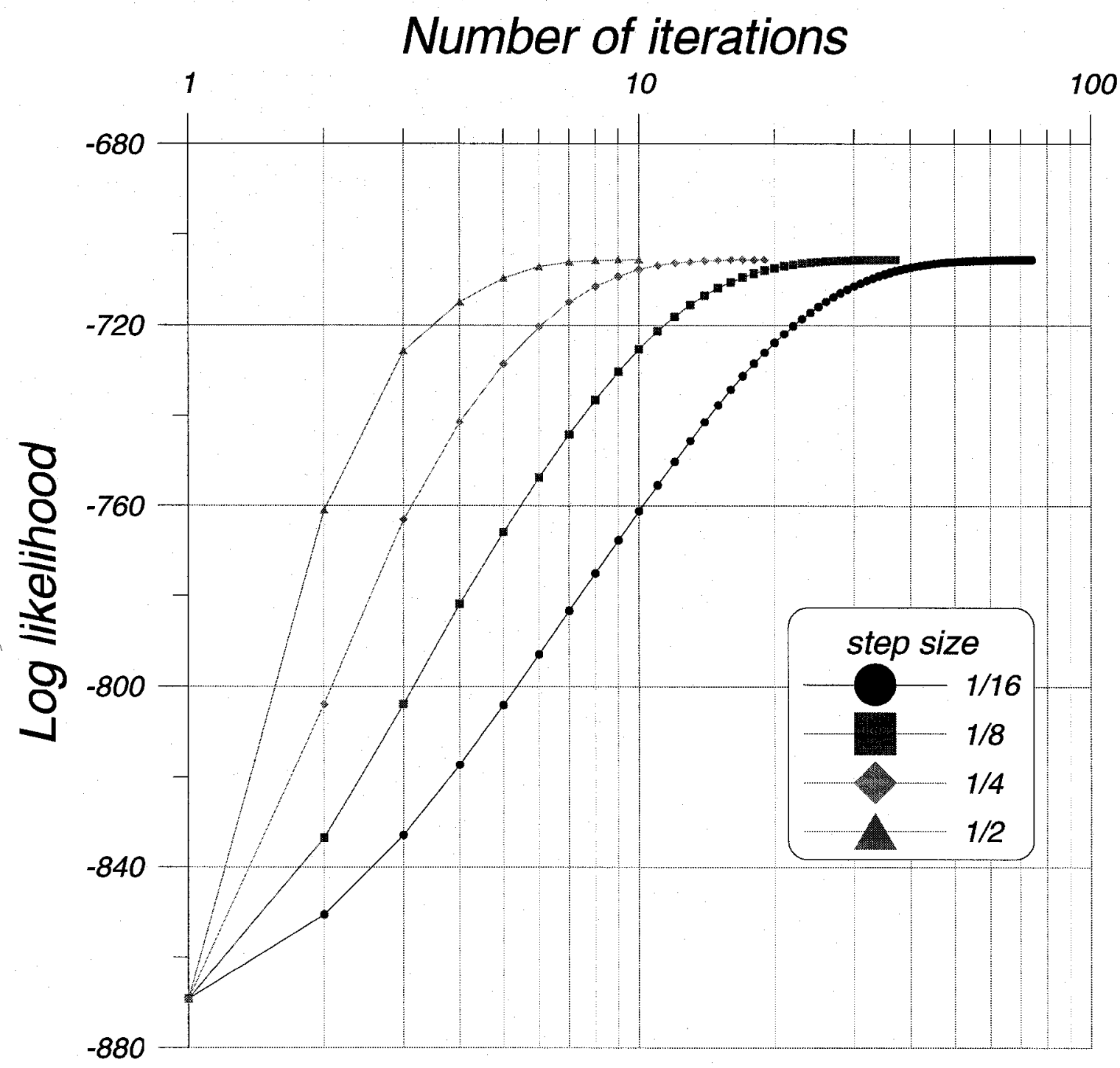

Figure 5-104: Log-likelihood values for all the step sizes in DFP algorithm responding to a new way of calculating the initial Hessian matrix adopted from $\mathrm{BHHH}-2$ algorithm (exp. 6) 


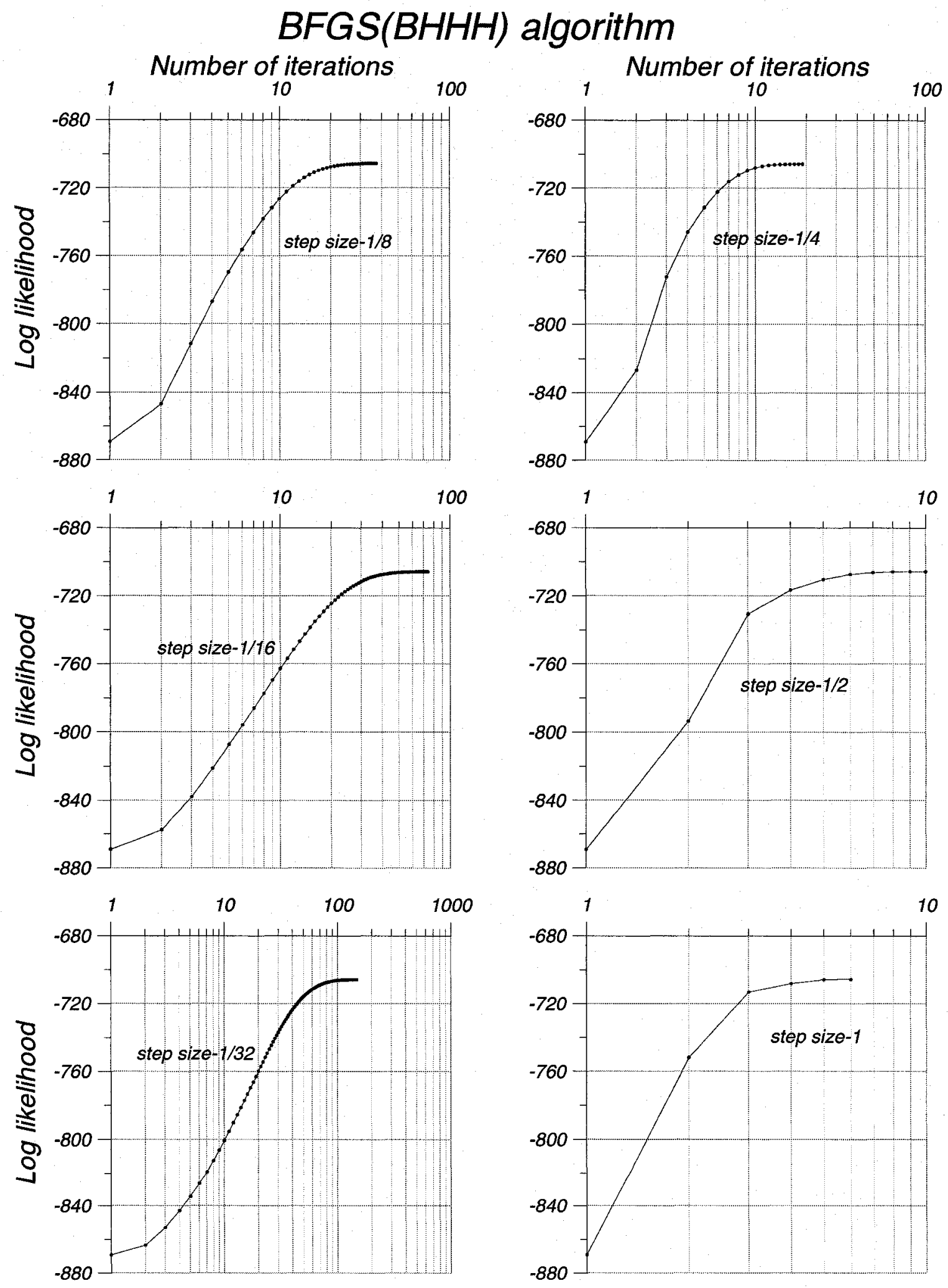

Figure 5-105: Log-likelihood values for each step size in BFGS algorithm responding to a new way of calculating the initial Hessian matrix adopted from $\mathrm{BHHH}$ algorithm (exp. 6) 


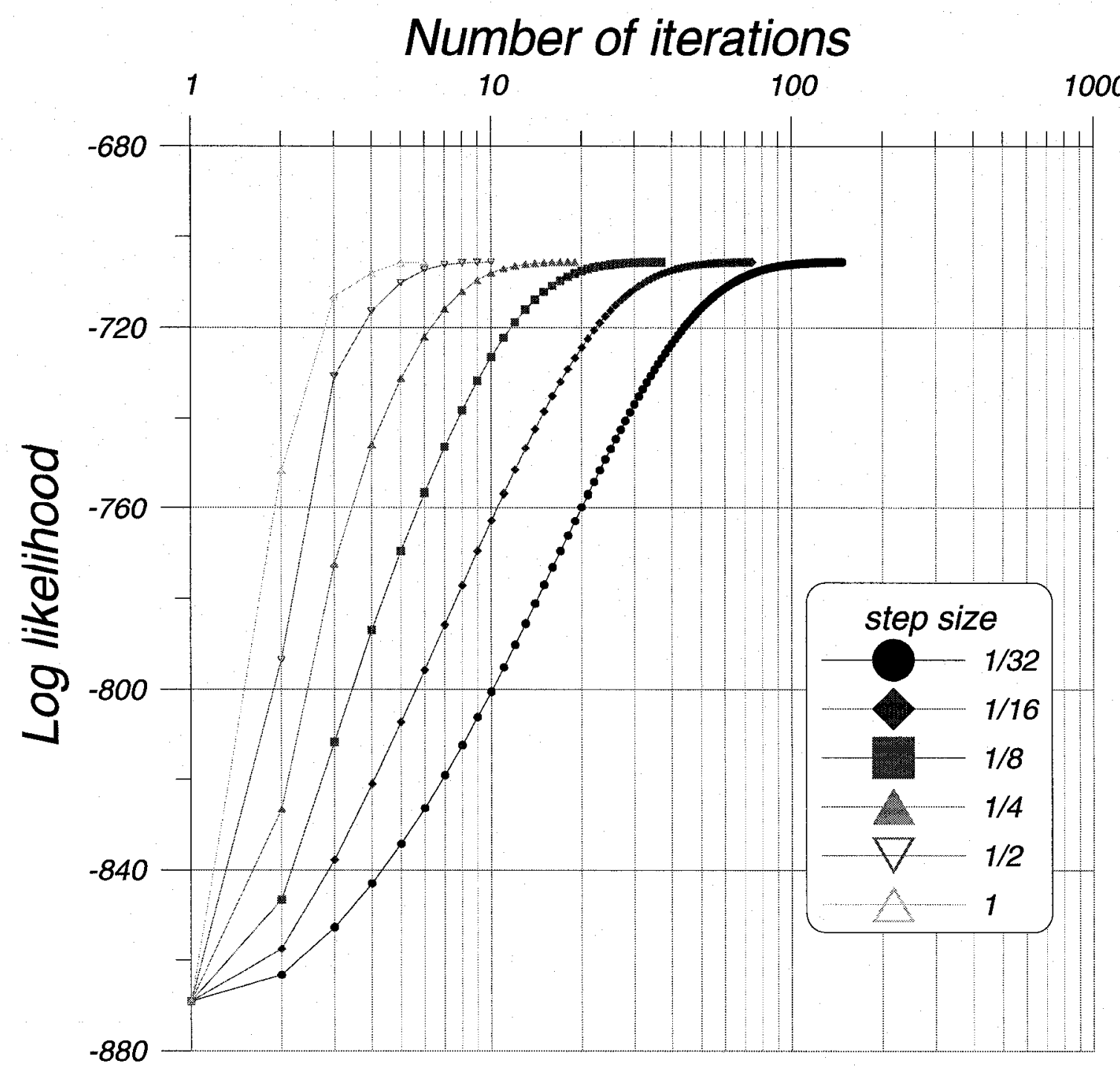

Figure 5-106: Log-likelihood values for all the step sizes in BFGS algorithm responding to a new way of calculating the initial Hessian matrix adopted from $\mathrm{BHHH}$ algorithm (exp. 6) 


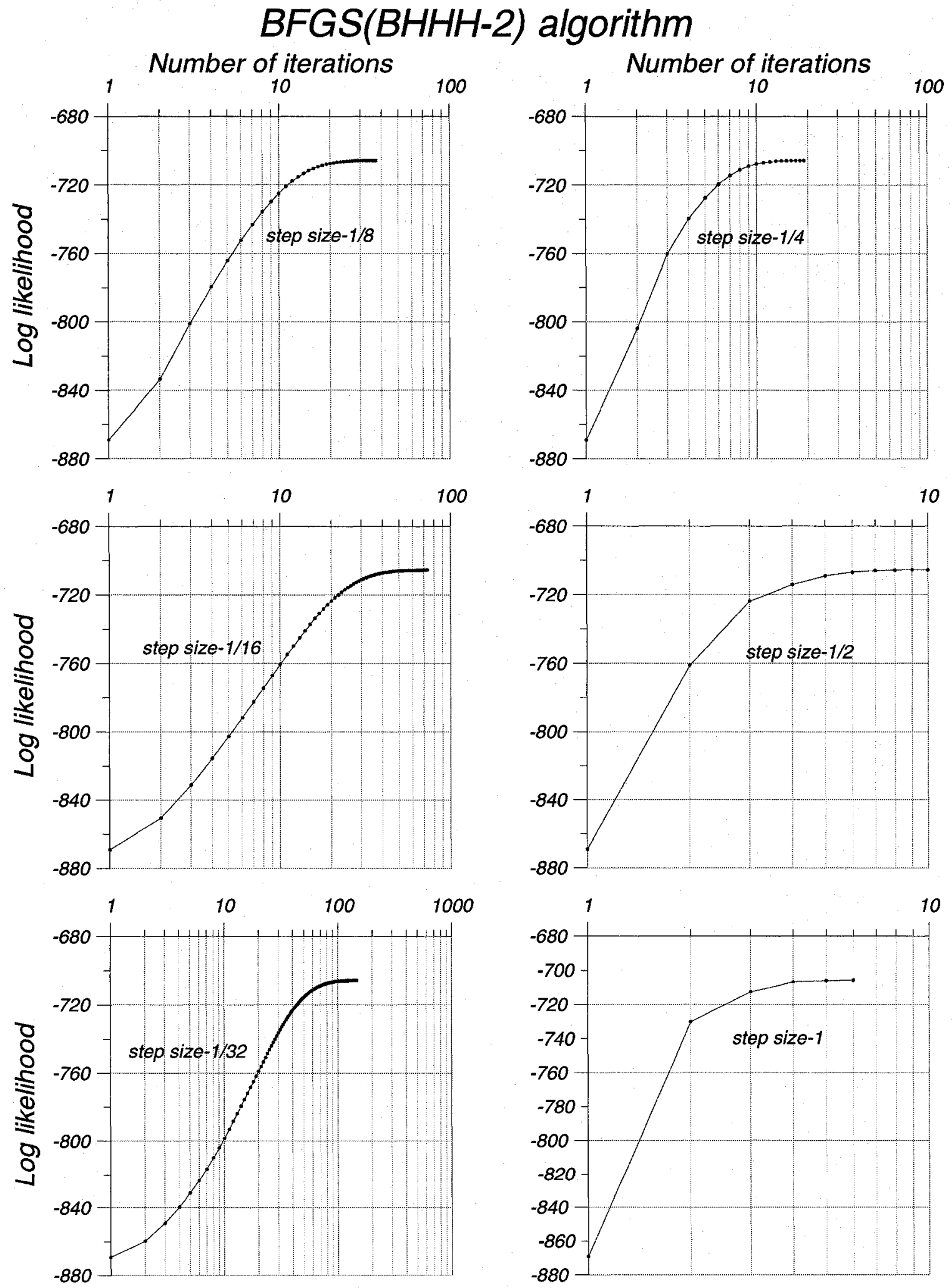

Figure 5-107: Log-likelihood values for each step size in BFGS algorithm responding to a new way of calculating the initial Hessian matrix adopted from BHHH-2 algorithm (exp. 6) 


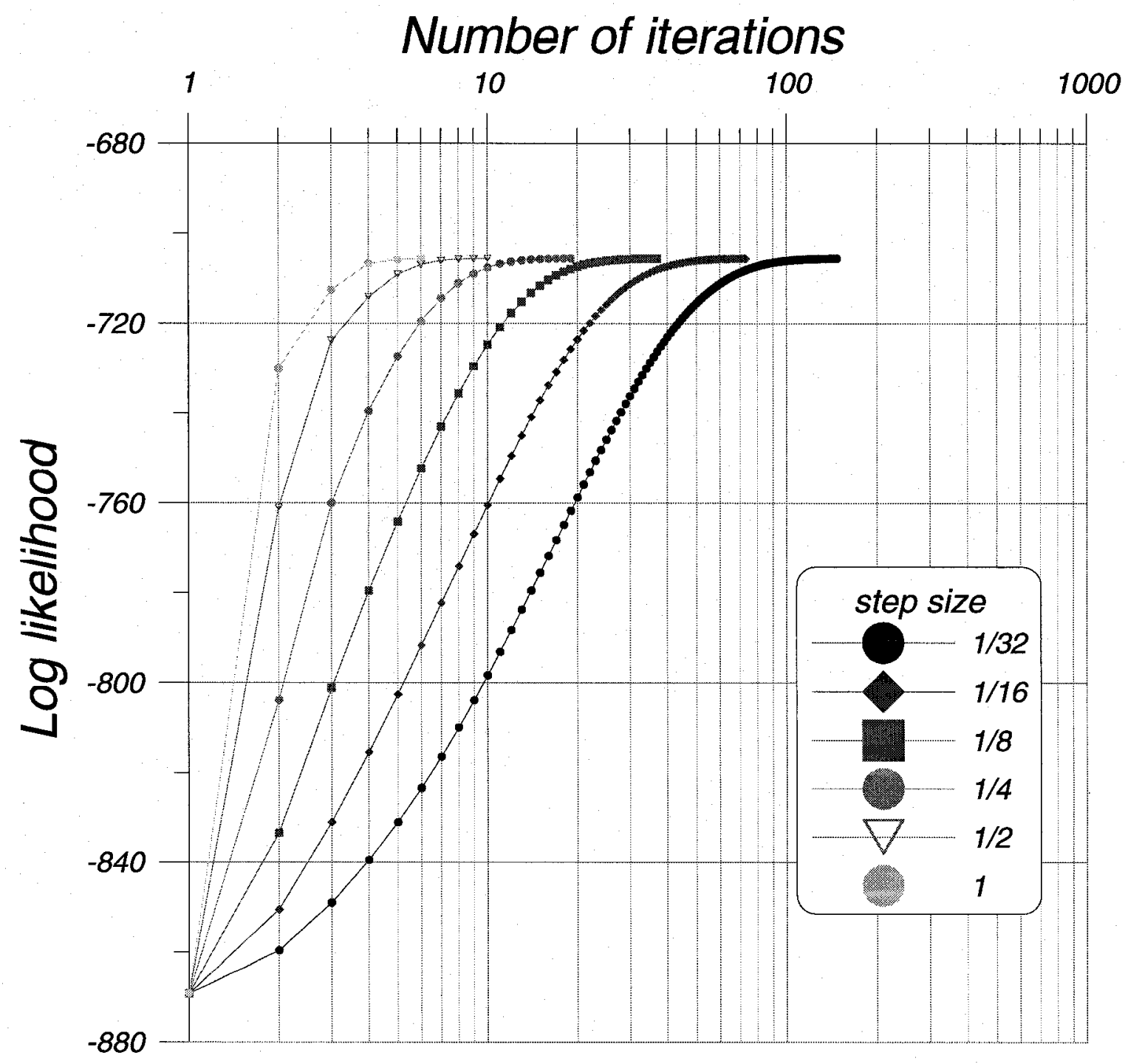

Figure 5-108: Log-likelihood values for all the step sizes in BFGS algorithm responding to a new way of calculating the initial Hessian matrix adopted from BHHH-2 algorithm (exp. 6) 


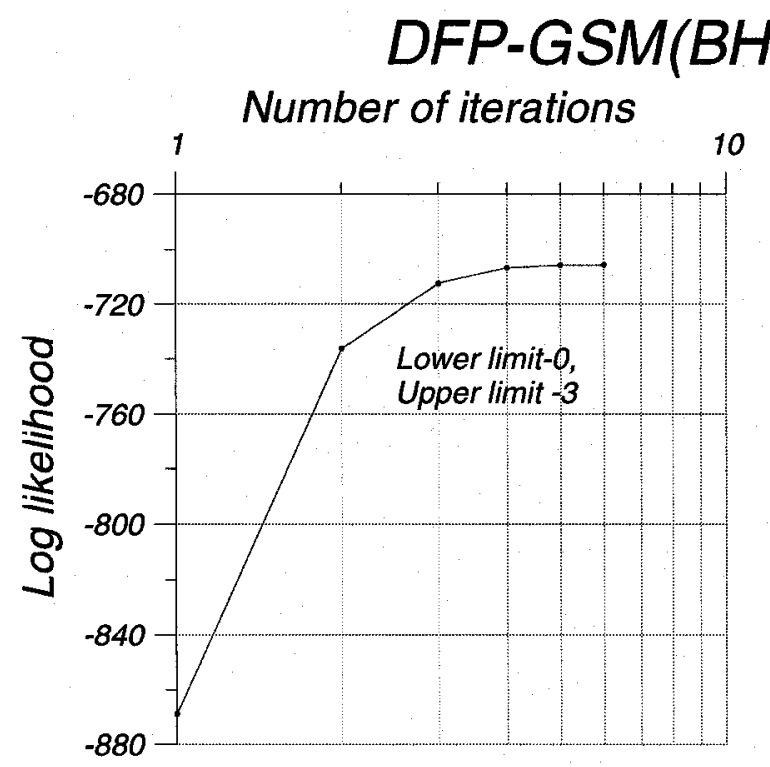

\section{) algorithm}
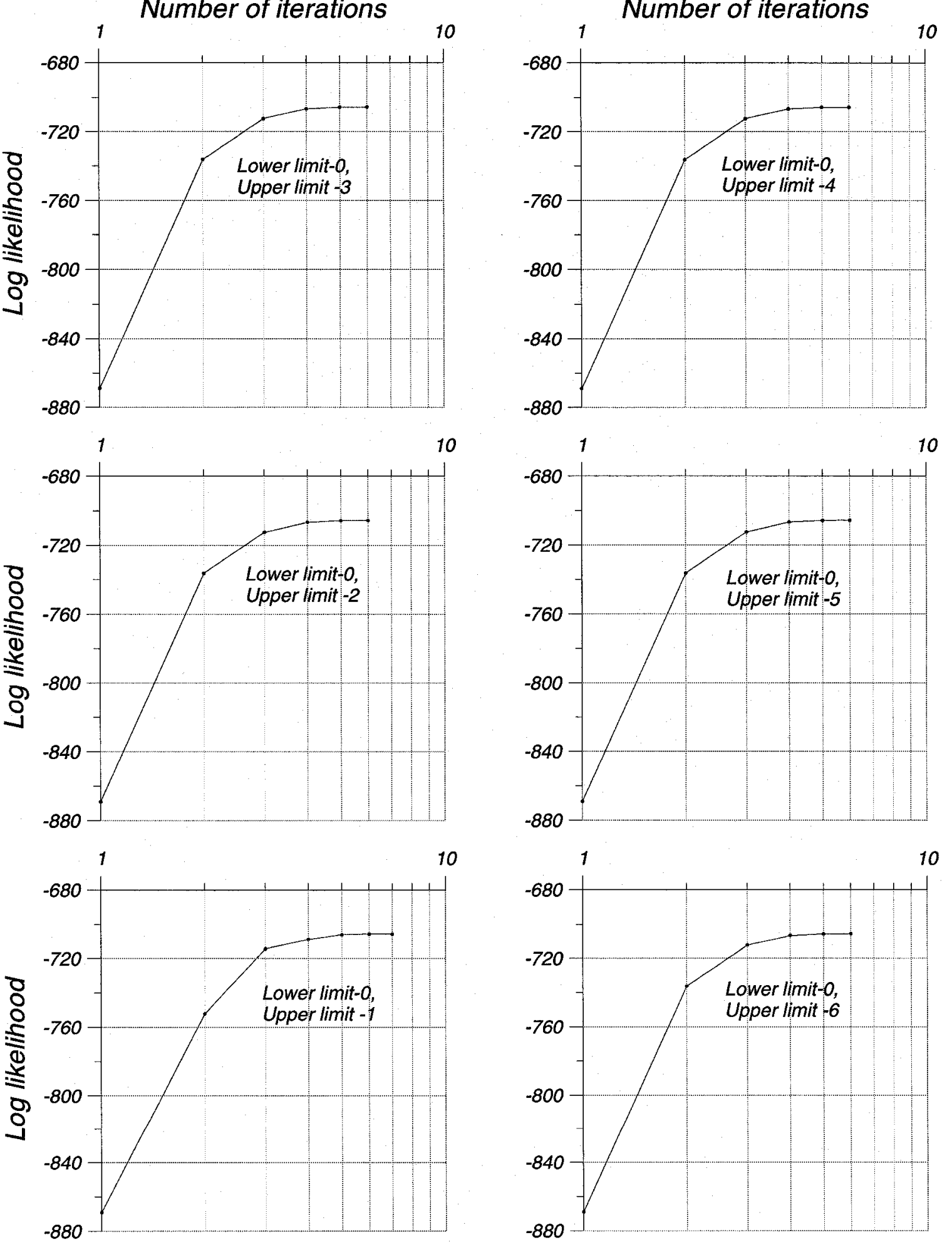

Figure 5-109: Log-likelihood values for the first six uncertain intervals in DFP-GSM algorithm responding to a new way of calculating the initial Hessian matrix adopted from BHHH algorithm(exp. 6) 

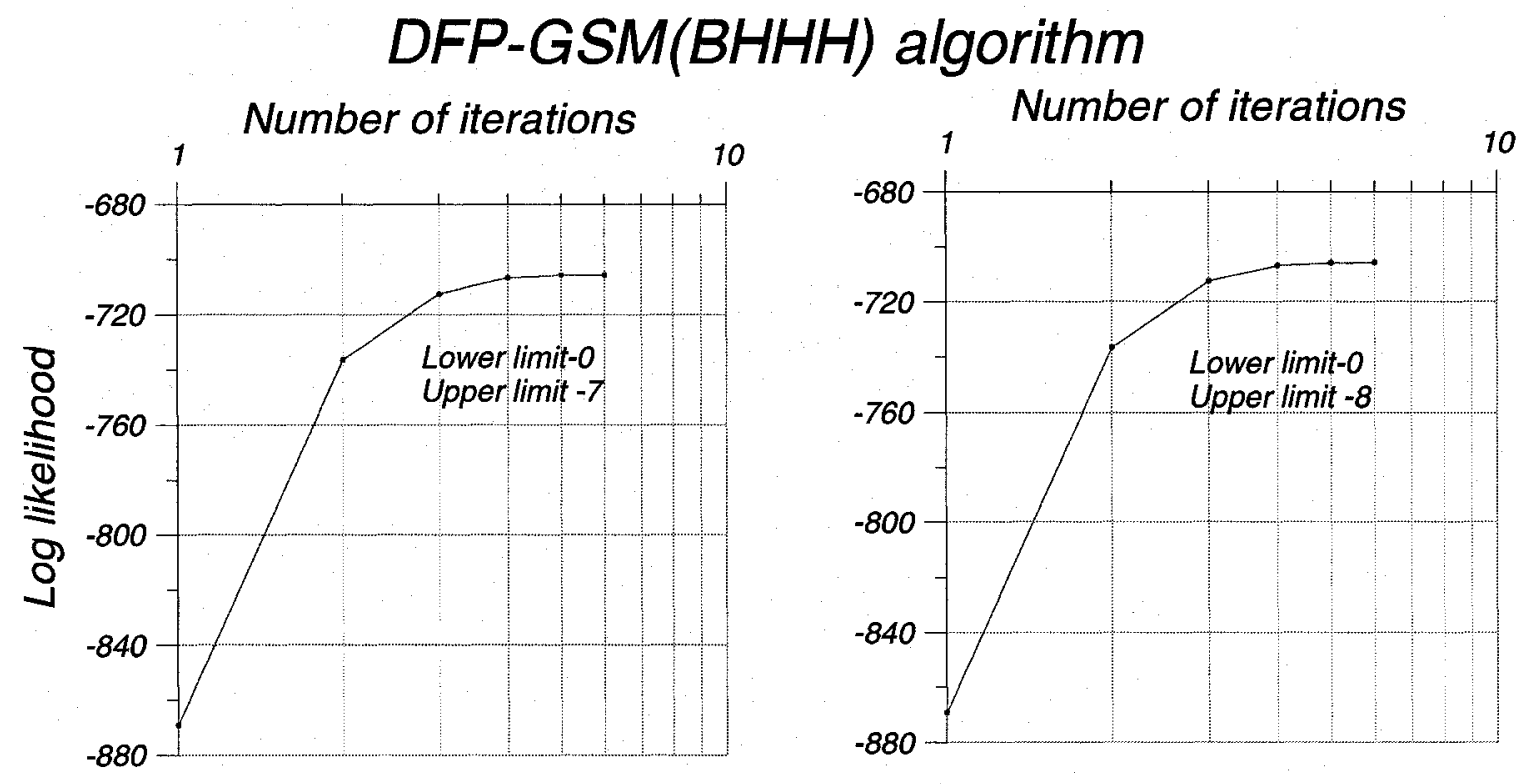

Figure 5-110: Log-likelihood values for the last two uncertain intervals in DFP-GSM algorithm responding to a new way of calculating the initial Hessian matrix adopted from $\mathrm{BHHH}$ algorithm (exp. 6) 


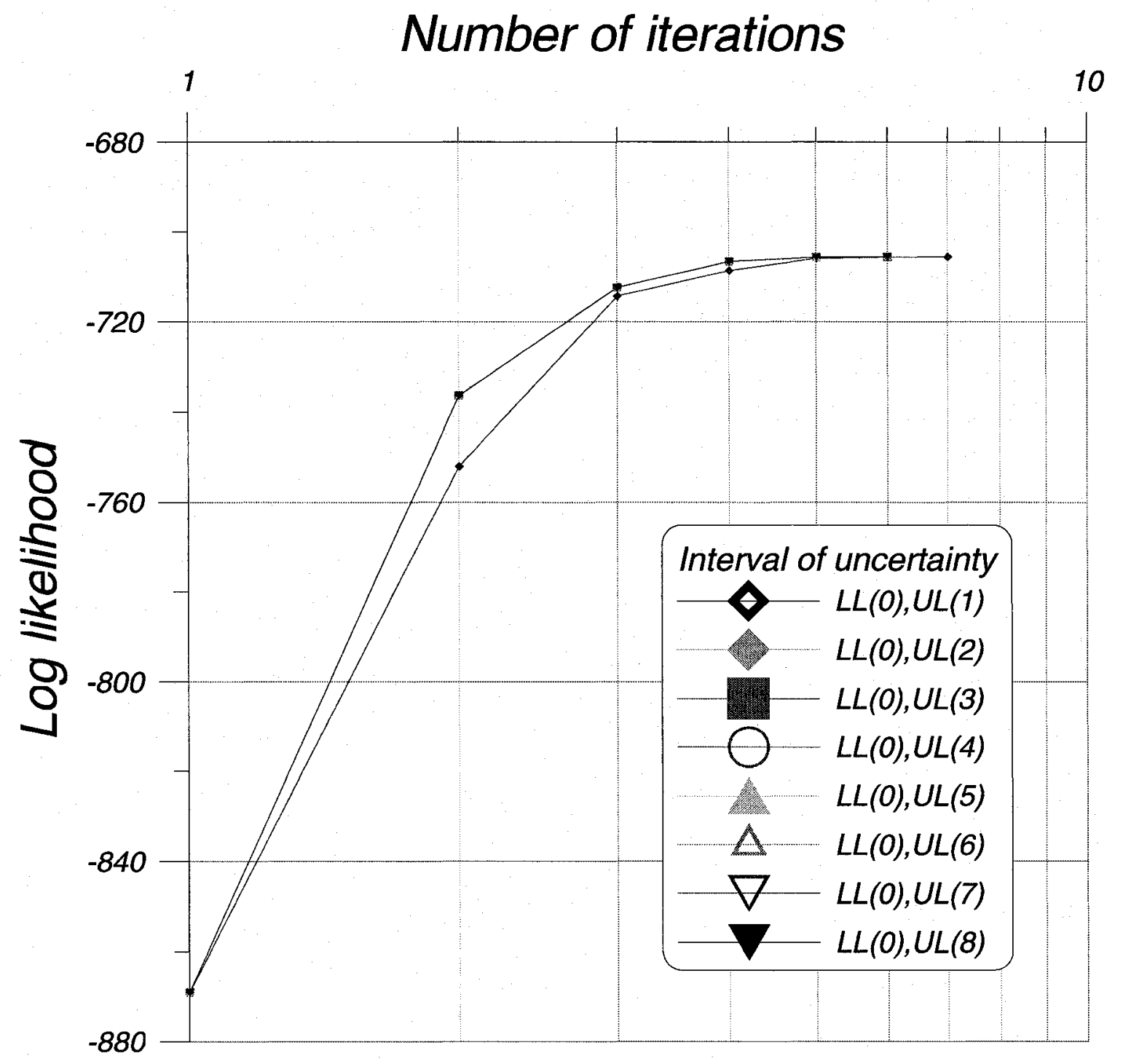

Figure 5-111: Log-likelihood values for the all uncertain intervals in DFP-GSM algorithm responding to a new way of calculating the initial Hessian matrix adopted from BHHH algorithm (exp. 6) 


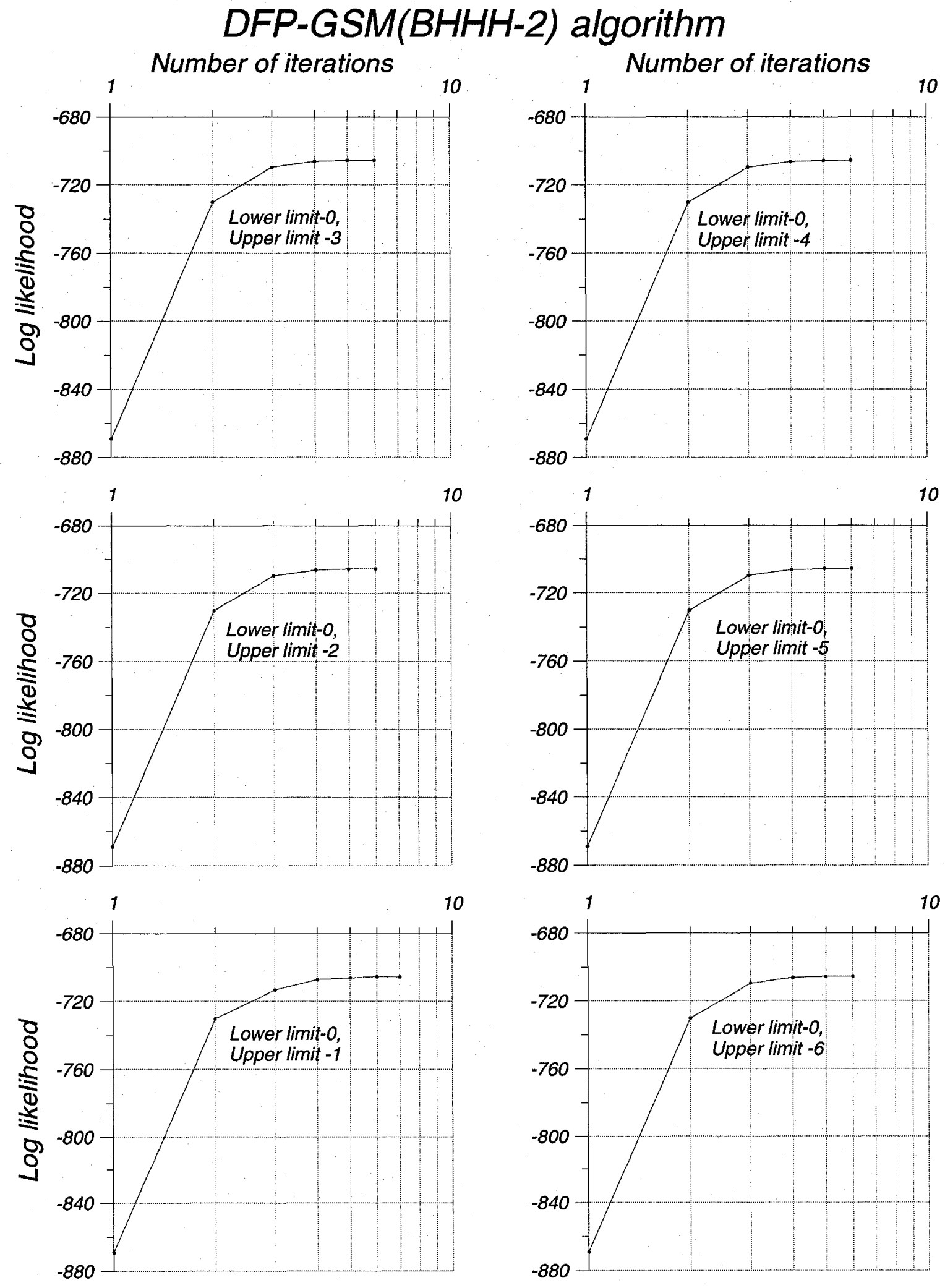

Figure 5-112: Log-likelihood values for the first six uncertain intervals in DFP-GSM algorithmresponding to a new way of calculating the initial Hessian matrix adopted from BHHH-2 algorithm (exp. 6) 

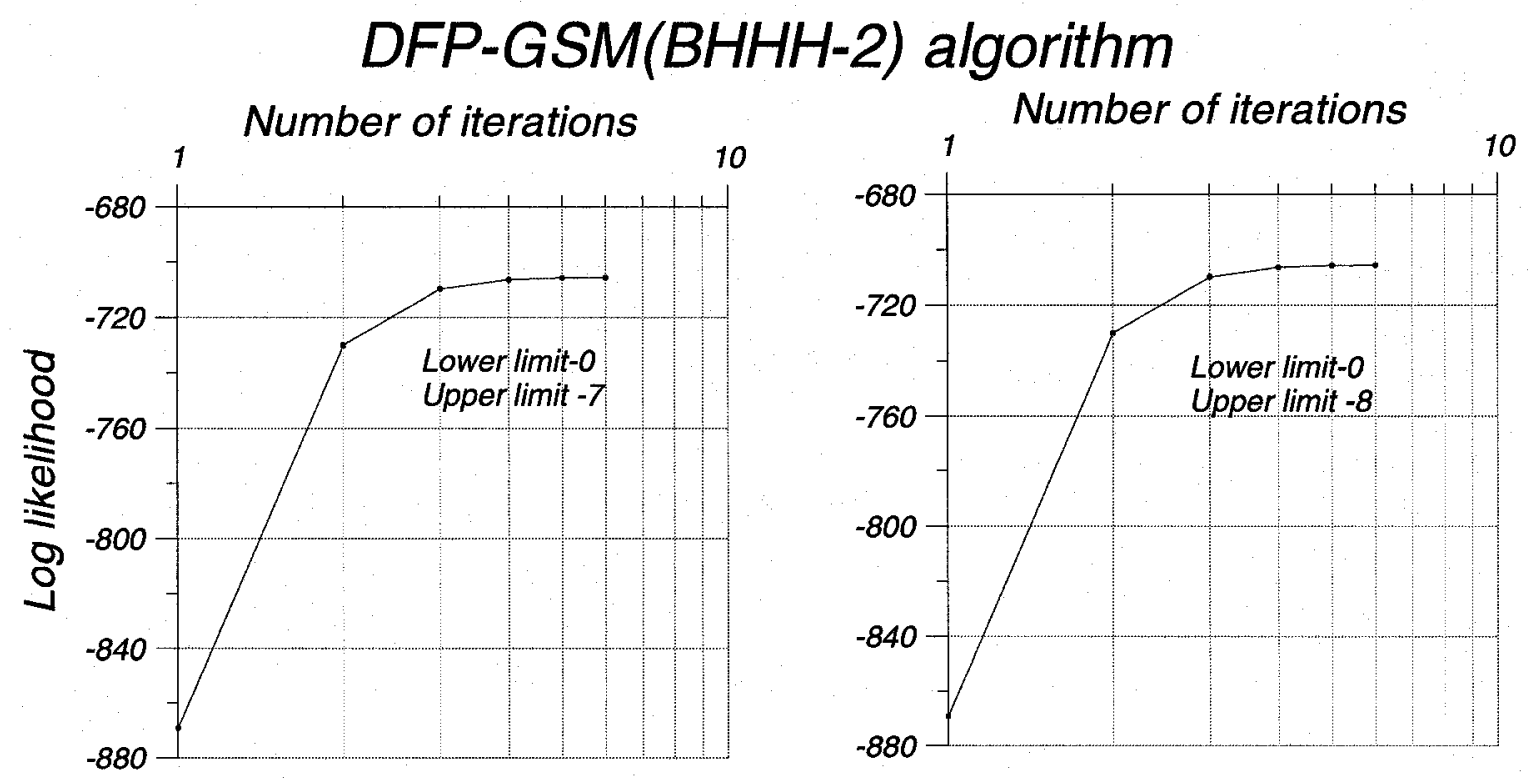

Figure 5-113: Log-likelihood values for the last two uncertain intervals in DFP-GSM algorithm responding to a new way of calculating the initial Hessian matrix adopted from BHHH-2 algorithm (exp. 6) 


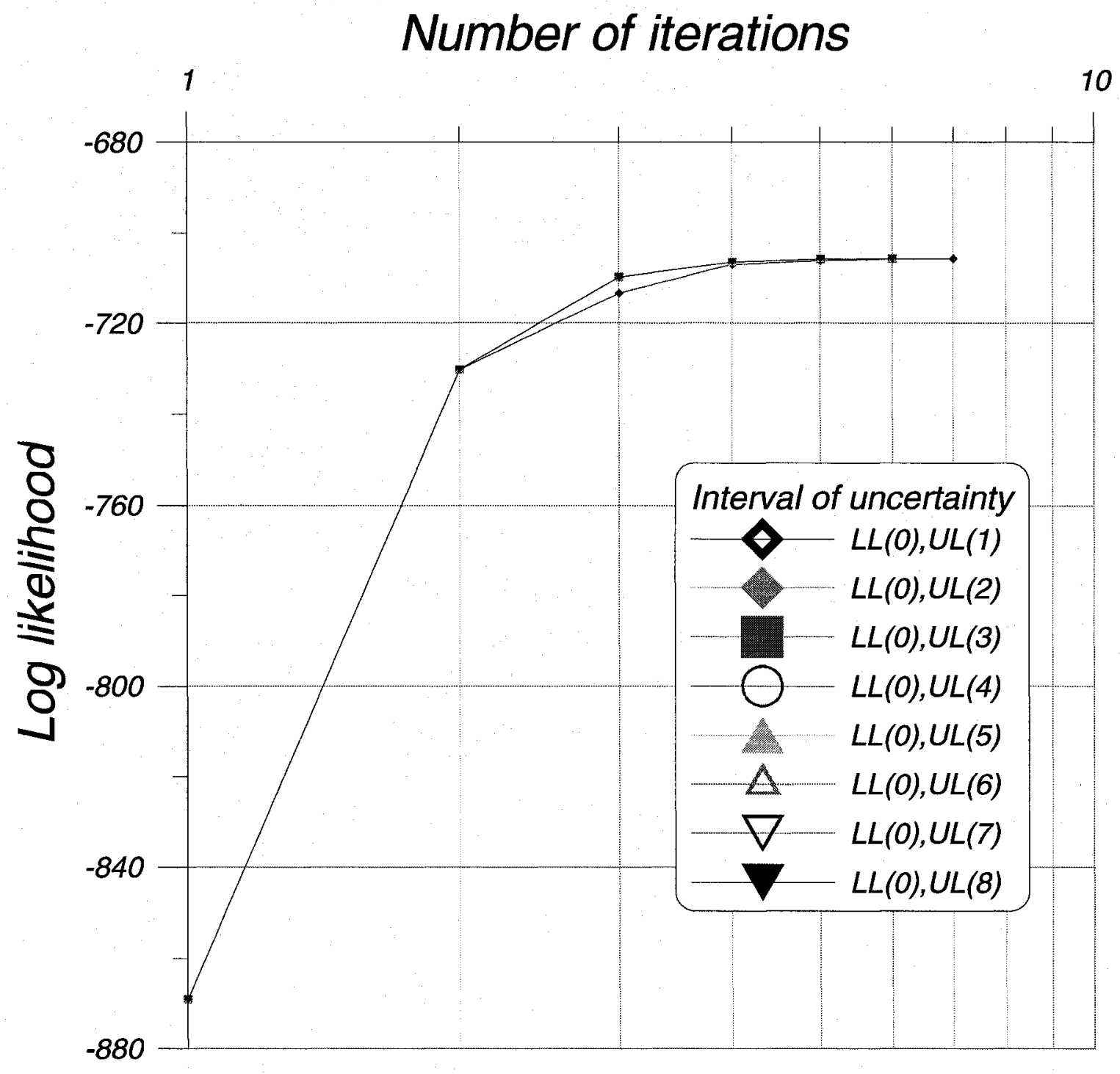

Figure 5-114: Log-likelihood values for the all uncertain intervals in DFP-GSM algorithm responding to a new way of calculating the initial Hessian matrix adopted from BHHH-2 algorithm (exp. 6) 


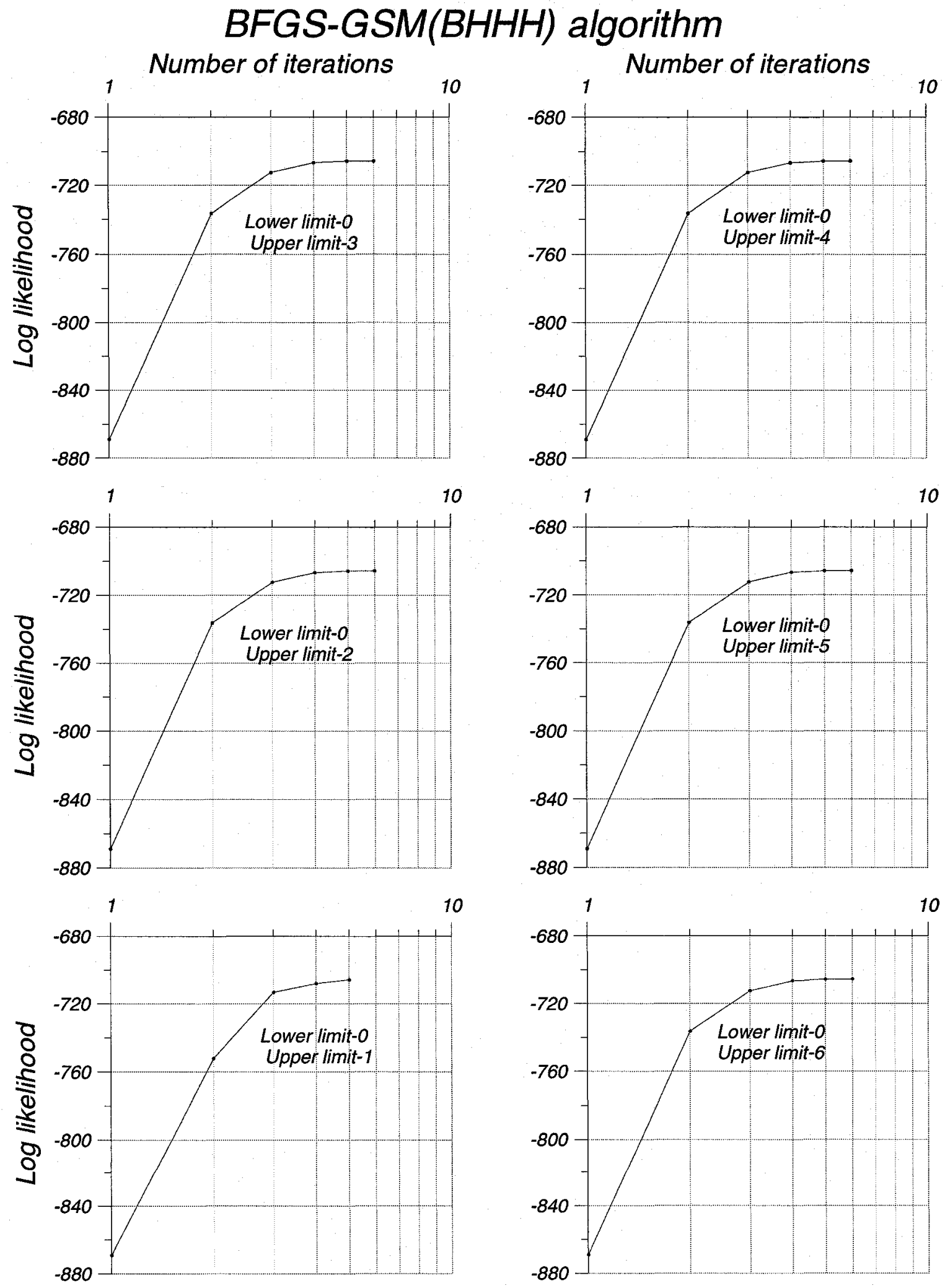

Figure 5-115: Log-likelihood values for the first six uncertain intervals in BFGS-GSM algorithm responding to a new way of calculating the initial Hessian matrix adopted from BHHH algorithm (exp. 6) 

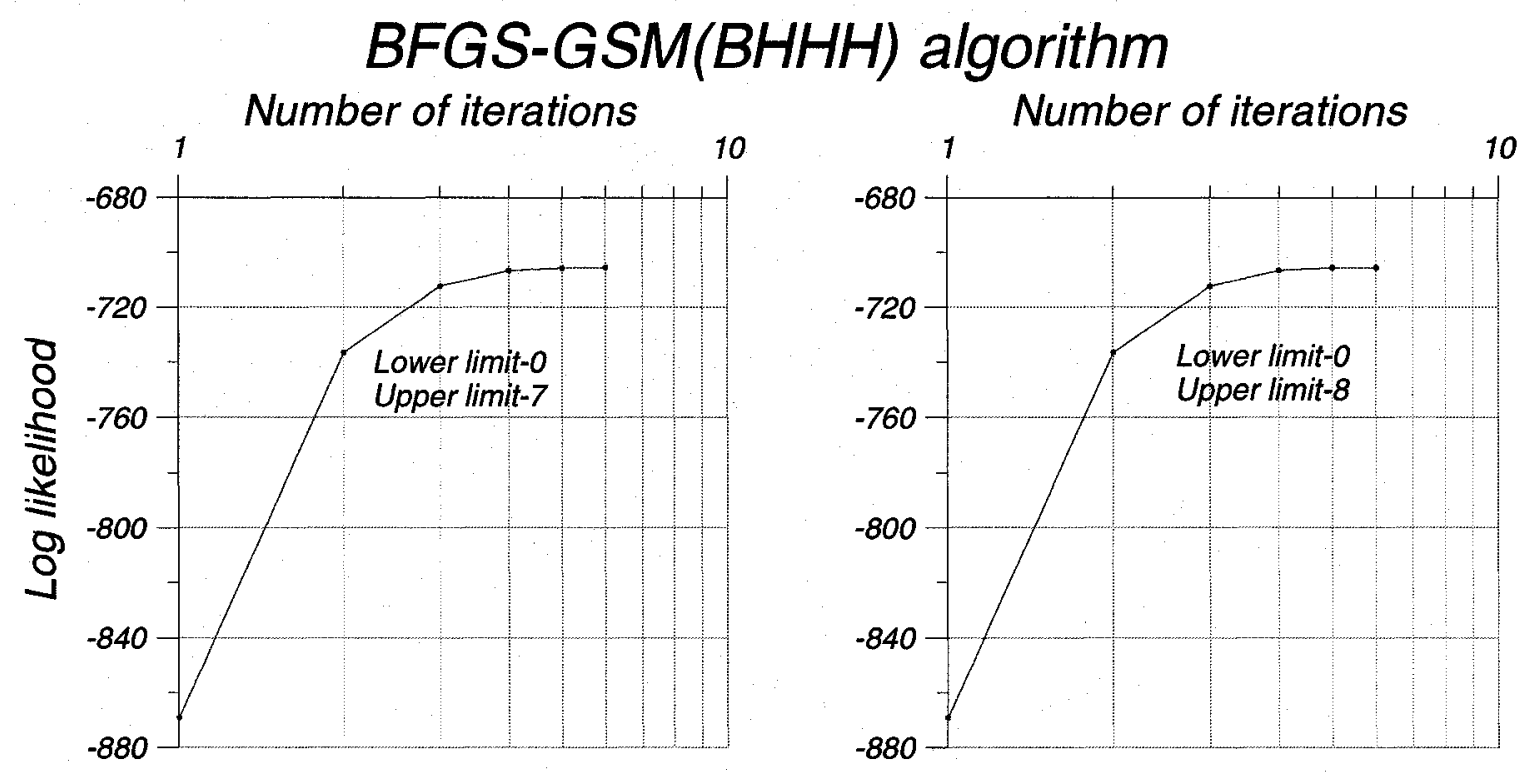

Figure 5-116: Log-likelihood values for the last two uncertain intervals in BFGS-GSM algorithm responding to a new way of calculating the initial Hessian matrix adopted from BHHH algorithm (exp. 6) 


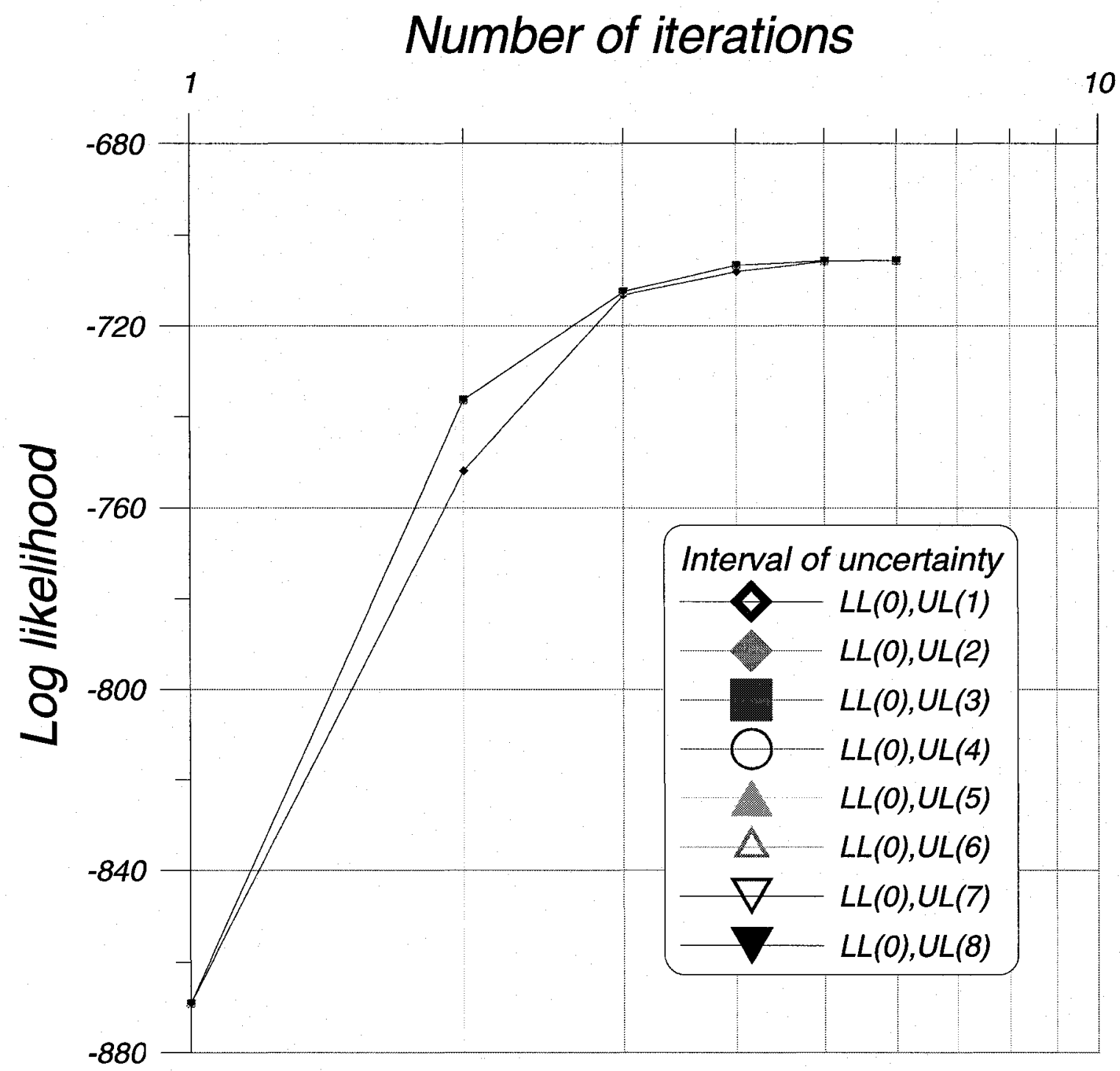

Figure 5-117: Log-likelihood values for the all uncertain intervals in BFGS-GSM algorithm responding to a new way of calculating the initial Hessian matrix adopted from $\mathrm{BHHH}$ algorithm (exp. 6) 


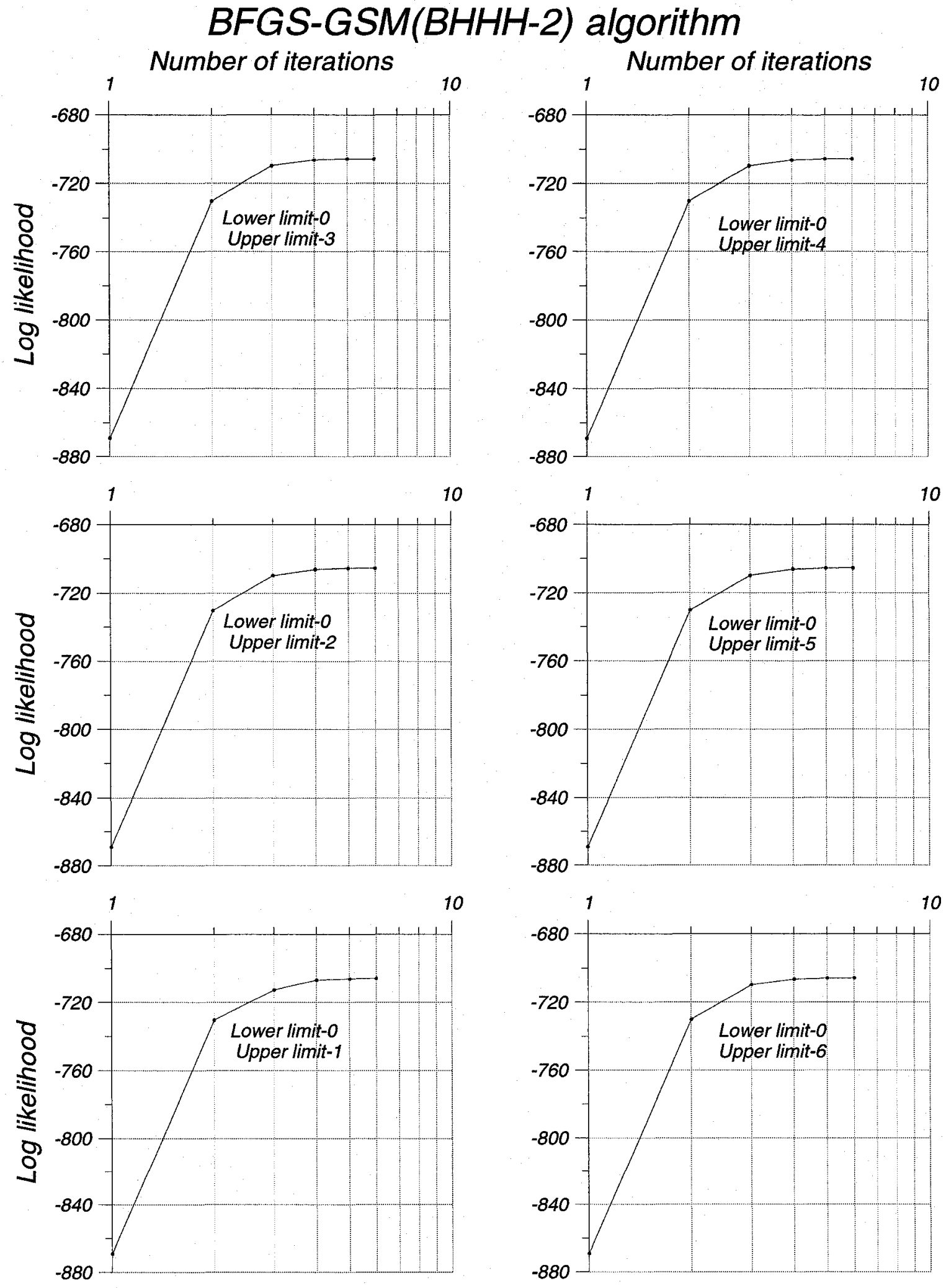

Figure 5-118: Log-likelihood values for the first six uncertain intervals in BFGS-GSM algorithm responding to a new way of calculating the initial Hessian matrix adopted fromBHHH-2 algorithm (exp. 6) 

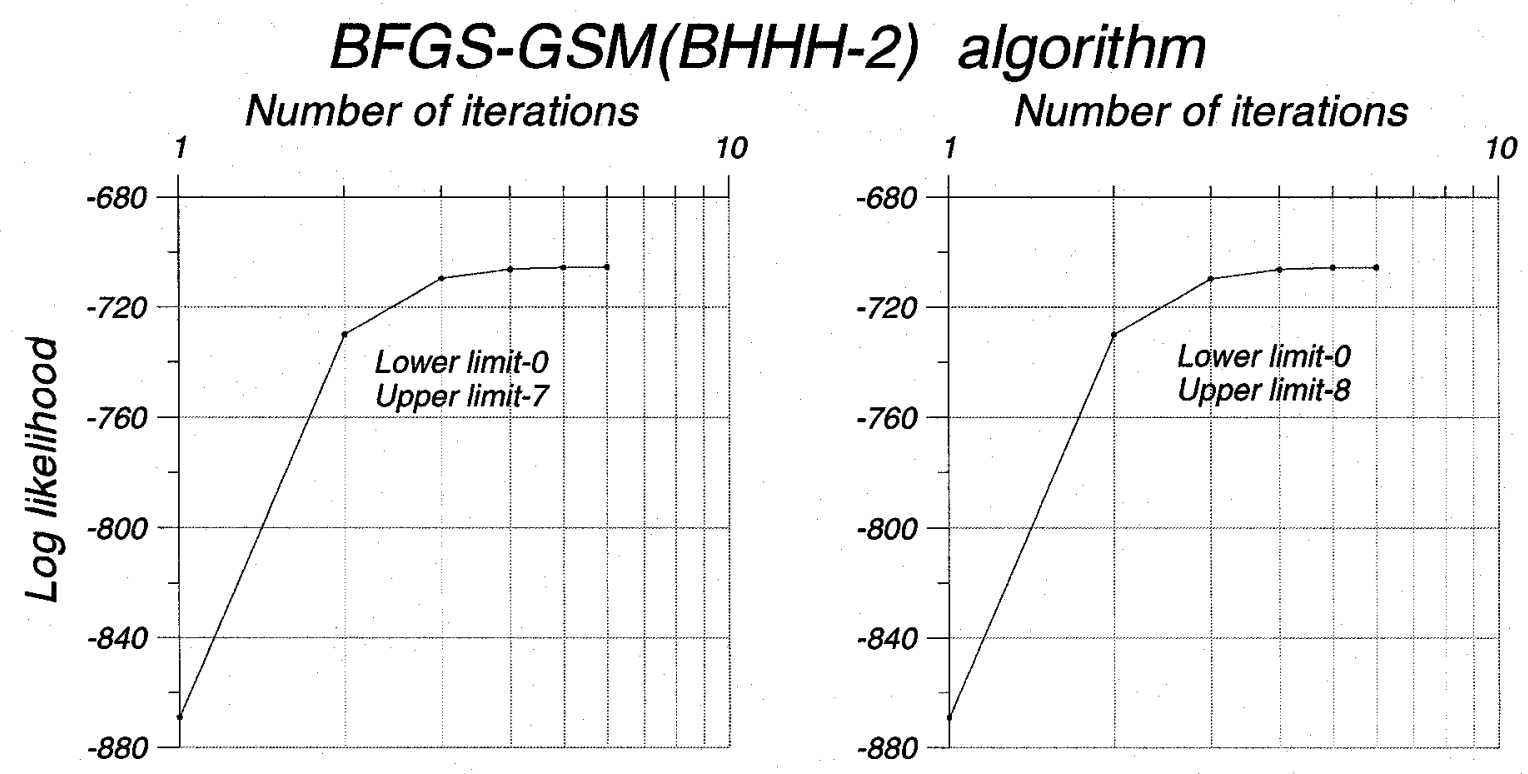

Figure 5-119: Log-likelihood values for the last two uncertain intervals in BFGS-GSM algorithm responding to a new way of calculating the initial Hessian matrix adopted from BHHH-2 algorithm (exp. 6) 


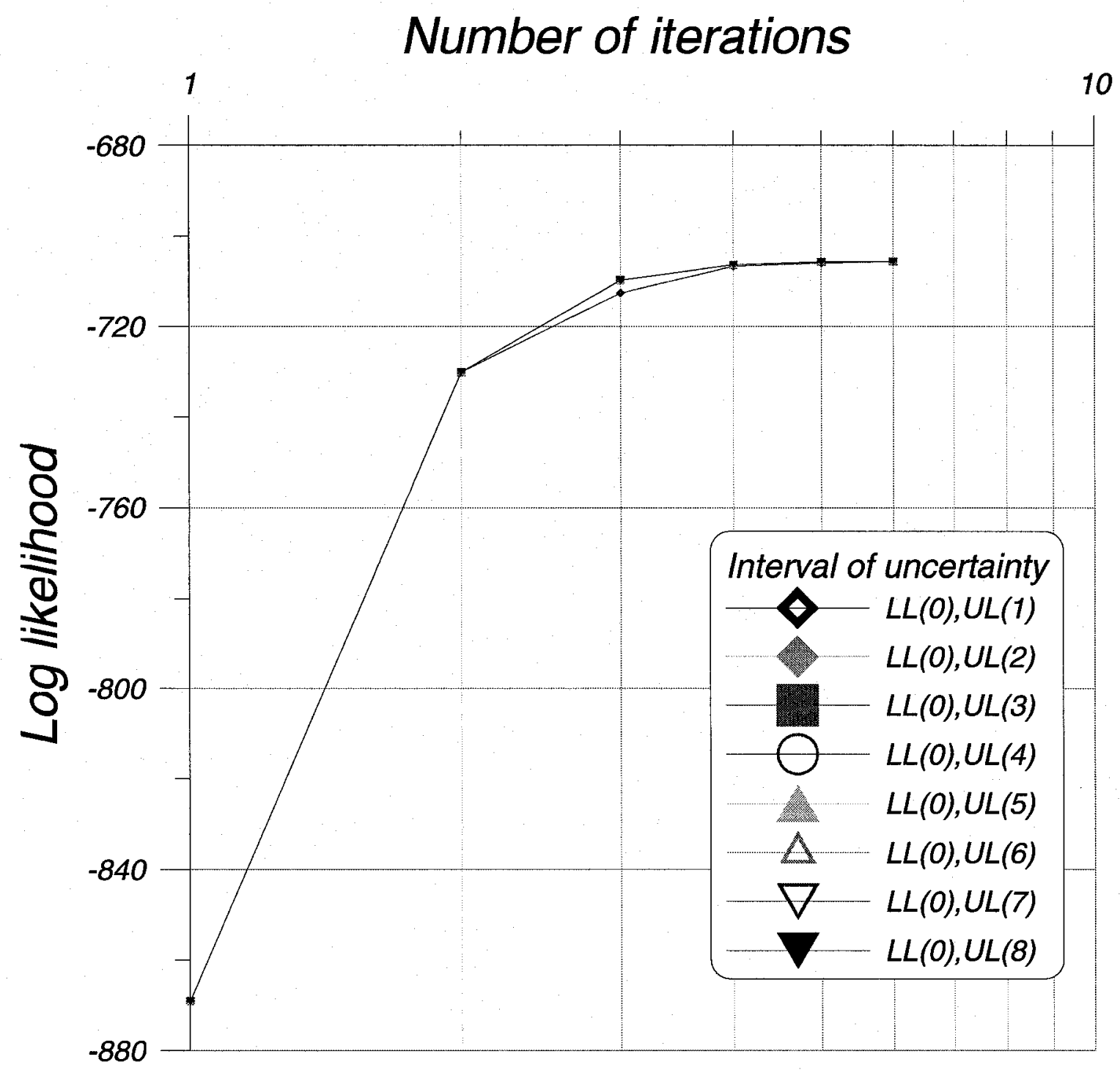

Figure 5-120: Log-likelihood values for the all uncertain intervals in BFGS-GSM algorithm responding to a new way of calculating the initial Hessian matrix adopted from BHHH-2 algorithm (exp. 6) 


\subsection{Comparison of estimation performance on the basis of each algorithm}

In the following three sections, different estimation performance achieved by applying different standard of estimation factors is compared, with an emphasis on operational behaviour of each algorithm. The estimation factors considered here are convergence criterion (5.5.1), initial starting points (5.5.2), and initial Hessian matrix (5.5.3).

\subsubsection{Effect of convergence criterion on estimation performance}

As shown in figure 5-121, the results show that with increased accuracy of convergence criterion, estimation performance deteriorates for the first four algorithms with all step sizes in terms of the number of iterations (see e, $\mathrm{f}, \mathrm{g}, \mathrm{h}$ of figure 5-121) and convergence time (see $\mathrm{i}, \mathrm{j}, \mathrm{k}, 1$ of figure 5-121). Exp 1-1 $\left(C R=10^{-4}\right)$ used a convergence criterion that is different in mathematical expression from Exp 1-2 and it is excluded from this comparison. CR represents a convergence criterion.

In addition, as depicted in figure 5-122, for the first two of four algorithms, a similar result is observed (see e, $\mathrm{f}, \mathrm{i}, \mathrm{j}$ of figure 5-122). Specifically, in the last two algorithms (i.e., DFP-GSM and BFGS-GSM), the pattern obtained with increasing accuracy of convergence criterion estimation performance that deteriorates does not hold for some uncertain intervals. 


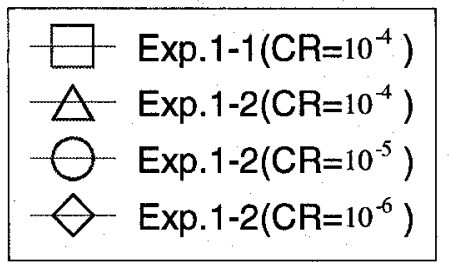

Newton Raphson

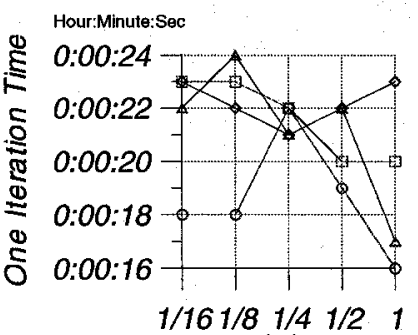

(a)

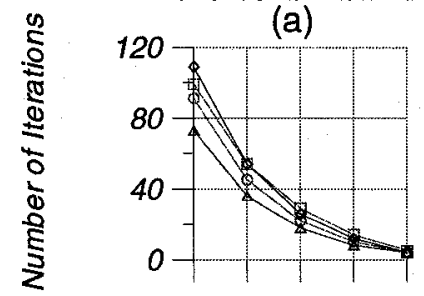

$\begin{array}{llll}1 / 161 / 8 & 1 / 4 & 1 / 2 \quad 1\end{array}$

(e)

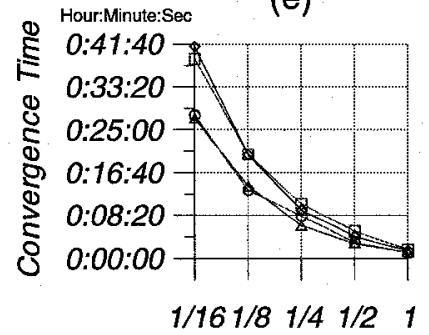

(i)

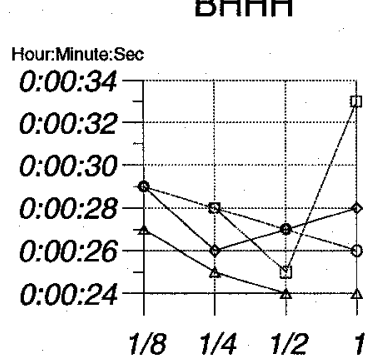

(b)

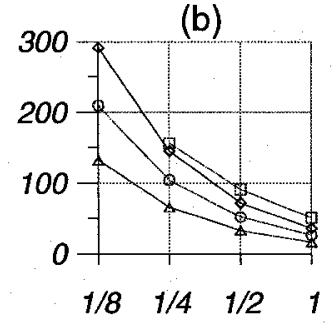

(f)

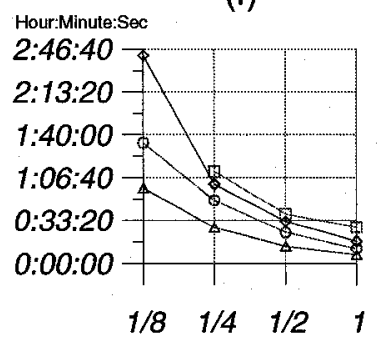

(j)
BHHH-2

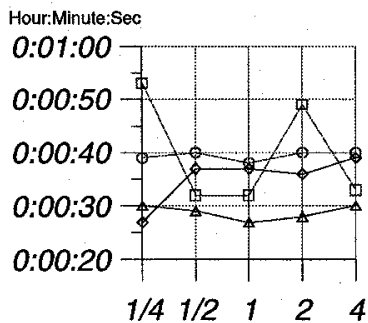

(c)

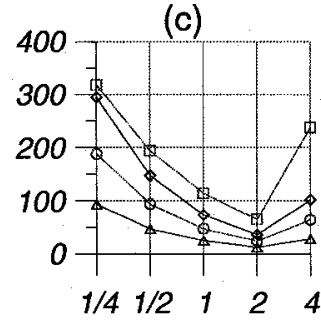

(g)

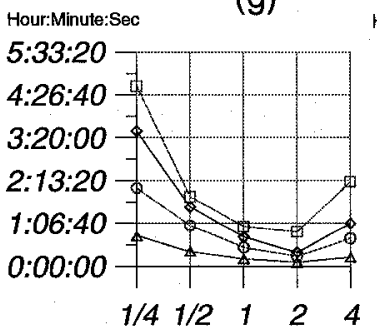

(k)
Steepest Ascent

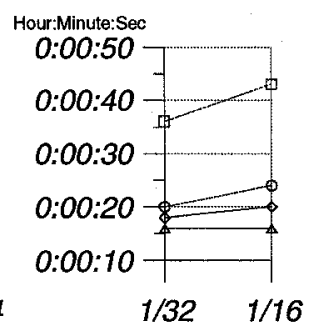

(d)

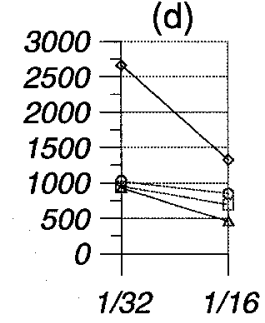

(h)

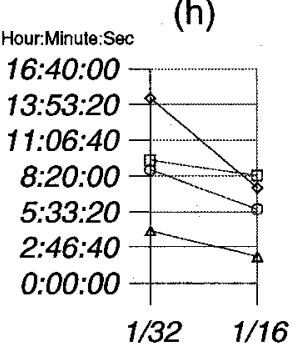

(I)

Figure 5-121: Variation of performance caused by different convergence criterion (1) 


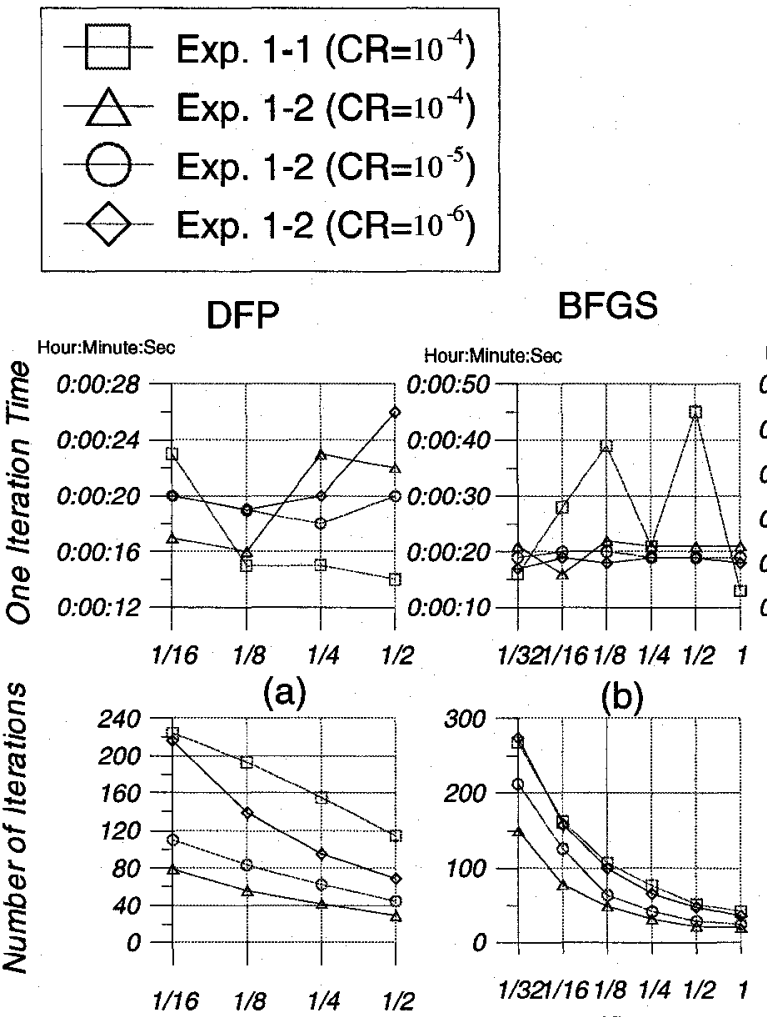

(e)

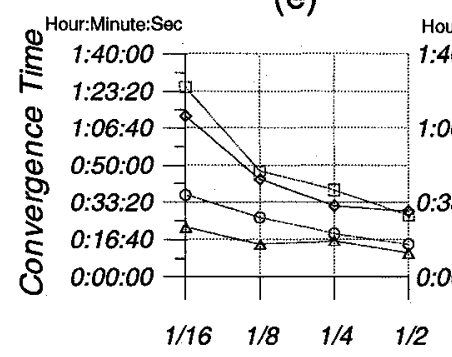

(i) (f)

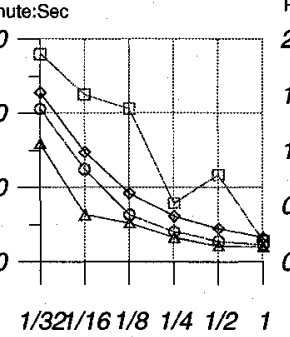

(j)

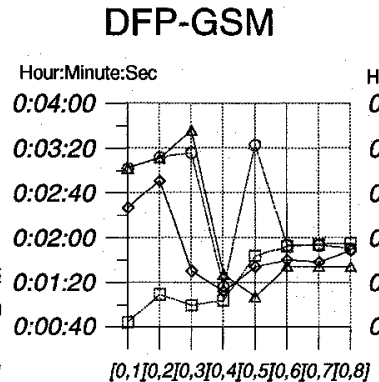

(c)

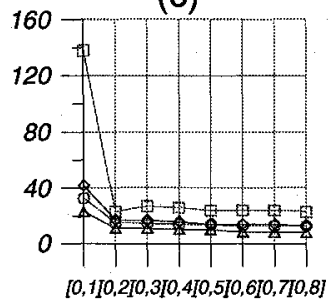

(g)

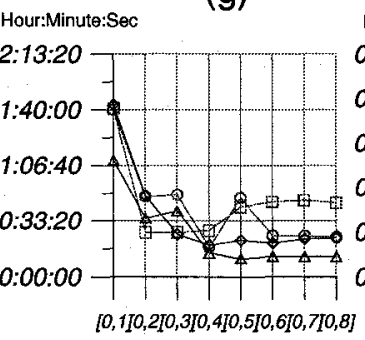

(k)
BFGS-GSM

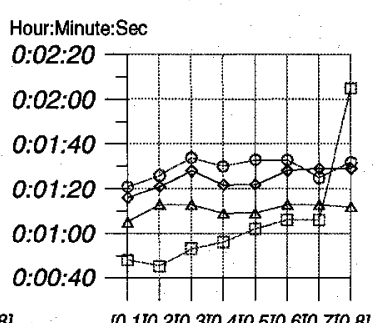

(d)

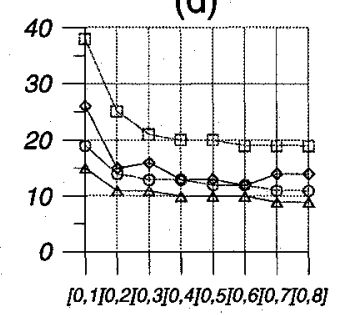

(h)

Hour:Minute:Sec

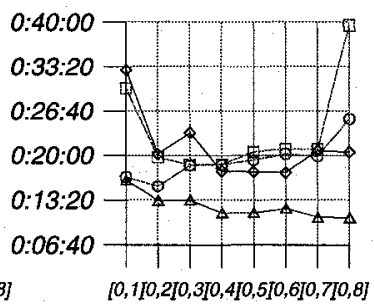

(I)

Figure 5-122: Variation of performance caused by different convergence criterion (2) 


\subsubsection{Effect of initial starting points on estimation performance}

Two different sets of initial starting parameters are applied in model estimation in order to see different estimation performance. As can be observed in figures 5-123 and 5-124, a vector of initial starting points made a difference in estimation performance. Also, the differences change diversely according to both step size (see e, f, g, h, i, j, k, l of figure 5123 and e, f, i, j of figure 5-124) and uncertain intervals (see g, h, k, 1 of figure 5-124).

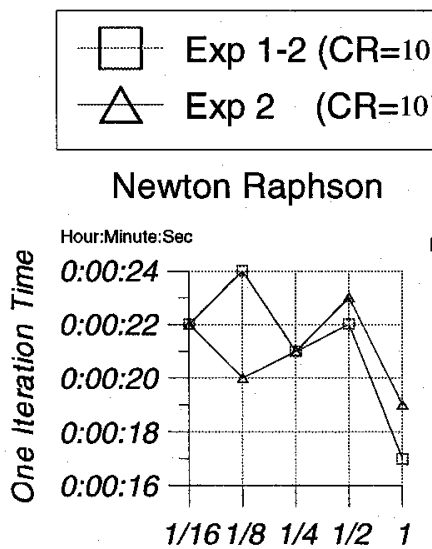

(a)

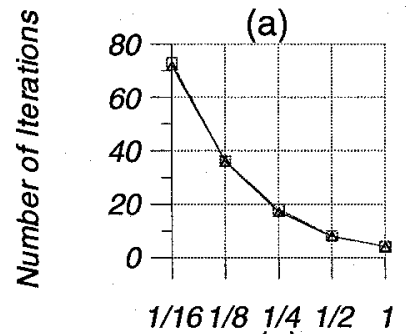

(e)

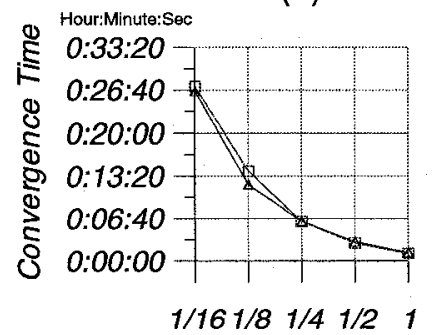

(i)

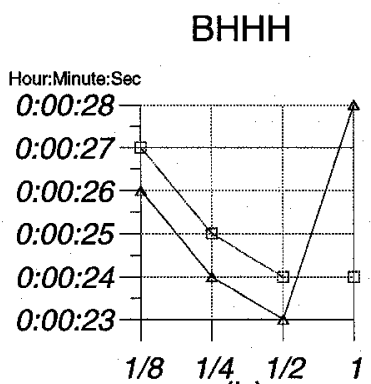

(b)

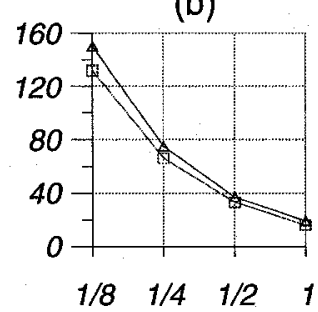

(f)

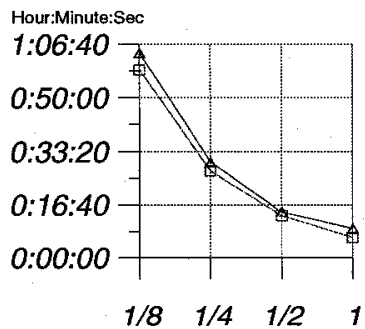

(j)

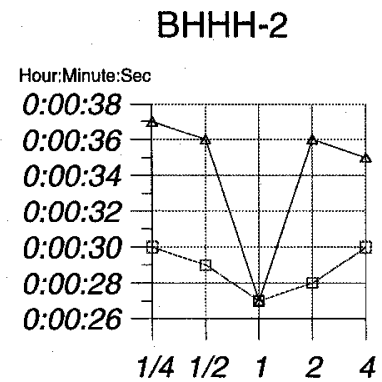

(c)

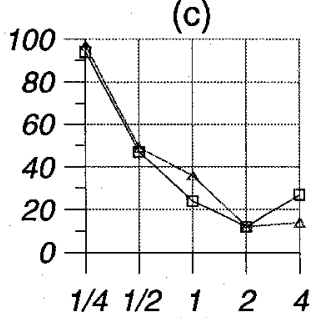

(g)

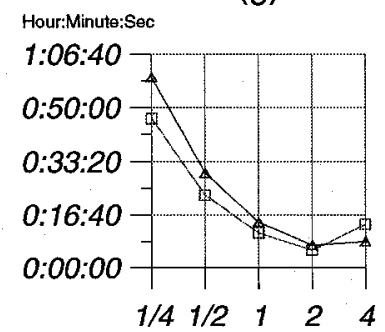

(k)

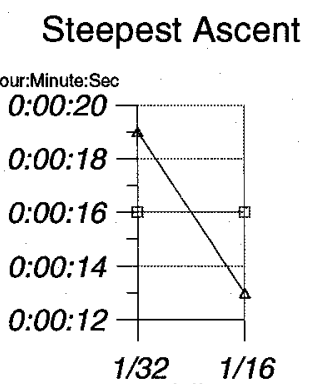

(d)

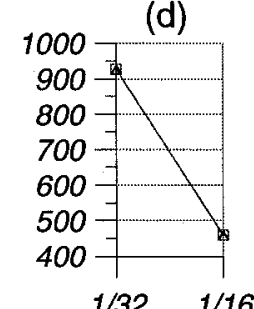

(h)

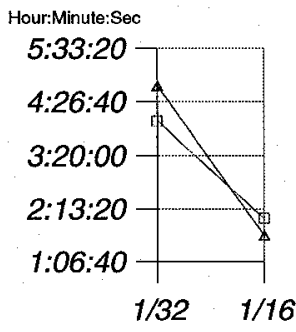

(I)

Figure 5-123: Variation of performance caused by different initial starting points (1) 


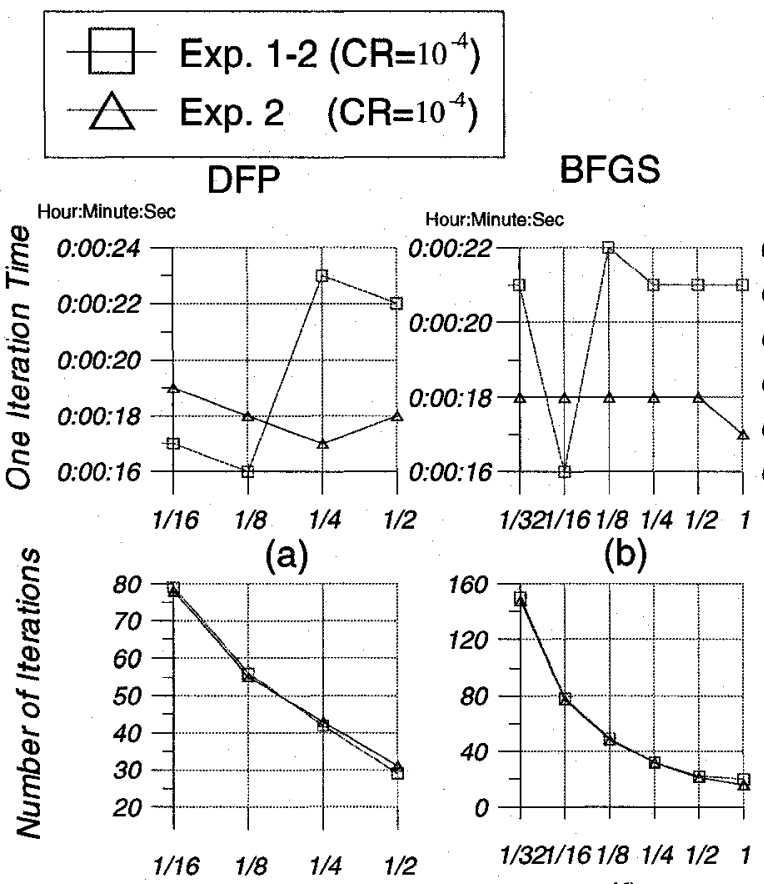

(e)

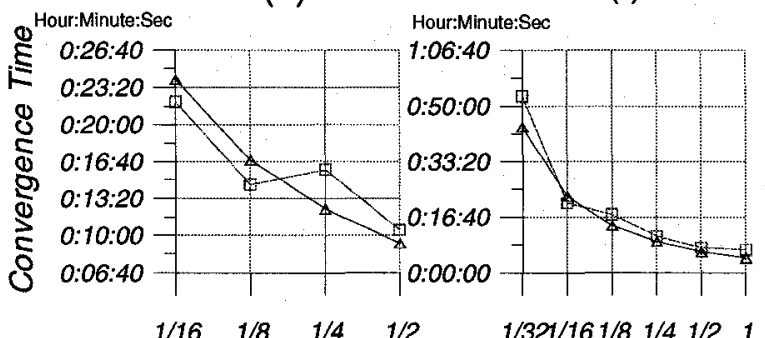

(i)

(j)

\section{DFP-GSM}

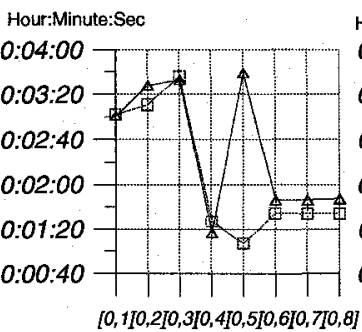

(c)

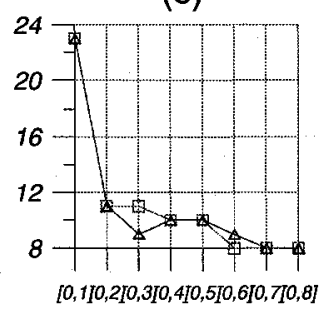

(g)

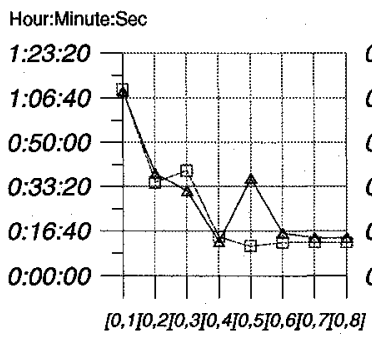

(k)

\section{BFGS-GSM}

Hour:Minute:Sec

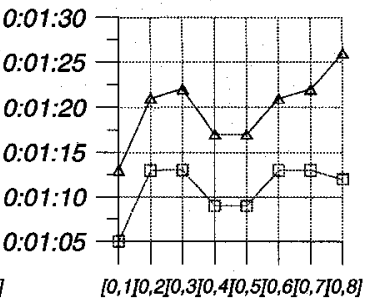

(d)

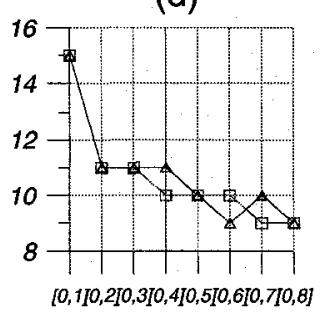

(h)

Hour:Minute:Sec

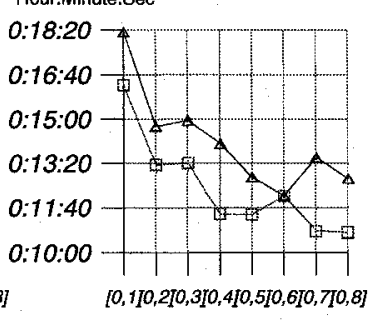

(I)

Figure 5-124: Variation of performance caused by different initial starting points (2) 


\subsubsection{Effect of initial Hessian matrix on estimation performance}

As presented in figure 5-125, the effect of adopting a different initial Hessian matrix calculated from using both $\mathrm{BHHH}$ and BHHH-2 methods is investigated for four algorithms. These four algorithms use an identity matrix at the initial stage of estimation in general routine. The results show an improvement in estimation performance for all step sizes and uncertain intervals in terms of the number of iterations and convergence time (see e, f, g, h, i, j, k, l of figure 5-125).
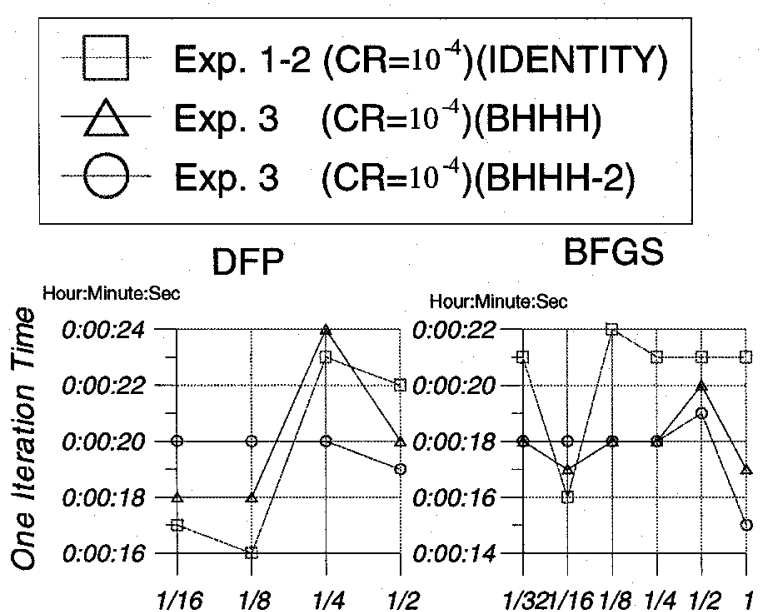

(a)

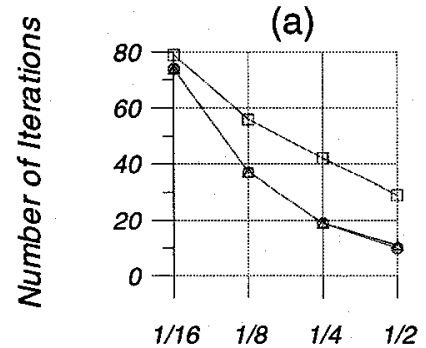

(e)

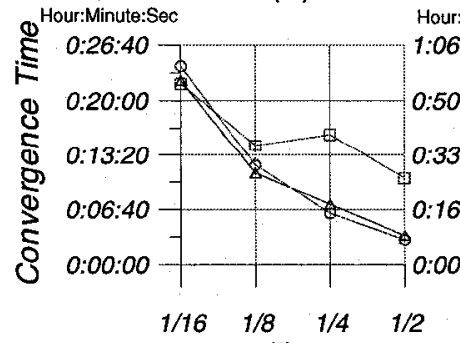

(i)

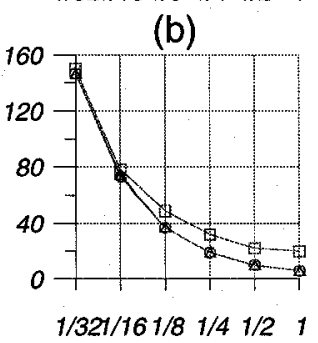

(f)

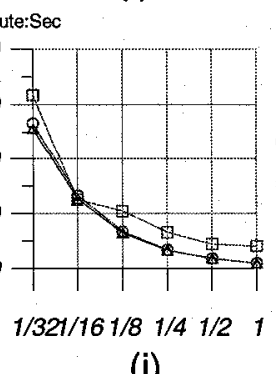

(j)
DFP-GSM

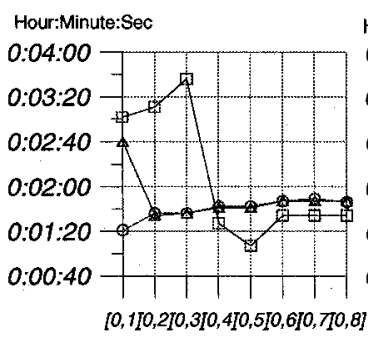

(c)

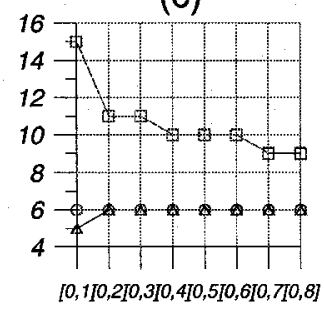

(g)

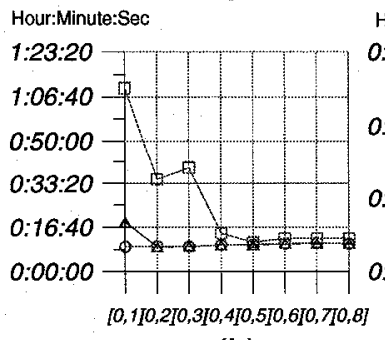

(k)
BFGS-GSM

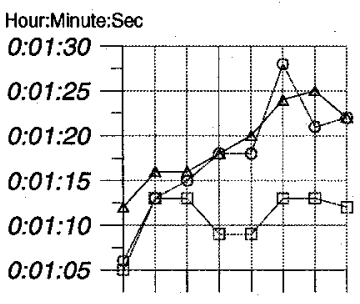

(d)

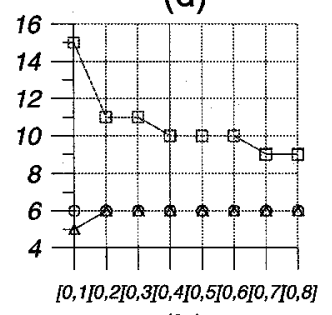

(h)

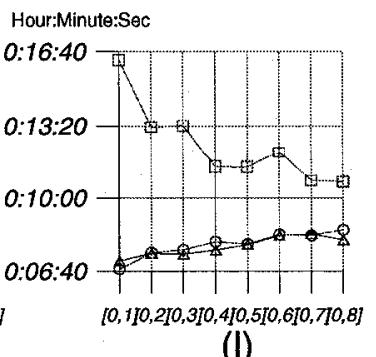

(I)

Figure 5-125: Variation of performance caused by different initial Hessian matrix 


\section{Chapter 6 Summary and Conclusions}

\subsection{Summary}

This section presents a summary of the results obtained from a set of experimental estimations of the multinomial logit model that is estimated using eight different estimation algorithms, and highlights lessons learned.

Discrete choice analysis has been of interest to researchers in many diverse disciplines who wished to predict choice behaviour of consumers. Therefore, there have been many applications to solve those problems. Particularly, in transportation fields, there have also been many applications of discrete choice modelling techniques to predict the behaviour of users of transportation systems or services. However, almost all works associated with discrete choice modelling focused only on the interpretation of the estimated results or concentrated on finding some useful meaning to support transportation policy or to establish a new one. Furthermore, almost all these types of discrete choice models were estimated using commercial statistical packages. In the earlier generation of discrete choice modelling, it was common practice to follow instructions of a selected specific computer package. However, as the choice model specification becomes more complicated, researchers working in the modelling field are challenged to generate the necessary computer code. However, there is little help available from the transportation planning literature. Some literatures on this can be found in econometric fields (Belsley, 1979; Belsley, 1980; Bunch, 1988) but they are not directly related to the concerns of transportation modeller. Also, these studies have a different theoretical base and it is difficult to apply the computer code to transportation modelling. 
In order to overcome this hurdle, this thesis research can be used as a guide by transportation modellers. They can use the eight algorithms with their own data and compare results reported in this thesis.

The following is a summary of the experimental estimations conducted in this thesis. The experiments are categorized according to factors that are important in estimation performance. To assess estimation algorithms themselves, the differences between eight algorithms are compared in terms of three performance measures in experiment 1.

- In experiment 1 , employing the first convergence criterion, Newton Raphson is the best estimation algorithm in terms of all three performance measures in spite of its difficulty of mathematical manipulation to calculate the real Hessian matrix of the given log-likelihood function. For the rest algorithms using approximate Hessian matrix, DFP-GSM and BFGS-GSM show stable performance in terms of both the number of iterations and the convergence time irrespective of the choice of any uncertain interval. BHHH and BHHH-2, DFP and BFGS show very diverse estimation performance, depending on the selection of the different step sizes at the initial stage of estimation.

To find out the factors that dominate model estimation performance, the following subjects are of major interest in this thesis research: the convergence criterion, initial guessing of starting points, initial Hessian matrix. 
- The first category considered in experimental estimation is to adopt convergence criterion that is different in both its mathematical expression and its degree of stopping precision as given in table 5-2. The tests on using the first category were carried out as experiments 1-4. The purpose of these experiments was to show the evidence of changing estimation performance, to compare them in terms of performance measures, and to recognize the convergence criterion as an important factor dominating the performance of estimation process.

- As an initial guess of the starting value of a vector of parameter, the value of $\beta_{k}=\left[\beta_{1}=0, \beta_{2}=0, \beta_{3}=0, \cdots, \beta_{9}=0\right]^{\prime}$ is used for all experiments 1-4.

- The first convergence criterion is most widely used as a stopping criterion. It is sourced form Ben-Akiva and Lerman (1985) and its mathematical expression is $\left[\frac{1}{k} \sum_{k=1}^{k}\left(\beta_{t+1, k}-\beta_{t, k}\right)^{2}\right]^{1 / 2}<10^{-4}$

- The second, third and fourth convergence criteria have the following common mathematical expression $G_{t}^{\prime}\left(-H_{t}\right)^{-1} G_{t}<C R$, It was suggested by Belsley (1979). In this thesis, by changing a CR gradually to other more stringent values such as $10^{-4}, 10^{-5}$ and $10^{-6}$, we show the fact that the changes of model estimation performance result from a change of CR.

- The first six algorithms (i.e., (1)Newton Raphson, (2BHHH, (3)BHHH-2, 
(4) Steepest Ascent, (5DFP, (6BFGS) out of eight algorithms considered in this thesis are estimated using various step sizes throughout the experiments $1-4$ and only the converged runs are summarized and compared.

- The last two algorithms (i.e., (7DFP-GSM, (8BFGS-GSM) out of eight algorithms use the golden-section line search (GSM) method instead of choosing a step size manually before the estimation procedure begins. The GSM method finds an optimal value of step size automatically in each iteration, with which the log-likelihood function is maximized in every iteration process.

- To use the GSM in line search work, we should assign the interval of uncertainty, in which the optimal step size is investigated automatically and it is used in every iteration estimation procedure. In the context of this thesis, eight different uncertain intervals are proposed and the experimental estimation is run using each of them. Typically, there are no limitations in choosing uncertain intervals, if a researcher chooses the interval having a distance of between 0 and a certain value over 0 (Bazaraa et al., 1999).

- From results of experiments 1-4, we identify several facts as follows: (1) In spite of using the same model specification (i.e., using the same multinomial logit choice function, the same utility functions for each alternatives) and common data set, there exist large or small differences among eight estimations algorithms in terms of three performance measures. (2) Depending on the step size, large 
variations are generated in the estimation performance among eight different algorithms or even within the same algorithm. In conclusion, the step size chosen by a researcher at an initial stage of model estimation can be an important control factor controlling the entire estimation performance while the model is under estimation. (3) As opposed to choosing a step size randomly as required in the first six estimation algorithms, in this thesis research, a new algorithm employs a golden section line search method (GSM) for finding the optimal step size automatically in every iteration. The two algorithms tried, DFP-GSM and BFGSGSM, show a stable estimation performance in terms of measures used regardless of using any uncertain intervals. By using this method, a researcher can minimize the risk of choosing a bad step size, which can happen in the case of first six estimation algorithms.

- The facts observed from a comparison of experiment 1 with experiment 2 are as followings: (1) To consider a case of applying the same level of stopping precision but different mathematical expression of stopping criterion, we apply $G_{t}^{\prime}\left(-H_{t}\right)^{-1} G_{t}<10^{-4}$ as a stopping criterion of experiment 2, and apply $\left[\frac{1}{k} \sum_{k=1}^{k}\left(\beta_{t+1, k}-\beta_{t, k}\right)^{2}\right]^{1 / 2}<10^{-4}$ as a stopping criterion of experiment 1 . (2) The result of experiment 2 employing the second type of stopping criterion shows a better estimation performance in terms of three performance measures used in this thesis: less time in one iteration, smaller number of iterations and faster convergence time. (3) This means that different types of stopping criteria can lead 
to different estimation results and show a different estimation performance, even in the same modelling situation.

- Observations drawn from the comparisons of experiments 2,3 and 4 are as follows. In this case, the same mathematical expression of stopping criterion is used but different stopping precision is employed. (1) To consider a case of applying a different stopping precision, we apply $G_{t}^{\prime}\left(-H_{t}\right)^{-1} G_{t}<10^{-4}$ as a stopping precision for experiment 2 , apply $G_{t}^{\prime}\left(-H_{t}\right)^{-1} G_{t}<10^{-5}$ as a stopping precision for experiment 3 , and apply $G_{t}^{\prime}\left(-H_{t}\right)^{-1} G_{t}<10^{-6}$ as a stopping precision for experiment 4 . (2) The results obtained with increasing precision of stopping criterion (i.e., experiment 2- experiment 3- experiment 4), the performance degenerates in terms of performance measures: taking more time in one iteration, more iterations before converging, and taking more time before convergence. (3) The reason for this phenomenon can be attributed to the fact that applying the more stringent stopping criterion necessitates more calculation time to satisfy the more stringent stopping criterion before terminating iteration procedures. (4) This result implies the fact that a stopping precision applied in the same stopping criterion is another control measure having a big impact on model estimation performance.

- The second category is to use a different initial guess of the parameter estimates of model. This is the subject of experiment 5 . 
- Here, the stopping criterion is $G_{t}^{\prime}\left(-H_{t}\right)^{-1} G_{t}<10^{-4}$.

- The main concern of experiment 5 is to recognize the importance of initial guess of starting points and to show an example of experimental estimation results reflecting the fact that the estimation performance can be affected by use of different starting points.

- Two types of strategies of initial guess of starting points were used.

- The first one is $\beta_{k}=\left[\beta_{1}=-0.1, \beta_{2}=-0.1, \beta_{3}=0, \cdots, \beta_{9}=0\right]^{\prime}$; It is applied to the first four estimation algorithms.

- The second is $\beta_{k}=\left[\beta_{1}=-0.01, \beta_{2}=-0.1, \beta_{3}=0, \cdots, \beta_{9}=0\right]^{\prime}$; this one is applied to the last four estimation algorithms.

- Although a new idea of finding good initial starting points is not proposed, it is important to know that changing initial starting points can result in different estimation performance and that the development of a new systematic way of guessing starting points guaranteeing a better operational performance would be a useful future research topic.

- Overall, the results obtained from experimental estimation are as follows: (1) Starting log-likelihood values are different from other experiments due to 
adopting new sets of starting points. (2) In the case of Steepest Algorithm, the convergence time greatly decreases compared to the results of other experiments. The improvement of estimation performance means that if a researcher can specify properly parameter's characteristics based on good prior knowledge (e.g., sign or magnitude of parameter estimates) or if a researcher can develop new scientific way of guessing starting points, estimation performance may be improved. (3) For BHHH-2 and Steepest Ascent algorithms, the converged loglikelihood values are somewhat different from the values of other algorithms, but the reason is not clear in this thesis.

The third experimental category is on employing a different way of calculating the initial Hessian matrix instead of using identity matrix. This activity is carried out in experiment 6.

- The applied convergence criterion is $G_{t}^{\prime}\left(-H_{t}\right)^{-1} G_{t}<10^{-4}$.

- The applied initial guess of starting points of a vector of parameters is $\beta_{k}=\left[\beta_{1}=0, \beta_{2}=0, \beta_{3}=0, \cdots, \beta_{9}=0\right]^{\prime}$.

- The third category is only applied to algorithms which basically use an identity matrix as an initial Hessian matrix in the model estimation procedures (i.e., DFP, BFGS, DFG-GSM, BFGS-GSM) because the first four algorithm do not use a way of guessing initial Hessian matrix at the initial stage of estimation. 
- An innovative way of getting a new initial Hessian matrix is possible by incorporating the method of both $\mathrm{BHHH}$ and $\mathrm{BHHH}-2$ for getting an initial Hessian matrix in the first iteration.

- By adding codes associated with the calculation of the initial Hessian matrix used in both BHHH and BHHH-2 algorithms to general estimation procedures of the above four algorithms, new estimation codes that differ only in the way of getting initial Hessian matrix were written. They are named as DFP (BHHH), BFGS (BHHH-2) and DFP-GSM (BHHH), BFGS-GSM (BHHH-2).

- An important lesson lessened from a given series of experimental estimations is to know that applying a different way of guessing initial Hessian matrix can make a difference in estimation performance assessed according to specified measures.

- In the context of this thesis research, the results of experiment 6 are in general better than that of experiment 2 . These use the same condition of stopping criterion, but differ in terms of the method for acquiring the initial Hessian matrix. It means that a better way of guessing the initial Hessian matrix, unlike the general procedures (i.e., an identity matrix as an initial Hessian matrix), would improve the estimation performance such as convergence time or the number of iterations. This issue can be another topic with potential to generate useful research results in econometric modelling. 
- The results obtained from experiment 6 are as follows: (1) In term of the single iteration time, there is no big difference between experiment 2 and experiment 6 . However, when using a new routine for experiment 6 , the single iteration time becomes similar among runs irrespective of using both different step size (in the case of DFP (BHHH), BFGS (BHHH) and DFP (BHHH-2), BFGS (BHHH-2)) and different uncertain intervals (in the case of DFP-GSM (BHHH), BFGS-GSM (BHHH) and DFP-GSM (BHHH-2), BFGS-GSM (BHHH-2)). (2) As for the number of iterations, the new routine used in experiment 6 shows better performance (i.e., a decrease in the number of iterations). (3) In term of the convergence time, there is a big difference between experiment 2 and experiment 6. The experiment 6 takes much less convergence time than that of experiment 2 . (4) The reason for this phenomenon is that using a more accurate initial Hessian matrix, which can be calculated and input into the routine of estimation procedure instead of using an identity matrix, would decrease the iteration time needed to reach the final Hessian matrix of when the estimation is terminated.

\subsection{Conclusions}

The multinomial logit model is the test model of the entire experimental estimation in this thesis research and the comparison of the eight different estimation algorithms pedagogically important in discrete mode choice modelling is the main subject of this thesis. The eight algorithms are crucial for a researcher to be able to conduct modelling with his/her own tailor-made code. Furthermore, the understanding of estimation procedures of discrete choice model is an asset to a researcher. From the experience 
gained in model estimation involving eight different algorithms and the log-likelihood estimator, the following conclusions are drawn.

(1) The multinomial logit (MNL) is the "standard" for all other discrete choice models such as probit, mixed logit, and generalized extreme value (GEV) models.

(2) The real mode choice data (540 observations) proved to be an asset in modelling and analyses.

(3) The maximum likelihood method (ML) used as an estimator is intuitive in its conceptual meaning.

(4) Visual Basic Application (VBA) provided in EXCEL proved to be very appropriate for developing code for the eight estimation algorithms.

(5) Newton Raphson is the best among all algorithms tested in this thesis research. A complex feature of this algorithm is the calculation of the real Hessian matrix of the given log-likelihood function. It is fully compensatory in its estimation performance compared to its competitors in order to overcome the complexity of its calculations. However, this thesis research has demonstrated that the calculation of the real Hessian for Newton Raphson is straightforward with the tailor-made code. 
(6) The second best algorithms are not clear, but DFP and BFGS are well positioned. These quasi-newton methods show similar performance due to the similarity in calculating and updating Hessian matrix. Updating rule of Hessian matrix of this quasi-newton method differentiates these two algorithms from other algorithms. BFGS is slightly more complex in Hessian updating formula than that of DFP.

(7) The worst algorithm is the Steepest Ascent method in terms of all performance measures. The reason can be attributed to its worst mechanism of finding a new vector of parameter with which the log-likelihood function's value is updated in each iteration.

(8) By incorporating the golden section line search method, two new estimation algorithms, called DFP-GSM and BFGS-GSM, are developed and tested for the first time in this thesis research. These perform better than DFP and BFGS.

(9) Important factors identified through six experimental estimations are as follows:

- The extent of closeness of each Hessian matrix of eight algorithms dominates the correctness of estimation results and makes a difference in performance between algorithms. The most correct Hessian matrix is that of Newton Raphson and other seven of algorithms proposed in this thesis research use approximated Hessian matrix in their estimation routine.

- A new method for calculation of the initial Hessian matrix used in this research 
proved to be beneficial.

- Selecting a step size is an important element when using the custom-built code. This research provides insights about this issue.

- Convergence criterion is another important factor dominating the entire estimation performance.

\subsection{Recommendations}

(1) Tailor-made coding of given specific modelling situation can be very helpful to appreciate and understand the details of the estimation procedures.

(2) In a small-size modelling, Newton-Raphson is strongly recommended. It is fast and absolutely correct. In such situation, the complexity of mathematical manipulations does not become an issue.

(3) In the case of handling huge data, such as in transportation demand modelling, especially in discrete choice modelling, the use of approximate Hessian methods (BHHH, BHHH-2, DFP, BFGS, DFP-GSM, BFGS-GSM) is recommended in order to save cost and efforts associated with the model estimation process. However, in the context of this thesis research, almost all mathematical treatments of Newton Raphson was clear, not difficult, and straightforward. Consequently, the Newton Raphson method should therefore be reconsidered as a 
competitive candidate even in huge scale modelling works.

(4) Understanding estimation algorithms is critical in cases where a researcher develops a new advanced model that is not supported by commercial statistical packages. Specifically, in the current new generation of discrete choice modelling activities, these technique and knowledge of coding of given modelling situation are essential.

\subsection{Future work}

(1) New trials of incorporating a new line-search method (non-linear programming method) could be useful for discovering a new estimation algorithm.

(2) A new systematic way of guessing starting points should be investigated. Such a new method will be an improvement over the current state-of-practice of guessing initial starting points.

(3) The use of the same experimental setting reported in this thesis for other discrete choice models (i.e., nested logit model, GEV model, and so on) could be useful for understanding operational characteristic according to the different model specifications. 


\section{References}

Bazaraa, Mokhtar S.; Sherali, Hanif D.; Shetty, C.M. (1999). Nonlinear Programming: Theory and Algorithms, John Wiley \& Sons, NY.

Belsley, David A. (1979). "On the computational competitiveness of full-information maximum-likelihood and three-stage least-squares in the estimation of nonlinear, simultaneous-equations models." Journal of Econometrics, 9, 315-342.

Belsley, David A. (1980). "On the efficient computation of the nonlinear full-information maximum-likelihood estimator." Journal of Econometrics, 14, 203-225.

Ben-Akiva, M. and Lermans, S. R. (1985). Discrete Choice Analysis Theory and Application to Travel Demand. MIT, MA.

Ben-Akiva, Moshe; McFadden, Daniel; Abe, Makoto; Böckenholt, Ulf; Bolduc, Denis; Gopinath, Dinesh; Morikawa, Takayuki; Ramaswamy, Venkatram; Rao, Vithala; Revelt, David; Steinberg, Dan (1997). "Modelling methods for discrete choice analysis." Marketing Letters, 8(3), 273-286.

Ben-Akiva, Moshe; McFadden, Daniel; Train, Kenneth; Walker, Joan; Bhat, Chandra; Bierlaire, Michel; Bolduc, Denis; Boersch-Supan, Axel; Brownstone, David; Bunch, David S.; Daly, Andrew; de Palma, Andre; Gopinath, Dinesh; Karlstrom, Anders; Munizaga, Marcela A. (2002). "Hybrid choice models: progress and challenges." Marketing Letters, 13(3), 163-175.

Bhat, Chandra R. (1998a). "Accommodating flexible substitution patterns in multidimensional choice modelling: formulation and application to travel mode and departure time choice." Transportation Research Part B, 32(7), 455-466.

Bhat, Chandra R. (1998b). "Accommodating variations in responsiveness to level-of-service measures in travel mode choice modelling." Transportation Research Part A, 32(7), 495 507.

Bhat, Chandra R. (1995). "A heteroscedastic extreme value model of intercity travel mode choice." Transportation Research Part B, 29(6), 471-483. 
Bhat, Chandra R. (2000). "A multi-level cross-classified model for discrete response variables." Transportation Research Part B, 34, 567-582.

Bhat, Chandra R. (1997). "Covariance heterogeneity in nested logit models: econometric structure and application to intercity travel." Transportation Research Part B, 31(1), 471483.

Bhat, Chandra R. (2001). "Quasi-random maximum simulated likelihood estimation of the mixed multinomial logit model." Transportation Research Part B, 35, 677-693.

Bhat, Chandra R. and Zhao, Huimin (2002). "The spatial analysis of activity stop generation." Transportation Research Part B, 36, 557-575.

Brownstone, David; Bunch, David; Train, Kenneth (2000). "Joint mixed logit models of stated and revealed preferences for alternative-fuel vehicles." Transportation Research Part B, 34, 315-338.

Brownstone, David and Train, Kenneth (1999). "Forecasting new product penetration with flexible substitution patterns.” Journal of Econometrics, 89, 109-129.

Bunch, David S. (1988). "A comparison of algorithms for maximum likelihood estimation of choice models." Journal of Econometrics, 38, 145-167.

Grapher User's Guide (2000). Graphing Software for Scientists and Engineers. Golden Software, Inc. U.S.A

Garrido, Rodrigo A. and Mahmassani, Hani S. (2000). "Forecasting freight transportation demand with the space-time multinomial probit model." Transportation Research Part B, 34, 403-418.

Greene, William H. (2000). Econometric Analysis. $4^{\text {th }}$ edn, Prentice Hall, Upper Saddle River, NJ.

Harvey, Greig (1987). "Airport choice in a multiple airport region." Transportation Research Part A, 21A (6), 439-449. 
Harvey, Greig (1986). "Study of airport access mode choice." Journal of Transportation Engineering, 112 (5) 525-545.

Hensher, David A.; Rose, John M.; Greene, William H. (2005). Applied Choice Analysis: A Primer. Cambridge University, NY.

Hess, Stephane and Polak, John W. (2005). "Mixed logit modelling of airport choice in multi-airport regions." Journal of Air Transport Management, 11, 59-68

Hunt, John Douglas and Abraham, John Edward (2004). "Stated response examination of factors influencing commercial movement route choice behaviour." Journal of Transportation Engineering, 72-77.

Koppelman, Frank S. and Bhat, Chandra (2006). A Self Instructing Course in Mode Choice Modelling: Multinomial and Nested Logit Models. U.S. Department of Transportation Federal Transit Administration.

Lee, Hyeong B. (1999). Hangeul Excel 2000, Cyber press, Seoul.

Lee, Hyeong B. (2000). Hangeul Excel 2000 VBA, Cyber press, Seoul.

Lee, Hyeong B. (1998). Visual Basic 6, Cyber press, Seoul.

Liu, Yu-Hsin and Mahmassani, Hani S. (2000). "Global maximum likelihood estimation procedure for multinomial probit (MNP) model parameters." Transportation Research Part B, 34, 419-449.

McFadden, D. (1974). "Conditional logit analysis of qualitative choice behaviour." Frontiers in Econometrics, P.Zarembka, ed. Academic Press, New York, 105-142.

Ohashi, Hiroshi; Kim, Tae-Seung; Oum, Tae Hoon; Yu, Chunyan (2005). "Choice of air cargo transshipment airport: an application to air cargo traffic to/from Northeast Asia." Journal of Air Transport Management, 11, 149-159. 
Psaraki, Voula and Abacoumkin, Costas (2002). "Access mode choice for relocated airports: the new Athens international airport." Journal of Air Transport Management, 8, 89-98.

Revelt, David and Train, Kenneth (1998). "Mixed logit with repeated choice: households' choices of appliance efficiency level." Review of Economics and Statistics, 80(4), 647657.

Roh, Hyukjae (2001). "Mode choice behaviour in Ground travel for kimpo international airport users: case of kimpo international airport passenger terminal." M.C.P thesis, Seoul National University, Seoul.

Sandor, Zsolt and Train, Kenneth (2004). "Quasi-random simulation of discrete choice models." Transportation Research Part B 38, 313-327.

Train, Kenneth (2003). Discrete Choice Methods with Simulation. Cambridge University, NY.

Washington, Simon p.; Karlaftis, Matthew G.; Mannering, Fred L. (2003). Statistical and Econometric Methods for Transportation Data Analysis, Chapman \& Hall/CRC, NY. 
Appendix A: An Analytical Derivative of the Log-likelihood Function 


\section{A.1: Representative utility functions of all five alternatives}

$V($ Car $)=\beta_{1} T C_{C}+\beta_{2} T T_{C}+\beta_{3}$ Accom $+\beta_{4} \operatorname{Sexm}+\beta_{5}$ Age340

$V($ Taxi $)=\beta_{1} T C_{T}+\beta_{2} T T_{T}+\beta_{6}$ Tasc

$V($ Subway $)=\beta_{1} T C_{S}+\beta_{2} T T_{S}+\beta_{7}$ Sasc

$V($ Bus $)=\beta_{1} T C_{B}+\beta_{2} T T_{B}+\beta_{8}$ Basc

$V($ Limousine $)=\beta_{1} T C_{L}+\beta_{2} T T_{L}+\beta_{9}$ Lasc

\section{A.2: Multinomial logit choice probability function}

$P_{n}(i)=\frac{e^{V_{n i}}}{\sum_{j} e^{V_{n j}}}$

\section{A.3: Likelihood function}

$L\left(\beta_{1}, \beta_{2}, \cdots, \beta_{8}, \beta_{9}\right)=\prod_{N} \prod_{i} P_{n}(i)^{y_{i n}}=\prod_{N}\left[P_{n}(C)^{y_{C n}} \cdot P_{n}(T)^{y_{T n}} \cdot P_{n}(S)^{\gamma_{S n}} \cdot P_{n}(B)^{y_{B n}} \cdot P_{n}(L)^{y_{L n}}\right]$

\section{A.4: Log-likelihood function}

$$
\begin{aligned}
& L L\left(\beta_{1}, \beta_{2}, \cdots, \beta_{8}, \beta_{9}\right)=\sum_{n=1}^{N}\left[y_{C n} \ln \left(\frac{e^{V(\text { Car })}}{e^{V(\text { Car })}+e^{V(\text { Taxi })}+e^{V(\text { Subway })}+e^{V(\text { Bus })}+e^{V(\text { Limousine })}}\right)\right. \\
& +y_{T h} \ln \left(\frac{e^{V(\text { (Taxi) }}}{e^{V(\text { Car })}+e^{V(\text { Taxi })}+e^{V(\text { Subway })}+e^{V(\text { (Bus) })}+e^{V(\text { Limousine })}}\right) \\
& +y_{S n} \ln \left(\frac{e^{V(\text { Subway })}}{e^{V(\text { (Car) }}+e^{V(\text { Taxi })}+e^{V(\text { Subway })}+e^{V(\text { Bus })}+e^{V(\text { Limousine })}}\right) \\
& +y_{B n} \ln \left(\frac{e^{V(\text { Bus })}}{e^{V(\text { Car })}+e^{V(\text { Taxi })}+e^{V(\text { (Subway) }}+e^{V(\text { Bus })}+e^{V(\text { Limousine })}}\right) \\
& \left.+y_{L n} \ln \left(\frac{e^{V(\text { Limujin })}}{e^{V(\text { Car })}+e^{V(\text { Taxi })}+e^{V(\text { Subway })}+e^{V(\text { (Bus) }}+e^{V(\text { Limousine })}}\right)\right]
\end{aligned}
$$

\section{A.5: The derivation of a gradient vector formulas specified to given choice problem}

The gradient of the log-likelihood function is a vector of the first derivatives of $L L\left(\beta_{1}, \beta_{2}, \cdots, \beta_{k}\right)$ with respect to each parameter, which is a key factor to determine the 
direction to go in iteration method. It is a calculation mechanism used to estimate the model's parameters. Its role and functional form in each iteration rule of eight different algorithms appear in chapter 3. For more details, please see that chapter. In this appendix, as an essential part of writing a computer code of estimation procedure, we should derive the first order partial derivatives of the log-likelihood function specified in this thesis.

The important considerations in making a specific computer code of discrete mode choice model are to have a clear understanding of model estimation procedures and to identify the key factors having an important role in estimation. A probabilistic choice function and its log-likelihood function are an important part of choice modelling. Furthermore, mathematical expansion of the log-likelihood function for obtaining a gradient vector and the Hessian matrix of the given log-likelihood function are critical to make an estimation computer code. The most important part of writing a computer estimation code is to find a clear relationship between the formulas derived and the data used in estimation. Particularly, in discrete mode choice model, the form of choice model function appears to be simple but finding a relationship between the data and the formulas is not easy to find. In addition, the expansion of given log-likelihood function is computationally extensive and costly. Careful expansion is an asset to do this.

In this thesis, the final formulas of a gradient vector and the Hessian matrix are derived from the given log-likelihood function by using partial derivatives in analytical terms.

The first partial derivative with respect to the generic variables, $\beta_{1}, \beta_{2}$, is derived and it takes the form shown below: 
1) The first partial derivative with respect to $\beta_{1}$ is

$$
\begin{aligned}
\frac{\partial L L\left(\beta_{1}, \beta_{2}, \cdots, \beta_{8}, \beta_{9}\right)}{\partial \beta_{1}} & =\sum_{n=1}^{N}\left[y_{C n} \frac{1}{P n(C)_{n}}\left(\frac{\partial P n(C)_{n}}{\partial \beta_{1}}\right)+y_{T n} \frac{1}{P n(T)_{n}}\left(\frac{\partial P n(T)_{n}}{\partial \beta_{1}}\right)\right. \\
& \left.+y_{S n} \frac{1}{P n(S)_{n}}\left(\frac{\partial P n(S)_{n}}{\partial \beta_{1}}\right)+y_{B n} \frac{1}{P n(B)_{n}}\left(\frac{\partial P n(B)_{n}}{\partial \beta_{1}}\right)+y_{L n} \frac{1}{P n(L)_{n}}\left(\frac{\partial P n(L)_{n}}{\partial \beta_{1}}\right)\right]
\end{aligned}
$$

where

$$
\begin{aligned}
& \frac{1}{P n(C)}\left(\frac{\partial P n(C)}{\partial \beta_{1}}\right)=\left(T C_{C n}-T C_{T n}\right) P n(T)_{n}+\left(T C_{C n}-T C_{S n}\right) P n(S)_{n}+\left(T C_{C n}-T C_{B n}\right) P n(B)_{n}+\left(T C_{C n}-T C_{L n}\right) P n(L)_{n} \\
& \frac{1}{P n(T)}\left(\frac{\partial P n(T)}{\partial \beta_{I}}\right)=\left(T C_{T n}-T C_{C n}\right) P n(C)_{n}+\left(T C_{T n}-T C_{S n}\right) P n(S)_{n}+\left(T C_{T_{n}}-T C_{B n}\right) P n(B)_{n}+\left(T C_{T n}-T C_{L n}\right) P n(L)_{n} \\
& \frac{1}{P n(S)}\left(\frac{\partial P n(S)}{\partial \beta_{1}}\right)=\left(T C_{S n}-T C_{C n}\right) P n(C)_{n}+\left(T C_{S n}-T C_{T n}\right) P n(T)_{n}+\left(T C_{S n}-T C_{B n}\right) P n(B)_{n}+\left(T C_{S n}-T C_{L n}\right) P n(L)_{n} \\
& \frac{1}{P n(B)}\left(\frac{\partial P n(B)}{\partial \beta_{1}}\right)=\left(T C_{B n}-T C_{C n}\right) P n\left(C_{n}+\left(T C_{B n}-T C_{T n}\right) P n(T)_{n}+\left(T C_{B n}-T C_{S n}\right) P n(S)_{n}+\left(T C_{B n}-T C_{L n}\right) P n(L)_{n}\right. \\
& \frac{1}{P n(L)}\left(\frac{\partial P n(L)}{\partial \beta_{1}}\right)=\left(T C_{L n}-T C_{C n}\right) P n\left(C_{n}+\left(T C_{L n}-T C_{T n}\right) P n(T)_{n}+\left(T C_{L n}-T C_{S n}\right) P n\left(S_{n}+\left(T C_{L n}-T C_{B n}\right) P n\left(B h_{n}\right.\right.\right.
\end{aligned}
$$

2) The first partial derivative with respect to $\beta_{2}$ is

$$
\begin{aligned}
\frac{\partial L L\left(\beta_{1}, \beta_{2}, \cdots, \beta_{8}, \beta_{9}\right)}{\partial \beta_{2}} & =\sum_{n=1}^{N}\left[y_{C n} \frac{1}{P n(C)}\left(\frac{\partial P n(C)}{\partial \beta_{2}}\right)+y_{T n} \frac{1}{P n(T)}\left(\frac{\partial P n(T)}{\partial \beta_{2}}\right)\right. \\
& \left.+y_{S n} \frac{1}{P n(S)}\left(\frac{\partial P n(S)}{\partial \beta_{2}}\right)+y_{B n} \frac{1}{P n(B)}\left(\frac{\partial P n(B)}{\partial \beta_{2}}\right)+y_{L n} \frac{1}{P n(L)}\left(\frac{\partial P n(L)}{\partial \beta_{2}}\right)\right]
\end{aligned}
$$

where

$$
\begin{aligned}
& \frac{1}{P n(C)}\left(\frac{\partial P n(C)}{\partial \beta_{2}}\right)=\left(T T_{C n}-T T_{T n}\right) P n(T)_{n}+\left(T T_{C n}-T T_{S n}\right) P n(S)_{n}+\left(T T_{C n}-T T_{B n}\right) P n(B)_{n}+\left(T T_{C n}-T T_{L n}\right) P n(L)_{n} \\
& \frac{1}{P n(T)}\left(\frac{\partial P n(T)}{\partial \beta_{2}}\right)=\left(T T_{T n}-T T_{C n}\right) P n(C)_{n}+\left(T T_{T n}-T T_{S n}\right) P n(S)_{n}+\left(T T_{T_{n}}-T T_{B n}\right) P n(B)_{n}+\left(T T_{T n}-T T_{L n}\right) P n(L)_{n} \\
& \frac{1}{P n(S)}\left(\frac{\partial P n(S)}{\partial \beta_{2}}\right)=\left(T T_{S n}-T T_{C n}\right) P n(C)_{n}+\left(T T_{S n}-T T_{T n}\right) P n(T)_{n}+\left(T T_{S n}-T T_{B n}\right) P n(B)_{n}+\left(T T_{S n}-T T_{L n}\right) P n(L)_{n} \\
& \frac{1}{P n(B)}\left(\frac{\partial P n(B)}{\partial \beta_{2}}\right)=\left(T T_{B n}-T T_{C n}\right) P n(C)_{n}+\left(T T_{B n}-T T_{T n}\right) P n(T)_{n}+\left(T T_{B n}-T T_{S n}\right) P n(S)_{n}+\left(T T_{B n}-T T_{L n}\right) P n(L)_{n} \\
& \frac{1}{P n(L)}\left(\frac{\partial P n(L)}{\partial \beta_{2}}\right)=\left(T T_{L n}-T T_{C n}\right) P n(C)_{n}+\left(T T_{L n}-T T_{T n}\right) P n(T)_{n}+\left(T T_{L n}-T T_{S n}\right) P n(S)_{n}+\left(T T_{L n}-T T_{B n}\right) P n(B)_{n}
\end{aligned}
$$


The first partial derivatives with respect to the alternative-specific variable parameters, $\beta_{3}, \beta_{4}, \beta_{5}$ are derived. Their functional forms are presented below:

1) The first partial derivative with respect to $\beta_{3}$ is

$$
\begin{aligned}
\frac{\partial L L\left(\beta_{1}, \beta_{2}, \cdots, \beta_{8}, \beta_{9}\right)}{\partial \beta_{3}} & =\sum_{n=1}^{N}\left[y_{C n} \frac{1}{P n(C)}\left(\frac{\partial P n(C)}{\partial \beta_{3}}\right)+y_{T n} \frac{1}{P n(T)}\left(\frac{\partial P n(T)}{\partial \beta_{3}}\right)\right. \\
& \left.+y_{S n} \frac{1}{P n(S)}\left(\frac{\partial P n(S)}{\partial \beta_{3}}\right)+y_{B n} \frac{1}{P n(B)}\left(\frac{\partial P n(B)}{\partial \beta_{3}}\right)+y_{L n} \frac{1}{P n(L)}\left(\frac{\partial P n(L)}{\partial \beta_{3}}\right)\right]
\end{aligned}
$$

where

$$
\begin{aligned}
& \frac{\partial P n(C)}{\partial \beta_{3}}=\frac{A c c o m \times e^{V(\text { Car })}\left(e^{V(\text { Car })}+e^{V(\text { (Taxi })}+e^{V(\text { Subway })}+e^{V(\text { Bus })}+e^{V(\text { Limousin } e)}\right)-e^{V(\text { Car })}\left(\text { Accom } \times e^{V(\text { Car })}\right)}{\left(e^{V(\text { Car })}+e^{V(\text { Taxi })}+e^{V(\text { Subway })}+e^{V(\text { Bus })}+e^{V(\text { Limousine })}\right)^{2}} \\
& =\frac{A c c o m \times e^{V(\text { Car })}\left(e^{V(\text { Taxi })}+e^{V(\text { Subway })}+e^{V(\text { Bus })}+e^{V(\text { Limousin } e)}\right)}{\left(e^{V(\text { Car })}+e^{V(\text { Taxi })}+e^{V(\text { Subway })}+e^{V(\text { Bus })}+e^{V(\text { Limousin } e)}\right)^{2}} \\
& \frac{1}{P n(C)}\left(\frac{\partial P n(C)}{\partial \beta_{3}}\right)=\frac{A c c o m\left(e^{V(\text { Taxi })}+e^{V(\text { Subway })}+e^{V(\text { Bus })}+e^{V(\text { Limousine })}\right)}{\left(e^{V(\text { Car })}+e^{V(\text { Taxi })}+e^{V(\text { Subway })}+e^{V(\text { Bus })}+e^{V(\text { Limousine })}\right)} \\
& =\operatorname{Accom}[\operatorname{Pn}(T)+\operatorname{Pn}(S)+P n(B)+P n(L)] \\
& \frac{\partial \operatorname{Pn}(T)}{\partial \beta_{3}}=\frac{0-e^{V(\text { Taxi })}\left(\text { Accom } \times e^{V(\text { Car })}\right)}{\left(e^{V(\text { Car })}+e^{V(\text { Taxi })}+e^{V(\text { Subway })}+e^{V(\text { Bus })}+e^{V(\text { Limousin } e)}\right)^{2}} \\
& \frac{1}{P n(T)}\left(\frac{\partial P n(T)}{\partial \beta_{3}}\right)=\frac{- \text { Accom } \times e^{V(\text { Car })}}{\left(e^{V(\text { Car })}+e^{V(\text { Taxi })}+e^{V(\text { Subway })}+e^{V(\text { Bus })}+e^{V(\text { Limousine })}\right)} \\
& =-\operatorname{Accom}[\operatorname{Pn}(C)] \\
& \frac{\partial P n(S)}{\partial \beta_{3}}=\frac{O-e^{V(\text { Subway })}\left(\text { Accom } \times e^{V(\text { Car })}\right)}{\left(e^{V(\text { Car })}+e^{V(\text { Taxi })}+e^{V(\text { Subway })}+e^{V(\text { Bus })}+e^{V(\text { Limousin })}\right)^{2}} \\
& \frac{1}{P n(S)}\left(\frac{\partial P n(S)}{\partial \beta_{3}}\right)=\frac{-\left(\text { Accome } e^{V(\text { Car })}\right)}{\left(e^{V(\text { Car })}+e^{V(\text { Taxi })}+e^{V(\text { Subway })}+e^{V(\text { Bus })}+e^{V(\text { Limousine })}\right)} \\
& =-\operatorname{Accom}[\operatorname{Pn}(C)]
\end{aligned}
$$




$$
\begin{aligned}
& \frac{\partial P n(B)}{\partial \beta_{3}}=\frac{0-e^{V(\text { Bus })}\left(\text { Accom } \times e^{V(\text { Car })}\right)}{\left(e^{V(\text { Car })}+e^{V(\text { Taxi })}+e^{V(\text { Subway })}+e^{V(\text { Bus })}+e^{V(\text { Limousine })}\right)^{2}} \\
& \begin{aligned}
\frac{1}{P n(B)}\left(\frac{\partial P n(B)}{\partial \beta_{3}}\right) & =\frac{-\left(\text { Accom } \times e^{V(\text { Car })}\right)}{\left(e^{V(\text { Car })}+e^{V(\text { Taxi })}+e^{V(\text { Subway })}+e^{V(\text { Bus })}+e^{V(\text { Limousine })}\right)} \\
& =- \text { Accom }[P n(C)]
\end{aligned} \\
& \begin{aligned}
& \frac{\partial P n(L)}{\partial \beta_{3}}=\frac{0-e^{V(\text { Limousine }}\left(\text { Accom } \times e^{V(\text { Car })}\right)}{\left(e^{V(\text { Car })}+e^{V(\text { Taxi })}+e^{V(\text { Subway })}+e^{V(\text { Bus })}+e^{V(\text { Limousine })}\right)^{2}} \\
& \frac{1}{P n(L)}\left(\frac{\partial P n(L)}{\partial \beta_{3}}\right)= \frac{-\left(\text { Accom } \times e^{V(\text { Car })}\right)}{\left(e^{V(\text { Car })}+e^{V(\text { Taxi })}+e^{V(\text { Subway })}+e^{V(\text { Bus })}+e^{V(\text { Limousine })}\right)} \\
&=- \text { Accom }[\text { Pn }(C)]
\end{aligned}
\end{aligned}
$$

2) The first partial derivative with respect to $\beta_{4}$ is

$$
\begin{aligned}
\frac{\partial L L\left(\beta_{1}, \beta_{2}, \cdots, \beta_{8}, \beta_{9}\right)}{\partial \beta_{4}} & =\sum_{n=1}^{N}\left[y_{C n} \frac{1}{P n(C)}\left(\frac{\partial P n(C)}{\partial \beta_{4}}\right)+y_{T n} \frac{1}{P n(T)}\left(\frac{\partial P n(T)}{\partial \beta_{4}}\right)\right. \\
& \left.+y_{S n} \frac{1}{P n(S)}\left(\frac{\partial P n(S)}{\partial \beta_{4}}\right)+y_{B n} \frac{1}{P n(B)}\left(\frac{\partial P n(B)}{\partial \beta_{4}}\right)+y_{L n} \frac{1}{P n(L)}\left(\frac{\partial P n(L)}{\partial \beta_{4}}\right)\right]
\end{aligned}
$$

where

$$
\begin{aligned}
& \frac{\partial P n(C)}{\partial \beta_{4}}=\frac{\operatorname{Sexm} \times e^{V(\text { Car })}\left(e^{V(\text { Car })}+e^{V(\text { Taxi })}+e^{V(\text { Subway })}+e^{V(\text { Bus })}+e^{V(\text { Limousin } e)}\right)-e^{V(\text { Car })}\left(\operatorname{Sexm} \times e^{V(\text { Car })}\right)}{\left(e^{V(\text { Car })}+e^{V(\text { Taxi })}+e^{V(\text { Subway })}+e^{V(\text { Bus })}+e^{V(\text { Limousin } e)}\right)^{2}}
\end{aligned}
$$

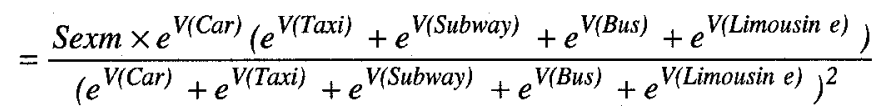

$$
\begin{aligned}
& \frac{1}{\operatorname{Pn}(C)}\left(\frac{\partial P n(C)}{\partial \beta_{4}}\right)=\frac{\operatorname{Sexm}\left(e^{V(\text { Taxi })}+e^{V(\text { Subway })}+e^{V(\text { Bus })}+e^{V(\text { Limousine })}\right)}{\left(e^{V(\text { Car })}+e^{V(\text { Taxi })}+e^{V(\text { Subway })}+e^{V(\text { Bus })}+e^{V(\text { Limousine })}\right)} \\
& =\operatorname{Sexm}[\operatorname{Pn}(T)+\operatorname{Pn}(S)+\operatorname{Pn}(B)+P n(L)]
\end{aligned}
$$$$
\frac{\partial P n(T)}{\partial \beta_{4}}=\frac{O-e^{V(\text { Taxi })}\left(\text { Sexm } \times e^{V(\text { Car })}\right)}{\left(e^{V(\text { Car })}+e^{V(\text { Taxi })}+e^{V(\text { Subway })}+e^{V(\text { Bus })}+e^{V(\text { Limousin } e)}\right)^{2}}
$$$$
\frac{1}{P n(T)}\left(\frac{\partial P n(T)}{\partial \beta_{4}}\right)=\frac{-\operatorname{Sexm} \times e^{V(\text { Car })}}{\left(e^{V(\text { Car })}+e^{V(\text { Taxi })}+e^{V(\text { Subway })}+e^{V(\text { Bus })}+e^{V(\text { Limousine })}\right)}
$$$$
=-\operatorname{Sexm}[\operatorname{Pn}(C)]
$$ 


$$
\begin{aligned}
& \frac{\partial P n(S)}{\partial \beta_{4}}=\frac{0-e^{V(\text { Subway })}\left(\text { Sexm } \times e^{V(\text { Car })}\right)}{\left(e^{V(\text { Car })}+e^{V(\text { Taxi })}+e^{V(\text { Subway })}+e^{V(\text { Bus })}+e^{V(\text { Limousine })}\right)^{2}} \\
& \frac{1}{P n(S)}\left(\frac{\partial P n(S)}{\partial \beta_{4}}\right)=\frac{-\left(\text { Sexm } \times e^{V(\text { Car })}\right)}{\left(e^{V(\text { Car })}+e^{V(\text { Taxi })}+e^{V(\text { Subway })}+e^{V(\text { Bus })}+e^{V(\text { Limousin } e)}\right)} \\
& =-\operatorname{Sexm}[\operatorname{Pn}(C)] \\
& \frac{\partial P n(B)}{\partial \beta_{4}}=\frac{0-e^{V(\text { Bus })}\left(\operatorname{Sexm} \times e^{V(\text { Car })}\right)}{\left(e^{V(\text { Car })}+e^{V(\text { Taxi })}+e^{V(\text { Subway })}+e^{V(\text { Bus })}+e^{V(\text { Limousin } e)}\right)^{2}} \\
& \frac{1}{P n(B)}\left(\frac{\partial P n(B)}{\partial \beta_{4}}\right)=\frac{-\left(\text { Sexm } \times e^{V(\text { Car })}\right)}{\left(e^{V(\text { Car })}+e^{V(\text { Taxi })}+e^{V(\text { Subway })}+e^{V(\text { Bus })}+e^{V(\text { Limousine })}\right)} \\
& =-\operatorname{Sexm}[\operatorname{Pn}(C)] \\
& \frac{\partial \operatorname{Pn}(L)}{\partial \beta_{4}}=\frac{0-e^{V(\text { limousin } e)}\left(\operatorname{Sexm} \times e^{V(\text { Car })}\right)}{\left(e^{V(\text { Car })}+e^{V(\text { Taxi })}+e^{V(\text { Subway })}+e^{V(\text { Bus })}+e^{V(\text { Limousin } e)}\right)^{2}} \\
& \frac{1}{P n(L)}\left(\frac{\partial P n(L)}{\partial \beta_{4}}\right)=\frac{-\left(\text { Sexm } \times e^{V(\text { Car })}\right)}{\left(e^{V(\text { Car })}+e^{V(\text { Taxi })}+e^{V(\text { Subway })}+e^{V(\text { Bus })}+e^{V(\text { Limousine })}\right)} \\
& =-\operatorname{Sexm}[\operatorname{Pn}(C)]
\end{aligned}
$$

3) The first partial derivative with respect $\beta_{5}$ is

$$
\begin{aligned}
\frac{\partial L L\left(\beta_{1}, \beta_{2}, \cdots, \beta_{8}, \beta_{9}\right)}{\partial \beta_{5}} & =\sum_{n=1}^{N}\left[y_{C n} \frac{1}{P n(C)}\left(\frac{\partial P n(C)}{\partial \beta_{5}}\right)+y_{T n} \frac{1}{P n(T)}\left(\frac{\partial P n(T)}{\partial \beta_{5}}\right)\right. \\
& \left.+y_{S n} \frac{1}{P n(S)}\left(\frac{\partial P n(S)}{\partial \beta_{5}}\right)+y_{B n} \frac{1}{P n(B)}\left(\frac{\partial P n(B)}{\partial \beta_{5}}\right)+y_{L n} \frac{1}{P n(L)}\left(\frac{\partial P n(L)}{\partial \beta_{5}}\right)\right]
\end{aligned}
$$

where

$$
\begin{aligned}
& \frac{\partial P n(C)}{\partial \beta_{5}}=\frac{A g e 340 \times e^{V(\text { Car })}\left(e^{V(\text { Car })}+e^{V(\text { Taxi })}+e^{V(\text { Subway })}+e^{V(\text { Bus })}+e^{V(\text { Limousine })}\right)-e^{V(\text { Car })}\left(\text { Age340 } \times e^{V(\text { Car })}\right)}{\left(e^{V(\text { Car })}+e^{V(\text { Taxi })}+e^{V(\text { Subway })}+e^{V(\text { Bus })}+e^{V(\text { Limousine })}\right)^{2}} \\
& =\frac{\text { Age } 340 \times e^{V(\text { Car })}\left(e^{V(\text { Taxi })}+e^{V(\text { Subway })}+e^{V(\text { Bus })}+e^{V(\text { Limousine })}\right)}{\left(e^{V(\text { Car })}+e^{V(\text { Taxi })}+e^{V(\text { Subway })}+e^{V(\text { Bus })}+e^{V(\text { Limousin })}\right)^{2}} \\
& \frac{1}{P n(C)}\left(\frac{\partial P n(C)}{\partial \beta_{5}}\right)=\frac{A g e 340\left(e^{V(\text { Taxi })}+e^{V(\text { Subway })}+e^{V(\text { Bus })}+e^{V(\text { Limousine })}\right)}{\left(e^{V(\text { Car })}+e^{V(\text { Taxi })}+e^{V(\text { Subway })}+e^{V(\text { Bus })}+e^{V(\text { Limousine })}\right)} \\
& =\operatorname{Age340}[\operatorname{Pn}(T)+\operatorname{Pn}(S)+\operatorname{Pn}(B)+\operatorname{Pn}(L)]
\end{aligned}
$$




$$
\begin{aligned}
& \frac{\partial P n(T)}{\partial \beta_{5}}=\frac{0-e^{V(\text { Taxi })}\left(\text { Age } 340 \times e^{V(\text { Car })}\right)}{\left(e^{V(\text { Car })}+e^{V(\text { Taxi })}+e^{V(\text { Subway })}+e^{V(\text { Bus })}+e^{V(\text { Limousine })}\right)^{2}} \\
& \frac{1}{\operatorname{Pn}(T)}\left(\frac{\partial P n(T)}{\partial \beta_{5}}\right)=\frac{-A g e 340 \times e^{V(\text { Car })}}{\left(e^{V(\text { Car })}+e^{V(\text { Taxi })}+e^{V(\text { Subway })}+e^{V(\text { Bus })}+e^{V(\text { Limousine })}\right)} \\
& =-\operatorname{Age340[Pn}(\mathrm{C})] \\
& \frac{\partial P n(S)}{\partial \beta_{5}}=\frac{0-e^{V(\text { Subway })}\left(\text { Age } 340 \times e^{V(\text { Car })}\right)}{\left(e^{V(\text { Car })}+e^{V(\text { Taxi })}+e^{V(\text { Subway })}+e^{V(\text { Bus })}+e^{V(\text { Limousine })}\right)^{2}} \\
& \frac{1}{\operatorname{Pn}(S)}\left(\frac{\partial P n(S)}{\partial \beta_{5}}\right)=\frac{-\left(\text { Age } 340 \times e^{V(\text { Car })}\right)}{\left(e^{V(\text { Car })}+e^{V(\text { Taxi })}+e^{V(\text { Subway })}+e^{V(\text { Bus })}+e^{V(\text { Limousine })}\right)} \\
& =-A g e 340[P n(C)] \\
& \frac{\partial P n(B)}{\partial \beta_{5}}=\frac{0-e^{V(\text { Bus })}\left(\text { Age } 340 \times e^{V(\text { Car })}\right)}{\left(e^{V(\text { Car })}+e^{V(\text { Taxi })}+e^{V(\text { Subway })}+e^{V(\text { Bus })}+e^{V(\text { Limousine })}\right)^{2}} \\
& \frac{1}{\operatorname{Pn}(B)}\left(\frac{\partial \operatorname{Pn}(B)}{\partial \beta_{5}}\right)=\frac{-\left(\text { Age } 340 \times e^{V(\text { Car })}\right)}{\left(e^{V(\text { Car })}+e^{V(\text { Taxi })}+e^{V(\text { Subway })}+e^{V(\text { Bus })}+e^{V(\text { Limousine })}\right)} \\
& =-\operatorname{Age} 340[\operatorname{Pn}(C)] \\
& \frac{\partial P n(L)}{\partial \beta_{5}}=\frac{0-e^{V(\text { Limousin } e)}\left(\text { Age } 340 \times e^{V(\text { Car })}\right)}{\left(e^{V(\text { Car })}+e^{V(\text { Taxi })}+e^{V(\text { Subway })}+e^{V(\text { Bus })}+e^{V(\text { Limousin } e)}\right)^{2}} \\
& \frac{1}{\operatorname{Pn}(L)}\left(\frac{\partial P n(L)}{\partial \beta_{5}}\right)=\frac{-\left(\text { Age } 340 \times e^{V(\text { Car })}\right)}{\left(e^{V(\text { Car })}+e^{V(\text { Taxi })}+e^{V(\text { Subway })}+e^{V(\text { Bus })}+e^{V(\text { Limousine })}\right)}
\end{aligned}
$$

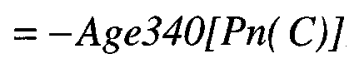

The first partial derivatives with respect to the alternative-specific-constants, $\beta_{6}, \beta_{7}, \beta_{8}, \beta_{9}$ are as follows:

1) The first partial derivatives with respect to $\beta_{6}$ is 


$$
\begin{aligned}
\frac{\partial L L\left(\beta_{1}, \beta_{2}, \cdots, \beta_{8}, \beta_{9}\right)}{\partial \beta_{6}} & =\sum_{n=1}^{N}\left[y_{C n} \frac{1}{P n(C)}\left(\frac{\partial P n(C)}{\partial \beta_{6}}\right)+y_{T n} \frac{1}{P n(T)}\left(\frac{\partial P n(T)}{\partial \beta_{6}}\right)\right. \\
& \left.+y_{S n} \frac{1}{P n(S)}\left(\frac{\partial P n(S)}{\partial \beta_{6}}\right)+y_{B n} \frac{1}{P n(B)}\left(\frac{\partial P n(B)}{\partial \beta_{6}}\right)+y_{L n} \frac{1}{P n(L)}\left(\frac{\partial P n(L)}{\partial \beta_{6}}\right)\right]
\end{aligned}
$$

where

$$
\begin{aligned}
& \frac{\partial P n(C)}{\partial \beta_{6}}=\frac{0-e^{V(\text { Car })}\left(e^{V(\text { Taxi })}\right)}{\left(e^{V(\text { Car })}+e^{V(\text { Taxi })}+e^{V(\text { Subway })}+e^{V(\text { Bus })}+e^{V(\text { Limousin } e)}\right)^{2}} \\
& \begin{aligned}
\frac{1}{P n(C)}\left(\frac{\partial P n(C)}{\partial \beta_{6}}\right) & =\frac{-\left(e^{V(\text { Taxi })}\right)}{\left(e^{V(\text { Car })}+e^{V(\text { Taxi })}+e^{V(\text { Subway })}+e^{V(\text { Bus })}+e^{V(\text { Limousin } e)}\right)} \\
& =-P n(T)
\end{aligned}
\end{aligned}
$$

$$
\begin{aligned}
& \frac{\partial P n(T)}{\partial \beta_{6}}=\frac{e^{V(\text { Taxi })}\left(e^{V(\text { Car })}+e^{V(\text { Taxi })}+e^{V(\text { Subway })}+e^{V(\text { Bus })}+e^{V(\text { Limousin } e)}\right)-\left(e^{V(\text { Taxi })}\right)^{2}}{\left(e^{V(\text { Car })}+e^{V(\text { Taxi })}+e^{V(\text { Subway })}+e^{V(\text { Bus })}+e^{V(\text { Limousin } e)}\right)^{2}} \\
& \begin{aligned}
\frac{1}{P n(T)}\left(\frac{\partial P n(T)}{\partial \beta_{6}}\right) & =\frac{\left(e^{V(\text { Car })}+e^{V(\text { Subway })}+e^{V(\text { Bus })}+e^{V(\text { Limousin } e)}\right)}{\left(e^{V(\text { Car })}+e^{V(\text { Taxi })}+e^{V(\text { Subway })}+e^{V(\text { Bus })}+e^{V(\text { Limousin } e)}\right)} \\
& =[\operatorname{Pn}(C)+\operatorname{Pn}(S)+\operatorname{Pn}(\text { B })+\operatorname{Pn}(L)]
\end{aligned}
\end{aligned}
$$

$$
\begin{aligned}
& \frac{\partial P n(S)}{\partial \beta_{6}}=\frac{0-e^{V(\text { Subway })}\left(e^{V(\text { (Taxi })}\right)}{\left(e^{V(\text { Car })}+e^{V(\text { Taxi })}+e^{V(\text { Subway })}+e^{V(\text { Bus })}+e^{V(\text { Limousine })}\right)^{2}} \\
& \begin{aligned}
\frac{1}{P n(S)}\left(\frac{\partial P n(S)}{\partial \beta_{6}}\right) & =\frac{-e^{V(\text { Taxi })}}{\left(e^{V(\text { Car })}+e^{V(\text { Taxi })}+e^{V(\text { Subway })}+e^{V(\text { Bus })}+e^{V(\text { Limousine })}\right)} \\
& =-P n(T)
\end{aligned}
\end{aligned}
$$

$$
\begin{aligned}
& \frac{\partial P n(B)}{\partial \beta_{6}}=\frac{0-e^{V(\text { Bus })}\left(e^{V(\text { Taxi })}\right)}{\left(e^{V(\text { Car })}+e^{V(\text { Taxi })}+e^{V(\text { Subway })}+e^{V(\text { Bus })}+e^{V(\text { Limousine })}\right)^{2}} \\
& \begin{aligned}
\frac{1}{P n(B)}\left(\frac{\partial P n(B)}{\partial \beta_{6}}\right) & =\frac{-e^{V(\text { Taxi })}}{\left(e^{V(\text { Car })}+e^{V(\text { Taxi })}+e^{V(\text { Subway })}+e^{V(\text { Bus })}+e^{V(\text { Limousine })}\right)} \\
& =-P n(T)
\end{aligned}
\end{aligned}
$$$$
\frac{\partial P n(L)}{\partial \beta_{6}}=\frac{0-e^{V(\text { Limousine })}\left(e^{V(\text { Taxi })}\right)}{\left(e^{V(\text { Car })}+e^{V(\text { Taxi })}+e^{V(\text { Subway })}+e^{V(\text { Bus })}+e^{V(\text { Limousine })}\right)^{2}}
$$ 


$$
\begin{aligned}
\frac{1}{P n(L)}\left(\frac{\partial P n(L)}{\partial \beta_{6}}\right) & =\frac{-e^{V(\text { Taxi })}}{\left(e^{V(\text { Car })}+e^{V(\text { Taxi })}+e^{V(\text { Subway })}+e^{V(\text { Bus })}+e^{V(\text { Limousine })}\right)} \\
& =-P n(T)
\end{aligned}
$$

2) The first partial derivative with respect to $\beta_{7}$ is

$$
\begin{aligned}
\frac{\partial L L\left(\beta_{1}, \beta_{2}, \cdots, \beta_{8}, \beta_{9}\right)}{\partial \beta_{7}} & =\sum_{n=1}^{N}\left[y_{C n} \frac{1}{P n(C)}\left(\frac{\partial P n(C)}{\partial \beta_{7}}\right)+y_{T n} \frac{1}{P n(T)}\left(\frac{\partial P n(T)}{\partial \beta_{7}}\right)\right. \\
& \left.+y_{S n} \frac{1}{P n(S)}\left(\frac{\partial P n(S)}{\partial \beta_{7}}\right)+y_{B n} \frac{1}{P n(B)}\left(\frac{\partial P n(B)}{\partial \beta_{7}}\right)+y_{L n} \frac{1}{P n(L)}\left(\frac{\partial P n(L)}{\partial \beta_{7}}\right)\right]
\end{aligned}
$$

where

$$
\begin{aligned}
& \frac{\partial P n(C)}{\partial \beta_{7}}=\frac{0-e^{V(\text { Car })}\left(e^{V(\text { Subway })}\right)}{\left(e^{V(\text { Car })}+e^{V(\text { Taxi })}+e^{V(\text { Subway })}+e^{V(\text { Bus })}+e^{V(\text { Limousin })}\right)^{2}} \\
& \begin{aligned}
\frac{1}{P n(C)}\left(\frac{\partial P n(C)}{\partial \beta_{7}}\right) & =\frac{-e^{V(\text { Subway })}}{\left(e^{V(\text { Car })}+e^{V(\text { Taxi })}+e^{V(\text { Subway })}+e^{V(\text { Bus })}+e^{V(\text { Limousine })}\right)} \\
& =-P n(S)
\end{aligned}
\end{aligned}
$$

$$
\begin{aligned}
& \frac{\partial P n(T)}{\partial \beta_{7}}=\frac{0-e^{V(\text { Taxi })}\left(e^{V(\text { Subway })}\right)}{\left(e^{V(\text { Car })}+e^{V(\text { Taxi })}+e^{V(\text { Subway })}+e^{V(\text { Bus })}+e^{V(\text { Limousine })}\right)^{2}} \\
& \begin{aligned}
\frac{1}{P n(T)}\left(\frac{\partial P n(T)}{\partial \beta_{7}}\right) & =\frac{-e^{V(\text { Subway })}}{\left(e^{V(\text { Car })}+e^{V(\text { Taxi })}+e^{V(\text { Subway })}+e^{V(\text { Bus })}+e^{V(\text { Limousine })}\right)} \\
& =-P n(S)
\end{aligned}
\end{aligned}
$$$$
\frac{\partial P n(S)}{\partial \beta_{7}}=\frac{e^{V(\text { Subway })}\left(e^{V(\text { Car })}+e^{V(\text { Taxi })}+e^{V(\text { Subway })}+e^{V(\text { Bus })}+e^{V(\text { Limousine })}\right)-e^{V(\text { Subway })} e^{V(\text { Subway })}}{\left(e^{V(\text { Car })}+e^{V(\text { Taxi })}+e^{V(\text { Subway })}+e^{V(\text { Bus })}+e^{V(\text { Limousine })}\right)^{2}}
$$$$
\frac{1}{P n(S)}\left(\frac{\partial P n(S)}{\partial \beta_{7}}\right)=\frac{\left(e^{V(\text { Car })}+e^{V(\text { Taxi })}+e^{V(\text { Bus })}+e^{(\text {Limousine })}\right)}{\left(e^{V(\text { Car })}+e^{V(\text { Taxi })}+e^{V(\text { Subway })}+e^{V(\text { Bus })}+e^{V(\text { Limousine })}\right)}
$$$$
=[P n(C)+P n(T)+P n(B)+P n(L)]
$$$$
\frac{\partial \operatorname{Pn}(B)}{\partial \beta_{7}}=\frac{0-e^{V(\text { Bus })}\left(e^{V(\text { Subway })}\right)}{\left(e^{V(\text { Car })}+e^{V(\text { Taxi })}+e^{V(\text { Subway })}+e^{V(\text { Bus })}+e^{V(\text { Limousine })}\right)^{2}}
$$ 


$$
\begin{aligned}
& \frac{1}{P n(B)}\left(\frac{\partial P n(B)}{\partial \beta_{7}}\right)=\frac{-e^{V(\text { Subway })}}{\left(e^{V(\text { Car })}+e^{V(\text { Taxi })}+e^{V(\text { Subway })}+e^{V(\text { Bus })}+e^{V(\text { Limousin })}\right)} \\
& =-P n(S) \\
& \begin{aligned}
& \frac{\partial P n(L)}{\partial \beta_{7}}=\frac{0-e^{V(\text { Limousine })}\left(e^{V(\text { Subway })}\right)}{\left(e^{V(\text { Car })}+e^{V(\text { Taxi })}+e^{V(\text { Subway })}+e^{V(\text { Bus })}+e^{V(\text { Limousine })}\right)^{2}} \\
& \frac{1}{P n(L)}\left(\frac{\partial P n(L)}{\partial \beta_{7}}\right)=\frac{-e^{V(\text { Subway })}}{\left(e^{V(\text { Car })}+e^{V(\text { Taxi })}+e^{V(\text { Subway })}+e^{V(\text { Bus })}+e^{V(\text { Limousine })}\right)} \\
&=-P n(S)
\end{aligned}
\end{aligned}
$$

3) The first partial derivative with respect to $\beta_{8}$ is

$$
\begin{aligned}
\frac{\partial L L\left(\beta_{1}, \beta_{2}, \cdots, \beta_{8}, \beta_{9}\right)}{\partial \beta_{8}} & =\sum_{n=1}^{N}\left[y_{C n} \frac{1}{P n(C)}\left(\frac{\partial P n(C)}{\partial \beta_{8}}\right)+y_{T n} \frac{1}{P n(T)}\left(\frac{\partial P n(T)}{\partial \beta_{8}}\right)\right. \\
& \left.+y_{S n} \frac{1}{P n(S)}\left(\frac{\partial P n(S)}{\partial \beta_{8}}\right)+y_{B n} \frac{1}{P n(B)}\left(\frac{\partial P n(B)}{\partial \beta_{8}}\right)+y_{L n} \frac{1}{P n(L)}\left(\frac{\partial P n(L)}{\partial \beta_{8}}\right)\right]
\end{aligned}
$$

where

$$
\begin{aligned}
& \frac{\partial \operatorname{Pn}(C)}{\partial \beta_{8}}=\frac{0-e^{V(\text { Car })}\left(e^{V(\text { Bus })}\right)}{\left(e^{V(\text { Car })}+e^{V(\text { Taxi })}+e^{V(\text { Subway })}+e^{V(\text { Bus })}+e^{V(\text { Limousine })}\right)^{2}} \\
& \frac{1}{P n(C)}\left(\frac{\partial P n(C)}{\partial \beta_{8}}\right)=\frac{-e^{V(\text { Bus })}}{\left(e^{V(\text { Car })}+e^{V(\text { Taxi })}+e^{V(\text { Subway })}+e^{V(\text { Bus })}+e^{V(\text { Limousine })}\right)} \\
& =-P n(B) \\
& \frac{\partial P n(T)}{\partial \beta_{8}}=\frac{0-e^{V(\text { Taxi })}\left(e^{V(\text { Bus })}\right)}{\left(e^{V(\text { Car })}+e^{V(\text { Taxi })}+e^{V(\text { Subway })}+e^{V(\text { Bus })}+e^{V(\text { Limousine })}\right)^{2}} \\
& \frac{1}{P n(T)}\left(\frac{\partial P n(T)}{\partial \beta_{8}}\right)=\frac{-e^{V(B u s)}}{\left(e^{V(\text { Car })}+e^{V(\text { Taxi })}+e^{V(\text { Subway })}+e^{V(\text { Bus })}+e^{V(\text { Limousine })}\right)} \\
& =-P n(B) \\
& \frac{\partial P n(S)}{\partial \beta_{8}}=\frac{0-e^{V(\text { Subway })}\left(e^{V(\text { Bus })}\right)}{\left(e^{V(\text { Car })}+e^{V(\text { Taxi })}+e^{V(\text { Subway })}+e^{V(\text { Bus })}+e^{V(\text { Limousine })}\right)^{2}}
\end{aligned}
$$




$$
\begin{aligned}
& \frac{1}{P n(S)}\left(\frac{\partial P n(S)}{\partial \beta_{8}}\right)=\frac{-e^{V(\text { Bus })}}{\left(e^{V(\text { Car })}+e^{V(\text { Taxi })}+e^{V(\text { Subway })}+e^{V(\text { Bus })}+e^{V(\text { Limousine })}\right)} \\
& =-P n(B) \\
& \frac{\partial P n(B)}{\partial \beta_{8}}=\frac{e^{V(\text { Bus })}\left(e^{V(\text { Car })}+e^{V(\text { Taxi })}+e^{V(\text { Subway })}+e^{V(\text { Bus })}+e^{V(\text { Limousine })}\right)-e^{V(\text { Bus })} e^{V(\text { Bus })}}{\left(e^{V(\text { Car })}+e^{V(\text { Taxi })}+e^{V(\text { Subway })}+e^{V(\text { Bus })}+e^{V(\text { Limousine })}\right)^{2}} \\
& \frac{1}{P n(B)}\left(\frac{\partial \operatorname{Pn}(B)}{\partial \beta_{8}}\right)=\frac{\left(e^{V(\text { Car })}+e^{V(\text { Taxi })}+e^{V(\text { Subway })}+e^{V(\text { Limousine })}\right)}{\left(e^{V(\text { Car })}+e^{V(\text { Taxi })}+e^{V(\text { Subway })}+e^{V(\text { Bus })}+e^{V(\text { Limousine })}\right)} \\
& =[P n(C)+P n(T)+P n(S)+P n(L)] \\
& \frac{\partial \operatorname{Pn}(L)}{\partial \beta_{8}}=\frac{0-e^{V(\text { limousine })}\left(e^{V(\text { Bus })}\right)}{\left(e^{V(\text { Car })}+e^{V(\text { Taxi })}+e^{V(\text { Subway })}+e^{V(\text { Bus })}+e^{V(\text { Limousine })}\right)^{2}} \\
& \frac{1}{P n(L)}\left(\frac{\partial P n(L)}{\partial \beta_{8}}\right)=\frac{-e^{V(\text { Bus })}}{\left(e^{V(\text { Car })}+e^{V(\text { Taxi })}+e^{V(\text { Subway })}+e^{V(\text { Bus })}+e^{V(\text { Limousine })}\right)} \\
& =-\operatorname{Pn}(B)
\end{aligned}
$$

4) The first partial derivatives with respect to $\beta_{9}$ is

$$
\begin{aligned}
\frac{\partial L L\left(\beta_{1}, \beta_{2}, \cdots, \beta_{8}, \beta_{9}\right)}{\partial \beta_{9}} & =\sum_{n=1}^{N}\left[y_{C n} \frac{1}{P n(C)}\left(\frac{\partial P n(C)}{\partial \beta_{9}}\right)+y_{T n} \frac{1}{P n(T)}\left(\frac{\partial P n(T)}{\partial \beta_{9}}\right)\right. \\
& \left.+y_{S n} \frac{1}{P n(S)}\left(\frac{\partial P n(S)}{\partial \beta_{9}}\right)+y_{B n} \frac{1}{P n(B)}\left(\frac{\partial P n(B)}{\partial \beta_{9}}\right)+y_{L n} \frac{1}{P n(L)}\left(\frac{\partial P n(L)}{\partial \beta_{9}}\right)\right]
\end{aligned}
$$

where

$$
\begin{aligned}
& \frac{\partial P n(C)}{\partial \beta_{9}}=\frac{0-e^{V(\text { Car })}\left(e^{V(\text { Limousine })}\right)}{\left(e^{V(\text { Car })}+e^{V(\text { Taxi })}+e^{V(\text { Subway })}+e^{V(\text { Bus })}+e^{V(\text { Limousine })}\right)^{2}} \\
& \frac{1}{P n(C)}\left(\frac{\partial P n(C)}{\partial \beta_{9}}\right)=\frac{-e^{V(\text { Limousine })}}{\left(e^{V(\text { Car })}+e^{V(\text { Taxi })}+e^{V(\text { Subway })}+e^{V(\text { Bus })}+e^{V(\text { Limousine })}\right)} \\
& =-P n(L) \\
& \frac{\partial P n(T)}{\partial \beta_{9}}=\frac{0-e^{V(\text { Taxi })}\left(e^{V(\text { Limousine })}\right)}{\left(e^{V(\text { Car })}+e^{V(\text { Taxi })}+e^{V(\text { Subway })}+e^{V(\text { Bus })}+e^{V(\text { Limousine })}\right)^{2}}
\end{aligned}
$$




$$
\begin{aligned}
& \frac{1}{P n(T)}\left(\frac{\partial P n(T)}{\partial \beta_{9}}\right)=\frac{-e^{V(\text { Limousine })}}{\left(e^{V(\text { Car })}+e^{V(\text { Taxi })}+e^{V(\text { Subway })}+e^{V(\text { Bus })}+e^{V(\text { Limousine })}\right)} \\
& =-P n(L)
\end{aligned}
$$

Using all the first partial derivatives functions derived above, real gradient values can be calculated in numerical way within the estimation process, and thus a gradient vector of log-likelihood function can be summarized in a vector form as given below. The gradient is a column vector. In the context of this thesis, it has 9 dimensions (i.e., 9 explanatory variables). It can be written as below: 


$$
G_{t}=\left(\frac{\partial L L(\beta)}{\partial \beta_{k}}\right)=\left(\begin{array}{c}
\frac{\partial L L\left(\beta_{1}, \cdots, \beta_{9}\right)}{\partial \beta_{1}} \\
\frac{\partial L L\left(\beta_{1}, \cdots, \beta_{9}\right)}{\partial \beta_{2}} \\
\vdots \\
\frac{\partial L L\left(\beta_{1}, \cdots, \beta_{9}\right)}{\partial \beta_{8}} \\
\frac{\partial L L\left(\beta_{1}, \cdots, \beta_{9}\right)}{\partial \beta_{9}}
\end{array}\right)
$$

Finally, the mathematical expression of a gradient vector can be transformed into computer code based on the interpretation implied in the above formula expansion and its numerical value is continuously changed during iterations until the process is terminated.

\section{A.6: The derivation of the Hessian matrix formulas specified to given choice problem}

To get a real Hessian matrix of given log-likelihood function, which is only used in Newton Raphson algorithm and contains full information of the sample used in discrete choice modelling, a researcher (or modeller) needs to derive the second-order partial derivatives of the log-likelihood function analytically. In fact, the process involved is extremely burdensome and computationally intensive. In spite of this difficulty associated with mathematical manipulation of the second partial derivatives, the real Hessian matrix obtained analytically and used in the combination of the Newton Raphson algorithm shows the best estimation performance in discrete mode choice modelling compared to any other algorithm using approximate Hessian matrix. Based on the knowledge of symmetry of the Hessian matrix for diagonal, only for the off-diagonal elements the second-order partial derivatives are applied. The following parts describe the derivation 
process of the second-order partial derivatives of log-likelihood function for the offdiagonal elements of the Hessian matrix:

1) The second order partial derivative with respect to $\beta_{1} \beta_{1}$ is

$$
\begin{aligned}
\frac{\partial^{2} L L\left(\beta_{1}, \beta_{2}, \cdots, \beta_{8}, \beta_{9}\right)}{\partial \beta_{1} \partial \beta_{1}}= & \sum_{n=1}^{N}\left[y_{C n} \frac{\partial}{\partial \beta_{1}}\left[\frac{1}{P n\left(C_{h}\right.}\left(\frac{\partial P n(C)_{h}}{\partial \beta_{1}}\right)\right]+y_{T n} \frac{\partial}{\partial \beta_{1}}\left[\frac{1}{P n(T)_{h}}\left(\frac{\partial P n\left(T_{h}\right.}{\partial \beta_{1}}\right)\right]+y_{S n} \frac{\partial}{\partial \beta_{1}}\left[\frac{1}{P n(S)}\left(\frac{\partial P n(S)_{h}}{\partial \beta_{1}}\right)\right]+\right. \\
& y_{B n} \frac{\partial}{\partial \beta_{1}}\left[\frac{1}{P n(B h}\left(\frac{\partial P n(B)}{\partial \beta_{1}}\right)\right]+y_{L n} \frac{\partial}{\partial \beta_{1}}\left[\frac{1}{P n(L)}\left(\frac{\partial P n(L)}{\partial \beta_{1}}\right)\right] J
\end{aligned}
$$

where

$$
\begin{aligned}
& \frac{1}{P n(C)}\left(\frac{\partial P n(C)}{\partial \beta_{1}}\right)=\left(T C_{C}-T C_{T}\right) P n(T)+\left(T C_{C}-T C_{S}\right) P n(S)+\left(T C_{C}-T C_{B}\right) P n(B)+\left(T C_{C}-T C_{L}\right) P n(L) \\
& \frac{1}{P n(T)}\left(\frac{\partial P n(T)}{\partial \beta_{1}}\right)=\left(T C_{T}-T C_{C}\right) P n(C)+\left(T C_{T}-T C_{S}\right) P n(S)+\left(T C_{T}-T C_{B}\right) P n(B)+\left(T C_{T}-T C_{L}\right) P n(L) \\
& \frac{1}{P n(S)}\left(\frac{\partial P n(S)}{\partial \beta_{1}}\right)=\left(T C_{S}-T C_{C}\right) P n(C)+\left(T C_{S}-T C_{T}\right) P n(T)+\left(T C_{S}-T C_{B}\right) P n(B)+\left(T C_{S}-T C_{L}\right) P n(L) \\
& \frac{1}{P n(B)}\left(\frac{\partial P n(B)}{\partial \beta_{1}}\right)=\left(T C_{B}-T C_{C}\right) P n(C)+\left(T C_{B}-T C_{T}\right) P n(T)+\left(T C_{B}-T C_{S}\right) P n(S)+\left(T C_{B}-T C_{L}\right) P n(L) \\
& \frac{1}{P n(L)}\left(\frac{\partial P n(L)}{\partial \beta_{1}}\right)=\left(T C_{L}-T C_{C}\right) P n(C)+\left(T C_{L}-T C_{T}\right) P n(T)+\left(T C_{L}-T C_{S}\right) P n(S)+\left(T C_{L}-T C_{B}\right) P n(B) \\
& \frac{\partial P n(C)}{\partial \beta_{I}}=\left(T C_{C}-T C_{T}\right) P n(C) P n(T)+\left(T C_{C}-T C_{S}\right) P n(C) P n(S) \\
& +\left(T C_{C}-T C_{B}\right) \operatorname{Pn}(C) P n(B)+\left(T C_{C}-T C_{L}\right) \operatorname{Pn}(C) P n(L) \\
& \frac{\partial P n(T)}{\partial \beta_{1}}=\left(T C_{T}-T C_{C}\right) P n(T) P n(C)+\left(T C_{T}-T C_{S}\right) P n(T) P n(S) \\
& +\left(T C_{T}-T C_{B}\right) \operatorname{Pn}(T) P n(B)+\left(T C_{T}-T C_{L}\right) \operatorname{Pn}(T) P n(L) \\
& \frac{\partial P n(S)}{\partial \beta_{I}}=\left(T C_{S}-T C_{C}\right) P n(S) P n(C)+\left(T C_{S}-T C_{T}\right) P n(S) P n(T) \\
& +\left(T C_{S}-T C_{B}\right) \operatorname{Pn}(S) P n(B)+\left(T C_{S}-T C_{L}\right) \operatorname{Pn}(S) P n(L) \\
& \frac{\partial P n(B)}{\partial \beta_{1}}=\left(T C_{B}-T C_{C}\right) P n(B) P n(C)+\left(T C_{B}-T C_{T}\right) P n(B) P n(T) \\
& +\left(T C_{B}-T C_{S}\right) \operatorname{Pn}(B) P n(S)+\left(T C_{B}-T C_{L}\right) P n(B) P n(L) \\
& \frac{\partial P n(L)}{\partial \beta_{1}}=\left(T C_{L}-T C_{C}\right) P n(L) P n(C)+\left(T C_{L}-T C_{T}\right) P n(L) P n(T) \\
& +\left(T C_{L}-T C_{S}\right) \operatorname{Pn}(L) \operatorname{Pn}(S)+\left(T C_{L}-T C_{B}\right) \operatorname{Pn}(L) P n(B)
\end{aligned}
$$




$$
\begin{aligned}
& \frac{\partial}{\partial \beta_{I}}\left[\frac{1}{P n(C)}\left(\frac{\partial P n(C)}{\partial \beta_{1}}\right)\right]=\left(T C_{C}-T C_{T}\right) \frac{\partial P n(T)}{\partial \beta_{1}}+\left(T C_{C}-T C_{S}\right) \frac{\partial P n(S)}{\partial \beta_{1}}+\left(T C_{C}-T C_{B}\right) \frac{\partial P n(B)}{\partial \beta_{1}}+\left(T C_{C}-T C_{L}\right) \frac{\partial P n(L)}{\partial \beta_{1}} \\
& \frac{\partial}{\partial \beta_{1}}\left[\frac{1}{P n(C)}\left(\frac{\partial P n(C)}{\partial \beta_{1}}\right)\right]=\left(T C_{C}-T C_{T}\right) P n(T)\left(\left(T C C_{T}-T C_{C}\right) P n(C)+\left(T C_{T}-T C_{S}\right) P n(S)\right. \\
& \left.+\left(T C_{T}-T C_{B}\right) \operatorname{Pn}(B)+\left(T C_{T}-T C_{L}\right) \operatorname{Pn}(L)\right] \\
& +\left(T C_{C}-T C_{S}\right) P n(S)\left[\left(T C_{S}-T C_{C}\right) P n(C)+\left(T C_{S}-T C_{T}\right) P n(T)\right. \\
& \left.+\left(T C_{S}-T C_{B}\right) P n(B)+\left(T C_{S}-T C_{L}\right) P n(L)\right] \\
& +\left(T C_{C}-T C_{B}\right) \operatorname{Pn}(B)\left[\left(T C_{B}-T C_{C}\right) P n(C)+\left(T C_{B}-T C_{T}\right) P n(T)\right. \\
& \left.+\left(T C_{B}-T C_{S}\right) P n(S)+\left(T C_{B}-T C_{L}\right) P n(L)\right] \\
& +\left(T C_{C}-T C_{L}\right) P n(L)\left[\left(T C_{L}-T C_{C}\right) P n(C)+\left(T C_{L}-T C_{T}\right) P n(T)\right. \\
& \left.+\left(T C_{L}-T C_{S}\right) P n(S)+\left(T C_{L}-T C_{B}\right) P n(B)\right]
\end{aligned}
$$

$$
\begin{aligned}
& \frac{\partial}{\partial \beta_{I}}\left[\frac{1}{P n(C)} \frac{\partial P n(C)}{\partial \beta_{1}}\right]=-P n(T) P n(C)\left(T C_{C}-T C_{T}\right)^{2}+P n(T) P n(S)\left(T C_{T}-T C_{S}\right)\left(T C_{C}-T C_{T}\right) \\
& +P n(T) P n(B)\left(T C_{T}-T C_{B}\right)\left(T C_{C}-T C_{T}\right)+P n(T) P n(L)\left(T C_{T}-T C_{L}\right)\left(T C_{C}-T C_{T}\right) \\
& -P n(S) P n(C)\left(T C_{C}-T C_{S}\right)^{2}+\operatorname{Pn}(S) P n(T)\left(T C_{C}-T C_{S}\right)\left(T C_{S}-T C_{T}\right) \\
& +\operatorname{Pn}(S) P n(B)\left(T C_{C}-T C_{S}\right)\left(T C_{S}-T C_{B}\right)+P n(S) P n(L)\left(T C_{C}-T C_{S}\right)\left(T C_{S}-T C_{L}\right) \\
& -P n(B) P n(C)\left(T C_{C}-T C_{B}\right)^{2}+P n(B) P n(T)\left(T C_{C}-T C_{B}\right)\left(T C_{B}-T C_{T}\right) \\
& +\operatorname{Pn}(B) P n(S)\left(T C_{C}-T C_{B}\right)\left(T C_{B}-T C_{S}\right)+\operatorname{Pn}(B) P n(L)\left(T C_{C}-T C_{B}\right)\left(T C_{B}-T C_{L}\right) \\
& -P n(L) P n(C)\left(T C_{C}-T C_{L}\right)^{2}+P n(L) P n(T)\left(T C_{C}-T C_{L}\right)\left(T C_{L}-T C_{T}\right) \\
& +P n(L) P n(S)\left(T C_{C}-T C_{L}\right)\left(T C_{L}-T C_{S}\right)+P n(L) P n(B)\left(T C_{C}-T C_{L}\right)\left(T C_{L}-T C_{B}\right)
\end{aligned}
$$

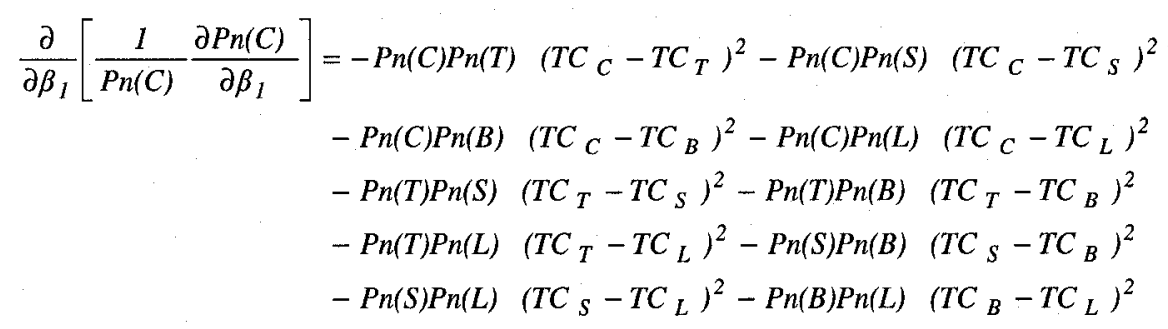

$$
\begin{aligned}
& \frac{1}{P n(T)}\left(\frac{\partial P n(T)}{\partial \beta_{1}}\right)=\left(T C_{T}-T C_{C}\right) P n(C)+\left(T C_{T}-T C_{S}\right) P n(S)+\left(T C_{T}-T C_{B}\right) P n(B)+\left(T C_{T}-T C_{L}\right) P n(L) \\
& \frac{1}{\partial \beta_{1}}\left[\frac{1}{P n(T)}\left(\frac{\partial P n(T)}{\partial \beta_{1}}\right)\right]=\left(T C_{T}-T C_{C}\right) \frac{\partial P n(C)}{\partial \beta_{1}}+\left(T C_{T}-T C_{S}\right) \frac{\partial P n(S)}{\partial \beta_{1}}+\left(T C_{T}-T C_{B}\right) \frac{\partial P n(B)}{\partial \beta_{1}}+\left(T C_{T}-T C_{L}\right) \frac{\partial P n(L)}{\partial \beta_{I}}
\end{aligned}
$$




$$
\begin{aligned}
& \frac{1}{\partial \beta_{1}}\left[\frac{1}{P n(T)}\left(\frac{\partial P n(T)}{\partial \beta_{1}}\right)\right]=\left(T C_{T}-T C_{C}\right)\left(\left(T C_{C}-T C_{T}\right) P n(C) P n(T)+\left(T C_{C}-T C_{S}\right) \operatorname{Pn}(C) P n(S)\right. \\
& \left.+\left(T C_{C}-T C_{B}\right) P n(C) P n(B)+\left(T C_{C}-T C_{L}\right) P n(C) P n(L)\right] \\
& +\left(T C_{T}-T C_{S}\right)\left[\left(T C_{S}-T C_{C}\right) P n(S) P n(C)+\left(T C_{S}-T C_{T}\right) P n(S) P n(T)\right. \\
& \left.+\left(T C_{S}-T C_{B}\right) \operatorname{Pn}(S) \operatorname{Pn}(B)+\left(T C_{S}-T C_{L}\right) \operatorname{Pn}(S) P n(L)\right] \\
& +\left(T C_{T}-T C_{B}\right)\left[\left(T C_{B}-T C_{C}\right) P n(B) P n(C)+\left(T C_{B}-T C_{T}\right) P n(B) P n(T)\right. \\
& \left.+\left(T C_{B}-T C_{S}\right) \operatorname{Pn}(B) \operatorname{Pn}(S)+\left(T C_{B}-T C_{L}\right) \operatorname{Pn}(B) P n(L)\right] \\
& +\left(T C_{T}-T C_{L}\right)\left[\left(T C_{L}-T C_{C}\right) \operatorname{Pn}(L) P n(C)+\left(T C_{L}-T C_{T}\right) \operatorname{Pn}(L) P n(T)\right. \\
& \left.+\left(T C_{L}-T C_{S}\right) P n(L) P n(S)+\left(T C_{L}-T C_{B}\right) P n(L) P n(B)\right]
\end{aligned}
$$

$$
\begin{aligned}
& \frac{1}{\partial \beta_{1}}\left[\frac{1}{P n(T)}\left(\frac{\partial P n(T)}{\partial \beta_{1}}\right)\right]=-P n(C) P n\left(T \nmid T C_{C}-T C_{T}\right)^{2}-P n(C) P n\left(S \nmid T C_{C}-T C_{S}\right)^{2} \\
& -P n(C) P n\left(B \nmid T C_{C}-T C_{B}\right)^{2}-P n(C) P n\left(L \backslash T C_{C}-T C_{L}\right)^{2} \\
& -\operatorname{Pn}(T) P n\left(S \gamma T C_{T}-T C_{S}\right)^{2}-P n(T) P n\left(B \gamma T C_{T}-T C_{B}\right)^{2} \\
& -P n(T) P n\left(L \chi T C_{T}-T C_{L}\right)^{2}-P n(S) P n\left(B \chi T C_{S}-T C_{B}\right)^{2} \\
& -\operatorname{Pn}(S) P n\left(L \backslash T C_{S}-T C_{L}\right)^{2}-P n(B) P n\left(L \ell T C_{B}-T C_{L}\right)^{2}
\end{aligned}
$$

$$
\begin{aligned}
& \left.\frac{1}{\partial \beta_{1}}\left[\frac{1}{P n(T)}\left(\frac{\partial P n(S)}{\partial \beta_{1}}\right)\right]=-P n(C) P n(T)\left(T C_{C}-T C_{T}\right)^{2}-P n(C) P n(S) T C_{C}-T C_{S}\right)^{2} \\
& -\operatorname{Pn}(C) \operatorname{Pn}(B)\left(T C_{C}-T C_{B}\right)^{2}-P n(C) P n(L)\left(T C_{C}-T C_{L}\right)^{2} \\
& \left.-\operatorname{Pn}(T) P n(S)\left(T C_{T}-T C_{S}\right)^{2}-P n(T) P n(B) T C_{T}-T C_{B}\right)^{2} \\
& -P n(T) P n(L)\left(T C_{T}-T C_{L}\right)^{2}-P n(S) P n(B)\left(T C_{S}-T C_{B}\right)^{2} \\
& -P n(S) P n(L)\left(T C_{S}-T C_{L}\right)^{2}-P n(B) P n(L)\left(T C_{B}-T C_{L}\right)^{2} \\
& \frac{1}{\partial \beta_{1}}\left[\frac{1}{P n(T)}\left(\frac{\partial P n(B)}{\partial \beta_{1}}\right)\right]=-P n(C) P n(T)\left(T C_{C}-T C_{T}\right)^{2}-P n(C) P n(S)\left(T C_{C}-T C_{S}\right)^{2} \\
& -P n(C) P n(B)\left(T C_{C}-T C_{B}\right)^{2}-P n(C) P n(L)\left(T C_{C}-T C_{L}\right)^{2} \\
& -P n(T) P n(S)\left(T C_{T}-T C_{S}\right)^{2}-P n(T) P n(B)\left(T C_{T}-T C_{B}\right)^{2} \\
& -P n(T) P n(L)\left(T C_{T}-T C_{L}\right)^{2}-P n(S) P n(B)\left(T C_{S}-T C_{B}\right)^{2} \\
& -\operatorname{Pn}(S) P n(L)\left(T C_{S}-T C_{L}\right)^{2}-P n(B) P n(L)\left(T C_{B}-T C_{L}\right)^{2}
\end{aligned}
$$




$$
\begin{aligned}
\frac{1}{\partial \beta_{1}}\left[\frac{1}{P n(T)}\left(\frac{\partial P n(B)}{\partial \beta_{I}}\right)\right]= & -\operatorname{Pn}(C) P n(T)\left(T C_{C}-T C_{T}\right)^{2}-\operatorname{Pn}(C) P n(S)\left(T C_{C}-T C_{S}\right)^{2} \\
& -\operatorname{Pn}(C) P n(B)\left(T C_{C}-T C_{B}\right)^{2}-\operatorname{Pn}(C) P n(L)\left(T C_{C}-T C_{L}\right)^{2} \\
& -\operatorname{Pn}(T) P n(S)\left(T C_{T}-T C_{S}\right)^{2}-\operatorname{Pn}(T) P n(B)\left(T C_{T}-T C_{B}\right)^{2} \\
& -\operatorname{Pn}(T) P n(L)\left(T C_{T}-T C_{L}\right)^{2}-\operatorname{Pn}(S) P n(B)\left(T C_{S}-T C_{B}\right)^{2} \\
& -\operatorname{Pn}(S) P n(L)\left(T C_{S}-T C_{L}\right)^{2}-\operatorname{Pn}(B) P n(L)\left(T C_{B}-T C_{L}\right)^{2}
\end{aligned}
$$

2) The second order partial derivative with respect to $\beta_{1} \beta_{2}$ is

$$
\begin{gathered}
\frac{\partial^{2} L L\left(\beta_{1}, \beta_{2}, \cdots, \beta_{8}, \beta_{9}\right)}{\partial \beta_{2} \partial \beta_{1}}=\sum_{n=1}^{N}\left[y_{C n} \frac{\partial}{\partial \beta_{2}}\left[\frac{1}{P n(C h}\left(\frac{\partial P n\left(C_{h}\right.}{\partial \beta_{1}}\right)\right]+y_{T n} \frac{\partial}{\partial \beta_{2}}\left[\frac{1}{P n\left(T_{h}\right.}\left(\frac{\partial P n(T)}{\partial \beta_{1}}\right)\right]+y_{S n} \frac{\partial}{\partial \beta_{2}}\left[\frac{1}{P n(S)}\left(\frac{\partial P n\left(S_{h}\right.}{\partial \beta_{1}}\right)\right]+\right. \\
\left.y_{B n} \frac{\partial}{\partial \beta_{2}}\left[\frac{1}{P n(B)}\left(\frac{\partial P n\left(B_{h}\right.}{\partial \beta_{1}}\right)\right]+y_{L n} \frac{\partial}{\partial \beta_{2}}\left[\frac{1}{P n(L)}\left(\frac{\partial P n(L)}{\partial \beta_{1}}\right)\right]\right]
\end{gathered}
$$

where

$$
\begin{aligned}
& \frac{1}{P n(C)}\left(\frac{\partial P n(C)}{\partial \beta_{1}}\right)=\left(T C_{C}-\right.\left.T C_{T}\right) P n(T)+\left(T C_{C}-T C_{S}\right) P n(S)+\left(T C_{C}-T C_{B}\right) P n(B)+\left(T C_{C}-T C_{L}\right) P n(L) \\
& \frac{\partial}{\partial \beta_{2}}\left[\frac{1}{P n(C)}\left(\frac{\partial P n(C)}{\partial \beta_{1}}\right)\right]=\left(T C_{C}-T C_{r}\right) \frac{\partial P n(T)}{\partial \beta_{2}}+\left(T C_{C}-T C_{S}\right) \frac{\partial P n(S)}{\partial \beta_{2}}+\left(T C_{C}-T C_{B}\right) \frac{\partial P n(B)}{\partial \beta_{2}}+\left(T C_{C}-T C_{L}\right) \frac{\partial P n(L)}{\partial \beta_{2}} \\
& \frac{\partial}{\partial \beta_{2}}\left[\frac{1}{P n(C)}\left(\frac{\partial P n(C)}{\partial \beta_{1}}\right)\right]=\left(T C_{C}-T C_{T}\right)\left[\left(T T_{T}-T T_{C}\right) P n(T) P n(C)+\left(T T_{T}-T T_{S}\right) P n(T) P n(S)\right. \\
&\left.+\left(T T_{T}-T T_{B}\right) P n(T) P n(B)+\left(T T_{T}-T T_{L}\right) P n(T) P n(L)\right] \\
&+\left(T C_{C}-T C_{S}\right)\left[\left(T T_{S}-T T_{C}\right) P n(S) P n(C)+\left(T T_{S}-T T_{T}\right) P n(S) P n(T)\right. \\
&\left.+\left(T T_{S}-T T_{B}\right) P n(S) P n(B)+\left(T T_{S}-T T_{L}\right) P n(S) P n(L)\right] \\
&+\left(T C_{C}-T C_{B}\right)\left[\left(T T_{B}-T T_{C}\right) P n(B) P n(C)+\left(T T_{B}-T T_{T}\right) P n(B) P n(T)\right. \\
&\left.+\left(T T_{B}-T T_{S}\right) P n(B) P n(S)+\left(T T_{B}-T T_{L}\right) P n(B) P n(L)\right] \\
&+\left(T C_{C}-T C_{L}\right)\left[\left(T T_{L}-T T_{C}\right) P n(L) P n(C)+\left(T T_{L}-T T_{T}\right) P n(L) P n(T)\right. \\
&\left.+\left(T T_{L}-T T_{S}\right) P n(L) P n(S)+\left(T T_{L}-T T_{B}\right) P n(L) P n(B)\right]
\end{aligned}
$$




$$
\begin{aligned}
\frac{\partial}{\partial \beta_{2}}\left[\frac{1}{P n(C)}\left(\frac{\partial P n(C)}{\partial \beta_{I}}\right)\right] & =-\left(T C_{C}-T C_{T}\right)\left(T T_{C}-T T_{T}\right) \operatorname{Pn}(C) P n(T)-\left(T C_{C}-T C_{S}\right)\left(T T_{C}-T T_{S}\right) \operatorname{Pn}(C) P n(S) \\
& -\left(T C_{C}-T C_{B}\right)\left(T T_{C}-T T_{B}\right) \operatorname{Pn}(C) P n(B)-\left(T C_{C}-T C_{L}\right)\left(T T_{C}-T T_{L}\right) P n(C) P n(L) \\
& -\left(T C_{T}-T C_{S}\right)\left(T T_{T}-T T_{S}\right) \operatorname{Pn}(T) P n(S)-\left(T C_{T}-T C_{B}\right)\left(T T_{T}-T T_{B}\right) P n(T) P n(B) \\
& -\left(T C_{T}-T C_{L}\right)\left(T T_{T}-T T_{L}\right) \operatorname{Pn}(T) P n(L)-\left(T C_{S}-T C_{B}\right)\left(T T_{S}-T T_{B}\right) P n(S) P n(B) \\
& -\left(T C_{S}-T C_{L}\right)\left(T T_{S}-T T_{L}\right) \operatorname{Pn}(S) P n(L)-\left(T C_{B}-T C_{L}\right)\left(T T_{B}-T T_{L}\right) P n(B) P n(L)
\end{aligned}
$$

$$
\begin{aligned}
\frac{1}{P n(T)}\left(\frac{\partial P n(T)}{\partial \beta_{1}}\right)=\left(T C_{T}-\right. & \left.T C_{C}\right) P n(C)+\left(T C_{T}-T C_{S}\right) P n(S)+\left(T C_{T}-T C_{B}\right) P n(B)+\left(T C_{T}-T C_{L}\right) P n(L) \\
\frac{1}{\partial \beta_{2}}\left[\frac{1}{P n(T)} \frac{\partial P n(T)}{\partial \beta_{1}}\right]=\left(T C_{T}-\right. & \left.T C_{C}\right) \frac{\partial P n(C)}{\partial \beta_{2}}+\left(T C_{T}-T C_{S}\right) \frac{\partial P n(S)}{\partial \beta_{2}}+\left(T C_{T}-T C_{B}\right) \frac{\partial P n(B)}{\partial \beta_{2}}+\left(T C_{T}-T C_{L}\right) \frac{\partial P n(L)}{\partial \beta_{2}} \\
\frac{\partial}{\partial \beta_{2}}\left[\frac{1}{P n(T)}\left(\frac{\partial P n(T)}{\partial \beta_{1}}\right)\right]= & \left(T C_{T}-T C_{C}\right)\left[\left(T T_{C}-T T_{T}\right) P n(C) P n(T)+\left(T T_{C}-T T_{S}\right) P n(C) P n(S)\right. \\
& \left.+\left(T T_{C}-T T_{B}\right) P n(C) P n(B)+\left(T T_{C}-T T_{L}\right) P n(C) P n(L)\right] \\
& +\left(T C_{T}-T C_{S}\right)\left[\left(T T_{S}-T T_{C}\right) P n(S) P n(C)+\left(T T_{S}-T T_{T}\right) P n(S) P n(T)\right. \\
& \left.+\left(T T_{S}-T T_{B}\right) P n(S) P n(B)+\left(T T_{S}-T T_{L}\right) P n(S) P n(L)\right] \\
& +\left(T C_{T}-T C_{B}\right)\left[\left(T T_{B}-T T_{C}\right) P n(B) P n(C)+\left(T T_{B}-T T_{T}\right) P n(B) P n(T)\right. \\
& \left.+\left(T T_{B}-T T_{S}\right) P n(B) P n(S)+\left(T T_{B}-T T_{L}\right) P n(B) P n(L)\right] \\
& +\left(T C_{T}-T C_{L}\right)\left[\left(T T_{L}-T T_{C}\right) P n(L) P n(C)+\left(T T_{L}-T T_{T}\right) P n(L) P n(T)\right. \\
& \left.+\left(T T_{L}-T T_{S}\right) P n(L) P n(S)+\left(T T_{L}-T T_{B}\right) P n(L) P n(B)\right]
\end{aligned}
$$

$$
\begin{aligned}
\frac{\partial}{\partial \beta_{2}}\left[\frac{1}{\operatorname{Pn}(T)}\left(\frac{\partial P n(T)}{\partial \beta_{1}}\right)\right] & =-\left(T C_{C}-T C_{T}\right)\left(T T_{C}-T T_{T}\right) \operatorname{Pn}(C) \operatorname{Pn}(T)-\left(T C_{C}-T C_{S}\right)\left(T T_{C}-T T_{S}\right) \operatorname{Pn}(C) \operatorname{Pn}(S) \\
& -\left(T C_{C}-T C_{B}\right)\left(T T_{C}-T T_{B}\right) \operatorname{Pn}(C) \operatorname{Pn}(B)-\left(T C_{C}-T C_{L}\right)\left(T T_{C}-T T_{L}\right) \operatorname{Pn}(C) \operatorname{Pn}(L) \\
& -\left(T C_{T}-T C_{S}\right)\left(T T_{T}-T T_{S}\right) \operatorname{Pn}(T) \operatorname{Pn}(S)-\left(T C_{T}-T C_{B}\right)\left(T T_{T}-T T_{B}\right) \operatorname{Pn}(T) \operatorname{Pn}(B) \\
& -\left(T C_{T}-T C_{L}\right)\left(T T_{T}-T T_{L}\right) \operatorname{Pn}(T) \operatorname{Pn}(L)-\left(T C_{S}-T C_{B}\right)\left(T T_{S}-T T_{B}\right) \operatorname{Pn}(S) \operatorname{Pn}(B) \\
& -\left(T C_{S}-T C_{L}\right)\left(T T_{S}-T T_{L}\right) \operatorname{Pn}(S) \operatorname{Pn}(L)-\left(T C_{B}-T C_{L}\right)\left(T T_{B}-T T_{L}\right) \operatorname{Pn}(B) \operatorname{Pn}(L)
\end{aligned}
$$




$$
\begin{aligned}
& \frac{\partial}{\partial \beta_{2}}\left[\frac{1}{P n(S)}\left(\frac{\partial P n(S)}{\partial \beta_{1}}\right)\right]=-\left(T C_{C}-T C_{T}\right)\left(T T_{C}-T T_{T}\right) \operatorname{Pn}(C) P n(T)-\left(T C_{C}-T C_{S}\right)\left(T T_{C}-T T_{S}\right) \operatorname{Pn}(C) \operatorname{Pn}(S) \\
& -\left(T C_{C}-T C_{B}\right)\left(T T_{C}-T T_{B}\right) P n(C) P n(B)-\left(T C_{C}-T C_{L}\right)\left(T T_{C}-T T_{L}\right) P n(C) P n(L) \\
& -\left(T C_{T}-T C_{S}\right)\left(T T_{T}-T T_{S}\right) P n(T) P n(S)-\left(T C_{T}-T C_{B}\right)\left(T T_{T}-T T_{B}\right) P n(T) P n(B) \\
& -\left(T C_{T}-T C_{L}\right)\left(T T_{T}-T T_{L}\right) P n(T) P n(L)-\left(T C_{S}-T C_{B}\right)\left(T T_{S}-T T_{B}\right) P n(S) P n(B) \\
& -\left(T C_{S}-T C_{L}\right)\left(T T_{S}-T T_{L}\right) P n(S) P n(L)-\left(T C_{B}-T C_{L}\right)\left(T T_{B}-T T_{L}\right) P n(B) P n(L) \\
& \frac{\partial}{\partial \beta_{2}}\left[\frac{1}{P n(S)}\left(\frac{\partial P n(B)}{\partial \beta_{1}}\right)\right]=-\left(T C_{C}-T C_{T}\right)\left(T T_{C}-T T_{T}\right) P n(C) P n(T)-\left(T C_{C}-T C_{S}\right)\left(T T_{C}-T T_{S}\right) P n(C) P n(S) \\
& -\left(T C_{C}-T C_{B}\right)\left(T T_{C}-T T_{B}\right) \operatorname{Pn}(C) P n(B)-\left(T C_{C}-T C_{L}\right)\left(T T_{C}-T T_{L}\right) P n(C) P n(L) \\
& -\left(T C_{T}-T C_{S}\right)\left(T T_{T}-T T_{S}\right) \operatorname{Pn}(T) P n(S)-\left(T C_{T}-T C_{B}\right)\left(T T_{T}-T T_{B}\right) \operatorname{Pn}(T) P n(B) \\
& -\left(T C_{T}-T C_{L}\right)\left(T T_{T}-T T_{L}\right) P n(T) P n(L)-\left(T C_{S}-T C_{B}\right)\left(T T_{S}-T T_{B}\right) \operatorname{Pn}(S) P n(B) \\
& -\left(T C_{S}-T C_{L}\right)\left(T T_{S}-T T_{L}\right) \operatorname{Pn}(S) \operatorname{Pn}(L)-\left(T C_{B}-T C_{L}\right)\left(T T_{B}-T T_{L}\right) \operatorname{Pn}(B) \operatorname{Pn}(L) \\
& \frac{\partial}{\partial \beta_{2}}\left[\frac{1}{P n(L)}\left(\frac{\partial P n(L)}{\partial \beta_{1}}\right)\right]=-\left(T C_{C}-T C_{T}\right)\left(T T_{C}-T T_{T}\right) P n(C) P n(T)-\left(T C_{C}-T C_{S}\right)\left(T T_{C}-T T_{S}\right) P n(C) P n(S) \\
& -\left(T C_{C}-T C_{B}\right)\left(T T_{C}-T T_{B}\right) P n(C) P n(B)-\left(T C_{C}-T C_{L}\right)\left(T T_{C}-T T_{L}\right) P n(C) P n(L) \\
& -\left(T C_{T}-T C_{S}\right)\left(T T_{T}-T T_{S}\right) \operatorname{Pn}(T) P n(S)-\left(T C_{T}-T C_{B}\right)\left(T T_{T}-T T_{B}\right) \operatorname{Pn}(T) \operatorname{Pn}(B) \\
& -\left(T C_{T}-T C_{L}\right)\left(T T_{T}-T T_{L}\right) P n(T) P n(L)-\left(T C_{S}-T C_{B}\right)\left(T T_{S}-T T_{B}\right) P n(S) P n(B) \\
& -\left(T C_{S}-T C_{L}\right)\left(T T_{S}-T T_{L}\right) \operatorname{Pn}(S) P n(L)-\left(T C_{B}-T C_{L}\right)\left(T T_{B}-T T_{L}\right) P n(B) P n(L)
\end{aligned}
$$

3) The second order partial derivatives with respect to $\beta_{1} \beta_{3}$ is

$$
\begin{aligned}
\frac{\partial^{2} L L\left(\beta_{1}, \beta_{2}, \cdots, \beta_{8}, \beta_{9}\right)}{\partial \beta_{3} \partial \beta_{1}}= & \sum_{n=1}^{N}\left[y_{C n} \frac{\partial}{\partial \beta_{3}}\left[\frac{1}{P n(C)_{n}}\left(\frac{\partial P n(C)_{n}}{\partial \beta_{1}}\right)\right]+y_{T n} \frac{\partial}{\partial \beta_{3}}\left[\frac{1}{P n(T)_{n}}\left(\frac{\partial P n(T)_{n}}{\partial \beta_{1}}\right)\right]+y_{S n} \frac{\partial}{\partial \beta_{3}}\left[\frac{1}{P n(S)_{n}}\left(\frac{\partial P n(S)_{n}}{\partial \beta_{1}}\right)\right]+\right. \\
& \left.y_{B n} \frac{\partial}{\partial \beta_{3}}\left[\frac{1}{P n(B)_{n}}\left(\frac{\partial P n(B)_{n}}{\partial \beta_{1}}\right)\right]+y_{L n} \frac{\partial}{\partial \beta_{3}}\left[\frac{1}{P n(L)_{n}}\left(\frac{\partial P n(L)_{n}}{\partial \beta_{1}}\right)\right]\right]
\end{aligned}
$$

where

$$
\begin{aligned}
& \frac{1}{P n(C)}\left(\frac{\partial P n(C)}{\partial \beta_{1}}\right)=\left(T C_{C}-T C_{T}\right) P n(T)+\left(T C_{C}-T C_{S}\right) P n(S)+\left(T C_{C}-T C_{B}\right) P n(B)+\left(T C_{C}-T C_{L}\right) P n(L) \\
& \frac{1}{\partial \beta_{3}}\left[\frac{1}{P n(C)}\left(\frac{\partial P n(C)}{\partial \beta_{1}}\right)\right]=\left(T C_{C}-T C_{T}\right) \frac{\partial P n(T)}{\partial \beta_{3}}+\left(T C_{C}-T C_{S}\right) \frac{\partial P n(S)}{\partial \beta_{3}}+\left(T C_{C}-T C_{B}\right) \frac{\partial P n(B)}{\partial \beta_{3}}+\left(T C_{C}-T C_{L}\right) \frac{\partial P n(L)}{\partial \beta_{3}} \\
& \frac{\partial P n(C)}{\partial \beta_{3}}=\operatorname{AccomPn}(C)[P n(T)+P n(S)+P n(B)+P n(L)]
\end{aligned}
$$




$$
\begin{aligned}
& \frac{\partial P n(T)}{\partial \beta_{3}}=-\operatorname{AccomPn}(T) P n(C) \\
& \frac{\partial P n(S)}{\partial \beta_{3}}=-A \operatorname{comPn}(S) P n(C) \\
& \frac{\partial P n(B)}{\partial \beta_{3}}=-A c c o m P n(B) P n(C) \\
& \frac{\partial P n(L)}{\partial \beta_{3}}=-\operatorname{AccomPn}(L) P n(C) \\
& \frac{1}{\partial \beta_{3}}\left[\frac{1}{\operatorname{Pn}(C)}\left(\frac{\partial P n(C)}{\partial \beta_{1}}\right)\right]=-\operatorname{Accom}\left(T C_{C}-T C_{T}\right) \operatorname{Pn}(C) P n(T)-\operatorname{Accom}\left(T C_{C}-T C_{S}\right) \operatorname{Pn}(C) \operatorname{Pn}(S) \\
& -\operatorname{Accom}\left(T C_{C}-T C_{B}\right) \operatorname{Pn}(C) P n(B)-\operatorname{Accom}\left(T C_{C}-T C_{L}\right) \operatorname{Pn}(C) P n(L) \\
& \frac{I}{P n(T)}\left(\frac{\partial P n(T)}{\partial \beta_{1}}\right)=\left(T C_{T}-T C_{C}\right) P n(C)+\left(T C_{T}-T C_{S}\right) P n(S)+\left(T C_{T}-T C_{B}\right) P n(B)+\left(T C_{T}-T C_{L}\right) P n(L) \\
& \frac{1}{\partial \beta_{3}}\left[\frac{1}{P n(T)}\left(\frac{\partial P n(T)}{\partial \beta_{1}}\right)\right]=\left(T C_{T}-T C_{C}\right) \frac{\partial P n(C)}{\partial \beta_{3}}+\left(T C_{T}-T C_{S}\right) \frac{\partial P n(S)}{\partial \beta_{3}}+\left(T C_{T}-T C_{B}\right) \frac{\partial P n(B)}{\partial \beta_{3}}+\left(T C_{T}-T C_{L}\right) \frac{\partial P n(L)}{\partial \beta_{3}} \\
& \frac{1}{\partial \beta_{3}}\left[\frac{1}{P n(T)}\left(\frac{\partial P n(T)}{\partial \beta_{1}}\right)\right]=-\operatorname{Accom}\left(T C_{C}-T C_{T}\right) P n(C) P n(T)-\operatorname{Accom}\left(T C_{C}-T C_{S}\right) P n(C) P n(S) \\
& -\operatorname{Accom}\left(T C_{C}-T C_{B}\right) \operatorname{Pn}(C) P n(B)-\operatorname{Accom}\left(T C_{C}-T C_{L}\right) \operatorname{Pn}(C) \operatorname{Pn}(L) \\
& \frac{1}{P n(S)}\left(\frac{\partial P n(S)}{\partial \beta_{1}}\right)=\left(T C_{S}-T C_{C}\right) P n(C)+\left(T C_{S}-T C_{T}\right) P n(T)+\left(T C_{S}-T C_{B}\right) P n(B)+\left(T C_{S}-T C_{L}\right) P n(L) \\
& \frac{1}{\partial \beta_{3}}\left[\frac{1}{P n(S)}\left(\frac{\partial P n(S)}{\partial \beta_{1}}\right)\right]=-\operatorname{Accom}\left(T C_{C}-T C_{T}\right) \operatorname{Pn}(C) P n(T)-\operatorname{Accom}\left(T C_{C}-T C_{S}\right) \operatorname{Pn}(C) P n(S) \\
& -\operatorname{Accom}\left(T C_{C}-T C_{B}\right) \operatorname{Pn}(C) P n(B)-\operatorname{Accom}\left(T C_{C}-T C_{L}\right) \operatorname{Pn}(C) P n(L) \\
& \frac{1}{P n(B)}\left(\frac{\partial P n(B)}{\partial \beta_{1}}\right)=\left(T C_{B}-T C_{C}\right) P n(C)+\left(T C_{B}-T C_{T}\right) P n(T)+\left(T C_{B}-T C_{S}\right) P n(S)+\left(T C_{B}-T C_{L}\right) P n(L) \\
& \frac{1}{\partial \beta_{3}}\left[\frac{1}{\operatorname{Pn}(B)}\left(\frac{\partial P n(B)}{\partial \beta_{1}}\right)\right]=-\operatorname{Accom}\left(T C_{C}-T C_{T}\right) \operatorname{Pn}(C) P n(T)-\operatorname{Accom}\left(T C_{C}-T C_{S}\right) \operatorname{Pn}(C) P n(S) \\
& -\operatorname{Accom}\left(T C_{C}-T C_{B}\right) P n(C) P n(B)-\operatorname{Accom}\left(T C_{C}-T C_{L}\right) P n(C) P n(L) \\
& \frac{1}{P n(L)}\left(\frac{\partial P n(L)}{\partial \beta_{1}}\right)=\left(T C_{L}-T C_{C}\right) P n(C)+\left(T C_{L}-T C_{T}\right) P n(T)+\left(T C_{L}-T C_{S}\right) P n(S)+\left(T C_{L}-T C_{B}\right) P n(B)
\end{aligned}
$$


$\begin{aligned} \frac{1}{\partial \beta_{3}}\left[\frac{1}{P n(L)}\left(\frac{\partial P n(L)}{\partial \beta_{1}}\right)\right] & =-\operatorname{Accom}\left(T C_{C}-T C_{T}\right) \operatorname{Pn}(C) P n(T)-\operatorname{Accom}\left(T C_{C}-T C_{S}\right) \operatorname{Pn}(C) P n(S) \\ & -\operatorname{Accom}\left(T C_{C}-T C_{B}\right) \operatorname{Pn}(C) P n(B)-\operatorname{Accom}\left(T C_{C}-T C_{L}\right) \operatorname{Pn}(C) P n(L)\end{aligned}$

4) The second order partial derivative with respect to $\beta_{1} \beta_{4}$ is

$$
\begin{aligned}
\frac{\partial^{2} L L\left(\beta_{1}, \beta_{2}, \cdots, \beta_{8}, \beta_{9}\right)}{\partial \beta_{4} \partial \beta_{1}}= & \sum_{n=1}^{N}\left[y_{C n} \frac{\partial}{\partial \beta_{4}}\left[\frac{1}{P n(C)_{h}}\left(\frac{\partial P n(C)_{h}}{\partial \beta_{1}}\right)\right]+y_{T n} \frac{\partial}{\partial \beta_{4}}\left[\frac{1}{P n(T)_{h}}\left(\frac{\partial P n(T)_{h}}{\partial \beta_{1}}\right)\right]+y_{S n} \frac{\partial}{\partial \beta_{4}}\left[\frac{1}{P n(S)_{h}}\left(\frac{\partial P n(S)_{h}}{\partial \beta_{1}}\right)\right]+\right. \\
& y_{B n} \frac{\partial}{\partial \beta_{4}}\left[\frac{1}{P n(B h}\left(\frac{\partial P n\left(B h_{h}\right.}{\partial \beta_{1}}\right)\right]+y_{L n} \frac{\partial}{\partial \beta_{4}}\left[\frac{1}{P n\left(L h_{h}\right.}\left(\frac{\partial P n(L h}{\partial \beta_{1}}\right)\right] l
\end{aligned}
$$

where

$$
\begin{aligned}
& \frac{1}{\partial \beta_{4}}\left[\frac{1}{\operatorname{Pn}(C)}\left(\frac{\partial P n(C)}{\partial \beta_{1}}\right)\right]=-\operatorname{Sexm}\left(T C_{C}-T C_{T}\right) \operatorname{Pn}(C) \operatorname{Pn}(T)-\operatorname{Sexm}\left(T C_{C}-T C_{S}\right) \operatorname{Pn}(C) \operatorname{Pn}(S) \\
& -\operatorname{Sexm}\left(T C_{C}-T C_{L}\right) \operatorname{Pn}(C) \operatorname{Pn}(B)-\operatorname{Sexm}\left(T C_{C}-T C_{L}\right) \operatorname{Pn}(C) \operatorname{Pn}(L) \\
& \frac{1}{\partial \beta_{4}}\left[\frac{1}{\operatorname{Pn}(T)}\left(\frac{\partial P n(T)}{\partial \beta_{1}}\right)\right]=-\operatorname{Sexm}\left(T C_{C}-T C_{T}\right) \operatorname{Pn}(C) P n(T)-\operatorname{Sexm}\left(T C_{C}-T C_{S}\right) \operatorname{Pn}(C) P n(S) \\
& -\operatorname{Sexm}\left(T C_{C}-T C_{L}\right) \operatorname{Pn}(C) \operatorname{Pn}(B)-\operatorname{Sexm}\left(T C_{C}-T C_{L}\right) \operatorname{Pn}(C) P n(L) \\
& \frac{1}{\partial \beta_{4}}\left[\frac{1}{P n(S)}\left(\frac{\partial P n(S)}{\partial \beta_{1}}\right)\right]=-\operatorname{Sexm}\left(T C_{C}-T C_{T}\right) \operatorname{Pn}(C) P n(T)-\operatorname{Sexm}\left(T C_{C}-T C_{S}\right) \operatorname{Pn}(C) \operatorname{Pn}(S) \\
& -\operatorname{Sexm}\left(T C_{C}-T C_{L}\right) \operatorname{Pn}(C) P n(B)-\operatorname{Sexm}\left(T C_{C}-T C_{L}\right) \operatorname{Pn}(C) P n(L) \\
& \frac{1}{\partial \beta_{4}}\left[\frac{1}{\operatorname{Pn}(B)}\left(\frac{\partial P n(B)}{\partial \beta_{1}}\right)\right]=-\operatorname{Sexm}\left(T C_{C}-T C_{T}\right) \operatorname{Pn}(C) \operatorname{Pn}(T)-\operatorname{Sexm}\left(T C_{C}-T C_{S}\right) \operatorname{Pn}(C) \operatorname{Pn}(S) \\
& -\operatorname{Sexm}\left(T C_{C}-T C_{L}\right) \operatorname{Pn}(C) \operatorname{Pn}(B)-\operatorname{Sexm}\left(T C_{C}-T C_{L}\right) \operatorname{Pn}(C) \operatorname{Pn}(L) \\
& \frac{1}{\partial \beta_{4}}\left[\frac{1}{P n(L)}\left(\frac{\partial P n(L)}{\partial \beta_{1}}\right)\right]=-\operatorname{Sexm}\left(T C_{C}-T C_{T}\right) \operatorname{Pn}(C) \operatorname{Pn}(T)-\operatorname{Sexm}\left(T C_{C}-T C_{S}\right) \operatorname{Pn}(C) \operatorname{Pn}(S) \\
& -\operatorname{Sexm}\left(T C_{C}-T C_{L}\right) \operatorname{Pn}(C) P n(B)-\operatorname{Sexm}\left(T C_{C}-T C_{L}\right) \operatorname{Pn}(C) P n(L) \\
& \frac{\partial P n(C)}{\partial \beta_{4}}=\operatorname{Sexm} \times \operatorname{Pn}(C)[P n(T)+P n(S)+P n(B)+P n(L)] \\
& \frac{\partial P n(T)}{\partial \beta_{4}}=-\operatorname{Sexm} \times \operatorname{Pn}(T)[P n(C)] \\
& \frac{\partial P n(S)}{\partial \beta_{4}}=-\operatorname{Sexm} \times \operatorname{Pn}(S)[\operatorname{Pn}(C)]
\end{aligned}
$$




$$
\begin{aligned}
& \frac{\partial P n(C)}{\partial \beta_{4}}=-\operatorname{Sexm} \times \operatorname{Pn}(B)[P n(C)] \\
& \frac{\partial P n(C)}{\partial \beta_{4}}=-\operatorname{Sexm} \times \operatorname{Pn}(L)[\operatorname{Pn}(C)]
\end{aligned}
$$

5) The second order partial derivative with respect to $\beta_{1} \beta_{5}$ is

$$
\begin{gathered}
\frac{\partial^{2} L L\left(\beta_{1}, \beta_{2}, \cdots, \beta_{8}, \beta_{9}\right)}{\partial \beta_{5} \partial \beta_{1}}=\sum_{n=1}^{N} l y_{C n} \frac{\partial}{\partial \beta_{5}}\left[\frac{1}{P n\left(C_{h}\right.}\left(\frac{\partial P n\left(C_{h}\right.}{\partial \beta_{1}}\right)\right]+y_{T n} \frac{\partial}{\partial \beta_{5}}\left[\frac{1}{P n\left(T_{h}\right.}\left(\frac{\partial P n\left(T_{h}\right.}{\partial \beta_{1}}\right)\right]+y_{S n} \frac{\partial}{\partial \beta_{5}}\left[\frac{1}{P n\left(S_{h}\right.}\left(\frac{\partial P n\left(S_{h}\right.}{\partial \beta_{1}}\right)\right]+ \\
y_{B n} \frac{\partial}{\partial \beta_{5}}\left[\frac{1}{P n\left(B_{h}\right.}\left(\frac{\partial P n\left(B h_{h}\right.}{\partial \beta_{1}}\right)\right]+y_{L n} \frac{\partial}{\partial \beta_{5}}\left[\frac{1}{P n\left(L L_{h}\right.}\left(\frac{\partial P n\left(L_{h}\right.}{\partial \beta_{1}}\right)\right] J
\end{gathered}
$$

where

$$
\begin{aligned}
& \frac{1}{\partial \beta_{5}}\left[\frac{1}{P n(C)}\left(\frac{\partial P n(C)}{\partial \beta_{1}}\right)\right]=-A g e 340\left(T C_{C}-T C_{T}\right) P n(C) P n(T)-A g e 340\left(T C_{C}-T C_{S}\right) P n(C) P n(S) \\
& \text { - Age340(TC } \left.C-T C_{B}\right) P n(C) P n(B)-\operatorname{Age340}_{C}\left(T C_{C}-T C_{L}\right) \operatorname{Pn}(C) P n(L) \\
& \frac{1}{\partial \beta_{5}}\left[\frac{1}{P n(T)}\left(\frac{\partial P n(T)}{\partial \beta_{1}}\right)\right]=-A g e 340\left(T C_{C}-T C_{T}\right) P n(C) P n(T)-A g e 340\left(T C_{C}-T C_{S}\right) P n(C) P n(S) \\
& -\operatorname{Age340}\left(T C_{C}-T C_{B}\right) \operatorname{Pn}(C) P n(B)-\operatorname{Age340}\left(T C_{C}-T C_{L}\right) \operatorname{Pn}(C) P n(L) \\
& \frac{1}{\partial \beta_{5}}\left[\frac{1}{P n(S)}\left(\frac{\partial P n(S)}{\partial \beta_{I}}\right)\right]=-\operatorname{Age340}\left(T C_{C}-T C_{T}\right) \operatorname{Pn}(C) P n(T)-\operatorname{Age340}\left(T C_{C}-T C_{S}\right) P n(C) P n(S) \\
& -\operatorname{Age340}\left(T C_{C}-T C_{B}\right) P n(C) P n(B)-\operatorname{Age340}\left(T C_{C}-T C_{L}\right) P n(C) P n(L) \\
& \left.\frac{1}{\partial \beta_{5}}\left[\frac{1}{P n(B)}\left(\frac{\partial P n(B)}{\partial \beta_{1}}\right)\right]=-\operatorname{Age340(TC} C_{C}-T C_{T}\right) \operatorname{Pn}(C) P n(T)-\operatorname{Age340}\left(T C_{C}-T C_{S}\right) \operatorname{Pn}(C) P n(S)
\end{aligned}
$$

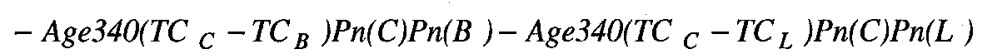

$$
\begin{aligned}
& \left.\left.\frac{1}{\partial \beta_{5}}\left[\frac{1}{P n(L)}\left(\frac{\partial P n(L)}{\partial \beta_{1}}\right)\right]=-\operatorname{Age340(TC} C_{C}-T C_{T}\right) \operatorname{Pn}(C) P n(T)-\operatorname{Age340(TC} C_{C}-T C_{S}\right) \operatorname{Pn}(C) P n(S) \\
& \left.-\operatorname{Age340}_{C}-T C_{B}\right) P n(C) P n(B)-\operatorname{Age340}\left(T C_{C}-T C_{L}\right) P n(C) P n(L) \\
& \frac{\partial P n(C)}{\partial \beta_{5}}=A g e 340 P n(C)[P n(T)+P n(S)+P n(B)+P n(L)] \\
& \frac{\partial P n(T)}{\partial \beta_{5}}=-\operatorname{Age} 340 P n(T) P n(C)
\end{aligned}
$$




$$
\begin{aligned}
& \frac{\partial P n(S)}{\partial \beta_{5}}=-A g e 340 P n(S) P n(C) \\
& \frac{\partial P n(S)}{\partial \beta_{5}}=-A g e 340 P n(B) P n(C) \\
& \frac{\partial P n(L)}{\partial \beta_{5}}=-A g e 340 P n(L) P n(C)
\end{aligned}
$$

6) The second order partial derivative with respect to $\beta_{1} \beta_{6}$ is

$$
\begin{aligned}
\frac{\partial^{2} L L\left(\beta_{1}, \beta_{2}, \cdots, \beta_{8}, \beta_{9}\right)}{\partial \beta_{6} \partial \beta_{1}}= & \sum_{n=1}^{N}\left[y_{C n} \frac{\partial}{\partial \beta_{6}}\left[\frac{1}{P n(C)_{n}}\left(\frac{\partial P n(C)_{n}}{\partial \beta_{1}}\right)\right]+y_{T n} \frac{\partial}{\partial \beta_{6}}\left[\frac{1}{P n(T)_{n}}\left(\frac{\partial P n(T)_{n}}{\partial \beta_{1}}\right)\right]+y_{S n} \frac{\partial}{\partial \beta_{6}}\left[\frac{1}{P n(S)_{n}}\left(\frac{\partial P n(S)_{n}}{\partial \beta_{1}}\right)\right]+\right. \\
& \left.y_{B n} \frac{\partial}{\partial \beta_{6}}\left[\frac{1}{P n(B)_{n}}\left(\frac{\partial P n(B)_{n}}{\partial \beta_{1}}\right)\right]+y_{L n} \frac{\partial}{\partial \beta_{6}}\left[\frac{1}{P n(L)_{n}}\left(\frac{\partial P n(L)_{n}}{\partial \beta_{1}}\right)\right]\right]
\end{aligned}
$$

where

$$
\begin{aligned}
& \frac{1}{P n(C)}\left(\frac{\partial P n(C)}{\partial \beta_{1}}\right)=\left(T C_{C}-T C_{T}\right) P n(T)+\left(T C_{C}-T C_{S}\right) P n(S)+\left(T C_{C}-T C_{B}\right) P n(B)+\left(T C_{C}-T C_{L}\right) P n(L) \\
& \frac{1}{\partial \beta_{6}}\left[\frac{1}{P n(C)}\left(\frac{\partial P n(C)}{\partial \beta_{1}}\right)\right]=-\left(T C_{T}-T C_{C}\right) P n(T) P n(C)-\left(T C_{T}-T C_{S}\right) P n(T) P n(S) \\
& -\left(T C_{T}-T C_{B}\right) P n(T) P n(B)-\left(T C_{T}-T C_{L}\right) P n(T) P n(L) \\
& \frac{1}{P n(T)}\left(\frac{\partial P n(T)}{\partial \beta_{1}}\right)=\left(T C_{T}-T C_{C}\right) P n(C)+\left(T C_{T}-T C_{S}\right) P n(S)+\left(T C_{T}-T C_{B}\right) P n(B)+\left(T C_{T}-T C_{L}\right) P n(L) \\
& \frac{1}{\partial \beta_{6}}\left[\frac{1}{P n(T)}\left(\frac{\partial P n(T)}{\partial \beta_{1}}\right)\right]=-\left(T C_{T}-T C_{C}\right) P n(T) P n(C)-\left(T C_{T}-T C_{S}\right) P n(T) P n(S) \\
& -\left(T C_{T}-T C_{B}\right) P n(T) P n(B)-\left(T C_{T}-T C_{L}\right) \operatorname{Pn}(T) \operatorname{Pn}(L) \\
& \frac{1}{P n(S)}\left(\frac{\partial P n(S)}{\partial \beta_{1}}\right)=\left(T C_{S}-T C_{C}\right) P n(C)+\left(T C_{S}-T C_{T}\right) P n(T)+\left(T C_{S}-T C_{B}\right) P n(B)+\left(T C_{S}-T C_{L}\right) P n(L) \\
& \frac{1}{\partial \beta_{6}}\left[\frac{1}{P n(S)}\left(\frac{\partial P n(S)}{\partial \beta_{1}}\right)\right]=-\left(T C_{T}-T C_{C}\right) P n(T) P n(C)-\left(T C_{T}-T C_{S}\right) P n(T) P n(S) \\
& -\left(T C_{T}-T C_{B}\right) P n(T) P n(B)-\left(T C_{T}-T C_{L}\right) P n(T) P n(L) \\
& \frac{1}{P n(B)}\left(\frac{\partial P n(B)}{\partial \beta_{I}}\right)=\left(T C_{B}-T C_{C}\right) P n(C)+\left(T C_{B}-T C_{T}\right) P n(T)+\left(T C_{B}-T C_{S}\right) P n(S)+\left(T C_{B}-T C_{L}\right) P n(L) \\
& \frac{1}{\partial \beta_{6}}\left[\frac{1}{P n(B)}\left(\frac{\partial P n(B)}{\partial \beta_{1}}\right)\right]=-\left(T C_{T}-T C_{C}\right) \operatorname{Pn}(T) P n(C)-\left(T C_{T}-T C_{S}\right) \operatorname{Pn}(T) P n(S) \\
& -\left(T C_{T}-T C_{B}\right) \operatorname{Pn}(T) P n(B)-\left(T C_{T}-T C_{L}\right) P n(T) P n(L)
\end{aligned}
$$




$$
\begin{aligned}
& \frac{1}{P n(L)}\left(\frac{\partial P n(L)}{\partial \beta_{1}}\right)=\left(T C_{L}-T C_{C}\right) P n(C)+\left(T C_{L}-T C_{T}\right) P n(T)+\left(T C_{L}-T C_{S}\right) P n(S)+\left(T C_{L}-T C_{B}\right) P n(B) \\
& \frac{1}{\partial \beta_{6}}\left[\frac{1}{\operatorname{Pn}(B)}\left(\frac{\partial P n(B)}{\partial \beta_{1}}\right)\right]=-\left(T C_{T}-T C_{C}\right) \operatorname{Pn}(T) P n(C)-\left(T C_{T}-T C_{S}\right) \operatorname{Pn}(T) P n(S) \\
& -\left(T C_{T}-T C_{B}\right) \operatorname{Pn}(T) P n(B)-\left(T C_{T}-T C_{L}\right) \operatorname{Pn}(T) P n(L)
\end{aligned}
$$

$$
\begin{aligned}
& \frac{\partial P n(C)}{\partial \beta_{6}}=-P n(C) P n(T) \\
& \frac{\partial P n(T)}{\partial \beta_{6}}=P n(T)[P n(C)+P n(S)+P n(B)+P n(L)] \\
& \frac{\partial P n(S)}{\partial \beta_{6}}=-P n(S) P n(T) \\
& \frac{\partial P n(B)}{\partial \beta_{6}}=-P n(B) P n(T) \\
& \frac{\partial P n(L)}{\partial \beta_{6}}=-P n(L) P n(T)
\end{aligned}
$$

7) The second order partial derivative with respect to $\beta_{1} \beta_{7}$ is

$$
\begin{aligned}
\frac{\partial^{2} L L\left(\beta_{1}, \beta_{2}, \cdots, \beta_{8}, \beta_{9}\right)}{\partial \beta_{7} \partial \beta_{1}}= & \sum_{n=1}^{N}\left[y_{C n} \frac{\partial}{\partial \beta_{7}}\left[\frac{1}{P n\left(C_{h}\right.}\left(\frac{\partial P n\left(C_{h}\right.}{\partial \beta_{1}}\right)\right]+y_{T n} \frac{\partial}{\partial \beta_{7}}\left[\frac{1}{P n\left(T_{h}\right.}\left(\frac{\partial P n\left(T_{h}\right.}{\partial \beta_{1}}\right)\right]+y_{S n} \frac{\partial}{\partial \beta_{7}}\left[\frac{1}{P n\left(S_{h}\right.}\left(\frac{\partial P n\left(S_{h}\right.}{\partial \beta_{1}}\right)\right]+\right. \\
& \left.y_{B n} \frac{\partial}{\partial \beta_{7}}\left[\frac{1}{P n\left(B_{h}\right.}\left(\frac{\partial P n(B h}{\partial \beta_{1}}\right)\right]+y_{L n} \frac{\partial}{\partial \beta_{7}}\left[\frac{1}{P n(L h}\left(\frac{\partial P n(L h}{\partial \beta_{1}}\right)\right]\right]
\end{aligned}
$$

where

$$
\begin{aligned}
\frac{1}{\partial \beta_{7}}\left[\frac{1}{P n(C)}\left(\frac{\partial P n(C)}{\partial \beta_{1}}\right)\right]= & -\left(T C_{S}-T C_{C}\right) \operatorname{Pn}(S) P n(C)-\left(T C_{S}-T C_{T}\right) \operatorname{Pn}(S) P n(T) \\
& -\left(T C_{S}-T C_{B}\right) \operatorname{Pn}(S) P n(B)-\left(T C_{S}-T C_{L}\right) \operatorname{Pn}(S) P n(L) \\
\frac{1}{\partial \beta_{7}}\left[\frac{1}{P n(T)}\left(\frac{\partial P n(T)}{\partial \beta_{1}}\right)\right]= & -\left(T C_{S}-T C_{C}\right) \operatorname{Pn}(S) P n(C)-\left(T C_{S}-T C_{T}\right) \operatorname{Pn}(S) P n(T) \\
& -\left(T C_{S}-T C_{B}\right) \operatorname{Pn}(S) P n(B)-\left(T C_{S}-T C_{L}\right) \operatorname{Pn}(S) P n(L) \\
\frac{1}{\partial \beta_{7}}\left[\frac{1}{\operatorname{Pn}(S)}\left(\frac{\partial P n(S)}{\partial \beta_{1}}\right)\right]= & -\left(T C_{S}-T C_{C}\right) \operatorname{Pn}(S) P n(C)-\left(T C_{S}-T C_{T}\right) \operatorname{Pn}(S) P n(T) \\
& -\left(T C_{S}-T C_{B}\right) \operatorname{Pn}(S) P n(B)-\left(T C_{S}-T C_{L}\right) \operatorname{Pn}(S) P n(L)
\end{aligned}
$$




$$
\begin{aligned}
& \frac{1}{\partial \beta_{7}}\left[\frac{1}{P n(S)}\left(\frac{\partial P n(S)}{\partial \beta_{1}}\right)\right]=-\left(T C_{S}-T C_{C}\right) P n(S) P n(C)-\left(T C_{S}-T C_{T}\right) P n(S) P n(T) \\
& -\left(T C_{S}-T C_{B}\right) P n(S) P n(B)-\left(T C_{S}-T C_{L}\right) \operatorname{Pn}(S) P n(L) \\
& \frac{1}{\partial \beta_{7}}\left[\frac{1}{\operatorname{Pn}(L)}\left(\frac{\partial \operatorname{Pn}(L)}{\partial \beta_{1}}\right)\right]=-\left(T C_{S}-T C_{C}\right) \operatorname{Pn}(S) P n(C)-\left(T C_{S}-T C_{T}\right) \operatorname{Pn}(S) \operatorname{Pn}(T) \\
& -\left(T C_{S}-T C_{B}\right) \operatorname{Pn}(S) \operatorname{Pn}(B)-\left(T C_{S}-T C_{L}\right) \operatorname{Pn}(S) \operatorname{Pn}(L) \\
& \frac{\partial P n(C)}{\partial \beta_{7}}=-\operatorname{Pn}(C) \operatorname{Pn}(S) \\
& \frac{\partial P n(T)}{\partial \beta_{7}}=-P n(T) P n(S) \\
& \frac{\partial P n(S)}{\partial \beta_{7}}=P n(S)[P n(C)+P n(T)+P n(B)+P n(L)] \\
& \frac{\partial P n(B)}{\partial \beta_{7}}=-P n(B) P n(S) \\
& \frac{\partial P n(L)}{\partial \beta_{7}}=-P n(L) P n(S)
\end{aligned}
$$

8) The second order partial derivative with respect to $\beta_{1} \beta_{8}$ is

$$
\begin{aligned}
\frac{\partial^{2} L L\left(\beta_{1}, \beta_{2}, \cdots, \beta_{8}, \beta_{9}\right)}{\partial \beta_{8} \partial \beta_{I}}= & \sum_{n=1}^{N}\left(y_{C n} \frac{\partial}{\partial \beta_{8}}\left[\frac{1}{P n(C)_{n}}\left(\frac{\partial P n(C)_{n}}{\partial \beta_{1}}\right)\right]+y_{T n} \frac{\partial}{\partial \beta_{8}}\left[\frac{1}{P n(T)_{n}}\left(\frac{\partial P n(T)_{n}}{\partial \beta_{I}}\right)\right]+y_{S_{n}} \frac{\partial}{\partial \beta_{8}}\left[\frac{1}{P n(S)_{n}}\left(\frac{\partial P n(S)_{n}}{\partial \beta_{I}}\right)\right]+\right. \\
& \left.y_{B n} \frac{\partial}{\partial \beta_{8}}\left[\frac{1}{P n(B)_{n}}\left(\frac{\partial P n(B)_{n}}{\partial \beta_{1}}\right)\right]+y_{L n} \frac{\partial}{\partial \beta_{8}}\left[\frac{1}{P n(L)_{n}}\left(\frac{\partial P n(L)_{n}}{\partial \beta_{1}}\right)\right]\right]
\end{aligned}
$$

where

$$
\begin{aligned}
\frac{1}{\partial \beta_{8}}\left[\frac{1}{P n(C)}\left(\frac{\partial P n(C)}{\partial \beta_{1}}\right)\right]= & -\left(T C_{B}-T C_{C}\right) \operatorname{Pn}(B) \operatorname{Pn}(C)-\left(T C_{B}-T C_{T}\right) \operatorname{Pn}(B) \operatorname{Pn}(T) \\
& -\left(T C_{B}-T C_{S}\right) \operatorname{Pn}(B) \operatorname{Pn}(S)-\left(T C_{B}-T C_{L}\right) \operatorname{Pn}(B) \operatorname{Pn}(L) \\
\frac{1}{\partial \beta_{8}}\left[\frac{1}{\operatorname{Pn}(T)}\left(\frac{\partial P n(T)}{\partial \beta_{I}}\right)\right]= & -\left(T C_{B}-T C_{C}\right) \operatorname{Pn}(B) \operatorname{Pn}(C)-\left(T C_{B}-T C_{T}\right) \operatorname{Pn}(B) \operatorname{Pn}(T) \\
& -\left(T C_{B}-T C_{S}\right) \operatorname{Pn}(B) \operatorname{Pn}(S)-\left(T C_{B}-T C_{L}\right) \operatorname{Pn}(B) \operatorname{Pn}(L)
\end{aligned}
$$




$$
\begin{aligned}
& \frac{1}{\partial \beta_{8}}\left[\frac{1}{P n(S)}\left(\frac{\partial P n(S)}{\partial \beta_{1}}\right)\right]=-\left(T C_{B}-T C_{C}\right) \operatorname{Pn}(B) P n(C)-\left(T C_{B}-T C_{T}\right) \operatorname{Pn}(B) \operatorname{Pn}(T) \\
& -\left(T C_{B}-T C_{S}\right) \operatorname{Pn}(B) \operatorname{Pn}(S)-\left(T C_{B}-T C_{L}\right) \operatorname{Pn}(B) \operatorname{Pn}(L) \\
& \frac{1}{\partial \beta_{8}}\left[\frac{1}{P n(B)}\left(\frac{\partial P n(B)}{\partial \beta_{1}}\right)\right]=-\left(T C_{B}-T C_{C}\right) \operatorname{Pn}(B) \operatorname{Pn}(C)-\left(T C_{B}-T C_{T}\right) \operatorname{Pn}(B) \operatorname{Pn}(T) \\
& -\left(T C_{B}-T C_{S}\right) \operatorname{Pn}(B) \operatorname{Pn}(S)-\left(T C_{B}-T C_{L}\right) \operatorname{Pn}(B) \operatorname{Pn}(L) \\
& \frac{1}{\partial \beta_{8}}\left[\frac{1}{\operatorname{Pn}(L)}\left(\frac{\partial P n(L)}{\partial \beta_{I}}\right)\right]=-\left(T C_{B}-T C_{C}\right) \operatorname{Pn}(B) P n(C)-\left(T C_{B}-T C_{T}\right) \operatorname{Pn}(B) P n(T) \\
& -\left(T C_{B}-T C_{S}\right) \operatorname{Pn}(B) \operatorname{Pn}(S)-\left(T C_{B}-T C_{L}\right) \operatorname{Pn}(B) \operatorname{Pn}(L) \\
& \frac{\partial \operatorname{Pn}(C)}{\partial \beta_{8}}=-\operatorname{Pn}(C) \operatorname{Pn}(B) \\
& \frac{\partial P n(T)}{\partial \beta_{8}}=-P n(T) P n(B) \\
& \frac{\partial P n(S)}{\partial \beta_{8}}=-P n(S) P n(B) \\
& \frac{\partial P n(B)}{\partial \beta_{8}}=P n(B)[P n(C)+P n(T)+P n(S)+P n(L)] \\
& \frac{\partial P n(L)}{\partial \beta_{8}}=-P n(L) P n(B)
\end{aligned}
$$

9) The second order partial derivative with respect to $\beta_{1} \beta_{9}$ is

$$
\begin{aligned}
\frac{\partial^{2} L L\left(\beta_{1}, \beta_{2}, \cdots, \beta_{8}, \beta_{9}\right)}{\partial \beta_{9} \partial \beta_{I}}= & \sum_{n=1}^{N} l y_{C n} \frac{\partial}{\partial \beta_{9}}\left[\frac{1}{P n(C)_{n}}\left(\frac{\partial P n(C)_{n}}{\partial \beta_{1}}\right)\right]+y_{T n} \frac{\partial}{\partial \beta_{9}}\left[\frac{1}{P n(T)_{n}}\left(\frac{\partial P n(T)_{n}}{\partial \beta_{1}}\right)\right]+y_{S n} \frac{\partial}{\partial \beta_{9}}\left[\frac{1}{P n(S)_{n}}\left(\frac{\partial P n(S)_{n}}{\partial \beta_{1}}\right)\right]+ \\
& y_{B n} \frac{\partial}{\partial \beta_{9}}\left[\frac{1}{P n(B)_{n}}\left(\frac{\partial P n(B)_{n}}{\partial \beta_{1}}\right)\right]+y_{L n} \frac{\partial}{\partial \beta_{9}}\left[\frac{1}{P n(L)_{n}}\left(\frac{\partial P n(L)_{n}}{\partial \beta_{1}}\right)\right] J
\end{aligned}
$$

where

$$
\begin{aligned}
\frac{1}{\partial \beta_{9}}\left[\frac{1}{P n(C)}\left(\frac{\partial P n(C)}{\partial \beta_{1}}\right)\right] & =-\left(T C_{L}-T C_{C}\right) \operatorname{Pn}(L) \operatorname{Pn}(C)-\left(T C_{L}-T C_{T}\right) \operatorname{Pn}(L) \operatorname{Pn}(T) \\
& -\left(T C_{L}-T C_{S}\right) \operatorname{Pn}(L) \operatorname{Pn}(S)-\left(T C_{L}-T C_{B}\right) \operatorname{Pn}(L) \operatorname{Pn}(B)
\end{aligned}
$$




$$
\begin{aligned}
& \frac{1}{\partial \beta_{9}}\left[\frac{1}{P n(C)}\left(\frac{\partial P n(C)}{\partial \beta_{1}}\right)\right]=-\left(T C_{L}-T C_{C}\right) P n(L) P n(C)-\left(T C_{L}-T C_{T}\right) P n(L) P n(T) \\
& -\left(T C_{L}-T C_{S}\right) \operatorname{Pn}(L) P n(S)-\left(T C_{L}-T C_{B}\right) P n(L) P n(B) \\
& \frac{1}{\partial \beta_{9}}\left[\frac{1}{P n(S)}\left(\frac{\partial P n(S)}{\partial \beta_{I}}\right)\right]=-\left(T C_{L}-T C_{C}\right) P n(L) P n(C)-\left(T C_{L}-T C_{T}\right) P n(L) P n(T) \\
& -\left(T C_{L}-T C_{S}\right) P n(L) P n(S)-\left(T C_{L}-T C_{B}\right) \operatorname{Pn}(L) P n(B) \\
& \frac{1}{\partial \beta_{9}}\left[\frac{1}{P n(B)}\left(\frac{\partial P n(B)}{\partial \beta_{1}}\right)\right]=-\left(T C_{L}-T C_{C}\right) P n(L) P n(C)-\left(T C_{L}-T C_{T}\right) \operatorname{Pn}(L) P n(T) \\
& -\left(T C_{L}-T C_{S}\right) P n(L) P n(S)-\left(T C_{L}-T C_{B}\right) P n(L) P n(B) \\
& \frac{1}{\partial \beta_{9}}\left[\frac{1}{P n(L)}\left(\frac{\partial P n(L)}{\partial \beta_{1}}\right)\right]=-\left(T C_{L}-T C_{C}\right) \operatorname{Pn}(L) P n(C)-\left(T C_{L}-T C_{T}\right) \operatorname{Pn}(L) P n(T) \\
& -\left(T C_{L}-T C_{S}\right) \operatorname{Pn}(L) P n(S)-\left(T C_{L}-T C_{B}\right) \operatorname{Pn}(L) P n(B)
\end{aligned}
$$

$\frac{\partial P n(C)}{\partial \beta_{9}}=-P n(C) P n(L)$

$\frac{\partial P n(T)}{\partial \beta_{9}}=-P n(T) P n(L)$

$\frac{\partial P n(S)}{\partial \beta_{9}}=-P n(S) P n(L)$

$\frac{\partial P n(B)}{\partial \beta_{9}}=-P n(B) P n(L)$

$\frac{\partial P n(L)}{\partial \beta_{9}}=\operatorname{Pn}(L)[\operatorname{Pn}(C)+\operatorname{Pn}(T)+\operatorname{Pn}(S)+\operatorname{Pn}(B)]$

11) The second order partial derivative with respect to $\beta_{2} \beta_{2}$ is

$$
\begin{aligned}
\frac{\partial^{2} L L\left(\beta_{1}, \beta_{2}, \cdots, \beta_{8}, \beta_{9}\right)}{\partial \beta_{2} \partial \beta_{2}}= & \sum_{n=1}^{N}\left[y_{C n} \frac{\partial}{\partial \beta_{2}}\left[\frac{1}{P n(C)_{n}}\left(\frac{\partial P n(C)_{n}}{\partial \beta_{2}}\right)\right]+y_{T n} \frac{\partial}{\partial \beta_{2}}\left[\frac{1}{P n(T)_{n}}\left(\frac{\partial P n(T)_{n}}{\partial \beta_{2}}\right)\right]+y_{S n} \frac{\partial}{\partial \beta_{2}}\left[\frac{1}{P n(S)_{n}}\left(\frac{\partial P n(S)_{n}}{\partial \beta_{2}}\right)\right]+\right. \\
& \left.y_{B n} \frac{\partial}{\partial \beta_{2}}\left[\frac{1}{P n(B)_{n}}\left(\frac{\partial P n(B)_{n}}{\partial \beta_{2}}\right)\right]+y_{L n} \frac{\partial}{\partial \beta_{2}}\left[\frac{1}{P n(L)_{n}}\left(\frac{\partial P n(L)_{n}}{\partial \beta_{2}}\right)\right]\right]
\end{aligned}
$$

where 


$$
\begin{aligned}
\frac{1}{\partial \beta_{2}}\left[\frac{1}{\operatorname{Pn}(C)}\left(\frac{\partial P n(C)}{\partial \beta_{2}}\right)\right]= & -\left(T T_{C}-T T_{T}\right)^{2} \operatorname{Pn}(T) \operatorname{Pn}(C)-\left(T T_{C}-T T_{S}\right)^{2} \operatorname{Pn}(S) \operatorname{Pn}(C) \\
& -\left(T T_{C}-T T_{B}\right)^{2} \operatorname{Pn}(B) \operatorname{Pn}(C)-\left(T T_{C}-T T_{L}\right)^{2} \operatorname{Pn}(L) \operatorname{Pn}(C) \\
& -\left(T T_{T}-T T_{S}\right)^{2} \operatorname{Pn}(T) \operatorname{Pn}(S)-\left(T T_{T}-T T_{B}\right)^{2} \operatorname{Pn}(B) \operatorname{Pn}(T) \\
& -\left(T T_{T}-T T_{L}\right)^{2} \operatorname{Pn}(L) \operatorname{Pn}(T)-\left(T T_{S}-T T_{B}\right)^{2} \operatorname{Pn}(B) \operatorname{Pn}(S) \\
& -\left(T T_{S}-T T_{L}\right)^{2} \operatorname{Pn}(L) \operatorname{Pn}(S)-\left(T T_{B}-T T_{L}\right)^{2} \operatorname{Pn}(L) \operatorname{Pn}(B)
\end{aligned}
$$$$
\frac{1}{\partial \beta_{2}}\left[\frac{1}{P n(T)}\left(\frac{\partial P n(T)}{\partial \beta_{2}}\right)\right]=-\left(T T_{C}-T T_{T}\right)^{2} P n(T) P n(C)-\left(T T_{C}-T T_{S}\right)^{2} \operatorname{Pn}(S) P n(C)
$$$$
-\left(T T_{C}-T T_{B}\right)^{2} \operatorname{Pn}(B) \operatorname{Pn}(C)-\left(T T_{C}-T T_{L}\right)^{2} \operatorname{Pn}(L) P n(C)
$$$$
-\left(T T_{T}-T T_{S}\right)^{2} \operatorname{Pn}(T) P n(S)-\left(T T_{T}-T T_{B}\right)^{2} \operatorname{Pn}(B) P n(T)
$$$$
-\left(T T_{T}-T T_{L}\right)^{2} \operatorname{Pn}(L) P n(T)-\left(T T_{S}-T T_{B}\right)^{2} \operatorname{Pn}(B) P n(S)
$$$$
-\left(T T_{S}-T T_{L}\right)^{2} \operatorname{Pn}(L) \operatorname{Pn}(S)-\left(T T_{B}-T T_{L}\right)^{2} \operatorname{Pn}(L) P n(B)
$$

$$
\begin{aligned}
\frac{1}{\partial \beta_{2}}\left[\frac{1}{\operatorname{Pn}(S)}\left(\frac{\partial P n(S)}{\partial \beta_{2}}\right)\right]= & -\left(T T_{C}-T T_{T}\right)^{2} \operatorname{Pn}(T) \operatorname{Pn}(C)-\left(T T_{C}-T T_{S}\right)^{2} \operatorname{Pn}(S) \operatorname{Pn}(C) \\
& -\left(T T_{C}-T T_{B}\right)^{2} \operatorname{Pn}(B) \operatorname{Pn}(C)-\left(T T_{C}-T T_{L}\right)^{2} \operatorname{Pn}(L) \operatorname{Pn}(C) \\
& -\left(T T_{T}-T T_{S}\right)^{2} \operatorname{Pn}(T) \operatorname{Pn}(S)-\left(T T_{T}-T T_{B}\right)^{2} \operatorname{Pn}(B) \operatorname{Pn}(T) \\
& -\left(T T_{T}-T T_{L}\right)^{2} \operatorname{Pn}(L) \operatorname{Pn}(T)-\left(T T_{S}-T T_{B}\right)^{2} \operatorname{Pn}(B) \operatorname{Pn}(S) \\
& -\left(T T_{S}-T T_{L}\right)^{2} \operatorname{Pn}(L) \operatorname{Pn}(S)-\left(T T_{B}-T T_{L}\right)^{2} \operatorname{Pn}(L) \operatorname{Pn}(B)
\end{aligned}
$$

$$
\begin{aligned}
\frac{1}{\partial \beta_{2}}\left[\frac{1}{\operatorname{Pn}(B)}\left(\frac{\partial P n(B)}{\partial \beta_{2}}\right)\right]= & -\left(T T_{C}-T T_{T}\right)^{2} \operatorname{Pn}(T) \operatorname{Pn}(C)-\left(T T_{C}-T T_{S}\right)^{2} \operatorname{Pn}(S) \operatorname{Pn}(C) \\
& -\left(T T_{C}-T T_{B}\right)^{2} \operatorname{Pn}(B) \operatorname{Pn}(C)-\left(T T_{C}-T T_{L}\right)^{2} \operatorname{Pn}(L) \operatorname{Pn}(C) \\
& -\left(T T_{T}-T T_{S}\right)^{2} \operatorname{Pn}(T) \operatorname{Pn}(S)-\left(T T_{T}-T T_{B}\right)^{2} \operatorname{Pn}(B) \operatorname{Pn}(T) \\
& -\left(T T_{T}-T T_{L}\right)^{2} \operatorname{Pn}(L) \operatorname{Pn}(T)-\left(T T_{S}-T T_{B}\right)^{2} \operatorname{Pn}(B) \operatorname{Pn}(S) \\
& -\left(T T_{S}-T T_{L}\right)^{2} \operatorname{Pn}(L) \operatorname{Pn}(S)-\left(T T_{B}-T T_{L}\right)^{2} \operatorname{Pn}(L) \operatorname{Pn}(B)
\end{aligned}
$$

$$
\begin{aligned}
\frac{1}{\partial \beta_{2}}\left[\frac{1}{\operatorname{Pn}(L)}\left(\frac{\partial \operatorname{Pn}(L)}{\partial \beta_{2}}\right)\right]= & -\left(T T_{C}-T T_{T}\right)^{2} \operatorname{Pn}(T) \operatorname{Pn}(C)-\left(T T_{C}-T T_{S}\right)^{2} \operatorname{Pn}(S) \operatorname{Pn}(C) \\
& -\left(T T_{C}-T T_{B}\right)^{2} \operatorname{Pn}(B) \operatorname{Pn}(C)-\left(T T_{C}-T T_{L}\right)^{2} \operatorname{Pn}(L) \operatorname{Pn}(C) \\
& -\left(T T_{T}-T T_{S}\right)^{2} \operatorname{Pn}(T) \operatorname{Pn}(S)-\left(T T_{T}-T T_{B}\right)^{2} \operatorname{Pn}(B) \operatorname{Pn}(T) \\
& -\left(T T_{T}-T T_{L}\right)^{2} \operatorname{Pn}(L) \operatorname{Pn}(T)-\left(T T_{S}-T T_{B}\right)^{2} \operatorname{Pn}(B) \operatorname{Pn}(S) \\
& -\left(T T_{S}-T T_{L}\right)^{2} \operatorname{Pn}(L) \operatorname{Pn}(S)-\left(T T_{B}-T T_{L}\right)^{2} \operatorname{Pn}(L) \operatorname{Pn}(B)
\end{aligned}
$$




$$
\begin{aligned}
\frac{\partial P n(C)}{\partial \beta_{2}} & =\left(T T_{C}-T T_{T}\right) \operatorname{Pn}(C) P n(T)+\left(T T_{C}-T T_{S}\right) \operatorname{Pn}(C) P n(S) \\
& +\left(T T_{C}-T T_{B}\right) \operatorname{Pn}(C) P n(B)+\left(T T_{C}-T T_{L}\right) \operatorname{Pn}(C) P n(L)
\end{aligned}
$$

$$
\begin{aligned}
\frac{\partial P n(T)}{\partial \beta_{2}} & =\left(T T_{T}-T T_{C}\right) P n(T) P n(C)+\left(T T_{T}-T T_{S}\right) P n(T) P n(S) \\
& +\left(T T_{T}-T T_{B}\right) P n(T) P n(B)+\left(T T_{T}-T T_{L}\right) P n(T) P n(L)
\end{aligned}
$$

$$
\begin{aligned}
\frac{\partial P n(S)}{\partial \beta_{2}} & =\left(T T_{S}-T T_{C}\right) P n(S) P n(C)+\left(T T_{S}-T T_{T}\right) \operatorname{Pn}(S) P n(T) \\
& +\left(T T_{S}-T T_{B}\right) \operatorname{Pn}(S) P n(B)+\left(T T_{S}-T T_{L}\right) P n(S) P n(L)
\end{aligned}
$$

$$
\begin{aligned}
\frac{\partial P n(B)}{\partial \beta_{2}} & =\left(T T_{B}-T T_{C}\right) \operatorname{Pn}(B) P n(C)+\left(T T_{B}-T T_{T}\right) \operatorname{Pn}(B) P n(T) \\
& +\left(T T_{B}-T T_{S}\right) P n(B) P n(S)+\left(T T_{B}-T T_{L}\right) P n(B) P n(L)
\end{aligned}
$$

$$
\begin{aligned}
\frac{\partial P n(L)}{\partial \beta_{2}} & =\left(T T_{L}-T T_{C}\right) \operatorname{Pn}(L) P n(C)+\left(T T_{L}-T T_{T}\right) \operatorname{Pn}(L) P n(T) \\
& +\left(T T_{L}-T T_{S}\right) \operatorname{Pn}(L) P n(S)+\left(T T_{L}-T T_{B}\right) P n(L) P n(B)
\end{aligned}
$$

12) The second order partial derivative with respect to $\beta_{2} \beta_{3}$ is

$$
\begin{aligned}
& \frac{\partial^{2} L L\left(\beta_{1}, \beta_{2}, \cdots, \beta_{8}, \beta_{9}\right)}{\partial \beta_{3} \partial \beta_{2}}=\sum_{n=1}^{N}\left[y_{C n} \frac{\partial}{\partial \beta_{3}}\left[\frac{1}{P n(C)_{h}}\left(\frac{\partial P n\left(C_{h}\right.}{\partial \beta_{2}}\right)\right]+y_{T n} \frac{\partial}{\partial \beta_{3}}\left[\frac{1}{P n\left(T_{h}\right.}\left(\frac{\partial P n\left(T_{h}\right.}{\partial \beta_{2}}\right)\right]+y_{S n} \frac{\partial}{\partial \beta_{3}}\left[\frac{1}{P n(S)_{h}}\left(\frac{\partial P n\left(S h_{h}\right.}{\partial \beta_{2}}\right)\right]+\right. \\
& \left.y_{B n} \frac{\partial}{\partial \beta_{3}}\left[\frac{1}{P n(B h}\left(\frac{\partial P n(B)_{h}}{\partial \beta_{2}}\right)\right]+y_{L n} \frac{\partial}{\partial \beta_{3}}\left[\frac{1}{P n(L h}\left(\frac{\partial P n(L)}{\partial \beta_{2}}\right)\right]\right] \\
& \frac{1}{\partial \beta_{3}}\left[\frac{1}{\operatorname{Pn}(C)}\left(\frac{\partial P n(C)}{\partial \beta_{2}}\right)\right]=-\operatorname{Accom}\left(T T_{C}-T T_{T}\right) \operatorname{Pn}(C) P n(T)-\operatorname{Accom}\left(T T_{C}-T T_{S}\right) \operatorname{Pn}(C) P n(S) \\
& -\operatorname{Accom}\left(T T_{C}-T T_{B}\right) P n(C) P n(B)-\operatorname{Accom}\left(T T_{C}-T T_{L}\right) P n(C) P n(L) \\
& \frac{1}{\partial \beta_{3}}\left[\frac{1}{P n(T)}\left(\frac{\partial P n(T)}{\partial \beta_{2}}\right)\right]=-\operatorname{Accom}\left(T T_{C}-T T_{T}\right) P n(C) P n(T)-A c c o m\left(T T_{C}-T T_{S}\right) P n(C) P n(S) \\
& -\operatorname{Accom}\left(T T_{C}-T T_{B}\right) P n(C) P n(B)-\operatorname{Accom}\left(T T_{C}-T T_{L}\right) P n(C) P n(L)
\end{aligned}
$$




$$
\begin{aligned}
& \frac{1}{\partial \beta_{3}}\left[\frac{1}{\operatorname{Pn}(S)}\left(\frac{\partial P n(S)}{\partial \beta_{2}}\right)\right]=-\operatorname{Accom}\left(T T_{C}-T T_{T}\right) \operatorname{Pn}(C) P n(T)-\operatorname{Accom}\left(T T_{C}-T T_{S}\right) \operatorname{Pn}(C) P n(S) \\
& -\operatorname{Accom}\left(T T_{C}-T T_{B}\right) \operatorname{Pn}(C) P n(B)-\operatorname{Accom}\left(T T_{C}-T T_{L}\right) \operatorname{Pn}(C) P n(L) \\
& \frac{1}{\partial \beta_{3}}\left[\frac{1}{P n(B)}\left(\frac{\partial P n(B)}{\partial \beta_{2}}\right)\right]=-\operatorname{Accom}\left(T T_{C}-T T_{T}\right) \operatorname{Pn}(C) P n(T)-A c c o m\left(T T_{C}-T T_{S}\right) P n(C) P n(S) \\
& -\operatorname{Accom}\left(T T_{C}-T T_{B}\right) P n(C) P n(B)-\operatorname{Accom}\left(T T_{C}-T T_{L}\right) P n(C) P n(L) \\
& \frac{1}{\partial \beta_{3}}\left[\frac{1}{P n(L)}\left(\frac{\partial P n(L)}{\partial \beta_{2}}\right)\right]=-\operatorname{Accom}\left(T T_{C}-T T_{T}\right) \operatorname{Pn}(C) P n(T)-\operatorname{Accom}\left(T T_{C}-T T_{S}\right) \operatorname{Pn}(C) P n(S) \\
& -\operatorname{Accom}\left(T T_{C}-T T_{B}\right) P n(C) P n(B)-\operatorname{Accom}\left(T T_{C}-T T_{L}\right) P n(C) P n(L) \\
& \frac{\partial P n(C)}{\partial \beta_{3}}=A c c o m P n(C)[P n(T)+P n(S)+P n(B)+P n(L)] \\
& \frac{\partial P n(T)}{\partial \beta_{3}}=-A c c o m P n(T)[P n(C)] \\
& \frac{\partial P n(S)}{\partial \beta_{3}}=-A c c o m P n(S)[P n(C)] \\
& \frac{\partial \operatorname{Pn}(B)}{\partial \beta_{3}}=-\operatorname{AccomPn}(B)[\operatorname{Pn}(C)] \\
& \frac{\partial P n(L)}{\partial \beta_{3}}=-\operatorname{AccomPn}(L)[\operatorname{Pn}(C)]
\end{aligned}
$$

13) The second order partial derivative with respect to $\beta_{2} \beta_{4}$ is

$$
\begin{aligned}
\frac{\partial^{2} L L\left(\beta_{1}, \beta_{2}, \cdots, \beta_{8}, \beta_{9}\right)}{\partial \beta_{4} \partial \beta_{2}}= & \sum_{n=1}^{N}\left[y_{C n} \frac{\partial}{\partial \beta_{4}}\left[\frac{1}{P n(C)_{n}}\left(\frac{\partial P n(C)_{n}}{\partial \beta_{2}}\right)\right]+y_{T n} \frac{\partial}{\partial \beta_{4}}\left[\frac{1}{P n(T)_{n}}\left(\frac{\partial P n(T)_{n}}{\partial \beta_{2}}\right)\right]+y_{S n} \frac{\partial}{\partial \beta_{4}}\left[\frac{1}{P n(S)_{n}}\left(\frac{\partial P n(S)_{n}}{\partial \beta_{2}}\right)\right]+\right. \\
& \left.y_{B n} \frac{\partial}{\partial \beta_{4}}\left[\frac{1}{P n(B)_{n}}\left(\frac{\partial P n(B)_{n}}{\partial \beta_{2}}\right)\right]+y_{L n} \frac{\partial}{\partial \beta_{4}}\left[\frac{1}{P n(L)_{n}}\left(\frac{\partial P n(L)_{n}}{\partial \beta_{2}}\right)\right]\right]
\end{aligned}
$$

where

$$
\begin{aligned}
\frac{1}{\partial \beta_{4}}\left[\frac{1}{\operatorname{Pn}(C)}\left(\frac{\partial^{2} P n(C)}{\partial \beta_{2}}\right)\right]= & -\operatorname{Sexm}\left(T T_{C}-T T_{T}\right) \operatorname{Pn}(C) \operatorname{Pn}(T)-\operatorname{Sexm}\left(T T_{C}-T T_{S}\right) \operatorname{Pn}(C) P n(S) \\
& -\operatorname{Sexm}\left(T T_{C}-T T_{B}\right) \operatorname{Pn}(C) \operatorname{Pn}(B)-\operatorname{Sexm}\left(T T_{C}-T T_{L}\right) \operatorname{Pn}(C) \operatorname{Pn}(L) \\
\frac{1}{\partial \beta_{4}}\left[\frac{1}{\operatorname{Pn}(T)}\left(\frac{\partial^{2} P n(T)}{\partial \beta_{2}}\right)\right]= & -\operatorname{Sexm}\left(T T_{C}-T T_{T}\right) \operatorname{Pn}(C) \operatorname{Pn}(T)-\operatorname{Sexm}\left(T T_{C}-T T_{S}\right) \operatorname{Pn}(C) P n(S) \\
& -\operatorname{Sexm}\left(T T_{C}-T T_{B}\right) \operatorname{Pn}(C) P n(B)-\operatorname{Sexm}\left(T T_{C}-T T_{L}\right) \operatorname{Pn}(C) P n(L)
\end{aligned}
$$




$$
\begin{aligned}
& \frac{1}{\partial \beta_{4}}\left[\frac{1}{P n(S)}\left(\frac{\partial^{2} P n(S)}{\partial \beta_{2}}\right)\right]=-\operatorname{Sexm}\left(T T_{C}-T T_{T}\right) \operatorname{Pn}(C) \operatorname{Pn}(T)-\operatorname{Sexm}\left(T T_{C}-T T_{S}\right) \operatorname{Pn}(C) \operatorname{Pn}(S) \\
& -\operatorname{Sexm}\left(T T_{C}-T T_{B}\right) P n(C) P n(B)-\operatorname{Sexm}\left(T T_{C}-T T_{L}\right) \operatorname{Pn}(C) P n(L) \\
& \frac{1}{\partial \beta_{4}}\left[\frac{1}{P n(B)}\left(\frac{\partial^{2} P n(B)}{\partial \beta_{2}}\right)\right]=-\operatorname{Sexm}\left(T T_{C}-T T_{T}\right) \operatorname{Pn}(C) P n(T)-\operatorname{Sexm}\left(T T_{C}-T T_{S}\right) P n(C) P n(S) \\
& -\operatorname{Sexm}\left(T T_{C}-T T_{B}\right) \operatorname{Pn}(C) P n(B)-\operatorname{Sexm}\left(T T_{C}-T T_{L}\right) \operatorname{Pn}(C) P n(L) \\
& \frac{1}{\partial \beta_{4}}\left[\frac{1}{P n(L)}\left(\frac{\partial^{2} P n(L)}{\partial \beta_{2}}\right)\right]=-\operatorname{Sexm}\left(T T_{C}-T T_{T}\right) P n(C) P n(T)-\operatorname{Sexm}\left(T T_{C}-T T_{S}\right) P n(C) P n(S) \\
& -\operatorname{Sexm}\left(T T_{C}-T T_{B}\right) \operatorname{Pn}(C) \operatorname{Pn}(B)-\operatorname{Sexm}\left(T T_{C}-T T_{L}\right) \operatorname{Pn}(C) \operatorname{Pn}(L) \\
& \frac{\partial P n(C)}{\partial \beta_{4}}=\operatorname{SexmPn}(C)[P n(T)+P n(S)+P n(B)+\operatorname{Pn}(L)] \\
& \frac{\partial P n(T)}{\partial \beta_{4}}=-\operatorname{SexmPn}(T)[\operatorname{Pn}(C)] \\
& \frac{\partial P n(S)}{\partial \beta_{4}}=-\operatorname{SexmPn}(S)[\operatorname{Pn}(C)] \\
& \frac{\partial P n(B)}{\partial \beta_{4}}=-\operatorname{SexmPn}(B)[\operatorname{Pn}(C)] \\
& \frac{\partial P n(L)}{\partial \beta_{4}}=-\operatorname{SexmPn}(L)[\operatorname{Pn}(C)]
\end{aligned}
$$

14) The second order partial derivative with respect to $\beta_{2} \beta_{5}$ is

$$
\begin{aligned}
& \frac{\partial^{2} L L\left(\beta_{1}, \beta_{2}, \cdots, \beta_{8}, \beta_{9}\right)}{\partial \beta_{5} \partial \beta_{2}}=\sum_{n=1}^{N}\left[y_{C n} \frac{\partial}{\partial \beta_{5}}\left[\frac{1}{P n(C)_{n}}\left(\frac{\partial P n(C)_{n}}{\partial \beta_{2}}\right)\right]+y_{T n} \frac{\partial}{\partial \beta_{5}}\left[\frac{1}{P n(T)_{n}}\left(\frac{\partial P n(T)_{n}}{\partial \beta_{2}}\right)\right]+y_{S n} \frac{\partial}{\partial \beta_{5}}\left[\frac{1}{P n(S)_{n}}\left(\frac{\partial P n(S)_{n}}{\partial \beta_{2}}\right)\right]+\right. \\
& \left.y_{B n} \frac{\partial}{\partial \beta_{5}}\left[\frac{1}{P n(B)_{n}}\left(\frac{\partial P n(B)_{n}}{\partial \beta_{2}}\right)\right]+y_{L n} \frac{\partial}{\partial \beta_{5}}\left[\frac{1}{P n(L)_{n}}\left(\frac{\partial P n(L)_{n}}{\partial \beta_{2}}\right)\right]\right] \\
& \frac{1}{\partial \beta_{5}}\left[\frac{1}{P n(C)}\left(\frac{\partial P n(C)}{\partial \beta_{2}}\right)\right]=-A g e 340\left(T T_{C}-T T_{T}\right) P n(C) P n(T)-A g e 340\left(T T_{C}-T T_{S}\right) P n(C) P n(S) \\
& -A_{g e 340\left(T T_{C}-T T_{B}\right) P n(C) P n(B)-A g e 340\left(T T_{C}-T T_{L}\right) P n(C) P n(L)}
\end{aligned}
$$




$$
\left.\begin{array}{rl}
\frac{1}{\partial \beta_{5}}\left[\frac{1}{P n(T)}\left(\frac{\partial P n(T)}{\partial \beta_{2}}\right)\right]= & -A g e 340\left(T T_{C}-T T_{T}\right) P n(C) P n(T)-A g e 340\left(T T_{C}-T T_{S}\right) P n(C) P n(S) \\
& -A g e 340\left(T T_{C}-T T_{B}\right) P n(C) P n(B)-A g e 340\left(T T_{C}-T T_{L}\right) P n(C) P n(L)
\end{array}\right)
$$

15) The second order partial derivative with respect to $\beta_{2} \beta_{6}$ is

$$
\begin{gathered}
\frac{\partial^{2} L L\left(\beta_{1}, \beta_{2}, \cdots, \beta_{8}, \beta_{9}\right)}{\partial \beta_{6} \partial \beta_{2}}=\sum_{n=1}^{N}\left[y_{C_{n}} \frac{\partial}{\partial \beta_{6}}\left[\frac{1}{P n(C)_{n}}\left(\frac{\partial P n\left(C_{n}\right.}{\partial \beta_{2}}\right)\right]+y_{T n} \frac{\partial}{\partial \beta_{6}}\left[\frac{1}{P n(T)_{n}}\left(\frac{\partial P n(T)_{n}}{\partial \beta_{2}}\right)\right]+y_{S n} \frac{\partial}{\partial \beta_{6}}\left[\frac{1}{P n(S)_{n}}\left(\frac{\partial P n(S)_{n}}{\partial \beta_{2}}\right)\right]+\right. \\
y_{B n} \frac{\partial}{\partial \beta_{6}}\left[\frac{1}{P n(B)_{n}}\left(\frac{\partial P n(B)_{n}}{\partial \beta_{2}}\right)\right]+y_{L n} \frac{\partial}{\partial \beta_{6}}\left[\frac{1}{P n(L)_{n}}\left(\frac{\partial P n(L)_{n}}{\partial \beta_{2}}\right)\right] J
\end{gathered}
$$

where 


$$
\begin{aligned}
& \frac{1}{\partial \beta_{6}}\left[\frac{1}{P n(C)}\left(\frac{\partial P n(C)}{\partial \beta_{2}}\right)\right]=-\left(T T_{T}-T T_{C}\right) P n(T) P n(C)-\left(T T_{T}-T T_{S}\right) P n(T) P n(S) \\
& -\left(T T_{T}-T T_{B}\right) \operatorname{Pn}(T) \operatorname{Pn}(B)-\left(T T_{T}-T T_{L}\right) \operatorname{Pn}(T) \operatorname{Pn}(L) \\
& \frac{1}{\partial \beta_{6}}\left[\frac{1}{P n(T)}\left(\frac{\partial P n(T)}{\partial \beta_{2}}\right)\right]=-\left(T T_{T}-T T_{C}\right) P n(T) P n(C)-\left(T T_{T}-T T_{S}\right) P n(T) P n(S) \\
& -\left(T T_{T}-T T_{B}\right) P n(T) P n(B)-\left(T T_{T}-T T_{L}\right) P n(T) P n(L) \\
& \frac{1}{\partial \beta_{6}}\left[\frac{1}{P n(S)}\left(\frac{\partial P n(S)}{\partial \beta_{2}}\right)\right]=-\left(T T_{T}-T T_{C}\right) \operatorname{Pn}(T) P n(C)-\left(T T_{T}-T T_{S}\right) \operatorname{Pn}(T) P n(S) \\
& -\left(T T_{T}-T T_{B}\right) P n(T) P n(B)-\left(T T_{T}-T T_{L}\right) P n(T) P n(L) \\
& \frac{1}{\partial \beta_{6}}\left[\frac{1}{P n(B)}\left(\frac{\partial P n(B)}{\partial \beta_{2}}\right)\right]=-\left(T T_{T}-T T_{C}\right) \operatorname{Pn}(T) P n(C)-\left(T T_{T}-T T_{S}\right) \operatorname{Pn}(T) P n(S) \\
& -\left(T T_{T}-T T_{B}\right) \operatorname{Pn}(T) \operatorname{Pn}(B)-\left(T T_{T}-T T_{L}\right) \operatorname{Pn}(T) \operatorname{Pn}(L) \\
& \frac{1}{\partial \beta_{6}}\left[\frac{1}{P n(L)}\left(\frac{\partial P n(L)}{\partial \beta_{2}}\right)\right]=-\left(T T_{T}-T T_{C}\right) P n(T) P n(C)-\left(T T_{T}-T T_{S}\right) P n(T) P n(S) \\
& -\left(T T_{T}-T T_{B}\right) \operatorname{Pn}(T) P n(B)-\left(T T_{T}-T T_{L}\right) \operatorname{Pn}(T) P n(L) \\
& \frac{\partial P n(C)}{\partial \beta_{6}}=-P n(C) P n(T) \\
& \frac{\partial P n(T)}{\partial \beta_{6}}=P n(T)[P n(C)+P n(S)+P n(B)+P n(L)] \\
& \frac{\partial P n(S)}{\partial \beta_{6}}=-P n(S) P n(T) \\
& \frac{\partial P n(B)}{\partial \beta_{6}}=-P n(B) P n(T) \\
& \frac{\partial P n(L)}{\partial \beta_{6}}=-P n(L) P n(T)
\end{aligned}
$$

16) The second order partial derivative with respect to $\beta_{2} \beta_{7}$ is

$$
\begin{gathered}
\frac{\partial^{2} L L\left(\beta_{1}, \beta_{2}, \cdots, \beta_{8}, \beta_{9}\right)}{\partial \beta_{7} \partial \beta_{2}}=\sum_{n=1}^{N}\left[y_{C n} \frac{\partial}{\partial \beta_{7}}\left[\frac{1}{P n\left(C_{h}\right.}\left(\frac{\partial P n\left(C_{h}\right.}{\partial \beta_{2}}\right)\right]+y_{T n} \frac{\partial}{\partial \beta_{7}}\left[\frac{1}{P n\left(T_{h}\right.}\left(\frac{\partial P n\left(T_{h}\right.}{\partial \beta_{2}}\right)\right]+y_{S n} \frac{\partial}{\partial \beta_{7}}\left[\frac{1}{P n\left(S_{h}\right.}\left(\frac{\partial P n\left(S_{h}\right.}{\partial \beta_{2}}\right)\right]+\right. \\
y_{B n} \frac{\partial}{\partial \beta_{7}}\left[\frac{1}{P n(B)}\left(\frac{\partial P n(B h}{\partial \beta_{h}}\right)\right]+y_{L n} \frac{\partial}{\partial \beta_{7}}\left[\frac{1}{P n(L h}\left(\frac{\partial P n(L h}{\partial \beta_{2}}\right)\right] l
\end{gathered}
$$

where 


$$
\begin{aligned}
& \frac{1}{\partial \beta_{7}}\left[\frac{1}{P n(C)}\left(\frac{\partial P n(C)}{\partial \beta_{2}}\right)\right]=-\left(T T_{S}-T T_{C}\right) P n(S) P n(C)-\left(T T_{S}-T T_{T}\right) P n(S) P n(T) \\
& -\left(T T_{S}-T T_{B}\right) \operatorname{Pn}(S) \operatorname{Pn}(B)-\left(T T_{S}-T T_{L}\right) \operatorname{Pn}(S) \operatorname{Pn}(L) \\
& \frac{1}{\partial \beta_{7}}\left[\frac{1}{P n(T)}\left(\frac{\partial P n(T)}{\partial \beta_{2}}\right)\right]=-\left(T T_{S}-T T_{C}\right) P n(S) P n(C)-\left(T T_{S}-T T_{T}\right) P n(S) P n(T) \\
& -\left(T T_{S}-T T_{B}\right) \operatorname{Pn}(S) P n(B)-\left(T T_{S}-T T_{L}\right) \operatorname{Pn}(S) P n(L) \\
& \frac{1}{\partial \beta_{7}}\left[\frac{1}{P n(S)}\left(\frac{\partial P n(S)}{\partial \beta_{2}}\right)\right]=-\left(T T_{S}-T T_{C}\right) P n(S) P n(C)-\left(T T_{S}-T T_{T}\right) P n(S) P n(T) \\
& -\left(T T_{S}-T T_{B}\right) P n(S) P n(B)-\left(T T_{S}-T T_{L}\right) P n(S) P n(L) \\
& \frac{1}{\partial \beta_{7}}\left[\frac{1}{P n(B)}\left(\frac{\partial P n(B)}{\partial \beta_{2}}\right)\right]=-\left(T T_{S}-T T_{C}\right) P n(S) P n(C)-\left(T T_{S}-T T_{T}\right) P n(S) P n(T) \\
& -\left(T T_{S}-T T_{B}\right) P n(S) P n(B)-\left(T T_{S}-T T_{L}\right) P n(S) P n(L) \\
& \frac{1}{\partial \beta_{7}}\left[\frac{1}{\operatorname{Pn}(L)}\left(\frac{\partial P n(L)}{\partial \beta_{2}}\right)\right]=-\left(T T_{S}-T T_{C}\right) \operatorname{Pn}(S) P n(C)-\left(T T_{S}-T T_{T}\right) \operatorname{Pn}(S) P n(T) \\
& -\left(T T_{S}-T T_{B}\right) \operatorname{Pn}(S) \operatorname{Pn}(B)-\left(T T_{S}-T T_{L}\right) \operatorname{Pn}(S) \operatorname{Pn}(L) \\
& \frac{\partial P n(C)}{\partial \beta_{7}}=-P n(C) P n(S) \\
& \frac{\partial P n(T)}{\partial \beta_{7}}=-P n(T) P n(S) \\
& \frac{\partial P n(S)}{\partial \beta_{7}}=P n(S)[P n(C)+P n(T)+P n(B)+P n(L)] \\
& \frac{\partial P n(B)}{\partial \beta_{7}}=-P n(B) P n(S) \\
& \frac{\partial P n(L)}{\partial \beta_{7}}=-P n(L) P n(S)
\end{aligned}
$$

17) The second order partial derivative with respect to $\beta_{2} \beta_{8}$ is

$$
\begin{aligned}
\frac{\partial^{2} L L\left(\beta_{1}, \beta_{2}, \cdots, \beta_{8}, \beta_{9}\right)}{\partial \beta_{8} \partial \beta_{2}}= & \sum_{n=1}^{N}\left[y_{C n} \frac{\partial}{\partial \beta_{8}}\left[\frac{1}{P n(C)_{n}}\left(\frac{\partial P n(C)_{n}}{\partial \beta_{2}}\right)\right]+y_{T n} \frac{\partial}{\partial \beta_{8}}\left[\frac{1}{P n(T)_{n}}\left(\frac{\partial P n(T)_{n}}{\partial \beta_{2}}\right)\right]+y_{S n} \frac{\partial}{\partial \beta_{8}}\left[\frac{1}{P n(S)_{n}}\left(\frac{\partial P n(S)_{n}}{\partial \beta_{2}}\right)\right]+\right. \\
& \left.y_{B n} \frac{\partial}{\partial \beta_{8}}\left[\frac{1}{P n(B)_{n}}\left(\frac{\partial P n(B)_{n}}{\partial \beta_{2}}\right)\right]+y_{L n} \frac{\partial}{\partial \beta_{8}}\left[\frac{1}{P n(L)_{n}}\left(\frac{\partial P n(L)_{n}}{\partial \beta_{2}}\right)\right]\right]
\end{aligned}
$$

where 


$$
\begin{aligned}
\frac{1}{\partial \beta_{8}}\left[\frac{1}{\operatorname{Pn}(C)}\left(\frac{\partial P n(C)}{\partial \beta_{2}}\right)\right]= & -\left(T T_{B}-T T_{C}\right) \operatorname{Pn}(B) \operatorname{Pn}(C)-\left(T T_{B}-T T_{T}\right) \operatorname{Pn}(B) \operatorname{Pn}(T) \\
& -\left(T T_{B}-T T_{S}\right) \operatorname{Pn}(B) \operatorname{Pn}(S)-\left(T T_{B}-T T_{L}\right) \operatorname{Pn}(B) \operatorname{Pn}(L)
\end{aligned}
$$

$$
\begin{aligned}
\frac{1}{\partial \beta_{8}}\left[\frac{1}{\operatorname{Pn}(T)}\left(\frac{\partial P n(T)}{\partial \beta_{2}}\right)\right]= & -\left(T T_{B}-T T_{C}\right) \operatorname{Pn}(B) \operatorname{Pn}(C)-\left(T T_{B}-T T_{T}\right) \operatorname{Pn}(B) \operatorname{Pn}(T) \\
& -\left(T T_{B}-T T_{S}\right) \operatorname{Pn}(B) \operatorname{Pn}(S)-\left(T T_{B}-T T_{L}\right) \operatorname{Pn}(B) \operatorname{Pn}(L)
\end{aligned}
$$

$$
\begin{aligned}
\frac{1}{\partial \beta_{8}}\left[\frac{1}{P n(S)}\left(\frac{\partial P n(S)}{\partial \beta_{2}}\right)\right] & =-\left(T T_{B}-T T_{C}\right) \operatorname{Pn}(B) \operatorname{Pn}(C)-\left(T T_{B}-T T_{T}\right) \operatorname{Pn}(B) \operatorname{Pn}(T) \\
& -\left(T T_{B}-T T_{S}\right) \operatorname{Pn}(B) \operatorname{Pn}(S)-\left(T T_{B}-T T_{L}\right) \operatorname{Pn}(B) \operatorname{Pn}(L)
\end{aligned}
$$

$$
\begin{aligned}
\frac{1}{\partial \beta_{8}}\left[\frac{1}{\operatorname{Pn}(B)}\left(\frac{\partial P n(B)}{\partial \beta_{2}}\right)\right]= & -\left(T T_{B}-T T_{C}\right) \operatorname{Pn}(B) \operatorname{Pn}(C)-\left(T T_{B}-T T_{T}\right) \operatorname{Pn}(B) P n(T) \\
& -\left(T T_{B}-T T_{S}\right) \operatorname{Pn}(B) \operatorname{Pn}(S)-\left(T T_{B}-T T_{L}\right) \operatorname{Pn}(B) P n(L)
\end{aligned}
$$

$$
\begin{aligned}
\frac{1}{\partial \beta_{8}}\left[\frac{1}{\operatorname{Pn}(L)}\left(\frac{\partial P n(L)}{\partial \beta_{2}}\right)\right] & =-\left(T T_{B}-T T_{C}\right) \operatorname{Pn}(B) \operatorname{Pn}(C)-\left(T T_{B}-T T_{T}\right) \operatorname{Pn}(B) \operatorname{Pn}(T) \\
& -\left(T T_{B}-T T_{S}\right) \operatorname{Pn}(B) \operatorname{Pn}(S)-\left(T T_{B}-T T_{L}\right) \operatorname{Pn}(B) \operatorname{Pn}(L)
\end{aligned}
$$

$$
\begin{aligned}
& \frac{\partial P n(C)}{\partial \beta_{8}}=-P n(C) P n(B) \\
& \frac{\partial P n(T)}{\partial \beta_{8}}=-P n(T) P n(B) \\
& \frac{\partial P n(S)}{\partial \beta_{8}}=-P n(S) P n(B) \\
& \frac{\partial P n(B)}{\partial \beta_{8}}=\operatorname{Pn}(B)[P n(C)+P n(T)+P n(S)+P n(L)] \\
& \frac{\partial P n(L)}{\partial \beta_{8}}=-P n(L) P n(B)
\end{aligned}
$$

18) The second order partial derivative with respect to $\beta_{2} \beta_{9}$ is

$$
\begin{gathered}
\frac{\partial^{2} L L\left(\beta_{1}, \beta_{2}, \cdots, \beta_{8}, \beta_{9}\right)}{\partial \beta_{9} \partial \beta_{2}}=\sum_{n=l}^{N}\left[y_{C_{n}} \frac{\partial}{\partial \beta_{9}}\left[\frac{1}{P n(C)_{n}}\left(\frac{\partial P n\left(C_{n}\right.}{\partial \beta_{2}}\right)\right]+y_{T n} \frac{\partial}{\partial \beta_{9}}\left[\frac{1}{P n(T)_{n}}\left(\frac{\partial P n(T)_{n}}{\partial \beta_{2}}\right)\right]+y_{s n} \frac{\partial}{\partial \beta_{9}}\left[\frac{1}{P n(S)_{n}}\left(\frac{\partial P n(S)_{n}}{\partial \beta_{2}}\right)\right]+\right. \\
y_{B n} \frac{\partial}{\partial \beta_{9}}\left[\frac{1}{P n(B)_{n}}\left(\frac{\partial P n(B)_{n}}{\partial \beta_{2}}\right)\right]+y_{L n} \frac{\partial}{\partial \beta_{9}}\left[\frac{1}{P n(L)_{n}}\left(\frac{\partial P n(L)_{n}}{\partial \beta_{2}}\right)\right] J
\end{gathered}
$$

where 


$$
\begin{aligned}
& \frac{1}{\partial \beta_{9}}\left[\frac{1}{P n(C)}\left(\frac{\partial P n(C)}{\partial \beta_{2}}\right)\right]=-\left(T T_{L}-T T_{C}\right) \operatorname{Pn}(L) P n(C)-\left(T T_{L}-T T_{T}\right) \operatorname{Pn}(L) P n(T) \\
& -\left(T T_{L}-T T_{S}\right) P n(L) P n(S)-\left(T T_{L}-T T_{B}\right) P n(L) P n(B) \\
& \frac{1}{\partial \beta_{9}}\left[\frac{1}{P n(T)}\left(\frac{\partial P n(T)}{\partial \beta_{2}}\right)\right]=-\left(T T_{L}-T T_{C}\right) P n(L) P n(C)-\left(T T_{L}-T T_{T}\right) P n(L) P n(T) \\
& -\left(T T_{L}-T T_{S}\right) P n(L) P n(S)-\left(T T_{L}-T T_{B}\right) \operatorname{Pn}(L) P n(B) \\
& \frac{1}{\partial \beta_{9}}\left[\frac{1}{P n(S)}\left(\frac{\partial P n(S)}{\partial \beta_{2}}\right)\right]=-\left(T T_{L}-T T_{C}\right) P n(L) P n(C)-\left(T T_{L}-T T_{T}\right) P n(L) P n(T) \\
& -\left(T T_{L}-T T_{S}\right) P n(L) P n(S)-\left(T T_{L}-T T_{B}\right) P n(L) P n(B) \\
& \frac{1}{\partial \beta_{9}}\left[\frac{1}{\operatorname{Pn}(B)}\left(\frac{\partial P n(B)}{\partial \beta_{2}}\right)\right]=-\left(T T_{L}-T T_{C}\right) P n(L) P n(C)-\left(T T_{L}-T T_{T}\right) P n(L) P n(T) \\
& -\left(T T_{L}-T T_{S}\right) P n(L) P n(S)-\left(T T_{L}-T T_{B}\right) P n(L) P n(B) \\
& \frac{1}{\partial \beta_{9}}\left[\frac{1}{P n(L)}\left(\frac{\partial P n(L)}{\partial \beta_{2}}\right)\right]=-\left(T T_{L}-T T_{C}\right) \operatorname{Pn}(L) P n(C)-\left(T T_{L}-T T_{T}\right) \operatorname{Pn}(L) P n(T) \\
& -\left(T T_{L}-T T_{S}\right) \operatorname{Pn}(L) P n(S)-\left(T T_{L}-T T_{B}\right) P n(L) P n(B) \\
& \frac{\partial P n(C)}{\partial \beta_{9}}=-P n(C) P n(L) \\
& \frac{\partial P n(T)}{\partial \beta_{9}}=-P n(T) P n(L) \\
& \frac{\partial P n(S)}{\partial \beta_{9}}=-P n(S) P n(L) \\
& \frac{\partial P n(B)}{\partial \beta_{9}}=-P n(B) P n(L) \\
& \frac{\partial P n(L)}{\partial \beta_{9}}=P n(L)[P n(C)+P n(T)+P n(S)+P n(B)]
\end{aligned}
$$

19) The second order partial derivative with respect to $\beta_{3} \beta_{3}$ is

$$
\begin{gathered}
\frac{\partial^{2} L L\left(\beta_{1}, \beta_{2}, \cdots, \beta_{8}, \beta_{9}\right)}{\partial \beta_{3} \partial \beta_{3}}= \\
\sum_{n=1}^{N}\left[y_{C n} \frac{\partial}{\partial \beta_{3}}\left[\frac{1}{P n(C)_{n}}\left(\frac{\partial P n(C)_{n}}{\partial \beta_{3}}\right)\right]+y_{T_{n}} \frac{\partial}{\partial \beta_{3}}\left[\frac{1}{P n(T)_{n}}\left(\frac{\partial P n(T)_{n}}{\partial \beta_{3}}\right)\right]+y_{S n} \frac{\partial}{\partial \beta_{3}}\left[\frac{1}{P n(S)_{n}}\left(\frac{\partial P n(S)_{n}}{\partial \beta_{3}}\right)\right]+\right. \\
y_{B n} \frac{\partial}{\partial \beta_{3}}\left[\frac{1}{P n(B)_{n}}\left(\frac{\partial P n(B)_{n}}{\partial \beta_{3}}\right)\right]+y_{L n} \frac{\partial}{\partial \beta_{3}}\left[\frac{1}{P n(L)_{n}}\left(\frac{\partial P n(L)_{n}}{\partial \beta_{3}}\right)\right] I
\end{gathered}
$$


where

$$
\begin{aligned}
& \left.\frac{1}{P n(C)}\left(\frac{\partial P n(C)}{\partial \beta_{3}}\right)=\frac{\operatorname{Accom}\left(e^{V(\text { Taxi })}+e^{V(\text { Subway })}+e^{V(\text { Bus })}+e^{V(\text { Limousine })}\right)}{\left(e^{V(\text { Car })}+e^{V(\text { Taxi })}+e^{V(\text { Subway })}+e^{V(\text { Bus })}+e^{V(\text { Limousin })}\right)}\right) \\
& =\operatorname{Accom}[P n(T)+P n(S)+P n(B)+P n(L)] \\
& \frac{1}{\partial \beta_{3}}\left[\frac{1}{P n(C)}\left(\frac{\partial P n(C)}{\partial \beta_{3}}\right)\right]=A c c o m\left[\frac{\partial P n(T)}{\partial \beta_{3}}+\frac{\partial P n(S)}{\partial \beta_{3}}+\frac{\partial P n(B)}{\partial \beta_{3}}+\frac{\partial P n(L)}{\partial \beta_{3}}\right] \\
& =\operatorname{Accom}[-\operatorname{AccomPn}(C) P n(T)-\operatorname{AccomPn}(C) P n(S) \\
& \text { - } \operatorname{AccomPn}(C) P n(B)-\operatorname{AccomPn}(C) P n(L)] \\
& =-A c c o m^{2} \times P n(C)[P n(T)+P n(S)+P n(B)+P n(L)] \\
& \frac{1}{\partial \beta_{3}}\left[\frac{1}{P n(T)}\left(\frac{\partial P n(T)}{\partial \beta_{3}}\right)\right]=-\operatorname{Accom}\left[\frac{\partial P n(C)}{\partial \beta_{3}}\right] \\
& =-\operatorname{Accom}[\operatorname{AccomPn}(C)[P n(T)+P n(S)+P n(B)+P n(L)]] \\
& =-A_{c c o m}{ }^{2} \times P n(C)[P n(T)+P n(S)+P n(B)+P n(L)] \\
& \frac{1}{\partial \beta_{3}}\left[\frac{1}{P n(S)}\left(\frac{\partial P n(S)}{\partial \beta_{3}}\right)\right]=-\operatorname{Accom}\left[\frac{\partial P n(C)}{\partial \beta_{3}}\right] \\
& =-\operatorname{Accom}[\operatorname{Acco} m P n(C)[P n(T)+P n(S)+P n(B)+P n(L)]] \\
& =-A_{c c o m}{ }^{2} \times P n(C)[P n(T)+P n(S)+P n(B)+P n(L)] \\
& \frac{1}{\partial \beta_{3}}\left[\frac{1}{P n(B)}\left(\frac{\partial P n(B)}{\partial \beta_{3}}\right)\right]=-\operatorname{Accom}\left[\frac{\partial P n(C)}{\partial \beta_{3}}\right] \\
& =-\operatorname{Accom}[\operatorname{Acco} m P n(C)[P n(T)+P n(S)+P n(B)+P n(L)]] \\
& =-A c c o m^{2} \times P n(C)[P n(T)+P n(S)+P n(B)+P n(L)] \\
& \frac{1}{\partial \beta_{3}}\left[\frac{1}{\operatorname{Pn}(L)}\left(\frac{\partial \operatorname{Pn}(L)}{\partial \beta_{3}}\right)\right]=-\operatorname{Accom}\left[\frac{\partial \operatorname{Pn}(C)}{\partial \beta_{3}}\right] \\
& =-\operatorname{Accom}[\operatorname{Acco} m P n(C)[P n(T)+P n(S)+P n(B)+P n(L)]] \\
& =-A c c o m^{2} \times P n(C)[P n(T)+P n(S)+P n(B)+P n(L)] \\
& \frac{\partial P n(C)}{\partial \beta_{3}}=A c c o m P n(C)[P n(T)+P n(S)+P n(B)+P n(L)] \\
& \frac{\partial P n(T)}{\partial \beta_{3}}=-\operatorname{AccomPn}(C) P n(T) \\
& \frac{\partial P n(S)}{\partial \beta_{3}}=-A \operatorname{comPn}(C) P n(S) \\
& \frac{\partial P n(B)}{\partial \beta_{3}}=-A \operatorname{comPn}(C) P n(B)
\end{aligned}
$$


$\frac{\partial \operatorname{Pn}(L)}{\partial \beta_{3}}=-\operatorname{AccomPn}(C) \operatorname{Pn}(L)$

20) The second order partial derivative with respect to $\beta_{3} \beta_{4}$ is

$$
\begin{aligned}
\frac{\partial^{2} L L\left(\beta_{1}, \beta_{2}, \cdots, \beta_{8}, \beta_{9}\right)}{\partial \beta_{4} \partial \beta_{3}}= & \sum_{n=1}^{N} l y_{C n} \frac{\partial}{\partial \beta_{4}}\left[\frac{1}{P n(C)_{n}}\left(\frac{\partial P n(C)_{n}}{\partial \beta_{3}}\right)\right]+y_{T n} \frac{\partial}{\partial \beta_{4}}\left[\frac{1}{P n(T)_{n}}\left(\frac{\partial P n(T)_{n}}{\partial \beta_{3}}\right)\right]+y_{S n} \frac{\partial}{\partial \beta_{4}}\left[\frac{1}{P n(S)_{n}}\left(\frac{\partial P n(S)_{n}}{\partial \beta_{3}}\right)\right]+ \\
& \left.y_{B n} \frac{\partial}{\partial \beta_{4}}\left[\frac{1}{P n(B)_{n}}\left(\frac{\partial P n(B)_{n}}{\partial \beta_{3}}\right)\right]+y_{L n} \frac{\partial}{\partial \beta_{4}}\left[\frac{1}{P n(L)_{n}}\left(\frac{\partial P n(L)_{n}}{\partial \beta_{3}}\right)\right]\right]
\end{aligned}
$$

where

$$
\begin{aligned}
& \frac{1}{P n(C)}\left(\frac{\partial P n(C)}{\partial \beta_{3}}\right)=\frac{A c c o m\left(e^{V(\text { Taxi })}+e^{V(\text { (Subway })}+e^{V(\text { Bus })}+e^{V(\text { Limousine })}\right)}{\left(e^{V(\text { Car })}+e^{V(\text { Taxi })}+e^{V(\text { Subway })}+e^{V(\text { Bus })}+e^{V(\text { Limousine })}\right)} \\
& =\operatorname{Accom}[P n(T)+P n(S)+P n(B)+P n(L)] \\
& \frac{I}{\partial \beta_{4}}\left[\frac{1}{P n(C)}\left(\frac{\partial P n(C)}{\partial \beta_{3}}\right)\right]=\operatorname{Accom}\left[\frac{\partial P n(T)}{\partial \beta_{4}}+\frac{\partial P n(S)}{\partial \beta_{4}}+\frac{\partial P n(B)}{\partial \beta_{4}}+\frac{\partial P n(L)}{\partial \beta_{4}}\right] \\
& =\operatorname{Accom}[-\operatorname{SexmPn}(C) \operatorname{Pn}(T)-\operatorname{Sexm}(C) \operatorname{Pn}(S) \\
& \text { - } \operatorname{SexmPn}(C) P n(B)-\operatorname{SexmPn}(C) P n(L)] \\
& =-\operatorname{Accom} \times \operatorname{Sexm} \times \operatorname{Pn}(C)[P n(T)+P n(S)+P n(B)+P n(L)] \\
& \frac{1}{\partial \beta_{4}}\left[\frac{1}{P n(T)}\left(\frac{\partial P n(T)}{\partial \beta_{3}}\right)\right]=-\operatorname{Accom}\left[\frac{\partial P n(C)}{\partial \beta_{4}}\right] \\
& =-\operatorname{Accom}[\operatorname{Sexm} P n(C)[P n(T)+P n(S)+P n(B)+P n(L)]] \\
& =-A c c o m \times \operatorname{Sexm} \times P n(C)[P n(T)+P n(S)+P n(B)+P n(L)] \\
& \frac{1}{\partial \beta_{4}}\left[\frac{1}{P n(S)}\left(\frac{\partial P n(S)}{\partial \beta_{3}}\right)\right]=-\operatorname{Accom}\left[\frac{\partial P n(C)}{\partial \beta_{4}}\right] \\
& =-\operatorname{Accom}[\operatorname{Sexm} P n(C)[P n(T)+P n(S)+P n(B)+P n(L)]] \\
& =-A c c o m \times \operatorname{Sexm} \times P n(C)[P n(T)+P n(S)+P n(B)+P n(L)] \\
& \frac{1}{\partial \beta_{4}}\left[\frac{1}{P n(B)}\left(\frac{\partial P n(B)}{\partial \beta_{3}}\right)\right]=-\operatorname{Accom}\left[\frac{\partial P n(C)}{\partial \beta_{4}}\right] \\
& =-A c c o m[\operatorname{Sexm} P n(C)[P n(T)+P n(S)+P n(B)+P n(L)]] \\
& =-A c c o m \times \operatorname{Sexm} \times P n(C)[P n(T)+P n(S)+P n(B)+P n(L)] \\
& \frac{1}{\partial \beta_{4}}\left[\frac{1}{P n(L)}\left(\frac{\partial P n(L)}{\partial \beta_{3}}\right)\right]=-\operatorname{Accom}\left[\frac{\partial P n(C)}{\partial \beta_{4}}\right] \\
& =-\operatorname{Accom}[\operatorname{SexmPn}(C)[P n(T)+P n(S)+P n(B)+P n(L)]] \\
& =-A c c o m \times \operatorname{Sexm} \times P n(C)[P n(T)+P n(S)+P n(B)+P n(L)]
\end{aligned}
$$




$$
\begin{aligned}
& \frac{\partial P n(C)}{\partial \beta_{4}}=\operatorname{Sexm} \times \operatorname{Pn}(C)[\operatorname{Pn}(T)+\operatorname{Pn}(S)+\operatorname{Pn}(B)+\operatorname{Pn}(L)] \\
& \frac{\partial P n(T)}{\partial \beta_{4}}=-\operatorname{Sexm} \times \operatorname{Pn}(C) P n(T) \\
& \frac{\partial P n(S)}{\partial \beta_{4}}=-\operatorname{Sexm} \times \operatorname{Pn}(C) \operatorname{Pn}(S) \\
& \frac{\partial P n(B)}{\partial \beta_{4}}=-\operatorname{Sexm} \times \operatorname{Pn}(C) \operatorname{Pn}(B) \\
& \frac{\partial P n(L)}{\partial \beta_{4}}=-\operatorname{Sexm} \times \operatorname{Pn}(C) P n(L)
\end{aligned}
$$

21) The second order partial derivative with respect to $\beta_{3} \beta_{5}$ is

$$
\begin{gathered}
\frac{\partial^{2} L L\left(\beta_{1}, \beta_{2}, \cdots, \beta_{8}, \beta_{9}\right)}{\partial \beta_{5} \partial \beta_{3}}=\sum_{n=1}^{N}\left[y_{C n} \frac{\partial}{\partial \beta_{5}}\left[\frac{1}{P n(C)_{n}}\left(\frac{\partial P n(C)_{n}}{\partial \beta_{3}}\right)\right]+y_{T n} \frac{\partial}{\partial \beta_{5}}\left[\frac{1}{P n(T)_{n}}\left(\frac{\partial P n(T)_{n}}{\partial \beta_{3}}\right)\right]+y_{S n} \frac{\partial}{\partial \beta_{5}}\left[\frac{1}{P n(S)_{n}}\left(\frac{\partial P n(S)_{n}}{\partial \beta_{3}}\right)\right]+\right. \\
\left.y_{B n} \frac{\partial}{\partial \beta_{5}}\left[\frac{1}{P n(B)_{n}}\left(\frac{\partial P n(B)_{n}}{\partial \beta_{3}}\right)\right]+y_{L n} \frac{\partial}{\partial \beta_{5}}\left[\frac{1}{P n(L)_{n}}\left(\frac{\partial P n(L)_{n}}{\partial \beta_{3}}\right)\right]\right]
\end{gathered}
$$

where

$$
\begin{aligned}
& \frac{1}{P n(C)}\left(\frac{\partial P n(C)}{\partial \beta_{3}}\right)=\frac{\operatorname{Accom}\left(e^{V(\text { Taxi })}+e^{V(\text { Subway })}+e^{V(\text { Bus })}+e^{V(\text { Limousine })}\right)}{\left(e^{V(\text { Car })}+e^{V(\text { Taxi })}+e^{V(\text { Subway })}+e^{V(\text { Bus })}+e^{V(\text { Limousine })}\right)} \\
& =\operatorname{Accom}[P n(T)+P n(S)+P n(B)+P n(L)] \\
& \frac{1}{\partial \beta_{5}}\left[\frac{1}{P n(C)}\left(\frac{\partial P n(C)}{\partial \beta_{3}}\right)\right]=\operatorname{Accom}\left[\frac{\partial P n(T)}{\partial \beta_{5}}+\frac{\partial P n(S)}{\partial \beta_{5}}+\frac{\partial P n(B)}{\partial \beta_{5}}+\frac{\partial P n(L)}{\partial \beta_{5}}\right] \\
& =\operatorname{Accom}[-\operatorname{Age340Pn}(C) P n(T)-\operatorname{Age340}(C) P n(S) \\
& \text { - Age340Pn(C)Pn(B)-Age340Pn(C)Pn(L)] } \\
& =-A c c o m \times A g e 340 \times P n(C)[P n(T)+P n(S)+P n(B)+P n(L)] \\
& \frac{1}{\partial \beta_{5}}\left[\frac{1}{P n(T)}\left(\frac{\partial P n(T)}{\partial \beta_{3}}\right)\right]=-\operatorname{Accom}\left[\frac{\partial P n(C)}{\partial \beta_{5}}\right] \\
& =-A c c o m[A g e 340 P n(C)[P n(T)+P n(S)+P n(B)+P n(L)]] \\
& =-A c c o m \times A g e 340 \times P n(C)[P n(T)+P n(S)+P n(B)+P n(L)] \\
& \frac{1}{\partial \beta_{5}}\left[\frac{1}{P n(S)}\left(\frac{\partial P n(S)}{\partial \beta_{3}}\right)\right]=-\operatorname{Accom}\left[\frac{\partial P n(C)}{\partial \beta_{5}}\right] \\
& =- \text { Accom[Age3 40Pn }(C)[P n(T)+P n(S)+P n(B)+P n(L)]] \\
& =-A c c o m \times A g e 340 \times P n(C)[P n(T)+P n(S)+P n(B)+P n(L)]
\end{aligned}
$$




$$
\begin{aligned}
& \frac{1}{\partial \beta_{5}}\left[\frac{1}{P n(B)}\left(\frac{\partial P n(B)}{\partial \beta_{3}}\right)\right]=-A c \operatorname{com}\left[\frac{\partial P n(C)}{\partial \beta_{5}}\right] \\
& =-\operatorname{Accom}[\text { Age 340Pn }(C)[P n(T)+P n(S)+P n(B)+P n(L)]] \\
& =-A c c o m \times A g e 340 \times P n(C)[P n(T)+P n(S)+P n(B)+P n(L)] \\
& \frac{1}{\partial \beta_{5}}\left[\frac{1}{\operatorname{Pn}(L)}\left(\frac{\partial P n(L)}{\partial \beta_{3}}\right)\right]=-\operatorname{Accom}\left[\frac{\partial \operatorname{Pn}(C)}{\partial \beta_{5}}\right] \\
& =-\operatorname{Accom}[\text { Age 3 40Pn }(C)[P n(T)+P n(S)+P n(B)+P n(L)]] \\
& =-A c c o m \times A g e 340 \times P n(C)[P n(T)+P n(S)+P n(B)+P n(L)] \\
& \frac{\partial P n(C)}{\partial \beta_{5}}=\operatorname{Age} 340 P n(C)(P n(T)+P n(S)+P n(B)+P n(L)) \\
& \frac{\partial P n(T)}{\partial \beta_{5}}=-\operatorname{Age} 340 \operatorname{Pn}(C) P n(T) \\
& \frac{\partial P n(S)}{\partial \beta_{5}}=-A g e 340 P n(C) P n(S) \\
& \frac{\partial P n(B)}{\partial \beta_{5}}=-A g e 340 P n(C) P n(B) \\
& \frac{\partial P n(L)}{\partial \beta_{5}}=-A g e 340 P n(C) P n(L)
\end{aligned}
$$

22) The second order partial derivative with respect to $\beta_{3} \beta_{6}$ is

$$
\begin{aligned}
\frac{\partial^{2} L L\left(\beta_{1}, \beta_{2}, \cdots, \beta_{8}, \beta_{9}\right)}{\partial \beta_{6} \partial \beta_{3}}= & \sum_{n=1}^{N}\left[y_{C n} \frac{\partial}{\partial \beta_{6}}\left[\frac{1}{P n\left(C_{h}\right.}\left(\frac{\partial P n\left(C_{h}\right.}{\partial \beta_{3}}\right)\right]+y_{T n} \frac{\partial}{\partial \beta_{6}}\left[\frac{1}{P n(T)_{h}}\left(\frac{\partial P n(T)_{h}}{\partial \beta_{3}}\right)\right]+y_{S n} \frac{\partial}{\partial \beta_{6}}\left[\frac{1}{P n\left(S_{h}\right.}\left(\frac{\partial P n\left(S_{h}\right.}{\partial \beta_{3}}\right)\right]+\right. \\
& \left.y_{B n} \frac{\partial}{\partial \beta_{6}}\left[\frac{1}{P n(B)}\left(\frac{\partial P n(B)_{h}}{\partial \beta_{3}}\right)\right]+y_{L n} \frac{\partial}{\partial \beta_{6}}\left[\frac{1}{P n(L)_{h}}\left(\frac{\partial P n(L)_{h}}{\partial \beta_{3}}\right)\right]\right]
\end{aligned}
$$

Where

$$
\begin{aligned}
& \frac{1}{P n(C)}\left(\frac{\partial P n(C)}{\partial \beta_{3}}\right)=\frac{A c c o m\left(e^{V(\text { Taxi })}+e^{V(\text { Subway })}+e^{V(\text { Bus })}+e^{V(\text { Limousine })}\right)}{\left(e^{V(\text { Car })}+e^{V(\text { Taxi })}+e^{V(\text { Subway })}+e^{V(\text { Bus })}+e^{V(\text { Limousine })}\right)} \\
& =\operatorname{Accom}[P n(T)+P n(S)+P n(B)+P n(L)] \\
& \frac{1}{\partial \beta_{6}}\left[\frac{1}{P n(C)}\left(\frac{\partial P n(C)}{\partial \beta_{3}}\right)\right]=A \operatorname{ccom}\left[\frac{\partial P n(T)}{\partial \beta_{6}}+\frac{\partial P n(S)}{\partial \beta_{6}}+\frac{\partial P n(B)}{\partial \beta_{6}}+\frac{\partial P n(L)}{\partial \beta_{6}}\right] \\
& =\operatorname{Accom}[\operatorname{Pn}(T)[P n(C)+P n(S)+P n(B)+P n(L)] \\
& \text { - } P n(S) P n(T)-P n(B) P n(T)-P n(L) P n(T)] \\
& =\operatorname{Accom}[\operatorname{Pn}(T) P n(C)]
\end{aligned}
$$




$$
\begin{aligned}
& \frac{1}{\partial \beta_{6}}\left[\frac{1}{P n(T)}\left(\frac{\partial P n(T)}{\partial \beta_{3}}\right)\right]=-\operatorname{Accom}\left[\frac{\partial P n(C)}{\partial \beta_{6}}\right] \\
& =-\operatorname{Accom}[-\operatorname{Pn}(C) P n(T)]=\operatorname{Accom}[\operatorname{Pn}(T) P n(C)] \\
& \frac{1}{\partial \beta_{6}}\left[\frac{1}{P n(S)}\left(\frac{\partial P n(S)}{\partial \beta_{3}}\right)\right]=-\operatorname{Accom}\left[\frac{\partial P n(C)}{\partial \beta_{6}}\right] \\
& =-\operatorname{Accom}[-\operatorname{Pn}(C) P n(T)]=\operatorname{Accom}[\operatorname{Pn}(T) P n(C)] \\
& \frac{1}{\partial \beta_{6}}\left[\frac{1}{P n(B)}\left(\frac{\partial P n(B)}{\partial \beta_{3}}\right)\right]=-\operatorname{Accom}\left[\frac{\partial P n(C)}{\partial \beta_{6}}\right] \\
& =-\operatorname{Accom}[-\operatorname{Pn}(C) P n(T)]=\operatorname{Accom}[\operatorname{Pn}(T) P n(C)] \\
& \frac{1}{\partial \beta_{6}}\left[\frac{1}{P n(L)}\left(\frac{\partial P n(L)}{\partial \beta_{3}}\right)\right]=-\operatorname{Accom}\left[\frac{\partial P n(C)}{\partial \beta_{6}}\right] \\
& =-\operatorname{Accom}[-\operatorname{Pn}(C) P n(T)]=\operatorname{Accom}[P n(T) P n(C)] \\
& \frac{\partial \operatorname{Pn}(C)}{\partial \beta_{6}}=\frac{0-e^{V(\text { Car })}\left(e^{V(\text { Taxi })}\right)}{\left(e^{V(\text { Car })}+e^{V(\text { (Taxi })}+e^{V(\text { Subway })}+e^{V(\text { Bus })}+e^{V(\text { Limousine })}\right)^{2}}=-\operatorname{Pn}(C) \operatorname{Pn}(T) \\
& \frac{\partial \operatorname{Pn}(T)}{\partial \beta_{6}}=\frac{e^{V(\text { Taxi })}\left(e^{V(\text { Car })}+e^{V(\text { Subway })}+e^{V(\text { Bus })}+e^{V(\text { Limousine })}\right)}{\left(e^{V(\text { Car })}+e^{V(\text { Taxi })}+e^{V(\text { Subway })}+e^{V(\text { Bus })}+e^{V(\text { Limousine })}\right)^{2}} \\
& =P n(T)(P n(C)+P n(S)+P n(B)+P n(L)) \\
& \frac{\partial P n(S)}{\partial \beta_{6}}=\frac{0-e^{V(\text { Subway })}\left(e^{V(\text { Taxi })}\right)}{\left(e^{V(\text { Car })}+e^{V(\text { Taxi })}+e^{V(\text { Subway })}+e^{V(\text { Bus })}+e^{V(\text { Limousine })}\right)^{2}}=-\operatorname{Pn}(\operatorname{S}) \operatorname{Pn}(T) \\
& \frac{\partial P n(B)}{\partial \beta_{6}}=\frac{0-e^{V(\text { Bus })}\left(e^{V(\text { Taxi })}\right)}{\left(e^{V(\text { Car })}+e^{V(\text { Taxi })}+e^{V(\text { Subway })}+e^{V(\text { Bus })}+e^{V(\text { Limousine })}\right)^{2}}=-\operatorname{Pn}(B) \operatorname{Pn}(T) \\
& \frac{\partial P n(L)}{\partial \beta_{6}}=\frac{0-e^{V(\text { limousine })}\left(e^{V(\text { Taxi })}\right)}{\left(e^{V(\text { Car })}+e^{V(\text { Taxi })}+e^{V(\text { Subway })}+e^{V(\text { Bus })}+e^{V(\text { Limousine })}\right)^{2}}=-\operatorname{Pn}(L) \operatorname{Pn}(T)
\end{aligned}
$$

23) The second order partial derivative with respect to $\beta_{3} \beta_{7}$ is

$$
\begin{aligned}
\frac{\partial^{2} L L\left(\beta_{1}, \beta_{2}, \cdots, \beta_{8}, \beta_{9}\right)}{\partial \beta_{7} \partial \beta_{3}}= & \sum_{n=1}^{N} l y_{C n} \frac{\partial}{\partial \beta_{7}}\left[\frac{1}{P n\left(C_{h}\right.}\left(\frac{\partial P n\left(C_{h}\right.}{\partial \beta_{3}}\right)\right]+y_{T n} \frac{\partial}{\partial \beta_{7}}\left[\frac{1}{P n\left(T_{h}\right.}\left(\frac{\partial P n(T)_{h}}{\partial \beta_{3}}\right)\right]+y_{S n} \frac{\partial}{\partial \beta_{7}}\left[\frac{1}{P n\left(S_{h}\right.}\left(\frac{\partial P n\left(S_{h}\right.}{\partial \beta_{3}}\right)\right]+ \\
& y_{B n} \frac{\partial}{\partial \beta_{7}}\left[\frac{1}{P n\left(B B_{h}\right.}\left(\frac{\partial P n(B h}{\partial \beta_{3}}\right)\right]+y_{L n} \frac{\partial}{\partial \beta_{7}}\left[\frac{1}{P n(L h}\left(\frac{\partial P n\left(L_{h}\right.}{\partial \beta_{3}}\right)\right] 1
\end{aligned}
$$


where

$$
\begin{aligned}
& \frac{1}{P n(C)}\left(\frac{\partial P n(C)}{\partial \beta_{3}}\right)=\frac{A c c o m\left(e^{V(\text { Taxi })}+e^{V(\text { Subway })}+e^{V(\text { Bus })}+e^{V(\text { Limousine })}\right)}{\left(e^{V(\text { Car })}+e^{V(\text { Taxi })}+e^{V(\text { Subway })}+e^{V(\text { Bus })}+e^{V(\text { Limousine })}\right)} \\
& =\operatorname{Accom}[P n(T)+P n(S)+P n(B)+P n(L)] \\
& \frac{1}{\partial \beta_{7}}\left[\frac{1}{P n(C)}\left(\frac{\partial P n(C)}{\partial \beta_{3}}\right)\right]=\operatorname{Accom}\left[\frac{\partial P n(T)}{\partial \beta_{7}}+\frac{\partial P n(S)}{\partial \beta_{7}}+\frac{\partial P n(B)}{\partial \beta_{7}}+\frac{\partial P n(L)}{\partial \beta_{7}}\right] \\
& =\operatorname{Accom}[-P n(T) P n(S)+P n(S)[P n(C)+P n(T)+P n(B)+P n(L)] \\
& \text { - } P n(B) P n(S)-P n(L) P n(S)] \\
& =\operatorname{Accom}[\operatorname{Pn}(S) P n(C)] \\
& \frac{1}{\partial \beta_{7}}\left[\frac{1}{P n(T)}\left(\frac{\partial P n(T)}{\partial \beta_{3}}\right)\right]=-\operatorname{Accom}\left[\frac{\partial P n(C)}{\partial \beta_{7}}\right] \\
& =-\operatorname{Accom}[-\operatorname{Pn}(C) \operatorname{Pn}(S)]=\operatorname{Accom}[\operatorname{Pn}(S) \operatorname{Pn}(C)] \\
& \frac{1}{\partial \beta_{7}}\left[\frac{1}{P n(S)}\left(\frac{\partial P n(S)}{\partial \beta_{3}}\right)\right]=-\operatorname{Accom}\left[\frac{\partial P n(C)}{\partial \beta_{7}}\right] \\
& =-\operatorname{Accom}[-\operatorname{Pn}(C) \operatorname{Pn}(S)]=\operatorname{Accom}[\operatorname{Pn}(S) \operatorname{Pn}(C)] \\
& \frac{I}{\partial \beta_{7}}\left[\frac{1}{P n(L)}\left(\frac{\partial P n(B)}{\partial \beta_{3}}\right)\right]=-\operatorname{Accom}\left[\frac{\partial P n(C)}{\partial \beta_{7}}\right] \\
& =-\operatorname{Accom}[-\operatorname{Pn}(C) P n(S)]=\operatorname{Accom}[\operatorname{Pn}(S) P n(C)] \\
& \frac{1}{\partial \beta_{7}}\left[\frac{1}{P n(L)}\left(\frac{\partial P n(L)}{\partial \beta_{3}}\right)\right]=-\operatorname{Accom}\left[\frac{\partial P n(C)}{\partial \beta_{7}}\right] \\
& =-\operatorname{Accom}[-\operatorname{Pn}(C) P n(S)]=\operatorname{Accom}[\operatorname{Pn}(S) P n(C)] \\
& \frac{\partial P n(C)}{\partial \beta_{7}}=-P n(C) P n(S) \\
& \frac{\partial P n(T)}{\partial \beta_{7}}=-P n(T) P n(S) \\
& \frac{\partial P n(S)}{\partial \beta_{7}}=P n(S)(P n(C)+P n(T)+P n(B)+P n(L)) \\
& \frac{\partial P n(B)}{\partial \beta_{7}}=-P n(B) P n(S) \\
& \frac{\partial P n(L)}{\partial \beta_{7}}=-P n(L) P n(S)
\end{aligned}
$$

24) The second order partial derivative with respect to $\beta_{3} \beta_{8}$ is 


$$
\begin{gathered}
\frac{\partial^{2} L L\left(\beta_{1}, \beta_{2}, \cdots, \beta_{8}, \beta_{9}\right)}{\partial \beta_{8} \partial \beta_{3}}=\sum_{n=1}^{N}\left[y_{C n} \frac{\partial}{\partial \beta_{8}}\left[\frac{1}{P n\left(C_{h}\right.}\left(\frac{\partial P n\left(C_{h}\right.}{\partial \beta_{3}}\right)\right]+y_{T n} \frac{\partial}{\partial \beta_{8}}\left[\frac{1}{P n\left(T_{h}\right.}\left(\frac{\partial P n\left(T_{h}\right.}{\partial \beta_{3}}\right)\right]+y_{S n} \frac{\partial}{\partial \beta_{8}}\left[\frac{1}{P n\left(S_{h}\right.}\left(\frac{\partial P n\left(S S_{h}\right.}{\partial \beta_{3}}\right)\right]+\right. \\
\left.y_{B n} \frac{\partial}{\partial \beta_{8}}\left[\frac{1}{P n\left(B B_{h}\right.}\left(\frac{\partial P n\left(B h_{h}\right.}{\partial \beta_{3}}\right)\right]+y_{L n} \frac{\partial}{\partial \beta_{8}}\left[\frac{1}{P n(L h}\left(\frac{\partial P n\left(L_{h}\right.}{\partial \beta_{3}}\right)\right]\right]
\end{gathered}
$$

where

$$
\begin{aligned}
& \frac{1}{P n(C)}\left(\frac{\partial P n(C)}{\partial \beta_{3}}\right)=\frac{A c c o m\left(e^{V(\text { Taxi })}+e^{V(\text { Subway })}+e^{V(\text { Bus })}+e^{V(\text { Limousine })}\right)}{\left(e^{V(\text { Car })}+e^{V(\text { Taxi })}+e^{V(\text { Subway })}+e^{V(\text { Bus })}+e^{V(\text { Limousine })}\right)} \\
& =\operatorname{Accom}[P n(T)+P n(S)+P n(B)+P n(L)] \\
& \frac{1}{\partial \beta_{8}}\left[\frac{1}{P n(C)}\left(\frac{\partial P n(C)}{\partial \beta_{3}}\right)\right]=\operatorname{Accom}\left[\frac{\partial P n(T)}{\partial \beta_{8}}+\frac{\partial P n(S)}{\partial \beta_{8}}+\frac{\partial P n(B)}{\partial \beta_{8}}+\frac{\partial P n(L)}{\partial \beta_{8}}\right] \\
& =\operatorname{Accom}[-P n(B) P n(T)-P n(B) P n(S)+P n(B)[P n(C)+P n(T)+P n(S)+P n(L)] \\
& \text { - } P n(B) P n(L)] \\
& =\operatorname{Accom}[\operatorname{Pn}(B) \operatorname{Pn}(C)]
\end{aligned}
$$$$
\frac{1}{\partial \beta_{8}}\left[\frac{1}{P n(T)}\left(\frac{\partial P n(T)}{\partial \beta_{3}}\right)\right]=-\operatorname{Accom}\left[\frac{\partial P n(C)}{\partial \beta_{8}}\right]
$$$$
=-\operatorname{Accom}[-\operatorname{Pn}(C) P n(B)]=\operatorname{Accom}[P n(B) P n(C)]
$$$$
\frac{1}{\partial \beta_{8}}\left[\frac{1}{\operatorname{Pn}(S)}\left(\frac{\partial P n(S)}{\partial \beta_{3}}\right)\right]=-\operatorname{Accom}\left[\frac{\partial P n(C)}{\partial \beta_{8}}\right]
$$$$
=-\operatorname{Accom}[-\operatorname{Pn}(C) \operatorname{Pn}(B)]=\operatorname{Accom}[\operatorname{Pn}(B) P n(C)]
$$$$
\frac{1}{\partial \beta_{8}}\left[\frac{1}{P n(L)}\left(\frac{\partial P n(B)}{\partial \beta_{3}}\right)\right]=-\operatorname{Accom}\left[\frac{\partial P n(C)}{\partial \beta_{8}}\right]
$$$$
=-\operatorname{Accom}[-\operatorname{Pn}(C) \operatorname{Pn}(B)]=\operatorname{Accom}[\operatorname{Pn}(B) P n(C)]
$$$$
\frac{1}{\partial \beta_{8}}\left[\frac{1}{P n(L)}\left(\frac{\partial P n(L)}{\partial \beta_{3}}\right)\right]=-\operatorname{Accom}\left[\frac{\partial P n(C)}{\partial \beta_{8}}\right]
$$$$
=-\operatorname{Accom}[-\operatorname{Pn}(C) P n(B)]=\operatorname{Accom}[P n(B) P n(C)]
$$$$
\frac{\partial P n(C)}{\partial \beta_{8}}=\frac{0-e^{V(\text { Car })}\left(e^{V(\text { Bus })}\right)}{\left(e^{V(\text { Car })}+e^{V(\text { Taxi })}+e^{V(\text { Subway })}+e^{V(\text { Bus })}+e^{V(\text { Limousine })}\right)^{2}}=-\operatorname{Pn}(C) \operatorname{Pn}(B)
$$$$
\frac{\partial \operatorname{Pn}(T)}{\partial \beta_{8}}=\frac{0-e^{V(\text { Taxi })}\left(e^{V(\text { Bus })}\right)}{\left(e^{V(\text { Car })}+e^{V(\text { Taxi })}+e^{V(\text { Subway })}+e^{V(\text { Bus })}+e^{V(\text { Limousine })}\right)^{2}}=-\operatorname{Pn}(T) \operatorname{Pn}(B)
$$$$
\frac{\partial P n(S)}{\partial \beta_{8}}=\frac{0-e^{V(\text { Subway })}\left(e^{V(\text { Bus })}\right)}{\left(e^{V(\text { Car })}+e^{V(\text { Taxi })}+e^{V(\text { Subway })}+e^{V(\text { Bus })}+e^{V(\text { Limousine })}\right)^{2}}=-\operatorname{Pn}(\operatorname{S}) \operatorname{Pn}(B)
$$ 


$$
\begin{aligned}
\frac{\partial P n(B)}{\partial \beta_{8}} & =\frac{e^{V(B u s)}\left(e^{V(\text { Car })}+e^{V(\text { Taxi })}+e^{V(\text { Subway })}+e^{V(\text { Bus })}+e^{V(\text { Limousin } e)}\right)-e^{V(B u s)} e^{V(B u s)}}{\left(e^{V(\text { Car })}+e^{V(\text { Taxi })}+e^{V(\text { Subway })}+e^{V(B u s)}+e^{V(\text { Limousin } e)}\right)^{2}} \\
& =\operatorname{Pn}(B)[\operatorname{Pn}(C)+\operatorname{Pn}(T)+P n(S)+P n(L)]
\end{aligned}
$$

$\frac{\partial \operatorname{Pn}(L)}{\partial \beta_{8}}=\frac{0-e^{V(\text { limousine })}\left(e^{V(\text { Bus })}\right)}{\left(e^{V(\text { Car })}+e^{V(\text { (Taxi })}+e^{V(\text { Subway })}+e^{V(\text { Bus })}+e^{V(\text { Limousine })}\right)^{2}}=-\operatorname{Pn}($ L $) \operatorname{Pn}(B)$

25) The second order partial derivative with respect to $\beta_{3} \beta_{9}$ is

$$
\begin{gathered}
\frac{\partial^{2} L L\left(\beta_{1}, \beta_{2}, \cdots, \beta_{8}, \beta_{9}\right)}{\partial \beta_{9} \partial \beta_{3}}=\sum_{n=1}^{N}\left[y_{C n} \frac{\partial}{\partial \beta_{9}}\left[\frac{1}{P n\left(C_{h}\right.}\left(\frac{\partial P n\left(C_{h}\right.}{\partial \beta_{3}}\right)\right]+y_{T n} \frac{\partial}{\partial \beta_{9}}\left[\frac{1}{P n(T h}\left(\frac{\partial P n\left(T_{h}\right.}{\partial \beta_{3}}\right)\right]+y_{S n} \frac{\partial}{\partial \beta_{9}}\left[\frac{1}{P n\left(S_{h}\right.}\left(\frac{\partial P n\left(S_{h}\right.}{\partial \beta_{3}}\right)\right]+\right. \\
\left.y_{B n} \frac{\partial}{\partial \beta_{9}}\left[\frac{1}{P n(B h}\left(\frac{\partial P n\left(B_{h}\right.}{\partial \beta_{3}}\right)\right]+y_{L n} \frac{\partial}{\partial \beta_{9}}\left[\frac{1}{P n(L h}\left(\frac{\partial P n\left(L_{h}\right.}{\partial \beta_{3}}\right)\right]\right]
\end{gathered}
$$

Where

$$
\begin{aligned}
& \frac{1}{P n(C)}\left(\frac{\partial P n(C)}{\partial \beta_{3}}\right)=\frac{A c c o m\left(e^{V(\text { Taxi })}+e^{V(\text { Subway })}+e^{V(\text { Bus })}+e^{V(\text { Limousine })}\right)}{\left(e^{V(\text { (Car) }}+e^{V(\text { Taxi })}+e^{V(\text { Subway })}+e^{V(\text { Bus })}+e^{V(\text { Limousine })}\right)} \\
& =\operatorname{Accom}[P n(T)+P n(S)+P n(B)+P n(L)] \\
& \frac{1}{\partial \beta_{9}}\left[\frac{1}{P n(C)}\left(\frac{\partial P n(C)}{\partial \beta_{3}}\right)\right]=\operatorname{Accom}\left[\frac{\partial P n(T)}{\partial \beta_{9}}+\frac{\partial P n(S)}{\partial \beta_{9}}+\frac{\partial P n(B)}{\partial \beta_{9}}+\frac{\partial P n(L)}{\partial \beta_{9}}\right] \\
& =\operatorname{Accom}[-P n(L) P n(T)-P n(L) P n(S)-P n(L) P n(B) \\
& +P n(L)[P n(C)+P n(T)+P n(S)+P n(B)]] \\
& =\operatorname{Accom}[\operatorname{Pn}(L) P n(C)] \\
& \frac{1}{\partial \beta_{9}}\left[\frac{1}{P n(T)}\left(\frac{\partial P n(T)}{\partial \beta_{3}}\right)\right]=-A c c o m\left[\frac{\partial P n(C)}{\partial \beta_{9}}\right] \\
& =-\operatorname{Accom}[-\operatorname{Pn}(L) P n(C)]=\operatorname{Accom}[\operatorname{Pn}(L) P n(C)] \\
& \frac{1}{\partial \beta_{9}}\left[\frac{1}{P n(S)}\left(\frac{\partial P n(S)}{\partial \beta_{3}}\right)\right]=-\operatorname{Accom}\left[\frac{\partial P n(C)}{\partial \beta_{9}}\right] \\
& =-\operatorname{Accom}[-\operatorname{Pn}(L) P n(C)]=\operatorname{Accom}[\operatorname{Pn}(L) P n(C)] \\
& \frac{1}{\partial \beta_{9}}\left[\frac{1}{\operatorname{Pn}(B)}\left(\frac{\partial \operatorname{Pn}(B)}{\partial \beta_{3}}\right)\right]=-\operatorname{Accom}\left[\frac{\partial \operatorname{Pn}(C)}{\partial \beta_{9}}\right] \\
& =-\operatorname{Accom}[-\operatorname{Pn}(L) P n(C)]=\operatorname{Accom}[\operatorname{Pn}(L) P n(C)] \\
& \frac{1}{\partial \beta_{9}}\left[\frac{1}{\operatorname{Pn}(L)}\left(\frac{\partial P n(L)}{\partial \beta_{3}}\right)\right]=-\operatorname{Accom}\left[\frac{\partial P n(C)}{\partial \beta_{9}}\right] \\
& =-\operatorname{Accom}[-\operatorname{Pn}(L) P n(C)]=\operatorname{Accom}[P n(L) P n(C)]
\end{aligned}
$$




$$
\begin{aligned}
& \frac{\partial P n(C)}{\partial \beta_{9}}=\frac{0-e^{V(\text { Car })}\left(e^{V(\text { Limousine })}\right)}{\left(e^{V(\text { Car })}+e^{V(\text { (Taxi })}+e^{V(\text { Subway })}+e^{V(\text { Bus })}+e^{V(\text { Limousine })}\right)^{2}}=-\operatorname{Pn}(L) \operatorname{Pn}(C) \\
& \frac{\partial P n(T)}{\partial \beta_{9}}=\frac{0-e^{V(\text { Taxi })}\left(e^{V(\text { Limousine })}\right)}{\left(e^{V(\text { Car })}+e^{V(\text { Taxi })}+e^{V(\text { Subway })}+e^{V(\text { Bus })}+e^{V(\text { Limousine })}\right)^{2}}=-\operatorname{Pn}(L) \operatorname{Pn}(T) \\
& \frac{\partial P n(S)}{\partial \beta_{9}}=\frac{0-e^{V(\text { Subway })}\left(e^{V(\text { Limousine })}\right)}{\left(e^{V(\text { Car })}+e^{V(\text { Taxi })}+e^{V(\text { Subway })}+e^{V(\text { Bus })}+e^{V(\text { Limousine })}\right)^{2}}=-\operatorname{Pn}(L) \operatorname{Pn}(S) \\
& \frac{\partial P n(B)}{\partial \beta_{9}}=\frac{0-e^{V(\text { Bus })}\left(e^{V(\text { Limousine })}\right)}{\left(e^{V(\text { Car })}+e^{V(\text { Taxi })}+e^{V(\text { Subway })}+e^{V(\text { Bus })}+e^{V(\text { Limousine })}\right)^{2}}=-\operatorname{Pn}(L) \operatorname{Pn}(B) \\
& \frac{\partial P n(L)}{\partial \beta_{9}}=\frac{e^{V(\text { Limousine })}\left(e^{V(\text { Car })}+e^{V(\text { Taxi })}+e^{V(\text { Subway })}+e^{V(\text { Bus })}+e^{V(\text { Limousine })}\right)-e^{V(\text { limujin })} e^{V(\text { Limousine })}}{\left(e^{V(\text { Car })}+e^{V(\text { Taxi })}+e^{V(\text { Subway })}+e^{V(B u s)}+e^{V(\text { Limousine })}\right)^{2}} \\
& =P n(L)[P n(C)+P n(T)+P n(S)+P n(B)]
\end{aligned}
$$

26) The second order partial derivative with respect to $\beta_{4} \beta_{4}$ is

$$
\begin{aligned}
\frac{\partial^{2} L L\left(\beta_{1}, \beta_{2}, \cdots, \beta_{8}, \beta_{9}\right)}{\partial \beta_{4} \partial \beta_{4}}= & \sum_{n=1}^{N}\left[y_{C n} \frac{\partial}{\partial \beta_{4}}\left[\frac{1}{P n(C)_{n}}\left(\frac{\partial P_{n}(C)_{n}}{\partial \beta_{4}}\right)\right]+y_{T_{n}} \frac{\partial}{\partial \beta_{4}}\left[\frac{1}{P n(T)_{n}}\left(\frac{\partial P n(T)_{n}}{\partial \beta_{4}}\right)\right]+y_{S n} \frac{\partial}{\partial \beta_{4}}\left[\frac{1}{P n(S)_{n}}\left(\frac{\partial P n(S)_{n}}{\partial \beta_{4}}\right)\right]+\right. \\
& y_{B n} \frac{\partial}{\partial \beta_{4}}\left[\frac{1}{P n(B)_{n}}\left(\frac{\partial P n(B)_{n}}{\partial \beta_{4}}\right)\right]+y_{L n} \frac{\partial}{\partial \beta_{4}}\left[\frac{1}{P n(L)_{n}}\left(\frac{\partial P n(L)_{n}}{\partial \beta_{4}}\right)\right] J
\end{aligned}
$$

where

$$
\begin{aligned}
& \frac{1}{\operatorname{Pn}(C)}\left(\frac{\partial \operatorname{Pn}(C)}{\partial \beta_{4}}\right)=\frac{\operatorname{Sexm}\left(e^{V(\text { Taxi })}+e^{V(\text { Subway })}+e^{V(\text { Bus })}+e^{V(\text { Limousin } e)}\right)}{\left(e^{V(\text { Car })}+e^{V(\text { Taxi })}+e^{V(\text { Subway })}+e^{V(\text { Bus })}+e^{V(\text { Limousin } e)}\right)} \\
& =\operatorname{Sexm}[\operatorname{Pn}(T)+\operatorname{Pn}(S)+\operatorname{Pn}(B)+\operatorname{Pn}(L)] \\
& \frac{1}{\partial \beta_{4}}\left[\frac{1}{P n(C)}\left(\frac{\partial P n(C)}{\partial \beta_{4}}\right)\right]=\operatorname{Sexm}\left[\frac{\partial P n(T)}{\partial \beta_{4}}+\frac{\partial P n(S)}{\partial \beta_{4}}+\frac{\partial P n(B)}{\partial \beta_{4}}+\frac{\partial P n(L)}{\partial \beta_{4}}\right] \\
& =\operatorname{Sexm}[-\operatorname{Sexm} \times \operatorname{Pn}(T) \times \operatorname{Pn}(C)-\operatorname{Sexm} \times \operatorname{Pn}(S) \times \operatorname{Pn}(C) \\
& -\operatorname{Sexm} \times \operatorname{Pn}(B) \times \operatorname{Pn}(C)-\operatorname{Sexm} \times \operatorname{Pn}(L) \times \operatorname{Pn}(C)] \\
& =-\operatorname{Sexm}^{2} \times \operatorname{Pn}(C)[\operatorname{Pn}(\bar{T})+\operatorname{Pn}(S)+P n(B)+\operatorname{Pn}(L)]
\end{aligned}
$$




$$
\begin{aligned}
& \frac{1}{P n(T)}\left(\frac{\partial P n(T)}{\partial \beta_{4}}\right)=\frac{-\operatorname{Sexm} \times e^{V(\text { Car })}}{\left(e^{V(\text { Car })}+e^{V(\text { Taxi })}+e^{V(\text { Subway })}+e^{V(\text { Bus })}+e^{V(\text { Limousine })}\right)} \\
& =-\operatorname{Sexm}[\operatorname{Pn}(C)] \\
& \frac{1}{\partial \beta_{4}}\left[\frac{1}{P n(T)}\left(\frac{\partial P n(T)}{\partial \beta_{4}}\right)\right]=-\operatorname{Sexm}\left[\frac{\partial P n(C)}{\partial \beta_{4}}\right] \\
& =-\operatorname{Sexm}^{2} \times \operatorname{Pn}(C)[P n(T)+P n(S)+P n(B)+P n(L)] \\
& \frac{1}{\partial \beta_{4}}\left[\frac{1}{P n(S)}\left(\frac{\partial P n(S)}{\partial \beta_{4}}\right)\right]=-\operatorname{Sexm}\left[\frac{\partial P n(C)}{\partial \beta_{4}}\right] \\
& =-\operatorname{Sexm}^{2} \times \operatorname{Pn}(C)[\operatorname{Pn}(T)+P n(S)+P n(B)+P n(L)] \\
& \frac{1}{\partial \beta_{4}}\left[\frac{1}{\operatorname{Pn}(B)}\left(\frac{\partial \operatorname{Pn}(B)}{\partial \beta_{4}}\right)\right]=-\operatorname{Sexm}\left[\frac{\partial \operatorname{Pn}(C)}{\partial \beta_{4}}\right] \\
& =-\operatorname{Sexm}^{2} \times P n(C)[P n(T)+P n(S)+P n(B)+P n(L)] \\
& \frac{1}{\partial \beta_{4}}\left[\frac{1}{P n(L)}\left(\frac{\partial P n(L)}{\partial \beta_{4}}\right)\right]=-\operatorname{Sexm}\left[\frac{\partial P n(C)}{\partial \beta_{4}}\right] \\
& =-\operatorname{Sexm}^{2} \times P n(C)[P n(T)+P n(S)+P n(B)+P n(L)] \\
& \frac{\partial P n(C)}{\partial \beta_{4}}=\frac{\operatorname{Sexm} \times e^{V(\text { Car })}\left(e^{V(\text { Car })}+e^{V(\text { Taxi })}+e^{V(\text { Subway })}+e^{V(\text { Bus })}+e^{V(\text { Limousin e })}\right)-e^{V(\text { (Car })}\left(\text { Sexm } \times e^{V(\text { Car })}\right)}{\left(e^{V(\text { Car })}+e^{V(\text { (Taxi })}+e^{V(\text { Subway })}+e^{V(\text { Bus })}+e^{V(\text { Limousin e) })}\right)^{2}} \\
& =\frac{\operatorname{Sexm} \times e^{V(\text { Car })}\left(e^{V(\text { Taxi })}+e^{V(\text { Subway })}+e^{V(\text { Bus })}+e^{V(\text { Limousin } e)}\right)}{\left(e^{V(\text { Car })}+e^{V(\text { Taxi) }}+e^{V(\text { Subway })}+e^{V(\text { Bus })}+e^{V(\text { Limousin } e)}\right)^{2}} \\
& =\operatorname{Sexm} \times \operatorname{Pn}(C)[P n(T)+P n(S)+P n(B)+P n(L)] \\
& \frac{\partial P n(T)}{\partial \beta_{4}}=\frac{0-e^{V(\text { Taxi })}\left(\text { Sexm } \times e^{V(\text { Car })}\right)}{\left(e^{V(\text { Car })}+e^{V(\text { Taxi })}+e^{V(\text { Subway })}+e^{V(\text { Bus })}+e^{V(\text { Limousin } e)}\right)^{2}} \\
& =-\operatorname{Sexm} \times \operatorname{Pn}(T) \times \operatorname{Pn}(C) \\
& \frac{\partial P n(S)}{\partial \beta_{4}}=\frac{0-e^{V(\text { Subway })}\left(\operatorname{Sexm} \times e^{V(\text { Car })}\right)}{\left(e^{V(\text { Car })}+e^{V(\text { Taxi })}+e^{V(\text { Subway })}+e^{V(\text { Bus })}+e^{V(\text { Limousine })}\right)^{2}} \\
& =-\operatorname{Sexm} \times \operatorname{Pn}(S) \times \operatorname{Pn}(C)
\end{aligned}
$$




$$
\begin{aligned}
\frac{\partial P n(B)}{\partial \beta_{4}} & =\frac{0-e^{V(\text { Bus })}\left(\operatorname{Sexm} \times e^{V(\text { Car })}\right)}{\left(e^{V(\text { Car })}+e^{V(\text { Taxi })}+e^{V(\text { Subway })}+e^{V(\text { Bus })}+e^{V(\text { Limousine })}\right)^{2}} \\
& =-\operatorname{Sexm} \times \operatorname{Pn}(B) \times P n(C) \\
\frac{\partial P n(L)}{\partial \beta_{4}} & =\frac{0-e^{V(\text { Limousine })}\left(\text { Sexm } \times e^{V(\text { Car })}\right)}{\left(e^{V(\text { Car })}+e^{V(\text { Taxi })}+e^{V(\text { Subway })}+e^{V(\text { Bus })}+e^{V(\text { Limousine })}\right)^{2}} \\
& =-\operatorname{Sexm} \times \operatorname{Pn}(L) \times \operatorname{Pn}(C)
\end{aligned}
$$

27) The second order partial derivative with respect to $\beta_{4} \beta_{5}$ is

$$
\begin{gathered}
\frac{\partial^{2} L L\left(\beta_{1}, \beta_{2}, \cdots, \beta_{8}, \beta_{9}\right)}{\partial \beta_{5} \partial \beta_{4}}=\sum_{n=l}^{N}\left[y_{C n} \frac{\partial}{\partial \beta_{5}}\left[\frac{1}{P n\left(C_{h}\right.}\left(\frac{\partial P n\left(C_{h}\right.}{\partial \beta_{4}}\right)\right]+y_{T n} \frac{\partial}{\partial \beta_{5}}\left[\frac{1}{P n\left(T_{h}\right.}\left(\frac{\partial P n\left(T_{h}\right.}{\partial \beta_{4}}\right)\right]+y_{S n} \frac{\partial}{\partial \beta_{5}}\left[\frac{1}{P n\left(S_{h}\right.}\left(\frac{\partial P n\left(S_{h}\right.}{\partial \beta_{4}}\right)\right]+\right. \\
\left.y_{B n} \frac{\partial}{\partial \beta_{5}}\left[\frac{1}{P n\left(B B_{h}\right.}\left(\frac{\partial P n\left(B_{h}\right.}{\partial \beta_{4}}\right)\right]+y_{L n} \frac{\partial}{\partial \beta_{5}}\left[\frac{1}{P n(L h}\left(\frac{\partial P n(L h}{\partial \beta_{4}}\right)\right]\right]
\end{gathered}
$$

where

$$
\begin{aligned}
& \frac{1}{\operatorname{Pn}(C)}\left(\frac{\partial \operatorname{Pn}(C)}{\partial \beta_{4}}\right)=\frac{\operatorname{Sexm}\left(e^{V(\text { Taxi })}+e^{V(\text { Subway })}+e^{V(\text { Bus })}+e^{V(\text { Limousin } e)}\right)}{\left(e^{V(\text { Car })}+e^{V(\text { Taxi })}+e^{V(\text { Subway })}+e^{V(\text { Bus })}+e^{V(\text { Limousin } e)}\right)} \\
& =\operatorname{Sexm}[\operatorname{Pn}(T)+\operatorname{Pn}(S)+\operatorname{Pn}(B)+\operatorname{Pn}(L)] \\
& \frac{1}{\partial \beta_{5}}\left[\frac{1}{P n(C)}\left(\frac{\partial P n(C)}{\partial \beta_{4}}\right)\right]=\operatorname{Sexm}\left[\frac{\partial P n(T)}{\partial \beta_{5}}+\frac{\partial P n(S)}{\partial \beta_{5}}+\frac{\partial P n(B)}{\partial \beta_{5}}+\frac{\partial P n(L)}{\partial \beta_{5}}\right] \\
& =\text { Sexm I -Age340Pn(C)Pn(T) - Age340Pn(C)Pn(S) } \\
& \text { - Age340Pn }(C) P n(B)-A g e 340 P n(C) P n(L)] \\
& =-\operatorname{Sexm} \times A g e 340 \times P n(C)[P n(T)+P n(S)+P n(B)+P n(L)] \\
& \frac{1}{\partial \beta_{5}}\left[\frac{1}{P n(T)}\left(\frac{\partial P n(T)}{\partial \beta_{4}}\right)\right]=-\operatorname{Sexm}\left[\frac{\partial P n(C)}{\partial \beta_{5}}\right] \\
& =-\operatorname{Sexm} \times A g e 340 \times P n(C)[P n(T)+P n(S)+P n(B)+P n(L)] \\
& \frac{1}{\partial \beta_{5}}\left[\frac{1}{P n(S)}\left(\frac{\partial P n(S)}{\partial \beta_{4}}\right)\right]=-\operatorname{Sexm}\left[\frac{\partial P n(C)}{\partial \beta_{5}}\right] \\
& =-\operatorname{Sexm} \times A g e 340 \times P n(C)[P n(T)+P n(S)+P n(B)+P n(L)]
\end{aligned}
$$




$$
\begin{aligned}
& \frac{1}{\partial \beta_{5}}\left[\frac{1}{P n(B)}\left(\frac{\partial P n(B)}{\partial \beta_{4}}\right)\right]=-\operatorname{Sexm}\left[\frac{\partial P n(C)}{\partial \beta_{5}}\right] \\
& =-\operatorname{Sexm} \times \operatorname{Age} 340 \times P n(C)[P n(T)+P n(S)+P n(B)+P n(L)] \\
& \frac{1}{\partial \beta_{5}}\left[\frac{1}{P n(L)}\left(\frac{\partial P n(L)}{\partial \beta_{4}}\right)\right]=-\operatorname{Sexm}\left[\frac{\partial P n(C)}{\partial \beta_{5}}\right] \\
& =-\operatorname{Sexm} \times A g e 340 \times P n(C)[P n(T)+P n(S)+P n(B)+P n(L)] \\
& \frac{\partial P n(C)}{\partial \beta_{5}}=A g e 340 \times P n(C)[P n(T)+P n(S)+P n(B)+P n(L)] \\
& \frac{\partial P n(T)}{\partial \beta_{5}}=-\operatorname{Age} 340 P n(C) P n(T) \\
& \frac{\partial P n(S)}{\partial \beta_{5}}=-A g e 340 P n(C) P n(S) \\
& \frac{\partial P n(B)}{\partial \beta_{5}}=-A g e 340 P n(C) P n(B) \\
& \frac{\partial P n(L)}{\partial \beta_{5}}=-A g e 340 P n(C) P n(L)
\end{aligned}
$$

27) The second order partial derivative with respect to $\beta_{4} \beta_{6}$ is

$$
\begin{aligned}
\frac{\partial^{2} L L\left(\beta_{l}, \beta_{2}, \cdots, \beta_{8}, \beta_{9}\right)}{\partial \beta_{6} \partial \beta_{4}}= & \sum_{n=1}^{N}\left[y_{C n} \frac{\partial}{\partial \beta_{6}}\left[\frac{1}{P n\left(C_{h}\right.}\left(\frac{\partial P n\left(C_{h}\right.}{\partial \beta_{4}}\right)\right]+y_{T n} \frac{\partial}{\partial \beta_{6}}\left[\frac{1}{P n\left(T_{h}\right.}\left(\frac{\partial P_{n}\left(T_{h}\right.}{\partial \beta_{4}}\right)\right]+y_{S n} \frac{\partial}{\partial \beta_{6}}\left[\frac{1}{P n\left(S_{h}\right.}\left(\frac{\partial P n\left(S_{h}\right.}{\partial \beta_{4}}\right)\right]+\right. \\
& \left.y_{B n} \frac{\partial}{\partial \beta_{6}}\left[\frac{1}{P n(B h}\left(\frac{\partial P n\left(B_{h}\right.}{\partial \beta_{4}}\right)\right]+y_{L n} \frac{\partial}{\partial \beta_{6}}\left[\frac{1}{P n(L h}\left(\frac{\partial P n(L)_{h}}{\partial \beta_{4}}\right)\right]\right]
\end{aligned}
$$

where

$$
\begin{aligned}
\frac{1}{P n(C)}\left(\frac{\partial P n(C)}{\partial \beta_{4}}\right) & =\frac{\operatorname{Sexm}\left(e^{V(\text { Taxi })}+e^{V(\text { Subway })}+e^{V(\text { Bus })}+e^{V(\text { Limousine })}\right)}{\left(e^{V(\text { Car })}+e^{V(\text { Taxi })}+e^{V(\text { Subway })}+e^{V(\text { Bus })}+e^{V(\text { Limousine })}\right)} \\
& =\operatorname{Sexm}[\operatorname{Pn}(T)+\operatorname{Pn}(S)+\operatorname{Pn}(B)+\operatorname{Pn}(L)]
\end{aligned}
$$




$$
\begin{aligned}
& \frac{1}{\partial \beta_{6}}\left[\frac{1}{P n(C)}\left(\frac{\partial P n(C)}{\partial \beta_{4}}\right)\right]=\operatorname{Sexm}\left[\frac{\partial P n(T)}{\partial \beta_{6}}+\frac{\partial P n(S)}{\partial \beta_{6}}+\frac{\partial P n(B)}{\partial \beta_{6}}+\frac{\partial P n(L)}{\partial \beta_{6}}\right] \\
& =\operatorname{Sexm}[\operatorname{Pn}(T \gamma P n(C)+P n(S)+P n(B)+P n(L))-P n(S) P n(T) \\
& \text { - } P n(B) P n(T)-P n(L) P n(T) \\
& =\operatorname{Sexm} \times \operatorname{Pn}(T) \times \operatorname{Pn}(C) \\
& \frac{1}{\partial \beta_{6}}\left[\frac{1}{P n(T)}\left(\frac{\partial P n(T)}{\partial \beta_{4}}\right)\right]=-\operatorname{Sexm}\left[\frac{\partial P n(C)}{\partial \beta_{6}}\right] \\
& =\operatorname{Sexm} \times \operatorname{Pn}(C) \times \operatorname{Pn}(T) \\
& \frac{1}{\partial \beta_{6}}\left[\frac{1}{P n(S)}\left(\frac{\partial P n(S)}{\partial \beta_{4}}\right)\right]=-\operatorname{Sexm}\left[\frac{\partial P n(C)}{\partial \beta_{6}}\right] \\
& =\operatorname{Sexm} \times \operatorname{Pn}(C) \times \operatorname{Pn}(T) \\
& \frac{1}{\partial \beta_{6}}\left[\frac{1}{P n(B)}\left(\frac{\partial P n(B)}{\partial \beta_{4}}\right)\right]=-\operatorname{Sexm}\left[\frac{\partial P n(C)}{\partial \beta_{6}}\right] \\
& =\operatorname{Sexm} \times \operatorname{Pn}(C) \times \operatorname{Pn}(T) \\
& \frac{1}{\partial \beta_{6}}\left[\frac{1}{\operatorname{Pn}(L)}\left(\frac{\partial \operatorname{Pn}(L)}{\partial \beta_{4}}\right)\right]=-\operatorname{Sexm}\left[\frac{\partial \operatorname{Pn}(C)}{\partial \beta_{6}}\right] \\
& =\operatorname{Sexm} \times \operatorname{Pn}(C) \times \operatorname{Pn}(T) \\
& \frac{\partial P n(C)}{\partial \beta_{6}}=-P n(C) P n(T) \\
& \frac{\partial P n(T)}{\partial \beta_{6}}=P n(T)(P n(C)+P n(S)+P n(B)+P n(L)) \\
& \frac{\partial P n(S)}{\partial \beta_{6}}=-P n(S) P n(T) \\
& \frac{\partial P n(B)}{\partial \beta_{6}}=-\operatorname{Pn}(B) \operatorname{Pn}(T) \\
& \frac{\partial P n(L)}{\partial \beta_{6}}=-P n(L) P n(T)
\end{aligned}
$$

28) The second order partial derivative with respect to $\beta_{4} \beta_{7}$ is 


$$
\begin{gathered}
\frac{\partial^{2} L L\left(\beta_{1}, \beta_{2}, \cdots, \beta_{8}, \beta_{9}\right)}{\partial \beta_{7} \partial \beta_{4}}=\sum_{n=1}^{N}\left[y_{C n} \frac{\partial}{\partial \beta_{7}}\left[\frac{1}{P n\left(C_{h}\right.}\left(\frac{\partial P n\left(C_{h}\right.}{\partial \beta_{4}}\right)\right]+y_{T n} \frac{\partial}{\partial \beta_{7}}\left[\frac{1}{P n(T)_{n}}\left(\frac{\partial P n\left(T_{h}\right.}{\partial \beta_{4}}\right)\right]+y_{S n} \frac{\partial}{\partial \beta_{7}}\left[\frac{1}{P n\left(S_{h}\right.}\left(\frac{\partial P n\left(S_{h}\right.}{\partial \beta_{4}}\right)\right]+\right. \\
y_{B n} \frac{\partial}{\partial \beta_{7}}\left[\frac{1}{P n(B)_{h}}\left(\frac{\partial P n\left(B_{h}\right.}{\partial \beta_{4}}\right)\right]+y_{L n} \frac{\partial}{\partial \beta_{7}}\left[\frac{1}{P n\left(L L_{h}\right.}\left(\frac{\partial P n\left(L h_{h}\right.}{\partial \beta_{4}}\right)\right] J
\end{gathered}
$$

where

$$
\begin{aligned}
& \frac{1}{P n(C)}\left(\frac{\partial P n(C)}{\partial \beta_{4}}\right)=\frac{\operatorname{Sexm}\left(e^{V(\text { Taxi })}+e^{V(\text { Subway })}+e^{V(\text { Bus })}+e^{V(\text { Limousine })}\right)}{\left(e^{V(\text { Car })}+e^{V(\text { Taxi })}+e^{V(\text { Subway })}+e^{V(\text { Bus })}+e^{V(\text { Limousine })}\right)} \\
& =\operatorname{Sexm}[\operatorname{Pn}(T)+\operatorname{Pn}(S)+\operatorname{Pn}(B)+\operatorname{Pn}(L)] \\
& \frac{1}{\partial \beta_{7}}\left[\frac{1}{P n(C)}\left(\frac{\partial P n(C)}{\partial \beta_{4}}\right)\right]=\operatorname{Sexm}\left[\frac{\partial P n(T)}{\partial \beta_{7}}+\frac{\partial P n(S)}{\partial \beta_{7}}+\frac{\partial P n(B)}{\partial \beta_{7}}+\frac{\partial P n(L)}{\partial \beta_{7}}\right] \\
& =\operatorname{Sexm}[-P n(T) P n(S)+P n(S)[P n(C)+P n(T)+P n(B)+P n(L) \\
& \text { - } P n(B) P n(S)-P n(L) P n(S)] \\
& =\operatorname{Sexm} \times \operatorname{Pn}(S) \times \operatorname{Pn}(C) \\
& \frac{1}{\partial \beta_{7}}\left[\frac{1}{P n(T)}\left(\frac{\partial P n(T)}{\partial \beta_{4}}\right)\right]=-\operatorname{Sexm}\left[\frac{\partial P n(C)}{\partial \beta_{7}}\right] \\
& =\operatorname{Sexm} \times \operatorname{Pn}(S) \times \operatorname{Pn}(C) \\
& \frac{1}{\partial \beta_{7}}\left[\frac{1}{P n(S)}\left(\frac{\partial P n(S)}{\partial \beta_{4}}\right)\right]=-\operatorname{Sexm}\left[\frac{\partial P n(C)}{\partial \beta_{7}}\right] \\
& =\operatorname{Sexm} \times \operatorname{Pn}(S) \times \operatorname{Pn}(C) \\
& \frac{1}{\partial \beta_{7}}\left[\frac{1}{P n(B)}\left(\frac{\partial P n(B)}{\partial \beta_{4}}\right)\right]=-\operatorname{Sexm}\left[\frac{\partial P n(C)}{\partial \beta_{7}}\right] \\
& =\operatorname{Sexm} \times \operatorname{Pn}(S) \times \operatorname{Pn}(C) \\
& \frac{1}{\partial \beta_{7}}\left[\frac{1}{P n(L)}\left(\frac{\partial P n(L)}{\partial \beta_{4}}\right)\right]=-\operatorname{Sexm}\left[\frac{\partial P n(C)}{\partial \beta_{7}}\right] \\
& =\operatorname{Sexm} \times \operatorname{Pn}(S) \times P n(C) \\
& \frac{\partial P n(C)}{\partial \beta_{7}}=-P n(S) P n(C) \\
& \frac{\partial P n(T)}{\partial \beta_{7}}=-P n(T) P n(S)
\end{aligned}
$$




$$
\begin{aligned}
& \frac{\partial P n(S)}{\partial \beta_{7}}=P n(S)[P n(C)+P n(T)+P n(B)+P n(L)] \\
& \frac{\partial P n(B)}{\partial \beta_{7}}=-P n(B) P n(S) \\
& \frac{\partial P n(L)}{\partial \beta_{7}}=-P n(L) P n(S)
\end{aligned}
$$

29) The second order partial derivative with respect to $\beta_{4} \beta_{8}$ is

$$
\begin{aligned}
\frac{\partial^{2} L L\left(\beta_{1}, \beta_{2}, \cdots, \beta_{8}, \beta_{g}\right)}{\partial \beta_{8} \partial \beta_{4}}= & \sum_{n=1}^{N}\left[y_{C n} \frac{\partial}{\partial \beta_{8}}\left[\frac{1}{P n\left(C_{h}\right.}\left(\frac{\partial P n\left(C_{h}\right.}{\partial \beta_{4}}\right)\right]+y_{T n} \frac{\partial}{\partial \beta_{8}}\left[\frac{1}{P n\left(T_{h}\right.}\left(\frac{\partial P n\left(T_{h}\right.}{\partial \beta_{4}}\right)\right]+y_{S n} \frac{\partial}{\partial \beta_{8}}\left[\frac{1}{P n\left(S_{h}\right.}\left(\frac{\partial P n\left(S_{h}\right.}{\partial \beta_{4}}\right)\right]+\right. \\
& \left.y_{B n} \frac{\partial}{\partial \beta_{8}}\left[\frac{1}{P n\left(B_{h}\right.}\left(\frac{\partial P n\left(B_{h}\right.}{\partial \beta_{4}}\right)\right]+y_{L n} \frac{\partial}{\partial \beta_{8}}\left[\frac{1}{P n\left(L_{h}\right.}\left(\frac{\partial P n\left(L_{h}\right.}{\partial \beta_{4}}\right)\right]\right]
\end{aligned}
$$

where

$$
\begin{aligned}
\frac{1}{\operatorname{Pn}(C)}\left(\frac{\partial P n(C)}{\partial \beta_{4}}\right) & =\frac{\operatorname{Sexm}\left(e^{V(\text { Taxi })}+e^{V(\text { Subway })}+e^{V(\text { Bus })}+e^{V(\text { Limousine })}\right)}{\left(e^{V(\text { Car })}+e^{V(\text { Taxi })}+e^{V(\text { Subway })}+e^{V(\text { Bus })}+e^{V(\text { Limousine })}\right)} \\
& =\operatorname{Sexm}[\operatorname{Pn}(T)+\operatorname{Pn}(S)+\operatorname{Pn}(B)+\operatorname{Pn}(L)]
\end{aligned}
$$$$
\begin{aligned}
\frac{1}{\partial \beta_{8}}\left[\frac{1}{\operatorname{Pn}(C)}\left(\frac{\partial P n(C)}{\partial \beta_{4}}\right)\right] & =\operatorname{Sexm}\left[\frac{\partial P n(T)}{\partial \beta_{8}}+\frac{\partial P n(S)}{\partial \beta_{8}}+\frac{\partial P n(B)}{\partial \beta_{8}}+\frac{\partial P n(L)}{\partial \beta_{8}}\right] \\
& =\operatorname{Sexm}[-\operatorname{Pn}(T) \operatorname{Pn}(B)-\operatorname{Pn}(S) \operatorname{Pn}(B)+\operatorname{Pn}(B)[\operatorname{Pn}(C)+\operatorname{Pn}(T) \\
& +\operatorname{Pn}(S)+\operatorname{Pn}(L)]-\operatorname{Pn}(L) \operatorname{Pn}(B)] \\
& =\operatorname{Sexm} \times \operatorname{Pn}(B) \times \operatorname{Pn}(C)
\end{aligned}
$$

$$
\begin{aligned}
\frac{1}{\partial \beta_{8}}\left[\frac{1}{\operatorname{Pn}(T)}\left(\frac{\partial P n(T)}{\partial \beta_{4}}\right)\right] & =-\operatorname{Sexm}\left[\frac{\partial \operatorname{Pn}(C)}{\partial \beta_{8}}\right] \\
& =\operatorname{Sexm} \times \operatorname{Pn}(B) \times \operatorname{Pn}(C)
\end{aligned}
$$

$$
\begin{aligned}
\frac{1}{\partial \beta_{8}}\left[\frac{1}{\operatorname{Pn}(S)}\left(\frac{\partial \operatorname{Pn}(S)}{\partial \beta_{4}}\right)\right] & =-\operatorname{Sexm}\left[\frac{\partial \operatorname{Pn}(C)}{\partial \beta_{8}}\right] \\
& =\operatorname{Sexm} \times \operatorname{Pn}(B) \times \operatorname{Pn}(C)
\end{aligned}
$$

$$
\begin{aligned}
\frac{1}{\partial \beta_{8}}\left[\frac{1}{\operatorname{Pn}(B)}\left(\frac{\partial \operatorname{Pn}(B)}{\partial \beta_{4}}\right)\right] & =-\operatorname{Sexm}\left[\frac{\partial \operatorname{Pn}(C)}{\partial \beta_{8}}\right] \\
& =\operatorname{Sexm} \times \operatorname{Pn}(B) \times \operatorname{Pn}(C)
\end{aligned}
$$




$$
\begin{aligned}
\frac{1}{\partial \beta_{8}}\left[\frac{1}{\operatorname{Pn}(L)}\left(\frac{\partial \operatorname{Pn}(L)}{\partial \beta_{4}}\right)\right] & =-\operatorname{Sexm}\left[\frac{\partial \operatorname{Pn}(C)}{\partial \beta_{8}}\right] \\
& =\operatorname{Sexm} \times \operatorname{Pn}(B) \times \operatorname{Pn}(C)
\end{aligned}
$$

$$
\begin{aligned}
& \frac{\partial P n(C)}{\partial \beta_{8}}=-P n(C) P n(B) \\
& \frac{\partial P n(T)}{\partial \beta_{8}}=-P n(T) P n(B) \\
& \frac{\partial P n(S)}{\partial \beta_{8}}=-P n(S) P n(B) \\
& \frac{\partial P n(B)}{\partial \beta_{8}}=P n(B)[P n(C)+P n(T)+P n(S)+P n(L)] \\
& \frac{\partial P n(L)}{\partial \beta_{8}}=-P n(L) P n(B)
\end{aligned}
$$

30) The second order partial derivative with respect to $\beta_{4} \beta_{9}$ is

$$
\begin{aligned}
\frac{\partial^{2} L L\left(\beta_{1}, \beta_{2}, \cdots, \beta_{8}, \beta_{9}\right)}{\partial \beta_{9} \partial \beta_{4}}= & \sum_{n=1}^{N}\left[y_{C n} \frac{\partial}{\partial \beta_{9}}\left[\frac{1}{P n\left(C C_{h}\right.}\left(\frac{\partial P n\left(C_{h}\right.}{\partial \beta_{4}}\right)\right]+y_{y_{n}} \frac{\partial}{\partial \beta_{9}}\left[\frac{1}{P n\left(T_{h}\right.}\left(\frac{\partial P n\left(T_{h}\right)}{\partial \beta_{4}}\right)\right]+y_{S n} \frac{\partial}{\partial \beta_{9}}\left[\frac{1}{P n\left(S_{h}\right)}\left(\frac{\partial P n\left(S_{h}\right)}{\partial \beta_{4}}\right)\right]+\right. \\
& \left.y_{B n} \frac{\partial}{\partial \beta_{9}}\left[\frac{1}{P n\left(B h_{h}\right.}\left(\frac{\partial P n\left(B_{h}\right.}{\partial \beta_{4}}\right)\right]+y_{L n} \frac{\partial}{\partial \beta_{9}}\left[\frac{1}{P n\left(L_{h}\right.}\left(\frac{\partial P n\left(L_{h}\right.}{\partial \beta_{4}}\right)\right]\right]
\end{aligned}
$$

where

$$
\begin{aligned}
& \frac{1}{P n(C)}\left(\frac{\partial P n(C)}{\partial \beta_{4}}\right)=\frac{\operatorname{Sexm}\left(e^{V(\text { Taxi })}+e^{V(\text { Subway })}+e^{V(\text { Bus })}+e^{V(\text { Limousine })}\right)}{\left(e^{V(\text { Car })}+e^{V(\text { Taxi })}+e^{V(\text { Subway })}+e^{V(\text { Bus })}+e^{V(\text { Limousine })}\right)} \\
& =\operatorname{Sexm}[\operatorname{Pn}(T)+P n(S)+P n(B)+P n(L)] \\
& \begin{aligned}
\frac{1}{\partial \beta_{9}}\left[\frac{1}{\operatorname{Pn}(C)}\left(\frac{\partial P n(C)}{\partial \beta_{4}}\right)\right]= & \operatorname{Sexm}\left[\frac{\partial P n(T)}{\partial \beta_{9}}+\frac{\partial P n(S)}{\partial \beta_{9}}+\frac{\partial P n(B)}{\partial \beta_{9}}+\frac{\partial P n(L)}{\partial \beta_{9}}\right] \\
= & \operatorname{Sexm}[-\operatorname{Pn}(L) \operatorname{Pn}(T)-\operatorname{Pn}(L) P n(S)-P n(L) P n(B)+P n(L)[ \\
& P n(C)+\operatorname{Pn}(T)+\operatorname{Pn}(S)+\operatorname{Pn}(B)] \\
= & \operatorname{Sexm} \times \operatorname{Pn}(L) \times \operatorname{Pn}(C)
\end{aligned}
\end{aligned}
$$




$$
\begin{aligned}
& \frac{1}{\partial \beta_{9}}\left[\frac{1}{P n(T)}\left(\frac{\partial P n(T)}{\partial \beta_{4}}\right)\right]=-\operatorname{Sexm}\left[\frac{\partial P n(C)}{\partial \beta_{9}}\right] \\
& =\operatorname{Sexm} \times \operatorname{Pn}(L) \times \operatorname{Pn}(C) \\
& \frac{1}{\partial \beta_{9}}\left[\frac{1}{P n(S)}\left(\frac{\partial P n(S)}{\partial \beta_{4}}\right)\right]=-\operatorname{Sexm}\left[\frac{\partial P n(C)}{\partial \beta_{9}}\right] \\
& =\operatorname{Sexm} \times \operatorname{Pn}(L) \times \operatorname{Pn}(C) \\
& \frac{1}{\partial \beta_{9}}\left[\frac{1}{P n(S)}\left(\frac{\partial P n(S)}{\partial \beta_{4}}\right)\right]=-\operatorname{Sexm}\left[\frac{\partial P n(C)}{\partial \beta_{9}}\right] \\
& =\operatorname{Sexm} \times \operatorname{Pn}(L) \times P n(C) \\
& \frac{1}{\partial \beta_{9}}\left[\frac{1}{P n(B)}\left(\frac{\partial P n(B)}{\partial \beta_{4}}\right)\right]=-\operatorname{Sexm}\left[\frac{\partial P n(C)}{\partial \beta_{9}}\right] \\
& =\operatorname{Sexm} \times \operatorname{Pn}(L) \times \operatorname{Pn}(C) \\
& \frac{1}{\partial \beta_{9}}\left[\frac{1}{P n(L)}\left(\frac{\partial P n(L)}{\partial \beta_{4}}\right)\right]=-\operatorname{Sexm}\left[\frac{\partial P n(C)}{\partial \beta_{9}}\right] \\
& =\operatorname{Sexm} \times \operatorname{Pn}(L) \times \operatorname{Pn}(C) \\
& \frac{\partial P n(C)}{\partial \beta_{9}}=-P n(L) P n(C) \\
& \frac{\partial P n(T)}{\partial \beta_{9}}=-P n(L) P n(T) \\
& \frac{\partial P n(S)}{\partial \beta_{9}}=-P n(L) P n(S) \\
& \frac{\partial P n(B)}{\partial \beta_{9}}=-P n(L) P n(B) \\
& \frac{\partial P n(L)}{\partial \beta_{9}}=P n(L)[P n(C)+P n(T)+P n(S)+P n(B)]
\end{aligned}
$$

31) The second order partial derivative with respect to $\beta_{5} \beta_{5}$ is

$$
\begin{aligned}
\frac{\partial^{2} L L\left(\beta_{1}, \beta_{2}, \cdots, \beta_{8}, \beta_{9}\right)}{\partial \beta_{5} \partial \beta_{5}}= & \sum_{n=1}^{N} l y_{C n} \frac{\partial}{\partial \beta_{5}}\left[\frac{1}{P n\left(C_{h}\right.}\left(\frac{\partial P n\left(C_{h}\right.}{\partial \beta_{5}}\right)\right]+y_{T n} \frac{\partial}{\partial \beta_{5}}\left[\frac{1}{P n\left(T_{h}\right.}\left(\frac{\partial P n(T)_{h}}{\partial \beta_{5}}\right)\right]+y_{S n} \frac{\partial}{\partial \beta_{5}}\left[\frac{1}{P n(S)}\left(\frac{\partial P n\left(S_{h}\right.}{\partial \beta_{5}}\right)\right]+ \\
& \left.y_{B n} \frac{\partial}{\partial \beta_{5}}\left[\frac{1}{P n\left(B_{h}\right.}\left(\frac{\partial P n(B)_{h}}{\partial \beta_{5}}\right)\right]+y_{L n} \frac{\partial}{\partial \beta_{5}}\left[\frac{1}{P n\left(L h_{h}\right.}\left(\frac{\partial P n\left(L_{h}\right.}{\partial \beta_{5}}\right)\right]\right]
\end{aligned}
$$


where

$$
\begin{aligned}
& \frac{1}{P n(C)}\left(\frac{\partial P n(C)}{\partial \beta_{5}}\right)=\frac{A g e 340\left(e^{V(\text { Taxi })}+e^{V(\text { Subway })}+e^{V(\text { Bus })}+e^{V(\text { Limousine })}\right)}{\left(e^{V(\text { Car })}+e^{V(\text { Taxi })}+e^{V(\text { Subway })}+e^{V(\text { Bus })}+e^{V(\text { Limousine })}\right)} \\
& =\operatorname{Age} 340[P n(T)+P n(S)+P n(B)+P n(L)] \\
& \frac{1}{\partial \beta_{5}}\left[\frac{1}{P n(C)}\left(\frac{\partial P n(C)}{\partial \beta_{5}}\right)\right]=A g e 340\left[\frac{\partial P n(T)}{\partial \beta_{5}}+\frac{\partial P n(S)}{\partial \beta_{5}}+\frac{\partial P n(B)}{\partial \beta_{5}}+\frac{\partial P n(L)}{\partial \beta_{5}}\right] \\
& =A g e 340[-A g e 340 P n(C) P n(T)-A g e 340 P n(C) P n(S) \\
& \text { - Age340Pn(C)Pn(B)-Age340Pn(C)Pn(L)] } \\
& =-A g e 340^{2} \operatorname{Pn}(C)[P n(T)+P n(S)+P n(B)+P n(L)] \\
& \frac{1}{\partial \beta_{5}}\left[\frac{1}{\operatorname{Pn}(T)}\left(\frac{\partial P n(T)}{\partial \beta_{5}}\right)\right]=-A g e 340\left[\frac{\partial P n(C)}{\partial \beta_{5}}\right] \\
& =-A g e 340^{2} \times P n(C)[P n(T)+P n(S)+P n(B)+P n(L)] \\
& \frac{1}{\partial \beta_{5}}\left[\frac{1}{P n(S)}\left(\frac{\partial P n(S)}{\partial \beta_{5}}\right)\right]=-A g e 340\left[\frac{\partial P n(C)}{\partial \beta_{5}}\right] \\
& =-A g e 340^{2} \times P n(C)[P n(T)+P n(S)+P n(B)+P n(L)] \\
& \frac{1}{\partial \beta_{5}}\left[\frac{1}{P n(B)}\left(\frac{\partial P n(B)}{\partial \beta_{5}}\right)\right]=-A g e 340\left[\frac{\partial P n(C)}{\partial \beta_{5}}\right] \\
& =-A g e 340^{2} \times P n(C)[P n(T)+P n(S)+P n(B)+P n(L)] \\
& \frac{1}{\partial \beta_{5}}\left[\frac{1}{P n(L)}\left(\frac{\partial P n(L)}{\partial \beta_{5}}\right)\right]=-A g e 340\left[\frac{\partial P n(C)}{\partial \beta_{5}}\right] \\
& =-A g e 340^{2} \times P n(C)[P n(T)+P n(S)+P n(B)+P n(L)] \\
& \frac{\partial P n(C)}{\partial \beta_{5}}=A g e 340 P n(C)[P n(T)+P n(S)+P n(B)+P n(L)] \\
& \frac{\partial P n(T)}{\partial \beta_{5}}=-A g e 340 \operatorname{Pn}(C) \operatorname{Pn}(T) \\
& \frac{\partial P n(S)}{\partial \beta_{5}}=-A g e 340 P n(C) P n(S) \\
& \frac{\partial P n(B)}{\partial \beta_{5}}=-A g e 340 P n(C) P n(B)
\end{aligned}
$$


$\frac{\partial P n(L)}{\partial \beta_{5}}=-\operatorname{Age340Pn}(C) P n(L)$

32) The second order partial derivative with respect to $\beta_{6} \beta_{5}$ is

$$
\begin{aligned}
\frac{\partial^{2} L L\left(\beta_{1}, \beta_{2}, \cdots, \beta_{8}, \beta_{9}\right)}{\partial \beta_{6} \partial \beta_{5}}= & \sum_{n=1}^{N}\left[y_{C n} \frac{\partial}{\partial \beta_{6}}\left[\frac{1}{P n\left(C C_{h}\right.}\left(\frac{\partial P n\left(C_{h}\right.}{\partial \beta_{5}}\right)\right]+y_{T n} \frac{\partial}{\partial \beta_{6}}\left[\frac{1}{P n(T h}\left(\frac{\partial P n(T)_{h}}{\partial \beta_{5}}\right)\right]+y_{S n} \frac{\partial}{\partial \beta_{6}}\left[\frac{1}{P n\left(S_{h}\right.}\left(\frac{\partial P n(S)_{h}}{\partial \beta_{5}}\right)\right]+\right. \\
& \left.y_{B n} \frac{\partial}{\partial \beta_{6}}\left[\frac{1}{P n(B)_{h}}\left(\frac{\partial P n\left(B_{h}\right.}{\partial \beta_{5}}\right)\right]+y_{L n} \frac{\partial}{\partial \beta_{6}}\left[\frac{1}{P n\left(L h_{h}\right.}\left(\frac{\partial P n\left(L_{h}\right.}{\partial \beta_{5}}\right)\right]\right]
\end{aligned}
$$

Where

$$
\begin{aligned}
\frac{1}{P n(C)}\left(\frac{\partial P n(C)}{\partial \beta_{5}}\right)= & \left.\frac{\text { Age } 340\left(e^{V(\text { Taxi })}+e^{V(\text { Subway })}+e^{V(\text { Bus })}+e^{V(\text { Limousine })}\right)}{\left(e^{V(\text { Car })}+e^{V(\text { Taxi })}+e^{V(\text { Subway })}+e^{V(B u s)}+e^{V(\text { Limousine })}\right.}\right) \\
= & A g e 340[P n(T)+P n(S)+P n(B)+P n(L)] \\
\frac{1}{\partial \beta_{6}}\left[\frac{1}{P n(C)}\left(\frac{\partial P n(C)}{\partial \beta_{5}}\right)\right]= & A g e 340\left[\frac{\partial P n(T)}{\partial \beta_{6}}+\frac{\partial P n(S)}{\partial \beta_{6}}+\frac{\partial P n(B)}{\partial \beta_{6}}+\frac{\partial P n(L)}{\partial \beta_{6}}\right] \\
& =A g e 340[P n(T)[P n(C)+P n(S)+P n(B)+P n(L)] \\
& -P n(S) P n(T)-P n(B) P n(T)-P n(L) P n(T)] \\
& =A g e 340 \times P n(T) \times P n(C)
\end{aligned}
$$$$
\frac{1}{\partial \beta_{6}}\left[\frac{1}{P n(T)}\left(\frac{\partial P n(T)}{\partial \beta_{5}}\right)\right]=-A g e 340\left[\frac{\partial P n(C)}{\partial \beta_{6}}\right]
$$$$
=\operatorname{Age} 340 \times P n(T) \times P n(C)
$$

$$
\begin{aligned}
\frac{1}{\partial \beta_{6}}\left[\frac{1}{P n(S)}\left(\frac{\partial P n(S)}{\partial \beta_{5}}\right)\right] & =-A g e 340\left[\frac{\partial P n(C)}{\partial \beta_{6}}\right] \\
& =A g e 340 \times P n(T) \times P n(C)
\end{aligned}
$$

$$
\begin{aligned}
\frac{1}{\partial \beta_{6}}\left[\frac{1}{P n(B)}\left(\frac{\partial P n(B)}{\partial \beta_{5}}\right)\right] & =-A g e 340\left[\frac{\partial P n(C)}{\partial \beta_{6}}\right] \\
& =A g e 340 \times P n(T) \times P n(C)
\end{aligned}
$$

$$
\begin{aligned}
\frac{1}{\partial \beta_{6}}\left[\frac{1}{P n(L)}\left(\frac{\partial P n(L)}{\partial \beta_{5}}\right)\right] & =-A g e 340\left[\frac{\partial P n(C)}{\partial \beta_{6}}\right] \\
& =A g e 340 \times P n(T) \times P n(C)
\end{aligned}
$$




$$
\begin{aligned}
& \frac{\partial P n(C)}{\partial \beta_{6}}=-P n(C) P n(T) \\
& \frac{\partial P n(T)}{\partial \beta_{6}}=P n(T)(P n(C)+P n(S)+P n(B)+P n(L)) \\
& \frac{\partial P n(S)}{\partial \beta_{6}}=-P n(S) P n(T) \\
& \frac{\partial P n(B)}{\partial \beta_{6}}=-P n(B) P n(T) \\
& \frac{\partial P n(L)}{\partial \beta_{6}}=-P n(L) P n(T)
\end{aligned}
$$

33) The second order partial derivative with respect to $\beta_{7} \beta_{5}$ is

$$
\begin{gathered}
\frac{\partial^{2} L L\left(\beta_{1}, \beta_{2}, \cdots, \beta_{8}, \beta_{9}\right)}{\partial \beta_{7} \partial \beta_{5}}=\sum_{n=1}^{N}\left[y_{C n} \frac{\partial}{\partial \beta_{7}}\left[\frac{1}{P n\left(C_{n}\right.}\left(\frac{\partial P n(C)_{n}}{\partial \beta_{5}}\right)\right]+y_{T_{n}} \frac{\partial}{\partial \beta_{7}}\left[\frac{1}{P n(T)_{n}}\left(\frac{\partial P n(T)_{n}}{\partial \beta_{5}}\right)\right]+y_{S n} \frac{\partial}{\partial \beta_{7}}\left[\frac{1}{P n(S)_{h}}\left(\frac{\partial P n\left(S_{h}\right.}{\partial \beta_{5}}\right)\right]+\right. \\
y_{B n} \frac{\partial}{\partial \beta_{7}}\left[\frac{1}{P n\left(B b_{h}\right.}\left(\frac{\partial P n(B)_{n}}{\partial \beta_{5}}\right)\right]+y_{L n} \frac{\partial}{\partial \beta_{7}}\left[\frac{1}{P n\left(L h_{n}\right.}\left(\frac{\partial P n(L)_{n}}{\partial \beta_{5}}\right)\right] I
\end{gathered}
$$

where

$$
\begin{aligned}
& \frac{1}{\operatorname{Pn}(C)}\left(\frac{\partial P n(C)}{\partial \beta_{5}}\right)=\frac{A g e 340\left(e^{V(\text { Taxi })}+e^{V(\text { Subway })}+e^{V(\text { Bus })}+e^{V(\text { Limousine })}\right)}{\left(e^{V(\text { Car })}+e^{V(\text { Taxi })}+e^{V(\text { Subway })}+e^{V(\text { Bus })}+e^{V(\text { Limousine })}\right)} \\
& =\operatorname{Age} 340[P n(T)+P n(S)+P n(B)+P n(L)] \\
& \frac{1}{\partial \beta_{7}}\left[\frac{1}{P n(C)}\left(\frac{\partial P n(C)}{\partial \beta_{5}}\right)\right]=A g e 340\left[\frac{\partial P n(T)}{\partial \beta_{7}}+\frac{\partial P n(S)}{\partial \beta_{7}}+\frac{\partial P n(B)}{\partial \beta_{7}}+\frac{\partial P n(L)}{\partial \beta_{7}}\right] \\
& =A g e 340[-P n(T) P n(S)+P n(S)[P n(C)+P n(T) \\
& +P n(B)+P n(L)]-P n(B) P n(S)-P n(L) P n(S)] \\
& =\operatorname{Age} 340 \times P n(S) \times P n(C)
\end{aligned}
$$

$$
\begin{aligned}
\frac{1}{\partial \beta_{7}}\left[\frac{1}{P n(T)}\left(\frac{\partial P n(T)}{\partial \beta_{5}}\right)\right] & =-A g e 340\left[\frac{\partial P n(C)}{\partial \beta_{7}}\right] \\
& =A g e 340 \times P n(S) \times P n(C) \\
\frac{1}{\partial \beta_{7}}\left[\frac{1}{P n(S)}\left(\frac{\partial P n(S)}{\partial \beta_{5}}\right)\right] & =-A g e 340\left[\frac{\partial P n(C)}{\partial \beta_{7}}\right] \\
& =A g e 340 \times P n(S) \times P n(C)
\end{aligned}
$$




$$
\begin{aligned}
& \frac{1}{\partial \beta_{7}}\left[\frac{1}{P n(B)}\left(\frac{\partial P n(B)}{\partial \beta_{5}}\right)\right]=-A g e 340\left[\frac{\partial P n(C)}{\partial \beta_{7}}\right] \\
& =A g e 340 \times P n(S) \times P n(C) \\
& \frac{1}{\partial \beta_{7}}\left[\frac{1}{P n(L)}\left(\frac{\partial P n(L)}{\partial \beta_{5}}\right)\right]=-A g e 340\left[\frac{\partial P n(C)}{\partial \beta_{7}}\right] \\
& =A g e 340 \times P n(S) \times P n(C) \\
& \frac{\partial P n(C)}{\partial \beta_{7}}=-P n(S) P n(C) \\
& \frac{\partial P n(T)}{\partial \beta_{7}}=-P n(T) P n(S) \\
& \frac{\partial P n(S)}{\partial \beta_{7}}=P n(S)[P n(C)+P n(T)+P n(B)+P n(L)] \\
& \frac{\partial P n(B)}{\partial \beta_{7}}=-P n(B) P n(S) \\
& \frac{\partial P n(L)}{\partial \beta_{7}}=-P n(L) P n(S)
\end{aligned}
$$

34) The second order partial derivative with respect to $\beta_{8} \beta_{5}$ is

$$
\begin{aligned}
\frac{\partial^{2} L L\left(\beta_{l}, \beta_{2}, \cdots, \beta_{8}, \beta_{9}\right)}{\partial \beta_{8} \partial \beta_{5}}= & \sum_{n=1}^{N}\left[y_{C n} \frac{\partial}{\partial \beta_{8}}\left[\frac{1}{P n\left(C_{h}\right.}\left(\frac{\partial P n\left(C_{h}\right.}{\partial \beta_{5}}\right)\right]+y_{n n} \frac{\partial}{\partial \beta_{8}}\left[\frac{1}{P n\left(T_{h}\right.}\left(\frac{\partial P_{n}\left(T_{h}\right.}{\partial \beta_{5}}\right)\right]+y_{S n} \frac{\partial}{\partial \beta_{8}}\left[\frac{1}{P n\left(S_{h}\right.}\left(\frac{\partial P n\left(S_{h}\right.}{\partial \beta_{5}}\right)\right]+\right. \\
& \left.y_{B n} \frac{\partial}{\partial \beta_{8}}\left[\frac{1}{P n(B h}\left(\frac{\partial P n\left(B_{h}\right.}{\partial \beta_{5}}\right)\right]+y_{L n} \frac{\partial}{\partial \beta_{8}}\left[\frac{1}{P n(L h}\left(\frac{\partial P n(L h}{\partial \beta_{5}}\right)\right]\right]
\end{aligned}
$$

where

$$
\begin{aligned}
\frac{1}{P n(C)}\left(\frac{\partial P n(C)}{\partial \beta_{5}}\right)= & \frac{A g e 340\left(e^{V(\text { Taxi })}+e^{V(\text { Subway })}+e^{V(\text { Bus })}+e^{V(\text { Limousine })}\right)}{\left(e^{V(\text { Car })}+e^{V(\text { Taxi })}+e^{V(\text { Subway })}+e^{V(\text { Bus })}+e^{V(\text { Limousine })}\right)} \\
= & A g e 340[\operatorname{Pn}(T)+P n(S)+P n(B)+P n(L)] \\
\frac{1}{\partial \beta_{8}}\left[\frac{1}{P n(C)}\left(\frac{\partial P n(C)}{\partial \beta_{5}}\right)\right]= & A g e 340\left[\frac{\partial P n(T)}{\partial \beta_{8}}+\frac{\partial P n(S)}{\partial \beta_{8}}+\frac{\partial P n(B)}{\partial \beta_{8}}+\frac{\partial P n(L)}{\partial \beta_{8}}\right] \\
= & A g e 340[-\operatorname{Pn}(T) \operatorname{Pn}(B)-\operatorname{Pn}(S) P n(B) \\
& +\operatorname{Pn}(B)[\operatorname{Pn}(C)+\operatorname{Pn}(T)+\operatorname{Pn}(S)+\operatorname{Pn}(L)]-\operatorname{Pn}(L) \operatorname{Pn}(B)] \\
= & A g e 340 \times P n(B) \times P n(C)
\end{aligned}
$$




$$
\begin{gathered}
\frac{1}{\partial \beta_{8}}\left[\frac{1}{P n(T)}\left(\frac{\partial P n(T)}{\partial \beta_{5}}\right)\right]=-A g e 340\left[\frac{\partial P n(C)}{\partial \beta_{8}}\right] \\
=A g e 340 \times P n(B) \times P n(C) \\
\frac{1}{\partial \beta_{8}}\left[\frac{1}{P n(S)}\left(\frac{\partial P n(S)}{\partial \beta_{5}}\right)\right]=-A g e 340\left[\frac{\partial P n(C)}{\partial \beta_{8}}\right] \\
=A g e 340 \times P n(B) \times P n(C) \\
\frac{1}{\partial \beta_{8}}\left[\frac{1}{P n(B)}\left(\frac{\partial P n(B)}{\partial \beta_{5}}\right)\right]=-A g e 340\left[\frac{\partial P n(C)}{\partial \beta_{8}}\right] \\
=A g e 340 \times P n(B) \times P n(C) \\
\frac{1}{\partial \beta_{8}}\left[\frac{1}{P n(L)}\left(\frac{\partial P n(L)}{\partial \beta_{5}}\right)\right]=-A g e 340\left[\frac{\partial P n(C)}{\partial \beta_{8}}\right] \\
=A g e 340 \times P n(B) \times P n(C) \\
\frac{\partial P n(C)}{\partial \beta_{8}}=-P n(C) P n(B) \\
\frac{\partial P n(T)}{\partial \beta_{8}}=-P n(T) P n(B) \\
\frac{\partial P n(S)}{\partial \beta_{8}}=-P n(S) P n(B)
\end{gathered}
$$

35) The second order partial derivative with respect to $\beta_{9} \beta_{5}$ is

$$
\begin{aligned}
\frac{\partial^{2} L L\left(\beta_{1}, \beta_{2}, \cdots, \beta_{8}, \beta_{9}\right)}{\partial \beta_{9} \partial \beta_{5}}= & \sum_{n=1}^{N} I y_{C n} \frac{\partial}{\partial \beta_{9}}\left[\frac{1}{P n(C)}\left(\frac{\partial P n\left(C_{h}\right.}{\partial \beta_{5}}\right)\right]+y_{T n} \frac{\partial}{\partial \beta_{9}}\left[\frac{1}{P n\left(T_{h}\right.}\left(\frac{\partial P n\left(T_{h}\right.}{\partial \beta_{5}}\right)\right]+y_{S n} \frac{\partial}{\partial \beta_{9}}\left[\frac{1}{P n\left(S_{h}\right.}\left(\frac{\partial P n\left(S_{h}\right.}{\partial \beta_{5}}\right)\right]+ \\
& \left.y_{B n} \frac{\partial}{\partial \beta_{9}}\left[\frac{1}{P n\left(B B_{h}\right.}\left(\frac{\partial P n\left(B_{h}\right.}{\partial \beta_{5}}\right)\right]+y_{L n} \frac{\partial}{\partial \beta_{9}}\left[\frac{1}{P n(L h}\left(\frac{\partial P n(L h}{\partial \beta_{5}}\right)\right]\right]
\end{aligned}
$$

where 


$$
\begin{aligned}
& \frac{1}{P n(C)}\left(\frac{\partial P n(C)}{\partial \beta_{5}}\right)=\frac{A g e 340\left(e^{V(\text { Taxi })}+e^{V(\text { Subway })}+e^{V(\text { Bus })}+e^{V(\text { Limousin } e)}\right)}{\left(e^{V(\text { Car })}+e^{V(\text { Taxi })}+e^{V(\text { Subway })}+e^{V(\text { Bus })}+e^{V(\text { Limousin } e)}\right)} \\
& =\operatorname{Age} 340[P n(T)+P n(S)+P n(B)+P n(L)] \\
& \frac{1}{\partial \beta_{9}}\left[\frac{1}{P n(C)}\left(\frac{\partial P n(C)}{\partial \beta_{5}}\right)\right]=A g e 340\left[\frac{\partial P n(T)}{\partial \beta_{9}}+\frac{\partial P n(S)}{\partial \beta_{9}}+\frac{\partial P n(B)}{\partial \beta_{9}}+\frac{\partial P n(L)}{\partial \beta_{9}}\right] \\
& =A g e 340[-P n(L) P n(T)-P n(L) P n(S)-P n(L) P n(B) \\
& +P n(L)[P n(C)+P n(T)+P n(S)+P n(B)]] \\
& =A g e 340 \times P n(L) \times P n(C) \\
& \frac{1}{\partial \beta_{9}}\left[\frac{1}{P n(T)}\left(\frac{\partial P n(T)}{\partial \beta_{5}}\right)\right]=-A g e 340\left[\frac{\partial P n(C)}{\partial \beta_{9}}\right] \\
& =A g e 340 \times P n(L) \times P n(C) \\
& \frac{1}{\partial \beta_{9}}\left[\frac{1}{P n(S)}\left(\frac{\partial P n(S)}{\partial \beta_{5}}\right)\right]=-A g e 340\left[\frac{\partial P n(C)}{\partial \beta_{9}}\right] \\
& =A g e 340 \times P n(L) \times P n(C) \\
& \frac{1}{\partial \beta_{9}}\left[\frac{1}{P n(B)}\left(\frac{\partial P n(B)}{\partial \beta_{5}}\right)\right]=-A g e 340\left[\frac{\partial P n(C)}{\partial \beta_{9}}\right] \\
& =\operatorname{Age} 340 \times P n(L) \times P n(C) \\
& \frac{1}{\partial \beta_{9}}\left[\frac{1}{P n(L)}\left(\frac{\partial P n(L)}{\partial \beta_{5}}\right)\right]=-A g e 340\left[\frac{\partial P n(C)}{\partial \beta_{9}}\right] \\
& =\operatorname{Age} 340 \times P n(L) \times P n(C) \\
& \frac{\partial P n(C)}{\partial \beta_{9}}=-P n(L) P n(C) \\
& \frac{\partial P n(T)}{\partial \beta_{9}}=-P n(L) P n(T) \\
& \frac{\partial P n(S)}{\partial \beta_{9}}=-P n(L) P n(S) \\
& \frac{\partial P n(B)}{\partial \beta_{9}}=-P n(L) P n(B) \\
& \frac{\partial P n(L)}{\partial \beta_{9}}=P n(L)[P n(C)+P n(T)+P n(S)+P n(B)]
\end{aligned}
$$


36) The second order partial derivative with respect to $\beta_{6} \beta_{6}$ is

$$
\begin{aligned}
\frac{\partial^{2} L L\left(\beta_{1}, \beta_{2}, \cdots, \beta_{8}, \beta_{9}\right)}{\partial \beta_{6} \partial \beta_{6}}= & \sum_{n=1}^{N}\left[y_{C n} \frac{\partial}{\partial \beta_{6}}\left[\frac{1}{P n\left(C_{h}\right.}\left(\frac{\partial P n\left(C_{h}\right.}{\partial \beta_{6}}\right)\right]+y_{T n} \frac{\partial}{\partial \beta_{6}}\left[\frac{1}{P n\left(T_{h}\right.}\left(\frac{\partial P n(T)_{h}}{\partial \beta_{6}}\right)\right]+y_{S n} \frac{\partial}{\partial \beta_{6}}\left[\frac{1}{P n\left(S_{h}\right.}\left(\frac{\partial P n\left(S_{h}\right.}{\partial \beta_{6}}\right)\right]+\right. \\
& \left.y_{B n} \frac{\partial}{\partial \beta_{6}}\left[\frac{1}{P n\left(B h_{n}\right.}\left(\frac{\partial P n(B)_{h}}{\partial \beta_{6}}\right)\right]+y_{L n} \frac{\partial}{\partial \beta_{6}}\left[\frac{1}{P n\left(L L_{n}\right.}\left(\frac{\partial P n(L)}{\partial \beta_{h}}\right)\right]\right]
\end{aligned}
$$

where

$$
\begin{aligned}
& \frac{1}{P n(C)}\left(\frac{\partial P n(C)}{\partial \beta_{6}}\right)=\frac{-\left(e^{V(\text { (Taxi })}\right)}{\left(e^{V(\text { Car })}+e^{V(\text { Taxi })}+e^{V(\text { Subway })}+e^{V(\text { Bus })}+e^{V(\text { Limousine })}\right)} \\
& =-P n(T) \\
& \frac{1}{\partial \beta_{6}}\left[\frac{1}{P n(C)}\left(\frac{\partial P n(C)}{\partial \beta_{6}}\right)\right]=-\frac{\partial P n(T)}{\partial \beta_{6}} \\
& =-P n(T)[P n(C)+P n(S)+P n(B)+P n(L)] \\
& \frac{1}{\partial \beta_{6}}\left[\frac{1}{P n(T)}\left(\frac{\partial P n(T)}{\partial \beta_{6}}\right)\right]=\left[\frac{\partial P n(C)}{\partial \beta_{6}}+\frac{\partial P n(S)}{\partial \beta_{6}}+\frac{\partial P n(B)}{\partial \beta_{6}}+\frac{\partial P n(L)}{\partial \beta_{6}}\right] \\
& =[-P n(C) P n(T)-P n(S) P n(T)-P n(B) P n(T)-P n(L) P n(T] \\
& =-P n(T)[P n(G+P n(S)+P n(B)+P n(L)] \\
& \frac{1}{\partial \beta_{6}}\left[\frac{1}{P n(S)}\left(\frac{\partial P n(S)}{\partial \beta_{6}}\right)\right]=-\frac{\partial P n(T)}{\partial \beta_{6}} \\
& =-P n(T)[P n(C)+P n(S)+P n(B)+P n(L)] \\
& \frac{1}{\partial \beta_{6}}\left[\frac{1}{P n(B)}\left(\frac{\partial P n(B)}{\partial \beta_{6}}\right)\right]=-\frac{\partial P n(T)}{\partial \beta_{6}} \\
& =-P n(T)[P n(C)+P n(S)+P n(B)+P n(L)] \\
& \frac{1}{\partial \beta_{6}}\left[\frac{1}{P n(L)}\left(\frac{\partial P n(L)}{\partial \beta_{6}}\right)\right]=-\frac{\partial P n(T)}{\partial \beta_{6}} \\
& =-P n(T)[P n(C)+P n(S)+P n(B)+P n(L)] \\
& \frac{\partial P n(C)}{\partial \beta_{6}}=-P n(C) P n(T)
\end{aligned}
$$




$$
\begin{aligned}
& \frac{\partial P n(T)}{\partial \beta_{6}}=P n(T)[P n(C)+P n(S)+P n(B)+P n(L)] \\
& \frac{\partial P n(S)}{\partial \beta_{6}}=-P n(S) P n(T) \\
& \frac{\partial P n(B)}{\partial \beta_{6}}=-P n(B) P n(T) \\
& \frac{\partial P n(L)}{\partial \beta_{6}}=-P n(L) P n(T)
\end{aligned}
$$

37) The second order partial derivative with respect to $\beta_{6} \beta_{7}$ is

$$
\begin{aligned}
\frac{\partial^{2} L L\left(\beta_{l}, \beta_{2}, \cdots, \beta_{8}, \beta_{9}\right)}{\partial \beta_{7} \partial \beta_{6}}= & \sum_{n=1}^{N} l y_{C n} \frac{\partial}{\partial \beta_{7}}\left[\frac{1}{P n\left(C_{h}\right.}\left(\frac{\partial P_{n}\left(C_{h}\right.}{\partial \beta_{6}}\right)\right]+y_{T n} \frac{\partial}{\partial \beta_{7}}\left[\frac{1}{P n\left(T_{h}\right.}\left(\frac{\partial P_{n}\left(T_{h}\right.}{\partial \beta_{6}}\right)\right]+y_{S n} \frac{\partial}{\partial \beta_{7}}\left[\frac{1}{P n\left(S_{h}\right.}\left(\frac{\partial P n\left(S_{h}\right.}{\partial \beta_{6}}\right)\right]+ \\
& \left.y_{B n} \frac{\partial}{\partial \beta_{7}}\left[\frac{1}{P n(B h}\left(\frac{\partial P n\left(B h_{h}\right.}{\partial \beta_{6}}\right)\right]+y_{L n} \frac{\partial}{\partial \beta_{7}}\left[\frac{1}{P n(L h}\left(\frac{\partial P n\left(L h_{h}\right.}{\partial \beta_{6}}\right)\right]\right]
\end{aligned}
$$

where

$$
\begin{aligned}
\frac{1}{P n(C)}\left(\frac{\partial P n(C)}{\partial \beta_{6}}\right)=\frac{-\left(e^{V(\text { Taxi })}\right)}{\left(e^{V(\text { Car })}+e^{V(\text { Taxi })}+e^{V(\text { Subway })}+e^{V(\text { Bus })}+e^{V(\text { Limousine })}\right)} & =-P n(T) \\
\frac{1}{\partial \beta_{7}}\left[\frac{1}{P n(C)}\left(\frac{\partial P n(C)}{\partial \beta_{6}}\right)\right] & =-\frac{\partial P n(T)}{\partial \beta_{7}} \\
& =P n(T) \times P n(S) \\
& =[-P n(S) P n(C)+P n(S)[P n(C)+P n(T)+P n(B)+P n(L) \\
\frac{1}{\partial \beta_{7}}\left[\frac{1}{P n(T)}\left(\frac{\partial P n(T)}{\partial \beta_{6}}\right)\right]= & {\left[\frac{\partial P n(C)}{\partial \beta_{7}}+\frac{\partial P n(S)}{\partial \beta_{7}}+\frac{\partial P n(B)}{\partial \beta_{7}}+\frac{\partial P n(L)}{\partial \beta_{7}}\right] } \\
= & P n(T) \times P n(S) \\
\frac{1}{\partial \beta_{7}}\left[\frac{1}{P n(S)}\left(\frac{\partial P n(S)}{\partial \beta_{6}}\right)\right] & =-\frac{\partial P n(T)}{\partial \beta_{7}} \\
& =\operatorname{Pn}(T) \times P n(S)
\end{aligned}
$$




$$
\begin{aligned}
\frac{1}{\partial \beta_{7}}\left[\frac{1}{P n(B)}\left(\frac{\partial P n(B)}{\partial \beta_{6}}\right)\right] & =-\frac{\partial P n(T)}{\partial \beta_{7}} \\
& =P n(T) \times P n(S) \\
\frac{1}{\partial \beta_{7}}\left[\frac{1}{P n(L)}\left(\frac{\partial P n(L)}{\partial \beta_{6}}\right)\right] & =-\frac{\partial P n(T)}{\partial \beta_{7}} \\
& =P n(T) \times P n(S)
\end{aligned}
$$

$\frac{\partial P n(C)}{\partial \beta_{7}}=-P n(S) P n(C)$

$\frac{\partial P n(T)}{\partial \beta_{7}}=-P n(T) P n(S)$

$\frac{\partial P n(S)}{\partial \beta_{7}}=P n(S)[P n(C)+P n(T)+P n(B)+P n(L)]$

$\frac{\partial P n(B)}{\partial \beta_{7}}=-P n(B) P n(S)$

$\frac{\partial P n(L)}{\partial \beta_{7}}=-P n(L) P n(S)$

38) The second order partial derivative with respect to $\beta_{6} \beta_{8}$ is

$$
\begin{aligned}
\frac{\partial^{2} L L\left(\beta_{1}, \beta_{2}, \cdots, \beta_{8}, \beta_{9}\right)}{\partial \beta_{8} \partial \beta_{6}}= & \sum_{n=1}^{N} l y_{C n} \frac{\partial}{\partial \beta_{8}}\left[\frac{1}{P n\left(C_{h}\right.}\left(\frac{\partial P n\left(C_{h}\right.}{\partial \beta_{6}}\right)\right]+y_{T n} \frac{\partial}{\partial \beta_{8}}\left[\frac{1}{P n(T h}\left(\frac{\partial P n\left(T_{h}\right.}{\partial \beta_{6}}\right)\right]+y_{S n} \frac{\partial}{\partial \beta_{8}}\left[\frac{1}{P n(S h}\left(\frac{\partial P n\left(S_{h}\right.}{\partial \beta_{6}}\right)\right]+ \\
& y_{B n} \frac{\partial}{\partial \beta_{8}}\left[\frac{1}{P n\left(B h_{h}\right.}\left(\frac{\partial P n\left(B h_{h}\right.}{\partial \beta_{6}}\right)\right]+y_{L n} \frac{\partial}{\partial \beta_{8}}\left[\frac{1}{P n(L h}\left(\frac{\partial P n\left(L_{h}\right.}{\partial \beta_{6}}\right)\right] J
\end{aligned}
$$

where

$$
\begin{aligned}
& \frac{1}{P n(C)}\left(\frac{\partial P n(C)}{\partial \beta_{6}}\right)=\frac{-\left(e^{V(\text { Taxi })}\right)}{\left(e^{V(\text { Car })}+e^{V(\text { Taxi })}+e^{V(\text { Subway })}+e^{V(\text { Bus })}+e^{V(\text { Limousin } e)}\right)} \\
& =-P n(T) \\
& \frac{1}{\partial \beta_{8}}\left[\frac{1}{P n(C)}\left(\frac{\partial P n(C)}{\partial \beta_{6}}\right)\right]=-\frac{\partial P n(T)}{\partial \beta_{8}}=\operatorname{Pn}(T) P n(B)
\end{aligned}
$$




$$
\begin{aligned}
\begin{aligned}
\frac{1}{\partial \beta_{8}}\left[\frac{1}{P n(T)}\left(\frac{\partial P n(T)}{\partial \beta_{6}}\right)\right]= & {\left[\frac{\partial P n(C)}{\partial \beta_{8}}+\frac{\partial P n(S)}{\partial \beta_{8}}+\frac{\partial P n(B)}{\partial \beta_{8}}+\frac{\partial P n(L)}{\partial \beta_{8}}\right] } \\
= & {[-P n(C) P n(B)-P n(S) P n(B)+P n(B)[P n(C)+P n(T)} \\
& +P n(S)+P n(L)]-P n(L) P n(B)] \\
& =P n(T) P n(B)
\end{aligned} \\
\begin{aligned}
\frac{1}{\partial \beta_{8}}\left[\frac{1}{P n(S)}\left(\frac{\partial P n(S)}{\partial \beta_{6}}\right)\right]= & -\frac{\partial P n(T)}{\partial \beta_{8}}=P n(T) P n(B)
\end{aligned} \\
\begin{aligned}
& \frac{1}{\partial \beta_{8}}\left[\frac{1}{P n(B)}\left(\frac{\partial P n(B)}{\partial \beta_{6}}\right)\right]=-\frac{\partial P n(T)}{\partial \beta_{8}}=P n(T) P n(B) \\
& \frac{1}{\partial \beta_{8}}\left[\frac{1}{P n(L)}\left(\frac{\partial P n(L)}{\partial \beta_{6}}\right)\right]=-\frac{\partial P n(T)}{\partial \beta_{8}}=P n(T) P n(B)
\end{aligned} \\
\frac{\partial P n(C)}{\partial \beta_{8}}=-P n(C) P n(B) \\
\frac{\partial P n(T)}{\partial \beta_{8}}=-P n(T) P n(B) \\
\begin{array}{l}
\frac{\partial P n(S)}{\partial \beta_{8}}=-P n(S) P n(B) \\
\frac{\partial P n(B)}{\partial \beta_{8}}=P n(B)[P n(C)+P n(T)+P n(S)+P n(L)]
\end{array} \\
\frac{\partial P n(L)}{\partial \beta_{8}}=-P n(L) P n(B)
\end{aligned}
$$

39) The second order partial derivative with respect to $\beta_{6} \beta_{9}$ is

$$
\begin{aligned}
\frac{\partial^{2} L L\left(\beta_{1}, \beta_{2}, \cdots, \beta_{8}, \beta_{9}\right)}{\partial \beta_{9} \partial \beta_{6}}= & \sum_{n=1}^{N} l y_{C n} \frac{\partial}{\partial \beta_{9}}\left[\frac{1}{P n\left(C_{h}\right.}\left(\frac{\partial P n\left(C_{h}\right.}{\partial \beta_{6}}\right)\right]+y_{T n} \frac{\partial}{\partial \beta_{9}}\left[\frac{1}{P n(T)_{h}}\left(\frac{\partial P n\left(T_{h}\right.}{\partial \beta_{6}}\right)\right]+y_{S n} \frac{\partial}{\partial \beta_{9}}\left[\frac{1}{P n\left(S_{h}\right.}\left(\frac{\partial P n\left(S_{h}\right.}{\partial \beta_{6}}\right)\right]+ \\
& y_{B n} \frac{\partial}{\partial \beta_{9}}\left[\frac{1}{P n\left(B h_{h}\right.}\left(\frac{\partial P n\left(B h_{h}\right.}{\partial \beta_{6}}\right)\right]+y_{L n} \frac{\partial}{\partial \beta_{9}}\left[\frac{1}{P n\left(L_{h}\right.}\left(\frac{\partial P n\left(L h_{h}\right.}{\partial \beta_{6}}\right)\right] J
\end{aligned}
$$

where 


$$
\begin{aligned}
& \frac{1}{P n(C)}\left(\frac{\partial P n(C)}{\partial \beta_{6}}\right)=\frac{-\left(e^{V(\text { Taxi })}\right)}{\left(e^{V(\text { Car })}+e^{V(\text { Taxi })}+e^{V(\text { Subway })}+e^{V(\text { Bus })}+e^{V(\text { Limousine })}\right)} \\
& =-\operatorname{Pn}(T) \\
& \frac{1}{\partial \beta_{9}}\left[\frac{1}{P n(C)}\left(\frac{\partial P n(C)}{\partial \beta_{6}}\right)\right]=-\frac{\partial P n(T)}{\partial \beta_{9}}=P n(L) P n(T) \\
& \frac{1}{\partial \beta_{9}}\left[\frac{1}{P n(T)}\left(\frac{\partial P n(T)}{\partial \beta_{6}}\right)\right]=\left[\frac{\partial P n(C)}{\partial \beta_{9}}+\frac{\partial P n(S)}{\partial \beta_{9}}+\frac{\partial P n(B)}{\partial \beta_{9}}+\frac{\partial P n(L)}{\partial \beta_{9}}\right] \\
& =[-P n(L) P n(C)-P n(L) P n(S)-P n(L) P n(B, \\
& +P n(L)[P n(G+P n(T)+P n(S)+P n(B)]] \\
& =\operatorname{Pn}(L) P n(T) \\
& \frac{1}{\partial \beta_{9}}\left[\frac{1}{P n(S)}\left(\frac{\partial P n(S)}{\partial \beta_{6}}\right)\right]=-\frac{\partial P n(T)}{\partial \beta_{9}}=\operatorname{Pn}(L) P n(T) \\
& \frac{1}{\partial \beta_{9}}\left[\frac{1}{P n(B)}\left(\frac{\partial P n(B)}{\partial \beta_{6}}\right)\right]=-\frac{\partial P n(T)}{\partial \beta_{9}}=P n(L) P n(T) \\
& \frac{1}{\partial \beta_{9}}\left[\frac{1}{P n(L)}\left(\frac{\partial P n(L)}{\partial \beta_{6}}\right)\right]=-\frac{\partial P n(T)}{\partial \beta_{9}}=\operatorname{Pn}(L) P n(T) \\
& \frac{\partial P n(C)}{\partial \beta_{9}}=-P n(L) P n(C) \\
& \frac{\partial P n(T)}{\partial \beta_{9}}=-P n(L) P n(T) \\
& \frac{\partial P n(S)}{\partial \beta_{9}}=-P n(L) P n(S) \\
& \frac{\partial P n(B)}{\partial \beta_{9}}=-P n(L) P n(B) \\
& \frac{\partial P n(L)}{\partial \beta_{9}}=P n(L)[P n(C)+P n(T)+P n(S)+P n(B)]
\end{aligned}
$$

40) The second order partial derivative with respect to $\beta_{7} \beta_{7}$ is 


$$
\begin{aligned}
\frac{\partial^{2} L L\left(\beta_{1}, \beta_{2}, \cdots, \beta_{8}, \beta_{9}\right)}{\partial \beta_{7} \partial \beta_{7}}= & \sum_{n=1}^{N}\left[y_{C n} \frac{\partial}{\partial \beta_{7}}\left[\frac{1}{P n\left(C_{h}\right.}\left(\frac{\partial P n\left(C_{h}\right.}{\partial \beta_{7}}\right)\right]+y_{T n} \frac{\partial}{\partial \beta_{7}}\left[\frac{1}{P n\left(T h_{h}\right.}\left(\frac{\partial P n\left(T_{h}\right.}{\partial \beta_{7}}\right)\right]+y_{S n} \frac{\partial}{\partial \beta_{7}}\left[\frac{1}{P n\left(S_{h}\right.}\left(\frac{\partial P n\left(S_{h}\right.}{\partial \beta_{7}}\right)\right]+\right. \\
& y_{B n} \frac{\partial}{\partial \beta_{7}}\left[\frac{1}{P n\left(B_{h}\right.}\left(\frac{\partial P n\left(B h_{h}\right.}{\partial \beta_{7}}\right)\right]+y_{L n} \frac{\partial}{\partial \beta_{7}}\left[\frac{1}{P n\left(L h_{h}\right.}\left(\frac{\partial P n\left(L_{h}\right.}{\partial \beta_{7}}\right)\right] J
\end{aligned}
$$

where

$$
\begin{aligned}
& \frac{1}{P n(C)}\left(\frac{\partial P n(C)}{\partial \beta_{7}}\right)=\frac{-e^{V(\text { Subway })}}{\left(e^{V(\text { Car })}+e^{V(\text { Taxi })}+e^{V(\text { Subway })}+e^{V(\text { Bus })}+e^{V(\text { Limousine })}\right)} \\
& =-\operatorname{Pn}(S) \\
& \frac{1}{\partial \beta_{7}}\left[\frac{1}{P n(C)}\left(\frac{\partial P n(C)}{\partial \beta_{7}}\right)\right]=-\frac{\partial P n(S)}{\partial \beta_{7}}=-P n(S)[P n(C)+P n(T)+P n(B)+P n(L)] \\
& \frac{1}{\partial \beta_{7}}\left[\frac{1}{P n(T)}\left(\frac{\partial P n(T)}{\partial \beta_{7}}\right)\right]=-\frac{\partial P n(S)}{\partial \beta_{7}}=-P n(S)[P n(C)+P n(T)+P n(B)+P n(L)] \\
& \frac{1}{P n(S)}\left(\frac{\partial P n(S)}{\partial \beta_{7}}\right)=\frac{\left(e^{V(\text { Car })}+e^{V(\text { Taxi })}+e^{V(\text { Bus })}+e^{V(\text { Limousin } e)}\right)}{\left(e^{V(\text { Car })}+e^{V(\text { Taxi })}+e^{V(\text { Subway })}+e^{V(\text { Bus })}+e^{V(\text { Limousin } e)}\right)} \\
& =[P n(C)+P n(T)+P n(B)+P n(L)] \\
& \frac{1}{\partial \beta_{7}}\left[\frac{1}{P n(S)}\left(\frac{\partial P n(S)}{\partial \beta_{7}}\right)\right]=\left[\frac{\partial P n(C)}{\partial \beta_{7}}+\frac{\partial P n(T)}{\partial \beta_{7}}+\frac{\partial P n(B)}{\partial \beta_{7}}+\frac{\partial P n(L)}{\partial \beta_{7}}\right] \\
& =[-\operatorname{Pn}(C) P n(S)-P n(T) P n(S)-P n(B) P n(S)-P n(L) P n(S)] \\
& =-P n(S)[P n(C)+P n(T)+P n(B)+P n(L)] \\
& \frac{1}{\partial \beta_{7}}\left[\frac{1}{P n(B)}\left(\frac{\partial P n(B)}{\partial \beta_{7}}\right)\right]=-\frac{\partial P n(S)}{\partial \beta_{7}}=-P n(S)[P n(C)+P n(T)+P n(B)+P n(L)] \\
& \frac{1}{\partial \beta_{7}}\left[\frac{1}{P n(L)}\left(\frac{\partial P n(L)}{\partial \beta_{7}}\right)\right]=-\frac{\partial P n(S)}{\partial \beta_{7}}=-P n(S)[P n(C)+P n(T)+P n(B)+P n(L)] \\
& \frac{\partial P n(C)}{\partial \beta_{7}}=-P n(C) P n(S) \\
& \frac{\partial P n(T)}{\partial \beta_{7}}=-P n(T) P n(S)
\end{aligned}
$$




$$
\begin{aligned}
& \frac{\partial P n(S)}{\partial \beta_{7}}=P n(S)[P n(C)+P n(T)+P n(B)+P n(L)] \\
& \frac{\partial P n(B)}{\partial \beta_{7}}=-P n(B) P n(S) \\
& \frac{\partial P n(L)}{\partial \beta_{7}}=-P n(L) P n(S)
\end{aligned}
$$

41) The second order partial derivative with respect to $\beta_{7} \beta_{8}$ is

$$
\begin{aligned}
\frac{\partial^{2} L L\left(\beta_{1}, \beta_{2}, \cdots, \beta_{8}, \beta_{9}\right)}{\partial \beta_{8} \partial \beta_{7}}= & \sum_{n=1}^{N}\left[y_{C n} \frac{\partial}{\partial \beta_{8}}\left[\frac{1}{P n\left(C_{h}\right.}\left(\frac{\partial P n\left(C_{h}\right.}{\partial \beta_{7}}\right)\right]+y_{T n} \frac{\partial}{\partial \beta_{8}}\left[\frac{1}{P n(T h}\left(\frac{\partial P n\left(T_{h}\right.}{\partial \beta_{7}}\right)\right]+y_{S n} \frac{\partial}{\partial \beta_{8}}\left[\frac{1}{P n\left(S_{h}\right.}\left(\frac{\partial P n\left(S_{h}\right.}{\partial \beta_{7}}\right)\right]+\right. \\
& \left.y_{B n} \frac{\partial}{\partial \beta_{8}}\left[\frac{1}{P n(B)_{h}}\left(\frac{\partial P n\left(B_{h}\right.}{\partial \beta_{7}}\right)\right]+y_{L n} \frac{\partial}{\partial \beta_{8}}\left[\frac{1}{P n\left(L L_{h}\right.}\left(\frac{\partial P n\left(L h_{h}\right.}{\partial \beta_{7}}\right)\right]\right]
\end{aligned}
$$

where

$$
\begin{aligned}
& \left.\frac{1}{\operatorname{Pn}(C)}\left(\frac{\partial P n(C)}{\partial \beta_{7}}\right)=\frac{-e^{V(\text { Subway })}}{\left(e^{V(\text { Car })}+e^{V(\text { Taxi })}+e^{V(\text { Subway })}+e^{V(\text { Bus })}+e^{V(\text { Limjousine })}\right.}\right) \\
& =-P n(S) \\
& \frac{1}{\partial \beta_{8}}\left[\frac{1}{P n(C)}\left(\frac{\partial P n(C)}{\partial \beta_{7}}\right)\right]=-\frac{\partial P n(S)}{\partial \beta_{8}}=\operatorname{Pn}(S) P n(B) \\
& \frac{1}{\partial \beta_{8}}\left[\frac{1}{P n(T)}\left(\frac{\partial P n(T)}{\partial \beta_{7}}\right)\right]=-\frac{\partial P n(S)}{\partial \beta_{8}}=\operatorname{Pn}(S) P n(B) \\
& \frac{1}{\partial \beta_{8}}\left[\frac{1}{P n(S)}\left(\frac{\partial P n(S)}{\partial \beta_{7}}\right)\right]=\left[\frac{\partial P n(C)}{\partial \beta_{8}}+\frac{\partial P n(T)}{\partial \beta_{8}}+\frac{\partial P n(B)}{\partial \beta_{8}}+\frac{\partial P n(L)}{\partial \beta_{8}}\right] \\
& =[-P n(C) P n(B)-P n(T) P n(B) \\
& +P n(B)[P n(C)+P n(T)+P n(S)+P n(L)]-P n(L) P n(B)] \\
& =\operatorname{Pn}(B) \operatorname{Pn}(S) \\
& \frac{I}{\partial \beta_{8}}\left[\frac{I}{P n(L)}\left(\frac{\partial P n(B)}{\partial \beta_{7}}\right)\right]=-\frac{\partial P n(S)}{\partial \beta_{8}}=\operatorname{Pn}(S) P n(B) \\
& \frac{1}{\partial \beta_{8}}\left[\frac{1}{P n(L)}\left(\frac{\partial P n(L)}{\partial \beta_{7}}\right)\right]=-\frac{\partial P n(S)}{\partial \beta_{8}}=\operatorname{Pn}(S) P n(B)
\end{aligned}
$$




$$
\begin{aligned}
& \frac{\partial P n(C)}{\partial \beta_{8}}=-P n(C) P n(B) \\
& \frac{\partial P n(T)}{\partial \beta_{8}}=-P n(T) P n(B) \\
& \frac{\partial P n(S)}{\partial \beta_{8}}=-P n(S) P n(B) \\
& \frac{\partial P n(B)}{\partial \beta_{8}}=\operatorname{Pn}(B)[P n(C)+P n(T)+P n(S)+P n(L)] \\
& \frac{\partial P n(L)}{\partial \beta_{8}}=-P n(L) P n(B)
\end{aligned}
$$

42) The second order partial derivative with respect to $\beta_{7} \beta_{9}$ is

$$
\begin{gathered}
\frac{\partial^{2} L L\left(\beta_{1}, \beta_{2}, \cdots, \beta_{8}, \beta_{9}\right)}{\partial \beta_{9} \partial \beta_{7}}=\sum_{n=l}^{N} l y_{C n} \frac{\partial}{\partial \beta_{9}}\left[\frac{1}{P n\left(C_{h}\right.}\left(\frac{\partial P n\left(C_{h}\right.}{\partial \beta_{7}}\right)\right]+y_{T n} \frac{\partial}{\partial \beta_{9}}\left[\frac{1}{P n\left(T_{h}\right.}\left(\frac{\partial P n\left(T_{h}\right.}{\partial \beta_{7}}\right)\right]+y_{S n} \frac{\partial}{\partial \beta_{9}}\left[\frac{1}{P n\left(S_{h}\right.}\left(\frac{\partial P n\left(S_{h}\right.}{\partial \beta_{7}}\right)\right]+ \\
y_{B n} \frac{\partial}{\partial \beta_{9}}\left[\frac{1}{P n\left(B_{h}\right.}\left(\frac{\partial P n\left(B_{h}\right.}{\partial \beta_{7}}\right)\right]+y_{L n} \frac{\partial}{\partial \beta_{9}}\left[\frac{1}{P n\left(L_{h}\right.}\left(\frac{\partial P n\left(L h_{h}\right.}{\partial \beta_{7}}\right)\right] J
\end{gathered}
$$

where

$$
\begin{aligned}
& \frac{1}{\operatorname{Pn}(C)}\left(\frac{\partial P n(C)}{\partial \beta_{7}}\right)=\frac{-e^{V(\text { Subway })}}{\left(e^{V(\text { Car })}+e^{V(\text { (Taxi })}+e^{V(\text { Subway })}+e^{V(\text { Bus })}+e^{V(\text { Limousin } e)}\right)} \\
& =-\operatorname{Pn}(S) \\
& \frac{1}{\partial \beta_{9}}\left[\frac{1}{P n(C)}\left(\frac{\partial P n(C)}{\partial \beta_{7}}\right)\right]=-\frac{\partial P n(S)}{\partial \beta_{9}}=\operatorname{Pn}(L) P n(S) \\
& \frac{1}{\partial \beta_{9}}\left[\frac{1}{P n(T)}\left(\frac{\partial P n(T)}{\partial \beta_{7}}\right)\right]=-\frac{\partial P n(S)}{\partial \beta_{9}}=\operatorname{Pn}(L) P n(S) \\
& \frac{1}{\partial \beta_{9}}\left[\frac{1}{P n(S)}\left(\frac{\partial P n(S)}{\partial \beta_{7}}\right)\right]=\left[\frac{\partial P n(C)}{\partial \beta_{9}}+\frac{\partial P n(T)}{\partial \beta_{9}}+\frac{\partial P n(B)}{\partial \beta_{9}}+\frac{\partial P n(L)}{\partial \beta_{9}}\right] \\
& =[-P n(L) P n(C)-P n(L) P n(T)-P n(L) P n(B) \\
& +P n(L)[P n(C)+P n(T)+P n(S)+P n(B)]] \\
& =\operatorname{Pn}(L) P n(S)
\end{aligned}
$$




$$
\begin{aligned}
& \frac{1}{\partial \beta_{9}}\left[\frac{1}{P n(B)}\left(\frac{\partial P n(B)}{\partial \beta_{7}}\right)\right]=-\frac{\partial P n(S)}{\partial \beta_{9}}=P n(L) P n(S) \\
& \frac{1}{\partial \beta_{9}}\left[\frac{1}{P n(L)}\left(\frac{\partial P n(L)}{\partial \beta_{7}}\right)\right]=-\frac{\partial P n(S)}{\partial \beta_{9}}=P n(L) P n(S) \\
& \frac{\partial P n(C)}{\partial \beta_{9}}=-P n(L) P n(C) \\
& \frac{\partial P n(T)}{\partial \beta_{9}}=-P n(L) P n(T) \\
& \frac{\partial P n(S)}{\partial \beta_{9}}=-P n(L) P n(S) \\
& \frac{\partial P n(B)}{\partial \beta_{9}}=-P n(L) P n(B) \\
& \frac{\partial P n(L)}{\partial \beta_{9}}=P n(L)[P n(C)+P n(T)+P n(S)+P n(B)]
\end{aligned}
$$

43) The second order partial derivative with respect to $\beta_{8} \beta_{8}$ is

$$
\begin{aligned}
\frac{\partial^{2} L L\left(\beta_{1}, \beta_{2}, \cdots, \beta_{8}, \beta_{9}\right)}{\partial \beta_{8} \partial \beta_{8}}= & \sum_{n=1}^{N}\left[y_{C n} \frac{\partial}{\partial \beta_{8}}\left[\frac{1}{P n(C h}\left(\frac{\partial P n\left(C_{h}\right.}{\partial \beta_{8}}\right)\right]+y_{T n} \frac{\partial}{\partial \beta_{8}}\left[\frac{1}{P n(T h}\left(\frac{\partial P n\left(T_{h}\right.}{\partial \beta_{8}}\right)\right]+y_{S n} \frac{\partial}{\partial \beta_{8}}\left[\frac{1}{P n\left(S_{h}\right.}\left(\frac{\partial P n\left(S_{h}\right.}{\partial \beta_{8}}\right)\right]+\right. \\
& \left.y_{B n} \frac{\partial}{\partial \beta_{8}}\left[\frac{1}{P n(B)}\left(\frac{\partial P n(B h}{\partial \beta_{8}}\right)\right]+y_{L n} \frac{\partial}{\partial \beta_{8}}\left[\frac{1}{P n(L h}\left(\frac{\partial P n\left(L h_{h}\right.}{\partial \beta_{8}}\right)\right]\right]
\end{aligned}
$$

where

$$
\begin{aligned}
& \frac{1}{\partial \beta_{8}}\left[\frac{1}{P n(C)}\left(\frac{\partial P n(C)}{\partial \beta_{8}}\right)\right]=-\frac{\partial P n(B)}{\partial \beta_{8}}=-P n(B)[P n(C)+P n(T)+P n(S)+P n(L)] \\
& \frac{1}{\partial \beta_{8}}\left[\frac{1}{P n(T)}\left(\frac{\partial P n(T)}{\partial \beta_{8}}\right)\right]=-\frac{\partial P n(B)}{\partial \beta_{8}}=-P n(B)[P n(C)+P n(T)+P n(S)+P n(L)] \\
& \frac{1}{\partial \beta_{8}}\left[\frac{1}{P n(S)}\left(\frac{\partial P n(S)}{\partial \beta_{8}}\right)\right]=-\frac{\partial P n(B)}{\partial \beta_{8}}=-P n(B)[P n(C)+P n(T)+P n(S)+P n(L)]
\end{aligned}
$$




$$
\begin{aligned}
& \begin{aligned}
\frac{1}{\partial \beta_{8}}\left[\frac{I}{P n(B)}\left(\frac{\partial P n(B)}{\partial \beta_{8}}\right)\right] & =\left[\frac{\partial P n(C)}{\partial \beta_{8}}+\frac{\partial P n(T)}{\partial \beta_{8}}+\frac{\partial P n(S)}{\partial \beta_{8}}+\frac{\partial P n(L)}{\partial \beta_{8}}\right] \\
& =[-P n(C) P n(B)-P n(T) P n(B)-P n(S) P n(B)-P n(L) P n(B)] \\
& =-P n(B)[P n(C)+P n(T)+P n(S)+P n(L)]
\end{aligned} \\
& \begin{aligned}
\frac{1}{\partial \beta_{8}}\left[\frac{1}{P n(L)}\left(\frac{\partial P n(L)}{\partial \beta_{8}}\right)\right]=-\frac{\partial P n(B)}{\partial \beta_{8}}=-P n(B)[P n(C)+P n(T)+P n(S)+P n(L)] \\
\frac{\partial P n(C)}{\partial \beta_{8}}=-P n(C) P n(B)
\end{aligned} \\
& \frac{\partial P n(T)}{\partial \beta_{8}}=-P n(T) P n(B) \\
& \frac{\partial P n(S)}{\partial \beta_{8}}=-P n(S) P n(B) \\
& \frac{\partial P n(B)}{\partial \beta_{8}}=P n(B)[P n(C)+P n(T)+P n(S)+P n(L)] \\
& \frac{\partial P n(L)}{\partial \beta_{8}}=-P n(L) P n(B)
\end{aligned}
$$

44) The second order partial derivative with respect to $\beta_{8} \beta_{9}$ is

$$
\begin{aligned}
\frac{\partial^{2} L L\left(\beta_{1}, \beta_{2}, \cdots, \beta_{8}, \beta_{9}\right)}{\partial \beta_{9} \partial \beta_{8}}= & \sum_{n=1}^{N} I y_{C n} \frac{\partial}{\partial \beta_{9}}\left[\frac{1}{P n\left(C_{h}\right.}\left(\frac{\partial P n\left(C_{h}\right.}{\partial \beta_{8}}\right)\right]+y_{T n} \frac{\partial}{\partial \beta_{9}}\left[\frac{1}{P n\left(T_{h}\right.}\left(\frac{\partial P n\left(T_{h}\right.}{\partial \beta_{8}}\right)\right]+y_{S n} \frac{\partial}{\partial \beta_{9}}\left[\frac{1}{P n\left(S_{h}\right.}\left(\frac{\partial P n\left(S_{h}\right.}{\partial \beta_{8}}\right)\right]+ \\
& \left.y_{B n} \frac{\partial}{\partial \beta_{9}}\left[\frac{1}{P n\left(B h_{h}\right.}\left(\frac{\partial P n\left(B h_{h}\right.}{\partial \beta_{8}}\right)\right]+y_{L n} \frac{\partial}{\partial \beta_{9}}\left[\frac{1}{P n\left(L L_{h}\right.}\left(\frac{\partial P n\left(L_{h}\right.}{\partial \beta_{8}}\right)\right]\right]
\end{aligned}
$$

where

$$
\begin{aligned}
& \frac{1}{\partial \beta_{9}}\left[\frac{1}{P n(C)}\left(\frac{\partial P n(C)}{\partial \beta_{8}}\right)\right]=-\frac{\partial P n(B)}{\partial \beta_{9}}=P n(L) P n(B) \\
& \frac{1}{\partial \beta_{9}}\left[\frac{1}{P n(T)}\left(\frac{\partial P n(T)}{\partial \beta_{8}}\right)\right]=-\frac{\partial P n(B)}{\partial \beta_{9}}=P n(L) P n(B) \\
& \frac{1}{\partial \beta_{9}}\left[\frac{1}{P n(S)}\left(\frac{\partial P n(S)}{\partial \beta_{8}}\right)\right]=-\frac{\partial P n(B)}{\partial \beta_{9}}=P n(L) P n(B)
\end{aligned}
$$




$$
\begin{aligned}
& \frac{1}{\partial \beta_{9}}\left[\frac{1}{P n(B)}\left(\frac{\partial P n(B)}{\partial \beta_{8}}\right)\right]=\left[\frac{\partial P n(C)}{\partial \beta_{9}}+\frac{\partial P n(T)}{\partial \beta_{9}}+\frac{\partial P n(S)}{\partial \beta_{9}}+\frac{\partial P n(L)}{\partial \beta_{9}}\right] \\
& =[-P n(L) P n(C)-P n(L) P n(T)-P n(L) P n(S) \\
& +P n(L)[P n(C)+P n(T)+P n(S)+P n(B)]] \\
& =P n(L) P n(B) \\
& \frac{1}{\partial \beta_{9}}\left[\frac{1}{P n(L)}\left(\frac{\partial P n(L)}{\partial \beta_{8}}\right)\right]=-\frac{\partial P n(B)}{\partial \beta_{9}}=P n(L) P n(B) \\
& \frac{\partial P n(C)}{\partial \beta_{9}}=-P n(L) P n(C) \\
& \frac{\partial P n(T)}{\partial \beta_{9}}=-P n(L) P n(T) \\
& \frac{\partial P n(S)}{\partial \beta_{9}}=-P n(L) P n(S) \\
& \frac{\partial P n(B)}{\partial \beta_{9}}=-P n(L) P n(B) \\
& \frac{\partial P n(L)}{\partial \beta_{9}}=P n(L)[P n(C)+P n(T)+P n(S)+P n(B)]
\end{aligned}
$$

45) The second order partial derivative with respect to $\beta_{9} \beta_{9}$ is

$$
\begin{aligned}
\frac{\partial^{2} L L\left(\beta_{1}, \beta_{2}, \cdots, \beta_{8}, \beta_{9}\right)}{\partial \beta_{9} \partial \beta_{9}}= & \sum_{n=1}^{N}\left[y_{C n} \frac{\partial}{\partial \beta_{9}}\left[\frac{1}{P n\left(C_{h}\right.}\left(\frac{\partial P n\left(C_{h}\right.}{\partial \beta_{9}}\right)\right]+y_{T n} \frac{\partial}{\partial \beta_{9}}\left[\frac{1}{P n\left(T_{h}\right.}\left(\frac{\partial P_{n}\left(T_{h}\right.}{\partial \beta_{9}}\right)\right]+y_{S n} \frac{\partial}{\partial \beta_{9}}\left[\frac{1}{P n\left(S_{h}\right.}\left(\frac{\partial P n\left(S_{h}\right.}{\partial \beta_{9}}\right)\right]+\right. \\
& \left.y_{B n} \frac{\partial}{\partial \beta_{9}}\left[\frac{1}{P n(B h}\left(\frac{\partial P n(B)_{h}}{\partial \beta_{9}}\right)\right]+y_{L n} \frac{\partial}{\partial \beta_{9}}\left[\frac{1}{P n(L h}\left(\frac{\partial P n\left(L_{h}\right.}{\partial \beta_{9}}\right)\right]\right]
\end{aligned}
$$

where

$$
\begin{aligned}
& \frac{\partial P n(C)}{\partial \beta_{9}}=\frac{O-e^{V(\text { Car })}\left(e^{V(\text { Limousine })}\right)}{\left(e^{V(\text { Car })}+e^{V(\text { Taxi })}+e^{V(\text { Subway })}+e^{V(\text { Bus })}+e^{V(\text { Limousine })}\right)^{2}} \\
& \frac{1}{\partial \beta_{9}}\left[\frac{1}{P n(C)}\left(\frac{\partial P n(C)}{\partial \beta_{9}}\right)\right]=-\frac{\partial P n(L)}{\partial \beta_{9}}=-P n(L)[P n(C)+P n(T)+P n(S)+P n(B)] \\
& \frac{1}{\partial \beta_{9}}\left[\frac{1}{P n(T)}\left(\frac{\partial P n(T)}{\partial \beta_{9}}\right)\right]=-\frac{\partial P n(L)}{\partial \beta_{9}}=-\operatorname{Pn}(L)[\operatorname{Pn}(C)+P n(T)+P n(S)+P n(B)]
\end{aligned}
$$




$$
\begin{aligned}
& \frac{1}{\partial \beta_{9}}\left[\frac{1}{P n(S)}\left(\frac{\partial P n(S)}{\partial \beta_{9}}\right)\right]=-\frac{\partial P n(L)}{\partial \beta_{9}}=-P n(L)[P n(C)+P n(T)+P n(S)+P n(B)] \\
& \frac{1}{\partial \beta_{9}}\left[\frac{1}{P n(B)}\left(\frac{\partial P n(B)}{\partial \beta_{9}}\right)\right]=-\frac{\partial P n(L)}{\partial \beta_{9}}=-P n(L)[P n(C)+P n(T)+P n(S)+P n(B)] \\
& \frac{1}{\partial \beta_{9}}\left[\frac{1}{P n(L)}\left(\frac{\partial P n(L)}{\partial \beta_{9}}\right)\right]=\left[\frac{\partial P n(C)}{\partial \beta_{9}}+\frac{\partial P n(T)}{\partial \beta_{9}}+\frac{\partial P n(S)}{\partial \beta_{9}}+\frac{\partial P n(B)}{\partial \beta_{9}}\right] \\
& =[-P n(C) P n(L)-P n(T) P n(L)-P n(S) P n(L)-P n(B) P n(L)] \\
& \frac{\partial P n(C)}{\partial \beta_{9}}=-P n(C) P n(L)[P n(C)+P n(T)+P n(S)+P n(B)] \\
& \frac{\partial P n(T)}{\partial \beta_{9}}=-P n(T) P n(L) \\
& \frac{\partial P n(S)}{\partial \beta_{9}}=-P n(S) P n(L) \\
& \frac{\partial P n(B)}{\partial \beta_{9}}=-P n(B) P n(L) \\
& \frac{\partial P n(L)}{\partial \beta_{9}}=P n(L)[P n(C)+P n(T)+P n(S)+P n(B)]
\end{aligned}
$$


Appendix B: Eight Estimation Algorithms (Visual Basic Application) (The computer codes provided in appendix $B$ are of experiment 1 ) 


\section{B.1: Newton Raphson (NR) algorithm}

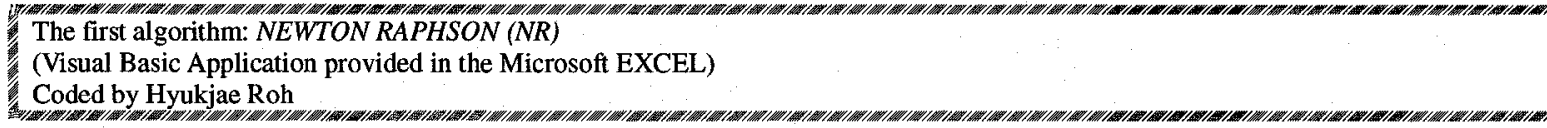

\section{Private Sub NEWTON RAPHSON_CLICKO)}

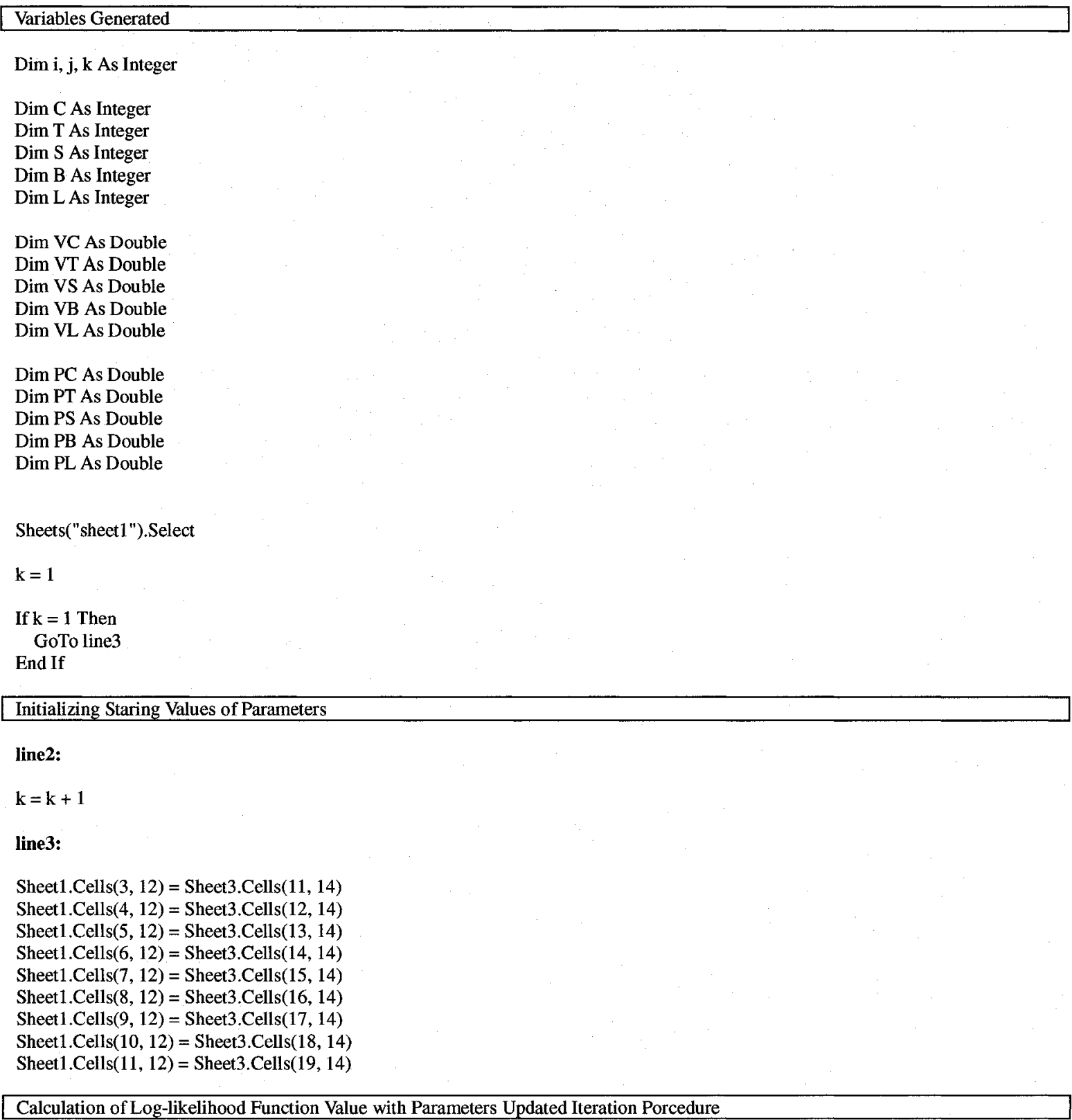

For $\mathrm{j}=3$ To 2698 Step 5

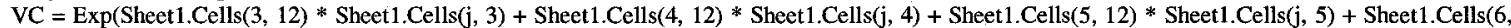
12) * Sheet1.Cells $(\mathrm{j}, 6)+$ Sheet1.Cells $(7,12) *$ Sheet $1 . \operatorname{Cells}(\mathrm{j}, 7))$

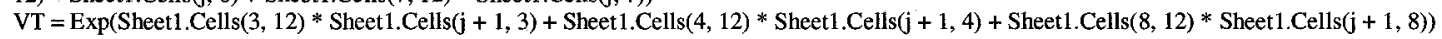

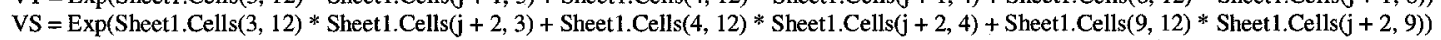

VB $=\operatorname{Exp}($ Sheet 1.Cells $(3,12) *$ Sheet1.Cells $(j+3,3)+$ Sheet1.Cells $(4,12) *$ Sheet1.Cells $(j+3,4)+$ Sheet 1.Cells $(10,12) *$ Sheet1.Cells $(j+3,10))$

$\mathrm{VL}=\operatorname{Exp}(\operatorname{Sheet} 1 . \operatorname{Cells}(3,12) * \operatorname{Sheet1.Cells}(\mathrm{j}+4,3)+\operatorname{Sheet1.Cells}(4,12) * \operatorname{Sheet1.Cells~}(\mathrm{j}+4,4)+\operatorname{Sheet} 1 . \operatorname{Cell} s(11,12) * \operatorname{Sheet} 1 . \operatorname{Cells}(\mathrm{j}+4,11))$

$\mathrm{PC}=\mathrm{VC} /(\mathrm{VC}+\mathrm{VT}+\mathrm{VS}+\mathrm{VB}+\mathrm{VL})$ 


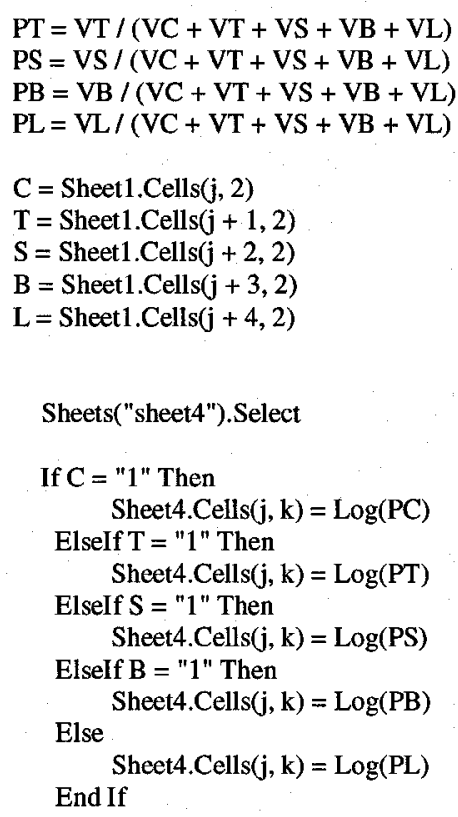

Next $\mathbf{j}$

For $\mathrm{i}=3$ To 2698 Step 5

Sheets("sheet2").Select

Utility Function Value for all alternatives' model specifications

$\mathrm{VC}=\operatorname{Exp}(\operatorname{Sheet1.Cells}(3,12) *$ Sheet1.Cells $(\mathrm{i}, 3)+\operatorname{Sheet1.Cells}(4,12) *$ Sheet1.Cells $(\mathrm{i}, 4)$

+ Sheet1.Cells $(5,12) *$ Sheet1.Cells $(1,5)+\operatorname{Sheet1.Cells~}(6,12) * \operatorname{Sheet1.Cells(i,6)}+\operatorname{Sheet1.Cells(7,12)} *$ Sheet 1.Cells $(i, 7))$

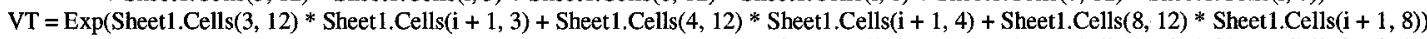

$\mathrm{VS}=\operatorname{Exp}(\operatorname{Sheet1} 1 . \operatorname{Cells}(3,12) *$ Sheet1.Cells $(\mathrm{i}+2,3)+\operatorname{Sheet} 1 . \operatorname{Cells}(4,12) *$ Sheet1.Cells $(\mathrm{i}+2,4)+\operatorname{Sheet1.Cells~}(9,12) *$ Sheet1.Cells $(\mathrm{i}+2,9))$

VB $=\operatorname{Exp}(\operatorname{Sheet1} . \operatorname{Cells}(3,12) *$ Sheet1.Cells $(i+3,3)+\operatorname{Sheet} 1 . \operatorname{Cells}(4,12) *$ Sheet1.Cells $(i+3,4)+$ Sheet1.Cells $(10,12) *$ Sheet1.Cells $(i+3,10))$

$\mathrm{VL}=\operatorname{Exp}($ Sheet $1 . C e l l s(3,12) *$ Sheet1.Cells $(\mathrm{i}+4,3)+$ Sheet1.Cells $(4,12) *$ Sheet1.Cells $(i+4,4)+$ Sheet1.Cells $(11,12) *$ Sheet1.Cells $(i+4,11))$

Mode Choice Probability of each observations in Logit probability model

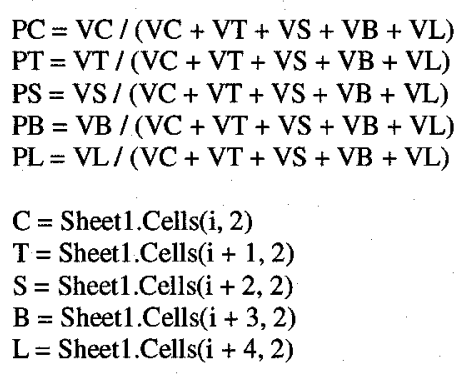

First-order Derivative with respect to $\beta_{1}$

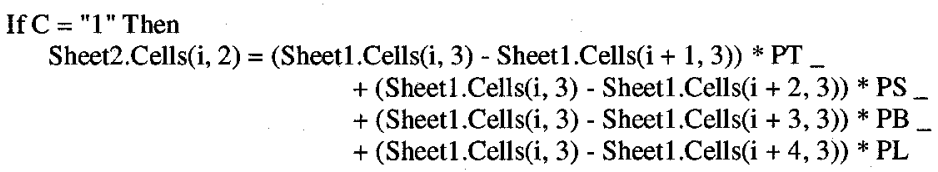


$+($ Sheet1.Cells $(\mathbf{i}+1,3)-$ Sheet1.Cells $(\mathbf{i}+2,3)) * \mathrm{PS}_{-}$ $+($ Sheet1.Cells $(i+1,3)$ - Sheet1.Cells $(i+3,3)) *$ PB $+($ Sheet1.Cells $(i+1,3)-$ Sheet1.Cells $(i+4,3)) *$ PL

ElseIf $S=$ "1" Then

Sheet2.Cells $(i, 2)=($ Sheet1 $. C e l l s(i+2,3)-$ Sheet 1.Cells $(i, 3)) * P C$

$+($ Sheet1.Cells $(\mathbf{i}+2,3)$ - Sheet 1.Cells $(\mathbf{i}+1,3)) *$ PT

$+($ Sheet1.Cells $(\mathrm{i}+2,3)$ - Sheet1.Cells $(\mathrm{i}+3,3)) * \mathrm{~PB}_{-}^{-}$

$+($ Sheet 1.Cells $(\mathrm{i}+2,3)-$ Sheet 1. Cells $(\mathrm{i}+4,3)) * \mathrm{PL}$.

ElseIf B = "1" Then

Sheet2.Cells $(i, 2)=($ Sheet1. Cells $(i+3,3)-$ Sheet 1.Cells $(i, 3)) * P C$

$+($ Sheet1.Cells $(i+3,3)$ - Sheet1.Cells $(i+1,3)) * P T$

$+($ Sheet1.Cells $(\mathrm{i}+3,3)-$ Sheet1.Cells $(\mathrm{i}+2,3)) * \mathrm{PS}_{-}$

$+($ Sheet1.Cells $(\mathrm{i}+3,3)-$ Sheet1.Cells $(\mathrm{i}+4,3)) * \mathrm{PL}$

Else

Sheet2.Cells $(i, 2)=($ Sheet1.Cells $(i+4,3)-$ Sheet1.Cells $(i, 3)) * P C$

$+($ Sheet1.Cells $(i+4,3)-$ Sheet1.Cells $(i+1,3)) * P_{-}$ $+($ Sheet1.Cells $(\mathrm{i}+4,3)$ - Sheet1.Cells $(\mathrm{i}+2,3)) * \mathrm{PS}_{-}$

$+($ Sheet1.Cells $(\mathrm{i}+4,3)-$ Sheet1.Cells $(\mathrm{i}+3,3)) * \mathrm{~PB}$

End If

First-order Derivative with respect to $\beta_{2}$

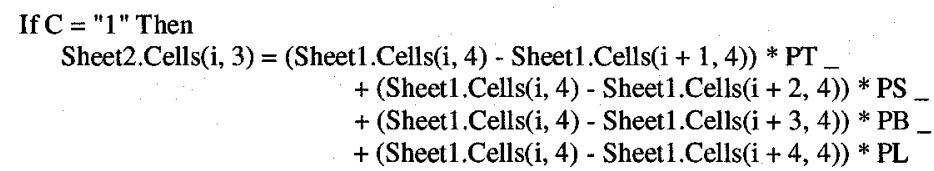

Elself $\mathrm{T}=$ "1" Then

Sheet2.Cells $(\mathrm{i}, 3)=($ Sheet1.Cells $(\mathbf{i}+1,4)-$ Sheet 1.Cells $(\mathrm{i}, 4)) * \mathrm{PC}$

$+($ Sheet1.Cells $(\mathrm{i}+1,4)-$ Sheet1.Cells $(\mathrm{i}+\overline{2}, 4)) *$ PS

$+($ Sheet1.Cells $(\mathrm{i}+1,4)-$ Sheet1.Cells $(\mathrm{i}+3,4)) *$ PB

$+($ Sheet1.Cells $(\mathrm{i}+1,4)$ - Sheet1.Cells $(\mathrm{i}+4,4)) * \mathrm{PL}$

Elself $S=$ " 1 " Then

Sheet2.Cells $(\mathrm{i}, 3)=($ Sheet1.Cells $(\mathrm{i}+2,4)-$ Sheet1.Cells $(\mathrm{i}, 4)) * \mathrm{PC}$

$+($ Sheet1.Cells $(\mathrm{i}+2,4)-$ Sheet1.Cells $(\mathrm{i}+1,4)) * \mathrm{PT}$

$+($ Sheet1.Cells $(\mathrm{i}+2,4)$ - Sheet1.Cells $(\mathrm{i}+3,4)) * \mathrm{~PB}$

$+($ Sheet1.Cells $(\mathrm{i}+2,4)$ - Sheet1.Cells $(\mathrm{i}+4,4)) * \mathrm{PL}$

Elself $B=$ " 1 " Then

Sheet2.Cells $(\mathrm{i}, 3)=($ Sheet1.Cells $(\mathrm{i}+3,4)-$ Sheet1.Cells $(\mathrm{i}, 4)) * \mathrm{PC}$

$+($ Sheet1.Cells $(i+3,4)-$ Sheet1.Cells $(i+1,4)) * P_{-}$

$+($ Sheet1.Cells $(\mathrm{i}+3,4)-$ Sheet1.Cells $(\mathrm{i}+2,4)) * \mathrm{PS}$

$+($ Sheet1.Cells $(\mathrm{i}+3,4)-$ Sheet1.Cells $(\mathrm{i}+4,4)) * \mathrm{PL}$

Else

Sheet2.Cells $(\mathbf{i}, 3)=($ Sheet1.Cells $(i+4,4)-$ Sheet1.Cells $(\mathbf{i}, 4)) *$ PC $+($ Sheet 1.Cells $(i+4,4)-$ Sheet1.Cells $(i+1,4)) *$ PT $+($ Sheet1.Cells $(\mathrm{i}+4,4)-$ Sheet 1. Cells $(\mathrm{i}+2,4)) * \mathrm{PS}_{-}^{-}$

$+($ Sheet1.Cells $(i+4,4)-$ Sheet1.Cells $(i+3,4)) *$ PB

End If

First-order Derivative with respect to $\beta_{3}$

If $\mathrm{C}=$ " 1 " Then

Sheet2.Cells $(\mathbf{i}, 4)=$ Sheet $1 . C e l l s(i, 5) *(P T+P S+P B+P L)$

Else

Sheet2.Cells $(\mathbf{i}, 4)=-$ Sheet1.Cells $(\mathbf{i}, 5) *$ PC

End If 


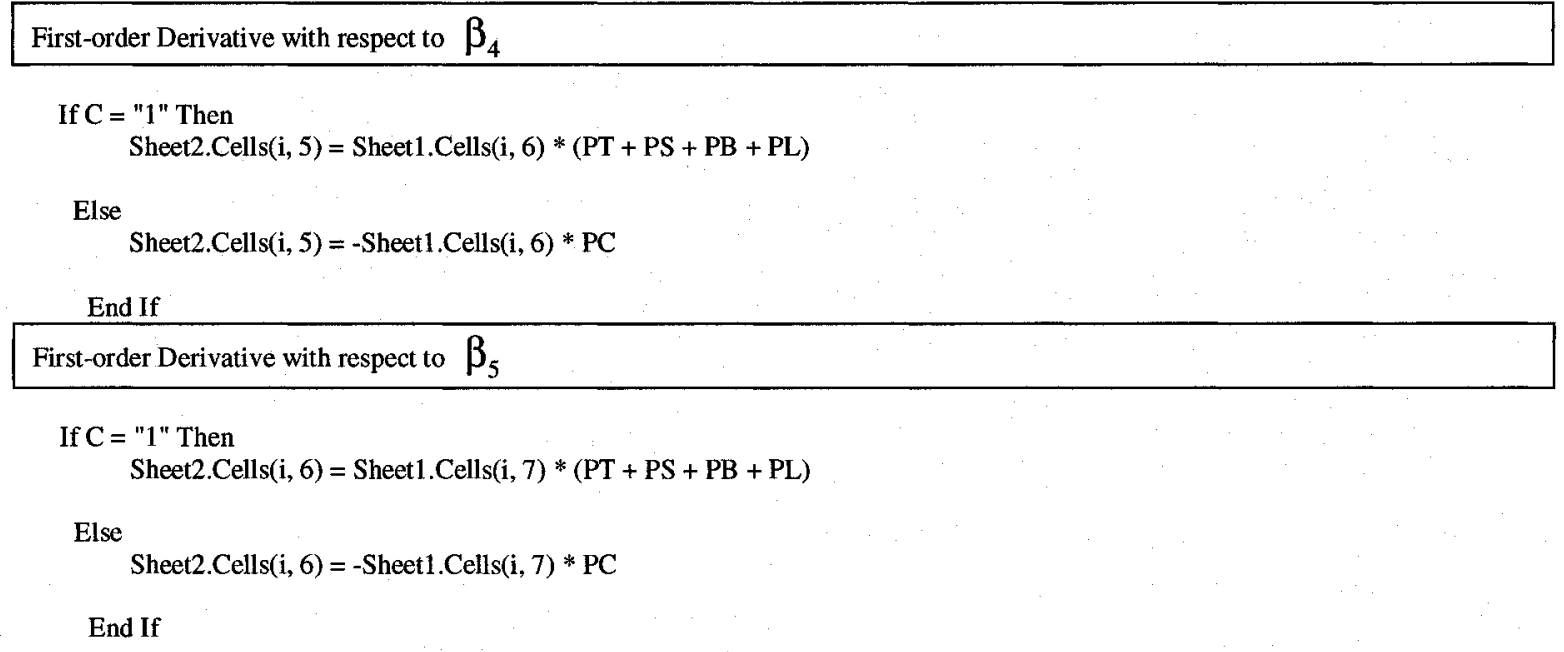

First-order Derivative with respect to $\beta_{6}$

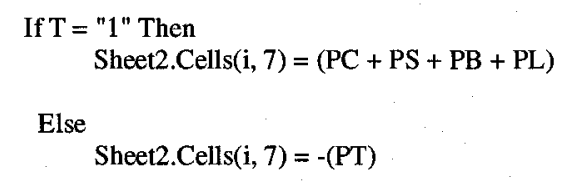

End If

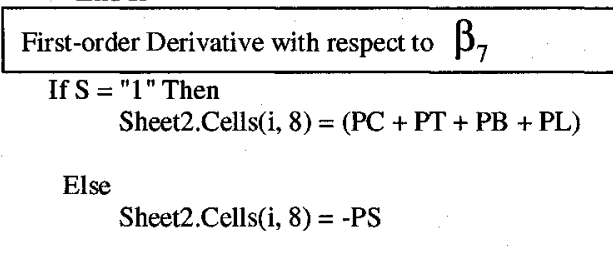

End If

First-order Derivative with respect to $\beta_{8}$
If $B=$ " 1 " Then
Sheet2.Cells $(\mathrm{i}, 9)=(\mathrm{PC}+\mathrm{PT}+\mathrm{PS}+\mathrm{PL})$

Else

Sheet2.Cells $(\mathrm{i}, 9)=-\mathrm{PB}$

End If

First-order Derivative with respect to $\beta_{9}$

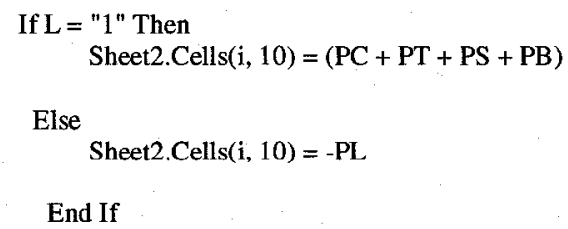

End If

Second-order Derivative with respect to $\beta_{1} \beta_{1}$
Sheet2.Cells $(\mathrm{i}, 12)=-\mathrm{PC} * \mathrm{PT} *(\operatorname{Sheet} 1 . \operatorname{Cells}(\mathrm{i}, 3)-$ Sheet1.Cells $(\mathrm{i}+1,3)) \wedge 2$
- PC * PS * (Sheet1.Cells $(\mathrm{i}, 3)-$ Sheet1.Cells $(\mathrm{i}+2,3))^{\wedge} 2$

- PC * PB * (Sheet1.Cells $(\mathrm{i}, 3)-$ Sheet1.Cells $(\mathrm{i}+3,3))^{\wedge} 2_{-}^{-}$ 
- PC * PL * (Sheet1.Cells $(\mathrm{i}, 3)-$ Sheet1.Cells $(\mathrm{i}+4,3))^{\wedge} 2$

- PT * PS * (Sheet1.Cells $(i+1,3)-$ Sheet1.Cells $(i+2,3))^{\wedge} 2_{-}$

$-\mathrm{PT} * \mathrm{~PB} *(\text { Sheet1.Cells }(\mathrm{i}+1,3)-\text { Sheet1.Cells }(\mathrm{i}+3,3))^{\wedge} 2-$

- PT * PL * (Sheet1.Cells $(i+1,3)$ - Sheet1.Cells $(i+4,3))^{\wedge} 2$

- PS * PB * (Sheet1.Cells $(\mathrm{i}+2,3)-$ Sheet1.Cells $(\mathrm{i}+3,3))^{\wedge} 2^{2}-$

-PS * PL * (Sheet1.Cells $(\mathrm{i}+2,3)-$ Sheet1.Cells $(\mathrm{i}+4,3))^{\wedge} 2$

- PB * PL * (Sheet1.Cells $(\mathrm{i}+3,3)-$ Sheet1.Cells $(\mathrm{i}+4,3))^{\wedge} 2^{-}$

Second-order Derivative with respect to $\beta_{1} \beta_{2}$

Sheet2.Cells $(i, 13)=-P C *$ PT * $($ Sheet1.Cells $(i, 3)-$ Sheet1.Cells $(i+1,3)) *($ Sheet1.Cells $(i, 4)-$ Sheet1.Cells $(i+1,4))$

- PC * PS * (Sheet1.Cells $(i, 3)-$ Sheet1.Cells $(i+2,3)) *($ Sheet1.Cells $(i, 4)-$ Sheet1.Cells $(i+2,4))$

- PC * PB * (Sheet1.Cells $(i, 3)-$ Sheet1.Cells $(i+3,3)) *($ Sheet1.Cells $(i, 4)-$ Sheet 1.Cells $(i+3,4))$

- PC * PL* (Sheet1.Cells $(\mathrm{i}, 3)-$ Sheet1.Cells $(\mathrm{i}+4,3)) *($ Sheet1.Cells $(i, 4)-$ Sheet1.Cells $(i+4,4))$

- PT * PS * (Sheet1.Cells $(\mathrm{i}+1,3)$ - Sheet1.Cells $(\mathbf{i}+2,3)) *($ Sheet1.Cells $(i+1,4)-$ Sheet1.Cells $(i+2,4))$

- PT * PB * (Sheet1.Cells $(i+1,3)-$ Sheet1.Cells $(i+3,3)) *($ Sheet1.Cells $(i+1,4)-$ Sheet1.Cells $(i+3,4))$

- PT * PL * (Sheet1.Cells $(i+1,3)-$ Sheet 1.Cells $(i+4,3)) *($ Sheet1.Cells $(i+1,4)-$ Sheet1.Cells $(i+4,4))$

- PS * PB * (Sheet1.Cells $(\mathrm{i}+2,3)-$ Sheet1.Cells $(\mathrm{i}+3,3)) *($ Sheet1.Cells $(\mathrm{i}+2,4)-$ Sheet1.Cells $(\mathrm{i}+3,4))$

- PS * PL * (Sheet1.Cells $(i+2,3)$ - Sheet 1.Cells $(i+4,3)) *($ Sheet1.Cells $(i+2,4)-$ Sheet1.Cells $(i+4,4))$

- PB * PL * (Sheet1.Cells $(\mathrm{i}+3,3)$ - Sheet1.Cells $(\mathrm{i}+4,3)) *($ Sheet1.Cells $(\mathrm{i}+3,4)$ - Sheet 1.Cells $(\mathrm{i}+4,4))$

Second-order Derivative with respect to $\beta_{1} \beta_{3}$

Sheet2.Cells $(\mathrm{i}, 14)=-$ Sheet1.Cells $(\mathrm{i}, 5) *($ Sheet1.Cells(i, 3) - Sheet1.Cells $(\mathrm{i}+1,3)) * \mathrm{PC} * \mathrm{PT}$

- Sheet1.Cells $(\mathrm{i}, 5) *($ Sheet1.Cells $(\mathrm{i}, 3)$ - Sheet1.Cells $(\mathrm{i}+2,3)) * \mathrm{PC} * \mathrm{PS}$

- Sheet1.Cells $(\mathrm{i}, 5) *($ Sheet1.Cells $(\mathrm{i}, 3)$ - Sheet1.Cells $(\mathrm{i}+3,3)) * \mathrm{PC} * \mathrm{~PB}_{-}$

- Sheet1.Cells(i, 5) * (Sheet1.Cells(i, 3) - Sheet1.Cells(i + 4, 3)) * PC * PL

Second-order Derivative with respect to $\beta_{1} \beta_{4}$

Sheet2.Cells $(\mathrm{i}, 15)=-$ Sheet1.Cells $(\mathrm{i}, 6) *($ Sheet1.Cells(i, 3) - Sheet1.Cells $(\mathrm{i}+1,3)) * \mathrm{PC} * \mathbf{P T}$

- Sheet1.Cells $(\mathbf{i}, 6) *($ Sheet1.Cells $(\mathrm{i}, 3)-$ Sheet1.Cells $(i+2,3)) *$ PC *PS

- Sheet1.Cells $(\mathbf{i}, 6) *($ Sheet1.Cells $(\mathrm{i}, 3)$ - Sheet1.Cells $(\mathrm{i}+3,3)) * \mathrm{PC} * \mathrm{~PB}$

- Sheet1.Cells(i, 6) * (Sheet1.Cells(i, 3) - Sheet1.Cells $(\mathrm{i}+4,3)) *$ PC *PL

Second-order Derivative with respect to $\beta_{1} \beta_{5}$

Sheet2.Cells $(\mathrm{i}, 16)=-$ Sheet1.Cells $(\mathrm{i}, 7) *($ Sheet1.Cells(i, 3) - Sheet1.Cells $(\mathrm{i}+1,3)) * \mathrm{PC} * \mathrm{PT}$

- Sheet1.Cells(i, 7$) *($ Sheet1.Cells $(\mathrm{i}, 3)$ - Sheet1.Cells $(\mathrm{i}+2,3)) * \mathrm{PC} * \mathrm{PS}$

- Sheet1.Cells $(\mathbf{i}, 7) *($ Sheet1.Cells $(\mathrm{i}, 3)$ - Sheet1.Cells $(\mathrm{i}+3,3)) * \mathrm{PC} * \mathrm{~PB}$

- Sheet1.Cells(i, 7) * (Sheet1.Cells(i, 3) - Sheet1.Cells $(\mathrm{i}+4,3)) *$ PC * PL

Second-order Derivative with respect to $\beta_{1} \beta_{6}$

Sheet2.Cells $(\mathrm{i}, 17)=-($ Sheet1.Cells $(\mathrm{i}+1,3)-$ Sheet 1. Cells $(\mathrm{i}, 3)) * \mathrm{PT} * \mathrm{PC}$

- (Sheet1.Cells $(\mathrm{i}+1,3)-$ Sheet1.Cells $(\mathrm{i}+2,3)) *$ PT * PS

- (Sheet1.Cells $(\mathrm{i}+1,3)-$ Sheet1.Cells $(\mathrm{i}+3,3)) * \mathrm{PT} * \mathrm{~PB}$

- (Sheet1.Cells $(\mathrm{i}+1,3)$ - Sheet 1.Cells $(\mathrm{i}+4,3)) * \mathrm{PT} * \mathrm{PL}$

Second-order Derivative with respect to $\beta_{1} \beta_{7}$

Sheet2.Cells(i, 18) $=-($ Sheet1.Cells $(\mathbf{i}+2,3)-$ Sheet1.Cells $(\mathbf{i}, 3)) *$ PS * PC

- (Sheet1.Cells $(i+2,3)$ - Sheet1.Cells $(i+1,3)) *$ PS * PT

- (Sheet1.Cells $(\mathrm{i}+2,3)-$ Sheet1.Cells $(\mathrm{i}+3,3)) * \mathrm{PS} * \mathrm{~PB}_{-}$

$-($ Sheet1.Cells $(i+2,3)-$ Sheet1.Cells $(i+4,3)) *$ PS * PL

Second-order Derivative with respect to $\beta_{1} \beta_{7}$

Sheet2.Cells $(\mathrm{i}, 19)=-($ Sheet1.Cells $(\mathrm{i}+3,3)-$ Sheet1.Cells $(\mathrm{i}, 3)) * \mathrm{~PB} * \mathrm{PC}$

$-($ Sheet1.Cells $(\mathrm{i}+3,3)-$ Sheet1.Cells $(\mathrm{i}+1,3)) * \mathrm{~PB} * \mathrm{PT}$

- $($ Sheet $1 . \operatorname{Cells}(\mathrm{i}+3,3)-$ Sheet1.Cells $(\mathrm{i}+2,3)) * \mathrm{~PB} * \mathrm{PS}_{-}^{-}$ 
- (Sheet1.Cells $(i+3,3)-$ Sheet1.Cells $(i+4,3)) *$ PB * PL

Second-order Derivative with respect to $\beta_{1} \beta_{9}$

Sheet2.Cells $(\mathrm{i}, 20)=-($ Sheet1.Cells $(\mathrm{i}+4,3)-$ Sheet $1 . \operatorname{Cells}(\mathrm{i}, 3)) * \mathrm{PL} * \mathrm{PC}$

- $($ Sheet 1. Cells $(\mathrm{i}+4,3)-$ Sheet1.Cells $(\mathrm{i}+1,3)) * \mathrm{PL} * \mathrm{PT}$

- (Sheet1.Cells $(\mathrm{i}+4,3)$ - Sheet1.Cells $(\mathrm{i}+2,3)) * \mathrm{PL}^{*} \mathrm{PS}_{-}$

$-($ Sheet1.Cells $(\mathrm{i}+4,3)-$ Sheet1.Cells $(\mathrm{i}+3,3)) * \mathrm{PL} * \mathrm{~PB}$

Second-order Derivative with respect to $\beta_{2} \beta_{2}$

Sheet2.Cells $(\mathrm{i}, 21)=-\mathrm{PC} * \mathbf{P T} *(\text { Sheet1.Cells }(\mathrm{i}, 4)-\text { Sheet } 1 \text {.Cells }(\mathrm{i}+1,4))^{\wedge} 2$

- PC * PS * (Sheet1.Cells $(i, 4)$ - Sheet1.Cells $(i+2,4))^{\wedge} 2$

- PC * PB * (Sheet1.Cells $(\mathrm{i}, 4)$ - Sheet1.Cells $(\mathrm{i}+3,4))^{\wedge} 2$ -

- PC * PL * (Sheet1.Cells $(\mathrm{i}, 4)-$ Sheet1.Cells $(\mathrm{i}+4,4))^{\wedge} 2$

- PT * PS * (Sheet1.Cells $(i+1,4)$ - Sheet1.Cells $(i+2,4))^{\wedge} 2$

- PT * PB * (Sheet1.Cells $(\mathrm{i}+1,4)-$ Sheet1.Cells $(\mathrm{i}+3,4)) \wedge 2_{-}$

- PT * PL * (Sheet1.Cells $(\mathrm{i}+1,4)-$ Sheet1.Cells $(\mathrm{i}+4,4))^{\wedge} 2$ -

- PS * PB * (Sheet1.Cells $(i+2,4)-$ Sheet1.Cells $(i+3,4)) \wedge{ }^{\wedge}$ -

- PS * PL * (Sheet1.Cells $(\mathrm{i}+2,4)$ - Sheet1.Cells $(\mathrm{i}+4,4))^{\wedge} 2_{-}$

- PB * PL * (Sheet1.Cells $(\mathrm{i}+3,4)$ - Sheet1.Cells $(\mathrm{i}+4,4))^{\wedge} 2$

Second-order Derivative with respect to $\beta_{2} \beta_{3}$

Sheet2.Cells $(\mathrm{i}, 22)=-$ Sheet1.Cells $(\mathrm{i}, 5) *($ Sheet1.Cells $(\mathbf{i}, 4)-$ Sheet1.Cells $(\mathrm{i}+1,4)) * \mathrm{PC} * \mathrm{PT}$

- Sheet1.Cells $(i, 5) *($ Sheet1.Cells $(i, 4)$ - Sheet1.Cells $(i+2,4)) * P C * P S$

- Sheet1.Cells $(i, 5) *($ Sheet1.Cells $(i, 4)$ - Sheet1.Cells $(i+3,4)) * P C * P B$

- Sheet1.Cells(i, 5) * (Sheet1.Cells(i, 4) - Sheet1.Cells(i + 4, 4)) * PC * PL

Second-order Derivative with respect to $\beta_{2} \beta_{4}$

Sheet2.Cells $(\mathbf{i}, 23)=-$ Sheet1.Cells $(\mathbf{i}, 6) *($ Sheet1.Cells $(\mathrm{i}, 4)-$ Sheet1.Cells $(\mathrm{i}+1,4)) * \mathrm{PC} * \mathrm{PT}$

- Sheet1.Cells $(\mathrm{i}, 6) *($ Sheet1.Cells $(\mathrm{i}, 4)-$ Sheet1.Cells $(\mathrm{i}+2,4)) * \mathrm{PC} * \mathrm{PS}$ -

- Sheet1.Cells $(\mathrm{i}, 6) *($ Sheet1.Cells $(\mathrm{i}, 4)$ - Sheet1.Cells $(\mathrm{i}+3,4)) * \mathrm{PC} * \mathrm{~PB}$ -

- Sheet1.Cells $(\mathrm{i}, 6) *($ Sheet1.Cells $(\mathrm{i}, 4)-$ Sheet1.Cells $(\mathrm{i}+4,4)) * \mathrm{PC} * \mathrm{PL}$

Second-order Derivative with respect to $\beta_{2} \beta_{5}$

Sheet2.Cells(i, 24) $=-$ Sheet1.Cells $(\mathbf{i}, 7) *($ Sheet1.Cells $(\mathrm{i}, 4)-$ Sheet1.Cells $(\mathbf{i}+1,4)) * \mathrm{PC} * \mathrm{PT}$

- Sheet1.Cells $(\mathrm{i}, 7) *($ Sheet1.Cells $(\mathrm{i}, 4)-$ Sheet1.Cells $(\mathrm{i}+2,4)) * \mathrm{PC} * \mathrm{PS}$

- Sheet1.Cells $(\mathrm{i}, 7) *($ Sheet1.Cells $(\mathrm{i}, 4)$ - Sheet1.Cells $(\mathrm{i}+3,4)) * \mathrm{PC} * \mathrm{~PB}$ -

- Sheet1.Cells $(\mathrm{i}, 7) *($ Sheet1.Cells $(\mathbf{i}, 4)-$ Sheet1.Cells $(\mathrm{i}+4,4)) * \mathrm{PC} * \mathrm{PL}$

Second-order Derivative with respect to $\beta_{2} \beta_{6}$

Sheet2.Cells $(\mathbf{i}, 25)=-($ Sheet 1 Cells $(\mathrm{i}+1,4)-$ Sheet $1 . C e l l s(i, 4)) * P T * P C$

- $($ Sheet1.Cells $(\mathbf{i}+1,4)-$ Sheet1.Cells $(\mathbf{i}+2,4)) * \mathbf{P T} * \mathbf{P S}$

- (Sheet1.Cells $(i+1,4)-$ Sheet1.Cells $(i+3,4)) * P^{2} * P^{-}$

- (Sheet1.Cells $(\mathrm{i}+1,4)-$ Sheet1.Cells $(\mathrm{i}+4,4)) * \mathrm{PT} * \mathrm{PL}$

Second-order Derivative with respect to $\beta_{2} \beta_{7}$

Sheet2.Cells $(\mathrm{i}, 26)=-($ Sheet1.Cells $(\mathrm{i}+2,4)-$ Sheet1.Cells $(\mathrm{i}, 4)) * \mathrm{PS} * \mathrm{PC}$

- $($ Sheet1.Cells $(\mathrm{i}+2,4)$ - Sheet1.Cells $(\mathrm{i}+1,4)) * \mathrm{PS} * \mathrm{PT}$

- (Sheet1.Cells $(\mathbf{i}+2,4)$ - Sheet1.Cells $(\mathbf{i}+3,4)) * \mathbf{P S} * \mathbf{P B}_{-}$

- (Sheet1.Cells $(i+2,3)-$ Sheet1.Cells $(i+4,4)) *$ PS * PL

Second-order Derivative with respect to $\beta_{2} \beta_{8}$

Sheet2.Cells $(\mathrm{i}, 27)=-($ Sheet1.Cells $(\mathrm{i}+3,4)-$ Sheet1.Cells $(\mathrm{i}, 4)) *$ PB $*$ PC

$-($ Sheet1.Cells $(i+3,4)-$ Sheet1.Cells $(i+1,4)) *{ }_{\text {PB }} * P_{-}$ 
- $($ Sheet1.Cells $(\mathrm{i}+3,4)-$ Sheet 1.Cells $(\mathrm{i}+2,4)) * \mathrm{~PB} * \mathrm{PS}$

$-($ Sheet1.Cells $(\mathrm{i}+3,4)-$ Sheet1.Cells $(\mathrm{i}+4,4)) * \mathrm{~PB} * \mathrm{PL}$

Second-order Derivative with respect to $\beta_{2} \beta_{9}$

Sheet2.Cells $(i, 28)=-($ Sheet1.Cells $(i+4,4)-$ Sheet1.Cells $(i, 4)) * P L * P C$

- (Sheet1.Cells $(\mathbf{i}+4,4)$ - Sheet 1.Cells $(\mathrm{i}+1,4)) * \mathbf{P L}^{*} * \mathrm{PT}$

- (Sheet1.Cells $(i+4,4)$ - Sheet1.Cells $(i+2,4)) *$ PL * PS

$-($ Sheet1.Cells $(\mathrm{i}+4,4)-$ Sheet1.Cells $(\mathrm{i}+3,4)) * \mathrm{PL} * \mathrm{~PB}$

Second-order Derivative with respect to $\beta_{3} \beta_{3}$

Sheet2.Cells $(\mathbf{i}, 29)=-$ Sheet 1.Cells $(\mathbf{i}, 5) \wedge 2 * \mathrm{PC} *(\mathrm{PT}+\mathrm{PS}+\mathrm{PB}+\mathrm{PL})$

Second-order Derivative with respect to $\beta_{3} \beta_{4}$

Sheet2.Cells $(\mathrm{i}, 30)=-$ Sheet1.Cells $(\mathrm{i}, 5) *$ Sheet1.Cells $(\mathrm{i}, 6) * \mathrm{PC} *(\mathrm{PT}+\mathrm{PS}+\mathrm{PB}+\mathrm{PL})$

Second-order Derivative with respect to $\beta_{3} \beta_{5}$

Sheet2.Cells $(\mathrm{i}, 31)=-$ Sheet1.Cells $(\mathbf{i}, 5) *$ Sheet1.Cells $(\mathrm{i}, 7) * \mathrm{PC} *(\mathrm{PT}+\mathrm{PS}+\mathrm{PB}+\mathrm{PL})$

Second-order Derivative with respect to $\beta_{3} \beta_{6}$

Sheet2.Cells(i, 32) $=$ Sheet1.Cells $(\mathrm{i}, 5) * \mathbf{P T} *$ PC

Second-order Derivative with respect to $\beta_{3} \beta_{7}$

Sheet2.Cells(i, 33) $=$ Sheet1.Cells $(\mathrm{i}, 5) *$ PS * PC

Second-order Derivative with respect to $\beta_{3} \beta_{8}$

Sheet2.Cells $(\mathbf{i}, 34)=$ Sheet1.Cells $(\mathrm{i}, 5) * \mathrm{~PB} * \mathrm{PC}$

Second-order Derivative with respect to $\beta_{3} \beta_{9}$

Sheet2.Cells(i, 35) $=$ Sheet1.Cells $(\mathrm{i}, 5) * \mathrm{PL} * \mathrm{PC}$

Second-order Derivative with respect to $\beta_{4} \beta_{4}$

Sheet2.Cells $(\mathbf{i}, 36)=-$ Sheet1.Cells $(\mathrm{i}, 6)^{\wedge} 2 * \mathrm{PC} *(\mathrm{PT}+\mathrm{PS}+\mathrm{PB}+\mathrm{PL})$

Second-order Derivative with respect to $\beta_{4} \beta_{5}$

Sheet2.Cells $(\mathbf{i}, 37)=-$ Sheet1.Cells $(\mathbf{i}, 6) *$ Sheet1.Cells $(\mathbf{i}, 7) * \mathrm{PC} *(\mathrm{PT}+\mathrm{PS}+\mathrm{PB}+\mathrm{PL})$

Second-order Derivative with respect to $\beta_{4} \beta_{6}$

Sheet2.Cells(i, 38) $=$ Sheet 1.Cells $(\mathrm{i}, 6) * \mathrm{PT} * \mathrm{PC}$

Second-order Derivative with respect to $\beta_{4} \beta_{7}$

Sheet2.Cells $(\mathrm{i}, 39)=$ Sheet 1. Cells $(\mathrm{i}, 6) * \mathrm{PS} * \mathrm{PC}$

Second-order Derivative with respect to $\beta_{4} \beta_{8}$

Sheet2.Cells $(i, 40)=$ Sheet 1.Cells $(i, 6) * P B * P C$

Second-order Derivative with respect to $\beta_{4} \beta_{9}$

Sheet2.Cells $(\mathrm{i}, 41)=$ Sheet1.Cells $(\mathrm{i}, 6) * \mathrm{PL} * \mathrm{PC}$

Second-order Derivative with respect to $\beta_{5} \beta_{5}$

Sheet2.Cells $(\mathrm{i}, 42)=-$ Sheet1.Cells $(\mathrm{i}, 7)^{\wedge} 2{ }^{*} \mathrm{PC} *(\mathrm{PT}+\mathrm{PS}+\mathrm{PB}+\mathrm{PL})$

Second-order Derivative with respect to $\beta_{5} \beta_{6}$

Sheet2.Cells $(i, 43)=$ Sheet1.Cells $(i, 7) *$ PT * PC 
Second-order Derivative with respect to $\beta_{5} \beta_{7}$

Sheet2.Cells $(\mathrm{i}, 44)=$ Sheet1.Cells $(\mathrm{i}, 7) * \mathrm{PS} * \mathrm{PC}$

Second-order Derivative with respect to $\beta_{5} \beta_{8}$

Sheet2.Cells $(\mathrm{i}, 45)=$ Sheet1.Cells $(\mathrm{i}, 7) * \mathrm{~PB} * \mathrm{PC}$

Second-order Derivative with respect to $\beta_{5} \beta_{9}$

Sheet2.Cells $(\mathrm{i}, 46)=$ Sheet1.Cells $(\mathrm{i}, 7) * \mathrm{PL} * \mathrm{PC}$

Second-order Derivative with respect to $\beta_{6} \beta_{6}$

Sheet2.Cells $(\mathrm{i}, 47)=-\mathrm{PT} *(\mathrm{PC}+\mathrm{PS}+\mathrm{PB}+\mathrm{PL})$

Second-order Derivative with respect to $\beta_{6} \beta_{7}$

Sheet2.Cells $(\mathrm{i}, 48)=\mathrm{PS} * \mathrm{PT}$

Second-order Derivative with respect to $\beta_{6} \beta_{8}$

Sheet2.Cells $(\mathrm{i}, 49)=\mathrm{PB} * \mathrm{PT}$

Second-order Derivative with respect to $\beta_{6} \beta_{9}$

Sheet2.Cells $(\mathrm{i}, 50)=\mathrm{PL} * \mathrm{PT}$

Second-order Derivative with respect to $\beta_{7} \beta$.

Sheet2.Cells $(\mathrm{i}, 51)=-\mathrm{PS} *(\mathrm{PC}+\mathrm{PT}+\mathrm{PB}+\mathrm{PL})$

Second-order Derivative with respect to $\beta_{7} \beta_{8}$

Sheet2.Cells $(\mathrm{i}, 52)=\mathrm{PB} * \mathrm{PS}$

Second-order Derivative with respect to $\beta_{7} \beta_{9}$

Sheet 2. Cells $(i, 53)=$ PL * PS

Second-order Derivative with respect to $\beta_{8} \beta_{8}$

Sheet2.Cells $(\mathrm{i}, 54)=-\mathrm{PB} *(\mathrm{PC}+\mathrm{PT}+\mathrm{PS}+\mathrm{PL})$

Second-order Derivative with respect to $\beta_{8} \beta_{9}$

Sheet2.Cells $(\mathrm{i}, 55)=\mathrm{PL} * \mathrm{~PB}$

Second-order Derivative with respect to $\beta_{9} \beta_{9}$

Sheet2.Cells(i, 56) $=-\mathrm{PL} *(\mathrm{PC}+\mathrm{PT}+\mathrm{PS}+\mathrm{PB})$

Next $\mathrm{i}$

Obtaining Gradient components calculated, summation of all observation and averaging it

For $\mathbf{j}=2$ To 56 Step 1

Sheet2.Cells $(2703, \mathrm{j})$.FormulaR1C1 $="=\mathrm{SUM}(\mathrm{R}[-2700] \mathrm{C}: \mathrm{R}[-1] \mathrm{C}) / 540 "$

Next $\mathbf{j}$

Sheets("sheet3").Select

Calculating Hessian Matrix and times -1: $\left(-H_{t}\right)$ 
Sheet3.Range("A1") = -Sheet2.Range("L2703").Value: Sheet3.Range("B1") = -Sheet2.Range("M2703").Value Sheet3.Range("C1") = -Sheet2.Range("N2703").Value: Sheet3.Range("D1") = -Sheet2.Range("O2703").Value Sheet3.Range("E1") = -Sheet2.Range("P2703").Value: Sheet3.Range("F1") = -Sheet2.Range("Q2703"). Value Sheet3.Range("G1") = -Sheet2.Range("R2703").Value: Sheet3.Range("H1") = -Sheet2.Range("S2703").Value Sheet3.Range("I1") = -Sheet2.Range("T2703").Value:

Sheet3.Range("A2") = -Sheet2.Range("M2703").Value: Sheet3.Range("B2") = -Sheet2.Range("U2703").Value Sheet3.Range("C2") = -Sheet2.Range("V2703").Value: Sheet3.Range("D2") = -Sheet2.Range("W2703").Value Sheet3.Range("E2") = -Sheet2.Range("X2703").Value: Sheet3.Range("F2") = -Sheet2.Range("Y2703").Value Sheet3.Range("G2") = -Sheet2.Range("Z2703").Value: Sheet3.Range("H2") = -Sheet2.Range("AA2703").Value Sheet3.Range("12") = -Sheet2.Range("AB2703").Value

Sheet3.Range("A3") = -Sheet2.Range("N2703").Value: Sheet3.Range("B3") = -Sheet2.Range("V2703").Value Sheet3.Range("C3") = -Sheet2.Range("AC2703"). Value: Sheet3.Range("D3") = -Sheet2.Range("AD2703").Value Sheet3.Range("E3") = -Sheet2.Range("AE2703").Value: Sheet3.Range("F3") = -Sheet2.Range("AF2703").Value Sheet3.Range("G3") = -Sheet2.Range("AG2703").Value: Sheet3.Range("H3") = -Sheet2.Range("AH2703").Value Sheet3.Range("I3") = -Sheet2.Range("AI2703"). Value

Sheet3.Range("A4") = -Sheet2.Range("O2703").Value: Sheet3.Range("B4") = -Sheet2.Range("W2703").Value Sheet3.Range("C4") = -Sheet2.Range("AD2703").Value: Sheet3.Range("D4") = -Sheet2.Range("AJ2703").Value Sheet3.Range("E4") = -Sheet2.Range("AK2703").Value: Sheet3.Range("F4") = -Sheet2.Range("AL2703").Value Sheet3.Range("G4") = -Sheet2.Range("AM2703").Value: Sheet3.Range("H4") = -Sheet2.Range("AN2703"). Value Sheet3.Range("I4") = -Sheet2.Range("AO2703"). Value

Sheet3.Range("A5") = -Sheet2.Range("P2703").Value: Sheet3.Range("B5") = -Sheet2.Range("X2703").Value Sheet3.Range("C5") = -Sheet2.Range ("AE2703").Value: Sheet3.Range("D5") = -Sheet2.Range("AK2703").Value Sheet3.Range("E5") = -Sheet2.Range("AP2703").Value: Sheet3.Range("F5") = -Sheet2.Range("AQ2703").Value Sheet3.Range("G5") = -Sheet2.Range("AR2703").Value: Sheet3.Range("H5") = -Sheet2.Range("AS2703").Value Sheet3.Range("I5") = -Sheet2.Range("AT2703").Value

Sheet3.Range("A6") = -Sheet2.Range("Q2703").Value: Sheet3.Range("B6") = -Sheet2.Range("Y2703").Value Sheet3.Range("C6") = -Sheet2.Range("AF2703"). Value: Sheet3.Range("D6") = -Sheet2.Range("AL2703").Value Sheet3.Range("E6") = -Sheet2.Range("AQ2703").Value: Sheet3.Range("F6") = -Sheet2.Range("AU2703").Value Sheet3.Range("G6") = -Sheet2.Range("AV2703").Value: Sheet3.Range("H6") = -Sheet2.Range("AW2703"). Value Sheet3.Range("16") = -Sheet2.Range("AX2703").Value

Sheet3.Range("A7") = -Sheet2.Range("R2703").Value: Sheet3.Range("B7") = -Sheet2.Range("Z2703").Value Sheet3.Range("C7") =-Sheet2.Range("AG2703").Value: Sheet3.Range("D7") = -Sheet2.Range("AM2703").Value Sheet3.Range("E7") = -Sheet2.Range("AR2703").Value: Sheet3.Range("F7") = -Sheet2.Range("AV2703").Value Sheet3.Range("G7") = -Sheet2.Range("AY2703").Value: Sheet3.Range("H7") = -Sheet2.Range("AZ2703").Value Sheet3.Range("I7") = -Sheet2.Range("BA2703").Value

Sheet3.Range("A8") = -Sheet2.Range("S2703").Value: Sheet3.Range("B8") = -Sheet2.Range("AA2703").Value Sheet3.Range("C8") = -Sheet2.Range("AH2703").Value: Sheet3.Range("D8") = -Sheet2.Range("AN2703").Value Sheet3.Range("E8") = -Sheet2.Range("AS2703").Value: Sheet3.Range("F8") = -Sheet2.Range("AW2703").Value Sheet3.Range("G8") = -Sheet2.Range("AZ2703").Value: Sheet3.Range("H8") = -Sheet2.Range("BB2703").Value Sheet3.Range("I8") = -Sheet2.Range("BC2703").Value

Sheet3.Range("A9") = -Sheet2.Range("T2703").Value: Sheet3.Range("B9") = -Sheet2.Range("AB2703").Value Sheet3.Range("C9") = -Sheet2.Range("AI2703").Value: Sheet3.Range("D9") = -Sheet2.Range("AO2703").Value Sheet3.Range("E9") = -Sheet2.Range("AT2703").Value: Sheet3.Range("F9") = -Sheet2.Range("AX2703").Value Sheet3.Range("G9") = -Sheet2.Range("BA2703"). Value: Sheet3.Range("H9") = -Sheet2.Range("BC2703").Value Sheet3.Range("I9") = -Sheet2.Range("BD2703").Value

Obtaining the iverse of Hessian matrix: $\left(-H_{t}\right)^{-1}$

Sheet3.Range("A11:I19").FormulaArray = "=(MINVERSE(A1:I9))"

Obtaining the gradient value of Log-likelihood function: $\boldsymbol{G}_{\boldsymbol{t}}$

'For $\mathrm{i}=11$ To 19 Step 1

Sheet 3. Cells $(11,11)=$ Sheet $2 \cdot$ Cells $(2703,2)$

Sheet 3 .Cells $(12,11)=$ Sheet 2 .Cells $(2703,3)$

Sheet 3. Cells $(13,11)=$ Sheet 2. Cells $(2703,4)$

Sheet 3. Cells $(14,11)=$ Sheet 2. Cells $(2703,5)$

Sheet $3 . \operatorname{Cells}(15,11)=$ Sheet 2 .Cells $(2703,6)$

Sheet 3. Cells $(16,11)=$ Sheet2.Cells $(2703,7)$ 
Sheet3.Cells $(17,11)=$ Sheet $2 \cdot \operatorname{Cells}(2703,8)$

Sheet 3 .Cells $(18,11)=$ Sheet 2 .Cells $(2703,9)$

Sheet 3. Cells $(19,11)=$ Sheet $2 \cdot \operatorname{Cells}(2703,10)$

'Next i

Calculating parameters value: $\beta_{t+1}=\beta_{t}+\lambda_{t} \Delta_{t}=\beta_{t}+\lambda_{t} W_{t} G_{t}=\beta_{t}+\lambda_{t}\left(-H_{t}^{-1}\right) G_{t}$

Sheet3.Range("M11:M19").FormulaArray = "=MMULT(A11:I19,K11:K19)"

Sheet3.Cells $(11,14)=$ Sheet1.Cells $(3,12)+$ Sheet3.Cells $(11,13)$

Sheet 3. Cells $(12,14)=$ Sheet 1. Cells $(4,12)+$ Sheet 3. Cells $(12,13)$

Sheet3.Cells $(13,14)=$ Sheet1.Cells $(5,12)+$ Sheet 3 .Cells $(13,13)$

Sheet3.Cells $(14,14)=$ Sheet1.Cells $(6,12)+$ Sheet3.Cells $(14,13)$

Sheet3.Cells $(15,14)=$ Sheet 1 .Cells $(7,12)+$ Sheet3.Cells $(15,13)$

Sheet3.Cells $(16,14)=$ Sheet 1. Cells $(8,12)+$ Sheet $3 \cdot \operatorname{Cells}(16,13)$

Sheet3.Cells $(17,14)=$ Sheet 1 .Cells $(9,12)+$ Sheet3.Cells $(17,13)$

Sheet3.Cells $(18,14)=$ Sheet 1 .Cells $(10,12)+$ Sheet $3 . C e l l s(18,13)$

Sheet $3 \cdot$ Cells $(19,14)=$ Sheet 1 .Cells $(11,12)+$ Sheet $3 \cdot$ Cells $(19,13)$

Checking the Criterion for stopping: $\left[\frac{1}{k} \sum_{k=1}^{k}\left(\beta_{t+1, k}-\beta_{t, k}\right)^{2}\right]^{1 / 2}<C R$

Sheet3.Cells $(21,12)=(($

(Sheet3.Cells $(11,14)-$ Sheet1.Cells $(3,12))^{\wedge} 2+(\text { Sheet3.Cells }(12,14)-\text { Sheet1.Cells }(4,12))^{\wedge} 2$ $+(\text { Sheet3.Cells }(13,14)-\text { Sheet1.Cells }(5,12))^{\wedge} 2+(\operatorname{Sheet3.Cells}(14,14)-\text { Sheet1.Cells }(6,12))^{\wedge} 2$ $+(\text { Sheet3.Cells }(15,14)-\text { Sheet1.Cells }(7,12))^{\wedge} 2+(\text { Sheet3.Cells }(16,14)-\text { Sheet1.Cells }(8,12))^{\wedge} 2$ $+($ Sheet3.Cells $(17,14)-$ Sheet1.Cells $(9,12)) \wedge 2+(\text { Sheet3.Cells }(18,14)-\text { Sheet1.Cells }(10,12))^{\wedge} \overline{2}-$ $+(\text { Sheet3.Cells }(19,14)-\text { Sheet1.Cells }(11,12))^{\wedge} 2_{-}$

$$
)^{*}(1 / 9)\right)^{\wedge}(0.5)
$$

If Sheet3.Cells $(21,12)<10^{\wedge}(-4)$ Then

GoTo line1

Else

GoTo line2

End If

line1:

Summation of Log-likelihood function value

For $\mathrm{j}=1$ To 5 Step 1

Sheet4.Cells $(2703, \mathrm{j}) \cdot$ FormulaR1C1 $=$ "=SUM(R[-2700]C:R[-1]C)"

Next $j$

End Sub 


\section{B.2: BHHH- algorithm}

The second algorithm : BHHH [Berndt, Hall, Hall, and Hausman]
(Visual Basic Application provided in the Microsof EXCEL)
Coded by Hyukjae Roh

Coded by Hyukjae Roh

Private Sub BHHH_Click()

Variables Generated

Dim i, J, K As Integer

Dim C As Integer

Dim $T$ As Integer

Dim S As Integer

Dim B As Integer

Dim 1 As Integer

Dim VC As Double

Dim VT As Double

Dim VS As Double

Dim VB As Double

Dim VL As Double

Dim PC As Double

Dim PT As Double

Dim PS As Double

Dim PB As Double

Dim PL As Double

$K=1$

If $\mathbf{K}=1$ Then

GoTo line3

End If

Sheets("sheet1").Select

Initializing Staring Values of Parameters

line2:

$\mathbf{K}=\mathbf{K}+\mathbf{1}$

line3:

Sheet1.Cells $(3,12)=$ Sheet 3. Cells $(11,14)$

Sheet1.Cells $(4,12)=$ Sheet3.Cells $(12,14)$

Sheet1.Cells $(5,12)=$ Sheet 3. Cells $(13,14)$

Sheet1.Cells $(6,12)=$ Sheet $3 \cdot$ Cells $(14,14)$

Sheet1.Cells $(7,12)=$ Sheet 3. Cells $(15,14)$

Sheet1.Cells $(8,12)=$ Sheet 3. Cells $(16,14)$

Sheet1.Cells $(9,12)=$ Sheet3.Cells $(17,14)$

Sheet1.Cells $(10,12)=$ Sheet 3. Cells $(18,14)$

Sheet1.Cells $(11,12)=$ Sheet3.Cells $(19,14)$

Calculation of the Log-likelihood function value

For $J=3$ To 2698 Step 5

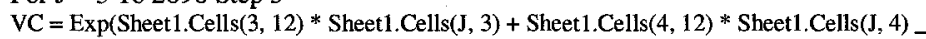

+ Sheet1.Cells $(5,12) *$ Sheet1.Cells $(J, 5)+$ Sheet1.Cells $(6,12) *$ Sheetl Cells $(J, 6)+$ Sheet1.Cells $(7,12) *$ Sheet1.Cells $(J, 7))$

$\mathrm{VT}=\operatorname{Exp}(\operatorname{Sheet1.Cells}(3,12) * \operatorname{Sheet1.Cells}(\mathrm{J}+1,3)+\operatorname{Sheet1.Cells}(4,12) * \operatorname{Sheet1.Cells}(J+1,4)+\operatorname{Sheet1.Cells}(8,12) * \operatorname{Sheet} 1 . \operatorname{Cells}(\mathrm{J}+1,8))$

VS $=\operatorname{Exp}(\operatorname{Sheet1.Cells}(3,12) * \operatorname{Sheet1.Cells}(J+2,3)+\operatorname{Sheet~1.Cells~}(4,12) *$ Sheet1.Cells $(J+2,4)+\operatorname{Sheet1}$.Cells $(9,12) * \operatorname{Sheet} 1$. Cells $(J+2,9))$

$\mathrm{VB}=\operatorname{Exp}(\operatorname{Sheet1.Cells}(3,12) *$ Sheet1.Cells $(J+3,3)+\operatorname{Sheet1.Cells~}(4,12) *$ Sheet1.Cells $(J+3,4)+\operatorname{Sheet1.Cells}(10,12) * \operatorname{Sheet} 1 . \operatorname{Cells}(\mathrm{J}+3,10))$

$\mathrm{VL}=\operatorname{Exp}(\operatorname{Sheet1.Cells}(3,12) *$ Sheet1.Cells $(J+4,3)+\operatorname{Sheet1.Cells}(4,12) *$ Sheet1.Cells $(J+4,4)+$ Sheet 1.Cells $(11,12) * \operatorname{Sheet} 1 . C e l l s(J+4,11))$

$\mathrm{PC}=\mathrm{VC} /(\mathrm{VC}+\mathrm{VT}+\mathrm{VS}+\mathrm{VB}+\mathrm{VL})$

$\mathrm{PT}=\mathrm{VT} /(\mathrm{VC}+\mathrm{VT}+\mathrm{VS}+\mathrm{VB}+\mathrm{VL})$ 


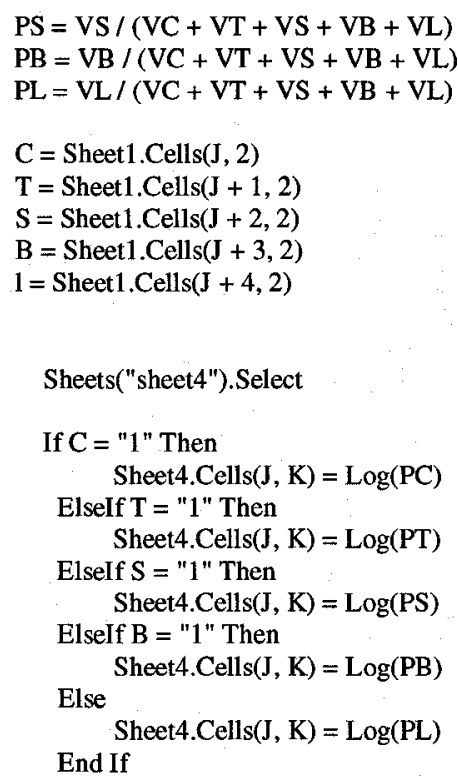

Next J

For $\mathrm{i}=3$ To 2698 Step 5

Sheets("sheet2").Select

Utility Function Value for all alternatives' model specifications

$\mathrm{vC}=\operatorname{Exp}($ Sheet $1 . C e l l s(3,12) *$ Sheet1.Cells $(\mathbf{i}, 3)+\operatorname{Sheet1.Cells~}(4,12) *$ Sheet 1.Cells $(\mathrm{i}, 4)$

+ Sheet 1.Cells $(5,12) *$ Sheet1.Cells $(i, 5)+$ Sheet1.Cells $(6,12) *$ Sheet1.Cells $(i, 6)+$ Sheet1.Cells $(7,12) *$ Sheet 1.Cells $(i, 7))$

$\mathrm{VT}=\operatorname{Exp}(\operatorname{Sheet} 1 . \operatorname{Cells}(3,12) * \operatorname{Sheet} 1$. Cells $(i+1,3)+\operatorname{Sheet1.Cells}(4,12) *$ Sheet1.Cells $(\mathrm{i}+1,4)+\operatorname{Sheet1.Cells}(8,12) *$ Sheet 1. Cells $(\mathrm{i}+1,8))$

VS $=\operatorname{Exp}($ Sheet1.Cells $(3,12) *$ Sheet1.Cells $(i+2,3)+$ Sheet1.Cells $(4,12) *$ Sheet1.Cells $(i+2,4)+$ Sheet1.Cells $(9,12) *$ Sheet1.Cells $(i+2,9))$

$\mathrm{VB}=\operatorname{Exp}($ Sheet1.Cells $(3,12) *$ Sheet1.Cells $(\mathbf{i}+3,3)+$ Sheet 1.Cells $(4,12) *$ Sheet1.Cells $(\mathrm{i}+3,4)+$ Sheet1.Cells $(10,12) *$ Sheet1.Cells $(\mathbf{i}+3,10))$

$\mathrm{VL}=\operatorname{Exp}(\operatorname{Sheet1.Cells}(3,12) *$ Sheet1.Cells $(\mathrm{i}+4,3)+\operatorname{Sheet} 1 . \operatorname{Cells}(4,12) *$ Sheet1.Cells $(i+4,4)+\operatorname{Sheet1} \cdot \operatorname{Cells}(11,12) * \operatorname{Sheet} 1 . \operatorname{Cells}(\mathrm{i}+4,11))$

Mode Choice Probability of each observations in Logit probability model

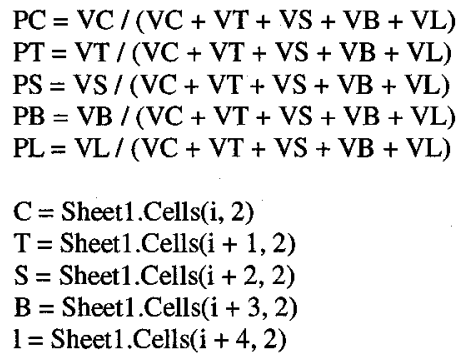

First-order Derivative with respect to $\beta_{1}$

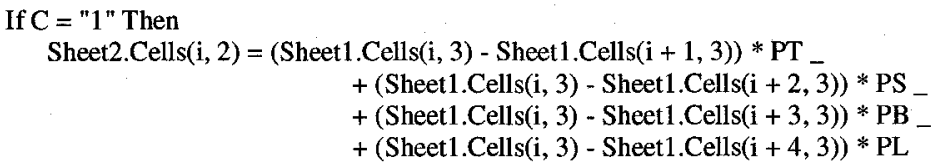


$+($ Sheet1.Cells $(i+1,3)-$ Sheet1.Cells $(i+4,3)) * P L$

Elself $S=$ " 1 " Then

Sheet2.Cells $(\mathrm{i}, 2)=($ Sheet1.Cells $(\mathrm{i}+2,3)-$ Sheet1.Cells $(\mathrm{i}, 3)) * \mathrm{PC}$

$+($ Sheet1.Cells $(\mathrm{i}+2,3)-$ Sheet1.Cells $(\mathrm{i}+\overline{1}, 3)) * \mathrm{PT}$

$+($ Sheet1.Cells $(i+2,3)$ - Sheet1.Cells $(i+3,3)) *$ PB

$+($ Sheet1.Cells $(\mathrm{i}+2,3)-$ Sheet1.Cells $(\mathrm{i}+4,3)) * \mathbf{P L}$

Elself $\mathrm{B}=$ " 1 " Then

Sheet2.Cells $(i, 2)=($ Sheet1.Cells $(i+3,3)-$ Sheet1.Cells $(i, 3)) * P C$

$+($ Sheet1.Cells $(\mathbf{i}+3,3)-$ Sheet1.Cells $(\mathbf{i}+1,3)) *$ PT

$+($ Sheet1.Cells $(\mathrm{i}+3,3)$ - Sheet1.Cells $(\mathrm{i}+2,3)) * \mathrm{PS}_{-}^{-}$

$+($ Sheet1.Cells $(\mathrm{i}+3,3)-$ Sheet1.Cells $(\mathrm{i}+4,3)) * \mathrm{PL}$

Else

Sheet2.Cells $(\mathrm{i}, 2)=($ Sheet 1.Cells $(\mathrm{i}+4,3)-$ Sheet1.Cells $(\mathrm{i}, 3)) * \mathrm{PC}$

$+($ Sheet1.Cells $(i+4,3)$ - Sheet1.Cells $(i+1,3)) *$ PT $+($ Sheet1.Cells $(\mathbf{i}+4,3)-$ Sheet 1. Cells $(i+2,3)) *$ PS $+($ Sheet1.Cells $(\mathrm{i}+4,3)-$ Sheet1.Cells $(\mathrm{i}+3,3)) *$ PB

End If

First-order Derivative with respect to $\beta_{2}$

If $\mathrm{C}=$ "1" Then

Sheet2.Cells $(\mathrm{i}, 3)=($ Sheet 1. Cells $(\mathrm{i}, 4)-$ Sheet1.Cells $(\mathrm{i}+1,4)) * \mathrm{PT}$

$+($ Sheet1.Cells $(i, 4)-$ Sheet1.Cells $(i+2,4)) *$ PS

$+($ Sheet1.Cells $(\mathrm{i}, 4)-$ Sheet1.Cells $(\mathrm{i}+3,4)) * \mathrm{~PB}$

$+($ Sheet1.Cells $(\mathrm{i}, 4)$ - Sheet1.Cells $(\mathrm{i}+4,4)) * \mathrm{PL}$

Elself $\mathrm{T}=$ " 1 " Then

Sheet2.Cells $(\mathrm{i}, 3)=($ Sheet 1 .Cells $(\mathrm{i}+1,4)-$ Sheet 1.Cells $(\mathrm{i}, 4)) * \mathrm{PC}$

$+($ Sheet1.Cells $(\mathrm{i}+1,4)-$ Sheet1.Cells $(\mathrm{i}+2,4)) * \mathrm{PS}$

$+($ Sheet1.Cells $(\mathrm{i}+1,4)-$ Sheet1.Cells $(\mathrm{i}+3,4)) * \mathrm{~PB}_{-}$

$+($ Sheet1.Cells $(\mathrm{i}+1,4)-$ Sheet1.Cells $(\mathrm{i}+4,4)) * \mathrm{PL}$

Elself $S=$ " 1 " Then

Sheet2.Cells $(\mathrm{i}, 3)=($ Sheet1.Cells $(\mathrm{i}+2,4)-$ Sheet1.Cells $(\mathrm{i}, 4)) * \mathrm{PC}$

$+($ Sheet1.Cells $(\mathrm{i}+2,4)$ - Sheet1.Cells $(\mathrm{i}+1,4)) * \mathrm{PT}$ $+($ Sheet1.Cells $(i+2,4)-$ Sheet1.Cells $(i+3,4)) *$ PB

$+($ Sheet1.Cells $(\mathrm{i}+2,4)-$ Sheet1.Cells $(\mathrm{i}+4,4)) * \mathrm{PL}$

ElseIf B = " 1 " Then

Sheet2.Cells $(\mathrm{i}, 3)=($ Sheet1.Cells $(\mathrm{i}+3,4)-$ Sheet1.Cells $(\mathrm{i}, 4)) * \mathrm{PC}$

$+($ Sheet 1.Cells $(\mathrm{i}+3,4)$ - Sheet1.Cells $(\mathrm{i}+1,4)) *$ PT

$+($ Sheet1.Cells $(\mathrm{i}+3,4)$ - Sheet1.Cells $(\mathrm{i}+2,4)) * \mathrm{PS}_{-}^{-}$

$+($ Sheet1.Cells $(\mathrm{i}+3,4)-$ Sheet1.Cells $(\mathrm{i}+4,4)) * \mathrm{PL}$

Else

Sheet2.Cells $(\mathbf{i}, 3)=($ Sheet1.Cells $(\mathbf{i}+4,4)-$ Sheet1.Cells $(\mathbf{i}, 4)) * \mathbf{P C}$

$+($ Sheet1.Cells $(i+4,4)$ - Sheet1.Cells $(i+1,4)) * P_{-}$

$+($ Sheet1.Cells $(i+4,4)-$ Sheet1.Cells $(i+2,4)) *$ PS

$+($ Sheet1.Cells $(i+4,4)-$ Sheet1.Cells $(i+3,4)) *$ PB

End If

First-order Derivative with respect to $\beta_{3}$

If $\mathrm{C}=$ " 1 " Then

Sheet2.Cells $(i, 4)=$ Sheet1.Cells $(i, 5) *(P T+P S+P B+P L)$

Else

Sheet2.Cells $(\mathbf{i}, 4)=-$ Sheet1.Cells $(\mathrm{i}, 5) * \mathrm{PC}$

End If 


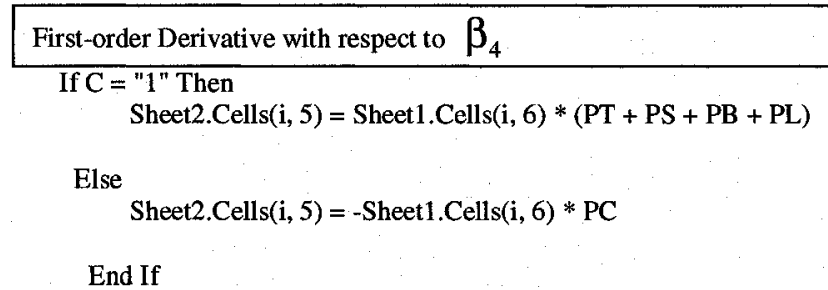

First-order Derivative with respect to $\beta_{5}$

If $\mathrm{C}=$ " 1 " Then

Sheet2.Cells $(\mathrm{i}, 6)=$ Sheet1.Cells $(\mathrm{i}, 7) *(\mathrm{PT}+\mathrm{PS}+\mathrm{PB}+\mathrm{PL})$

Else

Sheet2.Cells $(\mathrm{i}, 6)=-$ Sheet $1 . C e l l s(i, 7) *$ PC

End If

First-order Derivative with respect to $\beta_{6}$

If $\mathrm{T}=$ "1" Then

Sheet2.Cells $(\mathrm{i}, 7)=(\mathrm{PC}+\mathrm{PS}+\mathrm{PB}+\mathrm{PL})$

Else

Sheet 2.Cells $(\mathrm{i}, 7)=-(\mathrm{PT})$

End If
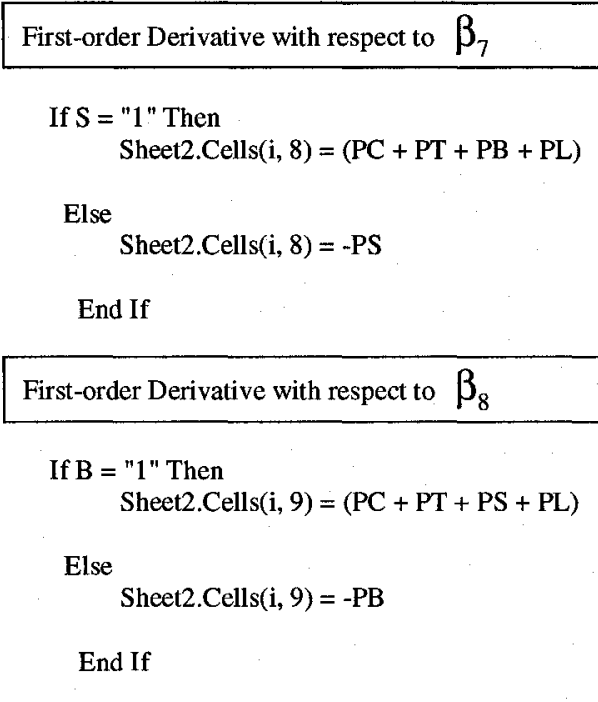

First-order Derivative with respect to $\beta_{9}$

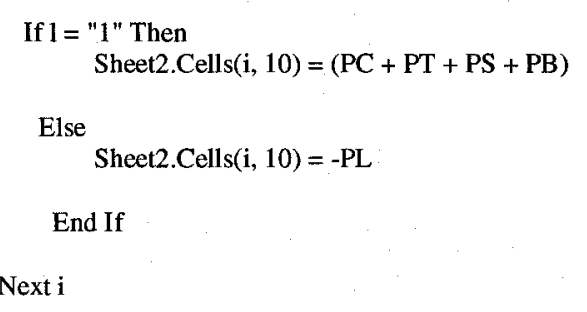


Arranging the data set for good to calculate and in simple form

For $\mathrm{i}=4$ To 542 Step 1

Sheet2.Range(Cells $(i, 2)$, Cells $(i+3,10)) \cdot$ Delete Shift: $=x l U p$

Next i

Obtaining outer product of each gradient value for all observations : $\left(G_{t n}\right)\left(G_{t n}\right)^{\prime}$

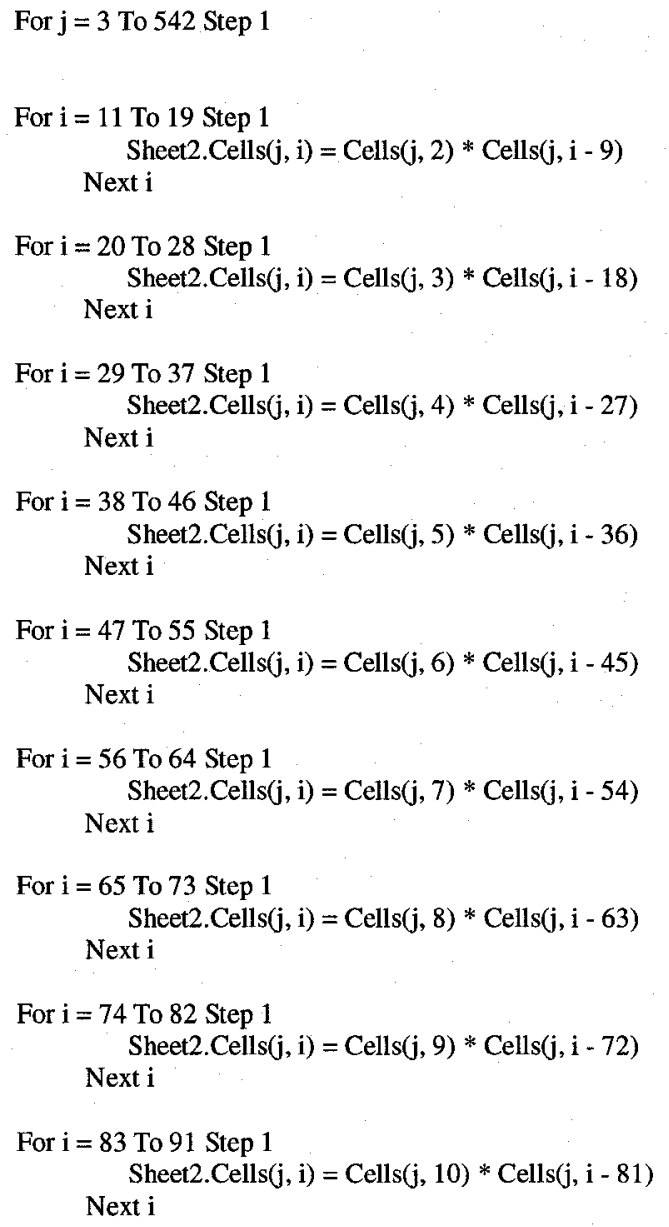

Next J

Summation of outer product of each observation gradient value and deviding the summation value with total observation number(540)

For J = 2 To 91 Step 1

Sheet2.Cells(543, J).FormulaR1C1 $=$ "=SUM(R[-540]C:R[-1]C)/540"

Next J

Sheets("sheet3").Select 
Obtaing the approximate Hessian matrix: $H_{t A P}^{B H H H}$

For $\mathrm{j}=1$ To 1 Step 1

For $\mathrm{i}=11$ To 19 Step 1

Sheet 3. Cells $(\mathrm{j}, \mathrm{i}-10)=$ Sheet 2 .Cells $(543, \mathrm{i})$.Value Next i

For $\mathrm{i}=20$ To 28 Step 1

Sheet3.Cells $(j+1, i-19)=$ Sheet 2 .Cells $(543, i)$.Value

Next $\mathrm{i}$

For $\mathrm{i}=29$ To 37 Step 1

Sheet3.Cells $(j+2, i-28)=$ Sheet $2 . \operatorname{Cells}(543, i)$.Value

Next i

For $\mathrm{i}=38$ To 46 Step 1

Sheet3.Cells $(j+3, i-37)=$ Sheet 2 .Cells $(543, i)$. Value

Next i

For $\mathrm{i}=47$ To 55 Step 1

Sheet3.Cells $(j+4, i-46)=$ Sheet $2 \cdot \operatorname{Cells}(543, i)$. Value

Next i

For $\mathrm{i}=56$ To 64 Step 1

Sheet $3 \cdot \operatorname{Cells}(j+5, i-55)=$ Sheet 2 .Cells $(543, i)$.Value

Next i

For $\mathrm{i}=65$ To 73 Step 1

Sheet3.Cells $(j+6, i-64)=$ Sheet2.Cells $(543, i)$.Value

Next $\mathrm{i}$

For $i=74$ To 82 Step 1

Sheet3.Cells $(j+7, i-73)=$ Sheet2.Cells $(543, i)$.Value

Next i

For $\mathbf{i}=83$ To 91 Step 1

Sheet3.Cells $(j+8, i-82)=$ Sheet 2 .Cells $(543, i)$.Value

Next i

Next J

Obtaing the inverse of approximate Hessian matrix: $\left(H_{t A P}^{B H H H}\right)^{-1}$

Sheet3.Range("A11:I19").FormulaArray = "=(MINVERSE(A1:I9))"

Reproduced with permission of the copyright owner. Further reproduction prohibited without permission. 
Obtaing the gradient value: $G_{t}$

For $\mathrm{i}=11$ To 19 Step 1

Sheet3.Cells $(\mathrm{i}, 11)=$ Sheet 2. Cells $(543, \mathrm{i}-9)$.Value

Next $\mathrm{i}$

Calculating parameter estimates: $\beta_{t+1}=\beta_{t}+\lambda_{t} \Delta_{t}=\beta_{t}+\lambda_{t} W_{t} G_{t}=\beta_{t}+\lambda_{t}\left(H_{t A P}^{B H H H}\right)^{-1} G_{t}$

Sheet3.Range("M11:M19").FormulaArray = "=MMULT(A11:I19,K11:K19)"

Sheet3.Cells $(11,14)=$ Sheet1.Cells $(3,12)+$ Sheet3.Cells $(11,13)$

Sheet3.Cells $(12,14)=$ Sheet 1 .Cells $(4,12)+$ Sheet 3 .Cells $(12,13)$

Sheet 3. Cells $(13,14)=$ Sheet 1 .Cells $(5,12)+$ Sheet 3 .Cells $(13,13)$

Sheet3.Cells $(14,14)=$ Sheet 1. Cells $(6,12)+$ Sheet3.Cells $(14,13)$

Sheet3.Cells $(15,14)=$ Sheet1.Cells $(7,12)+$ Sheet 3 .Cells $(15,13)$

Sheet3.Cells $(16,14)=$ Sheet 1 .Cells $(8,12)+$ Sheet 3 .Cells $(16,13)$

Sheet3.Cells $(17,14)=$ Sheet 1 .Cells $(9,12)+$ Sheet $3 \cdot \operatorname{Cells}(17,13)$

Sheet3.Cells $(18,14)=$ Sheet 1. Cells $(10,12)+$ Sheet3.Cells $(18,13)$

Sheet3.Cells $(19,14)=$ Sheet 1. Cells $(11,12)+$ Sheet3.Cells $(19,13)$

Checking the Criterion for stopping: $\left[\frac{1}{k} \sum_{k=1}^{k}\left(\beta_{t+1, k}-\beta_{t, k}\right)^{2}\right]^{1 / 2}<C R$

Sheet 3 .Cells $(21,12)=(($

(Sheet3.Cells $(11,14)-$ Sheet1.Cells $(3,12))^{\wedge} 2+(\operatorname{Sheet3.Cells~}(12,14)-\text { Sheet1.Cells }(4,12))^{\wedge} 2$ $+($ Sheet3.Cells $(13,14)-$ Sheet 1.Cells $(5,12)) \wedge 2+($ Sheet3.Cells $(14,14)-$ Sheet1.Cells $(6,12)) \wedge 2$ $+(\text { Sheet3.Cells }(15,14)-\text { Sheet1.Cells }(7,12))^{\wedge} 2+(\text { Sheet3.Cells }(16,14)-\text { Sheet1.Cells }(8,12))^{\wedge} 2$ $+(\text { Sheet3.Cells }(17,14)-\text { Sheet1.Cells }(9,12))^{\wedge} 2+(\operatorname{Sheet3.Cells}(18,14)-\operatorname{Sheet1.Cells}(10,12))^{\wedge} 2$ $+(\text { Sheet3.Cells }(19,14)-\text { Sheet1.Cells }(11,12))^{\wedge} 2_{-}$

If Sheet3.Cells $(21,12)<10^{\wedge}(-4)$ Then

$$
)^{*}(1 / 9)\right)^{\wedge}(0.5)
$$

GoTo line1

Else

GoTo line2

End If

line1:

End Sub 


\section{B.3: BHHH-2 algorithm}

The third algorithm : $\mathrm{BHHH}-2$

(Visual Basic Application provided in the Microsoft EXCEL)

Coded by Hyukjae Roh

Private Sub BHHH-2_ClickO

Variables Generated

Dim i As Integer

Dim C As Integer

Dim T As Integer

Dim S As Integer

Dim B As Integer

Dim 1 As Integer

Dim VC As Double

Dim VT As Double

Dim VS As Double

Dim VB As Double

Dim VL As Double

Dim PC As Double

Dim PT As Double

Dim PS As Double

Dim PB As Double

Dim PL As Double

$\mathrm{k}=1$

If $\mathrm{k}=1$ Then

GoTo line3:

End If

Sheets("sheet1").Select

Initializing Staring Values of Parameters

line2:

$\mathrm{k}=\mathrm{k}+1$

line3:

Sheet1.Cells $(3,12)=$ Sheet 3 .Cells $(11,14)$

Sheet 1 .Cells $(4,12)=$ Sheet 3 .Cells $(12,14)$

Sheet 1 .Cells $(5,12)=$ Sheet 3. Cells $(13,14)$

Sheet 1 .Cells $(6,12)=$ Sheet 3 .Cells $(14,14)$

Sheet 1 .Cells $(7,12)=$ Sheet 3 .Cells $(15,14)$

Sheet 1 .Cells $(8,12)=$ Sheet 3 .Cells $(16,14)$

Sheet 1.Cells $(9,12)=$ Sheet 3. Cells $(17,14)$

Sheet 1 .Cells $(10,12)=$ Sheet 3 .Cells $(18,14)$

Sheet 1 . Cells $(11,12)=$ Sheet $3 \cdot \operatorname{Cells}(19,14)$

Calculation of the Log-likelihood function value

For $\mathrm{J}=3$ To 2698 Step 5

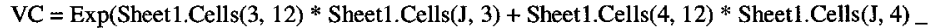

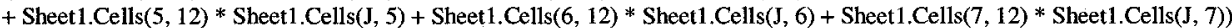

$\mathrm{VT}=\operatorname{Exp}(\operatorname{Sheet1.Cells}(3,12) *$ Sheet1.Cells $(\mathrm{J}+1,3)+\operatorname{Sheet1.Cells}(4,12) * \operatorname{Sheet1.Cells}(\mathrm{J}+1,4)+\operatorname{Sheet1.Cells~}(8,12) * \operatorname{Sheet} 1 . \operatorname{Cells}(\mathrm{J}+1,8))$

VS $=\operatorname{Exp}(\operatorname{Sheet1} . C e l l s(3,12) *$ Sheet1.Cells $(\mathrm{J}+2,3)+\operatorname{Sheet1.Cells~}(4,12) * \operatorname{Sheet1}$.Cells $(\mathrm{J}+2,4)+\operatorname{Sheet1.Cells~}(9,12) * \operatorname{Sheet1.Cells~}(\mathrm{J}+2,9))$

$\mathrm{VB}=\operatorname{Exp}(\operatorname{Sheet1.Cells}(3,12) *$ Sheet1.Cells $(\mathrm{J}+3,3)+\operatorname{Sheet1.Cells~}(4,12) *$ Sheet1.Cells $(\mathrm{J}+3,4)+$ Sheet1.Cells $(10,12) * \operatorname{Sheet} 1 . C e l l s(J+3,10))$

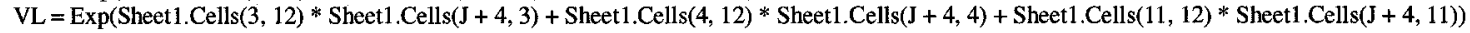




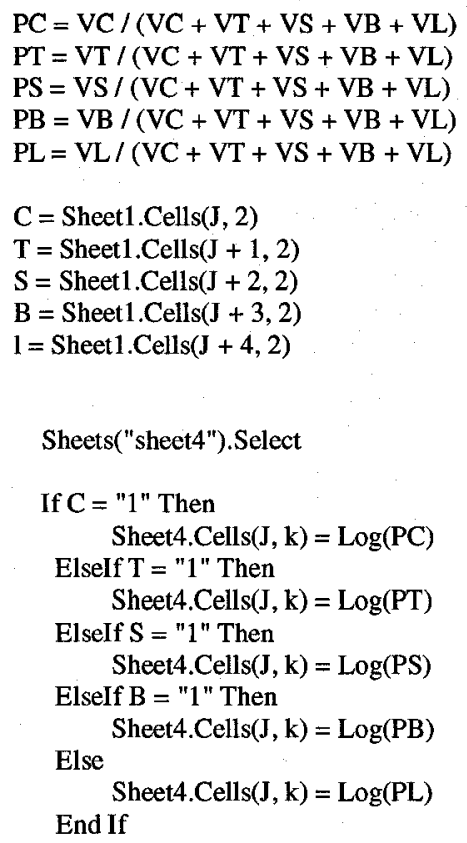

Mode Choice Probability of each observations in Logit probability model

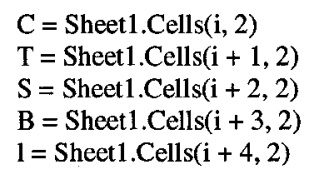

First-order Derivative with respect to $\beta_{1}$

If $\mathrm{C}=$ " 1 " Then

Sheet2.Cells $(\mathbf{i}, 2)=($ Sheet1.Cells $(i, 3)-$ Sheet1.Cells $(i+1,3)) *$ PT

$+($ Sheet1.Cells $(i, 3)-$ Sheet1.Cells $(i+2, \overline{3})) *$ PS

$+($ Sheet 1.Cells $(\mathrm{i}, 3)$ - Sheet 1.Cells $(\mathrm{i}+3,3)) * \mathrm{~PB}$

$+($ Sheet1.Cells $(i, 3)-$ Sheet1.Cells $(i+4,3)) *$ PL 


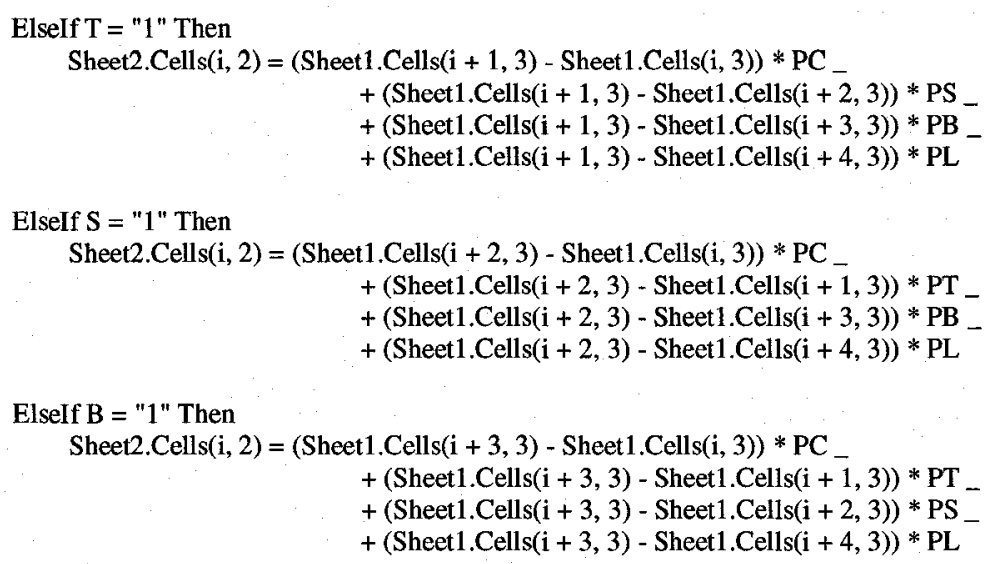

Else

Sheet2.Cells $(i, 2)=($ Sheet1.Cells $(i+4,3)-$ Sheet1.Cells $(i, 3)) * P C$ $+($ Sheet 1.Cells $(i+4,3)-$ Sheet 1.Cells $(i+1,3)) *$ PT $+($ Sheet1.Cells $(\mathrm{i}+4,3)$ - Sheet1.Cells $(\mathbf{i}+2,3)) * \mathrm{PS}^{-}$ $+($ Sheet1.Cells $(\mathrm{i}+4,3)-$ Sheet1.Cells $(\mathrm{i}+3,3)) *$ PB

End If

First-order Derivative with respect to $\beta_{2}$

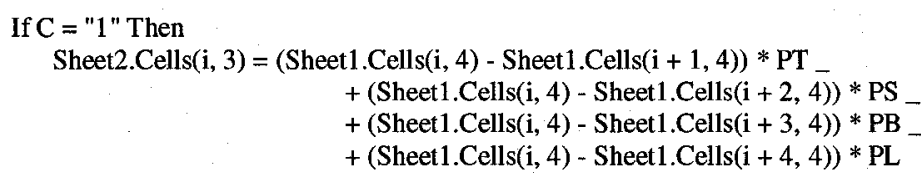

ElseIf $\mathrm{T}=$ " 1 " Then

Sheet2.Cells $(\mathbf{i}, 3)=($ Sheet1.Cells $(i+1,4)-$ Sheet1.Cells $(i, 4)) * P C$

$+($ Sheet1.Cells $(\mathbf{i}+1,4)-$ Sheet1.Cells $(\mathrm{i}+2,4)) * \mathrm{PS}_{-}$

$+($ Sheet1.Cells $(\mathrm{i}+1,4)-$ Sheet1.Cells $(\mathrm{i}+3,4)) * \mathrm{~PB}$

$+($ Sheet1.Cells $(\mathrm{i}+1,4)-$ Sheet1.Cells $(\mathrm{i}+4,4)) * \mathrm{PL}$

Elself $\mathrm{S}=$ "1" Then

Sheet2.Cells $(\mathbf{i}, 3)=($ Sheet1.Cells $(i+2,4)-$ Sheet1.Cells $(i, 4)) * P C$

$+($ Sheet 1.Cells $(\mathrm{i}+2,4)-$ Sheet 1.Cells $(\mathrm{i}+1,4)) * \mathrm{PT}$

$+($ Sheet1.Cells $(\mathrm{i}+2,4)$ - Sheet1.Cells $(\mathrm{i}+3,4)) * \mathrm{~PB}$

$+($ Sheet1.Cells $(\mathrm{i}+2,4)$ - Sheet1.Cells $(\mathrm{i}+4,4)) * \mathrm{PL}$

ElseIf B = " 1 " Then

Sheet2.Cells $(i, 3)=($ Sheet1.Cells $(i+3,4)-$ Sheet 1. Cells $(i, 4)) * P C$

$+($ Sheet1.Cells $(i+3,4)-$ Sheet1.Cells $(i+1,4)) * P T$ $+($ Sheet1.Cells $(i+3,4)-$ Sheet1.Cells $(i+2,4)) *$ PS

$+($ Sheet1.Cells $(\mathrm{i}+3,4)-$ Sheet1.Cells $(i+4,4)) *$ PL

Else

Sheet2.Cells $(\mathrm{i}, 3)=($ Sheet1.Cells $(\mathrm{i}+4,4)-$ Sheet 1. Cells $(\mathrm{i}, 4)) * \mathrm{PC}$

$+($ Sheet 1.Cells $(\mathrm{i}+4,4)-$ Sheet 1 Cells $(\mathrm{i}+1,4)) * \mathrm{PT}$

$+($ Sheet1.Cells $(\mathrm{i}+4,4)-$ Sheet1.Cells $(\mathrm{i}+2,4)) * \mathrm{PS}$ -

$+($ Sheet1.Cells $(\mathrm{i}+4,4)$ - Sheet1.Cells $(\mathrm{i}+3,4)) * \mathrm{~PB}$

End If

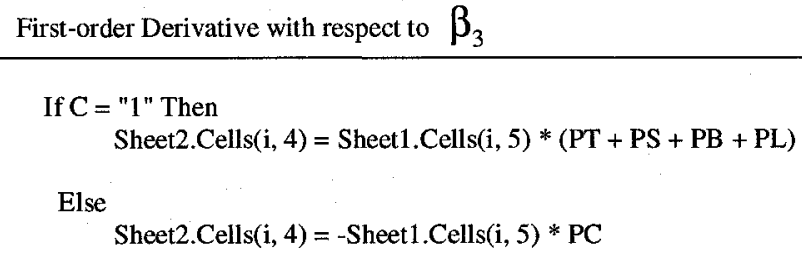


End If

First-order Derivative with respect to $\beta_{4}$

If $\mathbf{C}=$ " 1 " Then

Sheet2.Cells $(i, 5)=$ Sheet1.Cells $(i, 6) *(P T+P S+P B+P L)$

Else

Sheet2.Cells $(\mathbf{i}, 5)=-$ Sheet1.Cells $(\mathbf{i}, 6) *$ PC

End If

First-order Derivative with respect to $\beta_{5}$

If $\mathrm{C}=$ " 1 " Then

Sheet2.Cells $(\mathbf{i}, 6)=$ Sheet1.Cells $(\mathrm{i}, 7) *(\mathrm{PT}+\mathrm{PS}+\mathrm{PB}+\mathrm{PL})$

Else

Sheet2.Cells $(\mathrm{i}, 6)=-$ Sheet1.Cells $(\mathrm{i}, 7) *$ PC

End If

First-order Derivative with respect to $\beta_{6}$

If $\mathrm{T}=$ " 1 " Then

Sheet2.Cells $(i, 7)=(P C+P S+P B+P L)$

Else

Sheet 2. Cells $(\mathrm{i}, 7)=-(\mathrm{PT})$

End If

First-order Derivative with respect to $\beta_{7}$

If $S=$ "1" Then

Sheet2.Cells $(\mathrm{i}, 8)=(\mathrm{PC}+\mathrm{PT}+\mathrm{PB}+\mathrm{PL})$

Else

Sheet2.Cells $(\mathrm{i}, 8)=-\mathrm{PS}$

End If

First-order Derivative with respect to $\beta_{8}$

If $B=$ "1" Then

Sheet2.Cells $(\mathrm{i}, 9)=(\mathrm{PC}+\mathrm{PT}+\mathrm{PS}+\mathrm{PL})$

Else

Sheet2.Cells $(\mathbf{i}, 9)=-\mathrm{PB}$

End If

First-order Derivative with respect to $\beta_{9}$

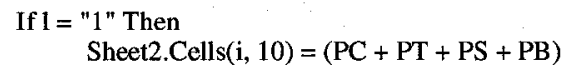

Else

Sheet2.Cells $(\mathbf{i}, 10)=-\mathrm{PL}$

End If

Next i

Arrange the data set for good to calculate and in simple form 
For $i=4$ To 542 Step 1

Sheet2.Range(Cells(i, 2), Cells $(i+3,10))$.Delete Shift:=xlUp

Next i

Obtaining Gradient components calculated, summation of all observation and averaging value of it $G_{t}$

For $\mathrm{J}=2$ To 10 Step 1

Sheet2.Cells $(543, \mathrm{~J})$. FormulaR1C1 = "=SUM(R[-540]C:R[-1]C)/540 "

Next J

Subtracting the mean gradient of all observations from gradient value of each observation $\left(G_{t n}-G_{t}\right)$

For $\mathrm{j}=2$ To 10 Step 1

For $\mathrm{i}=3$ To 542 Step 1

Sheet2.Cells $(i, j)=$ Sheet $2 . \operatorname{Cell} s(i, j)-\operatorname{Sheet} 2 . \operatorname{Cells}(543, j)$

Next i

Next J

Obtaining outer product of each gradient value for all observations : $\left(G_{t n}-G_{t}\right)\left(G_{t n}-G_{t}\right)^{\prime}$

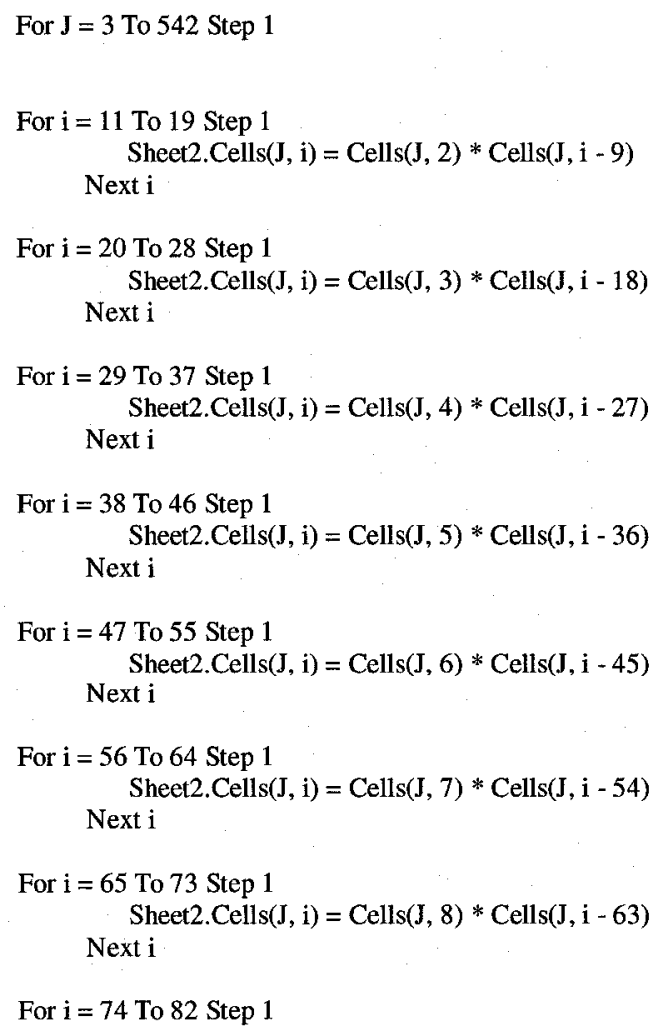




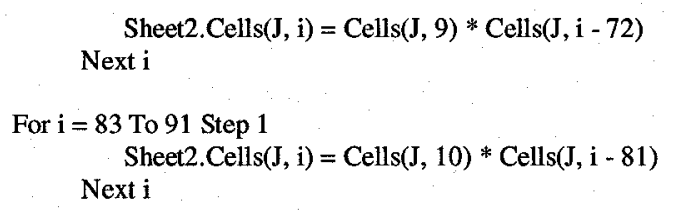

Next J

\begin{tabular}{|l} 
Summation of outer product of each observation gradient value and averaging the summation value: \\
$\sum_{i=1}^{N} \frac{\left(G_{t n}-G_{t}\right)\left(G_{t n}-G_{t}\right)^{\prime}}{N}$
\end{tabular}

For $\mathbf{J}=11$ To 91 Step 1

Sheet2.Cells(543, J).FormulaR1C1 = "=SUM(R[-540]C:R[-1]C)/540"

Next J

Sheets("sheet3").Select

Obtaining the approximate Hessian matrix : $H_{t A P}^{B H H H-2}$

For $\mathrm{j}=1$ To 1 Step 1

For $\mathrm{i}=11$ To 19 Step 1

Next $\mathbf{i}$

Sheet3.Cells $(j, i-10)=$ Sheet2.Cells $(543, i)$.Value

For $\mathrm{i}=20$ To 28 Step 1

Sheet $3 \cdot \operatorname{Cells}(j+1, i-19)=\operatorname{Sheet} 2 \cdot \operatorname{Cell} s(543, i)$.Value

Next i

For $\mathrm{i}=29$ To 37 Step 1

Sheet 3. Cells $(j+2, i-28)=$ Sheet $2 \cdot$ Cells $(543, i)$. Value

Next i

For $\mathrm{i}=38$ To 46 Step 1

Sheet $3 \cdot \operatorname{Cells}(j+3, i-37)=$ Sheet $2 \cdot \operatorname{Cells}(543, i)$.Value

Next $\mathbf{i}$

For $\mathrm{i}=47$ To 55 Step 1

Sheet3.Cells $(j+4, i-46)=$ Sheet2.Cells $(543$, i). Value

Next $\mathrm{i}$

For $\mathrm{i}=56$ To 64 Step 1

Sheet 3. Cells $(j+5, i-55)=$ Sheet $2 \cdot$ Cells $(543, i)$.Value

Next $\mathrm{i}$

For $\mathrm{i}=65$ To 73 Step 1

Sheet3.Cells $(j+6, i-64)=$ Sheet 2 .Cells $(543, i)$.Value 
Next $\mathrm{i}$

For $\mathrm{i}=74$ To 82 Step 1

Sheet 3 .Cells $(j+7, i-73)=$ Sheet $2 \cdot \operatorname{Cell} s(543, i)$.Value

Next i

For $\mathrm{i}=83$ To 91 Step 1

Sheet 3 .Cells $(j+8, i-82)=$ Sheet 2 .Cells $(543, i)$.Value

Next i

Next J

Obtaining the inverse matrix of approximate Hessian matrix $:\left(H_{t A P}^{B H H H-2}\right)^{-1}$

Sheet3.Range("A11:I19").FormulaArray = "=(MINVERSE(A1:I9))"

Obtaing the gradient value: $G_{t}$

For $\mathrm{i}=11$ To 19 Step 1

Sheet 3. Cells $(i, 11)=$ Sheet $2 \cdot$ Cells $(543, i-9)$.Value

Next i

Parameter estimates: $\beta_{t+1}=\beta_{t}+\lambda_{t} \Delta_{t}=\beta_{t}+\lambda_{t} W_{t} G_{t}=\beta_{t}+\lambda\left(H_{t A P}^{B H H H-2}\right)^{-1} G_{t}$

Sheet3.Range("M11:M19").FormulaArray = "=2*MMULT(A11:I19,K11:K19)"

Sheet3.Cells $(11,14)=$ Sheet 1. Cells $(3,12)+$ Sheet 3. Cells $(11,13)$

Sheet3.Cells $(12,14)=$ Sheet 1 .Cells $(4,12)+$ Sheet 3 .Cells $(12,13)$

Sheet3.Cells $(13,14)=$ Sheet1.Cells $(5,12)+$ Sheet 3. Cells $(13,13)$

Sheet3.Cells $(14,14)=$ Sheet 1 .Cells $(6,12)+$ Sheet 3 .Cells $(14,13)$

Sheet3.Cells $(15,14)=$ Sheet1.Cells $(7,12)+$ Sheet3.Cells $(15,13)$

Sheet3.Cells $(16,14)=$ Sheet 1. Cells $(8,12)+$ Sheet 3. Cells $(16,13)$

Sheet3.Cells $(17,14)=$ Sheet1 $\operatorname{Cells}(9,12)+$ Sheet 3. Cells $(17,13)$

Sheet 3 .Cells $(18,14)=$ Sheet 1 .Cells $(10,12)+$ Sheet 3. Cells $(18,13)$

Sheet 3 .Cells $(19,14)=$ Sheet 1 .Cells $(11,12)+$ Sheet 3. Cells $(19,13)$

Checking the Criterion for stopping: $\left[\frac{1}{k} \sum_{k=1}^{k}\left(\beta_{t+1, k}-\beta_{t, k}\right)^{2}\right]^{1 / 2}<C R$

Sheet3.Cells $(21,12)=(($

(Sheet3.Cells $(11,14)-$ Sheet1.Cells $(3,12))^{\wedge} 2+(\text { Sheet3.Cells }(12,14)-\text { Sheet1.Cells }(4,12))^{\wedge} 2$ $+(\text { Sheet3.Cells }(13,14)-\text { Sheet1.Cells }(5,12))^{\wedge} 2+(\text { Sheet3.Cells }(14,14)-\text { Sheet1.Cells }(6,12))^{\wedge} 2-$ $+(\text { Sheet3.Cells }(15,14)-\text { Sheet 1.Cells }(7,12))^{\wedge} 2+(\text { Sheet3.Cells }(16,14)-\text { Sheet 1.Cells }(8,12))^{\wedge} 2$

$+(\text { Sheet3.Cells }(17,14)-\text { Sheet1.Cells }(9,12))^{\wedge} 2+(\text { Sheet3.Cells }(18,14)-\text { Sheet1.Cells }(10,12))^{\wedge} 2-$ 
$+(\text { Sheet3.Cells }(19,14)-\text { Sheet 1.Cells }(11,12))^{\wedge} 2$

$$
)^{*}(1 / 9)\right)^{\wedge}(0.5)
$$

If Sheet3.Cells $(21,12)<10^{\wedge}(-4)$ Then

GoTo line1

Else

GoTo line2

End If

line1:

End Sub 


\section{B.4: Steepest Ascent (SA) algorithm}

The fourth algorithm: Stepest Ascent $(\mathrm{SA})$
(Visual Basic Application provided in the Microsoft EXCEL)
Coded by Hyukjae Roh

Private Sub SteepestAscent_Click()

Variables Generated

$$
\begin{aligned}
& \text { Dim i, } \mathbf{j}, \mathbf{k} \text { As Integer } \\
& \text { Dim C As Integer } \\
& \text { Dim T As Integer } \\
& \text { Dim S As Integer } \\
& \text { Dim B As Integer } \\
& \text { Dim L As Integer } \\
& \text { Dim VC As Double } \\
& \text { Dim VT As Double } \\
& \text { Dim VS As Double } \\
& \text { Dim VB As Double } \\
& \text { Dim VL As Double } \\
& \text { Dim PC As Double } \\
& \text { Dim PT As Double } \\
& \text { Dim PS As Double } \\
& \text { Dim PB As Double } \\
& \text { Dim PL As Double } \\
& \\
& \text { k }=1 \\
& \text { If } k=1 \text { Then } \\
& \text { GoTo line3 } \\
& \text { End If }
\end{aligned}
$$

Sheets("sheet1").Select

Initializing Staring Values of Parameters

line2:

$\mathrm{k}=\mathrm{k}+1$

line3:

Sheet1.Cells $(3,12)=$ Sheet $3 \cdot \operatorname{Cells}(11,14)$

Sheet1.Cells $(4,12)=$ Sheet 3. Cells $(12,14)$

Sheet1.Cells $(5,12)=$ Sheet3.Cells $(13,14)$

Sheet1.Cells $(6,12)=$ Sheet 3 .Cells $(14,14)$

Sheet1.Cells $(7,12)=$ Sheet $3 \cdot \operatorname{Cells}(15,14)$

Sheet 1 .Cells $(8,12)=$ Sheet 3 .Cells $(16,14)$

Sheet1.Cells $(9,12)=$ Sheet $3 \cdot \operatorname{Cells}(17,14)$

Sheet 1 .Cells $(10,12)=$ Sheet 3 .Cells $(18,14)$

Sheet 1 .Cells $(11,12)=$ Sheet 3. Cells $(19,14)$

Adding worksheets for calculation of Log-likelihood function value (sheet 1)

If $256-\mathrm{k}>=0$ Then

Sheets("sheet1").Select

For $\mathrm{j}=3$ To 2698 Step 5 
$\mathrm{VC}=\operatorname{Exp}($ Sheet1.Cells $(3,12) *$ Sheet1 Cells $(\mathrm{j}, 3)+$ Sheet1.Cells $(4,12) *$ Sheet1 .Cells $(\mathrm{j}, 4)$

+ Sheet1.Cells $(5,12) *$ Sheet1.Cells $(\mathrm{j}, 5)+\operatorname{Sheet1.Cells}(6,12) *$ Sheet1.Cells $(\mathrm{j}, 6)+\operatorname{Sheet1.Cells~}(7,12) *$ Sheet 1. Cells $(\mathrm{j}, 7))$

$\mathrm{VT}=\operatorname{Exp}(\operatorname{Sheet1.Cells}(3,12) *$ Sheet1.Cells $(\mathrm{j}+1,3)+\operatorname{Sheet1.Cells}(4,12) *$ Sheet1.Cells $(\mathrm{j}+1,4)+\operatorname{Sheet1.Cells}(8,12) * \operatorname{Sheet1.Cells}(\mathrm{j}+1,8))$ $V S=\operatorname{Exp}(\operatorname{Sheet1.Cells~}(3,12) *$ Sheet1.Cells $(j+2,3)+\operatorname{Sheet1.Cells~}(4,12) *$ Sheet1.Cells $(j+2,4)+\operatorname{Sheet} 1 . \operatorname{Cell} s(9,12) * \operatorname{Sheet} 1 . \operatorname{Cells}(j+2,9))$

VB $=\operatorname{Exp}($ Sheet 1.Cells $(3,12) *$ Sheet1.Cells $(j+3,3)+$ Sheet1.Cells $(4,12) *$ Sheet1.Cells $(j+3,4)+$ Sheet 1.Cells $(10,12) *$ Sheet1.Cells $(j+3,10))$

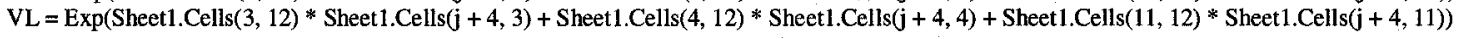

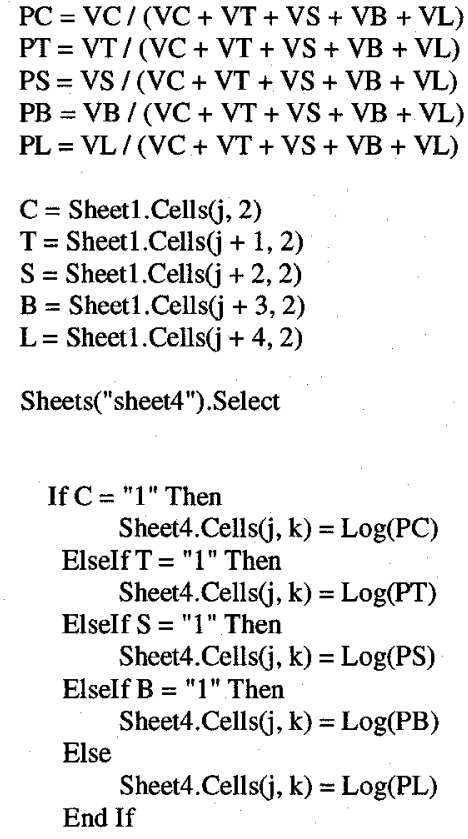

Sheets("sheet4").Select

Next $j$

Adding worksheets for calculation of Log-likelihood function value (sheet 2)

ElseIf $\mathrm{k}-256<=256$ Then

Sheets("sheet1").Select

For $\mathrm{j}=3$ To 2698 Step 5

VC $=\operatorname{Exp}($ Sheet 1.Cells $(3,12) *$ Sheet1.Cells $(\mathrm{j}, 3)+$ Sheet1.Cells $(4,12) *$ Sheet1.Cells $(\mathrm{j}, 4)$

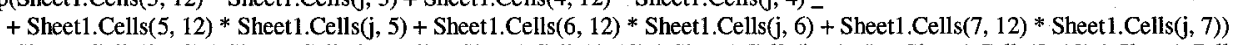

VT $=\operatorname{Exp}(\operatorname{Sheet} 1 . C e l l s(3,12) *$ Sheet1.Cells $(j+1,3)+$ Sheet1.Cells $(4,12) *$ Sheet1.Cells $(j+1,4)+$ Sheet 1.Cells $(8,12) *$ Sheet1.Cells $(j+1,8))$

$\mathrm{VS}=\operatorname{Exp}($ Sheet 1.Cells $(3,12) *$ Sheet1.Cells $(j+2,3)+$ Sheet1.Cells $(4,12) *$ Sheetl.Cells $(j+2,4)+$ Sheet1.Cells $(9,12) *$ Sheet1.Cells $(j+2,9))$

VB $=\operatorname{Exp}(\operatorname{Sheet1.Cells}(3,12) *$ Sheet1.Cells $(j+3,3)+$ Sheet1.Cells $(4,12) *$ Sheet1.Cells $(j+3,4)+$ Sheet 1. Cells $(10,12) *$ Sheet 1. Cells $(j+3,10))$

$\mathrm{VL}=\operatorname{Exp}(\operatorname{Sheet1.Cells}(3,12) *$ Sheet1.Cells $(\mathrm{j}+4,3)+\operatorname{Sheet1.Cells~}(4,12) *$ Sheet1.Cells $(\mathrm{j}+4,4)+\operatorname{Sheet} 1 . \operatorname{Cell} \mathrm{s}(11,12) * \operatorname{Sheet} 1 . \operatorname{Cell} \mathrm{s}(\mathrm{j}+4,11))$

$\mathrm{PC}=\mathrm{VC} /(\mathrm{VC}+\mathrm{VT}+\mathrm{VS}+\mathrm{VB}+\mathrm{VL})$

$\mathrm{PT}=\mathrm{VT} /(\mathrm{VC}+\mathrm{VT}+\mathrm{VS}+\mathrm{VB}+\mathrm{VL})$

$\mathrm{PS}=\mathrm{VS} /(\mathrm{VC}+\mathrm{VT}+\mathrm{VS}+\mathrm{VB}+\mathrm{VL})$

$\mathrm{PB}=\mathrm{VB} /(\mathrm{VC}+\mathrm{VT}+\mathrm{VS}+\mathrm{VB}+\mathrm{VL})$

$\mathrm{PL}=\mathrm{VL} /(\mathrm{VC}+\mathrm{VT}+\mathrm{VS}+\mathrm{VB}+\mathrm{VL})$

$\mathrm{C}=$ Sheet1. Cells $(\mathbf{j}, 2)$

$\mathrm{T}=$ Sheet1.Cells $(\mathrm{j}+1,2)$

$\mathrm{S}=$ Sheet1.Cells $(\mathrm{j}+2,2)$

$B=$ Sheet 1. Cells $(j+3,2)$

$\mathrm{L}=$ Sheet1.Cells $(\mathrm{j}+4,2)$

Sheets("sheet5").Select

$$
\begin{aligned}
& \text { If } \mathrm{C}=\text { " } 1 \text { " Then } \\
& \text { Sheet5.Cells }(\mathrm{j}, \mathrm{k}-256)=\log (\mathrm{PC}) \\
& \text { ElseIf } \mathrm{T}=\text { "1" Then } \\
& \text { Sheet5.Cells }(\mathrm{j}, \mathrm{k}-256)=\log (\mathrm{PT}) \\
& \text { Elself } \mathrm{S}=\text { "1" Then }
\end{aligned}
$$




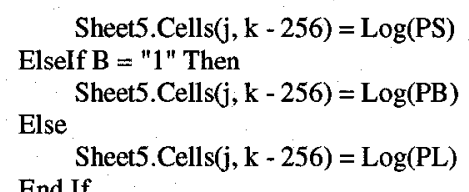

Next $\mathbf{j}$

Adding worksheets for calculation of Log-likelihood function value (sheet 3)

Elself $\mathrm{k}-512<=256$ Then

Sheets("sheet1").Select

For $\mathbf{j}=3$ To 2698 Step 5

$\mathrm{VC}=\operatorname{Exp}($ Sheet1.Cells $(3,12) *$ Sheet1.Cells $(\mathrm{j}, 3)+$ Sheet1.Cells $(4,12) *$ Sheet1.Cells $(\mathrm{j}, 4)$

+ Sheet1.Cells $(5,12) *$ Sheet1.Cells $(\mathfrak{j}, 5)+\operatorname{Sheet1.Cells}(6,12) * \operatorname{Sheet1.Cells~}(\mathrm{j}, 6)+\operatorname{Sheet1.Cells}(7,12) *$ Sheet 1. Cells $(\mathfrak{j}, 7))$

$\mathrm{VT}=\operatorname{Exp}(\operatorname{Sheet1}$.Cells $(3,12) *$ Sheet1.Cells $(j+1,3)+\operatorname{Sheet1.Cells~}(4,12) *$ Sheet1.Cells $(j+1,4)+\operatorname{Sheet1} 1$ Cells $(8,12) * \operatorname{Sheet1}$.Cells $(j+1,8))$

VS $=\operatorname{Exp}(\operatorname{Sheet1} \cdot \operatorname{Cells}(3,12) *$ Sheet1.Cells $(j+2,3)+$ Sheet1.Cells $(4,12) *$ Sheet1.Cells $(j+2,4)+$ Sheet1.Cells $(9,12) *$ Sheet1.Cells $(j+2,9))$

$\mathrm{VB}=\operatorname{Exp}(\operatorname{Sheet} 1$. Cells $(3,12) *$ Sheet1.Cells $(j+3,3)+\operatorname{Sheet1}$.Cells $(4,12) *$ Sheet1.Cells $(j+3,4)+\operatorname{Sheet1.Cells~}(10,12) *$ Sheet1.Cells $(j+3,10))$

$\mathrm{VL}=\operatorname{Exp}(\operatorname{Sheet1} . \operatorname{Cells}(3,12) *$ Sheet1.Cells $(j+4,3)+$ Sheet1.Cells $(4,12) *$ Sheet1.Cells $(j+4,4)+$ Sheet1.Cells $(11,12) *$ Sheet 1.Cells $(j+4,11))$

$\mathrm{PC}=\mathrm{VC} /(\mathrm{VC}+\mathrm{VT}+\mathrm{VS}+\mathrm{VB}+\mathrm{VL})$

$\mathrm{PT}=\mathrm{VT} /(\mathrm{VC}+\mathrm{VT}+\mathrm{VS}+\mathrm{VB}+\mathrm{VL})$

$\mathrm{PS}=\mathrm{VS} /(\mathrm{VC}+\mathrm{VT}+\mathrm{VS}+\mathrm{VB}+\mathrm{VL})$

$\mathrm{PB}=\mathrm{VB} /(\mathrm{VC}+\mathrm{VT}+\mathrm{VS}+\mathrm{VB}+\mathrm{VL})$

$P L=V L /(V C+V T+V S+V B+V L)$

$\mathrm{C}=$ Sheet $1 . \operatorname{Cells}(\mathrm{j}, 2)$

$\mathbf{T}=$ Sheet 1 Cells $(j+1,2)$

$\mathrm{S}=$ Sheet1.Cells $(\mathrm{j}+2,2)$

$B=$ Sheet1.Cells $(j+3,2)$

$\mathrm{L}=$ Sheet1.Cells $(\mathrm{j}+4,2)$

Sheets("sheet6").Select

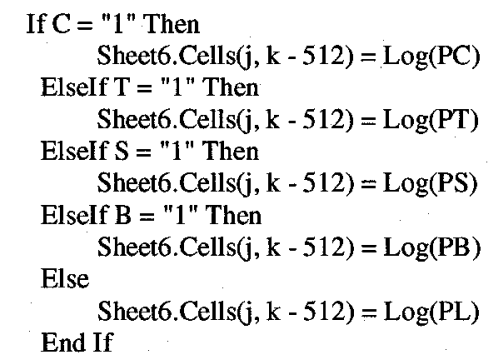

Next $\mathbf{j}$

Elself $\mathrm{k}-768<=256$ Then

Adding worksheets for calculation of Log-likelihood function value (sheet 4)

Sheets("sheet1").Select

For $\mathbf{j}=3$ To 2698 Step 5

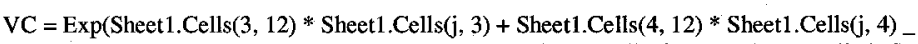
+ Sheet1.Cells $(5,12) *$ Sheet1.Cells $(\mathrm{j}, 5)+$ Sheet1.Cells $(6,12) *$ Sheet1.Cells $(\mathrm{j}, 6)+$ Sheet1.Cells $(7,12) *$ Sheet1.Cells $(\mathrm{j}, 7))$

VT $=\operatorname{Exp}(\operatorname{Sheet1} \operatorname{Cells}(3,12) *$ Sheet 1 Cells $(j+1,3)+$ Sheet1.Cells $(4,12) *$ Sheet1.Cells $(j+1,4)+$ Sheet 1. Cells $(8,12) *$ Sheet $1 . C$ ells $(j+1,8))$

VS $=\operatorname{Exp}($ Sheet1.Cells $(3,12) *$ Sheet1.Cells $(j+2,3)+$ Sheet1.Cells $(4,12) *$ Sheet1.Cells $(j+2,4)+$ Sheet1.Cells $(9,12) *$ Sheet $1 . C e l l s(j+2,9))$

$\mathrm{VB}=\operatorname{Exp}(\operatorname{Sheet} 1 . \operatorname{Cells}(3,12) * \operatorname{Sheet1.Cells}(\mathrm{j}+3,3)+\operatorname{Sheet1.Cells}(4,12) * \operatorname{Sheet1.Cells}(\mathrm{j}+3,4)+\operatorname{Sheet} 1 . \operatorname{Cell} \mathrm{s}(10,12) * \operatorname{Sheet} 1 . \operatorname{Cells}(\mathrm{j}+3,10))$

$\mathrm{VL}=\operatorname{Exp}(\operatorname{Sheet1} . \operatorname{Cells}(3,12) *$ Sheet1.Cells $(\mathrm{j}+4,3)+\operatorname{Sheet1.Cells}(4,12) * \operatorname{Sheet1.Cells~}(\mathrm{j}+4,4)+\operatorname{Sheet} 1 . \operatorname{Cells}(11,12) * \operatorname{Sheet} 1 . \operatorname{Cells}(\mathrm{j}+4,11))$ 


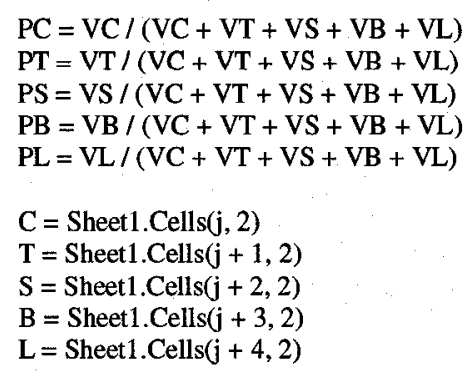

Sheets("sheet7").Select

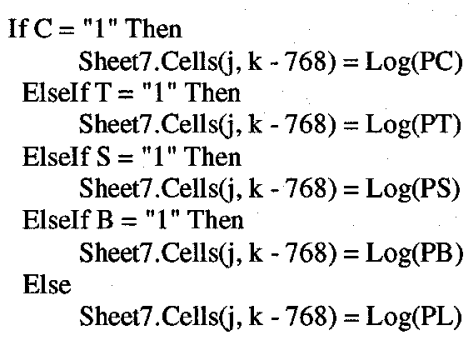

Next $\mathbf{j}$

End If

Utility Function Value for all alternatives' model specifications

For $\mathrm{i}=3$ To 2698 Step 5

Sheets("sheet2").Select

$\mathrm{vC}=\operatorname{Exp}(\operatorname{Sheet} 1 . \operatorname{Cell} \mathrm{s}(3,12) *$ Sheet1.Cells $(\mathrm{i}, 3)+\operatorname{Sheet1.Cells}(4,12) *$ Sheet1.Cells $(\mathrm{i}, 4)$

+ Sheet1.Cells $(5,12) *$ Sheet1.Cells $(i, 5)+$ Sheet1.Cells $(6,12) *$ Sheet1.Cells $(i, 6)+\operatorname{Sheet1.Cells~}(7,12) *$ Sheet1.Cells $(i, 7))$

$\mathrm{vT}=\operatorname{Exp}(\operatorname{Sheet1}$.Cells $(3,12) *$ Sheet1.Cells $(i+1,3)+\operatorname{Sheet} 1 . \operatorname{Cells}(4,12) * \operatorname{Sheet1.Cells~}(i+1,4)+\operatorname{Sheet1.Cells~}(8,12) *$ Sheet $1 . C e l l s(i+1,8))$

VS $=\operatorname{Exp}($ Sheetl.Cells $(3,12) *$ Sheet1.Cells $(i+2,3)+$ Sheet1.Cells $(4,12) *$ Sheet1.Cells $(i+2,4)+$ Sheet1.Cells $(9,12) *$ Sheet 1.Cells $(i+2,9))$

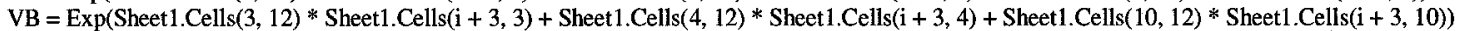

$\mathrm{VL}=\operatorname{Exp}(\operatorname{Sheet} 1 . C e l l s(3,12) *$ Sheet1.Cells $(\mathrm{i}+4,3)+\operatorname{Sheet1.Cells}(4,12) *$ Sheet1.Cells $(i+4,4)+\operatorname{Sheet1}$.Cells $(11,12) *$ Sheet1.Cells $(\mathbf{i}+4,11))$

Mode Choice Probability of each observations in Logit probability model

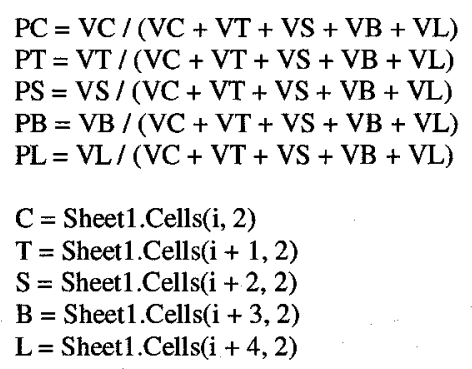

First-order Derivative with respect to $\beta_{1}$

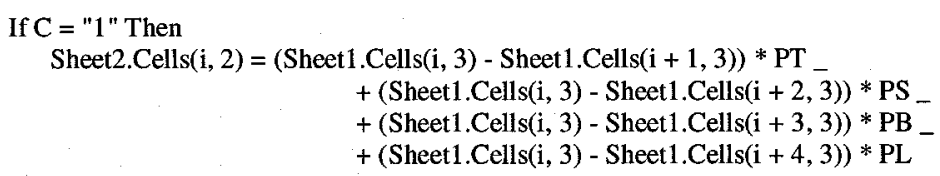


$+($ Sheet1.Cells $(\mathrm{i}+1,3)-$ Sheet1.Cells $(\mathrm{i}+2,3)) *$ PS

+ (Sheet1.Cells $(\mathrm{i}+1,3)$ - Sheet1.Cells $(\mathrm{i}+3,3)) * \mathrm{~PB}$

$+($ Sheet1.Cells $(\mathrm{i}+1,3)-$ Sheet1.Cells $(\mathrm{i}+4,3)) * \mathrm{PL}$

Elself $S=$ "1" Then

Sheet2.Cells $(i, 2)=($ Sheet1.Cells $(i+2,3)-$ Sheet 1.Cells $(i, 3)) * P C$

$+($ Sheet1.Cells $(\mathrm{i}+2,3)$ - Sheet1.Cells $(\mathrm{i}+1,3)) * \mathrm{PT}$ -

$+($ Sheet1.Cells $(i+2,3)-$ Sheet1.Cells $(i+3,3)) *$ PB -

$+($ Sheet1.Cells $(\mathrm{i}+2,3)-$ Sheet1.Cells $(\mathrm{i}+4,3)) * \mathrm{PL}$

Elself $B=$ "1" Then

Sheet2.Cells $(i, 2)=($ Sheet1.Cells $(\mathrm{i}+3,3)-$ Sheet1.Cells $(\mathrm{i}, 3)) * \mathrm{PC}$

$+($ Sheet1.Cells $(i+3,3)-$ Sheet1.Cells $(i+1,3)) *$ PT

$+($ Sheet1.Cells $(\mathrm{i}+3,3)-$ Sheet1.Cells $(\mathrm{i}+2,3)) * \mathrm{PS}_{-}^{-}$

$+($ Sheet1.Cells $(i+3,3)-$ Sheet1.Cells $(i+4,3)) *$ PL

Else

Sheet2.Cells $(\mathbf{i}, 2)=($ Sheet1.Cells $(i+4,3)-$ Sheet1.Cells $(i, 3)) * P C$

$+($ Sheet1.Cells $(i+4,3)-$ Sheet1.Cells $(i+1,3)) * P T$

$+($ Sheet1.Cells $(\mathrm{i}+4,3)-$ Sheet1.Cells $(\mathrm{i}+2,3)) *$ PS

$+($ Sheet1.Cells $(\mathrm{i}+4,3)-$ Sheet1.Cells $(\mathrm{i}+3,3)) * \mathrm{~PB}$

End If

First-order Derivative with respect to $\beta_{2}$

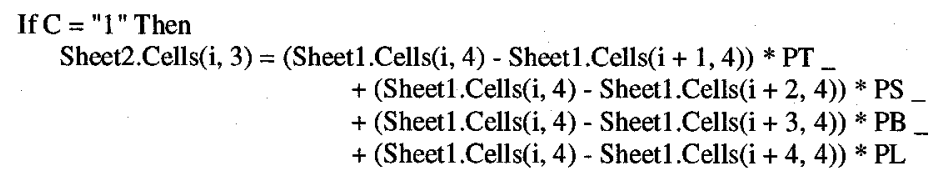

Elself $\mathrm{T}=$ "1" Then

Sheet2.Cells $(\mathbf{i}, 3)=($ Sheet1.Cells $(\mathrm{i}+1,4)-$ Sheet1.Cells $(\mathrm{i}, 4)) * \mathrm{PC}$

$+($ Sheet1.Cells $(i+1,4)-$ Sheet1.Cells $(i+2,4)) *$ PS

$+($ Sheet 1.Cells $(\mathbf{i}+1,4)-$ Sheet 1.Cells $(\mathbf{i}+3,4)) * \mathbf{P B}$

$+($ Sheet1.Cells $(\mathrm{i}+1,4)-$ Sheet1.Cells $(\mathrm{i}+4,4)) * \mathrm{PL}$

Elself $S=$ "1" Then

Sheet2.Cells $(i, 3)=($ Sheet1.Cells $(i+2,4)-$ Sheet1.Cells $(i, 4)) * P C$

$+($ Sheet 1.Cells $(\mathrm{i}+2,4)-$ Sheet 1.Cells $(\mathrm{i}+1,4)) * \mathrm{PT}$

$+($ Sheet1.Cells $(\mathrm{i}+2,4)-$ Sheet1.Cells $(\mathrm{i}+3,4)) * \mathrm{~PB}_{-}^{-}$

$+($ Sheet1.Cells $(i+2,4)-$ Sheet1.Cells $(i+4,4)) *$ PL

Elself $B=$ "1" Then

Sheet2.Cells $(\mathrm{i}, 3)=($ Sheet1.Cells $(\mathrm{i}+3,4)-$ Sheet 1 .Cells $(\mathrm{i}, 4)) * \mathrm{PC}$

$+($ Sheet 1.Cells $(i+3,4)-$ Sheet $1 . C e l l s(i+1,4)) * P T$ $+($ Sheet1.Cells $(\mathbf{i}+3,4)-$ Sheet 1.Cells $(\mathbf{i}+2,4)) *$ PS

$+($ Sheet 1.Cells $(i+3,4)-$ Sheet 1 .Cells $(i+4,4)) * P L$

Else

Sheet2.Cells $(\mathrm{i}, 3)=($ Sheet 1.Cells $(\mathrm{i}+4,4)-$ Sheet1.Cells $(\mathrm{i}, 4)) *$ PC

$+($ Sheet1.Cells $(\mathrm{i}+4,4)-$ Sheet 1.Cells $(\mathrm{i}+1,4)) * \mathrm{PT}$

$+($ Sheet1.Cells $(i+4,4)$ - Sheet1.Cells $(i+2,4)) *$ PS $_{-}^{-}$

$+($ Sheet1.Cells $(i+4,4)-$ Sheet1.Cells $(i+3,4)) *$ PB

End If

First-order Derivative with respect to $\beta_{3}$

If $\mathrm{C}=$ " 1 " Then

Sheet2.Cells $(\mathbf{i}, 4)=$ Sheet1.Cells $(\mathbf{i}, 5) *(P T+P S+P B+P L)$

Else

Sheet2.Cells $(i, 4)=-$ Sheet $1 . C e l l s(i, 5) * P C$

End If 
First-order Derivative with respect to $\beta_{4}$

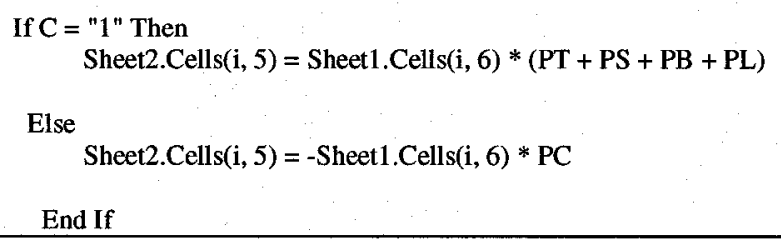

End If

First-order Derivative with respect to $\beta_{6}$

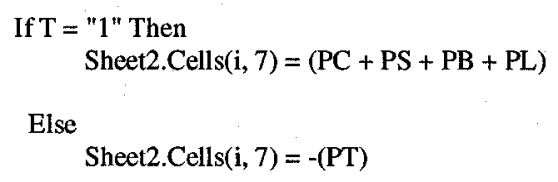

End If

First-order Derivative with respect to $\beta_{7}$

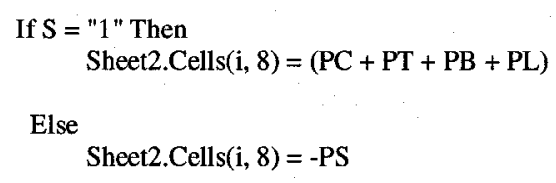

End If

First-order Derivative with respect to $\beta_{8}$

If $B=$ " 1 " Then

Sheet2.Cells $(\mathrm{i}, 9)=(\mathrm{PC}+\mathrm{PT}+\mathrm{PS}+\mathrm{PL})$

Else

Sheet2.Cells $(i, 9)=-P B$

End If

First-order Derivative with respect to $\beta_{9}$

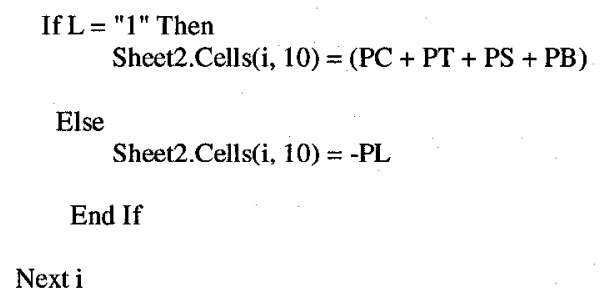


Next i

Obtaining Gradient components calculated, summation of all observation and averaging it

For $\mathbf{j}=2$ To 10 Step 1

Sheet2.Cells $(543, \mathrm{j})$.FormulaR1C1 $=$ "=SUM(R[-540]C:R[-1]C)/540"

Next $\mathbf{j}$

Sheets("sheet3").Select

Obtaining the gradient value of Log-likelihood function: $G_{t}$

For $\mathbf{i}=11$ To 19 Step 1

Sheet 3. Cells $(i, 11)=$ Sheet2.Cells $(543, i-9)$.Value

Next $\mathrm{i}$

Calculating delta: $\delta=\lambda \Delta_{t}=W_{t} G_{t}=\lambda I_{t}^{S A} G_{t}$

Range("M11:M19").FormulaArray = "=MMULT(A11:I19,K11:K19)"

Calculating parameters value: $\beta_{t+1}=\beta_{t}+\lambda \Delta_{t}=\beta_{t}+\lambda W_{t} G_{t}=\beta_{t}+\lambda I_{t}^{S A} G_{t}$

For $\mathrm{i}=11$ To 19 Step 1

Sheet3.Cells(i, 14) $=$ Sheet1.Cells $(i-8,12)+(1 / 18) *$ Sheet3.Cells $(i, 13)$

Next i

Checking the Criterion for stopping: $\left[\frac{1}{k} \sum_{k=1}^{k}\left(\beta_{t+1, k}-\beta_{t, k}\right)^{2}\right]^{1 / 2}<C R$

Sheet3.Cells $(21,12)=(($

$(\text { Sheet3.Cells }(11,14)-\text { Sheet1.Cells }(3,12))^{\wedge} 2+(\text { Sheet3.Cells }(12,14)-\text { Sheet1.Cells }(4,12))^{\wedge} 2$ $+(\text { Sheet3.Cells }(13,14)-\text { Sheet1.Cells }(5,12))^{\wedge} 2+(\text { Sheet3.Cells }(14,14)-\text { Sheet1.Cells }(6,12))^{\wedge} 2$

$+(\text { Sheet3.Cells }(15,14)-\text { Sheet1.Cells }(7,12))^{\wedge} 2+(\operatorname{Sheet3.Cells}(16,14)-\text { Sheet1.Cells }(8,12))^{\wedge} 2$

$+(\text { Sheet3.Cells }(17,14)-\text { Sheet 1.Cells }(9,12))^{\wedge} 2+(\operatorname{Sheet3.Cells}(18,14)-\text { Sheet1.Cells }(10,12))^{\wedge} 2-$

$+(\text { Sheet3.Cells }(19,14)-\text { Sheet1.Cells }(11,12))^{\wedge} 2$

)$\left.^{*}(1 / 9)\right)^{\wedge}(0.5)$

If Sheet3.Cells $(21,12)<10^{\wedge}(-4)$ Then

GoTo line1

Else

GoTo line2

End If

line1:

End Sub 


\section{B.5: DFP algorithm}

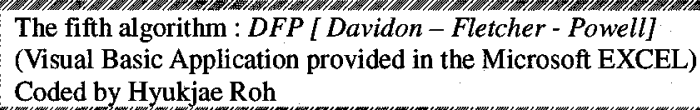

(Visual Basic Application provided in the Microsoft EXCEL)

Coded by Hyukjae Roh

Private Sub DFP_CLICK()

Variables Generated
Dim i, j, k As Integer
Dim C As Integer
Dim T As Integer
Dim S As Integer
Dim B As Integer
Dim L As Integer
Dim VC As Double
Dim VT As Double
Dim VS As Double
Dim VB As Double
Dim VL As Double
Dim PC As Double
Dim PT As Double
Dim PS As Double
Dim PB As Double
Dim PL As Double

Sheets("sheet1").Select

$\mathbf{k}=1$

If $k=1$ Then

GoTo line3

End If

Initializing Staring Values of Parameters

line2:

$\mathbf{k}=\mathbf{k}+1$

line3:

Sheet1.Cells $(3,12)=$ Sheet 3. Cells $(11,16)$

Sheet 1 .Cells $(4,12)=$ Sheet 3. Cells $(12,16)$

Sheet 1.Cells $(5,12)=$ Sheet3.Cells $(13,16)$

Sheet 1 Cells $(6,12)=$ Sheet 3 Cells $(14,16)$

Sheet 1 .Cells $(7,12)=$ Sheet 3 .Cells $(15,16)$

Sheet 1.Cells $(8,12)=$ Sheet 3. Cells $(16,16)$

Sheet 1. Cells $(9,12)=$ Sheet3.Cells $(17,16)$

Sheet 1. Cells $(10,12)=$ Sheet 3 .Cells $(18,16)$

Sheet1.Cells $(11,12)=$ Sheet 3. Cells $(19,16)$ 
Adding worksheets for calculation of Log-likelihood function value (sheet 1)

\author{
If $256-k>=0$ Then \\ Sheets("sheet1").Select
}

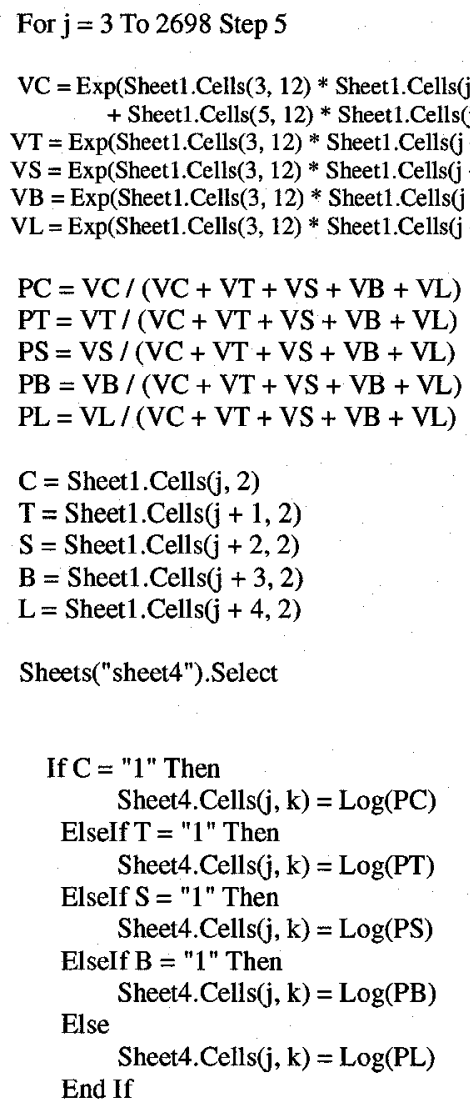

Sheets("sheet4").Select

Next $j$

Adding worksheets for calculation of Log-likelihood function value (sheet 2)

Elself $\mathrm{k}-256<=256$ Then

Sheets("sheet1").Select

For $\mathbf{j}=3$ To 2698 Step 5

$\mathrm{VC}=\operatorname{Exp}(\operatorname{Sheet} 1 . \operatorname{Cells}(3,12) *$ Sheet1.Cells $(\mathbf{j}, 3)+\operatorname{Sheet} 1 . \operatorname{Cells}(4,12) *$ Sheet1.Cells $(\mathrm{j}, 4)$

+ Sheet1.Cells $(5,12) *$ Sheet1.Cells(j, 5) + Sheet1.Cells $(6,12) *$ Sheet1.Cells(j, 6) + Sheet1.Cells $(7,12) *$ Sheet1.Cells $(\mathrm{j}, 7))$

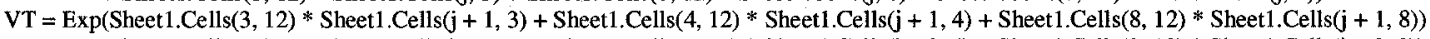

VS $=\operatorname{Exp}(\operatorname{Sheet1} 1$ Cells $(3,12) *$ Sheet1.Cells $(j+2,3)+$ Sheet1.Cells $(4,12) * \operatorname{Sheet1.Cells~}(j+2,4)+$ Sheet1.Cells $(9,12) *$ Sheet1.Cells $(j+2,9))$

VB $=\operatorname{Exp}(\operatorname{Sheet1} 1 . C e l l s(3,12) *$ Sheet1.Cells $(j+3,3)+\operatorname{Sheet1.Cells~}(4,12) *$ Sheet1.Cells $(j+3,4)+$ Sheet1.Cells $(10,12) *$ Sheet1.Cells $(j+3,10))$

$\mathrm{VL}=\operatorname{Exp}(\operatorname{Sheet1}$.Cells $(3,12) *$ Sheet1.Cells $(\mathrm{j}+4,3)+\operatorname{Sheet} 1 . \operatorname{Cells}(4,12) *$ Sheet1.Cells $(\mathrm{j}+4,4)+$ Sheet $1 . C e l l s(11,12) *$ Sheet1.Cells $(j+4,11))$

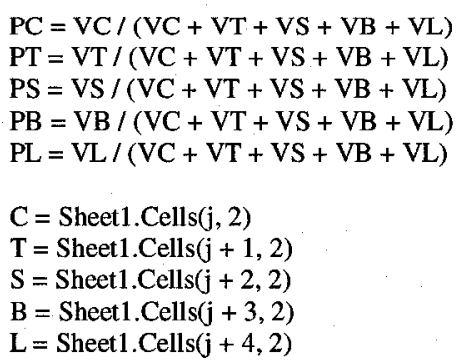


Sheets("sheet5").Select

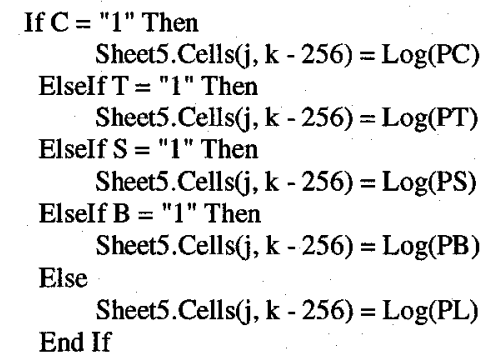

Next $\mathbf{j}$

Adding worksheets for calculation of Log-likelihood function value (sheet 3)

ElseIf $\mathrm{k}-512<=256$ Then

Sheets("sheet1").Select

For $\mathbf{j}=3$ To 2698 Step 5

$\mathrm{vC}=\operatorname{Exp}(\operatorname{Sheet1.Cells}(3,12) * \operatorname{Sheet1.Cells~}(\mathfrak{j}, 3)+\operatorname{Sheet1.Cells}(4,12) * \operatorname{Sheet1} 1$ Cells $(\mathrm{j}, 4)$

+ Sheet1.Cells $(5,12) *$ Sheet1.Cells $(\mathrm{j}, 5)+$ Sheet1.Cells $(6,12) *$ Sheet1.Cells $(\mathrm{j}, 6)+$ Sheet1.Cells $(7,12) *$ Sheet1.Cells $(\mathrm{j}, 7))$

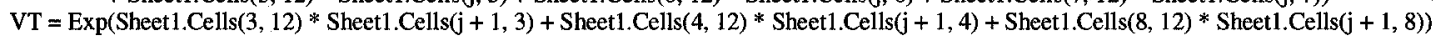

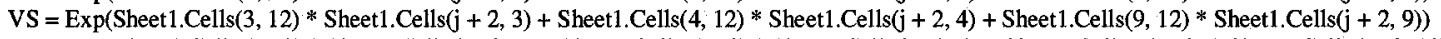

VB $=\operatorname{Exp}($ Sheet1.Cells $(3,12) *$ Sheet1.Cells $(j+3,3)+\operatorname{Sheet1.Cells~}(4,12) *$ Sheet1.Cells $(j+3,4)+$ Sheet1.Cells $(10,12) *$ Sheet1.Cells $(j+3,10))$

$\mathrm{VL}=\operatorname{Exp}(\operatorname{Sheet1.Cells}(3,12) *$ Sheet1.Cells $(j+4,3)+\operatorname{Sheet} 1 . C e l 1 s(4,12) *$ Sheet1.Cells $(j+4,4)+$ Sheet1.Cells $(11,12) *$ Sheet $1 . \operatorname{Cells}(j+4,11))$

$\mathrm{PC}=\mathrm{VC} /(\mathrm{VC}+\mathrm{VT}+\mathrm{VS}+\mathrm{VB}+\mathrm{VL})$

$\mathrm{PT}=\mathrm{VT} /(\mathrm{VC}+\mathrm{VT}+\mathrm{VS}+\mathrm{VB}+\mathrm{VL})$

$\mathrm{PS}=\mathrm{VS} /(\mathrm{VC}+\mathrm{VT}+\mathrm{VS}+\mathrm{VB}+\mathrm{VL})$

$\mathrm{PB}=\mathrm{VB} /(\mathrm{VC}+\mathrm{VT}+\mathrm{VS}+\mathrm{VB}+\mathrm{VL})$

$\mathrm{PL}=\mathrm{VL} /(\mathrm{VC}+\mathrm{VT}+\mathrm{VS}+\mathrm{VB}+\mathrm{VL})$

$\mathrm{C}=$ Sheet $1 . \operatorname{Cells}(\mathrm{j}, 2)$

$\mathbf{T}=$ Sheet 1 .Cells $(\mathrm{j}+1,2)$

$\mathrm{S}=$ Sheet 1 .Cells $(\mathrm{j}+2,2)$

$\mathrm{B}=$ Sheet1.Cells $(\mathrm{j}+3,2)$

$\mathbf{L}=$ Sheet 1 .Cells $(j+4,2)$

Sheets("sheet6").Select

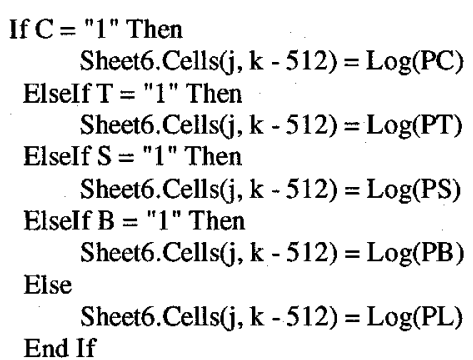

Next $\mathbf{j}$

Adding worksheets for calculation of Log-likelihood function value (sheet 4)

Elself $\mathrm{k}-768<=256$ Then

Sheets("sheet1").Select

For $\mathrm{j}=3$ To 2698 Step 5

$\mathrm{vC}=\operatorname{Exp}(\operatorname{Sheet} 1 . \operatorname{Cells}(3,12) * \operatorname{Sheet} 1 . \operatorname{Cells}(\mathrm{j}, 3)+\operatorname{Sheet} 1 . \operatorname{Cell} \mathrm{s}(4,12) * \operatorname{Sheet} 1 . \operatorname{Cells}(\mathrm{j}, 4)$ 
+ Sheet1.Cells $(5,12) *$ Sheet1.Cells $(\mathbf{j}, 5)+\operatorname{Sheet1.Cells~}(6,12) *$ Sheet1.Cells $(\mathrm{j}, 6)+\operatorname{Sheet1.Cells~}(7,12) * \operatorname{Sheet} 1 . \operatorname{Cells}(\mathrm{j}, 7))$

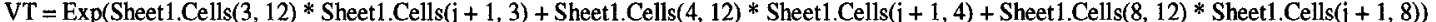
VS $=\operatorname{Exp}($ Sheet1.Cells $(3,12) *$ Sheet1.Cells $(j+2,3)+$ Sheet1.Cells $(4,12) *$ Sheet1.Cells $(j+2,4)+\operatorname{Sheet1.Cells}(9,12) *$ Sheet1.Cells $(j+2,9))$ $\mathrm{VB}=\operatorname{Exp}(\operatorname{Sheet1.Cells}(3,12) * \operatorname{Sheet1.Cells}(\mathrm{j}+3,3)+\operatorname{Sheet1.Cells}(4,12) * \operatorname{Sheet1.Cells}(\mathrm{j}+3,4)+\operatorname{Sheet1.Cells}(10,12) * \operatorname{Sheet} 1 . \operatorname{Cells}(\mathrm{j}+3,10))$

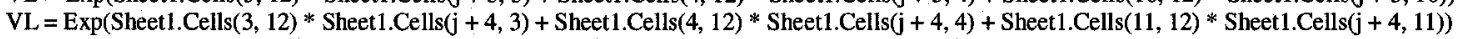

$\mathrm{PC}=\mathrm{VC} /(\mathrm{VC}+\mathrm{VT}+\mathrm{VS}+\mathrm{VB}+\mathrm{VL})$

$\mathrm{PT}=\mathrm{VT} /(\mathrm{VC}+\mathrm{VT}+\mathrm{VS}+\mathrm{VB}+\mathrm{VL})$

$\mathrm{PS}=\mathrm{VS} /(\mathrm{VC}+\mathrm{VT}+\mathrm{VS}+\mathrm{VB}+\mathrm{VL})$

$\mathrm{PB}=\mathrm{VB} /(\mathrm{VC}+\mathrm{VT}+\mathrm{VS}+\mathrm{VB}+\mathrm{VL})$

$\mathrm{PL}=\mathrm{VL} /(\mathrm{VC}+\mathrm{VT}+\mathrm{VS}+\mathrm{VB}+\mathrm{VL})$

$\mathrm{C}=$ Sheet1.Cells $(\mathrm{j}, 2)$

$\mathrm{T}=$ Sheet 1 Cells $(\mathrm{j}+1,2)$

$S=$ Sheet1.Cells $(j+2,2)$

B = Sheet1.Cells $(j+3,2)$

$\mathbf{L}=$ Sheet1.Cells $(\mathbf{j}+4,2)$

Sheets("sheet7").Select

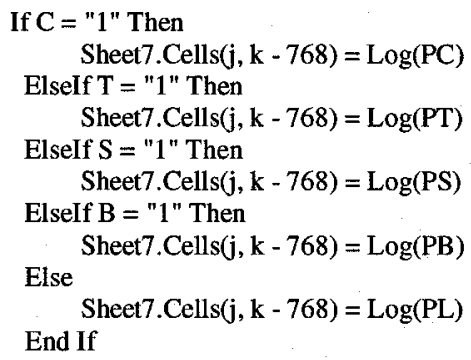

Next j

End If

Utility Function Value for all alternatives' model specifications

For $\mathrm{i}=3$ To 2698 Step 5

Sheets("sheet2").Select

$\mathrm{VC}=\operatorname{Exp}(\operatorname{Sheet} 1 . \operatorname{Cells}(3,12) *$ Sheet1.Cells $(\mathrm{i}, 3)+\operatorname{Sheet} 1 . \operatorname{Cells}(4,12) *$ Sheet 1. Cells $(\mathrm{i}, 4)$

+ Sheet1.Cells $(5,12) *$ Sheet1.Cells $(i, 5)+$ Sheet1.Cells $(6,12) *$ Sheet1.Cells $(1,6)+$ Sheet1.Cells $(7,12) *$ Sheet1.Cells $(i, 7))$

VT $=\operatorname{Exp}(\operatorname{Sheet1}$.Cells $(3,12) *$ Sheet1.Cells $(i+1,3)+\operatorname{Sheet1.Cells~}(4,12) *$ Sheet1.Cells $(i+1,4)+$ Sheet1.Cells $(8,12) *$ Sheet 1. Cells $(i+1,8))$

$\mathrm{VS}=\operatorname{Exp}(\operatorname{Sheet1} . \operatorname{Cells}(3,12) * \operatorname{Sheet} 1 . \operatorname{Cells}(\mathrm{i}+2,3)+\operatorname{Sheet} 1 . \operatorname{Cells}(4,12) * \operatorname{Sheet} 1 . \operatorname{Cells}(\mathrm{i}+2,4)+\operatorname{Sheet1.Cells}(9,12) * \operatorname{Sheet} 1 . \operatorname{Cells}(\mathrm{i}+2,9))$

$\mathrm{VB}=\operatorname{Exp}($ Sheet1.Cells $(3,12) *$ Sheet1.Cells $(\mathrm{i}+3,3)+$ Sheet 1.Cells $(4,12) *$ Sheet1.Cells $(\mathbf{i}+3,4)+$ Sheet1.Cells $(10,12) *$ Sheet1.Cells $(\mathrm{i}+3,10))$

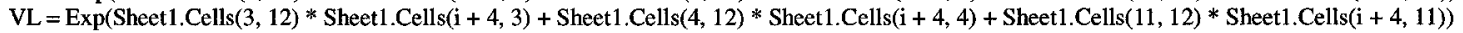

Mode Choice Probability of each observations with Logit probability model

$\mathrm{PC}=\mathrm{VC} /(\mathrm{VC}+\mathrm{VT}+\mathrm{VS}+\mathrm{VB}+\mathrm{VL})$

$\mathrm{PT}=\mathrm{VT} /(\mathrm{VC}+\mathrm{VT}+\mathrm{VS}+\mathrm{VB}+\mathrm{VL})$

$\mathrm{PS}=\mathrm{VS} /(\mathrm{VC}+\mathrm{VT}+\mathrm{VS}+\mathrm{VB}+\mathrm{VL})$

$\mathrm{PB}=\mathrm{VB} /(\mathrm{VC}+\mathrm{VT}+\mathrm{VS}+\mathrm{VB}+\mathrm{VL})$

$\mathrm{PL}=\mathrm{VL} /(\mathrm{VC}+\mathrm{VT}+\mathrm{VS}+\mathrm{VB}+\mathrm{VL})$

$\mathrm{C}=$ Sheet1.Cells $(\mathbf{i}, 2)$

$\mathrm{T}=$ Sheet 1. Cells $(\mathrm{i}+1,2)$

$\mathrm{S}=$ Sheet 1. Cells $(\mathrm{i}+2,2)$

$\mathrm{B}=$ Sheet 1 .Cells $(\mathrm{i}+3,2)$

$\mathrm{L}=$ Sheet 1.Cells $(\mathrm{i}+4,2)$

First-order Derivative with respect to $\beta_{1}$ 


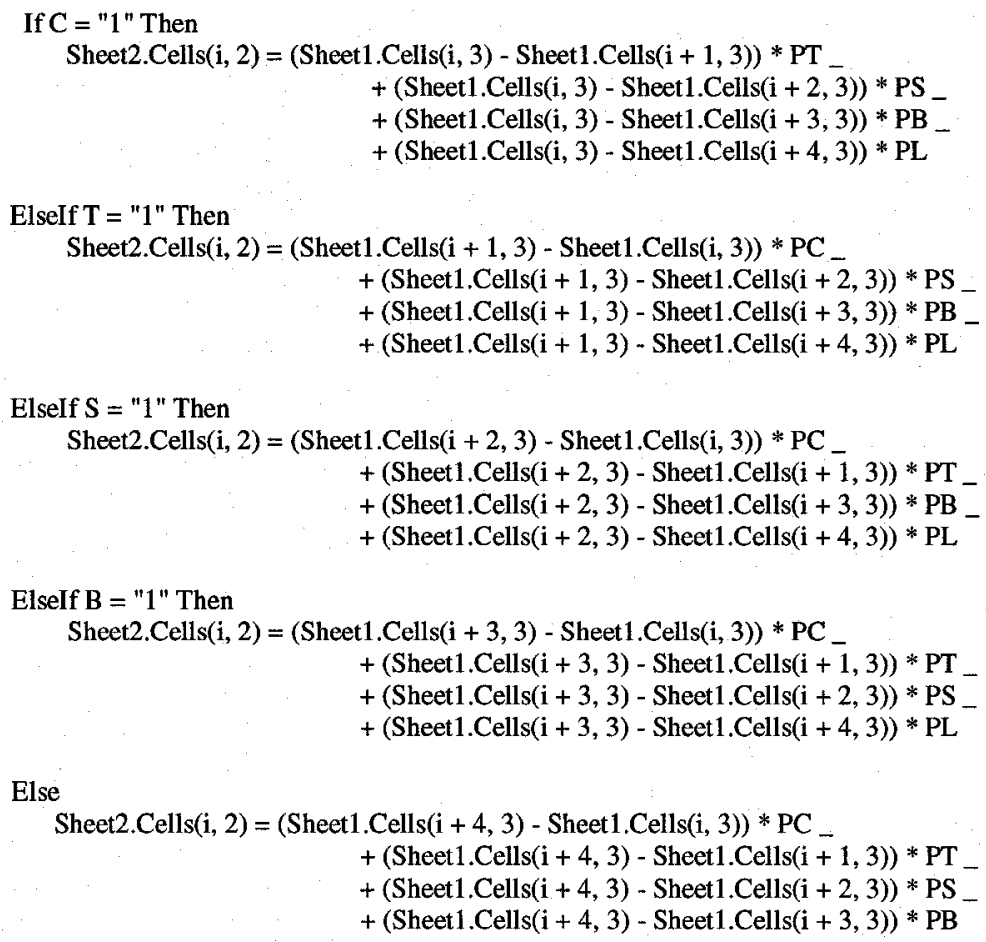

End If

First-order Derivative with respect to $\beta_{2}$

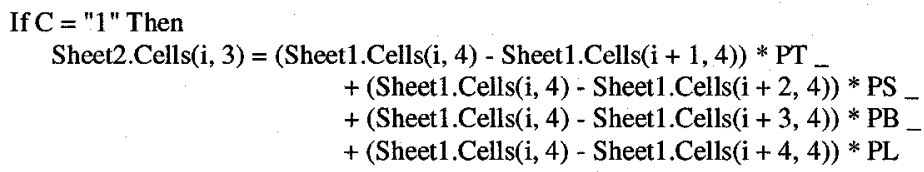

End If 
First-order Derivative with respect to $\beta_{3}$

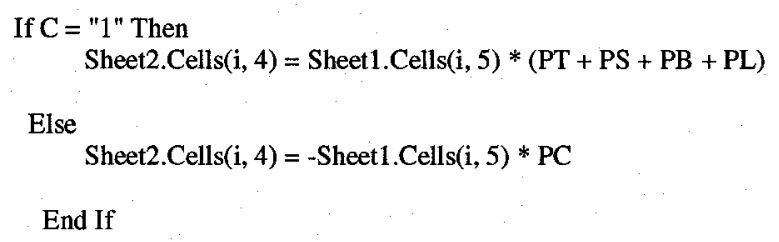

End If

First-order Derivative with respect to $\beta_{4}$

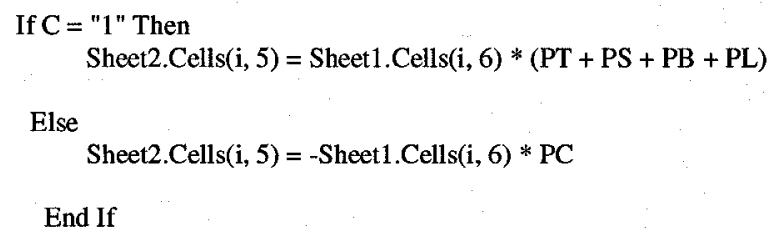

End If 
First-order Derivative with respect to $\beta_{9}$

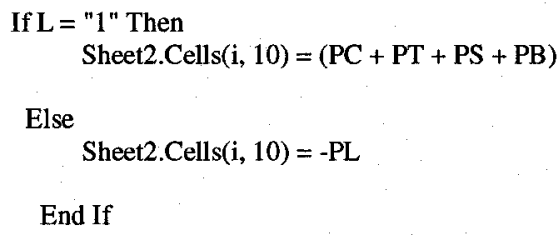

For $\mathbf{j}=2$ To 10 Step 1

Next $\mathbf{j}$

Sheets("sheet3").Select

Obtaining Gradient components calculated : $G_{t}$

For $\mathrm{i}=11$ To 19 Step 1

Sheet3.Cells $(\mathrm{i}, 11)=$ Sheet 2. Cells $(2703, \mathrm{i}-9)$

Next $\mathbf{i}$

Calculating delta : $\delta_{t}=\lambda \Delta_{t}=\lambda W_{t} G_{t}=\lambda\left(-H_{t A P}^{D F P}\right) G_{t}$

Sheet3.Range("M11:M19").FormulaArray = "=-(1/16)*(MMULT(A1:I9,K11:K19))"

Parameter estimates : $\beta_{t+1}=\beta_{t}+\delta_{t}=\beta_{t}+\lambda_{t} \Delta_{t}=\beta_{t}+\lambda_{t} W_{t} G_{t}=\beta_{t}+\lambda_{t}\left(-H_{t A P}^{D F P}\right) G_{t}$

Sheet3.Cells $(11,16)=$ Sheet 1 Cells $(3,12)+$ Sheet 3 .Cells $(11,13)$

Sheet3.Cells $(12,16)=$ Sheet1.Cells $(4,12)+$ Sheet3.Cells $(12,13)$

Sheet3.Cells $(13,16)=$ Sheet1.Cells $(5,12)+$ Sheet 3. Cells $(13,13)$

Sheet3.Cells $(14,16)=$ Sheet 1. Cells $(6,12)+$ Sheet $3 \cdot \operatorname{Cells}(14,13)$

Sheet3.Cells $(15,16)=$ Sheet 1. Cells $(7,12)+$ Sheet $3 . \operatorname{Cells}(15,13)$

Sheet3.Cells $(16,16)=$ Sheet1.Cells $(8,12)+$ Sheet3.Cells $(16,13)$

Sheet3.Cells $(17,16)=$ Sheet1.Cells $(9,12)+$ Sheet $3 . \operatorname{Cells}(17,13)$

Sheet 3 .Cells $(18,16)=$ Sheet 1 Cells $(10,12)+$ Sheet 3. Cells $(18,13)$

Sheet3.Cells $(19,16)=$ Sheet1.Cells $(11,12)+$ Sheet3.Cells $(19,13)$

Checking the Criterion for stopping: $\left[\frac{1}{k} \sum_{k=1}^{k}\left(\beta_{t+1, k}-\beta_{t, k}\right)^{2}\right]^{1 / 2}<C R$ 
Sheet3.Cells $(21,12)=\left(C_{-}\right.$

$(\text { Sheet3.Cells }(11,16)-\text { Sheet1.Cells }(3,12))^{\wedge} 2+(\text { Sheet3.Cells }(12,16)-\text { Sheet 1.Cells }(4,12))^{\wedge} 2$ $+(\text { Sheet3.Cells }(13,16)-\operatorname{Sheet1.Cells}(5,12))^{\wedge} 2+(\operatorname{Sheet3.Cells}(14,16)-\operatorname{Sheet1.Cells}(6,12))^{\wedge} 2$ $+(\operatorname{Sheet3.Cells}(15,16)-\text { Sheet 1.Cells }(7,12))^{\wedge} 2+(\operatorname{Sheet3.Cells}(16,16)-\operatorname{Sheet1.Cells}(8,12))^{\wedge} 2$ $+(\text { Sheet3.Cells }(17,16)-\text { Sheet1.Cells }(9,12))^{\wedge} 2+(\operatorname{Sheet3.Cells}(18,16)-\text { Sheet1.Cells }(10,12))^{\wedge} 2$ $+(\text { Sheet3.Cells }(19,16)-\text { Sheet1.Cells }(11,12))^{\wedge} 2$.

$$
) *(1 / 9))^{\wedge}(0.5)
$$

If Sheet $3 . \operatorname{Cells}(21,12)<10^{\wedge}(-4)$ Then

$$
\text { GoTo line1 }
$$

Else

Starting the procedure for obtaining updating approximate hessan

Calculating: $G_{t+1}$

First-order Derivative with respect to $\beta$

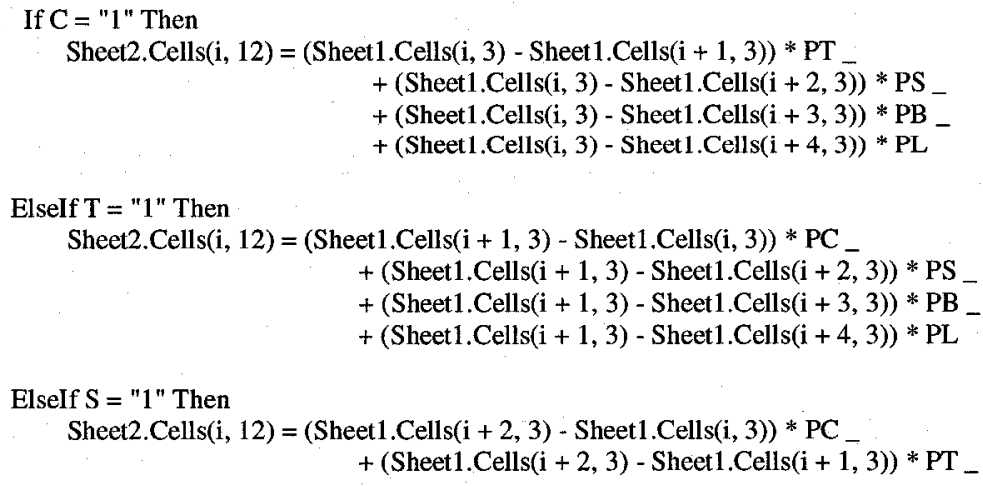


$+($ Sheet1.Cells $(\mathrm{i}+2,3)-$ Sheet1.Cells $(\mathrm{i}+3,3)) * \mathrm{~PB}$ $+($ Sheet1.Cells $(\mathrm{i}+2,3)-$ Sheet1.Cells $(\mathrm{i}+4,3)) * \mathrm{PL}$

ElseIf B = "1" Then

Sheet2.Cells $(\mathrm{i}, 12)=($ Sheet1.Cells $(\mathrm{i}+3,3)-$ Sheet1.Cells $(\mathrm{i}, 3)) * \mathrm{PC}$

$+($ Sheet1.Cells $(\mathrm{i}+3,3)$ - Sheet1.Cells $(\mathrm{i}+1,3)) * \mathrm{PT}$

$+($ Sheet1.Cells $(\mathrm{i}+3,3)$ - Sheet1.Cells $(\mathrm{i}+2,3)) * \mathrm{PS}_{-}$

$+($ Sheet1.Cells $(\mathrm{i}+3,3)-$ Sheet1.Cells $(\mathrm{i}+4,3)) * \mathrm{PL}$

Else

Sheet2.Cells $(i, 12)=($ Sheet1.Cells $(i+4,3)-$ Sheet1.Cells $(i, 3)) * P C$

$+($ Sheet1.Cells $(\mathrm{i}+4,3)-$ Sheet1.Cells $(\mathrm{i}+1,3)) * \mathrm{PT}$ $+($ Sheet1.Cells $(\mathbf{i}+4,3)$ - Sheet1.Cells $(i+2,3)) *$ PS

$+($ Sheet1.Cells $(\mathrm{i}+4,3)-$ Sheet1.Cells $(\mathrm{i}+3,3)) * \mathrm{~PB}$

End If

First-order Derivative with respect to $\beta_{2}$

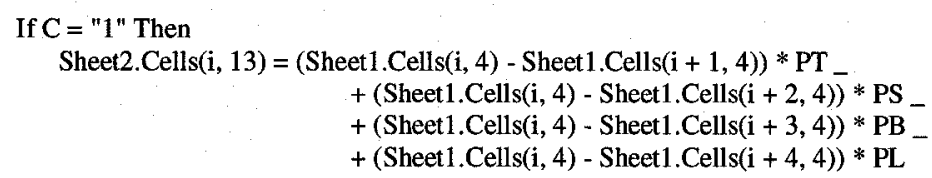

End If

First-order Derivative with respect to $\beta_{3}$

If $\mathrm{C}=$ " 1 " Then

Sheet2.Cells $(i, 14)=$ Sheet1.Cells $(i, 5) *(P T+P S+P B+P L)$

Else

Sheet2.Cells $(i, 14)=-$ Sheet 1 .Cells $(i, 5) *$ PC

End If

First-order Derivative with respect to $\beta_{4}$ 
For $j=12$ To 20 Step 1

Sheet2.Cells $(2703, \mathrm{j})$.FormulaR1C1 = "=-SUM(R[-2700]C:R[-1]C)/540"

Next $j$

Sheets("sheet3").Select

Obtaining gradient: $G_{t+1}$

For $\mathrm{i}=1$ To 9 Step 1

Sheet3.Cells $(i, 11)=$ Sheet2.Cells $(2703, i+11)$

Next i

Calculating gamma: $\gamma_{t}=G_{t+1}-G_{t}$

Sheet3.Cells $(11,18)=$ Sheet 3. Cells $(1,11)-$ Sheet3.Cells $(11,11)$

Sheet3.Cells $(12,18)=$ Sheet3.Cells $(2,11)-$ Sheet3.Cells $(12,11)$

Sheet3.Cells $(13,18)=$ Sheet3.Cells $(3,11)-$ Sheet3.Cells $(13,11)$

Sheet3.Cells $(14,18)=$ Sheet3.Cells $(4,11)-$ Sheet3.Cells $(14,11)$

Sheet 3 .Cells $(15,18)=$ Sheet 3 .Cells $(5,11)-$ Sheet 3 .Cells $(15,11)$

Sheet3.Cells $(16,18)=$ Sheet3.Cells $(6,11)-$ Sheet3.Cells $(16,11)$

Sheet3.Cells $(17,18)=$ Sheet3.Cells $(7,11)-$ Sheet3.Cells $(17,11)$

Sheet3.Cells $(18,18)=$ Sheet3.Cells $(8,11)-$ Sheet3.Cells $(18,11)$

Sheet3.Cells $(19,18)=$ Sheet3.Cells $(9,11)-$ Sheet3.Cells $(19,11)$

Transpose matrix of a vector of gamma: $\gamma_{t}$

Sheet 3 .Cells $(28,11)=$ Sheet 3 .Cells $(11,18)$

Sheet3.Cells $(28,12)=$ Sheet3.Cells $(12,18)$

Sheet3.Cells $(28,13)=$ Sheet 3 .Cells $(13,18)$

Sheet3.Cells $(28,14)=$ Sheet 3 .Cells $(14,18)$

Sheet3.Cells $(28,15)=$ Sheet3.Cells $(15,18)$

Sheet3.Cells $(28,16)=$ Sheet $3 \cdot \operatorname{Cells}(16,18)$

Sheet 3. Cells $(28,17)=$ Sheet 3 .Cells $(17,18)$

Sheet 3 .Cells $(28,18)=$ Sheet 3 .Cells $(18,18)$

Sheet 3. Cells $(28,19)=$ Sheet 3. Cells $(19,18)$

Transpose matrix of a vector of delta: $\delta$

Sheet 3. Cells $(30,11)=$ Sheet 3. Cells $(11,13)$

Sheet 3. Cells $(30,12)=$ Sheet 3 .Cells $(12,13)$

Sheet 3. Cells $(30,13)=$ Sheet 3 .Cells $(13,13)$

Sheet 3 .Cells $(30,14)=$ Sheet 3 .Cells $(14,13)$

Sheet3.Cells $(30,15)=$ Sheet 3. Cells $(15,13)$

Sheet 3 .Cells $(30,16)=$ Sheet 3 .Cells $(16,13)$

Sheet3.Cells $(30,17)=$ Sheet 3 .Cells $(17,13)$

Sheet3.Cells $(30,18)=$ Sheet 3. Cells $(18,13)$

Sheet3.Cells $(30,19)=$ Sheet 3. Cells $(19,13)$ 
Calculating approximate hessian: $H_{t+1}^{D F P}=H_{t}^{D F P}+\frac{\delta_{t} \delta_{t}^{\prime}}{\delta_{t}^{\prime} \gamma_{t}}-\frac{H_{t}^{D F P} \gamma_{t} \gamma_{t}^{\prime} H_{t}^{D F P}}{\gamma_{t}^{\prime} H_{t}^{D F P} \gamma_{t}}$

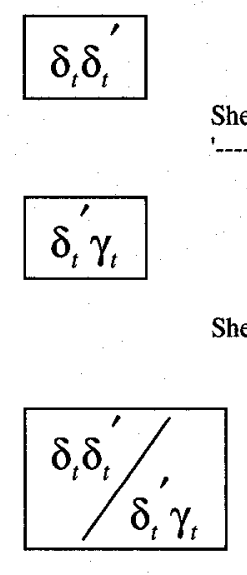

For $\mathrm{i}=33$ To 41 Step 1

For $\mathrm{j}=11$ To 19 Step 1

Sheet3.Cells $(\mathrm{i}, \mathrm{j}+10)=$ Sheet3.Cells $(\mathrm{i}, \mathrm{j}) /$ Sheet3.Cells $(43,12)$

Next i

$H_{t}^{D F P} \gamma_{t}$

Sheet3.Range("K45:K53").FormulaArray = "=MMULT(A1:I9,R11:R19)"

$H_{t}^{D F P} \gamma_{t} \gamma_{t}^{\prime}$

Sheet3.Range("M45:U53").FormulaArray = "=MMULT(K45:K53,K28:S28)"

$H_{t}^{D F P} \gamma_{t} \gamma_{t}^{\prime} H_{t}^{D F P}$

Sheet3.Range("K55:S63").FormulaArray = "=MMULT(M45:U53,A1:I9)"

$\gamma_{t}^{\prime} H_{t}^{D F P}$

Sheet3.Range("K66:S66").FormulaArray = "=MMULT(K28:S28,A1:19)"

$\gamma_{t}^{\prime} H_{t}^{D F P} \gamma_{t}$

Sheet3.Range("L67").FormulaArray = "=MMULT(K66:S66,R11:R19) "

$\frac{H_{t}^{D F P} \gamma_{t} \gamma_{t}^{\prime} H_{t}^{D F P}}{\gamma_{t}^{\prime} H_{t}^{D F P} \gamma_{t}}$

For $\mathrm{i}=55$ To 63 Step 1

For $\mathrm{j}=11$ To 19 Step 1 

Next $\mathbf{j}$

Sheet3.Cells $(\mathrm{i}, \mathrm{j}+10)=$ Sheet3.Cells $(\mathrm{i}, \mathrm{j}) /$ Sheet3.Cells $(67,12)$

Next $\mathrm{i}$

$H_{t}^{D F P}+\frac{\delta_{t} \delta_{t}^{\prime}}{\delta_{t}^{\prime} \gamma_{t}}-\frac{H_{t}^{D F P} \gamma_{t} \gamma_{t}^{\prime} H_{t}^{D F P}}{\gamma_{t}^{\prime} H_{t}^{D F P} \gamma_{t}}$

For $i=72$ To 80 Step 1

For $\mathbf{j}=11$ To 19 Step 1 Next $\mathbf{j}$ Sheet3.Cells $(i, j)=$ Sheet3.Cells $(\mathbf{i}-71, j-10)+$ Sheet3.Cells $(i-39, j+10)-\operatorname{Sheet} 3 . C e l l s(i-17, j+10)$

Next $\mathrm{i}$

Obtaining updated approximate hessain

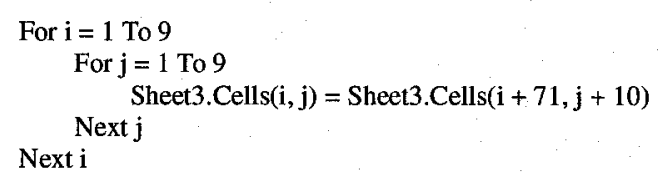

Ending the procedure for obtaining updating approximate hessan

\section{GoTo line2}

End If

line1

End Sub 


\section{B.6: BFGS algorithm}

The sixth algorithm: BFGS [ Broyden - Fletcher-Goldfarb-Shanno]

(Visual Basic Application provided in the Microsoft EXCEL)

Coded by Hyukjae Roh

Private Sub BFGS_CLICK()

Variables Generated

$\operatorname{Dim} \mathbf{i}, \mathbf{j}, \mathrm{k}$ As Integer

Dim C As Integer

Dim T As Integer

Dim S As Integer

Dim B As Integer

Dim L As Integer

Dim VC As Double

Dim VT As Double

Dim VS As Double

Dim VB As Double

Dim VL As Double

Dim PC As Double

Dim PT As Double

Dim PS As Double

Dim PB As Double

Dim PL As Double

Sheets("sheet1").Select

$\mathbf{k}=1$

If $k=1$ Then

GoTo line3

End If

Initializing Staring Values of Parameters

line2:

$\mathbf{k}=\mathbf{k}+1$

line3:

Sheet1.Cells $(3,12)=$ Sheet $3 . \operatorname{Cells}(11,16)$

Sheet1.Cells $(4,12)=$ Sheet 3 .Cells $(12,16)$

Sheet 1.Cells $(5,12)=$ Sheet $3 \cdot \operatorname{Cells}(13,16)$

Sheet1.Cells $(6,12)=$ Sheet3.Cells $(14,16)$

Sheet 1.Cells $(7,12)=$ Sheet 3 .Cells $(15,16)$

Sheet1.Cells $(8,12)=$ Sheet3.Cells $(16,16)$

Sheet1.Cells $(9,12)=$ Sheet3.Cells $(17,16)$

Sheet 1 .Cells $(10,12)=$ Sheet $3 \cdot$ Cells $(18,16)$

Sheet1.Cells $(11,12)=$ Sheet 3. Cells $(19,16)$

Adding worksheets for calculation of Log-likelihood function value (sheet 1) 
If $256-\mathrm{k}>=0$ Then

Sheets("sheet1").Select

For $\mathrm{j}=3$ To 2698 Step 5

$\mathrm{VC}=\operatorname{Exp}($ Sheet1.Cells $(3,12) *$ Sheet1.Cells $(\mathrm{j}, 3)+\operatorname{Sheet} 1 . C e l l s(4,12) *$ Sheet1.Cells $(\mathrm{j}, 4)$

+ Sheet1.Cells $(5,12) *$ Sheet1.Cells $(j, 5)+\operatorname{Sheet1.Cells(6,12)*Sheet1.Cells~}(\mathrm{j}, 6)+\operatorname{Sheet1.Cells~}(7,12) * \operatorname{Sheet1.Cells~}(\mathrm{j}, 7))$

VT $=\operatorname{Exp}(\operatorname{Sheet} 1 . C e l l s(3,12) *$ Sheet1.Cells $(j+1,3)+\operatorname{Sheet1.Cells~}(4,12) *$ Sheet1.Cells $(j+1,4)+\operatorname{Sheet1.Cells~}(8,12) *$ Sheet1.Cells $(j+1,8))$

VS $=\operatorname{Exp}($ Sheet1.Cells $(3,12) *$ Sheet1.Cells $(j+2,3)+$ Sheet1.Cells $(4,12) *$ Sheet1.Cells $(j+2,4)+$ Sheet1.Cells $(9,12) *$ Sheet1.Cells $(j+2,9))$

VB $=\operatorname{Exp}($ Sheet1.Cells $(3,12) *$ Sheet1.Cells $(j+3,3)+$ Sheet1.Cells $(4,12) *$ Sheet $1 . C e l l s(j+3,4)+$ Sheet1.Cells $(10,12) *$ Sheet1.Cells $(j+3,10))$

$\mathrm{VL}=\operatorname{Exp}(\operatorname{Sheet1.Cells~}(3,12) *$ Sheet1.Cells $(j+4,3)+\operatorname{Sheet} 1 . C e l l s(4,12) *$ Sheet1.Cells $(j+4,4)+$ Sheet1.Cells $(11,12) *$ Sheet 1.Cells $(j+4,11))$

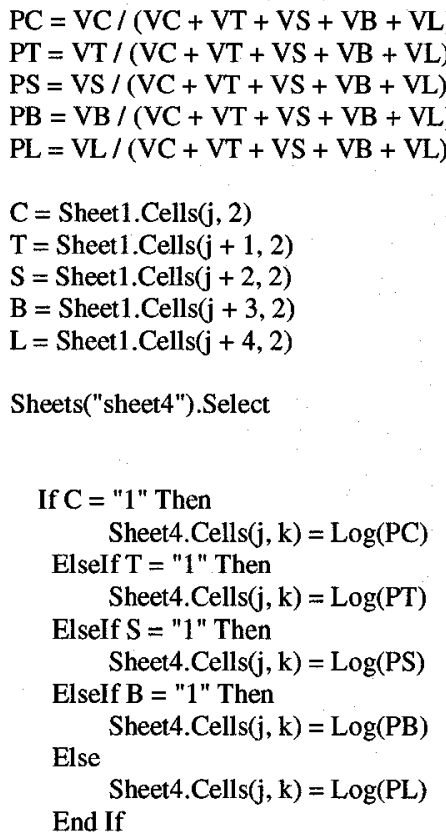

Next $j$

Adding worksheets for calculation of Log-likelihood function value (sheet 2)

Elself $\mathrm{k}-256<=256$ Then

Sheets("sheet1").Select

For $\mathbf{j}=3$ To 2698 Step 5

$\mathrm{VC}=\operatorname{Exp}(\operatorname{Sheet} 1 . \operatorname{Cells}(3,12) *$ Sheet1.Cells $(\mathrm{j}, 3)+\operatorname{Sheet} 1 . \operatorname{Cells}(4,12) *$ Sheet1.Cells $(\mathrm{j}, 4)$

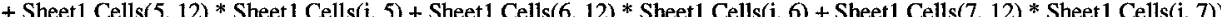

VT $=\operatorname{Exp}(\operatorname{Sheet1.Cells~}(3,12) *$ Sheet1.Cells $(j+1,3)+\operatorname{Sheet1.Cells~}(4,12) *$ Sheet1.Cells $(j+1,4)+\operatorname{Sheet1.Cells~}(8,12) * \operatorname{Sheet1} . \operatorname{Cells}(j+1,8))$

VS $=\operatorname{Exp}(\operatorname{Sheet} 1 . \operatorname{Cells}(3,12) * \operatorname{Sheet1.Cells~}(j+2,3)+\operatorname{Sheet1.Cells~}(4,12) * \operatorname{Sheet1.Cells~}(j+2,4)+\operatorname{Sheet1.Cells}(9,12) * \operatorname{Sheet} 1 . \operatorname{Cells}(j+2,9))$

VB $=\operatorname{Exp}(\operatorname{Sheet} 1 . C e l l s(3,12) *$ Sheet1.Cells $(j+3,3)+$ Sheet1.Cells $(4,12) *$ Sheet1.Cells $(j+3,4)+$ Sheet1.Cells $(10,12) * \operatorname{Sheet} 1 . C e l l s(j+3,10))$

$\mathrm{VL}=\operatorname{Exp}(\operatorname{Sheet1} . \operatorname{Cells}(3,12) *$ Sheet 1.Cells $(j+4,3)+\operatorname{Sheet} 1 . \operatorname{Cells}(4,12) *$ Sheet1.Cells $(j+4,4)+\operatorname{Sheet1.Cells~}(11,12) * \operatorname{Sheet} 1 . \operatorname{Cells}(j+4,11))$

$\mathrm{PC}=\mathrm{VC} /(\mathrm{VC}+\mathrm{VT}+\mathrm{VS}+\mathrm{VB}+\mathrm{VL})$

$\mathrm{PT}=\mathrm{VT} /(\mathrm{VC}+\mathrm{VT}+\mathrm{VS}+\mathrm{VB}+\mathrm{VL})$

$P S=V S /(V C+V T+V S+V B+V L)$

$\mathrm{PB}=\mathrm{VB} /(\mathrm{VC}+\mathrm{VT}+\mathrm{VS}+\mathrm{VB}+\mathrm{VL})$

$\mathrm{PL}=\mathrm{VL} /(\mathrm{VC}+\mathrm{VT}+\mathrm{VS}+\mathrm{VB}+\mathrm{VL})$

$\mathrm{C}=$ Sheet 1. Cells $(\mathrm{j}, 2)$

$\mathrm{T}=$ Sheet 1 Cells $(\mathrm{j}+1,2)$

$\mathrm{S}=$ Sheet1.Cells $(\mathrm{j}+2,2)$

B $=$ Sheet1.Cells $(j+3,2)$

$\mathrm{L}=$ Sheet1.Cells $(\mathrm{j}+4,2)$

Sheets("sheet5").Select 


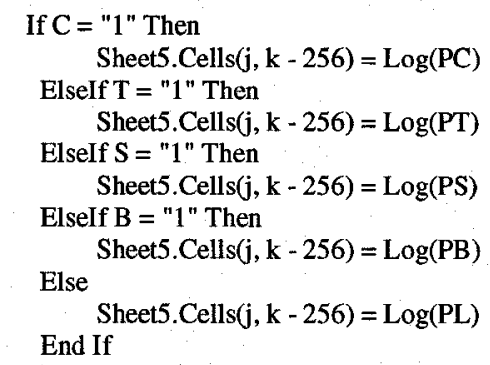

Next $\mathbf{j}$

Adding worksheets for calculation of Log-likelihood function value (sheet 3 )

Elself $\mathrm{k}-512<=256$ Then

Sheets("sheet1").Select

For $\mathrm{j}=3$ To 2698 Step 5

$\mathrm{VC}=\operatorname{Exp}(\operatorname{Sheet1} \cdot \operatorname{Cells}(3,12) * \operatorname{Sheet} 1 . \operatorname{Cells}(\mathrm{j}, 3)+\operatorname{Sheet1} \cdot \operatorname{Cells}(4,12) * \operatorname{Sheet} 1 . \operatorname{Cells}(\mathrm{j}, 4)$

+ Sheet1.Cells $(5,12) *$ Sheet1.Cells $(\mathrm{j}, 5)+$ Sheet1.Cells $(6,12) *$ Sheet1.Cells $(j, 6)+\operatorname{Sheet1.Cells~}(7,12) *$ Sheet 1.Cells $(\mathrm{j}, 7))$

VT $=\operatorname{Exp}(\operatorname{Sheet1} 1 . C e l l s(3,12) *$ Sheet1.Cells $(j+1,3)+\operatorname{Sheet1.Cells~}(4,12) *$ Sheet1.Cells $(j+1,4)+$ Sheet $1 . \operatorname{Cells}(8,12) *$ Sheet $1 . C e l l s(j+1,8))$

VS $=\operatorname{Exp}(\operatorname{Sheet1} 1$ Cells $(3,12) *$ Sheet1.Cells $(j+2,3)+\operatorname{Sheet1.Cells~}(4,12) *$ Sheet1.Cells $(j+2,4)+\operatorname{Sheet} 1 . \operatorname{Cell} s(9,12) *$ Sheet1.Cells $(j+2,9))$

VB $=\operatorname{Exp}(\operatorname{Sheet1.Cells}(3,12) *$ Sheet1.Cells $(j+3,3)+\operatorname{Sheet1.Cells~}(4,12) *$ Sheet1.Cells $(j+3,4)+$ Sheet1.Cells $(10,12) * \operatorname{Sheet1.Cells~}(j+3,10))$

$\mathrm{VL}=\operatorname{Exp}(\operatorname{Sheet1} . C e l l s(3,12) *$ Sheet1.Cells $(j+4,3)+\operatorname{Sheet} 1 . C e l l s(4,12) *$ Sheet1.Cells $(j+4,4)+\operatorname{Sheet1.Cells~}(11,12) * \operatorname{Sheet} 1 . \operatorname{Cell} s(j+4,11))$

$\mathrm{PC}=\mathrm{VC} /(\mathrm{VC}+\mathrm{VT}+\mathrm{VS}+\mathrm{VB}+\mathrm{VL})$

$\mathrm{PT}=\mathrm{VT} /(\mathrm{VC}+\mathrm{VT}+\mathrm{VS}+\mathrm{VB}+\mathrm{VL})$

$\mathrm{PS}=\mathrm{VS} /(\mathrm{VC}+\mathrm{VT}+\mathrm{VS}+\mathrm{VB}+\mathrm{VL})$

$\mathrm{PB}=\mathrm{VB} /(\mathrm{VC}+\mathrm{VT}+\mathrm{VS}+\mathrm{VB}+\mathrm{VL})$

$\mathrm{PL}=\mathrm{VL} /(\mathrm{VC}+\mathrm{VT}+\mathrm{VS}+\mathrm{VB}+\mathrm{VL})$

$\mathrm{C}=$ Sheet1.Cells $(\mathrm{j}, 2)$

$\mathrm{T}=$ Sheet1.Cells $(\mathrm{j}+1,2)$

$\mathrm{S}=$ Sheet 1 .Cells $(\mathrm{j}+2,2)$

$\mathrm{B}=$ Sheet 1 Cells $(\mathrm{j}+3,2)$

$\mathrm{L}=$ Sheet 1 .Cells $(\mathrm{j}+4,2)$

Sheets("sheet6").Select

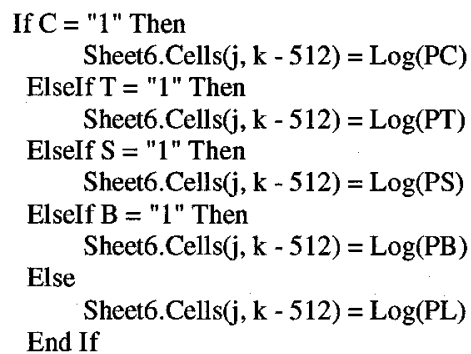

Next $\mathbf{j}$

Adding worksheets for calculation of Log-likelihood function value (sheet 4)

Elself $\mathbf{k}-768<=256$ Then

Sheets("sheet1").Select

For $\mathbf{j}=3$ To 2698 Step 5

$\mathrm{VC}=\operatorname{Exp}($ Sheet 1.Cells $(3,12) *$ Sheet1.Cells $(\mathrm{j}, 3)+\operatorname{Sheet1.Cells~}(4,12) *$ Sheet1.Cells $(\mathrm{j}, 4)$

+ Sheet1.Cells $(5,12) *$ Sheet1.Cells $(\mathfrak{j}, 5)+\operatorname{Sheet1.Cells~}(6,12) *$ Sheet1.Cells $(j, 6)+\operatorname{Sheet1.Cells~}(7,12) *$ Sheet1.Cells $(\mathfrak{j}, 7))$ 
VT $=\operatorname{Exp}(\operatorname{Sheet} 1$. Cells $(3,12) *$ Sheet1.Cells $(j+1,3)+\operatorname{Sheet1.Cells~}(4,12) *$ Sheet1.Cells $(j+1,4)+\operatorname{Sheet} 1 . C e l l s(8,12) * \operatorname{Sheet1.Cells~}(j+1,8))$ VS $=\operatorname{Exp}(\operatorname{Sheet1.Cells}(3,12) *$ Sheet1.Cells $(j+2,3)+$ Sheet1.Cells $(4,12) *$ Sheet1.Cells $(j+2,4)+$ Sheet 1.Cells $(9,12) * \operatorname{Sheet} 1 . C e l l s(j+2,9))$

VB $=\operatorname{Exp}($ Sheet 1.Cells $(3,12) *$ Sheet1.Cells $(j+3,3)+$ Sheet1.Cells $(4,12) *$ Sheet1.Cells $(j+3,4)+$ Sheet1.Cells $(10,12) *$ Sheet1.Cells $(j+3,10))$ $\mathrm{VL}=\operatorname{Exp}(\operatorname{Sheet1.Cells}(3,12) * \operatorname{Sheet1.Cells}(\mathbf{j}+4,3)+\operatorname{Sheet1.Cells}(4,12) * \operatorname{Sheet1.Cells}(\mathbf{j}+4,4)+\operatorname{Sheet} 1 . \operatorname{Cells}(11,12) * \operatorname{Sheet} 1 . \operatorname{Cells}(\mathrm{j}+4,11))$

$P C=V C /(V C+V T+V S+V B+V L)$

$\mathrm{PT}=\mathrm{VT} /(\mathrm{VC}+\mathrm{VT}+\mathrm{VS}+\mathrm{VB}+\mathrm{VL})$

$\mathrm{PS}=\mathrm{VS} /(\mathrm{VC}+\mathrm{VT}+\mathrm{VS}+\mathrm{VB}+\mathrm{VL})$

$\mathrm{PB}=\mathrm{VB} /(\mathrm{VC}+\mathrm{VT}+\mathrm{VS}+\mathrm{VB}+\mathrm{VL})$

$\mathrm{PL}=\mathrm{VL} /(\mathrm{VC}+\mathrm{VT}+\mathrm{VS}+\mathrm{VB}+\mathrm{VL})$

$\mathbf{C}=$ Sheet1.Cells $(\mathfrak{j}, 2)$

$T=$ Sheet1.Cells $(j+1,2)$

$\mathrm{S}=$ Sheet 1.Cells $(\mathrm{j}+2,2)$

$\mathrm{B}=$ Sheet 1 .Cells $(\mathfrak{j}+3,2)$

$\mathbf{L}=$ Sheet1.Cells $(j+4,2)$

Sheets("sheet7").Select

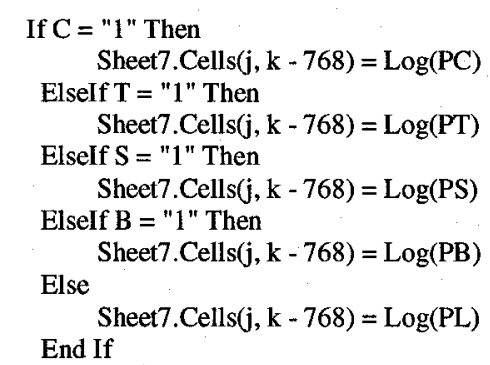

Next $\mathbf{j}$

End If

Utility Function Value for all alternatives' model specifications

For $\mathrm{i}=3$ To 2698 Step 5

Sheets("sheet2").Select

$\mathrm{VC}=\operatorname{Exp}($ Sheet1.Cells $(3,12) *$ Sheet1.Cells $(i, 3)+$ Sheet1.Cells $(4,12) *$ Sheet 1.Cells $(i, 4)$

+ Sheet 1.Cells $(5,12) *$ Sheet1.Cells $(i, 5)+$ Sheet1.Cells $(6,12) *$ Sheet1.Cells $(i, 6)+$ Sheet1.Cells $(7,12) *$ Sheet1.Cells $(i, 7))$

$\mathrm{VT}=\operatorname{Exp}(\operatorname{Sheet1.Cells}(3,12) * \operatorname{Sheet1.Cells}(\mathrm{i}+1,3)+\operatorname{Sheet1.Cells~}(4,12) *$ Sheet1.Cells $(\mathbf{i}+1,4)+\operatorname{Sheet} 1$. Cells $(8,12) * \operatorname{Sheet} 1$. Cells $(\mathrm{i}+1,8))$

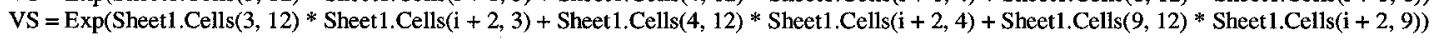

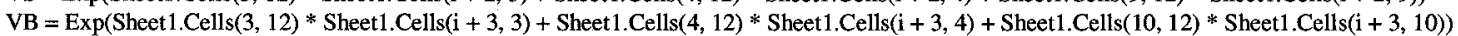

$\mathrm{VL}=\operatorname{Exp}(\operatorname{Sheet1.Cells}(3,12) *$ Sheet1.Cells $(\mathrm{i}+4,3)+\operatorname{Sheet1.Cells}(4,12) *$ Sheet 1.Cells $(\mathrm{i}+4,4)+$ Sheet1.Cells $(11,12) *$ Sheet1.Cells $(\mathrm{i}+4,11))$

Mode Choice Probability of each observations with Logit probability model

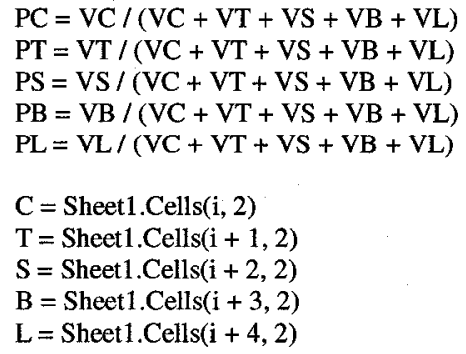

First-order Derivative with respect to $\beta_{1}$ 


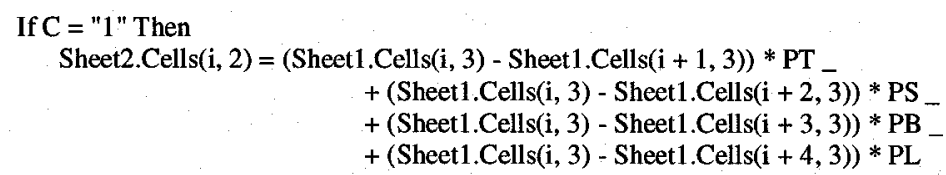

End If

First-order Derivative with respect to $\beta_{2}$

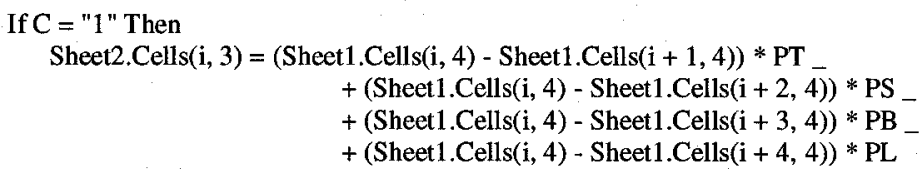


End If

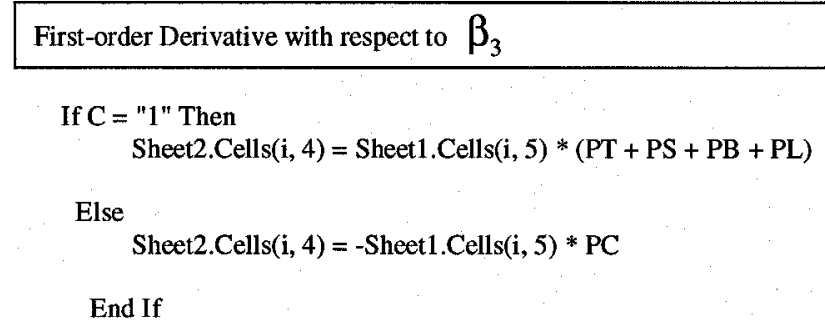

First-order Derivative with respect to $\beta_{4}$

If $\mathrm{C}=$ " 1 " Then

Sheet2.Cells $(\mathrm{i}, 5)=$ Sheet1.Cells $(\mathrm{i}, 6) *(\mathrm{PT}+\mathrm{PS}+\mathrm{PB}+\mathrm{PL})$

Else

Sheet2.Cells $(\mathrm{i}, 5)=-$ Sheet $1 . C e l l s(i, 6) *$ PC

End If

First-order Derivative with respect to $\beta_{5}$

If $\mathrm{C}=$ "1" Then

Sheet2.Cells $(\mathrm{i}, 6)=$ Sheet1 $\cdot$ Cells $(\mathrm{i}, 7) *(\mathrm{PT}+\mathrm{PS}+\mathrm{PB}+\mathrm{PL})$

Else

Sheet2.Cells $(\mathrm{i}, 6)=-$ Sheet1.Cells $(\mathrm{i}, 7) * \mathrm{PC}$

End If

First-order Derivative with respect to $\beta_{6}$

If $\mathrm{T}=$ " 1 " Then

Sheet2.Cells $(\mathbf{i}, 7)=(\mathrm{PC}+\mathrm{PS}+\mathrm{PB}+\mathrm{PL})$

Else

Sheet2.Cells $(\mathrm{i}, 7)=-(\mathrm{PT})$

End If

First-order Derivative with respect to $\beta_{7}$

If $S=$ " 1 " Then

Sheet2.Cells $(\mathrm{i}, 8)=(\mathrm{PC}+\mathrm{PT}+\mathrm{PB}+\mathrm{PL})$

Else

Sheet 2 .Cells $(\mathrm{i}, 8)=-\mathrm{PS}$

End If

First-order Derivative with respect to $\beta_{8}$

If $\mathrm{B}=$ " 1 " Then

Sheet2.Cells $(\mathrm{i}, 9)=(\mathrm{PC}+\mathrm{PT}+\mathrm{PS}+\mathrm{PL})$

Else

Sheet2.Cells $(i, 9)=-P B$ 
End If

First-order Derivative with respect to $\beta_{9}$

If $\mathrm{L}=$ " 1 " Then

Sheet2.Cells $(\mathbf{i}, 10)=(\mathrm{PC}+\mathrm{PT}+\mathrm{PS}+\mathrm{PB})$

Else

Sheet 2. Cells $(i, 10)=-P L$

End If

Next i

Summation of all observation and averaging value of it: $G_{t}$

For $\mathrm{j}=2$ To 10 Step 1

Sheet2.Cells $(2703, \mathrm{j})$.FormulaR1C1 = "=-SUM(R[-2700]C:R[-1]C)/540"

Next $j$

Sheets("sheet3").Select

Obtaining Gradient components calculated : $G_{t}$

For $\mathbf{i}=11$ To 19 Step 1

Sheet3.Cells $(i, 11)=$ Sheet $2 . \operatorname{Cells}(2703, i-9)$

Next $\mathrm{i}$

Calculating delta : $\delta_{t}=\lambda_{t} \Delta_{t}=\lambda_{t} W_{t} G_{t}=\lambda_{t}\left(-H_{t A P}^{B F G S}\right) G_{t}$

Sheet3.Range("M11:M19").FormulaArray = "=-(1/32)*(MMULT(A1:I9,K11:K19))"

Parameter estimates : $\beta_{t+1}=\beta_{t}+\delta_{t}=\beta_{t}+\lambda_{t} \Delta_{t}=\beta_{t}+\lambda_{t} W_{t} G_{t}=\beta_{t}+\lambda_{t}\left(-H_{t A P}^{B F G S}\right) G_{t}$

Sheet3.Cells $(11,16)=$ Sheet $1 . \operatorname{Cells}(3,12)+$ Sheet3.Cells $(11,13)$

Sheet3.Cells $(12,16)=$ Sheet1.Cells $(4,12)+$ Sheet 3 .Cells $(12,13)$

Sheet3.Cells $(13,16)=$ Sheet 1 .Cells $(5,12)+$ Sheet3.Cells $(13,13)$

Sheet3.Cells $(14,16)=$ Sheet1.Cells $(6,12)+$ Sheet3.Cells $(14,13)$

Sheet3.Cells $(15,16)=$ Sheet1.Cells $(7,12)+$ Sheet 3. Cells $(15,13)$

Sheet3.Cells $(16,16)=\operatorname{Sheet} 1 \cdot \operatorname{Cells}(8,12)+\operatorname{Sheet} 3 \cdot \operatorname{Cells}(16,13)$

Sheet3.Cells $(17,16)=$ Sheet1.Cells $(9,12)+$ Sheet3.Cells $(17,13)$

Sheet 3 .Cells $(18,16)=$ Sheet 1 .Cells $(10,12)+$ Sheet 3. Cells $(18,13)$

Sheet3.Cells $(19,16)=$ Sheet 1. Cells $(11,12)+$ Sheet 3. Cells $(19,13)$

Checking the Criterion for stopping: $\left[\frac{1}{k} \sum_{k=1}^{k}\left(\beta_{t+1, k}-\beta_{t, k}\right)^{2}\right]^{1 / 2}<C R$ 
Sheet 3. Cells $(21,12)=(($

$(\text { Sheet3.Cells }(11,16)-\text { Sheet1.Cells }(3,12))^{\wedge} 2+(\operatorname{Sheet3.Cells}(12,16)-\operatorname{Sheet1.Cells~}(4,12))^{\wedge} 2$ $+(\text { Sheet3.Cells }(13,16)-\text { Sheet 1.Cells }(5,12))^{\wedge} 2+(\text { Sheet3.Cells }(14,16)-\text { Sheet1.Cells }(6,12))^{\wedge} 2$ $+(\operatorname{Sheet3.Cells}(15,16)-\operatorname{Sheet1.Cells}(7,12))^{\wedge} 2+(\operatorname{Sheet3.Cells}(16,16)-\operatorname{Sheet1.Cells}(8,12))^{\wedge} 2$ $+(\text { Sheet3.Cells }(17,16)-\text { Sheet 1.Cells }(9,12))^{\wedge} 2+(\operatorname{Sheet3.Cells}(18,16)-\operatorname{Sheet1.Cells}(10,12))^{\wedge} 2$ $+(\text { Sheet3.Cells }(19,16)-\text { Sheet 1.Cells }(11,12))^{\wedge} 2$

$$
)^{*}(1 / 9)\right)^{\wedge}(0.5)
$$

If Sheet3.Cells $(21,12)<10^{\wedge}(-4)$ Then

GoTo line1

Else

Starting the procedure for obtaining updating approximate hessan

Calculating: $G_{t+1}$

For $\mathrm{i}=3$ To 2698 Step 5

Sheets("sheet2").Select

$\mathrm{vC}=\operatorname{Exp}($ Sheet3.Cells $(11,16) *$ Sheet1.Cells(i, 3) + Sheet3.Cells $(12,16) *$ Sheet1.Cells $(\mathbf{i}, 4)+$ Sheet3.Cells $(13,16) *$ Sheet1.Cells(i, 5) + Sheet3.Cells(14, 16) * Sheet1.Cells(i, 6) + Sheet3.Cells $(15,16) *$ Sheet1.Cells(i, 7))

VT $=$ Exp(Sheet3.Cells $(11,16) *$ Sheet1.Cells $(i+1,3)+$ Sheet3.Cells $(12,16) *$ Sheet1.Cells $(i+1,4)+$ Sheet3.Cells $(16,16)$ * Sheet1.Cells $(i+1,8)$ )

VS $=\operatorname{Exp}(\operatorname{Sheet3.Cells} s(11,16) *$ Sheet1.Cells $(i+2,3)+\operatorname{Sheet3.Cells}(12,16) *$ Sheet1.Cells $(i+2,4)+\operatorname{Sheet3.Cells}(17$ 16) * Sheet1.Cells $(\mathrm{i}+2,9))$

VB $=\operatorname{Exp}(\operatorname{Sheet3.Cells~}(11,16) *$ Sheet1.Cells $(\mathrm{i}+3,3)+\operatorname{Sheet3.Cells}(12,16) *$ Sheet1.Cells $(\mathrm{i}+3,4)+\operatorname{Sheet3.Cells} s(18,16)$

* Sheet1.Cells $(\mathrm{i}+3,10))$

$\mathrm{VL}=\operatorname{Exp}(\operatorname{Sheet3.Cells}(11,16) *$ Sheet1.Cells $(\mathrm{i}+4,3)+\operatorname{Sheet3.Cells}(12,16) *$ Sheet1.Cells $(i+4,4)+\operatorname{Sheet3.Cells}(19$ 16) * Sheet1.Cells $(i+4,11))$

$\mathrm{PC}=\mathrm{VC} /(\mathrm{VC}+\mathrm{VT}+\mathrm{VS}+\mathrm{VB}+\mathrm{VL})$

$\mathrm{PT}=\mathrm{VT} /(\mathrm{VC}+\mathrm{VT}+\mathrm{VS}+\mathrm{VB}+\mathrm{VL})$

$\mathrm{PS}=\mathrm{VS} /(\mathrm{VC}+\mathrm{VT}+\mathrm{VS}+\mathrm{VB}+\mathrm{VL})$

$\mathrm{PB}=\mathrm{VB} /(\mathrm{VC}+\mathrm{VT}+\mathrm{VS}+\mathrm{VB}+\mathrm{VL})$

$P L=V L /(V C+V T+V S+V B+V L)$

$\mathrm{C}=$ Sheet1.Cells $(\mathrm{i}, 2)$

$\mathrm{T}=$ Sheet 1 .Cells $(\mathrm{i}+1,2)$

$\mathrm{S}=$ Sheet 1 .Cells $(\mathrm{i}+2,2)$

$\mathbf{B}=$ Sheet 1 Cells $(\mathrm{i}+3,2)$

$\mathrm{L}=$ Sheet 1 .Cells $(\mathrm{i}+4,2)$

First-order Derivative with respect to $\beta$

\section{If $\mathrm{C}=$ " 1 " Then}

Sheet2.Cells $(\mathrm{i}, 12)=($ Sheet1.Cells $(\mathrm{i}, 3)-$ Sheet 1.Cells $(\mathrm{i}+1,3)) * \mathrm{PT}$

$+($ Sheet1.Cells $(\mathbf{i}, 3)-$ Sheet1.Cells $(\mathrm{i}+2,3)) *$ PS

$+($ Sheet1.Cells $(\mathbf{i}, 3)-$ Sheet 1.Cells $(\mathrm{i}+3,3)) * \mathrm{~PB}$

$+($ Sheet1.Cells $(\mathrm{i}, 3)-$ Sheet1.Cells $(\mathrm{i}+4,3)) * \mathrm{PL}$

Elself T $=$ "1" Then

Sheet2.Cells $(\mathbf{i}, 12)=($ Sheet1.Cells $(\mathbf{i}+1,3)-$ Sheet $1 . C e l l s(i, 3)) * P C$

$+($ Sheet1.Cells $(\mathrm{i}+1,3)$ - Sheet1.Cells $(\mathrm{i}+2,3)) * \mathrm{PS}$

$+($ Sheet1.Cells $(\mathrm{i}+1,3)$ - Sheet1.Cells $(\mathrm{i}+3,3)) * \mathrm{~PB}$

$+($ Sheet1.Cells $(\mathrm{i}+1,3)-$ Sheet1.Cells $(\mathrm{i}+4,3)) * \mathrm{PL}$

Elself $S=" 1 "$ Then

Sheet2.Cells $(\mathrm{i}, 12)=($ Sheet1.Cells $(\mathrm{i}+2,3)-$ Sheet1.Cells $(\mathrm{i}, 3)) * \mathrm{PC}_{-}$ 
$+($ Sheet 1.Cells $(\mathrm{i}+2,3)-$ Sheet1.Cells $(\mathrm{i}+1,3)) * \mathrm{PT}$ $+($ Sheet1.Cells $(\mathrm{i}+2,3)$ - Sheet1.Cells $(\mathrm{i}+3,3)) * \mathrm{~PB}-$ $+($ Sheet1.Cells $(i+2,3)-$ Sheet1.Cells $(i+4,3)) * P L$

ElseIf B = "1" Then

Sheet2.Cells $(\mathrm{i}, 12)=($ Sheet1.Cells $(\mathrm{i}+3,3)-$ Sheet1.Cells $(\mathrm{i}, 3)) * \mathrm{PC}$

$+($ Sheet1.Cells $(\mathrm{i}+3,3)-$ Sheet1.Cells $(\mathrm{i}+1,3)) * \mathrm{PT}$ $+($ Sheet1.Cells $(\mathrm{i}+3,3)$ - Sheet1.Cells $(\mathrm{i}+2,3)) * \mathrm{PS}_{-}^{-}$ $+($ Sheet1.Cells $(i+3,3)-$ Sheet1.Cells $(i+4,3)) *$ PL

Else

Sheet2.Cells $(i, 12)=($ Sheet1.Cells $(i+4,3)-$ Sheet1.Cells $(i, 3)) *$ PC $+($ Sheet1.Cells $(\mathrm{i}+4,3)-$ Sheet1.Cells $(\mathrm{i}+1,3)) * \mathrm{PT}$ $+($ Sheet1.Cells $(i+4,3)$ - Sheet1.Cells $(i+2,3)) * \mathrm{PS}_{-}$ $+($ Sheet1.Cells $(i+4,3)-$ Sheet1.Cells $(i+3,3)) *$ PB

End If

First-order Derivative with respect to $\beta_{2}$

If $\mathrm{C}=$ " 1 " Then

Sheet2.Cells $(i, 13)=($ Sheet1.Cells $(i, 4)-$ Sheet 1.Cells $(i+1,4)) *$ PT $+($ Sheet1.Cells $(i, 4)-$ Sheet1.Cells $(i+2,4)) *$ PS $+($ Sheet1.Cells $(\mathrm{i}, 4)$ - Sheet1.Cells $(\mathrm{i}+3,4)) * \mathrm{~PB}$ $+($ Sheet1.Cells $(\mathrm{i}, 4)-$ Sheet1.Cells $(\mathrm{i}+4,4)) * \mathrm{PL}$

Elself $\mathrm{T}=$ " 1 " Then

Sheet2.Cells $(\mathrm{i}, 13)=($ Sheet1.Cells $(\mathrm{i}+1,4)-$ Sheet1.Cells $(\mathrm{i}, 4)) *$ PC $+($ Sheet1.Cells $(\mathrm{i}+1,4)-$ Sheet1.Cells $(\mathrm{i}+2,4)) * \mathrm{PS}$ $+($ Sheet1.Cells $(i+1,4)-$ Sheet1.Cells $(i+3,4)) *$ PB $_{-}^{-}$ $+($ Sheet1.Cells $(\mathrm{i}+1,4)-$ Sheet1.Cells $(\mathrm{i}+4,4)) * \mathrm{PL}$

Elself $S=$ " 1 " Then

Sheet2.Cells $(\mathrm{i}, 13)=($ Sheet1.Cells $(\mathrm{i}+2,4)-$ Sheet1.Cells $(\mathrm{i}, 4)) * \mathrm{PC}$ $+($ Sheet1.Cells $(i+2,4)-$ Sheet1.Cells $(i+1,4)) *$ PT $+($ Sheet1.Cells $(\mathrm{i}+2,4)$ - Sheet1.Cells $(\mathrm{i}+3,4)) * \mathrm{~PB}$ $+($ Sheet1.Cells $(i+2,4)-$ Sheet1.Cells $(i+4,4)) *$ PL

Elself $B=$ " 1 " Then

Sheet2.Cells $(i, 13)=($ Sheet1.Cells $(i+3,4)-$ Sheet1.Cells $(i, 4)) *$ PC

$+($ Sheet1.Cells $(\mathrm{i}+3,4)$ - Sheet1.Cells $(\mathrm{i}+1,4)) * \mathrm{PT}$ $+($ Sheet1.Cells $(i+3,4)$ - Sheet1.Cells $(i+2,4)) *$ PS _ $+($ Sheet1.Cells $(i+3,4)-$ Sheet1.Cells $(i+4,4)) * \mathrm{PL}^{-}$

Else

Sheet2.Cells $(i, 13)=($ Sheet1.Cells $(i+4,4)-$ Sheet 1. Cells $(i, 4)) * P C$ $+($ Sheet1.Cells $(i+4,4)-$ Sheet1.Cells $(i+1,4)) * P T$ $+($ Sheet1.Cells $(i+4,4)-$ Sheet1.Cells $(i+2,4)) * P_{-}^{-}$ $+($ Sheet1.Cells $(\mathrm{i}+4,4)-$ Sheet1.Cells $(\mathrm{i}+3,4)) * \mathrm{~PB}^{-}$

End If

First-order Derivative with respect to $\beta_{3}$

If $\mathrm{C}=$ " 1 " Then

Sheet2.Cells $(\mathrm{i}, 14)=$ Sheet $1 . \operatorname{Cells}(\mathrm{i}, 5) *(\mathrm{PT}+\mathrm{PS}+\mathrm{PB}+\mathrm{PL})$

Else

Sheet2.Cells $(i, 14)=-$ Sheet1.Cells $(i, 5) *$ PC

End If

First-order Derivative with respect to $\beta_{4}$ 


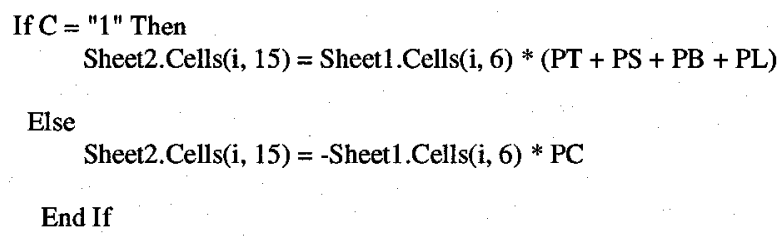

Summation and averaging gradient: $G_{t+1}$ 
For $\mathrm{j}=12$ To 20 Step 1

Sheet2.Cells $(2703, \mathrm{j})$.FormulaR1C1 = "=-SUM(R[-2700]C:R[-1]C)/540"

Next $j$

Sheets("sheet3").Select

Obtaining gradient: $G_{t+1}$

For $\mathbf{i}=1$ To 9 Step 1

Sheet3.Cells $(i, 11)=$ Sheet 2. eells $(2703, i+11)$

Next i

Calculating gamma: $\gamma_{t}=G_{t+1}-G_{t}$

Sheet3.Cells $(11,18)=$ Sheet3.Cells $(1,11)-$ Sheet3.Cells $(11,11)$

Sheet3.Cells $(12,18)=$ Sheet3.Cells $(2,11)-$ Sheet3.Cells $(12,11)$

Sheet3.Cells $(13,18)=$ Sheet3.Cells $(3,11)-$ Sheet3.Cells $(13,11)$

Sheet 3 .Cells $(14,18)=$ Sheet3.Cells $(4,11)-$ Sheet3.Cells $(14,11)$

Sheet3.Cells $(15,18)=$ Sheet3.Cells $(5,11)-$ Sheet3.Cells $(15,11)$

Sheet3.Cells $(16,18)=$ Sheet3.Cells $(6,11)-$ Sheet3.Cells $(16,11)$

Sheet3.Cells $(17,18)=$ Sheet3.Cells $(7,11)-$ Sheet3.Cells $(17,11)$

Sheet3.Cells $(18,18)=$ Sheet3.Cells $(8,11)-$ Sheet3.Cells $(18,11)$

Sheet3.Cells $(19,18)=$ Sheet3.Cells $(9,11)-$ Sheet3.Cells $(19,11)$

Calculating approximate hessian: $H_{t+1}^{B G F S}=H_{t}^{B F G S}+\frac{\delta_{t} \delta_{t}^{\prime}}{\delta_{t}^{\prime} \gamma_{t}}-\frac{H_{t}^{B F G S} \gamma_{t} \gamma_{t}^{\prime} H_{t}^{B F G S}}{\gamma_{t}^{\prime} H_{t}^{B F G S} \gamma_{t}}$

Transpose matrix of a vector of gamma: $\gamma_{t}$

Sheet3.Cells $(28,11)=$ Sheet 3 .Cells $(11,18)$

Sheet 3 .Cells $(28,12)=$ Sheet 3 .Cells $(12,18)$

Sheet3.Cells $(28,13)=$ Sheet 3 .Cells $(13,18)$

Sheet3.Cells $(28,14)=$ Sheet3.Cells $(14,18)$

Sheet3.Cells $(28,15)=$ Sheet 3 .Cells $(15,18)$

Sheet 3 .Cells $(28,16)=$ Sheet $3 \cdot$ Cells $(16,18)$

Sheet 3 .Cells $(28,17)=$ Sheet 3 .Cells $(17,18)$

Sheet 3 .Cells $(28,18)=$ Sheet 3 .Cells $(18,18)$

Sheet3.Cells $(28,19)=$ Sheet3.Cells $(19,18)$

Transpose matrix of a vector of delta: $\delta_{t}^{\prime}$

Sheet3.Cells $(30,11)=$ Sheet 3 .Cells $(11,13)$

Sheet 3 .Cells $(30,12)=$ Sheet $3 \cdot$ Cells $(12,13)$

Sheet3.Cells $(30,13)=$ Sheet 3. Cells $(13,13)$

Sheet 3. Cells $(30,14)=$ Sheet 3. Cells $(14,13)$ 
Sheet3.Cells $(30,15)=$ Sheet 3. Cells $(15,13)$

Sheet3.Cells $(30,16)=$ Sheet3.Cells $(16,13)$

Sheet3.Cells $(30,17)=$ Sheet 3 .Cells $(17,13)$

Sheet3.Cells $(30,18)=$ Sheet 3. Cells $(18,13)$

Sheet3.Cells $(30,19)=$ Sheet 3. Cells $(19,13)$

\section{$\delta_{t} \delta_{t}^{\prime}$}

Sheet3.Range("K33:S41").FormulaArray = "=MMULT(M11:M19,K30:S30)"

$\delta_{t}^{\prime} \gamma_{t}$

Sheet3.Range("L43").FormulaArray = "=MMULT(K30:S30,R11:R19)"

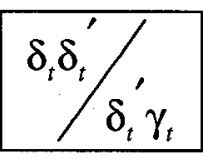

For $\mathbf{i}=33$ To 41 Step 1

For $\mathbf{j}=11$ To 19 Step 1 Next $j$ Sheet3.Cells $(i, j+10)=$ Sheet3.Cells $(i, j) /$ Sheet3.Cells $(43,12)$

Next $\mathrm{i}$

$H_{t}^{B F G S} \gamma_{t}$

Sheet3.Range("K45:K53").FormulaArray = "=MMULT(A1:I9,R11:R19)"

$$
H_{t}^{B F G S} \gamma_{t} \gamma_{t}^{\prime}
$$

Sheet3.Range("M45:U53").FormulaArray = "=MMULT(K45:K53,K28:S28)"

$$
H_{t}^{B F G S} \gamma_{t} \gamma_{t}^{\prime} H_{t}^{B F G S}
$$

Sheet3.Range("K55:S63").FormulaArray = "=MMULT(M45:U53,A1:I9)"

'--

$$
\gamma_{t}^{\prime} H_{t}^{B F G S}
$$

Sheet3.Range("K66:S66").FormulaArray = "=MMULT(K28:S28,A1:I9)"

$$
\gamma_{t}^{\prime} H_{t}^{B F G S} \gamma_{t}
$$

Sheet3.Range("L67").FormulaArray = "=MMULT(K66:S66,R11:R19) "

$$
\frac{H_{t}^{B F G S} \gamma_{t} \gamma_{t}^{\prime} H_{t}^{B F G S}}{\gamma_{t}^{\prime} H_{t}^{B F G S} \gamma_{t}}
$$

For $\mathrm{i}=55$ To 63 Step 1

For $\mathbf{j}=11$ To 19 Step 1 

Next $\mathbf{j}$

Sheet3.Cells $(i, j+10)=$ Sheet3.Cells $(i, j) /$ Sheet3.Cells $(67,12)$

Next i

$$
\begin{aligned}
& H_{t}^{B F G S}+\frac{\delta_{t} \delta_{t}^{\prime}}{\delta_{t}^{\prime} \gamma_{t}}-\frac{H_{t}^{B F G S} \gamma_{t} \gamma_{t}^{\prime} H_{t}^{B F G S}}{\gamma_{t}^{\prime} H_{t}^{B F G S} \gamma_{t}} \\
& \text { For } \mathrm{i}=72 \text { To } 80 \text { Step } 1 \\
& \text { For } \mathrm{j}=11 \text { To } 19 \text { Step } 1 \\
& \text { Next } \mathbf{j} \\
& \text { Sheet3.Cells }(\mathbf{i}, \mathbf{j})=\text { Sheet3.Cells }(\mathbf{i}-71, \mathbf{j}-10)+\text { Sheet } 3 . C e l l s(i-39, j+10)-\operatorname{Sheet} 3 . C e l l s(i-17, j+10)
\end{aligned}
$$

Next $\mathrm{i}$

Additional procedure for conducting BFGS method

$$
\left(\frac{1}{\delta_{t}^{\prime} \gamma_{t}}\right) \delta_{t}
$$

For $\mathbf{i}=11$ To 19 Step 1

Sheet 3. Cells $(i, 20)=$ Sheet $3 . \operatorname{Cell} s(i, 13) /$ Sheet 3. Cells $(43,12)$

Next i

$$
\left(\frac{1}{\gamma_{t}^{\prime} H_{B F G S} \gamma_{t}}\right) H_{B F G S} \gamma_{t}
$$

For $\mathrm{i}=11$ To 19 Step 1

Sheet3.Cells $(\mathrm{i}, 22)=$ Sheet3.Cells $(i+34,11) /$ Sheet 3. Cells $(67,12)$

Next i

$$
d_{t}=\left(\frac{1}{\delta_{t}^{\prime} \gamma_{t}}\right) \delta_{t}-\left(\frac{1}{\gamma_{t}^{\prime} H_{B F G S} \gamma_{t}}\right) H_{B F G S} \gamma_{t}
$$

$$
\text { For } \mathrm{i}=11 \text { To } 19 \text { Step } 1
$$

Sheet3.Cells $(\mathbf{i}, 24)=$ Sheet3.Cells $(\mathbf{i}, 20)-$ Sheet3.Cells $(\mathbf{i}, 22)$

Next $\mathrm{i}$

$$
d_{t}^{\prime}
$$

Sheet3.Cells $(84,11)=$ Sheet3.Cells $(11,24)$

Sheet3.Cells $(84,12)=$ Sheet 3 .Cells $(12,24)$

Sheet3.Cells $(84,13)=$ Sheet 3. Cells $(13,24)$

Sheet3.Cells $(84,14)=$ Sheet 3. Cells $(14,24)$ 
Sheet3.Cells $(84,15)=$ Sheet 3 .Cells $(15,24)$

Sheet3.Cells $(84,16)=$ Sheet3.Cells $(16,24)$

Sheet3.Cells $(84,17)=$ Sheet 3 .Cells $(17,24)$

Sheet 3 .Cells $(84,18)=$ Sheet 3 .Cells $(18,24)$

Sheet3.Cells $(84,19)=$ Sheet 3 .Cells $(19,24)$

$$
d_{t} d_{t}^{\prime}
$$

For $\mathbf{j}=11$ To 19 Step 1

For $\mathrm{i}=88$ To 96 Step 1 Next

Sheet3.Cells $(i, j)=$ Sheet3.Cells $(i-77,24) *$ Sheet3.Cells $(84, j)$

Next $\mathbf{j}$

$$
\begin{aligned}
& v d_{t} d_{t}^{\prime}=\left(\gamma_{t}^{\prime} H_{B F G S} \gamma_{t}\right) d_{t} d_{t}^{\prime} \\
& \text { For } \left.\mathrm{i}=\begin{array}{c}
100 \text { To } 108 \\
\text { For } \mathrm{j}=11 \text { To } 19 \\
\text { Sheet } 3 . C e l l s(\mathrm{i}, \mathrm{j})
\end{array}\right) \text { Sheet3.Cells }(\mathrm{i}-12, \mathrm{j}) * \text { Sheet3.Cells }(67,12) \\
& \text { Next } \mathrm{j}
\end{aligned}
$$

$\left(H_{B F G S}+\frac{\delta_{t} \delta_{t}^{\prime}}{\delta_{t}^{\prime} \gamma_{t}}-\frac{H_{B F G S} \gamma_{t} \gamma_{t}^{\prime} H_{B F G S}}{\gamma_{t}^{\prime} H_{B F G S} \gamma_{t}}\right)+v d_{t} d_{t}^{\prime}$

For $\mathrm{i}=111$ To 119 Step 1

For $j=11$ To 19 Step 1

Next j

$$
\text { Sheet3.Cells }(i, j)=\text { Sheet3.Cells }(i-39, j)+\operatorname{Sheet} 3 . \operatorname{Cells}(i-11, j)
$$

Next i

End of Additional procedure for conducting BFGS method

Obtaining updated approximate hessain

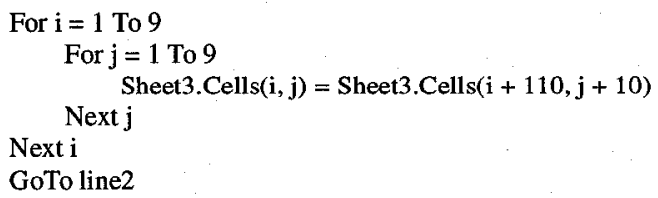

End If

line1:

End Sub 


\section{B.7: DFP-GSM algorithm}

The seventh algorithm: $D F P$-GSM

(Visual Basic Application provided in the Microsoft EXCEL)

Coded by Hyukjae Roh

Private Sub DFP-GSM_CLICK()

Variables Generated _ _ _ _

$\operatorname{Dim} \mathbf{i}, \mathbf{j}, \mathbf{k}, \mathrm{G}$ As Integer

Dim alfa As Double

Dim C As Integer

Dim $\mathrm{T}$ As Integer

Dim S As Integer

Dim B As Integer

Dim L As Integer

Dim VC As Double

Dim VT As Double

Dim VS As Double

Dim VB As Double

Dim VL As Double

Dim PC As Double

Dim PT As Double

Dim PS As Double

Dim PB As Double

Dim PL As Double

Sheets("sheet1").Select

$\mathrm{k}=1$

If $\mathrm{k}=1$ Then

End If

GoTo line3

Initializing Staring Values of Parameters

line2:

$\mathrm{k}=\mathrm{k}+\mathbf{1}$

line3:

Sheet1.Cells $(3,12)=$ Sheet $3 \cdot \operatorname{Cells}(11,16)$

Sheet1.Cells $(4,12)=$ Sheet 3 .Cells $(12,16)$

Sheet 1 .Cells $(5,12)=$ Sheet 3 .Cells $(13,16)$

Sheet1.Cells $(6,12)=$ Sheet $3 \cdot \operatorname{Cells}(14,16)$

Sheet1.Cells $(7,12)=$ Sheet3.Cells $(15,16)$

Sheet 1 .Cells $(8,12)=$ Sheet 3 .Cells $(16,16)$

Sheet1.Cells $(9,12)=$ Sheet $3 \cdot \operatorname{Cells}(17,16)$

Sheet1.Cells $(10,12)=$ Sheet 3 .Cells $(18,16)$

Sheet1.Cells $(11,12)=$ Sheet 3 .Cells $(19,16)$ 
Adding worksheets for calculation of Log-likelihood function value (sheet 1)

If $256-k>=0$ Then

Sheets("sheet1").Select

For $\mathrm{j}=3$ To 2698 Step 5

$\mathrm{VC}=\operatorname{Exp}(\operatorname{Sheet} 1 . \operatorname{Cells}(3,12) *$ Sheet1.Cells $(\mathrm{j}, 3)+$ Sheet1.Cells $(4,12) *$ Sheet1.Cells $(\mathrm{j}, 4)$

+ Sheet1.Cells $(5,12) *$ Sheet1.Cells(j, 5) + Sheet1.Cells $(6,12) *$ Sheet1.Cells(j, 6) + Sheet1.Cells $(7,12) *$ Sheet1.Cells(j, 7))

$\mathrm{VT}=\operatorname{Exp}(\operatorname{Sheet} 1 . \operatorname{Cells}(3,12) *$ Sheet1.Cells $(j+1,3)+\operatorname{Sheet1} . \operatorname{Cells}(4,12) *$ Sheet1.Cells $(j+1,4)+\operatorname{Sheet} 1 . \operatorname{Cells}(8,12) * \operatorname{Sheet1}$. Cells $(j+1,8))$

VS $=\operatorname{Exp}(\operatorname{Sheet} 1 . \operatorname{Cells}(3,12) *$ Sheet1.Cells $(j+2,3)+\operatorname{Sheet1.Cells~}(4,12) * \operatorname{Sheet1.Cells~}(j+2,4)+\operatorname{Sheet1} . \operatorname{Cell} s(9,12) * \operatorname{Sheet1} . \operatorname{Cells}(\mathrm{j}+2,9))$

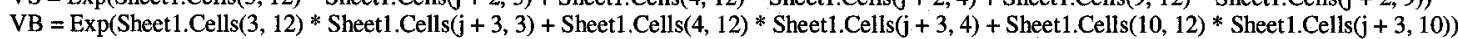

$\mathrm{VL}=\operatorname{Exp}(\operatorname{Sheet1.Cells}(3,12) *$ Sheet1.Cells $(j+4,3)+\operatorname{Sheet1.Cells~}(4,12) * \operatorname{Sheet1.Cells~}(j+4,4)+\operatorname{Sheet1.Cells~}(11,12) * \operatorname{Sheet1.Cells~}(j+4,11))$

$\mathrm{PC}=\mathrm{VC} /(\mathrm{VC}+\mathrm{VT}+\mathrm{VS}+\mathrm{VB}+\mathrm{VL})$

$\mathrm{PT}=\mathrm{VT} /(\mathrm{VC}+\mathrm{VT}+\mathrm{VS}+\mathrm{VB}+\mathrm{VL})$

$\mathrm{PS}=\mathrm{VS} /(\mathrm{VC}+\mathrm{VT}+\mathrm{VS}+\mathrm{VB}+\mathrm{VL})$

$\mathrm{PB}=\mathrm{VB} /(\mathrm{VC}+\mathrm{VT}+\mathrm{VS}+\mathrm{VB}+\mathrm{VL})$

$\mathrm{PL}=\mathrm{VL} /(\mathrm{VC}+\mathrm{VT}+\mathrm{VS}+\mathrm{VB}+\mathrm{VL})$

$\mathrm{C}=$ Sheet 1 .Cells $(\mathbf{j}, 2)$

$\mathrm{T}=$ Sheet1.Cells $(\mathfrak{j}+1,2)$

$\mathrm{S}=$ Sheet 1 .Cells $(\mathrm{j}+2,2)$

$\mathrm{B}=$ Sheet1.Cells $(\mathrm{j}+3,2)$

$\mathrm{L}=$ Sheet1.Cells $(\mathrm{j}+4,2)$

Sheets("sheet4").Select

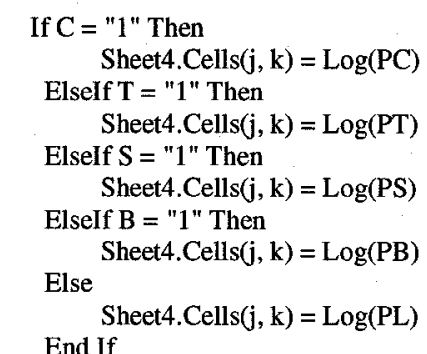

Next $\mathbf{j}$

Adding worksheets for calculation of Log-likelihood function value (sheet 2)

Elself $\mathrm{k}-256<=256$ Then

Sheets("sheet1").Select

For $\mathbf{j}=3$ To 2698 Step 5

$\mathrm{VC}=\operatorname{Exp}(\operatorname{Sheet1} 1$ Cells $(3,12) *$ Sheet1.Cells $(\mathrm{j}, 3)+\operatorname{Sheet1.Cells~}(4,12) * \operatorname{Sheet} 1 . \operatorname{Cells}(\mathrm{j}, 4)$ + Sheet1.Cells $(5,12) *$ Sheet1.Cells $(j, 5)+$ Sheet1.Cells $(6,12) *$ Sheet1.Cells $(j, 6)+$ Sheet1.Cells $(7,12) *$ Sheet1.Cells $(j ;, 7)$

VT $=\operatorname{Exp}(\operatorname{Sheet1} \cdot \operatorname{Cells}(3,12) *$ Sheet1.Cells $(j+1,3)+\operatorname{Sheet1.Cells~}(4,12) *$ Sheet1.Cells $(j+1,4)+$ Sheet1.Cells $(8,12) *$ Sheet1.Cells $(j+1,8))$

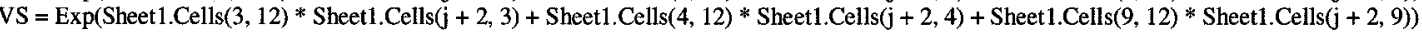

$\mathrm{VB}=\operatorname{Exp}(\operatorname{Sheet} 1 . \operatorname{Cells}(3,12) *$ Sheet1.Cells $(\mathrm{j}+3,3)+\operatorname{Sheet1.Cells~}(4,12) *$ Sheet1.Cells $(j+3,4)+\operatorname{Sheet} 1 . \operatorname{Cell} s(10,12) * \operatorname{Sheet} 1 . \operatorname{Cells}(\mathrm{j}+3,10))$

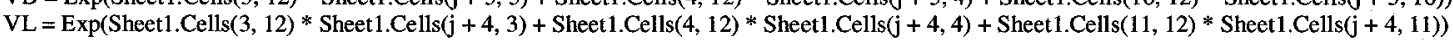

$\mathrm{PC}=\mathrm{VC} /(\mathrm{VC}+\mathrm{VT}+\mathrm{VS}+\mathrm{VB}+\mathrm{VL})$

$\mathrm{PT}=\mathrm{VT} /(\mathrm{VC}+\mathrm{VT}+\mathrm{VS}+\mathrm{VB}+\mathrm{VL})$

$\mathrm{PS}=\mathrm{VS} /(\mathrm{VC}+\mathrm{VT}+\mathrm{VS}+\mathrm{VB}+\mathrm{VL})$

$\mathrm{PB}=\mathrm{VB} /(\mathrm{VC}+\mathrm{VT}+\mathrm{VS}+\mathrm{VB}+\mathrm{VL})$

$\mathrm{PL}=\mathrm{VL} /(\mathrm{VC}+\mathrm{VT}+\mathrm{VS}+\mathrm{VB}+\mathrm{VL})$

$\mathrm{C}=$ Sheet 1. Cells $(\mathrm{j}, 2)$

$\mathrm{T}=$ Sheet 1 .Cells $(\mathrm{j}+1,2)$

$\mathrm{S}=$ Sheet1.Cells $(\mathrm{j}+2,2)$

$B=$ Sheet 1 Cells $(j+3,2)$

$\mathrm{L}=$ Sheet1.Cells $(\mathrm{j}+4,2)$ 
Sheets("sheet5").Select

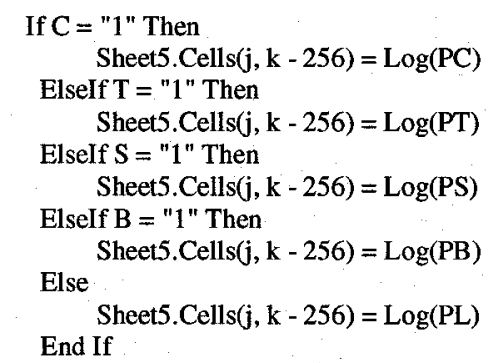

Next $\mathrm{j}$

Adding worksheets for calculation of Log-likelihood function value (sheet 3 )

ElseIf $\mathrm{k}-512<=256$ Then

Sheets("sheet1").Select

For $\mathrm{j}=3$ To 2698 Step 5

$\mathrm{VC}=\operatorname{Exp}(\operatorname{Sheet1} \cdot \operatorname{Cells}(3,12) * \operatorname{Sheet1.Cells(j,3)}+\operatorname{Sheet1.Cells}(4,12) * \operatorname{Sheet1.Cells~}(\mathbf{j}, 4)$ + Sheet1.Cells $(5,12) *$ Sheet1.Cells(j, 5) + Sheet1.Cells $(6,12) *$ Sheet1.Cells $(\mathrm{j}, 6)+$ Sheet1.Cells $(7,12) *$ Sheet1.Cells(j, 7$))$

VT $=\operatorname{Exp}(\operatorname{Sheet1} 1$ Cells $(3,12) *$ Sheet1.Cells $(j+1,3)+\operatorname{Sheet1} . \operatorname{Cells}(4,12) * \operatorname{Sheet1.Cells~}(j+1,4)+\operatorname{Sheet} 1 . \operatorname{Cells}(8,12) *$ Sheet1.Cells $(j+1,8))$

VS $=\operatorname{Exp}(\operatorname{Sheet1.Cells~}(3,12) *$ Sheet1.Cells $(j+2,3)+$ Sheet1.Cells $(4,12) *$ Sheet1.Cells $(j+2,4)+$ Sheet1.Cells $(9,12) * \operatorname{Sheet1.Cells~}(j+2,9))$

$\mathrm{VB}=\operatorname{Exp}(\operatorname{Sheet1} . \operatorname{Cells}(3,12) *$ Sheet1.Cells $(\mathrm{j}+3,3)+\operatorname{Sheet1} 1$ Cells $(4,12) *$ Sheet1.Cells $(j+3,4)+\operatorname{Sheet} 1 . \operatorname{Cell} s(10,12) *$ Sheet 1.Cells $(\mathrm{j}+3,10))$

$\mathrm{VL}=\operatorname{Exp}(\operatorname{Sheet1.Cells~}(3,12) *$ Sheet1.Cells $(j+4,3)+\operatorname{Sheet} 1 . \operatorname{Cells}(4,12) * \operatorname{Sheet1.Cells~}(j+4,4)+\operatorname{Sheet} 1 . \operatorname{Cells}(11,12) * \operatorname{Sheet1.Cells~}(j+4,11))$

$\mathrm{PC}=\mathrm{VC} /(\mathrm{VC}+\mathrm{VT}+\mathrm{VS}+\mathrm{VB}+\mathrm{VL})$

$\mathrm{PT}=\mathrm{VT} /(\mathrm{VC}+\mathrm{VT}+\mathrm{VS}+\mathrm{VB}+\mathrm{VL})$

$\mathrm{PS}=\mathrm{VS} /(\mathrm{VC}+\mathrm{VT}+\mathrm{VS}+\mathrm{VB}+\mathrm{VL})$

$\mathrm{PB}=\mathrm{VB} /(\mathrm{VC}+\mathrm{VT}+\mathrm{VS}+\mathrm{VB}+\mathrm{VL})$

$\mathrm{PL}=\mathrm{VL} /(\mathrm{VC}+\mathrm{VT}+\mathrm{VS}+\mathrm{VB}+\mathrm{VL})$

$\mathrm{C}=$ Sheet 1. Cells $(\mathrm{j}, 2)$

$\mathrm{T}=$ Sheet1.Cells $(\mathfrak{j}+1,2)$

$S=$ Sheet 1. Cells $(j+2,2)$

$\mathrm{B}=$ Sheet1.Cells $(\mathfrak{j}+3,2)$

$\mathrm{L}=$ Sheet1.Cells $(\mathfrak{j}+4,2)$

Sheets("sheet6").Select

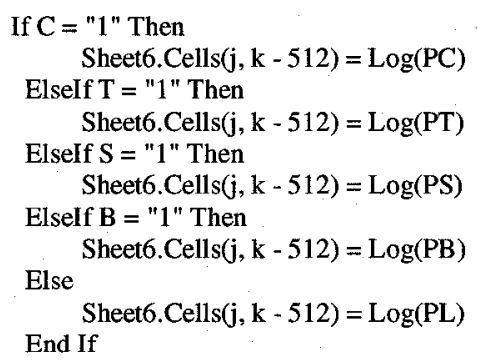

Next $\mathbf{j}$

Adding worksheets for calculation of Log-likelihood function value (sheet 4)

ElseIf $\mathrm{k}-768<=256$ Then

Sheets("sheet1").Select 
For $\mathbf{j}=3$ To 2698 Step 5

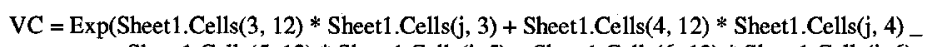

+ Sheet1.Cells $(5,12) *$ Sheet1.Cells $(j, 5)+$ Sheet1.Cells $(6,12) *$ Sheet1.Cells $(j, 6)+$ Sheet1.Cells $(7,12) *$ Sheet 1.Cells $(j, 7))$

VT $=\operatorname{Exp}($ Sheet1.Cells $(3,12) *$ Sheet1.Cells $(j+1,3)+$ Sheet1.Cells $(4,12) *$ Sheet1.Cells $(j+1,4)+$ Sheet1.Cells $(8,12) *$ Sheet1.Cells $(j+1,8))$

VS $=\operatorname{Exp}(\operatorname{Sheet1} \cdot \operatorname{Cells}(3,12) *$ Sheet1.Cells $(j+2,3)+\operatorname{Sheet1} \cdot \operatorname{Cells}(4,12) * \operatorname{Sheet1.Cells~}(j+2,4)+\operatorname{Sheet} 1 . \operatorname{Cells}(9,12) *$ Sheet1.Cells $(j+2,9))$

$\mathrm{VB}=\operatorname{Exp}(\operatorname{Sheet1}$.Cells $(3,12) *$ Sheet1.Cells $(j+3,3)+\operatorname{Sheet1.Cells~}(4,12) *$ Sheet1.Cells $(j+3,4)+$ Sheet1.Cells $(10,12) * \operatorname{Sheet1}$. Cells $(j+3,10))$

$\mathrm{VL}=\operatorname{Exp}(\operatorname{Sheet1.Cells}(3,12) *$ Sheet1.Cells $(j+4,3)+\operatorname{Sheet1.Cells~}(4,12) * \operatorname{Sheet1.Cells~}(j+4,4)+\operatorname{Sheet1} . \operatorname{Cells}(11,12) *$ Sheet 1.Cells $(j+4,11))$

$\mathrm{PC}=\mathrm{VC} /(\mathrm{VC}+\mathrm{VT}+\mathrm{VS}+\mathrm{VB}+\mathrm{VL})$

$\mathrm{PT}=\mathrm{VT} /(\mathrm{VC}+\mathrm{VT}+\mathrm{VS}+\mathrm{VB}+\mathrm{VL})$

$\mathrm{PS}=\mathrm{VS} /(\mathrm{VC}+\mathrm{VT}+\mathrm{VS}+\mathrm{VB}+\mathrm{VL})$

$\mathrm{PB}=\mathrm{VB} /(\mathrm{VC}+\mathrm{VT}+\mathrm{VS}+\mathrm{VB}+\mathrm{VL})$

$P L=V L /(V C+V T+V S+V B+V L)$

$\mathrm{C}=$ Sheet1.Cells $(\mathfrak{j}, 2)$

$\mathrm{T}=$ Sheet 1 .Cells $(\mathrm{j}+1,2)$

$\mathrm{S}=$ Sheet1.Cells $(\mathfrak{j}+2,2)$

$B=$ Sheet 1. Cells $(j+3,2)$

$\mathbf{L}=$ Sheet 1 .Cells $(\mathbf{j}+4,2)$

Sheets("sheet7").Select

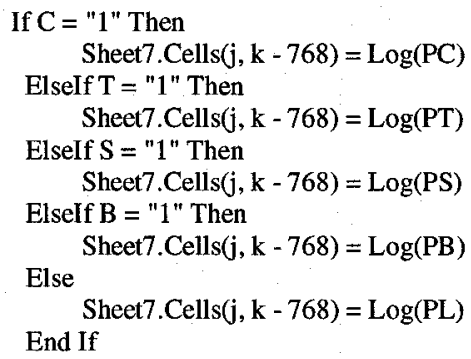

Next $\mathbf{j}$

End If

Utility Function Value for all alternatives' model specifications

For $\mathrm{i}=3$ To 2698 Step 5

Sheets("sheet2").Select

$\mathrm{VC}=\operatorname{Exp}($ Sheet1.Cells $(3,12) *$ Sheet1.Cells $(\mathbf{i}, 3)+$ Sheet1.Cells $(4,12) *$ Sheet1.Cells $(\mathrm{i}, 4)$

+ Sheet 1.Cells $(5,12) *$ Sheet1.Cells $(i, 5)+$ Sheet1.Cells $(6,12) *$ Sheet1.Cells $(i, 6)+$ Sheet1.Cells $(7,12) *$ Sheet1.Cells $(i, 7))$

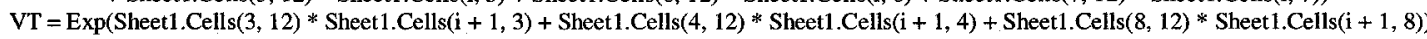

VS $=\operatorname{Exp}($ Sheet1 Cells $(3,12) *$ Sheet1.Cells $(i+2,3)+$ Sheet1.Cells $(4,12) *$ Sheet1.Cells $(i+2,4)+$ Sheet1.Cells $(9,12)$ * Sheet 1.Cells $(i+2,9))$

$\mathrm{VB}=\operatorname{Exp}(\operatorname{Sheet1.Cells}(3,12) *$ Sheet1.Cells $(\mathbf{i}+3,3)+\operatorname{Sheet} 1 . C e l l s(4,12) *$ Sheet1.Cells $(\mathrm{i}+3,4)+$ Sheet1.Cells $(10,12) *$ Sheet1.Cells $(\mathbf{i}+3,10))$

$\mathrm{VL}=\operatorname{Exp}(\operatorname{Sheet1.Cells}(3,12) *$ Sheet1.Cells $(i+4,3)+$ Sheet1.Cells $(4,12) *$ Sheet1.Cells $(i+4,4)+$ Sheet1.Cells $(11,12) *$ Sheet1.Cells $(i+4,11))$

Mode Choice Probability of each observations with Logit probability model

$\mathrm{PC}=\mathrm{VC} /(\mathrm{VC}+\mathrm{VT}+\mathrm{VS}+\mathrm{VB}+\mathrm{VL})$

$\mathrm{PT}=\mathrm{VT} /(\mathrm{VC}+\mathrm{VT}+\mathrm{VS}+\mathrm{VB}+\mathrm{VL})$

$P S=V S /(V C+V T+V S+V B+V L)$

$\mathrm{PB}=\mathrm{VB} /(\mathrm{VC}+\mathrm{VT}+\mathrm{VS}+\mathrm{VB}+\mathrm{VL})$

$\mathrm{PL}=\mathrm{VL} /(\mathrm{VC}+\mathrm{VT}+\mathrm{VS}+\mathrm{VB}+\mathrm{VL})$

$\mathrm{C}=$ Sheet 1. Cells $(\mathrm{i}, 2)$

$\mathrm{T}=$ Sheet 1 .Cells $(\mathrm{i}+1,2)$

$\mathrm{S}=$ Sheet1.Cells $(\mathrm{i}+2,2)$

$\mathrm{B}=$ Sheet 1 Cells $(\mathrm{i}+3,2)$

$\mathrm{L}=$ Sheet 1. Cells $(\mathrm{i}+4,2)$ 
First-order Derivative with respect to $\beta_{1}$

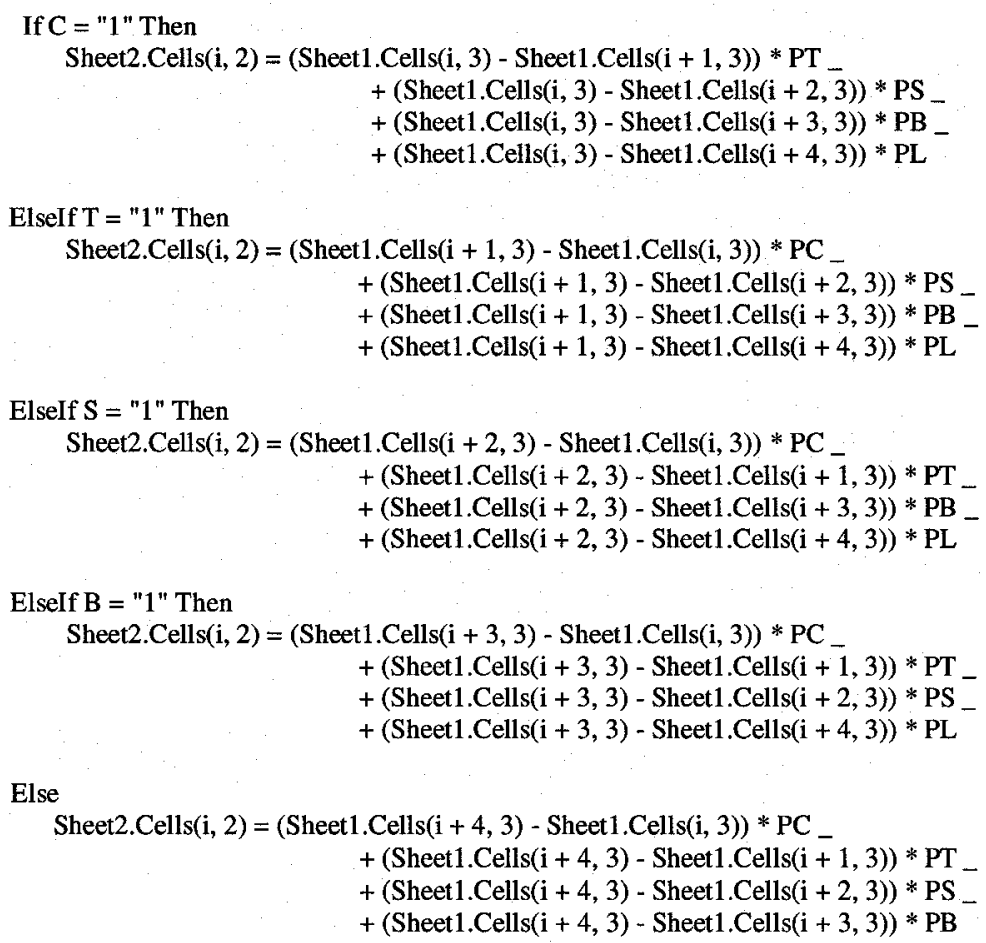

End If

First-order Derivative with respect to $\beta_{2}$

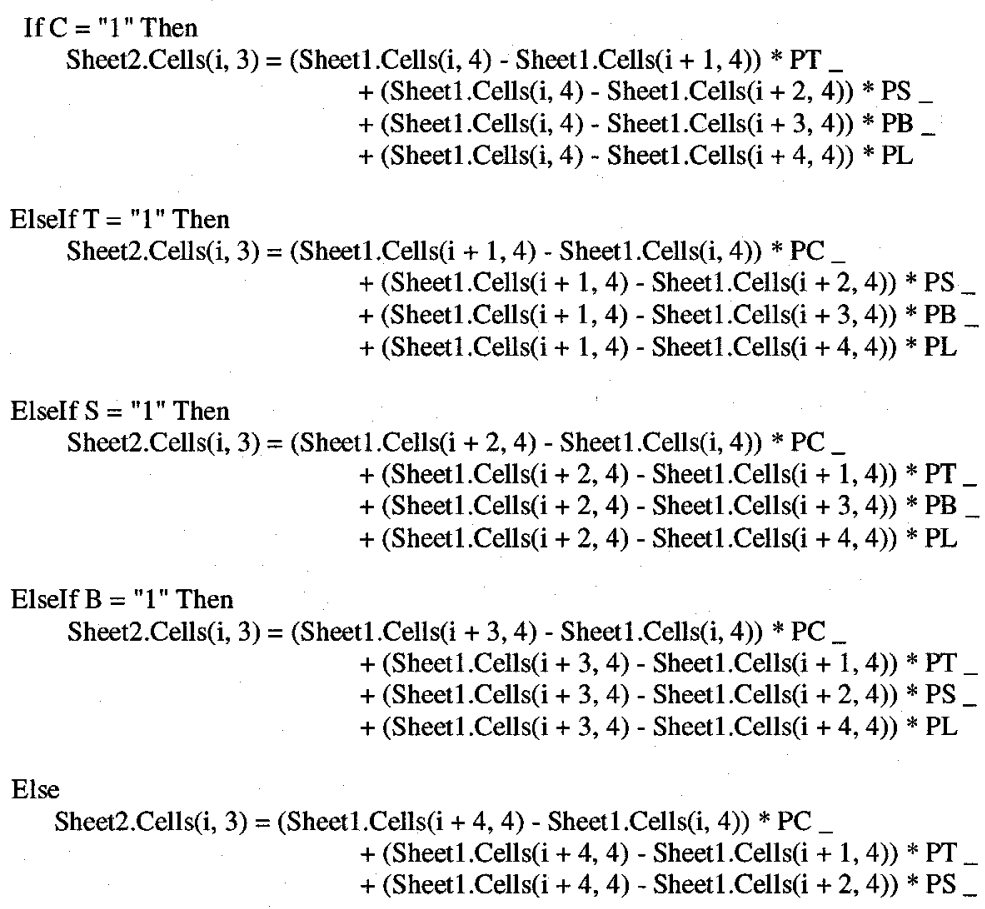


End If

First-order Derivative with respect to $\beta_{3}$

If $\mathrm{C}=$ " 1 " Then

Sheet2.Cells $(\mathrm{i}, 4)=$ Sheet1.Cells $(\mathrm{i}, 5) *(\mathrm{PT}+\mathrm{PS}+\mathrm{PB}+\mathrm{PL})$

Else

Sheet2.Cells $(i, 4)=-$ Sheet1.Cells $(\mathbf{i}, 5) *$ PC

End If

First-order Derivative with respect to $\beta_{4}$

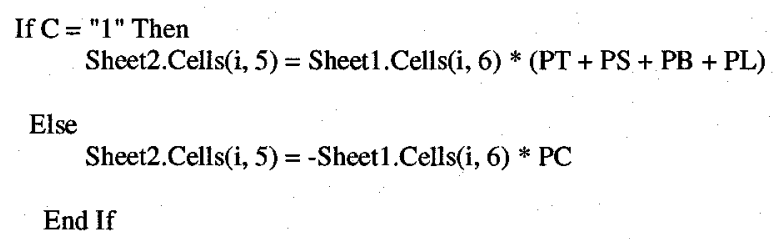

End If

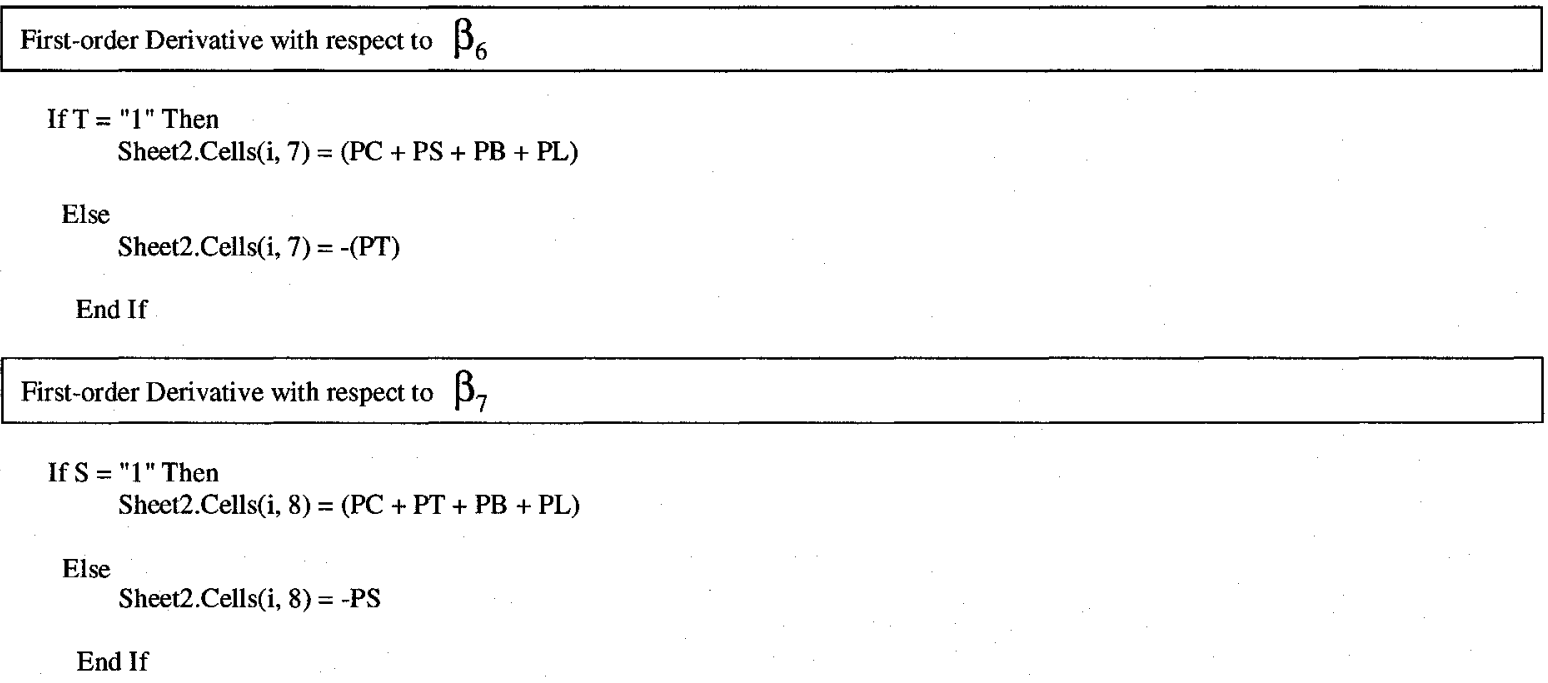

First-order Derivative with respect to $\beta_{8}$

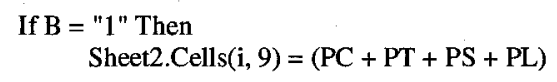


Sheet2.Cells $(i, 9)=-P B$

End If

First-order Derivative with respect to $\beta_{9}$

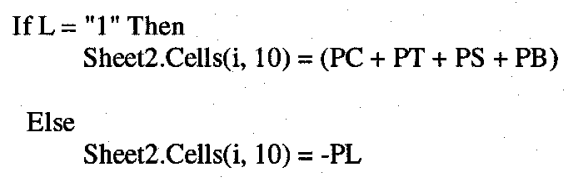

End If

Next $\mathrm{i}$

Summation of all observation and averaging it: $G_{t}$

For $\mathrm{j}=\mathbf{2}$ To 10 Step 1

Sheet2.Cells $(2703, \mathrm{j})$.FormulaR1C1 = "=-SUM(R[-2700]C:R[-1]C)/540"

Next $j$

Sheets("sheet3").Select

Obtaining Gradient components calculated : $G_{t}$

For $\mathrm{i}=11$ To 19 Step 1

Sheet3.Cells $(\mathbf{i}, 11)=$ Sheet2.Cells $(2703, \mathrm{i}-9)$

Next i

Calculating delta $: \delta_{t}=\lambda_{t-G S D} \Delta_{t}=\lambda_{t-G S D}\left(-H_{t A P}^{D F P-G S D}\right) G_{t}$

Sheet3.Range("M11:M19").FormulaArray = "=-(MMULT(A1:19,K11:K19))"

Starting or the Subroutine of Colden Section Method:-

Finding $\lambda_{t-G S D}$ by means of golden-section line search method

Sheets("sheet8").Select

$\mathrm{G}=1$

alfa $=0.618$

'boundary'

..................................... ould be changed....

Sheet8.Cells $(G+1,1)=$ Sheet $8 . C e l 1 s(4,10)$

$\ldots$

Sheet8.Cells $(\mathrm{G}+1,2)=$ Sheet8.Cells $(5,10)$

Sheet8.Cells $(G+1,3)=$ Sheet8.Cells $(G+1,1)+(1-$ alfa $) *($ Sheet8.Cells $(G+1,2)-$ Sheet8.Cells $(G+1,1))$

Sheet8.Cells $(G+1,4)=$ Sheet8.Cells $(G+1,1)+$ alfa * $($ Sheet8.Cells $(G+1,2)-$ Sheet8.Cells $(G+1,1))$ 
Evaluating Log-likelihood function to find the value, $\lambda_{t-G S D}$, to minimize $L L\left(\beta_{t}+\lambda_{t-G S D} \Delta_{t}\right)$ with golden-section line search method

Calculating function, $L L\left(\beta_{t}+\lambda_{t-G S D} \Delta_{t}\right)$, value for lower limit of the interval of uncertainty

Sheets("sheet8").Select

For $\mathrm{j}=3$ To 2698 Step 5

$\mathrm{VC}=\operatorname{Exp}((\operatorname{Sheet} 1 . \operatorname{Cells}(3,12)+\operatorname{Sheet} 8 \cdot \operatorname{Cells}(\mathrm{G}+1,3) *$ Sheet3.Cells $(11,13)) *$ Sheet1.Cells $(\mathrm{j}, 3)$

$+($ Sheet1.Cells $(4,12)+$ Sheet8.Cells $(G+1,3) *$ Sheet3.Cells $(12,13)) *$ Sheet1.Cells $(j, 4)$

$+($ Sheet 1.Cells $(5,12)+$ Sheet8.Cells $(G+1,3) *$ Sheet $3 . C e l l s(13,13)) *$ Sheet1.Cells $(j, 5)$ -

$+($ Sheet1.Cells $(6,12)+$ Sheet8.Cells $(\mathrm{G}+1,3) *$ Sheet3.Cells $(14,13)) *$ Sheet1.Cells $(\mathrm{j}, 6)$

$+(\operatorname{Sheet1}$.Cells $(7,12)+\operatorname{Sheet8.Cells}(\mathrm{G}+1,3) *$ Sheet3.Cells $(15,13)) *$ Sheet1.Cells $(\mathfrak{j}, 7))$

$\mathrm{VT}=\operatorname{Exp}((\operatorname{Sheet1} . \operatorname{Cells}(3,12)+\operatorname{Sheet8.Cells}(\mathrm{G}+1,3) *$ Sheet3.Cells $(11,13)) *$ Sheet1.Cells $(\mathrm{j}+1,3)$ $+($ Sheet1.Cells $(4,12)+$ Sheet8.Cells $(\mathrm{G}+1,3) *$ Sheet3.Cells $(12,13)) *$ Sheet1.Cells $(j+1,4)$

$+(\operatorname{Sheet1}$.Cells $(8,12)+$ Sheet8.Cells $(G+1,3) *$ Sheet3.Cells $(16,13)) *$ Sheet1.Cells $(j+1,8))$

VS $=\operatorname{Exp}((\operatorname{Sheet1}$. Cell $s(3,12)+\operatorname{Sheet} 8 . \operatorname{Cell} s(\mathrm{G}+1,3) *$ Sheet3.Cells $(11,13)) *$ Sheet1.Cells $(\mathrm{j}+2,3)$ $+($ Sheet1.Cells $(4,12)+$ Sheet8.Cells $(G+1,3) *$ Sheet3.Cells $(12,13)) *$ Sheet1.Cells $(j+2,4)-$ $+(\operatorname{Sheet} 1 . \operatorname{Cell} s(9,12)+\operatorname{Sheet} 8 . \operatorname{Cells}(\mathrm{G}+1,3) * \operatorname{Sheet} 3 \cdot \operatorname{Cells}(17,13)) * \operatorname{Sheet} 1 . \operatorname{Cells}(\mathrm{j}+2,9))$

$\mathrm{VB}=\operatorname{Exp}((\operatorname{Sheet} 1 . \operatorname{Cells}(3,12)+\operatorname{Sheet} 8 . \operatorname{Cells}(\mathrm{G}+1,3) *$ Sheet3.Cells $(11,13)) * \operatorname{Sheet1} \cdot \operatorname{Cells}(\mathrm{j}+3,3)$ $+($ Sheet1.Cells $(4,12)+$ Sheet8.Cells $(G+1,3) *$ Sheet3.Cells $(12,13)) *$ Sheet 1.Cells $(j+3,4)$ $+($ Sheet1.Cells $(10,12)+$ Sheet8.Cells $(G+1,3) *$ Sheet3.Cells $(18,13)) *$ Sheet1.Cells $(j+3,10))$

$\mathrm{VL}=\operatorname{Exp}((\operatorname{Sheet1.Cells}(3,12)+\operatorname{Sheet8.Cells}(\mathrm{G}+1,3) * \operatorname{Sheet} 3 . \operatorname{Cells}(11,13)) *$ Sheet1.Cells $(j+4,3)$ $+($ Sheet1.Cells $(4,12)+$ Sheet8.Cells $(\mathrm{G}+1,3) *$ Sheet3.Cells $(12,13)) *$ Sheet1.Cells $(\mathrm{j}+4,4)$ $+($ Sheet1.Cells $(11,12)+$ Sheet8.Cells $(G+1,3) *$ Sheet3.Cells $(19,13)) *$ Sheet1.Cells $(j+4,11))$

$\mathrm{PC}=\mathrm{VC} /(\mathrm{VC}+\mathrm{VT}+\mathrm{VS}+\mathrm{VB}+\mathrm{VL})$

$\mathrm{PT}=\mathrm{VT} /(\mathrm{VC}+\mathrm{VT}+\mathrm{VS}+\mathrm{VB}+\mathrm{VL})$

$\mathrm{PS}=\mathrm{VS} /(\mathrm{VC}+\mathrm{VT}+\mathrm{VS}+\mathrm{VB}+\mathrm{VL})$

$\mathrm{PB}=\mathrm{VB} /(\mathrm{VC}+\mathrm{VT}+\mathrm{VS}+\mathrm{VB}+\mathrm{VL})$

$\mathrm{PL}=\mathrm{VL} /(\mathrm{VC}+\mathrm{VT}+\mathrm{VS}+\mathrm{VB}+\mathrm{VL})$

$\mathrm{C}=$ Sheet1.Cells $(\mathrm{j}, 2)$

$T=$ Sheet 1. Cells $(j+1,2)$

$\mathrm{S}=$ Sheet 1 Cells $(\mathrm{j}+2,2)$

$B=$ Sheet 1.Cells $(\mathfrak{j}+3,2)$

$\mathrm{L}=$ Sheet 1.Cells $(\mathrm{j}+4,2)$

Sheets("sheet9").Select

-mathematical tratment for transforming the log-likelihood function into convex function->

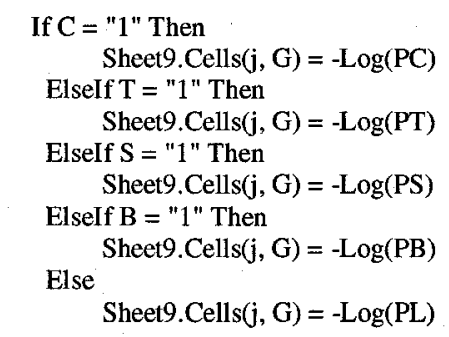


End If

Next $\mathbf{j}$

Sheet8.Cells $(G+1,5)=$ Sheet9.Cells $(2703, G)$

Calculating function, $L L\left(\beta_{t}+\lambda_{t-G S D} \Delta_{t}\right)$,value for upper limit of the interval of uncertainty

Sheets("sheet8").Select

For $\mathrm{j}=3$ To 2698 Step 5

$\mathrm{VC}=\operatorname{Exp}((\operatorname{Sheet} 1 . \operatorname{Cells}(3,12)+$ Sheet8.Cells $(\mathrm{G}+1,4) * \operatorname{Sheet} 3 . \operatorname{Cells}(11,13)) *$ Sheet1.Cells $(\mathrm{j}, 3)$ $+($ Sheet 1.Cells $(4,12)+$ Sheet8.Cells $(G+1,4) *$ Sheet3.Cells $(12,13)) *$ Sheet1.Cells $(j, 4)$ $+($ Sheet1.Cells $(5,12)+$ Sheet8.Cells $(\mathrm{G}+1,4) *$ Sheet3.Cells $(13,13)) *$ Sheet1.Cells $(j, 5)$ $+($ Sheet1.Cells $(6,12)+$ Sheet8.Cells $(G+1,4) *$ Sheet3.Cells $(14,13)) *$ Sheet1.Cells $(j, 6)$ $+($ Sheet1.Cells $(7,12)+$ Sheet8.Cells $(G+1,4) *$ Sheet3.Cells $(15,13)) *$ Sheet1.Cells $(j, 7))$

VT $=\operatorname{Exp}((\operatorname{Sheet1} \cdot \operatorname{Cells}(3,12)+\operatorname{Sheet8} \cdot \operatorname{Cells}(\mathrm{G}+1,4) *$ Sheet3.Cells $(11,13)) * \operatorname{Sheet1} \cdot \operatorname{Cells}(\mathrm{j}+1,3)$ $+($ Sheet1.Cells $(4,12)+$ Sheet8.Cells $(\mathrm{G}+1,4) *$ Sheet3.Cells $(12,13)) *$ Sheet1.Cells $(j+1,4)$ $+($ Sheet1.Cells $(8,12)+$ Sheet8.Cells $(G+1,4) *$ Sheet3.Cells $(16,13)) *$ Sheet1.Cells $(j+1,8))$

$\mathrm{VS}=\operatorname{Exp}(($ Sheet1.Cells $(3,12)+$ Sheet8.Cells $(\mathrm{G}+1,4) *$ Sheet3.Cells $(11,13)) *$ Sheet1.Cells $(\mathrm{j}+2,3)$ $+($ Sheet1.Cells $(4,12)+$ Sheet8.Cells $(\mathrm{G}+1,4) *$ Sheet3.Cells $(12,13)) *$ Sheet1.Cells $(j+2,4)$ $+($ Sheet1.Cells $(9,12)+$ Sheet8.Cells $(\mathrm{G}+1,4) *$ Sheet3.Cells $(17,13)) *$ Sheet1.Cells $(\mathrm{j}+2,9))$

$\mathrm{VB}=\operatorname{Exp}((\operatorname{Sheet} 1 . \operatorname{Cells}(3,12)+$ Sheet8.Cells $(\mathrm{G}+1,4) * \operatorname{Sheet} 3 \cdot \operatorname{Cells}(11,13)) *$ Sheet1.Cells $(\mathrm{j}+3,3)$ $+($ Sheet1.Cells $(4,12)+$ Sheet8.Cells $(\mathrm{G}+1,4) *$ Sheet3.Cells $(12,13)) *$ Sheet1.Cells $(\mathrm{j}+3,4)$ $+($ Sheet1.Cells $(10,12)+$ Sheet8.Cells $(G+1,4) *$ Sheet3.Cells $(18,13)) *$ Sheet1.Cells $(j+3,10))$

$\mathrm{VL}=\operatorname{Exp}((\operatorname{Sheet} 1 . \operatorname{Cells}(3,12)+$ Sheet8.Cells $(\mathrm{G}+1,4) * \operatorname{Sheet3.Cells}(11,13)) *$ Sheet1.Cells $(\mathrm{j}+4,3)$ $+($ Sheet1.Cells $(4,12)+$ Sheet8.Cells $(G+1,4) *$ Sheet3.Cells $(12,13)) *$ Sheet1.Cells $(j+4,4)$ $+($ Sheet1.Cells $(11,12)+$ Sheet8.Cells $(\mathrm{G}+1,4) *$ Sheet3.Cells $(19,13)) *$ Sheet1.Cells $(\mathrm{j}+4,1 \overline{1}))$

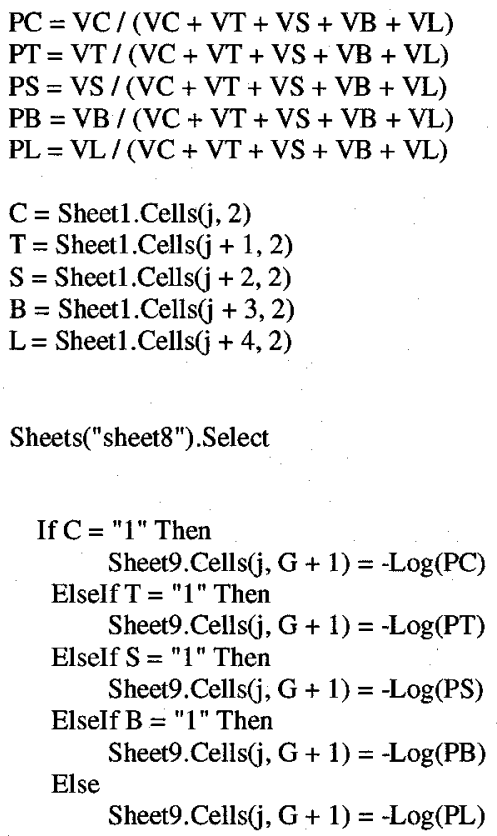


End If

Next j

Sheet8.Cells $(G+1,6)=$ Sheet9.Cells $(2703, G+1)$

line4:

Checking the stopping criterion

-could be changed/ the accuracy level of the stopping criterion $\rightarrow$

If Sheet8.Cells $(G+1,2)-$ Sheet8.Cells $(G+1,1)<0.01$ Then

GoTo line5

ElseIf Sheet8.Cells $(G+1,5)>$ Sheet8.Cells $(G+1,6)$ Then

Sheet8.Cells $(G+2,1)=$ Sheet8.Cells $(G+1,3)$

Sheet8.Cells $(G+2,2)=$ Sheet8.Cells $(G+1,2)$

Sheet8.Cells $(G+2,3)=$ Sheet8.Cells $(G+1,4)$

Sheet8.Cells $(G+2,4)=$ Sheet8.Cells $(G+2,1)+$ alfa $*($ Sheet8.Cells $(G+2,2)-$ Sheet8.Cells $(G+2,1))$

Sheet8.Cells $(G+2,5)=$ Sheet8.Cells $(G+1,6)$

Calculating function, $L L\left(\beta_{t}+\lambda_{t-G S D} \Delta_{t}\right)$,value of the new interval of uncertainty

Sheets("sheet8").Select

For $\mathbf{j}=3$ To 2698 Step 5

$\mathrm{VC}=\operatorname{Exp}((\operatorname{Sheet} 1 . \operatorname{Cells}(3,12)+\operatorname{Sheet8.Cells}(\mathrm{G}+2,4) *$ Sheet3.Cells $(11,13)) *$ Sheet1.Cells $(\mathfrak{j}, 3)$ $+($ Sheet1.Cells $(4,12)+$ Sheet8.Cells $(\mathrm{G}+2,4) *$ Sheet3.Cells(12, 13))* Sheet1.Cells $(\mathrm{j}, 4)$ $+($ Sheet1.Cells $(5,12)+$ Sheet8.Cells $(\mathrm{G}+2,4) *$ Sheet 3.Cells $(13,13)) *$ Sheet1.Cells $(j, 5)$

$+($ Sheet1.Cells $(6,12)+$ Sheet8.Cells $(G+2,4) *$ Sheet3.Cells $(14,13)) *$ Sheet1.Cells $(j, 6)$ -

$+($ Sheet1.Cells $(7,12)+$ Sheet8.Cells $(G+2,4) *$ Sheet3.Cells $(15,13)) *$ Sheet1.Cells(j, 7$))$

VT $=\operatorname{Exp}((\operatorname{Sheet1} . \operatorname{Cells}(3,12)+$ Sheet8.Cells $(\mathrm{G}+2,4) *$ Sheet3.Cells $(11,13)) *$ Sheet1.Cells $(\mathrm{j}+1,3)$ $+($ Sheet1.Cells $(4,12)+$ Sheet8.Cells $(G+2,4) *$ Sheet3.Cells $(12,13)) *$ Sheet1.Cells $(j+1,4)$ $+($ Sheet1.Cells $(8,12)+$ Sheet8.Cells $(\mathrm{G}+2,4) *$ Sheet3.Cells $(16,13)) *$ Sheet1.Cells $(j+1,8))$

VS $=\operatorname{Exp}(($ Sheet1.Cells $(3,12)+$ Sheet8.Cells $(G+2,4) *$ Sheet3.Cells $(11,13)) *$ Sheet 1.Cells $(j+2,3)$ $+($ Sheet1.Cells $(4,12)+$ Sheet8.Cells $(G+2,4) *$ Sheet3.Cells $(12,13)) *$ Sheet1.Cells $(j+2,4)$ $+($ Sheet1.Cells $(9,12)+$ Sheet8.Cells $(G+2,4) *$ Sheet3.Cells $(17,13)) *$ Sheet $1 . C e l l s(j+2,9))$

$\mathrm{VB}=\operatorname{Exp}((\operatorname{Sheet} 1 . \operatorname{Cells}(3,12)+$ Sheet8.Cells $(\mathrm{G}+2,4) *$ Sheet3.Cells $(11,13)) *$ Sheet1.Cells $(j+3,3)$ $+($ Sheet 1.Cells $(4,12)+$ Sheet8.Cells $(\mathrm{G}+2,4) *$ Sheet3.Cells $(12,13)) *$ Sheet1.Cells $(j+3,4)$ $+($ Sheet1.Cells $(10,12)+$ Sheet8.Cells $(G+2,4) *$ Sheet3.Cells $(18,13)) *$ Sheet1.Cells $(j+3,10))$

$\mathrm{VL}=\operatorname{Exp}((\operatorname{Sheet} 1 . \operatorname{Cells}(3,12)+\operatorname{Sheet} 8 \cdot \operatorname{Cells}(\mathrm{G}+2,4) * \operatorname{Sheet} 3 \cdot \operatorname{Cells}(11,13)) *$ Sheet1.Cells $(\mathrm{j}+4,3)$ $+($ Sheet1.Cells $(4,12)+$ Sheet8.Cells $(G+2,4) *$ Sheet3.Cells $(12,13)) *$ Sheet1.Cells $(j+4,4)$ $+($ Sheet1.Cells $(11,12)+$ Sheet8.Cells $(\mathrm{G}+2,4) *$ Sheet3.Cells $(19,13)) *$ Sheet1.Cells $(j+4,11))$ 


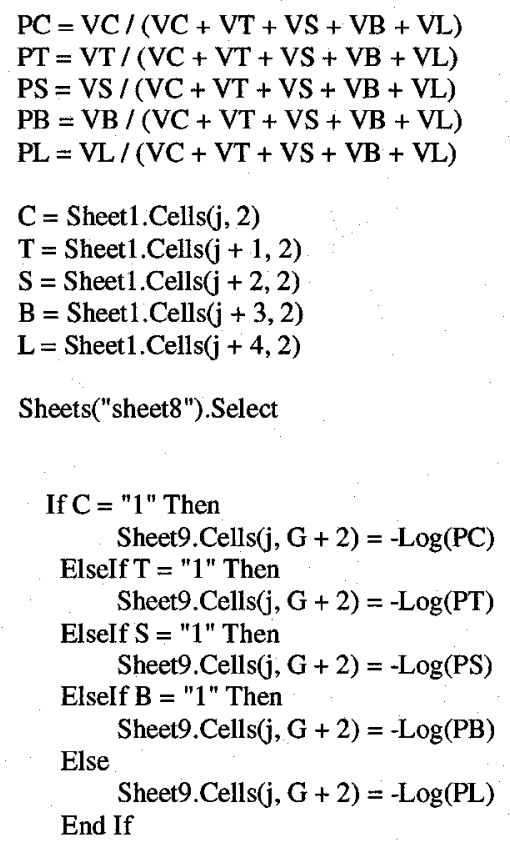

Next $\mathbf{j}$

Sheet8.Cells $(G+2,6)=$ Sheet9.Cells $(2703, G+2)$

Elself Sheet8.Cells $(G+1,5)<=$ Sheet8.Cells $(G+1,6)$ Then

Sheet8.Cells $(\mathrm{G}+2,1)=$ Sheet8.Cells $(\mathrm{G}+1,1)$

Sheet8.Cells $(G+2,2)=$ Sheet8.Cells $(G+1,4)$

Sheet8.Cells $(G+2,3)=\operatorname{Sheet8.Cells}(G+2,1)+(1-$ alfa $) *(\operatorname{Sheet} 8 . \operatorname{Cells}(G+2,2)-\operatorname{Sheet} 8 . \operatorname{Cells}(G+2,1))$

Sheet8.Cells $(\mathrm{G}+2,4)=$ Sheet8.Cells $(\mathrm{G}+1,3)$

Calculating function, $L L\left(\beta_{t}+\lambda_{t-G S D} \Delta_{t}\right)$,value of the new interval of uncertainty

Sheets("sheet8").Select

For $\mathrm{j}=3$ To 2698 Step 5

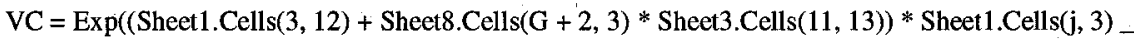

$+($ Sheet1.Cells $(4,12)+$ Sheet8.Cells $(G+2,3) *$ Sheet3.Cells $(12,13)) *$ Sheet1.Cells $(j, 4)$

$+($ Sheet1.Cells $(5,12)+$ Sheet8.Cells $(G+2,3) *$ Sheet3.Cells $(13,13)) *$ Sheet1.Cells $(\mathfrak{j}, 5)$

$+($ Sheet1.Cells $(6,12)+$ Sheet8.Cells $(G+2,3) *$ Sheet3.Cells $(14,13)) *$ Sheet1.Cells $(\mathbf{j}, 6)$

$+($ Sheet1.Cells $(7,12)+$ Sheet8.Cells $(G+2,3) *$ Sheet3.Cells $(15,13)) *$ Sheet1.Cells $(\mathfrak{j}, 7))$

$\mathrm{VT}=\operatorname{Exp}((\operatorname{Sheet1} . \operatorname{Cells}(3,12)+$ Sheet8.Cells $(\mathrm{G}+2,3) * \operatorname{Sheet} 3 . \operatorname{Cells}(11,13)) * \operatorname{Sheet1} \cdot \operatorname{Cells}(\mathrm{j}+1,3)$ $+($ Sheet1.Cells $(4,12)+$ Sheet8.Cells $(G+2,3) *$ Sheet3.Cells $(12,13)) *$ Sheet1.Cells $(j+1,4)$ $+($ Sheet1.Cells $(8,12)+$ Sheet8.Cells $(G+2,3) *$ Sheet3.Cells $(16,13)) *$ Sheet1.Cells $(j+1,8))$

VS $=\operatorname{Exp}((\operatorname{Sheet} 1 . \operatorname{Cells}(3,12)+\operatorname{Sheet8} \cdot \operatorname{Cells}(\mathrm{G}+2,3) * \operatorname{Sheet} 3 \cdot \operatorname{Cells}(11,13)) * \operatorname{Sheet} 1 . \operatorname{Cells}(\mathrm{j}+2,3)$ $+($ Sheet1.Cells $(4,12)+$ Sheet8.Cells $(G+2,3) *$ Sheet3.Cells $(12,13)) *$ Sheet $1 . C e l l s(j+2,4)$ $+($ Sheet1.Cells $(9,12)+$ Sheet8.Cells $(G+2,3) *$ Sheet3.Cells $(17,13)) *$ Sheet1.Cells $(j+2,9))$ 


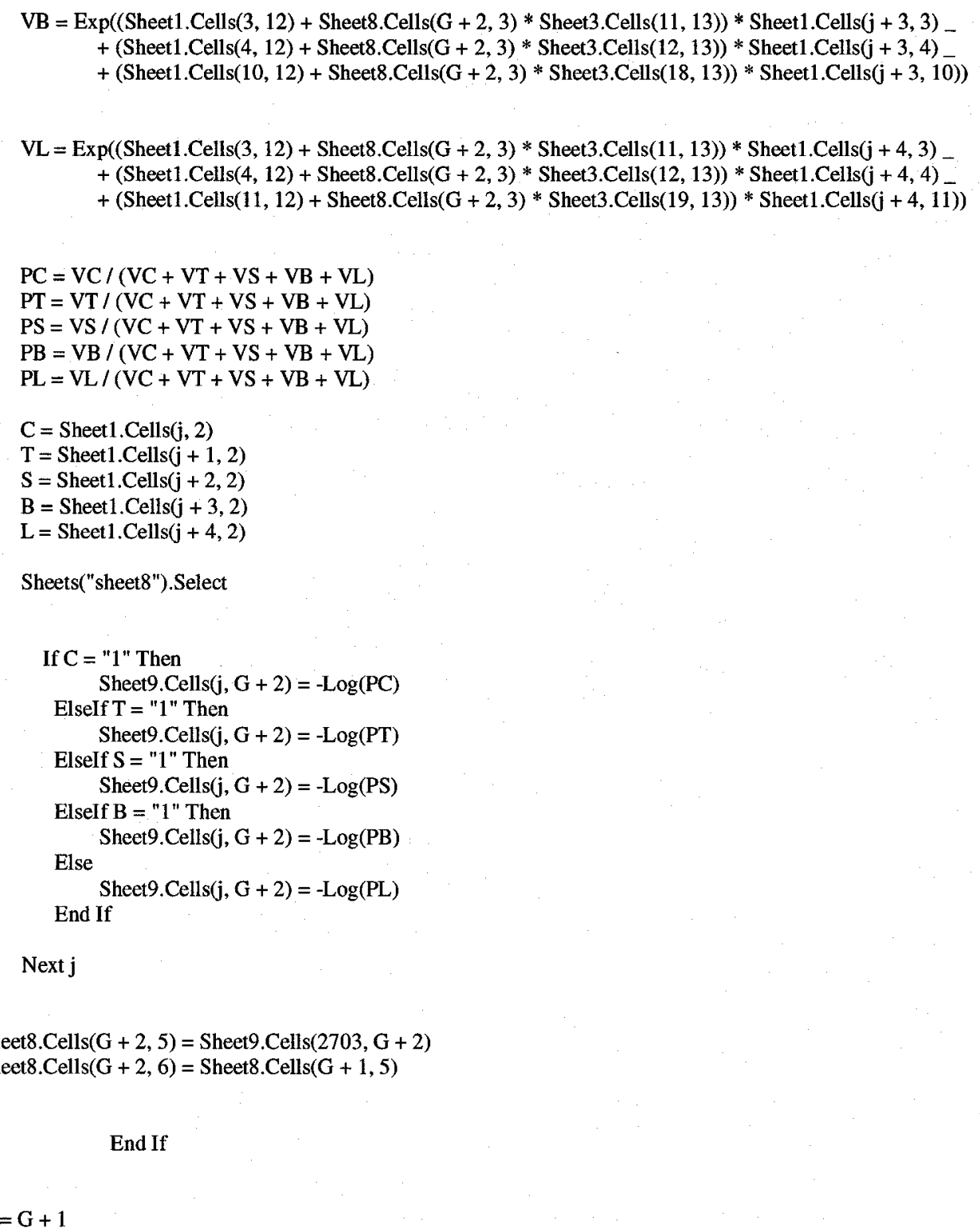

GoTo line4

line5:

Adopting $\lambda_{t-G S D}$ obtained from golden section method

For $\mathrm{i}=11$ To 19

Sheet3.Cells $(\mathrm{i}, 14)=(($ Sheet8.Cells $(G+1,2)+$ Sheet8.Cells $(G+1,1)) / 2) *$ Sheet3.Cells $(\mathrm{i}, 13)$

Next i

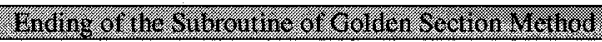


Parameter estimates : $\beta_{t+1}=\beta_{t}+\delta_{t}=\beta_{t}+\lambda_{t-G S D} \Delta_{t}=\beta_{t}+\lambda_{t-G S D} W_{t} G_{t}=\beta_{t}+\lambda_{t-G S D}\left(-H_{t A P}^{D F P-G S M}\right) G_{t}$

Sheet3.Cells $(11,16)=$ Sheet 1. Cells $(3,12)+$ Sheet $3 \cdot \operatorname{Cells}(11,14)$

Sheet3.Cells $(12,16)=$ Sheet1.Cells $(4,12)+$ Sheet3.Cells $(12,14)$

Sheet3.Cells $(13,16)=$ Sheet1.Cells $(5,12)+$ Sheet3.Cells $(13,14)$

Sheet3.Cells $(14,16)=$ Sheet 1 .Cells $(6,12)+$ Sheet3.Cells $(14,14)$

Sheet3.Cells $(15,16)=$ Sheet 1. Cells $(7,12)+$ Sheet 3 .Cells $(15,14)$

Sheet3.Cells $(16,16)=$ Sheet 1 .Cells $(8,12)+$ Sheet 3 . Cells $(16,14)$

Sheet3.Cells $(17,16)=$ Sheet 1 .Cells $(9,12)+$ Sheet3.Cells $(17,14)$

Sheet 3 .Cells $(18,16)=$ Sheet 1 .Cells $(10,12)+$ Sheet 3. Cells $(18,14)$

Sheet3.Cells $(19,16)=$ Sheet1.Cells $(11,12)+$ Sheet3.Cells $(19,14)$

Checking the Criterion for stopping: $\left[\frac{1}{k} \sum_{k=1}^{k}\left(\beta_{t+1, k}-\beta_{t, k}\right)^{2}\right]^{1 / 2}<C R$

Sheet3.Cells $(21,12)=\left(C_{-}\right.$

(Sheet3.Cells $(11,16)-$ Sheet1.Cells $(3,12))^{\wedge} 2+(\operatorname{Sheet3.Cells}(12,16)-\text { Sheet1.Cells }(4,12))^{\wedge} 2$

$+(\text { Sheet3.Cells }(13,16)-\operatorname{Sheet} 1 . \operatorname{Cell}(5,12))^{\wedge} 2+(\operatorname{Sheet} 3 \cdot \operatorname{Cells}(14,16)-\operatorname{Sheet1.Cells}(6,12))^{\wedge} 2$

$+(\text { Sheet3.Cells }(15,16)-\text { Sheet1.Cells }(7,12))^{\wedge} 2+(\text { Sheet3.Cells }(16,16)-\text { Sheet1.Cells }(8,12))^{\wedge} 2$

$+(\text { Sheet3.Cells }(17,16)-\text { Sheet1.Cells }(9,12))^{\wedge} 2+(\text { Sheet3.Cells }(18,16)-\text { Sheet1.Cells }(10,12))^{\wedge} 2$

$+(\text { Sheet } 3 . \text { Cells }(19,16)-\text { Sheet } 1 . C e l l s(11,12))^{\wedge} 2$

$$
)^{*}(1 / 9)\right)^{\wedge}(0.5)
$$

-could be changed......$\rightarrow$

If Sheet3.Cells $(21,12)<10^{\wedge}(-4)$ Then

GoTo line1

Else

Starting the procedure for obtaining updating approximate hessan

Calculating: $G_{t+1}$

For $\mathrm{i}=3$ To 2698 Step 5

Sheets("sheet2").Select

$\mathrm{VC}=\operatorname{Exp}($ Sheet3.Cells $(11, \quad 16) *$ Sheet1.Cells(i, 3)+Sheet3.Cells $(12, \quad 16) \quad * \quad$ Sheet1.Cells(i, 4$)$ + Sheet3.Cells $(13,16) *$ Sheet1.Cells(i, 5) + Sheet3.Cells $(14,16) *$ Sheet1.Cells $(\mathrm{i}, 6)+\operatorname{Sheet3.Cells}(15,16)$

* Sheetl.Cells(i, 7$)$ )

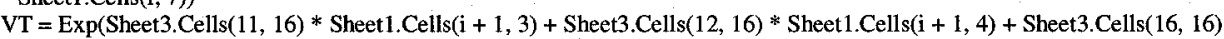

* Sheet1.Cells $(\mathrm{i}+1,8))$

vS $=\operatorname{Exp}($ Sheet3.Cells $(11,16) *$ Sheet1.Cells $(i+2,3)+$ Sheet3.Cells $(12,16) *$ Sheet1.Cells $(1+2,4)+\operatorname{Sheet} 3$. Cells $(17,16)$

* Sheet1.Cells $(\mathrm{i}+2,9))$

VB $=\operatorname{Exp}($ Sheet3.Cells $(11,16) *$ Sheet1.Cells $(i+3,3)+$ Sheet3.Cells $(12,16) *$ Sheet1.Cells $(i+3,4)+\operatorname{Sheet} 3 . C e l l s(18,16)$

$*$ Sheet1.Cells $(\mathrm{i}+3,10)$

$\mathrm{VL}=\operatorname{Exp}(\operatorname{Sheet} 3 . \operatorname{Cells}(11,16) *$ Sheet1.Cells $(\mathrm{i}+4,3)+\operatorname{Sheet3.Cells}(12,16) *$ Sheet1.Cells $(\mathrm{i}+4,4)+\operatorname{Sheet} 3 . \operatorname{Cells}(19,16)$

* Sheet1.Cells $(i+4,11))$

$\mathrm{PC}=\mathrm{VC} /(\mathrm{VC}+\mathrm{VT}+\mathrm{VS}+\mathrm{VB}+\mathrm{VL})$

$\mathrm{PT}=\mathrm{VT} /(\mathrm{VC}+\mathrm{VT}+\mathrm{VS}+\mathrm{VB}+\mathrm{VL})$

$\mathrm{PS}=\mathrm{VS} /(\mathrm{VC}+\mathrm{VT}+\mathrm{VS}+\mathrm{VB}+\mathrm{VL})$

$\mathrm{PB}=\mathrm{VB} /(\mathrm{VC}+\mathrm{VT}+\mathrm{VS}+\mathrm{VB}+\mathrm{VL})$

$\mathrm{PL}=\mathrm{VL} /(\mathrm{VC}+\mathrm{VT}+\mathrm{VS}+\mathrm{VB}+\mathrm{VL})$ 


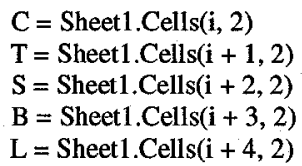

First-order Derivative with respect to $\beta_{1}$

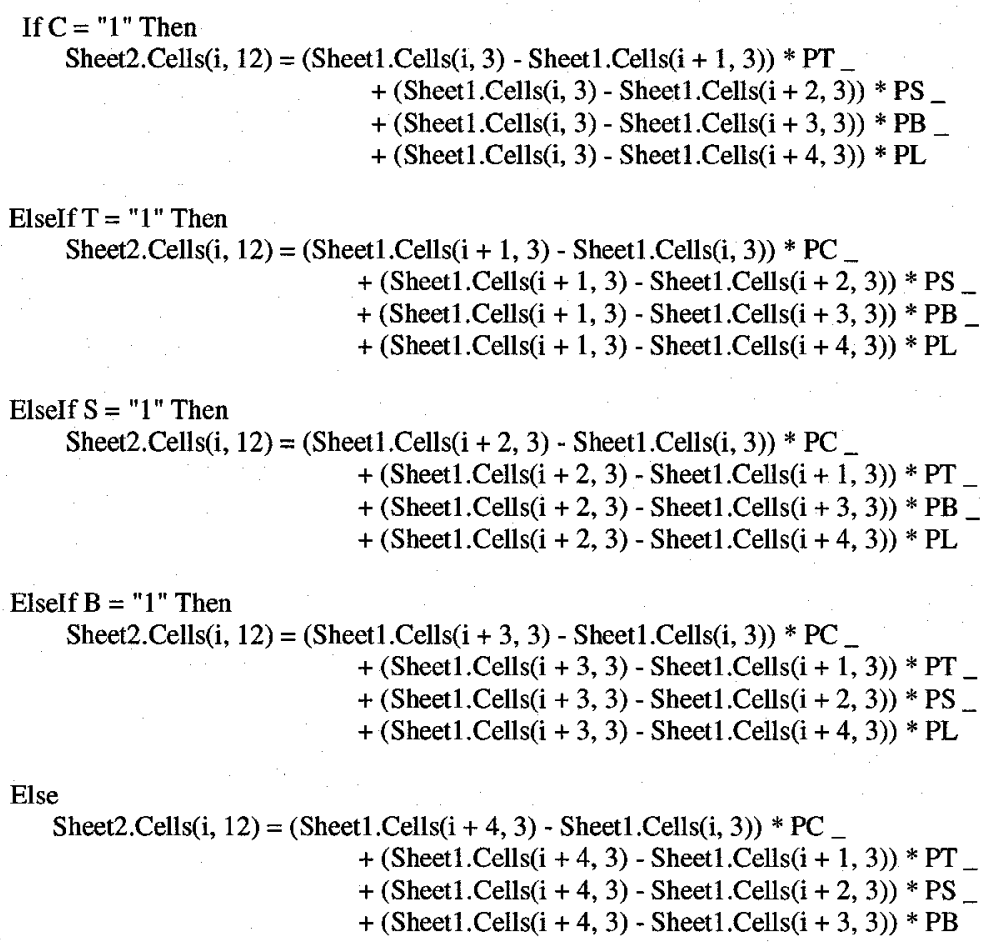

End If

First-order Derivative with respect to $\beta_{2}$

\section{If $\mathrm{C}=$ " 1 " Then}

Sheet2.Cells $(\mathrm{i}, 13)=($ Sheet 1.Cells $(\mathrm{i}, 4)-$ Sheet $1 . \operatorname{Cells}(\mathrm{i}+1,4)) * \mathrm{PT}$ $+($ Sheet1.Cells $(\mathrm{i}, 4)-$ Sheet1.Cells $(\mathrm{i}+2,4)) * \mathrm{PS}$ $+($ Sheet1.Cells $(\mathbf{i}, 4)-$ Sheet1.Cells $(\mathbf{i}+3,4)) *$ PB $+($ Sheet1.Cells $(\mathrm{i}, 4)-$ Sheet1.Cells $(\mathrm{i}+4,4)) * \mathrm{PL}$

Elself T $=$ "1" Then

Sheet2.Cells $(\mathbf{i}, 13)=($ Sheet1.Cells $(\mathbf{i}+1,4)-$ Sheet1.Cells $(\mathbf{i}, 4)) *$ PC $+($ Sheet1.Cells $(\mathrm{i}+1,4)$ - Sheet1.Cells $(\mathrm{i}+2,4)) *$ PS $+($ Sheet1.Cells $(\mathrm{i}+1,4) \cdot$ Sheet1.Cells $(\mathrm{i}+3,4)) * \mathrm{~PB}_{-}$ $+($ Sheet1.Cells $(\mathrm{i}+1,4)-$ Sheet1.Cells $(\mathrm{i}+4,4)) * \mathrm{PL}$

Elself $S=$ "1" Then

Sheet2.Cells $(\mathrm{i}, 13)=($ Sheet1.Cells $(\mathrm{i}+2,4)-$ Sheet1.Cells $(\mathrm{i}, 4)) * \mathrm{PC}$ $+($ Sheet1.Cells $(\mathrm{i}+2,4)-$ Sheet1.Cells $(\mathrm{i}+1,4)) * \mathrm{PT}$ $+($ Sheet1.Cells $(\mathbf{i}+2,4)-$ Sheet1.Cells $(\mathbf{i}+3,4)) * \mathrm{~PB}$ $+($ Sheet1.Cells $(i+2,4)-$ Sheet1.Cells $(i+4,4)) *$ PL 


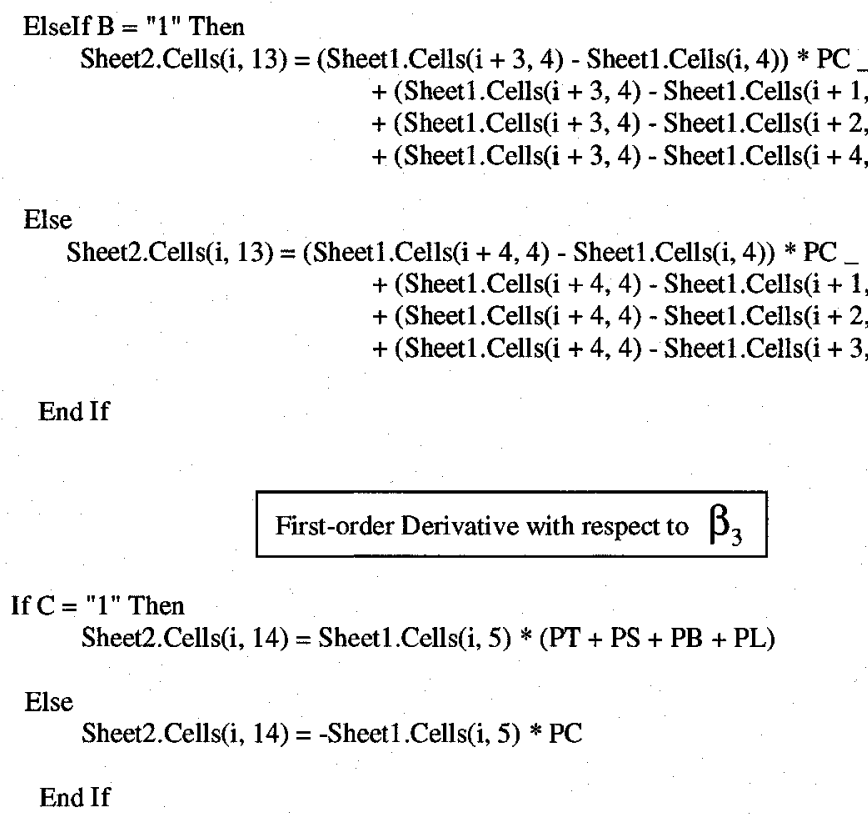

End If

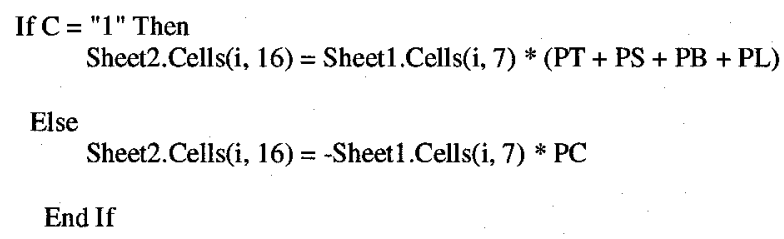




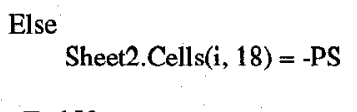

End If

First-order Derivative with respect to $\beta_{8}$

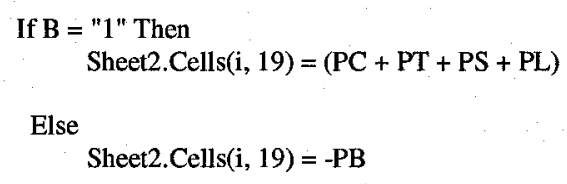

End If

\section{First-order Derivative with respect to $\beta_{9}$}

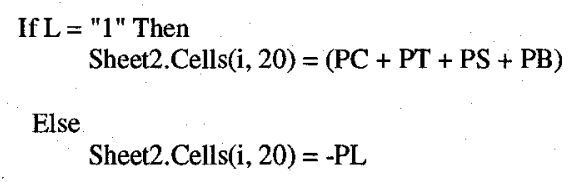

End If

Next i

Summation and averaging gradient: $G_{t+1}$

For $\mathrm{j}=12$ To 20 Step 1

Sheet2.Cells $(2703, \mathrm{j})$.FormulaR1C1 = "=-SUM(R[-2700]C:R[-1]C)/540"

Next $\mathbf{j}$

Sheets("sheet3").Select

Obtaining gradient: $G_{t+1}$

For $i=1$ To 9 Step 1

Sheet3.Cells $(i, 11)=$ Sheet2.Cells $(2703, i+11)$

Next i

Calculating gamma: $\gamma_{t}=G_{t+1}-G_{t}$

Sheet3.Cells $(11,18)=$ Sheet3.Cells $(1,11)-$ Sheet3.Cells $(11,11)$
Sheet3.Cells $(12,18)=$ Sheet3.Cells $(2,11)-$ Sheet3.Cells $(12,11)$
Sheet3.Cells $(13,18)=$ Sheet3.Cells $(3,11)-$ Sheet3.Cells $(13,11)$
Sheet3.Cells $(14,18)=$ Sheet3.Cells $(4,11)-$ Sheet3.Cells $(14,11)$
Sheet3.Cells $(15,18)=$ Sheet3.Cells $(5,11)-$ Sheet3.Cells $(15,11)$
Sheet3.Cells $(16,18)=$ Sheet3.Cells $(6,11)-$ Sheet3.Cells $(16,11)$
Sheet3.Cells $(17,18)=$ Sheet3.Cells $(7,11)-$ Sheet3.Cells $(17,11)$
Sheet3.Cells $(18,18)=$ Sheet3.Cells $(8,11)-$ Sheet3.Cells $(18,11)$
Sheet3.Cells $(19,18)=$ Sheet3.Cells $(9,11)-$ Sheet3.Cells $(19,11)$ 
Calculating approximate hessian: $H_{t+1}^{D F P-G S M}=H_{t}^{D F P-G S M}+\frac{\delta_{t} \delta_{t}^{\prime}}{\delta_{t}^{\prime} y_{t}}-\frac{H_{t}^{D F P-G S M} \gamma_{t} \gamma_{t}^{\prime} H_{t}^{D F P-G S M}}{\gamma_{t}^{\prime} H_{t}^{D F P-G S M} \gamma_{t}}$

Transpose matrix of a vector of gamma: $\gamma_{t}$

Sheet3.Cells $(28,11)=$ Sheet3.Cells $(11,18)$

Sheet3.Cells $(28,12)=$ Sheet3.Cells $(12,18)$

Sheet3.Cells $(28,13)=$ Sheet3.Cells $(13,18)$

Sheet 3 .Cells $(28,14)=$ Sheet 3 .Cells $(14,18)$

Sheet3.Cells $(28,15)=$ Sheet3.Cells $(15,18)$

Sheet3.Cells $(28,16)=$ Sheet 3. Cells $(16,18)$

Sheet3.Cells $(28,17)=$ Sheet3.Cells $(17,18)$

Sheet3.Cells $(28,18)=$ Sheet 3 .Cells $(18,18)$

Sheet 3 .Cells $(28,19)=$ Sheet 3. Cells $(19,18)$

Transpose matrix of a vector of delta: $\delta_{t}^{\prime}$

Sheet3.Cells $(30,11)=$ Sheet 3 .Cells $(11,14)$

Sheet 3. Cells $(30,12)=$ Sheet 3. Cells $(12,14)$

Sheet 3. Cells $(30,13)=$ Sheet 3. Cells $(13,14)$

Sheet3.Cells $(30,14)=$ Sheet 3 .Cells $(14,14)$

Sheet 3 .Cells $(30,15)=$ Sheet 3 .Cells $(15,14)$

Sheet 3 .Cells $(30,16)=$ Sheet 3 .Cells $(16,14)$

Sheet3.Cells $(30,17)=$ Sheet 3. Cells $(17,14)$

Sheet3.Cells $(30,18)=$ Sheet $3 \cdot \operatorname{Cells}(18,14)$

Sheet3.Cells $(30,19)=$ Sheet $3 \cdot$ Cells $(19,14)$

$\delta_{t} \delta_{t}$

Sheet3.Range("K33:S41").FormulaArray = "=MMULT(N11:N19,K30:S30)"

$\delta_{t}^{\prime} \gamma_{t}$

Sheet3.Range("L43").FormulaArray = "=MMULT(K30:S30,R11:R19)"

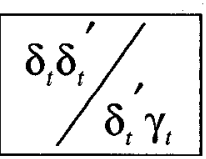

For $\mathrm{i}=33$ To 41 Step 1

For $\mathrm{j}=11$ To 19 Step 1

Next $j$

Sheet3.Cells $(\mathbf{i}, j+10)=$ Sheet3.Cells $(i, j) /$ Sheet $3 . \operatorname{Cells}(43,12)$

Next $\mathrm{i}$ 


$$
H_{t}^{D F P-G S M} \gamma_{t}
$$

Sheet3.Range("K45:K53").FormulaArray = "=MMULT(A1:I9,R11:R19)"

$$
H_{t}^{D F P \cdot G S M} \gamma_{t} \gamma_{t}^{\prime}
$$

Sheet3.Range("M45:U53").FormulaArray = "=MMULT(K45:K53,K28:S28)"

$$
H_{t}^{D F P-G S M} \gamma_{t} \gamma_{t}^{\prime} H_{t}^{D F P-G S M}
$$

Sheet3.Range("K55:S63").FormulaArray = "=MMULT(M45:U53,A1:19)" $-B 2$

$$
\gamma_{t}^{\prime} H_{t}^{D F P-G S M}
$$

Sheet3.Range("K66:S66").FormulaArray = "=MMULT(K28:S28,A1:19)"

$$
\gamma_{t}^{\prime} H_{t}^{D F P-G S M} \gamma_{t}
$$

Sheet3.Range("L67").FormulaArray = "=MMULT(K66:S66,R11:R19) "

$$
\frac{H_{t}^{D F P-G S M} \gamma_{t} \gamma_{t}^{\prime} H_{t}^{D F P-G S M}}{\gamma_{t}^{\prime} H_{t}^{D F P-G S M} \gamma_{t}}
$$

For $\mathrm{i}=55$ To 63 Step 1

For $\mathrm{j}=11$ To 19 Step 1 Next $\mathbf{j}$ Sheet3.Cells $(i, j+10)=$ Sheet3.Cells $(i, j) /$ Sheet3.Cells $(67,12)$

Next i

$$
H_{t}^{D F P-G S M}+\frac{\delta_{t} \delta_{t}^{\prime}}{\delta_{t}^{\prime} \gamma_{t}}-\frac{H_{t}^{D F P-G S M} \gamma_{t} \gamma_{t}^{\prime} H_{t}^{D F P-G S M}}{\gamma_{t}^{\prime} H_{t}^{D F P-G S M} \gamma_{t}}
$$

For $\mathbf{i}=72$ To 80 Step 1 
For $\mathrm{j}=11$ To 19 Step 1 Next j

Sheet3.Cells $(i, j)=$ Sheet3.Cells $(i-71, j-10)+\operatorname{Sheet} 3 \cdot \operatorname{Cells}(i-39, j+10)-\operatorname{Sheet} 3 \cdot \operatorname{Cells}(i-17, j+10)$

Next $\mathrm{i}$

Obtaining updated approximate hessain

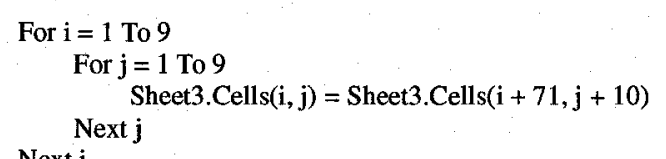

Next i

GoTo line2

End If

line1:

End Sub 


\section{B.8: BFGS-GSM algorithm}

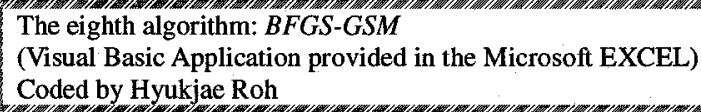

(Visual Basic Application provided in the Microsoft EXCEL)

Coded by Hyukjae Roh

Private Sub BFGS-GSM_CLICKO

Variables Generated

$\operatorname{Dim} \mathbf{i}, \mathbf{j}, \mathbf{k}, \mathrm{G}$ As Integer

Dim alfa As Double

Dim C As Integer

Dim T As Integer

Dim S As Integer

Dim B As Integer

Dim L As Integer

Dim VC As Double

Dim VT As Double

Dim VS As Double

Dim VB As Double

Dim VL As Double

Dim PC As Double

Dim PT As Double

Dim PS As Double

Dim PB As Double

Dim PL As Double

Sheets("sheet1").Select

$\mathrm{k}=1$
If $\mathrm{k}=1$ Then
GoTo line 3
End If

Initializing Staring Values of Parameters

line2:

$\mathrm{k}=\mathrm{k}+1$

line 3:

Sheet 1.Cells $(3,12)=$ Sheet3.Cells $(11,16)$

Sheet1.Cells $(4,12)=$ Sheet3.Cells $(12,16)$

Sheet1.Cells $(5,12)=$ Sheet 3. Cells $(13,16)$

Sheet1.Cells $(6,12)=$ Sheet3.Cells $(14,16)$

Sheet1.Cells $(7,12)=$ Sheet3.Cells $(15,16)$

Sheet1.Cells $(8,12)=$ Sheet3.Cells $(16,16)$

Sheet1.Cells $(9,12)=$ Sheet $3 \cdot \operatorname{Cells}(17,16)$

Sheet1.Cells $(10,12)=$ Sheet 3 .Cells $(18,16)$

Sheet1.Cells $(11,12)=$ Sheet 3. Cells $(19,16)$ 
Adding worksheets for calculation of Log-likelihood function value (sheet 1)

\author{
If $256 \cdot \mathrm{k}>=0$ Then \\ Sheets("sheet1").Select
}

For $\mathrm{j}=3$ To 2698 Step 5

$\mathrm{VC}=\operatorname{Exp}(\operatorname{Sheet} 1 . \operatorname{Cells}(3,12) *$ Sheet1.Cells $(\mathbf{j}, 3)+\operatorname{Sheet} 1 . \operatorname{Cells}(4,12) * \operatorname{Sheet} 1 . \operatorname{Cells}(\mathbf{j}, 4)$

+ Sheet1.Cells $(5,12) *$ Sheet1.Cells(j, 5) + Sheet1.Cells $(6,12) *$ Sheet1.Cells $(\mathrm{j}, 6)+$ Sheet1.Cells $(7,12) *$ Sheet1.Cells(j, 7))

VT $=\operatorname{Exp}(\operatorname{Sheet1.Cells}(3,12) *$ Sheet1.Cells $(j+1,3)+\operatorname{Sheet1.Cells~}(4,12) *$ Sheet1.Cells $(j+1,4)+\operatorname{Sheet1.Cells~}(8,12) * \operatorname{Sheet1}$.Cells $(j+1,8))$

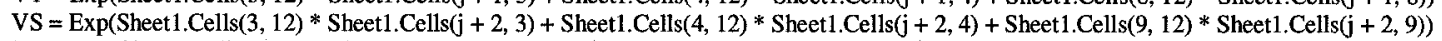

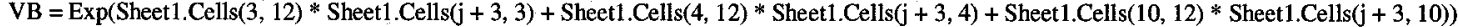

$\mathrm{VL}=\operatorname{Exp}(\operatorname{Sheet1.Cells}(3,12) *$ Sheet1.Cells $(j+4,3)+\operatorname{Sheet1.Cells~}(4,12) *$ Sheet1.Cells $(j+4,4)+\operatorname{Sheet} 1 . \operatorname{Cells}(11,12) * \operatorname{Sheet} 1 . \operatorname{Cells}(j+4,11))$

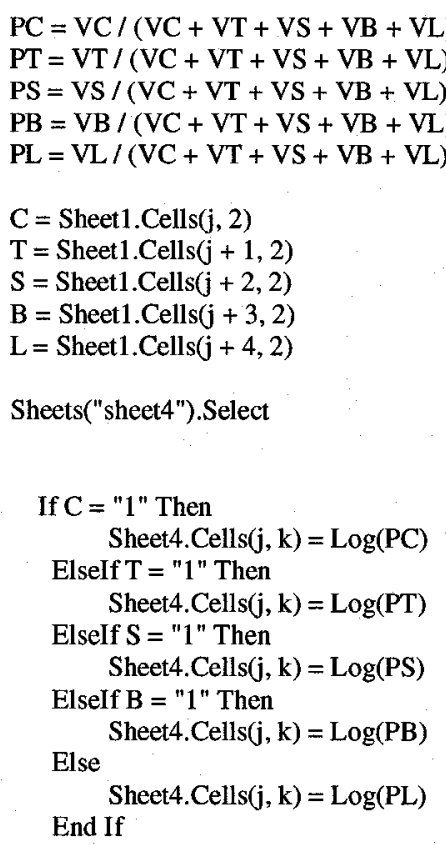

Next $\mathbf{j}$

Adding worksheets for calculation of Log-likelihood function value (sheet 2)

Elself $\mathrm{k}-256<=256$ Then

Sheets("sheet1").Select

For $\mathrm{j}=3$ To 2698 Step 5

$\mathrm{vC}=\operatorname{Exp}(\operatorname{Sheet} 1 . C e l l s(3,12) *$ Sheet1.Cells $(\mathrm{j}, 3)+\operatorname{Sheet} 1 . C e l l s(4,12) *$ Sheet1.Cells $(\mathrm{j}, 4)$

+ Sheet1.Cells $(5,12) *$ Sheet1.Cells(j, 5) + Sheet1.Cells $(6,12) *$ Sheet1.Cells(j, 6) + Sheet1.Cells $(7,12) *$ Sheet1.Cells(j, 7)

$\mathrm{VT}=\operatorname{Exp}(\operatorname{Sheet} 1$ Cells $(3,12) *$ Sheet1.Cells $(j+1,3)+$ Sheet1.Cells $(4,12) *$ Sheet1.Cells $(j+1,4)+\operatorname{Sheet1.Cells~}(8,12) * \operatorname{Sheet1.Cells~}(j+1,8))$

VS $=\operatorname{Exp}(\operatorname{Sheet1}$.Cells $(3,12) *$ Sheet1.Cells $(j+2,3)+\operatorname{Sheet1.Cells~}(4,12) *$ Sheet1.Cells $(j+2,4)+\operatorname{Sheet1.Cells~}(9,12) * \operatorname{Sheet1} . \operatorname{Cell} s(j+2,9))$

$\mathrm{VB}=\operatorname{Exp}(\operatorname{Sheet1.Cells}(3,12) * \operatorname{Sheet1} \cdot \operatorname{Cells}(\mathrm{j}+3,3)+\operatorname{Sheet1.Cells}(4,12) * \operatorname{Sheet1.Cells~}(j+3,4)+\operatorname{Sheet1} . \operatorname{Cells}(10,12) * \operatorname{Sheet} 1 . \operatorname{Cells}(\mathrm{j}+3,10))$

$V L=\operatorname{Exp}(\operatorname{Sheet1.Cells~}(3,12) *$ Sheet1.Cells $(j+4,3)+\operatorname{Sheet1.Cells~}(4,12) *$ Sheet1.Cells $(j+4,4)+\operatorname{Sheet} 1 . \operatorname{Cells}(11,12) * \operatorname{Sheet} 1 . \operatorname{Cells}(j+4,11))$

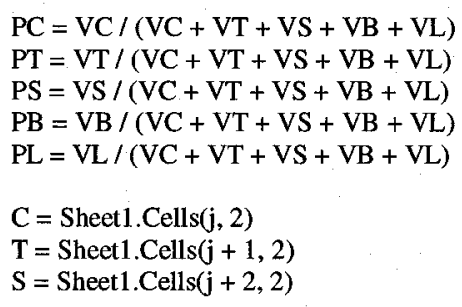


$B=$ Sheet1.Cells $(j+3,2)$

$\mathrm{L}=$ Sheet 1 .Cells $(\mathrm{j}+4,2)$

Sheets("sheet5").Select

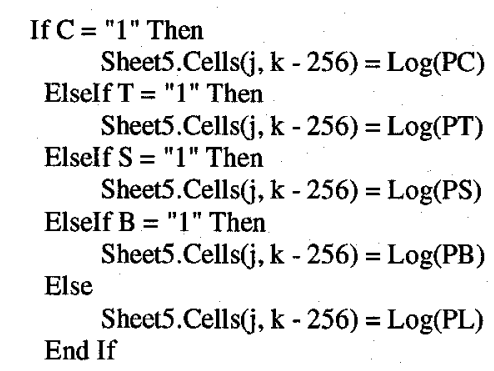

Next $\mathbf{j}$

Adding worksheets for calculation of Log-likelihood function value (sheet 3)

Elself $k-512<=256$ Then

Sheets("sheet1").Select

For $\mathbf{j}=3$ To 2698 Step 5

$\mathrm{VC}=\operatorname{Exp}(\operatorname{Sheet} 1 . \operatorname{Cells}(3,12) *$ Sheet1.Cells $(\mathfrak{j}, 3)+\operatorname{Sheet1.Cells~}(4,12) *$ Sheet1.Cells $(\mathbf{j}, 4)$

+ Sheet1.Cells $(5,12) *$ Sheet1.Cells $(\mathrm{j}, 5)+$ Sheet1.Cells $(6,12) *$ Sheet1.Cells $(\mathrm{j}, 6)+$ Sheet1.Cells $(7,12) *$ Sheet1.Cells $(\mathrm{j}, 7))$

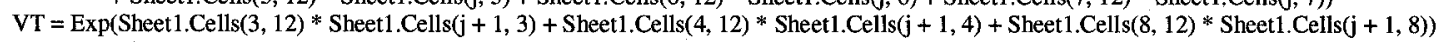

VS $=\operatorname{Exp}(\operatorname{Sheet1} 1$ Cells $(3,12) * \operatorname{Sheet1} 1$ Cells $(j+2,3)+\operatorname{Sheet1.Cells~}(4,12) * \operatorname{Sheet1.Cells~}(j+2,4)+\operatorname{Sheet1.Cells~}(9,12) * \operatorname{Sheet1.Cells~}(j+2,9))$

$\mathrm{VB}=\operatorname{Exp}(\operatorname{Sheet1} 1$ Cells $(3,12) *$ Sheet1.Cells $(j+3,3)+\operatorname{Sheet1.Cells~}(4,12) *$ Sheet1.Cells $(j+3,4)+$ Sheet1.Cells $(10,12) * \operatorname{Sheet} 1$. Cells $(j+3,10))$

$\mathrm{VL}=\operatorname{Exp}(\operatorname{Sheet} 1$. Cells $(3,12) *$ Sheet1.Cells $(j+4,3)+\operatorname{Sheet1.Cells~}(4,12) *$ Sheet1.Cells $(j+4,4)+$ Sheet1.Cells $(11,12) *$ Sheet 1.Cells $(j+4,11))$

$\mathrm{PC}=\mathrm{VC} /(\mathrm{VC}+\mathrm{VT}+\mathrm{VS}+\mathrm{VB}+\mathrm{VL})$

$\mathrm{PT}=\mathrm{VT} /(\mathrm{VC}+\mathrm{VT}+\mathrm{VS}+\mathrm{VB}+\mathrm{VL})$

$\mathrm{PS}=\mathrm{VS} /(\mathrm{VC}+\mathrm{VT}+\mathrm{VS}+\mathrm{VB}+\mathrm{VL})$

$\mathrm{PB}=\mathrm{VB} /(\mathrm{VC}+\mathrm{VT}+\mathrm{VS}+\mathrm{VB}+\mathrm{VL})$

$\mathrm{PL}=\mathrm{VL} /(\mathrm{VC}+\mathrm{VT}+\mathrm{VS}+\mathrm{VB}+\mathrm{VL})$

$\mathrm{C}=$ Sheet $1 . \operatorname{Cells}(\mathrm{j}, 2)$

$\mathrm{T}=$ Sheet1.Cells $(\mathrm{j}+1,2)$

$\mathrm{S}=$ Sheet1.Cells $(\mathrm{j}+2,2)$

$\mathrm{B}=$ Sheet 1. Cells $(\mathrm{j}+3,2)$

$\mathrm{L}=$ Sheet1.Cells $(\mathrm{j}+4,2)$

Sheets("sheet6").Select

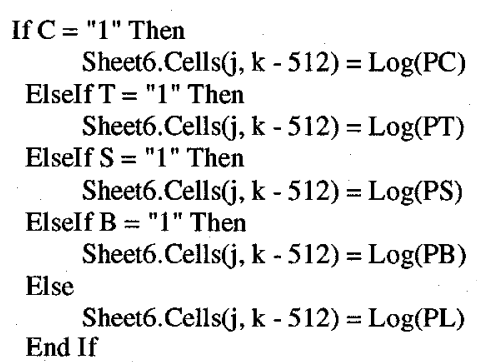

Next $\mathbf{j}$

Adding worksheets for calculation of Log-likelihood function value (sheet 4)

Elself $\mathrm{k}-768<=256$ Then

Sheets("sheet1").Select 
For $\mathrm{j}=3$ To 2698 Step 5

$\mathrm{vC}=\operatorname{Exp}(\operatorname{Sheet} 1 . \operatorname{Cells}(3,12) *$ Sheet1.Cells $(\mathrm{j}, 3)+\operatorname{Sheet} 1 . \operatorname{Cells}(4,12) * \operatorname{Sheet1.Cells(j,4)}$

+ Sheet1.Cells $(5,12) *$ Sheet1.Cells(j, 5) + Sheet1.Cells $(6,12) *$ Sheet1.Cells(j, 6) + Sheet1.Cells $(7,12) *$ Sheet1.Cells $(\mathrm{j}, 7))$

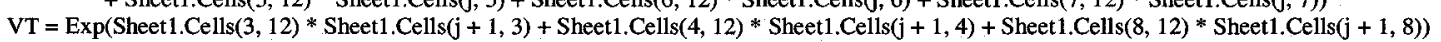

VS $=\operatorname{Exp}(\operatorname{Sheet1.Cel1s~}(3,12) *$ Sheet1.Cells $(j+2,3)+\operatorname{Sheet1.Cells~}(4,12) *$ Sheet1.Cells $(j+2,4)+\operatorname{Sheet1}$.Cells $(9,12) * \operatorname{Sheet1.Cells~}(j+2,9))$

$\mathrm{VB}=\operatorname{Exp}($ Sheet 1.Cells $(3,12) *$ Sheet1.Cells $(j+3,3)+$ Sheet1.Cells $(\mathbf{4}, 12) *$ Sheet1.Cells $(j+3,4)+$ Sheet1.Cells $(10,12) *$ Sheet $1 . C e l l s(j+3,10))$

$\mathrm{VL}=\operatorname{Exp}(\operatorname{Sheet1} . \operatorname{Cells}(3,12) *$ Sheet 1.Cells $(\mathrm{j}+4,3)+\operatorname{Sheet1.Cells}(4,12) *$ Sheet1.Cells $(j+4,4)+$ Sheet $1 . \operatorname{Cells}(11,12) *$ Sheet1.Cells $(j+4,11))$

$\mathrm{PC}=\mathrm{VC} /(\mathrm{VC}+\mathrm{VT}+\mathrm{VS}+\mathrm{VB}+\mathrm{VL})$

$\mathrm{PT}=\mathrm{VT} /(\mathrm{VC}+\mathrm{VT}+\mathrm{VS}+\mathrm{VB}+\mathrm{VL})$

$\mathrm{PS}=\mathrm{VS} /(\mathrm{VC}+\mathrm{VT}+\mathrm{VS}+\mathrm{VB}+\mathrm{VL})$

$\mathrm{PB}=\mathrm{VB} /(\mathrm{VC}+\mathrm{VT}+\mathrm{VS}+\mathrm{VB}+\mathrm{VL})$

$\mathrm{PL}=\mathrm{VL} /(\mathrm{VC}+\mathrm{VT}+\mathrm{VS}+\mathrm{VB}+\mathrm{VL})$

$\mathrm{C}=$ Sheet 1. Cells $(\mathrm{j}, 2)$

$\mathrm{T}=$ Sheet 1 .Cells $(\mathbf{j}+1,2)$

$\mathrm{S}=$ Sheet 1 .Cells $(\mathrm{j}+2,2)$

$B=$ Sheet1. Cells $(j+3,2)$

$\mathrm{L}=$ Sheet1.Cells $(\mathrm{j}+4,2)$

Sheets("sheet7").Select

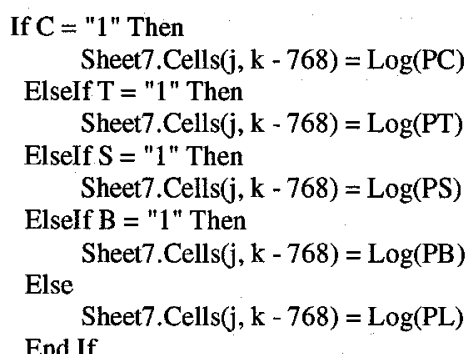

Next $j$

End If

Utility Function Value for all alternatives' model specifications

For $\mathrm{i}=3$ To 2698 Step 5

Sheets("sheet2").Select

$\mathrm{vC}=\operatorname{Exp}(\operatorname{Sheet1} . \operatorname{Cells}(3,12) *$ Sheet1.Cells $(\mathrm{i}, 3)+\operatorname{Sheet1} . \operatorname{Cell} \mathrm{s}(4,12) *$ Sheet1.Cells $(\mathrm{i}, 4)$

+ Sheet 1.Cells $(5,12) *$ Sheet1.Cells(i, 5) + Sheet1.Cells $(6,12) *$ Sheet1.Cells $(i, 6)+$ Sheetl.Cells $(7,12) *$ Sheet1.Cells $(i, 7))$

VT $=\operatorname{Exp}(\operatorname{Sheet1.Cells~}(3,12) *$ Sheet 1. Cells $(i+1,3)+$ Sheet1.Cells $(4,12) *$ Sheet1.Cells $(i+1,4)+$ Sheet1.Cells $(8,12) *$ Sheet1.Cells $(i+1,8))$

VS $=\operatorname{Exp}(\operatorname{Sheet1} 1$ Cells $(3,12) * \operatorname{Sheet1.Cells~}(i+2,3)+\operatorname{Sheet1.Cells~}(4,12) *$ Sheet1.Cells $(i+2,4)+\operatorname{Sheet1.Cells~}(9,12) * \operatorname{Sheet1} 1$.Cells $(i+2,9))$

$\mathrm{VB}=\operatorname{Exp}($ Sheet 1.Cells $(3,12) *$ Sheet1.Cells $(i+3,3)+$ Sheet 1.Cells $(4,12) *$ Sheet1.Cells $(i+3,4)+$ Sheet1.Cells $(10,12) *$ Sheet1.Cells $(i+3,10))$

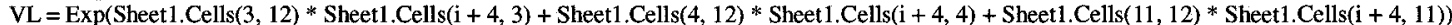

Mode Choice Probability of each observations with Logit probability model

$\mathrm{PC}=\mathrm{VC} /(\mathrm{VC}+\mathrm{VT}+\mathrm{VS}+\mathrm{VB}+\mathrm{VL})$

$\mathrm{PT}=\mathrm{VT} /(\mathrm{VC}+\mathrm{VT}+\mathrm{VS}+\mathrm{VB}+\mathrm{VL})$

$\mathrm{PS}=\mathrm{VS} /(\mathrm{VC}+\mathrm{VT}+\mathrm{VS}+\mathrm{VB}+\mathrm{VL})$

$\mathrm{PB}=\mathrm{VB} /(\mathrm{VC}+\mathrm{VT}+\mathrm{VS}+\mathrm{VB}+\mathrm{VL})$

$\mathrm{PL}=\mathrm{VL} /(\mathrm{VC}+\mathrm{VT}+\mathrm{VS}+\mathrm{VB}+\mathrm{VL})$

$\mathrm{C}=$ Sheet1.Cells $(\mathrm{i}, 2)$

$\mathrm{T}=$ Sheet 1 .Cells $(\mathrm{i}+1,2)$ 
$\mathrm{S}=$ Sheet 1 .Cells $(i+2,2)$

B $=$ Sheet 1. Cells $(\mathrm{i}+3,2)$

$\mathrm{L}=$ Sheet 1 .Cells $(i+4,2)$

First-order Derivative with respect to $\beta_{1}$

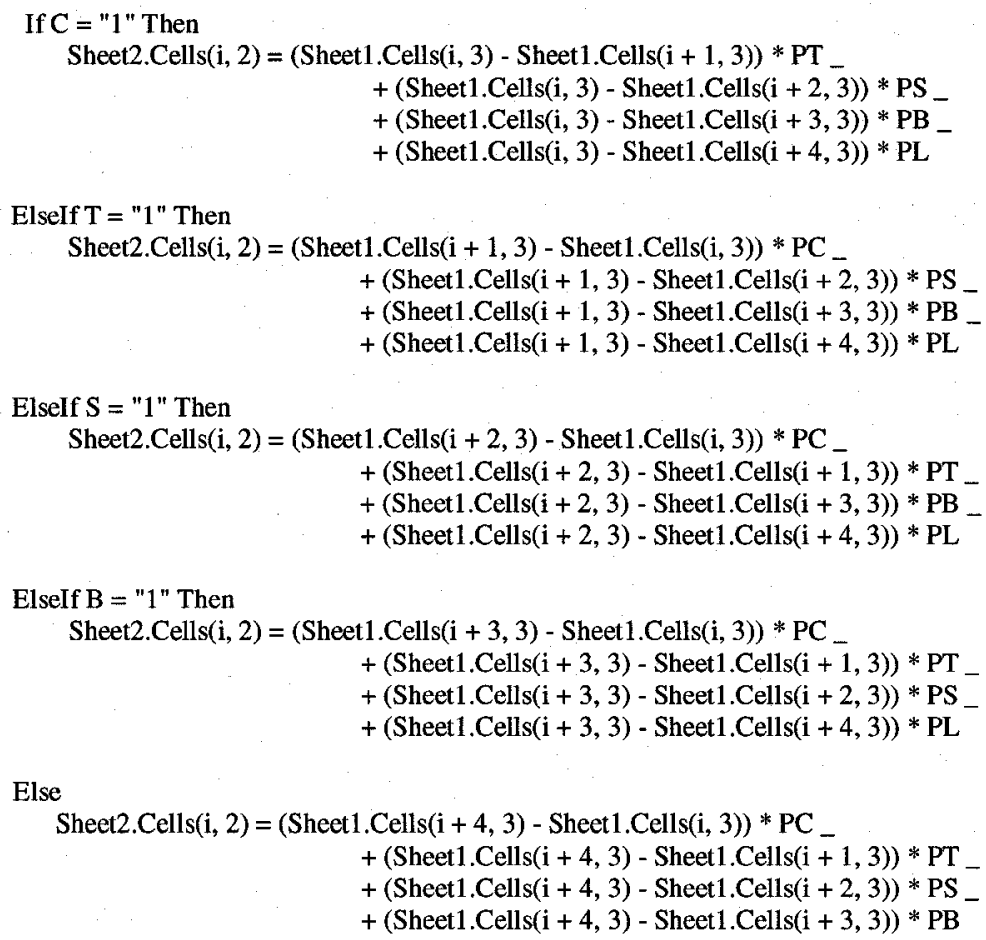

End If

First-order Derivative with respect to $\beta_{2}$

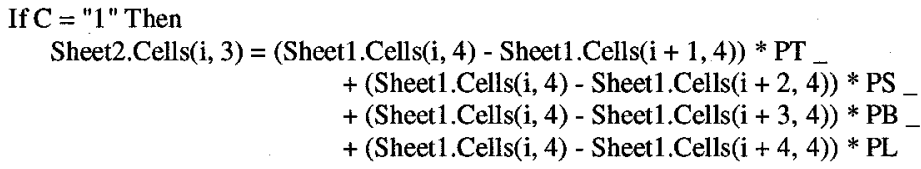


$+($ Sheet1.Cells $(\mathrm{i}+3,4)$ - Sheet1.Cells $(\mathrm{i}+1,4)) * \mathrm{PT}$

$+($ Sheet1.Cells $(\mathrm{i}+3,4)$ - Sheet1.Cells $(\mathrm{i}+2,4)) *$ PS

$+($ Sheet1.Cells $(\mathrm{i}+3,4)-$ Sheet1.Cells $(\mathrm{i}+4,4)) * \mathrm{PL}$

Else

Sheet2.Cells $(\mathrm{i}, 3)=($ Sheet1.Cells $(\mathrm{i}+4,4)-$ Sheet 1.Cells $(\mathrm{i}, 4)) * \mathrm{PC}$

$+($ Sheet1.Cells $(\mathrm{i}+4,4)$ - Sheet1.Cells $(\mathrm{i}+1,4)) *$ PT

$+($ Sheet 1. Cells $(\mathrm{i}+4,4)$ - Sheet1.Cells $(\mathrm{i}+2,4)) *$ PS

$+($ Sheet1.Cells $(\mathrm{i}+4,4)-$ Sheet1.Cells $(\mathrm{i}+3,4)) *$ PB

End If

First-order Derivative with respect to $\beta_{3}$

If $\mathrm{C}=$ "1" Then

Sheet2.Cells $(i, 4)=$ Sheet 1.Cells $(i, 5) *(P T+P S+P B+P L)$

Else

Sheet2.Cells $(i, 4)=-$ Sheet1.Cells(i, 5) * PC

End If

First-order Derivative with respect to $\beta_{4}$

If $\mathrm{C}=$ " 1 " Then

Sheet2.Cells $(\mathrm{i}, 5)=$ Sheet1.Cells $(\mathrm{i}, 6) *(\mathrm{PT}+\mathrm{PS}+\mathrm{PB}+\mathrm{PL})$

Else

Sheet2.Cells $(i, 5)=-$ Sheet1.Cells $(i, 6) * P C$

End If

First-order Derivative with respect to $\beta_{5}$

If $\mathrm{C}=$ " 1 " Then

Sheet2.Cells $(\mathbf{i}, 6)=$ Sheet1.Cells $(\mathrm{i}, 7) *(\mathrm{PT}+\mathrm{PS}+\mathrm{PB}+\mathrm{PL})$

Else

Sheet2.Cells $(\mathbf{i}, 6)=-$ Sheet1.Cells $(\mathrm{i}, 7) *$ PC

End If

First-order Derivative with respect to $\beta_{6}$

If T $=$ " 1 " Then

Sheet2.Cells $(\mathbf{i}, 7)=(\mathrm{PC}+\mathrm{PS}+\mathrm{PB}+\mathrm{PL})$

Else

Sheet2.Cells $(\mathbf{i}, 7)=-(\mathrm{PT})$

End If

First-order Derivative with respect to $\beta_{7}$

If $S=$ " 1 " Then

Sheet2.Cells $(i, 8)=(P C+P T+P B+P L)$

Reproduced with permission of the copyright owner. Further reproduction prohibited without permission. 
Else

Sheet2.Cells $(\mathrm{i}, 8)=-\mathrm{PS}$

End If

First-order Derivative with respect to $\beta_{8}$

If $\mathrm{B}=$ " 1 " Then

Sheet 2.Cells $(\mathbf{i}, 9)=(\mathrm{PC}+\mathrm{PT}+\mathrm{PS}+\mathrm{PL})$

Else

Sheet 2.Cells $(i, 9)=-P B$

End If

First-order Derivative with respect to $\beta_{9}$

If $\mathrm{L}=$ " 1 " Then

Sheet2.Cells $(\mathrm{i}, 10)=(\mathrm{PC}+\mathrm{PT}+\mathrm{PS}+\mathrm{PB})$

Else

Sheet2.Cells $(\mathrm{i}, 10)=-\mathrm{PL}$

End If

Next $\mathrm{i}$

Summation of all observation and averaging it: $G_{t}$

For $\mathbf{j}=2$ To 10 Step 1

Sheet2.Cells $(2703, \mathrm{j})$.FormulaR1C1 = "=-SUM(R[-2700]C:R[-1]C)/540"

Next $\mathbf{j}$

Sheets("sheet3").Select

Obtaining Gradient components calculated : $G_{t}$

For $\mathbf{i}=11$ To 19 Step 1

Sheet3.Cells $(i, 11)=$ Sheet 2. Cells $(2703, i-9)$

Next i

Calculating delta $: \delta_{t}=\lambda_{t-G S D} \Delta_{t}=\lambda_{t-G S D}\left(-H_{t A P}^{B F G S-G S D}\right) G_{t}$

Sheet3.Range("M11:M19").FormulaArray = "=-(MMULT(A1:19,K11:K19))"

Reproduced with permission of the copyright owner. Further reproduction prohibited without permission. 
Finding $\lambda_{t-G S D}$ by means of adopting golden-section line search method

Sheets("sheet8").Select

$\mathrm{G}=1$

alfa $=0.618$

'boundary'

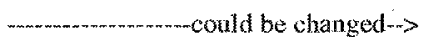

Sheet8.Cells $(G+1,1)=$ Sheet8.Cells $(4,10)$ could be changed $-\rightarrow$

Sheet8.Cells $(\mathrm{G}+1,2)=$ Sheet8.Cells $(5,10)$

Sheet8.Cells $(\mathrm{G}+1,3)=$ Sheet8.Cells $(\mathrm{G}+1,1)+(1$ - alfa $) *($ Sheet8.Cells $(\mathrm{G}+1,2)-$ Sheet8.Cells $(\mathrm{G}+1,1))$

Sheet8.Cells $(G+1,4)=$ Sheet8.Cells $(G+1,1)+$ alfa $*(\operatorname{Sheet8.Cells~}(G+1,2)-\operatorname{Sheet8.Cells~}(G+1,1))$

Evaluating Log-likelihood function to find the value, $\lambda_{t-G S D}$, to minimize $L L\left(\beta_{t}+\lambda_{t-G S D} \Delta_{t}\right)$ with golden-section line search method

Calculating function, $L L\left(\beta_{t}+\lambda_{t-G S D} \Delta_{t}\right)$, value for lower limit of the interval of uncertainty

Sheets("sheet8").Select

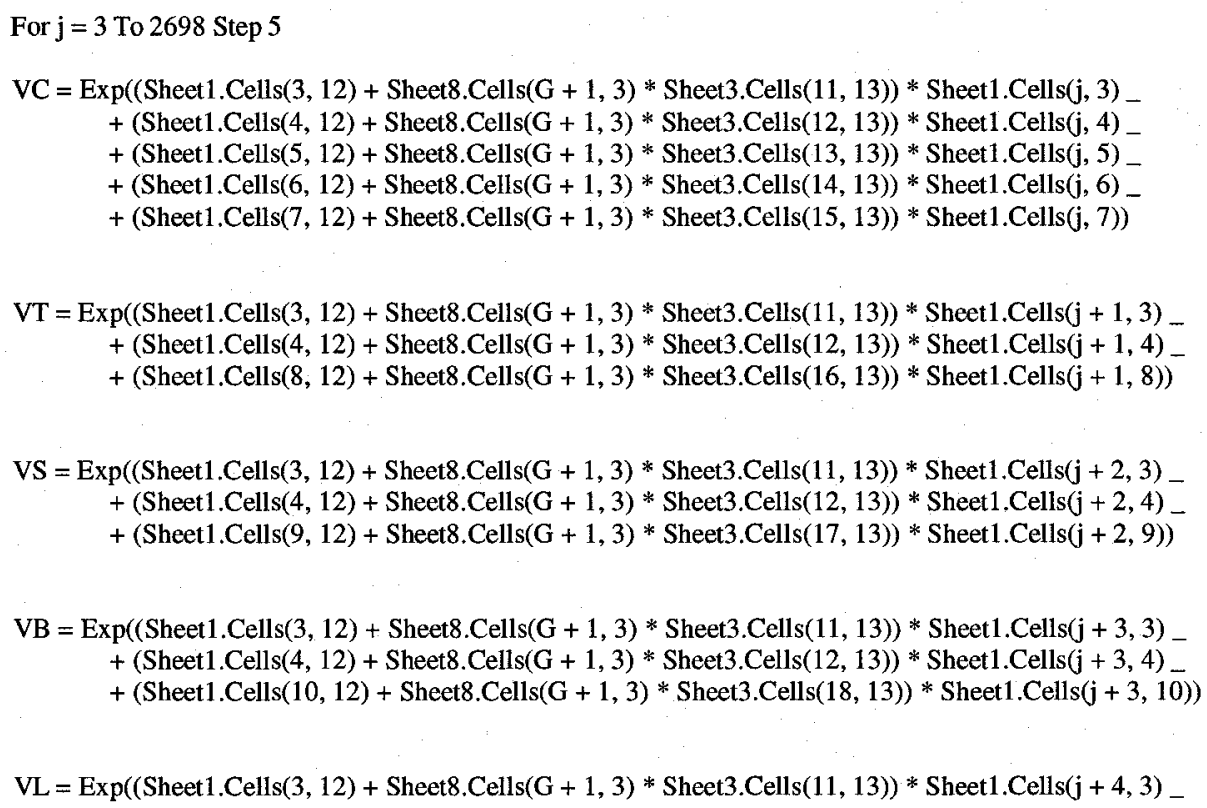


$+($ Sheet1.Cells $(4,12)+$ Sheet8.Cells $(G+1,3) *$ Sheet3.Cells $(12,13)) *$ Sheet1.Cells $(j+4,4)$

$+($ Sheet1.Cells $(11,12)+$ Sheet8.Cells $(G+1,3) *$ Sheet3.Cells $(19,13)) *$ Sheet1.Cells $(j+4,11))$

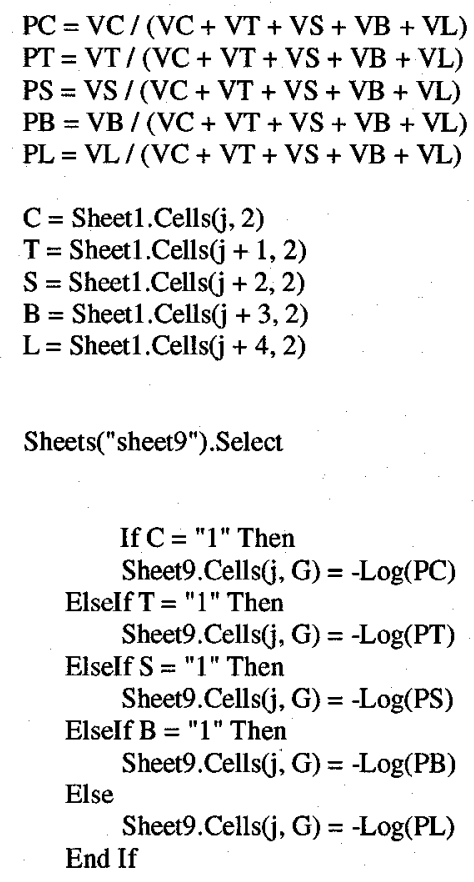

Sheets("sheet9").Select

Next $\mathbf{j}$

Sheet8.Cells $(G+1,5)=\operatorname{Sheet} 9 . \operatorname{Cells}(2703, G)$

Calculating function, $L L\left(\beta_{t}+\lambda_{t-G S D} \Delta_{t}\right)$,value for upper limit of the interval of uncertainty

Sheets("sheet8").Select

For $\mathrm{j}=3$ To 2698 Step 5

$\mathrm{VC}=\operatorname{Exp}((\operatorname{Sheet1.Cells}(3,12)+\operatorname{Sheet} 8 \cdot \operatorname{Cells}(\mathrm{G}+1,4) * \operatorname{Sheet} 3 . \operatorname{Cells}(11,13)) *$ Sheet1.Cells $(\mathrm{j}, 3)$ $+($ Sheet1.Cells $(4,12)+$ Sheet8.Cells $(G+1,4) *$ Sheet3.Cells $(12,13)) *$ Sheet1.Cells $(j, 4)$ _ $+($ Sheet1.Cells $(5,12)+$ Sheet8.Cells $(G+1,4) *$ Sheet3.Cells $(13,13)) *$ Sheet1.Cells $(j, 5)$ $+($ Sheet1.Cells $(6,12)+$ Sheet8.Cells $(\mathrm{G}+1,4) *$ Sheet3.Cells $(14,13)) *$ Sheet1.Cells $(\mathrm{j}, 6)$ $+($ Sheet1.Cells $(7,12)+$ Sheet8.Cells $(G+1,4) *$ Sheet3.Cells $(15,13)) *$ Sheet1.Cells $(j, 7))$

VT $=\operatorname{Exp}(($ Sheet1.Cells $(3,12)+$ Sheet8.Cells $(G+1,4) *$ Sheet3.Cells $(11,13)) *$ Sheet1.Cells $(j+1,3)$ $+($ Sheet1.Cells $(4,12)+$ Sheet8.Cells $(G+1,4) *$ Sheet3.Cells $(12,13)) *$ Sheet1.Cells $(j+1,4)$ $+($ Sheet1.Cells $(8,12)+$ Sheet8.Cells $(G+1,4) *$ Sheet3.Cells $(16,13)) *$ Sheet1.Cells $(j+1,8))$

VS $=\operatorname{Exp}(($ Sheet1.Cells $(3,12)+$ Sheet8.Cells $(\mathrm{G}+1,4) *$ Sheet3.Cells $(11,13)) *$ Sheet1.Cells $(j+2,3)$ $+($ Sheet1.Cells $(4,12)+$ Sheet8.Cells $(G+1,4) *$ Sheet3.Cells $(12,13)) *$ Sheet1.Cells $(j+2,4)$ $+($ Sheet1.Cells $(9,12)+$ Sheet8.Cells $(G+1,4) *$ Sheet3.Cells $(17,13)) *$ Sheet1.Cells $(j+2,9))$

$\mathrm{VB}=\operatorname{Exp}((\operatorname{Sheet} 1 . \operatorname{Cells}(3,12)+\operatorname{Sheet8.Cells}(\mathrm{G}+1,4) * \operatorname{Sheet} 3 . \operatorname{Cells}(11,13)) * \operatorname{Sheet} 1 . \operatorname{Cells}(\mathfrak{j}+3,3)$ $+($ Sheet1.Cells $(4,12)+$ Sheet8.Cells $(G+1,4) *$ Sheet3.Cells $(12,13)) *$ Sheet1.Cells $(j+3,4)$ $+($ Sheet1.Cells $(10,12)+$ Sheet8.Cells $(G+1,4) *$ Sheet3.Cells $(18,13)) *$ Sheet1.Cells $(j+3,1 \overline{0}))$

$\mathrm{VL}=\operatorname{Exp}(($ Sheet1.Cells $(3,12)+$ Sheet8.Cells $(\mathrm{G}+1,4) *$ Sheet3.Cells $(11,13)) *$ Sheet1.Cells $(j+4,3)$ $+($ Sheet1.Cells $(4,12)+$ Sheet8.Cells $(G+1,4) *$ Sheet3.Cells $(12,13)) *$ Sheet1.Cells $(j+4,4)$ 
$+($ Sheet1. Cells $(11,12)+$ Sheet8.Cells $(G+1,4) *$ Sheet3.Cells $(19,13)) *$ Sheet1.Cells $(j+4,11))$

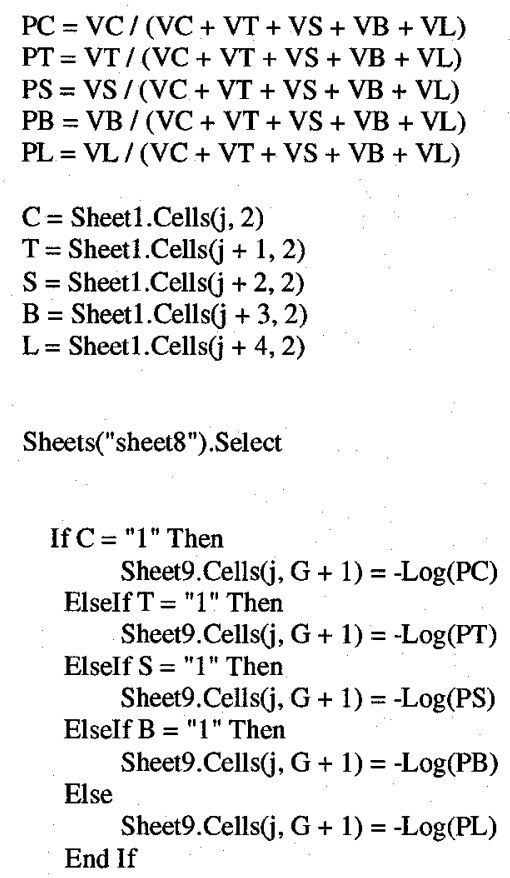

Next $j$

Sheet8.Cells $(G+1,6)=$ Sheet9.Cells $(2703, G+1)$

line4:

Checking the stopping criterion

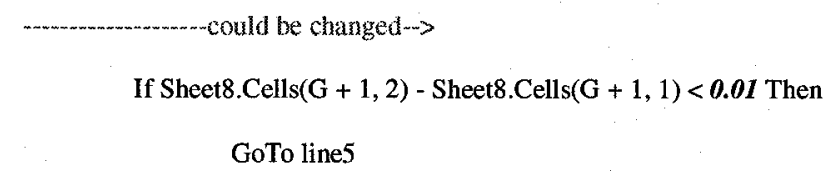

ElseIf Sheet8.Cells $(G+1,5)>$ Sheet8.Cells $(G+1,6)$ Then

Sheet8.Cells $(G+2,1)=$ Sheet8.Cells $(G+1,3)$

Sheet8.Cells $(G+2,2)=$ Sheet8.Cells $(G+1,2)$

Sheet8.Cells $(\mathrm{G}+2,3)=$ Sheet8.Cells $(\mathrm{G}+1,4)$

Sheet8.Cells $(G+2,4)=$ Sheet8.Cells $(G+2,1)+$ alfa $*($ Sheet8.Cells $(G+2,2)-$ Sheet8.Cells $(G+2,1))$

Sheet8.Cells $(\mathrm{G}+2,5)=$ Sheet8.Cells $(\mathrm{G}+1,6)$

Calculating function, $L L\left(\beta_{t}+\lambda_{t-G S D} \Delta_{t}\right)$, value of the new interval of uncertainty

Sheets("sheet8").Select 
For $\mathbf{j}=3$ To 2698 Step 5

$\mathrm{VC}=\operatorname{Exp}(($ Sheet1.Cells $(3,12)+\operatorname{Sheet} 8 . C \operatorname{Cells}(\mathrm{G}+2,4) *$ Sheet3.Cells $(11,13)) *$ Sheet1.Cells $(\mathrm{j}, 3)$ $+($ Sheet1.Cells $(4,12)+$ Sheet8.Cells $(\mathrm{G}+2,4) *$ Sheet3.Cells $(12,13)) *$ Sheet1.Cells $(\mathrm{j}, 4)$ $+($ Sheet1.Cells $(5,12)+$ Sheet8.Cells $(\mathrm{G}+2,4) *$ Sheet3.Cells $(13,13)) *$ Sheet1.Cells $(\mathrm{j}, 5)$ $+($ Sheet1.Cells $(6,12)+$ Sheet8.Cells $(G+2,4) *$ Sheet3.Cells $(14,13)) *$ Sheet1.Cells $(j, 6)$ $+($ Sheet1.Cells $(7,12)+$ Sheet8.Cells $(\mathrm{G}+2,4) *$ Sheet3.Cells $(15,13)) *$ Sheet1.Cells $(\mathfrak{j}, 7))$

$\mathrm{VT}=\operatorname{Exp}((\operatorname{Sheet1} . \operatorname{Cells}(3,12)+\operatorname{Sheet8.Cells}(\mathrm{G}+2,4) * \operatorname{Sheet} 3 . \operatorname{Cells}(11,13)) * \operatorname{Sheet} 1 . C e l l s(\mathrm{j}+1,3)$ $+($ Sheet1.Cells $(4,12)+$ Sheet8.Cells $(\mathrm{G}+2,4) *$ Sheet3.Cells $(12,13)) *$ Sheet1.Cells $(\mathrm{j}+1,4)$ $+($ Sheet1.Cells $(8,12)+$ Sheet8.Cells $(G+2,4) *$ Sheet3.Cells $(16,13)) *$ Sheet1.Cells $(j+1,8))$

VS $=\operatorname{Exp}(($ Sheet1.Cells $(3,12)+$ Sheet8.Cells $(G+2,4) *$ Sheet3.Cells $(11,13)) *$ Sheet1.Cells $(j+2,3)$ $+($ Sheet1.Cells $(4,12)+$ Sheet8.Cells $(\mathrm{G}+2,4) *$ Sheet3.Cells $(12,13)) *$ Sheet1.Cells $(\mathrm{j}+2,4)$ $+($ Sheet1.Cells $(9,12)+\operatorname{Sheet8.Cells}(\mathrm{G}+2,4) *$ Sheet3.Cells $(17,13)) *$ Sheet1.Cells $(\mathrm{j}+2,9))$

VB $=\operatorname{Exp}(($ Sheet1.Cells $(3,12)+$ Sheet8.Cel1s $(G+2,4) *$ Sheet3.Cells $(11,13)) *$ Sheet1.Cells $(j+3,3)$ $+($ Sheet1.Cells $(4,12)+$ Sheet8.Cells $(G+2,4) *$ Sheet3.Cells $(12,13)) *$ Sheet1.Cells $(j+3,4)$ $+($ Sheet1.Cells $(10,12)+$ Sheet8.Cells $(G+2,4) *$ Sheet3.Cells $(18,13)) *$ Sheet1.Cells $(\mathrm{j}+3,10))$

$\mathrm{VL}=\operatorname{Exp}((\operatorname{Sheet} 1 . \operatorname{Cells}(3,12)+\operatorname{Sheet} 8 . \operatorname{Cells}(\mathrm{G}+2,4) * \operatorname{Sheet} 3 \cdot \operatorname{Cells}(11,13)) * \operatorname{Sheet} 1 . \operatorname{Cell} s(\mathrm{j}+4,3)$ $+($ Sheet1.Cells $(4,12)+$ Sheet8.Cells $(G+2,4) *$ Sheet3.Cells $(12,13)) *$ Sheet1.Cells $(j+4,4)$ $+($ Sheet1.Cells $(11,12)+$ Sheet8.Cells $(G+2,4) *$ Sheet3.Cells $(19,13)) *$ Sheet1.Cells $(j+4,11))$

$\mathrm{PC}=\mathrm{VC} /(\mathrm{VC}+\mathrm{VT}+\mathrm{VS}+\mathrm{VB}+\mathrm{VL})$

$\mathrm{PT}=\mathrm{VT} /(\mathrm{VC}+\mathrm{VT}+\mathrm{VS}+\mathrm{VB}+\mathrm{VL})$

$P S=V S /(V C+V T+V S+V B+V L)$

$\mathrm{PB}=\mathrm{VB} /(\mathrm{VC}+\mathrm{VT}+\mathrm{VS}+\mathrm{VB}+\mathrm{VL})$

$\mathrm{PL}=\mathrm{VL} /(\mathrm{VC}+\mathrm{VT}+\mathrm{VS}+\mathrm{VB}+\mathrm{VL})$

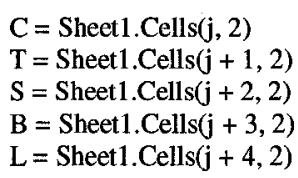

Sheets("sheet8").Select

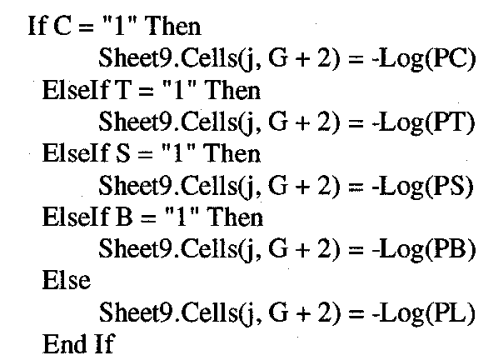

Next $\mathbf{j}$

Sheet8.Cells $(G+2,6)=$ Sheet9.Cells $(2703, G+2)$

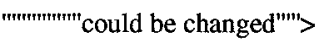

Elself Sheet8.Cells $(\mathrm{G}+1,5)<=$ Sheet8.Cells $(\mathrm{G}+1,6)$ Then

Sheet8.Cells $(G+2,1)=$ Sheet8.Cells $(G+1,1)$

Sheet8.Cells $(G+2,2)=$ Sheet $8 \cdot \operatorname{Cells}(G+1,4)$ 
1))

Sheet8.Cells $(\mathrm{G}+2,3)=$ Sheet8.Cells $(\mathrm{G}+2,1)+(1-$ alfa $) *($ Sheet8.Cells $(\mathrm{G}+2,2)-$ Sheet8.Cells $(\mathrm{G}+2$,

Sheet8.Cells $(G+2,4)=$ Sheet8.Cells $(G+1,3)$

Calculating function, $L L\left(\beta_{t}+\lambda_{t-G S D} \Delta_{t}\right)$, value of the new interval of uncertainty

Sheets("sheet8").Select

For $\mathbf{j}=3$ To 2698 Step 5

$\mathrm{VC}=\operatorname{Exp}((\operatorname{Sheet1.Cells}(3,12)+\operatorname{Sheet} 8 \cdot \operatorname{Cells}(\mathrm{G}+2,3) * \operatorname{Sheet} 3 . \operatorname{Cells}(11,13)) * \operatorname{Sheet1.Cells}(\mathrm{j}, 3)$ $+($ Sheet1.Cells $(4,12)+$ Sheet8.Cells $(G+2,3) *$ Sheet3.Cells $(12,13)) *$ Sheet1.Cells $(\mathbf{j}, 4)$ $+($ Sheet1.Cells $(5,12)+$ Sheet8.Cells $(G+2,3) *$ Sheet3.Cells $(13,13)) *$ Sheet1.Cells $(\mathbf{j}, 5) \ldots$ $+($ Sheet1.Cells $(6,12)+$ Sheet8.Cells $(G+2,3) *$ Sheet3.Cells $(14,13)) *$ Sheet1.Cells $(\mathbf{j}, 6)$ $+($ Sheet1.Cells $(7,12)+$ Sheet8.Cells $(G+2,3) *$ Sheet3.Cells $(15,13)) *$ Sheet1.Cells $(\mathbf{j}, 7))$

$\mathrm{VT}=\operatorname{Exp}((\operatorname{Sheet1} \cdot \operatorname{Cells}(3,12)+\operatorname{Sheet} 8 \cdot \operatorname{Cells}(\mathrm{G}+2,3) * \operatorname{Sheet} 3 \cdot \operatorname{Cells}(11,13)) * \operatorname{Sheet1} \cdot \operatorname{Cell}(\mathrm{j}+1,3)$ $+($ Sheet1.Cells $(4,12)+$ Sheet8.Cells $(G+2,3) *$ Sheet3.Cells $(12,13)) *$ Sheet1.Cells $(j+1,4)$ $+($ Sheet1.Cells $(8,12)+$ Sheet8.Cells $(\mathrm{G}+2,3) *$ Sheet3.Cells $(16,13)) *$ Sheet1.Cells $(\mathrm{j}+1,8))$

VS $=\operatorname{Exp}((\operatorname{Sheet1} . \operatorname{Cells}(3,12)+\operatorname{Sheet} 8 . \operatorname{Cells}(\mathrm{G}+2,3) * \operatorname{Sheet} 3 . \operatorname{Cells}(11,13)) * \operatorname{Sheet} 1 . \operatorname{Cells}(\mathrm{j}+2,3)$ $+($ Sheet1.Cells $(4,12)+$ Sheet8.Cells $(\mathrm{G}+2,3) *$ Sheet3.Cells $(12,13)) *$ Sheet $1 . C e l l s(j+2,4)$ $+($ Sheet1.Cells $(9,12)+$ Sheet8.Cells $(\mathrm{G}+2,3) *$ Sheet3.Cells $(17,13)) *$ Sheet1.Cells $(\mathrm{j}+2,9))$

VB $=\operatorname{Exp}(($ Sheet1.Cells $(3,12)+$ Sheet8.Cells $(G+2,3) *$ Sheet $3 . \operatorname{Cells}(11,13)) * \operatorname{Sheet} 1 . C e l l s(j+3,3)$ $+($ Sheet1.Cells $(4,12)+$ Sheet8.Cells $(\mathrm{G}+2,3) *$ Sheet3.Cells $(12,13)) *$ Sheet1.Cells $(\mathrm{j}+3,4)$ $+($ Sheet1.Cells $(10,12)+$ Sheet8.Cells $(\mathrm{G}+2,3) *$ Sheet3.Cells $(18,13)) *$ Sheet1.Cells $(\mathrm{j}+3,10))$

$\mathrm{VL}=\operatorname{Exp}(($ Sheet1.Cells $(3,12)+$ Sheet8.Cells $(\mathrm{G}+2,3) *$ Sheet3.Cells $(11,13)) *$ Sheet1.Cells $(j+4,3)$ $+($ Sheet1.Cells $(4,12)+$ Sheet8.Cells $(G+2,3) *$ Sheet3.Cells $(12,13)) *$ Sheet1.Cells $(j+4,4)$ + (Sheet1.Cells $(11,12)+$ Sheet8.Cells $(G+2,3) *$ Sheet3.Cells $(19,13)) *$ Sheet1.Cells $(j+4,11))$

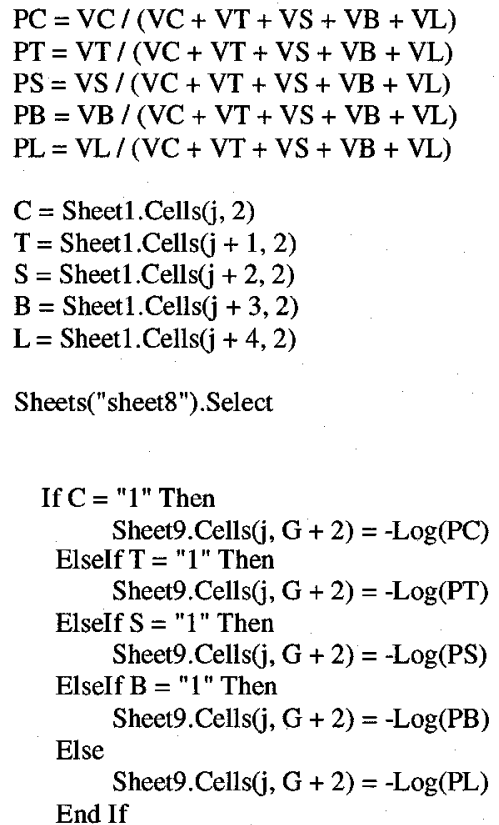

Next $\mathbf{j}$ 
Sheet8.Cells $(G+2,5)=$ Sheet9.Cells $(2703, G+2)$

Sheet8.Cells $(G+2,6)=$ Sheet8.Cells $(G+1,5)$

End If

$\mathrm{G}=\mathrm{G}+1$

GoTo line4

line5:

Adopting $\lambda_{t-G S D}$ obtained from golden section method

For $\mathrm{i}=11$ To 19

Sheet3.Cells $(\mathrm{i}, 14)=((\operatorname{Sheet8.Cells}(G+1,2)+\operatorname{Sheet8.Cells}(G+1,1)) / 2) *$ Sheet3.Cells $(\mathrm{i}, 13)$

Next i

Eriding or he subrouline or Golden Secion Method

Parameter estimates : $\beta_{t+1}=\beta_{t}+\delta_{t}=\beta_{t}+\lambda_{t-G S D} \Delta_{t}=\beta_{t}+\lambda_{t-G S D} W_{t} G_{t}=\beta_{t}+\lambda_{t-G S D}\left(-H_{t A P}^{B F G S-G S M}\right) G_{t}$

Sheet3.Cells $(11,16)=$ Sheet 1 Cells $(3,12)+$ Sheet3.Cells $(11,14)$

Sheet3.Cells $(12,16)=$ Sheet 1 Cells $(4,12)+$ Sheet $3 \cdot \operatorname{Cells}(12,14)$

Sheet3.Cells $(13,16)=$ Sheet1 $\operatorname{Cells}(5,12)+$ Sheet $3 \cdot \operatorname{Cells}(13,14)$

Sheet3.Cells $(14,16)=$ Sheet 1 .Cells $(6,12)+$ Sheet3.Cells $(14,14)$

Sheet 3 .Cells $(15,16)=$ Sheet 1 .Cells $(7,12)+$ Sheet $3 \cdot \operatorname{Cells}(15,14)$

Sheet3.Cells $(16,16)=$ Sheet1.Cells $(8,12)+$ Sheet3.Cells $(16,14)$

Sheet 3 .Cells $(17,16)=$ Sheet1.Cells $(9,12)+$ Sheet $3 \cdot \operatorname{Cells}(17,14)$

Sheet3.Cells $(18,16)=$ Sheet 1. Cells $(10,12)+$ Sheet 3. Cells $(18,14)$

Sheet3.Cells $(19,16)=$ Sheet 1 .Cells $(11,12)+$ Sheet 3. Cells $(19,14)$

Checking the Criterion for stopping: $\left[\frac{1}{k} \sum_{k=1}^{k}\left(\beta_{t+l, k}-\beta_{t, k}\right)^{2}\right]^{1 / 2}<C R$

Sheet 3. Cells $(21,12)=(($

$(\text { Sheet3.Cells }(11,16)-\text { Sheet1.Cells }(3,12))^{\wedge} 2+(\text { Sheet3.Cells }(12,16)-\text { Sheet1.Cells }(4,12))^{\wedge} 2$ $+(\text { Sheet3.Cells }(13,16)-\text { Sheet1.Cells }(5,12))^{\wedge} 2+(\text { Sheet3.Cells }(14,16)-\text { Sheet1.Cells }(6,12))^{\wedge} 2-$ $+(\text { Sheet3.Cells }(15,16)-\text { Sheet1.Cells }(7,12))^{\wedge} 2+(\text { Sheet3.Cells }(16,16)-\text { Sheet1.Cells }(8,12))^{\wedge} 2-$

$+(\text { Sheet3.Cells }(17,16)-\operatorname{Sheet} 1 . C e l l s(9,12))^{\wedge} 2+(\operatorname{Sheet3.Cells}(18,16)-\operatorname{Sheet1.Cells~}(10,12))^{\wedge} 2_{-}$

$+(\text { Sheet3.Cells }(19,16)-\text { Sheet1.Cells }(11,12))^{\wedge} 2$

could be changed $\rightarrow$

$$
)^{*}(1 / 9)\right)^{\wedge}(0.5)
$$

If Sheet3.Cells $(21,12)<10^{\wedge}(-4)$ Then

GoTo line1

Else

Starting the procedure for obtaining updating approximate hessan 
Calculating: $G_{t+1}$

For $\mathrm{i}=3$ To 2698 Step 5

Sheets("sheet2").Select

$\mathrm{vC}=\operatorname{Exp}($ Sheet3.Cells $(11,16) *$ Sheet 1.Cells(i, 3) + Sheet3.Cells $(12,16) *$ Sheet1.Cells $(i, 4)$

+ Sheet3.Cells $(13,16) *$ Sheet1.Cells(i, 5) + Sheet3.Cells(14, 16) * Sheet1.Cells(i, $\overline{6})+\operatorname{Sheet3.Cells(15,16)*}$ Sheet1.Cells(i, 7))

VT $=\operatorname{Exp}(\operatorname{Sheet} 3 . \operatorname{Cells}(11,16) *$ Sheet 1. Cells $(i+1,3)+\operatorname{Sheet3.Cells}(12,16) *$ Sheet1.Cells $(i+1,4)+\operatorname{Sheet} 3 . \operatorname{Cells}(16,16)$ * Sheet1.Cells $(i+1,8))$

VS $=$ Exp(Sheet3.Cells $(11,16) *$ Sheet1.Cells $(i+2,3)+\operatorname{Sheet3.Cells~}(12,16) *$ Sheet1.Cells $(i+2,4)+\operatorname{Sheet3.Cells~}(17,16)$ * Sheet1.Cells $(i+2,9))$

$\mathrm{VB}=\operatorname{Exp}($ Sheet3.Cells $(11,16) *$ Sheet1.Cells $(\mathrm{i}+3,3)+\operatorname{Sheet3.Cells}(12,16) *$ Sheet $1 . C e l l s(i+3,4)+\operatorname{Sheet} 3 . C e l l s(18,16)$ * Sheet1.Cells $(i+3,10))$

$\mathrm{VL}=\operatorname{Exp}(\operatorname{Sheet} 3 \cdot \operatorname{Cells}(11,16) *$ Sheet 1.Cells $(\mathrm{i}+4,3)+\operatorname{Sheet} 3 . \operatorname{Cells}(12,16) * \operatorname{Sheet} 1 . \operatorname{Cells}(\mathrm{i}+4,4)+\operatorname{Sheet} 3$. Cells $(19,16)$

* Sheet1.Cells $(\mathbf{i}+\mathbf{4}, 11))$

$\mathrm{PC}=\mathrm{VC} /(\mathrm{VC}+\mathrm{VT}+\mathrm{VS}+\mathrm{VB}+\mathrm{VL})$

$\mathrm{PT}=\mathrm{VT} /(\mathrm{VC}+\mathrm{VT}+\mathrm{VS}+\mathrm{VB}+\mathrm{VL})$

$\mathrm{PS}=\mathrm{VS} /(\mathrm{VC}+\mathrm{VT}+\mathrm{VS}+\mathrm{VB}+\mathrm{VL})$

$\mathrm{PB}=\mathrm{VB} /(\mathrm{VC}+\mathrm{VT}+\mathrm{VS}+\mathrm{VB}+\mathrm{VL})$

$\mathrm{PL}=\mathrm{VL} /(\mathrm{VC}+\mathrm{VT}+\mathrm{VS}+\mathrm{VB}+\mathrm{VL})$

$\mathrm{C}=$ Sheet 1. Cells $(\mathrm{i}, 2)$

$\mathrm{T}=$ Sheet1.Cells $(\mathrm{i}+1,2)$

$\mathrm{S}=$ Sheet1.Cells $(\mathrm{i}+2,2)$

$\mathrm{B}=$ Sheet1.Cells $(\mathrm{i}+3,2)$

$\mathrm{L}=$ Sheet1.Cells $(\mathrm{i}+4,2)$

First-order Derivative with respect to $\beta_{1}$

If $\mathrm{C}=$ " 1 " Then

Sheet2.Cells $(\mathrm{i}, 12)=($ Sheet1.Cells $(\mathrm{i}, 3)-$ Sheet 1.Cells $(\mathrm{i}+1,3)) * \mathrm{PT}$

$+($ Sheet1.Cells $(i, 3)-$ Sheet1.Cells $(i+2,3)) *$ PS

$+($ Sheet1.Cells $(\mathrm{i}, 3)-$ Sheet1.Cells $(\mathrm{i}+3,3)) * \mathrm{~PB}_{-}$

$+($ Sheet1.Cells $(i, 3)-$ Sheet1.Cells $(i+4,3)) * P L$

Elself $T=$ " 1 " Then

Sheet2.Cells $(\mathrm{i}, 12)=($ Sheet 1.Cells $(\mathrm{i}+1,3)-$ Sheet1.Cells $(\mathrm{i}, 3)) * \mathrm{PC}$

$+($ Sheet 1.Cells $(\mathrm{i}+1,3)$ - Sheet 1.Cells $(\mathrm{i}+2,3)) *$ PS

$+($ Sheet1.Cells $(\mathrm{i}+1,3)-$ Sheet1.Cells $(\mathrm{i}+3,3)) * \mathrm{~PB}$

$+($ Sheet1.Cells $(\mathrm{i}+1,3)-$ Sheet1.Cells $(\mathrm{i}+4,3)) * \mathrm{PL}$

Elself $S=$ "1" Then

Sheet2.Cells $(\mathbf{i}, 12)=($ Sheet1.Cells $(\mathrm{i}+2,3)-$ Sheet1.Cells $(\mathrm{i}, 3)) * \mathrm{PC}$

$+($ Sheet 1.Cells $(\mathrm{i}+2,3)-$ Sheet 1. Cells $(\mathrm{i}+1,3)) * \mathrm{PT}$ $+($ Sheet 1.Cells $(\mathrm{i}+2,3)-$ Sheet1.Cells $(\mathrm{i}+3,3)) * \mathrm{~PB}$

$+($ Sheet1.Cells $(\mathrm{i}+2,3)-$ Sheet1.Cells $(\mathrm{i}+4,3)) *$ PL

ElseIf $\mathbf{B}=$ "1" Then

Sheet2.Cells $(\mathrm{i}, 12)=($ Sheet1.Cells $(\mathrm{i}+3,3)-$ Sheet1.Cells $(\mathrm{i}, 3)) * \mathrm{PC}$

$+($ Sheet1.Cells $(\mathrm{i}+3,3)-$ Sheet1.Cells $(\mathrm{i}+1,3)) * \mathrm{PT}$

$+($ Sheet1.Cells $(i+3,3)$ - Sheet1.Cells $(i+2,3)) * \mathrm{PS}_{-}^{-}$

$+($ Sheet 1.Cells $(\mathrm{i}+3,3)-$ Sheet1.Cells $(\mathrm{i}+4,3)) * \mathrm{PL}$

Else

Sheet2.Cells $(\mathrm{i}, 12)=($ Sheet 1.Cells $(\mathrm{i}+4,3)-$ Sheet1.Cells $(\mathrm{i}, 3)) * \mathrm{PC}$

$+($ Sheet1.Cells $(\mathrm{i}+4,3)-$ Sheet1.Cells $(\mathrm{i}+\overline{1}, 3)) * \mathrm{PT}$

$+($ Sheet1.Cells $(\mathrm{i}+4,3)-$ Sheet1.Cells $(\mathrm{i}+2,3)) * \mathrm{PS}_{-}$ 
End If

First-order Derivative with respect to $\beta_{2}$

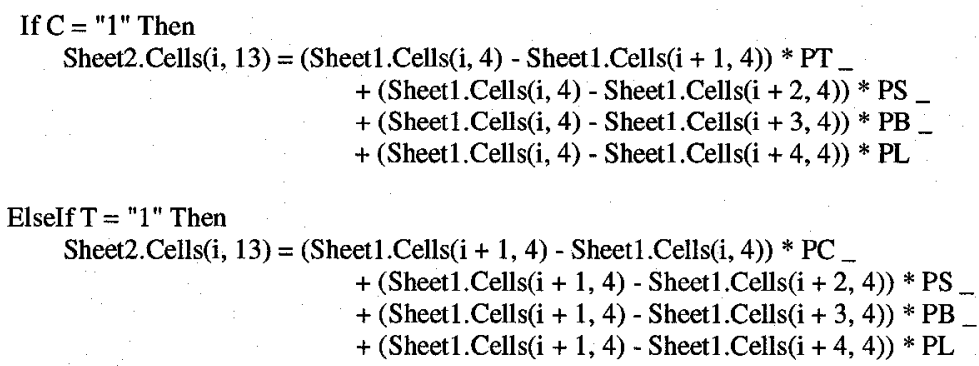

Else

Sheet2.Cells $(\mathrm{i}, 13)=($ Sheet1.Cells $(\mathrm{i}+4,4)-$ Sheet1.Cells $(\mathrm{i}, 4)) * \mathrm{PC}$ $+($ Sheet 1.Cells $(i+4,4)-$ Sheet1.Cells $(i+\overline{1}, 4)) * P T$ $+($ Sheet1.Cells $(i+4,4)-$ Sheet1.Cells $(i+2,4)) *$ PS $+($ Sheet1.Cells $(\mathrm{i}+4,4)-$ Sheet1.Cells $(\mathrm{i}+3,4)) *$ PB

End If

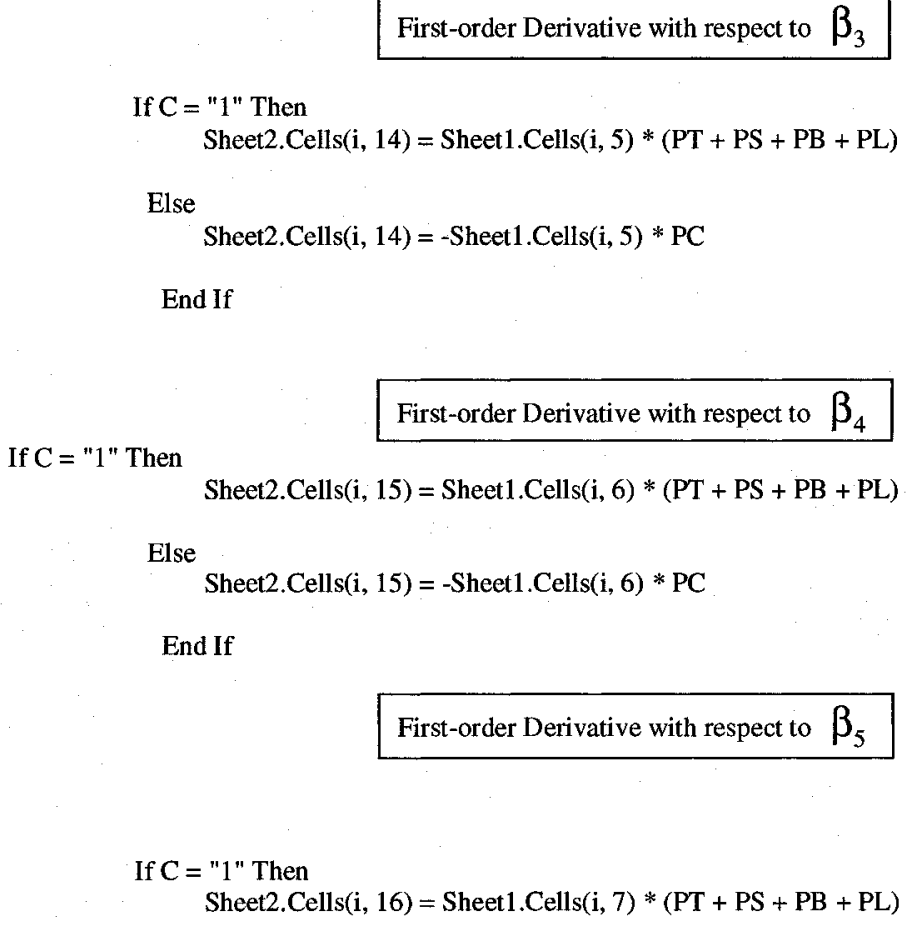

End If

If $\mathrm{C}=$ " 1 " Then

First-order Derivative with respect to $\beta_{4}$

Sheet2.Cells $(\mathbf{i}, 15)=$ Sheet1.Cells $(i, 6) *(P T+P S+P B+P L)$

Else

Sheet2.Cells $(\mathrm{i}, 15)=-$ Sheet1.Cells $(\mathrm{i}, 6) * \mathrm{PC}$

End If

First-order Derivative with respect to $\beta_{5}$

If $\mathrm{C}=$ "1" Then

Sheet2.Cells $(i, 16)=$ Sheet1.Cells $(i, 7) *(P T+P S+P B+P L)$ 
Else

Sheet2.Cells $(\mathrm{i}, 16)=-$ Sheet1.Cells $(\mathrm{i}, 7) * \mathrm{PC}$

End If

First-order Derivative with respect to $\beta_{6}$

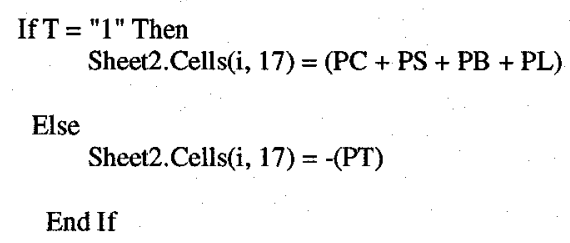

End If

First-order Derivative with respect to $\beta_{7}$

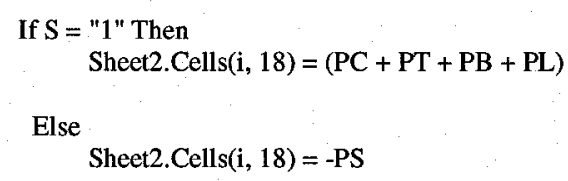


Obtaining gradient: $G_{t+1}$

For $\mathrm{i}=1$ To 9 Step 1

Sheet3.Cells $(i, 11)=$ Sheet2.Cells $(2703, i+11)$

Next i

Calculating gamma: $\gamma_{t}=G_{t+1}-G_{t}$

Sheet3.Cells $(11,18)=$ Sheet $3 \cdot \operatorname{Cells}(1,11)-$ Sheet3.Cells $(11,11)$

Sheet3.Cells $(12,18)=$ Sheet $3 . \operatorname{Cell} s(2,11)-$ Sheet3.Cells $(12,11)$

Sheet3.Cells $(13,18)=$ Sheet3.Cells $(3,11)-$ Sheet3.Cells $(13,11)$

Sheet3.Cells $(14,18)=$ Sheet 3. Cells $(4,11)-$ Sheet3.Cells $(14,11)$

Sheet3.Cells $(15,18)=$ Sheet3.Cells $(5,11)-$ Sheet3.Cells $(15,11)$

Sheet3.Cells $(16,18)=$ Sheet3.Cells $(6,11)-$ Sheet3.Cells $(16,11)$

Sheet3.Cells $(17,18)=$ Sheet3.Cells $(7,11)-$ Sheet3.Cells $(17,11)$

Sheet3.Cells $(18,18)=$ Sheet3.Cells $(8,11)-$ Sheet3.Cells $(18,11)$

Sheet3.Cells $(19,18)=$ Sheet3.Cells $(9,11)-$ Sheet3.Cells $(19,11)$

Calculating approximate hessian: $H_{t+1}^{B F G S-G S M}=H_{t}^{B F G S-G S M}+\frac{\delta_{t} \delta_{t}^{\prime}}{\delta_{t}^{\prime} \gamma_{t}}-\frac{H_{t}^{B F G S-G S M} \gamma_{t} \gamma_{t}^{\prime} H_{t}^{B F G S-G S M}}{\gamma_{t}^{\prime} H_{t}^{B F G-G S M} \gamma_{t}}$

Transpose matrix of a vector of gamma: $\gamma_{t}$

Sheet 3. Cells $(28,11)=$ Sheet 3 .Cells $(11,18)$

Sheet 3 .Cells $(28,12)=$ Sheet 3 .Cells $(12,18)$

Sheet 3. Cells $(28,13)=$ Sheet 3. Cells $(13,18)$

Sheet 3 .Cells $(28,14)=$ Sheet 3. Cells $(14,18)$

Sheet 3 .Cells $(28,15)=$ Sheet $3 \cdot$ Cells $(15,18)$

Sheet3.Cells $(28,16)=$ Sheet 3 .Cells $(16,18)$

Sheet 3 .Cells $(28,17)=$ Sheet 3. Cells $(17,18)$

Sheet 3 .Cells $(28,18)=$ Sheet 3 .Cells $(18,18)$

Sheet3.Cells $(28,19)=$ Sheet 3. Cells $(19,18)$

Transpose matrix of a vector of delta: $\delta_{t}$

Sheet 3 .Cells $(30,11)=$ Sheet 3. Cells $(11,14)$

Sheet3.Cells $(30,12)=$ Sheet 3 .Cells $(12,14)$

Sheet3.Cells $(30,13)=$ Sheet 3. Cells $(13,14)$

Sheet 3 .Cells $(30,14)=$ Sheet 3 .Cells $(14,14)$

Sheet3.Cells $(30,15)=$ Sheet 3 .Cells $(15,14)$

Sheet3.Cells $(30,16)=$ Sheet 3. Cells $(16,14)$

Sheet 3. Cells $(30,17)=$ Sheet 3 .Cells $(17,14)$

Sheet 3. Cells $(30,18)=$ Sheet 3 .Cells $(18,14)$

Sheet 3. Cells $(30,19)=$ Sheet 3. Cells $(19,14)$ 


\section{$\delta_{t} \delta_{t}^{\prime}$}

Sheet3.Range("K33:S41").FormulaArray = "=MMULT(N11:N19,K30:S30)".

$$
\delta_{t}^{\prime} \gamma_{t}
$$

Sheet3.Range("L43").FormulaArray = "=MMULT(K30:S30,R11:R19)"

$$
\delta_{t} \delta_{t}^{\prime} / \delta_{t}^{\prime} \gamma_{t}
$$

For $\mathrm{i}=33$ To 41 Step 1

For $\mathbf{j}=11$ To 19 Step 1 Next $\mathbf{j}$

Sheet3.Cells $(i, j+10)=$ Sheet3.Cells $(i, j) /$ Sheet3.Cells $(43,12)$

Next $\mathrm{i}$

$$
H_{t}^{B F G S-G S M} \gamma_{t}
$$

Sheet3.Range("K45:K53").FormulaArray = "=MMULT(A1:I9,R11:R19)"

$$
H_{t}^{B F G S-G S M} \gamma_{t} \gamma_{t}^{\prime}
$$

Sheet3.Range("M45:U53").FormulaArray = "=MMULT(K45:K53,K28:S28)"

$$
H_{t}^{B F G S-G S M} \gamma_{t} \gamma_{t}^{\prime} H_{t}^{B F G S-G S M}
$$

Sheet3.Range("K55:S63").FormulaArray = "=MMULT(M45:U53,A1:I9)"

$$
\gamma_{t}^{\prime} H_{t}^{B F G S-G S M}
$$

Sheet3.Range("K66:S66").FormulaArray = "=MMULT(K28:S28,A1:I9)"

$$
\gamma_{t}^{\prime} H_{t}^{B F G S-G S M} \gamma_{t}
$$


Sheet3.Range("L67").FormulaArray = "=MMULT(K66:S66,R11:R19) "

$$
\frac{H_{t}^{B F G S-G S M} \gamma_{t} \gamma_{t}^{\prime} H_{t}^{B F G S-G S M}}{\gamma_{t}^{\prime} H_{t}^{B F G S-G S M} \gamma_{t}}
$$

For $\mathrm{i}=55$ To 63 Step 1

For $\mathrm{j}=11$ To 19 Step 1

Next $j$ Sheet3.Cells $(i, j+10)=$ Sheet3.Cells $(i, j) /$ Sheet3.Cells $(67,12)$

Next $\mathrm{i}$

$H_{t}^{B F G S-G S M}+\frac{\delta_{t} \delta_{t}^{\prime}}{\delta_{t}^{\prime} \gamma_{t}}-\frac{H_{t}^{B F G S-G S M} \gamma_{t} \gamma_{t}^{\prime} H_{t}^{B F G S-G S M}}{\gamma_{t}^{\prime} H_{t}^{B F G S-G S M} \gamma_{t}}$

For $\mathrm{i}=72$ To 80 Step 1

For $\mathrm{j}=11$ To 19 Step 1 Next $j$

Sheet3.Cells $(i, j)=$ Sheet3.Cells $(i-71, j-10)+$ Sheet3.Cells $(i-39, j+10)-\operatorname{Sheet} 3 . C e l l s(i-17, j+10)$

Next i

Additional procedure for conducting BFGS method

$$
\left(\frac{1}{\delta_{t}^{\prime} \gamma_{t}}\right) \delta_{t}
$$

For $\mathrm{i}=11$ To 19 Step 1

Sheet3.Cells $(i, 20)=$ Sheet 3. Cells $(i, 14) /$ Sheet3.Cells $(43,12)$

Next i

$$
\left(\frac{1}{\gamma_{t} H_{t}^{B F G S-G S M} \gamma_{t}}\right) H_{t}^{B F G S-G S M} \gamma_{t}
$$

For $\mathbf{i}=11$ To 19 Step 1

Sheet3.Cells $(\mathbf{i}, 22)=$ Sheet $3 . \operatorname{Cell} s(\mathrm{i}+34,11) /$ Sheet $3 . \operatorname{Cells}(67,12)$

Next i 


$$
d_{t}=\left(\frac{1}{\delta_{t}^{\prime} \gamma_{t}}\right) \delta_{t}-\left(\frac{1}{\gamma_{t}^{\prime} H_{t}^{B F G S-G S M} \gamma_{t}}\right) H_{t}^{B F G S-G S M} \gamma_{t}
$$

For $\mathbf{i}=11$ To 19 Step 1

Sheet3.Cells(i, 24) = Sheet3.Cells $(i, 20)-$ Sheet3.Cells $(i, 22)$

Next i

$d_{t}^{\prime}$

Sheet3.Cells $(84,11)=$ Sheet 3 .Cells $(11,24)$

Sheet $3 \cdot$ Cells $(84,12)=$ Sheet $3 \cdot$ Cells $(12,24)$

Sheet3.Cells $(84,13)=$ Sheet 3 .Cells $(13,24)$

Sheet3.Cells $(84,14)=$ Sheet 3. Cells $(14,24)$

Sheet3.Cells $(84,15)=$ Sheet 3 .Cells $(15,24)$

Sheet3.Cells $(84,16)=$ Sheet 3 .Cells $(16,24)$

Sheet3.Cells $(84,17)=$ Sheet3.Cells $(17,24)$

Sheet3.Cells $(84,18)=$ Sheet 3. Cells $(18,24)$

Sheet3.Cells $(84,19)=$ Sheet3.Cells $(19,24)$

For $\mathbf{j}=11$ To 19 Step 1

For $\mathrm{i}=88$ To 96 Step 1

Sheet3.Cells(i, j) $=$ Sheet3.Cells $(i-77,24) *$ Sheet3.Cells $(84, j)$ Next i

Next $\mathbf{j}$

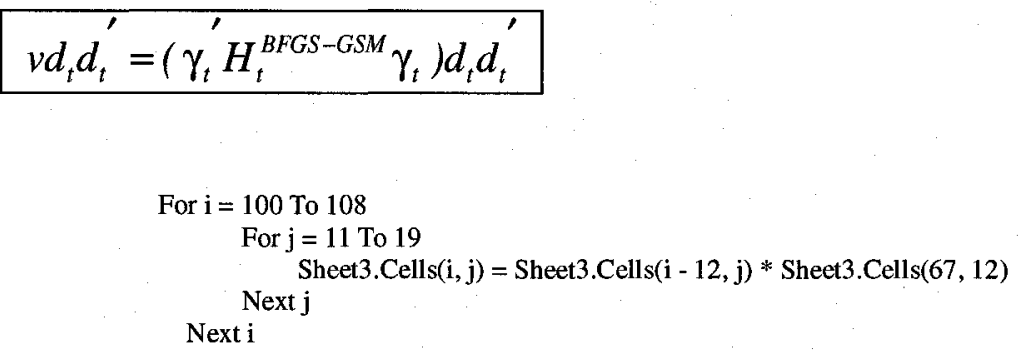

$\left(H_{B F G S}+\frac{\delta_{t} \delta_{t}^{\prime}}{\delta_{t}^{\prime} \gamma_{t}}-\frac{H_{B F G S} \gamma_{t} \gamma_{t}^{\prime} H_{B F G S}}{\gamma_{t}^{\prime} H_{B F G S} \gamma_{t}}\right)+v d_{t} d_{t}^{\prime}$

For $\mathrm{i}=111$ To 119 Step 1

For $\mathbf{j}=11$ To 19 Step 1 
Next $j$

Sheet3.Cells $(i, j)=$ Sheet3.Cells $(i-39, j)+$ Sheet3.Cells $(i-11, j)$

Next i

End of Additional procedure for conducting BFGS method

Obtaining updated approximate hessain

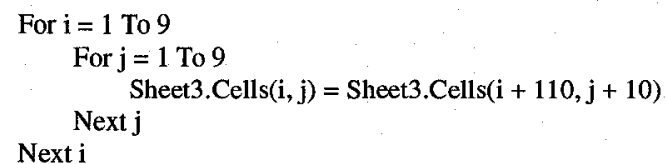

GoTo line2

End If

line1:

End Sub 
Appendix C: Data Used in Experimental Estimation (540 obsevations) 
C.1: Car alternative - 247 observations

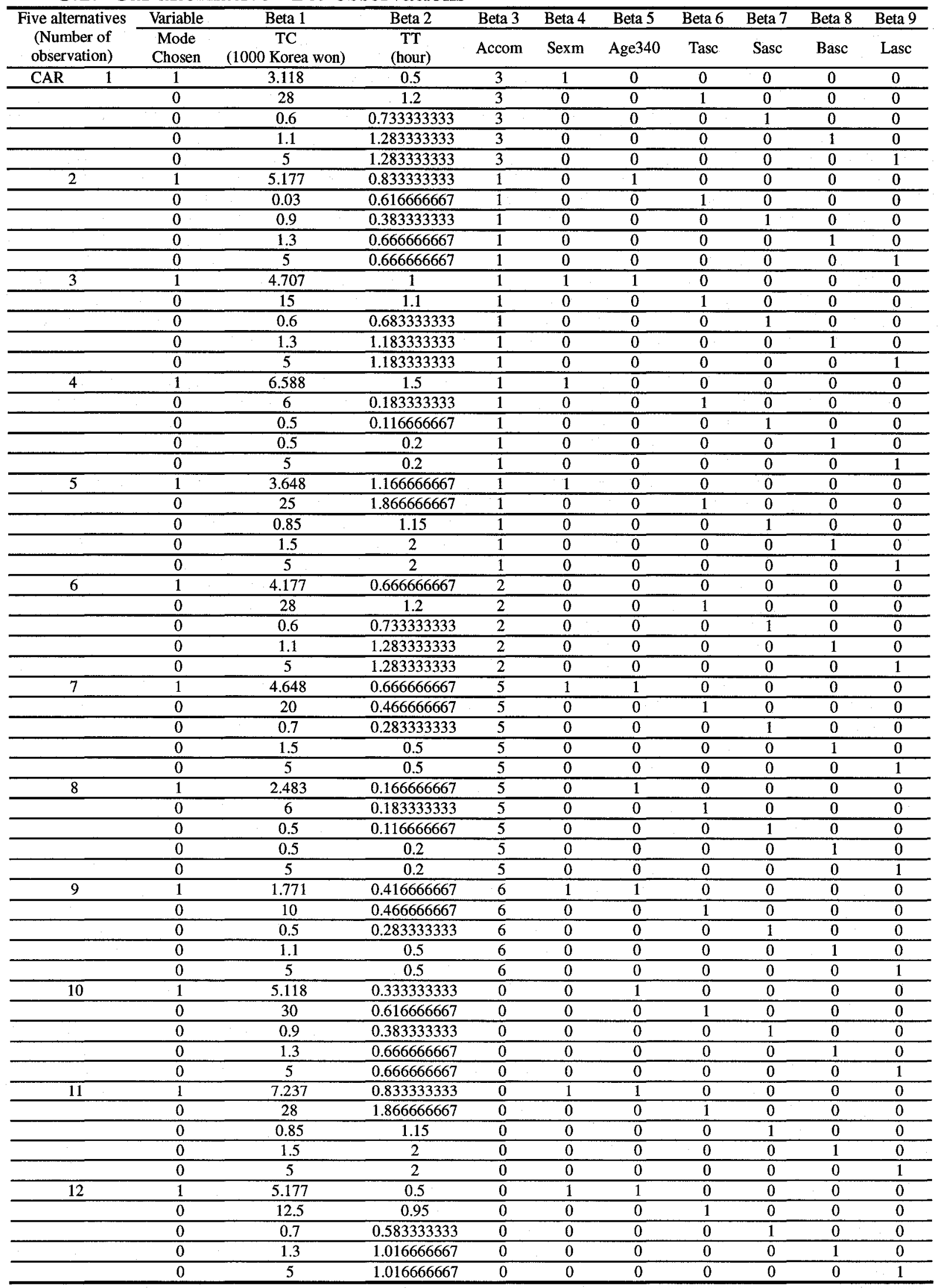




\begin{tabular}{|c|c|c|c|c|c|c|c|c|c|c|}
\hline \multirow{2}{*}{$\begin{array}{l}\text { Five alternatives } \\
\text { (Number of } \\
\text { observation) }\end{array}$} & Variable & Beta 1 & Beta 2 & Beta 3 & Beta 4 & Beta 5 & Beta 6 & Beta 7 & Beta 8 & Beta 9 \\
\hline & $\begin{array}{c}\text { Mode } \\
\text { Chosen }\end{array}$ & $\begin{array}{c}\text { TC } \\
\text { (1000 Korea won) } \\
\end{array}$ & $\begin{array}{c}\text { TT } \\
\text { (hour) }\end{array}$ & Accom & Sexm & Age340 & Tasc & Sasc & Basc & Lasc \\
\hline \multirow[t]{5}{*}{13} & 1 & 2.618 & 0.25 & 0 & 1 & 1 & 0 & 0 & 0 & 0 \\
\hline & 0 & 15 & 0.8166666667 & 0 & 0 & 0 & 1 & 0 & 0 & 0 \\
\hline & 0 & 0.8 & 0.5 & 0 & 0 & 0 & 0 & 1 & 0 & 0 \\
\hline & 0 & 1.4 & 0.8666666667 & 0 & 0 & 0 & 0 & 0 & 1 & 0 \\
\hline & 0 & 5 & 0.8666666667 & 0 & 0 & 0 & 0 & 0 & 0 & 1 \\
\hline \multirow[t]{5}{*}{14} & 1 & 5.188 & 0.333333333 & 1 & 1 & 1 & 0 & 0 & 0 & 0 \\
\hline & 0 & 10 & 0.5166666667 & 1 & 0 & 0 & 1 & 0 & 0 & 0 \\
\hline & 0 & 0.5 & 0.3166666667 & 1 & 0 & 0 & 0 & 1 & 0 & 0 \\
\hline & 0 & 1.1 & 0.5666666667 & 1 & 0 & 0 & 0 & 0 & 1 & 0 \\
\hline & 0 & 5 & 0.5666666667 & 1 & 0 & 0 & 0 & 0 & 0 & 1 \\
\hline \multirow[t]{5}{*}{15} & 1 & 75.177 & 1 & 0 & 1 & 1 & 0 & 0 & 0 & 0 \\
\hline & 0 & 30 & 0.6166666667 & 0 & 0 & 0 & 1 & 0 & 0 & 0 \\
\hline & 0 & 0.9 & 0.383333333 & 0 & 0 & 0 & 0 & 1 & 0 & 0 \\
\hline & 0 & 1.3 & 0.6666666667 & 0 & 0 & 0 & 0 & 0 & 1 & 0 \\
\hline & 0 & 5 & 0.666666667 & 1 & 0 & 0 & 0 & 0 & 0 & 1 \\
\hline \multirow[t]{5}{*}{16} & 1 & 5.707 & 0.75 & 3 & 1 & 0 & 0 & 0 & 0 & 0 \\
\hline & 0 & 20 & 1.433333333 & 3 & 0 & 0 & 1 & 0 & 0 & 0 \\
\hline & 0 & 0.6 & 0.883333333 & 3 & 0 & 0 & 0 & 1 & 0 & 0 \\
\hline & 0 & 1.6 & 1.533333333 & 3 & 0 & 0 & 0 & 0 & 1 & 0 \\
\hline & 0 & 5 & 1.533333333 & 3 & 0 & 0 & 0 & 0 & 0 & 1 \\
\hline \multirow[t]{5}{*}{17} & 1 & 4.118 & 0.333333333 & 0 & 1 & 1 & 0 & 0 & 0 & 0 \\
\hline & 0 & 6 & 0.183333333 & 0 & 0 & 0 & 1 & 0 & 0 & 0 \\
\hline & 0 & 0.5 & 0.116666667 & 0 & 0 & 0 & 0 & 1 & 0 & 0 \\
\hline & 0 & 0.5 & 0.2 & 0 & 0 & 0 & 0 & 0 & 1 & 0 \\
\hline & 0 & 5 & 0.2 & 0 & 0 & 0 & 0 & 0 & 0 & 1 \\
\hline \multirow[t]{5}{*}{18} & 1 & 76.51 & 0.6666666667 & 0 & 1 & 0 & 0 & 0 & 0 & 0 \\
\hline & 0 & 15 & 0.8166666667 & 0 & 0 & 0 & 1 & 0 & 0 & 0 \\
\hline & 0 & 0.6 & 0.5 & 0 & 0 & 0 & 0 & 1 & 0 & 0 \\
\hline & 0 & 1.5 & 0.8666666667 & 0 & 0 & 0 & 0 & 0 & 1 & 0 \\
\hline & 0 & 5 & 0.8666666667 & 0 & 0 & 0 & 0 & 0 & 0 & 1 \\
\hline \multirow[t]{5}{*}{19} & 1 & 4.648 & 1 & 1 & 0 & 0 & 0 & 0 & 0 & 0 \\
\hline & 0 & 20 & 0.4666666667 & 1 & 0 & 0 & 1 & 0 & 0 & 0 \\
\hline & 0 & 0.7 & 0.2833333333 & 1 & 0 & 0 & 0 & 1 & 0 & 0 \\
\hline & 0 & 1.5 & 0.5 & 1 & 0 & 0 & 0 & 0 & 1 & 0 \\
\hline & 0 & 5 & 0.5 & 1 & 0 & 0 & 0 & 0 & 0 & 1 \\
\hline \multirow[t]{5}{*}{20} & 1 & 6.978 & 0.666666667 & 4 & 1 & 0 & 0 & 0 & 0 & 0 \\
\hline & 0 & 10.618 & 1.333333333 & 4 & 0 & 0 & 1 & 0 & 0 & 0 \\
\hline & 0 & 0.8 & 0.833333333 & 4 & 0 & 0 & 0 & 1 & 0 & 0 \\
\hline & 0 & 1.3 & 1.433333333 & 4 & 0 & 0 & 0 & 0 & 1 & 0 \\
\hline & 0 & 5 & 1.433333333 & 4 & 0 & 0 & 0 & 0 & 0 & 1 \\
\hline \multirow[t]{5}{*}{21} & 1 & 6.377 & 0.3333333333 & 0 & 1 & 1 & 0 & 0 & 0 & 0 \\
\hline & 0 & 10 & 0.516666667 & 0 & 0 & 0 & 1 & 0 & 0 & 0 \\
\hline & 0 & 0.5 & 0.316666667 & 0 & 0 & 0 & 0 & 1 & 0 & 0 \\
\hline & 0 & 1.1 & 0.566666667 & 0 & 0 & 0 & 0 & 0 & 1 & 0 \\
\hline & 0 & 5 & 0.566666667 & 0 & 0 & 0 & 0 & 0 & 0 & 1 \\
\hline \multirow[t]{5}{*}{22} & 1 & 5.118 & 0.666666667 & 10 & 1 & 1 & 0 & 0 & 0 & 0 \\
\hline & 0 & 7 & 0.566666667 & 10 & 0 & 0 & 1 & 0 & 0 & 0 \\
\hline & 0 & 0.6 & 0.35 & 10 & 0 & 0 & 0 & 1 & 0 & 0 \\
\hline & 0 & 1.3 & 0.616666667 & 10 & 0 & 0 & 0 & 0 & 1 & 0 \\
\hline & 0 & 5 & 0.616666667 & 10 & 0 & 0 & 0 & 0 & 0 & 1 \\
\hline \multirow[t]{5}{*}{23} & 1 & 3.588 & 0.5 & 0 & 1 & 0 & 0 & 0 & 0 & 0 \\
\hline & 0 & 15 & 0.816666667 & 0 & 0 & 0 & 1 & 0 & 0 & 0 \\
\hline & 0 & 0.6 & 0.5 & 0 & 0 & 0 & 0 & 1 & 0 & 0 \\
\hline & 0 & 1.5 & 0.8666666667 & 0 & 0 & 0 & 0 & 0 & 1 & 0 \\
\hline & 0 & 5 & 0.8666666667 & 0 & 0 & 0 & 0 & 0 & 0 & 1 \\
\hline \multirow[t]{5}{*}{24} & 1 & 5.237 & 1 & 1 & 1 & 1 & 0 & 0 & 0 & 0 \\
\hline & 0 & 28 & 1.2 & 1 & 0 & 0 & 1 & 0 & 0 & 0 \\
\hline & 0 & 0.6 & 0.733333333 & 1 & 0 & 0 & 0 & 1 & 0 & 0 \\
\hline & 0 & 1.1 & 1.283333333 & 1 & 0 & 0 & 0 & 0 & 1 & 0 \\
\hline & 0 & 5 & 1.283333333 & 1 & 0 & 0 & 0 & 0 & 0 & 1 \\
\hline
\end{tabular}




\begin{tabular}{|c|c|c|c|c|c|c|c|c|c|c|}
\hline \multirow{2}{*}{$\begin{array}{c}\text { Five alternatives } \\
\text { (Number of } \\
\text { observation) }\end{array}$} & Variable & Beta 1 & Beta 2 & Beta 3 & Beta 4 & Beta 5 & Beta 6 & Beta 7 & Beta 8 & Beta 9 \\
\hline & $\begin{array}{l}\text { Mode } \\
\text { Chosen }\end{array}$ & $\begin{array}{c}\text { TC } \\
(1000 \text { Korea won) }\end{array}$ & $\begin{array}{c}\mathrm{TT} \\
\text { (hour) }\end{array}$ & Accom & Sexm & Age340 & Tasc & Sasc & Basc & Lasc \\
\hline \multirow[t]{5}{*}{25} & 1 & 16.936 & 2.5 & 0 & 1 & 1 & 0 & 0 & 0 & 0 \\
\hline & 0 & 53 & 2.0666666667 & 0 & 0 & 0 & 1 & 0 & 0 & 0 \\
\hline & 0 & 1 & 1.283333333 & 0 & 0 & 0 & 0 & 1 & 0 & 0 \\
\hline & 0 & 7.5 & 2.2 & 0 & 0 & 0 & 0 & 0 & 1 & 0 \\
\hline & 0 & 15 & 2.2 & 0 & 0 & 0 & 0 & 0 & 0 & 1 \\
\hline \multirow{5}{*}{26} & 1 & 2.271 & 1.5 & 5 & 1 & 0 & 0 & 0 & 0 & 0 \\
\hline & 0 & 8.468 & 1.05 & 5 & 0 & 0 & 1 & 0 & 0 & 0 \\
\hline & 0 & 1 & 0.65 & 5 & 0 & 0 & 0 & 1 & 0 & 0 \\
\hline & 0 & 1.3 & 1.133333333 & 5 & 0 & 0 & 0 & 0 & 1 & 0 \\
\hline & 0 & 5 & 1.133333333 & 5 & 0 & 0 & 0 & 0 & 0 & 1 \\
\hline \multirow[t]{5}{*}{27} & 1 & 2.618 & 0.5 & 1 & 1 & 0 & 0 & 0 & 0 & 0 \\
\hline & 0 & 15 & 0.8166666667 & 1 & 0 & 0 & 1 & 0 & 0 & 0 \\
\hline & 0 & 0.6 & 0.5 & 1 & 0 & 0 & 0 & 1 & 0 & 0 \\
\hline & 0 & 1.5 & 0.8666666667 & 1 & 0 & 0 & 0 & 0 & 1 & 0 \\
\hline & 0 & 5 & 0.8666666667 & 1 & 0 & 0 & 0 & 0 & 0 & 1 \\
\hline \multirow[t]{5}{*}{28} & 1 & 4.707 & 1 & 0 & 1 & 1 & 0 & 0 & 0 & 0 \\
\hline & 0 & 15 & 1.1 & 0 & 0 & 0 & 1 & 0 & 0 & 0 \\
\hline & 0 & 0.6 & 0.683333333 & 0 & 0 & 0 & 0 & 1 & 0 & 0 \\
\hline & 0 & 1.3 & 1.183333333 & 0 & 0 & 0 & 0 & 0 & 1 & 0 \\
\hline & 0 & 5 & 1.183333333 & 0 & 0 & 0 & 0 & 0 & 0 & 1 \\
\hline \multirow[t]{5}{*}{29.} & 1 & 8.415 & 1.6666666667 & 0 & 1 & 1 & 0 & 0 & 0 & 0 \\
\hline & 0 & 20 & 0.4666666667 & 0 & 0 & 0 & 1 & 0 & 0 & 0 \\
\hline & 0 & 0.7 & 0.2833333333 & 0 & 0 & 0 & 0 & 1 & 0 & 0 \\
\hline & 0 & 1.5 & 0.5 & 0 & 0 & 0 & 0 & 0 & 1 & 0 \\
\hline & 0 & 5 & 0.5 & 0 & 0 & 0 & 0 & 0 & 0 & 1 \\
\hline \multirow[t]{5}{*}{30} & 1 & 7.915 & 0.5 & 1 & 0 & 1 & 0 & 0 & 0 & 0 \\
\hline & 0 & 10 & 0.9 & 1 & 0 & 0 & 1 & 0 & 0 & 0 \\
\hline & 0 & 0.6 & 0.5666666667 & 1 & 0 & 0 & 0 & 1 & 0 & 0 \\
\hline & 0 & 1.3 & 0.9666666667 & 1 & 0 & 0 & 0 & 0 & 1 & 0 \\
\hline & 0 & 5 & 0.9666666667 & 1 & 0 & 0 & 0 & 0 & 0 & 1 \\
\hline \multirow[t]{5}{*}{31} & 1 & 5.177 & 0.6666666667 & 3 & 1 & 0 & 0 & 0 & 0 & 0 \\
\hline & 0 & 28 & 1.2 & 3 & 0 & 0 & 1 & 0 & 0 & 0 \\
\hline & 0 & 0.6 & 0.733333333 & 3 & 0 & 0 & 0 & 1 & 0 & 0 \\
\hline & 0 & 1.1 & 1.283333333 & 3 & 0 & 0 & 0 & 0 & 1 & 0 \\
\hline & 0 & 5 & 1.283333333 & 3 & 0 & 0 & 0 & 0 & 0 & 1 \\
\hline \multirow[t]{5}{*}{32} & 1 & 10.211 & 0.083333333 & 1 & 0 & 1 & 0 & 0 & 0 & 0 \\
\hline & 0 & 6 & 0.183333333 & 1 & 0 & 0 & 1 & 0 & 0 & 0 \\
\hline & 0 & 0.5 & 0.116666667 & 1 & 0 & 0 & 0 & 1 & 0 & 0 \\
\hline & 0 & 0.5 & 0.2 & 1 & 0 & 0 & 0 & 0 & 1 & 0 \\
\hline & 0 & 5 & 0.2 & 1 & 0 & 0 & 0 & 0 & 0 & 1 \\
\hline \multirow[t]{5}{*}{33} & 1 & 4.118 & 0.6666666667 & 1 & 1 & 0 & 0 & 0 & 0 & 0 \\
\hline & 0 & 12.052 & 1.533333333 & 1 & 0 & 0 & 1 & 0 & 0 & 0 \\
\hline & 0 & 0.6 & 0.95 & 1 & 0 & 0 & 0 & 1 & 0 & 0 \\
\hline & 0 & 5 & 1.633333333 & 1 & 0 & 0 & 0 & 0 & 1 & 0 \\
\hline & 0 & 5 & 1.633333333 & 1 & 0 & 0 & 0 & 0 & 0 & 1 \\
\hline \multirow[t]{5}{*}{34} & 1 & 8.415 & 0.6666666667 & 2 & 1 & 0 & 0 & 0 & 0 & 0 \\
\hline & 0 & 28 & 1.2 & 2 & 0 & 0 & 1 & 0 & 0 & 0 \\
\hline & 0 & 0.6 & 0.733333333 & 2 & 0 & 0 & 0 & 1 & 0 & 0 \\
\hline & 0 & 1.1 & 1.283333333 & 2 & 0 & 0 & 0 & 0 & 1 & 0 \\
\hline & 0 & 5 & 1.283333333 & 2 & 0 & 0 & 0 & 0 & 0 & 1 \\
\hline \multirow[t]{5}{*}{35} & 1 & 8.415 & 1 & 2 & 1 & 0 & 0 & 0 & 0 & 0 \\
\hline & 0 & 20 & 0.466666667 & 2 & 0 & 0 & 1 & 0 & 0 & 0 \\
\hline & 0 & 0.7 & 0.283333333 & 2 & 0 & 0 & 0 & 1 & 0 & 0 \\
\hline & 0 & 1.5 & 0.5 & 2 & 0 & 0 & 0 & 0 & 1 & 0 \\
\hline & 0 & 5 & 0.5 & 2 & 0 & 0 & 0 & 0 & 0 & 1 \\
\hline \multirow[t]{5}{*}{36} & 1 & 13.711 & 1.1666666667 & 2 & 1 & 0 & 0 & 0 & 0 & 0 \\
\hline & 0 & 20 & 0.4666666667 & 2 & 0 & 0 & 1 & 0 & 0 & 0 \\
\hline & 0 & 0.7 & 0.283333333 & 2 & 0 & 0 & 0 & 1 & 0 & 0 \\
\hline & 0 & 1.5 & 0.5 & 2 & 0 & 0 & 0 & 0 & 1 & 0 \\
\hline & 0 & 5 & 0.5 & 2 & 0 & 0 & 0 & 0 & 0 & 1 \\
\hline
\end{tabular}




\begin{tabular}{|c|c|c|c|c|c|c|c|c|c|c|}
\hline \multirow{2}{*}{$\begin{array}{c}\text { Five alternatives } \\
\text { (Number of } \\
\text { observation) }\end{array}$} & Variable & Beta 1 & Beta 2 & Beta 3 & Beta 4 & Beta 5 & Beta 6 & Beta 7 & Beta 8 & Beta 9 \\
\hline & $\begin{array}{l}\text { Mode } \\
\text { Chosen } \\
\end{array}$ & $\begin{array}{c}\text { TC } \\
\text { (1000 Korea won) }\end{array}$ & $\begin{array}{c}\text { TT } \\
\text { (hour) }\end{array}$ & Accom & Sexm & Age340 & Tasc & Sasc & Basc & Lasc \\
\hline \multirow[t]{5}{*}{37} & 1 & 16.889 & 1.6666666667 & 2 & 1 & 0 & 0 & 0 & 0 & 0 \\
\hline & 0 & 20 & 1.483333333 & 2 & 0 & 0 & 1 & 0 & 0 & 0 \\
\hline & 0 & 0.6 & 0.9166666667 & 2 & 0 & 0 & 0 & 1 & 0 & 0 \\
\hline & 0 & 1.6 & 1.583333333 & 2 & 0 & 0 & 0 & 0 & 1 & 0 \\
\hline & 0 & 5 & 1.583333333 & 2 & 0 & 0 & 0 & 0 & 0 & 1 \\
\hline \multirow[t]{5}{*}{38} & 1 & 4.707 & 0.833333333 & 3 & 1 & 0 & 0 & 0 & 0 & 0 \\
\hline & 0 & 28 & 1.2 & 3 & 0 & 0 & 1 & 0 & 0 & 0 \\
\hline & 0 & 0.6 & 0.733333333 & 3 & 0 & 0 & 0 & 1 & 0 & 0 \\
\hline & 0 & 1.1 & 1.283333333 & 3 & 0 & 0 & 0 & 0 & 1 & 0 \\
\hline & 0 & 5 & 1.283333333 & 3 & 0 & 0 & 0 & 0 & 0 & 1 \\
\hline \multirow[t]{5}{*}{39} & 1 & 1.588 & 1 & 2 & 1 & 1 & 0 & 0 & 0 & 0 \\
\hline & 0 & 28 & 1.2 & 2 & 0 & 0 & 1 & 0 & 0 & 0 \\
\hline & 0 & 0.6 & 0.733333333 & 2 & 0 & 0 & 0 & 1 & 0 & 0 \\
\hline & 0 & 1.1 & 1.283333333 & 2 & 0 & 0 & 0 & 0 & 1 & 0 \\
\hline & 0 & 5 & 1.283333333 & 2 & 0 & 0 & 0 & 0 & 0 & 1 \\
\hline \multirow[t]{5}{*}{40} & 1 & 7.885 & 1 & 0 & 1 & 1 & 0 & 0 & 0 & 0 \\
\hline & 0 & 12.052 & 1.533333333 & 0 & 0 & 0 & 1 & 0 & 0 & 0 \\
\hline & 0 & 0.6 & 0.95 & 0 & 0 & 0 & 0 & 1 & 0 & 0 \\
\hline & 0 & 5 & 1.633333333 & 0 & 0 & 0 & 0 & 0 & 1 & 0 \\
\hline & 0 & 5 & 1.633333333 & 0 & 0 & 0 & 0 & 0 & 0 & 1 \\
\hline \multirow[t]{5}{*}{41} & 1 & 2.118 & 1.5 & 1 & 0 & 1 & 0 & 0 & 0 & 0 \\
\hline & 0 & 20 & 0.466666667 & 1 & 0 & 1 & 1 & 0 & 0 & 0 \\
\hline & 0 & 0.7 & 0.283333333 & 1 & 0 & 1 & 0 & 1 & 0 & 0 \\
\hline & 0 & 1.5 & 0.5 & 1 & 0 & 1 & 0 & 0 & 1 & 0 \\
\hline & 0 & 5 & 0.5 & 1 & 0 & 1 & 0 & 0 & 0 & 1 \\
\hline \multirow[t]{5}{*}{42} & 1 & 4.358 & 1.5 & 2 & 1 & 1. & 0 & 0 & 0 & 0 \\
\hline & 0 & 13.127 & 1.666666667 & 2 & 0 & 0 & 1 & 0 & 0 & 0 \\
\hline & 0 & 0.6 & 1.033333333 & 2 & 0 & 0 & 0 & 1 & 0 & 0 \\
\hline & 0 & 2 & 1.8 & 2 & 0 & 0 & 0 & 0 & 1 & 0 \\
\hline & 0 & 5 & 1.8 & 2 & 0 & 0 & 0 & 0 & 0 & 1 \\
\hline \multirow[t]{5}{*}{43} & 1 & 2.588 & 0.5 & 2 & 1 & 0 & 0 & 0 & 0 & 0 \\
\hline & 0 & 10 & 0.516666667 & 2 & 0 & 0 & 1 & 0 & 0 & 0 \\
\hline & 0 & 0.5 & 0.316666667 & 2 & 0 & 0 & 0 & 1 & 0 & 0 \\
\hline & 0 & 1.1 & 0.566666667 & 2 & 0 & 0 & 0 & 0 & 1 & 0 \\
\hline & 0 & 5 & 0.566666667 & 2 & 0 & 0 & 0 & 0 & 0 & 1 \\
\hline \multirow[t]{5}{*}{44} & 1 & 4.237 & 0.666666667 & 2 & 1 & 1 & 0 & 0 & 0 & 0 \\
\hline & 0 & 28 & 1.2 & 2 & 0 & 0 & 1. & 0 & 0 & 0 \\
\hline & 0 & 0.6 & 0.733333333 & 2 & 0 & 0 & 0 & 1 & 0 & 0 \\
\hline & 0 & 1.1 & 1.283333333 & 2 & 0 & 0 & 0 & 0 & 1 & 0 \\
\hline & 0 & 5 & 1.283333333 & 2 & 0 & 0 & 0 & 0 & 0 & 1 \\
\hline \multirow[t]{5}{*}{45} & 1 & 4.677 & 0.666666667 & 0 & 1 & 0 & 0 & 0 & 0 & 0 \\
\hline & 0 & 28 & 1.2 & 0 & 0 & 0 & 1 & 0 & 0 & 0 \\
\hline & 0 & 0.6 & 0.733333333 & 0 & 0 & 0 & 0 & 1 & 0 & 0 \\
\hline & 0 & 1.1 & 1.283333333 & 0 & 0 & 0 & 0 & 0 & 1 & 0 \\
\hline & 0 & 5 & 1.283333333 & 0 & 0 & 0 & 0 & 0 & 0 & 1 \\
\hline \multirow[t]{5}{*}{46} & 1 & 3.177 & 0.6666666667 & 2 & 1 & 1 & 0 & 0 & 0 & 0 \\
\hline & 0 & 15 & 1.1 & 2 & 0 & 0 & 1 & 0 & 0 & 0 \\
\hline & 0 & 0.6 & 0.683333333 & 2 & 0 & 0 & 0 & 1 & 0 & 0 \\
\hline & 0 & 1.3 & 1.183333333 & 2 & 0 & 0 & 0 & 0 & 1 & 0 \\
\hline & 0 & 5 & 1.183333333 & 2 & 0 & 0 & 0 & 0 & 0 & 1 \\
\hline \multirow[t]{5}{*}{47} & 1 & 4.177 & 0.666666667 & 1 & 1 & 1 & 0 & 0 & 0 & 0 \\
\hline & 0 & 17 & 1.15 & 1 & 0 & 0 & 1 & 0 & 0 & 0 \\
\hline & 0 & 0.65 & 0.7 & 1 & 0 & 0 & 0 & 1 & 0 & 0 \\
\hline & 0 & 2 & 1.233333333 & 1 & 0 & 0 & 0 & 0 & 1 & 0 \\
\hline & 0 & 5 & 1.233333333 & 1 & 0 & 0 & 0 & 0 & 0 & 1 \\
\hline \multirow[t]{5}{*}{48} & 1 & 2.3 & 0.5 & 0 & 1 & 0 & 0 & 0 & 0 & 0 \\
\hline & 0 & 10 & 0.516666667 & 0 & 0 & 0 & 1 & 0 & 0 & 0 \\
\hline & 0 & 0.5 & 0.316666667 & 0 & 0 & 0 & 0 & 1 & 0 & 0 \\
\hline & 0 & 1.1 & 0.5666666667 & 0 & 0 & 0 & 0 & 0 & 1 & 0 \\
\hline & 0 & 5 & 0.566666667 & 0 & 0 & 0 & 0 & 0 & 0 & 1 \\
\hline
\end{tabular}




\begin{tabular}{|c|c|c|c|c|c|c|c|c|c|c|}
\hline \multirow{2}{*}{$\begin{array}{l}\text { Five alternatives } \\
\text { (Number of } \\
\text { observation) }\end{array}$} & Variable & Beta 1 & Beta 2 & Beta 3 & Beta 4 & Beta 5 & Beta 6 & Beta 7 & Beta 8 & Beta 9 \\
\hline & $\begin{array}{l}\text { Mode } \\
\text { Chosen }\end{array}$ & $\begin{array}{c}\text { TC } \\
\text { (1000 Korea won) } \\
\end{array}$ & $\begin{array}{c}\mathrm{TT} \\
\text { (hour) }\end{array}$ & Accom & Sexm & Age340 & Tasc & Sasc & Base & Lasc \\
\hline \multirow[t]{5}{*}{49} & 1 & 3.118 & 1 & 0 & 0 & 1 & 0 & 0 & 0 & 0 \\
\hline & 0 & 12.052 & 1.533333333 & 0 & 0 & 0 & 1 & 0 & 0 . & 0 \\
\hline & 0 & 0.6 & 0.95 & 0 & 0 & 0 & 0 & 1 & 0 & 0 \\
\hline & 0 & 5 & 1.633333333 & 0 & 0 & 0 & 0 & 0 & 1 & 0 \\
\hline & 0 & 5 & 1.633333333 & 0 & 0 & 0 & 0 & 0 & 0 & 1 \\
\hline \multirow[t]{5}{*}{50} & 1 & 4.648 & 0.6666666667 & 4 & 1 & 1 & 0 & 0 & 0 & 0 \\
\hline & 0 & 20 & 1.483333333 & 4 & 0 & 0 & 1 & 0 & 0 & 0 \\
\hline & 0 & 0.6 & 0.9166666667 & 4 & 0 & 0 & 0 & 1 & 0 & 0 \\
\hline & 0 & 1.6 & 1.583333333 & 4 & 0 & 0 & 0 & 0 & 1 & 0 \\
\hline & 0 & 5 & 1.583333333 & 4 & 0 & 0 & 0 & 0 & 0 & 1 \\
\hline \multirow[t]{5}{*}{51} & 1 & 4.677 & 0.333333333 & 5 & 1 & 0 & 0 & 0 & 0 & 0 \\
\hline & 0 & 11 & 0.7166666667 & 5 & 0 & 0 & 1 & 0 & 0 & 0 \\
\hline & 0 & 0.5 & 0.433333333 & 5 & 0 & 0 & 0 & 1 & 0 & 0 \\
\hline & 0 & 1.3 & 0.7666666667 & 5 & 0 & 0 & 0 & 0 & 1 & 0 \\
\hline & 0 & 5 & 0.7666666667 & 5 & 0 & 0 & 0 & 0 & 0 & 1 \\
\hline \multirow[t]{5}{*}{52} & 1 & 19.889 & 2.5 & 2 & 1 & 0 & 0 & 0 & 0 & 0 \\
\hline & 0 & 53 & 2.0666666667 & 2 & 0 & 0 & 1 & 0 & 0 & 0 \\
\hline & 0 & 1 & 1.283333333 & 2 & 0 & 0 & 0 & 1 & 0 & 0 \\
\hline & 0 & 7.5 & 2.2 & 2 & 0 & 0 & 0 & 0 & 1 & 0 \\
\hline & 0 & 15 & 2.2 & 2 & 0 & 0 & 0 & 0 & 0 & 1 \\
\hline \multirow[t]{5}{*}{53} & 1 & 7.415 & 1.5 & 1 & 1 & 0 & 0 & 0 & 0 & 0 \\
\hline & 0 & 20 & 0.466666667 & 1 & 0 & 0 & 1 & 0 & 0 & 0 \\
\hline & 0 & 0.7 & 0.283333333 & 1 & 0 & 0 & 0 & 1 & 0 & 0 \\
\hline & 0 & 1.5 & 0.5 & 1 & 0 & 0 & 0 & 0 & 1 & 0 \\
\hline & 0 & 5 & 0.5 & 1 & 0 & 0 & 0 & 0 & 0 & 1 \\
\hline \multirow[t]{5}{*}{54} & 1 & 2.618 & 0.666666667 & 1 & 1 & 1 & 0 & 0 & 0 & 0 \\
\hline & 0 & 28 & 1.2 & 1 & 0 & 0 & 1 & 0 & 0 & 0 \\
\hline & 0 & 0.6 & 0.733333333 & 1 & 0 & 0 & 0 & 1 & 0 & 0 \\
\hline & 0 & 1.1 & 1.283333333 & 1 & 0 & 0 & 0 & 0 & 1 & 0 \\
\hline & 0 & 5 & 1.283333333 & 1 & 0 & 0 & 0 & 0 & 0 & 1 \\
\hline \multirow[t]{5}{*}{55} & 1 & 2.8 & 0.6666666667 & 1 & 1 & 1 & 0 & 0 & 0 & 0 \\
\hline & 0 & 10 & 0.516666667 & 1 & 0 & 0 & 1 & 0 & 0 & 0 \\
\hline & 0 & 0.5 & 0.3166666667 & 1 & 0 & 0 & 0 & 1 & 0 & 0 \\
\hline & 0 & 1.1 & 0.5666666667 & 1 & 0 & 0 & 0 & 0 & 1 & 0 \\
\hline & 0 & 5 & 0.5666666667 & 1 & 0 & 0 & 0 & 0 & 0 & 1 \\
\hline \multirow[t]{5}{*}{56} & 1 & 2.483 & 0.5 & 0 & 1 & 0 & 0 & 0 & 0 & 0 \\
\hline & 0 & 15 & 0.8166666667 & 0 & 0 & 0 & 1 & 0 & 0 & 0 \\
\hline & 0 & 0.6 & 0.5 & 0 & 0 & 0 & 0 & 1 & 0 & 0 \\
\hline & 0 & 1.5 & 0.8666666667 & 0 & 0 & 0 & 0 & 0 & 1 & 0 \\
\hline & 0 & 5 & 0.8666666667 & 0 & 0 & 0 & 0 & 0 & 0 & 1 \\
\hline \multirow[t]{5}{*}{57} & 1 & 1.588 & 0.833333333 & 1 & 0 & 1 & 0 & 0 & 0 & 0 \\
\hline & 0 & 28 & 1.2 & 1 & 0 & 0 & 1 & 0 & 0 & 0 \\
\hline & 0 & 0.6 & 0.733333333 & 1 & 0 & 0 & 0 & 1 & 0 & 0 \\
\hline & 0 & 1.1 & 1.283333333 & 1 & 0 & 0 & 0 & 0 & 1 & 0 \\
\hline & 0 & 5 & 1.283333333 & 1 & 0 & 0 & 0 & 0 & 0 & 1 \\
\hline \multirow[t]{5}{*}{58} & 1 & 10.415 & 1 & 2 & 0 & 0 & 0 & 0 & 0 & 0 \\
\hline & 0 & 28 & 1.8666666667 & 2 & 0 & 0 & 1 & 0 & 0 & 0 \\
\hline & 0 & 0.85 & 1.15 & 2 & 0 & 0 & 0 & 1 & 0 & 0 \\
\hline & 0 & 1.5 & 2 & 2 & 0 & 0 & 0 & 0 & 1 & 0 \\
\hline & 0 & 5 & 2 & 2 & 0 & 0 & 0 & 0 & 0 & 1 \\
\hline \multirow[t]{5}{*}{59} & 1 & 2.118 & 1 & 1 & 1 & 1 & 0 & 0 & 0 & 0 \\
\hline & 0 & 10 & 0.85 & 1 & 0 & 0 & 1 & 0 & 0 & 0 \\
\hline & 0 & 0.6 & 0.533333333 & 1 & 0 & 0 & 0 & 1 & 0 & 0 \\
\hline & 0 & 1.1 & 0.9166666667 & 1 & 0 & 0 & 0 & 0 & 1 & 0 \\
\hline & 0 & 5 & 0.9166666667 & 1 & 0 & 0 & 0 & 0 & 0 & 1 \\
\hline \multirow[t]{5}{*}{60} & 1 & 6.237 & 0.6666666667 & 1 & 1 & 0 & 0 & 0 & 0 & 0 \\
\hline & 0 & 15 & 1.1 & 1 & 0 & 0 & 1 & 0 & 0 & 0 \\
\hline & 0 & 0.6 & 0.6833333333 & 1 & 0 & 0 & 0 & 1 & 0 & 0 \\
\hline & 0 & 1.3 & 1.183333333 & 1 & 0 & 0 & 0 & 0 & 1 & 0 \\
\hline & 0 & 5 & 1.183333333 & 1 & 0 & 0 & 0 & 0 & 0 & 1 \\
\hline
\end{tabular}




\begin{tabular}{|c|c|c|c|c|c|c|c|c|c|c|}
\hline \multirow{2}{*}{$\begin{array}{l}\text { Five alternatives } \\
\text { (Number of } \\
\text { observation) }\end{array}$} & Variable & Beta 1 & Beta 2 & Beta 3 & Beta 4 & Beta 5 & Beta 6 & Beta 7 & Beta 8 & Beta 9 \\
\hline & $\begin{array}{c}\text { Mode } \\
\text { Chosen }\end{array}$ & $\begin{array}{c}\text { TC } \\
\text { (1000 Korea won) } \\
\end{array}$ & $\begin{array}{c}\text { TT } \\
\text { (hour) }\end{array}$ & Accom & Sexm & Age340 & Tasc & Sasc & Basc & Lasc \\
\hline \multirow[t]{5}{*}{61} & 1 & 7.796 & 1.333333333 & 5 & 1 & 1 & 0 & 0 & 0 & 0 \\
\hline & 0 & 20 & 0.4666666667 & 5 & 0 & 0 & 1 & 0 & 0 & 0 \\
\hline & 0 & 0.7 & 0.283333333 & 5 & 0 & 0 & 0 & 1 & 0 & 0 \\
\hline & 0 & 1.5 & 0.5 & 5 & 0 & 0 & 0 & 0 & 1 & 0 \\
\hline & 0 & 5 & 0.5 & 5 & 0 & 0 & 0 & 0 & 0 & 1 \\
\hline \multirow[t]{5}{*}{62} & 1 & 5.296 & 0.666666667 & 4 & 1 & 0 & 0 & 0 & 0 & 0 \\
\hline & 0 & 12.5 & 0.95 & 4 & 0 & 0 & 1 & 0 & 0 & 0 \\
\hline & 0 & 0.7 & 0.583333333 & 4 & 0 & 0 & 0 & 1 & 0 & 0 \\
\hline & 0 & 1.3 & 1.0166666667 & 4 & 0 & 0 & 0 & 0 & 1 & 0 \\
\hline & 0 & 5 & 1.016666667 & 4 & 0 & 0 & 0 & 0 & 0 & 1 \\
\hline \multirow[t]{5}{*}{63} & 1 & 4.118 & 1 & 2 & 1 & 1 & 0 & 0 & 0 & 0 \\
\hline & 0 & 15 & 0.816666667 & 2 & 0 & 0 & 1 & 0 & 0 & 0 \\
\hline & 0 & 0.6 & 0.5 & 2 & 0 & 0 & 0 & 1 & 0 & 0 \\
\hline & 0 & 1.5 & 0.8666666667 & 2 & 0 & 0 & 0 & 0 & 1 & 0 \\
\hline & 0 & 5 & 0.866666667 & 2 & 0 & 0 & 0 & 0 & 0 & 1 \\
\hline \multirow[t]{5}{*}{64} & 1 & 4.677 & 0.5 & 1 & 1 & 1 & 0 & 0 & 0 & 0 \\
\hline & 0 & 28 & 1.2 & 1 & 0 & 0 & 1 & 0 & 0 & 0 \\
\hline & 0 & 0.6 & 0.733333333 & 1 & 0 & 0 & 0 & 1 & 0 & 0 \\
\hline & 0 & 1.1 & 1.283333333 & 1 & 0 & 0 & 0 & 0 & 1 & 0 \\
\hline & 0 & 5 & 1.283333333 & 1 & 0 & 0 & 0 & 0 & 0 & 1 \\
\hline \multirow[t]{5}{*}{65} & 1 & 16.889 & 2 & 0 & 1 & 1 & 0 & 0 & 0 & 0 \\
\hline & 0 & 53 & 2.0666666667 & 0 & 0 & 0 & 1 & 0 & 0 & 0 \\
\hline & 0 & 1 & 1.283333333 & 0 & 0 & 0 & 0 & 1 & 0 & 0 \\
\hline & 0 & 7.5 & 2.2 & 0 & 0 & 0 & 0 & 0 & 1 & 0 \\
\hline & 0 & 15 & 2.2 & 0 & 0 & 0 & 0 & 0 & 0 & 1 \\
\hline \multirow[t]{5}{*}{66} & 1 & 3.588 & 0.25 & 0 & 0 & 0 & 0 & 0 & 0 & 0 \\
\hline & 0 & 10 & 0.516666667 & 0 & 0 & 0 & 1 & 0 & 0 & 0 \\
\hline & 0 & 0.5 & 0.316666667 & 0 & 0 & 0 & 0 & 1 & 0 & 0 \\
\hline & 0 & 1.1 & 0.566666667 & 0 & 0 & 0 & 0 & 0 & 1 & 0 \\
\hline & 0 & 5 & 0.566666667 & 0 & 0 & 0 & 0 & 0 & 0 & 1 \\
\hline \multirow[t]{5}{*}{67} & 1 & 8.326 & 1 & 2 & 1 & 1 & 0 & 0 & 0 & 0 \\
\hline & 0 & 20 & 0.466666667 & 2 & 0 & 0 & 1 & 0 & 0 & 0 \\
\hline & 0 & 0.7 & 0.283333333 & 2 & 0 & 0 & 0 & 1 & 0 & 0 \\
\hline & 0 & 1.5 & 0.5 & 2 & 0 & 0 & 0 & 0 & 1 & 0 \\
\hline & 0 & 5 & 0.5 & 2 & 0 & 0 & 0 & 0 & 0 & 1 \\
\hline \multirow[t]{5}{*}{68} & 1 & 4.618 & 0.333333333 & 0 & 0 & 1 & 0 & 0 & 0 & 0 \\
\hline & 0 & 53 & 2.066666667 & 0 & 0 & 0 & 1 & 0 & 0 & 0 \\
\hline & 0 & 1 & 1.283333333 & 0 & 0 & 0 & 0 & 1 & 0 & 0 \\
\hline & 0 & 7.5 & 2.2 & 0 & 0 & 0 & 0 & 0 & 1 & 0 \\
\hline & 0 & 15 & 2.2 & 0 & 0 & 0 & 0 & 0 & 0 & 1 \\
\hline \multirow[t]{5}{*}{69} & 1 & 5.36 & 0.666666667 & 1 & 1 & 1 & 0 & 0 & 0 & 0 \\
\hline & 0 & 28 & 1.2 & 1 & 0 & 0 & 1 & 0 & 0 & 0 \\
\hline & 0 & 0.6 & 0.733333333 & 1 & 0 & 0 & 0 & 1 & 0 & 0 \\
\hline & 0 & 1.1 & 1.283333333 & 1 & 0 & 0 & 0 & 0 & 1 & 0 \\
\hline & 0 & 5 & 1.283333333 & 1 & 0 & 0 & 0 & 0 & 0 & 1 \\
\hline \multirow[t]{5}{*}{70} & 1 & 4.813 & 0.75 & 0 & 1 & 1 & 0 & 0 & 0 & 0 \\
\hline & 0 & 28 & 1.2 & 0 & 0 & 0 & 1 & 0 & 0 & 0 \\
\hline & 0 & 0.6 & 0.7333333333 & 0 & 0 & 0 & 0 & 1 & 0 & 0 \\
\hline & 0 & 1.1 & 1.283333333 & 0 & 0 & 0 & 0 & 0 & 1 & 0 \\
\hline & 0 & 5 & 1.283333333 & 0 & 0 & 0 & 0 & 0 & 0 & 1 \\
\hline \multirow[t]{5}{*}{71} & 1 & 6.296 & 1 & 1 & 1 & 1 & 0 & 0 & 0 & 0 \\
\hline & 0 & 28 & 1.2 & 1 & 0 & 0 & 1 & 0 & 0 & 0 \\
\hline & 0 & 0.6 & 0.733333333 & 1 & 0 & 0 & 0 & 1 & 0 & 0 \\
\hline & 0 & 1.1 & 1.283333333 & 1 & 0 & 0 & 0 & 0 & 1 & 0 \\
\hline & 0 & 5 & 1.2833333333 & 1 & 0 & 0 & 0 & 0 & 0 & 1 \\
\hline \multirow[t]{5}{*}{72} & 1 & 1.271 & 0.333333333 & 0 & 1 & 1 & 0 & 0 & 0 & 0 \\
\hline & 0 & 8.468 & 1.05 & 0 & 0 & 0 & 1 & 0 & 0 & 0 \\
\hline & 0 & 1 & 0.65 & 0 & 0 & 0 & 0 & 1 & 0 & 0 \\
\hline & 0 & 1.3 & 1.133333333 & 0 & 0 & 0 & 0 & 0 & 1 & 0 \\
\hline & 0 & 5 & 1.133333333 & 0 & 0 & 0 & 0 & 0 & 0 & 1 \\
\hline
\end{tabular}




\begin{tabular}{|c|c|c|c|c|c|c|c|c|c|c|}
\hline \multirow{2}{*}{$\begin{array}{c}\text { Five alternatives } \\
\text { (Number of } \\
\text { observation) }\end{array}$} & Variable & Beta 1 & Beta 2 & Beta 3 & Beta 4 & Beta 5 & Beta 6 & Beta 7 & Beta 8 & Beta 9 \\
\hline & $\begin{array}{l}\text { Mode } \\
\text { Chosen }\end{array}$ & $\begin{array}{c}\text { TC } \\
\text { (1000 Korea won) }\end{array}$ & $\begin{array}{c}\mathrm{TT} \\
\text { (hour) }\end{array}$ & Accom & Sexm & Age 340 & Tasc & Sasc & Basc & Lasc \\
\hline \multirow[t]{5}{*}{73} & 1 & 2.059 & 0.166666667 & 0 & 0 & 1 & 0 & 0 & 0 & 0 \\
\hline & 0 & 6 & 0.183333333 & 0 & 0 & 0 & 1 & 0 & 0 & 0 \\
\hline & 0 & 0.5 & 0.116666667 & 0 & 0 & 0 & 0 & 1 & 0 & 0 \\
\hline & 0 & 0.5 & 0.2 & 0 & 0 & 0 & 0 & 0 & 1 & 0 \\
\hline & 0 & 5 & 0.2 & 0 & 0 & 0 & 0 & 0 & 0 & 1 \\
\hline \multirow[t]{5}{*}{74} & 1 & 17.389 & 2.083333333 & 4 & 1 & 0 & 0 & 0 & 0 & 0 \\
\hline & 0 & 53 & 2.066666667 & 4 & 0 & 0 & 1 & 0 & 0 & 0 \\
\hline & 0 & 1 & 1.283333333 & 4 & 0 & 0 & 0 & 1 & 0 & 0 \\
\hline & 0 & 7.5 & 2.2 & 4 & 0 & 0 & 0 & 0 & 1 & 0 \\
\hline & 0 & 15 & 2.2 & 0 & 0 & 0 & 0 & 0 & 0 & 1 \\
\hline \multirow[t]{5}{*}{75} & 1 & 4.677 & 0.6666666667 & 0 & 1 & 0 & 0 & 0 & 0 & 0 \\
\hline & 0 & 28 & 1.2 & 0 & 0 & 0 & 1 & 0 & 0 & 0 \\
\hline & 0 & 0.6 & 0.733333333 & 0 & 0 & 0 & 0 & 1 & 0 & 0 \\
\hline & 0 & 1.1 & 1.283333333 & 0 & 0 & 0 & 0 & 0 & 1 & 0 \\
\hline & 0 & 5 & 1.283333333 & 0 & 0 & 0 & 0 & 0 & 0 . & 1 \\
\hline \multirow[t]{5}{*}{76} & 1 & 13.771 & 1.333333333 & 0 & 1 & 1 & 0 & 0 & 0 & 0 \\
\hline & 0 & 53 & 2.0666666667 & 0 & 0 & 0 & 1 & 0 & 0 & 0 \\
\hline & 0 & 1 & 1.283333333 & 0 & 0 & 0 & 0 & 1 & 0 & 0 \\
\hline & 0 & 7.5 & 2.2 & 0 & 0 & 0 & 0 & 0 & 1 & 0 \\
\hline & 0 & 15 & 2.2 & 0 & 0 & 0 & 0 & 0 & 0 & 1 \\
\hline \multirow[t]{5}{*}{77} & 1 & 4.177 & 1 & 1 & 1 & 1 & 0 & 0 & 0 & 0 \\
\hline & 0 & 30 & 0.616666667 & 1 & 0 & 0 & 1 & 0 & 0 & 0 \\
\hline & 0 & 0.9 & 0.383333333 & 1 & 0 & 0 & 0 & 1 & 0 & 0 \\
\hline & 0 & 1.3 & 0.666666667 & 1 & 0 & 0 & 0 & 0 & 1 & 0 \\
\hline & 0 & 5 & 0.6666666667 & 0 & 0 & 0 & 0 & 0 & 0 & 1 \\
\hline \multirow[t]{5}{*}{78} & 1 & 3.118 & 0.5 & 0 & 1 & 1 & 0 & 0 & 0 & 0 \\
\hline & 0 & 10 & 0.516666667 & 0 & 0 & 0 & 1 & 0 & 0 & 0 \\
\hline & 0 & 0.5 & 0.316666667 & 0 & 0 & 0 & 0 & 1 & 0 & 0 \\
\hline & 0 & 1.1 & 0.566666667 & 0 & 0 & 0 & 0 & 0 & 1 & 0 \\
\hline & 0 & 5 & 0.566666667 & 0 & 0 & 0 & 0 & 0 & 0 & 1 \\
\hline \multirow[t]{5}{*}{79} & 1 & 5.707 & 1 & 2 & 0 & 0 & 0 & 0 & 0 & 0 \\
\hline & 0 & 20 & 1.483333333 & 2 & 0 & 0 & 1 & 0 & 0 & 0 \\
\hline & 0 & 0.6 & 0.916666667 & 2 & 0 & 0 & 0 & 1 & 0 & 0 \\
\hline & 0 & 1.6 & 1.583333333 & 2 & 0 & 0 & 0 & 0 & 1 & 0 \\
\hline & 0 & 5 & 1.583333333 & 2 & 0 & 0 & 0 & 0 & 0 & 1 \\
\hline \multirow[t]{5}{*}{80} & 1 & 2.648 & 1.333333333 & 1 & 0 & 1 & 0 & 0 & 0 & 0 \\
\hline & 0 & 20 & 0.466666667 & 1 & 0 & 0 & 1 & 0 & 0 & 0 \\
\hline & 0 & 0.7 & 0.283333333 & 1 & 0 & 0 & 0 & 1 & 0 & 0 \\
\hline & 0 & 1.5 & 0.5 & 1 & 0 & 0 & 0 & 0 & 1 & 0 \\
\hline & 0 & 5 & 0.5 & 1 & 0 & 0 & 0 & 0 & 0 & 1 \\
\hline \multirow[t]{5}{*}{81} & 1 & 3.118 & 0.833333333 & 0 & 1 & 1 & 0 & 0 & 0 & 0 \\
\hline & 0 & 7.6 & 0.616666667 & 0 & 0 & 0 & 1 & 0 & 0 & 0 \\
\hline & 0 & 0.6 & 0.383333333 & 0 & 0 & 0 & 0 & 1 & 0 & 0 \\
\hline & 0 & 1.6 & 0.666666667 & 1 & 0 & 0 & 0 & 0 & 1 & 0 \\
\hline & 0 & 5 & 0.6666666667 & 1 & 0 & 0 & 0 & 0 & 0 & 1 \\
\hline \multirow[t]{5}{*}{82} & 1 & 4.177 & 1 & 0 & 1 & 1 & 0 & 0 & 0 & 0 \\
\hline & 0 & 12.5 & 0.95 & 0 & 0 & 0 & 1 & 0 & 0 & 0 \\
\hline & 0 & 0.7 & 0.583333333 & 0 & 0 & 0 & 0 & 1 & 0 & 0 \\
\hline & 0 & 1.3 & 1.016666667 & 0 & 0 & 0 & 0 & 0 & 1 & 0 \\
\hline & 0 & 5 & 1.016666667 & 0 & 0 & 0 & 0 & 0 & 0 & 1 \\
\hline \multirow[t]{5}{*}{83} & 1 & 4.118 & 0.666666667 & 0 & 1 & 1 & 0 & 0 & 0 & 0 \\
\hline & 0 & 10 & 0.516666667 & 0 & 0 & 0 & 1 & 0 & 0 & 0 \\
\hline & 0 & 0.5 & 0.316666667 & 0 & 0 & 0 & 0 & 1 & 0 & 0 \\
\hline & 0 & 1.1 & 0.566666667 & 0 & 0 & 0 & 0 & 0 & 1 & 0 \\
\hline & 0 & 5 & 0.566666667 & 0 & 0 & 0 & 0 & 0 & 0 & 1 \\
\hline \multirow[t]{5}{*}{84} & 1 & 4.677 & 0.833333333 & 1 & 1 & 1 & 0 & 0 & 0 & 0 \\
\hline & 0 & 30 & 0.616666667 & 1 & 0 & 0 & 1 & 0 & 0 & 0 \\
\hline & 0 & 0.9 & 0.383333333 & 1 & 0 & 0 & 0 & 1 & 0 & 0 \\
\hline & 0 & 1.3 & 0.666666667 & 1 & 0 & 0 & 0 & 0 & 1 & 0 \\
\hline & 0 & 5 & 0.666666667 & 1 & 0 & 0 & 0 & 0 & 0 & 1 \\
\hline
\end{tabular}




\begin{tabular}{|c|c|c|c|c|c|c|c|c|c|c|}
\hline \multirow{2}{*}{$\begin{array}{c}\text { Five alternatives } \\
\text { (Number of } \\
\text { observation) }\end{array}$} & Variable & Beta 1 & Beta 2 & Beta 3 & Beta 4 & Beta 5 & Beta 6 & Beta 7 & Beta 8 & Beta 9 \\
\hline & $\begin{array}{c}\text { Mode } \\
\text { Chosen }\end{array}$ & $\begin{array}{c}\text { TC } \\
\text { (1000 Korea won) }\end{array}$ & $\begin{array}{c}\mathrm{TT} \\
\text { (hour) }\end{array}$ & Accom & Sexm & Age340 & Tasc & Sasc & Basc & Lasc \\
\hline \multirow[t]{5}{*}{85} & 1 & 7.296 & 0.5 & 2 & 0 & 1 & 0 & 0 & 0 & 0 \\
\hline & 0 & 15 & 0.816666667 & 2 & 0 & 0 & 1 & 0 & 0 & 0 \\
\hline & 0 & 0.6 & 0.5 & 2 & 0 & 0 & 0 & 1 & 0 & 0 \\
\hline & 0 & 1.5 & 0.866666667 & 2 & 0 & 0 & 0 & 0 & 1 & 0 \\
\hline & 0 & 5 & 0.866666667 & 2 & 0 & 0 & 0 & 0 & 0 & 1 \\
\hline \multirow[t]{5}{*}{86} & 1 & 4.177 & 0.25 & 0 & 1 & 1 & 0 & 0 & 0 & 0 \\
\hline & 0 & 14 & 2.066666667 & 0 & 0 & 0 & 1 & 0 & 0 & 0 \\
\hline & 0 & 0.75 & 1.283333333 & 0 & 0 & 0 & 0 & 1 & 0 & 0 \\
\hline & 0 & 1.5 & 2.2 & 0 & 0 & 0 & 0 & 0 & 1 & 0 \\
\hline & 0 & 5 & 2.2 & 0 & 0 & 0 & 0 & 0 & 0 & 1 \\
\hline \multirow[t]{5}{*}{87} & 1 & 2.588 & 0.3333333333 & 0 & 1 & 1 & 0 & 0 & 0 & 0 \\
\hline & 0 & 10 & 0.516666667 & 0 & 0 & 0 & 1 & 0 & 0 & 0 \\
\hline & 0 & 0.5 & 0.316666667 & 0 & 0 & 0 & 0 & 1 & 0 & 0 \\
\hline & 0 & 1.1 & 0.566666667 & 0 & 0 & 0 & 0 & 0 & 1 & 0 \\
\hline & 0 & 5 & 0.566666667 & 0 & 0 & 0 & 0 & 0 & 0 & 1 \\
\hline \multirow[t]{5}{*}{88} & 1 & 4.677 & 1.333333333 & 0 & 0 & 0 & 0 & 0 & 0 & 0 \\
\hline & 0 & 28 & 1.2 & 0 & 0 & 0 & 1 & 0 & 0 & 0 \\
\hline & 0 & 0.6 & 0.733333333 & 0 & 0 & 0 & 0 & 1 & 0 & 0 \\
\hline & 0 & 1.1 & 1.2833333333 & 0 & 0 & 0 & 0 & 0 & 1 & 0 \\
\hline & 0 & 5 & 1.283333333 & 0 & 0 & 0 & 0 & 0 & 0 & 1 \\
\hline \multirow[t]{5}{*}{89} & 1 & 4.118 & 1.1666666667 & 0 & 1 & 1 & 0 & 0 & 0 & 0 \\
\hline & 0 & 12.052 & 1.533333333 & 0 & 0 & 0 & 1 & 0 & 0 & 0 \\
\hline & 0 & 0.6 & 0.95 & 0 & 0 & 0 & 0 & 1 & 0 & 0 \\
\hline & 0 & 5 & 1.633333333 & 0 & 0 & 0 & 0 & 0 & 1 & 0 \\
\hline & 0 & 5 & 1.633333333 & 0 & 0 & 0 & 0 & 0 & 0 & 1 \\
\hline \multirow[t]{5}{*}{90} & 1 & 5.237 & 1 & 0 & 1 & 1 & 0 & 0 & 0 & 0 \\
\hline & 0 & 13.127 & 1.666666667 & 0 & 0 & 0 & 1 & 0 & 0 & 0 \\
\hline & 0 & 0.6 & 1.033333333 & 0 & 0 & 0 & 0 & 1 & 0 & 0 \\
\hline & 0 & 2 & 1.8 & 0 & 0 & 0 & 0 & 0 & 1 & 0 \\
\hline & 0 & 5 & 1.8 & 0 & 0 & 0 & 0 & 0 & 0 & 1 \\
\hline \multirow[t]{5}{*}{91} & 1 & 5.237 & 1 & 0 & 1 & 1 & 0 & 0 & 0 & 0 \\
\hline & 0 & 7.5 & 1.1 & 0 & 0 & 0 & 1 & 0 & 0 & 0 \\
\hline & 0 & 0.7 & 0.6833333333 & 0 & 0 & 0 & 0 & 1 & 0 & 0 \\
\hline & 0 & 1.3 & 1.183333333 & 0 & 0 & 0 & 0 & 0 & 1 & 0 \\
\hline & 0 & 5 & 1.183333333 & 0 & 0 & 0 & 0 & 0 & 0 & 1 \\
\hline \multirow[t]{5}{*}{92} & 1 & 5.177 & 1 & 1 & 1 & 1 & 0 & 0 & 0 & 0 \\
\hline & 0 & 28 & 1.2 & 1 & 0 & 0 & 1 & 0 & 0 & 0 \\
\hline & 0 & 0.6 & 0.733333333 & 1 & 0 & 0 & 0 & 1 & 0 & 0 \\
\hline & 0 & 1.1 & 1.283333333 & 1 & 0 & 0 & 0 & 0 & 1 & 0 \\
\hline & 0 & 5 & 1.2833333333 & 0 & 0 & 0 & 0 & 0 & 0 & 1 \\
\hline \multirow[t]{5}{*}{93} & 1 & 3.648 & 1 & 0 & 1 & 1 & 0 & 0 & 0 & 0 \\
\hline & 0 & 28 & 1.2 & 0 & 0 & 0 & 1 & 0 & 0 & 0 \\
\hline & 0 & 0.6 & 0.733333333 & 0 & 0 & 0 & 0 & 1 & 0 & 0 \\
\hline & 0 & 1.1 & 1.283333333 & 0 & 0 & 0 & 0 & 0 & 1 & 0 \\
\hline & 0 & 5 & 1.2833333333 & 0 & 0 & 0 & 0 & 0 & 0 & 1 \\
\hline \multirow[t]{5}{*}{94} & 1 & 3.843 & 1 & 0 & 1 & 1 & 0 & 0 & 0 & 0 \\
\hline & 0 & 28 & 1.2 & 0 & 0 & 0 & 1 & 0 & 0 & 0 \\
\hline & 0 & 0.6 & 0.733333333 & 0 & 0 & 0 & 0 & 1 & 0 & 0 \\
\hline & 0 & 1.1 & 1.2833333333 & 0 & 0 & 0 & 0 & 0 & 1 & 0 \\
\hline & 0 & 5 & 1.2833333333 & 0 & 0 & 0 & 0 & 0 & 0 & 1 \\
\hline \multirow[t]{5}{*}{95} & 1 & 3.118 & 0.25 & 1 & 1 & 1 & 0 & 0 & 0 & 0 \\
\hline & 0 & 10 & 0.516666667 & 1 & 0 & 0 & 1 & 0 & 0 & 0 \\
\hline & 0 & 0.5 & 0.316666667 & 1 & 0 & 0 & 0 & 1 & 0 & 0 \\
\hline & 0 & 1.1 & 0.566666667 & 1 & 0 & 0 & 0 & 0 & 1 & 0 \\
\hline & 0 & 5 & 0.5666666667 & 1 & 0 & 0 & 0 & 0 & 0 & 1 \\
\hline \multirow[t]{5}{*}{96} & 1 & 6.237 & 0.6666666667 & 0 & 1 & 1 & 0 & 0 & 0 & 0 \\
\hline & 0 & 13.127 & 1.6666666667 & 0 & 0 & 0 & 1 & 0 & 0 & 0 \\
\hline & 0 & 0.6 & 1.033333333 & 0 & 0 & 0 & 0 & 1 & 0 & 0 \\
\hline & 0 & 2 & 1.8 & 0 & 0 & 0 & 0 & 0 & 1 & 0 \\
\hline & 0 & 5 & 1.8 & 0 & 0 & 0 & 0 & 0 & 0 & 1 \\
\hline
\end{tabular}




\begin{tabular}{|c|c|c|c|c|c|c|c|c|c|c|}
\hline \multirow{2}{*}{$\begin{array}{l}\text { Five alternatives } \\
\text { (Number of } \\
\text { observation) }\end{array}$} & Variable & Beta 1 & Beta 2 & Beta 3 & Beta 4 & Beta 5 & Beta 6 & Beta 7 & Beta 8 & Beta 9 \\
\hline & $\begin{array}{c}\text { Mode } \\
\text { Chosen }\end{array}$ & $\begin{array}{c}\text { TC } \\
\text { (1000 Korea won) }\end{array}$ & $\begin{array}{c}\mathrm{TT} \\
\text { (hour) }\end{array}$ & Accom & Sexm & Age340 & Tasc & Sasc & Basc & Lasc \\
\hline \multirow[t]{5}{*}{97} & 1 & 5.737 & 1.5 & 1 & 1 & 0 & 0 & 0 & 0 & 0 \\
\hline & 0 & 12 & 1.433333333 & 1 & 0 & 0 & 1 & 0 & 0 & 0 \\
\hline & $\overline{0}$ & 0.85 & 0.883333333 & 1 & 0 & 0 & $\overline{0}$ & $\overline{1}$ & $\overline{0}$ & 0 \\
\hline & 0 & 1.1 & 1.533333333 & 1 & 0 & 0 & 0 & 0 & 1 & 0 \\
\hline & 0 & 5 & 1.533333333 & 1 & 0 & 0 & 0 & 0 & 0 & 1 \\
\hline \multirow[t]{5}{*}{98} & 1 & 5.707 & 0.666666667 & 0 & 1 & 1 & 0 & 0 & 0 & 0 \\
\hline & 0 & 15 & 1.1 & 0 & 0 & 0 & 1 & 0 & 0 & 0 \\
\hline & 0 & 0.6 & 0.683333333 & 0 & 0 & 0 & 0 . & 1 & 0 & 0 \\
\hline & 0 & 1.3 & 1.183333333 & 0 & 0 & 0 & 0 & 0 & 1 & 0 \\
\hline & 0 & 5 & 1.183333333 & 0 & 0 & 0 & 0 & 0 & 0 & 1 \\
\hline \multirow[t]{5}{*}{99} & 1 & 5.177 & 0.666666667 & 2 & 1 & 1 & 0 & 0 & 0 & 0 \\
\hline & 0 & 28 & 1.2 & 2 & 0 & 0 & 1 & 0 & 0 & 0 \\
\hline & 0 & 0.6 & 0.733333333 & 2 & 0 & 0 & 0 & 1 & 0 & 0 \\
\hline & 0 & 1.1 & 1.283333333 & 2 & 0 & 0 & 0 & 0 & 1 & 0 \\
\hline & 0 & 5 & 1.283333333 & 2 & 0 & 0 & 0 & 0 & 0 & 1 \\
\hline \multirow[t]{5}{*}{100} & 1 & 4.118 & 0.333333333 & 2 & 0 & 1 & 0 & 0 & 0 & 0 \\
\hline & 0 & 10 & 0.85 & 2 & $\overline{0}$ & 0 & 1 & 0 & $\overline{0}$ & 0 \\
\hline & 0 & 0.6 & 0.533333333 & 2 & 0 & 0 & 0 & 1 & 0 & 0 \\
\hline & 0 & 1.1 & 0.9166666667 & 2 & 0 & 0 & $\overline{0}$ & 0 & 1 & 0 \\
\hline & 0 & 5 & 0.916666667 & 2 & 0 & 0 & 0 & 0 & 0 & 1 \\
\hline \multirow[t]{5}{*}{101} & 1 & 4.177 & 0.75 & 0 & 1 & 1 & 0 & 0 & 0 & 0 \\
\hline & 0 & 28 & 1.2 & 0 & 0 & 0 & 1 & 0 & 0 & 0 \\
\hline & 0 & 0.6 & 0.733333333 & 0 & 0 & 0 & 0 & 1 & 0 & 0 \\
\hline & 0 & 1.1 & 1.283333333 & 0 & 0 & 0 & 0 & 0 & 1 & 0 \\
\hline & 0 & 5 & 1.283333333 & 0 & 0 & 0 & 0 & 0 & 0 & 1 \\
\hline \multirow[t]{5}{*}{102} & 1 & 10.415 & 1.5 & 0 & 1 & 1 & 0 & 0 & 0 & 0 \\
\hline & 0 & 12 & 1.433333333 & 0 & 0 & 0 & 1 & 0 & 0 & 0 \\
\hline & 0 & 0.85 & 0.883333333 & 0 & 0 & 0 & 0 & 1 & 0 & 0 \\
\hline & 0 & 1.1 & 1.533333333 & 0 & 0 & 0 & 0 & 0 & 1 & 0 \\
\hline & 0 & 5 & 1.533333333 & 0 & 0 & 0 & 0 & 0 & 0 & 1 \\
\hline \multirow[t]{5}{*}{103} & 1 & 4.118 & 0.333333333 & 0 & 1 & 0 & 0 & 0 & 0 & 0 \\
\hline & 0 & 10 & 0.516666667 & 0 & 0 & 0 & 1 & 0 & 0 & 0 \\
\hline & 0 & 0.5 & 0.316666667 & 0 & 0 & 0 & 0 & 1 & 0 & 0 \\
\hline & 0 & 1.1 & 0.5666666667 & 0 & 0 & 0 & 0 & 0 & 1 & 0 \\
\hline & 0 & 5 & 0.566666667 & 0 & 0 & 0 & 0 & 0 & 0 & 1 \\
\hline \multirow[t]{5}{*}{104} & 1 & 5.207 & 0.833333333 & 0 & 1 & 1 & 0 & 0 & 0 & 0 \\
\hline & 0 & 28 & 1.2 & 0 & 0 & 0 & 1 & 0 & 0 & 0 \\
\hline & 0 & 0.6 & 0.733333333 & 0 & 0 & 0 & 0 & 1 & 0 & 0 \\
\hline & 0 & 1.1 & 1.283333333 & 0 & 0 & 0 & 0 & 0 & 1 & 0 \\
\hline & 0 & 5 & 1.283333333 & 0 & 0 & 0 & 0 & 0 & 0 & 1 \\
\hline \multirow[t]{5}{*}{105} & 1 & 5.237 & 0.666666667 & 0 & 1 & 0 & 0 & 0 & 0 & 0 \\
\hline & 0 & 13.127 & 1.666666667 & 0 & 0 & 0 & 1 & 0 & 0 & 0 \\
\hline & 0 & 0.6 & 1.033333333 & 0 & 0 & 0 & 0 & 1 & 0 & 0 \\
\hline & 0 & 2 & 1.8 & 0 & 0 & 0 & 0 & 0 & 1 & 0 \\
\hline & 0 & 5 & 1.8 & 0 & 0 & 0 & 0 & 0 & 0 & 1 \\
\hline \multirow[t]{5}{*}{106} & 1 & 4.707 & 1 & 2 & 1 & 1 & 0 & 0 & 0 & 0 \\
\hline & 0 & 28 & 1.2 & 2 & 0 & 0 & 1 & 0 & 0 & 0 \\
\hline & 0 & 0.6 & 0.733333333 & 2 & 0 & 0 & 0 & 1 & 0 & 0 \\
\hline & 0 & 1.1 & 1.283333333 & 2 & 0 & 0 & 0 & 0 & 1 & 0 \\
\hline & 0 & 5 & 1.283333333 & 2 & 0 & 0 & 0 & 0 & 0 & 1 \\
\hline \multirow[t]{5}{*}{107} & 1 & 6.177 & 1.166666667 & 3 & 1 & 1 & 0 & 0 & 0 & 0 \\
\hline & 0 & 30 & 0.616666667 & 3 & 0 & 0 & 1 & 0 & 0 & 0 \\
\hline & 0 & 0.9 & 0.383333333 & 3 & 0 & 0 & 0 & 1 & 0 & 0 \\
\hline & 0 & 1.3 & 0.666666667 & 3 & 0 & 0 & 0 & 0 & 1 & 0 \\
\hline & 0 & 5 & 0.666666667 & 3 & 0 & 0 & 0 & 0 & 0 & 1 \\
\hline \multirow[t]{5}{*}{108} & 1 & 3.059 & 0.5 & 4 & 1 & 1 & 0 & 0 & 0 & 0 \\
\hline & 0 & 10 & 0.466666667 & 4 & 0 & 0 & 1 & 0 & 0 & 0 \\
\hline & 0 & 0.5 & 0.283333333 & 4 & 0 & 0 & 0 & 1 & 0 & 0 \\
\hline & 0 & 1.1 & 0.5 & 4 & 0 & 0 & 0 & 0 & 1 & 0 \\
\hline & 0 & 5 & 0.5 & 4 & 0 & 0 & 0 & 0 & 0 & 1 \\
\hline
\end{tabular}




\begin{tabular}{|c|c|c|c|c|c|c|c|c|c|c|}
\hline \multirow{2}{*}{$\begin{array}{c}\text { Five alternatives } \\
\text { (Number of } \\
\text { observation) }\end{array}$} & Variable & Beta 1 & Beta 2 & Beta 3 & Beta 4 & Beta 5 & Beta 6 & Beta 7 & Beta 8 & Beta 9 \\
\hline & $\begin{array}{c}\text { Mode } \\
\text { Chosen }\end{array}$ & $\begin{array}{c}\text { TC } \\
\text { (1000 Korea won) }\end{array}$ & $\begin{array}{c}\text { TT } \\
\text { (hour) }\end{array}$ & Accom & Sexm & Age340 & Tasc & Sasc & Basc & Lasc \\
\hline \multirow[t]{5}{*}{109} & 1 & 18.83 & 2.5 & 1 & 1 & 1 & 0 & 0 & 0 & 0 \\
\hline & 0 & 53 & 2.0666666667 & 1 & 0 & 0 & 1 & 0 & 0 & 0 \\
\hline & 0 & 1 & 1.2833333333 & 1 & 0 & 0 & 0 & 1 & 0 & 0 \\
\hline & 0 & 7.5 & 2.2 & 1 & 0 & 0 & 0 & 0 & 1 & 0 \\
\hline & $\overline{0}$ & 15 & 2.2 & 1 & 0 & 0 & 0 & 0 & 0 & 1 \\
\hline \multirow[t]{5}{*}{110} & 1 & 2.377 & 0.5 & 2 & 1 & $\overline{1}$ & 0 & 0 & 0 & 0 \\
\hline & $\overline{0}$ & 10 & 0.516666667 & 2 & 0 & 0 & $\overline{1}$ & $\overline{0}$ & 0 & 0 \\
\hline & $\overline{0}$ & 0.5 & 0.3166666667 & 2 & $\overline{0}$ & 0 & 0 & 1 & 0 & 0 \\
\hline & 0 & 1.1 & 0.5666666667 & 2 & 0 & 0 & $\overline{0}$ & 0 & 1 & 0 \\
\hline & 0 & 5 & 0.5666666667 & 2 & $\overline{0}$ & 0 & 0 & 0 & 0 & 1 \\
\hline \multirow[t]{5}{*}{111} & 1 & 3.271 & 1 & 1 & 1 & 1 & 0 & $\overline{0}$ & 0 & 0 \\
\hline & 0 & 8.468 & 1.05 & 1 & 0 & 0 & 1 & 0 & 0 & 0 \\
\hline & 0 & 1 & 0.65 & 1 & 0 & $\overline{0}$ & 0 & 1 & 0 & 0 \\
\hline & 0 & 1.3 & 1.133333333 & 1 & $\overline{0}$ & $\overline{0}$ & $\overline{0}$ & 0 & 1 & $\overline{0}$ \\
\hline & 0 & 5 & 1.133333333 & 7 & 0 & 0 & 0 & 0 & 0 & $\overline{1}$ \\
\hline \multirow[t]{5}{*}{112} & 1 & 4.177 & 0.666666667 & 2 & 0 & 1 & 0 & 0 & 0 & 0 \\
\hline & 0 & 7.5 & 1.1 & 2 & 0 & 0 & 1 & 0 & 0 & 0 \\
\hline & 0 & 0.7 & 0.683333333 & 2 & 0 & 0 & 0 & 1 & 0 & 0 \\
\hline & 0 & 1.3 & 1.183333333 & 2 & 0 & 0 & 0 & 0 & 1 & 0 \\
\hline & 0 & 5 & 1.183333333 & 2 & 0 & 0 & 0 & 0 & 0 & 1 \\
\hline \multirow[t]{5}{*}{113} & 1 & 5.177 & 1 & 3 & 1 & 1 & 0 & 0 & 0 & 0 \\
\hline & 0 & 20 & 1.483333333 & 3 & 0 & 0 & 1 & 0 & 0 & 0 \\
\hline & 0 & 0.6 & 0.916666667 & 3 & 0 & 0 & 0 & 1 & 0 & 0 \\
\hline & 0 & 1.6 & 1.583333333 & 3 & 0 & 0 & 0 & 0 & 1 & 0 \\
\hline & 0 & 5 & 1.583333333 & 3 & 0 & 0 & 0 & 0 & 0 & 1 \\
\hline \multirow[t]{5}{*}{114} & 1 & 7.237 & 1 & 0 & 1 & 1 & 0 & 0 & 0 & 0 \\
\hline & 0 & 28 & 1.2 & 0 & 0 & 0 & 1 & 0 & 0 & 0 \\
\hline & 0 & 0.6 & 0.733333333 & 0 & 0 & 0 & 0 & 1 & 0 & 0 \\
\hline & 0 & 1.1 & 1.283333333 & 0 & 0 & 0 & 0 & 0 & $\frac{1}{1}$ & 0 \\
\hline & 0 & 5 & 1.283333333 & 0 & 0 & 0 & 0 & 0 & 0 & 1 \\
\hline \multirow[t]{5}{*}{115} & 1 & 4.177 & 1 & 1 & 1 & 1 & 0 & 0 & 0 & 0 \\
\hline & 0 & 28 & 1.2 & 1 & 0 & 0 & 1 & 0 & 0 & 0 \\
\hline & 0 & 0.6 & 0.733333333 & 1 & 0 & 0 & 0 & 1 & 0 & 0 \\
\hline & 0 & 1.1 & 1.283333333 & 1 & 0 & 0 & 0 & 0 & 1 & 0 \\
\hline & 0 & 5 & 1.283333333 & 1 & 0 & 0 & 0 & 0 & 0 & 1 \\
\hline \multirow[t]{5}{*}{116} & $\frac{1}{1}$ & 3.588 & 0.333333333 & 1 & 1 & 1 & 0 & 0 & 0 & 0 \\
\hline & 0 & 28 & 1.816666667 & 1 & 0 & 0 & 1 & 0 & 0 & 0 \\
\hline & 0 & 0.65 & 1.133333333 & 1 & 0 & 0 & 0 & 1 & 0 & 0 \\
\hline & 0 & 1 & 1.95 & 1 & 0 & 0 & 0 & 0 & 1 & 0 \\
\hline & 0 & 5 & 1.95 & 1 & 0 & 0 & 0 & 0 & $\overline{0}$ & 1 \\
\hline \multirow[t]{5}{*}{117} & 1 & 6.555 & 1.333333333 & 1 & 1 & 1 & 0 & 0 & 0 & 0 \\
\hline & 0 & 12 & 1.433333333 & 1 & 0 & 0 & 1 & 0 & 0 & 0 \\
\hline & 0 & 0.85 & 0.883333333 & 1 & 0 & 0 & 0 & 7 & 0 & 0 \\
\hline & 0 & 1.1 & 1.533333333 & 1 & 0 & 0 & 0 & 0 & 1 & 0 \\
\hline & 0 & 5 & 1.533333333 & 1 & 0 & 0 & 0 & 0 & 0 & 1 \\
\hline \multirow[t]{5}{*}{118} & 1 & 3.148 & 0.5 & 3 & 1 & 0 & 0 & 0 & 0 & 0 \\
\hline & 0 & 10 & 0.516666667 & 3 & 0 & 0 & 1 & 0 & 0 & 0 \\
\hline & 0 & 0.5 & 0.316666667 & 3 & 0 & 0 & 0 & 1 & 0 & 0 \\
\hline & 0 & 1.1 & 0.566666667 & 3 & 0 & 0 & 0 & 0 & 1 & 0 \\
\hline & 0 & 5 & 0.566666667 & 3 & 0 & 0 & 0 & 0 & 0 & 1 \\
\hline \multirow[t]{5}{*}{119} & 1 & 3.754 & 0.333333333 & 0 & 1 & 1 & 0 & 0 & 0 & 0 \\
\hline & 0 & 10 & 0.466666667 & 0 & 0 & 0 & 1 & 0 & 0 & 0 \\
\hline & 0 & 0.5 & 0.283333333 & 0 & 0 & 0 & 0 & 1 & 0 & 0 \\
\hline & 0 & 1.1 & 0.5 & 0 & 0 & 0 & 0 & 0 & 1 & 0 \\
\hline & 0 & 5 & 0.5 & 0 & 0 & 0 & 0 & 0 & 0 & 1 \\
\hline \multirow[t]{5}{*}{120} & 1 & 3.966 & 0.5 & 3 & 1 & 1 & 0 & 0 & 0 & 0 \\
\hline & 0 & 12.5 & 0.95 & 3 & 0 & 0 & 1 & 0 & 0 & 0 \\
\hline & 0 & 0.7 & 0.583333333 & 3 & 0 & 0 & 0 & 1 & 0 & 0 \\
\hline & 0 & 1.3 & 1.016666667 & 3 & 0 & 0 & 0 & 0 & 1 & 0 \\
\hline & 0 & 5 & 1.016666667 & 3 & 0 & 0 & 0 & 0 & 0 & 1 \\
\hline
\end{tabular}




\begin{tabular}{|c|c|c|c|c|c|c|c|c|c|c|}
\hline \multirow{2}{*}{$\begin{array}{c}\text { Five alternatives } \\
\text { (Number of } \\
\text { observation) }\end{array}$} & Variable & Beta 1 & Beta 2 & Beta 3 & Beta 4 & Beta 5 & Beta 6 & Beta 7 & Beta 8 & Beta 9 \\
\hline & $\begin{array}{c}\text { Mode } \\
\text { Chosen }\end{array}$ & $\begin{array}{c}\text { TC } \\
(1000 \text { Korea won) } \\
\end{array}$ & $\begin{array}{c}\text { TT } \\
\text { (hour) }\end{array}$ & Accom & Sexm & Age 340 & Tasc & Sasc & Basc & Lasc \\
\hline \multirow[t]{5}{*}{121} & 1 & 4.177 & 0.6666666667 & 0 & 1 & 1 & 0 & 0 & 0 & 0 \\
\hline & 0 . & 20 & 1.483333333 & 0 & 0 & 0 & 1 & 0 & 0 & 0 \\
\hline & 0 & 0.6 & 0.916666667 & 0 & 0 & 0 & 0 & 1 & 0 & 0 \\
\hline & 0 & 1.6 & 1.583333333 & 0 & 0 & 0 & 0 & 0 & 1 & 0 \\
\hline & 0 & 5 & 1.583333333 & 0 & 0 & 0 & 0 & 0 & 0 & 1 \\
\hline \multirow[t]{5}{*}{122} & 1 & 3.632 & 0.5 & 0 & 1 & 1 & 0 & 0 & 0 & 0 \\
\hline & 0 & 28 & 1.2 & 0 & 0 & 0 & 1 & 0 & 0 & 0 \\
\hline & 0 & 0.6 & 0.733333333 & 0 & 0 & 0 & 0 & 1 & 0 & 0 \\
\hline & 0 & 1.1 & 1.283333333 & 0 & 0 & 0 & 0 & 0 & 1 & 0 \\
\hline & 0 & 5 & 1.283333333 & 0 & 0 & 0 & $\mathbf{0}$ & 0 & 0 & 1 \\
\hline \multirow[t]{5}{*}{123} & 1 & 4.677 & 0.5 & 0 & 1 & 1 & 0 & 0 & 0 & 0 \\
\hline & 0 & 14 & 2.066666667 & 0 & 0 & 0 & 1 & 0 & 0 & 0 \\
\hline & 0 & 0.75 & 1.283333333 & 0 & 0 & 0 & 0 & 1 & 0 & 0 \\
\hline & 0 & 1.5 & 2.2 & 0 & 0 & 0 & 0 & 0 & 1 & 0 \\
\hline & 0 & 5 & 2.2 & 0 & 0 & 0 & 0 & 0 & 0 & 1 \\
\hline \multirow[t]{5}{*}{124} & 1 & 4.677 & 0.5 & 0 & 1 & 1 & 0 & 0 & 0 & 0 \\
\hline & 0 & 20 & 1.483333333 & 0 & 0 & 0 & 1 & 0 & 0 & 0 \\
\hline & 0 & 0.6 & 0.916666667 & 0 & 0 & 0 & 0 & 1 & 0 & 0 \\
\hline & 0 & 1.6 & 1.583333333 & 0 & 0 & 0 & 0 & 0 & 1 & 0 \\
\hline & 0 & 5 & 1.583333333 & 0 & 0 & 0 & 0 & 0 & 0 & 1 \\
\hline \multirow[t]{5}{*}{125} & 1 & 0.885 & 0.333333333 & 0 & 1 & 1 & 0 & 0 & 0 & 0 \\
\hline & 0 & 6 & 0.183333333 & 0 & 0 & 0 & 1 & 0 & 0 & 0 \\
\hline & 0 & 0.5 & 0.116666667 & 0 & 0 & 0 & 0 & 1 & 0 & 0 \\
\hline & 0 & 0.5 & 0.2 & 0 & 0 & 0 & 0 & 0 & 1 & 0 \\
\hline & 0 & 5 & 0.2 & 0 & 0 & 0 & 0 & 0 & 0 & 1 \\
\hline \multirow[t]{5}{*}{126} & 1 & 53.826 & 1.5 & 0 & 1 & 1 & 0 & 0 & 0 & 0 \\
\hline & 0 & 20 & 0.466666667 & 0 & 0 & 0 & 1 & 0 & 0 & 0 \\
\hline & 0 & 0.7 & 0.283333333 & 0 & 0 & 0 & 0 & 1 & 0 & 0 \\
\hline & 0 & 1.5 & 0.5 & 0 & 0 & 0 & 0 & 0 & 1 & 0 \\
\hline & 0 & 5 & 0.5 & 0 & 0 & 0 & 0 & 0 & 0 & 1 \\
\hline \multirow[t]{5}{*}{127} & 1 & 5.177 & 1.333333333 & 2 & 1 & 1 & 0 & 0 & 0 & 0 \\
\hline & 0 & 20 & 0.466666667 & 2 & 0 & 0 & 1 & 0 & 0 & 0 \\
\hline & 0 & 0.7 & 0.283333333 & 2 & 0 & 0 & 0 & 1 & 0 & 0 \\
\hline & 0 & 1.5 & 0.5 & 2 & 0 & 0 & 0 & 0 & 1 & 0 \\
\hline & 0 & 5 & 0.5 & 0 & 0 & 0 & 0 & 0 & 0 & 1 \\
\hline \multirow[t]{5}{*}{128} & 1 & 2.588 & 0.833333333 & 0 & 1 & 0 & 0 & 0 & 0 & 0 \\
\hline & 0 & 11 & 0.716666667 & 0 & 0 & 0 & 1 & 0 & 0 & 0 \\
\hline & 0 & 0.5 & 0.433333333 & 0 & 0 & 0 & 0 & 1 & 0 & 0 \\
\hline & 0 & 1.3 & 0.7666666667 & 0 & 0 & 0 & 0 & 0 & 1 & 0 \\
\hline & 0 & 5 & 0.766666667 & 0 & 0 & 0 & 0 & 0 & 0 & 1 \\
\hline \multirow[t]{5}{*}{129} & 1 & 2.059 & 0.333333333 & 1 & 1 & 1 & 0 & 0 & 0 & 0 \\
\hline & 0 & 10 & 0.516666667 & 1 & 0 & 0 & 1 & 0 & 0 & 0 \\
\hline & 0 & 0.5 & 0.316666667 & 1 & 0 & 0 & 0 & 1 & 0 & 0 \\
\hline & 0 & 1.1 & 0.566666667 & 1 & 0 & 0 & 0 & 0 & 1 & 0 \\
\hline & 0 & 5 & 0.5666666667 & 1 & 0 & 0 & 0 & 0 & 0 & 1 \\
\hline \multirow[t]{5}{*}{130} & 1 & 5.555 & 0.6666666667 & 0 & 1 & 0 & 0 & 0 & 0 & 0 \\
\hline & 0 & 20 & 1.483333333 & 0 & 0 & 0 & 1 & 0 & 0 & 0 \\
\hline & 0 & 0.6 & 0.9166666667 & 0 & 0 & 0 & 0 & 1 & 0 & 0 \\
\hline & 0 & 1.6 & 1.583333333 & 0 & 0 & 0 & 0 & 0 & 1 & 0 \\
\hline & 0 & 5 & 1.5833333333 & 0 & 0 & 0 & 0 & 0 & 0 & 1 \\
\hline \multirow[t]{5}{*}{131} & 1 & 20.889 & 2 & 2 & 1 & 1 & 0 & 0 & 0 & 0 \\
\hline & 0 & 53 & 2.066666667 & 2 & 0 & 0 & 1 & 0 & 0 & 0 \\
\hline & 0 & 1 & 1.283333333 & 2 & 0 & 0 & 0 & 1 & 0 & 0 \\
\hline & 0 & 7.5 & 2.2 & 2 & 0 & 0 & 0 & 0 & 1 & 0 \\
\hline & 0 & 15 & 2.2 & 2 & 0 . & 0 & 0 & 0 & 0 & 1 \\
\hline \multirow[t]{5}{*}{132} & 1 & 3.118 & 0.583333333 & 0 & 1 & 0 & 0 & 0 & 0 & 0 \\
\hline & 0 & 10 & 0.85 & 0 & 0 & 0 & 1 & 0 & 0 & 0 \\
\hline & 0 & 0.6 & 0.533333333 & 0 & 0 & 0 & 0 & 1 & 0 & 0 \\
\hline & 0 & 1.1 & 0.916666667 & 0 & 0 & 0 & 0 & 0 & 1 & 0 \\
\hline & 0 & 5 & 0.916666667 & 0 & 0 & 0 & 0 & 0 & 0 & 1 \\
\hline
\end{tabular}




\begin{tabular}{|c|c|c|c|c|c|c|c|c|c|c|}
\hline \multirow{2}{*}{$\begin{array}{c}\text { Five alternatives } \\
\text { (Number of } \\
\text { observation) }\end{array}$} & Variable & Beta 1 & Beta 2 & Beta 3 & Beta 4 & Beta 5 & Beta 6 & Beta 7 & Beta 8 & Beta 9 \\
\hline & $\begin{array}{l}\text { Mode } \\
\text { Chosen }\end{array}$ & $\begin{array}{c}\text { TC } \\
\text { (1000 Korea won) }\end{array}$ & $\begin{array}{c}\mathrm{TT} \\
\text { (hour) }\end{array}$ & Accom & Sexm & Age340 & Tasc & Sasc & Basc & Lasc \\
\hline \multirow[t]{5}{*}{133} & 1 & 3.648 & 0.666666667 & 1 & 1 & 1 & 0 & 0 & 0 & 0 \\
\hline & 0 & 28 & 1.2 & 1 & 0 & 0 & 1 & 0 & 0 & 0 \\
\hline & 0 & 0.6 & 0.733333333 & 1 & 0 & 0 & 0 & 1 & 0 & 0 \\
\hline & 0 & 1.1 & 1.283333333 & 1 & 0 & 0 & 0 & 0 & 1 & 0 \\
\hline & 0 & 5 & 1.283333333 & 1 & 0 & 0 & 0 & 0 & 0 & 1 \\
\hline \multirow[t]{5}{*}{134} & 1 & 3.648 & 1 & 4 & 1 & 1 & 0 & 0 & 0 & 0 \\
\hline & 0 & 20 & 1.483333333 & 4 & 0 & 0 & 1 & 0 & 0 & 0 \\
\hline & 0 & 0.6 & 0.916666667 & 4 & 0 & 0 & 0 & 1 & 0 & 0 \\
\hline & 0 & 1.6 & 1.583333333 & 4 & 0 & 0 & 0 & 0 & 1 & 0 \\
\hline & 0 & 5 & 1.583333333 & 4 & 0 & 0 & 0 & 0 & 0 & 1 \\
\hline \multirow[t]{5}{*}{135} & 1 & 3.618 & 0.666666667 & 3 & 1 & 1 & 0 & 0 & 0 & 0 \\
\hline & 0 & 12.052 & 1.533333333 & 3 & 0 & 0 & 1 & 0 & 0 & 0 \\
\hline & 0 & 0.6 & 0.95 & 3 & 0 & 0 & 0 & 1 & 0 & 0 \\
\hline & 0 & 5 & 1.633333333 & 3 & 0 & 0 & 0 & 0 & 1 & 0 \\
\hline & 0 & 5 & 1.633333333 & 3. & 0 & 0 & 0 & 0 & 0 & 1 \\
\hline \multirow[t]{5}{*}{136} & 1 & 5.707 & 1 & 2 & 1 & 0 & 0 & 0 & 0 & 0 \\
\hline & 0 & 20 & 1.483333333 & 2 & 0 & 0 & 1 & 0 & 0 & 0 \\
\hline & 0 & 0.6 & 0.916666667 & 2 & 0 & 0 & 0 & 1 & 0 & 0 \\
\hline & 0 & 1.6 & 1.583333333 & 2 & 0 & 0 & 0 & 0 & 1 & 0 \\
\hline & 0 & 5 & 1.583333333 & 2 & 0 & 0 & 0 & 0 & 0 & 1 \\
\hline \multirow[t]{5}{*}{137} & 1 & 2.588 & 0.416666667 & 1 & 1 & 1 & 0 & 0 & 0 & 0 \\
\hline & 0 & 6 & 0.183333333 & 1 & 0 & 0 & 1 & 0 & 0 & 0 \\
\hline & 0 & 0.5 & 0.116666667 & 1 & 0 & 0 & 0 & 1 & 0 & 0 \\
\hline & 0 & 0.5 & 0.2 & 1 & 0 & 0 & 0 & 0 & 1 & 0 \\
\hline & 0 & 5 & 0.2 & 1 & 0 & 0 & 0 & $\overline{0}$ & 0 & 1 \\
\hline \multirow[t]{5}{*}{138} & 1 & 2.588 & 0.5 & 1 & 1 & 1 & 0 & 0 & 0 & 0 \\
\hline & 0 & 10 & 0.516666667 & 1 & 0 & 0 & 1 & 0 & 0 & 0 \\
\hline & 0 & 0.5 & 0.316666667 & 1 & 0 & 0 & 0 & 1 & 0 & 0 \\
\hline & 0 & 1.1 & 0.566666667 & 1 & 0 & 0 & 0 & 0 & 1 & 0 \\
\hline & 0 & 5 & 0.566666667 & 1 & 0 & 0 & 0 & 0 & 0 & 1 \\
\hline \multirow[t]{5}{*}{139} & 1 & 2.088 & 0.5 & 2 & 1 & 0 & 0 & 0 & 0 & 0 \\
\hline & 0 & 10 & 0.85 & 2 & 0 & 0 & 1 & 0 & 0 & 0 \\
\hline & 0 & 0.6 & 0.533333333 & 2 & 0 & 0 & 0 & 1 & 0 & 0 \\
\hline & 0 & 1.1 & 0.916666667 & 2 & 0 & 0 & 0 & 0 & 1 & 0 \\
\hline & 0 & 5 & 0.916666667 & 2 & 0 & 0 & 0 & 0 & 0 & 1 \\
\hline \multirow[t]{5}{*}{140} & 1 & 6.237 & 1 & 2 & 1 & 1 & 0 & 0 & 0 & 0 \\
\hline & 0 & 17 & 1.15 & 2 & 0 & 0 & 1 & 0 & 0 & 0 \\
\hline & 0 & 0.65 & 0.7 & 2 & 0 & 0 & 0 & 1 & 0 & 0 \\
\hline & 0 & 2 & 1.233333333 & 2 & 0 & 0 & 0 & 0 & 1 & 0 \\
\hline & 0 & 5 & 1.233333333 & 2 & 0 & 0 & 0 & 0 & 0 & 1 \\
\hline \multirow[t]{5}{*}{141} & 1 & 18.689 & 3.5 & 2 & 0 & 1 & 0 & 0 & 0 & 0 \\
\hline & 0 & 53 & 2.066666667 & 2 & 0 & 0 & 1 & 0 & 0 & 0 \\
\hline & 0 & 1 & 1.283333333 & 2 & 0 & 0 & 0 & 1 & 0 & 0 \\
\hline & 0 & 7.5 & 2.2 & 2 & 0 & 0 & 0 & 0 & 1 & 0 \\
\hline & 0 & 15 & 2.2 & 2 & 0 & 0 & 0 & 0 & 0 & 1 \\
\hline \multirow[t]{5}{*}{142} & 1 & 2.618 & 1 & 0 & 1 & 0 & 0 & 0 & 0 & 0 \\
\hline & 0 & 15.5 & 1.333333333 & 0 & 0 & 0 & 1 & 0 & 0 & 0 \\
\hline & 0 & 0.8 & 0.833333333 & 0 & 0 & 0 & 0 & 1 & 0 & 0 \\
\hline & 0 & 1.6 & 1.433333333 & 0 & 0 & 0 & 0 & 0 & 1 & 0 \\
\hline & 0 & 5 & 1.433333333 & 0 & 0 & 0 & 0 & 0 & 0 & 1 \\
\hline \multirow[t]{5}{*}{143} & 1 & 2.688 & 0.333333333 & 0 & 1 & 1 & 0 & 0 & 0 & 0 \\
\hline & 0 & 6 & 0.183333333 & 0 & 0 & 0 & 1 & 0 & 0 & 0 \\
\hline & 0 & 0.5 & 0.116666667 & 0 & 0 & 0 & 0 & 1 & 0 & 0 \\
\hline & 0 & 0.5 & 0.2 & 0 & 0 & 0 & 0 & 0 & 1 & 0 \\
\hline & 0 & 5 & 0.2 & 0 & 0 & 0 & 0 & 0 & 0 & 1 \\
\hline \multirow[t]{5}{*}{144} & 1 & 4.707 & 0.583333333 & 1 & 1 & 1 & 0 & 0 & 0 & 0 \\
\hline & 0 & 15 & 1.1 & 1 & 0 & 0 & 1 & 0 & 0 & 0 \\
\hline & 0 & 0.6 & 0.683333333 & 1 & 0 & 0 & 0 & 1 & 0 & 0 \\
\hline & 0 & 1.3 & 1.183333333 & 1 & 0 & 0 & 0 & 0 & 1 & 0 \\
\hline & 0 & 5 & 1.183333333 & 1 & 0 & 0 & 0 & 0 & 0 & 1 \\
\hline
\end{tabular}




\begin{tabular}{|c|c|c|c|c|c|c|c|c|c|c|}
\hline \multirow{2}{*}{$\begin{array}{c}\text { Five alternatives } \\
\text { (Number of } \\
\text { observation) }\end{array}$} & Variable & Beta 1 & Beta 2 & Beta 3 & Beta 4 & Beta 5 & Beta 6 & Beta 7 & Beta 8 & Beta 9 \\
\hline & $\begin{array}{c}\text { Mode } \\
\text { Chosen }\end{array}$ & $\begin{array}{c}\text { TC } \\
\text { (1000 Korea won) } \\
\end{array}$ & $\begin{array}{c}\text { TT } \\
\text { (hour) }\end{array}$ & Accom & Sexm & Age340 & Tasc & Sasc & Basc & Lasc \\
\hline \multirow[t]{5}{*}{145} & 1 & 3.177 & 1.5 & 0 & 1 & 1 & 0 & 0 & 0 & 0 \\
\hline & 0 & 28 & 1.8666666667 & 0 & 0 & 0 & 1 & 0 & 0 & 0 \\
\hline & 0 & 0.85 & 1.15 & 0 & 0 & 0 & 0 & 1 & 0 & 0 \\
\hline & 0 & 1.5 & 2 & 0 & 0 & 0 & 0 & 0 & 1 & 0 \\
\hline & 0 & 5 & 2 & 0 & 0 & 0 & 0 & 0 & 0 & 1 \\
\hline \multirow[t]{5}{*}{146} & 1 & 3.707 & 0.833333333 & 1 & 1 & 1 & 0 & 0 & 0 & 0 \\
\hline & 0 & 12 & 1.433333333 & 1 & 0 & 0 & 1 & 0 & 0 & 0 \\
\hline & 0 & 0.85 & 0.8833333333 & 1 & 0 & 0 & 0 & 1 & 0 & 0 \\
\hline & 0 & 1.1 & 1.533333333 & 1 & 0 & 0 & 0 & 0 & 1 & 0 \\
\hline & 0. & 5 & 1.533333333 & 1 & 0 & 0 & 0 & 0 & 0 & 1 \\
\hline \multirow{5}{*}{147} & 1 & 3.618 & 1 & 4 & 1 & 0 & 0 & 0 & 0 & 0 \\
\hline & 0 & 10 & 0.616666667 & 4 & 0 & 0 & 1 & 0 & 0 & 0 \\
\hline & 0 & 0.6 & 0.383333333 & 4 & 0 & 0 & 0 & 1 & 0 & 0 \\
\hline & 0 & 1 & 0.6666666667 & 4 & 0 & 0 & 0 & 0 & 1 & 0 \\
\hline & 0 & 5 & 0.6666666667 & 4 & 0 & 0 & 0 & 0 & 0 & 1 \\
\hline \multirow[t]{5}{*}{148} & 1 & 6.508 & 1 & 3 & 0 & 0 & 0 & 0 & 0 & 0 \\
\hline & 0 & 12 & 1.433333333 & 3 & 0 & 0 & 1 & 0 & 0 & 0 \\
\hline & 0 & 0.85 & 0.883333333 & 3 & 0 & 0 & 0 & 1 & 0 & 0 \\
\hline & 0 & 1.1 & 1.533333333 & 3 & 0 & 0 & 0 & 0 & 1 & 0 \\
\hline & 0 & 5 & 1.533333333 & 3 & 0 & 0 & 0 & 0 & 0 & 1 \\
\hline \multirow[t]{5}{*}{149} & 1 & 3.177 & 1 & 3 & 0 & 0 & 0 & 0 & 0 & 0 \\
\hline & 0 & 28 & 1.2 & 3 & 0 & 0 & 1 & 0 & 0 & 0 \\
\hline & 0 & 0.6 & 0.733333333 & 3 & 0 & 0 & 0 & 1 & 0 & 0 \\
\hline & 0 & 1.1 & 1.283333333 & 3 & 0 & 0 & 0 & 0 & 1 & 0 \\
\hline & 0 & 5 & 1.283333333 & 3 & 0 & 0 & 0 & 0 & 0 & 1 \\
\hline \multirow[t]{5}{*}{150} & 1 & 3.677 & 1 & 2 & 0 & 1 & 0 & 0 & 0 & 0 \\
\hline & 0 & 12 & 1.433333333 & 2 & 0 & 0 & 1 & 0 & 0 & 0 \\
\hline & 0 & 0.9 & 0.883333333 & 2 & 0 & 0 & 0 & 1 & 0 & 0 \\
\hline & 0 & 1.1 & 1.533333333 & 2 & 0 & 0 & 0 & 0 & 1 & 0 \\
\hline & 0 & 5 & 1.533333333 & 2 & 0 & 0 & 0 & 0 & 0 & 1 \\
\hline \multirow[t]{5}{*}{151} & 1 & 2.118 & 4.6666666667 & 1 & 1 & 0 & 0 & 0 & 0 & 0 \\
\hline & 0 & 28 & 1.8666666667 & 1 & 0 & 0 & 1 & 0 & 0 & 0 \\
\hline & 0 & 0.85 & 1.15 & 1 & 0 & 0 & 0 & 1 & 0 & 0 \\
\hline & 0 & 1.5 & 2 & 1 & 0 & 0 & 0 & 0 & 1 & 0 \\
\hline & 0 & 5 & 2 & 1 & 0 & 0 & 0 & 0 & 0 & 1 \\
\hline \multirow[t]{5}{*}{152} & 1 & 4.237 & 2 & 1 & 0 & 1 & 0 & 0 & 0 & 0 \\
\hline & 0 & 12 & 1.433333333 & 1 & 0 & 0 & 1 & 0 & 0 & 0 \\
\hline & 0 & 0.85 & 0.883333333 & 1 & 0 & 0 & 0 & 1 & 0 & 0 \\
\hline & 0 & 1.1 & 1.533333333 & 1 & 0 & 0 & 0 & 0 & 1 & 0 \\
\hline & 0 & 5 & 1.533333333 & 1 & 0 & 0 & 0 & 0 & 0 & 1 \\
\hline \multirow[t]{5}{*}{153} & 1 & 4.237 & 0.6666666667 & 1 & 0 & 1 & 0 & 0 & 0 & 0 \\
\hline & 0 & 20 & 0.4666666667 & 1 & 0 & 0 & 1 & 0 & 0 & 0 \\
\hline & 0 & 0.7 & 0.283333333 & 1 & 0 & 0 & 0 & 1 & 0 & 0 \\
\hline & 0 & 1.5 & 0.5 & 1 & 0 & 0 & 0 & 0 & 1 & 0 \\
\hline & 0 & 5 & 0.5 & 1 & 0 & 0 & 0 & 0 & 0 & 1 \\
\hline \multirow[t]{5}{*}{154} & 1 & 3.118 & 0.3333333333 & 3 & 1 & 0 & 0 & 0 & 0 & 0 \\
\hline & 0 & 10 & 0.5166666667 & 3 & 0 & 0 & 1 & 0 & 0 & 0 \\
\hline & 0 & 0.5 & 0.3166666667 & 3 & 0 & 0 & 0 & 1 & 0 & 0 \\
\hline & 0 & 1.1 & 0.5666666667 & 3 & 0 & 0 & 0 & 0 & 1 & 0 \\
\hline & 0 & 5 & 0.5666666667 & 3 & 0 & 0 & 0 & 0 & 0 & 1 \\
\hline \multirow[t]{5}{*}{155} & 1 & 4.059 & 0.333333333 & 4 & 0 & 0 & 0 & 0 & 0 & 0 \\
\hline & 0 & 14 & 2.0666666667 & 4 & 0 & 0 & 1 & 0 & 0 & 0 \\
\hline & 0 & 0.75 & 1.283333333 & 4 & 0 & 0 & 0 & 1 & 0 & 0 \\
\hline & 0 & 1.5 & 2.2 & 4 & 0 & 0 & 0 & 0 & 1 & 0 \\
\hline & 0 & 5 & 2.2 & 4 & 0 & 0 & 0 & 0 & 0 & 1 \\
\hline \multirow[t]{5}{*}{156} & 1 & 3.177 & 0.5 & 1 & 0 & 1 & 0 & 0 & 0 & 0 \\
\hline & 0 & 10 & 0.9 & 1 & 0 & 0 & 1 & 0 & 0 & 0 \\
\hline & 0 & 0.6 & 0.5666666667 & 1 & 0 & 0 & 0 & 1 & 0 & 0 \\
\hline & 0 & 1.3 & 0.966666667 & 1 & 0 & 0 & 0 & 0 & 1 & 0 \\
\hline & 0 & 5 & 0.966666667 & 1 & 0 & 0 & 0 & 0 & 0 & 1 \\
\hline
\end{tabular}




\begin{tabular}{|c|c|c|c|c|c|c|c|c|c|c|}
\hline \multirow{2}{*}{$\begin{array}{c}\text { Five alternatives } \\
\text { (Number of } \\
\text { observation) }\end{array}$} & Variable & Beta 1 & Beta 2 & Beta 3 & Beta 4 & Beta 5 & Beta 6 & Beta 7 & Beta 8 & Beta 9 \\
\hline & $\begin{array}{l}\text { Mode } \\
\text { Chosen }\end{array}$ & $\begin{array}{c}\text { TC } \\
\text { (1000 Korea won) } \\
\end{array}$ & $\begin{array}{c}\mathrm{TT} \\
\text { (hour) }\end{array}$ & Accom & Sexm & Age 340 & Tasc & Sasc & Basc & Lasc \\
\hline \multirow[t]{5}{*}{157} & 1 & 6.237 & 1.5 & 3 & 0 & 0 & 0 & 0 & 0 & 0 \\
\hline & 0 & 20 & 0.466666667 & 3 & 0 & 0 & 1 & 0 & 0 & 0 \\
\hline & 0 & 0.7 & 0.283333333 & 3 & 0 & 0 & 0 & 1 & 0 & 0 \\
\hline & 0 & 1.5 & 0.5 & 3 & 0 & 0 & 0 & 0 & 1 & 0 \\
\hline & 0 & 5 & 0.5 & 3 & 0 & 0 & 0 & 0 & 0 & 1 \\
\hline \multirow[t]{5}{*}{158} & 1 & 4.177 & 1 & 1 & 1 & 0 & 0 & 0 & 0 & 0 \\
\hline & 0 & 12 & 1.433333333 & 1 & 0 & 0 & 1 & 0 & 0 & 0 \\
\hline & 0 & 0.9 & 0.883333333 & 1 & 0 & 0 & 0 & 1 & 0 & 0 \\
\hline & 0 & 1.1 & 1.533333333 & 1 & 0 & 0 & 0 & 0 & 1 & 0 \\
\hline & 0 & 5 & 1.5333333333 & 1 & 0 & 0 & 0 & 0 & 0 & 1 \\
\hline \multirow[t]{5}{*}{159} & 1 & 3.343 & 0.5 & 1 & 1 & 1 & 0 & 0 & 0 & 0 \\
\hline & 0 & 28 & 1.8666666667 & 1 & 0 & 0 & 1 & 0 & 0 & 0 \\
\hline & 0 & 0.85 & 1.15 & 1 & 0 & 0 & 0 & 1 & 0 & 0 \\
\hline & 0 & 1.5 & 2 & 1 & 0 & 0 & 0 & 0 & 1 & 0 \\
\hline & 0 & 5 & 2 & 1 & 0 & 0 & 0 & 0 & 0 & 1 \\
\hline \multirow[t]{5}{*}{160} & 1 & 3.389 & 0.833333333 & 1 & 1 & 0 & 0 & 0 & 0 & 0 \\
\hline & 0 & 28 & 1.2 & 1 & 0 & 0 & 1 & 0 & 0 & 0 \\
\hline & 0 & 0.6 & 0.733333333 & 1 & 0 & 0 & 0 & 1 & 0 & 0 \\
\hline & 0 & 1.1 & 1.283333333 & 1 & 0 & 0 & 0 & 0 & 1 & 0 \\
\hline & 0 & 5 & 1.283333333 & 1 & 0 & 0 & 0 & 0 & 0 & 1 \\
\hline \multirow[t]{5}{*}{161} & 1 & 14.3 & 3.5 & 2 & 0 & 1 & 0 & 0 & 0 & 0 \\
\hline & 0 & 53 & 2.066666667 & 2 & 0 & 0 & 1 & 0 & 0 & 0 \\
\hline & 0 & 1.1 & 1.283333333 & 2 & 0 & 0 & 0 & 1 & 0 & 0 \\
\hline & 0 & 7.5 & 2.2 & 2 & 0 & 0 & 0 & 0 & 1 & 0 \\
\hline & 0 & 15 & 2.2 & 2 & 0 & 0 & 0 & 0 & 0 & 1 \\
\hline \multirow[t]{5}{*}{162} & 1 & 7.437 & 1.5 & 3 & 1 & 1 & 0 & 0 & 0 & 0 \\
\hline & 0 & 12 & 1.433333333 & 3 & 0 & 0 & 1 & 0 & 0 & 0 \\
\hline & 0 & 0.85 & 0.883333333 & 3 & 0 & 0 & 0 & 1 & 0 & 0 \\
\hline & 0 & 1.1 & 1.533333333 & 3 & 0 & 0 & 0 & 0 & 1 & 0 \\
\hline & 0 & 5 & 1.5333333333 & 3 & 0 & 0 & 0 & 0 & 0 & 1 \\
\hline \multirow[t]{5}{*}{163} & 1 & 5.177 & 1 & 4 & 1 & 0 & 0 & 0 & 0 & 0 \\
\hline & 0 & 28 & 1.866666667 & 4 & 0 & 0 & 1 & 0 & 0 & 0 \\
\hline & 0 & 0.85 & 1.15 & 4 & 0 & 0 & 0 & 1 & 0 & 0 \\
\hline & 0 & 1.5 & 2 & 4 & 0 & 0 & 0 & 0 & 1 & 0 \\
\hline & 0 & 5 & 2 & 4 & 0 & 0 & 0 & 0 & 0 & 1 \\
\hline \multirow[t]{5}{*}{164} & 1 & 123.177 & 1.5 & 4 & 0 & 0 & 0 & 0 & 0 & 0 \\
\hline & 0 & 28 & 1.866666667 & 4 & 0 & 0 & 1 & 0 & 0 & 0 \\
\hline & 0 & 0.85 & 1.15 & 4 & 0 & 0 & 0 & 1 & 0 & 0 \\
\hline & 0 & 1.5 & 2 & 4 & 0 & 0 & 0 & 0 & 1 & 0 \\
\hline & 0 & 5 & 2 & 4 & 0 & 0 & 0 & 0 & 0 & 1 \\
\hline \multirow[t]{5}{*}{165} & 1 & 3.177 & 2 & 5 & 1 & 1 & 0 & 0 & 0 & 0 \\
\hline & 0 & 14 & 2,066666667 & 5 & 0 & 0 & 1 & 0 & 0 & 0 \\
\hline & 0 & 0.75 & 1.2833333333 & 5 & 0 & 0 & 0 & 1 & 0 & 0 \\
\hline & 0 & 1.5 & 2.2 & 5 & 0 & 0 & 0 & 0 & 1 & 0 \\
\hline & 0 & 5 & 2.2 & 5 & 0 & 0 & 0 & 0 & 0 & 1 \\
\hline \multirow[t]{5}{*}{166} & 1 & 3.51 & 0.5 & 4 & 1 & 1 & 0 & 0 & 0 & 0 \\
\hline & 0 & 15 & 0.816666667 & 4 & 0 & 0 & 1 & 0 & 0 & 0 \\
\hline & 0 & 0.6 & 0.5 & 4 & 0 & 0 & 0 & 1 & 0 & 0 \\
\hline & 0 & 1.5 & 0.8666666667 & 4 & 0 & 0 & 0 & 0 & 1 & 0 \\
\hline & 0 & 5 & 0.8666666667 & 4 & 0 & 0 & 0 & 0 & 0 & 1 \\
\hline \multirow[t]{5}{*}{167} & 1 & 5.296 & 0.666666667 & 1 & 1 & 1 & 0 & 0 & 0 & 0 \\
\hline & 0 & 28 & 1.2 & 1 & 0 & 0 & 1 & 0 & 0 & 0 \\
\hline & 0 & 0.6 & 0.733333333 & 1 & 0 & 0 & 0 & 1 & 0 & 0 \\
\hline & 0 & 1.1 & 1.283333333 & 1 & 0 & 0 & 0 & 0 & 1 & 0 \\
\hline & 0 & 5 & 1.283333333 & 1 & 0 & 0 & 0 & 0 & 0 & 1 \\
\hline \multirow[t]{5}{*}{168} & 1 & 3.436 & 0.5 & 5 & $\overline{0}$ & 1 & 0 & 0 & 0 & 0 \\
\hline & 0 & 7.6 & 0.616666667 & 5 & 0 & 0 & 1 & 0 & 0 & 0 \\
\hline & 0 & 0.6 & 0.383333333 & 5 & 0 & 0 & 0 & 1 & 0 & 0 \\
\hline & 0 & 1.6 & 0.666666667 & 5 & 0 & 0 & 0 & 0 & 1 & 0 \\
\hline & 0 & 5 & 0.666666667 & 5 & 0 & 0 & 0 & 0 & 0 & 1 \\
\hline
\end{tabular}




\begin{tabular}{|c|c|c|c|c|c|c|c|c|c|c|}
\hline \multirow{2}{*}{$\begin{array}{l}\text { Five alternatives } \\
\text { (Number of } \\
\text { observation) }\end{array}$} & Variable & Beta 1 & Beta 2 & Beta 3 & Beta 4 & Beta 5 & Beta 6 & Beta 7 & Beta 8 & Beta 9 \\
\hline & $\begin{array}{c}\text { Mode } \\
\text { Chosen }\end{array}$ & $\begin{array}{c}\text { TC } \\
\text { (1000 Korea won) }\end{array}$ & $\begin{array}{c}\mathrm{TT} \\
\text { (hour) }\end{array}$ & Accom & Sexm & Age340 & Tasc & Sasc & Basc & Lasc \\
\hline \multirow[t]{5}{*}{169} & 1 & 1.588 & 0.5 & 1 & 1 & 0 & 0 & 0 & 0 & 0 \\
\hline & 0 & 15 & 0.8166666667 & 1 & 0 & 0 & $T$ & 0 & 0 & 0 \\
\hline & 0 & 0.6 & 0.5 & 1 & 0 & 0 & 0 & 1 & 0 & 0 \\
\hline & 0 & 1.5 & 0.8666666667 & 1 & 0 & 0 & 0 & 0 & 1 & 0 \\
\hline & 0 & 5 & 0.8666666667 & 1 & 0 & 0 & 0 & 0 & 0 & 1 \\
\hline \multirow[t]{5}{*}{170} & 1 & 2.436 & 1 & 1 & 1 & 0 & 0 & $\overline{0}$ & $\overline{0}$ & $\overline{0}$ \\
\hline & 0 & 20 & 1.483333333 & 1 & 0 & 0 & 1 & $\overline{0}$ & $\overline{0}$ & $\overline{0}$ \\
\hline & 0 & 0.6 & 0.916666667 & 1 & 0 & 0 & $\overline{0}$ & $\overline{1}$ & $\overline{0}$ & 0 \\
\hline & $\overline{0}$ & 1.6 & 1.583333333 & $T$ & $\overline{0}$ & 0 & $\overline{0}$ & 0 & 1 & 0 \\
\hline & 0 & 5 & 1.583333333 & 1 & 0 & 0 & 0 & 0 & 0 & 1 \\
\hline \multirow[t]{5}{*}{171} & 1 & 2.118 & 1 & 0 & 1 & 1 . & 0 & 0 & 0 & 0 \\
\hline & 0 & 20 & 0.4666666667 & 0 & 0 & 0 & 1 & 0 & 0 & 0 \\
\hline & 0 & 0.7 & 0.283333333 & 0 & 0 & 0 & 0 & 1 & 0 & 0 \\
\hline & 0 & 1.5 & 0.5 & 0 & 0 & 0 & 0 & 0 & 1 & 0 \\
\hline & 0 & 5 & 0.5 & 0 & 0 & 0 & 0 & 0 & 0 & 1 \\
\hline \multirow[t]{5}{*}{172} & 1 & 3.389 & 0.5 & 1 & 0 & 0 & 0 & 0 & 0 & 0 \\
\hline & 0 & 15 & 0.816666667 & 1 & 0 & 0 & 1 & 0 & 0 & 0 \\
\hline & 0 & 0.6 & 0.5 & 1 & 0 & 0 & 0 & 1 & 0 & 0 \\
\hline & 0 & 1.5 & 0.866666667 & 1 & 0 & 0 & 0 & 0 & 1 & 0 \\
\hline & 0 & 5 & 0.866666667 & 1 & 0 & 0 & 0 & 0 & 0 & 1 \\
\hline \multirow[t]{5}{*}{173} & 1 & 2.648 & 0.416666667 & 0 & 1 & 1 & 0 & 0 & 0 & 0 \\
\hline & 0 & 10 & 0.516666667 & 0 & 0 & 0 & 1 & 0 & 0 & 0 \\
\hline & 0 & 0.5 & 0.316666667 & 0 & 0 & 0 & 0 & 1 & 0 & 0 \\
\hline & 0 & 1.1 & 0.566666667 & 1 & 0 & 0 & 0 & 0 & 1 & 0 \\
\hline & 0 & 5 & 0.566666667 & 1 & 0 & 0 & 0 & 0 & 0 & 1 \\
\hline \multirow[t]{5}{*}{174} & 1 & 3.177 & 0.5 & 0 & 0 & 0 & 0 & 0 & 0 & 0 \\
\hline & 0 & 12 & 1.433333333 & 0 & 0 & 0 & 1 & 0 & 0 & 0 \\
\hline & 0 & 0.9 & 0.883333333 & 0 & 0 & 0 & 0 & 1 & 0 & 0 \\
\hline & 0 & 1.1 & 1.533333333 & 0 & 0 & 0 & 0 & 0 & 1 & 0 \\
\hline & 0 & 5 & 1.533333333 & 0 & 0 & 0 & 0 & 0 & 0 & 1 \\
\hline \multirow[t]{5}{*}{175} & 1 & 5.555 & 0.666666667 & 3 & 0 & 1 & 0 & 0 & 0 & 0 \\
\hline & 0 & 12 & 1.433333333 & 3 & 0 & 0 & 1 & 0 & 0 & 0 \\
\hline & 0 & 0.85 & 0.883333333 & 3 & 0 & 0 & 0 & 1 & 0 & 0 \\
\hline & 0 & 1.1 & 1.533333333 & 3. & 0 & 0 & 0 & 0 & 1 & 0 \\
\hline & 0 & 5 & 1.533333333 & 3 & 0 & 0 & 0 & 0 & 0 & 1 \\
\hline \multirow[t]{5}{*}{176} & 1 & 4.177 & 0.833333333 & 4 & 0 & 0 & 0 & 0 & 0 & 0 \\
\hline & 0 & 12 & 1.433333333 & 4 & 0 & 0 & 1 & 0 & 0 & 0 \\
\hline & 0 & 0.9 & 0.883333333 & 4 & 0 & 0 & 0 & 1 & 0 & 0 \\
\hline & 0 & 1.1 & 1.533333333 & 4 & 0 & 0 & 0 & 0 & 1 & 0 \\
\hline & 0 & 5 & 1.533333333 & 4 & 0 & 0 & 0 & 0 & 0 & 1 \\
\hline \multirow[t]{5}{*}{177} & 1 & 0.423 & 0.25 & 0 & 1 & 0 & 0 & 0 & 0 & 0 \\
\hline & 0 & 6 & 0.183333333 & 0 & 0 & 0 & 1 & 0 & 0 & 0 \\
\hline & 0 & 0.5 & 0.1166666667 & 0 & 0 & 0 & 0 & 1 & 0 & 0 \\
\hline & 0 & 0.5 & 0.2 & 0 & 0 & 0 & 0 & 0 & 1 & 0 \\
\hline & 0 & 5 & 0.2 & 0 & 0 & 0 & 0 & 0 & 0 & 1 \\
\hline \multirow[t]{5}{*}{178} & 1 & 0.423 & 0.25 & 2 & 1 & 0 & 0 & 0 & 0 & 0 \\
\hline & 0 & 6 & 0.183333333 & 2 & 0 & 0 & 1 & 0 & 0 & 0 \\
\hline & 0 & 0.5 & 0.116666667 & 2 & 0 & 0 & 0 & 1 & 0 & 0 \\
\hline & 0 & 0.5 & 0.2 & 2 & 0 & 0 & 0 & 0 & 1 & 0 \\
\hline & 0 & 5 & 0.2 & 2 & 0 & 0 & 0 & 0 & 0 & 1 \\
\hline \multirow[t]{5}{*}{179} & 1 & 2.118 & 1 & 0 & 1 & 1 & 0 & 0 & 0 & 0 \\
\hline & 0 & 20 & 0.4666666667 & 0 & 0 & 0 & 1 & 0 & 0 & 0 \\
\hline & 0 & 0.7 & 0.283333333 & 0 & 0 & 0 & 0 & 1 & 0 & 0 \\
\hline & 0 & 1.5 & 0.5 & 0 & 0 & 0 & 0 & 0 & 1 & 0 \\
\hline & 0 & 5 & 0.5 & 0 & 0 & 0 & 0 & 0 & 0 & 1 \\
\hline \multirow[t]{5}{*}{180} & 1 & 3.177 & 0.6666666667 & 2 & 1 & 1 & 0 & 0 & 0 & 0 \\
\hline & 0 & 12 & 1.433333333 & 2 & 0 & 0 & 1 & 0 & 0 & 0 \\
\hline & 0 & 0.9 & 0.883333333 & 2 & 0 & 0 & 0 & 1 & 0 & 0 \\
\hline & 0 & 1.1 & 1.533333333 & 2 & 0 & 0 & 0 & 0 & 1 & 0 \\
\hline & 0 & 5 & 1.533333333 & 2 & 0 & 0 & 0 & 0 & 0 & 1 \\
\hline
\end{tabular}




\begin{tabular}{|c|c|c|c|c|c|c|c|c|c|c|}
\hline \multirow{2}{*}{$\begin{array}{c}\text { Five alternatives } \\
\text { (Number of } \\
\text { observation) }\end{array}$} & Variable & Beta 1 & Beta 2 & Beta 3 & Beta 4 & Beta 5 & Beta 6 & Beta 7 & Beta 8 & Beta 9 \\
\hline & $\begin{array}{c}\text { Mode } \\
\text { Chosen }\end{array}$ & $\begin{array}{c}\text { TC } \\
\text { (1000 Korea won) }\end{array}$ & $\begin{array}{c}\text { TT } \\
\text { (hour) }\end{array}$ & Accom & Sexm & Age 340 & Tasc & Sasc & Basc & Lasc \\
\hline \multirow[t]{5}{*}{181} & 1 & 12.593 & 1.5 & 4 & 1 & 0 & 0 & 0 & 0 & 0 \\
\hline & 0 & 20 & 1.483333333 & 4 & 0 & 0 & 1 & 0 & 0 & 0 \\
\hline & 0 & 0.6 & 0.9166666667 & 4 & 0 & 0 & 0 & 1 & 0 & 0 \\
\hline & 0 & 1.6 & 1.5833333333 & 4 & 0 & 0 & 0 & 0 & 1 & 0 \\
\hline & 0 & 5 & 1.5833333333 & 4 & 0 & 0 & 0 & 0 & 0 & 1 \\
\hline \multirow[t]{5}{*}{182} & 1 & 7.915 & 0.6666666667 & 6 & 0 & 1 & 0 & 0 & 0 & 0 \\
\hline & 0 & 10 & 0.6166666667 & 6 & 0 & 0 & 1 & 0 & 0 & 0 \\
\hline & 0 & 0.6 & 0.383333333 & 6 & 0 & 0 & 0 & 1 & 0 & 0 \\
\hline & 0 & 1 & 0.6666666667 & 6 & 0 & 0 & 0 & 0 & 1 & 0 \\
\hline & 0 & 5 & 0.666666667 & 6 & 0 & 0 & 0 & 0 & 0 & 1 \\
\hline \multirow[t]{5}{*}{183} & 1 & 2.588 & 0.166666667 & 4 & 0 & 0 & 0 & 0 & 0 & 0 \\
\hline & 0 & 6 & 0.183333333 & 4 & 0 & 0 & 1 & 0 & 0 & 0 \\
\hline & 0 & 0.5 & 0.116666667 & 4 & 0 & 0 & 0 & 1 & 0 & 0 \\
\hline & 0 & 0.5 & 0.2 & 4 & 0 & 0 & 0 & 0 & 1 & 0 \\
\hline & 0 & 5 & 0.2 & 4 & 0 & 0 & 0 & 0 & 0 & 1 \\
\hline \multirow[t]{5}{*}{184} & 1 & 3.118 & 0.5 & 3 & 0 & 1 & 0 & 0 & 0 & 0 \\
\hline & 0 & 10 & 0.5166666667 & 3 & 0 & 0 & 1 & 0 & 0 & 0 \\
\hline & 0 & 0.5 & 0.316666667 & 3 & 0 & 0 & 0 & 1 & 0 & 0 \\
\hline & 0 & 1.1 & 0.5666666667 & 3 & 0 & 0 & 0 & 0 & 1 & 0 \\
\hline & 0 & 5 & 0.5666666667 & 3 & 0 & 0 & 0 & 0 & 0 & 1 \\
\hline \multirow[t]{5}{*}{185} & 1 & 2.618 & 0.6666666667 & 2 & 1 & 1 & 0 & 0 & 0 & 0 \\
\hline & 0 & 20 & 0.466666667 & 2 & 0 & 0 & 1 & 0 & 0 & 0 \\
\hline & 0 & 0.7 & 0.283333333 & 2 & 0 & 0 & 0 & 1 & 0 & 0 \\
\hline & 0 & 1.5 & 0.5 & 2 & 0 & 0 & 0 & 0 & 1 & 0 \\
\hline & 0 & 5 & 0.5 & 2 & 0 & 0 & 0 & 0 & 0 & 1 \\
\hline \multirow[t]{5}{*}{186} & 1 & 9.296 & 1 & 4 & 0 & 1 & 0 & 0 & 0 & 0 \\
\hline & 0 & 28 & 1.866666667 & 4 & 0 & 0 & 1 & 0 & 0 & 0 \\
\hline & 0 & 0.85 & 1.15 & 4 & 0 & 0 & 0 & 1 & 0 & 0 \\
\hline & 0 & 1.5 & 2 & 4 & 0 & 0 & 0 & 0 & 1 & 0 \\
\hline & 0 & 5 & 2 & 4 & 0 & 0 & 0 & 0 & 0 & 1 \\
\hline \multirow[t]{5}{*}{187} & 1 & 4.588 & 0.666666667 & 3 & 0 & 1 & 0 & 0 & 0 & 0 \\
\hline & 0 & 11 & 0.716666667 & 3 & 0 & 0 & 1 & 0 & 0 & 0 \\
\hline & 0 & 0.5 & 0.433333333 & 3 & 0 & 0 & 0 & 1 & 0 & 0 \\
\hline & 0 & 1.3 & 0.7666666667 & 3 & 0 & 0 & 0 & 0 & 1 & 0 \\
\hline & 0 & 5 & 0.766666667 & 3 & 0 & 0 & 0 & 0 & 0 & 1 \\
\hline \multirow[t]{5}{*}{188} & 1 & 10.593 & 3 & 2 & 0 & 1 & 0 & 0 & 0 & 0 \\
\hline & 0 & 53 & 2.0666666667 & 2 & 0 & 0 & 1 & 0 & 0 & 0 \\
\hline & 0 & 1 & 1.283333333 & 2 & 0 & 0 & 0 & 1 & 0 & 0 \\
\hline & 0 & 7.5 & 2.2 & 2 & 0 & 0 & 0 & 0 & 1 & 0 \\
\hline & 0 & 15 & 2.2 & 2 & 0 & 0 & 0 & 0 & 0 & 1 \\
\hline \multirow[t]{5}{*}{189} & 1 & 3.177 & 0.6666666667 & 2 & 0 & 0 & 0 & 0 & 0 & 0 \\
\hline & 0 & 12 & 1.4333333333 & 2 & 0 & 0 & 1 & 0 & 0 & 0 \\
\hline & 0 & 0.9 & 0.883333333 & 2 & 0 & 0 & 0 & 1 & 0 & 0 \\
\hline & 0 & 1.1 & 1.533333333 & 2 & 0 & 0 & 0 & 0 & 1 & 0 \\
\hline & 0 & 5 & 1.533333333 & 2 & 0 & 0 & 0 & 0 & 0 & 1 \\
\hline \multirow[t]{5}{*}{190} & 1 & 3.389 & 0.6666666667 & 20 & 1 & 1 & 0 & 0 & 0 & 0 \\
\hline & 0 & 28 & 1.2 & 20 & 0 & 0 & 1 & 0 & 0 & 0 \\
\hline & 0 & 0.6 & 0.733333333 & 20 & 0 & 0 & 0 & 1 & 0 & 0 \\
\hline & 0 & 1.1 & 1.283333333 & 20 & 0 & 0 & 0 & 0 & 1 & 0 \\
\hline & 0 & 5 & 1.283333333 & 20 & 0 & 0 & 0 & 0 & 0 & 1 \\
\hline \multirow[t]{5}{*}{191} & 1 & 6.355 & 1.5 & 2 & 0 & 0 & 0 & 0 & 0 & 0 \\
\hline & 0 & 20 & 1.483333333 & 2 & 0 & 0 & 1 & 0 & 0 & 0 \\
\hline & 0 & 0.6 & 0.916666667 & 2 & 0 & 0 & 0 & 1 & 0 & 0 \\
\hline & 0 & 1.6 & 1.583333333 & 2 & 0 & 0 & 0 & 0 & 1 & 0 \\
\hline & 0 & 5 & 1.583333333 & 2 & 0 & 0 & 0 & 0 & 0 & 1 \\
\hline \multirow[t]{5}{*}{192} & 1 & 3.648 & 3 & 10 & 1 & 0 & 0 & 0 & 0 & 0 \\
\hline & 0 & 20 & 0.4666666667 & 10 & 0 & 0 & 1 & 0 & 0 & 0 \\
\hline & 0 & 0.7 & 0.2833333333 & 10 & 0 & 0 & 0 & 1 & 0 & 0 \\
\hline & 0 & 1.5 & 0.5 & 10 & 0 & 0 & 0 & 0 & 1 & 0 \\
\hline & 0 & 5 & 0.5 & 10 & 0 & 0 & 0 & 0 & 0 & 1 \\
\hline
\end{tabular}




\begin{tabular}{|c|c|c|c|c|c|c|c|c|c|c|}
\hline \multirow{2}{*}{$\begin{array}{c}\text { Five alternatives } \\
\text { (Number of } \\
\text { observation) }\end{array}$} & Variable & Beta 1 & Beta 2 & Beta 3 & Beta 4 & Beta 5 & Beta 6 & Beta 7 & Beta 8 & Beta 9 \\
\hline & $\begin{array}{l}\text { Mode } \\
\text { Chosen }\end{array}$ & $\begin{array}{c}\text { TC } \\
\text { (1000 Korea won) }\end{array}$ & $\begin{array}{c}\text { TT } \\
\text { (hour) }\end{array}$ & Accom & Sexm & Age340 & Tasc & Sasc & Basc & Lasc \\
\hline \multirow[t]{5}{*}{193} & 1 & 5.796 & 1 & 2 & 1 & 1 & 0 & 0 & 0 & 0 \\
\hline & 0 & 12 & 1.433333333 & 2 & 0 & 0 & 1 & 0 & 0 & 0 \\
\hline & 0 & 0.85 & 0.883333333 & 2 & 0 & 0 & 0 & $\overline{1}$ & 0 & 0 \\
\hline & 0 & 1.1 & 1.533333333 & 2 & 0 & 0 & 0 & 0 & 1 & 0 \\
\hline & 0 & 5 & 1.533333333 & 2 & 0 & 0 & 0 & 0 & 0 & 1 \\
\hline \multirow[t]{5}{*}{194} & 1 & 5.737 & 0.666666667 & 4 & 1 & 0 & 0 & 0 & 0 & 0 \\
\hline & 0 & 7.5 & 1.1 & 4 & 0 & 0 & 1 & 0 & 0 & 0 \\
\hline & 0 & 0.7 & 0.683333333 & 4 & 0 & 0 & 0 & 1 & 0 & 0 \\
\hline & 0 & 1.3 & 1.183333333 & 4 & 0 & 0 & 0 & 0 & 1 & 0 \\
\hline & 0 & 5 & 1.183333333 & 4 & 0 & 0 & 0 & 0 & 0 & 1 \\
\hline \multirow[t]{5}{*}{195} & 1 & 2.648 & 0.833333333 & 0 & 1 & 0 & 0 & 0 & 0 & 0 \\
\hline & 0 & 28 & 1.8666666667 & 0 & 0 & 0 & 1 & 0 & 0 & 0 \\
\hline & 0 & 0.85 & 1.15 & 0 & 0 & 0 & 0 & 1 & 0 & 0 \\
\hline & 0 & 1.5 & 2 & 0 & 0 & 0 & 0 & 0 & 1 & 0 \\
\hline & 0 & 5 & 2 & 0 & 0 & 0 & 0 & 0 & 0 & 1 \\
\hline \multirow[t]{5}{*}{196} & 1 & 2.059 & 0.5 & 3 & 1 & 0 & 0 & 0 & 0 & 0 \\
\hline & 0 & 6 & 0.183333333 & 3 & 0 & 0 & 1 & 0 & 0 & 0 \\
\hline & 0 & 0.5 & 0.116666667 & 3 & 0 & 0 & 0 & 1 & 0 & 0 \\
\hline & 0 & 0.5 & 0.2 & 3 & 0 & 0 & 0 & 0 & 1 & 0 \\
\hline & 0 & 5 & 0.2 & 3 & 0 & 0 & 0 & 0 & 0 & 1 \\
\hline \multirow[t]{5}{*}{197} & 1 & 7.237 & 1.5 & 2 & 0 & 0 & 0 & 0 & 0 & 0 \\
\hline & 0 & 20 & 0.4666666667 & 2 & 0 & 0 & 1 & 0 & 0 & 0 \\
\hline & 0 & 0.7 & 0.283333333 & 2 & 0 & 0 & 0 & 1 & 0 & 0 \\
\hline & 0 & 1.5 & 0.5 & 2 & 0 & 0 & 0 & 0 & 1 & 0 \\
\hline & 0 & 5 & 0.5 & 2 & 0 & 0 & 0 & 0 & 0 & 1 \\
\hline \multirow[t]{5}{*}{198} & 1 & 2.588 & 1 & 2 & 0 & 0 & 0 & 0 & 0 & 0 \\
\hline & 0 & 11 & 0.716666667 & 2 & 0 & 0 & 1 & 0 & 0 & 0 \\
\hline & 0 & 0.5 & 0.433333333 & 2 & 0 & 0 & 0 & 1 & 0 & 0 \\
\hline & 0 & 1.3 & 0.766666667 & 2 & 0 & 0 & 0 & 0 & 1 & 0 \\
\hline & 0 & 5 & 0.766666667 & 2 & 0 & 0 & 0 & 0 & 0 & 1 \\
\hline \multirow[t]{5}{*}{199} & 1 & 5.237 & 0.833333333 & 1 & 0 & 0 & 0 & 0 & 0 & 0 \\
\hline & 0 & 20 & 0.4666666667 & 1 & 0 & 0 & 1 & 0 & 0 & 0 \\
\hline & 0 & 0.7 & 0.283333333 & 1 & 0 & 0 & 0 & 1 & 0 & 0 \\
\hline & 0 & 1.5 & 0.5 & 1 & 0 & 0 & 0 & 0 & 1 & 0 \\
\hline & 0 & 5 & 0.5 & 1 & 0 & 0 & 0 & 0 & 0 & 1 \\
\hline \multirow[t]{5}{*}{200} & 1 & 1.271 & 0.6666666667 & 1 & 1 & 1 & 0 & 0 & 0 & 0 \\
\hline & 0 & 28 & 1.2 & 1 & 0 & 0 & 1 & 0 & 0 & 0 \\
\hline & 0 & 0.6 & 0.733333333 & 1 & 0 & 0 & 0 & 1 & 0 & 0 \\
\hline & 0 & 1.1 & 1.283333333 & 1 & 0 & 0 & 0 & 0 & 1 & 0 \\
\hline & 0 & 5 & 1.283333333 & 1 & 0 & 0 & 0 & 0 & 0 & 1 \\
\hline \multirow[t]{5}{*}{201} & 1 & 2.588 & 0.266666667 & 3 & 0 & 1 & 0 & 0 & 0 & 0 \\
\hline & 0 & 6 & 0.183333333 & 3 & 0 & 0 & 1 & 0 & 0 & 0 \\
\hline & 0 & 0.5 & 0.116666667 & 3 & 0 & 0 & 0 & 1 & 0 & 0 \\
\hline & 0 & 0.5 & 0.2 & 3 & 0 & 0 & 0 & 0 & 1 & 0 \\
\hline & 0 & 5 & 0.2 & 3 & 0 & 0 & 0 & 0 & 0 & 1 \\
\hline \multirow[t]{5}{*}{202} & 1 & 1.588 & 0.5 & 2 & 1 & 1 & 0 & 0 & 0 & 0 \\
\hline & 0 & 15 & 0.816666667 & 2 & 0 & 0 & 1 & 0 & 0 & 0 \\
\hline & 0 & 0.6 & 0.5 & 2 & 0 & 0 & 0 & 1 & 0 & 0 \\
\hline & 0 & 1.5 & 0.866666667 & 2 & 0 & 0 & 0 & 0 & 1 & 0 \\
\hline & 0 & 5 & 0.866666667 & 2 & 0 & 0 & 0 & 0 & 0 & 1 \\
\hline \multirow[t]{5}{*}{203} & 1 & 4.118 & 0.333333333 & 1 & 1 & 1 & 0 & 0 & 0 & 0 \\
\hline & 0 & 10 & 0.516666667 & 1 & 0 & 0 & 1 & 0 & 0 & 0 \\
\hline & 0 & 0.5 & 0.316666667 & 1 & 0 & 0 & 0 & 1 & 0 & 0 \\
\hline & 0 & 1.1 & 0.566666667 & 1 & 0 & 0 & 0 & 0 & 1 & 0 \\
\hline & 0 & 5 & 0.566666667 & 1 & 0 & 0 & 0 & 0 & 0 & 1 \\
\hline \multirow[t]{5}{*}{204} & 1 & 3.389 & 0.833333333 & 1 & 1 & 0 & 0 & 0 & 0 & 0 \\
\hline & 0 & 28 & 1.2 & 1 & 0 & 0 & 1 & 0 & 0 & 0 \\
\hline & 0 & 0.6 & 0.733333333 & 1 & 0 & 0 & 0 & 1 & 0 & 0 \\
\hline & 0 & 1.1 & 1.283333333 & 1 & 0 & 0 & 0 & 0 & 1 & 0 \\
\hline & 0 & 5 & 1.283333333 & 1 & 0 & 0 & 0 & 0 & 0 & 1 \\
\hline
\end{tabular}




\begin{tabular}{|c|c|c|c|c|c|c|c|c|c|c|}
\hline \multirow{2}{*}{$\begin{array}{c}\text { Five alternatives } \\
\text { (Number of } \\
\text { observation) }\end{array}$} & Variable & Beta 1 & Beta 2 & Beta 3 & Beta 4 & Beta 5 & Beta 6 & Beta 7 & Beta 8 & Beta 9 \\
\hline & $\begin{array}{l}\text { Mode } \\
\text { Chosen }\end{array}$ & $\begin{array}{c}\text { TC } \\
\text { (1000 Korea won) }\end{array}$ & $\begin{array}{c}\text { TT } \\
\text { (hour) }\end{array}$ & Accom & Sexm & Age 340 & Tasc & Sasc & Basc & Lasc \\
\hline \multirow[t]{5}{*}{205} & 1 & 2.118 & 0.6666666667 & 1 & 1 & 1 & 0 & 0 & 0 & 0 \\
\hline & 0 & 28 & 1.2 & 1 & 0 & 0 & 1 & 0 & 0 & 0 \\
\hline & 0 & 0.6 & 0.733333333 & 1 & 0 & 0 & 0 & 1 & 0 & 0 \\
\hline & 0 & 1.1 & 1.283333333 & 1 & 0 & 0 & 0 & 0 & 1 & 0 \\
\hline & 0 & 5 & 1.283333333 & 1 & 0 & 0 & 0 & 0 & 0 & 1 \\
\hline \multirow[t]{5}{*}{206} & 1 & 3.177 & 0.6666666667 & 1 & 0 & 0 & 0 & 0 & 0 & 0 \\
\hline & 0 & 28 & 1.2 & 1 & 0 & 0 & 1 & 0 & 0 & 0 \\
\hline & 0 & 0.6 & 0.733333333 & 1 & 0 & 0 & 0 & 1 & 0 & 0 \\
\hline & 0 & 1.1 & 1.283333333 & 1 & 0 & 0 & 0 & 0 & 1 & 0 \\
\hline & 0 & 5 & 1.283333333 & 1 & 0 & 0 & 0 & 0 & 0 & 1 \\
\hline \multirow[t]{5}{*}{207} & 1 & 2.118 & 0.666666667 & 1 & 0 & 0 & 0 & 0 & 0 & 0 \\
\hline & 0 & 15 & 0.816666667 & 1 & 0 & 0 & 1 & 0 & 0 & 0 \\
\hline & 0 & 0.8 & 0.5 & 1 & 0 & 0 & 0 & 1 & 0 & 0 \\
\hline & 0 & 1.4 & 0.8666666667 & 1 & 0 & 0 & 0 & 0 & 1 & 0 \\
\hline & 0 & 5 & 0.866666667 & 1 & 0 & 0 & 0 & 0 & 0 & 1 \\
\hline \multirow[t]{5}{*}{208} & 1 & 6.355 & 3 & 4 & 1 & 1 & 0 & 0 & 0 & 0 \\
\hline & 0 & 28 & 1.866666667 & 4 & 0 & 0 & 1 & 0 & 0 & 0 \\
\hline & 0 & 0.85 & 1.15 & 4 & 0 & 0 & 0 & 1 & 0 & 0 \\
\hline & 0 & 1.5 & 2 & 4 & 0 & 0 & 0 & 0 & 1 & 0 \\
\hline & 0 & 5 & 2 & 4 & 0 & 0 & 0 & 0 & 0 & 1 \\
\hline \multirow[t]{5}{*}{209} & 1 & 4.237 & 1 & 1 & 0 & 0 & 0 & 0 & 0 & 0 \\
\hline & 0 & 20 & 0.4666666667 & 1 & 0 & 0 & 1 & 0 & 0 & 0 \\
\hline & 0 & 0.7 & 0.283333333 & 1 & 0 & 0 & 0 & 1 & 0 & 0 \\
\hline & 0 & 1.5 & 0.5 & 1 & 0 & 0 & 0 & 0 & 1 & 0 \\
\hline & 0 & 5 & 0.5 & 1 & 0 & 0 & 0 & 0 & 0 & 1 \\
\hline \multirow[t]{5}{*}{210} & 1 & 2.588 & 0.6666666667 & 1 & 1 & 0 & 0 & 0 & 0 & 0 \\
\hline & 0 & 15 & 0.816666667 & 1 & 0 & 0 & 1 & 0 & 0 & 0 \\
\hline & 0 & 0.6 & 0.5 & 1 & 0 & 0 & 0 & 1 & 0 & 0 \\
\hline & 0 & 1.5 & 0.8666666667 & 1 & 0 & 0 & 0 & 0 & 1 & 0 \\
\hline & 0 & 5 & 0.8666666667 & 1 & 0 & 0 & 0 & 0 & 0 & 1 \\
\hline \multirow[t]{5}{*}{211} & 1 & 2.406 & 0.666666667 & 1 & 1 & 1 & 0 & 0 & 0 & 0 \\
\hline & 0 & 28 & 1.2 & 1 & 0 & 0 & 1 & 0 & 0 & 0 \\
\hline & 0 & 0.6 & 0.733333333 & 1 & 0 & 0 & 0 & 1 & 0 & 0 \\
\hline & 0 & 1.1 & 1.283333333 & 1 & 0 & 0 & 0 & 0 & 1 & 0 \\
\hline & 0 & 5 & 1.2833333333 & 1 & 0 & 0 & 0 & 0 & 0 & 1 \\
\hline \multirow[t]{5}{*}{212} & 1 & 4.237 & 1.666666667 & 2 & 0 & 0 & 0 & 0 & 0 & 0 \\
\hline & 0 & 12 & 1.433333333 & 2 & 0 & 0 & 1 & 0 & 0 & 0 \\
\hline & 0 & 0.85 & 0.883333333 & 2 & 0 & 0 & 0 & 1 & 0 & 0 \\
\hline & 0 & 1.1 & 1.533333333 & 2 & 0 & 0 & 0 & 0 & 1 & 0 \\
\hline & 0 & 5 & 1.5333333333 & 2 & 0 & 0 & 0 & 0 & 0 & 1 \\
\hline \multirow[t]{5}{*}{213} & 1 & 4.766 & 1 & 1 & 0 & 0 & 0 & 0 & 0 & 0 \\
\hline & 0 & 28 & 1.2 & 1 & 0 & 0 & 1 & 0 & 0 & 0 \\
\hline & 0 & 0.6 & 0.7333333333 & 1 & 0 & 0 & 0 & 1 & 0 & 0 \\
\hline & 0 & 1.1 & 1.283333333 & 1 & 0 & 0 & 0 & 0 & 1 & 0 \\
\hline & 0 & 5 & 1.283333333 & 1 & 0 & 0 & 0 & 0 & 0 & 1 \\
\hline \multirow[t]{5}{*}{214} & 1 & 2.118 & 1 & 3 & 1 & 0 & 0 & 0 & 0 & 0 \\
\hline & 0 & 7 & 0.5666666667 & 3 & 0 & 0 & 1 & 0 & 0 & 0 \\
\hline & 0 & 0.6 & 0.35 & 3 & 0 & 0 & 0 & 1 & 0 & 0 \\
\hline & 0 & 1.3 & 0.616666667 & 3 & 0 & 0 & 0 & 0 & 1 & 0 \\
\hline & 0 & 5 & 0.616666667 & 3 & 0 & 0 & 0 & 0 & 0 & 1 \\
\hline \multirow[t]{5}{*}{215} & 1 & 3.177 & 1 & 1 & 0 & 1 & 0 & 0 & 0 & 0 \\
\hline & 0 & 28 & 1.2 & 1 & 0 & 0 & 1 & 0 & 0 & 0 \\
\hline & 0 & 0.6 & 0.7333333333 & 1 & 0 & 0 & 0 & 1 & 0 & 0 \\
\hline & 0 & 1.1 & 1.283333333 & 1 & 0 & 0 & 0 & 0 & 1 & 0 \\
\hline & 0 & 5 & 1.283333333 & 1 & 0 & 0 & 0 & 0 & 0 & 1 \\
\hline \multirow[t]{5}{*}{216} & 1 & 4.648 & 1.6666666667 & 2 & 0 & 1 & 0 & 0 & 0 & 0 \\
\hline & 0 & 20 & 0.4666666667 & 2 & 0 & 0 & 1 & 0 & 0 & 0 \\
\hline & 0 & 0.7 & 0.283333333 & 2 & 0 & 0 & 0 & 1 & 0 & 0 \\
\hline & 0 & 1.5 & 0.5 & 2 & 0 & 0 & 0 & 0 & 1 & 0 \\
\hline & 0 & 5 & 0.5 & 2 & 0 & 0 & 0 & 0 & 0 & 1 \\
\hline
\end{tabular}




\begin{tabular}{|c|c|c|c|c|c|c|c|c|c|c|}
\hline \multirow{2}{*}{$\begin{array}{c}\text { Five alternatives } \\
\text { (Number of } \\
\text { observation) }\end{array}$} & Variable & Beta 1 & Beta 2 & Beta 3 & Beta 4 & Beta 5 & Beta 6 & Beta 7 & Beta 8 & Beta 9 \\
\hline & $\begin{array}{l}\text { Mode } \\
\text { Chosen }\end{array}$ & $\begin{array}{c}\text { TC } \\
\text { (1000 Korea won) }\end{array}$ & $\begin{array}{c}\mathrm{TT} \\
\text { (hour) }\end{array}$ & Accom & Sexm & Age340 & Tasc & Sasc & Basc & Lasc \\
\hline \multirow[t]{5}{*}{217} & 1 & 4.745 & 0.6666666667 & 2 & 0 & 1 & 0 & 0 & 0 & 0 \\
\hline & 0 & 10 & 0.4666666667 & 2 & 0 & 0 & 1 & 0 & 0 & 0 \\
\hline & 0 & 0.5 & 0.283333333 & 2 & 0 & 0 & 0 & 1 & 0 & 0 \\
\hline & 0 & 1.1 & 0.5 & 2 & 0 & 0 & 0 & 0 & 1 & 0 \\
\hline & 0 & 5 & 0.5 & 2 & 0 & 0 & 0 & 0 & 0 & 1 \\
\hline \multirow[t]{5}{*}{218} & 1 & 4.177 & 1 & 1 & 1 & 1 & 0 & 0 & 0 & 0 \\
\hline & 0 & 28 & 1.2 & 1 & 0 & 0 & 1 & 0 & 0 & 0 \\
\hline & 0 & 0.6 & 0.733333333 & 1 & 0 & 0 & 0 & 1 & 0 & 0 \\
\hline & 0 & 1.1 & 1.283333333 & 1 & 0 & 0 & 0 & 0 & 1 & 0 \\
\hline & 0 & 5 & 1.283333333 & 1 & 0 & 0 & 0 & 0 & 0 & 1 \\
\hline \multirow[t]{5}{*}{219} & 1 & 4.677 & 0.5 & 1 & 1 & 0 & 0 & 0 & 0 & 0 \\
\hline & 0 & 28 & 1.2 & 1 & 0 & 0 & 1 & 0 & 0 & 0 \\
\hline & 0 & 0.6 & 0.733333333 & 1 & 0 & 0 & 0 & 1 & 0 & 0 \\
\hline & 0 & 1.1 & 1.283333333 & 1 & 0 & 0 & 0 & 0 & 1 & 0 \\
\hline & 0 & 5 & 1.283333333 & 1 & 0 & 0 & 0 & 0 & 0 & 1 \\
\hline \multirow[t]{5}{*}{220} & 1 & 11.093 & 1.5 & 1 & 0 & 0 & 0 & 0 & 0 & 0 \\
\hline & 0 & 53 & 2.0666666667 & 1 & 0 & 0 & 1 & 0 & 0 & 0 \\
\hline & 0 & 1 & 1.283333333 & 1 & 0 & 0 & 0 & 1 & 0 & 0 \\
\hline & 0 & 7.5 & 2.2 & 1 & 0 & 0 & 0 & 0 & 1 & 0 \\
\hline & 0 & 15 & 2.2 & 1 & 0 & 0 & 0 & 0 & 0 & 1 \\
\hline \multirow[t]{5}{*}{221} & 1 & 3.618 & 1 & 1 & 1 & 0 & 0 & 0 & 0 & 0 \\
\hline & 0 & 15 & 0.816666667 & 1 & 0 & 0 & 1 & 0 & 0 & 0 \\
\hline & 0 & 0.8 & 0.5 & 1 & 0 & 0 & 0 & 1 & 0 & 0 \\
\hline & 0 & 1.4 & 0.866666667 & 1 & 0 & 0 & 0 & 0 & 1 & 0 \\
\hline & 0 & 5 & 0.8666666667 & 1 & 0 & 0 & 0 & 0 & 0 & 1 \\
\hline \multirow[t]{5}{*}{222} & 1 & 2.118 & 0.6666666667 & 4 & 1 & 1 & 0 & 0 & 0 & 0 \\
\hline & 0 & 7 & 0.566666667 & 4 & 0 & 0 & 1 & 0 & 0 & 0 \\
\hline & 0 & 0.6 & 0.35 & 4 & 0 & 0 & 0 & 1 & 0 & 0 \\
\hline & 0 & 1.3 & 0.6166666667 & 4 & 0 & 0 & 0 & 0 & 1 & 0 \\
\hline & 0 & 5 & 0.616666667 & 4 & 0 & 0 & 0 & 0 & 0 & 1 \\
\hline \multirow[t]{5}{*}{223} & 1 & 3.707 & 0.5 & 1 & 1 & 1 & 0 & 0 & 0 & 0 \\
\hline & 0 & 14 & 2.066666667 & 1 & 0 & 0 & 1 & 0 & 0 & 0 \\
\hline & 0 & 0.75 & 1.283333333 & 1 & 0 & 0 & 0 & 1 & 0 & 0 \\
\hline & 0 & 1.5 & 2.2 & 1 & 0 & 0 & 0 & 0 & 1 & 0 \\
\hline & 0 & 5 & 2.2 & 1 & 0 & 0 & 0 & 0 & 0 & 1 \\
\hline \multirow[t]{5}{*}{224} & 1 & 4.118 & 0.6666666667 & 1 & 1 & 0 & 0 & 0 & 0 & 0 \\
\hline & 0 & 28 & 1.2 & 1 & 0 & 0 & 1 & 0 & 0 & 0 \\
\hline & 0 & 0.6 & 0.733333333 & 1. & 0 & 0. & 0 & 1 & 0 & 0 \\
\hline & 0 & 1.1 & 1.283333333 & 1 & 0 & 0 & 0 & 0 & 1 & 0 \\
\hline & 0 & 5 & 1.283333333 & 1 & 0 & 0 & 0 & 0 & 0 & 1 \\
\hline \multirow[t]{5}{*}{225} & 1 & 19.9 & 3 & 1 & 0 & 0 & 0 & 0 & 0 & 0 \\
\hline & 0 & 53 & 2.0666666667 & 1 & 0 & 0 & 1 & 0 & 0 & 0 \\
\hline & 0 & 1 & 1.283333333 & 1 & 0 & 0 & 0 & 1 & 0 & 0 \\
\hline & 0 & 7.5 & 2.2 & 1 & 0 & 0 & 0 & 0 & 1 & 0 \\
\hline & 0 & 15 & 2.2 & 1 & 0 & 0 & 0 & 0 & 0 & 1 \\
\hline \multirow[t]{5}{*}{226} & 1 & 14.793 & 1 & 1 & 1 & 0 & 0 & 0 & 0 & 0 \\
\hline & 0 & 53 & 2.0666666667 & 1 & 0 & 0 & 1 & 0 & 0 & 0 \\
\hline & 0 & 1 & 1.283333333 & 1 & 0 & 0 & 0 & 1 & 0 & 0 \\
\hline & 0 & 7.5 & 2.2 & 1 & 0 & 0 & 0 & 0 & 1 & 0 \\
\hline & 0 & 15 & 2.2 & 1 & 0 & 0 & 0 & 0 & 0 & 1 \\
\hline \multirow[t]{5}{*}{227} & 1 & 12.093 & 2.333333333 & 6 & 1 & 0 & 0 & 0 & 0 & 0 \\
\hline & 0 & 53 & 2.0666666667 & 6 & 0 & 0 & 1 & 0 & 0 & 0 \\
\hline & 0 & 1 & 1.283333333 & 6 & 0 & 0 & 0 & 1 & 0 & 0 \\
\hline & 0 & 7.5 & 2.2 & 6 & 0 & 0 & 0 & 0 & 1 & 0 \\
\hline & 0 & 15 & 2.2 & 6 & 0 & 0 & 0 & 0 & 0 & 1 \\
\hline \multirow[t]{5}{*}{228} & 1 & 1.059 & 1.5 & 1 & 0 & 0 & 0 & 0 & 0 & 0 \\
\hline & 0 & 28 & 1.8666666667 & 1 & 0 & 0 & 1 & 0 & 0 & 0 \\
\hline & 0 & 0.85 & 1.15 & 1 & 0 & 0 & 0 & 1 & 0 & 0 \\
\hline & 0 & 1.5 & 2 & 1 & 0 & 0 & 0 & 0 & 1 & 0 \\
\hline & 0 & 5 & 2 & 1 & 0 & 0 & 0 & 0 & 0 & 1 \\
\hline
\end{tabular}




\begin{tabular}{|c|c|c|c|c|c|c|c|c|c|c|}
\hline \multirow{2}{*}{$\begin{array}{c}\text { Five alternatives } \\
\text { (Number of } \\
\text { observation) }\end{array}$} & Variable & Beta 1 & Beta 2 & Beta 3 & Beta 4 & Beta 5 & Beta 6 & Beta 7 & Beta 8 & Beta 9 \\
\hline & $\begin{array}{l}\text { Mode } \\
\text { Chosen }\end{array}$ & $\begin{array}{c}\text { TC } \\
\text { (1000 Korea won) }\end{array}$ & $\begin{array}{c}\text { TT } \\
\text { (hour) }\end{array}$ & Accom & Sexm & Age340 & Tasc & Sasc & Basc & Lasc \\
\hline \multirow[t]{5}{*}{229} & 1 & 2.118 & 0.416666667 & 4 & 0 & 0 & 0 & 0 & 0 & 0 \\
\hline & 0 & 7 & 0.566666667 & 4 & 0 & 0 & 1 & 0 & 0 & 0 \\
\hline & 0 & 0.6 & 0.35 & 4 & 0 & 0 & 0 & 1 & 0 & 0 \\
\hline & 0 & 1.3 & 0.616666667 & 4 & 0 & 0 & 0 & 0 & 1 & 0 \\
\hline & 0 . & 5 & 0.616666667 & 4 & 0 & 0 & 0 & 0 & 0 & 1 \\
\hline \multirow[t]{5}{*}{230} & 1 & 2.118 & 0.333333333 & 1 & 1 & 1 & 0 & 0 & 0 & 0 \\
\hline & 0 & 15 & 0.816666667 & 1 & 0 & 0 & 1 & 0 & 0 & 0 \\
\hline & 0 & 0.6 & 0.5 & 1 & 0 & 0 & 0 & 1 & 0 & 0 \\
\hline & 0 & 1.5 & 0.866666667 & 1 & 0 & 0 & 0 & 0 & 1 & 0 \\
\hline & 0 & 5 & 0.866666667 & 1 & 0 & 0 & 0 & 0 & 0 & 1 \\
\hline \multirow[t]{5}{*}{231} & 1 & 4.177 & 1 & 1 & 0 & 0 & 0 & 0 & 0 & 0 \\
\hline & 0 & 28 & 1.2 & 1 & 0 & 0 & 1 & 0 & 0 & 0 \\
\hline & 0 & 0.6 & 0.733333333 & 1 & 0 & 0 & 0 & 1 & 0 & 0 \\
\hline & 0 & 1.1 & 1.283333333 & 1 & 0 & 0 & 0 & 0 & 1 & 0 \\
\hline & 0 & 5 & 1.283333333 & 1 & 0 & 0 & 0 & 0 & 0 & 1 \\
\hline \multirow[t]{5}{*}{232} & 1 & 4.177 & 1 & 3 & 0 & 1 & 0 & 0 & 0 & 0 \\
\hline & 0 & 28 & 1.2 & 3 & 0 & 0 & 1 & 0 & 0 & 0 \\
\hline & 0 & 0.6 & 0.733333333 & 3 & 0 & 0 & 0 & 1 & 0 & 0 \\
\hline & 0 & 1.1 & 1.283333333 & 3 & 0 & 0 & 0 & 0 & 1 & 0 \\
\hline & 0 & 5 & 1.283333333 & 3 & 0 & 0 & 0 & 0 & 0 & 1 \\
\hline \multirow[t]{5}{*}{233} & 1 & 7.355 & 1 & 1 & 0 & 0 & 0 & 0 & 0 & 0 \\
\hline & 0 & 10 & 0.516666667 & 1 & 0 & 0 & 1 & 0 & 0 & 0 \\
\hline & 0 & 0.5 & 0.316666667 & 1 & 0 & 0 & 0 & 1 & 0 & 0 \\
\hline & 0 & 1.1 & 0.5666666667 & 1 & 0 & 0 & 0 & 0 & 1 & 0 \\
\hline & 0 & 5 & 0.566666667 & 1 & 0 & 0 & 0 & 0 & 0 & 1 \\
\hline \multirow[t]{5}{*}{234} & 1 & 5.177 & 0.833333333 & 3 & 1 & 1 & 0 & 0 & 0 & 0 \\
\hline & 0 & 12 & 1.433333333 & 3 & 0 & 0 & 1 & 0 & 0 & 0 \\
\hline & 0 & 0.9 & 0.883333333 & 3 & 0 & 0 & 0 & 1 & 0 & 0 \\
\hline & 0 & 1.1 & 1.533333333 & 3 & 0 & 0 & 0 & 0 & 1 & 0 \\
\hline & 0 & 5 & 1.533333333 & 3 & 0 & 0 & 0 & 0 & 0 & 1 \\
\hline \multirow[t]{5}{*}{235} & 1 & 6.296 & 1.5 & 4 & 0 & 1 & 0 & 0 & 0 & 0 \\
\hline & 0 & 20 & 0.4666666667 & 4 & 0 & 0 & 1 & 0 & 0 & 0 \\
\hline & 0 & 0.7 & 0.283333333 & 4 & 0 & 0 & 0 & 1 & 0 & 0 \\
\hline & 0 & 1.5 & 0.5 & 4 & 0 & 0 & 0 & 0 & 1 & 0 \\
\hline & 0 & 5 & 0.5 & 4 & 0 & 0 & 0 & 0 & 0 & 1 \\
\hline \multirow[t]{5}{*}{236} & 1 & 4.177 & 0.333333333 & 2 & 1 & 0 & 0 & 0 & 0 & 0 \\
\hline & 0 & 10 & 0.516666667 & 2 & 0 & 0 & 1 & 0 & 0 & 0 \\
\hline & 0 & 0.5 & 0.316666667 & 2 & 0 & 0 & 0 & 1 & 0 & 0 \\
\hline & 0 & 1.1 & 0.566666667 & 2 & 0 & 0 & 0 & 0 & 1 & 0 \\
\hline & 0 & 5 & 0.566666667 & 2 & 0 & 0 & 0 & 0 & 0 & 1 \\
\hline \multirow[t]{5}{*}{237} & 1 & 4.707 & 0.666666667 & 2 & 0 & 1 & 0 & 0 & 0 & 0 \\
\hline & 0 & 14 & 2.066666667 & 2 & 0 & 0 & 1 & 0 & 0 & 0 \\
\hline & 0 & 0.75 & 1.283333333 & 2 & 0 & 0 & 0 & 1 & 0 & 0 \\
\hline & 0 & 1.5 & 2.2 & 2 & 0 & 0 & 0 & 0 & 1 & 0 \\
\hline & 0 & 5 & 2,2 & 2 & 0 & 0 & 0 & 0 & 0 & 1 \\
\hline \multirow[t]{5}{*}{238} & 1 & 6.296 & 1.333333333 & 4 & 1 & 1 & 0 & 0 & 0 & 0 \\
\hline & 0 & 28 & 1.866666667 & 4 & 0 & 0 & 1 & 0 & 0 & 0 \\
\hline & 0 & 0.85 & 1.15 & 4 & 0 & 0 & 0 & 1 & 0 & 0 \\
\hline & 0 & 1.5 & 2 & 4 & 0 & 0 & 0 & 0 & 1 & 0 \\
\hline & 0 & 5 & 2 & 4 & 0 & 0 & 0 & 0 & 0 & 1 \\
\hline \multirow[t]{5}{*}{239} & 1 & 4.707 & 0.666666667 & 3 & 0 & 0 & 0 & 0 & 0 & 0 \\
\hline & 0 & 14 & 2.066666667 & 3 & 0 & 0 & 1 & 0 & 0 & 0 \\
\hline & 0 & 0.75 & 1.283333333 & 3 & 0 & 0 & 0 & 1 & 0 & 0 \\
\hline & 0 & 1.5 & 2.2 & 3 & 0 & 0 & 0 & 0 & 1 & 0 \\
\hline & 0 & 5 & 2.2 & 3 & 0 & 0 & 0 & 0 & 0 & 1 \\
\hline \multirow[t]{5}{*}{240} & 1 & 3.936 & 1 & 3 & 1 & 0 & 0 & 0 & 0 & 0 \\
\hline & 0 & 20 & 1.483333333 & 3 & 0 & 0 & 1 & 0 & 0 & 0 \\
\hline & 0 & 0.6 & 0.916666667 & 3 & 0 & 0 & 0 & 1 & 0 & 0 \\
\hline & 0 & 1.6 & 1.583333333 & 3 & 0 & 0 & 0 & 0 & 1 & 0 \\
\hline & 0 & 5 & 1.583333333 & 3 & 0 & 0 & 0 & 0 & 0 & 1 \\
\hline
\end{tabular}




\begin{tabular}{|c|c|c|c|c|c|c|c|c|c|c|}
\hline \multirow{2}{*}{$\begin{array}{c}\text { Five alternatives } \\
\text { (Number of } \\
\text { observation) }\end{array}$} & Variable & Beta 1 & Beta 2 & Beta 3 & Beta 4 & Beta 5 & Beta 6 & Beta 7 & Beta 8 & Beta 9 \\
\hline & $\begin{array}{c}\text { Mode } \\
\text { Chosen } \\
\end{array}$ & $\begin{array}{c}\text { TC } \\
\text { (1000 Korea won) } \\
\end{array}$ & $\begin{array}{c}\mathrm{TT} \\
\text { (hour) }\end{array}$ & Accom & Sexm & Age340 & Tasc & Sasc & Basc & Lasc \\
\hline \multirow[t]{5}{*}{241} & 1 & 4.436 & 1 & 2 & 0 & 1 & 0 & 0 & 0 & 0 \\
\hline & 0 & 7.6 & 0.616666667 & 2 & 0 & 0 & 1 & 0 & 0 & 0 \\
\hline & 0 & 0.6 & 0.383333333 & 2 & 0 & 0 & 0 & 1 & 0 & 0 \\
\hline & 0 & 1.6 & 0.666666667 & 2 & 0 & 0 & 0 & 0 & 1 & 0 \\
\hline & 0 & 5 & 0.666666667 & 2 & 0 & 0 & 0 & 0 & 0 & 1 \\
\hline \multirow[t]{5}{*}{242} & 1 & 4.118 & 0.5 & 1 & 0 & 1 & 0 & 0 & 0 & 0 \\
\hline & 0 & 10 & 0.9 & 1 & 0 & 0 & 1 & 0 & 0 & 0 \\
\hline & 0 & 0.6 & 0.566666667 & 1 & 0 & 0 & 0 & 1 & 0 & 0 \\
\hline & 0 & 1.3 & 0.9666666667 & 1 & 0 & 0 & 0 & 0 & 1 & 0 \\
\hline & 0 & 5 & 0.966666667 & 1 & 0 & 0 & 0 & 0 & 0 & 1 \\
\hline \multirow[t]{5}{*}{243} & 1 & 3.648 & 0.6666666667 & 2 & 1 & 0 & 0 & 0 & 0 & 0 \\
\hline & 0 & 28 & 1.2 & 2 & 0 & 0 & 1 & 0 & 0 & 0 \\
\hline & 0 & 0.6 & 0.733333333 & 2 & 0 & 0 & 0 & 1 & 0 & 0 \\
\hline & 0 & 1.1 & 1.283333333 & 2 & 0 & 0 & 0 & 0 & 1 & 0 \\
\hline & 0 & 5 & 1.283333333 & 2 & 0 & 0 & 0 & 0 & 0 & 1 \\
\hline \multirow[t]{5}{*}{244} & 1 & 8.248 & 1 & 1 & 1 & 1 & 0 & 0 & 0 & 0 \\
\hline & 0 & 20 & 0.466666667 & 1 & 0 & 0 & 1 & 0 & 0 & 0 \\
\hline & 0 & 0.7 & 0.283333333 & 1 & 0 & 0 & 0 & 1 & 0 & 0 \\
\hline & 0 & 1.5 & 0.5 & 1 & 0 & 0 & 0 & 0 & 1 & 0 \\
\hline & 0 & 5 & 0.5 & 1 & 0 & 0 & 0 & 0 & 0 & 1 \\
\hline \multirow[t]{5}{*}{245} & 1 & 2.059 & 0.4166666667 & 1 & 1 & 0 & 0 & 0 & 0 & 0 \\
\hline & 0 & 28 & 1.816666667 & 1 & 0 & 0 & 1 & 0 & 0 & 0 \\
\hline & 0 & 0.65 & 1.133333333 & 1 & 0 & 0 & 0 & 1 & 0 & 0 \\
\hline & 0 & 1 & 1.95 & 1 & 0 & 0 & 0 & 0 & 1 & 0 \\
\hline & 0 & 5 & 1.95 & 1 & 0 & 0 & 0 & 0 & 0 & 1 \\
\hline \multirow[t]{5}{*}{246} & 1 & 3.707 & 0.666666667 & 1 & 1 & 1 & 0 & 0 & 0 & 0 \\
\hline & 0 & 14 & 1.816666667 & 1 & 0 & 0 & 1 & 0 & 0 & 0 \\
\hline & 0 & 0.75 & 1.133333333 & 1 & 0 & 0 & 0 & 1 & 0 & 0 \\
\hline & 0 & 1.5 & 1.95 & 1 & 0 & 0 & 0 & 0 & 1 & 0 \\
\hline & 0 & 5 & 1.95 & 1 & 0 & 0 & 0 & 0 & 0 & 1 \\
\hline \multirow[t]{5}{*}{247} & 1 & 1.588 & 0.416666667 & 1 & 0 & 0 & 0 & 0 & 0 & 0 \\
\hline & 0 & 28 & 1.2 & 1 & 0 & 0 & 1 & 0 & 0 & 0 \\
\hline & 0 & 0.6 & 0.733333333 & 1 & 0 & 0 & 0 & 1 & 0 & 0 \\
\hline & 0 & 1.1 & 1.283333333 & 1 & 0 & 0 & 0 & 0 & 1 & 0 \\
\hline & 0 & 5 & 1.283333333 & 1 & 0 & 0 & 0 & 0 & 0 & 1 \\
\hline
\end{tabular}


C.2: Taxi alternative - 70 observations

\begin{tabular}{|c|c|c|c|c|c|c|c|c|c|c|}
\hline \multirow{2}{*}{$\begin{array}{l}\text { Five alternatives } \\
\text { (Number of } \\
\text { observation) }\end{array}$} & Variable & Beta 1 & Beta 2 & Beta 3 & Beta 4 & Beta 5 & Beta 6 & Beta 7 & Beta 8 & Beta 9 \\
\hline & $\begin{array}{l}\text { Mode } \\
\text { Chosen }\end{array}$ & $\begin{array}{c}\text { TC } \\
\text { (1000 Korea won) } \\
\end{array}$ & $\begin{array}{c}\mathrm{TT} \\
\text { (hour) }\end{array}$ & Accom & Sexm & Age 340 & Tasc & Sasc & Basc & Lasc \\
\hline \multirow[t]{5}{*}{$\begin{array}{ll}\text { TAXI } 1 \\
\end{array}$} & 0 & 8.017 & 1.15 & 1 & 0 & 0 & 0 & 0 & 0 & 0 \\
\hline & 1 & 15 & 0.6666666667 & 1 & 0 & 0 & 1 & 0 & 0 & 0 \\
\hline & 0 & 0.65 & 0.7 & 1 & 0 & 0 & 0 & 1 & 0 & 0 \\
\hline & 0 & 2 & 1.233333333 & 1 & 0 & 0 & 0 & 0 & 1 & 0 \\
\hline & 0 & 5 & 1.233333333 & 1 & 0 & 0 & 0 & 0 & 0 & 1 \\
\hline \multirow[t]{5}{*}{2} & 0 & 9.076 & 0.816666667 & 0 & 0 & 1 & 0 & 0 & 0 & 0 \\
\hline & 1 & 15 & 0.6666666667 & 0 & 0 & 0 & 1 & 0 & 0 & 0 \\
\hline & 0 & 0.6 & 0.5 & 0 & 0 & 0 & 0 & 1 & 0 & 0 \\
\hline & 0 & 1.5 & 0.8666666667 & 0 & 0 & 0 & 0 & 0 & 1 & 0 \\
\hline & 0 & 5 & 0.866666667 & 0 & 0 & 0 & 0 & 0 & 0 & 1 \\
\hline \multirow[t]{5}{*}{3} & 0 & 9.076 & 0.816666667 & 0 & 1 & 1 & 0 & 0 & 0 & 0 \\
\hline & 1 & 8 & 0.5 & 0 & 0 & 0 & 1 & 0 & 0 & 0 \\
\hline & 0 & 0.6 & 0.5 & 0 & 0 & 0 & 0 & 1 & 0 & 0 \\
\hline & 0 & 1.5 & 0.8666666667 & 0 & 0 & 0 & 0 & 0 & 1 & 0 \\
\hline & 0 & 5 & 0.866666667 & 0 & 0 & 0 & 0 & 0 & 0 & 1 \\
\hline \multirow[t]{5}{*}{4} & 0 & 8.78 & 0.4666666667 & 0 & 1 & 0 & 0 & 0 & 0 & 0 \\
\hline & 1 & 5 & 0.1666666667 & 0 & 0 & 0 & 1 & 0 & 0 & 0 \\
\hline & 0 & 0.5 & 0.283333333 & 0 & 0 & 0 & 0 & 1 & 0 & 0 \\
\hline & 0 & 1.1 & 0.5 & 0 & 0 & 0 & 0 & 0 & 1 & 0 \\
\hline & 0 & 5 & 0.5 & 0 & 0 & 0 & 0 & 0 & 0 & 1 \\
\hline \multirow[t]{5}{*}{5} & 0 & 5.18 & 0.183333333 & 0 & 1 & 0 & 0 & 0 & 0 & 0 \\
\hline & 1 & 1.7 & 0.083333333 & 0 & 0 & 0 & 1 & 0 & 0 & 0 \\
\hline & 0 & 0.5 & 0.116666667 & 0 & 0 & 0 & 0 & 1 & 0 & 0 \\
\hline & 0 & 0.5 & 0.2 & 0 & 0 & 0 & 0 & 0 & 1 & 0 \\
\hline & 0 & 5 & 0.2 & 0 & 0 & 0 & 0 & 0 & 0 & 1 \\
\hline \multirow[t]{5}{*}{6} & 0 & 8.18 & 0.85 & 1 & 1 & 1 & 0 & 0 & 0 & 0 \\
\hline & 1 & 10 & 0.5 & 1 & 0 & 0 & 1 & 0 & 0 & 0 \\
\hline & 0 & 0.6 & 0.533333333 & 1 & 0 & 0 & 0 & 1 & 0 & 0 \\
\hline & 0 & 1.1 & 0.916666667 & 1 & 0 & 0 & 0 & 0 & 1 & 0 \\
\hline & 0 & 5 & 0.916666667 & 1 & 0 & 0 & 0 & 0 & 0 & 1 \\
\hline \multirow[t]{5}{*}{7} & 0 & 9.18 & 1.2 & 0 & 0 & 0 & 0 & 0 & 0 & 0 \\
\hline & 1 & 13 & 0.5 & 0 & 0 & 0 & 1 & 0 & 0 & 0 \\
\hline & 0 & 0.6 & 0.733333333 & 0 & 0 & 0 & 0 & 1 & 0 & 0 \\
\hline & 0 & 1.1 & 1.283333333 & 0 & 0 & 0 & 0 & 0 & 1 & 0 \\
\hline & 0 & 5 & 1.283333333 & 0 & 0 & 0 & 0 & 0 & 0 & 1 \\
\hline \multirow[t]{5}{*}{8} & 0 & 13.18 & 1.866666667 & 1 & 0 & 0 & 0 & 0 & 0 & 0 \\
\hline & 1 & 23 & 0.833333333 & 1 & 0 & 0 & 1 & 0 & 0 & 0 \\
\hline & 0 & 0.85 & 1.15 & 1 & 0 & 0 & 0 & 1 & 0 & 0 \\
\hline & 0 & 1.5 & 2 & 1 & 0 & 0 & 0 & 0 & 1 & 0 \\
\hline & 0 & 5 & 2 & 1 & 0 & 0 & 0 & 0 & 0 & 1 \\
\hline \multirow[t]{5}{*}{9} & 0 & 7.735 & 2.0666666667 & 2 & 0 & 0 & 0 & 0 & 0 & 0 \\
\hline & 1 & 14 & 0.6666666667 & 2 & 0 & 0 & 1 & 0 & 0 & 0 \\
\hline & 0 & 0.75 & 1.283333333 & 2 & 0 & 0 & 0 & 1 & 0 & 0 \\
\hline & 0 & 1.5 & 2.2 & 2 & 0 & 0 & 0 & 0 & 1 & 0 \\
\hline & 0 & 5 & 2.2 & 2 & 0 & 0 & 0 & 0 & 0 & 1 \\
\hline \multirow[t]{5}{*}{10} & 0 & 7.1 & 1.1 & 0 & 1 & 1 & 0 & 0 & 0 & 0 \\
\hline & 1 & 15 & 1 & 0 & 0 & 0 & 1 & 0 & 0 & 0 \\
\hline & 0 & 0.6 & 0.6833333333 & 0 & 0 & 0 & 0 & 1 & 0 & 0 \\
\hline & 0 & 1.3 & 1.183333333 & 0 & 0 & 0 & 0 & 0 & 1 & 0 \\
\hline & 0 & 5 & 1.1833333333 & 0 & 0 & 0 & 0 & 0 & 0 & 1 \\
\hline \multirow[t]{5}{*}{11} & 0 & 8.017 & 1.15 & 0 & 1 & 1 & 0 & 0 & 0 & 0 \\
\hline & 1 & 18 & 0.666666667 & 0 & 0 & 0 & 1 & 0 & 0 & 0 \\
\hline & 0 & 0.65 & 0.7 & 0 & 0 & 0 & 0 & 1 & 0 & 0 \\
\hline & 0 & 2 & 1.233333333 & 0 & 0 & 0 & 0 & 0 & 1 & 0 \\
\hline & 0 & 5 & 1.233333333 & 0 & 0 & 0 & 0 & 0 & 0 & 1 \\
\hline \multirow[t]{5}{*}{12} & 0 & 13.18 & 1.866666667 & 1 & 1 & 0 & 0 & 0 & 0 & 0 \\
\hline & 1 & 25 & 1 & 1 & 0 & 0 & 1 & 0 & 0 & 0 \\
\hline & 0 & 0.85 & 1.15 & 1 & 0 & 0 & 0 & 1 & 0 & 0 \\
\hline & 0 & 1.5 & 2 & 1 & 0 & 0 & 0 & 0 & 1 & 0 \\
\hline & 0 & 5 & 2 & 1 & 0 & 0 & 0 & 0 & 0 & 1 \\
\hline
\end{tabular}




\begin{tabular}{|c|c|c|c|c|c|c|c|c|c|c|}
\hline \multirow{2}{*}{$\begin{array}{l}\text { Five alternatives } \\
\text { (Number of } \\
\text { observation) }\end{array}$} & Variable & Beta 1 & Beta 2 & Beta 3 & Beta 4 & Beta 5 & Beta 6 & Beta 7 & Beta 8 & Beta 9 \\
\hline & $\begin{array}{l}\text { Mode } \\
\text { Chosen }\end{array}$ & $\begin{array}{c}\text { TC } \\
\text { (1000 Korea won) }\end{array}$ & $\begin{array}{c}\mathrm{TT} \\
\text { (hour) }\end{array}$ & Accom & Sexm & Age 340 & Tasc & Sasc & Basc & Lasc \\
\hline \multirow[t]{5}{*}{13} & 0 & 7.18 & 0.5166666667 & 1 & 0 & 0 & 0 & 0 & 0 & 0 \\
\hline & 1 & 10 & 0.416666667 & 1 & 0 & 0 & 1 & 0 & 0 & 0 \\
\hline & 0 & 0.5 & 0.316666667 & 1 & 0 & 0 & 0 & 1 & 0 & 0 \\
\hline & 0 & 1.1 & 0.566666667 & 1 & 0 & 0 & 0 & 0 & 1 & 0 \\
\hline & 0 & 5 & 0.566666667 & 1 & 0 & 0 & 0 & 0 & 0 & 1 \\
\hline \multirow[t]{5}{*}{14} & 0 & 8.18 & 1.483333333 & 2 & 0 & 0 & 0 & 0 & 0 & 0 \\
\hline & 1 & 24 & 1.1666666667 & 2 & 0 & 0 & 1 & 0 & 0 & 0 \\
\hline & 0 & 0.6 & 0.916666667 & 2 & 0 & 0 & 0 & 1 & 0 & 0 \\
\hline & 0 & 1.6 & 1.583333333 & 2 & 0 & 0 & 0 & 0 & 1 & 0 \\
\hline & 0 & 5 & 1.583333333 & 2 & 0 & 0 & 0 & 0 & 0 & 1 \\
\hline \multirow[t]{5}{*}{15} & 0 & 8.18 & 1.483333333 & 0 & 1 & 0 & 0 & 0 & 0 & 0 \\
\hline & 1 & 15 & 1.5 & 0 & 0 & 0 & 1 & 0 & 0 & 0 \\
\hline & 0 & 0.6 & 0.916666667 & 0 & 0 & 0 & 0 & 1 & 0 & 0 \\
\hline & 0 & 1.6 & 1.583333333 & 0 & 0 & 0 & 0 & 0 & 1 & 0 \\
\hline & 0 & 5 & 1.583333333 & 0 & 0 & 0 & 0 & 0 & 0 & 1 \\
\hline \multirow[t]{5}{*}{16} & 0 & 8.017 & 1.15 & 1 & 0 & 0 & 0 & 0 & 0 & 0 \\
\hline & 1 & 16 & 0.583333333 & 1 & 0 & 0 & 1 & 0 & 0 & 0 \\
\hline & 0 & 0.65 & 0.7 & 1 & 0 & 0 & 0 & 1 & 0 & 0 \\
\hline & 0 & 2 & 1.233333333 & 1 & 0 & 0 & 0 & 0 & 1 & 0 \\
\hline & 0 & 5 & 1.233333333 & 1 & 0 & 0 & 0 & 0 & 0 & 1 \\
\hline \multirow[t]{5}{*}{17} & 0 & 9.18 & 1.2 & 1 & 0 & 0 & 0 & 0 & 0 & 0 \\
\hline & 1 & 12 & 0.5 & 1 & 0 & 0 & 1 & 0 & 0 & 0 \\
\hline & 0 & 0.6 & 0.733333333 & 1 & 0 & 0 & 0 & 1 & 0 & 0 \\
\hline & 0 & 1.1 & 1.283333333 & 1 & 0 & 0 & 0 & 0 & 1 & 0 \\
\hline & 0 & 5 & 1.283333333 & 1 & 0 & 0 & 0 & 0 & 0 & 1 \\
\hline \multirow[t]{5}{*}{18} & 0 & 9.18 & 1.2 & 0 & 1 & 1 & 0 & 0 & 0 & 0 \\
\hline & 1 & 16 & 0.5 & 0 & 0 & 0 & 1 & 0 & 0 & 0 \\
\hline & 0 & 0.6 & 0.733333333 & 0 & 0 & 0 & 0 & 1 & 0 & 0 \\
\hline & 0 & 1.1 & 1.283333333 & 0 & 0 & 0 & 0 & 0 & 1 & 0 \\
\hline & 0 & 5 & 1.283333333 & 0 & 0 & 0 & 0 & 0 & 0 & 1 \\
\hline \multirow[t]{5}{*}{19} & 0 & 7.18 & 0.516666667 & 0 & 1 & 1 & 0 & 0 & 0 & 0 \\
\hline & 1 & 9 & 0.666666667 & 0 & 0 & 0 & 1 & 0 & 0 & 0 \\
\hline & 0 & 0.5 & 0.316666667 & 0 & 0 & 0 & 0 & 1 & 0 & 0 \\
\hline & 0 & 1.1 & 0.566666667 & 0 & 0 & 0 & 0 & 0 & 1 & 0 \\
\hline & 0 & 5 & 0.566666667 & 0 & 0 & 0 & 0 & 0 & 0 & 1 \\
\hline \multirow[t]{5}{*}{20} & 0 & 8.476 & 0.95 & 1 & 1 & 1 & 0 & 0 & 0 & 0 \\
\hline & 1 & 13 & 0.6666666667 & 1 & 0 & 0 & 1 & 0 & 0 & 0 \\
\hline & 0 & 0.7 & 0.5833333333 & 1 & 0 & 0 & 0 & 1 & 0 & 0 \\
\hline & 0 & 1.3 & 1.016666667 & 1 & 0 & 0 & 0 & 0 & 1 & 0 \\
\hline & 0 & 5 & 1.016666667 & 1 & 0 & 0 & 0 & 0 & 0 & 1 \\
\hline \multirow[t]{5}{*}{21} & 0 & 5.298 & 0.616666667 & 0 & 1 & 1 & 0 & 0 & 0 & 0 \\
\hline & 1 & 10 & 0.333333333 & 0 & 0 & 0 & 1 & 0 & 0 & 0 \\
\hline & 0 & 0.6 & 0.383333333 & 0 & 0 & 0 & 0 & 1 & 0 & 0 \\
\hline & 0 & 1 & 0.666666667 & 0 & 0 & 0 & 0 & 0 & 1 & 0 \\
\hline & 0 & 5 & 0.666666667 & 0 & 0 & 0 & 0 & 0 & 0 & 1 \\
\hline \multirow[t]{5}{*}{22} & 0 & 6.18 & 0.9 & 0 & 0 & 0 & 0 & 0 & 0 & 0 \\
\hline & 1 & 8.4 & 0.5 & 0 & 0 & 0 & 1 & 0 & 0 & 0 \\
\hline & 0 & 0.6 & 0.566666667 & 0 & 0 & 0 & 0 & 1 & 0 & 0 \\
\hline & 0 & 1.3 & 0.966666667 & 0 & 0 & 0 & 0 & 0 & 1 & 0 \\
\hline & 0 & 5 & 0.966666667 & 0 & 0 & 0 & 0 & 0 & 0 & 1 \\
\hline \multirow[t]{5}{*}{23} & 0 & 6.357 & 0.716666667 & 0 & 1 & 1 & 0 & 0 & 0 & 0 \\
\hline & 1 & 11 & 0.5 & 0 & 0 & 0 & 1 & 0 & 0 & 0 \\
\hline & 0 & 0.5 & 0.433333333 & 0 & 0 & 0 & 0 & 1 & 0 & 0 \\
\hline & 0 & 1.3 & 0.766666667 & 0 & 0 & 0 & 0 & 0 & 1 & 0 \\
\hline & 0 & 5 & 0.766666667 & 0 & 0 & 0 & 0 & 0 & 0 & 1 \\
\hline \multirow[t]{5}{*}{24} & 0 & 7.18 & 0.516666667 & 0 & 1 & 1 & 0 & 0 & 0 & 0 \\
\hline & 1 & 10 & 0.333333333 & 0 & 0 & 0 & 1 & 0 & 0 & 0 \\
\hline & 0 & 0.5 & 0.316666667 & 0 & 0 & 0 & 0 & 1 & 0 & 0 \\
\hline & 0 & 1.1 & 0.566666667 & 0 & 0 & 0 & 0 & 0 & 1 & 0 \\
\hline & 0 & 5 & 0.566666667 & 0 & 0 & 0 & 0 & 0 & 0 & 1 \\
\hline
\end{tabular}




\begin{tabular}{|c|c|c|c|c|c|c|c|c|c|c|}
\hline \multirow{2}{*}{$\begin{array}{l}\text { Five alternatives } \\
\text { (Number of } \\
\text { observation) }\end{array}$} & Variable & Beta 1 & Beta 2 & Beta 3 & Beta 4 & Beta 5 & Beta 6 & Beta 7 & Beta 8 & Beta 9 \\
\hline & $\begin{array}{c}\text { Mode } \\
\text { Chosen }\end{array}$ & $\begin{array}{c}\text { TC } \\
\text { (1000 Korea won) }\end{array}$ & $\begin{array}{c}\text { TT } \\
\text { (hour) }\end{array}$ & Accom & Sexm & Age340 & Tasc & Sasc & Basc & Lasc \\
\hline \multirow[t]{5}{*}{25} & 0 & 8.78 & 0.4666666667 & 0 & 0 & 1 & 0 & 0 & 0 & 0 \\
\hline & 1 & 5.8 & 0.333333333 & 0 & 0 & 0 & 1 & 0 & 0 & 0 \\
\hline & 0 & 0.5 & 0.2833333333 & 0 & 0 & 0 & 0 & 1 & 0 & 0 \\
\hline & 0 & 1.1 & 0.5 & 0 & 0 & 0 & 0 & 0 & 1 & 0 \\
\hline & 0 & 5 & 0.5 & 0 & 0 & 0 & 0 & 0 & 0 & 1 \\
\hline \multirow[t]{5}{*}{26} & 0 & 5.18 & 0.183333333 & 2 & 0 & 0 & 0 & 0 & 0 & 0 \\
\hline & 1 & 6 & 0.5 & 2 & 0 & 0 & 1 & 0 & 0 & 0 \\
\hline & 0 & 0.5 & 0.1166666667 & 2 & 0 & 0 & 0 & 1 & 0 & 0 \\
\hline & 0 & 0.5 & 0.2 & 2 & 0 & 0 & 0 & 0 & 1 & 0 \\
\hline & 0 & 5 & 0.2 & 2 & 0 & 0 & 0 & 0 & 0 & 1 \\
\hline \multirow[t]{5}{*}{27} & 0 & 7.18 & 0.5166666667 & 5 & 0 & 0 & 0 & 0 & 0 & 0 \\
\hline & 1 & 10 & 0.5 & 5 & 0 & 0 & 1 & 0 & 0 & 0 \\
\hline & 0 & 0.5 & 0.316666667 & 5 & 0 & 0 & 0 & 1 & 0 & 0 \\
\hline & 0 & 1.1 & 0.5666666667 & 5 & 0 & 0 & 0 & 0 & 1 & 0 \\
\hline & 0 & 5 & 0.5666666667 & 5 & 0 & 0 & 0 & 0 & 0 & 1 \\
\hline \multirow[t]{5}{*}{28} & 0 & 9.076 & 0.816666667 & 5 & 0 & 1 & 0 & 0 & 0 & 0 \\
\hline & 1 & 15 & 0.5833333333 & 5 & 0 & 0 & 1 & 0 & 0 & 0 \\
\hline & 0 & 0.6 & 0.5 & 5 & 0 & 0 & 0 & 1 & 0 & 0 \\
\hline & 0 & 1.5 & 0.866666667 & 5 & 0 & 0 & 0 & 0 & 1 & 0 \\
\hline & 0 & 5 & 0.8666666667 & 5 & 0 & 0 & 0 & 0 & 0 & 1 \\
\hline \multirow[t]{5}{*}{29} & 0 & 9.076 & 0.816666667 & 2 & 0 & 0 & 0 & 0 & 0 & 0 \\
\hline & 1 & 9 & 0.5 & 2 & 0 & 0 & 1 & 0 & 0 & 0 \\
\hline & 0 & 0.6 & 0.5 & 2 & 0 & 0 & 0 & 1 & 0 & 0 \\
\hline & 0 & 1.5 & 0.866666667 & 2 & 0 & 0 & 0 & 0 & 1 & 0 \\
\hline & 0 & 5 & 0.866666667 & 2 & 0 & 0 & 0 & 0 & 0 & 1 \\
\hline \multirow[t]{5}{*}{30} & 0 & 5.298 & 0.5666666667 & 0 & 1 & 1 & 0 & 0 & 0 & 0 \\
\hline & 1 & 7 & 0.583333333 & 0 & 0 & 0 & 1 & 0 & 0 & 0 \\
\hline & 0 & 0.6 & 0.35 & 0 & 0 & 0 & 0 & 1 & 0 & 0 \\
\hline & 0 & 1.3 & 0.616666667 & 0 & 0 & 0 & 0 & 0 & 1 & 0 \\
\hline & 0 & 5 & 0.6166666667 & 0 & 0 & 0 & 0 & 0 & 0 & 1 \\
\hline \multirow[t]{5}{*}{31} & 0 & 8.017 & 1.15 & 1 & 0 & 0 & 0 & 0 & 0 & 0 \\
\hline & 1 & 15 & 0.5 & 1 & 0 & 0 & 1 & 0 & 0 & 0 \\
\hline & 0 & 0.65 & 0.7 & 1 & 0 & 0 & 0 & 1 & 0 & 0 \\
\hline & 0 & 2 & 1.233333333 & 1 & 0 & 0 & 0 & 0 & 1 & 0 \\
\hline & 0 & 5 & 1.233333333 & 1 & 0 & 0 & 0 & 0 & 0 & 1 \\
\hline \multirow[t]{5}{*}{32} & 0 & 8.18 & 0.85 & 2 & 0 & 0 & 0 & 0 & 0 & 0 \\
\hline & 1 & 12 & 0.666666667 & 2 & 0 & 0 & 1 & 0 & 0 & 0 \\
\hline & 0 & 0.6 & 0.533333333 & 2 & 0 & 0 & 0 & 1 & 0 & 0 \\
\hline & 0 & 1.1 & 0.916666667 & 2 & 0 & 0 & 0 & 0 & 1 & 0 \\
\hline & 0 & 5 & 0.916666667 & 2 & 0 & 0 & 0 & 0 & 0 & 1 \\
\hline \multirow[t]{5}{*}{33} & 0 & 5.18 & 0.183333333 & 1 & 1 & 0 & 0 & 0 & 0 & 0 \\
\hline & 1 & 5 & 0.333333333 & 1 & 0 & 0 & 1 & 0 & 0 & 0 \\
\hline & 0 & 0.5 & 0.116666667 & 1 & 0 & 0 & 0 & 1 & 0 & 0 \\
\hline & 0 & 0.5 & 0.2 & 1 & 0 & 0 & 0 & 0 & 1 & 0 \\
\hline & 0 & 5 & 0.2 & 1 & 0 & 0 & 0 & 0 & 0 & 1 \\
\hline \multirow[t]{5}{*}{34} & 0 & 8.18 & 0.85 & 0 & 0 & 1 & 0 & 0 & 0 & 0 \\
\hline & 1 & 9 & 0.5 & 0 & 0 & 0 & 1 & 0 & 0 & 0 \\
\hline & 0 & 0.6 & 0.533333333 & 0 & 0 & 0 & 0 & 1 & 0 & 0 \\
\hline & 0 & 1.1 & 0.9166666667 & 0 & 0 & 0 & 0 & 0 & 1 & 0 \\
\hline & 0 & 5 & 0.9166666667 & 0 & 0 & 0 & 0 & 0 & 0 & 1 \\
\hline \multirow[t]{5}{*}{35} & 0 & 8.017 & 1.15 & 0 & 0 & 0 & 0 & 0 & 0 & 0 \\
\hline & 1 & 17 & 0.666666667 & 0 & 0 & 0 & 1 & 0 & 0 & 0 \\
\hline & 0 & 0.65 & 0.7 & 0 & 0 & 0 & 0 & 1 & 0 & 0 \\
\hline & 0 & 2 & 1.233333333 & 0 & 0 & 0 & 0 & 0 & 1 & 0 \\
\hline & 0 & 5 & 1.233333333 & 0 & 0 & 0 & 0 & 0 & 0 & 1 \\
\hline \multirow[t]{5}{*}{36} & 0 & 5.18 & 0.183333333 & 3 & 1 & 0 & 0 & 0 & 0 & 0 \\
\hline & 1 & 1.8 & 0.1666666667 & 3 & 0 & 0 & 1 & 0 & 0 & 0 \\
\hline & 0 & 0.5 & 0.116666667 & 3 & 0 & 0 & 0 & 1 & 0 & 0 \\
\hline & 0 & 0.5 & 0.2 & 3 & 0 & 0 & 0 & 0 & 1 & 0 \\
\hline & 0 & 5 & 0.2 & 3 & 0 & 0 & 0 & 0 & 0 & 1 \\
\hline
\end{tabular}




\begin{tabular}{|c|c|c|c|c|c|c|c|c|c|c|}
\hline \multirow{2}{*}{$\begin{array}{c}\text { Five alternatives } \\
\text { (Number of } \\
\text { observation) }\end{array}$} & Variable & Beta 1 & Beta 2 & Beta 3 & Beta 4 & Beta 5 & Beta 6 & Beta 7 & Beta 8 & Beta 9 \\
\hline & $\begin{array}{c}\text { Mode } \\
\text { Chosen }\end{array}$ & $\begin{array}{c}\mathrm{TC} \\
\text { (1000 Korea won) }\end{array}$ & $\begin{array}{c}\text { TT } \\
\text { (hour) }\end{array}$ & Accom & Sexm & Age340 & Tasc & Sasc & Basc & Lasc \\
\hline \multirow[t]{5}{*}{37} & 0 & 8.18 & 1.483333333 & 0 & 0 & 0 & 0 & 0 & 0 & 0 \\
\hline & 1 & 20 & 0.666666667 & 0 & 0 & 0 & 1 & 0 & 0 & 0 \\
\hline & 0 & 0.6 & 0.916666667 & 0 & 0 & 0 & 0 & 1 & 0 & 0 \\
\hline & 0 & 1.6 & 1.583333333 & 0 & 0 & 0 & 0 & 0 & 1 & 0 \\
\hline & 0 & 5 & 1.583333333 & 0 & 0 & 0 & 0 & 0 & 0 & 1 \\
\hline \multirow[t]{5}{*}{38} & 0 & 8.476 & 0.95 & 0 & 1 & 0 & 0 & 0 & 0 & 0 \\
\hline & 1 & 12.5 & 0.583333333 & 0 & 0 & 0 & 1 & 0 & 0 & 0 \\
\hline & 0 & 0.7 & 0.583333333 & 0 & 0 & 0 & 0 & 1 & 0 & 0 \\
\hline & 0 & 1.3 & 1.016666667 & 0 & 0 & 0 & 0 & 0 & 1 & 0 \\
\hline & 0 & 5 & 1.016666667 & 0 & 0 & 0 & 0 & 0 & 0 & 1 \\
\hline \multirow[t]{5}{*}{39} & 0 & 9.18 & 1.2 & 0 & 0 & 1 & 0 & 0 & 0 & 0 \\
\hline & 1 & 28 & 0.5 & 0 & 0 & 0 & 1 & 0 & 0 & 0 \\
\hline & 0 & 0.6 & 0.733333333 & 0 & 0 & 0 & 0 & 1 & 0 & 0 \\
\hline & 0 & 1.1 & 1.283333333 & 0 & 0 & 0 & 0 & 0 & 1 & 0 \\
\hline & 0 & 5 & 1.283333333 & 0 & 0 & 0 & 0 & 0 & 0 & 1 \\
\hline \multirow[t]{5}{*}{40} & 0 & 7.18 & 0.616666667 & 0 & 0 & 0 & 0 & 0 & 0 & 0 \\
\hline & 1 & 30 & 1.666666667 & 0 & 0 & 0 & 1 & 0 & 0 & 0 \\
\hline & 0 & 0.9 & 0.383333333 & 0 & 0 & 0 & 0 & 1 & 0 & 0 \\
\hline & 0 & 1.3 & 0.666666667 & 0 & 0 & 0 & 0 & 0 & 1 & 0 \\
\hline & 0 & 5 & 0.666666667 & 0 & 0 & 0 & 0 & 0 & 0 & 1 \\
\hline \multirow[t]{5}{*}{41} & 0 & 9.18 & 1.2 & 2 & 1 & 0 & 0 & 0 & 0 & 0 \\
\hline & 1 & 15 & 0.5 & 2 & 0 & 0 & 1 & 0 & 0 & 0 \\
\hline & 0 & 0.6 & 0.733333333 & 2 & 0 & 0 & 0 & 1 & 0 & 0 \\
\hline & 0 & 1.1 & 1.2833333333 & 2 & 0 & 0 & 0 & 0 & 1 & 0 \\
\hline & 0 & 5 & 1.283333333 & 2 & 0 & 0 & 0 & 0 & 0 & 1 \\
\hline \multirow[t]{5}{*}{42} & 0 & 8.4 & 0.616666667 & 1 & 0 & 1 & 0 & 0 & 0 & 0 \\
\hline & 1 & 7.6 & 0.5 & 1 & 0 & 0 & 1 & 0 & 0 & 0 \\
\hline & 0 & 0.6 & 0.383333333 & 1 & 0 & 0 & 0 & 1 & 0 & 0 \\
\hline & 0 & 1.6 & 0.666666667 & 1 & 0 & 0 & 0 & 0 & 1 & 0 \\
\hline & 0 & 5 & 0.666666667 & 1 & 0 & 0 & 0 & 0 & 0 & 1 \\
\hline \multirow[t]{5}{*}{43} & 0 & 9.076 & 0.816666667 & 1 & 0 & 0 & 0 & 0 & 0 & 0 \\
\hline & 1 & 10 & 1 & 1 & 0 & 0 & 1 & 0 & 0 & 0 \\
\hline & 0 & 0.6 & 0.5 & 1 & 0 & 0 & 0 & 1 & 0 & 0 \\
\hline & 0 & 1.5 & 0.8666666667 & 1 & 0 & 0 & 0 & 0 & 1 & 0 \\
\hline & 0 & 5 & 0.8666666667 & 1 & 0 & 0 & 0 & 0 & 0 & 1 \\
\hline \multirow[t]{5}{*}{44} & 0 & 8.78 & 0.4666666667 & 0 & 1 & 1 & 0 & 0 & 0 & 0 \\
\hline & 1 & 10 & 1 & 0 & 0 & 0 & 1 & 0 & 0 & 0 \\
\hline & 0 & 0.5 & 0.283333333 & 0 & 0 & 0 & 0 & 1 & 0 & 0 \\
\hline & 0 & 1.1 & 0.5 & 0 & 0 & 0 & 0 & 0 & 1 & 0 \\
\hline & 0 & 5 & 0.5 & 0 & 0 & 0 & 0 & 0 & 0 & 1 \\
\hline \multirow[t]{5}{*}{45} & 0 & 5.18 & 0.183333333 & 0 & 0 & 1 & 0 & 0 & 0 & 0 \\
\hline & 1 & 3 & 0.333333333 & 0 & 0 & 0 & 1 & 0 & 0 & 0 \\
\hline & 0 & 0.5 & 0.1166666667 & 0 & 0 & 0 & 0 & 1 & 0 & 0 \\
\hline & 0 & 0.5 & 0.2 & 0 & 0 & 0 & 0 & 0 & 1 & 0 \\
\hline & 0 & 5 & 0.2 & 0 & 0 & 0 & 0 & 0 & 0 & 1 \\
\hline \multirow[t]{5}{*}{46} & 0 & 8.476 & 0.95 & 0 & 1 & 1 & 0 & 0 & 0 & 0 \\
\hline & 1 & 7.5 & 0.833333333 & 0 & 0 & 0 & 1 & 0 & 0 & 0 \\
\hline & 0 & 0.7 & 0.583333333 & 0 & 0 & 0 & 0 & 1 & 0 & 0 \\
\hline & 0 & 1.3 & 1.016666667 & 0 & 0 & 0 & 0 & 0 & 1 & 0 \\
\hline & 0 & 5 & 1.016666667 & 0 & 0 & 0 & 0 & 0 & 0 & 1 \\
\hline \multirow[t]{5}{*}{47} & 0 & 6.18 & 0.9 & 1 & 0 & 0 & 0 & 0 & 0 & 0 \\
\hline & 1 & 10 & 0.5 & 1 & 0 & 0 & 1 & 0 & 0 & 0 \\
\hline & 0 & 0.6 & 0.566666667 & 1 & 0 & 0 & 0 & 1 & 0 & 0 \\
\hline & 0 & 1.3 & 0.966666667 & 1 & 0 & 0 & 0 & 0 & 1 & 0 \\
\hline & 0 & 5 & 0.966666667 & 1 & 0 & 0 & 0 & 0 & 0 & 1 \\
\hline \multirow[t]{5}{*}{48} & 0 & 9.18 & 1.2 & 1 & 0 & 1 & 0 & 0 & 0 & 0 \\
\hline & 1 & 14 & 0.6666666667 & 1 & 0 & 0 & 1 & 0 & 0 & 0 \\
\hline & 0 & 0.6 & 0.733333333 & 1 & 0 & 0 & 0 & 1 & 0 & 0 \\
\hline & 0 & 1.1 & 1.283333333 & 1 & 0 & 0 & 0 & 0 & 1 & 0 \\
\hline & 0 & 5 & 1.283333333 & 1 & 0 & 0 & 0 & 0 & 0 & 1 \\
\hline
\end{tabular}




\begin{tabular}{|c|c|c|c|c|c|c|c|c|c|c|}
\hline \multirow{2}{*}{$\begin{array}{c}\text { Five alternatives } \\
\text { (Number of } \\
\text { observation) }\end{array}$} & Variable & Beta 1 & Beta 2 & Beta 3 & Beta 4 & Beta 5 & Beta 6 & Beta 7 & Beta 8 & Beta 9 \\
\hline & $\begin{array}{l}\text { Mode } \\
\text { Chosen }\end{array}$ & $\begin{array}{c}\text { TC } \\
\text { (1000 Korea won) } \\
\end{array}$ & $\begin{array}{c}\mathrm{TT} \\
\text { (hour) }\end{array}$ & Accom & Sexm & Age340 & Tasc & Sasc & Basc & Lasc \\
\hline \multirow[t]{5}{*}{49} & 0 & 6.18 & 0.9 & 1 & 0 & 1 & 0 & 0 & 0 & 0 \\
\hline & 1 & 8 & 0.833333333 & 1 & 0 & 0 & 1 & 0 & 0 & 0 \\
\hline & 0 & 0.6 & 0.5666666667 & 1 & 0 & 0 & 0 & 1 & 0 & 0 \\
\hline & 0 & 1.3 & 0.966666667 & 1 & 0 & 0 & 0 & 0 & 1 & 0 \\
\hline & 0 & 5 & 0.966666667 & 1 & 0 & 0 & 0 & 0 & 0 & 1 \\
\hline \multirow[t]{5}{*}{50} & 0 & 9.18 & 1.2 & 0 & 1 & 0 & 0 & 0 & 0 & 0 \\
\hline & 1 & 8 & 1 & 0 & 0 & 0 & 1 & 0 & 0 & 0 \\
\hline & 0 & 0.6 & 0.733333333 & 0 & 0 & 0 & 0 & 1 & 0 & 0 \\
\hline & 0 & 1.1 & 1.283333333 & 0 & 0 & 0 & 0 & 0 & 1 & 0 \\
\hline & 0 & 5 & 1.283333333 & 0 & 0 & 0 & 0 & 0 & 0 & 1 \\
\hline \multirow[t]{5}{*}{51} & 0 & 5.18 & 0.183333333 & 0 & 1 & 1 & 0 & 0 & 0 & 0 \\
\hline & 1 & 3 & 0.25 & 0 & 0 & 0 & 1 & 0 & 0 & 0 \\
\hline & 0 & 0.5 & 0.116666667 & 0 & 0 & 0 & 0 & 1 & 0 & 0 \\
\hline & 0 & 0.5 & 0.2 & 0 & 0 & 0 & $\mathbf{0}$ & 0 & 1 & 0 \\
\hline & 0 & 5 & 0.2 & 0 & 0 & 0 & 0 & 0 & 0 & 1 \\
\hline \multirow[t]{5}{*}{52} & 0 & 5.18 & 0.183333333 & 0 & 1 & 1 & 0 & 0 & 0 & 0 \\
\hline & 1 & 3.5 & 0.333333333 & 0 & 0 & 0 & 1 & 0 & 0 & 0 \\
\hline & 0 & 0.5 & 0.116666667 & 0 & 0 & 0 & 0 & 1 & 0 & 0 \\
\hline & 0 & 0.5 & 0.2 & 0 & 0 & 0 & 0 & 0 & 1 & 0 \\
\hline & 0 & 5 & 0.2 & 0 & 0 & 0 & 0 & 0 & 0 & 1 \\
\hline \multirow[t]{5}{*}{53} & 0 & 7.18 & 0.516666667 & 1 & 0 & 1 & 0 & 0 & 0 & 0 \\
\hline & 1 & 7 & 0.333333333 & 1 & 0 & 0 & 1 & 0 & 0 & 0 \\
\hline & 0 & 0.5 & 0.316666667 & 1 & 0 & 0 & 0 & 1 & 0 & 0 \\
\hline & 0 & 1.1 & 0.566666667 & 1 & 0 & 0. & 0 & 0 & 1 & 0 \\
\hline & 0 & 5 & 0.566666667 & 1 & 0 & 0 & 0 & 0 & 0 & 1 \\
\hline \multirow[t]{5}{*}{54} & 0 & 8.18 & 1.483333333 & 2 & 1 & 1 & 0 & 0 & 0 & 0 \\
\hline & 1. & 16.5 & 1 & 2 & 0 & 0 & 1 & 0 & 0 & 0 \\
\hline & 0 & 0.6 & 0.916666667 & 2 & 0 & 0 & 0 & 1 & 0 & 0 \\
\hline & 0 & 1.6 & 1.583333333 & 2 & 0 & 0 & 0 & 0 & 1 & 0 \\
\hline & 0 & 5 & 1.583333333 & 2 & 0 & 0 & 0 & 0 & 0 & 1 \\
\hline \multirow[t]{5}{*}{55} & 0 & 8.18 & 1.483333333 & 0 & 1 & 1 & 0 & 0 & 0 & 0 \\
\hline & 1 & 16 & 1 & 0 & 0 & 0 & 1 & 0 & 0 & 0 \\
\hline & 0 & 0.6 & 0.916666667 & 0 & 0 & 0 & 0 & 1 & 0 & 0 \\
\hline & 0 & 1.6 & 1.583333333 & 0 & 0 & 0 & 0 & 0 & 1 & 0 \\
\hline & 0 & 5 & 1.583333333 & 0 & 0 & 0 & 0 & 0 & 0 & 1 \\
\hline \multirow[t]{5}{*}{56} & 0 & 9.076 & 0.816666667 & 0 & 1 & 1 & 0 & 0 & 0 & 0 \\
\hline & 1 & 9 & 0.5 & 0 & 0 & 0 & 1 & 0 & 0 & 0 \\
\hline & 0 & 0.6 & 0.5 & 0 & 0 & 0 & 0 & 1 & 0 & 0 \\
\hline & 0 & 1.5 & 0.866666667 & 0 & 0 & 0 & 0 & 0 & 1 & 0 \\
\hline & 0 & 5 & 0.866666667 & 0 & 0 & 0 & 0 & 0 & 0 & 1 \\
\hline \multirow[t]{5}{*}{57} & 0 & 9.18 & 1.2 & 0 & 0 & 0 & 0 & 0 & 0 & 0 \\
\hline & 1 & 13.6 & 0.666666667 & 0 & 0 & 0 & 1 & 0 & 0 & 0 \\
\hline & 0 & 0.6 & 0.733333333 & 0 & 0 & 0 & 0 & 1 & 0 & 0 \\
\hline & 0 & 1.1 & 1.283333333 & 0 & 0 & 0 & 0 & 0 & 1 & 0 \\
\hline & 0 & 5 & 1.2833333333 & 0 & 0 & 0 & 0 & 0 & 0 & 1 \\
\hline \multirow[t]{5}{*}{58} & 0 & 8.18 & 1.483333333 & 2 & 0 & 0 & 0 & 0 & 0 & 0 \\
\hline & 1 & 22 & 0.5 & 2 & 0 & 0 & 1 & 0 & 0 & 0 \\
\hline & 0 & 0.6 & 0.916666667 & 2 & 0 & 0 & 0 & 1 & 0 & 0 \\
\hline & 0 & 3.5 & 1.583333333 & 2 & 0 & 0 & 0 & 0 & 1 & 0 \\
\hline & 0 & 5 & 1.583333333 & 2 & 0 & 0 & 0 & 0 & 0 & 1 \\
\hline \multirow[t]{5}{*}{59} & 0 & 8.18 & 1.483333333 & 2 & 0 & 0 & 0 & 0 & 0 & 0 \\
\hline & 1 & 22 & 0.5 & 2 & 0 & 0 & 1 & 0 & 0 & 0 \\
\hline & 0 & 0.6 & 0.916666667 & 2 & 0 & 0 & 0 & 1 & 0 & 0 \\
\hline & 0 & 3.5 & 1.583333333 & 2 & 0 & 0 & 0 & 0 & 1 & 0 \\
\hline & 0 & 5 & 1.583333333 & 2 & 0 & 0 & 0 & 0 & 0 & 1 \\
\hline \multirow[t]{5}{*}{60} & 0 & 5.18 & 0.183333333 & 0 & 0 & 0 & 0 & 0 & 0 & 0 \\
\hline & 1 & 2.1 & 0.166666667 & 0 & 0 & 0 & 1 & 0 & 0 & 0 \\
\hline & 0 & 0.5 & 0.116666667 & 0 & 0 & 0 & 0 & 1 & 0 & 0 \\
\hline & 0 & 0.5 & 0.2 & 0 & 0 & 0 & 0 & 0 & 1 & 0 \\
\hline & 0 & 5 & 0.2 & 0 & 0 & 0 & 0 & 0 & 0 & 1 \\
\hline
\end{tabular}




\begin{tabular}{|c|c|c|c|c|c|c|c|c|c|c|}
\hline \multirow{2}{*}{$\begin{array}{c}\text { Five alternatives } \\
\text { (Number of } \\
\text { observation) }\end{array}$} & Variable & Beta 1 & Beta 2 & Beta 3 & Beta 4 & Beta 5 & Beta 6 & Beta 7 & Beta 8 & Beta 9 \\
\hline & $\begin{array}{c}\text { Mode } \\
\text { Chosen }\end{array}$ & $\begin{array}{c}\text { TC } \\
\text { (1000 Korea won) }\end{array}$ & $\begin{array}{c}\text { TT } \\
\text { (hour) }\end{array}$ & Accom & Sexm & Age340 & Tasc & Sasc & Basc & Lasc \\
\hline \multirow[t]{5}{*}{61} & 0 & 9.076 & 0.816666667 & 0 & 0 & 0 & 0 & 0 & 0 & 0 \\
\hline & 1 & 12 & 0.333333333 & 0 & 0 & 0 & 1 & 0 & 0 & 0 \\
\hline & 0 & 0.6 & 0.5 & 0 & 0 & 0 & 0 & 1 & 0 & 0 \\
\hline & 0 & 1.5 & 0.866666667 & 0 & 0 & 0 & 0 & 0 & 1 & 0 \\
\hline & 0 & 5 & 0.8666666667 & 0 & 0 & 0 & 0 & 0 & 0 & 1 \\
\hline \multirow[t]{5}{*}{62} & 0 & 9.18 & 1.2 & 1 & 0 . & 0 & 0 & 0 & 0 & 0 \\
\hline & 1 & 12.5 & 0.5 & 1 & 0 & 0 & 1 & 0 & 0 & 0 \\
\hline & 0 & 0.6 & 0.733333333 & 1 & 0 & 0 & 0 & 1 & 0 & 0 \\
\hline & 0 & 1.1 & 1.283333333 & 1 & 0 & 0 & 0 & 0 & 1 & 0 \\
\hline & 0 & 5 & 1.283333333 & 1 & 0 & 0 & 0 & 0 & 0 & 1 \\
\hline \multirow[t]{5}{*}{63} & 0 & 9.076 & 0.816666667 & 2 & 0 & 1 & 0 & 0 & 0 & 0 \\
\hline & 1 & 12 & 0.6666666667 & 2 & 0 & 0 & 1 & 0 & 0 & 0 \\
\hline & 0 & 0.6 & 0.5 & 2 & 0 & 0 & 0 & 1 & 0 & 0 \\
\hline & 0 & 1.5 & 0.866666667 & 2 & 0 & 0 & 0 & 0 & 1 & 0 \\
\hline & 0 & 5 & 0.8666666667 & 2 & 0 & 0 & 0 & 0 & 0 & 1 \\
\hline \multirow[t]{5}{*}{64} & 0 & 7.18 & 0.516666667 & 0 & 0 & 1 & 0 & 0 & 0 & 0 \\
\hline & 1 & 10 & 0.666666667 & 0 & 0 & 0 & 1 & 0 & 0 & 0 \\
\hline & 0 & 0.5 & 0.316666667 & 0 & 0 & 0 & 0 & 1 & 0 & 0 \\
\hline & 0 & 1.1 & 0.566666667 & 0 & 0 & 0 & 0 & 0 & 1 & 0 \\
\hline & 0 & 5 & 0.566666667 & 0 & 0 & 0 & 0 & 0 & 0 & 1 \\
\hline \multirow[t]{5}{*}{65} & 0 & 5.18 & 0.183333333 & 1 & 0 & 0 & $\overline{0}$ & 0 & 0 & 0 \\
\hline & 1 & 4 & 0.333333333 & 1 & 0 & 0 & 1 & 0 & 0 & 0 \\
\hline & 0 & 0.5 & 0.116666667 & 1 & 0 & 0 & 0 & 1 & 0 & 0 \\
\hline & 0 & 0.5 & 0.2 & 1 & 0 & 0 & 0 & 0 & 1 & 0 \\
\hline & 0 & 5 & 0.2 & 1 & 0 & 0 & 0 & 0 & 0 & 1 \\
\hline \multirow[t]{5}{*}{66} & 0 & 9.18 & 1.2 & 0 & 0 & 1 & 0 & 0 & 0 & 0 \\
\hline & 1 & 14.4 & 0.916666667 & 0 & 0 & 0 & 1 & 0 & 0 & 0 \\
\hline & 0 & 0.6 & 0.733333333 & 0 & 0 & 0 & 0 & 1 & 0 & 0 \\
\hline & 0 & 1.1 & 1.283333333 & 0 & 0 & 0 & 0 & 0 & 1 & 0 \\
\hline & 0 & 5 & 1.283333333 & 0 & 0 & 0 & 0 & 0 & 0 & 1 \\
\hline \multirow[t]{5}{*}{67} & 0 & 9.18 & 1.2 & 0 & 1 & 0 & 0 & 0 & 0 & 0 \\
\hline & 1 & 15 & 0.333333333 & 0 & 0 & 0 & 1 & 0 & 0 & 0 \\
\hline & 0 & 0.6 & 0.733333333 & 0 & 0 & 0 & 0 & 1 & 0 & 0 \\
\hline & 0 & 1.1 & 1.283333333 & 0 & 0 & 0 & 0 & 0 & 1 & 0 \\
\hline & 0 & 5 & 1.283333333 & 0 & 0 & 0 & 0 & 0 & 0 & 1 \\
\hline \multirow[t]{5}{*}{68} & 0 & 8.18 & 0.85 & 0 & 0 & 1 & 0 & 0 . & 0 & 0 \\
\hline & 1 & 10 & 0.5 & 0 & 0 & 0 & 1 & 0 & 0 & 0 \\
\hline & 0 & 0.6 & 0.533333333 & 0 & 0 & 0 & 0 & 1 & 0 & 0 \\
\hline & 0 & 1.1 & 0.916666667 & 0 & 0 & 0 & 0 & 0 & 1 & 0 \\
\hline & 0 & 5 & 0.916666667 & 0 & 0 & 0 & 0 & 0 & 0 & 1 \\
\hline \multirow[t]{5}{*}{69} & 0 & 7.18 & 0.516666667 & 1 & 1 & 1 & 0 & 0 & 0 & 0 \\
\hline & 1 & 12.5 & 0.6666666667 & 1 & 0 & 0 & 1 & 0 & 0 & 0 \\
\hline & 0 & 0.5 & 0.3166666667 & 1 & 0 & 0 & 0 & 1 & 0 & 0 \\
\hline & 0 & 1.1 & 0.5666666667 & 1 & 0 & 0 & 0 & 0 & 1 & 0 \\
\hline & 0 & 5 & 0.566666667 & 1 & 0 & 0 & 0 & 0 & 0 & 1 \\
\hline \multirow[t]{5}{*}{70} & 0 & 18.18 & 2.0666666667 & 1 & 1 & 0 & 0 & 0 & 0 & 0 \\
\hline & 1 & 53 & 1.333333333 & 1 & 0 & 0 & 1 & 0 & 0 & 0 \\
\hline & 0 & 1 & 1.283333333 & 1 & 0 & 0 & 0 & 1 & 0 & 0 \\
\hline & 0 & 7.5 & 2.2 & 1 & 0 & 0 & 0 & 0 & 1 & 0 \\
\hline & 0 & 15 & 2.2 & 1 & 0 & 0 & 0 & 0 & 0 & 1 \\
\hline
\end{tabular}


C.3: Subway alternative -112 observations

\begin{tabular}{|c|c|c|c|c|c|c|c|c|c|c|}
\hline \multirow{2}{*}{$\begin{array}{c}\text { Five alternatives } \\
\text { (Number of } \\
\text { observation) }\end{array}$} & Variable & Beta 1 & Beta 2 & Beta 3 & Beta 4 & Beta 5 & Beta 6 & Beta 7 & Beta 8 & Beta 9 \\
\hline & $\begin{array}{l}\text { Mode } \\
\text { Chosen }\end{array}$ & $\begin{array}{c}\text { TC } \\
\text { (1000 Korea won) }\end{array}$ & $\begin{array}{c}\mathrm{TT} \\
\text { (hour) }\end{array}$ & Accom & Sexm & Age 340 & Tasc & Sasc & Basc & Lasc \\
\hline \multirow[t]{5}{*}{ SUBWAY 1} & 0 & 9.18 & 1.2 & 4 & 0 & 0 & 0 & 0 & 0 & 0 \\
\hline & 0 & 28 & 1.2 & 4 & 0 & 0 & 1 & 0 & 0 & 0 \\
\hline & 1 & 0.6 & 1.5 & 4 & 0 & 0 & 0 & 1 & 0 & 0 \\
\hline & 0 & 1.1 & 1.283333333 & 4 & 0 & 0 & 0 & 0 & 1 & 0 \\
\hline & 0 & 5 & 1.283333333 & 4 & 0 & 0 & 0 & 0 & 0 & 1 \\
\hline \multirow[t]{5}{*}{2} & 0 & 10.18 & 1.6666666667 & 3 & 0 & 0 & 0 & 0 & 0 & 0 \\
\hline & 0 & 13.127 & 1.666666667 & 3 & 0 & 0 & 1 & 0 & 0 & 0 \\
\hline & 1 & 0.6 & 2 & 3 & 0 & 0 & 0 & 1 & 0 & 0 \\
\hline & 0 & 2 & 1.8 & 3 & 0 & 0 & 0 & 0 & 1 & 0 \\
\hline & 0 & 5 & 1.8 & 3 & 0 & 0 & 0 & 0 & 0 & 1 \\
\hline \multirow[t]{5}{*}{3} & 0 & 8.18 & 1.533333333 & 1 & 0 & 1 & 0 & 0 & 0 & 0 \\
\hline & 0 & 12.052 & 1.533333333 & 1 & 0 & 0 & 1 & 0 & 0 & 0 \\
\hline & 1 & 0.6 & 1.5 & 1 & 0 & 0 & 0 & 1 & 0 & 0 \\
\hline & 0 & 5 & 1.633333333 & 1 & 0 & 0 & 0 & 0 & 1 & 0 \\
\hline & 0 & 5 & 1.633333333 & 1 & 0 & 0 & 0 & 0 & 0 & 1 \\
\hline \multirow[t]{5}{*}{4} & 0 & 6.357 & 0.716666667 & 1 & 0 & 0 & 0 & 0 & 0 & 0 \\
\hline & 0 & 11 & 0.716666667 & 1 & 0 & 0 & 1 & 0 & 0 & 0 \\
\hline & 1 & 0.5 & 1 & 1 & 0 & 0 & 0 & 1 & 0 & 0 \\
\hline & 0 & 1.3 & 0.766666667 & 1 & 0 & 0 & 0 & 0 & 1 & 0 \\
\hline & 0 & 5 & 0.766666667 & 1 & 0 & 0 & 0 & 0 & 0 & 1 \\
\hline \multirow[t]{5}{*}{5} & 0 & 9.18 & 1.2 & 1 & 0 & 0 & 0 & 0 & 0 & 0 \\
\hline & 0 & 28 & 1.2 & 1 & 0 & 0 & 1 & 0 & 0 & 0 \\
\hline & 1 & 1 & 1 & 1 & 0 & 0 & 0 & 1 & 0 & 0 \\
\hline & 0 & 1.1 & 1.283333333 & 1 & 0 & 0 & 0 & 0 & 1 & 0 \\
\hline & 0 & 5 & 1.283333333 & 1 & 0 & 0 & 0 & 0 & 0 & 1 \\
\hline \multirow[t]{5}{*}{6} & 0 & 6.18 & 0.9 & 0 & 0 & 0 & 0 & 0 & 0 & 0 \\
\hline & 0 & 10 & 0.9 & 0 & 0 & 0 & 1 & 0 & 0 & 0 \\
\hline & 1 & 0.6 & 1 & 0 & 0 & 0 & 0 & 1 & 0 & 0 \\
\hline & 0 & 1.3 & 0.9666666667 & 0 & 0 & 0 & 0 & 0 & 1 & 0 \\
\hline & 0 & 5 & 0.9666666667 & 0 & 0 & 0 & 0 & 0 & 0 & 1 \\
\hline \multirow[t]{5}{*}{7} & 0 & 8.18 & 1.533333333 & 0 & 0 & 1 & 0 & 0 & 0 & 0 \\
\hline & 0 & 12.052 & 1.533333333 & 0 & 0 & 0 & 1 & 0 & 0 & 0 \\
\hline & 1 & 0.6 & 1.166666667 & 0 & 0 & 0 & 0 & 1 & 0 & 0 \\
\hline & 0 & 5 & 1.633333333 & 0 & 0 & 0 & 0 & 0 & 1 & 0 \\
\hline & 0 & 5 & 1.633333333 & 0 & 0 & 0 & 0 & 0 & 0 & 1 \\
\hline \multirow[t]{5}{*}{8} & 0 & 5.298 & 0.566666667 & 1 & 0 & 0 & 0 & 0 & 0 & 0 \\
\hline & 0 & 7 & 0.566666667 & 1 & 0 & 0 & 1 & 0 & 0 & 0 \\
\hline & 1 & 0.6 & 1 & 1 & 0 & 0 & 0 & 1 & 0 & 0 \\
\hline & 0 & 1.3 & 0.616666667 & 1 & 0 & 0 & 0 & 0 & 1 & 0 \\
\hline & 0 & 5 & 0.616666667 & 1 & 0 & 0 & 0 & 0 & 0 & 1 \\
\hline \multirow[t]{5}{*}{9} & 0 & 13.18 & 1.866666667 & 1 & 1 & 1 & 0 & 0 & 0 & 0 \\
\hline & 0 & 28 & 1.866666667 & 1 & 0 & 0 & 1 & 0 & 0 & 0 \\
\hline & 1 & 0.85 & 1 & 1 & 0 & 0 & 0 & 1 & 0 & 0 \\
\hline & 0 & 1.5 & 2 & 1 & 0 & 0 & 0 & 0 & 1 & 0 \\
\hline & 0 & 5 & 2 & 1 & 0 & 0 & 0 & 0 & 0 & 1 \\
\hline \multirow[t]{5}{*}{10} & 0 & 8.18 & 1.433333333 & 0 & 1 & 1 & 0 & 0 & 0 & 0 \\
\hline & 0 & 20 & 1.433333333 & 0 & 0 & 0 & 1 & 0 & 0 & 0 \\
\hline & 1 & 0.6 & 0.833333333 & 0 & 0 & 0 & 0 & 1 & 0 & 0 \\
\hline & 0 & 1.6 & 1.533333333 & 0 & 0 & 0 & 0 & 0 & 1 & 0 \\
\hline & 0 & 5 & 1.533333333 & 0 & 0 & 0 & 0 & 0 & 0 & 1 \\
\hline \multirow[t]{5}{*}{11} & 0 & 9.18 & 1.2 & 1 & 0 & 1 & 0 & 0 & 0 & 0 \\
\hline & 0 & 28 & 1.2 & 1 & 0 & 0 & 1 & 0 & 0 & 0 \\
\hline & 1 & 0.6 & 1.666666667 & 1 & 0 & 0 & 0 & 1 & 0 & 0 \\
\hline & 0 & 1.1 & 1.283333333 & 1 & 0 & 0 & 0 & 0 & 1 & 0 \\
\hline & 0 & 5 & 1.283333333 & 1 & 0 & 0 & 0 & 0 & 0 & 1 \\
\hline \multirow[t]{5}{*}{12} & 0 & 8.18 & 1.483333333 & 0 & 1 & 0 & 0 & 0 & 0 & 0 \\
\hline & 0 & 20 & 1.483333333 & 0 & 0 & 0 & 1 & 0 & 0 & 0 \\
\hline & 1 & 0.6 & 1.666666667 & 0 & 0 & 0 & 0 & 1 & 0 & 0 \\
\hline & 0 & 1.6 & 1.583333333 & 0 & 0 & 0 & 0 & 0 & 1 & 0 \\
\hline & 0 & 5 & 1.583333333 & 0 & 0 & 0 & 0 & 0 & 0 & 1 \\
\hline
\end{tabular}




\begin{tabular}{|c|c|c|c|c|c|c|c|c|c|c|}
\hline \multirow{2}{*}{$\begin{array}{l}\text { Five alternatives } \\
\text { (Number of } \\
\text { observation) }\end{array}$} & Variable & Beta 1 & Beta 2 & Beta 3 & Beta 4 & Beta 5 & Beta 6 & Beta 7 & Beta 8 & Beta 9 \\
\hline & $\begin{array}{l}\text { Mode } \\
\text { Chosen }\end{array}$ & $\begin{array}{c}\mathrm{TC} \\
\text { (1000 Korea won) } \\
\end{array}$ & $\begin{array}{c}\mathrm{TT} \\
\text { (hour) }\end{array}$ & Accom & Sexm & Age340 & Tasc & Sasc & Basc & Lasc \\
\hline \multirow[t]{5}{*}{13} & 0 & 8.476 & 0.95 & 0 & 1 & 0 & 0 & 0 & 0 & 0 \\
\hline & 0 & 12.5 & 0.95 & 0 & 0 & 0 & 1 & 0 & 0 & 0 \\
\hline & 1 & 0.6 & 0.75 & 0 & 0 & 0 & 0 & 1 & 0 & 0 \\
\hline & 0 & 1.3 & 1.016666667 & 0 & 0 & 0 & 0 & 0 & 1 & 0 \\
\hline & 0 & 5 & 1.016666667 & 0 & 0 & 0 & 0 & 0 & 0 & 1 \\
\hline \multirow[t]{5}{*}{14} & 0 & 18.18 & 2.066666667 & 0 & 0 & 0 & 0 & 0 & 0 & 0 \\
\hline & 0 & 53 & 2.066666667 & 0 & 0 & 0 & 1 & 0 & 0 & 0 \\
\hline & 1 & 1 & 1.166666667 & 0 & 0 & 0 & 0 & 1 & 0 & 0 \\
\hline & 0 & 7.5 & 2.2 & 0 & 0 & 0 & 0 & 0 & 1 & 0 \\
\hline & 0 & 15 & 2.2 & 0 & 0 & 0 & 0 & 0 & 0 & 1 \\
\hline \multirow[t]{5}{*}{15} & 0 & 8.18 & 1.483333333 & 0 & 0 & 0 & 0 & 0 & 0 & 0 \\
\hline & 0 & 20 & 1.483333333 & 0 & 0 & 0 & 1 & 0 & 0 & 0 \\
\hline & 1 & 1.1 & 1.75 & 0 & 0 & 0 & 0 & 1 & 0 & 0 \\
\hline & 0 & 1.6 & 1.583333333 & 0 & 0 & 0 & 0 & 0 & 1 & 0 \\
\hline & 0 & 5 & 1.583333333 & 0 & 0 & 0 & 0 & 0 & 0 & 1 \\
\hline \multirow[t]{5}{*}{16} & 0 & 8.18 & 1.333333333 & 0 & 0 & 0 & 0 & 0 & 0 & 0 \\
\hline & 0 & 15.5 & 1.333333333 & 0 & 0 & 0 & 1 & 0 & 0 & 0 \\
\hline & 1 & 0.7 & 1.5 & 0 & 0 & 0 & 0 & 1 & 0 & 0 \\
\hline & 0 & 1.6 & 1.433333333 & 0 & 0 & 0 & 0 & 0 & 1 & 0 \\
\hline & 0 & 5 & 1.433333333 & 0 & 0 & 0 & 0 & 0 & 0 & 1 \\
\hline \multirow[t]{5}{*}{17} & 0 & 5.18 & 0.183333333 & 0 & 1 & 1 & 0 & 0 & 0 & 0 \\
\hline & 0. & 6 & 0.183333333 & 0 & 0 & 0 & 1 & 0 & 0 & 0 \\
\hline & 1 & 0.5 & 0.333333333 & 0 & 0 & 0 & 0 & 1 & 0 & 0 \\
\hline & 0 & 0.5 & 0.2 & 0 & 0 & 0 & 0 & 0 & 1 & 0 \\
\hline & 0 & 5 & 0.2 & 0 & 0 & 0 & 0 & 0 & 0 & 1 \\
\hline \multirow[t]{5}{*}{18} & 0 & 9.18 & 1.2 & 1 & 0 & 0 & 0 & 0 & 0 & 0 \\
\hline & 0 & 28 & 1.2 & 1 & 0 & 0 & 1 & 0 & 0 & 0 \\
\hline & 1 & 0.6 & 0.75 & 1 & 0 & 0 & 0 & 1 & 0 & 0 \\
\hline & 0 & 1.1 & 1.283333333 & 1 & 0 & 0 & 0 & 0 & 1 & 0 \\
\hline & 0 & 5 & 1.283333333 & 1 & 0 & 0 & 0 & 0 & 0 & 1 \\
\hline \multirow[t]{5}{*}{19} & 0 & 13.18 & 1.866666667 & 0 & 0 & 1 & 0 & 0 & 0 & 0 \\
\hline & 0 & 25 & 1.866666667 & 0 & 0 & 0 & 1 & 0 & 0 & 0 \\
\hline & 1 & 0.7 & 1.5 & 0 & 0 & 0 & 0 & 1 & 0 & 0 \\
\hline & 0 & 1.5 & 2 & 0 & 0 & 0 & 0 & 0 & 1 & 0 \\
\hline & 0 & 5 & 2 & 0 & 0 & 0 & 0 & 0 & 0 & 1 \\
\hline \multirow[t]{5}{*}{20} & 0 & 8.017 & 1.15 & 0 & 1 & 0 & 0 & 0 & 0 & 0 \\
\hline & 0 & 17 & 1.15 & 0 & 0 & 0 & 1 & 0 & 0 & 0 \\
\hline & 1 & 0.6 & 0.6666666667 & 0 & 0 & 0 & 0 & 1 & 0 & 0 \\
\hline & 0 & 2 & 1.233333333 & 0 & 0 & 0 & 0 & 0 & 1 & 0 \\
\hline & 0 & 5 & 1.233333333 & 0 & 0 & 0 & 0 & 0 & 0 & 1 \\
\hline \multirow[t]{5}{*}{21} & 0 & 7.18 & 0.516666667 & 0 & 0 & 0 & 0 & 0 & 0 & 0 \\
\hline & 0 & 10 & 0.516666667 & 0 & 0 & 0 & 1 & 0 & 0 & 0 \\
\hline & 1 & 0.5 & 1 & 0 & 0 & 0 & 0 & 1 & 0 & 0 \\
\hline & 0 & 1.1 & 0.5666666667 & 0 & 0 & 0 & 0 & 0 & 1 & 0 \\
\hline & 0 & 5 & 0.566666667 & 0 & 0 & 0 & 0 & 0 & 0 & 1 \\
\hline \multirow[t]{5}{*}{22} & 0 & 8.48 & 1.816666667 & 4 & 1 & 1 & 0 & 0 & 0 & 0 \\
\hline & 0 & 28 & 1.816666667 & 4 & 0 & 0 & 1 & 0 & 0 & 0 \\
\hline & 1 & 0.65 & 0.833333333 & 4 & 0 & 0 & 0 & 1 & 0 & 0 \\
\hline & 0 & 1 & 1.95 & 4 & 0 & 0 & 0 & 0 & 1 & 0 \\
\hline & 0 & 5 & 1.95 & 4 & 0 & 0 & 0 & 0 & 0 & 1 \\
\hline \multirow[t]{5}{*}{23} & 0 & 8.18 & 1.433333333 & 4 & 1 & 0 & 0 & 0 & 0 & 0 \\
\hline & 0 & 12 & 1.433333333 & 4 & 0 & 0 & 1 & 0 & 0 & 0 \\
\hline & 1 & 0.9 & 1.5 & 4 & 0 & 0 & 0 & 1 & 0 & 0 \\
\hline & 0 & 1.1 & 1.533333333 & 4 & 0 & 0 & 0 & 0 & 1 & 0 \\
\hline & 0 & 5 & 1.533333333 & 4 & 0 & 0 & 0 & 0 & 0 & 1 \\
\hline \multirow[t]{5}{*}{24} & 0 & 13.18 & 1.866666667 & 0 & 0 & 1 & 0 & 0 & 0 & 0 \\
\hline & 0 & 28 & 1.866666667 & 0 & 0 & 0 & 1 & 0 & 0 & 0 \\
\hline & 1 & 0.7 & 0.75 & 0 & 0 & 0 & 0 & 1 & 0 & 0 \\
\hline & 0 & 1.5 & 2 & 0 & 0 & 0 & 0 & 0 & 1 & 0 \\
\hline & 0 & 5 & 2 & 0 & 0 & 0 & 0 & 0 & 0 & 1 \\
\hline
\end{tabular}




\begin{tabular}{|c|c|c|c|c|c|c|c|c|c|c|}
\hline \multirow{2}{*}{$\begin{array}{c}\text { Five alternatives } \\
\text { (Number of } \\
\text { observation) }\end{array}$} & Variable & Beta 1 & Beta 2 & Beta 3 & Beta 4 & Beta 5 & Beta 6 & Beta 7 & Beta 8 & Beta 9 \\
\hline & $\begin{array}{c}\text { Mode } \\
\text { Chosen }\end{array}$ & $\begin{array}{c}\text { TC } \\
\text { (1000 Korea won) }\end{array}$ & $\begin{array}{c}\mathrm{TT} \\
\text { (hour) }\end{array}$ & Accom & Sexm & Age340 & Tase & Sasc & Basc & Lasc \\
\hline \multirow[t]{5}{*}{25} & 0 & 5.18 & 0.183333333 & 0 & 0 & 0 & 0 & 0 & 0 & 0 \\
\hline & 0 & 6 & 0.183333333 & 0 & 0 & 0 & $\overline{1}$ & 0 & 0 & 0 \\
\hline & 1 & 1 & 0.5 & 0 & 0 & 0 & 0 & 1 & 0 & 0 \\
\hline & 0 & 0.5 & 0.2 & 0 & 0 & 0 & 0 & 0 & 1 & 0 \\
\hline & 0 & 5 & 0.2 & 0 & 0 & 0 & 0 & 0 & 0 & 1 \\
\hline \multirow[t]{5}{*}{26} & 0 & 8.476 & 0.95 & 0 & 0 & 1 & 0 & 0 & 0 & 0 \\
\hline & 0 & 12.5 & 0.95 & 0 & 0 & 0 & 1 & 0 & 0 & 0 \\
\hline & 1 & 0.6 & 1 & 0 & 0 & 0 & 0 & 1 & 0 & 0 \\
\hline & 0 & 1.3 & 1.0166666667 & 0 & 0 & 0 & 0 & 0 & 1 & 0 \\
\hline & 0 & 5 & 1.0166666667 & 0 & 0 & 0 & 0 & 0 & 0 & 1 \\
\hline \multirow[t]{5}{*}{27} & 0 & 5.31 & 0.8166666667 & 1 & 0 & 0 & 0 & 0 & 0 & 0 \\
\hline & 0 & 15 & 0.8166666667 & 1 & 0 & 0 & 1 & 0 & 0 & 0 \\
\hline & 1 & 0.8 & 1.5 & 1 & 0 & 0 & 0 & 1 & 0 & 0 \\
\hline & 0 & 1.4 & 0.8666666667 & 1 & 0 & 0 & 0 & 0 & 1 & 0 \\
\hline & 0 & 5 & 0.8666666667 & 1 & 0 & 0 & 0 & 0 & 0 & 1 \\
\hline \multirow[t]{5}{*}{28} & 0 & 6.18 & 0.9 & 0 & 1 & 0 & 0 & 0 & 0 & 0 \\
\hline & 0 & 10 & 0.9 & 0 & 0 & 0 & 1 & 0 & 0 & 0 \\
\hline & 1 & 0.6 & 0.75 & 0 & 0 & 0 & 0 & 1 & 0 & 0 \\
\hline & 0 & 1.3 & 0.966666667 & 0 & 0 & 0 & 0 & 0 & 1 & 0 \\
\hline & 0 & 5 & 0.9666666667 & 0 & 0 & 0 & 0 & 0 & 0 & 1 \\
\hline \multirow[t]{5}{*}{29} & 0 & 8.18 & 0.85 & 2 & 1 & 0 & 0 & 0 & 0 & 0 \\
\hline & 0 & 10 & 0.85 & 2 & 0 & 0 & 1 & 0 & 0 & 0 \\
\hline & 1 & 0.65 & 1 & 2 & 0 & 0 & 0 & 1 & 0 & 0 \\
\hline & 0 & 1.1 & 0.916666667 & 2 & 0 & 0 & 0 & 0 & 1 & 0 \\
\hline & 0 & 5 & 0.916666667 & 2 & 0 & 0 & 0 & 0 & 0 & 1 \\
\hline \multirow[t]{5}{*}{30} & 0 & 8.78 & 0.466666667 & 5 & 1 & 0 & 0 & 0 & 0 & 0 \\
\hline & 0 & 10 & 0.466666667 & 5 & 0 & 0 & 1 & 0 & 0 & 0 \\
\hline & 1 & 0.5 & 0.333333333 & 5 & 0 & 0 & 0 & 1 & 0 & 0 \\
\hline & 0 & 1.1 & 0.5 & 5 & 0 & 0 & 0 & 0 & 1 & 0 \\
\hline & 0 & 5 & 0.5 & 5 & 0 & 0 & 0 & 0 & 0 & 1 \\
\hline \multirow[t]{5}{*}{31} & 0 & 7.18 & 0.5166666667 & 0 & 1 & 0 & 0 & 0 & 0 & 0 \\
\hline & 0 & 10 & 0.516666667 & 0 & 0 & 0 & 1 & 0 & 0 & 0 \\
\hline & 1 & 0.5 & 0.4166666667 & 0 & 0 & 0 & 0 & 1 & 0 & 0 \\
\hline & 0 & 1.1 & 0.5666666667 & 0 & 0 & 0 & 0 & 0 & 1 & 0 \\
\hline & 0 & 5 & 0.5666666667 & 0 & 0 & 0 & 0 & 0 & 0 & 1 \\
\hline \multirow[t]{5}{*}{32} & 0 & 8.18 & 0.85 & 0 & 1 & 0 & 0 & 0 & 0 & 0 \\
\hline & 0 & 10 & 0.85 & 0 & 0 & 0 & 1 & 0 & 0 & 0 \\
\hline & 1 & 0.6 & 1.333333333 & 0 & 0 & 0 & 0 & 1 & 0 & 0 \\
\hline & 0 & 1.1 & 0.9166666667 & 0 & 0 & 0 & 0 & 0 & 1 & 0 \\
\hline & 0 & 5 & 0.9166666667 & 0 & 0 & 0 & 0 & 0 & 0 & 1 \\
\hline \multirow[t]{5}{*}{33} & 0 & 8.18 & 1.3333333333 & 0 & 1 & 1 & 0 & 0 & 0 & 0 \\
\hline & 0 & 15.5 & 1.333333333 & 0 & 0 & 0 & 1 & 0 & 0 & 0 \\
\hline & 1 & 0.8 & 1.5 & 0 & 0 & 0 & 0 & 1 & 0 & 0 \\
\hline & 0 & 1.6 & 1.433333333 & 0 & 0 & 0 & 0 & 0 & 1 & 0 \\
\hline & 0 & 5 & 1.433333333 & 0 & 0 & 0 & 0 & 0 & 0 & 1 \\
\hline \multirow[t]{5}{*}{34} & 0 & 8.18 & 1.483333333 & 0 & 1 & 0 & 0 & 0 & 0 & 0 \\
\hline & 0 & 20 & 1.483333333 & 0 & 0 & 0 & 1 & 0 & 0 & 0 \\
\hline & 1 & 0.6 & 1.5 & 0 & 0 & 0 & 0 & 1 & 0 & 0 \\
\hline & 0 & 1.6 & 1.583333333 & 0 & 0 & 0 & 0 & 0 & 1 & 0 \\
\hline & 0 & 5 & 1.5833333333 & 0 & 0 & 0 & 0 & 0 & 0 & 1 \\
\hline \multirow[t]{5}{*}{35} & 0 & 8.48 & 1.8166666667 & 0 & 1 & 0 & 0 & 0 & 0 & 0 \\
\hline & 0 & 28 & 1.8166666667 & 0 & 0 & 0 & 1 & 0 & 0 & 0 \\
\hline & 1 & 0.65 & 1 & 0 & 0 & 0 & 0 & 1 & 0 & 0 \\
\hline & 0 & 1 & 1.95 & 0 & 0 & 0 & 0 & 0 & 1 & 0 \\
\hline & 0 & 5 & 1.95 & 0 & 0 & 0 & 0 & 0 & 0 & 1 \\
\hline \multirow[t]{5}{*}{36} & 0 & 7.18 & 0.5166666667 & 0 & 1 & 0 & 0 & 0 & 0 & 0 \\
\hline & 0 & 10 & 0.516666667 & 0 & 0 & 0 & 1 & 0 & 0 & 0 \\
\hline & 1 & 0.6 & 0.666666667 & 0 & 0 & 0 & 0 & 1 & 0 & 0 \\
\hline & 0 & 1.1 & 0.566666667 & 0 & 0 & 0 & 0 & 0 & 1 & 0 \\
\hline & 0 & 5 & 0.566666667 & 0 & 0 & 0 & 0 & 0 & 0 & 1 \\
\hline
\end{tabular}




\begin{tabular}{|c|c|c|c|c|c|c|c|c|c|c|}
\hline \multirow{2}{*}{$\begin{array}{l}\text { Five alternatives } \\
\text { (Number of } \\
\text { observation) }\end{array}$} & Variable & Beta 1 & Beta 2 & Beta 3 & Beta 4 & Beta 5 & Beta 6 & Beta 7 & Beta 8 & Beta 9 \\
\hline & $\begin{array}{c}\text { Mode } \\
\text { Chosen }\end{array}$ & $\begin{array}{c}\text { TC } \\
\text { (1000 Korea won) } \\
\end{array}$ & $\begin{array}{c}\text { TT } \\
\text { (hour) }\end{array}$ & Accom & Sexm & Age340 & Tasc & Sasc & Basc & Lasc \\
\hline \multirow[t]{5}{*}{37} & 0 & 9.026 & 0.4666666667 & 0 & 1 & 1 & 0 & 0 & 0 & 0 \\
\hline & 0 & 20 & 0.466666667 & 0 & 0 & 0 & 1 & 0 & 0 & 0 \\
\hline & 1 & 0.7 & 1.5 & 0 & 0 & 0 & 0 & 1 & 0 & 0 \\
\hline & 0 & 1.5 & 0.5 & 0 & 0 & 0 & 0 & 0 & 1 & 0 \\
\hline & 0 & 5 & 0.5 & 0 & 0 & 0 & 0 & 0 & 0 & 1 \\
\hline \multirow[t]{5}{*}{38} & 0 & 10.18 & 1.6666666667 & 0 & 1 & 1 & 0 & 0 & 0 & 0 \\
\hline & 0 & 13.127 & 1.6666666667 & 0 & 0 & 0 & 1 & 0 & 0 & 0 \\
\hline & 1 & 0.6 & 1.6666666667 & 0 & 0 & 0 & 0 & 1 & 0 & 0 \\
\hline & 0 & 2 & 1.8 & 0 & 0 & 0 & 0 & 0 & 1 & 0 \\
\hline & 0 & 5 & 1.8 & 0 & 0 & 0 & 0 & 0 & 0 & 1 \\
\hline \multirow[t]{5}{*}{39} & 0 & 8.476 & 0.95 & 0 & 1 & 1 & 0 & 0 & 0 & 0 \\
\hline & 0 & 12.5 & 0.95 & 0 & 0 & 0 & 1 & 0 & 0 & 0 \\
\hline & 1 & 0.6 & 1.1666666667 & 0 & 0 & 0 & 0 & 1 & 0 & 0 \\
\hline & 0 & 1.3 & 1.0166666667 & 0 & 0 & 0 & 0 & 0 & 1 & 0 \\
\hline & 0 & 5 & 1.0166666667 & 0 & 0 & 0 & 0 & 0 & 0 & 1 \\
\hline \multirow[t]{5}{*}{40} & 0 & 5.18 & 0.183333333 & 1 & 0 & 0 & 0 & 0 & 0 & 0 \\
\hline & 0 & 6 & 0.183333333 & 1 & 0 & 0 & 1 & 0 & 0 & 0 \\
\hline & 1 & 0.5 & 0.333333333 & 1 & 0 & 0 & 0 & 1 & 0 & 0 \\
\hline & 0 & 0.5 & 0.2 & 1 & 0 & 0 & 0 & 0 & 1 & 0 \\
\hline & 0 & 5 & 0.2 & 1 & 0 & 0 & 0 & 0 & 0 & 1 \\
\hline \multirow[t]{5}{*}{41} & 0 & 8.4 & 0.616666667 & 2 & 1 & 1 & 0 & 0 & 0 & 0 \\
\hline & 0 & 7.6 & 0.616666667 & 2 & 0 & 0 & 1 & 0 & 0 & 0 \\
\hline & 1 & 0.6 & 0.5 & 2 & 0 & 0 & 0 & 1 & 0 & 0 \\
\hline & 0 & 1.6 & 0.666666667 & 2 & 0 & 0 & 0 & 0 & 1 & 0 \\
\hline & 0 & 5 & 0.666666667 & 2 & 0 & 0 & 0 & 0 & 0 & 1 \\
\hline \multirow[t]{5}{*}{42} & 0 & 5.18 & 0.183333333 & 1 & 1 & 0 & 0 & 0 & 0 & 0 \\
\hline & 0 & 6 & 0.183333333 & 1 & 0 & 0 & 1 & 0 & 0 & 0 \\
\hline & 1 & 0.5 & 0.5 & 1 & 0 & 0 & 0 & 1 & 0 & 0 \\
\hline & 0 & 0.5 & 0.2 & 1 & 0 & 0 & 0 & 0 & 1 & 0 \\
\hline & 0 & 5 & 0.2 & 1 & 0 & 0 & 0 & 0 & 0 & 1 \\
\hline \multirow[t]{5}{*}{43} & 0 & 9.026 & 0.4666666667 & 3 & 0 & 1 & 0 & 0 & 0 & 0 \\
\hline & 0 & 20 & 0.4666666667 & 3 & 0 & 0 & 1 & 0 & 0 & 0 \\
\hline & 1 & 0.65 & 0.666666667 & 3 & 0 & 0 & 0 & 1 & 0 & 0 \\
\hline & 0 & 1.5 & 0.5 & 3 & 0 & 0 & 0 & 0 & 1 & 0 \\
\hline & 0 & 5 & 0.5 & 3 & 0 & 0 & 0 & 0 & 0 & 1 \\
\hline \multirow[t]{5}{*}{44} & 0 & 7.18 & 0.5166666667 & 1 & 0 & 0 & 0 & 0 & 0 & 0 \\
\hline & 0 & 10 & 0.516666667 & 1 & 0 & 0 & 1 & 0 & 0 & 0 \\
\hline & 1 & 0.5 & 0.5 & 1 & 0 & 0 & 0 & 1 & 0 & 0 \\
\hline & 0 & 1.1 & 0.566666667 & 1 & 0 & 0 & 0 & 0 & 1 & 0 \\
\hline & 0 & 5 & 0.566666667 & 1 & 0 & 0 & 0 & 0 & 0 & 1 \\
\hline \multirow[t]{5}{*}{45} & 0 & 8.78 & 0.466666667 & 0 & 1 & 1 & 0 & 0 & 0 & 0 \\
\hline & 0 & 10 & 0.4666666667 & 0 & 0 & 0 & 1 & 0 & 0 & 0 \\
\hline & 1 & 0.5 & 0.333333333 & 0 & 0 & 0 & 0 & 1 & 0 & 0 \\
\hline & 0 & 1.1 & 0.5 & 0 & 0 & 0 & 0 & 0 & 1 & 0 \\
\hline & 0 & 5 & 0.5 & 0 & 0 & 0 & 0 & 0 & 0 & 1 \\
\hline \multirow[t]{5}{*}{46} & 0 & 8.18 & 1.483333333 & 2 & 0 & 0 & 0 & 0 & 0 & 0 \\
\hline & 0 & 20 & 1.483333333 & 2 & 0 & 0 & 1 & 0 & 0 & 0 \\
\hline & 1 & 0.6 & 1.5 & 2 & 0 & 0 & 0 & 1 & 0 & 0 \\
\hline & 0 & 1.6 & 1.583333333 & 2 & 0 & 0 & 0 & 0 & 1 & 0 \\
\hline & 0 & 5 & 1.583333333 & 2 & 0 & 0 & 0 & 0 & 0 & 1 \\
\hline \multirow[t]{5}{*}{47} & 0 & 8.476 & 0.95 & 2 & 1 & 1 & 0 & 0 & 0 & 0 \\
\hline & 0 & 12.5 & 0.95 & 2 & 0 & 0 & 1 & 0 & 0 & 0 \\
\hline & 1 & 0.5 & 0.75 & 2 & 0 & 0 & 0 & 1 & 0 & 0 \\
\hline & 0 & 1.3 & 1.0166666667 & 2 & 0 & 0 & 0 & 0 & 1 & 0 \\
\hline & 0 & 5 & 1.0166666667 & 2 & 0 & 0 & 0 & 0 & 0 & 1 \\
\hline \multirow[t]{5}{*}{48} & 0 & 8.18 & 1.4833333333 & 0 & 1 & 1 & 0 & 0 & 0 & 0 \\
\hline & 0 & 20 & 1.483333333 & 0 & 0 & 0 & 1 & 0 & 0 & 0 \\
\hline & 1 & 0.6 & 1.5 & 0 & 0 & 0 & 0 & 1 & 0 & 0 \\
\hline & 0 & 1.6 & 1.583333333 & 0 & 0 & 0 & 0 & 0 & 1 & 0 \\
\hline & 0 & 5 & 1.583333333 & 0 & 0 & 0 & 0 & 0 & 0 & 1 \\
\hline
\end{tabular}




\begin{tabular}{|c|c|c|c|c|c|c|c|c|c|c|}
\hline \multirow{2}{*}{$\begin{array}{c}\text { Five alternatives } \\
\text { (Number of } \\
\text { observation) }\end{array}$} & Variable & Beta 1 & Beta 2 & Beta 3 & Beta 4 & Beta 5 & Beta 6 & Beta 7 & Beta 8 & Beta 9 \\
\hline & $\begin{array}{l}\text { Mode } \\
\text { Chosen }\end{array}$ & $\begin{array}{c}\text { TC } \\
\text { (1000 Korea won) }\end{array}$ & $\begin{array}{c}\text { TT } \\
\text { (hour) }\end{array}$ & Accom & Sexm & Age 340 & Tasc & Sasc & Basc & Lasc \\
\hline \multirow[t]{5}{*}{49} & 0 & 8.18 & 1.333333333 & 2 & 1 & 0 & 0 & 0 & 0 & 0 \\
\hline & 0 & 15.5 & 1.333333333 & 2 & 0 & 0 & 1 & 0 & 0 & 0 \\
\hline & 1 & 0.6 & 1.25 & 2 & 0 & 0 & 0 & 1 & 0 & 0 \\
\hline & 0 & 1.6 & 1.433333333 & 2 & 0 & 0 & 0 & 0 & 1 & 0 \\
\hline & 0 & 5 & 1.433333333 & 2 & 0 & 0 & 0 & 0 & 0 & 1 \\
\hline \multirow[t]{5}{*}{50} & 0 & 9.076 & 0.816666667 & 4 & 0 & 0 & 0 & 0 & 0 & 0 \\
\hline & 0 & 15 & 0.816666667 & 4 & 0 & 0 & 1 & 0 & 0 & 0 \\
\hline & 1 & 0.6 & 1.333333333 & 4 & 0 & 0 & 0 & 1 & 0 & 0 \\
\hline & 0 & 1.5 & 0.866666667 & 4 & 0 & 0 & 0 & 0 & 1 & 0 \\
\hline & 0 & 5 & 0.8666666667 & 4 & 0 & 0 & 0 & 0 & 0 & 1 \\
\hline \multirow[t]{5}{*}{51} & 0 & 7.18 & 0.516666667 & 0 & 0 & 0 & 0 & 0 & 0 & 0 \\
\hline & 0 & 10 & 0.516666667 & 0 & 0 & 0 & 1 & 0 & 0 & 0 \\
\hline & 1 & 0.5 & 0.5 & 0 & 0 & 0 & 0 & 1 & 0 & 0 \\
\hline & 0 & 1.1 & 0.566666667 & 0 & 0 & 0 & 0 & 0 & 1 & 0 \\
\hline & 0 & 5 & 0.566666667 & 0 & 0 & 0 & 0 & 0 & 0 & 1 \\
\hline \multirow[t]{5}{*}{52} & 0 & 8.18 & 1.483333333 & 0 & 1 & 1 & 0 & 0 & 0 & 0 \\
\hline & 0 & 20 & 1.483333333 & 0 & 0 & 0 & 1 & 0 & 0 & 0 \\
\hline & 1 & 1 & 1.5 & 0 & 0 & 0 & 0 & 1 & 0 & 0 \\
\hline & 0 & 1.6 & 1.583333333 & 0 & 0 & 0 & 0 & 0 & 1 & 0 . \\
\hline & 0 & 5 & 1.583333333 & 0 & 0 & 0 & 0 & 0 & 0 & 1 \\
\hline \multirow[t]{5}{*}{53} & 0 & 7.1 & 1.1 & 1 & 0 & 0 & 0 & 0 & 0 & 0 \\
\hline & 0 & 15 & 1.1 & 1 & 0 & 0 & 1 & 0 & 0 & 0 \\
\hline & 1 & 0.55 & 1.5 & 1 & 0 & 0 & 0 & 1 & 0 & 0 \\
\hline & 0 & 1.3 & 1.183333333 & 1 & 0 & 0 & 0 & 0 & 1 & 0 \\
\hline & 0 & 5 & 1.183333333 & 1 & 0 & 0 & 0 & 0 & 0 & 1 \\
\hline \multirow[t]{5}{*}{54} & 0 & 5.18 & 0.183333333 & 0 & 1 & 0 & 0 & 0 & 0 & 0 \\
\hline & 0 & 6 & 0.183333333 & 0 & 0 & 0 & 1 & 0 & 0 & 0 \\
\hline & 1 & 0.5 & 0.333333333 & 0 & 0 & 0 & 0 & 1 & 0 & 0 \\
\hline & 0 & 0.5 & 0.2 & 0 & 0 & 0 & 0 & 0 & 1 & 0 \\
\hline & 0 & 5 & 0.2 & 0 & 0 & 0 & 0 & 0 & 0 & 1 \\
\hline \multirow[t]{5}{*}{55} & 0 & 6.18 & 0.9 & 0 & 0 & 1 & 0 & 0 & 0 & 0 \\
\hline & 0 & 10 & 0.9 & 0 & 0 & 0 & 1 & 0 & 0 & 0 \\
\hline & 1 & 0.6 & 1 & 0 & 0 & 0 & 0 & 1 & 0 & 0 \\
\hline & 0 & 1.3 & 0.966666667 & 0 & 0 & 0 & 0 & 0 & 1 & 0 \\
\hline & 0 & 5 & 0.966666667 & 0 & 0 & 0 & 0 & 0 & 0 & 1 \\
\hline \multirow[t]{5}{*}{56} & 0 & 8.476 & 0.95 & 0 & 1 & 0 & 0 & 0 & 0 & 0 \\
\hline & 0 & 12.5 & 0.95 & 0 & 0 & 0 & 1 & 0 & 0 & 0 \\
\hline & 1 & 0.8 & 1.333333333 & 0 & 0 & 0 & 0 & 1 & 0 & 0 \\
\hline & 0 & 1.3 & 1.016666667 & 0 & 0 & 0 & 0 & 0 & 1 & 0 \\
\hline & 0 & 5 & 1.016666667 & 0 & 0 & 0 & 0 & 0 & 0 & 1 \\
\hline \multirow[t]{5}{*}{57} & 0 & 8.18 & 0.85 & 1 & 0 & 1 & 0 & 0 & 0 & 0 \\
\hline & 0 & 10 & 0.85 & 1 & 0 & 0 & 1 & 0 & 0 & 0 \\
\hline & 1 & 0.5 & 1 & 1 & 0 & 0 & 0 & 1 & 0 & 0 \\
\hline & 0 & 1.1 & 0.916666667 & 1 & 0 & 0 & 0 & 0 & 1 & 0 \\
\hline & 0 & 5 & 0.916666667 & 1 & 0 & 0 & 0 & 0 & 0 & 1 \\
\hline \multirow[t]{5}{*}{58} & 0 & 8.476 & 0.95 & 0 & 0 & 0 & 0 & 0 & 0 & 0 \\
\hline & 0 & 12.5 & 0.95 & 0 & 0 & 0 & 1 & 0 & 0 & 0 \\
\hline & 1 & 0.6 & 1.083333333 & 0 & 0 & 0 & 0 & 1 & 0 & 0 \\
\hline & 0 & 1.3 & 1.016666667 & 0 & 0 & 0 & 0 & 0 & 1 & 0 \\
\hline & 0 & 5 & 1.016666667 & 0 & 0 & 0 & 0 & 0 & 0 & 1 \\
\hline \multirow[t]{5}{*}{59} & 0 & 8.18 & 1.483333333 & 0 & 1 & 1 & 0 & 0 & 0 & 0 \\
\hline & 0 & 20 & 1.483333333 & 0 & 0 & 0 & 1 & 0 & 0 & 0 \\
\hline & 1 & 1.2 & 1.5 & 0 & 0 & 0 & 0 & 1 & 0 & 0 \\
\hline & 0 & 1.6 & 1.583333333 & 0 & 0 & 0 & 0 & 0 & 1 & 0 \\
\hline & 0 & 5 & 1.583333333 & 0 & 0 & 0 & 0 & 0 & 0 & 1 \\
\hline \multirow[t]{5}{*}{60} & 0 & 9.18 & 1.2 & 0 & 1 & 1 & 0 & 0 & 0 & 0 \\
\hline & 0 & 28 & 1.2 & 0 & 0 & 0 & 1 & 0 & 0 & 0 \\
\hline & 1 & 0.6 & 1.5 & 0 & 0 & 0 & 0 & 1 & 0 & 0 \\
\hline & 0 & 1.1 & 1.283333333 & 0 & 0 & 0 & 0 & 0 & 1 & 0 \\
\hline & 0 & 5 & 1.283333333 & 0 & 0 & 0 & 0 & 0 & 0 & 1 \\
\hline
\end{tabular}




\begin{tabular}{|c|c|c|c|c|c|c|c|c|c|c|}
\hline \multirow{2}{*}{$\begin{array}{c}\text { Five alternatives } \\
\text { (Number of } \\
\text { observation) }\end{array}$} & Variable & Beta 1 & Beta 2 & Beta 3 & Beta 4 & Beta 5 & Beta 6 & Beta 7 & Beta 8 & Beta 9 \\
\hline & $\begin{array}{l}\text { Mode } \\
\text { Chosen }\end{array}$ & $\begin{array}{c}\text { TC } \\
\text { (1000 Korea won) }\end{array}$ & $\begin{array}{c}\text { TT } \\
\text { (hour) }\end{array}$ & Accom & Sexm & Age340 & Tasc & Sasc & Basc & Lasc \\
\hline \multirow[t]{5}{*}{61} & 0 & 6.18 & 0.9 & 1 & 0 & 1 & 0 & 0 & 0 & 0 \\
\hline & 0 & 10 & 0.9 & 1 & 0 & 0 & 1 & 0 & 0 & 0 \\
\hline & 1 & 0.6 & 0.75 & 1 & 0 & 0 & 0 & 1 & 0 & 0 \\
\hline & 0 & 1.3 & 0.966666667 & 1 & 0 & 0 & 0 & 0 & 1 & 0 \\
\hline & 0 & 5 & 0.966666667 & 1 & 0 & 0 & 0 & 0 & 0 & 1 \\
\hline \multirow[t]{5}{*}{62} & 0 & 6.357 & 0.716666667 & 0 & 1 & 0 & 0 & 0 & 0 & 0 \\
\hline & 0 & 11 & 0.716666667 & 0 & 0 & 0 & 1 & 0 & 0 & 0 \\
\hline & 1 & 0.5 & 1 & 0 & 0 & 0 & 0 & 1 & 0 & 0 \\
\hline & 0 & 1.3 & 0.766666667 & 0 & 0 & 0 & 0 & 0 & 1 & 0 \\
\hline & 0 & 5 & 0.766666667 & 0 & 0 & 0 & 0 & 0 & 0 & 1 \\
\hline \multirow[t]{5}{*}{63} & 0 & 10.6 & 1.433333333 & 0 & 1 & 0 & 0 & 0 & 0 & 0 \\
\hline & 0 & 12 & 1.433333333 & 0 & 0 & 0 & 1 & 0 & 0 & 0 \\
\hline & 1 & 0.85 & 1.333333333 & 0 & 0 & 0 & 0 & 1 & 0 & 0 \\
\hline & 0 & 1.1 & 1.533333333 & 0 & 0 & 0 & 0 & 0 & 1 & 0 \\
\hline & 0 & 5 & 1.533333333 & 0 & 0 & 0 & 0 & 0 & 0 & 1 \\
\hline \multirow[t]{5}{*}{64} & 0 & 8.017 & 0.95 & 1 & 1 & 0 & 0 & 0 & 0 & 0 \\
\hline & 0 & 17 & 0.95 & 1 & 0 & 0 & 1 & 0 & 0 & 0 \\
\hline & 1 & 0.8 & 1 & 1 & 0 & 0 & 0 & 1 & 0 & 0 \\
\hline & 0 & 2 & 1.016666667 & 1 & 0 & 0 & 0 & 0 & 1 & 0 \\
\hline & 0 & 5 & 1.016666667 & 1 & 0 & 0 & 0 & 0 & 0 & 1 \\
\hline \multirow[t]{5}{*}{65} & 0 & 10.18 & 1.666666667 & 2 & 1 & 0 & 0 & 0 & 0 & 0 \\
\hline & 0 & 13.127 & 1.666666667 & 2 & 0 & 0 & 1 & 0 & 0 & 0 \\
\hline & 1 & 0.8 & 1.333333333 & 2 & 0 & 0 & 0 & 1 & 0 & 0 \\
\hline & 0 & 2 & 1.8 & 2 & 0 & 0 & 0 & 0 & 1 & 0 \\
\hline & 0 & 5 & 1.8 & 2 & 0 & 0 & 0 & 0 & 0 & 1 \\
\hline \multirow[t]{5}{*}{66} & 0 & 8.476 & 0.95 & 3 & 1 & 0 & 0 & 0 & 0 & 0 \\
\hline & 0 & 12.5 & 0.95 & 3 & 0 & 0 & 1 & 0 & 0 & 0 \\
\hline & 1 & 0.8 & 1 & 3 & 0 & 0 & 0 & 1 & 0 & 0 \\
\hline & 0 & 1.3 & 1.016666667 & 3 & 0 & 0 . & 0 & 0 & 1 & 0 \\
\hline & 0 & 5 & 1.016666667 & 3 & 0 & 0 & 0 & 0 & 0 & 1 \\
\hline \multirow[t]{5}{*}{67} & 0 & 8.476 & 0.95 & 0 & 1 & 1 & 0 & 0 & 0 & 0 \\
\hline & 0 & 12.5 & 0.95 & 0 & 0 & 0 & 1 & 0 & 0 & 0 \\
\hline & 1 & 0.7 & 1 & 0 & 0 & 0 & 0 & 1 & 0 & 0 \\
\hline & 0 & 1.3 & 1.016666667 & 0 & 0 & 0 & 0 & 0 & 1 & 0 \\
\hline & 0 & 5 & 1.016666667 & 0 & 0 & 0 & 0 & 0 & 0 & 1 \\
\hline \multirow[t]{5}{*}{68} & 0 & 8.78 & 0.4666666667 & 0 & 0 & 0 & 0 & 0 & 0 & 0 \\
\hline & 0 & 10 & 0.4666666667 & 0 & 0 & 0 & 1 & 0 & 0 & 0 \\
\hline & 1 & 0.5 & 0.333333333 & 0 & 0 & 0 & 0 & 1 & 0 & 0 \\
\hline & 0 & 1.1 & 0.5 & 0 & 0 & 0 & 0 & 0 & 1 & 0 \\
\hline & 0 & 5 & 0.5 & 0 & 0 & 0 & 0 & 0 & 0 & 1 \\
\hline \multirow[t]{5}{*}{69} & 0 & 8.18 & 1.533333333 & 1 & 1 & 0 & 0 & 0 & 0 & 0 \\
\hline & 0 & 12.052 & 1.533333333 & 1 & 0 & 0 & 1 & 0 & 0 & 0 \\
\hline & 1 & 0.7 & 1 & 1 & 0 & 0 & 0 & 1 & 0 & 0 \\
\hline & 0 & 5 & 1.633333333 & 1 & 0 & 0 & 0 & 0 & 1 & 0 \\
\hline & 0 & 5 & 1.633333333 & 1 & 0 & 0 & 0 & 0 & 0 & 1 \\
\hline \multirow[t]{5}{*}{70} & 0 & 10.18 & 1.666666667 & 0 & 0 & 0 & 0 & 0 & 0 & 0 \\
\hline & 0 & 13.127 & 1.666666667 & 0 & 0 & 0 & 1 & 0 & 0 & 0 \\
\hline & 1 & 0.6 & 1 & 0 & 0 & 0 & 0 & 1 & 0 & 0 \\
\hline & 0 & 2 & 1.8 & 0 & 0 & 0 & 0 & 0 & 1 & 0 \\
\hline & 0 & 5 & 1.8 & 0 & 0 & 0 & 0 & 0 & 0 & 1 \\
\hline \multirow[t]{5}{*}{71} & 0 & 8.18 & 1.533333333 & 0 & 0 & 0 & 0 & 0 & 0 & 0 \\
\hline & 0 & 12.052 & 1.533333333 & 0 & 0 & 0 & 1 & 0 & 0 & 0 \\
\hline & 1 & 0.6 & 1 & 0 & 0 & 0 . & 0 & 1 & 0 & 0 \\
\hline & 0 & 5 & 1.633333333 & 0 & 0 & 0 & 0 & 0 & 1 & 0 \\
\hline & 0 & 5 & 1.633333333 & 0 & 0 & 0 & 0 & 0 & 0 & 1 \\
\hline \multirow[t]{5}{*}{72} & 0 & 8.476 & 0.95 & 0 & 0 & 0 & 0 & 0 & 0 & 0 \\
\hline & 0 & 12.5 & 0.95 & 0 & 0 & 0 & 1 & 0 & 0 & 0 \\
\hline & 1 & 0.7 & 1 & 0 & 0 & 0 & 0 & 1 & 0 & 0 \\
\hline & 0 & 1.3 & 1.016666667 & 0 & 0 & 0 & 0 & 0 & 1 & 0 \\
\hline & 0 & 5 & 1.016666667 & 0 & 0 & 0 & 0 & 0 & 0 & 1 \\
\hline
\end{tabular}




\begin{tabular}{|c|c|c|c|c|c|c|c|c|c|c|}
\hline \multirow{2}{*}{$\begin{array}{c}\text { Five alternatives } \\
\text { (Number of } \\
\text { observation) }\end{array}$} & Variable & Beta 1 & Beta 2 & Beta 3 & Beta 4 & Beta 5 & Beta 6 & Beta 7 & Beta 8 & Beta 9 \\
\hline & $\begin{array}{l}\text { Mode } \\
\text { Chosen }\end{array}$ & $\begin{array}{c}\text { TC } \\
\text { (1000 Korea won) }\end{array}$ & $\begin{array}{c}\text { TT } \\
\text { (hour) }\end{array}$ & Accom & Sexm & Age340 & Tasc & Sasc & Basc & Lasc \\
\hline \multirow[t]{5}{*}{73} & 0 & 8.476 & 0.95 & 0 & 1 & 0 & 0 & 0 & 0 & 0 \\
\hline & 0 & 12.5 & 0.95 & 0 & 0 & 0 & 1 & 0 & 0 & 0 \\
\hline & 1 & 0.7 & 1 & 0 & 0 & 0 & 0 & 1 & 0 & 0 \\
\hline & 0 & 1.3 & 1.016666667 & 0 & 0 & 0 & 0 & 0 & 1 & 0 \\
\hline & 0 & 5 & 1.016666667 & 0 & 0 & 0 & 0 & 0 & 0 & 1 \\
\hline \multirow[t]{5}{*}{74} & 0 & 9.076 & 0.816666667 & 1 & 0 & 0 & 0 & 0 & 0 & 0 \\
\hline & 0 & 15 & 0.816666667 & 1 & 0 & 0 & 1 & 0 & 0 & 0 \\
\hline & 1 & 0.7 & 0.833333333 & 1 & 0 & 0 & 0 & 1 & 0 & 0 \\
\hline & 0 & 1.5 & 0.8666666667 & 1 & 0 & 0 & 0 & 0 & 1 & 0 \\
\hline & 0 & 5 & 0.866666667 & 1 & 0 & 0 & 0 & 0 & 0 & 1 \\
\hline \multirow[t]{5}{*}{75} & 0 & 8.476 & 0.95 & 1 & 0 & 0 & 0 & 0 & 0 & 0 \\
\hline & 0 & 12.5 & 0.95 & 1 & 0 & 0 & 1 & 0 & 0 & 0 \\
\hline & 1 & 0.7 & 1 & 1 & 0 & 0 & 0 & 1 & 0 & 0 \\
\hline & 0 & 1.3 & 1.016666667 & 1 & 0 & 0 & 0 & 0 & 1 & 0 \\
\hline & 0 & 5 & 1.016666667 & 1 & 0 & 0 & 0 & 0 & 0 & 1 \\
\hline \multirow[t]{5}{*}{76} & 0 & 8.476 & 0.95 & 1 & 0 & 0 & 0 & 0 & 0 & 0 \\
\hline & 0 & 12.5 & 0.95 & 1 & 0 & 0 & 1 & 0 & 0 & 0 \\
\hline & 1 & 0.7 & 1 & 1 & 0 & 0 & 0 & 1 & 0 & 0 \\
\hline & 0 & 1.3 & 1.016666667 & 1 & 0 & 0 & 0 & 0 & 1 & 0 \\
\hline & 0 & 5 & 1.016666667 & 1 & 0 & 0 & 0 & 0 & 0 & 1 \\
\hline \multirow[t]{5}{*}{77} & 0 & 8.476 & 0.95 & 0 & 0 & 0 & 0 & 0 & 0 & 0 \\
\hline & 0 & 12.5 & 0.95 & 0 & 0 & 0 & 1. & 0 & 0 & 0 \\
\hline & 1 & 0.7 & 1 & 0 & 0 & 0 & 0 & 1 & 0 & 0 \\
\hline & 0 & 1.3 & 1.016666667 & 0 & 0 & 0 & 0 & 0 & 1 & 0 \\
\hline & 0 & 5 & 1.016666667 & 0 & 0 & 0 & 0 & 0 & 0 & 1 \\
\hline \multirow[t]{5}{*}{78} & 0 & 8.017 & 1.15 & 1 & 0 & 1 & 0 & 0 & 0 & 0 \\
\hline & 0 & 17 & 1.15 & 1 & 0 & 0 & 1 & 0 & 0 & 0 \\
\hline & 1 & 0.65 & 1 & 1 & 0 & 0 & 0 & 1 & 0 & 0 \\
\hline & 0 & 2 & 1.233333333 & 1 & 0 & 0 & 0 & 0 & 1 & 0 \\
\hline & 0 & 5 & 1.233333333 & 1 & 0 & 0 & 0 & 0 & 0 & 1 \\
\hline \multirow[t]{5}{*}{79} & 0 & 7.1 & 1.1 & 0 & 1 & 1 & 0 & 0 & 0 & 0 \\
\hline & 0 & 15 & 1.1 & 0 & 0 & 0 & 1 & 0 & 0 & 0 \\
\hline & 1 & 0.6 & 0.75 & 0 & 0 & 0 & 0 & 1 & 0 & 0 \\
\hline & 0 & 1.3 & 1.183333333 & 0 & 0 & 0 & 0 & 0 & 1 & 0 \\
\hline & 0 & 5 & 1.183333333 & 0 & 0 & 0 & 0 & 0 & 0 & 1 \\
\hline \multirow[t]{5}{*}{80} & 0 & 7.18 & 0.516666667 & 1 & 0 & 1 & 0 & 0 & 0 & 0 \\
\hline & 0 & 10 & 0.516666667 & 1 & 0 & 0 & 1 & 0 & 0 & 0 \\
\hline & 1 & 0.5 & 0.5 & 1 & 0 & 0 & 0 & 1 & 0 & 0 \\
\hline & 0 & 1.1 & 0.566666667 & 1 & 0 & 0 & 0 & 0. & 1 & 0 \\
\hline & 0 & 5 & 0.566666667 & 1 & 0 & 0 & 0 & 0 & 0 & 1 \\
\hline \multirow[t]{5}{*}{81} & 0 & 9.026 & 0.466666667 & 1 & 1 & 0 & 0 & 0 & 0 & 0 \\
\hline & 0 & 20 & 0.466666667 & 1 & 0 & 0 & 1 & 0 & 0 & 0 \\
\hline & 1 & 0.9 & $\begin{array}{r}1.5 \\
\end{array}$ & 1 & 0 & 0 & 0 & 1 & 0 & 0 \\
\hline & 0 & 1.5 & 0.5 & 1 & 0 & 0 & 0 & 0 & 1 & 0 \\
\hline & 0 & 5 & 0.5 & 1 & 0 & 0 & 0 & 0 & 0 & 1 \\
\hline \multirow[t]{5}{*}{82} & 0 & 7.18 & 0.516666667 & 0 & 0 & 0 & 0 & 0 & 0 & 0 \\
\hline & 0 & 10 & 0.516666667 & 0 & 0 & 0 & 1 & 0 & 0 & 0 \\
\hline & 1 & 0.6 & 0.666666667 & 0 & 0 & 0 & 0 & 1 & 0 & 0 \\
\hline & 0 & 1.1 & 0.566666667 & 0 & 0 & 0 & 0 & 0 & 1 & 0 \\
\hline & 0 & 5 & 0.566666667 & 0 & 0 & 0 & 0 & 0 & 0 & 1 \\
\hline \multirow[t]{5}{*}{83} & 0 & 9.18 & 1.2 & 2 & 1 & 1 & 0 & 0 & 0 & 0 \\
\hline & 0 & 28 & 1.2 & 2 & 0 & 0 & 1 & 0 & 0 & 0 \\
\hline & 1 & 0.6 & 1 & 2 & 0 & 0 & 0 & 1 & 0 & 0 \\
\hline & 0 & 1.1 & 1.283333333 & 2 & 0 & 0 & 0 & 0 & 1 & 0 \\
\hline & 0 & 5 & 1.283333333 & 2 & 0 & 0 & 0 & 0 & 0 & 1 \\
\hline \multirow[t]{5}{*}{84} & 0 & 8.476 & 0.95 & 0 & 0 & 1 & 0 & 0 & 0 & 0 \\
\hline & 0 & 12.5 & 0.95 & 0 & 0 & 0 & 1 & 0 & 0 & 0 \\
\hline & 1 & 0.6 & 1 & 0 & 0 & 0 & 0 & 1 & 0 & 0 \\
\hline & 0 & 1.3 & 1.016666667 & 0 & 0 & 0 & 0 & 0 & 1 & 0 \\
\hline & 0 & 5 & 1.016666667 & 0 & 0 & 0 & 0 & 0 & 0 & 1 \\
\hline
\end{tabular}




\begin{tabular}{|c|c|c|c|c|c|c|c|c|c|c|}
\hline \multirow{2}{*}{$\begin{array}{l}\text { Five alternatives } \\
\text { (Number of } \\
\text { observation) }\end{array}$} & Variable & Beta 1 & Beta 2 & Beta 3 & Beta 4 & Beta 5 & Beta 6 & Beta 7 & Beta 8 & Beta 9 \\
\hline & $\begin{array}{l}\text { Mode } \\
\text { Chosen }\end{array}$ & $\begin{array}{c}\text { TC } \\
\text { (1000 Korea won) } \\
\end{array}$ & $\begin{array}{c}\mathrm{TT} \\
\text { (hour) } \\
\end{array}$ & Accom & Sexm & Age340 & Tasc & Sasc & Basc & Lasc \\
\hline \multirow[t]{5}{*}{85} & 0 & 7.735 & 2.066666667 & 0 & 1 & 1 & 0 & 0 & 0 & 0 \\
\hline & 0 & 14 & 2.0666666667 & 0 & 0 & 0 & 1 & 0 & 0 & 0 \\
\hline & 1 & 0.75 & 0.833333333 & 0 & 0 & 0 & 0 & 1 & 0 & 0 \\
\hline & 0 & 1.5 & 2.2 & 0 & 0 & 0 & 0 & 0 & 1 & 0 \\
\hline & 0 & 5 & 2.2 & 0 & 0 & 0 & 0 & 0 & 0 & 1 \\
\hline \multirow[t]{5}{*}{86} & 0 & 7.18 & 0.516666667 & 1 & 0 & 1 & 0 & 0 & 0 & 0 \\
\hline & 0 & 10 & 0.5166666667 & 1 & 0 & 0 & 1 & 0 & 0 & 0 \\
\hline & 1 & 0.5 & 0.583333333 & 1 & 0 & 0 & 0 & 1 & 0 & 0 \\
\hline & 0 & 1.1 & 0.566666667 & 1 & 0 & 0 & 0 & 0 & 1 & 0 \\
\hline & 0 & 5 & 0.566666667 & 1 & 0 & 0 & 0 & 0 & 0 & 1 \\
\hline \multirow[t]{5}{*}{87} & 0 & 7.18 & 0.516666667 & 0 & 1 & 0. & 0 & 0 & 0 & 0 \\
\hline & 0 & 10 & 0.516666667 & 0 & 0 & 0 & 1 & 0 & 0 & 0 \\
\hline & 1 & 0.5 & 0.5 & 0 & 0 & 0 & 0 & 1 & 0 & 0 \\
\hline & 0 & 1.1 & 0.566666667 & 0 & 0 & 0 & 0 & 0 & 1 & 0 \\
\hline & 0 & 5 & 0.566666667 & 0 & 0 & 0 & 0 & 0 & 0 & 1 \\
\hline \multirow[t]{5}{*}{88} & 0 & 6.18 & 0.9 & 1 & 1 & 1 & 0 & 0 & 0 & 0 \\
\hline & 0 & 10 & 0.9 & 1 & 0 & 0 & 1 & 0 & 0 & 0 \\
\hline & 1 & 0.6 & 0.75 & 1 & 0 & 0 & 0 & 1 & 0 & 0 \\
\hline & 0 & 1.3 & 0.966666667 & 1 & 0 & 0 & 0 & 0 & 1 & 0 \\
\hline & 0 & 5 & 0.966666667 & 1 & 0 & 0 & 0 & 0 & 0 & 1 \\
\hline \multirow[t]{5}{*}{89} & 0 & 8.18 & 1.533333333 & 0 & 0 & 1 & 0 & 0 & 0 & 0 \\
\hline & 0 & 12.052 & 1.533333333 & 0 & 0 & 0 & 1 & 0 & 0 & 0 \\
\hline & 1 & 0.7 & 1.333333333 & 0 & 0 & 0 & 0 & 1 & 0 & 0 \\
\hline & 0 & 5 & 1.633333333 & 0 & 0 & 0 & 0 & 0 & 1 & 0 \\
\hline & 0 & 5 & 1.633333333 & 0 & 0 & 0 & 0 & 0 & 0 & 1 \\
\hline \multirow[t]{5}{*}{90} & 0 & 9.18 & 1.2 & 1 & 1 & 1 & 0 & 0 & 0 & 0 \\
\hline & 0 & 28 & 1.2 & 1 & 0 & 0 & 1 & 0 & 0 & 0 \\
\hline & 1 & 0.6 & 1 & 1 & 0 & 0 & 0 & 1 & 0 & 0 \\
\hline & 0 & 1.1 & 1.283333333 & 1 & 0 & 0 & 0 & 0 & 1 & 0 \\
\hline & 0 & 5 & 1.283333333 & 1 & 0 & 0 & 0 & 0 & 0 & 1 \\
\hline \multirow[t]{5}{*}{91} & 0 & 8.476 & 0.95 & 1 & 1 & 1 & 0 & 0 & 0 & 0 \\
\hline & 0 & 12.5 & 0.95 & 1 & 0 & 0 & 1 & 0 & 0 & 0 \\
\hline & 1 & 0.6 & 0.75 & 1 & 0 & 0 & 0 & 1 & 0 & 0 \\
\hline & 0 & 1.3 & 1.016666667 & 1 & 0 & 0 & 0 & 0 & 1 & 0 \\
\hline & 0 & 5 & 1.016666667 & 1 & 0 & 0 & 0 & 0 & 0 & 1 \\
\hline \multirow[t]{5}{*}{92} & 0 & 9.076 & 0.816666667 & 0 & 1 & 1 & 0 & 0 & 0 & 0 \\
\hline & 0 & 15 & 0.816666667 & 0 & 0 & 0 & 1 & 0 & 0 & 0 \\
\hline & 1 & 0.6 & 0.833333333 & 0 & 0 & 0 & 0 & 1 & 0 & 0 \\
\hline & 0 & 1.5 & 0.8666666667 & 0 & 0 & 0 & 0 & 0 & 1 & 0 \\
\hline & 0 & 5 & 0.8666666667 & 0 & 0 & 0 & 0 & 0 & 0 & 1 \\
\hline \multirow[t]{5}{*}{93} & 0 & 5.18 & 0.183333333 & 0 & 1 & 0 & 0 & 0 & 0 & 0 \\
\hline & 0 & 6 & 0.183333333 & 0 & 0 & 0 & 1 & 0 & 0 & 0 \\
\hline & 1 & 0.5 & 0.25 & 0 & 0 & 0 & 0 & 1 & 0 & 0 \\
\hline & 0 & 0.5 & 0.2 & 0 & 0 & 0 & 0 & 0 & 1 & 0 \\
\hline & 0 & 5 & 0.2 & 0 & 0 & 0 & 0 & 0 & 0 & 1 \\
\hline \multirow[t]{5}{*}{94} & 0 & 8.4 & 0.616666667 & 2 & 0 & 1 & 0 & 0 & 0 & 0 \\
\hline & 0 & 7.6 & 0.616666667 & 2 & 0 & 0 & 1 & 0 & 0 & 0 \\
\hline & 1 & 0.6 & 1 & 2 & 0 & 0 & 0 & 1 & 0 & 0 \\
\hline & 0 & 1.6 & 0.6666666667 & 2 & 0 & 0 & 0 & 0 & 1 & 0 \\
\hline & 0 & 5 & 0.6666666667 & 2 & 0 & 0 & 0 & 0 & 0 & 1 \\
\hline \multirow[t]{5}{*}{95} & 0 & 9.026 & 0.4666666667 & 0 & 0 & 0 & 0 & 0 & 0 & 0 \\
\hline & 0 & 20 & 0.466666667 & 0 & 0 & 0 & 1 & 0 & 0 & 0 \\
\hline & 1 & 0.55 & 1.5 & 0 & 0 & 0 & 0 & 1 & 0 & 0 \\
\hline & 0 & 1.5 & 0.5 & 0 & 0 & 0 & 0 & 0 & 1 & 0 \\
\hline & 0 & 5 & 0.5 & 0 & 0 & 0 & 0 & 0 & 0 & 1 \\
\hline \multirow[t]{5}{*}{96} & $\overline{0}$ & 8.18 & 0.85 & 0 & 1 & 0 & 0 & 0 & 0 & 0 \\
\hline & 0 & 10 & 0.85 & 0 & 0 & 0 & 1 & 0 & 0 & 0 \\
\hline & 1 & 0.6 & 1 & 0 & 0 & 0 & 0 & 1 & 0 & 0 \\
\hline & 0 & 1.1 & 0.916666667 & 0 & 0 & 0 & 0 & 0 & 1 & 0 \\
\hline & 0 & 5 & 0.916666667 & 0 & 0 & 0 & 0 & 0 & 0 & 1 \\
\hline
\end{tabular}




\begin{tabular}{|c|c|c|c|c|c|c|c|c|c|c|}
\hline \multirow{2}{*}{$\begin{array}{c}\text { Five alternatives } \\
\text { (Number of } \\
\text { observation) }\end{array}$} & Variable & Beta 1 & Beta 2 & Beta 3 & Beta 4 & Beta 5 & Beta 6 & Beta 7 & Beta 8 & Beta 9 \\
\hline & $\begin{array}{c}\text { Mode } \\
\text { Chosen }\end{array}$ & $\begin{array}{c}\text { TC } \\
\text { (1000 Korea won) }\end{array}$ & $\begin{array}{c}\text { TT } \\
\text { (hour) }\end{array}$ & Accom & Sexm & Age 340 & Tasc & Sasc & Basc & Lasc \\
\hline \multirow[t]{5}{*}{97} & 0 & 7.18 & 0.516666667 & 0 & 1 & 0 & 0 & 0 & 0 & 0 \\
\hline & 0 & 10 & 0.5166666667 & 0 & 0 & 0 & 1 & 0 & 0 & 0 \\
\hline & 1 & 0.6 & 0.5 & 0 & 0 & 0 & 0 & 1 & 0 & 0 \\
\hline & 0 & 1.1 & 0.566666667 & 0 & 0 & 0 & 0 & 0 & 1 & 0 \\
\hline & 0 & 5 & 0.5666666667 & 0 & 0 & 0 & 0 & 0 & 0 & 1 \\
\hline \multirow[t]{5}{*}{98} & 0 & 5.298 & 0.566666667 & 0 & 1 & 0 & 0 & 0 & 0 & 0 \\
\hline & 0 & 7 & 0.566666667 & 0 & 0 & 0 & 1 & 0 & 0 & 0 \\
\hline & 1 & 0.6 & 1 & 0 & 0 & 0 & 0 & 1 & 0 & 0 \\
\hline & 0 & 1.3 & 0.616666667 & 0 & 0 & 0 & 0 & 0 & 1 & 0 \\
\hline & 0 & 5 & 0.616666667 & 0 & 0 & 0 & 0 & 0 & 0 & 1 \\
\hline \multirow[t]{5}{*}{99} & 0 & 8.18 & 0.85 & 2 & 1 & 1 & 0 & 0 & 0 & 0 \\
\hline & 0 & 10 & 0.85 & 2 & 0 & 0 & 1 & 0 & 0 & 0 \\
\hline & 1 & 0.5 & 0.75 & 2 & 0 & 0 & 0 & 1 & 0 & 0 \\
\hline & 0 & 1.1 & 0.916666667 & 2 & 0 & 0 & 0 & 0 & 1 & 0 \\
\hline & 0 & 5 & 0.916666667 & 2 & 0 & 0 & 0 & 0 & 0 & 1 \\
\hline \multirow[t]{5}{*}{100} & 0 & 13.18 & 1.866666667 & 0 & 1 & 0 & 0 & 0 & 0 & 0 \\
\hline & 0 & 28 & 1.866666667 & 0 & 0 & 0 & 1 & 0 & 0 & 0 \\
\hline & 1 & 0.6 & 1 & 0 & 0 & 0 & 0 & 1 & 0 & 0 \\
\hline & 0 & 1.5 & 2 & 0 & 0 & 0 & 0 & 0 & 1 & 0 \\
\hline & 0 & 5 & 2 & 0 & 0 & 0 & 0 & 0 & 0 & 1 \\
\hline \multirow[t]{5}{*}{101} & 0 & 8.18 & 0.85 & 0 & 0 & 0 & 0 & 0 & 0 & 0 \\
\hline & 0 & 10 & 0.85 & 0 & 0 & 0 & 1 & 0 & 0 & 0 \\
\hline & 1 & 0.6 & 0.6666666667 & 0 & 0 & 0 & 0 & 1 & 0 & 0 \\
\hline & 0 & 1.1 & 0.916666667 & 0 & 0 & 0 & 0 & 0 & 1 & 0 \\
\hline & 0 & 5 & 0.916666667 & 0 & 0 & 0 & 0 & 0 & 0 & 1 \\
\hline \multirow[t]{5}{*}{102} & 0 & 8.18 & 1.433333333 & 2 & 0 & 0 & 0 & 0 & 0 & 0 \\
\hline & 0 & 12 & 1.433333333 & 2 & 0 & 0 & 1 & 0 & 0 & 0 \\
\hline & 1 & 0.6 & 1 & 2 & 0 & 0 & 0 & 1 & 0 & 0 \\
\hline & 0 & 1.1 & 1.533333333 & 2 & 0 & 0 & 0 & 0 & 1 & 0 \\
\hline & 0 & 5 & 1.533333333 & 2 & 0 & 0 & 0 & 0 & 0 & 1 \\
\hline \multirow[t]{5}{*}{103} & 0 & 8.18 & 1.533333333 & 0 & 1 & 0 & 0 & 0 & 0 & 0 \\
\hline & 0 & 12.052 & 1.533333333 & 0 & 0 & 0 & 1 & 0 & 0 & 0 \\
\hline & 1 & 0.7 & 1 & 0 & 0 & 0 & 0 & 1 & 0 & 0 \\
\hline & 0 & 5 & 1.633333333 & 0 & 0 & 0 & 0 & 0 & 1 & 0 \\
\hline & 0 & 5 & 1.633333333 & 0 & 0 & 0 & 0 & 0 & 0 & 1 \\
\hline \multirow[t]{5}{*}{104} & 0 & 9.18 & 1.2 & 0 & 1 & 1 & 0 & 0 & 0 & 0 \\
\hline & 0 & 28 & 1.2 & 0 & 0 & 0 & 1 & 0 & 0 & 0 \\
\hline & 1 & 0.6 & 1 & 0 & 0 & 0 & 0 & 1 & 0 & 0 \\
\hline & 0 & 1.1 & 1.283333333 & 0 & 0 & 0 & 0 & 0 & 1 & 0 \\
\hline & 0 & 5 & 1.283333333 & 0 & 0 & 0 & 0 & 0 & 0 & 1 \\
\hline \multirow[t]{5}{*}{105} & 0 & 8.18 & 1.533333333 & 1 & 0 & 0 & 0 & 0 & 0 & 0 \\
\hline & 0 & 12.052 & 1.533333333 & 1 & 0 & 0 & 1 & 0 & 0 & 0 \\
\hline & 1 & 0.6 & 1.166666667 & 1 & 0 & 0 & 0 & 1 & 0 & 0 \\
\hline & 0 & 5 & 1.633333333 & 1 & 0 & 0 & 0 & 0 & 1 & 0 \\
\hline & 0 & 5 & 1.633333333 & 1 & 0 & 0 & 0 & 0 & 0 & 1 \\
\hline \multirow[t]{5}{*}{106} & 0 & 9.18 & 1.2 & 1 & 1 & 0 & 0 & 0 & 0 & 0 \\
\hline & 0 & 28 & 1.2 & 1 & 0 & 0 & 1 & 0 & 0 & 0 \\
\hline & 1 & 0.6 & 1.333333333 & 1 & 0 & 0 & 0 & 1 & 0 & 0 \\
\hline & 0 & 1.1 & 1.283333333 & 1 & 0 & 0 & 0 & 0 & 1 & 0 \\
\hline & 0 & 5 & 1.283333333 & 1 & 0 & 0 . & 0 & 0 & 0 & 1 \\
\hline \multirow[t]{5}{*}{107} & 0 & 8.476 & 0.95 & 0 & 1 & 0 & 0 & 0 & 0 & 0 \\
\hline & 0 & 12.5 & 0.95 & 0 & 0 & 0 & 1 & 0 & 0 & 0 \\
\hline & 1 & 0.6 & 0.833333333 & 0 & 0 & 0 & 0 & 1 & 0 & 0 \\
\hline & 0 & 1.3 & 1.016666667 & 0 & 0 & 0 & 0 & 0 & 1 & 0 \\
\hline & 0 & 5 & 1.016666667 & 0 & 0 & 0 & 0 & 0 & 0 & 1 \\
\hline \multirow[t]{5}{*}{108} & 0 & 7.18 & 0.516666667 & 1 & 1 & 0 & 0 & 0 & 0 & 0 \\
\hline & 0 & 10 & 0.516666667 & 1 & 0 & 0 & 1 & 0 & 0 & 0 \\
\hline & 1 & 0.6 & 0.75 & 1 & 0 & 0 & 0 & 1 & 0 & 0 \\
\hline & 0 & 1.1 & 0.566666667 & 1 & 0 & 0 & 0 & 0 & 1 & 0 \\
\hline & 0 & 5 & 0.566666667 & 1 & 0 & 0 & 0 & 0 & 0 & 1 \\
\hline
\end{tabular}




\begin{tabular}{|c|c|c|c|c|c|c|c|c|c|c|}
\hline \multirow{2}{*}{$\begin{array}{c}\text { Five alternatives } \\
\text { (Number of } \\
\text { observation) }\end{array}$} & Variable & Beta 1 & Beta 2 & Beta 3 & Beta 4 & Beta 5 & Beta 6 & Beta 7 & Beta 8 & Beta 9 \\
\hline & $\begin{array}{c}\text { Mode } \\
\text { Chosen }\end{array}$ & $\begin{array}{c}\text { TC } \\
\text { (1000 Korea won) }\end{array}$ & $\begin{array}{c}\mathrm{TT} \\
\text { (hour) }\end{array}$ & Accom & Sexm & Age 340 & Tasc & Sasc & Basc & Lasc \\
\hline \multirow[t]{5}{*}{109} & 0 & 5.18 & 0.183333333 & 0 & 0 & 0 & 0 & 0 & 0 & 0 \\
\hline & 0 & 6 & 0.183333333 & 0 & 0 & 0 & 1 & 0 & 0 & 0 \\
\hline & 1 & 0.5 & 0.166666667 & 0 & 0 & 0 & 0 & 1 & 0 & 0 \\
\hline & 0 & 0.5 & 0.2 & 0 & 0 & 0 & 0 & 0 & 1 & 0 \\
\hline & 0 & 5 & 0.2 & 0 & 0 & 0 & 0 & 0 & 0 & 1 \\
\hline \multirow[t]{5}{*}{110} & 0 & 8.18 & 1.533333333 & 1 & 0 & 0 & 0 & 0 & 0 & 0 \\
\hline & 0 & 12.052 & 1.533333333 & 1 & 0 & 0 & 1 & 0 & 0 & 0 \\
\hline & 1 & 0.6 & 1.5 & 1 & 0 & 0 & 0 & 1 & 0 & 0 \\
\hline & 0 & 5 & 1.633333333 & 1 & 0 & 0 & 0 & 0 & 1 & 0 \\
\hline & 0 & 5 & 1.633333333 & 1 & 0 & 0 & 0 & 0 & 0 & 1 \\
\hline \multirow[t]{5}{*}{111} & 0 & 8.476 & 0.95 & 0 & 1 & 0 & 0 & 0 & 0 & 0 \\
\hline & 0 & 12.5 & 0.95 & 0 & 0 & 0 & 1 & 0 . & 0 & 0 \\
\hline & 1 & 0.65 & 1 & 0 & 0 & 0 & 0 & 1 & 0 & 0 \\
\hline & 0 & 1.3 & 1.016666667 & 0 & 0 & 0 & 0 & 0 & 1 & 0 \\
\hline & 0 & 5 & 1.016666667 & 0 & 0 & 0 & 0 & 0 & 0 & 1 \\
\hline \multirow[t]{5}{*}{112} & 0 . & 7.18 & 0.516666667 & 0 & 1 & 0 & 0 & 0 & 0 & 0 \\
\hline & 0 & 10 & 0.516666667 & 0 & 0 & 0 & 1 & 0 & 0 & 0 \\
\hline & 1 & 0.5 & 0.5 & 0 & 0 & 0 & 0 & 1 & 0 & 0 \\
\hline & 0 & 1.1 & 0.566666667 & 0 & 0 & 0 & 0 & 0 & 1 & 0 \\
\hline & 0 & 5 & 0.5666666667 & 0 & 0 & 0 & 0 & 0 & 0 & 1 \\
\hline
\end{tabular}


C.4: Bus alternative -73 observations

\begin{tabular}{|c|c|c|c|c|c|c|c|c|c|c|}
\hline \multirow{2}{*}{$\begin{array}{c}\text { Five alternatives } \\
\text { (Number of } \\
\text { observation) }\end{array}$} & Variable & Beta 1 & Beta 2 & Beta 3 & Beta 4 & Beta 5 & Beta 6 & Beta 7 & Beta 8 & Beta 9 \\
\hline & $\begin{array}{c}\text { Mode } \\
\text { Chosen }\end{array}$ & $\begin{array}{c}\text { TC } \\
\text { (1000 Korea won) }\end{array}$ & $\begin{array}{c}\text { TT } \\
\text { (hour) }\end{array}$ & Accom & Sexm & Age 340 & Tasc & Sasc & Basc & Lasc \\
\hline \multirow[t]{5}{*}{ BUS } & 0 & 8.18 & 1.8166666667 & 1 & 1 & 1 & 0 & 0 & 0 & 0 \\
\hline & 0 & 12 & 1.816666667 & 1 & 0 & 0 & 1 & 0 & 0 & 0 \\
\hline & 0 & 0.9 & 1.133333333 & 1 & 0 & 0 & 0 & 1 & 0 & 0 \\
\hline & 1 & 1 & 1 & 1 & 0 & 0 & 0 & 0 & 1 & 0 \\
\hline & 0 & 5 & 1.95 & 1 & 0 & 0 & 0 & 0 & 0 & 1 \\
\hline \multirow[t]{5}{*}{2} & 0 & 10.6 & 1.433333333 & 0 & 1 & 1 & 0 & 0 & 0 & 0 \\
\hline & 0 & 12 & 1.433333333 & 0 & 0 & 0 & 1 & 0 & 0 & 0 \\
\hline & 0 & 0.85 & 0.883333333 & 0 & 0 & 0 & 0 & 1 & 0 & 0 \\
\hline & 1 & 1 & 1.166666667 & 0 & 0 & 0 & 0 & 0 & 1 & 0 \\
\hline & 0 & 5 & 1.533333333 & 0 & 0 & 0 & 0 & 0 & 0 & 1 \\
\hline \multirow[t]{5}{*}{3} & 0 & 8.78 & 0.466666667 & 0 & 0 & 0 & 0 & 0 & 0 & 0 \\
\hline & 0 & 10 & 0.466666667 & 0 & 0 & 0 & 1 & 0 & 0 & 0 \\
\hline & 0 & 0.5 & 0.283333333 & 0 & 0 & 0 & 0 & 1 & 0 & 0 \\
\hline & 1 & 0.5 & 0.166666667 & 0 & 0 & 0 & 0 & 0 & 1 & 0 \\
\hline & 0 & 5 & 0.5 & 0 & 0 & 0 & 0 & 0 & 0 & 1 \\
\hline \multirow[t]{5}{*}{4} & 0 & 6.18 & 0.9 & 0 & 0 & 0 & 0 & 0 & 0 & 0 \\
\hline & 0 & 10 & 0.9 & 0 & 0 & 0 & 1 & 0 & 0 & 0 \\
\hline & 0 & 0.6 & 0.566666667 & 0 & 0 & 0 & 0 & 1 & 0 & 0 \\
\hline & 1 & 1.3 & 0.6666666667 & 0 & 0 & 0 & 0 & 0 & 1 & 0 \\
\hline & 0 & 5 & 0.9666666667 & 0 & 0 & 0 & 0 & 0 & 0 & 1 \\
\hline \multirow[t]{5}{*}{5} & 0 & 5.18 & 0.183333333 & 1 & 0 & 0 & 0 & 0 & 0 & 0 \\
\hline & 0 & 6 & 0.183333333 & 1 & 0 & 0 & 1 & 0 & 0 & 0 \\
\hline & 0 & 0.5 & 0.116666667 & 1 & 0 & 0 & 0 & 1 & 0 & 0 \\
\hline & 1 & 0.5 & 0.5 & 1 & 0 & 0 & 0 & 0 & 1 & 0 \\
\hline & 0 & 5 & 0.2 & 1 & 0 & 0 & 0 & 0 & 0 & 1 \\
\hline \multirow[t]{5}{*}{6} & 0 & 8.18 & 1.483333333 & 1 & 1 & 0 & 0 & 0 & 0 & 0 \\
\hline & 0 & 22 & 1.483333333 & 1 & 0 & 0 & 1 & 0 & 0 & 0 \\
\hline & 0 & 0.6 & 0.916666667 & 1 & 0 & 0 & 0 & 1 & 0 & 0 \\
\hline & 1 & 3.5 & 1.6666666667 & 1 & 0 & 0 & 0 & 0 & 1 & 0 \\
\hline & 0 & 5 & 1.583333333 & 1 & 0 & 0 & 0 & 0 & 0 & 1 \\
\hline \multirow[t]{5}{*}{7} & 0 & 9.18 & 1.2 & 40 & 1 & 0 & 0 & 0 & 0 & 0 \\
\hline & 0 & 28 & 1.2 & 40 & 0 & 0 & 1 & 0 & 0 & 0 \\
\hline & 0 & 0.6 & 0.733333333 & 40 & 0 & 0 & 0 & 1 & 0 & 0 \\
\hline & 1 & 1 & 0.666666667 & 40 & 0 & 0 & 0 & 0 & 1 & 0 \\
\hline & 0 & 5 & 1.283333333 & 40 & 0 & 0 & 0 & 0 & 0 & 1 \\
\hline \multirow[t]{5}{*}{8} & 0 & 6.357 & 0.7166666667 & 0 & 0 & 1 & 0 & 0 & 0 & 0 \\
\hline & 0 & 11 & 0.716666667 & 0 & 0 & 0 & 1 & 0 & 0 & 0 \\
\hline & 0 & 0.5 & 0.433333333 & 0 & 0 & 0 & 0 & 1 & 0 & 0 \\
\hline & 1 & 1.3 & 0.416666667 & 0 & 0 & 0 & 0 & 0 & 1 & 0 \\
\hline & 0 & 5 & 0.766666667 & 0 & 0 & 0 & 0 & 0 & 0 & 1 \\
\hline \multirow[t]{5}{*}{9} & 0 & 8.48 & 1.816666667 & 0 & 1 & 1 & 0 & 0 & 0 & 0 \\
\hline & 0 & 28 & 1.816666667 & 0 & 0 & 0 & 1 & 0 & 0 & 0 \\
\hline & 0 & 0.65 & 1.133333333 & 0 & 0 & 0 & 0 & 1 & 0 & 0 \\
\hline & 1 & 1 & 0.416666667 & 0 & 0 & 0 & 0 & 0 & 1 & 0 \\
\hline & 0 & 5 & 1.95 & 0 & 0 & 0 & 0 & 0 & 0 & 1 \\
\hline \multirow[t]{5}{*}{10} & 0 & 5.18 & 0.183333333 & 0 & 0 & 0 & 0 & 0 & 0 & 0 \\
\hline & 0 & 6 & 0.183333333 & 0 & 0 & 0 & 1 & 0 & 0 & 0 \\
\hline & 0 & 0.5 & 0.116666667 & 0 & 0 & 0 & 0 & 1 & 0 & 0 \\
\hline & 1 & 0.5 & 0.333333333 & 0 & 0 & 0 & 0 & 0 & 1 & 0 \\
\hline & 0 & 5 & 0.2 & 0 & 0 & 0 & 0 & 0 & 0 & 1 \\
\hline \multirow[t]{5}{*}{11} & 0 & 6.18 & 0.9 & 0 & 0 & 1 & 0 & 0 & 0 & 0 \\
\hline & 0 & 10 & 0.9 & 0 & 0 & 0 & 1 & 0 & 0 & 0 \\
\hline & 0 & 0.6 & 0.566666667 & 0 & 0 & 0 & 0 & 1 & 0 & 0 \\
\hline & 1 & 1.6 & 1.333333333 & 0 & 0 & 0 & 0 & 0 & 1 & 0 \\
\hline & 0 & 5 & 0.966666667 & 0 & 0 & 0 & 0 & 0 & 0 & 1 \\
\hline \multirow[t]{5}{*}{12} & 0 & 6.18 & 0.9 & 11 & 0 & 0 & 0 & 0 & 0 & 0 \\
\hline & 0 & 10 & 0.9 & 11 & 0 & 0 & 1 & 0 & 0 & 0 \\
\hline & 0 & 0.6 & 0.566666667 & 11 & 0 & 0 & 0 & 1 & 0 & 0 \\
\hline & 1 & 1.3 & 0.833333333 & 11 & 0 & 0 & 0 & 0 & 1 & 0 \\
\hline & 0 & 5 & 0.966666667 & 11 & 0 & 0 & 0 & 0 & 0 & 1 \\
\hline
\end{tabular}




\begin{tabular}{|c|c|c|c|c|c|c|c|c|c|c|}
\hline \multirow{2}{*}{$\begin{array}{l}\text { Five alternatives } \\
\text { (Number of } \\
\text { observation) }\end{array}$} & Variable & Beta 1 & Beta 2 & Beta 3 & Beta 4 & Beta 5 & Beta 6 & Beta 7 & Beta 8 & Beta 9 \\
\hline & $\begin{array}{l}\text { Mode } \\
\text { Chosen }\end{array}$ & $\begin{array}{c}\text { TC } \\
\text { (1000 Korea won) }\end{array}$ & $\begin{array}{c}\text { TT } \\
\text { (hour) }\end{array}$ & Accom & Sexm & Age340 & Tasc & Sasc & Basc & Lasc \\
\hline \multirow[t]{5}{*}{13} & 0 & 9.076 & 0.816666667 & 0 & 0 & 1 & 0 & 0 & 0 & 0 \\
\hline & 0 & 15 & 0.8 & 0 & 0 & 0 & 1 & 0 & 0 & 0 \\
\hline & 0 & 0.6 & 0.5 & 0 & 0 & 0 & 0 & 1 & 0 & 0 \\
\hline & 1 & 1.8 & 1 & 0 & 0 & 0 & 0 & 0 & 1 & 0 \\
\hline & 0 & 5 & 0.866666667 & 0 & 0 & 0 & 0 & 0 & 0 & 1 \\
\hline \multirow[t]{5}{*}{14} & 0 & 8.78 & 0.466666667 & 0 & 1 & 0 & 0 & 0 & 0 & 0 \\
\hline & 0 & 10 & 0.466666667 & 0 & 0 & 0 & 1 & 0 & 0 & 0 \\
\hline & 0 & 0.5 & 0.283333333 & 0 & 0 & 0 & 0 & 1 & 0 & 0 \\
\hline & 1 & 1.1 & 0.666666667 & 0 & 0 & 0 & 0 & 0 & 1 & 0 \\
\hline & 0 & 5 & 0.5 & 0 & 0 & 0 & 0 & 0 & 0 & 1 \\
\hline \multirow[t]{5}{*}{15} & 0 & 18.18 & 2.066666667 & 1 & 1 & 0 & 0 & 0 & 0 & 0 \\
\hline & 0 & 53 & 2.066666667 & 1 & 0 & 0 & 1 & 0 & 0 & 0 \\
\hline & 0 & 1 & 1.283333333 & 1 & 0 & 0 & 0 & 1 & 0 & 0 \\
\hline & 1 & 14.5 & 3.083333333 & 1 & 0 & 0 & 0 & 0 & 1 & 0 \\
\hline & 0 & 5 & 2.2 & 1 & 0 & 0 & 0 & 0 & 0 & 1 \\
\hline \multirow[t]{5}{*}{16} & 0 & 7.18 & 0.516666667 & 0 & 0 & 1 & 0 & 0 & 0 & 0 \\
\hline & 0 & 10 & 0.516666667 & 0 & 0 & 0 & 1 & 0 & 0 & 0 \\
\hline & 0 & 0.5 & 0.316666667 & 0 & 0 & 0 & 0 & 1 & 0 & 0 \\
\hline & 1 & 1 & 0.416666667 & 0 & 0 & 0 & 0 & 0 & 1 & 0 \\
\hline & 0 & 5 & 0.566666667 & 0 & 0 & 0 & 0 & 0 & 0 & 1 \\
\hline \multirow[t]{5}{*}{17} & 0 & 18.18 & 2.066666667 & 1 & 0 & 1 & 0 & 0 & 0 & 0 \\
\hline & 0 & 53 & 2.066666667 & 1 & 0 & 0 & 1 & 0 & 0 & 0 \\
\hline & 0 & 1 & 1.283333333 & 1 & 0 & 0 & 0 & 1 & 0 & 0 \\
\hline & 1 & 7.5 & 2 & 1 & 0 & 0 & 0 & 0 & 1 & 0 \\
\hline & 0 & 5 & 2.2 & 1 & 0 & 0 & 0 & 0 & 0 & 1 \\
\hline \multirow[t]{5}{*}{18} & 0 & 7,18 & 0.516666667 & 0 & 0 & 1 & 0 & 0 & 0 & 0 \\
\hline & 0 & 10 & 0.5166666667 & 0 & 0 & 0 & 1 & 0 & 0 & 0 \\
\hline & 0 & 0.5 & 0.316666667 & 0 & 0 & 0 & 0 & 1 & 0 & 0 \\
\hline & 1 & 9.3 & 2.6666666667 & 0 & 0 & 0 & 0 & 0 & 1 & 0 \\
\hline & 0 & 5 & 0.566666667 & 0 & 0 & 0 & 0 & 0 & 0 & 1 \\
\hline \multirow[t]{5}{*}{19} & 0 & 7.18 & 0.516666667 & 2 & 1 & 0 & 0 & 0 & 0 & 0 \\
\hline & 0 & 10 & 0.516666667 & 2 & 0 & 0 & 1 & 0 & 0 & 0 \\
\hline & 0 & 0.5 & 0.316666667 & 2 & 0 & 0 & 0 & 1 & 0 & 0 \\
\hline & 1 & 1.3 & 0.6666666667 & 2 & 0 & 0 & 0 & 0 & 1 & 0 \\
\hline & 0 & 5 & 0.5666666667 & 2 & 0 & 0 & 0 & 0 & 0 & 1 \\
\hline \multirow[t]{5}{*}{20} & 0 & 8.18 & 1.533333333 & 0 & 1 & 0 & 0 & 0 & 0 & 0 \\
\hline & 0 & 12.052 & 1.533333333 & 0 & 0 & 0 & 1 & 0 & 0 & 0 \\
\hline & 0 & 0.6 & 0.95 & 0 & 0 & 0 & 0 & 1 & 0 & 0 \\
\hline & 1 & 5 & 0.6666666667 & 0 & 0 & 0 & 0 & 0 & 1 & 0 \\
\hline & 0 & 5 & 1.633333333 & $\overline{0}$ & 0 & 0 & 0 & 0 & 0 & 1 \\
\hline \multirow[t]{5}{*}{21} & 0 & 9.18 & 1.2 & 5 & 1 & 1 & 0 & 0 & 0 & 0 \\
\hline & 0 & 28 & 1.2 & 5 & 0 & 0 & 1 & 0 & 0 & 0 \\
\hline & 0 & 0.6 & 1.283333333 & 5 & 0 & 0 & 0 & 1 & 0 & 0 \\
\hline & 1 & 14.5 & 0.666666667 & 5 & 0 & 0 & 0 & 0 & 1 & 0 \\
\hline & 0 & 5 & 1.283333333 & 5 & 0 & 0 & 0 & 0 & 0 & 1 \\
\hline \multirow[t]{5}{*}{22} & 0 & 5.31 & 0.816666667 & 3 & 0 & 1 & 0 & 0 & 0 & 0 \\
\hline & 0 & 15 & 0.816666667 & 3 & 0 & 0 & 1 & 0 & 0 & 0 \\
\hline & 0 & 0.8 & 0.5 & 3 & 0 & 0 & 0 & 1 & 0 & 0 \\
\hline & 1 & 5 & 2 & 3 & 0 & 0 & 0 & 0 & 1 & 0 \\
\hline & 0 & 5 & 0.866666667 & 3 & 0 & 0 & 0 & 0 & 0 & 1 \\
\hline \multirow[t]{5}{*}{23} & 0 & 9.18 & 1.2 & 0 & 1 & 1 & 0 & 0 & 0 & 0 \\
\hline & 0 & 28 & 1.2 & 0 & 0 & 0 & 1 & 0 & 0 & 0 \\
\hline & 0 & 0.6 & 0.733333333 & 0 & 0 & 0 & 0 & 1 & 0 & 0 \\
\hline & 1 & 1.3 & 0.5 & 0 & 0 & 0 & 0 & 0 & 1 & 0 \\
\hline & 0 & 5 & 1.283333333 & 0 & 0 & 0 & 0 & 0 & 0 & 1 \\
\hline \multirow[t]{5}{*}{24} & 0 & 9.18 & 1.2 & 0 & 1 & 1 & 0 & 0 & 0 & 0 \\
\hline & 0 & 28 & 1.2 & 0 & 0 & 0 & 1 & 0 & 0 & 0 \\
\hline & 0 & 0.6 & 0.733333333 & 0 & 0 & 0 & 0 & 1 & 0 & 0 \\
\hline & 1 & 1.3 & 0.6666666667 & 0 & 0 & 0 & 0 & 0 & 1 & 0 \\
\hline & 0 & 5 & 1.283333333 & 0 & 0 & 0 & 0 & 0 & 0 & 1 \\
\hline
\end{tabular}




\begin{tabular}{|c|c|c|c|c|c|c|c|c|c|c|}
\hline \multirow{2}{*}{$\begin{array}{l}\text { Five alternatives } \\
\text { (Number of } \\
\text { observation) }\end{array}$} & Variable & Beta 1 & Beta 2 & Beta 3 & Beta 4 & Beta 5 & Beta 6 & Beta 7 & Beta 8 & Beta 9 \\
\hline & $\begin{array}{c}\text { Mode } \\
\text { Chosen }\end{array}$ & $\begin{array}{c}\text { TC } \\
\text { (1000 Korea won) }\end{array}$ & $\begin{array}{c}\text { TT } \\
\text { (hour) }\end{array}$ & Accom & Sexm & Age340 & Tasc & Sasc & Basc & Lasc \\
\hline \multirow[t]{5}{*}{25} & 0 & 8.476 & 0.95 & 0 & 0 & 0 & 0 & 0 & 0 & 0 \\
\hline & 0 & 12.5 & 0.95 & 0 & 0 & 0 & 1 & 0 & 0 & 0 \\
\hline & 0 & 0.7 & 0.583333333 & 0 & 0 & 0 & 0 & 1 & 0 & 0 \\
\hline & 1 & 1.1 & 0.833333333 & 0 & 0 & 0 & 0 & 0 & 1 & 0 \\
\hline & 0 & 5 & 1.0166666667 & 0 & 0 & 0 & 0 & 0 & 0 & 1 \\
\hline \multirow[t]{5}{*}{26} & 0 & 7.1 & 1.1 & 0 & 0 & 1 & 0 & 0 & 0 & 0 \\
\hline & 0 & 15 & 1.1 & 0 & 0 & 0 & 1 & 0 & 0 & 0 \\
\hline & 0 & 0.6 & 0.683333333 & 0 & 0 & 0 & 0 & 1 & 0 & 0 \\
\hline & 1 & 1.3 & 1 & 0 & 0 & 0 & 0 & 0 & 1 & 0 \\
\hline & 0 & 5 & 1.183333333 & 0 & 0 & 0 & 0 & 0 & 0 & 1 \\
\hline \multirow[t]{5}{*}{27} & 0 & 8.476 & 0.95 & 0 & 1 & 1 & 0 & 0 & 0 & 0 \\
\hline & 0 & 12.5 & 0.95 & 0 & 0 & 0 & 1 & 0 & 0 & 0 \\
\hline & 0 & 0.7 & 0.583333333 & 0 & 0 & 0 & 0 & 1 & 0 & 0 \\
\hline & 1 & 1 & 1 & 0 & 0 & 0 & 0 & 0 & 1 & 0 \\
\hline & 0 & 5 & 1.0166666667 & 0 & 0 & 0 & 0 & 0 & 0 & 1 \\
\hline \multirow[t]{5}{*}{28} & 0 & 7.18 & 0.5166666667 & 0 & 0 & 1 & 0 & 0 & 0 & 0 \\
\hline & 0 & 10 & 0.516666667 & 0 & 0 & 0 & 1 & 0 & 0 & 0 \\
\hline & 0 & 0.5 & 0.316666667 & 0 & 0 & 0 & 0 & 1 & 0 & 0 \\
\hline & 1 & 1.3 & 0.5 & 0 & 0 & 0 & 0 & 0 & 1 & 0 \\
\hline & 0 & 5 & 0.566666667 & 0 & 0 & 0 & 0 & 0 & 0 & 1 \\
\hline \multirow[t]{5}{*}{29} & 0 & 8.476 & 0.95 & 0 & 1 & 0 & 0 & 0 & 0 & 0 \\
\hline & 0 & 12.5 & 0.95 & 0 & 0 & 0 & 1 & 0 & 0 & 0 \\
\hline & 0 & 0.7 & 0.5833333333 & 0 & 0 & 0 & 0 & 1 & 0 & 0 \\
\hline & 1 & 1.3 & 0.666666667 & 0 & 0 & 0 & 0 & 0 & 1 & 0 \\
\hline & 0 & 5 & 1.016666667 & 0 & 0 & 0 & 0 & 0 & 0 & 1 \\
\hline \multirow[t]{5}{*}{30} & 0 & 8.18 & 1.4833333333 & 1 & 0 & 1 & 0 & 0 & 0 & 0 \\
\hline & 0 & 20 & 1.4833333333 & 1 & 0 & 0 & 1 & 0 & 0 & 0 \\
\hline & 0 & 0.6 & 0.9166666667 & 1 & 0 & 0 & 0 & 1 & 0 & 0 \\
\hline & 1 & 2 & 1.333333333 & 1 & 0 & 0 & 0 & 0 & 1 & 0 \\
\hline & 0 & 5 & 1.5833333333 & 1 & 0 & 0 & 0 & 0 & 0 & 1 \\
\hline \multirow[t]{5}{*}{31} & 0 & 8.78 & 0.466666667 & 1 & 1 & 0 & 0 & 0 & 0 & 0 \\
\hline & 0 & 10 & 0.466666667 & 1 & 0 & 0 & 1 & 0 & 0 & 0 \\
\hline & 0 & 0.5 & 0.283333333 & 1 & 0 & 0 & 0 & 1 & 0 & 0 \\
\hline & 1 & 2 & 1 & 1 & 0 & 0 & 0 & 0 & 1 & 0 \\
\hline & 0 & 5 & 0.5 & 1 & 0 & 0 & 0 & 0 & 0 & 1 \\
\hline \multirow[t]{5}{*}{32} & 0 & 7.18 & 0.5166666667 & 0 & 0 & 1 & 0 & 0 & 0 & 0 \\
\hline & 0 & 10 & 0.516666667 & 0 & 0 & 0 & 1 & 0 & 0 & 0 \\
\hline & 0 & 0.5 & 0.316666667 & 0 & 0 & 0 & 0 & 1 & 0 & 0 \\
\hline & 1 & 1.5 & 0.833333333 & 0 & 0 & 0 & 0 & 0 & 1 & 0 \\
\hline & 0 & 5 & 0.5666666667 & 0 & 0 & 0 & 0 & 0 & 0 & 1 \\
\hline \multirow[t]{5}{*}{33} & 0 & 13.18 & 1.866666667 & 1 & 0 & 0 & 0 & 0 & 0 & 0 \\
\hline & 0 & 28 & 1.866666667 & 1 & 0 & 0 & 1 & 0 & 0 & 0 \\
\hline & 0 & 0.85 & 1.15 & 1 & 0 & 0 & 0 & 1 & 0 & 0 \\
\hline & 1 & 1.5 & 2 & 1 & 0 & 0 & 0 & 0 & 1 & 0 \\
\hline & 0 & 5 & 2 & 1 & 0 & 0 & 0 & 0 & 0 & 1 \\
\hline \multirow[t]{5}{*}{34} & 0 & 9.076 & 0.816666667 & 1 & 0 & 1 & 0 & 0 & 0 & 0 \\
\hline & 0 & 15 & 0.8166666667 & 1 & 0 & 0 & 1 & 0 & 0 & 0 \\
\hline & 0 & 0.6 & 0.5 & 1 & 0 & 0 & 0 & 1 & 0 & 0 \\
\hline & 1 & 1.5 & 1 & 1 & 0 & 0 & 0 & 0 & 1 & 0 \\
\hline & 0 & 5 & 0.8666666667 & 1 & 0 & 0 & 0 & 0 & 0 & 1 \\
\hline \multirow[t]{5}{*}{35} & 0 & 8.78 & 0.4666666667 & 1 & 1 & 0 & 0 & 0 & 0 & 0 \\
\hline & 0 & 10 & 0.4666666667 & 1 & 0 & 0 & 1 & 0 & 0 & 0 \\
\hline & 0 & 0.5 & 0.283333333 & 1 & 0 & 0 & 0 & 1 & 0 & 0 \\
\hline & 1 & 1 & 1 & 1 & 0 & 0 & 0 & 0 & 1 & 0 \\
\hline & 0 & 5 & 0.5 & 1 & 0 & 0 & 0 & 0 & 0 & 1 \\
\hline \multirow[t]{5}{*}{36} & 0 & 5.18 & 0.183333333 & 1 & 1 & 0 & 0 & 0 & 0 & 0 \\
\hline & 0 & 6 & 0.183333333 & 1 & 0 & 0 & 1 & 0 & 0 & 0 \\
\hline & 0 & 0.5 & 0.116666667 & 1 & 0 & 0 & 0 & 1 & 0 & 0 \\
\hline & 1 & 0.5 & 0.333333333 & 1 & 0 & 0 & 0 & 0 & 1 & 0 \\
\hline & 0 & 5 & 0.2 & 1 & 0 & 0 & 0 & 0 & 0 & 1 \\
\hline
\end{tabular}




\begin{tabular}{|c|c|c|c|c|c|c|c|c|c|c|}
\hline \multirow{2}{*}{$\begin{array}{c}\text { Five alternatives } \\
\text { (Number of } \\
\text { observation) }\end{array}$} & Variable & Beta 1 & Beta 2 & Beta 3 & Beta 4 & Beta 5 & Beta 6 & Beta 7 & Beta 8 & Beta 9 \\
\hline & $\begin{array}{c}\text { Mode } \\
\text { Chosen } \\
\end{array}$ & $\begin{array}{c}\text { TC } \\
\text { (1000 Korea won) } \\
\end{array}$ & $\begin{array}{c}\text { TT } \\
\text { (hour) } \\
\end{array}$ & Accom & Sexm & Age340 & Tasc & Sasc & Basc & Lasc \\
\hline \multirow[t]{5}{*}{37} & 0 & 8.78 & 0.466666667 & 1 & 0 & 1 & 0 & 0 & 0 & 0 \\
\hline & 0 & 10 & 0.466666667 & 1 & 0 & 0 & 1 & 0 & 0 & 0 \\
\hline & 0 & 0.5 & 0.283333333 & 1 & 0 & 0 & 0 & 1 & 0 & 0 \\
\hline & 1 & 1.5 & 1 & 1 & 0 & 0 & 0 & 0 & 1 & 0 \\
\hline & 0 & 5 & 0.5 & 1 & 0 & 0 & 0 & 0 & 0 & 1 \\
\hline \multirow[t]{5}{*}{38} & 0 & 8.18 & 0.85 & 1 & 0 & 0 & 0 & 0 & 0 & 0 \\
\hline & 0 & 10 & 0.85 & 1 & 0 & 0 & 1 & 0 & 0 & 0 \\
\hline & 0 & 0.6 & 0.533333333 & 1 & 0 & 0 & 0 & 1 & 0 & 0 \\
\hline & 1 & 2.5 & 1.333333333 & 1 & 0 & 0 & 0 & 0 & 1 & 0 \\
\hline & 0 & 5 & 0.916666667 & 1 & 0 & 0 & 0 & 0 & 0 & 1 \\
\hline \multirow[t]{5}{*}{39} & 0 & 7.735 & 2.066666667 & 1 & 0 & 1 & 0 & 0 & 0 & 0 \\
\hline & 0 & 14 & 2.066666667 & 1 & 0 & 0 & 1 & 0 & 0 & 0 \\
\hline & 0 & 0.75 & 1.283333333 & 1 & 0 & 0 & 0 & 1 & 0 & 0 \\
\hline & 1 & 1.5 & 0.833333333 & 1 & 0 & 0 & 0 & 0 & 1 & 0 \\
\hline & 0 & 5 & 2.2 & 1 & 0 & 0 & 0 & 0 & 0 & 1 \\
\hline \multirow[t]{5}{*}{40} & 0 & 9.076 & 0.816666667 & 0 & 1 & 0 & 0 & 0 & 0 & 0 \\
\hline & 0 & 15 & 0.816666667 & 0 & 0 & 0 & 1 & 0 & 0 & 0 \\
\hline & 0 & 0.6 & 0.5 & 0 & 0 & 0 & 0 & 1 & 0 & 0 \\
\hline & 1 & 1.6 & 1.333333333 & 0 & 0 & 0 & 0 & 0 & 1 & 0 \\
\hline & 0 & 5 & 0.866666667 & 0 & 0 & 0 & 0 & 0 & 0 & 1 \\
\hline \multirow[t]{5}{*}{41} & 0 & 7.735 & 2.066666667 & 0 & 1 & 0 & 0 & 0 & 0 & 0 \\
\hline & 0 & 14 & 2.066666667 & 0 & 0 & 0 & 1 & 0 & 0 & 0 \\
\hline & 0 & 0.75 & 1.283333333 & 0 & 0 & 0 & 0 & 1 & 0 & 0 \\
\hline & 1 & 2.1 & 1.5 & 0 & 0 & 0 & 0 & 0 & 1 & 0 \\
\hline & 0 & 5 & 2.2 & 0 & 0 & 0 & 0 & 0 & 0 & 1 \\
\hline \multirow[t]{5}{*}{42} & 0 & 7.18 & 0.516666667 & 0 & 1 & 1 & 0 & 0 & 0 & 0 \\
\hline & 0 & 10 & 0.516666667 & 0 & 0 & 0 & 1 & 0 & 0 & 0 \\
\hline & 0 & 0.5 & 0.3166666667 & 0 & 0 & 0 & 0 & 1 & 0 & 0 \\
\hline & 1 & 1.6 & 0.583333333 & 0 & 0 & 0 & 0 & 0 & 1 & 0 \\
\hline & 0 & 5 & 0.566666667 & 0 & 0 & 0 & 0 & 0 & 0 & 1 \\
\hline \multirow[t]{5}{*}{43} & 0 & 8.18 & 1.483333333 & 0 & 1 & 1 & 0 & 0 & 0 & 0 \\
\hline & 0 & 20 & 1.483333333 & 0 & 0 & 0 & 1 & 0 & 0 & 0 \\
\hline & 0 & 0.6 & 0.916666667 & 0 & 0 & 0 & 0 & 1 & 0 & 0 \\
\hline & 1 & 1.6 & 1.333333333 & 0 & 0 & 0 & 0 & 0 & 1 & 0 \\
\hline & 0 & 5 & 1.583333333 & 0 & 0 & 0 & 0 & 0 & 0 & 1 \\
\hline \multirow[t]{5}{*}{44} & 0 & 8.78 & 0.466666667 & 0 & 1 & 1 & 0 & 0 & 0 & 0 \\
\hline & 0 & 10 & 0.466666667 & 0 & 0 & 0 & 1 & 0 & 0 & 0 \\
\hline & 0 & 0.5 & 0.283333333 & 0 & 0 & 0 & 0 & 1 & 0 & 0 \\
\hline & 1 & 0.5 & 0.333333333 & 0 & 0 & 0 & 0 & 0 & 1 & 0 \\
\hline & 0 & 5 & 0.5 & 0 & 0 & 0 & 0 & 0 & 0 & 1 \\
\hline \multirow[t]{5}{*}{45} & 0 & 8.476 & 0.95 & 0 & 1 & 1 & 0 & 0 & 0 & 0 \\
\hline & 0 & 12.5 & 0.95 & 0 & 0 & 0 & 1 & 0 & 0 & 0 \\
\hline & 0 & 0.7 & 0.583333333 & 0 & 0 & 0 & 0 & 1 & 0 & 0 \\
\hline & 1 & 1.5 & 1 & 0 & 0 & 0 & 0 & 0 & 1 & 0 \\
\hline & 0 & 5 & 1.0166666667 & 0 & 0 & 0 & 0 & 0 & 0 & 1 \\
\hline \multirow[t]{5}{*}{46} & 0 & 7.18 & 0.5166666667 & 0 & 1 & 0 & 0 & 0 & 0 & 0 \\
\hline & 0 & 10 & 0.5166666667 & 0 & 0 & 0 & 1 & 0 & 0 & 0 \\
\hline & 0 & 0.5 & 0.3166666667 & 0 & 0 & 0 & 0 & 1 & 0 & 0 \\
\hline & 1 & 1.1 & 0.583333333 & 0 & 0 & 0 & 0 & 0 & 1 & 0 \\
\hline & 0 & 5 & 0.566666667 & 0 & 0 & 0 & 0 & 0 & 0 & 1 \\
\hline \multirow[t]{5}{*}{47} & 0 & 8.017 & 1.15 & 0 & 1 & 1 & 0 & 0 & 0 & 0 \\
\hline & 0 & 17 & 1.15 & 0 & 0 & 0 & 1 & 0 & 0 & 0 \\
\hline & 0 & 0.65 & 0.7 & 0 & 0 & 0 & 0 & 1 & 0 & 0 \\
\hline & 1 & 2 & 1.166666667 & 0 & 0 & 0 & 0 & 0 & 1 & 0 \\
\hline & 0 & 5 & 1.233333333 & 0 & 0 & 0 & 0 & 0 & 0 & 1 \\
\hline \multirow[t]{5}{*}{48} & 0 & 9.18 & 1.2 & 0 & 0 & 0 & 0 & 0 & 0 & 0 \\
\hline & 0 & 28 & 1.2 & 0 & 0 & 0 & 1 & 0 & 0 & 0 \\
\hline & 0 & 0.6 & 0.733333333 & 0 & 0 & 0 & 0 & 1 & 0 & 0 \\
\hline & 1 & 1.3 & 0.666666667 & 0 & 0 & 0 & 0 & 0 & 1 & 0 \\
\hline & 0 & 5 & 1.283333333 & 0 & 0 & 0 & 0 & 0 & 0 & 1 \\
\hline
\end{tabular}




\begin{tabular}{|c|c|c|c|c|c|c|c|c|c|c|}
\hline \multirow{2}{*}{$\begin{array}{l}\text { Five alternatives } \\
\text { (Number of } \\
\text { observation) }\end{array}$} & Variable & Beta 1 & Beta 2 & Beta 3 & Beta 4 & Beta 5 & Beta 6 & Beta 7 & Beta 8 & Beta 9 \\
\hline & $\begin{array}{l}\text { Mode } \\
\text { Chosen }\end{array}$ & $\begin{array}{c}\text { TC } \\
\text { (1000 Korea won) } \\
\end{array}$ & $\begin{array}{c}\mathrm{TT} \\
\text { (hour) }\end{array}$ & Accom & Sexm & Age340 & Tasc & Sasc & Basc & Lasc \\
\hline \multirow[t]{5}{*}{49} & 0 & 8.18 & 1.533333333 & 0 & 1 & 0 & 0 & 0 & 0 & 0 \\
\hline & 0 & 12.052 & 1.533333333 & 0 & 0 & 0 & 1 & 0 & 0 & 0 \\
\hline & 0 & 0.6 & 0.95 & 0 & 0 & 0 & 0 & 1 & 0 & 0 \\
\hline & 1 & 5 & 2 & 0 & 0 & 0 & 0 & 0 & 1 & 0 \\
\hline & 0 & 5 & 1.633333333 & 0 & 0 & 0 & 0 & 0 & 0 & 1 \\
\hline \multirow[t]{5}{*}{50} & 0 & 9.18 & 1.2 & 2 & 1 & 0 & 0 & 0 & 0 & 0 \\
\hline & 0 & 28 & 1.2 & 2 & 0 & 0 & 1 & 0 & 0 & 0 \\
\hline & 0 & 0.6 & 0.733333333 & 2 & 0 & 0 & 0 & 1 & 0 & 0 \\
\hline & 1 & 5 & 2 & 2 & 0 & 0 & 0 & 0 & 1 & 0 \\
\hline & 0 & 5 & 1.283333333 & 2 & 0 & 0 & 0 & 0 & 0 & 1 \\
\hline \multirow[t]{5}{*}{51} & 0 & 9.076 & 0.816666667 & 0 & 1 & 0 & 0 & 0 & 0 & 0 \\
\hline & 0 & 15 & 0.816666667 & 0 & 0 & 0 & 1 & 0 & 0 & 0 \\
\hline & 0 & 0.6 & 0.5 & 0 & 0 & 0 & 0 & 1 & 0 & 0 \\
\hline & 1 & 1.1 & 0.666666667 & 0 & 0 & 0 & $\overline{0}$ & 0 & 1 & 0 \\
\hline & 0 & 5 & 0.866666667 & 0 & 0 & 0 & 0 & 0 & 0 & 1 \\
\hline \multirow[t]{5}{*}{52} & 0 & 8.476 & 0.95 & 1 & 0 & 0 & 0 & 0 & 0 & 0 \\
\hline & 0 & 12.5 & 0.95 & 1 & 0 & 0 & 1 & 0 & 0 & 0 \\
\hline & 0 & 0.7 & 0.583333333 & 1 & 0 & 0 & 0 & 1 & 0 & 0 \\
\hline & 1 & 1.1 & 1 & 1 & 0 & 0 & 0 & 0 & 1 & 0 \\
\hline & 0 & 5 & 1.016666667 & 1 & 0 & 0 & 0 & 0 & 0 & 1 \\
\hline \multirow[t]{5}{*}{53} & 0 & 7.18 & 0.516666667 & 0 & 1 & 1 & 0 & 0 & 0 & 0 \\
\hline & 0 & 10 & 0.516666667 & 0 & 0 & 0 & 1 & 0 & 0 & 0 \\
\hline & 0 & 0.5 & 0.316666667 & 0 & 0 & 0 & 0 & 1 & 0 & 0 \\
\hline & 1 & 1.1 & 0.583333333 & 0 & 0 & 0 & 0 & 0 & 1 & 0 \\
\hline & 0 & 5 & 0.566666667 & 0 & 0 & 0 & 0 & 0 & 0 & 1 \\
\hline \multirow[t]{5}{*}{54} & 0 & 7.735 & 2.066666667 & 0 & 0 & 1 & 0 & 0 & 0 & 0 \\
\hline & 0 & 14 & 2.0666666667 & 0 & 0 & 0 & 1 & 0 & 0 & 0 \\
\hline & 0 & 0.75 & 1.283333333 & 0 & 0 & 0 & 0 & 1 & 0 & 0 \\
\hline & 1 & 1.1 & 0.6666666667 & 0 & 0 & 0 & 0 & 0 & 1 & 0 \\
\hline & 0 & 5 & 2.2 & 0 & 0 & 0 & 0 & 0 & 0 & 1 \\
\hline \multirow[t]{5}{*}{55} & 0 & 10.6 & 1.433333333 & 0 & 1 & 1 & 0 & 0 & 0 & 0 \\
\hline & 0 & 12 & 1.433333333 & 0 & 0 & 0 & 1 & 0 & 0 & 0 \\
\hline & 0 & 0.85 & 0.883333333 & 0 & 0 & 0 & 0 & 1 & 0 & 0 \\
\hline & 1 & 5 & 1.5 & 0 & 0 & 0 & 0 & 0 & 1 & 0 \\
\hline & 0 & 5 & 1.533333333 & 0 & 0 & 0 & 0 & 0 & 0 & 1 \\
\hline \multirow[t]{5}{*}{56} & 0 & 8.48 & 1.816666667 & 0 & 1 & 0 & 0 & 0 & 0 & 0 \\
\hline & 0 & 28 & 1.816666667 & 0 & 0 & 0 & 1 & 0 & 0 & 0 \\
\hline & 0 & 0.65 & 1.133333333 & 0 & 0 & 0 & 0 & 1 & 0 & 0 \\
\hline & 1 & 1 & 0.5 & 0 & 0 & 0 & 0 & 0 & 1 & 0 \\
\hline & 0 & 5 & 1.95 & 0 & 0 & 0 & 0 & 0 & 0 & 1 \\
\hline \multirow[t]{5}{*}{57} & 0 & 5.18 & 0.183333333 & 2 & 1 & 0 & 0 & 0 & 0 & 0 \\
\hline & 0 & 6 & 0.183333333 & 2 & 0 & 0 & 1 & 0 & 0 & 0 \\
\hline & 0 & 0.5 & 0.116666667 & 2 & 0 & 0 & 0 & 1 & 0 & 0 \\
\hline & 1 & 1 & 0.5 & 2 & 0 & 0 & 0 & 0 & 1 & 0 \\
\hline & 0 & 5 & 0.2 & 2 & 0 & 0 & 0 & 0 & 0 & 1 \\
\hline \multirow[t]{5}{*}{58} & 0 & 5.18 & 0.183333333 & 0 & 1 & 0 & 0 & 0 & 0 & 0 \\
\hline & 0 & 6 & 0.183333333 & 0 & 0 & 0 & 1 & 0 & 0 & 0 \\
\hline & 0 & 0.5 & 0.116666667 & 0 & 0 & 0 & 0 & 1 & 0 & 0 \\
\hline & 1 & 0.5 & 0.5 & 0 & 0 & 0 & 0 & 0 & 1 & 0 \\
\hline & 0 & 5 & 0.2 & 0 & 0 & 0 & 0 & 0 & 0 & 1 \\
\hline \multirow[t]{5}{*}{59} & 0 & 9.18 & 1.2 & 0 & 0 & 0 & 0 & 0 & 0 & 0 \\
\hline & 0 & 28 & 1.2 & 0 & 0 & 0 & 1 & 0 & 0 & 0 \\
\hline & 0 & 0.6 & 0.733333333 & 0 & 0 & 0 & 0 & 1 & 0 & 0 \\
\hline & 1 & 1.1 & 1 & 0 & 0 & 0 & 0 & 0 & 1 & 0 \\
\hline & 0 & 5 & 1.283333333 & 0 & 0 & 0 & 0 & 0 & 0 & 1 \\
\hline \multirow[t]{5}{*}{60} & 0 & 7.735 & 2.0666666667 & 0 & 1 & 1 & 0 & 0 & 0 & 0 \\
\hline & 0 & 14 & 2.066666667 & 0 & 0 & 0 & 1 & 0 & 0 & 0 \\
\hline & 0 & 0.75 & 1.283333333 & 0 & 0 & 0 & 0 & 1 & 0 & 0 \\
\hline & 1 & 1 & 0.5 & 0 & 0 & 0 & 0 & 0 & 1 & 0 \\
\hline & 0 & 5 & 2.2 & 0 & 0 & 0 & 0 & 0 & 0 & 1 \\
\hline
\end{tabular}




\begin{tabular}{|c|c|c|c|c|c|c|c|c|c|c|}
\hline \multirow{2}{*}{$\begin{array}{l}\text { Five alternatives } \\
\text { (Number of } \\
\text { observation) }\end{array}$} & Variable & Beta 1 & Beta 2 & Beta 3 & Beta 4 & Beta 5 & Beta 6 & Beta 7 & Beta 8 & Beta 9 \\
\hline & $\begin{array}{c}\text { Mode } \\
\text { Chosen }\end{array}$ & $\begin{array}{c}\text { TC } \\
\text { (1000 Korea won) }\end{array}$ & $\begin{array}{c}\text { TT } \\
\text { (hour) }\end{array}$ & Accom & Sexm & Age 340 & Tasc & Sasc & Basc & Lasc \\
\hline \multirow[t]{5}{*}{61} & 0 & 10.6 & 1.433333333 & 1 & 1 & 1 & 0 & 0 & 0 & 0 \\
\hline & 0 & 12 & 1.433333333 & 1 & 0 & 0 & 1 & 0 & 0 & 0 \\
\hline & 0 & 0.85 & 0.883333333 & 1 & 0 & 0 & 0 & 1 & 0 & 0 \\
\hline & 1 & 1.1 & 0.25 & 1 & 0 & 0 & 0 & 0 & 1 & 0 \\
\hline & 0 & 5 & 1.533333333 & 1 & 0 & 0 & 0 & 0 & 0 & 1 \\
\hline \multirow[t]{5}{*}{62} & 0 & 8.476 & 0.95 & 1 & 0 & 1 & 0 & 0 & 0 & 0 \\
\hline & 0 & 12.5 & 0.95 & 1 & 0 & 0 & 1 & 0 & 0 & 0 \\
\hline & 0 & 0.7 & 0.583333333 & 1 & 0 & 0 & 0 & 1 & 0 & 0 \\
\hline & 1 & 1.1 & 0.6666666667 & 1 & 0 & 0 & 0 & 0 & 1 & 0 \\
\hline & 0 & 5 & 1.0166666667 & 1 & 0 & 0 & 0 & 0 & 0 & 1 \\
\hline \multirow[t]{5}{*}{63} & 0 & 7.735 & 2.0666666667 & 0 & 1 & 0 & 0 & 0 & 0 & 0 \\
\hline & 0 & 14 & 2.0666666667 & 0 & 0 & 0 & 1 & 0 & 0 & 0 \\
\hline & 0 & 0.75 & 1.2833333333 & 0 & 0 & 0 & 0 & 1 & 0 & 0 \\
\hline & 1 & 2.1 & 1 & 0 & 0 & 0 & 0 & 0 & 1 & 0 \\
\hline & 0 & 5 & 2.2 & 0 & 0 & 0 & 0 & 0 & 0 & 1 \\
\hline \multirow[t]{5}{*}{64} & 0 & 7.735 & 2.0666666667 & 0 & 0 & 1 & 0 & 0 & 0 & 0 \\
\hline & 0 & 14 & 2.066666667 & 0 & 0 & 0 & 1 & 0 & 0 & 0 \\
\hline & 0 & 0.75 & 1.283333333 & 0 & 0 & 0 & 0 & 1 & 0 & 0 \\
\hline & 1 & 1 & 0.25 & 0 & 0 & 0 & 0 & 0 & 1 & 0 \\
\hline & 0 & 5 & 2.2 & 0 & 0 & 0 & 0 & 0 & 0 & 1 \\
\hline \multirow[t]{5}{*}{65} & 0 & 7.735 & 2.0666666667 & 0 & 0 & 1 & 0 & 0 & 0 & 0 \\
\hline & 0 & 14 & 2.066666667 & 0 & 0 & 0 & 1 & 0 & 0 & 0 \\
\hline & 0 & 0.75 & 1.2833333333 & 0 & 0 & 0 & 0 & 1 & 0 & 0 \\
\hline & 1 & 1 & 0.4166666667 & 0 & 0 & 0 & 0 & 0 & 1 & 0 \\
\hline & 0 & 5 & 2.2 & 0 & 0 & 0 & 0 & 0 & 0 & 1 \\
\hline \multirow[t]{5}{*}{66} & 0 & 5.298 & 0.616666667 & 0 & 0 & 1 & 0 & 0 & 0 & 0 \\
\hline & 0 & 10 & 0.616666667 & 0 & 0 & 0 & 1 & 0 & 0 & 0 \\
\hline & 0 & 0.6 & 0.383333333 & 0 & 0 & 0 & 0 & 1 & 0 & 0 \\
\hline & 1 & 1 & 0.333333333 & 0 & 0 & 0 & 0 & 0 & 1 & 0 \\
\hline & 0 & 5 & 0.6666666667 & 0 & 0 & 0 & 0 & 0 & 0 & 1 \\
\hline \multirow[t]{5}{*}{67} & 0 & 7.735 & 2.0666666667 & 0 & 0 & 0 & 0 & 0 & 0 & 0 \\
\hline & 0 & 14 & 2.0666666667 & 0 & 0 & 0 & 1 & 0 & 0 & 0 \\
\hline & 0 & 0.75 & 1.2833333333 & 0 & 0 & 0 & 0 & 1 & 0 & 0 \\
\hline & 1 & 1.5 & 1 & 0 & 0 & 0 & 0 & 0 & 1 & 0 \\
\hline & 0 & 5 & 2.2 & 0 & 0 & 0 & 0 & 0 & 0 & 1 \\
\hline \multirow[t]{5}{*}{68} & 0 & 7.18 & 0.5166666667 & 0 & 1 & 0 & 0 & 0 & 0 & 0 \\
\hline & 0 & 10 & 0.5166666667 & 0 & 0 & 0 & 1 & 0 & 0 & 0 \\
\hline & 0 & 0.5 & 0.3166666667 & 0 & 0 & 0 & 0 & 1 & 0 & 0 \\
\hline & 1 & 1 & 0.833333333 & 0 & 0 & 0 & 0 & 0 & 1 & 0 \\
\hline & 0 & 5 & 0.5666666667 & 0 & 0 & 0 & 0 & 0 & 0 & 1 \\
\hline \multirow[t]{5}{*}{69} & 0 & 10.18 & 1.6666666667 & 1 & 1 & 0 & 0 & 0 & 0 & 0 \\
\hline & 0 & 13.127 & 1.666666667 & 1 & 0 & 0 & 1 & 0 & 0 & 0 \\
\hline & 0 & 0.6 & 1.033333333 & 1 & 0 & 0 & 0 & 1 & 0 & 0 \\
\hline & 1 & 2 & 1.333333333 & 1 & 0 & 0 & 0 & 0 & 1 & 0 \\
\hline & 0 & 5 & 1.8 & 1 & 0 & 0 & 0 & 0 & 0 & 1 \\
\hline \multirow[t]{5}{*}{70} & 0 & 5.18 & 0.183333333 & 1 & 0 & 0 & 0 & 0 & 0 & 0 \\
\hline & 0 & 6 & 0.183333333 & 1 & 0 & 0 & 1 & 0 & 0 & 0 \\
\hline & 0 & 0.5 & 0.1166666667 & 1 & 0 & 0 & 0 & 1 & 0 & 0 \\
\hline & 1 & 0.5 & 0.5 & 1 & 0 & 0 & 0 & 0 & 1 & 0 \\
\hline & 0 & 5 & 0.2 & 1 & 0 & 0 & 0 & 0 & 0 & 1 \\
\hline \multirow[t]{5}{*}{71} & 0 & 9.18 & 1.2 & 0 & 1 & 0 & 0 & 0 & 0 & 0 \\
\hline & 0 & 28 & 1.2 & 0 & 0 & 0 & 1 & 0 & 0 & 0 \\
\hline & 0 & 0.6 & 0.733333333 & 0 & 0 & 0 & 0 & 1 & 0 & 0 \\
\hline & 1 & 1.8 & 1.166666667 & 0 & 0 & 0 & 0 & 0 & 1 & 0 \\
\hline & 0 & 5 & 1.283333333 & 0 & 0 & 0 & 0 & 0 & 0 & 1 \\
\hline \multirow[t]{5}{*}{72} & 0 & 8.18 & 1.333333333 & 1 & 0 & 0 & 0 & 0 & 0 & 0 \\
\hline & 0 & 15.5 & 1.333333333 & 1 & 0 & 0 & 1 & 0 & 0 & 0 \\
\hline & 0 & 0.8 & 0.833333333 & 1 & 0 & 0 & 0 & 1 & 0 & 0 \\
\hline & 1 & 1.6 & 2 & 1 & 0 & 0 & 0 & 0 & 1 & 0 \\
\hline & 0 & 5 & 1.433333333 & 1 & 0 & 0 & 0 & 0 & 0 & 1 \\
\hline
\end{tabular}




\begin{tabular}{ccccccccccc}
\hline $\begin{array}{c}\text { Five alternatives } \\
\text { (Number of } \\
\text { observation) }\end{array}$ & Variable & Beta 1 & Beta 2 & Beta 3 & Beta 4 & Beta 5 & Beta 6 & Beta 7 & Beta 8 & Beta 9 \\
\hline & $\begin{array}{c}\text { Mode } \\
\text { Chosen }\end{array}$ & $\begin{array}{c}\text { TC } \\
(1000 \text { Korea won) }\end{array}$ & $\begin{array}{c}\text { TT } \\
\text { (hour) }\end{array}$ & Accom & Sexm & Age340 & Tasc & Sasc & Basc & Lasc \\
\hline 73 & 0 & 5.31 & 0.816666667 & 1 & 0 & 0 & 0 & 0 & 0 & 0 \\
\hline & 0 & 15 & 0.816666667 & 1 & 0 & 0 & 1 & 0 & 0 & 0 \\
\hline & 0 & 0.8 & 0.5 & 1 & 0 & 0 & 0 & 1 & 0 & 0 \\
\hline & 1 & 1.4 & 0.666666667 & 1 & 0 & 0 & 0 & 0 & 1 & 0 \\
\hline & 0 & 5 & 0.866666667 & 1 & 0 & 0 & 0 & 0 & 0 & 1 \\
\hline
\end{tabular}


C.5: Limousine alternative - 38 observations

\begin{tabular}{|c|c|c|c|c|c|c|c|c|c|c|}
\hline \multirow{2}{*}{$\begin{array}{c}\text { Five alternatives } \\
\text { (Number of } \\
\text { observation) }\end{array}$} & Variable & Beta 1 & Beta 2 & Beta 3 & Beta 4 & Beta 5 & Beta 6 & Beta 7 & Beta 8 & Beta 9 \\
\hline & $\begin{array}{l}\text { Mode } \\
\text { Chosen }\end{array}$ & $\begin{array}{c}\mathrm{TC} \\
(1000 \text { Korea won) }\end{array}$ & $\begin{array}{c}\text { TT } \\
\text { (hour) }\end{array}$ & Accom & Sexm & Age340 & Tasc & Sasc & Basc & Lasc \\
\hline \multirow[t]{5}{*}{ LIMOUSINE 1} & 0 & 9.18 & 1.2 & 1 & 0 & 0 & 0 & 0 & 0 & 0 \\
\hline & 0 & 28 & 1.2 & 1 & 0 & 0 & 1 & 0 & 0 & 0 \\
\hline & 0 & 0.6 & 0.733333333 & 1 & 0 & 0 & 0 & 1 & 0 & 0 \\
\hline & 0 & 1.1 & 1.283333333 & 1 & 0 & 0 & 0 & 0 & 1 & 0 \\
\hline & 1 & 5 & 0.833333333 & 1 & 0 & 0 & $\overline{0}$ & 0 & 0 & 1 \\
\hline \multirow[t]{5}{*}{2} & 0 & 9.18 & 1.2 & 0 & 1 & 0 & 0 & 0 & 0 & 0 \\
\hline & 0 & 28 & 1.2 & 0 & 0 & 0 & 1 & 0 & 0 & 0 \\
\hline & 0 & 0.6 & 0.733333333 & 0 & 0 & 0 . & 0 & 1 & 0 & 0 \\
\hline & 0 & 1.1 & 1.283333333 & 0 & 0 & 0 & 0 & 0 & 1 & 0 \\
\hline & 1 & 5 & 1 & 0 & 0 & 0 & 0 & 0 & 0 & 1 \\
\hline \multirow[t]{5}{*}{3} & 0 & 13.18 & 1.866666667 & 0 & 0 & 1 & 0 & 0 & 0 & 0 \\
\hline & 0 & 28 & 1.866666667 & 0 & 0 & 0 & 1 & 0 & 0 & 0 \\
\hline & 0 & 0.85 & 1.15 & 0 & 0 & 0 & 0 & 1 & 0 & 0 \\
\hline & 0 & 1.5 & 2 & 0 & 0 & 0 & 0 & 0 & 1 & 0 \\
\hline & 1 & 5 & 0.75 & 0 & 0 & 0 & 0 & 0 & 0 & 1 \\
\hline \multirow[t]{5}{*}{4} & 0 & 9.18 & 1.2 & 0 & 1 & 1 & 0 & 0 & 0 & 0 \\
\hline & 0 & 28 & 1.2 & 0 & 0 & 0 & 1 & 0 & 0 & 0 \\
\hline & 0 & 0.6 & 0.733333333 & 0 & 0 & 0 & 0 & 1 & 0 & 0 \\
\hline & 0 & 1.1 & 1.283333333 & 0 & 0 & 0 & 0 & 0 & 1 & 0 \\
\hline & 1 & 5 & 0.75 & 0 & 0 & 0 & 0 & 0 & 0 & 1 \\
\hline \multirow[t]{5}{*}{5} & 0 & 10.6 & 1.433333333 & 0 & 1 & 0 & 0 & 0 & 0 & 0 \\
\hline & 0 & 12 & 1.433333333 & 0 & 0 & 0 & 1 & 0 & 0 & 0 \\
\hline & 0 & 0.85 & 0.883333333 & 0 & 0 & 0 & 0 & 1 & 0 & 0 \\
\hline & 0 & 1.1 & 1.533333333 & 0 & 0 & 0 & 0 & 0 & 1 & 0 \\
\hline & 1 & 5 & 1.5 & 0 & 0 & 0 & 0 & 0 & 0 & 1 \\
\hline \multirow[t]{5}{*}{6} & 0 & 9.026 & 0.4666666667 & 1 & 0 & 0 & 0 & 0 & 0 & 0 \\
\hline & 0 & 20 & 0.4666666667 & 1 & 0 & 0 & 1 & 0 & 0 & 0 \\
\hline & 0 & 0.7 & 0.283333333 & 1 & 0 & 0 & 0 & 1 & 0 & 0 \\
\hline & 0 & 1.5 & 0.5 & 1 & 0 & 0 & 0 & 0 & 1 & 0 \\
\hline & 1 & 6 & 1.5 & 1 & 0 & 0 & $\overline{0}$ & 0 & 0 & 1 \\
\hline \multirow[t]{5}{*}{7} & 0 & 8.476 & 0.95 & 2 & 1 & 0 & 0 & 0 & 0 & 0 \\
\hline & 0 & 12.5 & 0.95 & 2 & 0 & 0 & 1 & 0 & 0 & 0 \\
\hline & 0 & 0.7 & 0.583333333 & 2 & 0 & 0 & 0 & 1 & 0 & 0 \\
\hline & 0 & 1.3 & 1.016666667 & 2 & 0 & 0 & 0 & 0 & 1 & 0 \\
\hline & 1 & 3.5 & 1 & 2 & 0 & 0 & 0 & 0 & 0 & 1 \\
\hline \multirow[t]{5}{*}{8} & 0 & 18.18 & 2.066666667 & 3 & 1 & 0 & 0 & 0 & 0 & 0 \\
\hline & 0 & 53 & 2.066666667 & 3 & 0 & 0 & 1 & 0 & 0 & 0 \\
\hline & 0 & 1 & 1.283333333 & 3 & 0 & 0 & 0 & 1 & 0 & 0 \\
\hline & 0 & 7.5 & 2.2 & 3 & 0 & 0 & 0 & 0 & 1 & 0 \\
\hline & 1 & 9 & 3 & 3 & 0 & 0 & 0 & 0 & 0 & 1 \\
\hline \multirow[t]{5}{*}{9} & 0 & 5.31 & 0.816666667 & 0 & 1 & 0 & 0 & 0 & 0 & 0 \\
\hline & 0 & 15 & 0.816666667 & 0 & 0 & 0 & 1 & 0 & 0 & 0 \\
\hline & 0 & 0.8 & 0.5 & 0 & 0 & 0 & 0 & 1 & 0 & 0 \\
\hline & 0 & 1.4 & 0.8666666667 & 0 & 0 & 0 & 0 & 0 & 1 & 0 \\
\hline & 1 & 5 & 1 & 0 & 0 & 0 & 0 & 0 & 0 & 1 \\
\hline \multirow[t]{5}{*}{10} & 0 & 13.18 & 1.866666667 & 0 & 0 & 1 & 0 & 0 & 0 & 0 \\
\hline & 0 & 28 & 1.866666667 & 0 & 0 & 0 & 1 & 0 & 0 & 0 \\
\hline & 0 & 0.85 & 1.15 & 0 & 0 & 0 & 0 & 1 & 0 & 0 \\
\hline & 0 & 1.5 & 2 & 0 & 0 & 0 & 0 & 0 & 1 & 0 \\
\hline & 1 & 5 & 0.833333333 & 0 & 0 & 0 & 0 & 0 & 0 & 1 \\
\hline \multirow[t]{5}{*}{11} & 0 & 8.18 & 1.483333333 & 0 & 0 & 1 & 0 & 0 & 0 & 0 \\
\hline & 0 & 20 & 1.483333333 & 0 & 0 & 0 & 1 & 0 & 0 & 0 \\
\hline & 0 & 0.6 & 0.916666667 & 0 & 0 & 0 & 0 & 1 & 0 & 0 \\
\hline & 0 & 1.6 & 1.583333333 & 0 & 0 & 0 & 0 & 0 & 1 & 0 \\
\hline & 1 & 5 & 1 & 0 & 0 & 0 & 0 & 0 & 0 & 1 \\
\hline \multirow[t]{5}{*}{12} & 0 & 10.6 & 1.433333333 & 0 & 1 & 1 & 0 & 0 & 0 & 0 \\
\hline & 0 & 12 & 1.433333333 & 0 & 0 & 0 & 1 & 0 & 0 & 0 \\
\hline & 0 & 0.85 & 0.883333333 & 0 & 0 & 0 & 0 & 1 & 0 & 0 \\
\hline & 0 & 1.1 & 1.533333333 & 0 & 0 & 0 & 0 & 0 & 1 & 0 \\
\hline & 1 & 5 & 1.5 & 0 & 0 & 0 & 0 & 0 & 0 & 1 \\
\hline
\end{tabular}




\begin{tabular}{|c|c|c|c|c|c|c|c|c|c|c|}
\hline \multirow{2}{*}{$\begin{array}{l}\text { Five alternatives } \\
\text { (Number of } \\
\text { observation) }\end{array}$} & Variable & Beta 1 & Beta 2 & Beta 3 & Beta 4 & Beta 5 & Beta 6 & Beta 7 & Beta 8 & Beta 9 \\
\hline & $\begin{array}{c}\text { Mode } \\
\text { Chosen }\end{array}$ & $\begin{array}{c}\text { TC } \\
\text { (1000 Korea won) }\end{array}$ & $\begin{array}{c}\mathrm{TT} \\
\text { (hour) }\end{array}$ & Accom & Sexm & Age340 & Tasc & Sasc & Basc & Lasc \\
\hline \multirow[t]{5}{*}{13} & 0 & 9.18 & 1.2 & 0 & 0 & 0 & 0 & 0 & 0 & 0 \\
\hline & 0 & 28 & 1.2 & 0 & 0 & 0 & 1 & 0 & 0 & 0 \\
\hline & 0 & 0.6 & 0.733333333 & 0 & 0 & 0 & 0 & 1 & 0 & 0 \\
\hline & 0 & 1.1 & 1.2833333333 & 0 & 0 & 0 & 0 & 0 & 1 & 0 \\
\hline & 1 & 5 & 0.75 & 0 & 0 & 0 & 0 & 0 & 0 & 1 \\
\hline \multirow[t]{5}{*}{14} & 0 & 8.18 & 1.483333333 & 0 & 0 & 1 & 0 & 0 & 0 & 0 \\
\hline & 0 & 20 & 1.483333333 & 0 & 0 & 0 & 1 & 0 & 0 & 0 \\
\hline & 0 & 0.6 & 0.916666667 & 0 & 0 & 0 & 0 & 1 & 0 & 0 \\
\hline & 0 & 1.6 & 1.583333333 & 0 & 0 & 0 & 0 & 0 & 1 & 0 \\
\hline & 1 & 5 & 0.75 & 0 & 0 & 0 & 0 & 0 & 0 & 1 \\
\hline \multirow[t]{5}{*}{15} & 0 & 9.18 & 1.2 & 4 & 1 & 0 & 0 & 0 & 0 & 0 \\
\hline & 0 & 28 & 1.2 & 4 & 0 & 0 & 1 & 0 & 0 & 0 \\
\hline & 0 & 0.6 & 0.733333333 & 4 & 0 & 0 & 0 & 1 & 0 & 0 \\
\hline & 0 & 1.1 & 1.283333333 & 4 & 0 & 0 & 0 & 0 & 1 & 0 \\
\hline & 1 & 5 & 1 & 4 & 0 & 0 & 0 & 0 & 0 & 1 \\
\hline \multirow[t]{5}{*}{16} & 0 & 13.18 & 1.8666666667 & 0 & 0 & 1 & 0 & 0 & 0 & 0 \\
\hline & 0 & 28 & 1.8666666667 & 0 & 0 & 0 & 1 & 0 & 0 & 0 \\
\hline & 0 & 0.85 & 1.15 & 0 & 0 & 0 & 0 & 1 & 0 & 0 \\
\hline & 0 & 1.5 & 2 & 0 & 0 & 0 & 0 & 0 & 1 & 0 \\
\hline & 1 & 5 & 0.666666667 & 0 & 0 & 0 & 0 & 0 & 0 & 1 \\
\hline \multirow[t]{5}{*}{17} & 0 & 9.026 & 0.466666667 & 2 & 0 & 0 & 0 & 0 & 0 & 0 \\
\hline & 0 & 20 & 0.466666667 & 2 & 0 & 0 & 1 & 0 & 0 & 0 \\
\hline & 0 & 0.7 & 0.283333333 & 2 & 0 & 0 & 0 & 1 & 0 & 0 \\
\hline & 0 & 1.5 & 0.5 & 2 & 0 & 0 & 0 & 0 & 1 & 0 \\
\hline & 1 & 5 & 0.833333333 & 2 & 0 & 0 & 0 & 0 & 0 & 1 \\
\hline \multirow[t]{5}{*}{18} & 0 & 9.026 & 0.466666667 & 0 & 1 & 1 & 0 & 0 & 0 & 0 \\
\hline & 0 & 20 & 0.466666667 & 0 & 0 & 0 & 1 & 0 & 0 & 0 \\
\hline & 0 & 0.7 & 0.283333333 & 0 & 0 & 0 & 0 & 1 & 0 & 0 \\
\hline & 0 & 1.5 & 0.5 & 0 & 0 & 0 & 0 & 0 & 1 & 0 \\
\hline & 1 & 2.5 & 1.1666666667 & 0 & 0 & 0 & 0 & 0 & 0 & 1 \\
\hline \multirow[t]{5}{*}{19} & 0 & 18.18 & 2.0666666667 & 0 & 1 & 1 & 0 & 0 & 0 & 0 \\
\hline & 0 & 53 & 2.0666666667 & 0 & 0 & 0 & 1 & 0 & 0 & 0 \\
\hline & 0 & 1 & 1.283333333 & 0 & 0 & 0 & $\overline{0}$ & 1 & 0 & 0 \\
\hline & 0 & 7.5 & 2.2 & 0 & 0 & 0 & 0 & 0 & 1 & 0 \\
\hline & 1 & 15 & 4 & 0 & 0 & 0 & 0 & 0 & 0 & 1 \\
\hline \multirow[t]{5}{*}{20} & 0 & 18.18 & 2.0666666667 & 0 & 1 & 1 & 0 & 0 & 0 & 0 \\
\hline & 0 & 53 & 2.066666667 & 0 & 0 & 0 & 1 & 0 & 0 & 0 \\
\hline & 0 & 1 & 1.283333333 & 0 & 0 & 0 & 0 & 1 & 0 & 0 \\
\hline & 0 & 7.5 & 2.2 & 0 & 0 & 0 & 0 & 0 & 1 & 0 \\
\hline & 1 & 9.3 & 2 & 0 & 0 & 0 & 0 & 0 & 0 & 1 \\
\hline \multirow[t]{5}{*}{21} & 0 & 18.18 & 2.066666667 & 0 & 1 & 1 & 0 & 0 & 0 & 0 \\
\hline & 0 & 53 & 2.066666667 & 0 & 0 & 0 & 1 & 0 & 0 & 0 \\
\hline & 0 & 1 & 1.283333333 & 0 & 0 & 0 & 0 & 1 & 0 & 0 \\
\hline & 0 & 7.5 & 2.2 & 0 & 0 & 0 & 0 & 0 & 1 & 0 \\
\hline & 1 & 7.9 & 1.666666667 & 0 & 0 & 0 & 0 & 0 & 0 & 1 \\
\hline \multirow[t]{5}{*}{22} & 0 & 8.18 & 1.483333333 & 0 & 1 & 0 & 0 & 0 & 0 & 0 \\
\hline & 0 & 20 & 1.483333333 & 0 & 0 & 0 & 1 & 0 & 0 & 0 \\
\hline & 0 & 0.6 & 0.916666667 & 0 & 0 & 0 & 0 & 1 & 0 & 0 \\
\hline & 0 & 1.6 & 1.583333333 & 0 & 0 & 0 & 0 & 0 & 1 & 0 \\
\hline & 1 & 5 & 1 & 0 & 0 & 0 & 0 & 0 & 0 & 1 \\
\hline \multirow[t]{5}{*}{23} & 0 & 8.476 & 0.95 & 0 & 1 & 1 & 0 & 0 & 0 & 0 \\
\hline & 0 & 12.5 & 0.95 & 0 & 0 & 0 & 1 & 0 & 0 & 0 \\
\hline & 0 & 0.7 & 0.583333333 & 0 & 0 & 0 & 0 & 1 & 0 & 0 \\
\hline & 0 & 1.3 & 1.0166666667 & 0 & 0 & 0 & 0 & 0 & 1 & 0 \\
\hline & 1 & 5 & 0.6666666667 & 0 & 0 & 0 & 0 & 0 & 0 & 1 \\
\hline \multirow[t]{5}{*}{24} & 0 & 10.6 & 1.433333333 & 0 & 1 & 1 & 0 & 0 & 0 & 0 \\
\hline & 0 & 12 & 1.433333333 & 0 & 0 & 0 & 1 & 0 & 0 & 0 \\
\hline & 0 & 0.85 & 0.883333333 & 0 & 0 & 0 & 0 & 1 & 0 & 0 \\
\hline & 0 & 1.1 & 1.533333333 & 0 & 0 & 0 & 0 & 0 & 1 & 0 \\
\hline & 1 & 5 & 1.166666667 & 0 & 0 & 0 & 0 & 0 & 0 & 1 \\
\hline
\end{tabular}




\begin{tabular}{|c|c|c|c|c|c|c|c|c|c|c|}
\hline \multirow{2}{*}{$\begin{array}{c}\text { Five alternatives } \\
\text { (Number of } \\
\text { observation) }\end{array}$} & Variable & Beta 1 & Beta 2 & Beta 3 & Beta 4 & Beta 5 & Beta 6 & Beta 7 & Beta 8 & Beta 9 \\
\hline & $\begin{array}{c}\text { Mode } \\
\text { Chosen }\end{array}$ & $\begin{array}{c}\text { TC } \\
\text { (1000 Korea won) } \\
\end{array}$ & $\begin{array}{c}\mathrm{TT} \\
\text { (hour) }\end{array}$ & Accom & Sexm & Age340 & Tasc & Sasc & Basc & Lasc \\
\hline \multirow[t]{5}{*}{25} & 0 & 9.026 & 0.466666667 & 0 & 1 & 1 & 0 & 0 & 0 & 0 \\
\hline & 0 & 20 & 0.466666667 & 0 & 0 & 0 & 1 & 0 & 0 & 0 \\
\hline & 0 & 0.7 & 0.283333333 & 0 & 0 & 0 & 0 & 1 & 0 & 0 \\
\hline & 0 & 1.5 & 0.5 & 0 & 0 & 0 & 0 & 0 & 1 & 0 \\
\hline & 1 & 5.1 & 1.333333333 & 0 & 0 & 0 & 0 & 0 & 0 & 1 \\
\hline \multirow[t]{5}{*}{26} & 0 & 9.026 & 0.466666667 & 0 & 1 & 0 & 0 & 0 & 0 & 0 \\
\hline & 0 & 20 & 0.466666667 & 0 & 0 & 0 & 1 & 0 & 0 & 0 \\
\hline & 0 & 0.7 & 0.283333333 & 0 & 0 & 0 & 0 & 1 & 0 & 0 \\
\hline & 0 & 1.5 & 0.5 & 0 & 0 & 0 & 0 & 0 & 1 & 0 \\
\hline & 1 & 5 & 1.1666666667 & 0 & 0 & 0 & 0 & 0 & 0 & 1 \\
\hline \multirow[t]{5}{*}{27} & 0 & 18.18 & 2.0666666667 & 0 & 1 & 1 & 0 & 0 & 0 & 0 \\
\hline & 0 & 53 & 2.066666667 & 0 & 0 & 0 & 1 & 0 & 0 & 0 \\
\hline & 0 & 1 & 1.283333333 & 0 & 0 & 0 & 0 & 1 & 0 & 0 \\
\hline & 0 & 7.5 & 2.2 & 0 & 0 & 0 & 0 & 0 & 1 & 0 \\
\hline & 1 & 14.5 & 3.6666666667 & 0 & 0 & 0 & 0 & 0 & 0 & 1 \\
\hline \multirow[t]{5}{*}{28} & 0 & 13.18 & 1.8666666667 & 0 & 1 & 1 & 0 & 0 & 0 & 0 \\
\hline & 0 & 28 & 1.8666666667 & 0 & 0 & 0 & 1 & 0 & 0 & 0 \\
\hline & 0 & 0.85 & 1.15 & 0 & 0 & 0 & 0 & 1 & 0 & 0 \\
\hline & 0 & 1.5 & 2 & 0 & 0 & 0 & 0 & 0 & 1 & 0 \\
\hline & 1 & 5 & 0.833333333 & 0 & 0 & 0 & 0 & 0 & 0 & 1 \\
\hline \multirow[t]{5}{*}{29} & 0 & 13.18 & 1.8666666667 & 0 & 1 & 1 & 0 & 0 & 0 & 0 \\
\hline & 0 & 28 & 1.8666666667 & 0 & 0 & 0 & 1 & 0 & 0 & 0 \\
\hline & 0 & 0.85 & 1.15 & 0 & 0 & 0 & 0 & 1 & 0 & 0 \\
\hline & 0 & 1.5 & 2 & 0 & 0 & 0 & 0 & 0 & 1 & 0 \\
\hline & 1 & 5 & 1 & 0 & 0 & 0 & 0 & 0 & 0 & 1 \\
\hline \multirow[t]{5}{*}{30} & 0 & 10.6 & 1.433333333 & 0 & 1 & 1 & 0 & 0 & 0 & 0 \\
\hline & 0 & 12 & 1.433333333 & 0 & 0 & 0 & 1 & 0 & 0 & 0 \\
\hline & 0 & 0.85 & 0.883333333 & 0 & 0 & 0 & 0 & 1 & 0 & 0 \\
\hline & 0 & 1.1 & 1.533333333 & 0 & 0 & 0 & 0 & 0 & 1 & 0 \\
\hline & 1 & 5 & 0.833333333 & 0 & 0 & 0 & 0 & 0 & 0 & 1 \\
\hline \multirow[t]{5}{*}{31} & 0 & 9.026 & 0.466666667 & 0 & 1 & 0 & 0 & 0 & 0 & 0 \\
\hline & 0 & 20 & 0.466666667 & 0 & 0 & 0 & 1 & 0 & 0 & 0 \\
\hline & 0 & 0.7 & 0.2833333333 & 0 & 0 & 0 & 0 & 1 & 0 & 0 \\
\hline & 0 & 1.5 & 0.5 & 0 & 0 & 0 & 0 & 0 & 1 & 0 \\
\hline & 1 & 5 & 0.666666667 & 0 & 0 & 0 & 0 & 0 & 0 & 1 \\
\hline \multirow[t]{5}{*}{32} & 0 & 9.18 & 1.2 & 0 & 1 & 0 & 0 & 0 & 0 & 0 \\
\hline & 0 & 28 & 1.2 & 0 & 0 & 0 & 1 & 0 & 0 & 0 \\
\hline & 0 & 0.6 & 0.733333333 & 0 & 0 & 0 & 0 & 1 & 0 & 0 \\
\hline & 0 & 1.1 & 1.283333333 & 0 & 0 & 0 & 0 & 0 & 1 & 0 \\
\hline & 1 & 5 & 1 & 0 & 0 & 0 & 0 & 0 & 0 & 1 \\
\hline \multirow[t]{5}{*}{33} & 0 & 13.18 & 1.8666666667 & 1 & 1 & 0 & 0 & 0 & 0 & 0 \\
\hline & 0 & 28 & 1.866666667 & 1 & 0 & 0 & 1 & 0 & 0 & 0 \\
\hline & 0 & 0.85 & 1.15 & 1 & 0 & 0 & 0 & 1 & 0 & 0 \\
\hline & 0 & 1.5 & 2 & 1 & 0 & 0 & 0 & 0 & 1 & 0 \\
\hline & 1 & 5 & 1 & 1 & 0 & 0 & 0 & 0 & 0 & 1 \\
\hline \multirow[t]{5}{*}{34} & 0 & 10.6 & 1.433333333 & 1 & 1 & 1 & 0 & 0 & 0 & 0 \\
\hline & 0 & 12 & 1.433333333 & 1 & 0 & 0 & 1 & 0 & 0 & 0 \\
\hline & 0 & 0.85 & 0.883333333 & 1 & 0 & 0 & 0 & 1 & 0 & 0 \\
\hline & 0 & 1.1 & 1.533333333 & 1 & 0 & 0 & 0 & 0 & 1 & 0 \\
\hline & 1 & 5 & 1.5 & 1 & 0 & 0 & 0 & 0 & 0 & 1 \\
\hline \multirow[t]{5}{*}{35} & 0 & 18.18 & 2.0666666667 & 0 & 0 & 1 & 0 & 0 & 0 & 0 \\
\hline & 0 & 53 & 2.066666667 & 0 & 0 & 0 & 1 & 0 & 0 & 0 \\
\hline & 0 & 1 & 1.2833333333 & 0 & 0 & 0 & 0 & 1 & 0 & 0 \\
\hline & 0 & 7.5 & 2.2 & 0 & 0 & 0 & 0 & 0 & 1 & 0 \\
\hline & 1 & 15 & 3 & 0 & 0 & 0 & 0 & 0 & 0 & 1 \\
\hline \multirow[t]{5}{*}{36} & 0 & 7.18 & 1.483333333 & 1 & 1 & 0 & 0 & 0 & 0 & 0 \\
\hline & 0 & 10 & 1.483333333 & 1 & 0 & 0 & 1 & 0 & 0 & 0 \\
\hline & 0 & 0.5 & 0.916666667 & 1 & 0 & 0 & 0 & 1 & 0 & 0 \\
\hline & 0 & 1.1 & 1.583333333 & 1 & 0 & 0 & 0 & 0 & 1 & 0 \\
\hline & 1 & 5 & 1.166666667 & 1 & 0 & 0 & 0 & 0 & 0 & 1 \\
\hline
\end{tabular}




\begin{tabular}{|c|c|c|c|c|c|c|c|c|c|c|}
\hline \multirow{2}{*}{$\begin{array}{c}\text { Five alternatives } \\
\text { (Number of } \\
\text { observation) }\end{array}$} & Variable & Beta 1 & Beta 2 & Beta 3 & Beta 4 & Beta 5 & Beta 6 & Beta 7 & Beta 8 & Beta 9 \\
\hline & $\begin{array}{l}\text { Mode } \\
\text { Chosen }\end{array}$ & $\begin{array}{c}\text { TC } \\
\text { (1000 Korea won) }\end{array}$ & $\begin{array}{c}\mathrm{TT} \\
\text { (hour) }\end{array}$ & Accom & Sexm & Age 340 & Tasc & Sasc & Basc & Lasc \\
\hline \multirow[t]{5}{*}{37} & 0 & 9.18 & 1.2 & 0 & 1 & 0 & 0 & 0 & 0 & 0 \\
\hline & 0 & 28 & 1.2 & 0 & 0 & 0 & 1 & 0 & 0 & 0 \\
\hline & 0 & 0.6 & 0.733333333 & 0 & 0 & 0 & 0 & 1 & 0 & 0 \\
\hline & 0 & 1.1 & 1.283333333 & 0 & 0 & 0 & 0 & 0 & 1 & 0 \\
\hline & 1 & 2.5 & 0.833333333 & 0 & 0 & 0 & 0 & 0 & 0 & 1 \\
\hline \multirow[t]{5}{*}{38} & 0 & 18.18 & 2.066666667 & 3 & 1 & 0 & 0 & 0 & 0 & $\overline{0}$ \\
\hline & 0 & 53 & 2.066666667 & 3 & 0 & 0 & 1 & 0 & 0 & 0 \\
\hline & 0 & 1 & 1.283333333 & 3 & 0 & 0 & 0 & 1 & 0 & 0 \\
\hline & 0 & 7.5 & 2.2 & 3 & 0 & 0 & 0 & 0 & 1 & 0 \\
\hline & 1 & 15 & 5 & 3 & 0 & 0 & 0 & 0 & 0 & 1 \\
\hline
\end{tabular}

